
NATIONAL INSTITUTE OF STANDAROS \&

TECHNOLOGI

Research Information Center

Gathersburg, MD 20898 

United States Department of Commerce Technology Administration

National Institute of Standards and Technology

NISTIR 3999

\section{A REVIEW OF IRRADIATION EFFECTS \\ ON ORGANIC-MATRIX INSULATION}

N.J. Simon 


\section{A REVIEW OF IRRADIATION EFFECTS ON ORGANIC-MATRIX INSULATION}

\section{N.J. Simon}

Materials Reliability Division

Materials Science and Engineering Laboratory

National Institute of Standards and Technology

Boulder, CO 80303-3328

Sponsored by:

Department of Energy

Office of Fusion Energy

Washington, DC 20545

June 1993

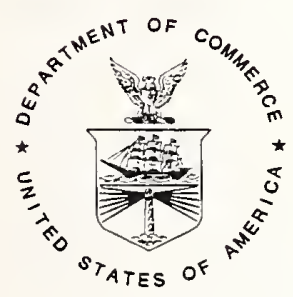

U.S. DEPARTMENT OF COMMERCE, Ronald H. Brown, Secretary NATIONAL INSTITUTE OF STANDARDS AND TECHNOLOGY, Arati Prabhakar, Director 

This review assesses the data base on epoxy and polyimide matrix insulation to determine whether organic electric insulation systems can be used in the toroidal field (TF) magnets of next generation fusion devices such as ITER* and TPX*. Owing to the difficulties of testing insulation under fusion reactor conditions, there is a considerable mismatch between the ITER requirements and the data that are currently available. For example, nearly all of the high-dose ( $5 \times 10^{7}$ to $10^{8} \mathrm{~Gy}$ ) data obtained on epoxy and polyimide matrix insulation employed gamma irradiation, electron irradiation, or reactor irradiation with a fast neutron fluence far below $10^{23} / \mathrm{m}^{2}$, the fluence expected for the insulation at the TF magnets, as set forth in ITER conceptual design documents. Also, the neutron spectrum did not contain a very high energy ( $E$ $\geq 5 \mathrm{MeV}$ ) component. Such data underestimate the actual damage that would be obtained with the neutron fluence and spectrum expected at a TF magnet. Experiments on a polyimide (Kapton) indicate that gamma or electron doses or mixed gamma and neutron reactor doses would have to be downgraded by a factor of up to ten to simulate fusion neutron doses. Even when neutrons did constitute a significant portion of the total dose, B-containing E-glass reinforcement was often used; therefore, excess damage from the ${ }^{10} \mathrm{~B}+\mathrm{n} \rightarrow{ }^{7} \mathrm{Li}$ $+\alpha$ reaction occurred near the glass-epoxy interface. This problem can easily be avoided by substituting $B$-free glass ( $R, S$, or $T$ types).

A major portion of the data base was obtained with ambient temperature testing and/or irradiation. However, ambient-temperature test results cannot be extrapolated to $4-\mathrm{K}$ conditions with confidence because cryogenic mechanical properties are more radiation sensitive. The few specimens irradiated at $4 \mathrm{~K}$ have almost always been subjected to ambient temperature warmup before cryogenic testing. Since warm-up after 4-K irradiation is a likely scenario for TF magnets, and explosive release of gas and heat from free radical reactions has been predicted, the data obtained under warm-up conditions are important. However, high-fluence tests with a fast neutron spectrum must be conducted to compare in-situ, 4-K properties with properties after warm-up. The few existing data indicate that post-irradiation mechanical properties 
measured after warm-up may have to be downgraded by a factor of about 2 to represent continuous, steady-state cryogenic conditions.

In general, the test methods have not simulated the stresses expected in ITER magnet insulation under operating conditions. Most of the data have been generated with three-point flexural bend tests, in which tensile stresses predominate along the outside bend radius, and the tensile properties of the reinforcement strongly affect the measured flexural strength. Interlaminar shear strength tests would assess interfacial properties, such as the matrixfiber bond, which are more vulnerable to radiation. In the ITER conceptual design, simultaneous shear and compression stresses are predicted to occur in the insulation, and adhesion of the turn-to-turn insulation to the conductor sheath alloy is also essential. Therefore, shear/compressive failure envelopes should be obtained. A true shear bond strength can be extrapolated from the failure envelope in simultaneous shear/compression testing.

Extensive ambient-temperature irradiation and testing, reinforced by some cryogenic results, have indicated that multifunctional or glycidyl amine resin systems such as TGDM* and TGPAP* may be more radiation-resistant than DGEBA* resin systems. Although low viscosity systems suitable for vacuum impregnation may be developed from these multifunctional epoxies, such systems have not yet been optimized for magnet fabrication. Isocyanate-epoxy systems have also shown promising radiation resistance, but have not been pursued for cryogenic magnet fabrication. Much effort has gone into DGEBA-based resins such as Orlitherm, and recent cryogenic irradiation testing by both the NET* team and CTD, Inc.* has emphasized DGEBA resins. DGEBA S-glass-reinforced composites begin to degrade in shear strength at a fast neutron fluence of about $1 \times 10^{22} / \mathrm{m}^{2}$, and at a fast neutron fluence of about $3 \times 10^{22} / \mathrm{m}^{2}$, they retain less than $50 \%$ of their unirradiated shear strength.

When mica reinforcement was used in addition to glass reinforcement, a high degree of gamma radiation resistance was observed with some proprietary, EPN* prepreg epoxy systems. However, the mica reinforcement data were obtained only at ambient temperature, with flexural tests on mica systems that were evidently flexible and wrappable, owing to their organic components. 
Fabrication of flexible mica insulation sheet that contains only inorganic components may not be achievable. The possible separation of mica layers, owing to either gas buildup between the layers or expansion under neutron irradiation, affects electrical integrity, since pores may exist in individual layers.

Owing to the limited amount of testing under radiation and stress conditions that simulate magnet operations, the cryogenic data base is inadequate to predict the 4-K performance of glass- or mica-reinforced epoxy or polyimide composites irradiated at $4 \mathrm{~K}$ to magnet fast neutron fluences of $1 \mathrm{x} 10^{23} / \mathrm{m}^{2}$. Several issues must be addressed to obtain an adequate ITER data base for the properties of insulation systems under irradiation:

(1) Neutron irradiation sources with a fast neutron, fusion-like spectrum need to be developed or restarted. Adequate 4-K irradiation (without overheating) and capability for larger-scale in situ stress application and testing must be a part of this development. The existing experimental space at the Garching reactor is very limited, and only one capsule-load (-13 mm diameter x $50 \mathrm{~mm}$ length) can be irradiated per week, for only 20 weeks in each year. Some cryogenic testing at neutron energies above about $5 \mathrm{MeV}$ must be carried out.

(2) A near-term effort should be made by polymer chemists to optimize glycidyl-amine (TGDM-type) and isocyanate epoxies for magnet fabrication in parallel with additional irradiation testing of DGEBA systems. Attempts should also be made to replace the commonly used hardener, MNA*, with other compounds that do not release as much gaseous product under irradiation, since gas buidup could be disadvantageous if warm-up occurs. Polyimide systems could be developed and tested if vacuum impregnation is not required.

(3) At present, interlaminar shear, shear/compression, and adhesion tests have not yet been used to generate an adequate data base under $4-\mathrm{K}$, fast neutron irradiation, although the latter two types of tests are now in preliminary development. Even these improved tests would produce only a screening data base. A considerable number of scale-up investigations, and 
the development of additional tests in which the appropriate stresses are applied to insulation while it is under irradiation are still necessary before design data can be generated.

Trade names are used in this report solely to provide complete identification of specimens for scientific purposes. Such identification of products neither constitutes nor implies endorsement of the products.

* ITER = International Thermonuclear Experimental Reactor

TPX = Tokamak Physics Experiment

TGDM = Tetraglycidyl diaminodiphenyl methane

TGPAP = Triglycidyl para-aminophenol

DGEBA = Diglycidyl ether of bisphenol A

NET = Next European Torus

CTD, Inc. = Composite Technology Development, Inc. (Contractor

for U.S. ITER Home Team)

EPN = Epoxy novolac

MNA = Methyl "nadic" anhydride 


\section{ACKNOWLEDGEMENTS}

The author is indebted to R.P. Reed for numerous discussions of the issues raised in this report, for a critical reading of the manuscript, and for encouragement to undertake the task of compiling and evaluating the data. R.J. Deal has extracted data from the references with care and has expertly and accurately prepared the large number of graphs in this publication. The efficiency and word-processing skills of P.K. Wright in preparing the manuscript are very gratefully acknowledged. A number of readers have assisted in removing errors and omissions from the text: they are J.B. Schutz, P.E. Fabian, D.R. Smith, M. Young, and M.E. DeWeese. Of course, the author is responsible for the imperfections that remain. Discussions with N.A. Munshi, H.W. Weber, D. Evans, H.K. Katheder, H. Gerstenberg, P. Bruzzone, F.W. Clinard, and M. Sawan have contributed greatly to the author's understanding of issues related to the use of organic insulation in fusion reactor magnets. S. Egusa, D. Evans, F.W. Clinard, W. Maurer, and R.P. Reed have generously provided copies of reports and documents. Finally, the author is grateful to M.M. Cohen of the Office of Fusion Energy for sponsoring this work. 
1. ASSESSMENT OF THE DATA BASE ON IRRADIATION OF EPOXY

INSULATION . . . . . . . . . . . . . . . . . . . . . . . . 1

1.1 INTRODUCTION . . . . . . . . . . . . . . . . . . . . . . . . . 1

1.2 COMPARISON OF THE ACTUAL DATA BASE WITH THE DATA BASE

NEEDED FOR ITER . . . . . . . . . . . . . . . . . . . . . . . . 2

1.3 EFFECT OF IRRADIATION SPECTRUM, SPECIES, AND

TEMPERATURE . . . . . . . . . . . . . . . . . . 10

1.3.1 Energy Spectrum . . . . . . . . . . . . . . . . 11

1.3.1.1 Neutron Spectrum ............... 11

1.3.1.2 Gamma Spectrum . . . . . . . . . . . . 22

1.3.2 Radiation Species . . . . . . . . . . . . . 22

1.3.2.1. Relative Neutron-Gamma Fluence . . . . . . 22

1.3.2.2. Neutron Damage Mechanisms . . . . . . . . 31

1.3.2.3 Gamma Ray Damage Mechanisms . . . . . . . . 35

1.3.2.4. Electron Damage Mechanisms . . . . . . . . 38

1.3.2.5. Experimental Comparisons of Neutron and Gamma Irradiation Damage . . . . . . . . . 40

1.3.2.6. Experimental Comparisons of Gamma and Electron Irradiation Damage . . . . . . . 46

1.3.3. Temperature . . . . . . . . . . . . . 48

1.3.3.1. Experimental Comparisons of Damage from Irradiation at Different Temperature . . . . 48

1.4. EFFECT OF TEST TEMPERATURE AND WARM-UP . . . . . . . . . . . . 55

1.4.1. Comparison of Damage from Tests at Different Temperatures .................. 55

1.4.2. Comparison of Damage from Tests with and without Warm-up . . . . . . . . . . . . . . . . . 64

1.4.2.1. Chemical Effects of Ambient Irradiation . . . . . . . . . . . . . . 64

1.4.2.2. Chemical Effects of Cryogenic Irradiation . . . . . . . . . . . . . . 67

1.4.2.3. Results of Tests Conducted with and without Warm-up . . . . . . . . . . . 68

1.5. TEST METHODS . . . . . . . . . . . . . . . . . 76

1.5.1. Sensitivity to Radiation Damage . . . . . . . . . 76

1.5.2. Shear Tests... . . . . . . . . . . . . 81

1.5.3. Shear/Compression Tests . . . . . . . . . . . 82

1.5.4. Adhesive-Bond Tests . . . . . . . . . . . 86

Figure 1.1 . . . . . . . . . . . . . . . . . . . . . 9

Figure 1.2 . . . . . . . . . . . . . . . . . . . . 12

Figure 1.3 . . . . . . . . . . . . . . . . . . . . 14

Figure 1.4 
Figure 1.5

Figure 1.6

Figure 1.7

Figure 1.8

Figure 1.9

Figure 1.10

Figure 1.11

Figure 1.12

Figure 1.13

Figure 1.14

Figure 1.15

Figure 1.16

Figure 1.17

Figure 1.18

Figure 1.19

Figure 1.20

Figure 1.21

Figure 1.22

Figure 1.23

Figure 1.24

Figure 1.25

Figure 1.26

Figure 1.27

Figure 1.28

Figure 1.29

Figure 1.30

Figure 1.31

Figure 1.32

Figure 1.33

Figure 1.34

Figure 1.35

Figure 1.36

Figure 1.37

Figure 1.38

2. AMBIENT TEMPERATURE IRRADIATION AND TESTING

2.1. EFFECT OF RESIN AND HARDENER TYPE: GLASS-REINFORCED RESINS

2.2. EFFECT OF RESIN AND HARDENER TYPE: NEAT RESINS . . . . . . . . 94

2.3. EFFECT OF VISCOSITY (PROPORTION OF HARDENER) . . . . . . . . . . 99

2.4. EFFECT OF CURING CONDITIONS . . . . . . . . . . . . . . . . . 100

2.5. EFFECT OF OXYGEN DURING IRRADIATION . . . . . . . . . . . . . 100

2.6. EFFECT OF INORGANIC FILLERS . . . . . . . . . . . . . . . . . 101

2.7. EFFECT OF COMBINED MICA- AND GLASS-REINFORCEMENT . . . . . . 103

2.8. EFFECT OF HIGH ENERGY NEUTRON IRRADIATION . . . . . . . . . . 105

2.9. EFFECT OF IRRADIATION SPECIES . . . . . . . . . . . . . . 106

2.9.1. Comparison of Electron and Gamma

Irradiation. 
2.9.2. Comparison of Neutron and Gamma

Irradiation . . . . . . . . . . . . . . . 107

2.10. RESIN SYSTEMS USED IN MAGNET INSULATION . . . . . . . . . 108

2.10.1. Resins Used for Superconducting Magnets . . . . . . . 108

2.10.2. Resins Used for Ambient Temperature Magnets . . . . . 108

2.11. MISCELLANEOUS AMBIENT TEMPERATURE TESTS . . . . . . . . . . . 111

2.11.1. Investigations of Resin Systems and Hardeners .. . . . . . . . . . . . . . 112

2.11.2. Fracture-Toughness Tests . . . . . . . . . . . 113

2.11.3. Comparison of 2- and 3-Dimensiona1

Reinforcement . . . . . . . . . . . . 113

Figure 2.1 . . . . . . . . . . . . . . . . . . . . . 88

Figure 2.2 . . . . . . . . . . . . . . . . . . . . . . 89

Figure 2.3 . . . . . . . . . . . . . . . . . . . . . . . 91

Figure 2.4 . . . . . . . . . . . . . . . . . . . . . . . 92

Figure 2.5 . . . . . . . . . . . . . . . . . . . . 104

3. AMBIENT-TEMPERATURE IRRADIATION WITH 77-K TESTING . . . . . . . . . . 115

3.1. COMPARISON OF 77-K TESTS WITH AMBIENT-TEMPERATURE

TESTS . . . . . . . . . . . . . . . . . . . . . . 115

3.2. SYSTEMATIC COMPARISONS OF RESIN SYSTEMS . . . . . . . . . . . 116

3.3. SYSTEMATIC COMPARISONS OF REINFORCEMENT TYPES . . . . . . . . 119

3.4. EFFECT OF IRRADIATION ENVIRONMENT . . . . . . . . . . . . . . 120

3.5. EFFECT OF IRRADIATION SPECIES . . . . . . . . . . . . . . . 121

Figure 3.1 . . . . . . . . . . . . . . . . . . . . 117

4. AMBIENT TEMPERATURE IRRADIATION WITH 4-K TESTING . . . . . . . . . . 123

5. 77-K IRRADIATION WITH AMBIENT TEMPERATURE TESTING . . . . . . . . . . 124

6. 77-K IRRADIATION WITH 77-K TESTING . . . . . . . . . . . 125

7. 20-K IRRADIATION WITH 77-K TESTING . . . . . . . . . . . 127

8. 4- TO 5-K IRRADIATION WITH 77-K TESTING, WITH AND WITHOUT
WARM-UP . . . . . . . . . . . . . . . . . . . . . . . 129

9. 4- TO 5-K IRRADIATION WITH 4-K TESTING WITHOUT WARM-UP . . . . . . 133

10. SHEAR/COMPRESSIVE TESTS AT AMBIENT TEMPERATURE . . . . . . . . . . . 135

10.1. UNIAXIAL LOADING . . . . . . . . . . . . . . . . . . . . 135

10.2. BIAXIAL LOADING . . . . . . . . . . . . . . . 135

11. SHEAR TESTS OF COMPOSITE/ALLOY ADHESION . . . . . . . . . . . . . . . 136

11.1. COMPARISON OF MECHANICAL AND CHEMICAL BONDING . . . . . . . 136

11.2. COMPARISON OF REINFORCEMENT TYPE . . . . . . . . . . . . . . 139

11.3. COMPARISON OF RESIN SYSTEMS . . . . . . . . . . . . . . . 143

11.3.1. Results at Low Doses. . . . . . . . . . . . 143

11.3.2. Results at High Doses . . . . . . . . . . . 143

Figure 11.1 . . . . . . . . . . . . . . . . . . . 137

Figure 11.2 . . . . . . . . . . . . . . . . . . . . . 140

Figure 11.3 . . . . . . . . . . . . . . . . . . . . . 142 
Figure 11.4

12. SUMMARY AND CONCLUSIONS: AMBIENT-TEMPERATURE IRRADIATION AND TESTING

AMBIENT-TEMPERATURE IRRADIATION AND

12.1. SUMMARY OF RESULTS . . . . . . . . . . . . . . . . 145

12.2. DISCUSSION . . . . . . . . . . . . . . . . . . . 148

13. SUMMARY AND CONCLUSIONS: CRYOGENIC DATA BASE . . . . . . . . . . . 150

13.1. CRYOGENIC TESTING: AMBIENT TEMPERATURE IRRADIATION . . . . . 150

13.2. CRYOGENIC TESTING: 77-K IRRADIATION . . . . . . . . . . . . 152

13.3. CRYOGENIC TESTING: 20-K IRRADIATION . . . . . . . . . . . . 153

13.4. CRYOGENIC TESTING: 4- OR 5-K IRRADIATION . . . . . . . . . . 153

13.5. DISCUSSION OF CRYOGENIC DATA BASE ON MAGNET RESINS: FURTHER INVESTIGATIONS

APPENDIX A: GRAPHS AND TABLES . . . . . . . . . . . . . . . . . . . . A-1

NOTES FOR GRAPHS AND SUPPLEMENTARY TABLES . . . . . . . . . . . . . . . . A-1

§2: Irradiation Graphs . . . . . . . . . . . . . . . . . . A.2-1

§2: Supplementary Tables . . . . . . . . . . . . . . . A.2-107

§3: Irradiation Graphs . . . . . . . . . . . . . . . . . . . . A.3-1

§3: Supplementary Tables . . . . . . . . . . . . . . A.3-19

\$4: Irradiation Graph . . . . . . . . . . . . . . . . . . . . . A.4-1

§5: Irradiation Graphs . . . . . . . . . . . . . . . . A.5-1

§5: Supplementary Tables . . . . . . . . . . . . . . A.5-5

§6: Irradiation Graphs . . . . . . . . . . . . . . . . A.6-1

§6: Supplementary Tables . . . . . . . . . . . . . . A.6-4

§7: Irradiation Graphs . . . . . . . . . . . . . . . . . A.7-1

§7: Supplementary Tables . . . . . . . . . . . . . A.7-6

§8: Irradiation Graphs . . . . . . . . . . . . . . . . . A.8-1

§8: Supplementary Tables . . . . . . . . . . . . . A.8-16

\$9: Irradiation Graphs . . . . . . . . . . . . . . . . . A.9-1

§9: Supplementary Tables . . . . . . . . . . . . . . . . . A.9-7

§10: Irradiation Graphs . . . . . . . . . . . . . . . . . . . . A.10-1

$\S 10$ : Supplementary Tables . . . . . . . . . . . . . A.10-4

§11: Irradiation Graphs . . . . . . . . . . . . . . . . . . . A.11-1

§11: Supplementary Tables . . . . . . . . . . . . . A.11-13

APPENDIX B: POLYIMIDES AND BISMALEIMIDES . . . . . . . . . . . . . B-1

B. INTRODUCTION . . . . . . . . . . . . . . . . . . . . . B-1

B.1. ASSESSMENT OF DATABASE ON IRRADIATION OF POLYIMIDE INSULATION B-1

B.1.1. Comparison of Neutron and Gamma Irradiation Damage . B-1

B.1.2. Comparison of Gamma and Electron Irradiation Damage . B-4

B.1.3. Effect of Irradiation Temperature . . . . . . . . . B-4

B.1.4 Effect of Oxygen During Irradiation . . . . . . . . B-5

B.1.5. Effect of Test Temperature with and without Warm-up . B-5

B.1.6. Effect of Annealing . . . . . . . . . . . . B-6

B.1.7. Effect of Glass-Reinforcement Parameters . . . . . B-7

B.1.8. Effect of Test Method . . . . . . . . . . . . . . . . B-8

B.2. COMPARISON OF POLYIMIDE AND EPOXY RADIATION RESISTANCE . . . B-9

B.2.1. Low-Dose Comparisons $\left(\leq 10^{7} \mathrm{~Gy}\right)$. . . . . . . . . . . B-9

B.2.2. High-Dose Comparisons $\left(-10^{8} \mathrm{~Gy}\right)$. . . . . . . . . . B-10

B.2.3. Polyimide Films... . . . . . . . . . . . . B-13 
B.3. SUMMARY OF IRRADIATION TESTING OF POLYIMIDES AND

BISMALEIMIDES . . . . . . . . . . . . . B-15

Figure B.1.1............................ B-2

$\S$ B.1. Irradiation Graphs and Supplementary Tables . . . . . . . . B.1-1

$\S$ B. 2. Irradiation Graphs . . . . . . . . . . . . . . . . . . . B.2-1

APPENDIX C: FATIGUE . . . . . . . . . . . . . . . . . . . C C-1

C. INTRODUCTION . . . . . . . . . . . . . . . . . . . . . . C-I

C.1. COMPRESSIVE FATIGUE TESTS .................. . C-1

C.2. SHEAR/COMPRESSIVE FATIGUE TESTS . . . . . . . . . . . . . C-2

C.3. TENSILE FATIGUE TESTS . . . . . . . . . . . . . . . . . . C-3

C.4. FLEXURAL FATIGUE TESTS . . . . . . . . . . . . . . . . C-4

C.5. SUMMARY OF FATIGUE RESULTS . . . . . . . . . . . . . . . C-4

§C.1. Irradiation Graphs and Supplementary Table . . . . . . . . C.1-1

$\S C .2$. Irradiation Graph and Supplementary Table . . . . . . . . . C.2-1

§C.3. Irradiation Graph and Supplementary Table . . . . . . . . . C.3-1

§C.4 Irradiation Graph and Supplementary Table . . . . . . . . C.4-1

REFERENCES . . . . . . . . . . . . . . . . . . . . R-1 
Figure 1.1. A comparison of degradation under neutron irradiation of the interlaminar shear strength of a resin reinforced with either $E$ glass or $T$ glass. The fabric type and finish and epoxy matrix were identical. Data from Okada et al. [1992]. (Supplementary Table

A. 7-1.) .

Figure 1.2. Calculated neutron spectra, at the toroidal field magnet, for two fusion reactor designs. Data from Hahn et al. [1986].

Figure 1.3. Neutron spectra in the winding pack of ITER for two calculations based on different nuclear data libraries. Data from Maki et al. [1991].

Figure 1.4. A normalized plot of a calculation of the ITER neutron spectrum at the front layer of the insulator of the TF magnet. These results pertain to a total thickness of $1 \mathrm{~m}$ between plasma and magnet, consisting of a $650-\mathrm{mm}$ blanket/shield (508 ferritic steel/50\% Li) and a $350-\mathrm{mm}$ double-wall Inconel vacuum vessel with WC balls, water-cooled. Data from Sawan [1993].

Figure 1.5. Normalized neutron irradiation spectra from three irradiation sources. The RTNS line extends above the scale. Data from Hahn et al. [1986].

Figure 1.6. Comparison of the neutron spectra produced in the IPNS source at two positions with a pure fission neutron spectrum. Data from Birtcher et al. [1982].

Figure 1.7. (a) Comparison of a calculated fusion spectrum in the TF magnet (inner blanket/shield of stainless steel and $\mathrm{BC}_{4}$ ) at two locations, $A$ and $B$, with the neutron spectrum produced in the Argonne National Laboratory Low Temperature Facility. Data from Abdou [1978]. (b) A calculated ITER spectrum at the TF magnet. Data from Sawan [1993]. (c) Fraction of flux above neutron energy [Abdou, 1978]. . . . 19

Figure 1.8. (a) Comparison of a calculated fusion spectrum with the neutron spectrum produced in the Garching FRM reactor. Data from Söll [1978]. (b) Comparison of a calculated fusion spectrum at the magnet with the neutron spectrum produced in the Garching FRM reactor. Data from Ullmaier [1976]. . . . . . . . . . . . . . . . . . . 20

Figure 1.9. A plot of the calculated gamma spectrum at the inboard leg of the ETF toroidal coil. Tabulated data from Van Konynenburg [1981] . . . . . . . . . . . . . . . . . . . . . . 23

Figure 1.10. Calculations of the gamma spectrum in the winding pack of ITER based on two nuclear data libraries. Data from Maki et al. [1991].

Figure 1.11. A calculation of the ITER gamma ray spectrum at the midplane of the inboard side of the TF magnet. Data from El-Guebaly [1990]. 
Figure 1.12. (a) Calculated flux versus depth in the magnet of a fusion reactor, from neutrons of energy $>8 \mathrm{MeV}$, gamma rays, and total neutrons. (b) Calculated dose, in the epoxy insulation, versus depth in the magnet of a fusion reactor, from neutrons, gamma rays, and both neutrons and gamma rays. Data from Abdou [1978].

Figure 1.13. Calculated total dose and gamma dose versus distance along the axis of a tokamak reactor. Data from Banford [1984].

Figure 1.14. Calculated neutron and gamma ray flux distributions for the ITER design versus distance along the axis. Data from Maki et al. [1991].

Figure 1.15. Schematic illustration of a cross-section curve versus energy for neutrons, showing resonance effects. Data from Leighton [1959].

Figure 1.16. Schematic illustration of main-chain and side-chain rupture of excited polymers, with the formation of free radicals.

Figure 1.17. Interaction mechanisms of gamma rays with matter, illustrating the dependence on the atomic number, $Z$, of the absorber. The Compton effect will dominate for the energy range of gamma rays expected at the magnet position of a tokamak fusion reactor. Data from Kircher [1964].

Figure 1.18. Schematic diagram of the Compton process, illustrating the scattered gamma ray and the recoil electron.

Figure 1.19. The ionization curve for electrons, illustrating the sizeable decrease in ionization for electrons at about $1 \mathrm{MeV}$ in energy. Data from Lapp and Andrews [1954].

Figure 1.20. Irradiation data comparing the effects of neutron, electron, and gamma irradiation on a fiber-glass reinforced TGDM (tetraglycidyl diaminodiphenyl methane) epoxy matrix cured with DDS (diaminodiphenyl sulphone). (a) Flexural tests at $295 \mathrm{~K}$. Data from Egusa et al. [1984a,b]. (b) Flexural tests at $77 \mathrm{~K}$. Data from Egusa et al. [1985a; 1987b]. The $\mathrm{H} 2$ irradiation thimble exposed specimens to a heavier dose of thermal neutrons than the "Rabbit" thimble. (Supplementary Table B.1-1.)

Figure 1.21. The chemical structure of an unmodified epoxy resin based upon bisphenol A.

Figure 1.22. The change in the histogram of the compressive strength of a neat resin caused by gamma irradiation. Data from Nishijima and Okada [1977; 1978].

Figure 1.23. The change in the histogram of the compressive strength caused by reactor irradiation comprising both neutrons and gamma rays, as indicated. Data from Nishijima and Okada [1977; 1978]. 
Figure 1.24. A comparison of flexural test results of a composite irradiated at both $5 \mathrm{~K}$ and ambient temperature with neutrons. See Figure 1.20 for a description of the composite. (a) Tested at $295 \mathrm{~K}$. (b) Tested at $77 \mathrm{~K}$. Data from Egusa et al. [1984a; 1987b]. (Supplementary Table B.1-1.)

Figure 1.25. A comparison of the shear strengths of three types of reinforced epoxy resins that were reactor-irradiated at both $4 \mathrm{~K}$ and at ambient temperature. See text for differences in the fast neutron spectrum in the two reactors. Data from Munshi [1991]. (Supplementary Tables A.3-3 and A.8-4.) . . . . . . . . . . . . . . . . . . . . . . 51

Figure 1.26. The chemical structures of two different types of resin systems tested by Munshi [1991] (Figure 1.25) and the most common multifunctional epoxy, TGDM.

Figure 1.27. The increased degradation from irradiation when oxygen is present, compared to irradiation carried out in vacuum. Data from Morgan et al. [1970]. (Supplementary Table A.2-1.) . . . . . . . . . 54

Figure 1.28. The increased degradation exhibited by flexural specimens tested at $77 \mathrm{~K}$ (b), compared with those tested at $295 \mathrm{~K}$ (a). Data from Egusa et al. [1988]. (Supplementary Table A.3-5.) See Figures B.2-14 and B.2-15 for initial strengths. . . . . . . . . . . . . . . . . . . 56

Figure 1.29. Data showing comparative flexural test results on the same composite systems at 295,77, and $4 \mathrm{~K}$. Data from Egusa and Hagiwara [1986] and Egusa [1990a]. (Supplementary Table A.3-2.) . . . . 58

Figure 1.30. (a) A comparison of a glass-reinforced epoxy composite tested in compression at both 77 and $4 \mathrm{~K}$. (b) A comparison of the same epoxy resin system tested in neat form at both 77 and $4 \mathrm{~K}$. Data from Takamura and Kato [1980]. (Supplementary Table A.8-3.) . . . . . . . 59

Figure 1.31. A comparison of two commercial laminates tested at both 300 and $75 \mathrm{~K}$ after neutron irradiation at $4 \mathrm{~K}$. Data from Tucker et al. [1985]. (Supplementary Table A.8-1.)................ . 63

Figure 1.32. Schematic examples of free-radical reactions in polymer chains: cross-linking, unsaturation, chain scission, and evolution of gas.

Figure 1.33. Chemical structure diagrams of four common hardeners, or curing agents: MNA, DDM, DDS, and TETA.

Figure 1.34. A comparison of 77-K compression test data on a DGEBA resin reinforced with a filler (a) or with fiber glass (b). The data in (a) were obtained by Coltman et al. [1979] after irradiation at $4.9 \mathrm{~K}$ and warm-up to ambient temperature; the data in (b) were obtained without warm-up by Takamura and Kato [1980]. The comparison was made by Banford [1984]. (Supplementary Tables A.8-2 and A.8-3.) . . . . . . 69 
Figure 1.35. A comparison of compression tests at $77 \mathrm{~K}$, with and without warm-up to ambient temperature, on specimens irradiated at $5 \mathrm{~K}$. The tests were made on a neat DGEBA resin cured with an aromatic amine. Data from Kato and Takamura [1979]. See also Takamura and Kato [1981]. (Supplementary Table A.8-3.)

Figure 1.36. Tensile test data comparing polytetrafluoroethylene irradiated, stored, and tested at $20 \mathrm{~K}$ (b) with the same polymer after warm-up to ambient temperature (c). Comparative ambient temperature properties without irradiation are shown in (a). Data from Van de Voorde [1973].

Figure 1.37. Comparison of gamma irradiation damage to a fiber-glass reinforced TGDM resin system with and without vacuum annealing at $180^{\circ} \mathrm{C}$. Data from Egusa et a1. [1985b]. (Supplementary Table B.1-1.) . . . . . . . 74

Figure 1.38. Fracture surfaces of an E-glass reinforced composite after neutron irradiation, with (a) and without (b) warm-up to ambient temperature. Reprinted with permission from Nishijima et al. [1988], p. 41, Plenum, New York.

Figure 1.39. The distribution of tensile, compressive, and interlaminar shear stresses in a three-point bend test.

Figure 1.40. The change of Young's modulus with gamma irradiation in different types of fiber-glass/epoxy composites. Irradiation was at ambient temperatures; flexural testing was carried out at 295 and $77 \mathrm{~K}$. Data from Egusa and Hagiwara [1986]. (Supplementary Table A.3-2.)

Figure 1.41. The change of the shear modulus with gamma irradiation in different types of fiber-glass/epoxy composites. Irradiation was at ambient temperatures; flexural testing was carried out at 295 and $77 \mathrm{~K}$. Data from Egusa and Hagiwara [1986]. (Supplementary Table A.3-2.) . . . . 79

Figure 1.42. The change of the flexural strength with gamma irradiation in different types of fiber-glass/epoxy composites. Irradiation was at ambient temperatures; flexural testing was carried out at 295 and $77 \mathrm{~K}$. Data from Egusa and Hagiwara [1986]. (Supplementary Table A.3-2.)

Figure 1.43. Dependence of the failure mode in a flexural test upon the span to thickness ratio, $\mathrm{L} / \mathrm{t}$.

Figure 1.44. Shear failure in a flexural test with $\mathrm{L} / \mathrm{t}=20$, where the specimen was machined at an angle of $45^{\circ}$ to the warp direction. The E-glass reinforced TGDM/DDS resin system was irradiated at ambient temperatures with $2-\mathrm{MeV}$ electrons and tested at 295 and $77 \mathrm{~K}$. Data from Egusa [1990a,b]. (Supplementary Table B.1-1.) . . . . . . . . . . 84

Figure 1.45. Tension failure in a flexural test with $\mathrm{L} / \mathrm{t}=20$, where the specimen was machined at an angle of $0^{\circ}$ to the warp direction. The E-glass reinforced TGDM/DDS resin system was irradiated at ambient temperatures with $2-\mathrm{MeV}$ electrons and tested at 295 and $77 \mathrm{~K}$. Data from Egusa [1990a,b]. (Supplementary Table B.1-1.). . . . . . . . 
Figure 2.1. The chemical structure of Epikote 812 and other resins investigated by Price and Sheldon [1965]. The structures of the resins DGEBA, EPN, and TGDM are given in Figure 1.26. . . . . . . . . . 88

Figure 2.2. The order of radiation resistance of epoxy groups, as determined by Price and Sheldon [1965].

Figure 2.3. The chemical structure of the resin Gft-VE-2025, investigated by Sheldon and Stapleton [1968].

Figure 2.4. The chemical structure of the resins TGPAP and DGA, investigated by Evans et al. [1970].

Figure 2.5. Viscosity of epoxy systems, with and without filler, as a function of temperature. Data from Brechna [1965].

Figure 3.1. The chemical structure of the resin DGEBF, investigated by Egusa et al. [1968].

Figure 11.1. Lap shear specimens. (a) Lipták et al. [1985].

(b) Imel et al. [1979]; Schmunk et al. [1981]. (c) Kernohan et al.

[1979]; Coltman et al. [1979].

Figure 11.2. Bond specimen, Brechna [1965]. . . . . . . . . . . . . 140

Figure 11.3. Lap shear specimen, Poehlchen et al. [1990]. . . . . . . . 142

Figure 11.4. Shear/compression specimen, McManamy et al. [1991b]. . . . 144

Figure A.2-1. Flexural strength at $295 \mathrm{~K}$ of FGR epoxy resins with MNA hardener after ambient gamma irradiation. Supplementary Table A.2-1. Data from Price and Sheldon [1965]. . . . . . . . . . . . A.2-1

Figure A.2-2. Tensile strength at $295 \mathrm{~K}$ of FGR epoxy resins with MNA hardener after ambient gamma irradiation. Supplementary Table A.2-1. Data from Price and Sheldon [1965]. . . . . . . . . . . A.2-2

Figure A.2-3. Flexural strength at $295 \mathrm{~K}$ of FGR TGDM resin with several hardeners after ambient gamma irradiation. Supplementary Table A.2-1. Data from Sheldon and Stapleton [1968]. . . . . . . . A.2-3

Figure A.2-4. Flexural strength at $295 \mathrm{~K}$ of FGR EPN resin with two hardeners after ambient gamma irradiation. Supplementary Table A.2-1. Data from Sheldon and Stapleton [1968]. . . . . . . . . . . A.2-4

Figure A.2-5. Flexural strength at $295 \mathrm{~K}$ of FGR DGEBA resin (MY 740) with several hardeners after ambient gamma irradiation. Supplementary Table A.2-1. Data from Sheldon and Stapleton [1968]. . A.2-5

Figure A.2-6. Flexural strength at $295 \mathrm{~K}$ of FGR DGEBA resin (RX 35) with several hardeners after ambient gamma irradiation. Supplementary Table A.2-1. Data from Sheldon and Stapleton [1968]. . . A.2-6 
Figure A.2-7. Flexural strength at $295 \mathrm{~K}$ of FGR cycloaliphatic resins with MNA hardener after ambient gamma irradiation.

Supplementary Table A.2-1. Data from Sheldon and Stapleton [1968].

Figure A.2-8. Flexural strength at $295 \mathrm{~K}$ of FGR epoxy ether resin with several hardeners after ambient gamma irradiation.

Supplementary Table A.2-1. Data from Sheldon and Stapleton [1968].

Figure A.2-9. Flexural strength at $295 \mathrm{~K}$ of FGR brominated epoxy resin with two hardeners after ambient gamma irradiation.

Supplementary Table A.2-1. Data from Sheldon and Stapleton [1968].

Figure A.2-10. Flexural strength at $295 \mathrm{~K}$ of FGR DGEBA resin with excess DDM hardener after ambient gamma irradiation. Supplementary

Table A.2-1. Data from Sheldon and Stapleton [1968].

Figure A.2-11. Flexural strength at $295 \mathrm{~K}$ of FGR DGEBA resin with DDM hardener and varied wetting agent after ambient gamma

irradiation. Supplementary Table A.2-1. Data from Sheldon and

Stapleton [1968].

Figure A.2-12. Flexural strength at $295 \mathrm{~K}$ of FGR DGEBA resin with DDM hardener and varied glass finish after ambient gamma irradiation. Supplementary Table A.2-1. Data from Sheldon and Stapleton [1968].

Figure A.2-13. Flexural strength at $295 \mathrm{~K}$ of FGR DGEBA resin with MNA hardener and varied glass finish after ambient gamma irradiation. Supplementary Table A.2-1. Data from Sheldon and Stapleton [1968].

Figure A.2-14. Flexural strength at $295 \mathrm{~K}$ of FGR epoxy resins with DDM hardener after ambient gamma irradiation. Supplementary Table A.2-1. Data from Evans et al. [1970].

Figure A.2-15. Flexural strength at $295 \mathrm{~K}$ of FGR epoxy resins with DDS hardener after ambient gamma irradiation. Supplementary Table A.2-1. Data from Evans et al. [1970].

Figure A.2-16. Flexural strength at $295 \mathrm{~K}$ of FGR epoxy resins with MNA hardener after ambient gamma irradiation. Supplementary Table A.2-1. Data from Evans et al. [1970].

Figure A.2-17. Flexural strength at $295 \mathrm{~K}$ of FGR TGDM resin with several hardeners after ambient gamma irradiation. Supplementary Table A.2-1. Data from Evans et al. [1970].

Figure A.2-18. Flexural strength at $295 \mathrm{~K}$ of FGR TGPAP resin with several hardeners after ambient gamma irradiation. Supplementary Table A.2-1. Data from Evans et al. [1970].

Figure A.2-19. Flexural strength at $295 \mathrm{~K}$ of FGR EPN resin with several hardeners after ambient gamma irradiation. Supplementary Table A.2-1. Data from Evans et al. [1970]. 
Figure A.2-20. Flexural strength at $295 \mathrm{~K}$ of FGR DGEBA resin with several hardeners after ambient gamma irradiation. Supplementary Table A.2-1. Data from Evans et al. [1970]. . . . . . . . . . . A.2-20

Figure A.2-21. Flexural strength at $295 \mathrm{~K}$ of FGR DGA resin with several hardeners after ambient gamma irradiation. Supplementary Table A.2-1. Data from Evans et al. [1970]. . . . . . . . . . . A.2-21

Figure A.2-22. Flexural strength at $295 \mathrm{~K}$ of FGR epoxy resins (MY 790 and MY 750) after ambient gamma irradiation. Supplementary Table A.2-1. Data from Evans et al. [1972a]. . . . . . . . . . A.2-22

Figure A.2-23. Flexural strength at $295 \mathrm{~K}$ of neat and FGR TGDM resin, MNA hardener after ambient reactor irradiation.

Supplementary Table A.2-2. Data from Schönbacher and StolarzIżycka [1979].

Figure A.2-24. Flexural strength at $295 \mathrm{~K}$ of FGR EPN resin with several hardeners after ambient reactor irradiation. Supplementary Table A.2-2. Data from Schönbacher and Stolarz-Iźycka [1979]. . . . A.2-24

Figure A.2-25. Flexural strength at $295 \mathrm{~K}$ of FGR DGEBA resin, MNA hardener, mica paper tape (Orlitherm) after ambient reactor irradiation. Supplementary Table A.2-2. Data from Schönbacher and Stolarz-Iźycka [1979] . . . . . . . . . . . . . . . . A.2-25

Figure A.2-26. Flexural strength at $295 \mathrm{~K}$ of neat and FGR DGEBA resin with MNA hardener after ambient reactor irradiation.

Supplementary Table A.2-2. Data from Schönbacher and StolarzIzycka [1979].

Figure A.2-27. Flexural strength at 295 K of FGR TGDM and bisphenol A-based resins after ambient electron irradiation. Supplementary Table A.2-3. Data from Hagiwara et al. [1985]. . . . . . . . . . A.2-27

Figure A.2-28. Interlaminar shear strength at $295 \mathrm{~K}$ of FGR TGDM resin, DDS hardener after ambient electron irradiation.

Supplementary Table A.2-3. Data from Hagiwara et al. [1985]. . . . . A.2-28

Figure A.2-29. Flexural strength at $295 \mathrm{~K}$ of FGR TGDM, EPN, and DGEBA resins, DDM hardener after ambient irradiation. No supplementary information. Data from Van de Voorde and Restat [1972]. . . . . . . . . . . . . . . . . . . . . . . . . A.2-29

Figure A.2-30. Flexural strength at $295 \mathrm{~K}$ of neat TGDM resin with several hardeners after ambient gamma irradiation. Supplementary Table A.2-1. Data from Morgan and Stapleton [1975] . . . . . . . . A.2-30

Figure A.2-31. Flexural strength at $295 \mathrm{~K}$ of neat DGEBA resin with several hardeners after ambient gamma irradiation. Supplementary Table A.2-1. Data from Morgan and Stapleton [1975] . . . . . . . . A.2-31

Figure A.2-32. Flexural strength at $295 \mathrm{~K}$ of neat TGDM resin with several hardeners after ambient gamma irradiation. Supplementary Table A.2-1. Data from Morgan and Stapleton [1975] . . . . . . . . A.2-32 
Figure A.2-33. Flexural strength at $295 \mathrm{~K}$ of neat DGEBA resin with several hardeners after ambient gamma irradiation. Supplementary Table A.2-1. Data from Morgan and Stapleton [1975].

Figure A.2-34. Flexural strength at $295 \mathrm{~K}$ of neat EPN resin with anhydride hardeners after ambient gamma irradiation. Supplementary Table A.2-1. Data from Morgan and Stapleton [1975].

Figure A.2-35. Flexural strength at $295 \mathrm{~K}$ of neat glycidyl ester resin with anhydride hardeners after ambient gamma irradiation.

Supplementary Table A.2-1. Data from Morgan and Stapleton [1975].

Figure A.2-36. Flexural strength at $295 \mathrm{~K}$ of neat TGDM and bisphenol A-based resins after ambient electron irradiation. Supplementary Table A.2-3. Data from Hagiwara et a1. [1985].

Figure A.2-37. Flexural strength at $295 \mathrm{~K}$ of neat epoxy resins with anhydride hardener after ambient reactor irradiation.

Supplementary Table A.2-4. Data from Pluym and Van de Voorde [1967] and Van de Voorde and Restat [1972].

Figure A.2-38. Flexural strength at $295 \mathrm{~K}$ of neat epoxy resins with aromatic amine hardener after ambient reactor irradiation.

Supplementary Table A.2-4. Data from Pluym and Van de Voorde [1967] and Van de Voorde and Restat [1972].

Figure A.2-39. Flexural strength at $295 \mathrm{~K}$ of neat DGEBA resin with several hardeners after ambient reactor irradiation. Supplementary Table A.2-4. Data from Pluym and Van de Voorde [1967] and Van de Voorde and Restat [1972].

Figure A.2-40. Flexural strength at $295 \mathrm{~K}$ of neat EPN resin with several hardeners after ambient reactor irradiation. Supplementary Table A.2-4. Data from Pluym and Van de Voorde [1967] and Van de Voorde and Restat [1972].

Figure A.2-41. Flexural strength at $295 \mathrm{~K}$ of neat EPN resin with anhydride hardeners after ambient reactor irradiation.

Supplementary Table A.2-4. Data from Pluym and Van de Voorde [1967].

Figure A.2-42. Flexural strength at $295 \mathrm{~K}$ of neat Araldite D resin with TETA hardener after ambient reactor irradiation. Supplementary Table A.2-2. Data from Schönbacher and Stolarz-Iżycka [1979]

Figure A.2-43. Flexural strength at $295 \mathrm{~K}$ of neat unidentified resin with several hardeners after ambient irradiation. No supplementary information. Data from Parkinson and Sisman [1971]

Figure A.2-44. Shear strength at $295 \mathrm{~K}$ of neat DGEBA resin with several hardeners after ambient gamma irradiation. Supplementary Table A.2-5. Data from Markley et al. [1969] 
Figure A.2-45. Shear strength at $295 \mathrm{~K}$ of neat DGEBA resin with ambient temperature hardeners after ambient gamma irradiation. Supplementary Table A.2-5. Data from Markley et al. [1969]. A. $2-45$

Figure A.2-46. Shear strength at $295 \mathrm{~K}$ of neat DGEBA resin with anhydride hardeners after ambient gamma irradiation. Supplementary Table A.2-5. Data from Markley et al. [1969]. . . . . . . . . . A.2-46

Figure A.2-47. Shear strength at $295 \mathrm{~K}$ of neat TGDM, TGPAP, and DGEBA resins with DAN hardener after ambient gamma irradiation. Supplementary Table A.2-5. Data from Markley et al. [1969].

Figure A.2-48. Shear strength at $295 \mathrm{~K}$ of neat EPN and DGEBA resins with eutectic amine hardener after ambient gamma irradiation. Supplementary Table A.2-5. Data from Markley et al. [1969]. A. $2-48$

Figure A.2-49. Shear strength at $295 \mathrm{~K}$ of neat TGDM and TGPAP resins with several hardeners after ambient gamma irradiation. Supplementary Table A.2-5. Data from Markley et a1. [1969].

Figure A.2-50. Flexural strength at $295 \mathrm{~K}$ of FGR TGDM resin with varied amount of MNA hardener after ambient gamma irradiation. Supplementary Table A.2-1. Data from Evans et a1. [1972a]. A. $2-50$

Figure A.2-51. Flexural strength at $295 \mathrm{~K}$ of neat TGDM resin with varied amount of MNA hardener after ambient gamma irradiation. Supplementary Table A.2-1. Data from Evans et al. [1972a]. . . . . A.2-51

Figure A.2-52. Flexural strength at $295 \mathrm{~K}$ of neat TGDM resin with varied cure conditions after ambient gamma irradiation. Supplementary Table A.2-1. Data from Evans et al. [1972a]. A. $2-52$

Figure A.2-53. Flexural strength at $295 \mathrm{~K}$ of neat EPN resin with varied cure conditions after ambient gamma irradiation. Supplementary Table A.2-1. Data from Evans et al. [1972a]. A. $2-53$

Figure A.2-54. Flexural strength at $295 \mathrm{~K}$ of neat TGDM resin with varied cure conditions after ambient gamma irradiation. Supplementary Table A.2-1. Data from Evans et al. [1972a]. A. $2-54$

Figure A.2-55. Flexural strength at $295 \mathrm{~K}$ of FGR EPN resin with varied cure conditions after ambient gamma irradiation. Supplementary Table A.2-1. Data from Evans et al. [1972a]. A. $2-55$

Figure A.2-56. Flexural strength at $295 \mathrm{~K}$ of neat DGEBA resin with DDM hardener and varied cure conditions after ambient gamma irradiation. Supplementary Table A.2-2. Data from Schönbacher and Stolarz-Iżycka [1979].

Figure A.2-57. Flexural strength at $295 \mathrm{~K}$ of neat and FGR DGEBA resin with MNA hardener and varied cure conditions after ambient reactor irradiation. Supplementary Table A.2-2. Data from Schönbacher and Stolarz-Iżycka [1979]. 
Figure A.2-58. Flexural strength at $295 \mathrm{~K}$ of neat TGDM resin with two hardeners after ambient gamma irradiation. Supplementary Table A.2-1. Data from Morgan et al. [1970].

Figure A.2-59. Flexural strength at $295 \mathrm{~K}$ of neat TGDM resin with two hardeners after ambient gamma irradiation in vacuum.

Supplementary Table A.2-1. Data from Morgan et al. [1970].

Figure A.2-60. Flexural strength at $295 \mathrm{~K}$ of neat EPN resin with two hardeners after ambient gamma irradiation. Supplementary Table A.2-1. Data from Morgan et al. [1970].

Figure A.2-61. Flexural strength at $295 \mathrm{~K}$ of neat EPN resin with two hardeners after ambient gamma irradiation in vacuum.

Supplementary Table A.2-1. Data from Morgan et al. [1970].

Figure A.2-62. Flexural strength at $295 \mathrm{~K}$ of neat DGEBA resin with three hardeners after ambient gamma irradiation. Supplementary

Table A.2-1. Data from Morgan et al. [1970].

Figure A.2-63. Flexural strength at $295 \mathrm{~K}$ of neat DGEBA resin with three hardeners after ambient gamma irradiation in vacuum. Supplementary Table A.2-1. Data from Morgan et al. [1970]. A. $2-63$

Figure A.2-64. Flexural strength at $295 \mathrm{~K}$ of DGEBA resin with DDM hardener and various fillers after ambient reactor irradiation.

Supplementary Table A.2-4. Data from Pluym and Van de Voorde [1967] and Van de Voorde and Restat [1972].

Figure A.2-65. Flexural strength at $295 \mathrm{~K}$ of DGEBA resin with two hardeners and silica filler after ambient reactor irradiation.

Supplementary Table A.2-4. Data from Pluym and Van de Voorde [1967]

Figure A.2-66. Flexural strength at $295 \mathrm{~K}$ of DGEBA resin with DDM hardener and silica fillers after ambient reactor irradiation. Supplementary Table A.2-4. Data from Pluym and Van de Voorde [1967].

Figure A.2-67. Flexural strength at $295 \mathrm{~K}$ of EPN and DGEBA resins with and without filler after ambient reactor irradiation. Supplementary Table A.2-2. Data from Schönbacher and StolarzIźycka [1979].

Figure A.2-68. Flexural strength at $295 \mathrm{~K}$ of bisphenol A resin with and without filler after ambient reactor irradiation. Supplementary Table A.2-2. Data from Schönbacher and Stolarz-Iżycka [1979]. . . . A.2-68

Figure A.2-69. Flexural strength at $295 \mathrm{~K}$ of DGEBA resin, acid anhydride hardener, with and without filler after ambient reactor irradiation. Supplementary Table A.2-2. Data from Schönbacher and Stolarz-Iźycka [1979]. 
Figure A.2-70. Flexural strength at $295 \mathrm{~K}$ of DGEBA resin, acid anhydride hardener, with and without filler after ambient reactor irradiation. Supplementary Table A.2-2. Data from Schönbacher and Stolarz-Iźycka [1979].

Figure A.2-71. Flexural strength at $295 \mathrm{~K}$ of DGEBA resin, MNA hardener, with and without filler after ambient reactor irradiation.

Supplementary Table A.2-2. Data from Schönbacher and StolarzIżycka [1979].

Figure A.2-72. Flexural strength at $295 \mathrm{~K}$ of proprietary resin, anhydride hardener, with silica and quartz fillers, after ambient reactor irradiation. Supplementary Table A.2-2. Data from Schönbacher and Stolarz-Iżycka [1979].

Figure A.2-73. Compressive strength at $295 \mathrm{~K}$ of DGEBA resin with alumina filler after ambient electron irradiation. Supplementary Table A.2-6. See text for discussion of possible error in dose determination. Data from Brechna [1965].

Figure A.2-74. Flexural strength at $295 \mathrm{~K}$ of neat and glass or mica reinforced bisphenol A resin after ambient reactor irradiation. Supplementary Table A.2-2. Data from Schönbacher and Stolarz-Iźycka [1979].

Figure A.2-75. Flexural strength at $295 \mathrm{~K}$ of FGR/mica proprietary resins after ambient reactor irradiation. Supplementary Table A.2-2. Data from Schönbacher and Stolarz-Iżycka [1979]. A. $2-75$

Figure A.2-76. Flexural strength at $295 \mathrm{~K}$ of FGR/mica solventless resins after ambient reactor irradiation. Supplementary Table A.2-7. Data from lipták et al. [1985]. . . . . . . . . . . . . A.2-76

Figure A.2-77. Flexural strength at $295 \mathrm{~K}$ of FGR/mica solventless, resin-rich systems after ambient reactor irradiation. Supplementary Table A.2-7. Data from Lipták et al. [1985]. . . . . . . . . . . A.2-77

Figure A.2-78. Flexural strength at $295 \mathrm{~K}$ of FGR DEGBA resin and DGEBA laminate (G-10) after ambient reactor irradiation.

Supplementary Table A.2-8. Data from Imel et al. [1979]. Total dose estimated from conversion provided by Imel et al. and aim glass fraction. . . . . . . . . . . . . . . . . . . . . A.2-78

Figure A.2-79. Compressive strength at $295 \mathrm{~K}$ of FGR bisphenol A and other resins after ambient $14-\mathrm{MeV}$ neutron irradiation. Supplementary Table A.2-9. Data from Iida and Sumita [1983]. . . . . A.2-79

Figure A.2-80. Compressive strength at $295 \mathrm{~K}$ of EPN resins with varied reinforcement, pre-preg fabrication after ambient $14-\mathrm{MeV}$ neutron irradiation. Supplementary Table A.2-9. Data from Iida and Sumita [1983]. 
Figure A.2-81. Maximum tear test load (see text) at $295 \mathrm{~K}$ of FGR epoxy laminate (Lamiverre A) after ambient electron or gamma irradiation. Supplementary Table A.2-10. Data from Nishiura et al. [1988a].

Figure A.2-82. Acoustic emission increase (see text) at $295 \mathrm{~K}$ of FGR epoxy laminate (Lamiverre A) after ambient electron or gamma irradiation. Supplementary Table A.2-10. Data from Nishiura et al. [1988a].

Figure A.2-83. Tensile strength at $295 \mathrm{~K}$ of FGR epoxy resin (Epomik) after ambient electron or gamma irradiation.

Supplementary Table A.2-10. Data from Nishiura et al. [1988b] A. $2-83$

Figure A.2-84. Interlaminar shear strength at $295 \mathrm{~K}$ of FGR epoxy laminates (Lamiverre A, Hoxan) after ambient neutron or gamma irradiation. Supplementary Table A.2-11. Data from Nishijima et al. [1988].

Figure A.2-85. Flexural strength at $295 \mathrm{~K}$ of proprietary resin after ambient gamma irradiation. Supplementary Table A.2-12. Data from Hacker et al. [1984].

Figure A.2-86. Flexural strength at $295 \mathrm{~K}$ of neat DGEBA and bisphenol A resins with anhydride hardeners after ambient reactor irradiation. Supplementary Table A.2-13. Data from Phillips et al. [1981].

Figure A.2-87. Flexural strength at $295 \mathrm{~K}$ of neat DGEBA and not characterized resins (includes isocyanate system) after ambient reactor irradiation. Supplementary Table A.2-7. Data from Lipták et al. [1985].

Figure A.2-88. Flexural strength at $295 \mathrm{~K}$ of FGR DGEBA, EPN, and not characterized resins (includes Orlitherm systems) after ambient reactor irradiation. Supplementary Table A.2-7. Data from Lipták et al. [1985].

Figure A.2-89. Flexural strength at $295 \mathrm{~K}$ of FGR bisphenol A-based resin with MNA hardener after ambient reactor irradiation. No supplementary information. Banford [1984].

Figure A.2-90. Tensile strength at $295 \mathrm{~K}$ of FGR laminate and DGEBA resin after ambient gamma irradiation. Supplementary Table A.2-5. Data from Markley et al. [1969]. A. $2-90$

Figure A.2-91. Compressive strength at $295 \mathrm{~K}$ of FGR laminate and DGEBA resin after ambient gamma irradiation. Supplementary Table A.2-5. Data from Markley et al. [1969].

Figure A.2-92. Shear strength at $295 \mathrm{~K}$ of DGEBA resin after ambient gamma irradiation. Supplementary Table A.2-5. Data from Markley et al. [1969]. 
Figure A.2-93. Flexural strength at $295 \mathrm{~K}$ of neat bisphenol A-based resin after ambient reactor irradiation. Supplementary Table A.2-4. Data from Pluym and Van de Voorde [1967].

Figure A.2-94. Tensile strength at $295 \mathrm{~K}$ of FGR bisphenol A resins after ambient gamma irradiation. Supplementary Table A.2-14. Data from Dahlerup-Peterson and Perrot [1979] and Dahlerup-Peterson [1980].

[Figure A.2-95. Flexural strength at $295 \mathrm{~K}$ of neat proprietary resins with several hardeners after ambient reactor irradiation. Supplementary Table A.2-2. Data from Schönbacher and StolarzIzycka [1979].

Figure A.2-96. Flexural strength at $295 \mathrm{~K}$ of neat proprietary resins with several hardeners after ambient reactor irradiation. Supplementary Table A.2-2. Data from Schönbacher and StolarzIzycka [1979].

Figure A.2-97. Flexural strength at $295 \mathrm{~K}$ of FGR proprietary resins after ambient reactor irradiation. Supplementary Table A.2-2. Data from Schönbacher and Stolarz-Iźycka [1979].

Figure A.2-98. Flexural strength at $295 \mathrm{~K}$ of FGR TGDM resin with MNA hardener after ambient irradiation. No supplementary information. Data from Van de Voorde and Restat [1972].

Figure A.2-99. Flexural strength at $295 \mathrm{~K}$ of FGR DGEBA resin with MNA hardener after ambient irradiation. No supplementary information. Data from Van de Voorde and Restat [1972].

Figure A.2-100. Flexural strength at $295 \mathrm{~K}$ of FGR EPN resin with mica reinforcement after ambient irradiation. No supplementary information. Data from Van de Voorde and Restat [1972].

Figure A.2-101. Flexural strength at $295 \mathrm{~K}$ of neat glycidyl ether/EPN resin with anhydride hardener after ambient irradiation. No supplementary information. Data from Van de Voorde and Restat [1972].

Figure A.2-102. Flexural strength at $295 \mathrm{~K}$ of neat cycloaliphatic resin with acid anhydride hardener after ambient irradiation. No supplementary information. Data from Van de Voorde and Restat [1972].

Figure A.2-103. Flexural strength at $295 \mathrm{~K}$ of neat DGEBA resin with acid anhydride hardener after ambient irradiation. No

supplementary information. Data from Van de Voorde and Restat [1972].

Figure A.2-104. Interlaminar fracture toughness at $295 \mathrm{~K}$ of FGR TGDM resin with DDS hardener after ambient electron irradiation. Supplementary Table A.2-15. Data from Takeda et al. [1989]. . . . A.2-104 
Figure A.2-105. Compressive strength at $295 \mathrm{~K}$ of FGR epoxy and BT resins and laminates (G-10CR, G-11CR) after ambient electron irradiation. Supplementary Table A.2-16. Data from Nishijima et al. [1990].

Figure A.2-106. Flexural strength at $295 \mathrm{~K}$ of FGR (3-D) bisphenol $A$ resin with aromatic amine hardener after ambient reactor irradiation. Supplementary Table A.2-17. Data from McManamy et al. [1990; 1991].

Figure A.3-1. Flexural strength at 77 and $295 \mathrm{~K}$ of FGR TGDM resin with DDS hardener after ambient electron irradiation. Supplementary Table A.3-1. Data from Egusa [1988].

Figure A.3-2. Flexural strength at 77 and $295 \mathrm{~K}$ of neat TGDM resin with DDS hardener after ambient electron irradiation. Supplementary Table A.3-1. Data from Egusa [1988].

Figure A.3-3. Tensile strength at $77 \mathrm{~K}$ of FGR bisphenol A resins after ambient gamma irradiation. Supplementary Table A.2-14. Data from Dahlerup-Peterson and Perrot [1979] and DahlerupPeterson [1980].

Figure A.3-4. Tensile strength at 77 and $295 \mathrm{~K}$ of FGR epoxy laminate (Lamiverre A) after ambient gamma irradiation.

Supplementary Table A.2-10. Data from Nishiura et al. [1988a]

Figure A.3-5. Flexural strength at $295 \mathrm{~K}$ of FGR TGDM and DGEBA resins and laminates (G-10CR, G-11CR) after ambient gamma irradiation. Supplementary Table A.3-2. Data from Egusa and Hagiwara [1986].

Figure A.3-6. Flexural strength at $77 \mathrm{~K}$ of FGR TGDM and DGEBA resins and laminates (G-10CR, G-11CR) after ambient gamma irradiation. Supplementary Table A.3-2. Data from Egusa and Hagiwara [1986].

Figure A.3-7. Shear strength at $77 \mathrm{~K}$ of FGR epoxy resins (including $G-11 C R$ ) after ambient reactor irradiation. Supplementary Table A.3-3. Data from Munshi [1991].

Figure A.3-8. Fracture resistance at $77 \mathrm{~K}$ of FGR epoxy resins (including G-11CR) after ambient reactor irradiation. Supplementary Table A.3-3. Data from Munshi [1991]. . . . . . . . . . . A.3-8

Figure A.3-9. Flexural strength at $77 \mathrm{~K}$ of FGR ( $\mathrm{E}$ and $\mathrm{T}$ glass) TGDM with DDS hardener after ambient gamma irradiation. Supplementary Table A.3-4. Data from Egusa [1991a].

Figure A.3-10. Flexural strength at $77 \mathrm{~K}$ of FGR (variable volume fraction) TGDM with DDS hardener after ambient gamma irradiation. Supplementary Table A.3-4. Data from Egusa [1991a]. 
Figure A.3-11. Flexural strength at $77 \mathrm{~K}$ and $295 \mathrm{~K}$ of FGR (variable volume fraction) DGEBF with DDM hardener after ambient electron irradiation. Supplementary Table A.3-5. Data from Egusa et al. [1988].

Figure A.3-12. Flexural strength at $77 \mathrm{~K}$ of FGR TGDM resin (variable thickness) with DDS hardener after ambient gamma irradiation.

Supplementary Table A.3-4. Data from Egusa [1991a]. . . . . . . . . . A.3-12

Figure A.3-13. Flexural strength at $77 \mathrm{~K}$ of FGR DGEBA resins (including G-10CR and G-11CR) after ambient gamma irradiation in air or argon. Supplementary Table A.3-4. Data from Egusa [1991a]. . A.3-13

Figure A.3-14. Maximum tear test load at $77 \mathrm{~K}$ of FGR epoxy laminate (Lamiverre A) after ambient gamma or electron irradiation.

Supplementary Table A.2-10. Data from Nishiura et al. [1988a]. . . . A.3-14

Figure A.3-15. Load at which acoustic emission count increases steeply at $77 \mathrm{~K}$ of FGR epoxy laminate (Lamiverre A) after ambient gamma or electron irradiation. Supplementary Table A.2-10. Data from Nishiura et al. [1988a]. . . . . . . . . . . . . . A.3-15

Figure A.3-16. Tensile strength at $77 \mathrm{~K}$ of FGR epoxy resin (Epomik) after ambient gamma or electron irradiation. Supplementary Table A.2-10. Data from Nishiura et al. [1988b]. . . . . . . . . . . A.3-16

Figure A.3-17. Flexural strength at $77 \mathrm{~K}$ of neat TGDM resin with DDS hardener after ambient gamma or electron irradiation.

Supplementary Table A.3-4. Data from Egusa [1991a]. . . . . . . . . A.3-17

Figure A.3-18. Flexural strength at $77 \mathrm{~K}$ of FGR TGDM resin with DDS hardener after ambient gamma or electron irradiation. Supplementary Table A.3-4. Data from Egusa [1991a]. . . . . . . . . A.3-18

Figure A.4-1. Flexural strength at $4 \mathrm{~K}$ of FGR TGDM and DGEBA resins (including G-10CR and G-IICR) after ambient gamma irradiation. Supplementary Table A.3-4. Data from Egusa [1990]. . . A.4-1

Figure A.5-1. Flexural strength at $295 \mathrm{~K}$ of a mixture of DGEBA resin with other components after $77-\mathrm{K}$ electron or ambient gamma irradiation. Supplementary Table A.5-1. Data from Evans et al. [1971]........................... A.5-1

Figure A.5-2. Flexural strength at $295 \mathrm{~K}$ of mixtures of DGEBA resin with other components after ambient gamma irradiation. Supplementary Table A.5-1. Data from Evans et a1. [1971].

Figure A.5-3. Flexural strength at $295 \mathrm{~K}$ of mixtures of DGEBA resin with other components after ambient gamma irradiation. (Expanded scale.) Supplementary Table A.5-1. Data from Evans et al. [1971]. . . A.5-3

Figure A.5-4. Flexural strength at $295 \mathrm{~K}$ of mixtures of DGEBA resin with other components after 77-K electron irradiation. Supplementary Table A.5-1. Data from Evans et al. [1971]. 
Figure A.6-1. Tensile strength at $77 \mathrm{~K}$ of FGR DGEBA resin (Orlitherm) after 77-K reactor irradiation. Supplementary Table A.6-1. Data

from Weber et al. [1983].

Figure A.6-2. Flexural strength at $77 \mathrm{~K}$ of neat aromatic, DGEBA, and other resin after $77-\mathrm{K}$ reactor irradiation. Supplementary Table

A.6-2. Data from Van de Voorde [1972].

Figure A.6-3. Flexural strength at unspecified temperature of neat epoxy resins irradiated at low temperature. No supplementary information. Data from Evans and Morgan [1982].

Figure A.7-1. Interlaminar shear strength at $77 \mathrm{~K}$ of FGR epoxy laminates (Lamiverre $\mathrm{A}$, Hoxan) after $20-\mathrm{K}$ reactor and $77-\mathrm{K}$ gamma irradiation. Supplementary Table A.2-11. Data from Nishijima et al. [1988].

Figure A.7-2. Interlaminar shear strength at $77 \mathrm{~K}$ of FGR epoxy and bismaleimide laminates after $20-\mathrm{K}$ reactor irradiation. Comparison of E- and T-glass reinforcement. Supplementary Table A.7-1. Data from Nishijima et al. [1991] and Okada et al. [1992].

Figure A.7-3. Interlaminar shear strength at $77 \mathrm{~K}$ of FGR (variable volume fraction) epoxy resin after $20-\mathrm{K}$ reactor irradiation. Supplementary Table A.7-1. Data from Okada et al. [1992].

Figure A.7-4. Flexural strength at $77 \mathrm{~K}$ of FGR (2- and 3-D) epoxy resin after 20-K reactor irradiation. Supplementary Table A.2-16. Data from Nishijima et al. [1990].

Figure A.7-5. Interlaminar shear strength at $77 \mathrm{~K}$ of FGR (2- and 3-D) epoxy resin after 20-K reactor irradiation. Supplementary Table A.2-16. Data from Nishijima et al. [1990].

Figure A.8-1. Flexural strength at 75 and $295 \mathrm{~K}$ of FGR DGEBA laminates (G-10CR, G-11CR) after $4-\mathrm{K}$ reactor irradiation.

Supplementary Table A.8-1. Data from Hurley et al. [1978; 1983].

Figure A.8-2. Compressive strength at 75 and $295 \mathrm{~K}$ of FGR DGEBA laminates (G-10CR, G-11CR) after $4-\mathrm{K}$ reactor irradiation.

Supplementary Table A.8-1. Data from Hurley et al. [1978; 1983].

Figure A.8-3. Flexural strength at 75 and $295 \mathrm{~K}$ of DGEBA resin with silica filler after $4-\mathrm{K}$ reactor irradiation. Supplementary Table A.8-1. Data from Hurley et al. [1978].

Figure A.8-4. Tensile strength at 75 and $295 \mathrm{~K}$ of DGEBA resin with silica filler after $4-\mathrm{K}$ reactor irradiation. Supplementary Table A.8-1. Data from Hurley et al. [1978].

Figure A.8-5. Flexural strength at $75 \mathrm{~K}$ of FGR DGEBA laminates (G-10CR, G-1ICR) after 4-K reactor irradiation. Supplementary Table A.8-1. Data from Tucker et al. [1985]. 
Figure A.8-6. Flexural strength at $77 \mathrm{~K}$ of DGEBA resins, filled, after 4-K reactor irradiation. Supplementary Table A.8-2. Data from Kernohan et al. [1979].

Figure A.8-7. Flexural strength at $77 \mathrm{~K}$ of DGEBA resins, filled, after 4-K reactor irradiation. Supplementary Table A.8-2. Data from Coltman et al. [1979].

Figure A.8-8. Compressive strength at $77 \mathrm{~K}$ of DGEBA resins, filled, after 4-K reactor irradiation. Supplementary Table A.8-2. Data from Kernohan et al. [1979].

Figure A.8-9. Compressive strength at $77 \mathrm{~K}$ of DGEBA resins, filled, after 4-K reactor irradiation. Supplementary Table A.8-2. Data from Coltman et al. [1979].

Figure A.8-10. Flexural strength at $77 \mathrm{~K}$ of FGR DGEBA laminate (G-10) after 4-K reactor irradiation. Supplementary Table A.8-2. Data from Kernohan et al. [1979].

Figure A.8-11. Flexural strength at $77 \mathrm{~K}$ of FGR DGEBA laminates (G-10CR, G-10-11CR) after 4-K reactor irradiation. Supplementary Table A.8-2. Data from Coltman et al. [1979] and Coltman and Klabunde [1983].

Figure A.8-12. Compressive strength at $77 \mathrm{~K}$ of FGR DGEBA laminate (G-10) after 4-K reactor irradiation. Supplementary Table A.8-2. Data from Kernohan et al. [1979].

Figure A.8-13. Compressive strength at $77 \mathrm{~K}$ of FGR DGEBA laminates (G-10CR, G-10-11CR) after 4-K reactor irradiation. Supplementary Table A.8-2. Data from Coltman et al. [1979] and Coltman and Klabunde [1983].

Figure A.8-14. Compressive strength at $77 \mathrm{~K}$ of DGEBA resin with various hardeners after $5-\mathrm{K}$ reactor irradiation. Supplementary Table A.8-3. Data from Kato and Takamura [1979]. . . . . . . . . . A.8-14

Figure A.8-15. Shear strength at $77 \mathrm{~K}$ of FGR epoxy resins after 4-K reactor irradiation. Supplementary Table A.8-4. Data from Munshi [1991].

Figure A.9-1. Compressive strength at 4 and $77 \mathrm{~K}$ of DGEBA resin with various hardeners after $5-\mathrm{K}$ reactor irradiation.

Supplementary Table A.8-3. Data from Takamura and Kato [1980]. . . . . A.9-1

Figure A.9-2. Compressive strength at 4 and $77 \mathrm{~K}$ of FGR DGEBA resin with acid anhydride hardener after $5-\mathrm{K}$ reactor irradiation. Supplementary Table A.8-3. Data from Takamura and Kato [1980]. . . . . A.9-2

Figure A.9-3. Flexural strength at $4 \mathrm{~K}$ of FGR epoxies and laminates (including $G-10 C R$ and $G-11 C R$ and isocyanate hardener) after $5-K$ reactor irradiation. Supplementary Table A.9-1. Data from Takamura and Kato [1984]. 
Figure A.9-4. Compressive strength at $4 \mathrm{~K}$ of FGR epoxies and laminates (including G-10CR and G-11CR and isocyanate hardener) after 5-K reactor irradiation. Supplementary Table A.9-1. Data from Takamura and Kato [1984].

Figure A.9-5. Tensile strength at $4 \mathrm{~K}$ of FGR epoxies and laminates (including G-10CR and G-11CR and isocyanate hardener) after $5-\mathrm{K}$ reactor irradiation. Supplementary Table A.9-1. Data from Takamura and Kato [1984].

Figure A.9-6. Flexural strength at $4 \mathrm{~K}$ of FGR DGEBA resin with two hardeners after 5-K reactor irradiation. Supplementary Table A.9-1. Data from Takamura and Kato [1981; 1984].

Figure A.10-1. Shear/compression failure envelope at $295 \mathrm{~K}$ of FGR DGEBA laminate (G-10CR) after ambient electron irradiation. Supplementary Table A.10-1. Data from Nishijima et al. [1990]. A. $10-1$

Figure A.10-2. Shear/compression failure envelope at $295 \mathrm{~K}$ of FGR epoxy resin after ambient electron irradiation. Supplementary Table A.10-1. Data from Nishijima et al. [1990]. A. $10-2$

Figure A.10-3. Shear strength with compressive load of $345 \mathrm{Mpa}$ at $295 \mathrm{~K}$ of FGR (3-D) epoxy resin after ambient reactor irradiation. Supplementary Table A.2-17. Data from McManamy et a1. [1990; 1991a].

Figure A.11-1. Shear strength at $295 \mathrm{~K}$ of FGR DGEBA resin (Orlitherm) to copper substrate, with and without primer, after ambient reactor irradiation. Supplementary Table A.2-7. Data from Lipták et al. [1985].

Figure A.11-2. Shear strength at $295 \mathrm{~K}$ of FGR DGEBA resin to copper substrate, with and without primer and sandblasting, after ambient reactor irradiation. Supplementary Table A.2-8. Data from Imel et a1. [1979]. Total dose estimated from conversion provided by Imel et al. and aim glass fraction. A. 11-2

Figure A.11-3. Shear strength at $77 \mathrm{~K}$ of FGR epoxy or BT resins to stainless steel substrate after ambient gamma irradiation. Supplementary Table A.11-1. Data from Egusa et al. [1992]. A. $11-3$

Figure A.11-4. Shear strength at $77 \mathrm{~K}$ of FGR and filled DGEBA resins to copper substrate (not abraded) after 4-K reactor irradiation. Supplementary Table A.8-2. Data from Kernohan et al. [1979].

Figure A.11-5. Shear strength at $295 \mathrm{~K}$ of FGR and filled DGEBA resins to sanded copper substrate after ambient electron irradiation. Supplementary Table A.2-6. See discussion in text of a possible error in dose determination. Data from Brechna [1965]. 
Figure A.11-6. Shear strength at $77 \mathrm{~K}$ of FGR DGEBA resin (Orlitherm) to stainless steel substrate after 4-K reactor

irradiation. Supplementary Table A.11-2. Data from Poehlchen

et al. [1990] and Pöhlchen [1992].

Figure A.11-7. Shear strength at $300 \mathrm{~K}$ of FGR DGEBA resin (Orlitherm) to stainless steel substrate after $4-\mathrm{K}$ reactor irradiation. Supplementary Table A.11-2. Data from Poehlchen et al. [1990] and Pöhlchen [1992].

Figure A.11-8. Shear strength at $4 \mathrm{~K}$ of FGR DGEBA resin (Orlitherm) to stainless steel substrate after $4-\mathrm{K}$ reactor irradiation. Supplementary Table A.11-2. Data from Poehlchen et al. [1990] and Pöhlchen [1992].

Figure A.11-9. Shear strength at $4 \mathrm{~K}$ of FGR DGEBA resin (Orlitherm) to stainless steel substrate after $4-\mathrm{K}$ reactor irradiation. Supplementary Table A.11-2 (except $n_{f}$ fluence $-10^{22} / \mathrm{m}^{2}$ ). Data from Katheder et al. [1992].

Figure A.11-10. Shear strength at $295 \mathrm{~K}$ of FGR DGEBA resin to copper substrate after ambient reactor irradiation. Supplementary

Table A.11-3. Data from Schmunk et al. [1981]. Dose determined from usual conversion factor.

Figure A.11-11. Shear strength at $77 \mathrm{~K}$ of FGR and filled DGEBA resins to copper substrate (abraded) after $4-\mathrm{K}$ reactor irradiation. Supplementary Table A.8-2. Data from Coltman et al. [1979].

Figure A.11-12. Shear strength at $295 \mathrm{~K}$ of FGR resin to copper substrate, sandblasted and primed, after ambient reactor irradiation. Supplementary Table A.2-17. Data from McManamy et a1. [1991b].

Figure B.1-1. Flexural strength at $77 \mathrm{~K}$ of FGR polyaminobismaleimide resin after ambient neutron (IPNS) or gamma irradiation. The spectrum at the "rabbit" irradiation thimble was described as degraded fission with a high energy component; that at $\mathrm{H} 2$ consisted of a large flux of low energy neutrons. Supplementary Table B.1-1. Data from Egusa et a1. [1987b]............. . B.1-1

Figure B.1-2. Elongation at $295 \mathrm{~K}$ of polyimide film after ambient 14-Mev neutron (RTNS) or gamma irradiation. Supplementary Table B.1-2. Data from Abe et al. [1987].

Figure B.1-3. Flexural strength at $77 \mathrm{~K}$ of FGR polyaminobismaleimide resin after ambient electron or gamma irradiation. Supplementary Table A.3-4. Data from Egusa et al. [1991a]. . . . . . B.1-3

Figure B.1-4. Shear strength at $77 \mathrm{~K}$ of FGR bismaleimide and polyimide resins after ambient or $4-\mathrm{K}$ reactor irradiation. Supplementary Tables A.3-3 and A.8-4. Data from Munshi [1991]. . . . B.1-4 
Figure B.1-5. Flexural strength at $295 \mathrm{~K}$ of FGR polyaminobismaleimide resin after ambient or 5-K neutron (IPNS) irradiation. The spectrum at both irradiation thimbles was described as degraded fission with a high energy component. Supplementary Table B.1-1.

Data from Egusa et a1. [1984a].

Figure B.1-6. Flexural strength at $77 \mathrm{~K}$ of FGR polyaminobismaleimide resin after ambient or $5-\mathrm{K}$ neutron (IPNS) irradiation. The spectrum at both irradiation thimbles was described as degraded fission with a high energy component. Supplementary Table B.1-1. Data from Egusa et al. [1987b].

Figure B.1-7. Flexural strength at $77 \mathrm{~K}$ of FGR polyimide resin after ambient gamma irradiation in either air or argon. Supplementary Table A.3-4. Data from Egusa et a1. [1991a]. . . . . . B.1-7

Figure B.1-8. Flexural strength at 77 and $295 \mathrm{~K}$ of FGR polyaminobismaleimide resin after ambient gamma irradiation. Supplementary Table A.3-1. Data from Egusa [1988]............... . B.1-8

Figure B.1-9. Flexural strength at 77 and $295 \mathrm{~K}$ of neat polyimide resin after ambient electron irradiation. Supplementary Table A.3-1. Data from Egusa [1988]. . . . . . . . . . . . . . . B.1-9

Figure B.1-10. Flexural strength at 77 and $295 \mathrm{~K}$ of FGR polyaminobismaleimide resin after ambient electron irradiation.

Supplementary Table B.1-1. Data from Egusa et al. [1985a].

Figure B.1-11. Compressive strength at 4 and 77 of polyimide (Vespel) resin after 5-K reactor irradiation. Supplementary Table A.8-3. Data from Takamura and Kato [1980].

Figure B.1-12. Flexural strength at $295 \mathrm{~K}$ of FGR polyaminobismaleimide resin, with and without vacuum anneal, after ambient gamma irradiation. Supplementary Table B.1-1. Data from Egusa et al. [1985b].

Figure B.1-13. Flexural strength at $77 \mathrm{~K}$ of FGR polyaminobismaleimide resin after ambient gamma irradiation. Comparison of E- and T-glass reinforcement. Supplementary Table A.3-4. Data from Egusa et a1. [1991a].

Figure B.1-14. Flexura1 strength at $77 \mathrm{~K}$ of FGR polyaminobismaleimide laminates after ambient gamma irradiation. Comparison of E- and S-2-glass reinforcement. Supplementary Table A.3-4.

Data from Egusa et a1. [1991a].

Figure B.1-15. Flexural strength at 77 and $295 \mathrm{~K}$ of FGR polyaminobismaleimide resin after ambient gamma irradiation. Specimen orientation at $0^{\circ}$ to warp. Supplementary Table B.1-1. Data from Egusa [1990a]. 
Figure B.1-16. Flexural strength at 77 and $295 \mathrm{~K}$ of FGR polyaminobismaleimide resin after ambient gamma irradiation. Specimen orientation at $45^{\circ}$ to warp. Supplementary Table B.1-1. Data from Egusa [1990a].

Figure B.1-17. Flexural strength at $295 \mathrm{~K}$ of FGR bismaleimide resins after ambient reactor irradiation. Supplementary Table A.2-17. Data from McManamy et a1. [1990; 1991a]. . . . . . . . . B.1-17

Figure B.1-18. Shear strength with compressive load of $345 \mathrm{Mpa}$ at $295 \mathrm{~K}$ of FGR bismaleimide resins after ambient reactor irradiation. Supplementary Table A.2-17. Data from McManamy et al. [1990; 1991a].

Figure B.2-1. Compressive strength at 4 and $77 \mathrm{~K}$ of FGR DGEBA and polyimide (Vespel) resins after $5-\mathrm{K}$ reactor irradiation. Supplementary Table A.8-3. Data from Takamura and Kato [1980]. . . B.2-1

Figure B.2-2. Flexural strength at $4 \mathrm{~K}$ of FGR epoxies and polyimide (TIL-G1000) resin after 5-K reactor irradiation. Supplementary

Table A.9-1. Data from Takamura and Kato [1984]. . . . . . . . . . B.2-2

Figure B.2-3. Tensile strength at $4 \mathrm{~K}$ of FGR epoxies and polyimide (TIL-G1000) resin after 5-K reactor irradiation. Supplementary Table A.9-1. Data from Takamura and Kato [1984]. . . . . . . . . B.2-3

Figure B.2-4. Compressive strength at $4 \mathrm{~K}$ of FGR epoxies and polyimide (TIL-G1000) resin after $5-\mathrm{K}$ reactor irradiation.

Supplementary Table A.9-1. Data from Takamura and Kato [1984]. . . . . B.2-4

Figure B.2-5. Compressive strength at $75 \mathrm{~K}$ of FGR DGEBA and bismaleimide laminates (G-10CR, G-11CR, NP-530, and Spaulrad) after 4-K reactor irradiation. Supplementary Table A.8-1. Data from Hurley et a1. [1978; 1983].

Figure B.2-6. Flexural strength at $75 \mathrm{~K}$ of FGR DGEBA and bismaleimide laminates (G-10CR, G-11CR, NP-530, and Spaulrad) after 4-K reactor irradiation. Supplementary Table A.8-1. Data from Hurley et al. [1978; 1983].

Figure B.2-7. Flexural strength at $300 \mathrm{~K}$ of FGR DGEBA and bismaleimide laminates (G-10CR, G-11CR, NP-530, and Spaulrad) after 4-K reactor irradiation. Supplementary Table A.8-1. Data from Tucker et al. [1985].

Figure B.2-8. Flexural strength at $75 \mathrm{~K}$ of FGR DGEBA and bismaleimide laminates (G-10CR, G-11CR, NP-530, and Spaulrad) after 4-K reactor irradiation. Supplementary Table A.8-1. Data from Tucker et al. [1985]. . . . . . . . . . . . . . . B.2-8

Figure B.2-9. Interlaminar shear strength at $77 \mathrm{~K}$ of FGR epoxy and bismaleimide laminates after $20-\mathrm{K}$ reactor irradiation. Supplementary Table A.7-1. Data from Okada et al. [1992]. 
Figure B.2-10. Flexural strength at $295 \mathrm{~K}$ of epoxy resins and polyimide after ambient electron irradiation. Supplementary

Table A.2-3. Data from Hagiwara et al. [1985].

Figure B.2-11. Flexural strength at $295 \mathrm{~K}$ of FGR TGDM and DGEBA and polyaminobismaleimide resins and laminates (G-10CR, G-11CR) after ambient gamma irradiation. Supplementary Table A.3-2. Data from Egusa and Hagiwara [1986].

Figure B.2-12. Flexural strength at $77 \mathrm{~K}$ of FGR TGDM and DGEBA and polyaminobismaleimide resins and laminates (G-10CR, G-11CR) after ambient gamma irradiation. Supplementary Table A.3-2. Data from Egusa and Hagiwara [1986].

Figure B.2-13. Flexural strength at $4 \mathrm{~K}$ of FGR TGDM and DGEBA and polyaminobismaleimide resins and laminates (G-10CR, G-11CR) after ambient gamma irradiation. Supplementary Table B.1-1. Data from Egusa [1990a].

Figure B.2-14. Flexural strength at $295 \mathrm{~K}$ of FGR BT/DGEBA, DGEBF, and TGDM resins after ambient electron irradiation. Supplementary Table A.3-5. Data from Egusa et al. [1988].

Figure B.2-15. Flexural strength at $77 \mathrm{~K}$ of FGR BT/DGEBA, DGEBF, and TGDM resins after ambient electron irradiation. Supplementary Table A.3-5. Data from Egusa et al. [1988].

Figure B.2-16. Flexural strength at $300 \mathrm{~K}$ of FGR polyaminobismaleimide laminates after $4-\mathrm{K}$ reactor irradiation. Supplementary Table A.8-2. Data from Coltman and Klabunde [1981].

Figure B.2-17. Flexural strength at $77 \mathrm{~K}$ of FGR polyaminobismaleimide laminates after $4-\mathrm{K}$ reactor irradiation. Supplementary Table A.8-2. Data from Coltman and Klabunde [1981].

Figure B.2-18. Compressive strength at $77 \mathrm{~K}$ of FGR polyaminobismaleimide laminates after $4-\mathrm{K}$ reactor irradiation. Supplementary Table A.8-2. Data from Coltman and Klabunde [1981].

Figure B.2-19. Flexural strength at $300 \mathrm{~K}$ of polyimide resin (Vespel) after 4-K reactor irradiation. Supplementary Table A.8-2. Data from Coltman and Klabunde [1981].

Figure B.2-20. Flexural strength at $77 \mathrm{~K}$ of polyimide resin (Vespel) after 4-K reactor irradiation. Supplementary Table A.8-2. Data from Coltman and Klabunde [1981].

Figure B.2-21. Compressive strength at $77 \mathrm{~K}$ of polyimide resin (Vespel) after 4-K reactor irradiation. Supplementary Table A.8-2. Data from Coltman and Klabunde [1981].

Figure B.2-22. Flexural strength at $295 \mathrm{~K}$ of neat and FGR polyimide resins after ambient reactor irradiation. Supplementary Table A.2-2. Data from Schönbacher and Stolarz-Iżycka [1979]. 
Figure B.2-23. Flexural strength at $295 \mathrm{~K}$ of FGR polyaminobismaleimide and epoxy resins after ambient reactor irradiation.

Supplementary Table A.2-17. Data from McManamy et al. [1991b]. . . . . B.2-23

Figure B.2-24. Shear strength at 77 K of FGR epoxy, bismaleimide, and polyimide resins after ambient reactor irradiation.

Supplementary Table A.3-3. Data from Munshi [1991].

Figure B.2-25. Fracture resistance at $77 \mathrm{~K}$ of FGR epoxy, bismaleimide, and polyimide resins after ambient reactor irradiation. Supplementary Table A.3-3. Data from Munshi [1991]. . . . . . . . . B.2-25

Figure B.2-26. Shear strength at $77 \mathrm{~K}$ of FGR epoxy, bismaleimide, and polyimide resins after $4-\mathrm{K}$ reactor irradiation. Supplementary Table A.8-4. Data from Munshi [1991].

Figure B.2-27. Flexural strength at $295 \mathrm{~K}$ of FGR bisphenol resin with MNA hardener, with and without Kapton reinforcement, after ambient reactor irradiation. No supplementary information. Banford [1984].

Figure B.2-28. Flexural strength at $295 \mathrm{~K}$ of polyimide film after ambient irradiation. No supplementary information. Data from Van de Voorde and Restat [1972].

Figure B.2-29. Tensile strength at $77 \mathrm{~K}$ of polyimide film (Kapton) after 5-K reactor irradiation. Supplementary Table A.9-1. Data from Takamura and Kato [1979; 1981]

Figure C.1-1. Fatigue 1ife at 295 and $77 \mathrm{~K}$ of FGR laminates (G-10 and G-11), FGR DGEBA resin, and FGR polyaminobismaleimide resin (Kerimid 601) after ambient reactor irradiation. Supplementary Table C.1-1. The total dose of gamma and fast neutron irradiation (E $>0.1 \mathrm{MeV}$ ) was estimated from the usual conversion rule, except for the Kerimid 601 doses from Becker et al. [1985], in which $3.2 \times 10^{15} / \mathrm{m}^{2}=1$ Gy was assumed. Data from Erez and Becker [1982] and Becker et al. [1985].

Figure C.2-1. Shear/compressive fatigue at $295 \mathrm{~K}$ of FGR polyaminobismaleimide laminate (Spaulrad S), BT, and epoxy after ambient reactor irradiation. Supplementary Table C.2-1. Data from McManamy et al. [1991a]. . . . . . . . . . . . . . . . C.2-1

Figure C.3-1. Fatigue life at 295 and $77 \mathrm{~K}$ of FGR laminate, G-11CR, after ambient ${ }^{60} \mathrm{Co}$ irradiation. Supplementary Table C.3-1. Data from Korukonda et al. [1983]. The percent of tensile strength is also shown ( $463 \mathrm{MPa}$ at $295 \mathrm{~K}, 932 \mathrm{MPa}$ at $77 \mathrm{~K}$ ). The number of cycles to crack initiation is plotted . . . . . . . . . . . . C.3-1.

Figure C.4-1. Flexural fatigue life at 4-K of neat bisphenol A-based resin (6861-2) after $-27-\mathrm{K}$ reactor irradiation. Supplementary Table C.4-1. Data from Nishijima et al. [1981]. . . . . . . . . . . . C.4-1 


\section{LIST OF SUPPLEMENTARY TABLES}

Page

Table A.2-1. Supplementary Information, Price and Sheldon

[1965] and other Rutherford Laboratory references.

A. $2-107$

Table A.2-2. Supplementary Information, Schönbacher and

Stolarz-Iźycka [1979].

A. $2-108$

Table A.2-3. Supplementary Information, Hagiwara et al.

[1985], Takeda et al. [1985

A. $2-109$

Table A.2-4. Supplementary Information, Pluym and

Van de Voorde [1967].

A. $2-110$

Table A.2-5. Supplementary Information, Markley et al.

[1969].

A. $2-111$

Table A.2-6. Supplementary Information, Brechna [1965] .

A. $2-112$

Table A.2-7. Supplementary Information, Lipták et a1. [1969]

A. $2-113$

Table A.2-8. Supplementary Information, Imel et al. [1979]

A. $2-114$

Table A.2-9. Supplementary Information, Iida and Sumita [1983]

A. $2-115$

Table A.2-10. Supplementary Information, Nishiura et a1. [1988a, b]

Table A.2-11. Supplementary Information, Nishijima et a1. [1988]

Table A.2-12. Supplementary Information, Hacker et al. [1984]

Table A.2-13. Supplementary Information, Phillips et al. [1984]

Table A.2-14. Supplementary Information, Dahlerup-

Peterson and Perrot [1979], Dahlerup-Peterson [1980]

A. $2-120$

Table A.2-15. Supplementary Information, Takeda et a1. [1989]

Table A.2-16. Supplementary Information, Nishijima et al. [1990]

Table A.2-17. Supplementary Information, McManamy et al. [1990, 1991a, b].

Table A.3-1. Supplementary Information, Egusa [1988]

A. $3-19$ 
Table A.3-2. Supplementary Information, Egusa and Hagiwara [1986].

Table A.3-3. Supplementary Information, Munshi [1991],

Munshi and Weber [1992]. . . . . . . . . . . . . . . A.3-21

Table A.3-4. Supplementary Information, Egusa [1991a] . . . . . A.3-22

Table A.3-5. Supplementary Information, Egusa et al. [1988] . . . . . . . . . . . . . . . . . A.3-23

Table A.5-1. Supplementary Information, Evans et al. [1971] . . . . . . . . . . . . . . . . A.5-5

Table A.6-1. Supplementary Information, Weber et al. [1983]

Table A.6-2. Supplementary Information, Van de Voorde [1972]

Table A.7-1. Supplementary Information, Nishijima et al. [1991], Okada et al. [1992] . . . . . . . . . . . . A.7-6

Table A.8-1. Supplementary Information, Hurley et al. [1978; 1983].................... . A.8-16

Table A.8-2. Supplementary Information, Kernohan et al. [1979], Coltman et al. [1979], Coltman and Kalubunde [1983]

Table A.8-3. Supplementary Information, Kato and Takamura [1979], Takamura and Kato [1979; 1980]

Table A.8-4. Supplementary Information, Munshi [1991;

Table A.9-1. Supplementary Information, Takamura and Kato [1984] . . . . . . . . . . . . . . . . . . . . A.9-7

Table A.10-1. Supplementary Information, Nishijima et al. [1990]

Table A.11-1. Supplementary Information, Egusa et al. [1992]

Table A.11-2. Supplementary Information, Poehlchen et al. [1990], Pöhlchen [1992].

Table A.11-3. Supplementary Information, Schmunk et a1.

Table B.1-1. Supplementary Information, Egusa et a1. 
Table B.1-2. Supplementary Information, Abe et al.

Table C.1-1. Supplementary Information, Erez and Becker

Table C.2-1. Supplementary Information, McManamy et al.

[1991a]... . . . . . . . . . . . . . . . C.2-2

Table C.3-1. Supplementary Information, Korukunda et al. [1983]

Table C.4-1. Supplementary Information, Nishijima et al. [1981]. 


\section{ASSESSMENT OF THE DATA BASE ON IRRADIATION OF EPOXY INSULATION}

\subsection{INTRODUCTION}

The objectives of this review are: (1) to provide a compilation of all the relevant mechanical property data on irradiation damage to epoxy-matrix electrical insulating materials; (2) to assess whether these data can be used to select superconducting magnet insulation for next-generation fusion devices, such as ITER and TPX; and (3) to determine what further data need to be obtained for the selection of insulation for ITER toroidal and poloidal magnets. Documents from the ITER conceptual design activity were used to determine the required radiation doses, mechanical properties, and fabrication methods.

This review includes a large amount of data on a complex subject. It is organized as follows: Section 1 provides a guide to assessing the data base with regard to the requirements of the ITER conceptual design. Sections 2 through 11 summarize the data, which are presented in graphs in the corresponding Appendices A.2 through A.11. The appendices also contain tables with supplementary information on specimen materials, test methods, and irradiation parameters. The data in $\S \S 2$ to 9 are arranged by the temperature of irradiation and the test temperature. These data were obtained on neat or filled resins, or on glass-reinforced resin systems by employing mainly flexure, tension, and compression tests. The results from a small number of tests in which simultaneous shear and compressive loading were employed are summarized in $\$ 10$. The results from tests in which the composites were bonded to metals are discussed in $\$ 11$. The conclusions regarding ambient temperature irradiation and testing are presented in $\$ 12$. The conclusions regarding the relatively small portion of the data base in which either or both irradiation and testing were conducted at cryogenic temperatures are presented in $\S 13$.

This review was intended to serve primarily as a guide to epoxy resin systems that would be suitable for the fabrication of magnets within the framework of the ITER conceptual design. The fabrication process considered during the conceptual design activity for magnet turn-to-turn insulation was vacuum impregnation of fiberglass with a low viscosity epoxy resin. However, data on epoxy resin systems that may not be ideal for vacuum impregnation are also 
provided in this review, because the ITER design is still evolving and other fabrication methods are under consideration. Also, even if most of the insulation were provided by a vacuum impregnation process, laminates manufactured with high pressure and other processes could be used in some parts of the magnet, perhaps for insulation to ground. Therefore, data on laminates are included in this review. For the same reasons, data on the irradiation resistance of polyimides and bismaleimides are presented in Appendix B, usually in comparison to data obtained for epoxy resins. The small amount of available data on fatigue properties after irradiation is discussed in Appendix $C$.

\subsection{COMPARISON OF THE ACTUAL DATA BASE WITH THE DATA BASE NEEDED FOR ITER}

To downselect one or more insulation systems for ITER magnets, data from screening tests must be employed, because test procedures to obtain design data have not yet been developed and validated. But even the screening data that are available are marginal for material downselection. This is because there is a considerable mismatch between an "ideal" data base that would apply directly to the ITER conceptual design requirements and the data that are actually available. An ideal data base would have the following characteristics:

(1) Specimens would be irradiated at $4 \mathrm{~K}$ in a neutron and gamma spectrum similar to that expected at the ITER magnet location at doses up to and somewhat above those allowed (Table 1.1). This dose is $5 \times 10^{7}$ Gy for the toroidal field (TF) magnet insulation, but the peak fast neutron fluence (E > $0.1 \mathrm{MeV}$ ) to the superconductor is $10^{23} / \mathrm{m}^{2}$ [Bottura et al., 1991; Tomabechi et al., 1991]. This fluence penetrates the insulation, and from the usual fluence-to-dose conversion rule* for $\mathrm{E}>0.1 \mathrm{MeV}$, the calculated fluence of

\footnotetext{
* A commonly used conversion rule for fast neutrons incident on fiber-glass-epoxy composites is $10^{16} / \mathrm{m}^{2}=$ $1 \mathrm{~Gy}$. The actual dose-to-fluence conversion factor depends on the exact molecular formula of the substance irradiated as well as the spectrum of the irradiating species. The approximate rule quoted above is not valid for very high energy neutrons: for example, a fluence of $10^{15} / \mathrm{m}^{2}$ of $14-\mathrm{MeV}$ neutrons is equivalent to a higher dose, 3.25 Gy, in Kapton [Abe et al., 1987], and Dell and Goland [1981] calculated an increase of about 5 in the 14 -MeV displacement cross section over that for a reactor spectrum for DGEBA epoxy. (1 Gy = $1 \mathrm{~J} / \mathrm{kg}$ of absorbed energy.) See Maurer [1985], Egusa et al. [1984a; 1985a; 1987a; 1987b] and Dell and Goland [1981] for further discussion of the range of conversion factors. Also note that a lower conversion value of $10^{15} / \mathrm{m}^{2}=0.44 \mathrm{~Gy}$, published by Van Konynenburg [1981] for $\mathrm{G}-10$, was based on hand calculations for the magnet neutron spectrum of the ETF. About $60 \%$ of the flux in the ETF spectrum is above $0.17 \mathrm{MeV}$; this figure is similar to some ITER spectrum calculations [El-Guebaly, 1990]. Because the conversion factor is usually not known precisely, it would be desirable if future radiation studies reported results in terms of the fast neutron fluence. This report discusses results in terms of dose, since that has been most commonly reported, but indicates the fraction of dose or fluence from fast neutrons whenever possible.
} 


\section{III.1.4. Radialion exposure}

In the radiation environment of the winding packs of the TF magnets, the insulators and the copper and superconductors will all experience some damage. From a survey of available data at the star of the Conceptual Design Activity (CDA), it was decided that the radiacion-damage levels listed in Table III-5 could reasomably be weterated by careful design and choice of materials.

TABLE III-5. LEVEIS OF RADIATION DAMAGE TO BE TOLERATED DURING THE OPERATIONAL LIFE OF THE MAGNET SYSTEMS

Insuiator dose (Gy)

Copper darnage (Apa)

Neutron fluence $\left(\mathrm{n} / \mathrm{m}^{2}\right)$

\section{$2 \times 10^{7}$ (average) $5 \times 10^{7}$ (peak, i.e. average - over one conductor width)}

$$
5 \times 10^{-4}
$$

$1 \times 10^{23}(\mathrm{E}>0.1 \mathrm{MeV})$

SHIELDING PERFORMANCE PARAMETERS (safety factors are included)

\begin{tabular}{|l|c|c||}
\hline Nuclear response & $\begin{array}{c}\text { Design } \\
\text { limit }\end{array}$ & $\begin{array}{c}\text { Calculated } \\
\text { value }\end{array}$ \\
\hline $\begin{array}{l}\text { Total nuclear heating in the toroidal field } \\
\text { coils }(\mathrm{kW})\end{array}$ & 55 & 57 \\
\hline Peak nuclear heating in the winding pack (kW/m $)$ & 5 & 1.8 \\
\hline Peak dose to the electrical insulator (Gy) & $5 \times 10^{7}$ & $5 \times 10^{7}$ \\
\hline $\begin{array}{l}\text { Peak fast neutron fluence (E > }>1 \text { MeV) to } \\
\text { superconductor }\left(\mathrm{n} / \mathrm{m}^{2}\right)\end{array}$ & $10^{23}$ & $6 \times 10^{22}$ \\
\hline $\begin{array}{l}\text { Peak displacement in the copper stabilizer (dpa) } \\
\text { Biological dose outside the cryostat, one day } \\
\text { after shutdown }(\mu \mathrm{Sv} / \mathrm{h})\end{array}$ & $6 \times 10^{-3}$ & $2.7 \times 10^{-3}$ \\
\hline
\end{tabular}

* A total thickness of $175 \mathrm{~cm}$ is assumed for the outboard first wall, blanket, shield and cryostat. 
$6 \times 10^{22} / \mathrm{m}^{2}$ is equal to a fast neutron dose of $6 \times 10^{7} \mathrm{~Gy}$. (The insulation thickness of at least $2 \mathrm{~mm}$ is adequate for energy absorption. For recoil protons with an energy of about $0.31 \mathrm{MeV}$, Egusa et al. [1987a] calculated a range in epoxy and polyimide resins of about $4 \mu \mathrm{m}$.) This apparent contradiction in total insulation dose also appears in recent ITER shield design calculations, such as those shown in Table 1.2. Since conversion factors vary with material, it would be preferable to state radiation requirements for insulation in terms of neutron and gamma fluence and spectrum. For example, recent $4-\mathrm{K}$ tests to a total dose of $5 \times 10^{7} \mathrm{~Gy}$ had a (reactor) fast neutron fluence of only $-10^{22} / \mathrm{m}^{2}$ [Katheder et al., 1992]. A memo on ITER shield design [El-Guebaly, 1990] gave a fast neutron fluence to the insulator of about a factor of 3 times the "peak" fluence of $2.4 \mathrm{x}$ $10^{22} / \mathrm{m}^{2}$, and a memo from M. Sawan [1993] set forth a fast neutron fluence of $3.6 \times 10^{22} / \mathrm{m}^{2}$ with a harder spectrum for a recent design. Therefore, it would be conservative to reactor-test insulation to at least $5 \times 10^{23} / \mathrm{m}^{2}$ at $4 \mathrm{~K}$.

(2) Specimens would be tested at $4 \mathrm{~K}$ without warm-up, and also after warm-up to ambient temperatures, since ITER and commercial reactors may undergo about 10 warm-ups [Henning and Miller, 1989]. Estimates of the number of annealings required to maintain a low enough resistivity in the copper stabilizer for the superconductor vary with shield design [El-Guebaly and Sawan, 1989]. Loss of coolant and other scenarios may also result in warm-up.

(3) Screening tests would characterize or simulate magnet insulation operating conditions of shear load limits of about $30 \mathrm{MPa}$, with and without accompanying compressive loads limited to about $450 \mathrm{MPa}$ [Henning, 1989]. Failure would be defined to be characteristic of failure under operating conditions; that is, if microcracking would cause a serious deterioration in magnet performance, the stress on the load curve where microcracking begins would be reported, as well as the breaking stress. Since loss of adhesion of insulation to the conductor sheath alloy constitutes failure in the conceptual design, compressive/shear tests of alloy-composite specimens would be conducted in addition to interlaminar shear tests of fiber glass-reinforced epoxy. Because screening tests can be conducted only on miniaturized specimens at present [e.g., Poehlchen et al., 1990], the scaleup for design data remains to be developed. 
Table 1.2. Results of Shielding Analysis for Tungsten Shield Configuration "C". (Data from Daenner et al. [1991]).

\begin{tabular}{|c|c|c|c|c|}
\hline & & Limit & $1 \mathrm{D}$ & $3 D *$ \\
\hline \multirow{3}{*}{$\begin{array}{l}\text { Peak dose to electrical insulator } \\
\left(10^{7} \mathrm{~Gy}\right)\end{array}$} & Inboard & & 4.9 & 4.2 \\
\hline & Recess & 5.0 & 3.5 & 1.8 \\
\hline & Divertor & & 13.5 & 2.7 \\
\hline \multirow{3}{*}{$\begin{array}{l}\text { Peak displacement damage to copper } \\
\text { stabilizer }\left(10^{-3} \mathrm{dpa}\right)\end{array}$} & Inboard & & 2.7 & - \\
\hline & Recess & 6.0 & 2.1 & - \\
\hline & Divertor & & 6.0 & - \\
\hline \multirow{3}{*}{$\begin{array}{l}\text { Peak fast fluence }(\mathrm{E}>0.1 \mathrm{MeV}) \text { to } \\
\text { superconductor }\left(10^{23} \mathrm{~m}^{-2}\right)\end{array}$} & Inboard & & 0.0 & 0.6 \\
\hline & Recess & 1.0 & 0.4 & 0.3 \\
\hline & Divertor & & 1.5 & 4.4 \\
\hline \multirow{3}{*}{$\begin{array}{l}\text { Peak heat deposition in winding } \\
\text { pack }\left(\mathrm{kW} / \mathrm{m}^{3}\right) \text { during Physics Phase }\end{array}$} & Inboard & & 1.4 & 1.0 \\
\hline & Recess & 6.0 & $1 . \bullet$ & 0.5 \\
\hline & Divertor & & 6.0 & 1.0 \\
\hline \multirow{6}{*}{$\begin{array}{l}\text { Integral heat deposition in } \\
\text { toroidal field coil system (kW) } \\
\text { during Physics Phase }\end{array}$} & Inboard & & 10.0 & 13.4 \\
\hline & Recess & & 4.2 & 3.5 \\
\hline & Outboard & & negl. & - \\
\hline & Top Div. & & 28.0 & 20.7 \\
\hline & Bot. Div. & & 20.0 & 22.0 \\
\hline & Total & 55.0 & 62.2 & 59.6 \\
\hline
\end{tabular}

* 3-dimensional results have statistical error of less than $10 \%$. 
(4) Fiberglass reinforcement of specimens would consist of $B$-free $R, S$, or $T$ glass, rather than $B$-containing $E$ glass, to avoid the ${ }^{10} \mathrm{~B}+\mathrm{n} \rightarrow{ }^{7} \mathrm{Li}+\alpha$ reaction that occurs with thermal neutrons. The $1.8 \mathrm{-MeV}$ alpha particles emitted in this reaction cause intense local damage at the glass-matrix interface [Klabunde and Coltman, 1983]. Helium and tritium are produced from ${ }^{10} \mathrm{~B}$ by high energy neutrons. (E glass contains about 2 to 3 mass of $\mathrm{B}$.)

(5) The epoxy matrix of specimens would consist of resin systems suitable for vacuum impregnation, since this has been the most common fabrication process for magnets insulated with an epoxy composite. This implies low resin viscosity near ambient temperature, a long pot life $(-24 \mathrm{~h})$, low surface tension, low shrinkage after solidification, and manageable hazards to workers under field conditions.

In contrast to this ideal data base, the actual data base, with a few exceptions, has the following characteristics:

(1) The most extensive irradiation studies, in which resin systems, hardeners, and other additives such as accelerators and flexibilizers were varied systematically, were carried out at ambient temperatures, usually with gamma or predominantly gamma radiation, and never with a fluence of fast neutrons $\left(\mathrm{E}>0.1 \mathrm{MeV}\right.$ ) of $7 \times 10^{22} / \mathrm{m}^{2}$ to $10^{23} / \mathrm{m}^{2}$ as allowed in the ITER conceptual design [e.g., Tomabechi et al., 1991; El-Guebaly, 1990]. Two experiments on polyimide resins suggest that gamma (or electron) doses may have to be downgraded by a factor of about 10 to simulate damage by high energy neutrons $(\$ 1.3 .2 .5)$. Irradiation data at $4 \mathrm{~K}$ that systematically compare resin and reinforcement systems are very scarce, and the 4-K irradiations do not meet the spectrum and dose requirements at the ITER TF coils. Furthermore, dose rate effects have not been investigated, although the total dose will accumulate from pulsed operations over the lifetime of ITER. Banford [1984] has suggested that since much of the ambient-temperature irradiation was carried out at a high dose rate $\left(-10^{4}-10^{5} \mathrm{~Gy} / \mathrm{h}\right)$, radiation resistance may appear higher than that which would be exhibited at a lower dose rate with more time for degradative chemical reactions to occur.

(2) Only limited testing has been carried out at $4 \mathrm{~K}$, and, as the review in $\$ 1.4 .2 .3$ below shows, experiments to test the effect of warm-up after high 
dose neutron irradiation have been done mainly with E-glass composites, so results were confounded by the alpha-particle damage that would not occur with B-free glass reinforcement. Also, tests have not been conducted on confined systems.

(3) The most common test has been the three-point bend test with a span length/specimen thickness ratio $>5$, so that flexure, including tensile and compressive properties, rather than interlaminar shear properties, was primarily measured [Reed et al., 1992; Becker, 1990; Nishijima et al., 1991]. Pure tension tests have also been done. Details of the load curves of shear, flexure, and tensile tests have rarely been reported; in one case, small load drops may have indicated microcracking, or may have resulted from other factors [Chabert and Marcou, 1989]. These features of the load curve were not presented in the first published account of the work [Poehlchen et al., 1990]. More recently, however, Katheder et al. [1992] reported that these load drops arose from discontinuous yielding of the stainless steel specimen fixture.

No cryogenic shear/compression tests have been carried out after specimen irradiation, although some ambient temperature tests have been conducted [Nishijima et al., 1990; McManamy et al., 1990; 1991a]. Such tests are now in a developmental stage [Okada and Nishijima, 1990; McManamy et al., 1990, 1991a; Simon et al., 1992; Drexler et al. 1992], and cryogenic irradiations of suitable specimens are planned. Scaling up of the results on miniaturized specimens has not yet been carried out; encouraging preliminary results by McManamy and Snook [1989] were limited to the case of shear strength accompanied by a 350-MPa compressive load. A small number of shear tests of bond adhesion have been carried out (\$11) but on a very limited number of resin and reinforcement systems, and usually at doses below ITER requirements. For example, Poehlchen et al. [1990] reported promising data obtained after a dose of $10^{7} \mathrm{~Gy}$, but many epoxy resin systems do not exhibit pronounced degradation until doses above $10^{7} \mathrm{~Gy}$. In all of these investigations, specimens were subjected to shear/compressive stresses only after irradiation; facilities to apply such stresses during irradiation have not yet been developed.

(4) Only a very limited amount of data is available for epoxy composites reinforced with $\mathrm{B}$-free glass. In some cases, since the data were obtained 
with gamma or electron radiation, the use of B-containing $E$ glass should not affect the results significantly. However, since the ${ }^{10} \mathrm{~B}+\mathrm{n} \rightarrow{ }^{7} \mathrm{Li}+\alpha$ reaction is so deleterious to composite strength, as Figure 1.1 of okada et al. [1992] indicates, much of the data obtained with neutron irradiation is not useful for a facility such as ITER in which B-free glass will be employed. This consideration limits the usefulness of much of the data reported from CERN (European Organization for Nuclear Research). For example, Schönbacher and Izacka-Stolarz [1979] obtained data with reactor radiation in which about 5\% of the dose was from fast neutrons and thermal neutrons were also present, but only E glass was used as a reinforcement. Most of the U.S. cryogenic irradiation data were also obtained on E-glass composites [e.g., Coltman and Klabunde, 1983; Tucker et al., 1985].

(5) Much of the data have been obtained with epoxy resins that are not suitable for vacuum impregnation of magnets, owing to the high viscosity of the resin. For example, the resin TGDM (tetraglycidyl diminodiphenyl methane or $\mathrm{N}, \mathrm{N}^{\prime}$-tetraepoxypropyl-4,4'-methylene-dianiline) is too viscous when employed with the usual aromatic hardeners. Also, the pot life of TGDM with aromatic hardners is usually too short for use in large-scale vacuum impregnation systems. Yet a large number of systematic tests were carried out at low temperatures with prepreg specimens of TGDM (also abbreviated as TGDDM) cured with aromatics [Takeda et a1., 1985; Egusa et a1., 1988; Egusa, 1988, 1990a, 1991a, 1991b]. The resin DGEBA (diglycidyl ether of bisphenol A) is normally less viscous and more suited for vacuum impregnation but fewer highdose, low temperature irradiation and test data are available for this resin. Furthermore, Pöhlchen [1992] has suggested that irradiation testing be carried out only on resins that have successfully been used by magnet manufacturers to impregnate large coils without excessive porosity or cracking. Only a small fraction of the data base pertains to such resins: for example, ambient data on magnet resins are presented in $\$ 2.10$ and cryogenic data on adhesively bonded magnet resins are discussed in $\$ 11$.

Thus, there is a large disparity between the ITER requirements as presented in the conceptual design documents and the data available on irradiation of epoxy insulations, although alternative ITER magnet designs now under consideration may ameliorate some aspects of this situation, such as radiation dose. The 


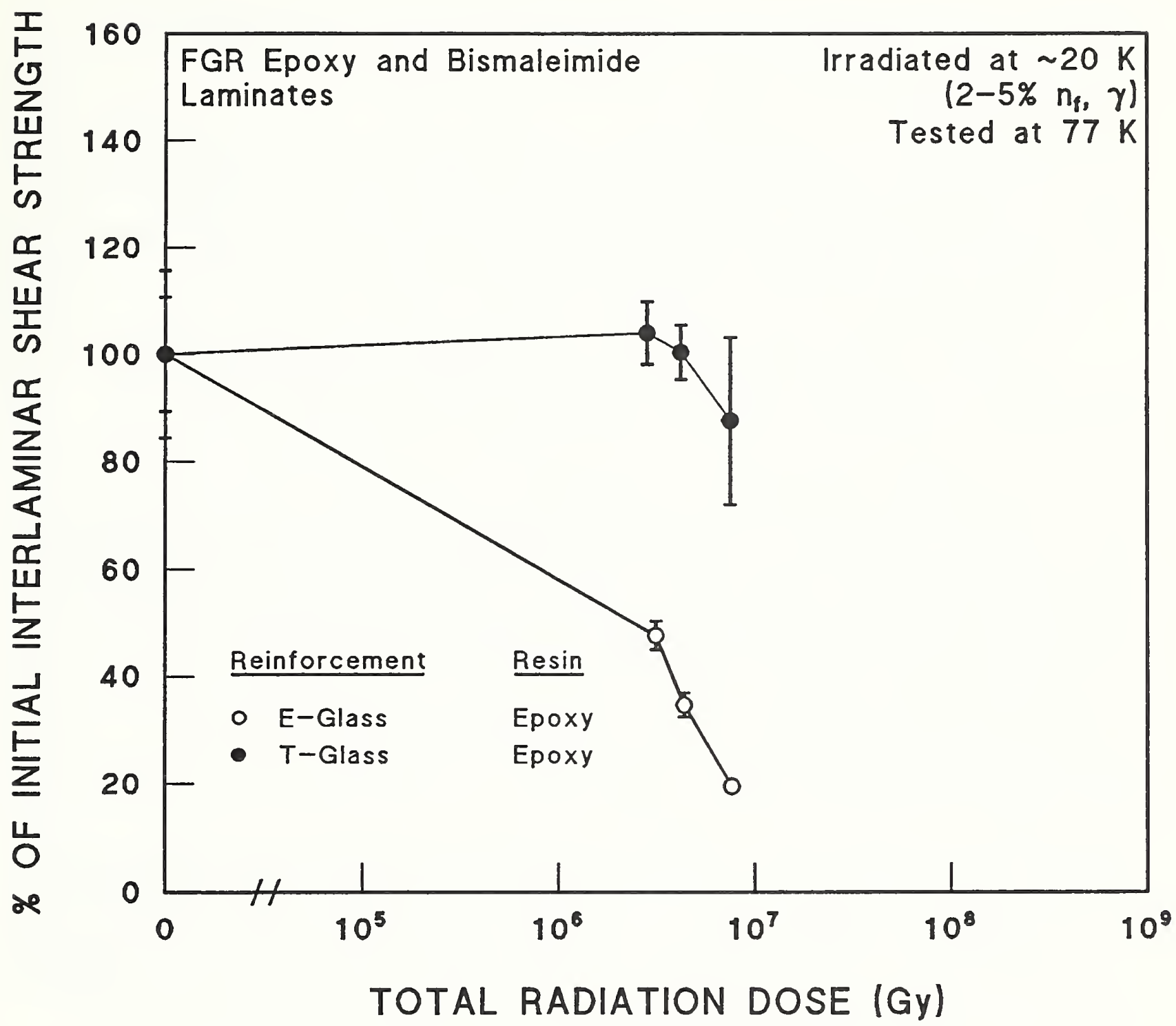

\begin{tabular}{lccc} 
"O" Strength, MPa & Glass Vol. Fr. & Supplier \\
\hline 0 & $120.6 \pm 18.9$ & $50 \%$ & -- \\
$-142.7 \pm 16.3$ & $50 \%$ & --
\end{tabular}

Comments: Identical fabric type, glass surface finish and epoxy matrix for E-Glass and T-Glass laminates.

Figure 1.1. A comparison of degradation under neutron irradiation of the interlaminar shear strength of a resin reinforced with either $\mathrm{E}$ glass or $\mathrm{T}$ glass. The fabric type and finish and epoxy matrix were identical. Data from Okada et al. [1992]. (Supplementary Table A.7-1.) 
objective of the balance of this introductory section is to discuss the following questions related to assessment of the database:

(1) Are results obtained with lower energy, fission neutrons applicable to insulation in TF coils, where a significant dose ( $\left.10^{8} \mathrm{~Gy}\right)$ arises from fast neutrons $(E>0.1 \mathrm{MeV})$ and a portion of this dose is from very high energy neutrons $(\mathrm{E}>5 \mathrm{MeV})$ ? $(\S 1.3 .1 .1)$

(2) Can gamma radiation simulate or be substituted for high-dose neutron radiation? Can electron radiation simulate gamma radiation? (\$1.3.1.2, $1.3 .1 .3,1.3 .2$ )

(3) Is it conservative to use results of ambient irradiation in an oxygencontaining atmosphere for design, or does cryogenic irradiation cause greater degradation even though oxygen is not present, because annealing and gas formation and diffusion cannot occur? (\$1.3.3)

(4) Can tests at 295 or $77 \mathrm{~K}$ be used to predict 4-K post-irradiation properties? (\$1.4.1)

(5) Will periodic warm-up to ambient temperatures anneal irradiation damage in insulation, as is known to occur for the defects in the copper stabilizer of the conductor, or will explosive release of gas, energy from free-radical reactions, stored energy from defects, or other detrimental effects occur? $(\S 1.4 .2)$

(6) Should new tests be used to assess the inter-dependence of shear and compressive properties of irradiated insulation and the adhesion of insulation to the conductor sheath alloy? ( $\$ 1.5)$

\subsection{EFFECT OF IRRADIATION SPECTRA, SPECIES, AND TEMPERATURE}

A mismatch exists between the irradiation used to obtain most of the data and the irradiation expected at TF magnets in fusion reactors in regard to three significant parameters: (1) the energy spectrum, (2) the species of irradiation, and (3) the temperature of irradiation. Clearly, the damage inflicted by more energetic neutrons cannot easily be extrapolated from tests 
with neutrons of lower energy, so the energy spectrum is an important parameter with regard to neutrons, although it is less significant for gamma rays ( $\$ 1.3 .1 .3)$. As discussed further below ( $\$ 1.3 .2)$, some types of damage inflicted by neutrons are expected to be different from the ionization damage caused by gamma rays and electrons. Thus, the data obtained with gamma rays and electrons may not be useful in predicting high energy neutron damage, although irradiation with gamma rays and electrons comprises much of the data base. (Gamma rays make up part of the irradiation dose at a fusion magnet.)

The temperature of irradiation may affect the nature and extent of damage, although data on this point are scarce, as will be seen below (\$1.3.3). Since a major component of organic insulator damage is chemical in nature, resulting from both ionization and the creation of free radicals, the availability of oxygen should influence the extent of damage ( $\$ 1.3 .3,2.5,5)$. Oxygen diffuses into the composite during irradiation at ambient temperatures, unless an inert atmosphere is provided, but is absent in an environment of liquid helium or liquid nitrogen. Furthermore, there is a delay in the production and release of gases generated during irradiation at low temperatures because diffusion is nearly nonexistent. Gases may be explosively released upon warmup after cryogenic irradiation, causing cracking, but may diffuse away without causing as much damage during irradiation at room temperature. These three factors of spectrum, species, and temperature are discussed below in more detail.

\subsubsection{Energy Spectrum}

\subsubsection{Neutron Spectrum}

Before irradiation spectra are compared to the spectra expected in a fusion device, the procedures and assumptions used to predict the fusion spectra will be discussed. Figure 1.2 shows the neutron spectra calculated for the magnet location for two older fusion reactor designs, STARFIRE, a commercial model, and MARS (Mirror Advanced Reactor Study) [Hahn et al., 1986]. (STARFIRE is a water-cooled, solid breeder $\left(\mathrm{LiAlO}_{2}\right)$ tokamak; MARS is a lead-lithium-cooled mirror machine.) The spectra shown are the result of calculations for the least amount of shielding between the plasma and the superconductor, a worst case. The units of the $y$ axis are flux per unit lethargy, or $d \phi / d(\operatorname{lnE})$, where 


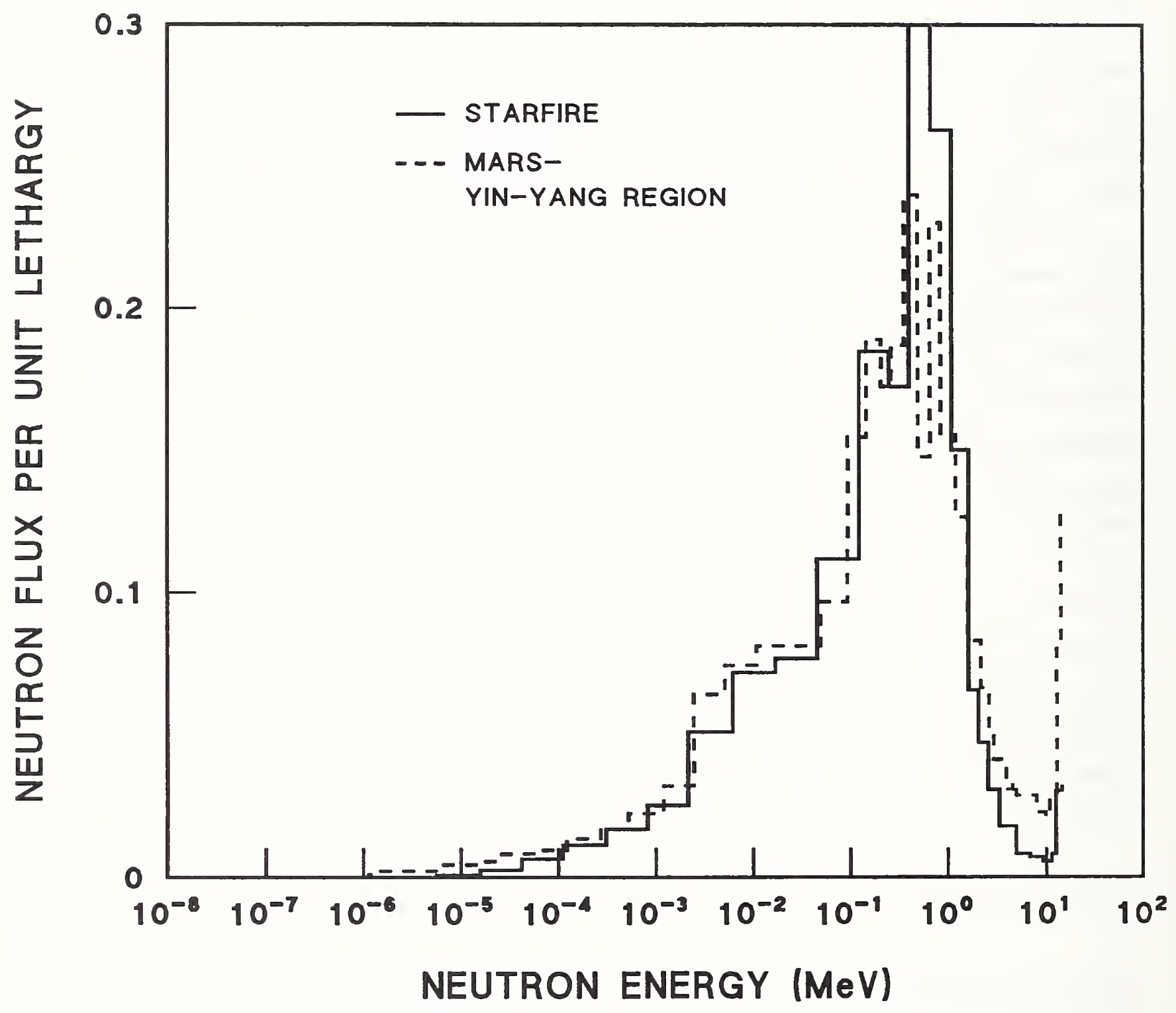

Figure 1.2. Calculated neutron spectra, at the toroidal field magnet, for two fusion reactor designs. Data from Hahn et al. [1986]. 
$\phi$ is flux (units of incident particles per area per unit time) and $\mathrm{E}$ is energy. In this normalized plot, the area under a section of the spectrum is directly proportional to the fraction of neutrons within the energy range contained in the section. Figure 1.3 is a similar spectrum plot for the ITER device, based on a shield design with stainless steel, water, and $\mathrm{B}_{4} \mathrm{C}$ [Maki et al., 1991]. The figure shows the results of two calculations based on different nuclear data libraries. The differences between the spectra for the two sets of nuclear data libraries are generally small above $1 \mathrm{keV}$. In the ITER conceptual design, the shielding consists of a stainless steel/water combination backed by a thin layer of $\mathrm{Pb} / \mathrm{B}_{4} \mathrm{C}$. The $300-\mathrm{mm}$, stainless-steel wall of the vacuum vessel is part of the shielding configuration [Tomabechi et al., 1991]. The two spectra shown in Figure 1.3 are based on calculations for stainless steel and water with additional $\mathrm{B}_{4} \mathrm{C}$, but not $\mathrm{Pb}$. However, additional data presented by Maki et al. indicate that the high energy part of the spectrum is similar for a shield containing $\mathrm{Pb}$ instead of $\mathrm{B}_{4} \mathrm{C}$. In any event, the $\mathrm{Pb}$ and $\mathrm{B}_{4} \mathrm{C}$ layer, and some of the stainless steel may be replaced by up to $200 \mathrm{~mm}$ of $\mathrm{W}$ to bring down the total neutron fluence to the superconductor and the nuclear heat deposited in the TF coil to the limits of $1 \times 10^{23} / \mathrm{m}^{2}(\mathrm{E}>0.1 \mathrm{MeV})$ and $5 \times 10^{7} \mathrm{~Gy}$, respectively [Daenner et al., 1991]. Furthermore, the calculations of energy spectrum, total fluence, and dose are always based upon somewhat idealized structural models, and often are 1- or 2dimensional rather than 3-dimensional calculations. Uncertainties in the nuclear database contribute to a basic uncertainty in the results and safety factors of about 1.5 to 3 are commonly applied to the predicted fluences and dose levels [Daenner et al., 1991]. Also, variations in the blanket/shield configurations of ITER lead to variations in the results. For comparison, Figure 1.4 shows the results of a recent calculation of a harder ITER magnet spectrum with $\mathrm{H}_{2} \mathrm{O}$ replaced by alloys in the blanket/shield. Shielding and blanket designs and methods of calculation of radiation fluence continue to be refined. Still, some general conclusions can be drawn when these predicted spectra are compared with the irradiation test spectra.

Figure 1.5 of Hahn et al. [1986] shows the normalized spectra of several neutron sources typical of those commonly used for irradiation testing. One source, TRIGA, is a fission reactor with about $39 \%$ of the neutron flux density at an energy above $0.1 \mathrm{MeV}$. (This percentage may vary somewhat with specimen 


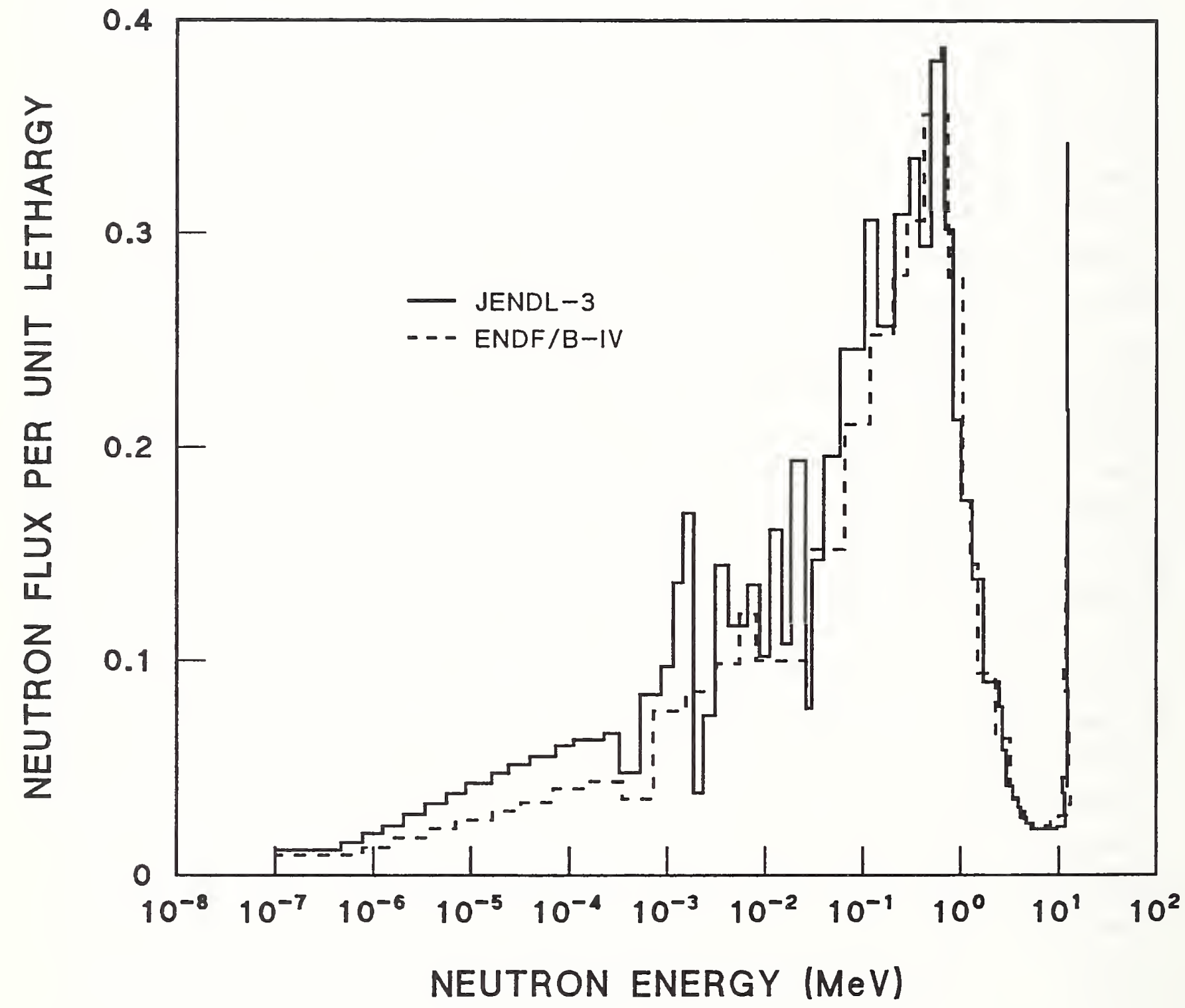

Figure 1.3. Neutron spectra in the winding pack of ITER for two calculations based on different nuclear data libraries. Data from Maki et al. [1991]. 


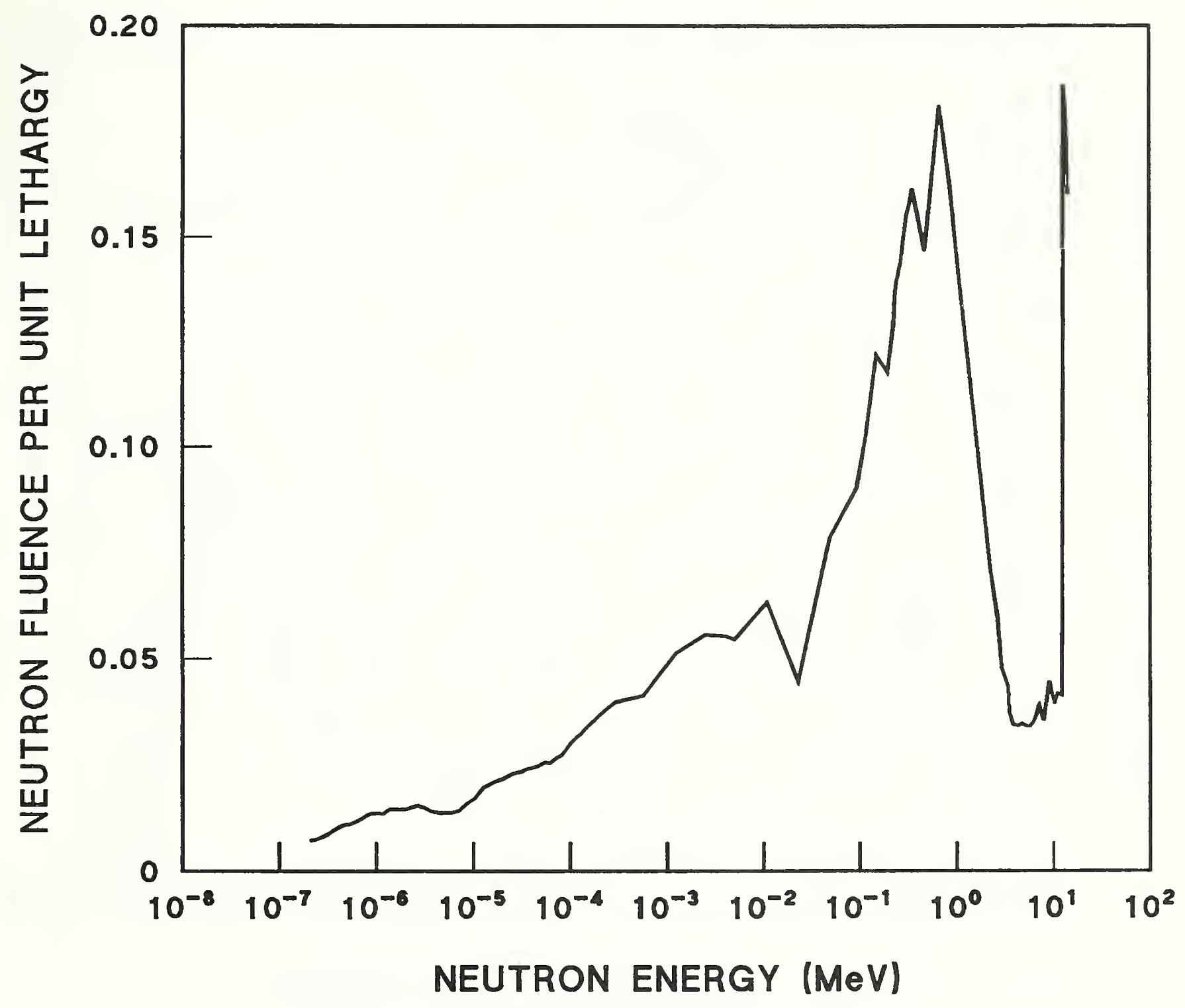

Figure 1.4. A normalized plot of a calculation of the ITER neutron spectrum at the front layer of the insulator of the TF magnet. These results pertain to a total thickness of $1 \mathrm{~m}$ between plasma and magnet, consisting of a $650-\mathrm{mm}$ blanket/shield (50\% ferritic steel/50\% Li) and a $350-\mathrm{mm}$ double-wall Inconel vacuum vessel with WC balls, water-cooled. Data from Sawan [1993]. 


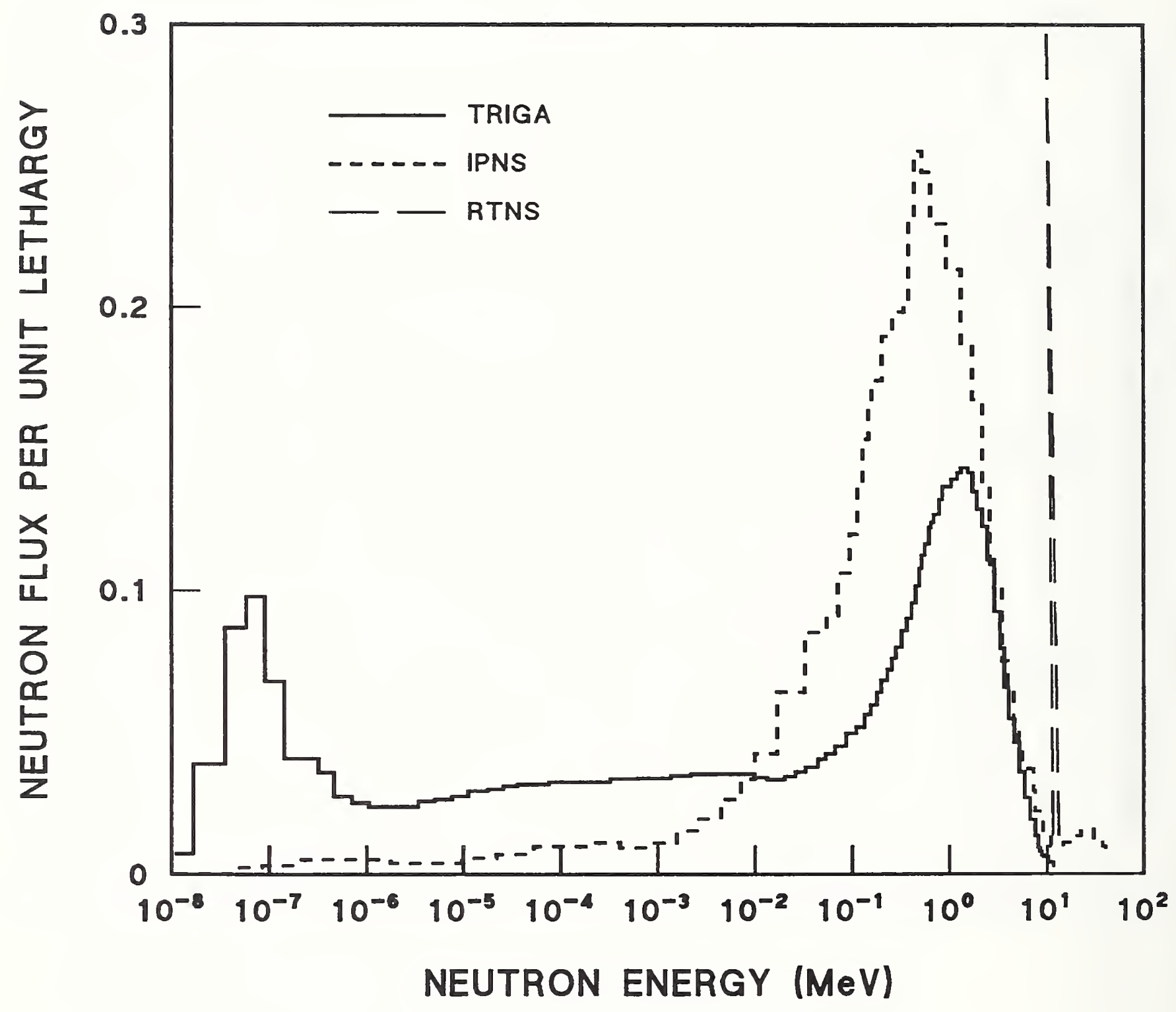

Figure 1.5. Normalized neutron irradiation spectra from three irradiation sources. The RTNS line extends above the scale. Data from Hahn et al. [1986]. 
location in the reactor.) Most neutron irradiation of insulation has been done in fission reactors. The IPNS (Intense Pulsed Neutron Source), located at Argonne National Laboratory, produced neutrons from a proton accelerator by spallation from a ${ }^{238} \mathrm{U}$ target [Birtcher et al., 1982]. In contrast to the TRIGA fission reactor, about 718 of the IPNS neutron flux had an energy above $0.1 \mathrm{MeV}$, and a comparison of Figure 1.5 with Figures 1.2 to 1.4 shows that the IPNS spectrum approximated the fusion spectrum more closely than the TRIGA fission spectrum. The RTNS (Rotating Target Neutron Source), located at Lawrence Livermore National Laboratory, produced a monoenergetic beam of 14$\mathrm{MeV}$ neutrons. Calculations from data given in Figures 1.2 to 1.4 show that about 50 to 638 of the fusion neutron spectrum has an energy above $0.1 \mathrm{MeV}$; hence, the IPNS, which had cryogenic irradiation capabilities, also had a spectrum closer to that projected for a fusion TF magnet. The IPNS, along with the RTNS, is no longer operational, but it was used for some of the data on epoxy composites obtained by Hurley et al. [1978; 1983], by Egusa et al. [1987a,b], and by others (see §8). Figure 1.6 highlights the difference between the IPNS spectrum and a fission spectrum. Figure 1.7a of Abdou [1978] is a comparison of the neutron spectrum from the Argonne National Laboratory Low Temperature Fast Flux Facility for $\mathrm{E}>0.08 \mathrm{MeV}$ with a typical calculated tokamak fusion spectrum at the TF coil. This shows a fairly close correspondence, except above $5 \mathrm{MeV}$. Note that the very high energy region of this spectrum agrees approximately with recent calculations for ITER, shown in Figure 1.7b. Figures 1.8a and 1.8b, from Söll [1978] and Ullmaier [1976], respectively, present similar calculations of the neutron spectrum at the magnet position of a fusion reactor in comparison to the spectrum from the research reactor in Garching-at-Munich. However, the calculated spectra do not represent recent reactor designs, and Figures 1.2 to 1.4 show that the high energy parts of the magnet spectra of the older reactor designs and the more recent ITER design are somewhat different. These figures are of interest because the Garching reactor is now the chief neutron source available with irradiation capability at $4 \mathrm{~K}$.

The very high energy spectrum (above about $5 \mathrm{MeV}$ ) requires further discussion. Abdou [1978] noted that his results (Figure 1.7a) showed that a typical fission spectrum can simulate a fusion spectrum well for energies below about $5 \mathrm{MeV}$. In fact, the fraction of neutrons above $0.1 \mathrm{MeV}$ for the fission spectrum is about 908 , whereas this fraction is about 608 for a tokamak 


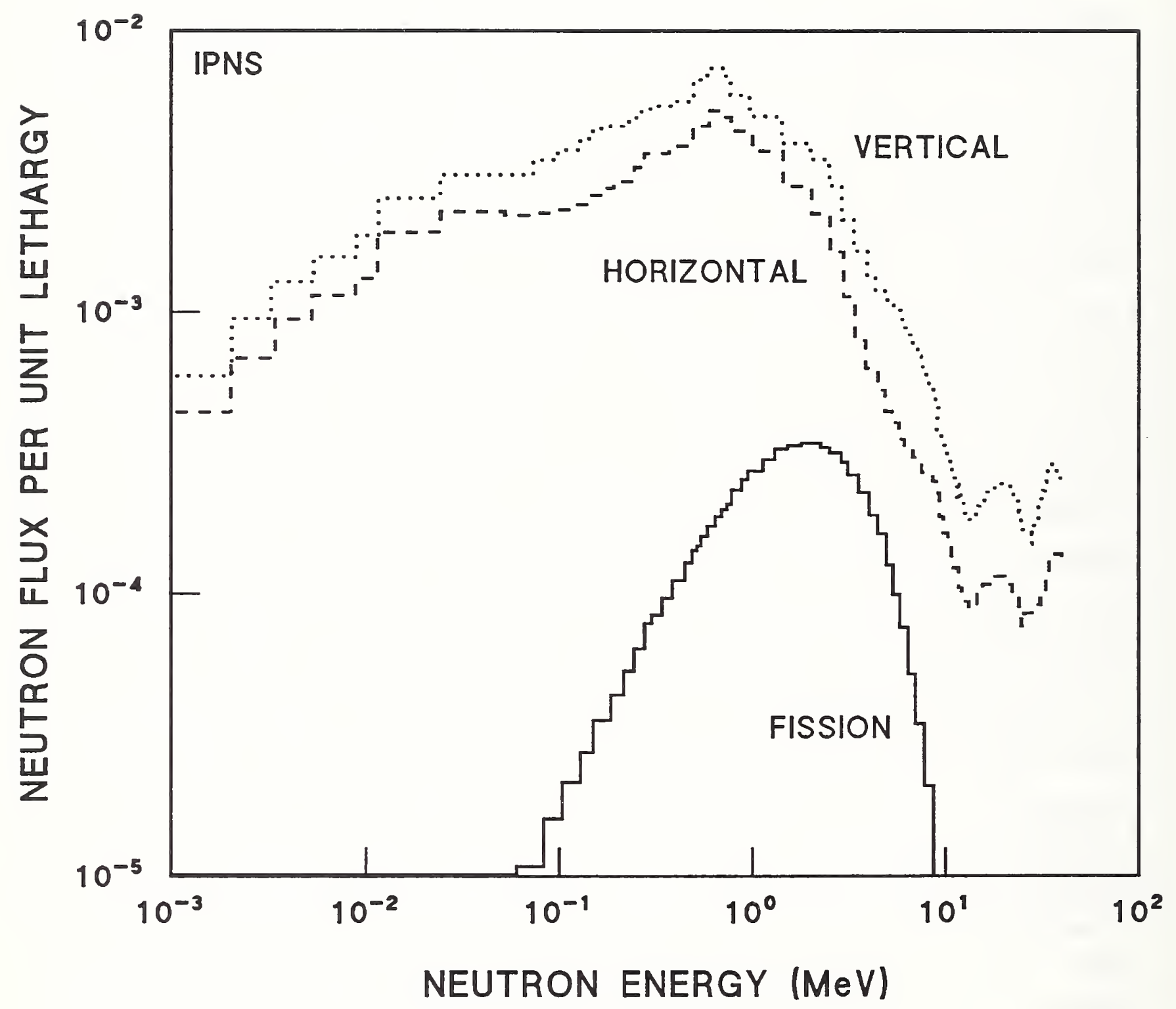

Figure 1.6. Comparison of the neutron spectra produced in the IPNS source at two positions with a pure fission neutron spectrum. Data from Birtcher et al. [1982]. 


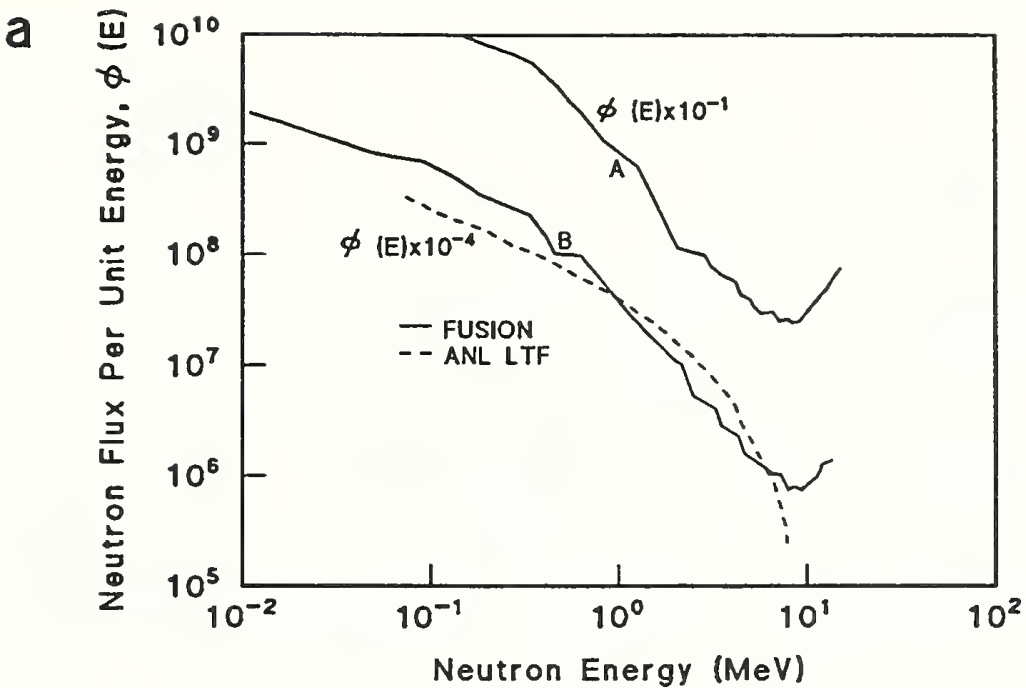

b

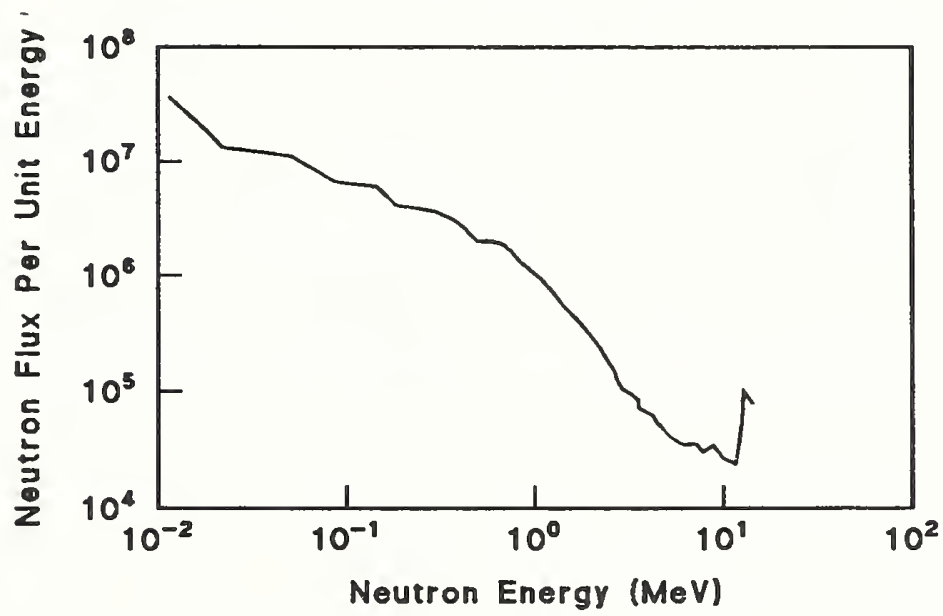

C

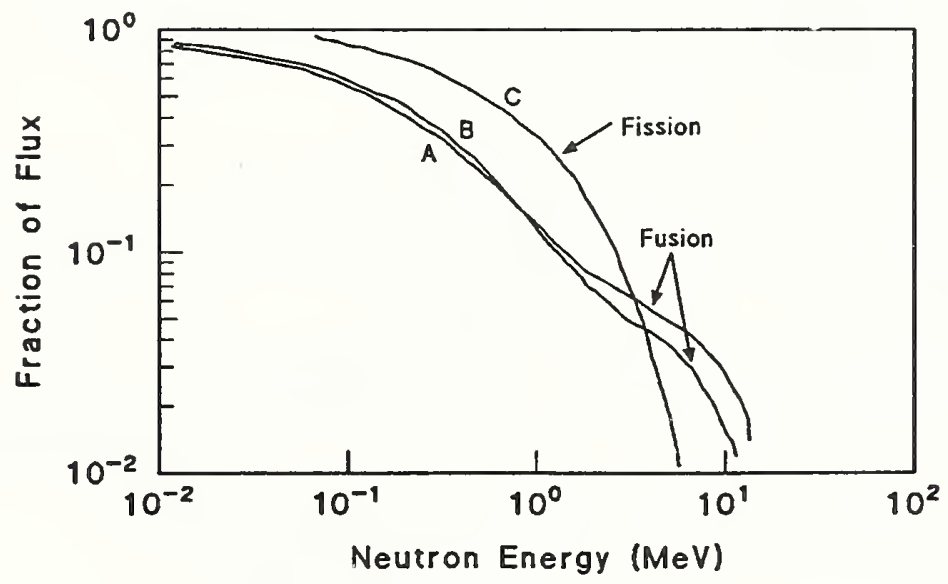

Figure 1.7. (a) Comparison of a calculated fusion spectrum at the TF magnet (inner blanket/shield of stainless steel and $\mathrm{BC}_{4}$ ) at two locations, $\mathrm{A}$ and $\mathrm{B}$, with the neutron spectrum produced in the Argonne National Laboratory Low Temperature Facility. Data from Abdou [1978]. (b) A calculated ITER spectrum at the TF magnet. Data from Sawan [1993]. (c) Fraction of flux above neutron energy [Abdou, 1978]. 
a

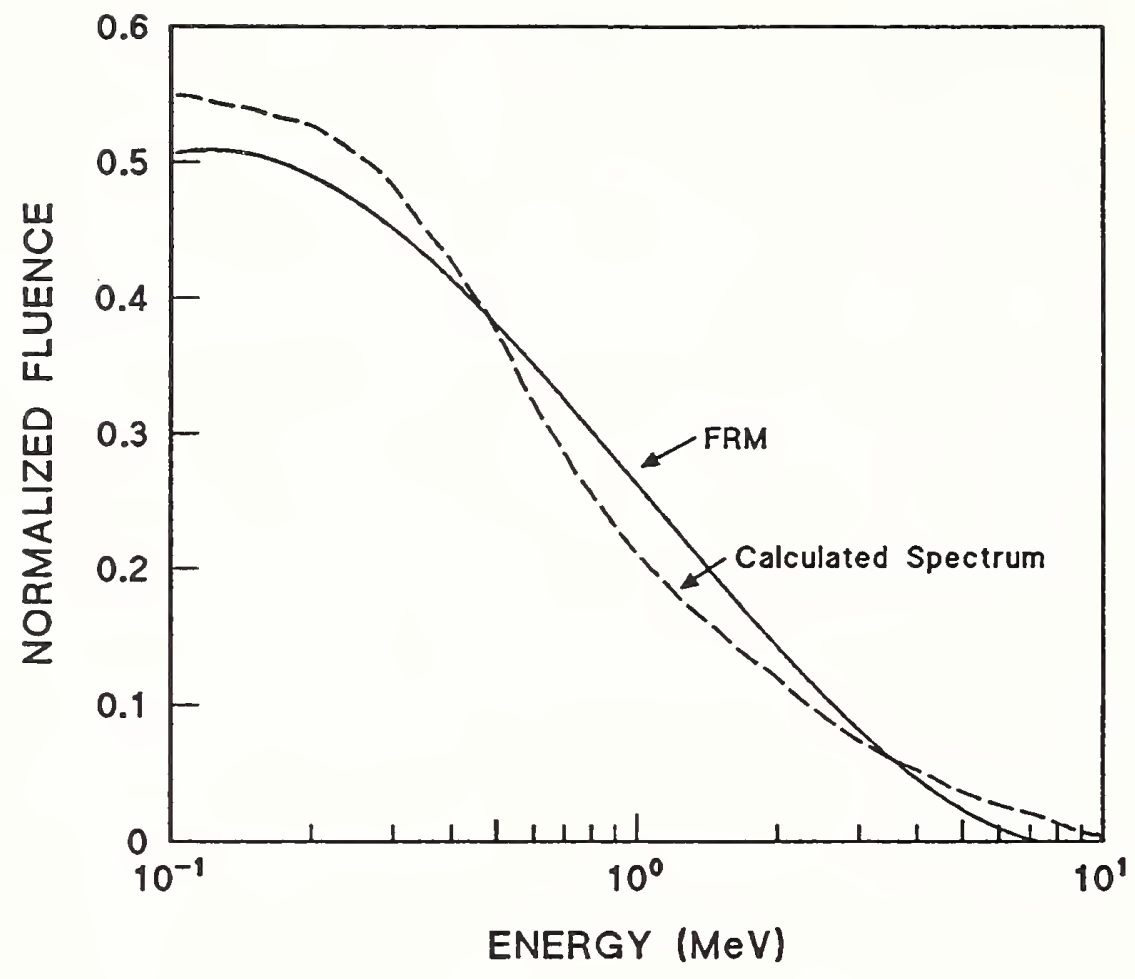

b

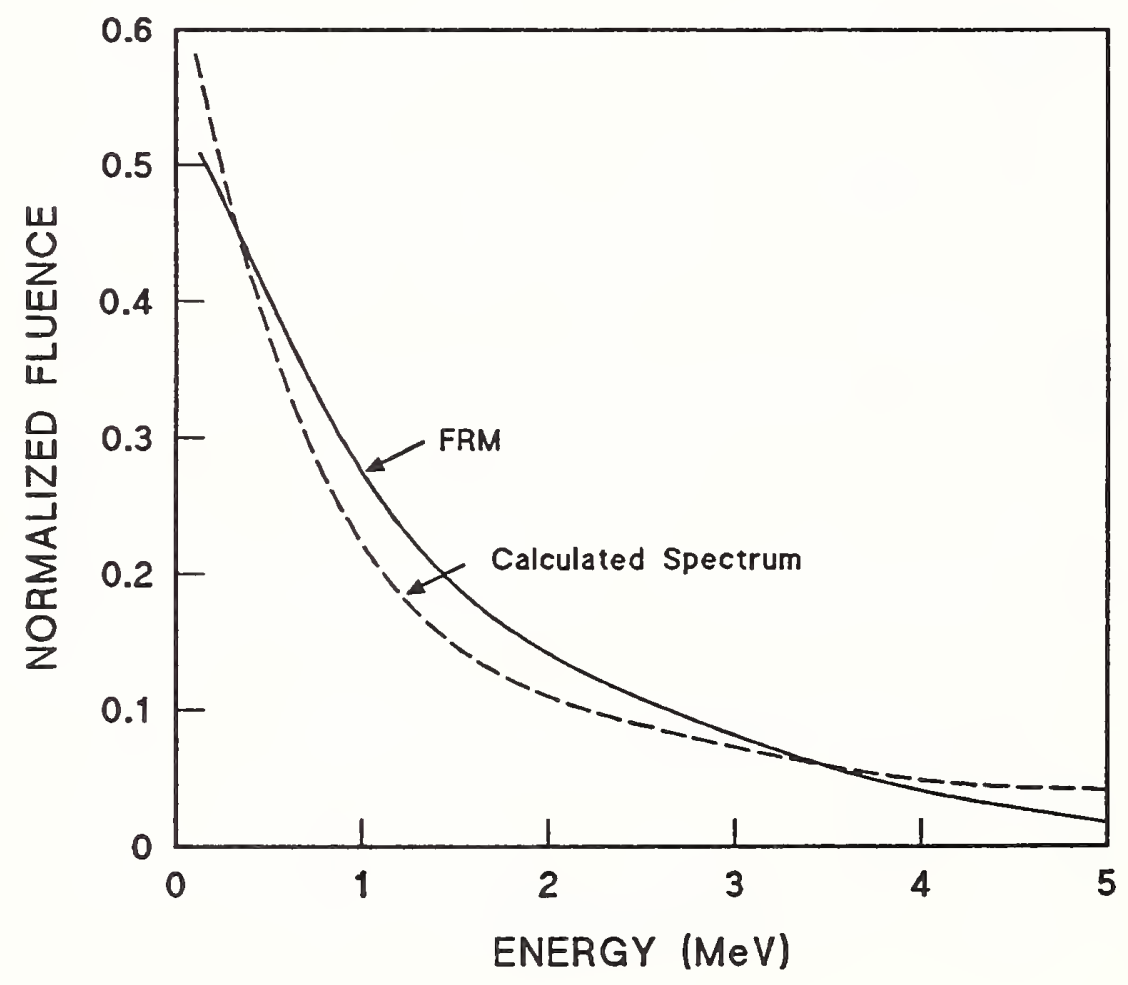

Figure 1.8. (a) Comparison of a calculated fusion spectrum with the neutron spectrum produced in the Garching FRM reactor. Data from Söll [1978]. (b) Comparison of a calculated fusion spectrum at the magnet with the neutron spectrum produced in the Garching FRM reactor. Data from U1lmaier [1976]. 
reactor magnet, as shown in Figure 1.7.c. (Hahn et al. [1986] estimated these fractions as 398 and $55 \%$ respectively.) But only about $1.5 \%$ of the fission neutron spectrum is above $5 \mathrm{MeV}$, and there are essentially no neutrons with energies above $8 \mathrm{MeV}$. According to Abdou, about $5 \%$ of the neutrons incident upon a TF magnet would have an energy above $5 \mathrm{MeV}$. Furthermore, several types of reactions in magnet materials, $(n, \alpha),(n, p)$, and $\left(n, n^{\prime} p\right)$, occur only at these high energies. Also, the recoil energies of the atoms impacted in these reactions are higher than those of atoms impacted in lower-energy reactions. Since both the types of reactions and the recoil energies are different, there may be fundamental differences between the type of damage produced by high and low energy neutrons. Thus, when insulation materials are tested in fission reactors, the total neutron fluence measured may not be equated to the expected total fluence at magnet locations of a fusion reactor such as ITER. In metals, the correlation of damage in tests with a known spectrum with expected fusion reactor damage is relatively well established. The displacements-per-atom (dpa) parameter can be used to make this correlation. At present, no correlation parameter has been developed for organic insulation, because most damage is either chemical in nature or involves the matrix-reinforcement interface. Only a limited number of insulation irradiations have been carried out with very high-energy neutrons. These irradiations (with IPNS and RTNS) are reviewed in $\$ 2.6$ and $\$ 3$; however, no comparisons have been made with irradiations of the same materials at lower neutron energies.

The problem of the very high energy neutron dose may be less severe if shielding is increased, because this will lower the fluence of neutrons at higher energies. However, the total blanket and shield thickness of stainless steel and $\mathrm{B}_{4} \mathrm{C}$ used to generate the predicted fusion curve in Figure $1.7 \mathrm{a}$ is $1 \mathrm{~m}$ (actual material thickness of about $0.87 \mathrm{~m}$ ), and the total corresponding material thickness in a typical ITER configuration (including high-atomic number first wall components) is comparable, about $0.9 \mathrm{~m}$ (Daenner et al., 1991; Maki et al., 1991]. As Figure 1.7b indicates, a significant fluence of very high energy neutrons is also expected in ITER TF magnets. Recent changes in blanket/shield materials during the EDA (Engineering Design Activity) of ITER have increased the fraction of the neutron fluence above $5 \mathrm{MeV}$ to about 78 [Sawan, 1993]. 
The literature has little discussion of the expected gamma ray fluence in tokamak reactors and few published examples of the expected spectra. Figure 1.9 is a normalized plot from tabular results presented by Van Konynenburg [1981] of the calculated gamma spectrum (in flux units) at the inboard leg of the ETF (Engineering Test Facility) toroidal coil. The shield, of stainless steel, boron, and water, was 0.82-m thick. Recent models based on ITER conceptual design parameters give a spectrum with a flatter appearance between 1 and $8 \mathrm{MeV}$, as shown in Figure 1.10 [Maki et al., 1991]. See also Figure 1.11 [E1-Guebaly, 1990]. Figure 1.10 shows the results of calculations with two different sets of nuclear data for a stainless steel/water $/ \mathrm{B}_{4} \mathrm{C}$ shield. Figures 1.9 to 1.11 also indicate the mean energy of ${ }^{60} \mathrm{Co}$, a gamma irradiation source commonly used for testing. However, some gamma irradiation test data have been obtained from fission reactor or spent fuel exposures; these sources produce a gamma spectrum with a range of energies rather than the discrete values of ${ }^{60} \mathrm{Co}$ sources. As discussed in $\$ 1.3 .2 .2$ below, the energy of an incident gamma ray is converted into the kinetic energy of a recoil electron through the Compton effect. The participating ion does not experience significant recoil, in contrast to the knocked-on atoms impacted in neutron irradiation. The recoil electron produced in the initial reaction event causes further ionization and excitation until all the energy is dissipated through a cascade of such events. Therefore, in contrast to damage from neutrons, gamma-ray damage is expected to be less dependent on the initial energy, and gamma irradiation doses can be equated to gamma fusion doses with higher reliability.

\subsubsection{Radiation Species}

\subsubsection{Relative Neutron-Gamma Fluence}

Figure 1.12a, from Abdou [1978], shows both the neutron and gamma ray fluxes as a function of depth at the inboard side of a toroidal magnet and Figure $1.12 \mathrm{~b}$ shows the radiation dose in epoxy insulators as a function of depth in the magnet. The neutron flux or dose is about an order of magnitude higher than the gamma flux or dose. Relative values of these fluxes and doses depend on the composition and thickness of material between the plasma and the 


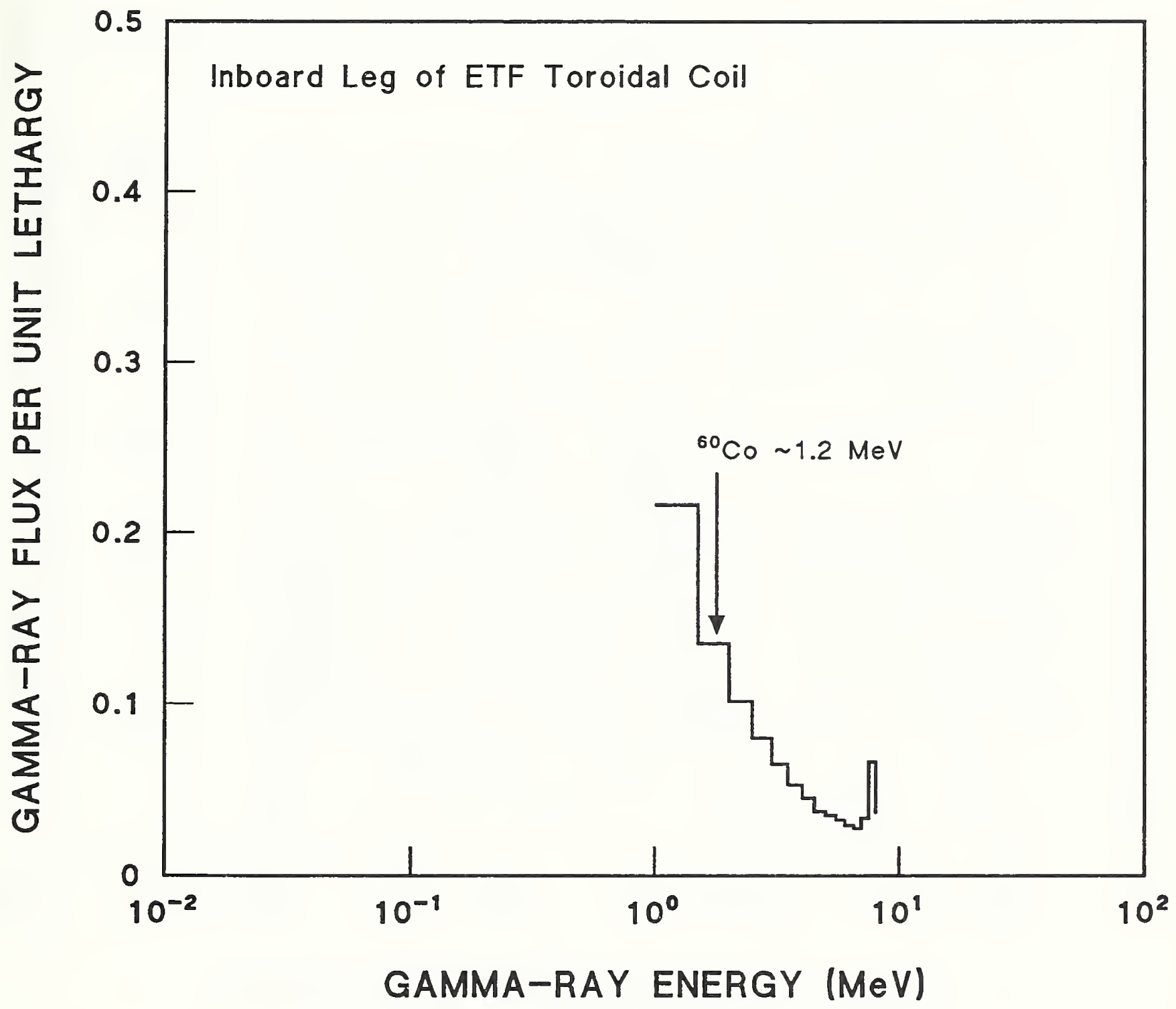

Figure 1.9. A plot of the calculated gamma spectrum at the inboard leg of the ETF toroidal coil. Tabulated data from Van Konynenburg [1981]. 


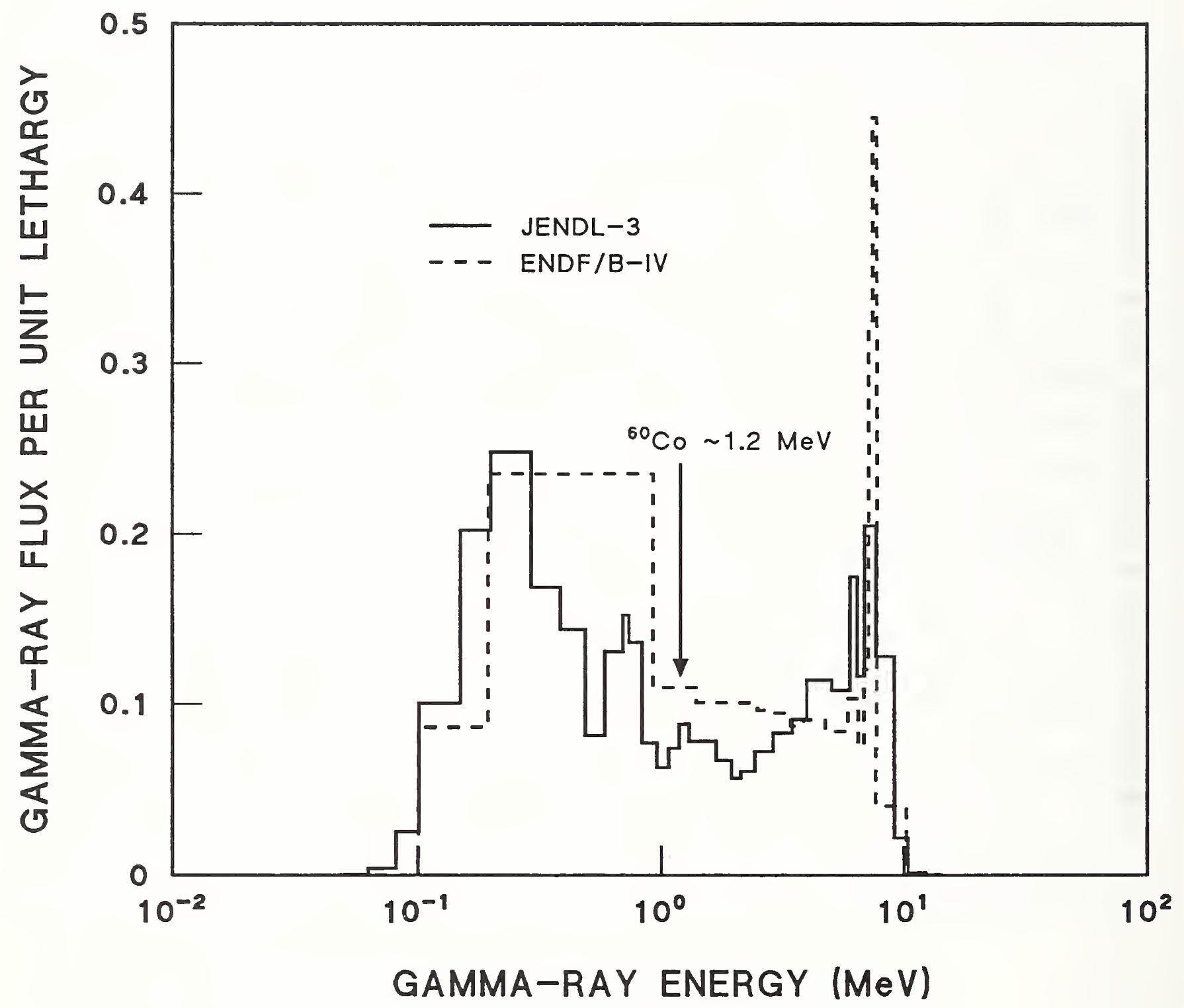

Figure 1.10. Calculations of the gamma spectrum in the winding pack of ITER based on two nuclear data libraries. Data from Maki et al. [1991]. 


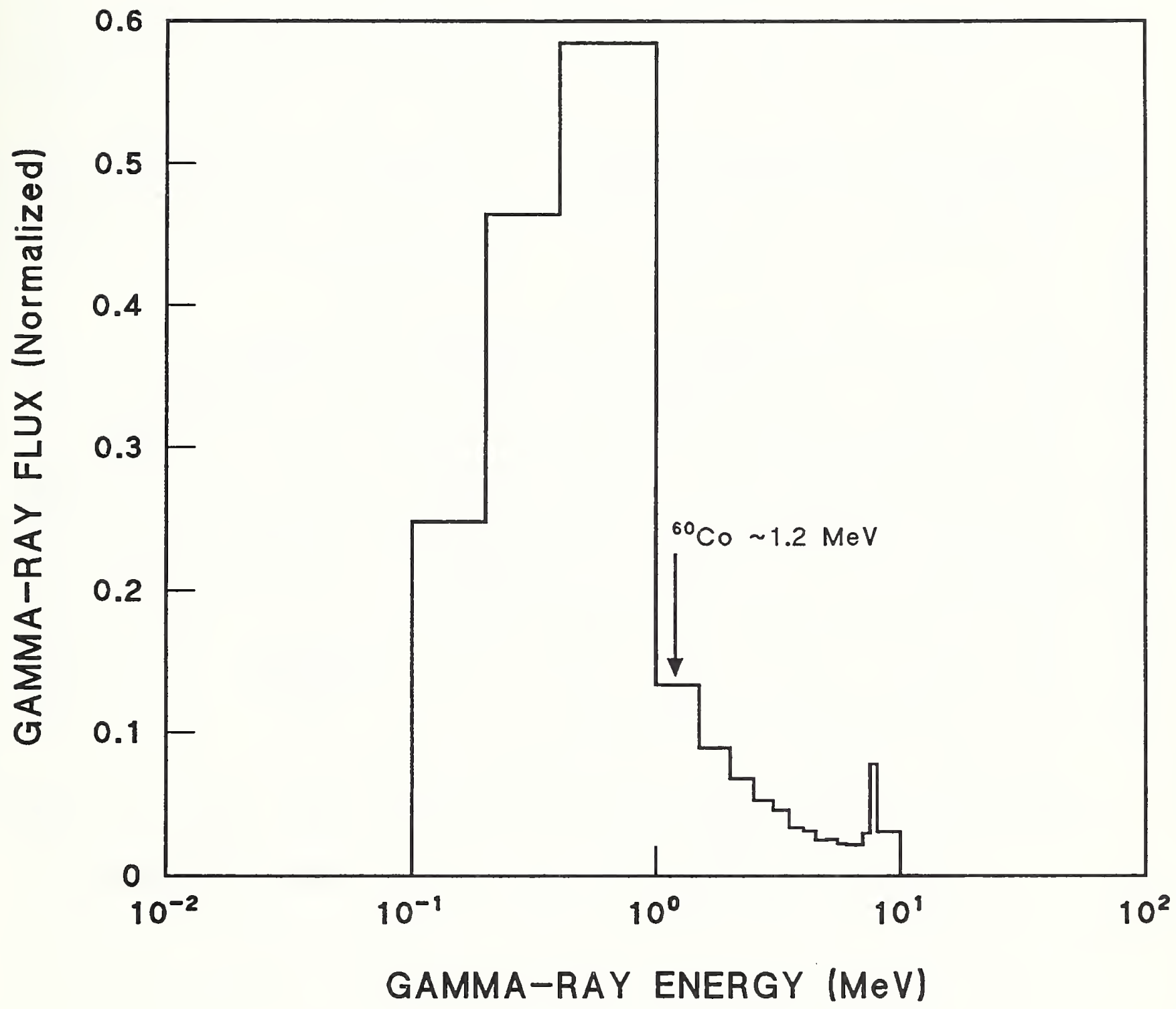

Figure 1.11. A calculation of the ITER gamma ray spectrum at the midplane of the inboard side of the TF magnet. Data from El-Guebaly [1990]. 

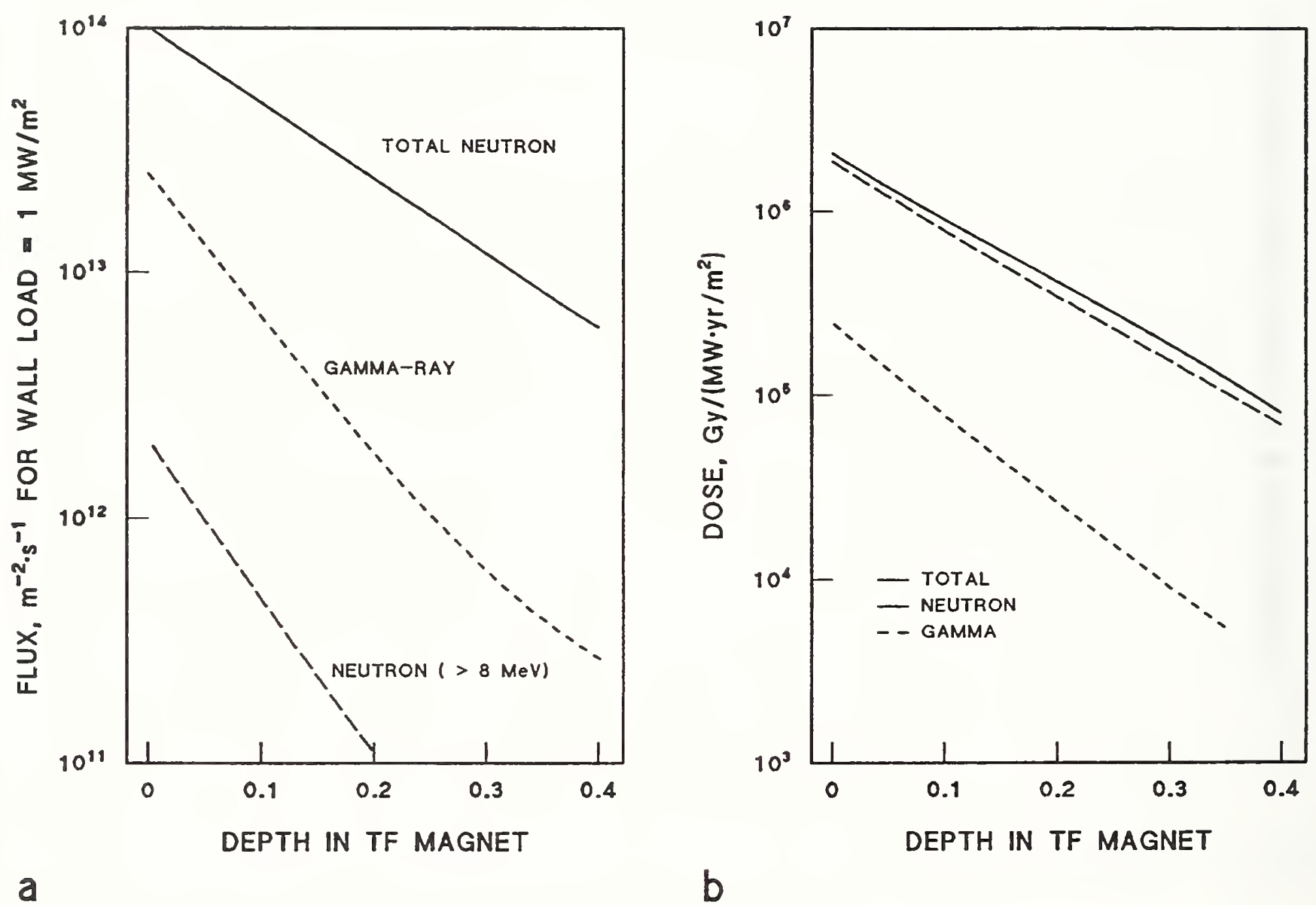

Figure 1.12. (a) Calculated flux versus depth in the magnet of a fusion reactor, from neutrons of energy $>8 \mathrm{MeV}$, gamma rays, and total neutrons. (b) Calculated dose, in the epoxy insulation, versus depth in the magnet of a fusion reactor, from neutrons, gamma rays, and both neutrons and gamma rays. Data from Abdou [1978]. 
magnet, since gamma irradiation originates from nuclear reactions and the decay of radioactive isotopes, which are nucleus-specific. For Abdou's calculation, the inner blanket and shield consisted of stainless steel and $\mathrm{B}_{4} \mathrm{C}$, and the physical thickness from the first wall to the inner edge of the toroidal field coil was about $1 \mathrm{~m}$, including gaps, with a net material thickness of about $0.87 \mathrm{~m}$. Figure 1.13, from a calculation for the Culham Mk II tokamak [Banford, 1984], shows a similar ratio of nearly 10 to 1 for the ratio of neutron to gamma dose in the magnet. The materials specified for the blanket and shield (stainless steel, $\mathrm{B}_{4} \mathrm{C}$, and $\mathrm{Pb}$ ) are similar to materials used in the ITER conceptual design.

As a design limit for shield performance, the ITER conceptual design allows a fast neutron fluence of $1 \times 10^{23} / \mathrm{m}^{2}$ to the superconductor and a peak dose of 5 $\mathrm{x} 10^{7}$ Gy to the magnet insulation, as indicated in Table 1.1 [Tomabechi et al., 1991; Bottura et al., 1991]. If the conversion rule, $1 \mathrm{~Gy}=10^{15} / \mathrm{m}^{2}$, is used, the fast neutron dose would equal $10^{8} \mathrm{~Gy}$. Since this neutron fluence will also pass through the insulation, it will experience a significant neutron dose, as the earlier results shown in Figures 1.12 and 1.13 indicate. Another ITER-based calculation gave a "peak" fast neutron fluence for the insulation of $2.4 \times 10^{22} / \mathrm{m}^{2}$, but suggested that a factor of 3 be applied to that result to account for the effect of assembly gaps and uncertainties in data evaluation and modeling [E1-Guebaly, 1990]. However, the predicted neutron-to-gamma ray ratio depends upon the materials and the models used in the calculations. Table 1.3, which gives data from four different calculations related to the ITER conceptual design [Maki et al., 1991], indicates that the neutron/gamma flux ratio can vary from as high as 5 to as low as 2.3. Another ITER design calculation gave a ratio of about 4 [E]Guebaly, 1990]. These ratios are lower than the factor of about 10 given in Figures 1.12 and 1.13. Figure 1.14 is a plot of the neutron and gamma flux throughout the ITER reactor, for the model of the first column in Table 1.3. The neutron/gamma ratio is 10 or greater at some locations, but falls off at the magnet position $(-260 \mathrm{~cm})$. Since the gamma radiation arises from nuclear reactions and the decay of radioisotopes, it can vary substantially with the materials present in different parts of the reactor. Fluence-to-dose conversion factors given by Van Konynenburg [1981] are similar for gamma rays and neutrons. 
Table 1.3. Calculated Neutron and Gamma Fluxes at the Toroidal Field Magnet, ITER Design. (Data from Maki et al. [1991].

\begin{tabular}{|c|c|c|c|c|}
\hline $\begin{array}{l}\text { Transport Cross Section } \\
\text { Data base } \\
\text { Reaction Cross Section }\end{array}$ & $\begin{array}{c}\text { GICX40 } \\
\text { ENDF } / \text { B-III.IV } \\
\text { GICX } 40\end{array}$ & $\begin{array}{l}\text { FUSION-J } 3 \\
\text { JENDL-3 } \\
\text { JENDL-3 }\end{array}$ & $\begin{array}{l}\text { FSXJ 3T2 } \\
\text { JENDL-3 } \\
\text { JENDL-3 }\end{array}$ & $\begin{array}{l}\text { VITAMIN-C } \\
\text { ENDF/B-IV } \\
\text { MACKLIB-IV }\end{array}$ \\
\hline \multicolumn{5}{|c|}{$\begin{array}{l}\frac{\text { Neutron flux }}{\text { Fast neutron }}\left(\mathrm{m}^{-2} \mathrm{~s}^{-1}\right) \\
\qquad\left(E_{\mathrm{n}}>0.1 \mathrm{MeV}\right)\end{array}$} \\
\hline $\begin{array}{l}\text { Winding Pack } \\
(\mathrm{R}: 257-259 \mathrm{~cm})\end{array}$ & $8.320 \mathrm{E}+13$ & $1.237 \mathrm{E}+14$ & $1.237 \mathrm{E}+14$ & $1.083 \mathrm{E}+14$ \\
\hline \multicolumn{5}{|c|}{$\begin{array}{l}\text { Total neutron } \\
\qquad\left(E_{\mathrm{n}}<14.1 \mathrm{MeV}\right)\end{array}$} \\
\hline $\begin{array}{l}\text { Winding Pack } \\
(\mathrm{R}: 257-259 \mathrm{~cm})\end{array}$ & $2.014 \mathrm{E}+14$ & $3.207 \mathrm{E}+14$ & $3.208 \mathrm{E}+14$ & $2.545 \mathrm{E}+14$ \\
\hline \multicolumn{5}{|c|}{ Total gamma ray flux $\left(\mathrm{m}^{-2} \mathrm{~s}^{-1}\right)$} \\
\hline $\begin{array}{l}\text { Winding Pack } \\
(\mathrm{R}: 257-259 \mathrm{~cm})\end{array}$ & $4.016 \mathrm{E}+13$ & $6.947 \mathrm{E}+13$ & $6.217 \mathrm{E}+13$ & $1.099 \mathrm{E}+14$ \\
\hline
\end{tabular}




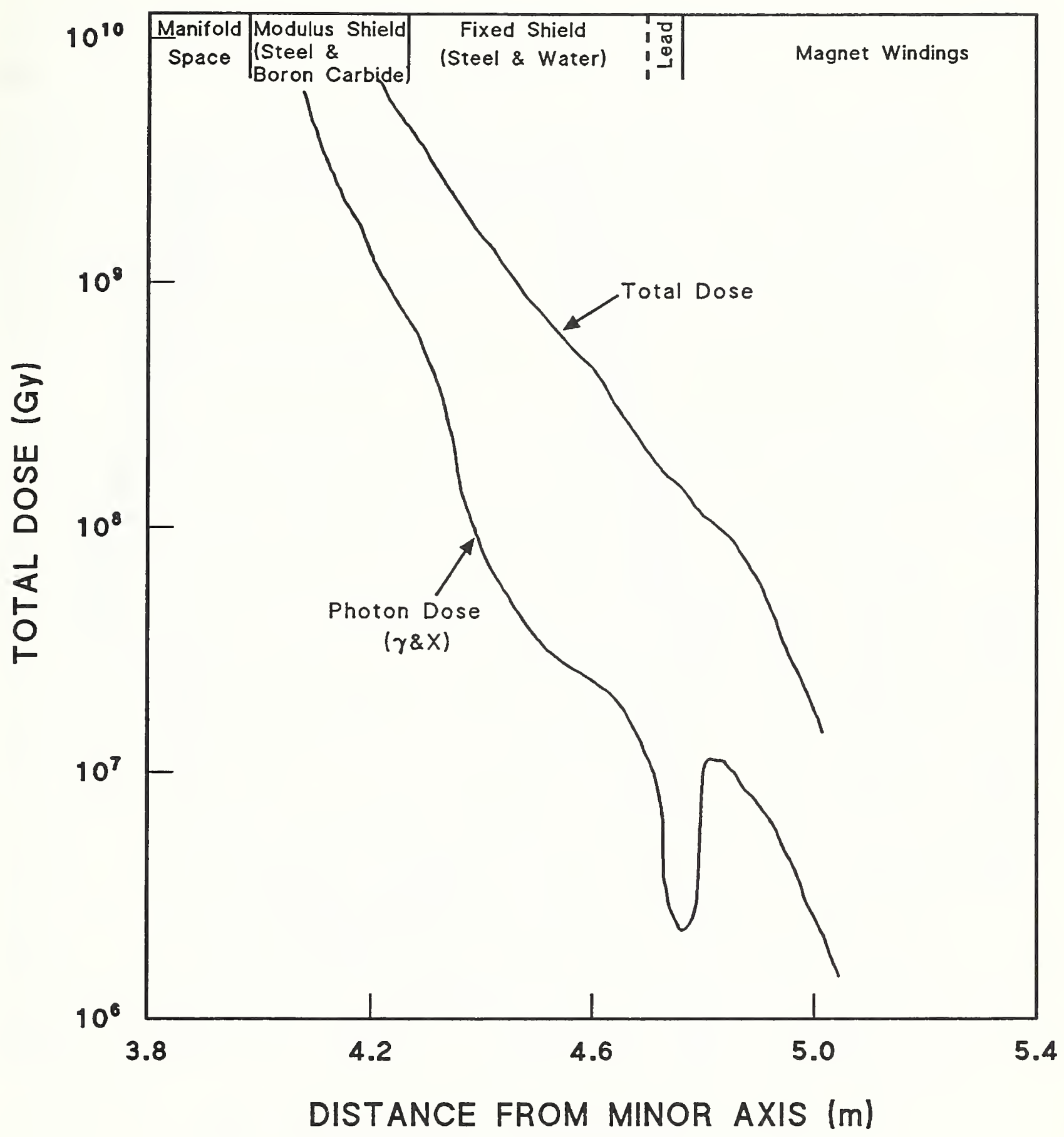

Figure 1.13. Calculated total dose and gamma dose versus distance along the axis of a tokamak reactor. Data from Banford [1984]. 


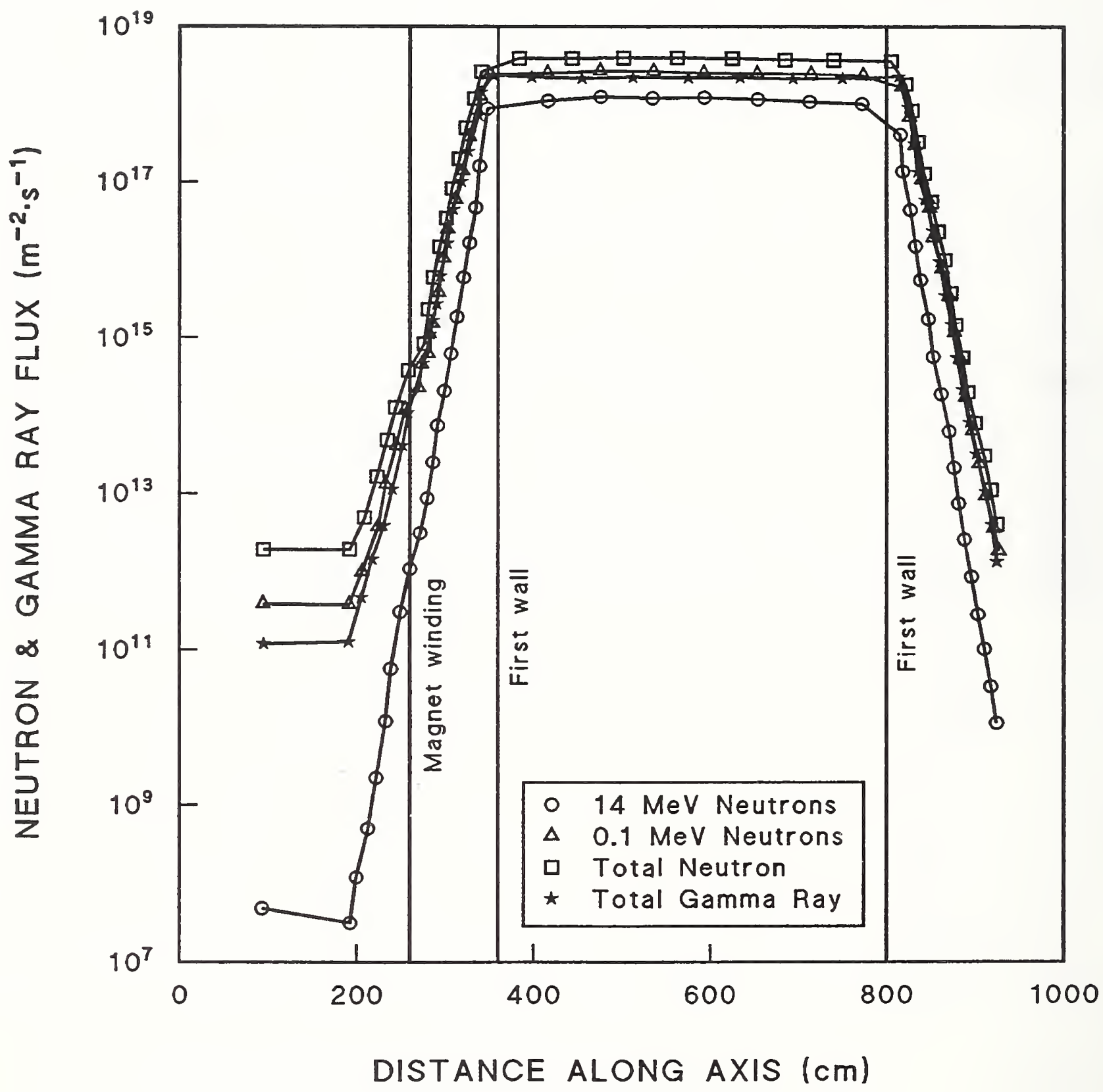

Figure 1.14. Calculated neutron and gamma ray flux distributions for the ITER design versus distance along the axis. Data from Maki et al. [1991]. 
Although neutron radiation will predominate at the TF magnets, there are very few high-dose neutron-irradiation data on epoxy composites reinforced with Bfree glasses. Instead, most high-dose data were obtained with radiation composed mainly of gamma rays, or with electron radiation. To assess the usefulness of irradiation data obtained from gamma and electron irradiation, differences and similarities in the damage mechanisms of different radiation species are discussed in the balance of this subsection. Also, the available experimental evidence on comparative species irradiation is reviewed.

\subsubsection{Neutron Damage Mechanisms}

Thermal neutrons (energy $\approx \mathrm{kT}=2.5 \times 10^{-8} \mathrm{MeV}$ ) interact with matter chiefly by transmutation reactions, $(n, \gamma)$, in which the neutron is captured by a nucleus and a gamma ray is emitted. Transmutation reactions can be induced by very low energy neutrons owing to the absence of a Coulomb, or charge, barrier; in fact, for neutrons of energy below $-10^{-3} \mathrm{MeV}$, the cross section for capture varies inversely with neutron velocity, and may attain a magnitude that is thousands of times the geometric cross section of the nucleus [Leighton, 1959]. At somewhat higher energies, up to about $10^{-2} \mathrm{MeV}$, very high cross sections for neutron capture again occur, as shown in Figure 1.15, at certain preferential or resonance energies that correspond to discrete energy levels within the nucleus. However, resonance absorption occurs chiefly in nuclei with mass numbers greater than 100, which excludes the $\mathrm{H}, \mathrm{C}$, $N$, and $O$ atoms contained in epoxy resins [Lapp and Andrews, 1954]. For example, the energy level of the lowest excited state of $\mathrm{C}$ is very high, -4 $\mathrm{MeV}$. The organic elements also have very small thermal neutron capture cross sections [Lapp and Andrews, 1954]. The one exception (in B-containing glass reinforcement) is ${ }^{10} \mathrm{~B}+\mathrm{n} \rightarrow{ }^{7} \mathrm{Li}+\alpha$, in which short-range alpha particles of energy about $1.8 \mathrm{MeV}$ are emitted and cause intense local damage. But, in general, transmutation reactions are not important in organic insulators below the relatively high energies of about $2 \mathrm{MeV}$. In this very high energy range, as noted above, charged particles can be emitted because the Coulomb barrier can be overcome. Thus, reactions such as $(n, \alpha),(n, p)$, and $\left(n, n^{\prime} p\right)$ may occur.

In the range below these very high energies, fast neutrons ( $E>0.1 \mathrm{MeV}$ ) and intermediate-energy neutrons produce radiation damage by direct collision with atoms. These "knocked-on" atoms have transferred kinetic energies that can 


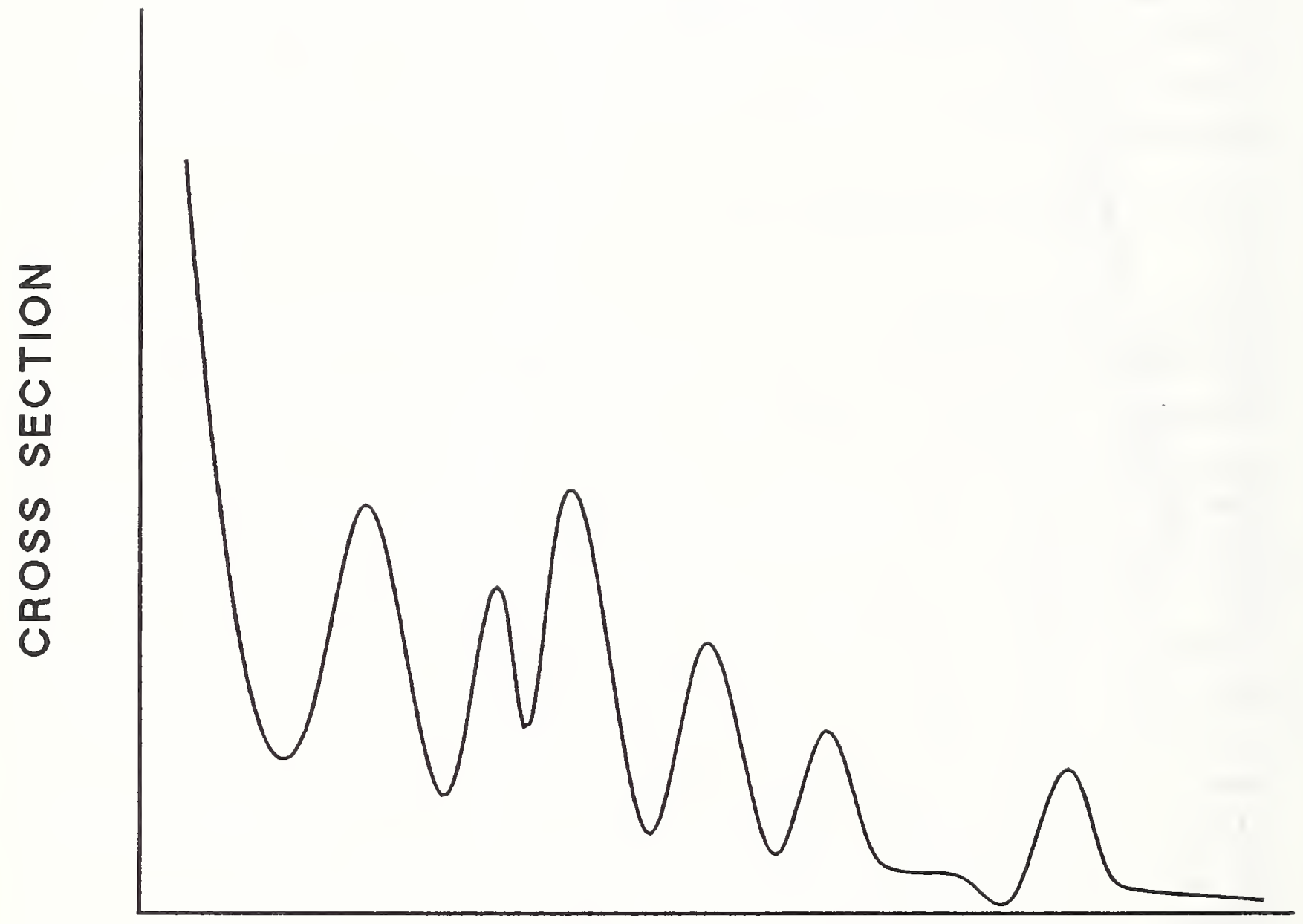

\section{ENERGY}

Figure 1.15. Schematic illustration of a cross-section curve versus energy for neutrons, showing resonance effects. Data from Leighton [1959]. 
cover a wide range of values. In organic insulators, the primary effect is the liberation of hydrogen ions, or protons. This occurs because the energy transferred in elastic collisions (hard-core approximation) is given by

$E_{\text {transferred }}=\left[(4 M) /(M+1)^{2}\right] \cdot E_{\text {incident }}$,

where $M$ is the relative atomic mass of the impacted atom, and the direction of recoil is in line with the direction of incidence. Since the mass of the hydrogen atom is nearly equal to the mass of the neutron, the ratio of transferred to incident energy is nearly equal to 1 (or $\frac{1}{2}$ if the angle of recoil is averaged). For $C$ atoms, however, this ratio is 0.28 and for 0 atoms, it is 0.21 . The minimum energy necessary to remove an atom from its lattice site, the threshold displacement energy, is about $25 \mathrm{eV}$ for most materials [Kircher, 1964].

The literature gives very little specific information about the primary damage produced in organic insulators by protons and other knocked-on atoms with kinetic energies up to several $\mathrm{MeV}$. In contrast, the chemical damage that occurs as a result of ionization or excitation has been extensively discussed. Since the ionization energy in organic materials is only about 10 to $12 \mathrm{eV}$ [Kircher, 1964] the impacted atom is easily ionized or an electron is excited into a bound orbit of higher energy. Electro-static interactions between the moving ion and stationary atoms further dissipate energy until the ion comes to rest. Excitation of polymer molecules of the epoxy can be brought about directly through interaction with the moving ions or it can take place indirectly by the generation of a polymer ion and its subsequent recombination with a thermal electron. Excitation of the polymer molecule is very likely to result in rupture of covalent bonds within the molecule, with the formation of free radicals (because the electrons in the bond were paired). Free radicals are very reactive and can initiate further chemical reactions. As indicated in Figure 1.16, either the main chain or a side chain of an epoxy polymer can be ruptured. This results in chain scission or in cross-linking and sometimes, following recombination of ions, evolution of gas. The chemical effects of free-radical reactions are discussed more fully below (\$1.4.2.1). The presence or absence of oxygen (as in liquid helium) affects the rate of free-radical reactions. As expected, ambient-temperature irradiation in a 


\section{i) Main-chain rupture}

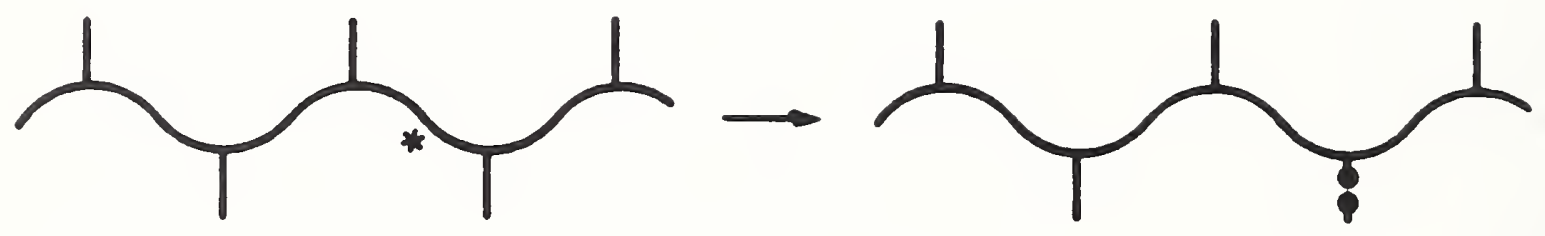

\section{ii) Side-chain rupture}

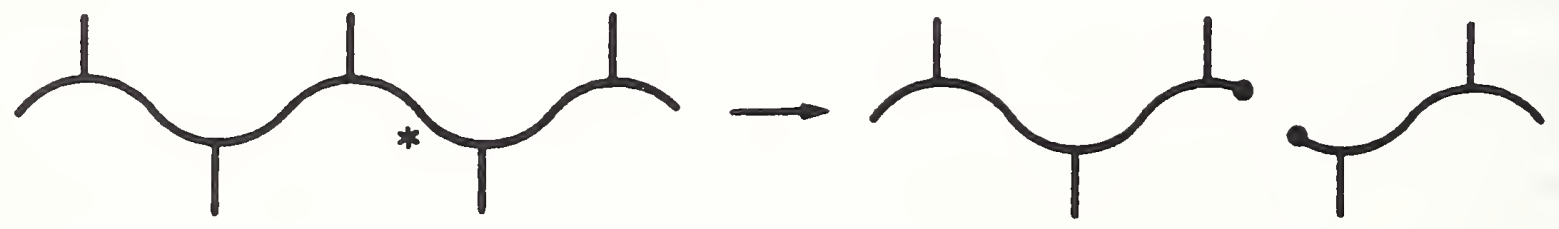

(energetic free radicals)

Figure 1.16. Schematic illustration of main-chain and side-chain rupture of excited polymers, with the formation of free radicals. 
vacuum has been shown to result in lower degradation than irradiation in air (Figures A. 2-58 to A.2-63, ${ }^{\star *} \S 2.5$ ).

\subsubsection{Gamma Ray Damage Mechanisms}

Figures 1.9 to 1.11 show that the energy of the expected gamma spectrum will range approximately from 0.1 to $10 \mathrm{MeV}$. The ${ }^{60} \mathrm{Co}$ sources used for many irradiation tests furnish gamma rays of energy 1.2 and $1.3 \mathrm{MeV}$. In these energy ranges, Figure 1.17 shows that the predominant interaction of gamma rays with organic matter will be the Compton process. (Gamma ray energies above about $12 \mathrm{MeV}$ are necessary for neutron production from collisions with most light nuclei [Fassò et al., 1984]). Note that the y axis of Figure 1.17 denotes the atomic number, $\mathrm{Z}$, of the nucleus; for organic insulators, $\mathrm{Z}$ is generally below 8 , and the Compton effect is dominant over a wider energy range than it is for materials with higher $Z$. In the Compton process, diagrammed in Figure 1.18, a gamma ray is incident upon a loosely bound, outer-shell electron, and is scattered by the electron, losing energy. The difference in energy of the incident and scattered gamma rays, less the energy required for ionization, is imparted to the electron, which is ejected with a recoil energy. A detailed consideration of the Compton process [Lapp and Andrews, 1954] shows that the percentage of energy loss is higher for incident gamma rays of higher energy; for example, a $0.1-\mathrm{MeV}$ gamma ray will loose about $15 \%$ of its energy but a 5-MeV gamma ray will loose about 90\%. (These numbers are correct for the case of right-angle scattering.) Note from Figure 1.18 that the energy lost by the gamma ray is taken up by the electron; the ion does not experience any significant change in kinetic energy. This contrasts with neutron irradiation, in which the knocked-on atoms receive an increase in kinetic energy that is roughly 20 to $100 \%$ of that of the incident neutron [Equation (1-1)]. Furthermore, the calculated cross sections for displacements of atoms caused by the energetic electrons produced in the Compton process were negligible [Dell and Goland, 1981]. Thus, although both neutron and gamma irradiation result in ionization and excitation, the displacement of atoms is absent in gamma irradiation.

\footnotetext{
* All of the figures containing the prefix A are located in the appendices A.2 to A.11 at the end of the report. Each figure caption contains a reference to a table, located in the same appendix section, that contains further information (when available) on irradiation, test, and specimen parameters.
} 


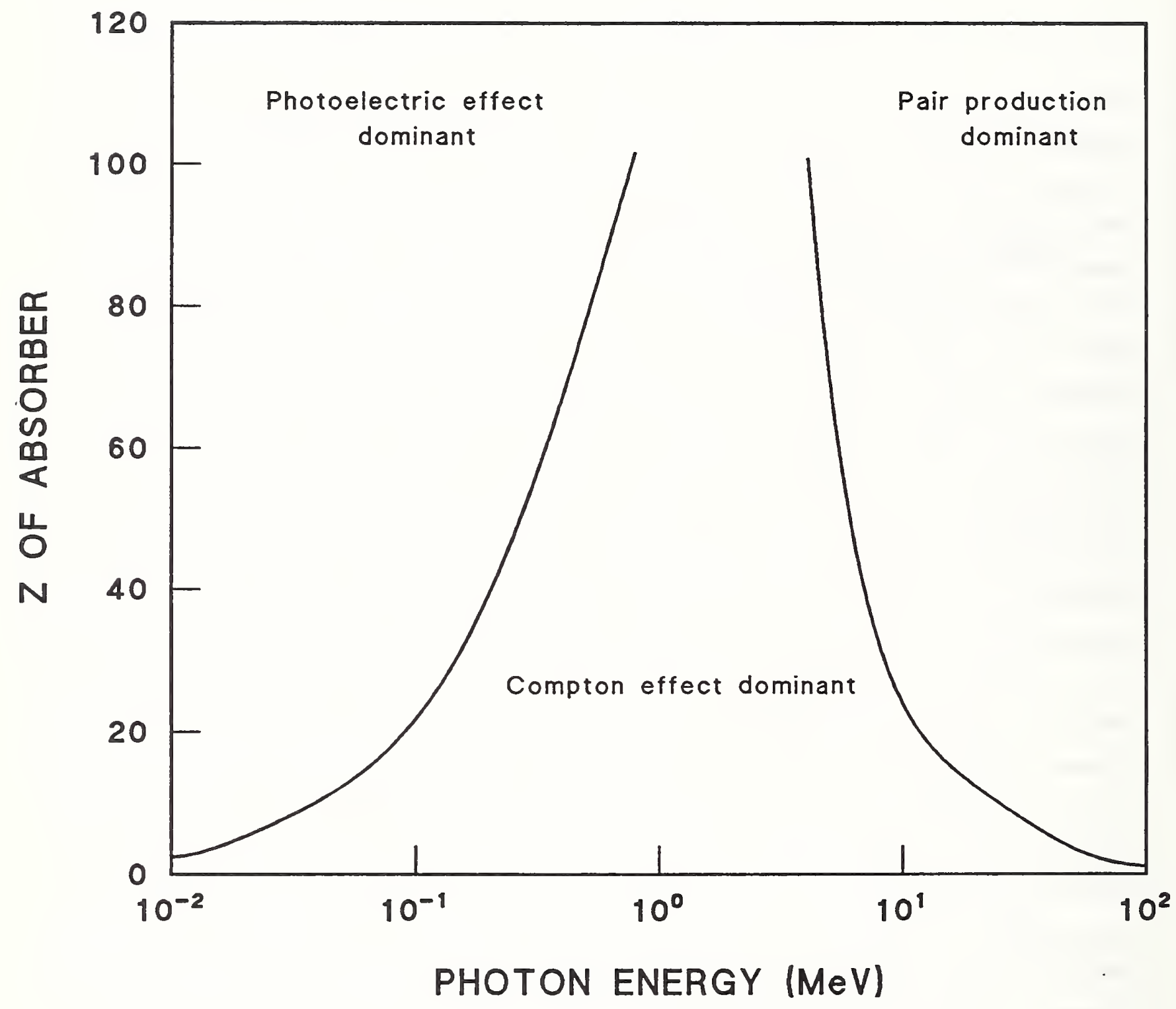

Figure 1.17. Interaction mechanisms of gamma rays with matter, illustrating the dependence on the atomic number, $Z$, of the absorber. The Compton effect will dominate for the energy range of gamma rays expected at the magnet position of a tokamak fusion reactor. Data from Kircher [1964]. 


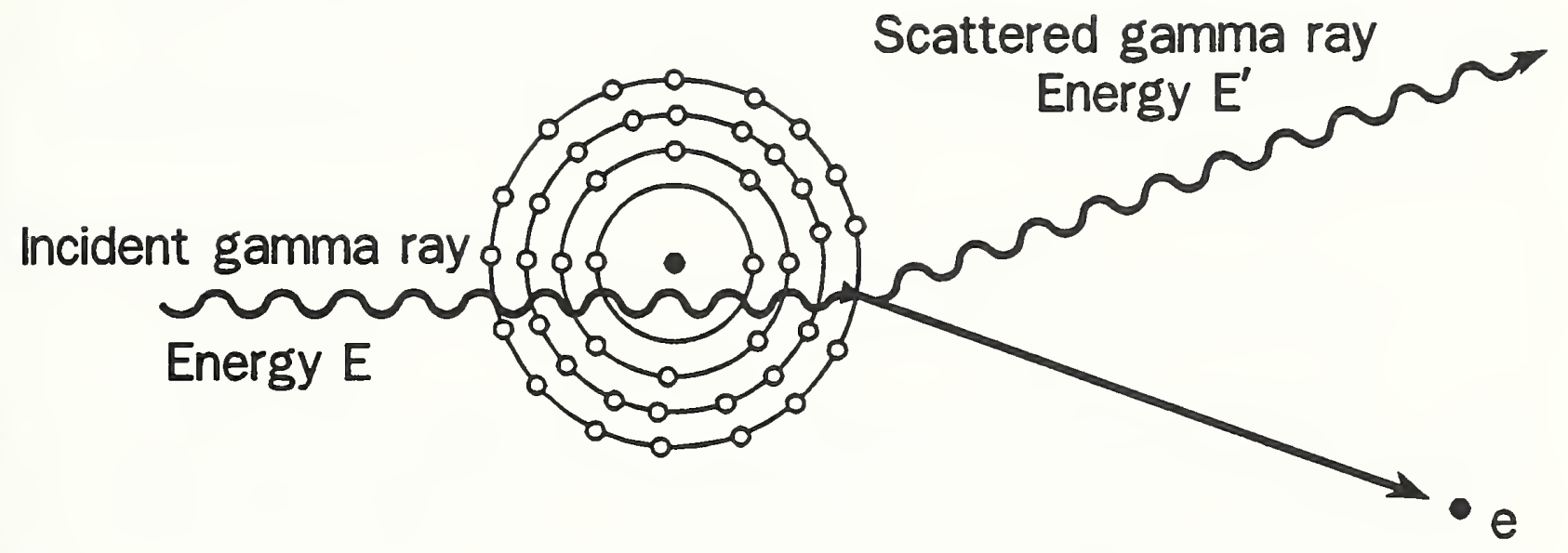

\section{Ion Kinetic Energy Unchanged}

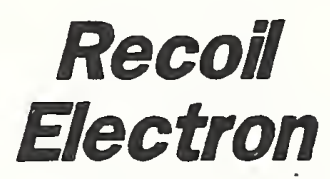

Figure 1.18. Schematic diagram of the Compton process, illustrating the scattered gamma ray and the recoil electron. 


\subsubsection{Electron Damage Mechanisms}

The damage resulting from electron irradiation is discussed because some of the irradiation data were obtained with this type of radiation. As shown in the section above, gamma irradiation produces energetic electrons which actually cause most of the changes in the irradiated material. Electrons lose energy in matter primarily by collisions yielding ionized or excited atoms, although a radiative collision process, bremsstrahlung, also occurs when electrons decelerate in matter, and gamma rays are subsequently produced. However, gamma production by this mechanism is important only at energies above several $\mathrm{MeV}$, for elements of high $\mathrm{Z}$. The ratio of bremsstrahlung to ionization events is

$$
\text { (Bremsstrahlung)/(Ionization) } \approx \mathrm{E}(\mathrm{MeV}) \cdot \mathrm{Z} / 800 \text {, }
$$

where the atomic number, $\mathrm{Z}$, is equal to the number of protons in the nucleus [Kircher, 1964]. For oxygen, the element with the highest $Z$ that occurs commonly in organic insulation, bremsstrahlung is only about 28 of ionization for an energy of $2 \mathrm{MeV}$, the level at which most of the electron irradiation experiments have been conducted [Egusa et al., 1988; Egusa, 1988, 1990a, $1991 \mathrm{a}, 1991 \mathrm{~b}]$.

Some electrons ejected during ionization will have enough energy to cause further ionization. Since the energy loss per ion pair formed is about $30 \mathrm{eV}$, a $2-\mathrm{MeV}$ electron will not lose much energy per ion pair. Furthermore, the effective interaction distance of the electric field of a "fast" electron $(-1-10 \mathrm{MeV})$ is actually narrower than that of a slow electron ( $\leq 0.1 \mathrm{MeV}$ ) which has a higher specific ionization, as shown in Figure 1.19 [Lapp and Andrews, 1954]. The energy that can be imparted to an orbital electron depends on the length of time that the moving electrori spends in its vicinity; thus, the effective interaction distance of a fast electron is actually less than that of a slow electron, and a fast electron may not transfer enough energy to cause ionization, as Figure 1.19 shows. In other words, a slow electron can be pictured as sweeping out a cylinder of ionization in a material that is larger than that of a fast electron. 


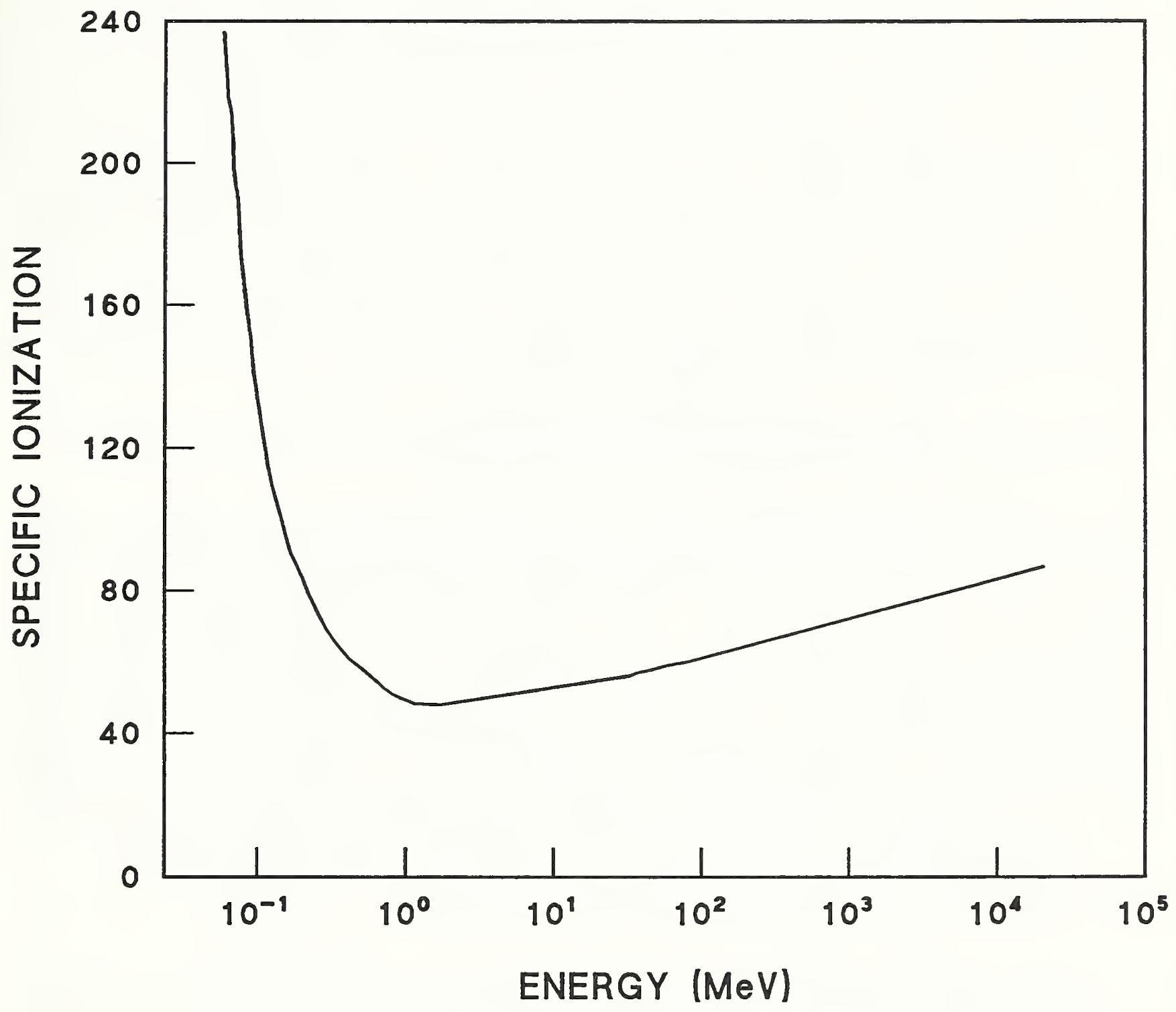

Figure 1.19. The ionization curve for electrons, illustrating the sizeable decrease in ionization for electrons at about $1 \mathrm{MeV}$ in energy. Data from Lapp and Andrews [1954]. 
1.3.2.5. Experimental Comparisons of Neutron and Gamma Irradiation Damage

Both neutrons and gamma rays transfer their energies to orbital electrons: the neutrons, through the liberation of knocked-on protons, which are charged and therefore ionize atoms, and the gamma rays, through the Compton effect. The ionization or excitation of atoms leads to covalent bond breakage and free radical reactions. However, the shorter range of protons, as shown in Table 1.4 for a light metal*** [Billington and Crawford, 1961], indicates that the distribution of damage can be quite different, so that neutron chemical damage may be confined to relatively discrete regions whereas gamma ray (or electron) damage may be more dispersed [van der Klein, 1975]. Also, the displacement of highly energetic protons within the composite may lead to structural damage.

Table 1.4. Range (in mm) of Heavy, Charged Particles in Aluminum. (Data from Billington \& Crawford [1961].)

\begin{tabular}{|l|c|c|c|c|}
\hline \multicolumn{5}{|c|}{ Incident Energy of Particle (MeV) } \\
\hline & 1 & 10 & 20 & 40 \\
\hline Protons & 0.013 & 0.62 & 2.2 & 7.2 \\
\hline Alpha Particles & $\ldots-$ & 0.059 & 0.185 & 0.592 \\
\hline Electrons & 1.48 & 22. & $-\ldots$ & $\ldots$ \\
\hline
\end{tabular}

* For purposes of comparison, the authors noted that the ranges of gamma rays and neutrons are of the order of $\mathrm{cm}$. For example, the range of $1-\mathrm{MeV}$ gamma rays is $5.95 \mathrm{~cm}$, from the reciprocal of the linear absorption coefficient, $0.168 \mathrm{~cm}^{-1}$ [Lapp and Andrews, 1954].

Unfortunately, experimental tests of the differences in the effects of neutron and gamma radiation are not plentiful. Egusa et al. have demonstrated that Eglass-reinforced epoxy insulation incurs greater degradation at a given dose level from neutron irradiation than from gamma irradiation. Figure 1.20 shows this increased degradation in the flexural strength of a plain-weave, glassreinforced TGDM resin at two test temperatures [Egusa et a1., 1984a, 1987b] . However, much of this degradation could be ascribed to the $(B, n) \rightarrow(\operatorname{Li}, \alpha)$ reaction, since the alpha particles and lithium atoms deposit an energy of

\footnotetext{
\#\# Comparative data for gamma rays, electrons, and protons are not readily available for organic materials. However, the $1-\mathrm{MeV}$ ranges for protons, alpha particles, and electrons in the polyimide Kapton are $19 \mu \mathrm{m}, 4.2$ $\mu \mathrm{m}$, and $3.3 \mathrm{~mm}$, respectively [Berger et al., 1992; Berger and Seltzer, 1982].
} 


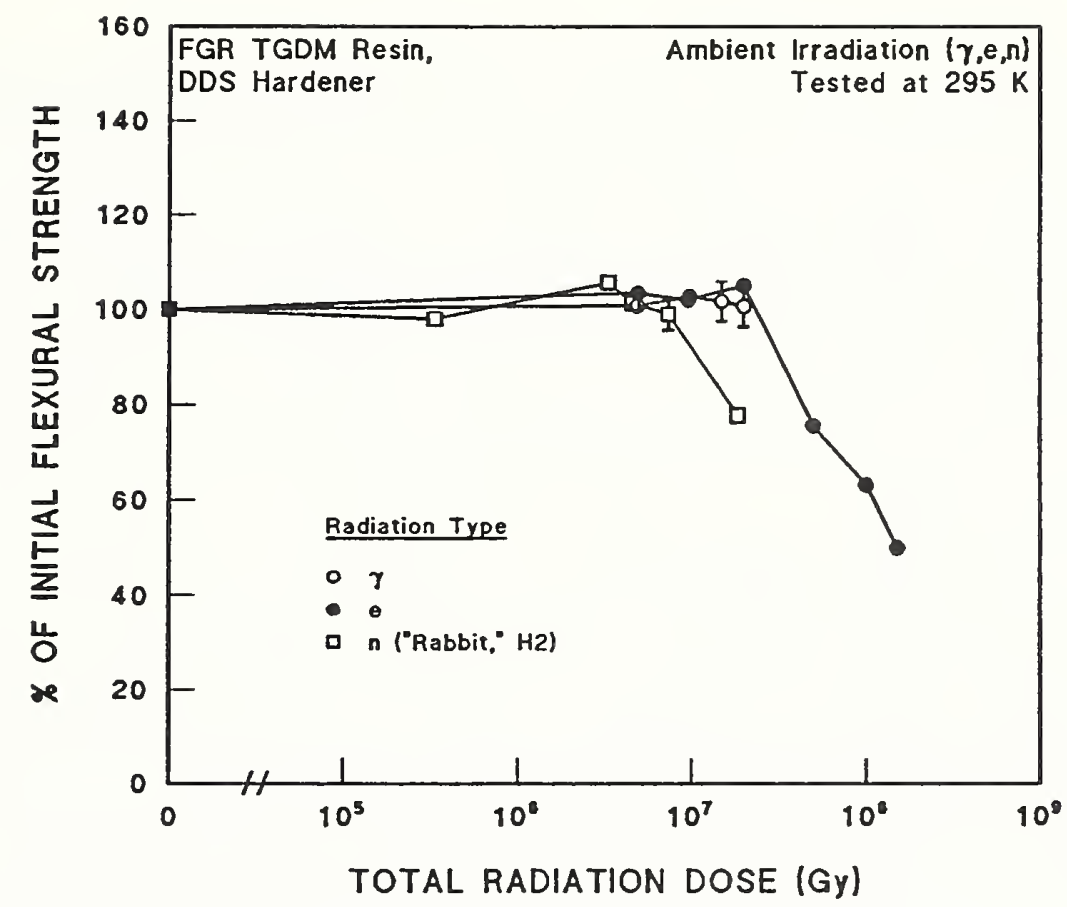

b

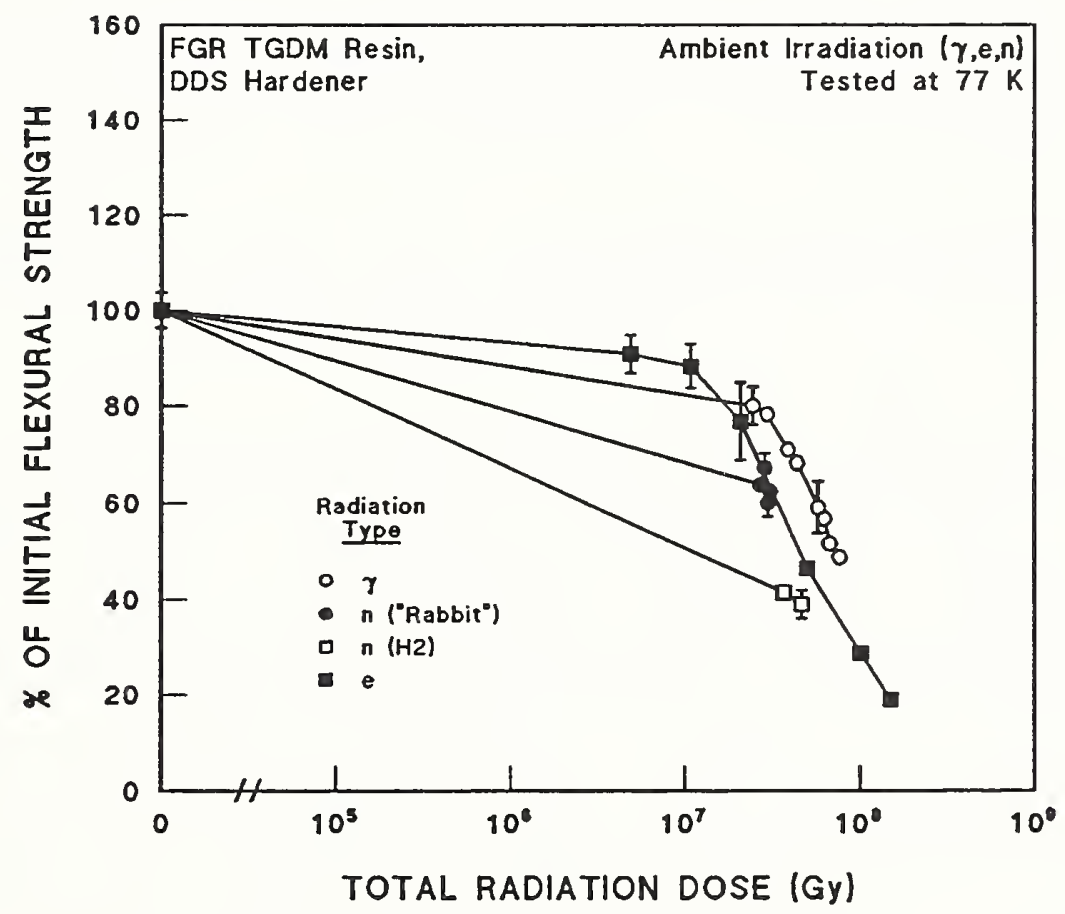

Figure 1.20. Irradiation data comparing the effects of neutron, electron, and gamma irradiation on a fiber-glass reinforced TGDM (tetraglycidyl diaminodiphenyl methane) epoxy matrix cured with DDS (diaminodiphenyl sulphone). (a) Flexural tests at $295 \mathrm{~K}$. Data from Egusa et al. [1984a,b]. (b) Flexural tests at $77 \mathrm{~K}$. Data from Egusa et al. [1985a; 1987b]. The H2 irradiation thimble exposed specimens to a heavier dose of thermal neutrons than the "Rabbit" thimble. (Supplementary Table B.1-1.) 
about $2.8 \mathrm{MeV}$ within a few $\mu \mathrm{m}$ of the boron-containing E-glass [Klabunde and Coltman, 1983; Nishijima et al., 1991]; thus, the glass-matrix interface would be considerably weakened. In fact, Egusa et al. [1987a; 1987b] reported calculations that indicated that the energy absorbed in these E-glass reactions added 40\% ("Rabbit" irradiation thimble) to 1600\% (H2 irradiation thimble) to the nominal dose. Furthermore, since the comparison was not carried out to $10^{8}$ Gy it is difficult to extrapolate to the ITER neutron dose from the data provided.

Experiments comparing the effects of both neutron and gamma irradiation on the commercial epoxy laminates Lamiverre $A$ and Hoxan also were conducted on Eglass-reinforced resin systems [Nishijima et al., 1988]. The interlaminar shear strength was measured for both laminates. The Lamiverre-A comparison was made with ambient-temperature testing after ambient-temperature irradiation (Figure A.2-84). The Hoxan comparison was based on a reactor (neutron) irradiation at $20 \mathrm{~K}$, gamma irradiation at $77 \mathrm{~K}$, and testing at $77 \mathrm{~K}$ (Figure A.7-1). Although the results in both cases indicate much greater degradation at $10^{7}$ Gy for neutron than for gamma irradiation, any intrinsic difference between the two types of radiation is again confounded by the reaction of thermal neutrons with boron in the E-glass reinforcement of both composites.

The tests comparing gamma and neutron irradiation made by Nishijima and Okada [1977; 1978] do not suffer from this problem since they were made on neat resin of bisphenol $\mathrm{A}$, with degree of polymerization, $\mathrm{n}$, of 8 to 11 (Figure 1.21). Compression tests at $77 \mathrm{~K}$ were made without specimen warm-up after either doses at $77 \mathrm{~K}$ up to $10^{6}$ Gy of ${ }^{60} \mathrm{Co}$-gamma irradiation or reactor fluences at $40 \mathrm{~K}$ up to $10^{20} / \mathrm{m}^{2}$ of neutron irradiation with $\mathrm{E}>0.1 \mathrm{MeV}$ (accompanied by up to $3 \times 10^{6}$ Gy of gamma irradiation). The gamma irradiations were carried out on 50 samples (per dose) that were sealed and kept in a vacuum. The 100 samples (per dose) that were reactor-irradiated at $40 \mathrm{~K}$ were immersed immediately in liquid nitrogen after irradiation. No other information was provided on the environment during irradiation. Histograms were constructed for the compressive strength, the compressive strain to failure, and the modulus obtained from the compression stress-strain curves. For either the zero-dose case or gamma irradiation at a dose of $10^{5} \mathrm{~Gy}$, the compressive strength obeyed a normal distribution with nearly identical mean 


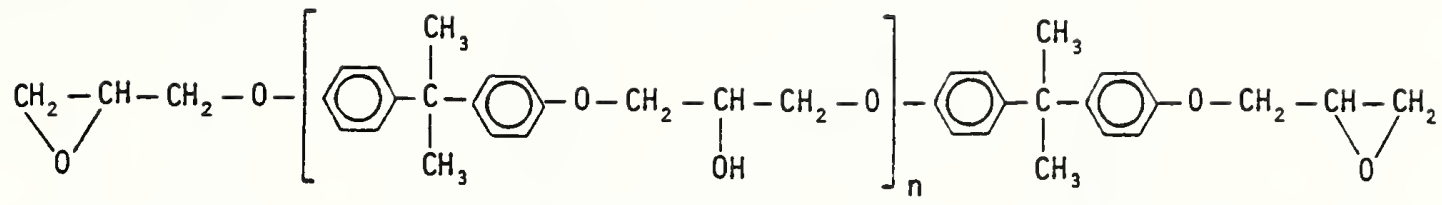

Figure 1.21. The chemical structure of an unmodified epoxy resin based upon bisphenol A.

values. But at $10^{6} \mathrm{~Gy}$, the distribution was no longer normal, the mean was shifted to a lower value, and a tail was exhibited at lower stresses (Figure 1.22). The results for the compressive strain to failure were similar, except that the mean was shifted to a lower value (compared to the zero-dose value) at $10^{5} \mathrm{~Gy}$ as well as at $10^{6} \mathrm{~Gy}$. The mean of the elastic modulus also decreased with increasing radiation dose, but a normal distribution was maintained. However, the width, or variance, of the distribution increased.

Different effects were observed for the specimens irradiated in the reactor. Before comparing these results, note that the highest reactor dose is equivalent to a gamma dose of $3 \times 10^{6} \mathrm{~Gy}$, which is three times that of the highest dose of pure ${ }^{60} \mathrm{Co}$ irradiation, plus a neutron dose of $10^{5}$ Gy that is a factor of 30 lower that the gamma dose (using the conversion factor 1 Gy = $10^{15}$ neutrons $/ \mathrm{m}^{2}$ ). Thus, the comparison is actually not between pure gamma and pure neutron irradiation, but between a pure gamma irradiation at $77 \mathrm{~K}$ and a higher-level gamma irradiation accompanied by a 30-times lower dose of neutron irradiation at $40 \mathrm{~K}$. Nevertheless, differences were observed when specimens irradiated under the two regimes were tested. Under reactor irradiation, the mean compressive strength decreased and the variance increased with increasing dose, as indicated in Figure 1.23. However, the distribution remained normal as the dose increased, rather than developing a tail at low strength levels as shown for pure gamma irradiation in Figure 1.22. This result is puzzling, since the reactor dose of gamma irradiation was three times that of the ${ }^{60} \mathrm{Co}$ irradiation. It is unlikely that the differences observed could be due to differences in the oxygen available in the radiation environment rather than to differences in radiation dose, 


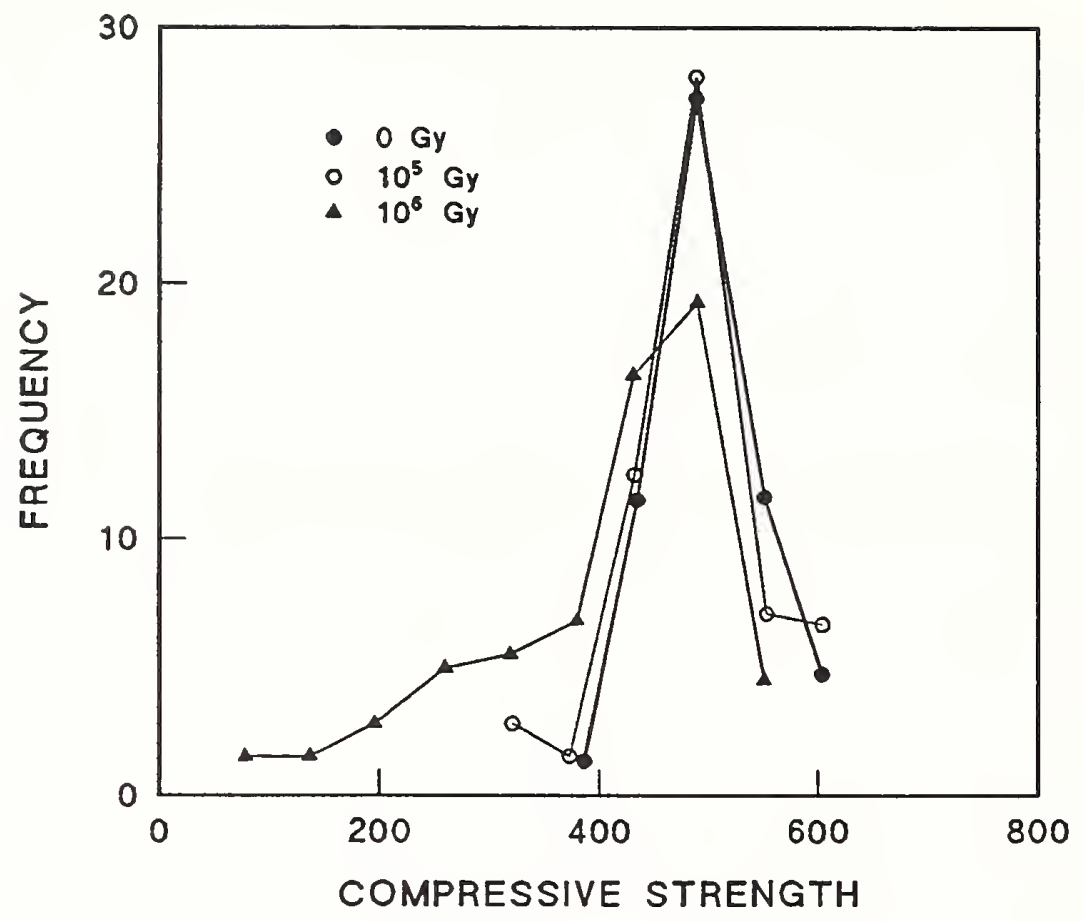

Figure 1.22. The change in the histogram of the compressive strength of a neat resin caused by gamma irradiation. Data from Nishijima and Okada [1977; 1978].

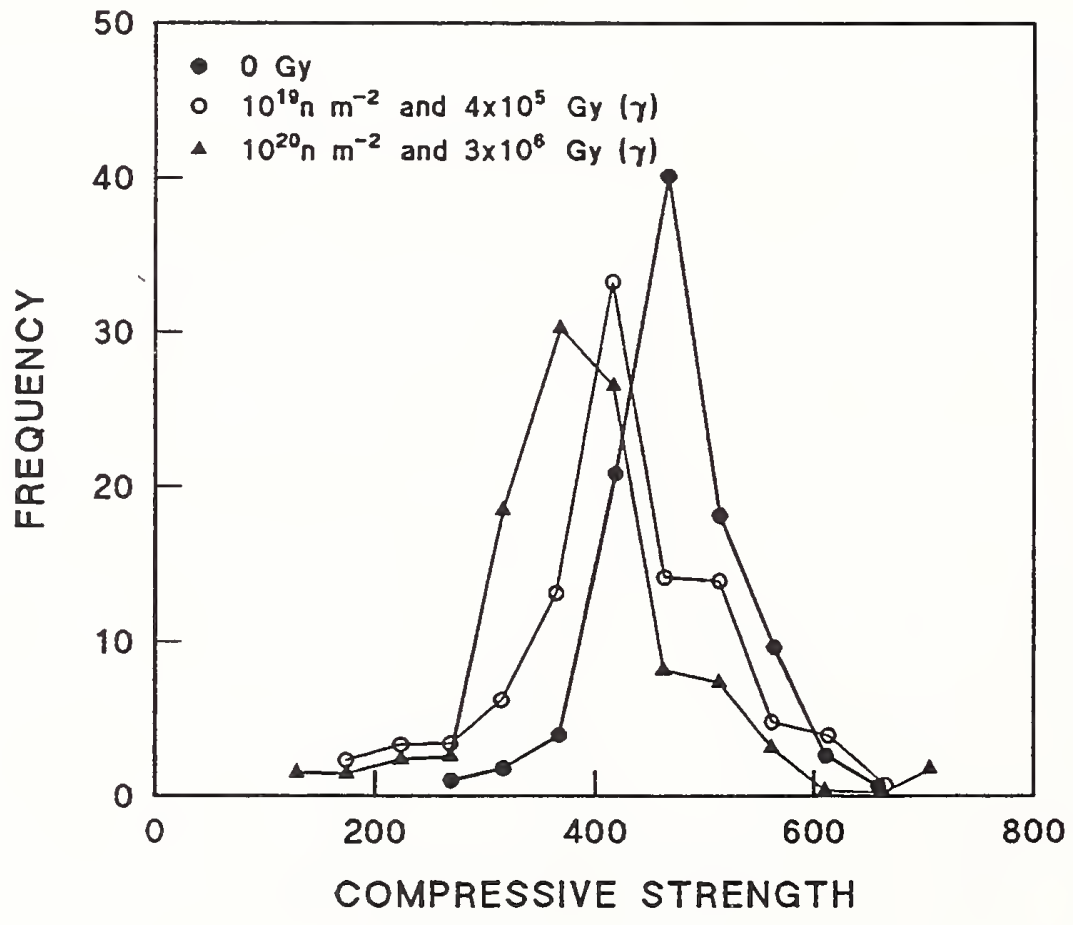

Figure 1.23. The change in the histogram of the compressive strength caused by reactor irradiation comprising both neutrons and gamma rays, as indicated. Data from Nishijima and Okada [1977; 1978]. 
spectrum, or species, because the samples were "sealed and kept in a vacuum to avoid oxygen attacking the polymer during irradiation" [Nishijima and Okada, 1978].

The distribution of values for the compressive strain to failure remained normal with increasing reactor irradiation dose, but the variance and mean value increased. As observed for pure gamma irradiation, the mean values of the elastic modulus decreased with increasing dose, but distributions remained normal. Since the elastic modulus, $\mathrm{E}$, is given by $\mathrm{E}=3 \rho \mathrm{RT} / \mathrm{M}_{\mathrm{c}}$ in viscoelastic theory, where $\rho$ is the density, $R$ is the gas constant, $T$ is the absolute temperature, and $M_{c}$ is the average molecular mass of a polymer chain, Nishijima and Okada suggested that both types of radiation result in increased $\mathrm{M}_{c}$ or increased cross-linking overall. But, because the width of the distribution was greater at the highest doses for both types of radiation, it appears that some chain degradation also occurs. Increased variance with dose was also observed for the compressive strength of reactor-irradiated specimens, in contrast to the more constant variance with dose of gammairradiated specimens. Nishijima and Okada therefore concluded that both cross-linking and chain scission occurred under neutron irradiation, although degradation (scission) dominated because the mean was shifted to a lower value. However, their conclusion was based upon viscoelastic theory, which is probably not a valid model for epoxy resins at low temperature; linear elastic properties have been reported in this temperature regime. Also, free-radical reactions such as cross-linking are inhibited at cryogenic temperatures.

Abe et al. [1987] compared the effects of neutron and gamma irradiation on a polyimide film (Kapton) at ambient temperature. This investigation is described in more detail in Appendix B.1.1. Tensile property measurements showed that a $1.1 \times 10^{7}-\mathrm{Gy}$ dose of $14 \mathrm{MeV}$ neutrons had the effect of a gammaray dose that was about eight times higher. Although this experiment was not carried out with epoxy resins, it indicates that a given absorbed dose of gamma irradiation should not be equated to the same absorbed dose of neutron irradiation. In fact, the experiment indicates that it would be conservative to downgrade gamma doses by a factor of about ten, to simulate high-energy neutron damage. An earlier, qualitative experiment on Kapton by Hurley et al. [1978] also supports downgrading. These authors found that Kapton irradiated by a high-energy (but not $14-\mathrm{MeV}$ ) neutron spectrum from IPNS exhibited the 
same strong discoloration at about $2 \times 10^{6}$ Gy that gamma-irradiated material exhibited at $1 \times 10^{8} \mathrm{~Gy}$. Environmental conditions were similar for both irradiations. Infrared spectra were found to be different for the gamma- and neutron-irradiated specimens. Although comparable experiments have not been done on epoxy systems, it would be conservative not to employ gamma radiation as the major part of a dose used to assess insulation for ITER since it probably will not comprise more than about $20 \%$ of the incident flux at the magnet and since it may be subject to downgrading by a substantial factor.

\subsubsection{Experimental Comparisons of Gamma and Electron Irradiation Damage}

The incident energies used in most gamma and electron irradiations of composites have ranged from about 1 to $2 \mathrm{MeV}$. Since the incident energies are similar, the ionization and excitation damage from gamma or electron irradiation should be similar, because the energy of the photon is ultimately transmitted to the material via the recoil electron produced in the Compton effect. Furthermore, there are no strong localizing effects as occur with neutron or alpha radiation. (There is a weaker localizing effect because lowenergy electrons sweep out a wider cylinder of interaction than high-energy electrons as they move through a material, as discussed in \$1.3.2.3 above.) In comparing irradiations carried out with gamma rays or electrons, however, the difference in penetration depth between the two types of radiation must not be overlooked. Table 1.4 shows that the range of a $1-\mathrm{MeV}$ electron in the light metal, Al, is $1.5 \mathrm{~mm}$. However, the range for a $1-\mathrm{MeV}$ gamma ray is $-6 \mathrm{~cm}$, based on a linear absorption coefficient of $0.168 \mathrm{~cm}^{-1}$ in A1 [Lapp and Andrews, 1954]. Charlesby [1972] stated that for electrons in the $\mathrm{MeV}$ range, an approximate penetration depth of $5 \mathrm{~mm}$ per $\mathrm{MeV}$ in polymer material of unit density can be used, whereas ${ }^{60} \mathrm{Co}$-gamma radiation ( $1.2 \mathrm{MeV}$ ) has more than 10 times this range.

On the other hand, the calculated linear rate of energy transfer, $-\mathrm{dE} / \mathrm{dx}$, is not very different for low energy electrons and ${ }^{60} \mathrm{Co}$ gamma rays in water, as shown by Table 1.5. The distance between the spurs (from secondary electrons) which make up the track also is the same for $1-\mathrm{MeV}$ electrons and ${ }^{60} \mathrm{Co}-$ gamma rays. (This parameter is considerably smaller for alpha particles and 
Table 1.5. Linear Energy Transfer and Mean Spur Separation for Different Radiations*. (Data from Van de Voorde [1970] and citations therein).

\begin{tabular}{|c|c|c|}
\hline Radiation & $-\mathrm{dE} / \mathrm{dx}, \mathrm{eV} / \mathrm{nm}$ & Spur Separation, nm \\
\hline \hline $0.01 \mathrm{MeV}$ electrons & 2.3 & 26 \\
\hline $0.1 \mathrm{MeV}$ electrons & 0.4 & 140 \\
\hline $1 \mathrm{MeV}$ electrons & 0.2 & 300 \\
\hline${ }^{60} \mathrm{Co} \gamma$ rays & 0.2 & 300 \\
\hline \hline $1 \mathrm{MeV}$ protons & 28 & 2.1 \\
\hline $10 \mathrm{MeV}$ protons & 4.7 & 13 \\
\hline \hline $1 \mathrm{MeV} \alpha$ particles & 264 & 0.23 \\
\hline $10 \mathrm{MeV} \alpha$ particles & 56 & 1.1 \\
\hline \hline
\end{tabular}

* Mean energy per spur taken as $60 \mathrm{eV}$

protons, which corresponds with the intense local damage produced by the $(B, n) \rightarrow(L i, \alpha)$ reaction and by knocked-on protons from neutron collisions.)

Despite the equivalence, in theory, of gamma and electron irradiation, considerable differences have been observed experimentally. However, if the irradiation is carried out in ambient air, different dose rates can cause a difference between the damage observed at a given dose for electron and gamma irradiation. The dose rate factor was used by Egusa [1991a] to explain the different results shown in Figure A.3-16 for neat resin specimens irradiated by either ${ }^{60} \mathrm{Co}$-gamma rays at $0.017 \mathrm{MGy} / \mathrm{h}$ or $2-\mathrm{MeV}$ electrons at $11.5 \mathrm{MGy} / \mathrm{h}$. Considerably more degradation was exhibited by the specimens irradiated with gamma rays. Since the bulky solid specimens were irradiated in air, regions near the surface could experience more oxidative degradation during the gamma ray irradiation because there was much more time for the oxygen to diffuse in. However, when glass-reinforced composites of the same TGDM resin system were irradiated with both gamma rays and electrons under the same conditions as the neat resins, no difference between the two types of irradiation was observed (Figure A.3-17). Egusa proposed that the wel1-known sensitivity of neat resin specimens to surface flaws (see also $\$ 1.4$ below) and the relative 
insensitivity of glass-reinforced composites to such flaws, could explain the dissimilarity in comparative electron-gamma irradiation results.

An effect opposite to that reported by Egusa on neat resins was reported by Nishiura et al. [1988a] in comparative irradiations of a commercial laminate. Both electron and gamma irradiations were performed at ambient temperature, to about $3.5 \times 10^{7} \mathrm{~Gy}$. More degradation from the electron irradiation than from the gamma irradiation was observed in both tear and acoustic emission tests conducted at $295 \mathrm{~K}(\$ 2.9)$ and at $77 \mathrm{~K}(\$ 3.5)$. The results are shown in Figures A.2-81 and A.2-82 (295 K) and Figures A.3-14 and A.3-15 (77 K). Differences in dose rate should not have caused the differences in radiation damage observed in these studies since the ${ }^{60} \mathrm{Co}$-gamma irradiation was performed more slowly than the electron irradiation, but the electron irradiation caused more degradation. Furthermore, when the dose rate of the 20-MeV electron irradiation was decreased, the damage remained the same. Cooling water was circulated to the electron-irradiated specimens. It appears that the only factor that could account for the difference is the higher energy level of the incident electrons. Incident electrons of higher energy level create more gamma rays and have a shorter interaction range than lower energy electrons ( $\$ 1.3 .2 .3)$. Whether either of these mechanisms affected the results is unknown. The different nature of the tests performed (tear and acoustic emission) could also be the cause of the discrepancy between these results and the flexural tests of Egusa et al. But the conclusion that can be drawn from the tests by Egusa and by Nishiura et al. is that the dose equivalence of electron and gamma irradiation of composites under all conditions remains unproven, and the two types of irradiation are not equivalent for neat resins, at least if the dose rates and incident energies are significantly different.

\subsubsection{Temperature}

1.3.3.1. Experimental Comparisons of Damage from Irradiation at Different Temperature

Comparisons of damage to identical specimens irradiated at different temperatures are not plentiful. For example, the experiments of Nishijima et al. [1988] on this point are confounded by the use of different temperatures 
to test specimens irradiated at ambient and cryogenic temperatures. Glassreinforced, TGDM epoxy specimens irradiated with neutrons at both $5 \mathrm{~K}$ and ambient temperature were tested at both 295 and $77 \mathrm{~K}$ by Egusa et al. [1984a; 1987b]. Figure 1.24 shows that the 295-K flexural test results were similar, up to the largest $5-\mathrm{K}$ dose of $2 \times 10^{7} \mathrm{~Gy}$. However, tests at $77 \mathrm{~K}$ indicated less damage from 5-K irradiation, followed by warm-up, than from ambient temperature irradiation. Since the irradiated composite was reinforced with E-glass, glass-epoxy interfacial damage from the $(B, n) \rightarrow(\mathrm{Li}, \alpha)$ reaction may have been the predominant contribution to failure. Such a failure mode is not relevant to the B-free glass reinforced composites that are expected to be used in future fusion reactors.

Comparative tests to examine the effects of the irradiation temperature have been done on B-free glass, however. Results of torsion tests of uniaxial, S-glass-reinforced rod specimens are given in Figure 1.25 [Munshi, 1991]. Specimens were irradiated at both 4 and $330 \mathrm{~K}$. The ambient-temperature irradiation was performed in a TRIGA reactor with about $22 \%$ of the dose from fast neutrons ( $E>0.1 \mathrm{MeV}) * * * * *$ and the remainder from gamma rays and lowerenergy neutrons [Munshi and Weber, 1992]. The 4-K irradiation was performed in the Garching FRM (Forschungsreaktor München), with about $20 \%$ of the dose from fast neutrons***** and about 50\% from gamma rays [Wallner et al., 1988; Gerstenberg and Gläser, 1992; Katheder et al., 1992; Munshi, 1992]. Both irradiation sources were fission reactors, so both neutron spectra were similar, although measurable differences in the fast neutron spectra have been reported [Weber et al., 1986]. The specimens irradiated at $4 \mathrm{~K}$ were warmed up before testing. The shear strength of these specimens at $76 \mathrm{~K}$ after a dose of about $2 \times 10^{8}$ Gy was found to be comparable for both irradiation temperatures for two resin types, an epoxy novolac (EPN) and a multifunctional epoxy, such as TGDM or TGPAP (triglycidyl p-amino phenol). (The chemical structures of EPN, TGDM, and DGEBA resins are diagrammed in Figure 1.26.) However, the deterioration in shear strength of the DGEBA resin was much greater when it was irradiated at $4 \mathrm{~K}$ than when it was irradiated at ambient temperature. Although DGEBA resin systems are commonly used for magnet impregnation because of their low viscosity (they are the basis, for example, of the Orlitherm

\footnotetext{
**** The fast-neutron percentage of the total dose was obtained from the numerical data provided on the total dose, the neutron flux or fluence, and the total irradiation time. See also Wallner et al. [1988]. The conversion rule for $\mathrm{E}>0.1 \mathrm{MeV}$ for epoxy, $1 \mathrm{~Gy}=10^{15} / \mathrm{m}^{2}$, was used.
} 
a

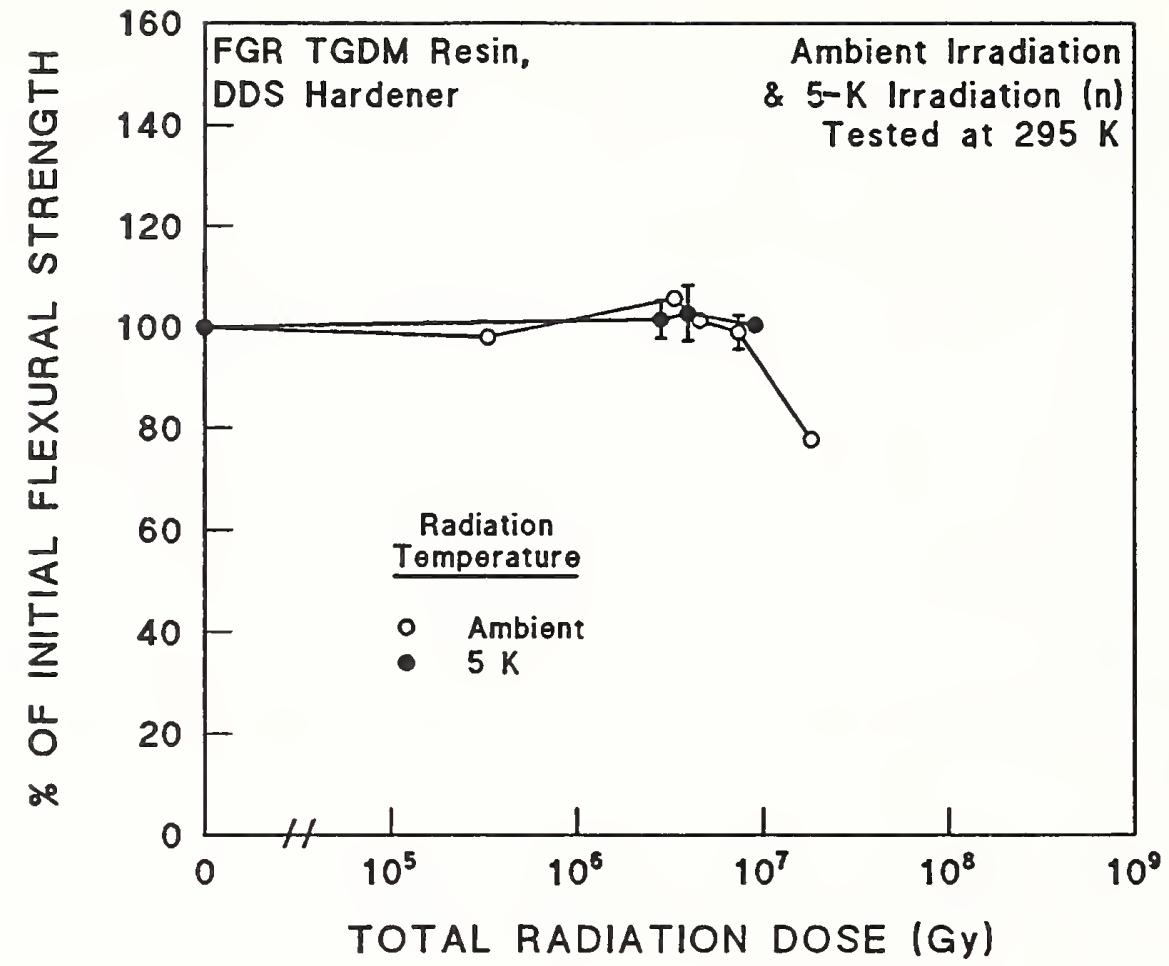

b

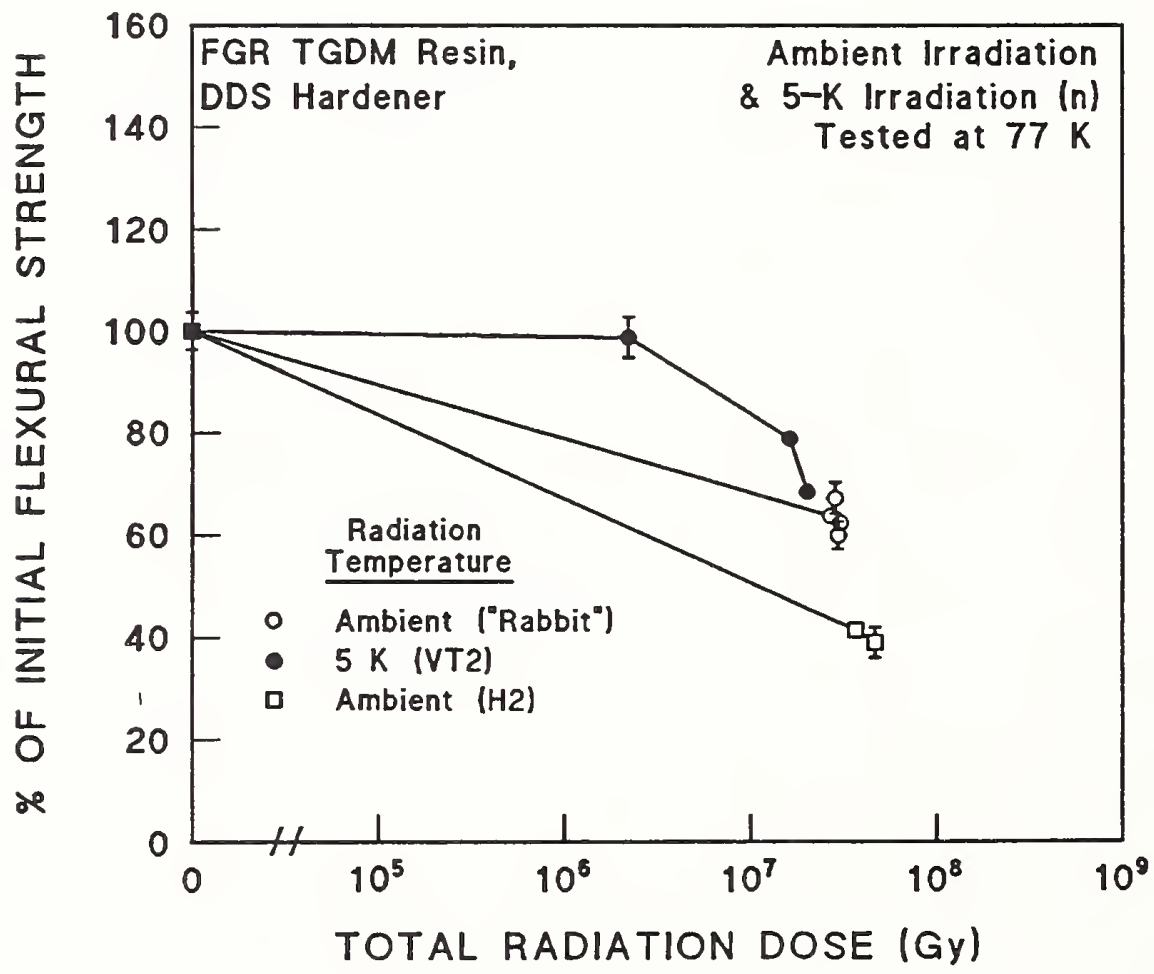

Figure 1.24. A comparison of flexural test results of a composite irradiated at both $5 \mathrm{~K}$ and ambient temperature with neutrons. See Figure 1.20 for a description of the composite. (a) Tested at $295 \mathrm{~K}$. (b) Tested at $77 \mathrm{~K}$. Data from Egusa et al. [1984a; 1987b]. (Supplementary Table B.1-1.) 


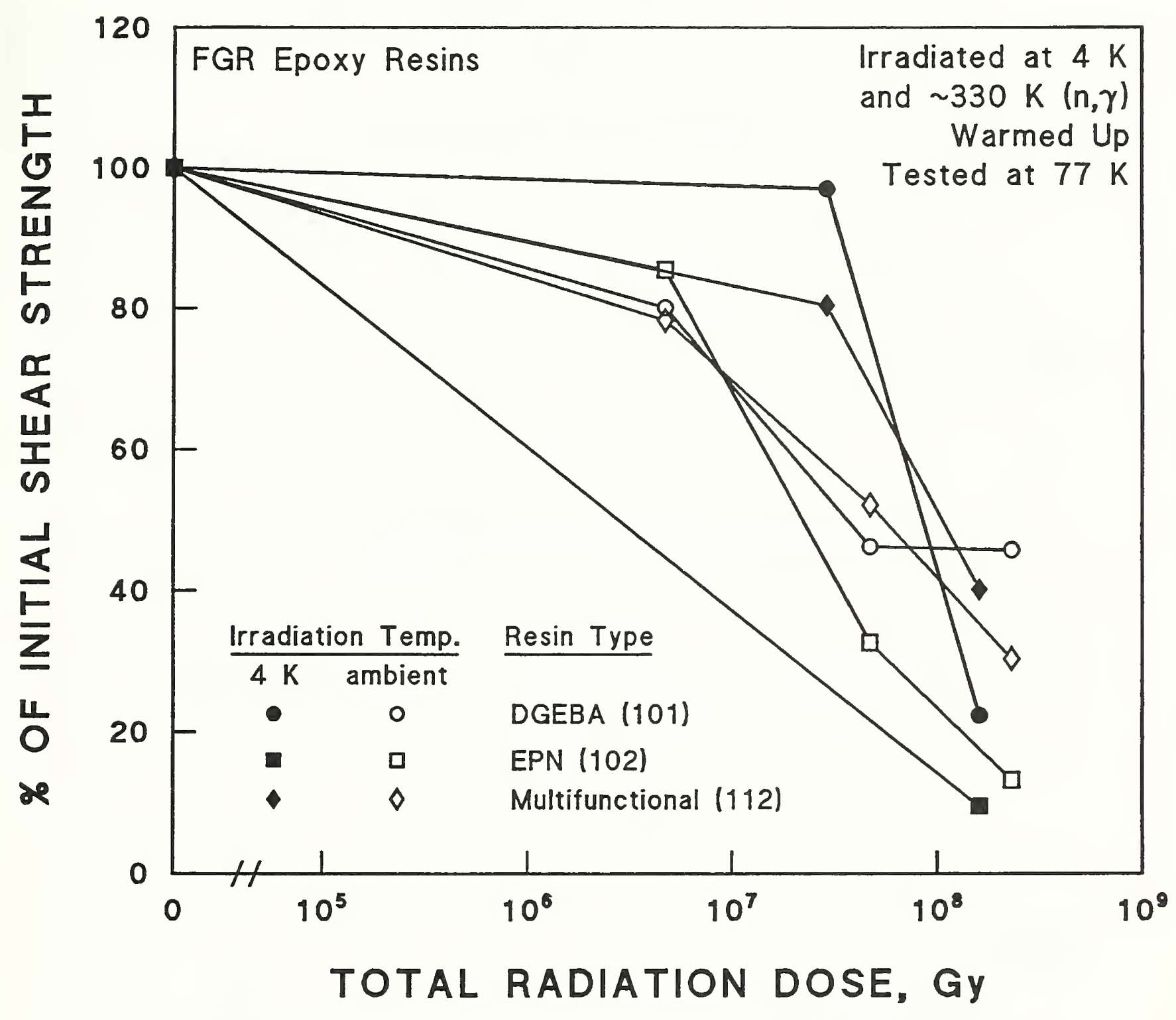

Figure 1.25. A comparison of the shear strengths of three types of reinforced epoxy resins that were reactor-irradiated at both $4 \mathrm{~K}$ and at ambient temperature. See text for differences in the fast neutron spectrum in the two reactors. Data from Munshi [1991]. (Supplementary Tables A.3-3 and A.8-4.) 


\section{DGEBA:}<smiles>CC(C)(c1ccc(OCC2CO2)cc1)c1ccc(OCC2CO2)cc1</smiles>

Diglycidyl Ether of Bisphenol A

\section{EPN:}<smiles>CCc1cccc(C(C)CC)c1OCC1COC(C)C1</smiles>

Polyglycidyl Ether of Phenol Formaldehyde Novolac

TGDM:<smiles>c1cc(N(CC2CO2)CC2CO2)ccc1CN(CC1CO1)CC1CO1</smiles>

Tetraglycidyl Diaminodiphenyl Methane

Figure 1.26. The chemical structures of two different types of resin systems tested by Munshi [1991] (Figure 1.25) and the most common multifunctional epoxy, TGDM. 
systems), this result indicates that it may not be conservative to extrapolate data from ambient-temperature irradiation of DGEBA magnet resins to $4 \mathrm{~K}$.

A comparison of Figure 1.25 with $1.24 \mathrm{~b}$ shows that in both experiments, the multifunctional epoxy (TGDM and CTD 112, respectively) exhibited less degradation after 4- or 5-K irradiation than after ambient irradiation, when testing was performed at $77 \mathrm{~K}$. However, the number of samples tested in both investigations was small, reproducibility of results has not been shown, and the multifunctional epoxy was compared to other epoxy types only in the experiment by Munshi. Therefore, it is premature to conclude that multifunctional epoxies are superior to EPN and DGEBA resins in cryogenic irradiation resistance. Nevertheless, these results highlight the need for further cryogenic irradiation testing of a variety of resin systems for purposes of optimization.

The results of Munshi on the increased degradation of a DGEBA resin system after cryogenic irradiation can be reconciled with results obtained by Morgan et al. [1970] on the increase in degradation in neat resins from irradiation when oxygen is present. Figure 1.27 shows that for a DGEBA resin with MNA or DDM (diamino diphenyl methane) as the hardener, degradation at a given dose is more severe when the gamma irradiation is carried out in air instead of in a vacuum. One might therefore expect that irradiation in a liquid inert gas such as helium would cause less degradation than ambient-temperature irradiation in air. However, the 4-K irradiated specimens of Munshi were warmed up before testing and frozen-in radicals would have reacted and frozenin gas would have diffused (see $\$ 1.4 .2$ below). Thus, a better comparison of the inert environment of cryogenic irradiation with vacuum or inert gas ambient irradiation would be obtained in specimens that were tested without warm-up.

Furthermore, Morgan et al. tested only neat resins, and Egusa [1991a] found that when composites, rather than neat resins, were irradiated either in ambient air or argon, no difference was found in flexure properties at $77 \mathrm{~K}$ (Figure A.3-13). The gamma irradiation dose from ${ }^{60} \mathrm{Co}$ in these tests ranged from about 6 to $9 \times 10^{7} \mathrm{~Gy}$. The commercial laminates G-10CR and G-11CR and a glass-reinforced DGEBA/DDM composite were tested. Although damage probably occurred at the specimen surface as a result of oxidative degradation after 

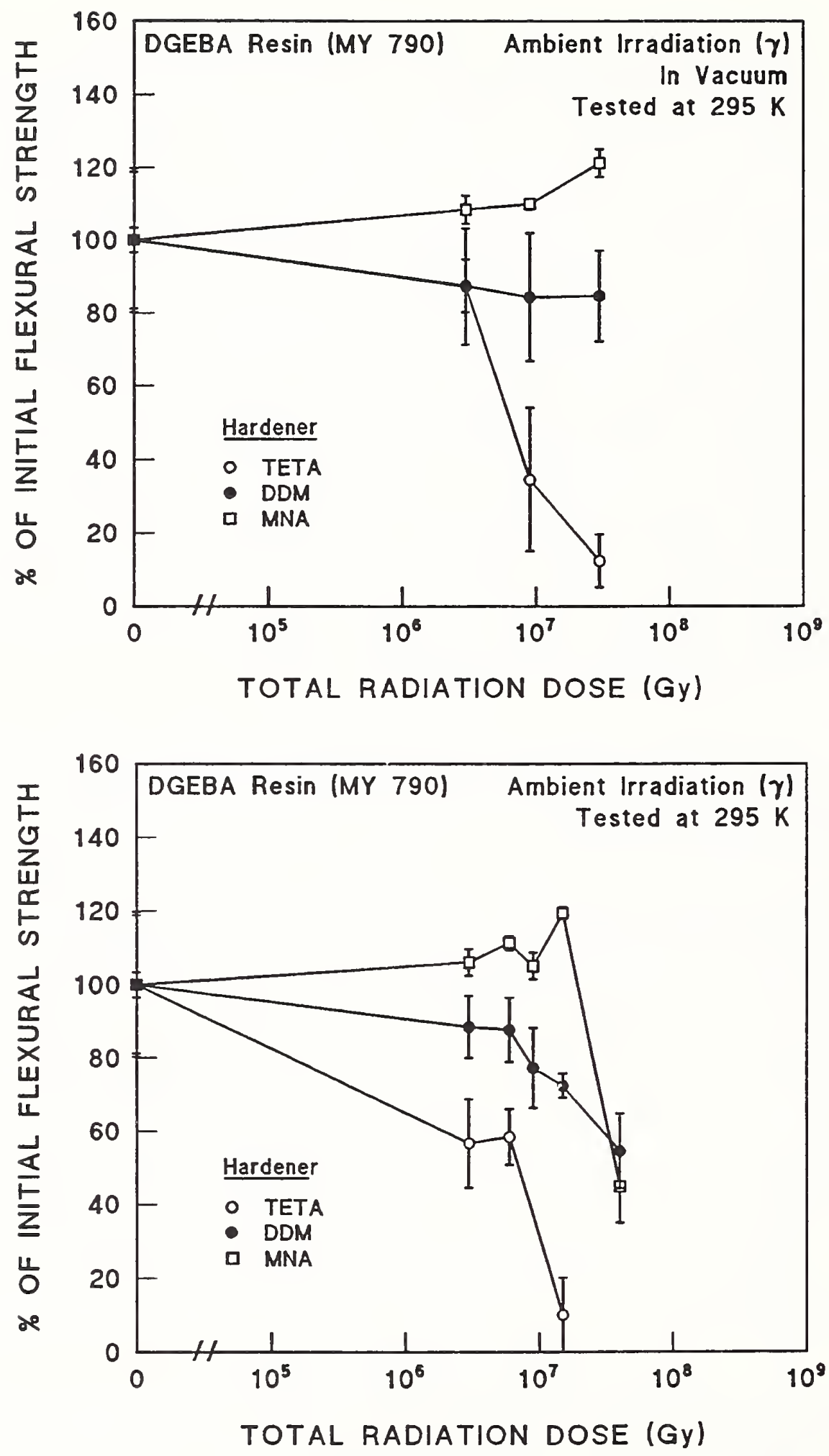

Figure 1.27. The increased degradation from irradiation when oxygen is present, compared to irradiation carried out in vacuum. Data from Morgan et al. [1970]. (Supplementary Table A.2-1.) 
irradiation in air, the flexural behavior of a composite is relatively insensitive to such surface flaws.

The use of neat resins probably explains the differences in flexural strength observed by Evans et al. [1971] on specimens irradiated in air and in liquid nitrogen. The differences are shown in Figures A.5-1 to A.5-4, and the experiment is discussed in $\$ 5$. The specimens were tested at ambient temperature after gamma irradiation in air or electron irradiation in flowing liquid nitrogen. However, there is a great deal of scatter in the results, inherent in neat resin tests, and also, owing to a lack of replication of the liquid nitrogen irradiations which were carried out on only one specimen of each type. Therefore, the conclusions of Evans et al., that the resin systems irradiated at $77 \mathrm{~K}$ showed less change than those irradiated in air, are not fully supported by all the data. Also, for a true comparison of the effects of temperature upon irradiation damage, inert atmospheres should be employed at both temperatures.

\subsection{EFFECT OF TEST TEMPERATURE AND WARM-UP}

\subsubsection{Comparison of Damage from Tests at Different Temperatures}

As noted above, many of the tests of irradiated insulation have been carried out at ambient temperature. Since the insulation for ITER must perform at $4 \mathrm{~K}$, the applicability of ambient and $77-\mathrm{K}$ screening tests to 4 -K properties is of interest. Figure 1.28 compares the results of $77-\mathrm{K}$ and ambient temperature tests [Egusa et al., 1988]. For two of three similarly reinforced resin systems irradiated with $2 \mathrm{-MeV}$ electrons at ambient temperature, the deterioration in flexural strength is more pronounced at $77 \mathrm{~K}$ for doses above about $5 \times 10^{7} \mathrm{~Gy}$. (The TGDM/DDM system does not exhibit deterioration at this dose at either temperature; this will be discussed further in §3.2.)

Additional data on one of these resin systems, DGEBF, also indicates increased degradation when testing is performed at $77 \mathrm{~K}$, in comparison with ambient temperature testing (Figure A.3-11, §3.2).

Egusa explained the difference in test temperature results of composites as follows. He had concluded from his earlier studies that the determining parameter in radiation damage was the ultimate strain of the composite matrix. 
a
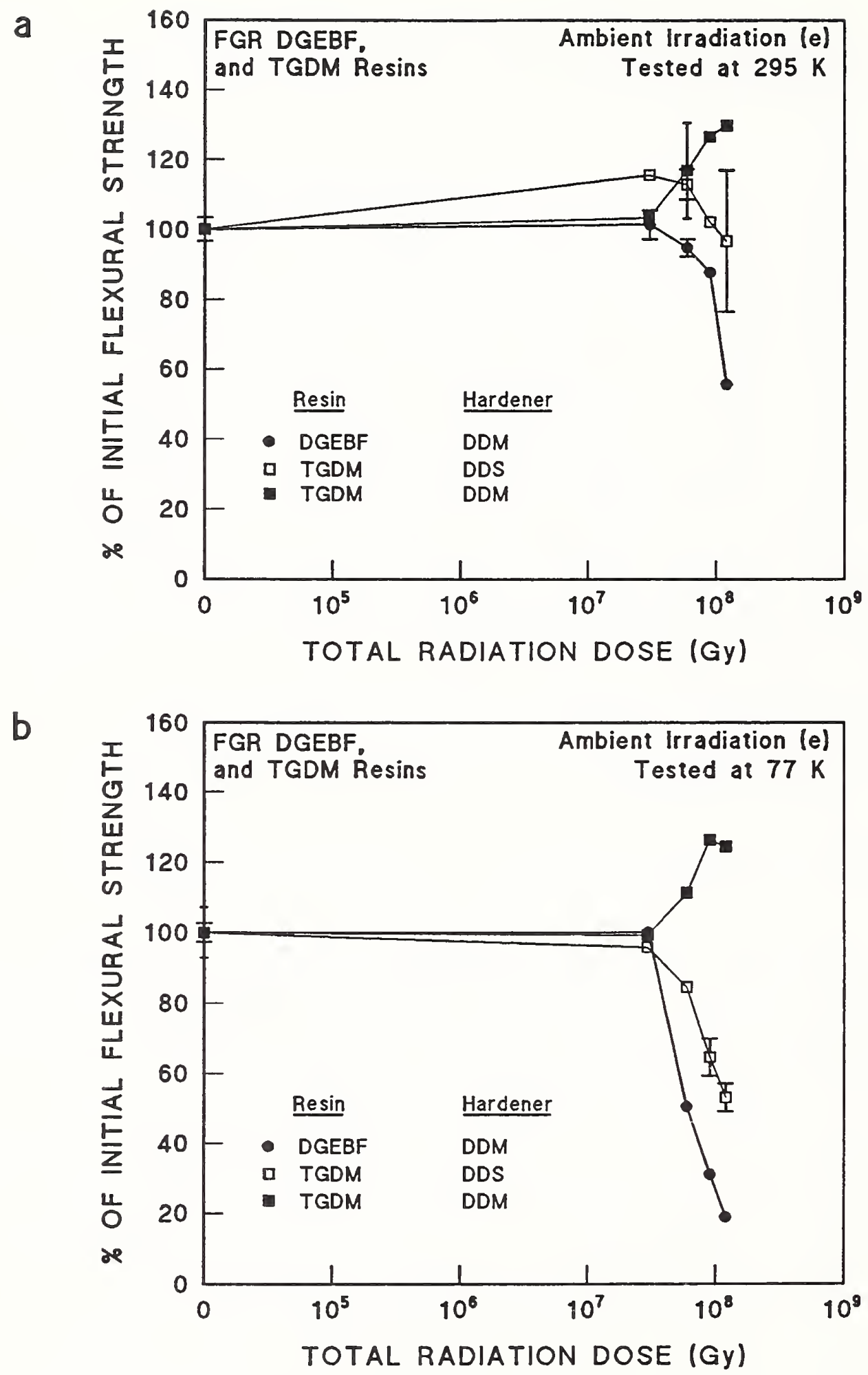

Figure 1.28. The increased degradation exhibited by flexural specimens tested at $77 \mathrm{~K}$ (b), compared with those tested at $295 \mathrm{~K}$ (a). Data from Egusa et al. [1988]. (Supplementary Table A.3-5.) See Figures B.2-14 and B.2-15 for initial strengths. 
As long as the matrix resin system was identical, Egusa found that the dosedependence of the flexural strength followed a similar pattern regardless of the type of reinforcing fiber or its surface treatment. If the matrix ultimate strain is hypothesized to be initially higher than the fiber ultimate strain, the strength of the composite will not be adversely affected by radiation until the matrix ultimate strain drops below that of the fiber. Figure 1.26 a shows that this occurs at a dose of about $9 \times 10^{7} \mathrm{~Gy}$. It also occurs upon cooling to $77 \mathrm{~K}$, because the matrix resin becomes more brittle, and, therefore, the composite is more radiation sensitive at $77 \mathrm{~K}$.

When composites with resin systems similar to those described above were tested at both 77 and $4 \mathrm{~K}$ after ambient gamma irradiation in air [Egusa, 1990a], the differences in degradation were much less pronounced than those observed between ambient temperature and 77-K testing [Egusa and Hagiwara, 1986]. Figure 1.29 shows the combined data from the two references at all three test temperatures. (Differences in behavior of the different resin systems are discussed below, in $\$ 2.1$ and 3.2.)

When Takamura and Kato [1980] tested a composite at both 77 and $4 \mathrm{~K}$, they also found little difference in radiation damage (Figure 1.28a). Composites were tested in compression, without warm-up to ambient temperatures, after combined neutron and gamma irradiation at $5 \mathrm{~K}$ to a dose of $1.1 \times 10^{7} \mathrm{~Gy}$. Both the results of Egusa et al. and those of Takamura and Kato indicate that extrapolation to $4 \mathrm{~K}$ of test results on composites at $77 \mathrm{~K}$ is more valid than the extrapolation to $4 \mathrm{~K}$ of test results at ambient temperature. However, Takamura and Kato [1980] also tested the same DGEBA resin used in the composite in neat form with various hardeners under the same conditions (Figure 1.30b). As discussed in $\$ 9$ (Figures A.9-1 and A.9-2), the relative percentage of the retained compressive strength at 4 and $77 \mathrm{~K}$ depended upon the hardener used, and some of the test-temperature differences were large. These results imply that $77-\mathrm{K}$ tests may not correctly indicate the 4-K retained strengths of neat resins.

In general, properties of composites improve significantly at $77 \mathrm{~K}$ in comparison with ambient values, but usually do not change as much between 77 and $4 \mathrm{~K}$. Higher strengths for composites at low temperatures result from the increased strength and stiffness of the resin matrix as the temperature 

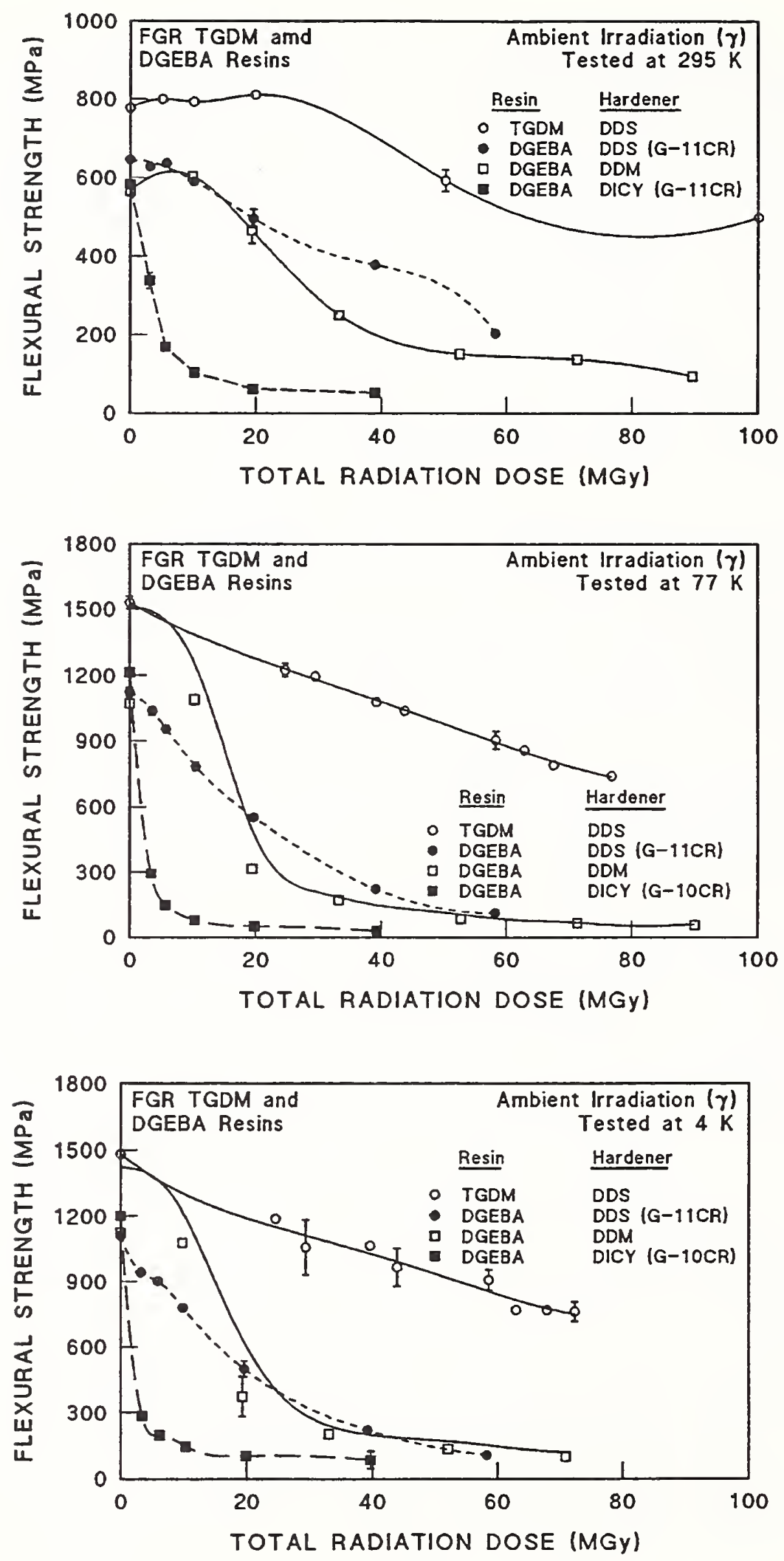

Figure 1.29. Data showing comparative flexural test results on the same composite systems at 295, 77, and 4 K. Data from Egusa and Hagiwara [1986] and Egusa [1990a]. (Supplementary Table A.3-2.) 
a
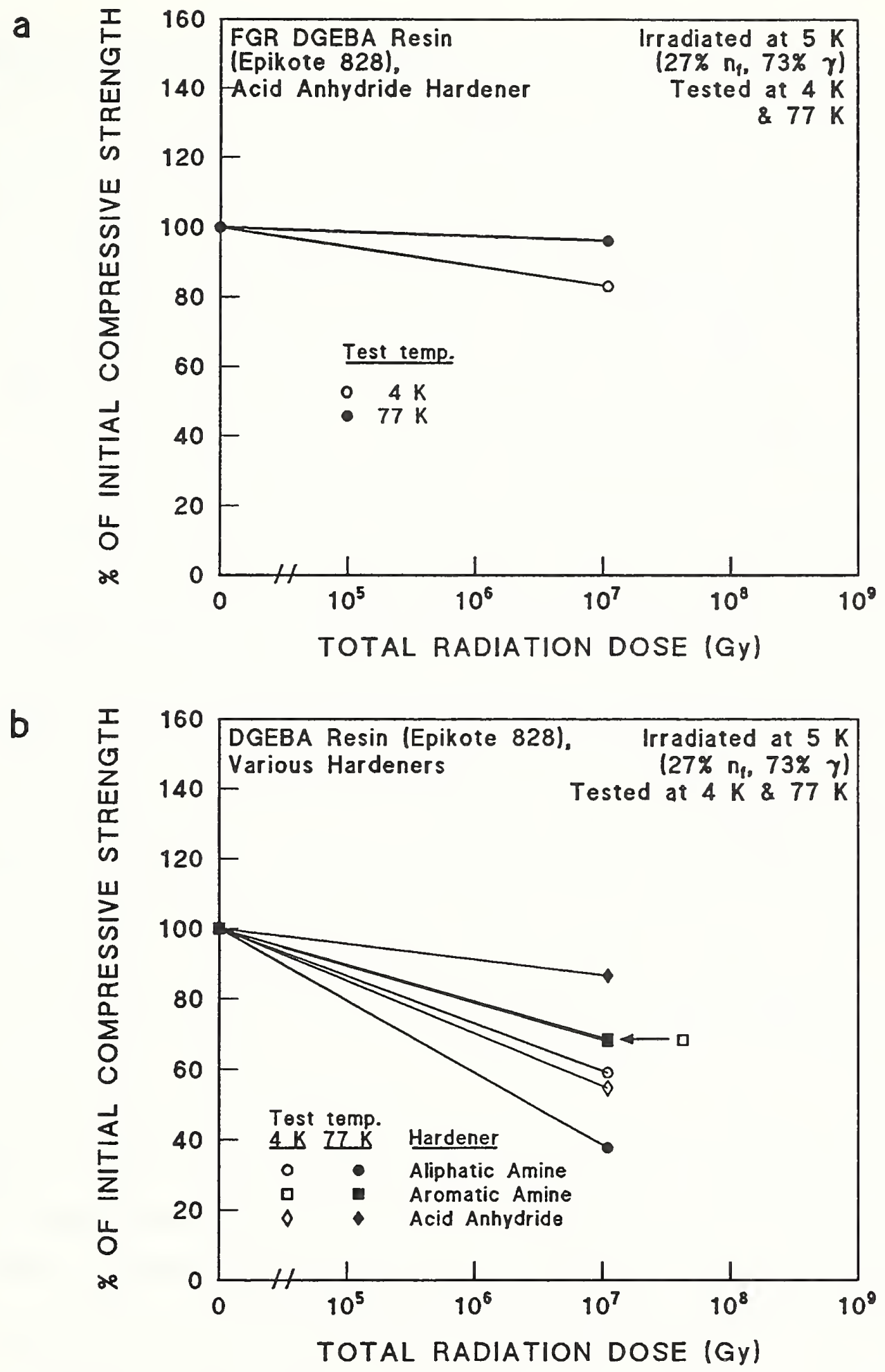

Figure 1.30. (a) A comparison of a glass-reinforced epoxy composite tested in compression at both 77 and $4 \mathrm{~K}$. (b) A comparison of the same epoxy resin system tested in neat form at both 77 and $4 \mathrm{~K}$. Data from Takamura and Kato [1980]. (Supplementary Table A.8-3.) 
decreases significantly below the glass transition temperature, which ranges from approximately 100 to $200^{\circ} \mathrm{C}$ for epoxies. The increased resin stiffness results from a decrease in mobility of the long polymer chains at lower temperatures. Increases in resin strength at low temperatures are associated with higher stiffness and with sufficient insensitivity to microcrack formation to permit larger elastic strains than at higher temperatures. (In general, there is little anelastic or plastic deformation of resins at low temperatures). Strength and stiffness increases may also be obtained, chemically, by increasing the cross-linking between long chain molecules, which also decreases chain mobility.

The increased cryogenic radiation sensitivity of composites, therefore, suggests that resin microcracking begins at lower strain levels or, alternatively, that the bond strength at fiber-matrix interfaces is reduced at low temperatures. Microcracks are expected to originate from the flaws or porosity formed from coalescence of radiation-induced defects. (Coalescence may occur during ambient-temperature irradiation or warm-up of specimens subsequently tested at cryogenic temperatures.) Fractures may also originate from debonding at the fiber-matrix interface. Interface debonding provides sites for microcrack formation and reduces the capability for load transfer through the matrix between the stronger, glass-fiber component of the composite. Reduction of the fiber-matrix bond strength also increases the difficulty of accommodating radiation-induced residual strains at the interface.

When the neat resin as well as the composite was tested in flexure at both ambient temperature and $77 \mathrm{~K}$, the neat resin did not exhibit the increased radiation sensitivity at $77 \mathrm{~K}$ that the reinforced resin showed [Egusa, 1988] . The resin system studied was TGDM/DDS (diamino diphenyl sulfone). Ambient temperature irradiation from $2-\mathrm{MeV}$ electrons or ${ }^{60} \mathrm{Co}$-gamma rays was administered in doses up to 1.4 to $1.6 \times 10^{7}$ Gy. Figure A.3-1 shows that the E-glass reinforced composite exhibited a sizable difference in radiation sensitivity at the two test temperatures, in accord with Figures 1.28 and 1.29, above. However, as Figure A.3-2 indicates, this difference was not observed in the neat resin. The difference in post-irradiation strength with test temperature is evidently correlated with the change in flexural strength with temperature of the materials tested by Egusa. The flexural strength of 
the TGDM/DDS composite was considerably larger at $77 \mathrm{~K}$ than at ambient temperature, but the strength of the neat resin did not change much with temperature. Since some neat resin systems do show significant increases in flexural strength at $77 \mathrm{~K}$ [Darr, Fabian, and Reed, 1992], the apparent radiation resistance of these neat resins could also vary significantly with the test temperature, as the resistance of the composites tested by Egusa did. Therefore, Egusa's results on one neat resin system may not apply to all such systems.

If it is true that a change in apparent radiation resistance with test temperature requires a significant change with temperature of the property that is measured, the results of several other groups are puzzling at first glance because they appear to contradict this principle. All of these groups tested composites at $77 \mathrm{~K}$ and ambient temperature, and in all cases, the property measured increased significantly at $77 \mathrm{~K}$ over its ambient temperature value. However, in general, no significant effect of the test temperature was observed. The following review of these experiments shows that in every case where little or no effect was observed, the irradiation was not carried out to a dose high enough to cause significant deterioration of the property measured. Therefore, no difference in the degradation with test temperature could be observed.

Tensile tests were made at both ambient temperature and at $77 \mathrm{~K}$ after ambient temperature, ${ }^{60} \mathrm{Co}$-gamma or $20 \mathrm{MeV}$ electron irradiation of a glass-cloth reinforced resin (Epomik) by Nishiura et al. [1988b]. For the case of gamma irradiation, a somewhat greater degree of degradation was observed for the 77-K tests than for the ambient-temperature tests, but essentially no effect of irradiation at either test temperature was observed with the electronirradiated specimens (Figures A.2-83 and A.3-16). However, the maximum dose was $10^{7} \mathrm{~Gy}$, and often, significant degradation of composites takes place only above this dose (e.g., Figure A.3-1). Therefore, unlike the investigations of Egusa, these experiments did not furnish a significant probe of the differences in apparent radiation resistance with test temperature.

The commercial laminate Lamiverre A also was tested in tension at both ambient temperature and $77 \mathrm{~K}$ after ambient temperature, ${ }^{60} \mathrm{Co}$-irradiation [Nishiura et al., 1988a]. Figure A.3-4 shows that no difference was observed, again 
perhaps because the dose of $2 \times 10^{7}$ Gy was below that required for significant degradation. Figure A.3-1 from Egusa [1988] shows that very little deterioration of a TGDM/DDS composite occurs at this dose for similar irradiation and test conditions. However, the resin system used in Lamiverre A was not reported.

Dahlerup-Peterson and Perrot [1979] (see also Dahlerup-Peterson [1980]) also tested composites in tension at both ambient temperature and $77 \mathrm{~K}$ after ambient temperature, ${ }^{60} \mathrm{Co}$-irradiation to $1.7 \times 10^{7} \mathrm{~Gy}$. Both of the two composites tested were made from a bisphenol-A resin system; one was unidirectionally reinforced with $\mathrm{E}$ glass, the other, a commercial material, was reinforced with E-glass cloth. A comparison of Figures A.2-94 and A.3-3 shows a slight deterioration in tensile strength of both composites at $77 \mathrm{~K}$, but no degradation when tested at ambient temperature. This result is in qualitative agreement with that of Egusa, but the magnitude of the effect is much smaller, again, probably because the irradiation was not carried out to a dose high enough to reveal significant degradation, and possible differences in degradation with the test temperature.

One other group measured the effect of test temperature. However, in contrast to the investigations discussed above, these irradiations were performed at 4 to $5 \mathrm{~K}$ with neutrons rather than at ambient temperature with gamma rays or electrons [Tucker et al., 1985]. The difference between results of flexural tests at 75 and $300 \mathrm{~K}$ after $4-\mathrm{K}$ neutron irradiation appeared to depend upon the resin system used. The commercial laminates tested, G-10CR and G-11CR, differ mainly in the resin system, since glass weave, finish, and glass volume fraction are similar. Figure 1.31 shows that the laminate G-10CR exhibited much more severe degradation when tested at $75 \mathrm{~K}$ than when tested at $300 \mathrm{~K}$, but there was much less degradation of G-11CR at the maximum dose, and the test temperature did not appear to be a significant factor. However, the maximum dose was about $3 \times 10^{6} \mathrm{~Gy}$, below the range at which significant degradation is exhibited by G-11CR (Figure 1.29). Therefore, again, irradiation was not carried out to a dose high enough to determine the effects of test temperature, and the results of Egusa on this subject were not contradicted. 

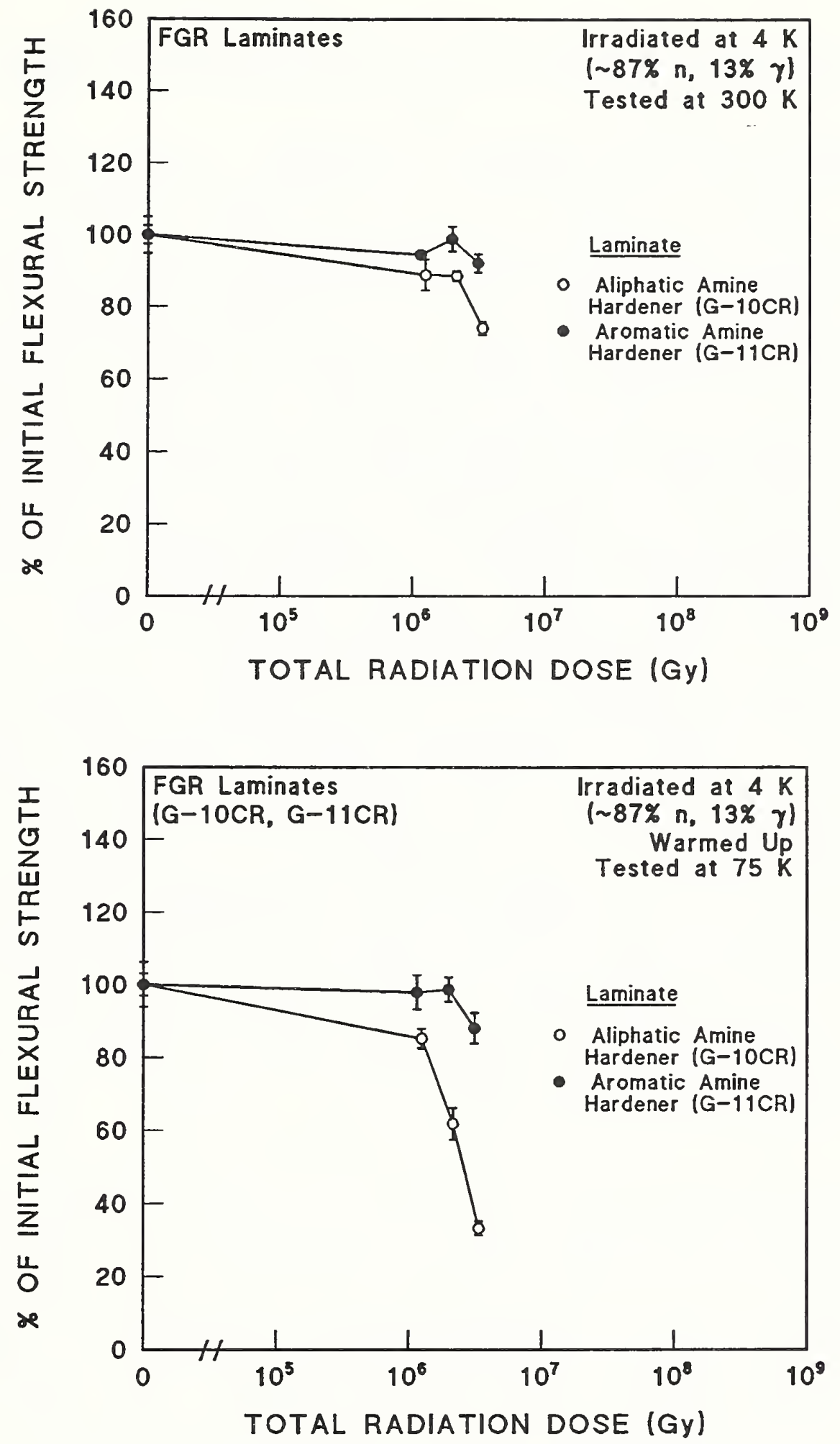

Figure 1.31. A comparison of two commercial laminates tested at both 300 and $75 \mathrm{~K}$ after neutron irradiation at $4 \mathrm{~K}$. Data from Tucker et al. [1985]. (Supplementary Table A.8-1.) 


\subsubsection{Comparison of Damage from Tests with and without Warm-up}

\subsubsection{Chemical Effects of Ambient Irradiation}

To understand the effects of specimen warm-up before testing, the chemistry of radiation damage must first be briefly discussed. Neutrons impart energy directly to impacted atoms by collision and generate high-energy protons in typical epoxies; protons, like gamma rays or electrons, can then interact with orbital electrons through Coulomb interactions. Like gamma rays and electrons, protons can ionize molecules or excite them to a higher energy level. The amount of energy carried by the proton or the irradiating species is generally more than sufficient to effect ionization $(-30 \mathrm{eV})$ or to break covalent bonds in epoxy molecules $(-5 \mathrm{eV})$ [Van de Voorde, 1970]. The effects of ionization, the removal of an electron from an atom in a molecule, have been discussed in the literature less frequently than the effects of cleaving covalent bonds. However, a table of the effects of ions in irradiated polyethylene includes both cross-linking and gas evolution, the major modes of damage produced after bond cleavage, when free radicals are formed [Van de Voorde, 1970]. Figure 1.16 shows the formation of free radicals as a result of division of main or side chain bonds. A free radical contains an electron that is unpaired with respect to its spin state; it is therefore very reactive. Examples of free radical reactions of epoxy polymers are shown in Figure 1.32; they include (1) cross-linking, (2) unsaturation, (3) degradation or chain scission, and (4) gas evolution.

The gases evolved from epoxy resins are mainly hydrogen, carbon monoxide, and carbon dioxide. Small amounts of ethane and methane are also produced. The amount evolved is expressed by the $G$ value, defined as the number of chemical changes produced by $100 \mathrm{eV}$ of absorbed energy. The relative $\mathrm{G}$ values of the gases produced depend upon the chemical type of both the resin and hardener, but most strongly upon the hardener [Evans and Morgan, 1982, 1984, and citations therein]. Table 1.6 shows the relative G-values for three major resin systems, each with three commonly used hardeners. Chemical structures of the three resin types listed in the table, DGEBA, EPN, and TGDM, were shown in Figure 1.26, along with the complete chemical name of the resin. Figure 1.33 shows the chemical structure of several common hardeners, including the three types used in the resin systems of Table 1.6. For all three resin 
i) Cross-linking: the formation of chemical bond between two polymeric chains
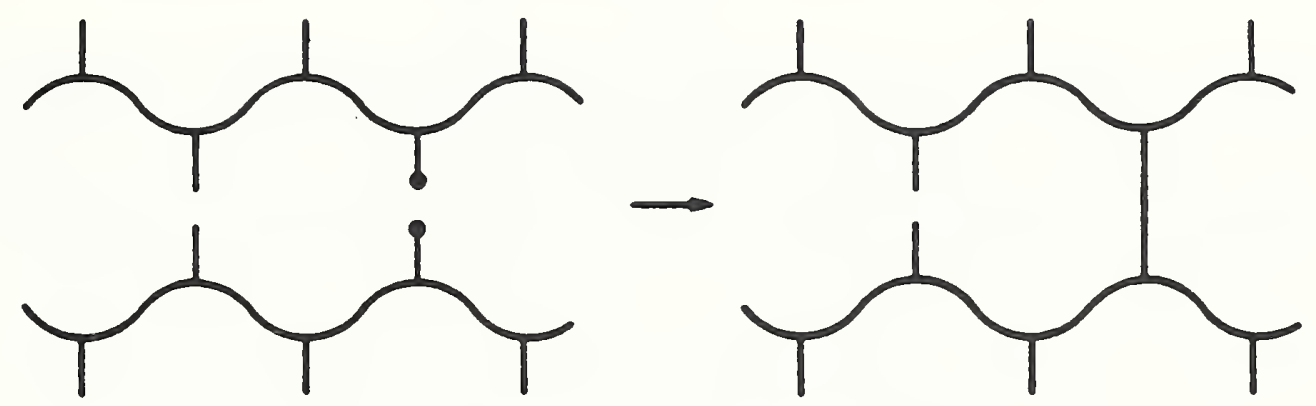

ii) Unsaturation: the formation of double chemical bonds within the polymer chain
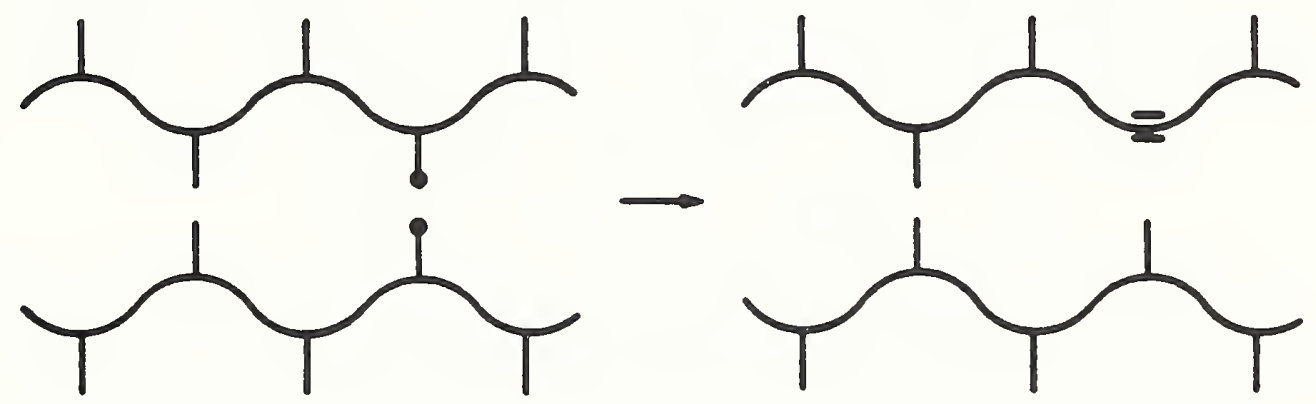

iii) Degradation: the fracturing of polymer molecules

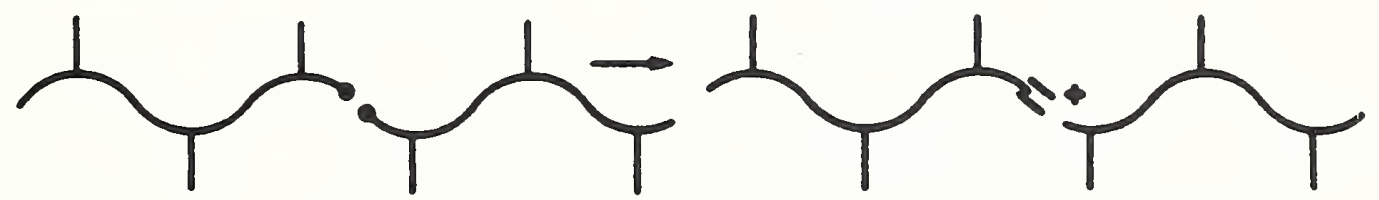

iv) Gas evolution: results in the rupture of small molecules from the polymer

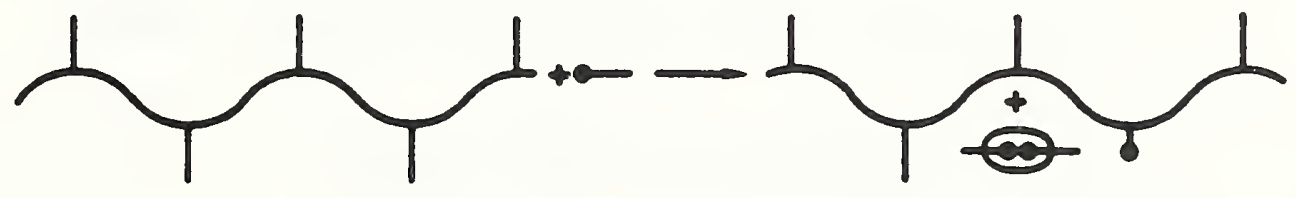

(gas)

Figure 1.32. Schematic examples of free-radical reactions in polymer chains: cross-linking, unsaturation, chain scission, and evolution of gas. 
<smiles>CCC1C2CCCC1C1C(=O)OC(=O)C21</smiles>

Methyl nadic anhydride (MNA)

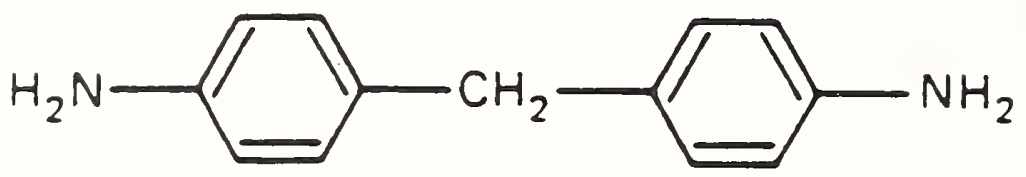

Diaminodiphenylmethane (MDA)

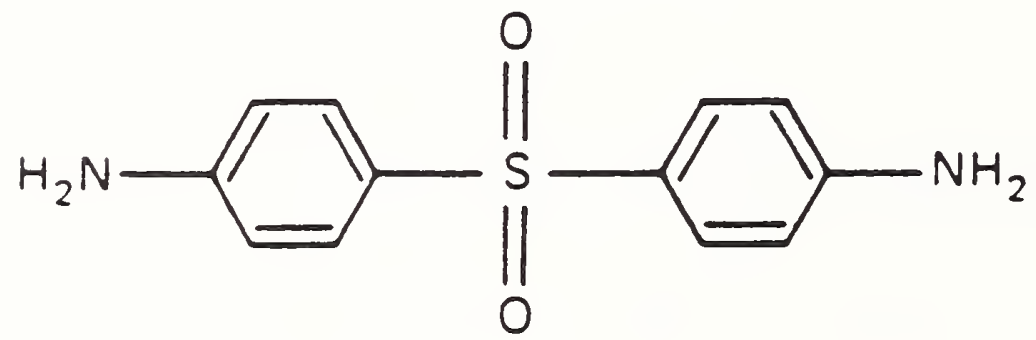

Diaminodiphenylsulfone (DDS)<smiles>NCCNCCNCCN</smiles>

Triethylenetetramine (TETA)

Figure 1.33. Chemical structure diagrams of four common hardeners, or curing agents: MNA, DDM, DDS, and TETA. 
Table 1.6. G Values* for Gases Evolved from Epoxy Resins by High Energy Radiation. (Data from Evans and Morgan [1984].)

\begin{tabular}{|c|c|c|c|c|c|c|c|c|c|}
\hline RESIN & \multicolumn{3}{|c|}{ DGEBA } & \multicolumn{3}{|c|}{ EPN } & \multicolumn{3}{|c|}{ TGDM } \\
\hline HARDENER & TETA & DDM & MNA & TETA & DDM & MNA & TETA & DDM & MNA \\
\hline Hydrogen & 0.21 & 0.11 & 0.10 & 0.21 & 0.10 & 0.10 & 0.23 & 0.10 & 0.14 \\
\hline $\begin{array}{l}\text { Carbon } \\
\text { Dioxide }\end{array}$ & 0.001 & 0.0014 & 0.35 & 0.001 & - & 0.33 & - & - & 0.38 \\
\hline $\begin{array}{l}\text { Carbon } \\
\text { Monoxide }\end{array}$ & 0.037 & 0.028 & 0.14 & 0.017 & 0.011 & 0.15 & 0.019 & 0.018 & 0.082 \\
\hline Ethane & 0.001 & 0.0015 & - & 0.0007 & - & - & - & 0.0003 & - \\
\hline Methane & 0.0037 & 0.0015 & - & 0.0026 & 0.0002 & - & 0.0022 & 0.0026 & - \\
\hline
\end{tabular}

* G Value $=$ No. of Chemical Changes $/ 100 \mathrm{eV}$ of absorbed radiation

types, the $G$ value for hydrogen with the aliphatic amine hardener TETA (triethylene tetramine) is about twice that obtained with the aromatic hardener DDM (diamino diphenyl methane). The $G$ value for hydrogen with the commonly used anhydride hardener, MNA, is similar to the G value obtained with the hardener DDM, but the MNA-cured resins also general carbon monoxide, from degradation of ester linkages, and carbon dioxide, from carboxylic acid groups [Evans and Morgan, 1984]. Thus, the largest volume of gas is generated from resins cured with anhydride agents. Since anhydride hardeners such as MNA significantly lower the viscosities in the resin system, this hardener is commonly used for vacuum impregnation. However, MNA-hardened systems may be less suited to cryogenic use (in a radiation environment) if warm-up will occur. If resin systems that generate a large volume of gas are used, provisions for removal of the gas upon warm-up must be considered.

\subsubsection{Chemical Effects of Cryogenic Irradiation}

All of the initial chemical reactions discussed in the preceeding section occur at cryogenic irradiation temperatures at about the same rate as at ambient temperatures. However, free radicals and ions are frozen into the polymer structure, because diffusion is restricted. Upon warm-up, gases may be formed and released, perhaps explosively in confined systems [Condit and Van Konynenburg, 1978, cited in Banford, 1984]. The resin may expand or crack 
as a result of the sudden release of gas formed after cryogenic irradiation, whereas the same volume of gas could have gradually diffused away during ambient irradiation without severe damage. Furthermore, the storage and release during warm-up of energy from atoms displaced during irradiation has not been investigated for organic materials, although stored energy phenomena are well-known in metals [Chaplin et al., 1989]. Thus, the use of ambient irradiation data for fusion reactor design is questionable. In the ITER conceptual design, 10 warm-up cycles are expected during the reactor lifetime, and 5 such cycles are expected during the lifetime of a commercial reactor [Henning and Miller, 1989]. These numbers may change as the shield design is modified [El-Guebaly and Sawan, 1989]. However, warm-up may also occur during loss of coolant and other circumstances.

\subsubsection{Results of Tests Conducted with and without Harm-up}

In a fusion reactor, the irradiated insulation must perform satisfactorily both before and after warm-up. However, most epoxy-based composites irradiated at low temperatures have been warmed up before testing. Furthermore, some warm-up phenomena may be especially severe in large, confined systems such as a TF magnet and may be unobservable in tests of sma11, unconfined specimens. Nevertheless, a few experiments have attempted to address the issue of warm-up. In addition, Banford [1984] has compared experimental results from two different laboratories in an attempt to answer this question. He compared the compression strength of E-glass-fiber reinforced Epikote 828 irradiated at $5 \mathrm{~K}$ and tested at $77 \mathrm{~K}$ [Takamura and Kato, 1980] with that of silica-filled Epikote 828 irradiated at $4.9 \mathrm{~K}$ and tested at $78 \mathrm{~K}$ after warm-up to $307 \mathrm{~K}$ [Coltman et al., 1979]. Epikote 828 is a DGEBA resin; it was used with an acid anhydride hardener by Takamura and Kato and with a modified aromatic polyamine hardener ("Z") by Coltman et al. Both sets of data are reproduced in Figure 1.34. Banford concluded that less degradation was seen in the tests without warm-up. However, as Figure 1.34 indicates, the specimens irradiated under both conditions experienced a combined neutron and gamma dose in fission reactors. Employing the usual conversion rule, the total " $L$ " dose in the experiments of Coltman et al. [2.4 $\times 10^{7}$ Gy $(\gamma) ; 2.2 \times 10^{5}$ Gy $\left.(n)\right]$ is probably more comparable to the dose of Takamura and Kato $\left[0.8 \times 10^{7} \mathrm{~Gy}(\gamma) ; 0.3 \times 10^{7} \mathrm{~Gy}(\mathrm{n})\right]$ than their "H" dose, 
a

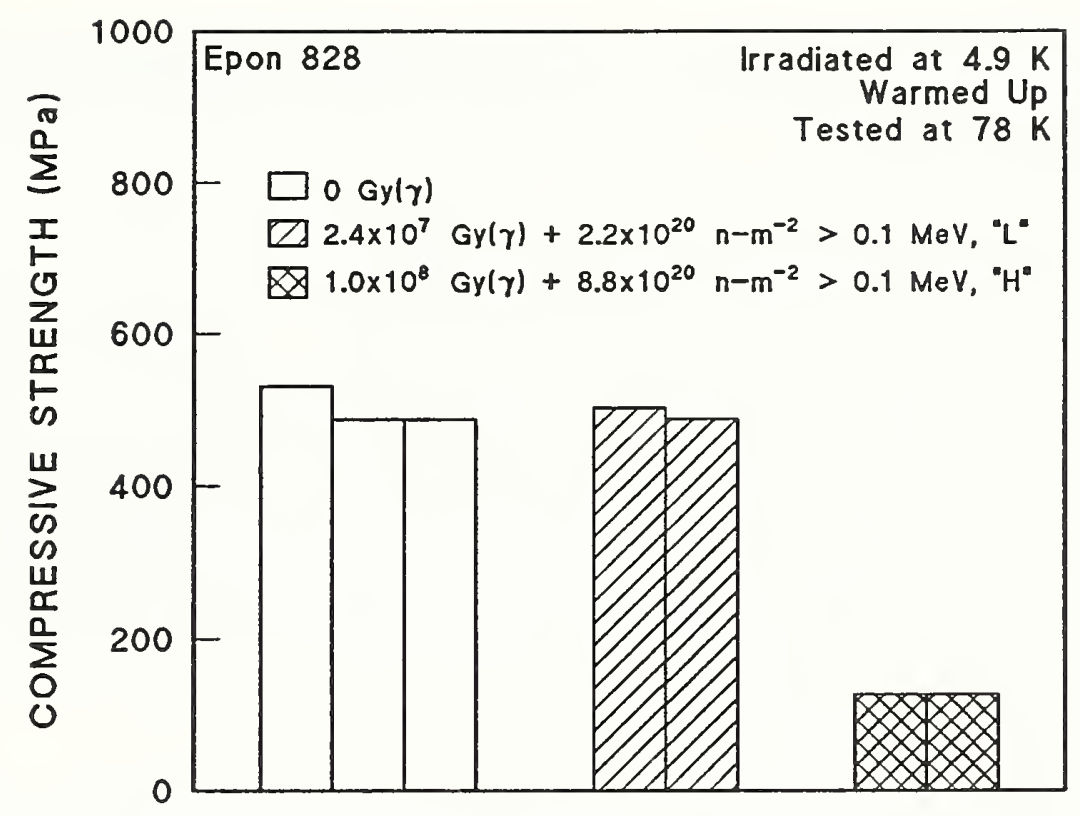

b

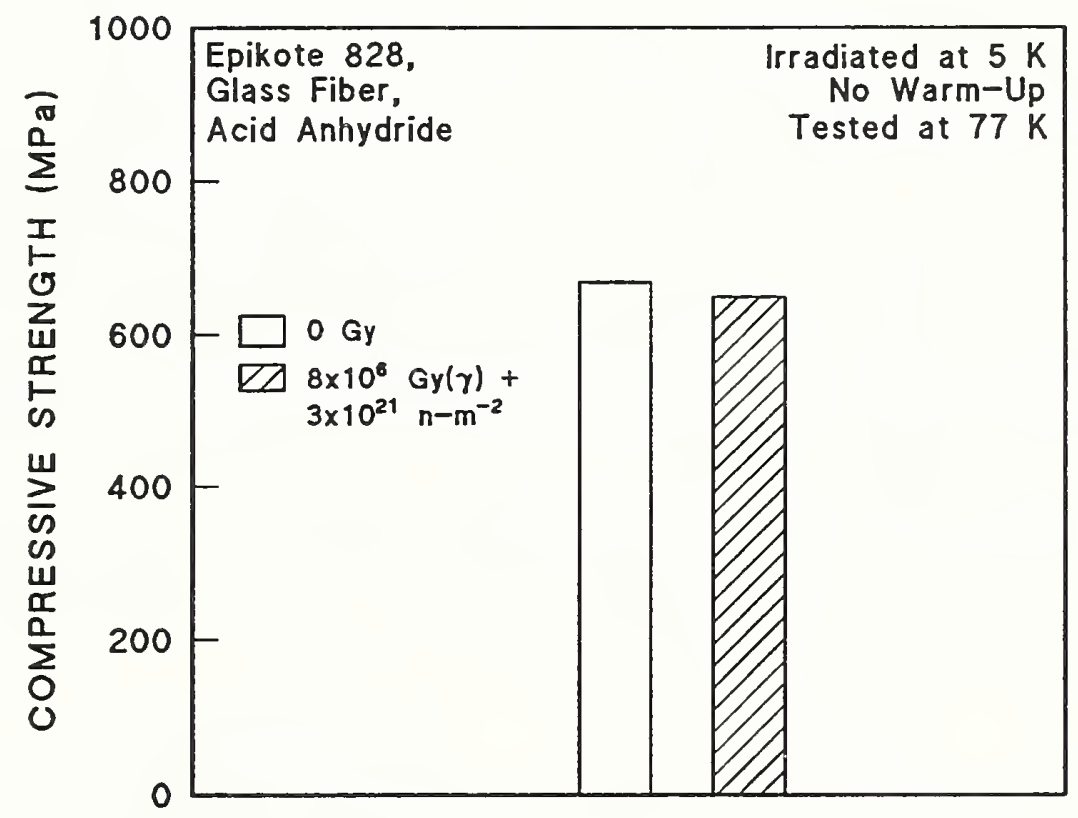

Figure 1.34. A comparison of 77-K compression test data on a DGEBA resin reinforced with a filler (a) or with fiber glass (b). The data in (a) were obtained by Coltman et al. [1979] after irradiation at $4.9 \mathrm{~K}$ and warm-up to ambient temperature; the data in (b) were obtained without warm-up by Takamura and Kato [1980]. The comparison was made by Banford [1984]. (Supplementary Tables A.8-2 and A.8-3.) 
which is $10^{8} \mathrm{~Gy}$ in total. If the " $\mathrm{L}$ " dose is used for comparison, the results of tests with and without warm-up are actually quite similar. Banford seems to have made the comparison with regard to the neutron dose only. In this case, the " $\mathrm{H}$ " neutron fluence of $0.88 \times 10^{21} / \mathrm{m}^{2}$ is more than a factor of three lower than the neutron fluence of Takamura and Kato, $3 \times 10^{21} / \mathrm{m}^{2}$. However, the gamma dose in the experiments of Coltman et al. clearly exceeds the neutron dose by a factor of 100 ; therefore, it probably governs the damage. Nevertheless, it is difficult to conclude anything on the effect of warm-up from the results of these two experiments, because a reactor neutron dose was present and only one of the specimen types compared was reinforced with B-containing glass.

Fortunately, Kato and Takamura [1979] carried out one series of tests in which the same neat DGEBA resin (Epikote 828 ), cured with an aromatic amine, was irradiated at $5 \mathrm{~K}$ and tested in compression at $77 \mathrm{~K}$ both with and without warm-up. (See also Takamura and Kato [1981].) The compressive strength was approximately a factor of two higher for the specimens that were warmed up before testing after irradiation at the highest dose of $1.1 \mathrm{x} 10^{7}$ Gy (Figure 1.35). Although it is not clear from the report of this work that the same aromatic amine hardener was used in the same amount in both sets of specimens tested with and without warm-up, and the highest dose of $1.1 \times 10^{7}$ Gy is below that proposed for ITER TF coils, perhaps the results are indicative of some degree of annealing, or recovery of radiation damage, in neat resins. Such warm-up results, in small, unconfined specimens, do not disprove more severe consequences of warm-up in larger, confined systems.

In contrast to these results on a neat epoxy resin, Van de Voorde [1973, and citations therein] reported that polytetrafluoroethylene irradiated at $20 \mathrm{~K}$ to $5 \times 10^{6}$ Gy retained good tensile strength when stored and tested at $20 \mathrm{~K}$, but when stored and tested at $300 \mathrm{~K}$, severe degradation occurred (Figure $1.36 \mathrm{~b}, \mathrm{c})$. However, the result cited by Van de Voorde does not completely take account of the improvement in properties at cryogenic temperatures from ambient temperature values. Thus, although the measured post-irradiation tensile strength at $300 \mathrm{~K}$ is very low compared to that measured at $20 \mathrm{~K}$, about 30 to 408 of the initial 300-K tensile strength was retained, based on data on nonirradiated specimens from another laboratory (Figure 1.36a). 


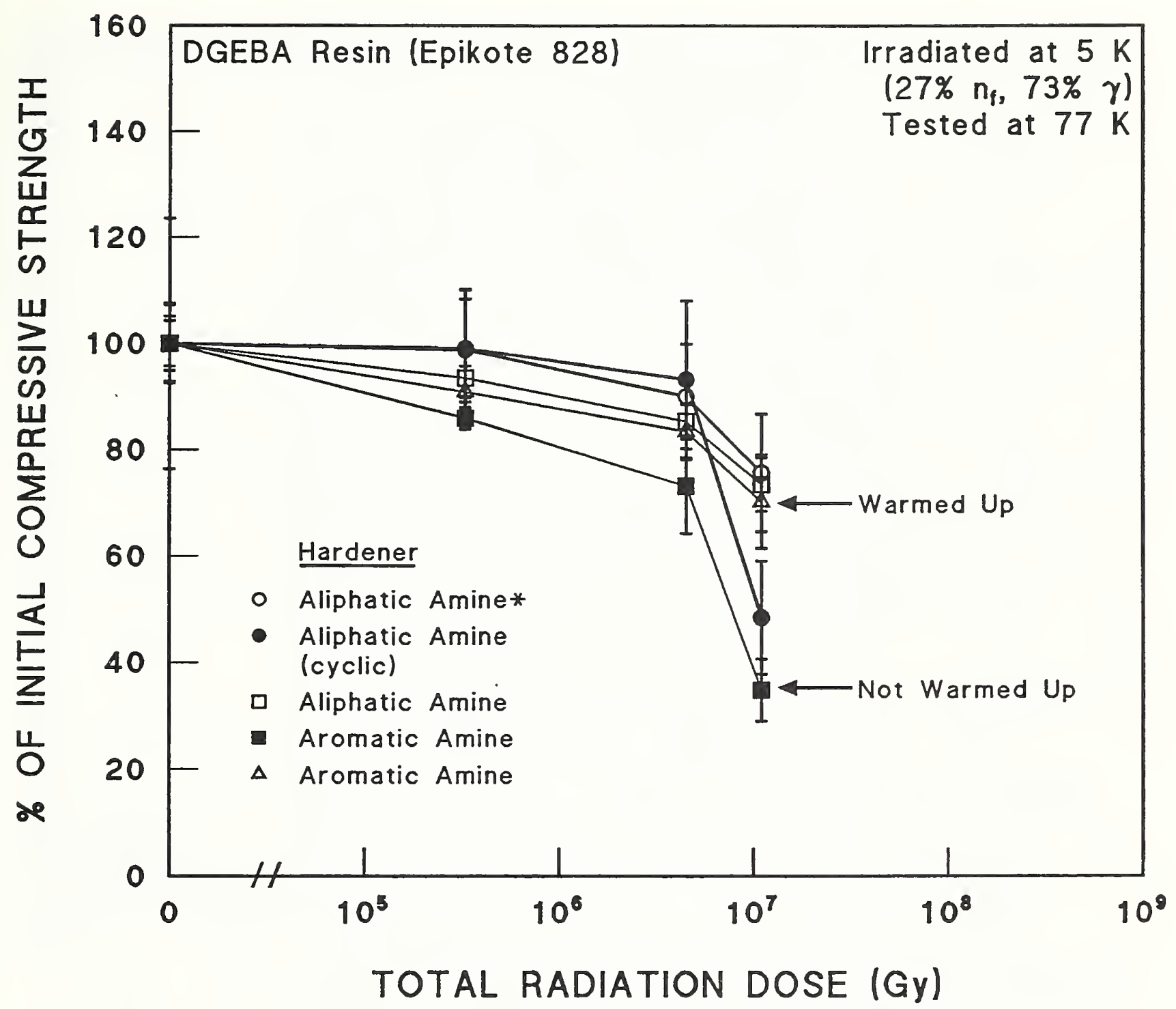

Supplier

Shell

Shell

Shell

Shell

Shell

* Filled

Figure 1.35. A comparison of compression tests at $77 \mathrm{~K}$, with and without warm-up to ambient temperature, on specimens irradiated at $5 \mathrm{~K}$. The tests were made on a neat DGEBA resin cured with an aromatic amine. Data from Kato and Takamura [1979]. See also Takamura and Kato [1981]. (Supplementary Table A. 8-3.) 


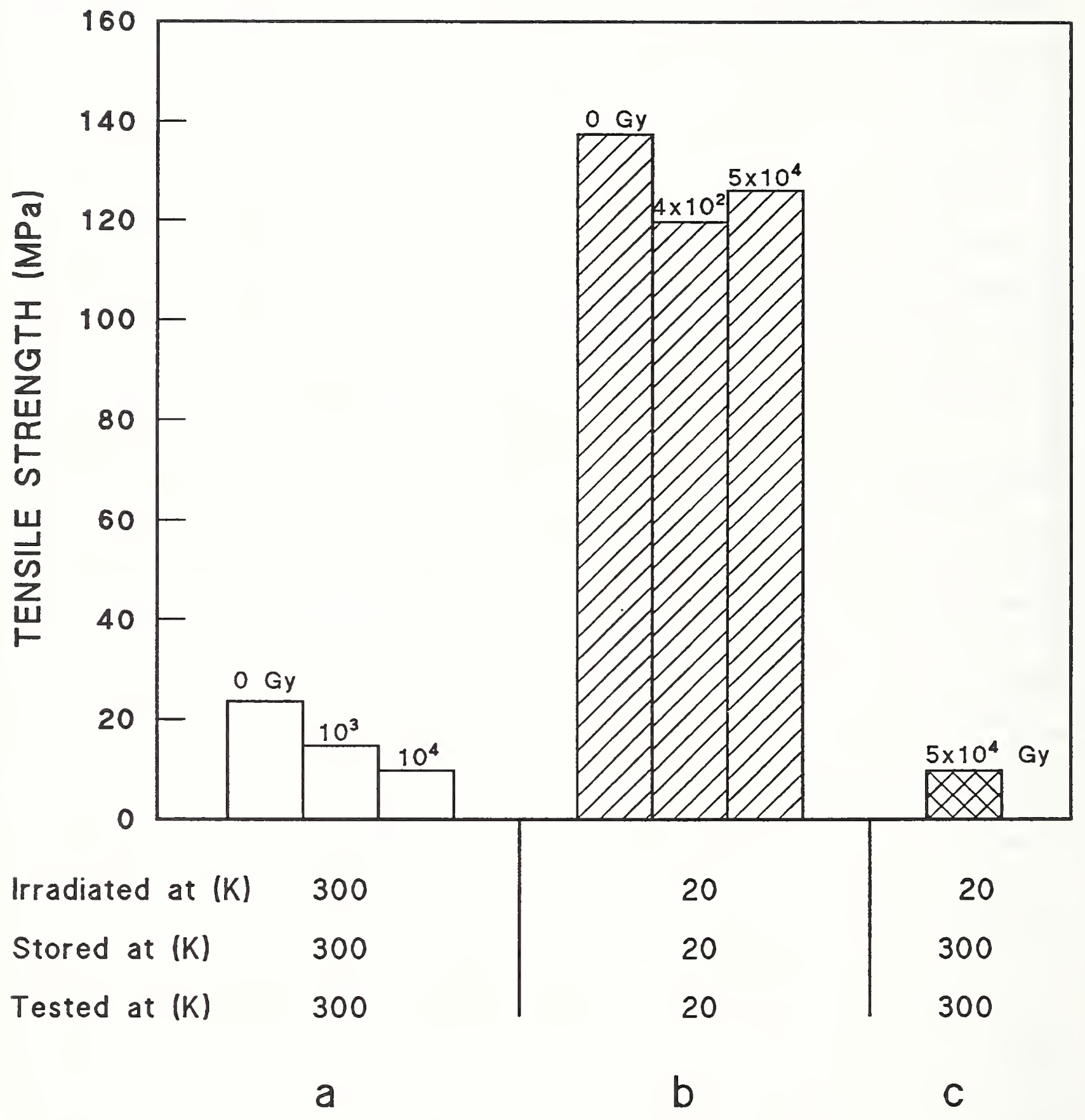

Figure 1.36. Tensile test data comparing polytetrafluoroethylene irradiated, stored, and tested at $20 \mathrm{~K}$ (b) with the same polymer after warm-up to ambient temperature (c). Comparative ambient temperature properties without irradiation are shown in (a). Data from Van de Voorde [1973]. 
Annealing of irradiation damage was also studied by Egusa et al. [1985b], but the irradiation was performed in air at ambient temperature and specimens were then heated to $180^{\circ} \mathrm{C}$ in vacuum for $2 \mathrm{~h}$. This temperature is higher that the glass transition temperature for most epoxies. The specimens were tested at ambient temperature. The flexural strength of E-glass-reinforced epoxy specimens irradiated to $2 \times 10^{7}$ Gy was about $30 \%$ below the strength of identically irradiated specimens not subjected to annealing (Figure 1.37). The annealing was thus considered to activate latent damage from the ${ }^{60} \mathrm{Co}$-gamma irradiation, rather than to cause recovery. Since the shear modulus was similarly affected by annealing, while the Young's modulus was unchanged, Egusa et al. hypothesized that the annealing activated radiation damage at the fiber-matrix interface, causing a decrease in the load transfer capacity at the interface. Other explanations are also possible. For example, products produced by the irradiation could migrate to the interface during the annealing and weaken it.

Nishijima et al. [1988] measured the 77-K interlaminar shear strength of E-glass-fiber-reinforced commercial laminates with and without warm-up at ambient temperature. The resin system was not identified. Table 1.7 shows that the warm-up degraded the strength by about 408 after combined gamma and neutron irradiation of 5 to $8 \mathrm{MGy}$ at $20 \mathrm{~K}$. Figure 1.38 shows the severe damage at the fiber-matrix interface after warm-up. Although these data show that warm-up or annealing activates latent damage, as do those of Egusa et al. at much higher temperatures of anneal, the results are in disagreement with the neat resin results of Takamura and Kato discussed above. The reason for the discrepancy is unclear, but both sets of data on composites have limited applicability to tokamak fusion reactors of the ITER design because E-glass will probably not be used in these machines, and the intense interfacial damage seen in Figure 1.38 probably is the result of localized alpha-particle emission from the $(B, n) \rightarrow(L i, \alpha)$ reaction. Thus, these two experiments on composites may chiefly indicate the effects of warm-up after alpha irradiation, whereas what is needed are studies of the effects of gamma and neutron irradiation with and without warm-up on composites reinforced with Bfree glass.

Such an experiment was recently carried out at the Garching reactor by Katheder et al. [1992]. Preliminary results indicated that warm-up improved 


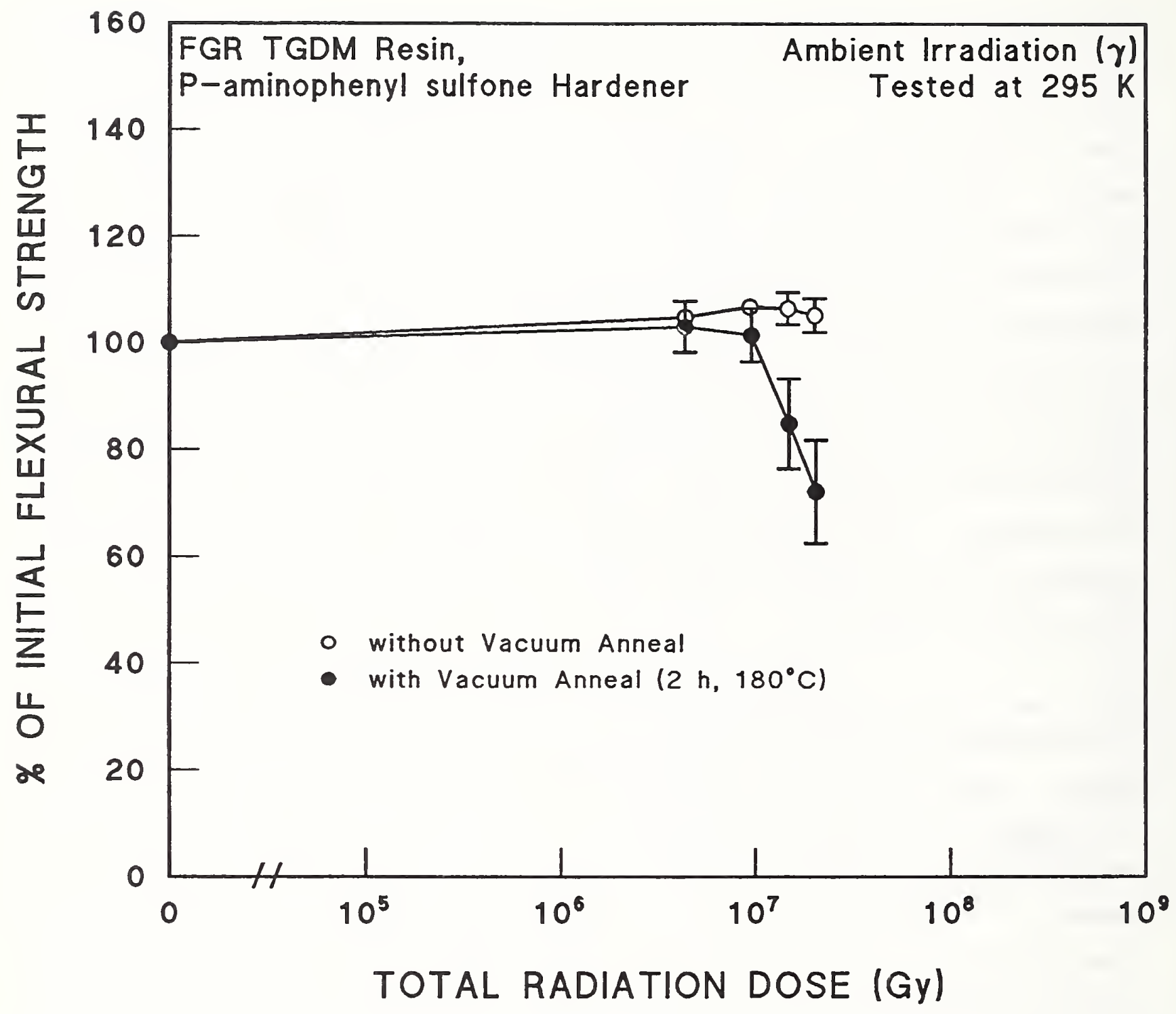

"O" Strength, MPa

- $745.0 \pm 0.0$

- $745.0 \pm 0.0$
Supplier

Sumitomo Bakelite

Co., Ltd.

Sumitomo Bakelite Co., Ltd. 

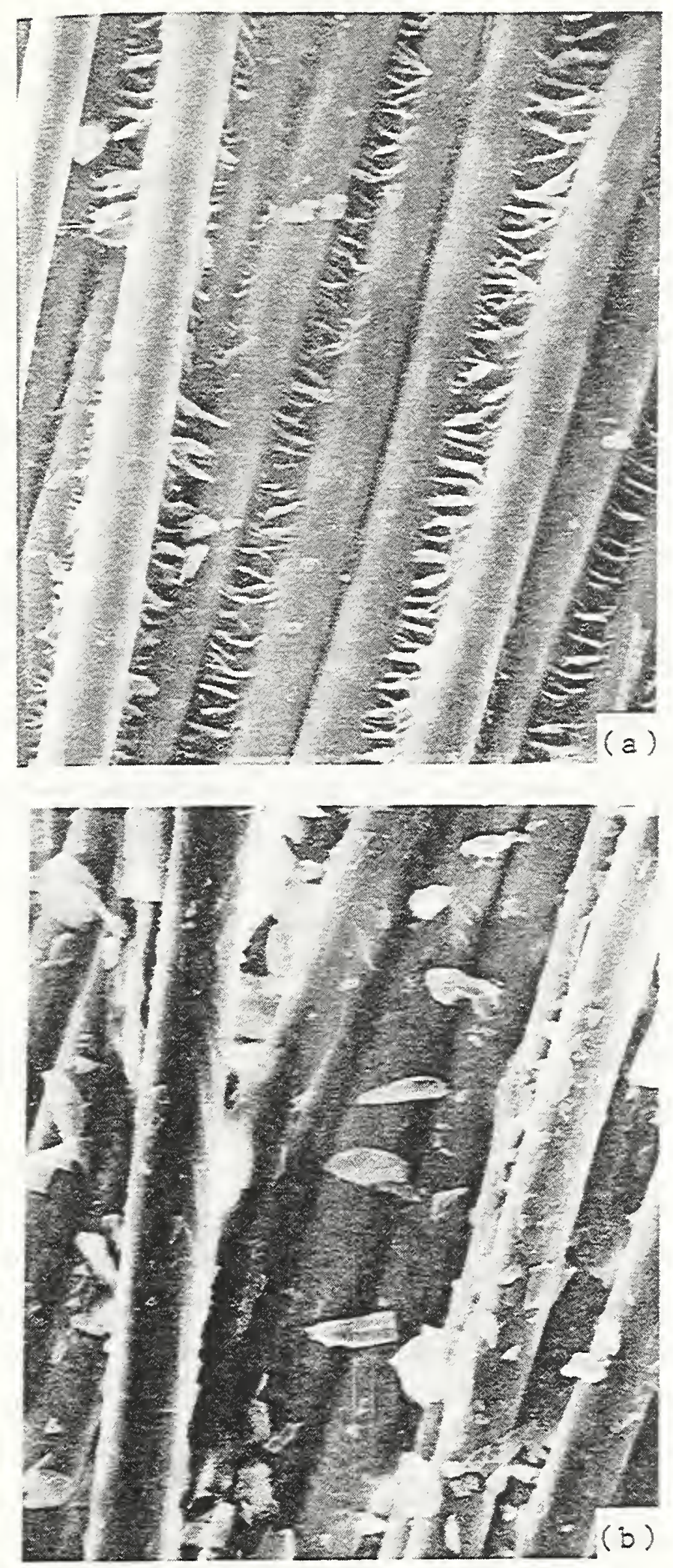

Figure 1.38. Fracture surfaces of an E-glass reinforced composite after neutron irradiation, with (a) and without (b) warm-up to ambient temperature. Reprinted with permission from Nishijima et al. [1988], p. 41, Plenum, New York. 
Table 1.7. Effect of Warmup to Ambient Temperature on Interlaminar Shear Strength (ILSS) Measured at $77 \mathrm{~K}$. (Data from Nishijima et al. [1988].)

\begin{tabular}{||c|l|c|c|}
\hline $\begin{array}{c}\text { Dose } \\
(\mathrm{n}, \gamma \text { Irradiation })\end{array}$ & Condition & ILSS, MPa & $\begin{array}{c}\text { Degradation Induced } \\
\text { by Warmup }\end{array}$ \\
\hline $\begin{array}{c}9.6 \times 10^{20} \mathrm{n} / \mathrm{m}^{2}, \\
8.4 \mathrm{MGy}\end{array}$ & without warmup & $7.6 \pm 0.53$ & \\
\cline { 2 - 5 } & with warmup & $4.9 \pm 0.2$ & 368 \\
\hline \hline $4.1 \times 10^{20} \mathrm{n} / \mathrm{m}^{2}$, & without warmup & $12.2 \pm 1.2$ & 388 \\
\cline { 2 - 5 } $5.1 \mathrm{MGy}$ & with warmup & $7.6 \pm 0.5$ & 3 \\
\hline
\end{tabular}

the shear bond strength to a stainless substrate of a DGEBA epoxy composite reinforced with $\mathrm{R}$ glass (see also \$11.2, Figure A.11-9). These results, together with those of Takamura and Kato, indicate that the post-irradiation strength of epoxy composites under steady-state cryogenic conditions cannot be conservatively predicted from studies in which warm-up occurred. Furthermore, although these results on small, unconfined specimens are encouraging, they do not rule out possible problems with swelling or pressure from gas release or explosive release of heat from free radical reactions or stored energy in larger, confined insulation systems.

\subsection{TEST METHODS}

\subsubsection{Sensitivity to Radiation Damage}

Three different composite properties of an E-glass reinforced TGDM/DDS resin system were measured by Egusa and Hagiwara [1986]: the Young's modulus, the shear modulus, and the flexural strength. The three-point bend test was used to assess these properties (Figure 1.39); the moduli were obtained by varying the span length and solving simultaneous equations. Figures 1.40 to 1.42 show the degradation of these three properties, at both ambient temperature and $77 \mathrm{~K}$, after ambient gamma irradiation from ${ }^{60} \mathrm{Co}$. The Young's modulus seemed to be a relatively insensitive marker of radiation damage, while the shear modulus appeared much more sensitive. Egusa [1990b] noted that the Young's modulus (E) of a composite could be calculated from a rule of mixtures

$$
E_{c}=V_{f} E_{f}+V_{m} E_{m}
$$




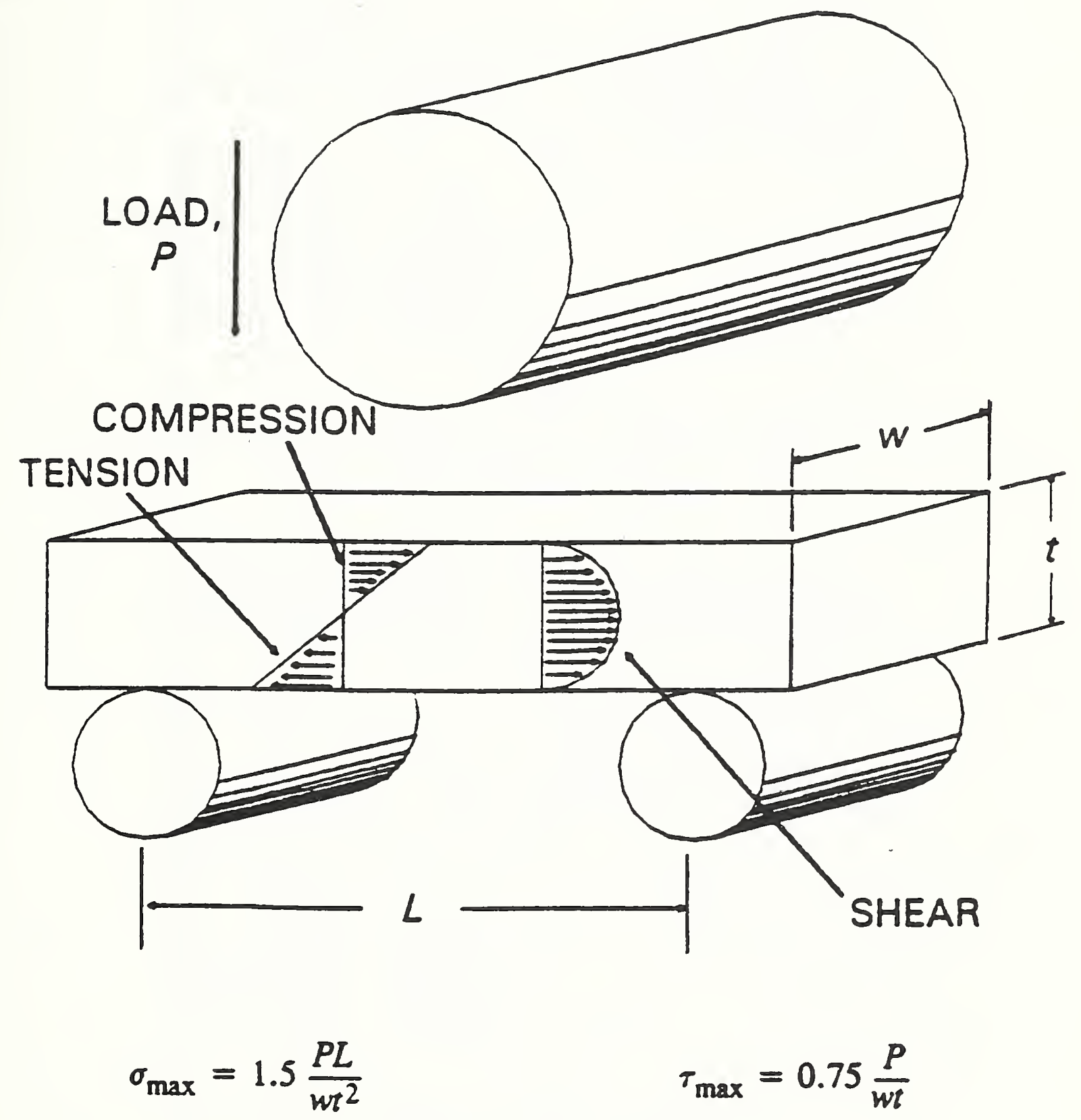

Figure 1.39. The distribution of tensile, compressive, and interlaminar shear stresses in a three-point bend test. 

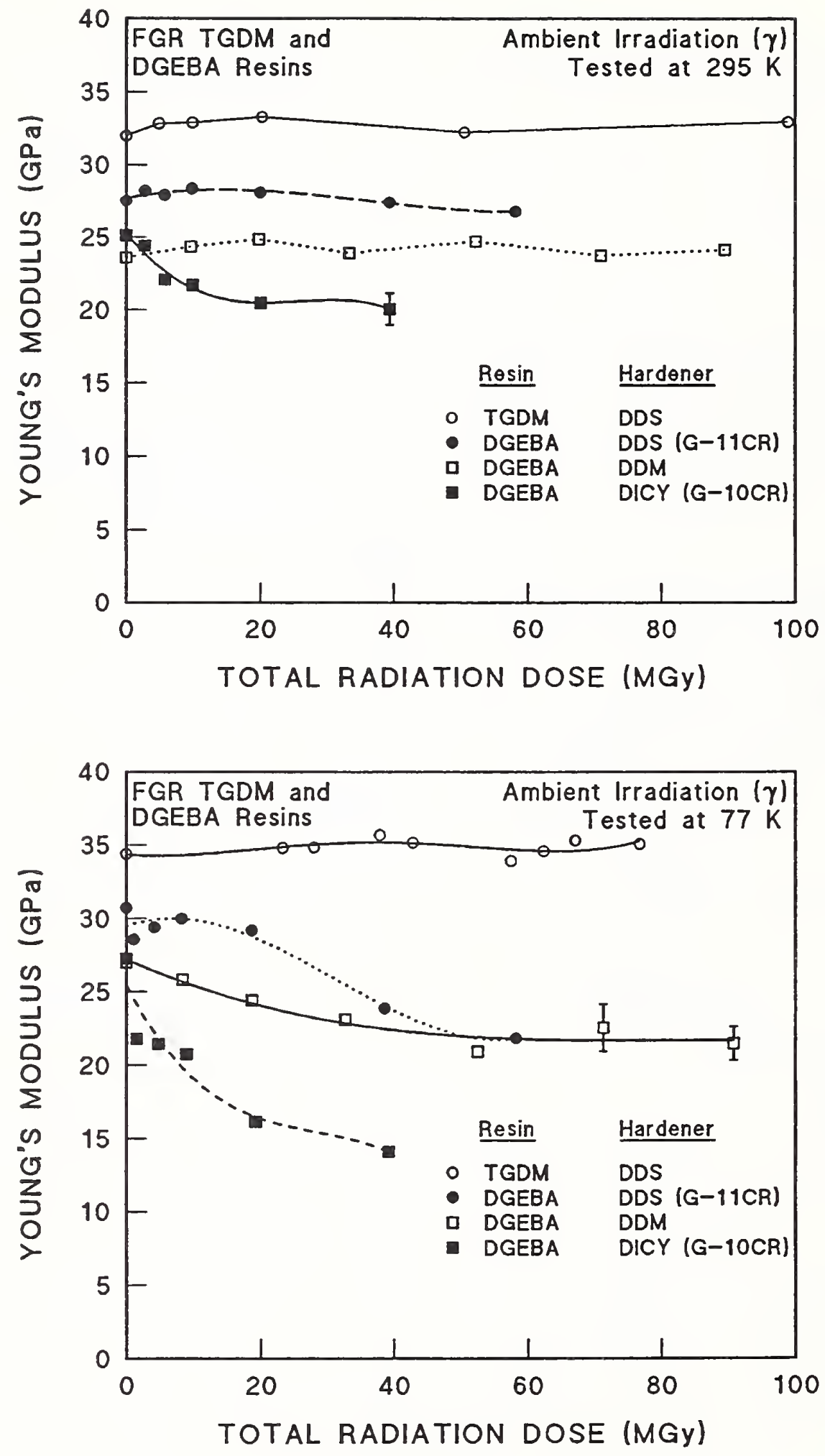

Figure 1.40. The change of Young's modulus with gamma irradiation in different types of fiber-glass/epoxy composites. Irradiation was at ambient temperatures; flexural testing was carried out at 295 and $77 \mathrm{~K}$. Data from Egusa and Hagiwara [1986]. (Supplementary Table A.3-2.) 

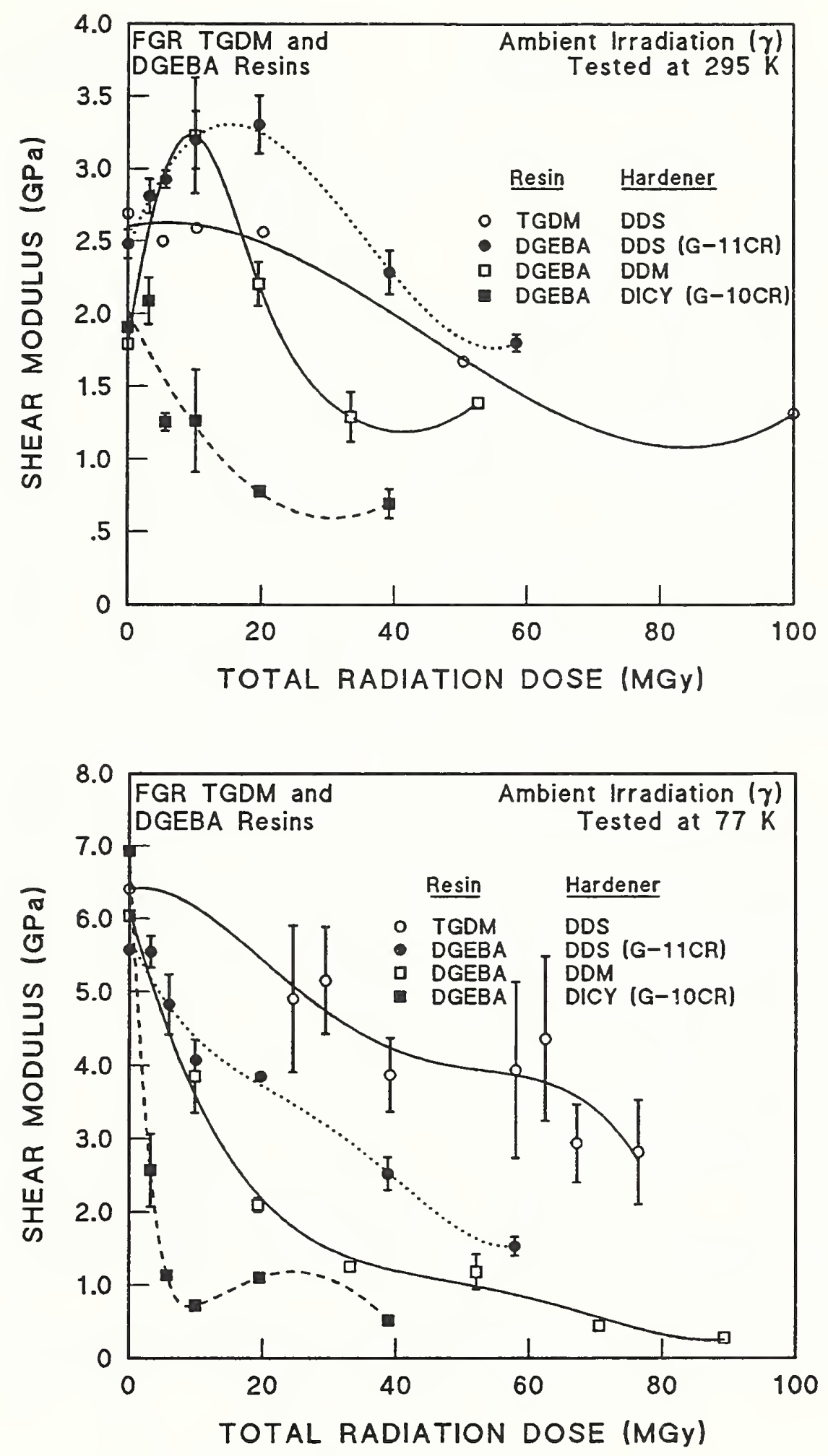

Figure 1.41. The change of the shear modulus with gamma irradiation in different types of fiber-glass/epoxy composites. Irradiation was at ambient temperatures; flexural testing was carried out at 295 and $77 \mathrm{~K}$. Data from Egusa and Hagiwara [1986]. (Supplementary Table A.3-2.) 

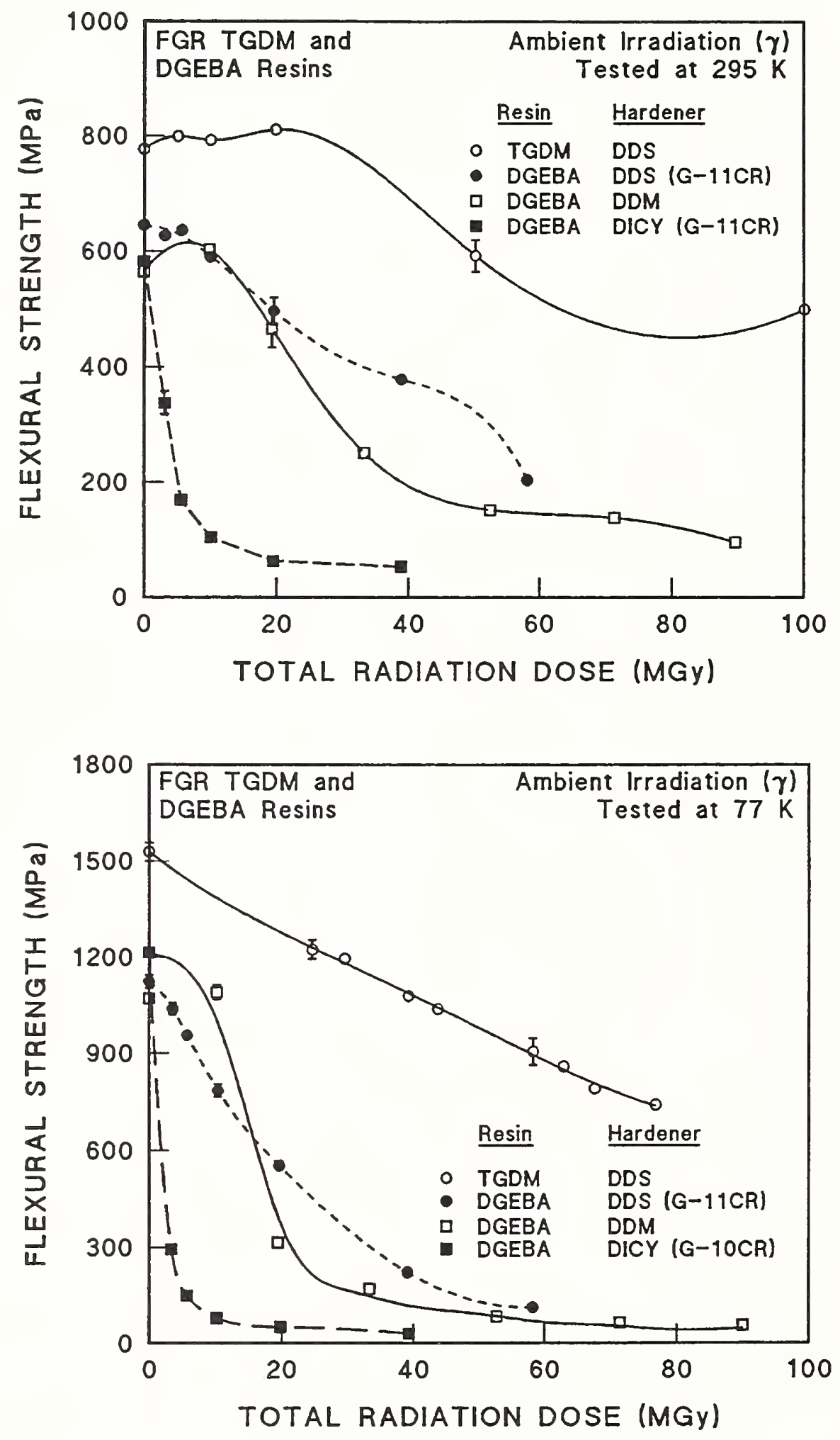

Figure 1.42. The change of the flexural strength with gamma irradiation in different types of fiber-glass/epoxy composites. Irradiation was at ambient temperatures; flexural testing was carried out at 295 and $77 \mathrm{~K}$. Data from Egusa and Hagiwara [1986]. (Supplementary Table A.3-2.) 
where $V$ is the volume fraction and the subscripts $c, f$, and $m$ represent the composite, the fiber, and the matrix, respectively. The interlaminar shear modulus (G) of a composite is approximated by the inverse rule of mixtures

$$
1 / G_{c}=V_{f} / G_{f}+V_{m} / G_{m}
$$

and, therefore, should also be unchanged because of the relation $G=E / 2 \cdot(1+\nu)$, where $\nu$ is the Poisson ratio [Egusa et al., 1984b]. However, as Egusa et al. noted, the underlying principle of the inverse rule of mixtures is that the shear modulus is actually determined by the part of the composite that is most compliant in shear. Thus, if the fiber-matrix interface is the component that is the most sensitive to radiation, the composite shear modulus will degrade even if the fiber and matrix stiffness and Young's modulus remain relatively unchanged. Separation or debonding between fiber and matrix has been optically observed before degradation of mechanical properties [Klabunde and Coltman, 1983].

Figure 1.42 shows that the flexural strength, like the shear modulus, also degrades monotonically with radiation dose, and appears to be a relatively sensitive marker of radiation damage. Because many authors did not report the shear modulus, and since shear modulus is inherently more difficult to define and measure than the strength in flexure, the flexural strength (or other reported strength parameter) has been chosen as the index of radiation damage in this review. Sometimes, tensile strength rather than flexural strength was measured in composite irradiation tests. Since this property chiefly reflects the tensile strength of the reinforcement, and since glass is much less subject to radiation damage than the epoxy matrix or the glass-matrix interface, this is a less sensitive parameter. Results given in $\$ 2.1$ demonstrate this, for an experiment in which both tensile and flexural strengths of a series of glass-reinforced resin systems were measured [Price and Sheldon, 1965].

\subsubsection{Shear Tests}

Although the flexural strength is usually more sensitive to radiation damage than tensile or compressive strength, flexural strength is not the best insulation property to study for ITER magnet design. The failure mode in a 
three-point bend test depends upon the span-to-thickness ratio $(\mathrm{L} / \mathrm{t})$ of the specimen, as shown in Figure 1.43. If the ratio is high, a tension failure occurs. Because this ratio is high in a flexural test, results are strongly influenced by tensile properties of the reinforcement, as Figure $1.43 \mathrm{~d}$ indicates. As the ratio decreases to an ideal value of about 5 (for many materials), a true interlaminar shear may be measured and correlated with interlaminar shear measured by other test methods [Reed et al., 1992]. (Extremely low ratios of $\mathrm{L} / \mathrm{t}$ also lead to inappropriate failure modes, shown in Figure 1.43.) The interlaminar shear is the composite property that is most relevant to the TF magnets of the ITER conceptual design. However, nearly all of the data reported in this review were obtained for $\mathrm{L} / \mathrm{t}$ ratios much higher than 5, and usually, the failure mode was not defined and reported. An exception are tests by Egusa [1990a,b] in which a specimen machined at an angle of $45^{\circ}$ to the warp direction failed in shear (Figure 1.43c), although $\mathrm{L} / \mathrm{t}$ was about 20. Specimens machined so the specimen axis was parallel to the warp showed a tension failure, like that depicted in Figure 1.43d. Figure 1.44 for the $45^{\circ}$ orientation, and Figure 1.45 for the $0^{\circ}$ orientation, show that the dose dependence of both types of failure was similar at $77 \mathrm{~K}$, but not at $295 \mathrm{~K}$. In general, however, the flexural test results reported in this review may not be representative of failures that would occur in a magnet under shear stresses predicted from the ITER conceptual design. The available information on test methods and specimen dimensions are reported in supplemental tables in the appendices.

\subsubsection{Shear/Compression Tests}

To meet magnet insulation requirements of the ITER conceptual design, tests have been developed in which combined shear and compressive forces are applied in a controlled manner. References to these new test methods may be found in $\S 1.2$. The relatively small amount of irradiation data that has been obtained under these test conditions is summarized in $\$ 10$. 


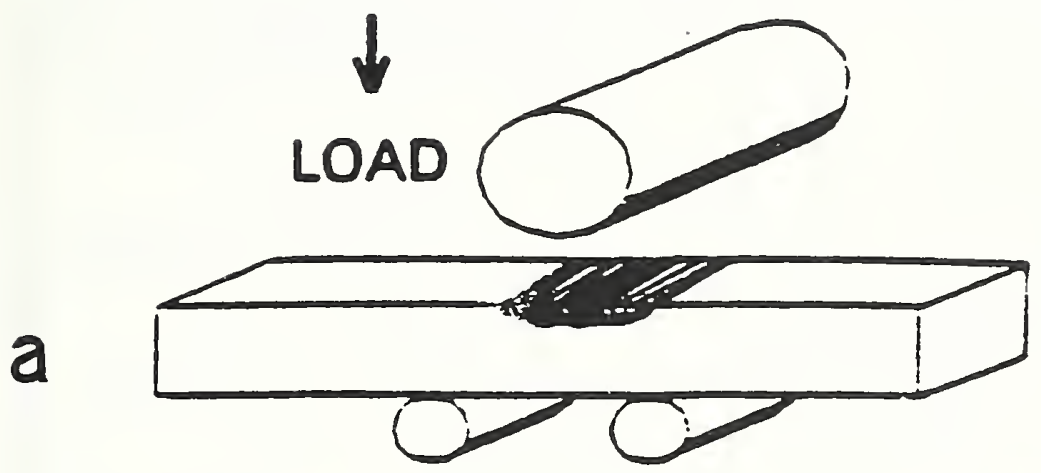

\section{Compressive failure}

b

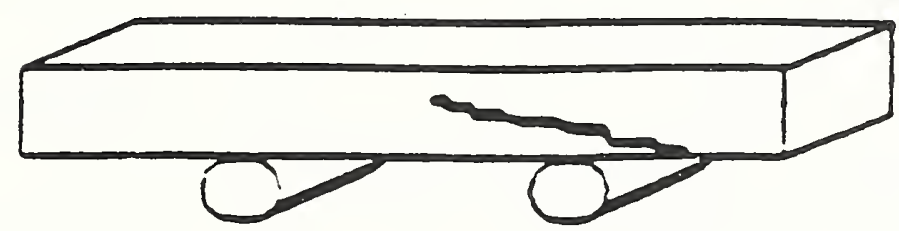

Diagonal shear failure Span too small

C

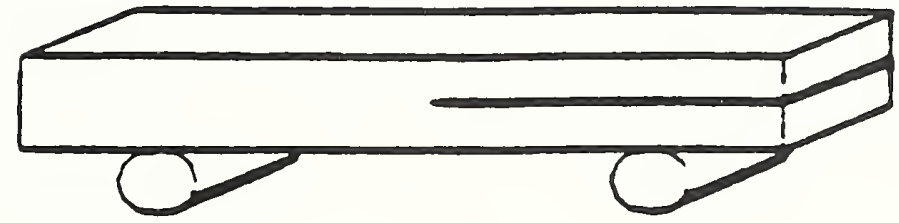

Intertaminar shear failure Span correct

\section{Tension failure}

d

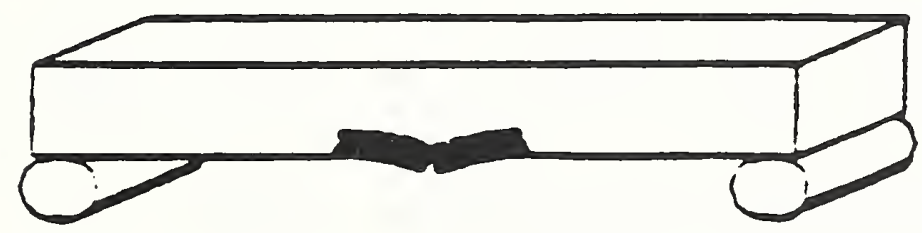

Figure 1.43. Dependence of the failure mode in a flexural test upon the span to thickness ratio, $L / t$. 


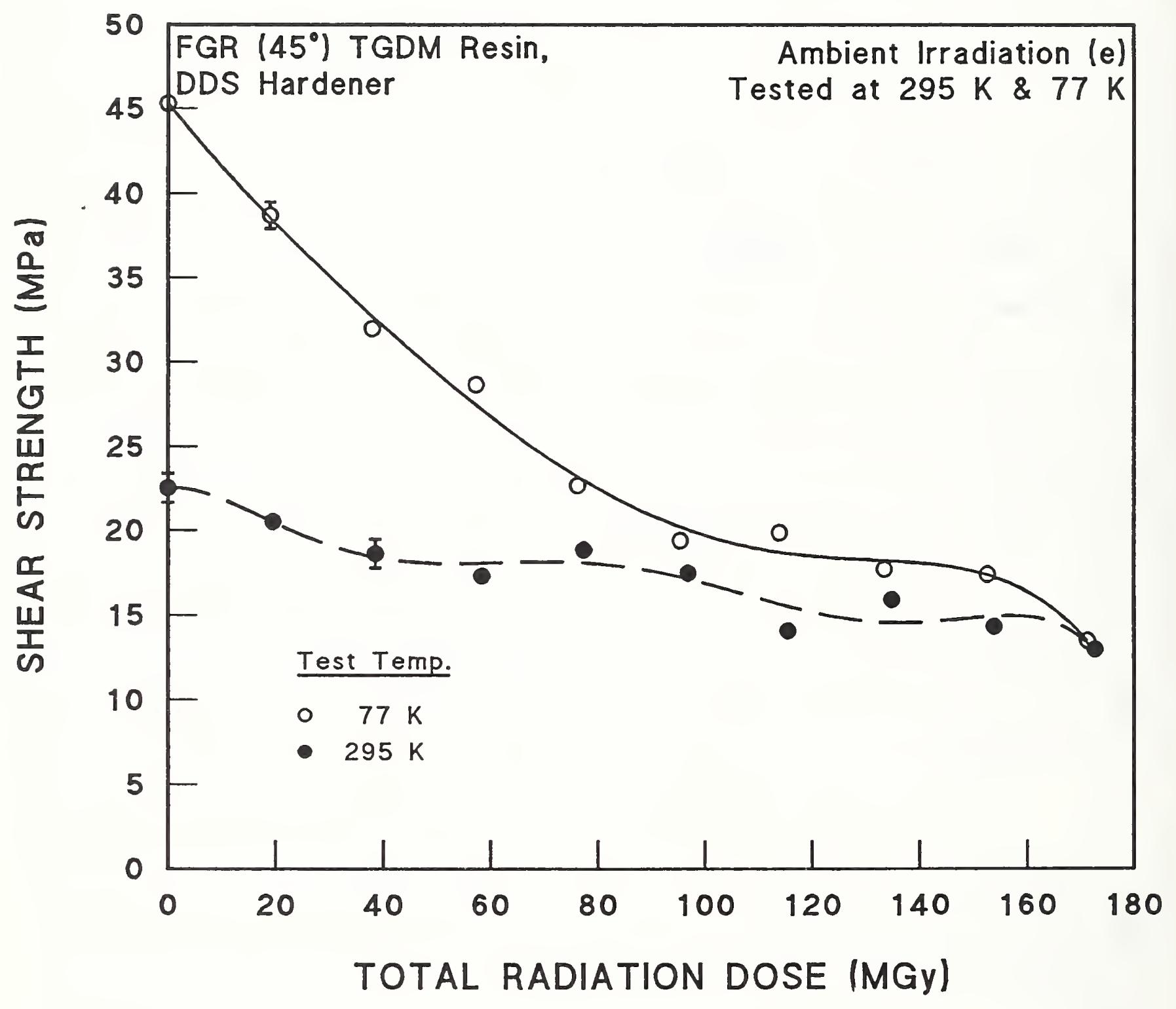

Figure 1.44. Shear failure in a flexural test with $L / t=20$, where the specimen was machined at an angle of $45^{\circ}$ to the warp direction. The E-glass reinforced TGDM/DDS resin system was irradiated at ambient temperatures with 2-MeV electrons and tested at 295 and $77 \mathrm{~K}$. Data from Egusa [1990a,b] . (Supplementary Table B.1-1.) 


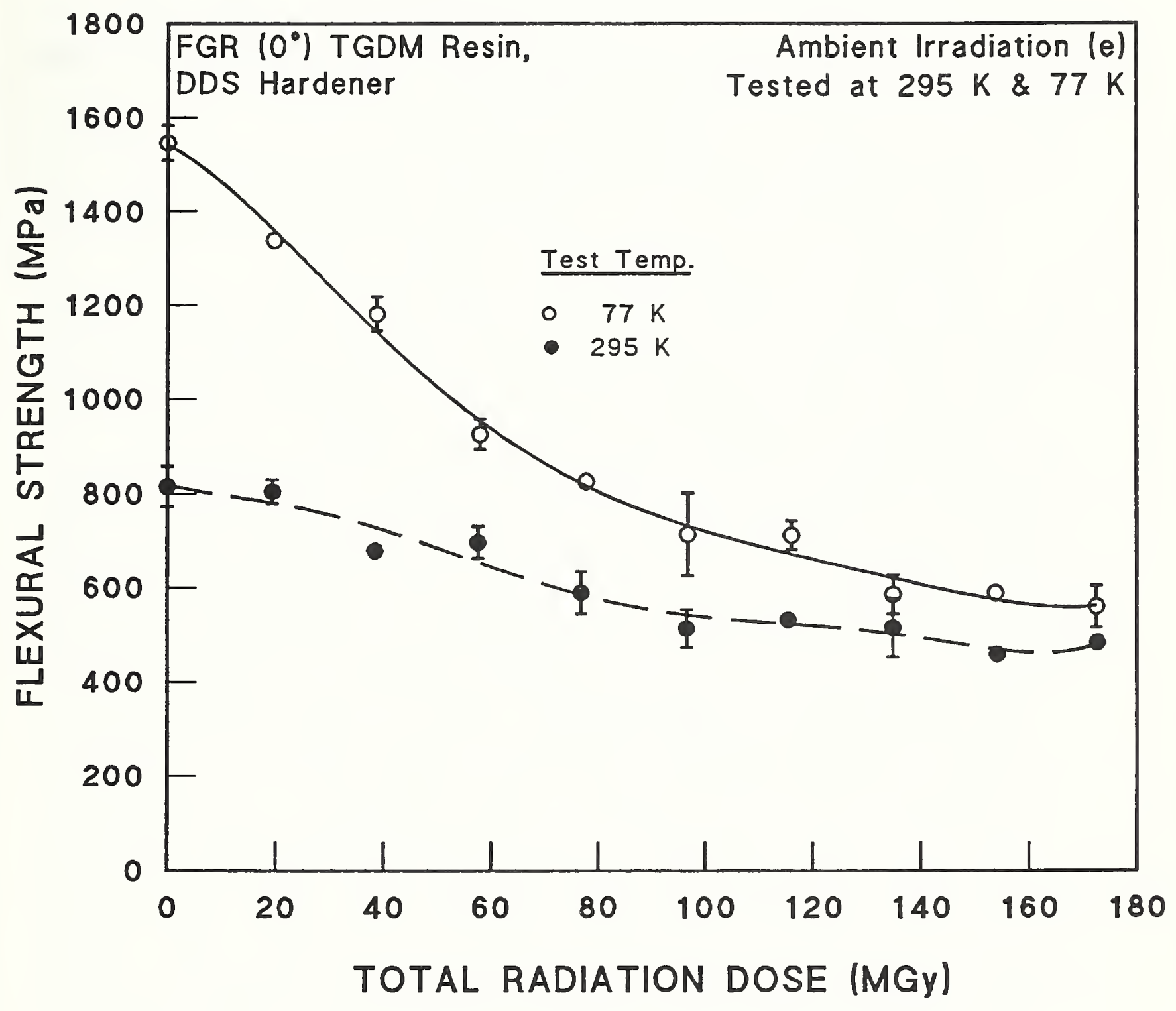

Figure 1.45. Tension failure in a flexural test with $L / t=20$, where the specimen was machined at an angle of $0^{\circ}$ to the warp direction. The E-glass reinforced TGDM/DDS resin system was irradiated at ambient temperatures with 2-MeV electrons and tested at 295 and $77 \mathrm{~K}$. Data from Egusa [1990a,b]. (Supplementary Table B.1-1.) 


\subsubsection{Adhesive-Bond Tests}

The ITER conceptual design requires integrity of the bond between the insulation and the conductor sheath alloy in the TF magnets. Again, very few tests have been conducted that simulate this operating requirement. They are summarized in $\$ 11$. 


\section{AMBIENT TEMPERATURE IRRADIATION AND TESTING}

\subsection{EFFECT OF RESIN AND HARDENER TYPE: GLASS-REINFORCED RESINS}

Nine different resin systems were prepared as E-glass-reinforced laminates and tested in both flexure and tension by Price and Sheldon [1965]. The chemical structures of the resins are diagrammed in Figure 2.1, except for TGDM, EPN, and DGEBA, which were diagrammed in Figure 1.26. The same hardener, MNA, and accelerator, BDMA (benzyl dimethylamine), were used with all resins. All were irradiated in air with doses up to $1.36 \times 10^{8}$ Gy with "mainly gamma" radiation from a spent reactor fuel element at Rutherford Laboratory, England. The test results are shown in Figures A.2-1 and A.2-2. The differences in performance are more apparent from the flexural-strength data (Figure A.2-1) than from the tensile-test data (Figure A.2-2), because the tensile strength of the composite mainly represents the tensile strength of the glass reinforcement, which was the same for all resin systems. The resin with the lowest retained flexural and tensile strength was Epikote 812. Epikote 812 has no aromatic structure, in contrast to all the other resins that were irradiated. Aromatic rings are thought to confer radiation stability because of their many energy levels, through which incident energy is gradually reduced to nearly zero without breaking covalent bonds. The resin DGEBA was the second lowest performer in percentage of retained tensile strength and the third lowest in retained flexural strength. The cycloaliphatic resins, ERL-4206, ERL-4201, and MS406, were intermediate in retained strength. The EPN resin, which retained about 908 of its strength, was among the better performers. The TGDM resin, which retained about $100 \%$ of its strength, performed best in both tests. Price and Sheldon postulated that the TGDM resin was superior because it contains nitrogen in addition to aromatic rings, and nitrogen has an electron-donating capability.

Structurally, the DGEBA resin was considered to be comparable to TGDM, except that the nitrogen atoms are replaced by oxygen atoms. Features of the chemical structure of the other resins were used by Price and Sheldon to explain their performance rankings. They concluded that epoxy groups have the order of stability shown in Figure 2.2 when cured with acid anhydrides. When $R$ in the figure has aromatic character, it has a significant stabilizing effect. 
812

$$
\underbrace{\mathrm{CH}_{2}}_{0}-\underset{\mathrm{OH}}{\mathrm{CH}}-\mathrm{CH}_{2}-\mathrm{O}-\mathrm{CH}_{2}-\underset{0}{\mathrm{CP}}-\mathrm{CH}_{2}^{\mathrm{C}}-\mathrm{O}-\mathrm{CH}_{2}
$$

\section{$\times 33 / 1189$}

$$
\text { 到 }
$$

Experimental

$$
{ }_{0}^{\mathrm{CH}_{2}}-\mathrm{CH}-\mathrm{CH}_{2}-0
$$

4206<smiles>C1CC([Ge]2CO2)C2OC12</smiles><smiles>CCC1CC2OC2CC1C(=O)OCC1CC2OC2C1</smiles>

406

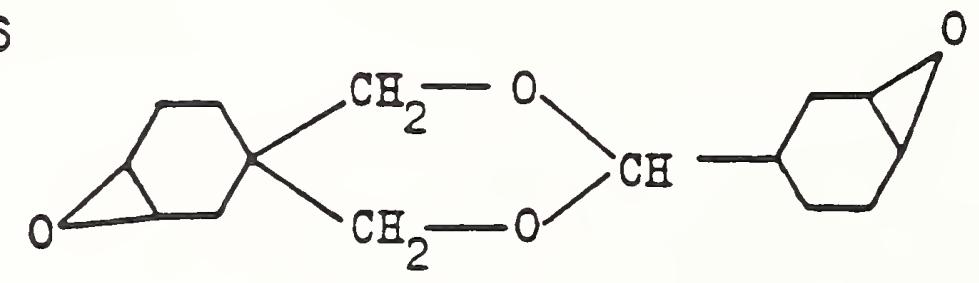

Figure 2.1. The chemical structure of Epikote 812 and other resins investigated by Price and Sheldon [1965]. The structures of the resins DGEBA, EPN, and TGDM are given in Figure 1.26. 


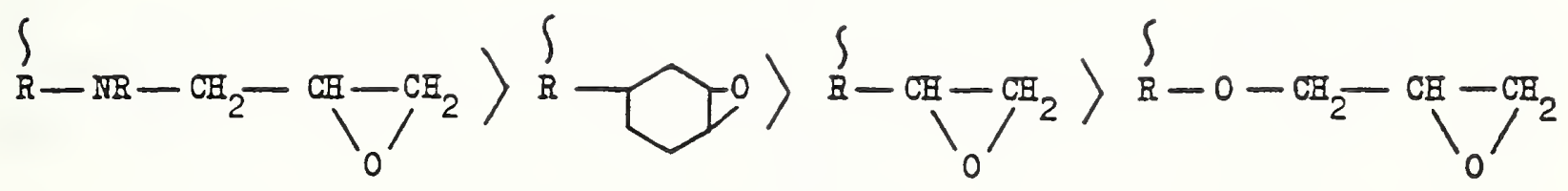

Figure 2.2. The order of radiation resistance of epoxy groups, as determined by Price and Sheldon [1965].

This experiment was an excellent systematic test of resin types because the same anhydride hardener (MNA) and accelerator (BDMA) were used with all resins. Furthermore, the use of E-glass reinforcement was probably not detrimental since most of the irradiation dose was from gamma rays. But because anhydride hardeners such as MNA form radiation-unstable ether and ester structures, Price and Sheldon suggested that systems cured with aromatic polyamines should also be studied to avoid this problem and to promote better radiation stability.

To investigate whether better radiation stability could be achieved, the three aromatic amine hardeners, DDS, DDM, and MPD (m-phenylene diamine) were compared to the acid anhydride hardener MNA in a later Rutherford study [Sheldon and Stapleton, 1968]. It was known from studies conducted on DGEBAbased resins that aromatic amines conferred superior radiation resistance [citations in Sheldon and Stapleton, 1968]. Some of the same resin systems previously tested by Price and Sheldon were similarly prepared as E-glassreinforced laminates and tested in flexure. All specimens were irradiated in air with gamma radiation doses up to $2 \times 10^{8}$ Gy. These results, shown in Figures A.2-3 to A.2-7, indicated that for TGDM, the order of radiation stability with the amine hardeners is:

$$
\mathrm{DDS} \approx \mathrm{MPD}>\mathrm{DDM}>\mathrm{MNA} \text {. }
$$


Although the differences between the first three hardeners that Sheldon and Stapleton reported are not large, all aromatic amine hardners appear to be somewhat superior to MNA. Systems prepared with the hardener MNA have better handling properties, however, such as lower viscosity. The EPN resin was not tested with MNA, and the DGEBA resin systems tested by Sheldon and Stapleton showed mixed results with MNA (Figures A.2-5 and A.2-6); in fact, results with DDS were lower or equivalent to results with MNA. Results for several cycloaliphatic resins cured with MNA are shown in Figure A.2-7; other data from Sheldon and Stapleton (not shown) on one of these resins indicated that DDM was somewhat inferior to MNA.

In addition to ranking the radiation stability of hardeners with different resin types, the results of Sheldon and Stapleton generally confirmed the order of radiation resistance reported by Price and Sheldon: TGDM > EPN > cycloaliphatic resins > DGEBA. Except for the EPN dose, however, the irradiation doses of $2 \times 10^{8}$ Gy were almost a factor of two higher. The poor radiation resistance of a fully saturated epoxy ether (Figure A.2-8) again showed the importance of aromatics in the base resin. This resin lacks aromatic groups, as shown in Figure 2.3. A brominated epoxy that was tested also had unsatisfactory radiation resistance (Figure A.2-9).

Sheldon and Stapleton varied a number of parameters of glass-reinforced DGEBA resin systems, but none of these variations raised the performance of this resin to the best levels achieved with TGDM and EPN. Figure A.2-10 shows the results of using variable amounts of the hardener DDM with a DGEBA resin. (RX 35 is a molecular distillation of a commercial product.) The tests showed that radiation resistance increased with increasing proportions of hardener (the usual stoichiometric amount is $27 \mathrm{phr}$, parts of hardener per 100 parts of resin), but the performance was still below that of most other systems tested. The use of silicone wetting agents in various proportions (Figure A.2-11) also did not change the performance of this resin system significantly. Variation of the glass fabric finish, including the use of a heat-cleaned glass with no coupling agent, also failed to improve the performance of a reinforced DGEBA resin system, at least with DDM as the hardener (Figure A.2-12). However, the same variations, with MNA as the hardener, did improve the performance at the highest dose (Figure A.2-13). The aminosilane and other coupling agents showed markedly better radiation resistance at $2 \times 10^{8}$ Gy than the heat- 


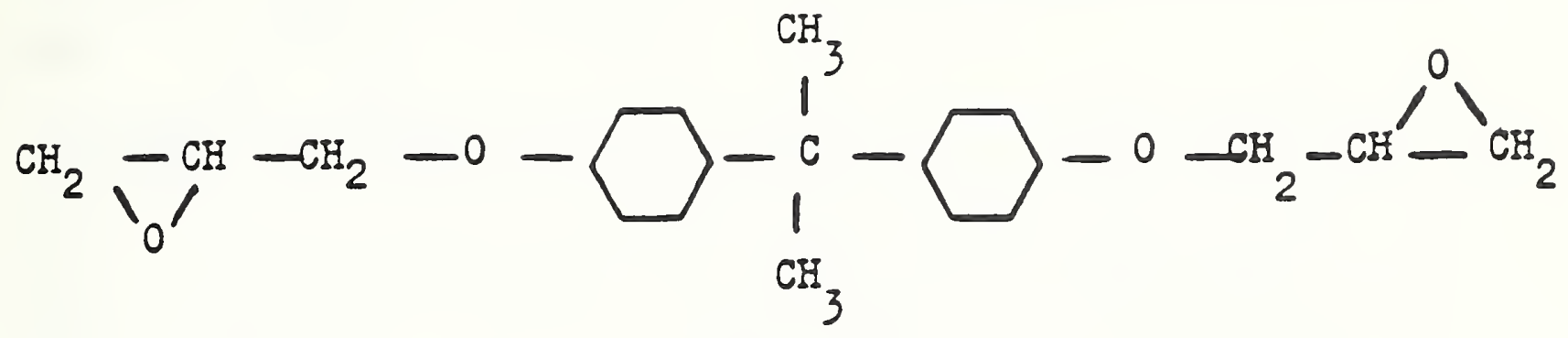

Figure 2.3. The chemical structure of the resin Gft-VE-2025, investigated by Sheldon and Stapleton [1968].

cleaned finish without a coupling agent. Since the flexural strength decreased with dose up to $1 \times 10^{8}$ Gy and then showed an increase at $2 \mathrm{x}$ $10^{8} \mathrm{~Gy}$, it would be desirable to confirm this unusual behavior with additional tests.

Systematic studies of resins and hardeners were repeated and extended by the Rutherford Laboratory group [Evans et al., 1970]. The resins DGEBA, EPN, DGA (diglycidyl aniline), TGPAP (triglycidyl p-amino phenol), and TGDM were studied with MNA, DDM, and DDS as hardeners. The chemical structures of TGPAP and DGA are shown in Figure 2.4. Glass-fabric laminate specimens and tests were similar to those of the earlier Rutherford Laboratory investigations, and irradiation was again in air at ambient temperature. Gamma radiation with a mean energy of $1 \mathrm{MeV}$ was used for doses up to $2 \times 10^{8} \mathrm{~Gy}$. Figures A.2-14 to A.2-16 show that for each hardener, the order of radiation stability is TGDM > TGPAP > EPN > DGEBA > DGA. The two glycidyl amines are considerably more stable than the glycidyl ethers. (Evans et al. reported TGPAP > TGDM, but their numerical results, especially at high doses, do not support this.) Figures A.2-17 to A.2-21, which compare the three hardeners for each resin (except for DGA, which cannot be cured with MNA), show that DDM and DDS generally give resin systems that are more stable toward radiation than MNA. However, DDM and DDS are probably not technically feasible for use in large- 
Triglycidyl para-aminophenol

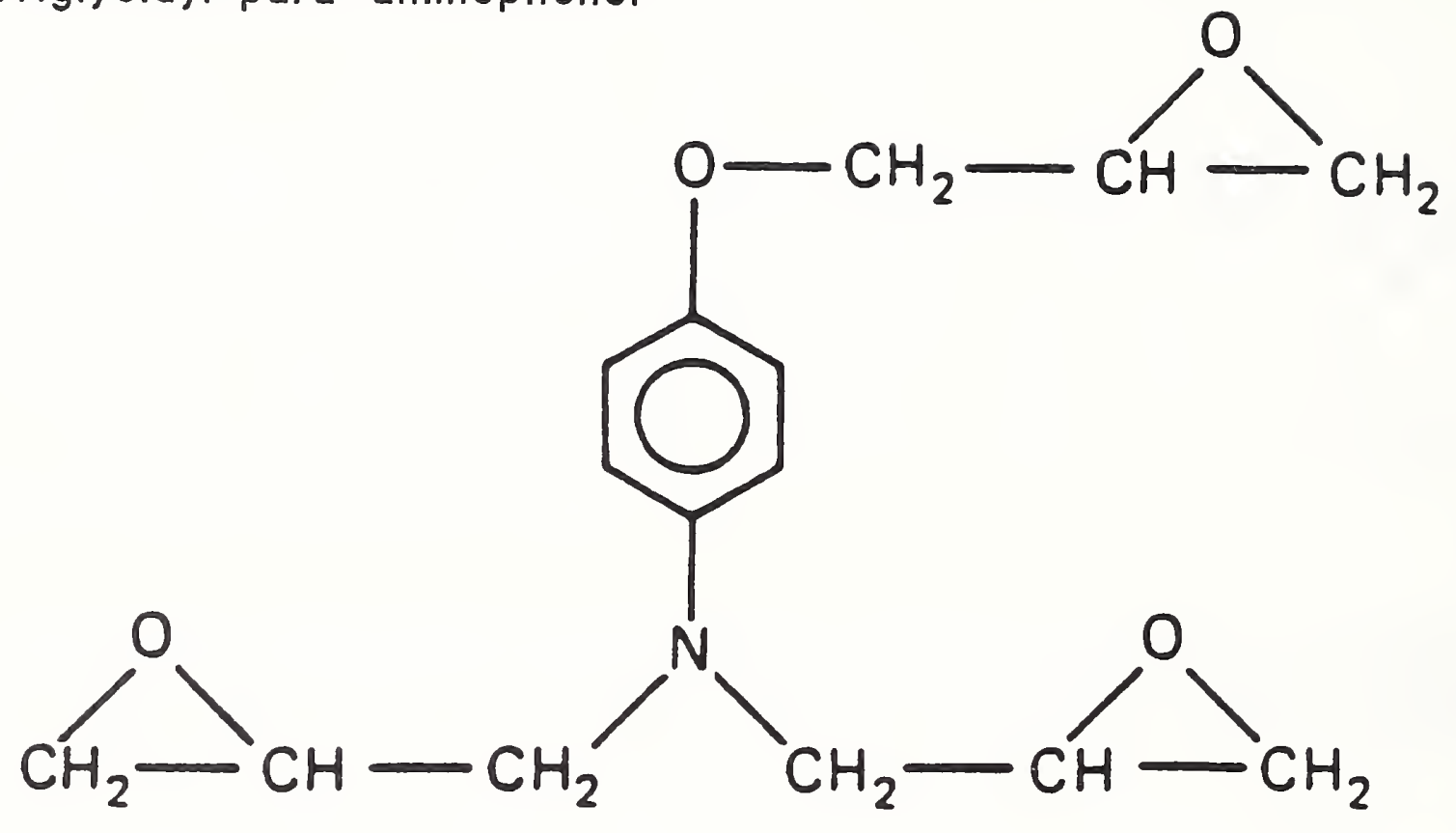

Diglycidyl aniline

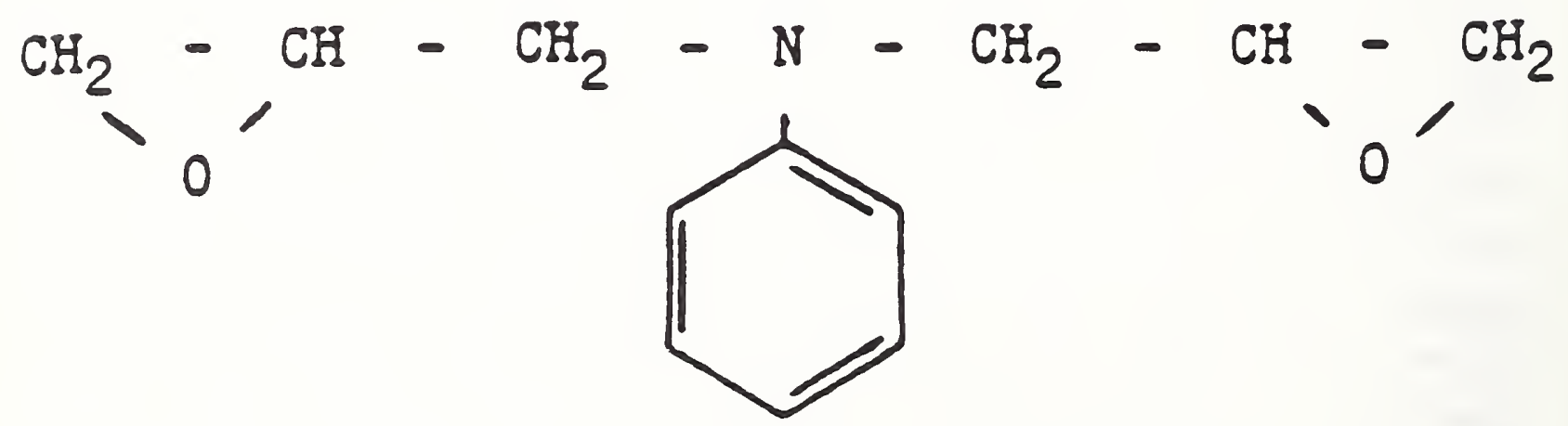

Figure 2.4. The chemical structure of the resins TGPAP and DGA, investigated by Evans et al. [1970]. 
scale magnet-potting or vacuum-impregnation operations owing to the short ambient-temperature pot life of their resin systems.

Results on two other glass-reinforced resin-hardener systems, prepared, irradiated, and tested in a similar manner to those described above, are given in Figure A.2-22 [Evans et al., 1972a]. At a gamma irradiation dose of $2 \mathrm{x}$ $10^{8} \mathrm{~Gy}$, these systems are inferior to the best of the other systems, TGPAP, TGDM, and EPN.

Schönbacher and Stolarz-Iżycka [1979], working at CERN, tested an extended series of resin systems. While these investigations, overall, were not as systematic as those of Pluym and Van de Voorde [1967] (\$2.2) and those carried out at the Rutherford Laboratories, the results generally confirmed the earlier work on the order of radiation stability for different resin types. Specimens were irradiated in a pool reactor with about $5 \%$ of the total dose from fast neutrons and a substantial gamma-ray component. The resin TGDM was not studied much by these investigators, but Figure A.2-23 shows retention of about 808 of flexural strength at a dose of about $8 \times 10^{7} \mathrm{~Gy}$, for a TGDM composite reinforced with silane-finish E-glass. At comparable doses, Figure A.2-24 shows, at best, retention of $60 \%$ of flexural strength for E-glassreinforced EPN resins. With the exception of one system, with a "special" silane finish, the data of Schönbacher and Stolarz-Izycka appear to indicate that the E-glass-reinforced DGEBA composites retain less than 608 of initial strength at this dose (Figure A.2-25). Tests on three of these proprietary systems (Orlitherm) were extended to a dose of $10^{8} \mathrm{~Gy}$. Results for two systems showed that they retained less than about $35 \%$ of initial strength; however, the third system with the "special" silane finish retained about $65 \%$ of initial strength. Data reported by Schönbacher and Stolarz-Iżycka on another DGEBA system (MY 740) with an MNA hardener and the accelerator DMP (2,4,6-tris[N,N-dimethylamino) methyl] phenol) appear to indicate similar radiation resistance to that found for the non-"special" Orlitherm systems (Figure A.2-26). Thus, the order of radiation stability for generic resin types, TGDM > EPN > DGEBA, is generally confirmed by the data of Schönbacher and Stolarz-Iźycka.

The resin system TGDM/DDS was compared to a bisphenol-A based epoxy with a DDM hardener by Hagiwara et al. [1985]. Both systems were reinforced with the 
same plain-weave, E-glass fiber and irradiated with $3-\mathrm{MeV}$ electrons to a dose up to $10^{8} \mathrm{~Gy}$. Flexural test results are shown in Figure A.2-27; results of interlaminar shear tests with a notched tensile specimen are given in Figure A.2-28 (for TGDM only). These results confirmed the superiority of TGDM systems to bisphenol-A based systems, such as DGEBA, and showed that the TGDM/DDS composite retained over $70 \%$ of the initial interlaminar shear strength. Details concerning the interlaminar shear tests, the fiber-glass weave, and the prepreg preparation of the TGDM/DDS laminate were given by Takeda et al. [1985].

Van de Voorde and Restat [1972] reported flexural test results on reinforced TGDM, EPN, and DGEBA resin systems irradiated to $10^{8} \mathrm{~Gy}$ (the radiation species was not reported). Figure A.2-29 shows that the order of radiation stability with the hardener, DDM, was TGDM > EPN > DGEBA.

\subsection{EFFECT OF RESIN AND HARDENER TYPE: NEAT RESINS}

Resin systems that were thought to be useful for impregnating magnet coils were tested as castings in a further series of experiments at Rutherford Laboratories [Morgan and Stapleton, 1975]. Gamma irradiation conditions and flexural test procedures were similar to those described above. The purpose of the tests was to systematically compare radiation resistance of a glycidyl ether resin, DGEBA (MY 740) with the tetraglycidyl amine resin, TGDM (MY 720). An EPN resin (EPN 1139) and a glycidyl ester resin (LMB 234) were also included in the test series for comparative purposes. Figures A.2-30 and A.2-31 provide a comparison of the glycidyl ether with the glycidyl amine. The figures show that all of the aromatic diamine-based hardeners produced radiation-resistant castings with the glycidyl amine that retained 1008 or better of initial flexural strength at doses up to $10^{8} \mathrm{~Gy}$. But, the same hardeners combined with the glycidyl ether resin showed very little retained strength at the highest doses near $10^{8} \mathrm{~Gy}$. In a second series of tests, the glycidyl amine was again combined with three of the aromatic diamine hardeners used previously, HY 219, HY 932, and H 121. In addition, another DDM-based aromatic diamine hardener, HY 830, and MNA and aliphatic anhydrides were tested. The aromatic amines were again superior in radiation resistance up to doses of about $7 \times 10^{7}$ Gy (Figure A.2-32). The glycidyl ether resin, in combination with aromatic amines, was again inferior in performance (Figure 
A.2-33). (HY 219 is a formulated aromatic amine containing DDM; HY 932 is a formulated aromatic diamine mixture, and $\mathrm{H} 121$ is m-phenylene diamine in Nmethyl-2-pyrrolidone.) None of the other resin systems tested with anhydride hardeners (Figures A.2-34 and A.2-35) exhibited satisfactory radiation resistance at doses of about 6 to $7 \times 10^{7} \mathrm{~Gy}$. This study by Morgan and Stapleton confirmed the value of the glycidyl amine (TGDM) resin. The authors stated that TGDM, in combination with the aromatic diamine HY 219 , provided a "versatile low viscosity resin system suitable for impregnating glass fiber reinforced components and capable of being cured over a wide temperature range including, with the use of a suitable accelerator, temperatures close to ambient."

The superior radiation resistance of a TGDM neat resin system in comparison to a bisphenol-A based system was also corroborated by 3-MeV electron irradiation studies conducted by Hagiwara et al. [1985] at doses to $10^{8}$ Gy. However, as Figure A.2-36 shows, different hardeners were used with each resin. The TGDM/DDS system is probably too viscous for vacuum impregnation, since it was prepared as a prepreg system when reinforcement was used [Takeda et al., 1985].

A series of resins was also tested in neat form by Pluym and Van de Voorde [1967] with combined neutron and gamma irradiation. The resins tested were based on the following chemical selection rules for radiation stability:

(1) Aromatic compounds are more stable than aliphatics, presumably because the resonating structures serve as an energy sink. The excitation energy can be slowly dissipated to thermal energy without rupture of bonds.

(2) Substituted aromatics are more stable than unsubstituted compounds, presumably because the side chain provides an easier way for the energy to flow into the ring.

(3) Branched chains are less stable than straight chains. If the polymer contains two side chains bound to one carbon atom, it will be degraded upon irradiation, owing to the presence of the quaternary $C$ atom.

(4) Small molecules are more stable than large molecules, because a small volume per molecule means less energy absorption per molecule. 
(5) Saturated molecules are more stable than unsaturated ones and a terminal unsaturation is less stable than an internal one.

(6) The order of decreasing stability for compounds is as follows: aromatics, aliphatics, ethers, alcohols, esters, and ketones. Acidic compounds are less stable than basic ones, as acids contain the -C-Olinkage which is particularly susceptible to radiation damage.

(7) Very rigid structures are most stable; flexibility in the main or side chains is to be avoided.

Citations are given by Pluym and Van de Voorde for the ambient-temperature irradiation testing of various polymers from which these rules were developed.

When these selection rules were applied to commercially available epoxy resins, Pluym and Van de Voorde noted that since quaternary $C$ atoms are present in epoxies, an improvement would occur if the methyl groups (Figure 1.19) were replaced with $\mathrm{H}$ atoms. A resin was found in which the methyl groups had been replaced, respectively, with an $\mathrm{H}$ atom and a polymer that had undergone a similar substitution (X33/1189). Also, a resin was found in which the $-\mathrm{C}-\mathrm{O}$ bond was replaced by a $-\mathrm{C}-\mathrm{N}-$ bond, and, in addition, no quaternary $\mathrm{C}$ atom was present (TGDM). It was not possible to increase the number of benzene rings and epoxy groups in the classical epoxy resin structure (Figure 1.21), but Pluym and Van de Voorde noted that two other types of resin, EPN (Figure 1.26) and EPP (not further identified), have these chemical attributes, and, in addition, quaternary $C$ atoms are absent. Therefore, these two resins were also tested. One cycloaliphatic epoxy, DY 032, was tested because cycloaliphatic resins have technical fabrication advantages such as long pot life, low viscosity, and rigid structure. However, a disadvantage is that these resins cannot be cured with aromatic resins.

The aromatic amines, DDM, DDS, and MPDA (metaphenylene-diamine), were selected as hardeners because they are more radiation resistant, owing to the resonance effect and their flexible structures (equivalent to a low heat-distortion point). Also, quaternary $C$ atoms and unsaturated bonds are absent, the molecules are small and rigid, and no branched chains or aliphatic structures are present. Since anhydride hardeners are extensively used in industry, because of their long pot life and ease of handling, they were also included 
in the testing program. The anhydrides investigated were phthalic anhydride (PA, HT 901), hexahydrophthalic anhydride (HPA), MNA, and a mixture (HY 905).

Figure A.2-37 shows the results of testing this series of neat resins with an acid anhydride hardener. Although all the resins have been degraded to less than $20 \%$ of their original strength at the highest doses of about $6 \times 10^{7} \mathrm{~Gy}$, at somewhat lower doses the superior performance of EPN, TGDM, and resin $\mathrm{X} 33 / 1189$ is evident, when compared to the cycloaliphatic resin and the DGEBA resin, Araldite F. (Araldite $F$ is identified as a DGEBA resin by a chemical structure diagram in the report by Pluym and Van de Voorde.) The relative superiority of EPN and TGDM are in agreement with results obtained at the Rutherford Laboratories. Figure A.2-38, which shows the performance of the series of resins cured with an aromatic amine hardener, once more shows some superiority of TGDM, X33/1189, and EPN at doses up to about $2 \times 10^{7} \mathrm{~Gy}$, but again, the strength of all the neat resin systems is below 208 of initial strength at about $6 \times 10^{7} \mathrm{~Gy}$.

From these data, the authors concluded that the order of neat resin radiation stability was EPN > TGDM > X33/1189 > DGEBA (Araldite F) > EPP > DY032, and that, in general, better radiation resistance was obtained with the aromatic amine hardeners. Figure A.2-39, which presents data on various hardeners with the classical DGEBA epoxy, shows that a resin used as hardener was best, followed by the aromatic amines, by $\mathrm{BF}_{3} \mathrm{MEA}$ (boron trifluoride monoethylamine), and then the anhydride mixture, HY 905. Figure A.2-40 gives the results of varying the hardener in EPN resin systems. In this case, the anhydride MNA gave better results than the aromatic amine DDS, and it was also the only hardener that lowered the viscosity of the system. The hardeners tested for the EPN resin were considered to be the only ones that could be used without serious practical difficulties.

Figure A.2-40 can be compared with Figure A.2-41 which shows a comparable series of tests on EPN neat resins with various acid anhydride hardeners and several additives such as the accelerators DY 062 and DY 063 [Schönbacher and Stolarz-Iźycka, 1979]. In both sets of results, good radiation resistance is demonstrated with an EPN/MNA system, but only up to a dose of about $2 \mathrm{x}$ $10^{7} \mathrm{~Gy}$. 
Other neat resin results from Schönbacher and Stolarz-Iżycka [1979] (Figure A.2-42) show that the aliphatic amine hardener TETA (triethylene tetramine, Figure 1.31) did not confer satisfactory radiation resistance when used with an Araldite D resin. Parkinson and Sisman [1971] presented comparative hardener data for an unspecified epoxy resin (Figure A.2-43). The results tended to confirm the ordering of aromatic amine $>$ acid anhydride $\geq$ aliphatic amine.

Markley et al. [1969] extended the work of Price and Sheldon [1965], Sheldon and Stapleton [1968], and others by testing the same basic set of resins with additional hardeners. Again, gamma irradiation was used; in these experiments, the source was ${ }^{60} \mathrm{Co}$. Most of the work was carried out with shear tests of neat resins. Figure A.2-44 shows that for DGEBA resins, the order of stability for hardeners is MDA > $841>$ DMB > OTOL > DAN, where MDA is the aromatic amine DDM, JD 841 is a eutectic amine compound, DMB is 3,3' dimethoxybenzidene, OTOL is 3, 3' dimethylbenzidine, and DAN is diaminonapthalene. Some ambient-temperature hardeners were also tested with DGEBA resins; results are shown in Figure A.2-45. Some of these compounds, the polyamide EM 308 and the zinc fluoroborate (8771), were actually better than two of the aromatic amines, OTOL and DAN. The radiation resistance of EM 308 was not reduced by the addition of PGE (phenylglycidyl ether). The compound TETA had poor radiation resistance, as noted above. Anhydride hardeners were also evaluated with DGEBA resins (Epon 1001 is a high molecular mass DGEBA); these results are shown in Figure A.2-46. Although comparative results with DDM and MNA differed from these that would be expected from the work of Sheldon and Stapleton, this may be due to the use of polypropylene glycol (P 400) as a flexibilizer with the MNA system. The combination of Epon 1001 with PA (phthalic anhydride) gave remarkably good radiation resistance, leading to the following overall ranking of hardeners for DGEBA resins:

$$
\begin{aligned}
& \text { 1001, } \mathrm{PA}>\mathrm{MDA}(\mathrm{DDM})>841>\mathrm{MNA}, \mathrm{P} 400>\mathrm{DMB}=8771= \\
& \text { EM } 308>\text { OTOL }>\text { DAN }=\text { TETA. }
\end{aligned}
$$

Markley et al. [1969] also compared the radiation resistance of glycidyl amine and EPN resins to that of DGEBA. Figure A.2-47 shows that when the same hardener, DAN, was used, the glycidyl amine resins TGPAP and TGDM were superior in radiation resistance to DGEBA. This result further confirms the 
work of Evans et a1. [1970]. Figure A.2-48 shows that the EPN resin was more radiation-resistant than the DGEBA resin when both were cured with JD 841 , a eutectic amine compound. The resin TGPAP was tested with several hardeners, and TGDM was also tested with MNA. These results, shown in Figure A.2-49, indicated that for glycidyl amines, $\mathrm{DAN}>$ OTOL $=841=\mathrm{MNA} \approx \mathrm{MDA}$ (DDM), a result that was quite different from that found for the DGEBA resins. Sheldon and Stapleton [1968] also found that aromatic amines gave better radiation resistance than MNA when used with TGDM, but that MNA was preferable when used with DGEBA (§2.1).

\subsection{EFFECT OF VISCOSITY (PROPORTION OF HARDENER)}

After initial investigations had indicated the superiority of the TGDM resin, a further study at the Rutherford Laboratory was directed toward lowering the viscosity of TGDM resin systems. Since MNA gives a relatively low viscosity when used as a hardener in resin systems, the proportion of MNA was systematically varied in TGDM glass-reinforced and neat resin systems to see whether the resulting systems with lower viscosity would still have a satisfactory resistance to radiation [Evans et al., 1972a]. Flexural specimens were prepared in a manner similar to those described above and were irradiated in air with gamma rays of mean energy $1 \mathrm{MeV}$. Test results are given in Figures A.2-50 and A.2-51 for a system with a viscosity considered to be low enough for vacuum impregnation of large systems $[2.7 \mathrm{~Pa} \cdot \mathrm{s}$ (27 P), at $24^{\circ} \mathrm{C}$ ]. (Hacker et al. [1984] suggested a viscosity of $0.2 \mathrm{~Pa} \cdot \mathrm{s}$ for vacuum impregnation of magnets, but allowed fabrication temperatures higher than $24^{\circ} \mathrm{C}$.) This system (180 parts per hundred of resin, pph) retained high flexural strength at $1.5 \times 10^{8}$ Gy in a glass-reinforced composite. Retained strengths of the neat resin systems at similar doses were considerably lower, as expected, and the replication of flexural test results for neat resins was poor, probably owing to their greater flaw sensitivity. The use of diglycidyl aniline to lower the viscosity reduced the radiation resistance of the glassreinforced system to unacceptable levels. Figure A.2-23 from Schönbacher and Stolarz-Iźycka [1979] shows the performance of a glass-reinforced TGDM/MNA system that was used in a magnet. The retained strength of this system above

$5 \times 10^{7}$ Gy was lower than the strengths of TGDM/MNA systems tested by Evans et a1., but the use of neutron irradiation with E-glass reinforcement could account for the difference. 


\subsection{EFFECT OF CURING CONDITIONS}

The radiation stability of DGEBA with MNA hardener was studied for several post-curing conditions: $4 \mathrm{~h}$ at $100^{\circ} \mathrm{C}, 10 \mathrm{~h}$ at $150^{\circ} \mathrm{C}$, and $10 \mathrm{~h}$ at $200^{\circ} \mathrm{C}$ [Evans et al., 1970]. The glass-reinforced laminate specimens and the irradiation and test conditions were similar to those described above ( $\$ 2.1$ ) for tests of different resin-hardener systems. At a gamma irradiation dose of $2 \times 10^{8} \mathrm{~Gy}$, no significant differences were detected in the radiation resistance. Further studies on the variation of post-curing conditions were carried out by Evans et al. [1972a] for two other resins, TGDM and EPN. These systems were tested in flexure, both as neat resins (Figures A.2-52 and A.2-53) and as glassreinforced laminates prepared in a similar manner to those described above (Figures A.2-54 and A.2-55). No significant differences were observed when cure conditions were varied, as indicated in the legends on the figures.

Schönbacher and Stolarz-Iżycka [1979] also varied post-curing conditions in two DGEBA-type resin systems (Figures A.2-56 and A.2-57). When the hardener DDM was used, comparative results given in Figure A.2-56 appeared to show some superiority of a longer, higher temperature post cure $\left(24 \mathrm{~h}, 120^{\circ} \mathrm{C}\right)$ at a dose of $1 \times 10^{7} \mathrm{~Gy}$. As is usual with measurements on neat resin, however, the standard deviations are large, which makes it difficult to draw conclusions. Also, at a higher dose of $5 \times 10^{7} \mathrm{~Gy}$, all specimens were broken before they could be tested. A cure under similar conditions $\left(24 \mathrm{~h}, 125^{\circ} \mathrm{C}\right)$ but with the hardener MNA (Figure A.2-57) showed that less than $10 \%$ of the initial strength was retained at $5 \times 10^{7} \mathrm{~Gy}$. These results were in agreement with those of Evans et al. [1970], which showed that extending the cure time did not improve the resistance of DGEBA-type resins to high radiation doses.

\subsection{EFFECT OF OXYGEN DURING IRRADIATION}

Peroxide radicals can form in the presence of oxygen and accelerate the breakdown of specimens irradiated in air. This predicted destabilizing effect of oxygen during irradiation was investigated by comparing test results from specimens sealed under vacuum in glass capsules with results from specimens irradiated in air [Morgan et al., 1970]. Preparation, gamma irradiation, and testing of flexural specimens were similar to those conditions described above for other studies at the Rutherford Laboratories. The resins studied were 
TGDM, EPN, and DGEBA. The hardeners were DDM, MNA, and TETA, an aliphatic amine. Figures A.2-58 to A.2-63 present the results. Note that the irradiations in air were carried out to higher doses than those in vacuum. Up to about $3 \times 10^{7} \mathrm{~Gy}$, TGDM and EPN did not appear to be very sensitive to the presence of oxygen. The DGEBA resin appeared much more sensitive, however. In fact, in vacuum, the performance of the DGEBA/DDM resin system improved, so that it was closer to that of TGDM and EPN. The radiation resistance of DGEBA with the aliphatic TETA hardener was expected to be poor.

\subsection{EFFECT OF INORGANIC FILLERS}

Replacement of part of the volume of resin with inorganic fillers increases radiation resistance significantly. Fillers also increase the elastic modulus, decrease the total shrinkage that occurs upon curing, lower the coefficient of thermal expansion, decrease the amount of water absorbed, and increase the thermal conductivity and the pot life [Pluym and Van de Voorde, 1967]. However, the increase in thermal conductivity at ambient temperature can change to a decrease at $4 \mathrm{~K}$ for fine-grained fillers owing to the acoustic mismatch between the filler and the epoxy [GESSS, 1974]. But, powdered inorganic fillers such as $\mathrm{Al}_{2} \mathrm{O}_{3}$ do not react with epoxy resins and, therefore, they do not shorten gel and cure times as they do when used with the polyester resins.

Filled resins are less suitable for vacuum impregnations because they have a high viscosity, and the particles may be filtered out by the small gaps between conductors or by fiberglass, if it is also used [GESSS, 1974]. Unfortunately, also, fine-grained fillers settle out of the fluid resin system more rapidly than larger-grained fillers do. Figure 2.5 from Brechna [1965] provides examples of the large increases in viscosity when fillers are added to DGEBA resin systems. Evans et al. [1972b] noted that when fibrous fillers were incorporated into resin systems in amounts sufficient to prevent lowtemperature cracking, a non-flowing paste was formed. If small particles were used instead as fillers, better flow properties were obtained, but in general these systems were not especially crack resistant. Also, the initial or unirradiated flexural strength is lower if a filler is used. 
Pluym and Van de Voorde [1967] investigated the effects of four main types of fillers upon radiation resistance: (1) metallic oxides, (2) silicates, (3) carbonates, and (4) sulfates. Representative fillers were chosen for testing from each of these groups as follows:

(1) aluminum oxide, for high thermal conductivity; magnesium oxide for purposes of comparison.

(2) silicon dioxide, for improved mechanical properties, inertness to acids and alkalis, and good suspension properties.

(3) graphite, known to increase radiation resistance in rubbers.

(4) barium sulfate, for its relatively minor effect on viscosity.

Figure A.2-64 shows the effect of adding these fillers to a DGEBA resin (Araldite F) used with an aromatic amine hardener, DDM. All of these fillers improved radiation resistance up to about $2 \times 10^{7}$ Gy, but at about $5 \times 10^{7}$ Gy, only alumina and silica gave promising results. However, additional data from Van de Voorde and Restat [1972] showed that the retained flexural strength of alumina-filled resin was poor at $-10^{8}$ Gy. Note also that the radiation resistance at $5 \times 10^{7} \mathrm{~Gy}$ is still poorer than those of the best fiber-glass reinforced epoxies such as EPN and TGDM (e.g., Figures A.2-23 and A.2-24), and that fiber-glass reinforcement gives a much higher initial strength.

Furthermore, Figure A.2-65 demonstrates that the effect of a change in hardener was still significant, even in a filled epoxy. Figure A.2-66 shows that the effects of filler grain size were not statistically significant at low doses, but that at about $5 \times 10^{7} \mathrm{~Gy}$, better resistance was obtained with the larger grain size of $40 \mu$.

Figures A.2-67 to A.2-71 also demonstrate that the addition of fillers such as silica $\left(\mathrm{SiO}_{2}\right)$ and dolomite $\left[\mathrm{CaMg}\left(\mathrm{CO}_{3}\right)_{2}\right]$ usually improved flexural strength after irradiation, compared with the neat resin [Schönbacher and StolarzIżycka, 1979]. Figure A.2-68 indicates that the resins with dolomite retained better irradiation resistance than those with silica, although the effect could be due to a difference in grain size, which was not reported. In Figures A.2-69 and A.2-70, however, the difference between silica and dolomite was not statistically significant. Additional results comparing a DGEBA resin with and without fillers are given in Figure A.2-71, but some of the fillers used were not specified. Figure A.2-72 shows that there was not much difference between the radiation resistance of alumina- and quartz-filled 
resin, up to a dose of about $2 \times 10^{7} \mathrm{~Gy}$, and that the alumina-filled system fell below 208 of the initial strength before a dose of $10^{8}$ Gy was reached. Data from Brechna [1965] on a DGEBA resin with alumina filler showed a much greater radiation resistance (Figure A.2-73) than indicated by the data of other authors. It is possible that the electron radiation doses reported by Brechna were too high by a factor of about $10^{2}$ because his results appear to disagree with other results reported in this subsection. Brechna's doses were determined calorimetrically whereas other authors used activation techniques. Brechna reported the viscosity versus temperature of DGEBA resins with and without fillers; these data are shown in Figure 2.5.

\subsection{EFFECT OF COMBINED MICA- AND GLASS-REINFORCEMENT}

Figure A.2-74 indicates that mica by itself was not a satisfactory substitute for a 708-glass reinforcement (whether the glass percentage pertained to mass or to volume was not reported). The percentage of mica reinforcement also was not reported. Combined mica/glass reinforcement systems were also irradiated with neutrons and gamma rays by Schönbacher and Stolarz-Iżycka [1979]; the results are shown in Figure A.2-75. The resins used were proprietary. The composition of Samica is: 11-188 E-glass, 50-758 mica, and 11-358 silicone binder. The Samicanit system exhibited outstanding radiation resistance at a dose of $10^{8} \mathrm{~Gy}$. These authors also listed initial flexural strengths for Samica reinforcements used with an EPN resin (Figure A.2-24), but did not provide irradiation data. The irradiation behavior of mica/glass reinforcements with a DGEBA resin, Orlitherm, was also studied by Schönbacher and Stolarz-Iźycka (Figure A.2-25). However, the addition of the mica-papertape to each of two types of glass-reinforced systems lowered the radiation resistance at doses of about $5 \times 10^{7}$ Gy. Figure A.2-101 (discussed in $\$ 2.11 .1$ below) also shows that the addition of mica tape lowered the percentage of initial flexural strength retained in a glass-reinforced EPN resin system, although the initial flexural strength was maintained to $5 \times 10^{7}$ Gy [Van de Voorde and Restat, 1972].

Lipták et al. [1985] also studied mica-reinforced epoxy insulation that had been used in high-voltage electrical machinery. In two cases, the insulation had been used for vacuum pressure impregnation (VPI), and in one case, the insulation was used in a magnet. Figures A.2-76 and A.2-77 show the results 


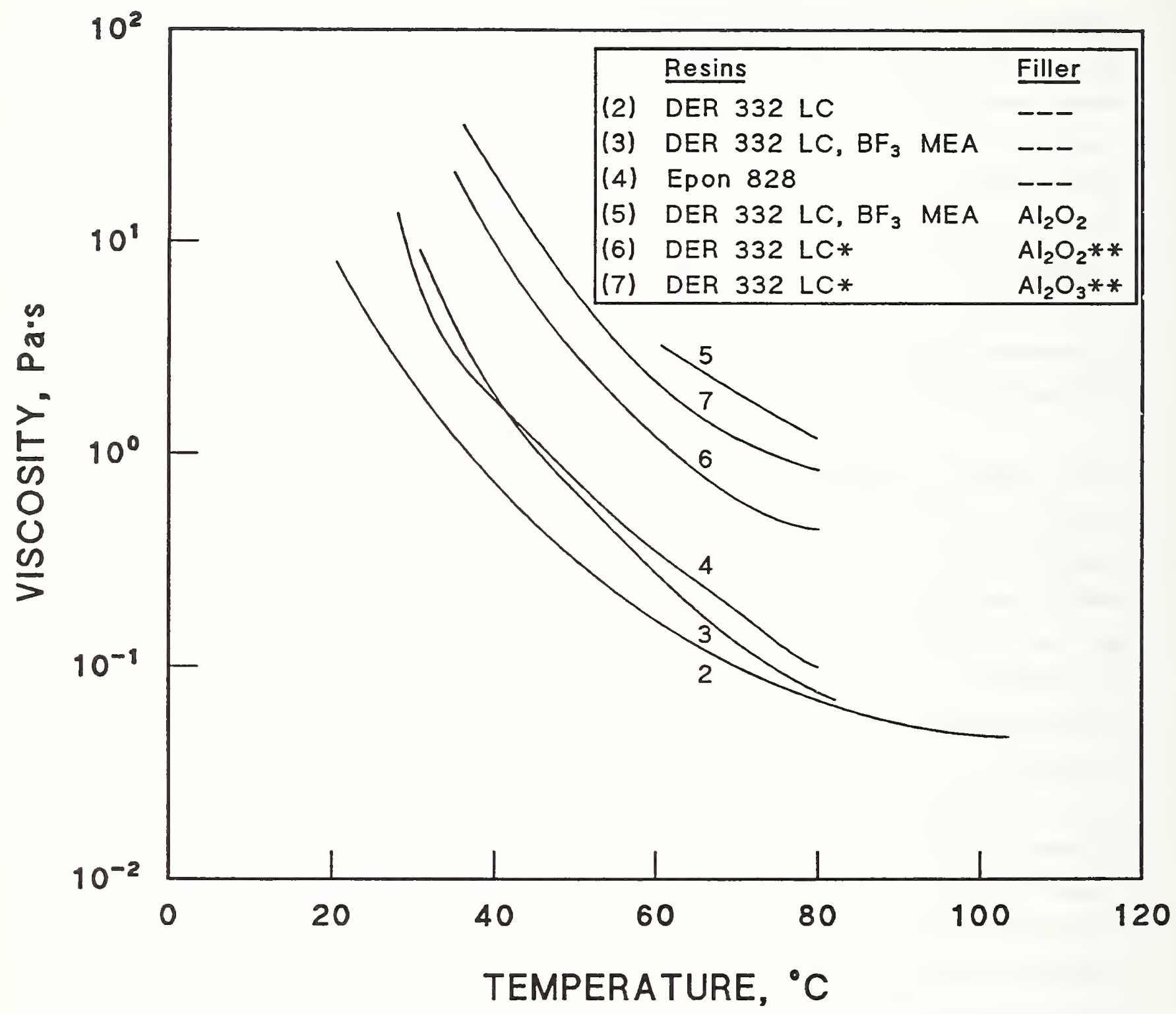

* Hardener: MPDA, DMA

* Thixotropic material also added, 1 ppm (of DER)

(6) $=100 \mathrm{ppm}$

$(7)=120 \mathrm{ppm}$

Figure 2.5. Viscosity of epoxy systems, with and without filler, as a function of temperature. Data from Brechna [1965]. 
of irradiating these systems with radiation composed primarily of gamma rays, at doses up to $10^{8} \mathrm{~Gy}$. The systems shown in Figure A.2-76 did not exhibit good flexural strength at the maximum dose. However, Figure A.2-77 shows that the retained strengths of Samicatherm used with resin-rich (prepreg) EPN systems were much higher, often 35 to $50 \%$ of the initial strength, and one system, Samicatherm flexible, exhibited $111 \%$ of the initial strength at $10^{8} \mathrm{~Gy}$. The authors noted that this result was surprising and stated that mica delamination was occasionally observed at doses above $10^{7} \mathrm{~Gy}$. They explained that the gases that were produced at their dose rates of $2 \mathrm{x}$ $10^{5} \mathrm{~Gy} / \mathrm{h}$ could not diffuse out quickly enough to prevent local increases of pressure within the sample. These localized regions of high pressure sometimes caused separations within the mica layer. Perhaps similar separations occurred more frequently when Schönbacher and Stolarz-Iżycka irradiated Samicatherm systems at a higher dose rate of $1-2 \times 10^{6} \mathrm{~Gy} / \mathrm{h}$. Figure A.2-75 shows that these authors observed much poorer radiation resistance of Samicatherm than Lipták et al. reported (Figure A.2-77). Many other factors could also account for the differences, however.

In summary, there are two isolated results in the ambient-temperature literature that indicate very good radiation resistance of mica/glass reinforced epoxy resin systems: Samicanit [Schönbacher and Stolarz-Iźycka, 1979] and Samicatherm (flexible) [Lipták et al., 1985]. Both systems contained E-glass, but were irradiated with an irradiation mix with a much lower neutron component than is expected for ITER TF magnets. Cryogenic irradiation and test data are not available at present for mica/glassreinforced epoxy resin systems, and some ambient temperature results have indicated lower radiation resistance when mica tape was added to fiber-glassreinforced systems.

\subsection{EFFECT OF HIGH ENERGY NEUTRON IRRADIATION}

A few investigators have used high energy neutrons to irradiate specimens at ambient temperature. The IPNS was used for irradiation of the commercial DGEBA laminate, G-10 and a glass-reinforced DGEBA resin (DOW 332) [Ime1 et al., 1979]. The spectrum of the IPNS facility is shown in Figures 1.4 and 1.5. Unfortunately, the irradiations were not carried out to very high doses (Figure A.2-78), so the lack of deterioration at $-10^{6}$ Gy is not informative, 
in comparison to a possible dose of $7 \times 10^{7}$ to $10^{8} \mathrm{~Gy}$ of neutrons with energies above $0.1 \mathrm{MeV}$ in accord with the ITER conceptual design (Table 1.1 , $\S 1.2)$. The somewhat better performance of the resin under vacuum conditions is in agreement with the results of Morgan et al. [1970], discussed in \$2.5.

Iida and Sumita [1983] used 14-MeV neutrons to irradiate various E-glass reinforced resins, some of which were used for the Japanese coil in the 4-K Large Coil Task (LCT) [Dresner et al., 1989]. (The use of E-glass results in production of $\mathrm{He}$ and tritium under fast neutron irradiation even though the thermal neutron reaction is not present.) The RTNS-II source at Lawrence Livermore National Laboratory was used for these irradiations. The resin systems had been previously irradiated to $10^{7}$ Gy with a ${ }^{60} \mathrm{Co}$ gamma source without exhibiting significant damage. None of the systems appeared to have been fabricated with vacuum impregnation techniques. The results of compression tests are given in Figure A.2-79 for the LCT resins and in Figure A.2-80 for EPN resins reinforced with various materials. No significant damage was observed for any of the systems, but the highest dose was only $5 \mathrm{x}$ $10^{6} \mathrm{~Gy}$. However, the $14-\mathrm{MeV}$ neutron fluence at the ITER TF magnets will be only a fraction of the total fast nuetron fluence so this result could be of use if supplemented with other data, to make up a total fast neutron fluence of about $10^{23} / \mathrm{m}^{2}$. Owing to the low total dose in this experiment, however, no distinctions were observed among the various systems tested, such as the isocyanate-hardened system.

\subsection{EFFECT OF IRRADIATION SPECIES}

\subsubsection{Comparison of Electron and Gamma Irradiation}

The differences between the effects of irradiation by gamma-rays and electrons on a commercial epoxy laminate, Lamiverre A, were measured by Nishiura et al. [1988a]. The laminate was reinforced with a plain weave glass with silane finish. After irradiation at about $3.5 \times 10^{7} \mathrm{~Gy}$, both tear and acousticemission tests showed that the material was more degraded by 20-MeV, ambient electron irradiation than by gamma irradiation from a ${ }^{60} \mathrm{Co}$ source, with energy of about $1.3 \mathrm{MeV}$ (Figures A.2-81 and A.2-82). The dose rate of the electron irradiation was much higher than that of the gamma irradiation; this indicates that oxygen diffusion should not be the major cause of the different response, 
because the specimens irradiated more slowly with gamma rays would be expected to exhibit more severe deterioration (\$2.5). Furthermore, lowering the dose rate of the electron irradiation did not lower the damage at a given dose. Cooling also should not have been a factor, because the specimens were cooled with circulating water at $290 \mathrm{~K}$ during electron irradiation, whereas the gamma irradiation was in ambient air. The authors concluded that the $20-\mathrm{MeV}$ electron irradiation was more damaging than the lower-energy gamma irradiation. Similar results were found with 77-K testing ( $\$ 3.5$ ); maximum doses were about $5 \times 10^{7} \mathrm{~Gy}$, but the difference between gamma-ray and electron irradiation was apparent at about $3 \times 10^{7} \mathrm{~Gy}$. (See also §1.3.2.5.)

Earlier results from this group [Nishiura et al., 1988b] on the tensile properties of fiber glass-reinforced epoxy (Epomik) showed less difference between the effects of electron and gamma irradiation, but the highest dose, $10^{7} \mathrm{~Gy}$, was lower than in the later tests, and, also, a different property was measured. The irradiation parameters were the same as those described above, except for the dose. The composite was prepared by impregnation, but a chemical description of the proprietary resin was not provided. These results are shown in Figure A.2-83.

\subsubsection{Comparison of Neutron and Gamma Irradiation}

A commercial E-glass-cloth reinforced epoxy laminate, Lamiverre A, was irradiated with both ${ }^{60} \mathrm{Co}$ gamma rays and neutrons from a reactor; the interlaminar shear strength was then measured [Nishijima et al., 1988]. Figure A.2-84 shows that the effects of gamma irradiation were much smaller than those of neutron irradiation, to a dose of about $10^{7} \mathrm{~Gy}$. (Results of gamma irradiation of a similar commercial laminate, Hoxan, were comparable to those for Lamiverre A.) However, most of the difference between neutron and gamma irradiation is probably due to the use of E-glass and the consequent $(B, n) \rightarrow(L i, \alpha)$ reaction. Thus, the results do not prove an intrinsic difference between neutron and gamma irradiation of B-free glass-reinforced epoxy resins, although such a difference may well exist (\$1.3.2.4). 


\subsection{RESIN SYSTEMS USED IN MAGNET INSULATION}

\subsubsection{Resins Used for Superconducting Magnets}

A commercial magnet resin was tested after irradiation by ${ }^{60} \mathrm{Co}$ gamma rays to a dose of $3 \times 10^{9} \mathrm{~Gy}$. Numerical test results were not reported, but the flexural strength was said to be unchanged after the irradiation [Hacker et a1., 1984]. This report is portrayed in Figure A.2-85. These data are included here even though complete numerical test results were not provided, because irradiation testing of resins suitable for impregnating large superconducting magnets is important and the resin was used by Siemens AG to fabricate the European coil for the LCT. Table 2.1 gives the viscosity as a function of time and temperature for the one-component resin system. Considering that the limiting maximum viscosity for impregnation is about $200 \mathrm{mPa} \cdot \mathrm{s}(200 \mathrm{cP})$, the pot life is about $90 \mathrm{~h}$ at $80^{\circ} \mathrm{C}$. The shrinkage in the solid phase was only 3.4 volume $\%$ (shrinkage in the liquid phase is irrelevant for vacuum impregnation). The thermal contraction between 293 and $4 \mathrm{~K}$ is about 1.18 . This is comparable to the contraction of other epoxies. The density is $1.19 \mathrm{~g} / \mathrm{cm}^{3}$. The curing temperature was $100^{\circ} \mathrm{C}$ for $200 \mathrm{~h}\left(\mathrm{~N}_{2}\right.$ atmosphere). Irradiation tests of resin systems used for the Japanese coil for the LCT were discussed above, in $\S 2.8$ (Figure A.2-79).

Table 2.1. Viscosity $(\mathrm{mPa} \cdot \mathrm{s})$ versus time (h) at 89,90 , and $100^{\circ} \mathrm{C}$ for the one-component resin system used to impregnate an LCT coil. (Data from Hacker et al. [1984].)

\begin{tabular}{|c|c|c|c|c|c|c|}
\hline${ }^{\circ} \mathrm{C} / \mathrm{h}$ & 0 & 10 & 20 & 50 & 70 & 90 \\
\hline \hline 80 & 30 & 30 & 30 & 35 & 65 & 200 \\
\hline 90 & 25 & 25 & 25 & 100 & 1000 & $\ldots$ \\
\hline 100 & 20 & 20 & 110 & $\ldots$ & $\ldots$ & $\ldots$ \\
\hline
\end{tabular}

\subsubsection{Resins Used for Ambient Temperature Magnets}

Other investigators have irradiated resins which have been used for commercial magnet insulation, but not in cryogenic systems. Phillips et al. [1981] investigated four resins based on bisphenol $A$ and one resin based on hydantoin-bisphenol A. Some of these resins had been used in large quantities 
in magnets at CERN (one MY 745 system was used in the Super Proton Synchroton); others had not been used in magnets, but had optimum properties for vacuum impregnation. Information on fracture energy, viscosity, and pot life was furnished for these resins (Table 2.2). Low fracture energy was linked to the use of MNA as a hardener. The results of reactor irradiation of these resins in neat form are given in Figure A.2-86. The resin system based on hydantoin (CY 362) had good mechanical properties, high fracture energy, and a lower tendency to creep than the other systems. However, its radiation resistance was very poor. Phillips et al. concluded that the mixed system based on EPN 1138, MY 745, and CY 221, and the classic bisphenol-A based resin CY 205 with HY 905 hardener (hexahydrophthalic anhydride) provided the best compromise in properties for impregnation of magnet coils that would operate in a radiation environment. The mixed system was used for vacuum impregnation of CERN Intersecting Storage Ring magnet coils [Beynel et al., 1982]. However, the mixed system retained only 1.58 of the initial strength at $5 \mathrm{x}$ $10^{7} \mathrm{~Gy}$, and specimens of the classic resin system were broken at that dose (before testing). Reinforced systems would show higher radiation resistance, however. Both MY 745 and CY 221 are liquid, modified resins based on bisphenol A.

Lipták et al. [1985] also investigated ambient-temperature commercial magnet resins. Their irradiation dose consisted mainly of gamma rays. Figure A.2-87 shows the results of flexure testing of neat resins up to $1 \times 10^{8}$ Gy; Figure A.2-88 gives the results for glass-reinforced resins tested to the same dose. The "class $\mathrm{H}$ " neat resin combination, containing both epoxy resin and isocyanate, retained 648 of the initial flexure strength at $10^{8} \mathrm{~Gy}$. The excellent mechanical properties were ascribed to strong cross-linking between molecular groups of the epoxy and isocyanates (molecules containing $-\mathrm{N}=\mathrm{C}=\mathrm{O}$ groups from which polyurethanes are derived). This resin system was developed for high temperature applications, and may not be suitable for vacuum impregnation of cryogenic magnets. The glass-reinforced resins were either commercial laminates (Vetronit, including G-10 and G-11 type products, and Delmat) or systems suitable for vacuum impregnation. Although the laminates were used in magnet construction, they did not generally show good radiation resistance, except for the EPN-glassmat combination. At $10^{8} \mathrm{~Gy}$, both nonlaminate, Orlitherm composites retained about $50 \%$ of their initial strength. 
Table 2.2. Impregnation systems: description and properties. (Data from Phillips et al. [1981].)

\begin{tabular}{|c|c|c|c|c|c|}
\hline Impregnation & \multicolumn{5}{|c|}{ TYPE } \\
\hline Components & 1 & $2 a$ & 3 & 4 & 5 \\
\hline Epoxy Resin & $\begin{array}{l}\text { EPN + } \\
\text { Bisphenol A, } \\
\text { modified } \\
\text { (EPN 1138), } \\
\text { (MY 745), } \\
\text { (CY 221) }\end{array}$ & $\begin{array}{l}\text { Bisphenol A, } \\
\text { modified } \\
\text { (MY 745) }\end{array}$ & $\begin{array}{l}\text { DGEBA } \\
(\text { CY } 205= \\
\text { Araldite F) }\end{array}$ & $\begin{array}{l}\text { DGEBA } \\
(\text { CY } 205= \\
\text { Araldite F) }\end{array}$ & $\begin{array}{l}\text { Hydantoin }+ \\
\text { Bisphenol A } \\
\text { modified } \\
\text { (CY) } 362\end{array}$ \\
\hline $\begin{array}{l}\text { Anhydride } \\
\text { hardener }\end{array}$ & $\begin{array}{l}\text { Special } \\
\text { formulated } \\
\text { product } \\
\text { (HY 905) }\end{array}$ & MNA (HY 906) & $\begin{array}{l}\text { Special } \\
\text { formulated } \\
\text { product } \\
\text { (HY 905) }\end{array}$ & MNA (HY 906) & $\begin{array}{l}\text { Special } \\
\text { formulated } \\
\text { product } \\
\text { (HY 905) }\end{array}$ \\
\hline Accelerator & $\begin{array}{l}\text { Ammonium } \\
\text { phenolate } \\
(X B \text { 2687) }\end{array}$ & $\begin{array}{l}\text { Ammonium } \\
\text { phenolate } \\
\text { (XB 2687) }\end{array}$ & $\begin{array}{l}\text { Amine } \\
\text { substituted } \\
\text { phenol, } \\
\text { modified } \\
\text { (DY 061) }\end{array}$ & $\begin{array}{l}\text { Amine } \\
\text { substituted } \\
\text { phenol } \\
\text { (DY 064) }\end{array}$ & $\begin{array}{l}\text { Ammonium } \\
\text { phenolate } \\
\text { (XB2687) }\end{array}$ \\
\hline $\begin{array}{l}\text { Additives (in } \\
\text { epoxy resins) }\end{array}$ & $\begin{array}{l}\text { Flexibilizing } \\
\text { component }+ \\
\text { small amount } \\
\text { of reactive } \\
\text { diluent }\end{array}$ & $\begin{array}{l}\text { Small amount } \\
\text { of reactive } \\
\text { diluent }\end{array}$ & None & None & None \\
\hline \multicolumn{6}{|l|}{$\begin{array}{l}\text { Properties of } \\
\text { the system }\end{array}$} \\
\hline \multicolumn{6}{|l|}{$\begin{array}{l}\text { Processing } \\
\text { properties } / \\
\text { reactivity } \\
\left.\text { data (at } C^{\circ}\right)\end{array}$} \\
\hline \multicolumn{6}{|l|}{$\begin{array}{l}\text { Viscosity } \\
\text { (initial), } \\
\mathrm{mPa} \cdot \mathrm{s}\end{array}$} \\
\hline 25 & $1600-1800$ & $1250-1450$ & $1600-1800$ & $1750-1950$ & $750-950$ \\
\hline 40 & $400-450$ & $350-400$ & $400-450$ & $420-480$ & $300-350$ \\
\hline 60 & $100-150$ & $80-130$ & $100-150$ & $120-170$ & $60-80$ \\
\hline \multicolumn{6}{|l|}{$\begin{array}{l}\text { Pot life } \\
\text { (time to } \\
\text { double } \\
\text { initial } \\
\text { viscosity), h }\end{array}$} \\
\hline 25 & $110-115$ & $140-145$ & $2-5$ & $25-30$ & $30-35$ \\
\hline 60 & $50-55$ & $55-60$ & $1-2$ & $5-10$ & $25-30$ \\
\hline 60 & $25-30$ & $15-20$ & $0.5-1$ & $1-3$ & $5-10$ \\
\hline \multicolumn{6}{|l|}{ Gel time, min } \\
\hline 140 & $30-35$ & $15-20$ & $4-6$ & $5-7$ & $3-5$ \\
\hline$\frac{\text { Fracture }}{\text { Energy, } \mathrm{J} / \mathrm{m}^{2}}$ & $115 \pm 5$ & $105 \pm 7$ & $131 \pm 6$ & $93 \pm 10$ & $140 \pm 5$ \\
\hline
\end{tabular}


The report by Liptak et al. contains additional information on mica reinforcement of magnet resin systems that is discussed in $\$ 2.9$.

Results of testing a JET (Joint European Torus) coil resin with glass tape reinforcement are given in Figure A.2-89. This system retained over $60 \%$ of the initial flexural strength at $1 \times 10^{8} \mathrm{~Gy}$. It consisted of a bisphenol-A based resin with MNA hardener [Banford, 1984]. The radiation species was not specified by Banford, and copies of the report cited were not available to this reviewer.

A DGEBA resin system with a polyamide hardener and a commercial laminate used in the Zero Gradient Synchrotron magnets at Argonne National Laboratory have also been irradiated. These composites were irradiated with ${ }^{60} \mathrm{Co}$ gamma rays and tested in tension and compression [Markley et al., 1969]. The results, shown in Figures A.2-90 and A.2-91, indicated that very little of the initial tensile strength was retained at $2 \times 10^{7}$ Gy and that compressive strength, as expected, was less sensitive to radiation. Shear tests were also carried out on the DGEBA resin system with disk specimens that were $19 \mathrm{~mm}$ in diameter and 1.6-mm thick. The shear results were the same for DGEBA/EM 308 with or without the addition of 30 phr (parts per hundred of resin) of phenylglycidyl ether (PGE) (Figure A.2-92). This additive lowered the viscosity but decreased the initial tensile strength. However, the value of tensile strength at the highest dose was about the same, with or without PGE.

Properties of some other resin systems used for magnets were discussed above (§2.1, Figures A.2-23 and A.2-25).

\subsection{MISCELLANEOUS AMBIENT-TEMPERATURE TESTS}

In the tests described in $\$ 2.1$ to 2.6 , one parameter of an epoxy composite, such as the hardener, the resin system, or the cure condition, was generally varied systematically while other parameters were kept constant. In contrast, most of the investigations described in this section were less systematic. Furthermore, in these studies comparisons were often made between systems that were not irradiated to the same final dose. 


\subsubsection{Investigations of Resin Systems and Hardeners}

The solid, unmodified bisphenol-A based resin Araldite B (Figure $1.19, \mathrm{n} \approx 2$ ) was tested with the commercial hardener HT 901 [Pluym and Van de Voorde, 1967]. The flexural strength fell to about $20 \%$ of the initial value at a dose of $5 \times 10^{7}$ Gy that consisted mainly of gamma irradiation (Figure A.2-93). Tensile strength data from the same Araldite $B / H T 901$ resin system were reported by Dahlerup-Peterson and Perrot [1979] (Figure A.2-94). These gamma irradiation data corroborated those of Pluym and Van de Voorde at a dose up to $1 \times 10^{7} \mathrm{~Gy}$. Parkinson and Sisman [1971] reported that Araldite B resin retained 50 to $80 \%$ of the initial shear strength at a dose of $10^{7} \mathrm{~Gy}$. The hardener and radiation species were not reported. A solid resin would be of less use than liquid resins in vacuum impregnation, but the results are presented here for completeness.

Results for several proprietary resins (Dobeckot), with several hardeners, are presented in Figure A.2-95 [Schönbacher and Stolarz-Iżycka, 1979]. For three of these systems, the data did not extend to the high doses expected for ITER TF magnets. The fourth system had lost much of its initial flexural strength at $3 \times 10^{7} \mathrm{~Gy}$. Data on other proprietary, chemically unspecified resins also obtained by Schönbacher and Stolarz-Iżycka are given in Figures A.2-96 and A.2-97. All of these systems either were not tested at high doses or had lost much of their initial strength by $5 \times 10^{7}$ or $1 \times 10^{8} \mathrm{~Gy}$.

Van de Voorde and Restat [1972] reported results of flexural tests on a series of resin systems irradiated with unspecified species, usually gamma source or reactor neutrons. Although these results are not the product of systematic studies, they generally confirmed the ordering discussed in $\$ 2.1$ and 2.2 . For example, Figure A.2-98 shows that at $1 \times 10^{8} \mathrm{~Gy}$, glass-reinforced TGDM retained more than $80 \%$ of the initial flexural strength when cured with MNA, whereas the retained strength of a DGEBA resin system (Araldite F), about $49 \%$, was not as high at this dose (Figure A.2-99). Unfortunately, irradiation of EPN systems was not carried out to quite such high doses for two of the three EPN systems shown in Figure A.2-100, but for the EPN system for which $10^{8} \mathrm{~Gy}$ was reached, retained strength was about 558 of the initial value. Figures A.2-101 to A.2-103 show results of irradiation and testing of three additional 
neat resin systems, none of which had high retained strength above 1 to $5 \mathrm{x}$ $10^{7} \mathrm{~Gy}$.

\subsubsection{Fracture-Toughness Tests}

A different property, the Mode II interlaminar fracture toughness ( $\mathrm{G}_{\text {IIC }}$ ) was investigated by Takeda et al. [1989]. End-notched, flexural specimens were used to measure this property. The composite, a plain-weave, E-glass reinforced TGDM resin cured with DDS, was irradiated to $9 \times 10^{7}$ Gy with $3-\mathrm{MeV}$ electrons. The authors stated that the radiation-induced degradation was more pronounced in the $G_{\text {IIC }}$ tests than in measurements of the interlaminar shear strength. Figure A.2-104, which shows the $G_{I I C}$ results, can be compared with Figure A.2-28, which shows the results of interlaminar tensile shear tests on the same material using a notched tensile specimen [Hagiwara et al., 1985]. The comparison shows that the interlaminar shear strength is less sensitive to radiation than the interlaminar fracture toughness. Scanning electron micrographs of the fracture surface after the $G_{\text {IIC }}$ tests showed debonding of the glass fibers from the matrix.

Toughness information on a series of resins is also given in Table 2.2.

\subsubsection{Comparison of 2- and 3-Dimensional Reinforcement}

Composites reinforced with a 3-dimensional glass fabric weave were irradiated at ambient temperature with 2-MeV electrons and tested in compression [Nishijima et al., 1990]. The commercial DGEBA laminates, G-10CR and G-11CR, were also tested for purposes of comparison. Figure A.2-105 shows the results of compression tests of specimens $5 \mathrm{~mm} \times 5 \mathrm{~mm} \times 1 \mathrm{~mm}$ in size at doses to $2.4 \times$ $10^{8} \mathrm{~Gy}$. The initial increases in compressive strength with irradiation dose were ascribed to radiation cross-linking. The recently developed 3dimensional composites, ZI-003 and ZI-005, had higher initial compressive strengths than the 2-dimensional laminates, and also showed better performance under irradiation. However, at normal volume fractions, compressive tests chiefly measure the resistance to compression of the glass weave rather than interfacial and matrix properties; therefore, it is not surprising that all composites tested exhibited very good retention of the initial compressive strength. Further comparative testing of 2- and 3-dimensional-reinforced 
composites was carried out by Nishijima et al. with combined shear and compressive loading. These results are discussed in $\S 10.1$. Other shear/compression test results on 3-dimensional weaves are given in $\$ 10.2$. Further discussion of three-dimensional reinforced composites and their properties can be found in a paper by Iwasaki et al. [1990]. The dielectric properties of 3-dimensional glass weaves may rule out their use for turn-toturn magnet insulation.

Flexural tests of a 3-dimensional, B-free glass weave were also conducted by McManamy et al. [1990; 1991a]. The proprietary bisphenol A epoxy resin was used with an aromatic amine hardener. The results of reactor irradiation by neutrons and gamma rays are shown in Figure A.2-106. They indicate a slight decrease in flexural strength at the intermediate dose of $4 \times 10^{7} \mathrm{~Gy}$, followed by recovery to strengths near the initial values at a dose about an order of magnitude higher. 


\section{AMBIENT-TEMPERATURE IRRADIATION WITH 77-K TESTING}

Egusa and coworkers conducted a series of systematic investigations in which tests at $77 \mathrm{~K}$ were used to evaluate the results of ambient-temperature irradiation. Testing at $77 \mathrm{~K}$ was compared with ambient-temperature testing to elucidate mechanisms of degradation by irradiation. The performance of different resin systems was also studied at $77 \mathrm{~K}$. In addition, the effects of varying the type of fabric, the glass volume fraction, the glass type, and the specimen thickness were examined. The effects of radiation environment were studied. Differences in the effects of neutron, gamma, and electron irradiation were also analyzed in tests at $77 \mathrm{~K}$. Although Egusa and coworkers have contributed most of the information on 77-K testing of irradiated epoxyglass composites, significant contributions from other investigators are also reviewed in this section.

\subsection{COMPARISON OF 77-K TESTS WITH AMBIENT TEMPERATURE TESTS}

As discussed in $\$ 1.4 .1$, the effects of ambient-temperature radiation upon composites are more pronounced when testing is conducted at $77 \mathrm{~K}$. Also, the flexural strength of a glass-reinforced epoxy composite at $77 \mathrm{~K}$ is about twice that at ambient temperature. Figure A.3-1 illustrates these effects for a plain-weave, E-glass fabric reinforcement of a TGDM/DDS rasin system [Egusa, 1988]. The specimens were irradiated to about $1.7 \times 10^{8} \mathrm{~Gy}$ with $2-\mathrm{MeV}$ electrons. In contrast, Figure A.3-2 shows that the flexural strength of the neat resin is not much changed at $77 \mathrm{~K}$ from the ambient temperature value and that the high-dose radiation degradation is also almost the same at the two temperatures. Comparison of both figures shows that the dose dependence (percentage change in property per increment in dose) of the composite is very similar to that of the neat resin, indicating that the changes in matrix strength with irradiation essentially determine the dose dependence of the composite strength. However, Egusa suggested that this conclusion was incorrect, because other experiments showed that the flexural strength of the same neat epoxy resin and the strength of a carbon-epoxy composite were not correlated at ambient temperature [Egusa, 1988]. Egusa proposed instead that the change under irradiation of the strain at failure (ultimate strain) determines the dose dependence of the composite strength. He explained his data in terms of two different failure modes: a matrix-failure mode and a 
fiber-failure mode, although he recognized that the matrix ultimate strain of a composite of glass-reinforced resin is not the same as that of the neat resin.

Dahlerup-Peterson and Perrot [1979] and Dahlerup-Peterson [1980] also compared results of testing glass-reinforced composites at $295 \mathrm{~K}$ with testing at $77 \mathrm{~K}$. These authors tested two bisphenol-A resin composites irradiated with gamma rays at ambient temperature (Figures A.2-95 and A.3-3). Comparison of the two figures shows that the tensile strengths of both composites remained nearly unchanged at $1.7 \times 10^{7}$ Gy at ambient temperature, and had decreased only a small amount at this dose when tested at $77 \mathrm{~K}$. This is not in disagreement with Egusa's results on composites because he also observed little degradation at comparable doses, near $2 \times 10^{7} \mathrm{~Gy}$, and because tensile tests are usually a less-sensitive index of radiation damage to composites than flexural tests (Figures A.2-1 and A.2-2, \$2.1). Nishiura et al. [1988a] also reported little difference in degradation from gamma irradiation when an epoxy laminate, Lamiverre A, was tested at both ambient temperature and $77 \mathrm{~K}$ (Figure A.3-4). Again, however, the highest dose was about $2 \times 10^{7} \mathrm{~Gy}$, below the dose range at which significant degradation was observed by Egusa. Also, like DahlerupPeterson and Perrot, Nishiura et al. measured the tensile strength, a composite parameter that is more closely related to the tensile strength of the glass reinforcement than to matrix or interfacial properties of the composite. Therefore, the tensile strength is less sensitive to radiation.

\subsection{SYSTEMATIC COMPARISONS OF RESIN SYSTEMS}

Four types of epoxy resin systems with similar satin-weave, E-glass reinforcement were irradiated at ambient temperature to $1.2 \times 10^{8}$ Gy with 2$\mathrm{MeV}$ electrons and tested at both ambient temperature and $77 \mathrm{~K}$ [Egusa et al., 1988]. The results, shown in Figure 1.26, indicated that radiation stability is given by: TGDM/DDM > TGDM/DDS > DGEBF/DDM where DGEBF is the diglycidyl ether of bisphenol $\mathrm{F}$ ( $\mathrm{CH}_{3}$ groups are replaced with $\mathrm{H}$ atoms as shown in Figure 3.1). Similar tests of DGEBF with a combination of both DDM and DAN (diamino-naphthalene) as hardener gave essentially the same results as for DGEBF/DDM. The dose dependence observed for TGDM/DDM was unusual, and Egusa et al. suggested that it could be associated with cross-linking reactions in the matrix resin and/or at the 


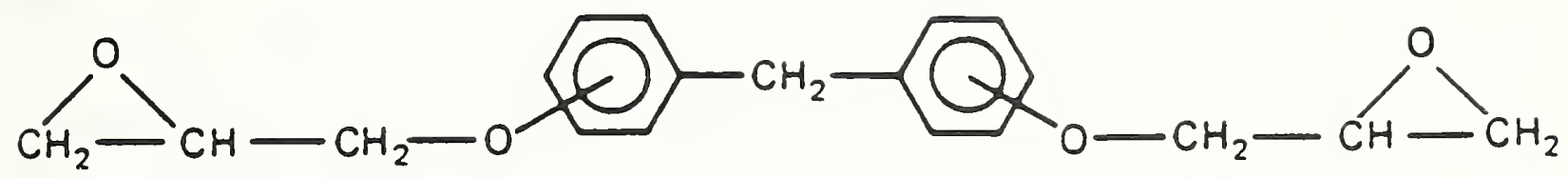

Figure 3.1. The chemical structure of the resin DGEBF, investigated by Egusa et al. [1968].

fiber-matrix interface, although no direct evidence for this was available. Evans et al. [1970] also observed some initial improvement in properties in a TGDM/DDM composite, but the improvement was not as pronounced as that reported by Egusa et al., and it did not extend to gamma doses above $10^{8} \mathrm{~Gy}$. Sheldon and Stapleton [1968] also observed a similar, though smaller effect, but it was noted for TGDM/DDS as well as for TGDM/DDM (Figure A.2-3). Both TGDM/DDS and TGDM/DDM are more suited to prepreg fabrication techniques than to vacuum impregnation.

Four different epoxy composites were irradiated at ambient temperature with ${ }^{60} \mathrm{Co}$-gamma rays and tested at both ambient temperature and $77 \mathrm{~K}$ (Figures A.3-5 and A.3-6) [Egusa and Hagiwara, 1986]. Doses ranged up to $9 \times 10^{7} \mathrm{~Gy}$. Two of the composites were prepared with E-glass plain-weave fabric and the resin systems, TGDM/DDS or DGEBA/DDM; the two others, G-10CR and G-11CR, were commercial laminates. The resin in G-10CR is a solid DGEBA epoxy cured with dicyanodiamide (DICY), and the resin in G-11CR is a liquid DGEBA resin cured with DDS. Both G-10CR and G-11CR have an E-glass, plain-weave fabric reinforcement, but their silane finish is different from that used in the prepared composites (see tables below the figures). Volume fractions are similar. At high doses, the figures show that all three DGEBA-type composites are considerably inferior to the TGDM/DDS system at both temperatures. This result extends some of the results of comparative ambient temperature tests of different resin types discussed in $\$ 2.1$ and 2.2 to cryogenic temperatures (see Figure A.7-1 also). 
Munshi [1991] also systematically compared several epoxy resin systems irradiated at ambient temperature in a reactor and tested at $76 \mathrm{~K}$. The specimens were reinforced with S-2 Glass. The highest dose consisted of a fast neutron fluence of $5 \times 10^{22} / \mathrm{m}^{2}\left(5 \times 10^{7}\right.$ Gy from the usual conversion factor) in a total dose of $2.3 \times 10^{8}$ Gy [Munshi and Weber, 1992]. Thus, the fast neutron dose $(\mathrm{E}>0.1 \mathrm{MeV})$ was about $22 \%$ of the total dose. The specimens were uniaxially reinforced rods tested in torsion to determine the shear strength [Kasen and Stoddard, 1986]. The fracture resistance was also measured. In this test, the crack length induced by pressing a sharp blade longitudinally into the rod specimen is measured, and used to compute a $G_{1 C}$ value. Figures A.3-7 and A.3-8 show the results of the shear and fracture resistance tests. The multifunctional epoxy (CTD-110) had the highest retained percentage of initial shear strength, but the anhydride-hardened DGEBA resin (CTD-101) retained nearly as high a percentage. The results for fracture resistance were similar, in that the multifunctional epoxy (analogous to TGDM or TGPAP) was just slightly better than the DGEBA resin system at the highest dose. Not all of the epoxies were tested for fracture resistance. The results for the G-11CR resin were poor for both properties measured. (The G-11CR resin was reinforced with S-2 Glass and prepared as a rod specimen for torsion testing, rather than as the commercial high-pressure laminate.) The results for G-11CR corroborate those of Egusa and Hagiwara (Figure A.3-6). They found that the flexural properties of G-1ICR laminates were degraded to a very low level by high radiation doses, as were other DGEBA composite systems that they tested. However, the retention of about $45 \%$ of the initial shear strength that Munshi reported for a second DGEBA system, CTD 101, was similar to that reported for the multifunctional system, CTD 110. This result is not in agreement with the 77-K tests of Egusa and Hagiwara or the trend of the ambient-temperature tests of the Rutherford group (\$2.1 and 2.2). Exact comparisons are not possible because the CTD resin systems are proprietary and the test methods are different.

The type of screening test conducted by Munshi is preferable to much of the other work in this field, especially when the requirements of the ITER conceptual design are considered. A non-B glass reinforcement was used, and the highest fast neutron fluence, $5 \times 10^{22} / \mathrm{m}^{2}$, was within a factor of two of the allowable ITER fluence at the conductor, $1 \times 10^{23} / \mathrm{m}^{2}$ [Tomabechi et al., 1991]. Also, specimens were tested in shear, rather than in flexure, which 
generates tensile and compressive stresses. Furthermore, viscosity and pot life information on the resins was presented (Table 3.1); this facilitates assessment of the resin systems for vacuum impregnation. A maximum viscosity of $0.2 \mathrm{~Pa} \cdot \mathrm{s}$ (200 cPoise) was suggested by Hacker et al. [1984] for large-scale vacuum impregnation; this indicates that the multi-functional epoxies would be marginal and that the DGEBA anhydride system would be useable at temperatures somewhat above $40^{\circ} \mathrm{C}$.

Table 3.1. Physical Properties of Resin Systems

\begin{tabular}{|c|c|c|c|}
\hline CTD System & Epoxy/Hardener & $\begin{array}{l}\text { Pot Life } \\
\left(\mathrm{h} ;{ }^{\circ} \mathrm{C}\right)\end{array}$ & $\begin{array}{l}\text { Viscosity } \\
\left(\mathrm{Pa} \cdot \mathrm{s} /{ }^{\circ} \mathrm{C}\right)\end{array}$ \\
\hline 100 & $\begin{array}{l}\text { DGEBA/Aromatic, Aliphatic } \\
\text { Amine }\end{array}$ & $6 ; 40$ & $1.10 / 40$ \\
\hline 101 & DGEBA/Anhydride & $60 ; 40$ & $0.40 / 40$ \\
\hline 102 & EPN/Anhydride & $50 ; 40$ & $0.40 / 40$ \\
\hline 110 & $\begin{array}{l}\text { Multifunctional/ } \\
\text { Aromatic Amine }\end{array}$ & $1 ; 135$ & $-0.3 / 135$ \\
\hline 112 & $\begin{array}{l}\text { Multifunctional/ } \\
\text { Aromatic Amine }\end{array}$ & $3 ; 135$ & $-0.3 / 135$ \\
\hline 501 & $\longrightarrow$ & $8 ; 25$ & $1.0 / 40$ \\
\hline G-11CR & DGEBA/DDS & $2 ; 120$ & NA \\
\hline
\end{tabular}

\subsection{SYSTEMATIC COMPARISONS OF REINFORCEMENT TYPES}

The resin system TGDM/DDS was held constant and the reinforcement fabric type was varied for composites irradiated in air with ${ }^{60} \mathrm{Co}$-gamma rays and tested in flexure at $77 \mathrm{~K}$ [Egusa, 1991a]. Figure A.3-9 shows that the initial strength is higher for less dense fabrics with a smaller fiber diameter and a lower fiber number per yarn, but despite this difference in the initial value, the dependence on irradiation dose is similar for all reinforcement types. All fabrics were plain weave. The type of glass fiber, E or T (B-free), also did not appear to affect either the initial strength or the gamma irradiation dose dependence significantly. However, at a high dose (1.8 x $10^{8}$ Gy) distinctions between reinforcements tended to disappear, within the statistical accuracy of the data. 
Egusa [1991a] also investigated the effects of the volume fraction of glass reinforcement in parallel with an investigation of the glass transition temperature of the resin. The degradation from gamma irradiation of reinforced TGDM resin systems that have different glass transition temperatures became similar at doses of about $5 \times 10^{6}$ Gy (Figure A.3-10). This suggests that radiation-induced cross-linking occurs in the resins with lower transition temperatures. The figure also shows that the initial strength was lower in a composite with a glass volume fraction of 558 , than in an otherwise identical composite with a volume fraction of 638 , although the difference in strength was only about 10\%. However, at high doses of gamma irradiation, about $10^{8} \mathrm{~Gy}$, this strength difference disappeared because the composite with the higher volume fraction of glass retained a lower percentage of initial strength.

In an earlier investigation [Egusa et a1., 1988], glass volume fraction was varied over a wider range, from 40 to 57\%, for a DGEBF resin system (Figure A.3-11). Again, at $77 \mathrm{~K}$ the initial strength advantage of the higher volume fraction had nearly disappeared at a dose of about $10^{8}$ Gy from 2-MeV electrons. Figure A.3-12 [Egusa, 1991a] shows that the specimen thickness affects the initial strength, as the volume fraction does, but then has little effect on the degradation of strength at a high irradiation dose. The initial superiority of the specimen that is only 1-mm thick disappears at gamma doses above about $10^{8} \mathrm{~Gy}$. Egusa proposed that the independence of dose dependence and high-dose strength from specimen thickness and various reinforcement parameters again provides support for a damage mechanism determined by the change in the matrix ultimate strain with irradiation.

\subsection{EFFECT OF IRRADIATION ENVIRONMENT}

A fiberglass-reinforced DGEBA/DDM epoxy composite and two commercial DGEBA resin laminates, described in $\$ 3.2$, were irradiated either in air or in argon with ${ }^{60} \mathrm{Co}$-gamma rays [Egusa, 1991a]. Figure A.3-13 shows that there was no significant difference in the results of flexural tests at $77 \mathrm{~K}$ after irradiation in either atmosphere. A composite is expected to be less sensitive to oxygen degradation during irradiation than a neat resin; hence, these results do not contradict those of Morgan et al. [1970] on neat DGEBA/DDM resins at ambient temperature ( $\$ 2.5)$. (The composite strength is 
relatively insensitive to surface defects caused by diffusion of oxygen during irradiation, whereas the neat resin is flaw-sensitive in failure.)

\subsection{EFTECT OF IRRADIATION SPECIES}

The differences between gamma and electron irradiation of a commercial epoxy laminate, Lamiverre A, were measured by Nishiura et al. [1988a]. The laminate is reinforced with a plain-weave glass with a silane finish. After irradiation at about $3.5 \times 10^{7} \mathrm{~Gy}$, both tear and acoustic emission tests showed that the material was more degraded by $20-\mathrm{MeV}$, ambient electron irradiation than by gamma irradiation from a ${ }^{60} \mathrm{Co}$ source, with an energy of about $1.3 \mathrm{MeV}$ (Figures A.3-14 and A.3-15). The dose rate of the electron irradiation was much higher than that of the gamma irradiation; this indicates that oxygen diffusion should not be the major cause of the difference, because the specimens irradiated more slowly with gamma rays would be expected to exhibit more severe deterioration (\$2.5). Furthermore, lowering the dose rate of the electron irradiation did not lower the damage at a given dose. Overheating should not have been a factor, because the specimens were cooled with circulating water at $290 \mathrm{~K}$ during the electron irradiation, whereas the gamma irradiation was in ambient air. The authors concluded that the 20-MeV electron irradiation was more damaging than the lower-energy gamma irradiation. Similar results were found with ambient-temperature testing (§2.9); maximum doses were about $5 \times 10^{7} \mathrm{~Gy}$, but the difference with radiation species was apparent at about $3 \times 10^{7} \mathrm{~Gy}$.

Earlier results from this group [Nishiura et al., 1988b] on the 77-K tensile properties of fiber glass-reinforced epoxy (Epomik) showed no significant difference between electron and gamma irradiation, but the highest dose, $10^{7} \mathrm{~Gy}$, was lower, and tensile properties are less sensitive to irradiation damage. The irradiation parameters were the same as those described above, except for the dose level. The composite was prepared by impregnation. These results are shown in Figure A.3-16.

Egusa [1991a] also investigated differences in the degradation of resins subjected to either ${ }^{60} \mathrm{Co}-$ gamma or $2-\mathrm{MeV}$ electron irradiation (Figure A.3-17). However, the effect he reported was opposite to that of Nishiura et al., because the gamma irradiation caused a larger initial degradation with dose of 
flexural properties of the TGDM/DDS neat resins tested. This effect was attributed to the lower dose rate of the gamma irradiation, which permitted oxidative degradation near the surface (\$1.3.3). However, when glassreinforced specimens of the same resin system were tested, no difference between the effects of gamma and electron irradiation was observed (Figure A.3-18). Egusa suggested that the neat resin specimens were much more sensitive to surface flaws. Since Nishiura et al. [1988a; 1988b] also tested composites, but found that electron irradiation caused more damage than gamma irradiation, perhaps the difference in results can be related to the higher energy of the electrons used for irradiation by Nishiura et al. The electron dose rates were similar in both experiments. 


\section{AMBIENT TEMPERATURE IRRADIATION WITH 4-K TESTING}

Data for ambient irradiation and $4-\mathrm{K}$ test conditions were reported by Egusa [1990a]. The performance of a TGDM/DDS system was compared to those of three different DGEBA resin systems (Figure A.4-1). The highest dose level of the ${ }^{60} \mathrm{Co}$ gamma irradiation was about $7 \times 10^{7} \mathrm{~Gy}$. Two of the DGEBA systems, G-10CR and G-11CR, are commercial laminates. All of the systems were reinforced with plain weave, E-glass. In accord with results at higher test temperatures after similar ambient-temperature irradiation (Figure 1.27), the three DGEBA systems were inferior in radiation resistance to the TGDM/DDS system. 


\section{77-K IRRADIATION WITH AMBIENT TEMPERATURE TESTING}

Evans et al. [1971] compared the effects of 1-MeV gamma irradiation in air with electron irradiation at $77 \mathrm{~K}$. Flexural tests on neat resin specimens were done at ambient temperature. These specimens were prepared in a mold rather than by machining, to avoid surface flaws. For the 77-K irradiations, no replicate specimens were tested, which may explain some of the differences in results from those usually obtained, since neat resin results usually show wide scatter, owing to their flaw-sensitivity (§1.4.1). Most of the tests were carried out on mixtures of various components with one commercial DGEBA resin (MY 740), but Figure A.5-1 shows comparative results with a different DGEBA system. Except for the three mixtures listed at the top of the legend in Figures A.5-2, A.5-3, and A.5-4, all of these resin systems had passed screening tests that indicated adequate resistance to thermal shock and all otherwise appeared promising for impregnating superconducting magnets. The authors concluded that, in general, the resin systems irradiated at $77 \mathrm{~K}$ and tested at ambient temperature showed less change than those both irradiated and tested at ambient temperature. This difference was attributed to the absence of oxygen in the 77-K irradiations, which were conducted in flowing liquid nitrogen. However, Figure A.5-1 shows that retention of flexural strength was higher in the material irradiated in air, and a comparison of Figure A.5-3 (an expansion of Figure A.5-2) with Figure A.5-4 shows that the authors' conclusions were not observed with all resin systems tested. Furthermore, this investigation may not be an ideal comparison of the effects of ambient temperature irradiation with 77-K irradiation, since the irradiation species at the two temperatures were not the same. Other experiments have indicated that gamma and electron irradiation may not be equivalent ( $\S \S 1.3 .2 .6,3.5$ ). 


\section{77-K IRRADIATION WITH 77-K TESTING}

A proprietary DGEBA magnet resin system, Orlitherm (EP 305) was used to vacuum-impregnate specimens reinforced with E-glass tape and, in some cases, polyimide foil (Kapton). The specimens were irradiated at $77 \mathrm{~K}$ in a reactor and tested in tension at $77 \mathrm{~K}$ without warm-up [Weber et al., 1983]. Fast neutrons ( $E>1 \mathrm{MeV}$ ) comprised about $33 \%$ of the neutron dose, which in turn was about 338 of the total dose. (The authors used the customary fluence-todose conversion rule.)

The results, shown in Figure A.6-1, indicated very good strength retention at $7.4 \times 10^{7} \mathrm{~Gy}$. However, dust-like particles were released from the specimens, upon tapping, beginning at a gamma dose of $1 \times 10^{7} \mathrm{~Gy}$, and swelling reached $5 \%$ at a gamma-dose level of $5 \times 10^{7} \mathrm{~Gy}$. (The gamma doses were accompanied by a neutron dose equal to about 508 of the gamma dose.) At a total dose of $1.5 \mathrm{x}$ $10^{8} \mathrm{~Gy}$, all of the test specimens were broken before mounting for testing could be completed. The authors noted that the tests, which measured tensile strength in the fiber direction, were not conservative for failure under magnet operating conditions and that shear and flexural tests would show greater degradation at the dose levels employed. Furthermore, because warm-up did not occur, additional degradation caused by gas formation and release did not take place. A comparison of these results for orlitherm composites with ambient temperature results (Figures A.2-25 and A.2-88) indicates that extension of ambient data to cryogenic conditions is not conservative, for the excellent results for several Orlitherm composites at $10^{8}$ Gy were not reproduced by Weber et al. However, the resin formulations may not have been exactly the same, since the manufacturer's type numbers were different. Also, the use of B-containing glass combined with a higher neutron dose may account for the discrepancy with ambient temperature results, since these were obtained with irradiation of high gamma ray content. Furthermore, although the use of Kapton foil may have weakened the reinforcement system (see Figure B.2-27), the results with the added foil were not worse that the results without it.

Three epoxy resins were irradiated at $77 \mathrm{~K}$ in a reactor (gamma dose $-67 \%$, neutron dose $-33 \%$ ) and tested in flexure and tension without warm-up at $77 \mathrm{~K}$ [Van de Voorde, 1972]. The flexural test results are shown in Figure A.6-2. 
All of the irradiated resins were found to have satisfactory resistance to thermal shock from 293 to $77 \mathrm{~K}$, including absence of fissures and internal stresses upon examination in polarized 1ight. One of the resins was identified only as "aromatic based," and the hardener was not reported. Although all resins showed good radiation resistance, the maximum dose of $1 \mathrm{x}$ $10^{7}$ Gy was below that at which severe degradation usually occurs and below the dose expected at ITER TF magnets.

The irradiation temperature, species, and test temperature were not specified for the data shown in Figure A.6-3 [Evans and Morgan, 1982]. However, the authors stated that all the resin systems were resistant to thermal shock. The chemical characteristics of the resin systems were not completely reported, but hardener type was given. One resin system type that apparently included some aromatic amine hardeners (Formulation 220) retained more than $100 \%$ of the initial flexural strength, but the maximum dose was only $1 \mathrm{x}$ $10^{7} \mathrm{~Gy}$. 


\section{20-K IRRADIATION WITH 77-K TESTING}

Figure A.7-1 shows the difference between results of neutron irradiation at about $20 \mathrm{~K}$ and gamma radiation at $77 \mathrm{~K}$ in the degradation of an E-glass reinforced commercial epoxy laminate, Hoxan [Nishijima et al., 1988]. The degradation of another commercial laminate, Lamiverre, was comparable at neutron doses near $10^{7} \mathrm{~Gy}$. Notched specimens were used to obtain the interlaminar shear strength (guillotine method). Actually, the comparison between neutron and gamma irradiation of Hoxan was not ideal because the neutron irradiation was accompanied by a gamma dose since the irradiation was done in a reactor. Furthermore, some of the difference between the effects of neutron and gamma irradiation can be attributed to the $(B, n) \rightarrow(L i, \alpha)$ reaction, discussed in $\$ 1.3 .2 .4$. But the larger change in properties under neutron irradiation, as compared with gamma irradiation, is probably one more indication that gamma doses may need to be downgraded somewhat to obtain the equivalent neutron irradiation damage. Comparisons of neutron- and gammairradiation damage are also discussed in $\$ 1.3 .2$.

Figure A.7-2 shows the effects of the B reaction in E glass again, compared to $B-f r e e ~ T$ glass, but note that the composite fabricated with another resin, bismaleimide (Appendix B), but also reinforced with E glass, does not exhibit the same degradation from alpha particles that the E-glass reinforced-epoxy composite does [Nishijima et al., 1991; Okada et al., 1992]. Figure A.7-3 shows that the volume fraction of E-glass-reinforced epoxy does not affect the dose dependence of degradation under combined gamma and neutron irradiation from a reactor at $-20 \mathrm{~K}$. It would be of more interest to examine the variation of volume fraction with B-free glass, in case the damage from alpha particles tended to obscure intrinsic volume fraction effects. However, Egusa [1991a] also found that volume fraction did not appear to strongly affect strength after irradiation, despite differences in test methods and irradiation species and dose ( $\$ 3.30)$.

Reactor irradiation at $20 \mathrm{~K}$ was also used by Nishijima et al. [1990] to compare the performance of a 3-dimensional glass weave reinforcement with the standard 2-dimensional weave. The resin system was not identified, but was reported to be the same for both types of reinforcement. The glass type (E or B-free) and its surface finish also were not described, but these parameters 
were the same for the two types of reinforcement. Figure A.7-4 shows the performance of both reinforcement types tested in flexure at $77 \mathrm{~K}$ without warm-up. At the maximum dose of $8 \times 10^{6} \mathrm{~Gy}$, the performance of the 3dimensional reinforcement is superior. The two types of reinforcement systems were also tested with a guillotine test [see, e.g., Becker, 1990] to obtain the interlaminar shear strength. The test was carried out at $77 \mathrm{~K}$ without warm-up. At the maximum dose of $1.5 \times 10^{7} \mathrm{~Gy}$, the performance of the 3-dimensional reinforcement system was again superior (Figure A.7-5). Both tests of the 3-dimensional material were also reported by Yasuda et al. [1988]. Three-dimensional materials may not have adequate dielectric properties for use in tokamak reactor coils, however [Okada, 1992]. 


\section{4- TO 5-K IRRADIATION WITH 77-K TESTING, WITH AND WITHOUT WARM-UP}

Commercial DGEBA epoxy laminates (G-10CR and G-1ICR) were irradiated at about $4 \mathrm{~K}$ in the IPNS facility, with a fast neutron dose ( $\mathrm{E}>0.1 \mathrm{MeV}$ ) of about $70 \%$ of the total neutron dose of $1.3 \times 10^{6}$ Gy [Hurley et al., 1978; 1983]. An additional gamma dose of less than $15 \%$ was not included in the total dose. The specimens were then warmed to room temperature and tested in flexure and compression at 295 and $75 \mathrm{~K}$ (Figures A.8-1 and A.8-2). A silica-filled DGEBA resin (Epon 828) with an aromatic hardener was similarly irradiated and tested in flexure and tension (Figures A.8-3 and A.8-4). All resin systems showed good retention of properties, but this was undoubtedly due to the low total dose. Since these data were obtained under conditions, including warm-up, that simulated magnet operating conditions better than most irradiation tests, it is unfortunate that the total dose was so far below that expected in a fusion reactor. The negligible effect of the test temperature on G-10CR results is in agreement with data of Egusa and Hagiwara [1986] in which such effects were not observed until higher doses of about $5 \times 10^{6}$ Gy were reached. However, the comparative test temperature data of Egusa were obtained after ambient temperature irradiations.

Further fast neutron irradiations at $4 \mathrm{~K}$ in the IPNS facility were carried out to slightly higher doses, about $4 \times 10^{6}$ Gy [Tucker et al., 1985]. The commercial laminates $G-10 C R$ and G-1ICR were again tested in flexure (Figure A.8-5). It is likely that the specimens were tested after warm-up, as in the earlier work, but this aspect of the test procedure was not reported. The G$10 C R$ exhibited considerable degradation at $4 \times 10^{6}$ Gy, but the G-11CR retained nearly $90 \%$ of the initial flexural strength. This divergent behavior probably arises from the difference in irradiation resistance of the hardeners used in the two systems. An aromatic amine is used to cure G-11CR, but G-10CR is cured with a less resistant aliphatic amine.

Filled DGEBA resins Epon 828 and Stycast 2850 FT blue were reactor-irradiated at $4.9 \mathrm{~K}$, warmed to ambient temperature, and tested at $77 \mathrm{~K}$ [Kernohan et al., 1979]. A glass-epoxy laminate, G-10CR, was similarly irradiated and tested. After about a third of the irradiation dose had been absorbed, the materials were warmed to ambient temperature and then cooled again for the remainder of 
the irradiation dose. Both flexural and compressive tests at $77 \mathrm{~K}$ were performed after a final warm-up.

The bulk shielding reactor (BSR) at ORNL (Oak Ridge National Laboratory) was used for the irradiation of these specimens. The major part of the dose was from gamma rays, because a cadmium shield was used, and cadmium converts most thermal neutrons to gamma rays by an $(n, \gamma)$ reaction. Since the maximum dose attained was about $2 \times 10^{7} \mathrm{~Gy}$, further irradiations of the same materials were carried out at ORNL (in a modified setup at the BSR) to a total dose of $10^{8}$ Gy [Coltman et al., 1979]. In the second series of irradiations, the ratio of the fast neutron flux $(E>0.1 \mathrm{MeV}$ ) to the gamma flux was increased by a factor of 17 . However, the total fast neutron fluence was still only $8.7 \times 10^{20} / \mathrm{m}^{2}$, which is $8.7 \times 10^{5}$ Gy if the conversion rule, $1 \mathrm{~Gy}=10^{15} / \mathrm{m}^{2}$, is used. Thus, the contribution of fast neutrons to the total dose of $10^{8}$ Gy was still very small compared to that of gamma rays. Nevertheless, a G-10CR with B-free glass was also tested, as was a G-11CR laminate.

Figures A.8-6 and A.8-7 present the flexural test results for the filled epoxies from the two irradiations and Figures A.8-8 and A.8-9 present the corresponding compressive test results. Figures A.8-10 and A.8-11 give the flexural test results on laminates for the two irradiations, and Figures A. 8-12 and A.8-13 give the compressive test results. Additional data at lower doses from a third set of ORNL irradiations of the same laminates [Coltman and Klabunde, 1983] has been added to Figures A.8-11 and A.8-13. These irradiations were also carried out at $4.9 \mathrm{~K}$ and the materials were again tested after warm-up to ambient temperature. A comparison of the odd- and even-numbered figures listed above shows that the additional fast neutron flux had no significant effect. (Odd-numbered figures pertain to irradiations with the added fast neutron flux.) Since the fast neutrons constituted a very small portion of the total dose, this result is not surprising. Both the flexural and the compressive test results at $10^{8}$ Gy showed that none of the DGEBA-based materials retained more than 10 to $20 \%$ of their initial strength. For the first irradiations at a dose of $2 \times 10^{6} \mathrm{~Gy}$, measurements of the specimen mass were made before and after irradiation [Long et al., 1979]. No measurable mass changes were found $(<0.2 \mathrm{mg}$ in specimens of mass 62 to $500 \mathrm{mg}$ ). 
Tucker et al. and Hurley et al. tested the same laminates previously irradiated by Coltman et al. in order to compare the effects of fast neutron irradiation with predominantly gamma irradiation. To make this comparison, Figure A.8-5 should be compared with Figure A.8-11. Unfortunately, the fast neutron irradiation doses do not extend to the higher levels at which significant degradation of G-11CR occurs. However, a comparison of results for G-10CR at about $3 \times 10^{6}$ Gy indicates that the neutron damage is more severe. Of course, these results are confounded by the use of $\mathrm{E}$ glass.

Kato and Takamura [1979] irradiated a DGEBA resin (Epikote 828) combined with various hardeners in a reactor at $5 \mathrm{~K}$. (See also Takamura and Kato [1981].) The neat resin systems were tested in compression at $77 \mathrm{~K}$ without warm-up, except for one system that was warmed up after the highest dose of $1.1 \mathrm{x}$ $10^{7}$ Gy (Figure A.8-14). The compressive strength was higher for the warmed-up set of specimens, as indicated in the figure. The percentage of retained strength of one group of filled specimens at $1.1 \times 10^{7}$ Gy was somewhat lower than that reported for a similar system (Epon 828-Z with silica) by Coltman et al. [1979] (Figure A.8-9). However, the specimens and tests were not identical, so exact agreement should not be expected. Also, the specimens of Coltman et al. were warmed up and Figure A.8-14 indicates that this procedure may increase the strength. Recent data on the shear strength of composite adhesive bonds also indicates that warm-up increases the apparent radiation resistance [Katheder et al., 1992].

Neutron irradiation at $5 \mathrm{~K}$ followed by flexural testing at $77 \mathrm{~K}$ was carried out by Egusa et al. [1987b] on a TGDM/DDM resin system reinforced by E glass. The specimens were warmed up before testing. These results were discussed in §1.3.3. As Figure 1.22a indicates, there was little difference between ambient-temperature test results after either ambient-temperature or 5-K neutron irradiation. However, the 5-K neutron irradiation was carried out only up to $2 \times 10^{7} \mathrm{~Gy}$, below the dose required in the ITER conceptual design. About $82 \%$ of the initial flexural strength was retained at this dose. The results of $77-\mathrm{K}$ tests (Figure $1.22 \mathrm{~b}$ ) indicated that $5-\mathrm{K}$ neutron irradiation was about as damaging as ambient-temperature irradiation at $2 \times 10^{7} \mathrm{~Gy}$. Again, the highest dose at $5 \mathrm{~K}$ was below that expected in ITER TF magnets. Also, the results may have been confounded by heavy alpha particle damage, owing to the use of glass containing B. 
More recently, Munshi [1991] compared three different resin systems reinforced with S-2 Glass after 4-K reactor irradiation to $1.6 \times 10^{8}$ Gy. The fast neutron flux $(E>0.1 \mathrm{MeV}$ ) in the reactor was about equal to both the thermal and the intermediate energy fluxes [Wallner et al., 1988], and the fast neutron fluence of $3.1 \times 10^{2}$ constituted about $20 \%$ of the total dose, from the usual conversion rule (\$1.33). The high energy portion of the neutron spectrum is shown in Figure 1.8. About $50 \%$ of the total dose is from gamma radiation [Katheder et al., 1992]. One of the resins tested was DGEBA, the second was EPN, and the third was a multifunctional epoxy similar to TGDM or TGPAP. The specimens were uniaxially-reinforced rods tested in torsion to determine the shear strength. Testing was carried out at $76 \mathrm{~K}$ after warm-up to ambient temperature. The results (Figure A.8-15) showed that the irradiation resistance of the multifunctional epoxy was better than that of the EPN and DGEBA resins at the highest dose of $1.6 \times 10^{8} \mathrm{~Gy}$. This agrees with the trend found in ambient-temperature irradiation and testing (\$2.1). Table 3.1 , which gives the viscosity and pot life of these resins, indicates that the DGEBA resin system would be the most suitable for vacuum impregnation. This resin system retained about 228 of the initial shear strength at $1.6 \times 10^{8} \mathrm{~Gy}$ and about 978 at $2.9 \times 10^{7} \mathrm{~Gy}$. 


\section{4- TO 5-K IRRADIATION WITH 4-K TESTING WITHOUT WARM-UP}

Takamura and Kato [1980] conducted in situ, 4-K compression tests of specimens that were reactor-irradiated at about $5 \mathrm{~K}$ to a combined neutron and gamma dose of $1.1 \times 10^{7} \mathrm{~Gy}$. Tests were also done at $77 \mathrm{~K}$. Neat resin results on a DGEBA resin (Epikote 828) with three different types of hardener are shown in Figure A.9-1. Perhaps because of the high scatter inherent in neat resin testing, no consistent trend of the results with test temperature is seen. The retained values for the aliphatic amine hardener are higher in 4-K tests, the retained values for the acid anhydride hardener are higher in 77-K tests, and the retained values are nearly the same in both 4 and 77-K tests for the aromatic hardener. However, Figure A.9-2 shows that when the DGEBA resin with an acid anhydride hardener was glass-reinforced, results similar to those for the neat resin were obtained. Therefore, the effect of test temperature may actually depend somewhat on the hardener. More testing would be necessary to substantiate this hypothesis.

Similar in-situ test methods were used in further irradiation studies by Takamura and Kato [1984] on glass-reinforced systems, but tensile and flexural test capabilities were added. The highest reactor dose was again $1.1 \mathrm{x}$ $10^{7} \mathrm{~Gy}$. The flexural, compressive, and tensile test results for a cycloaliphatic epoxy and an isocyanate-hardened epoxy and two commercial laminates, G-10CR and G-11CR, are shown in Figures A.9-3 to A.9-5. In accord with the results of Coltman et al. [1979] (\$8), the G-11CR with aromatic hardener performed better than the G-10CR. The isocyanate-hardened epoxy was superior in radiation resistance to all epoxies tested, however; its flexural strength increased to nearly 1208 of the initial value at $1.1 \times 10^{7} \mathrm{~Gy}$. But the initial flexural and tensile strengths of the isocyanate system (ISOX) were significantly lower than those of other epoxy systems. In ambient temperature testing, Lipták et al. [1985] found that a neat resin containing isocyanate retained 648 of the initial flexural strength at $1 \times 10^{8}$ Gy $(\S 2.10 .2)$.

In situ flexural tests of unidirectionally reinforced DGEBA specimens were also conducted by Takamura and Kato [1981a; 1984]. The irradiation and test conditions were the same as those discussed above. The results for two different hardeners are shown in Figure A.9-6. Both types of specimens had a 
high initial strength and both retained a high percentage of their initial strength at $1.1 \times 10^{7} \mathrm{~Gy}$. 


\subsection{UNIAXIAL LOADING}

A V-shaped, compressive test fixture was used to load two 1-mm thick specimens in simultaneous shear and compression [Nishijima et al., 1990]. The angle of the test fixture was varied to change the ratio of resolved shear to compressive stress and thus generate a failure envelope. Figure A.10-1 shows the decrease of the failure envelope to a smaller size as the radiation dose was increased for G-11CR, a commercial laminate. After a dose of $1.8 \times 10^{8} \mathrm{~Gy}$ of $2-\mathrm{MeV}$ electron irradiation at ambient temperature, the G-11CR laminate could not meet the test program criteria that required a compressive strength of $400 \mathrm{MPa}$, combined with a shear strength of $100 \mathrm{MPa}$. However, a composite prepared with a 3-dimensional weave (resin system not specified) exhibited much better performance, as shown in Figure A.10-2. The tests on both materials were conducted at ambient temperature. However, 3-dimensional weaves may have dielectric properties that make them unsuitable for turn-toturn magnet insulation [Okada, 1992].

\subsection{BIAXIAL LOADING}

In another approach to combined shear/compressive testing, two specimens were loaded in compression by a hydraulic fixture to a predetermined amount, and then a perpendicular shear force was applied until a specimen failed [McManamy et al., 1990; 1991a]. Figure A.10-3 shows the results of one test with a compressive load of $345 \mathrm{MPa}$. A 3-dimensional weave with B-free glass was used to reinforce the epoxy. The resin was not specified. At a dose of $3 x$ $10^{8} \mathrm{~Gy}$, of which about 428 was from neutrons, the specimens were found to have shear strengths that exceeded the initial value. Both testing and irradiation were performed at ambient temperature. 


\section{SHEAR TESTS OF COMPOSITE/ALLOY ADHESION}

Although insulation failure in a TF magnet in the ITER could occur within the composite, the adhesive bond between the composite and the conductor sheath alloy is also susceptible to radiation damage and failure. For a number of reasons, the data available on the radiation resistance of this bond do not match ITER conceptual design conditions. First, most data were obtained with a substrate of copper, which has substantially different surface oxidation properties from the steel and iron-nickel alloys that are candidates for the conductor sheath. Second, most data were obtained with E-glass-reinforced specimens rather than with B-free glass reinforcement. Third, many of the resin systems used for specimens are not suitable for vacuum impregnation. Fourth, none of the specimens were irradiated with fast neutrons to fluences of $7 \times 10^{22}$ to $10^{23} / \mathrm{m}^{2}$ Gy at $4 \mathrm{~K}$. Finally, tests were often carried out at ambient temperature on lap shear specimens for which the compressive component is not accurately known. (Irradiation tests using the shear/compressive test methodology discussed in $\$ 10$ have not yet been conducted under cryogenic conditions.)

Although none of the adhesive bond investigations meet all of the ITER conceptual design requirements, some information on the bond parameters that affect radiation resistance has been obtained. Preliminary data are available on the relative radiation resistance of chemical and mechanical bonding. Data are also available on the effects of the reinforcement type (filler or glass fiber) and resin type on radiation resistance.

\subsection{COMPARISON OF MECHANICAL AND CHEMICAL BONDING}

Lipták et al. [1985] showed that the initial shear strength of a copper substrate (Figure 11.1a) with E-glass-reinforced DGEBA epoxy is much higher if the surface is primed with DZ-80, a polyamide compound. However, at a dose of about $1.3 \times 10^{7} \mathrm{~Gy}$, the shear strength of the primed specimens had fallen to a value equivalent to that of the unprimed specimens (Figure A.11-1). The conditions of this experiment did not match ITER requirements in several respects. The neutron component of the dose was less than 5\%, and the irradiation and testing were carried out at ambient conditions. Furthermore, 
a

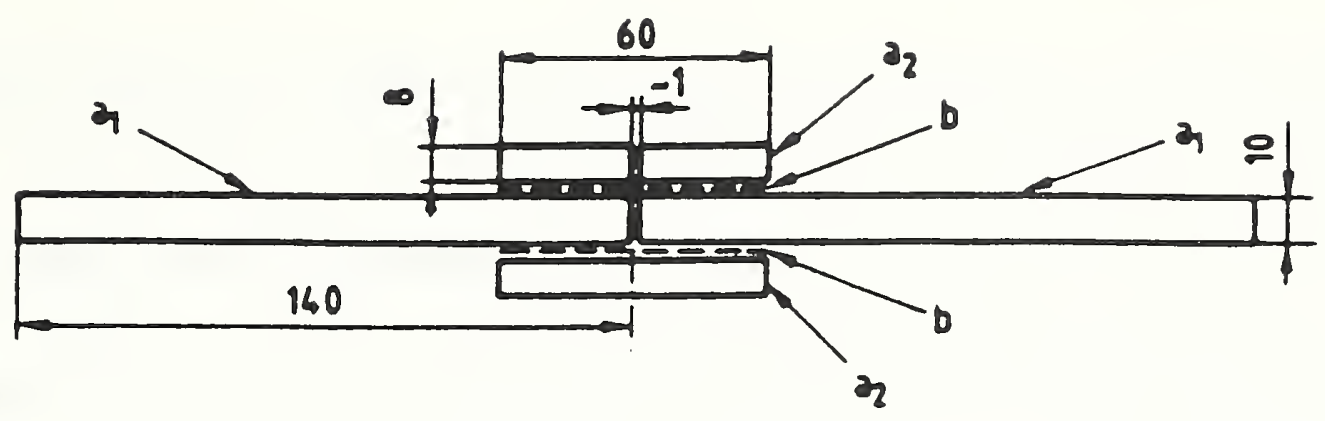

b
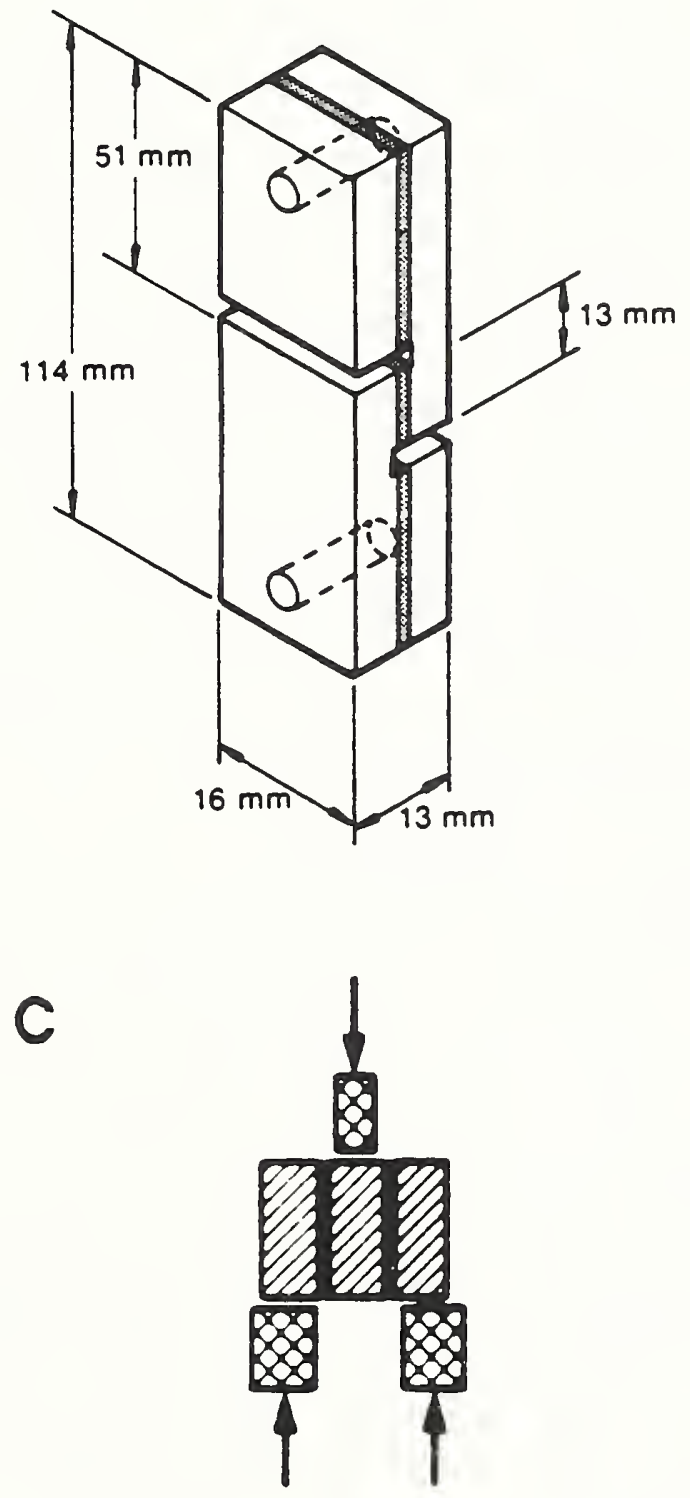

Figure 11.1. Lap shear specimens. (a) Lipták et a1. [1985]. (b) Imel et al. [1979]; Schmunk et a1. [1981]. (c) Kernohan et a1. [1979]; Coltman et al. [1979]. 
a copper surface cleaned by abrasives or chemical methods oxidizes faster than the alloys expected to be used for the conductor sheath in ITER; thus, the effects of a primer and other conditions of surface preparation may be quite different for ITER conductor alloys. (The preparation of the copper surface was not described by Lipták et al.) However, the experiment suggested that a chemical bond may be more vulnerable to radiation damage than a mechanical bond.

Imel et al. [1979] also examined the effects of priming a copper surface with DZ-80 under similar irradiation and test conditions (Figure A.11-2). Again, the highest shear strengths were obtained when both surfaces of a sandwich specimen (Figure 11.1b) were primed, and the initial shear strength of about $30 \mathrm{MPa}$ was similar to that reported by Liptak et al. The primed surfaces were compared with sandblasted, unprimed surfaces, but only up to a maximum dose of about $1.2 \times 10^{6} \mathrm{~Gy}$. Despite the low dose, far below ITER requirements, the data indicated that the lower adhesive strength of the mechanically prepared surface was retained after radiation to a greater degree than the higher strength of the primed surface. However, the shear strength of the primed specimens did not decline to the strength of the unprimed specimens, as occurred in the experiments of Lipták et al.

Further evidence of the greater deterioration of a chemical bond than of a mechanical bond was furnished by investigations of Egusa et al. [1992] on lap shear specimens. (See Figure 11.3 for specimen geometry, similar to that used by Poehlchen et al., below.) Figure A.11-3 shows that a one T-glass-BT (bismaleimide triazine) bonded composite had a higher initial bond strength to stainless steel at $77 \mathrm{~K}$ than a similar T-glass-BT bonded composite fabricated by another manufacturer or a T-glass-TGDM bonded composite. However, the initially stronger composite specimen retained only about $30 \%$ of its unirradiated strength at an ambient gamma irradiation dose of about $5 \mathrm{x}$ $10^{7} \mathrm{~Gy}$, whereas the strengths of the other two bonded specimens were nearly unchanged after irradiation to that dose. Egusa et al. suggested that the higher initial strength of about $50 \%$ of the one BT specimen was due to some degree of chemical bonding, in addition to the frictional or mechanical bonding strength, which was hypothesized to be about the same for all three composites. This chemical bond would then be more sensitive to irradiation, 
which primarily causes chemical changes in organic materials. However, as Egusa et al. noted, there was no direct evidence for this hypothesis.

\subsection{COMPARISON OF REINFORCEMIENT TYPE}

Two groups have tested the adhesion of both filled epoxies and fiberglassreinforced epoxies to copper substrates. Kernohan et al. [1979] reported that a glass-reinforced, B-staged epoxy exhibited lower radiation resistance than two types of filled DGEBA epoxies (Figure A.11-4), but Brechna [1965] found that bonds made with one of the same types of filled epoxy $\left(2850 \mathrm{FT} / \mathrm{Al}_{2} \mathrm{O}_{3}\right)$ had a poorer radiation resistance than bonds made with three glass-reinforced DGEBA systems (Figure A.11-5). The comparison by Brechna was more direct because only DGEBA resin systems were compared, but the test configuration, shown in Figure 11.2, was more complex and very different from those of Keronan et al. and other adhesive bond investigators (Figure 11.1c). Also, as noted above (\$2.6), the electron radiation doses of Brechna appear to disagree with those of other authors by a factor of about $10^{2}$, so his results are difficult to compare to other results on an absolute basis.

One group has directly compared the radiation resistance of $E$ glass with $B-$ free glass ( $R$ glass) when used in an adhesive bond; as expected, the B-free reinforcement gave higher shear strengths at $77 \mathrm{~K}$ after reactor irradiation at $4 \mathrm{~K}$ [Poehlchen et al., 1990; Pöhlchen, 1992]. Also, as Figure A.11-6 indicates, the initial (zero-dose) strength of the R-glass system without flexibilizer was retained at the maximum dose of $10^{7} \mathrm{~Gy}$. However, E glass with a normal finish, with and without Kapton reinforcement, was compared to $R$ glass that had undergone a heat treatment at $700^{\circ} \mathrm{C}$ that removed the surface finish. The magnet resin system Orlitherm EP 311 was used in these tests. This is a DGEBA resin with an MNA hardener and about $5 \%$ flexibilizer. Figure A.11-6 presents the results of the comparative 77-K shear tests conducted after warm-up; results with heat-treated $R$ glass at other test temperatures, 4 and $300 \mathrm{~K}$, are given in Figures A.11-7 and A.11-8. These figures indicate improvement in shear strength when the test temperature was decreased to $4 \mathrm{~K}$. However, although the mean shear strength after irradiation at $4 \mathrm{~K}$ was about $140 \mathrm{MPa}$, both the total dose and the fast-neutron fluence were below those 

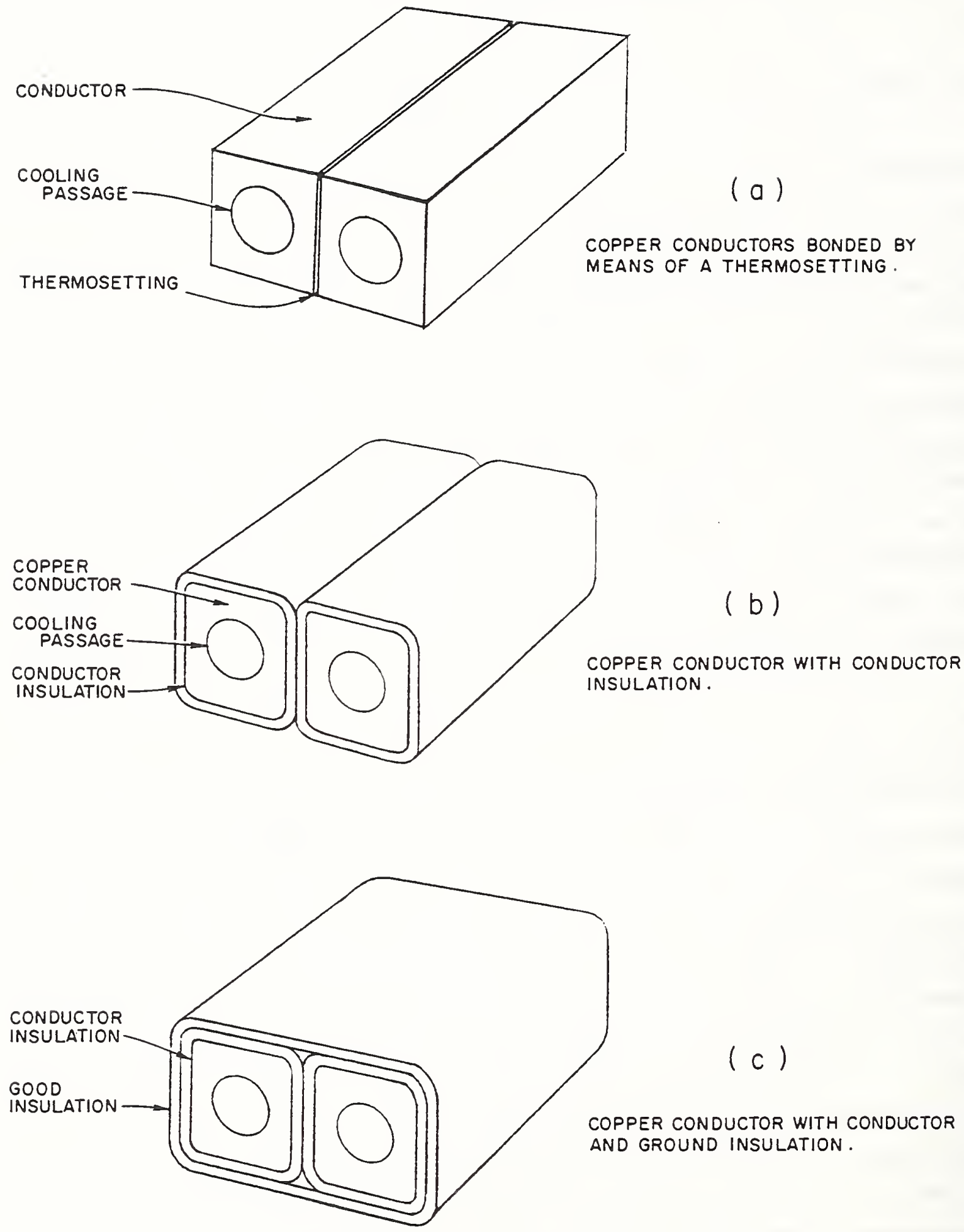

Figure 11.2. Bond specimen, Brechna [1965]. 
required by the ITER conceptual design ( $\$ 1.3 .3)$. (The reported total dose was increased by a factor of two, to $10^{7} \mathrm{~Gy}$, in the second report of these investigations [Pöhlchen, 1992].)

The lap shear specimen configuration used with these tests is shown in Figure 11.3. Some calculations on the homogeneity of shear stresses in this specimen and the degree of compressive stress accompanying the shear stress were described by Fardi et a1. [1989]. A disadvantage of this specimen (termed the NET, Next European Torus, specimen) is that the compressive stresses are not measured, but have to be calculated and may amount to $15 \%$ or more of the applied shear stress. Thus, the test may not measure pure shear, but, rather, gives a data point at one unknown locus on a shear/compression failure envelope. (An example of a shear/compression failure envelope is depicted in Figure A.10-1.)

More recently, tests have been made with this lap shear specimen in a cryostat that allows in-situ 4-K measurements after 4-K irradiation [Katheder et al., 1992]. This apparatus permits a comparison of post-irradiation damage with and without warm-up to ambient temperature, with the same irradiation source used by Poehlchen et al. Figure A.11-9 shows that specimens that were warmed up had significantly higher shear strengths than specimens tested in situ. These results are in accord with 77-K compression test results of Takamura and Kato (Figure A.8-14) and indicate that some annealing of damage occurred. The specimens tested by Katheder et al. were reinforced with desized $\mathrm{R}$ glass interleaved with Kapton, a polyimide film. The resin used was a proprietary DGEBA/MNA system, Orlitherm. The reactor irradiation dose of $5 \times 10^{7}$ Gy was composed of about 508 gamma rays and $20 \%$ fast neutrons ( $E>0.1 \mathrm{MeV}$ ). (The fast neutron fluence from the 9-h irradiation was about $10^{22} / \mathrm{m}^{2}$, or $10^{7}$ Gy with the usual conversion factor.) These results indicate that the use of cryogenic irradiation and test data in which specimens were warmed up is not conservative, because post-irradiation strengths under steady-state 4-K conditions could be about a factor of two lower. Other reinforcement systems, for which irradiation data were not yet available, exhibited higher initial shear strengths than the Kapton-interleaved system. 


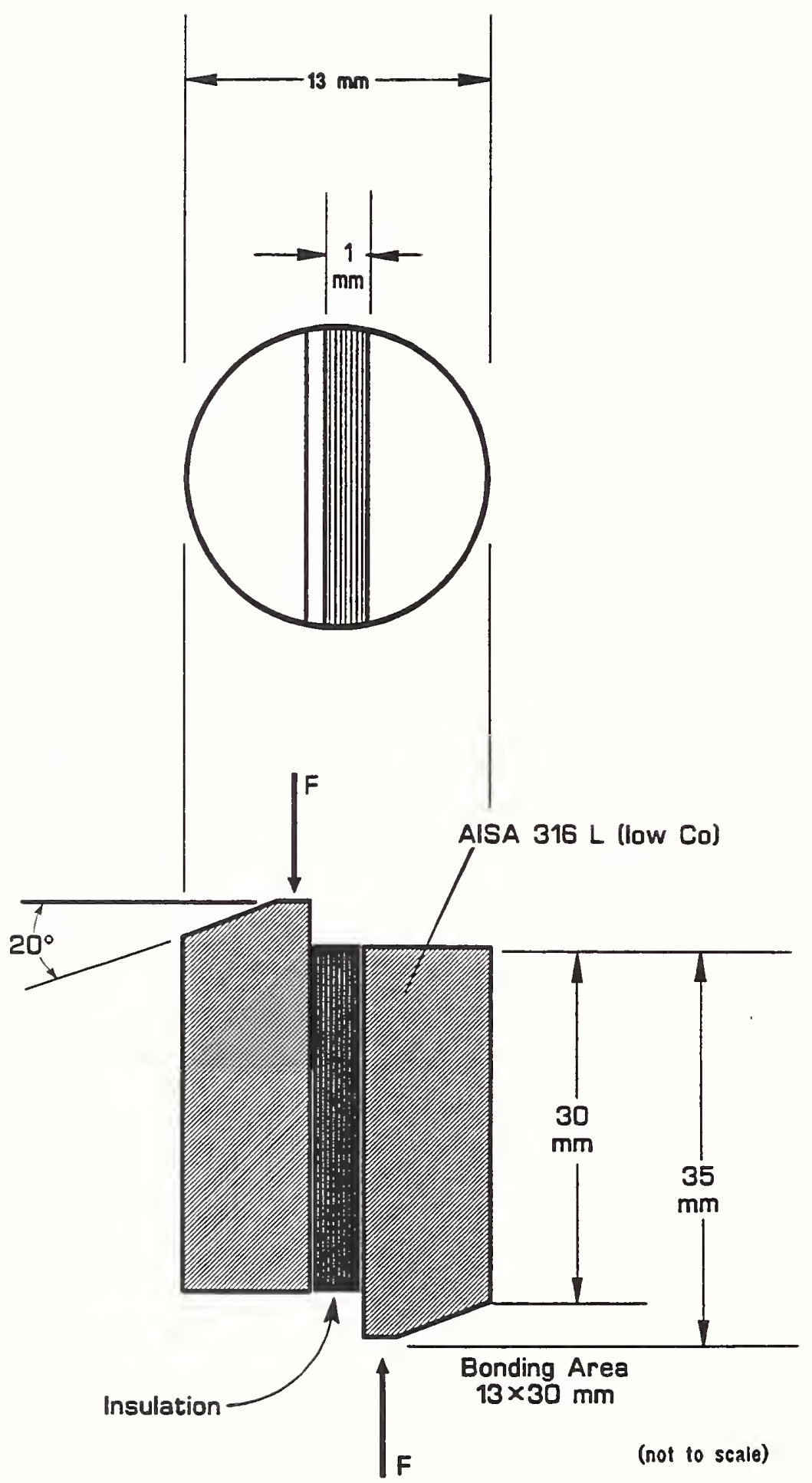

Figure 11.3. Lap shear specimen, Poehlchen et al. [1990]. 


\subsection{COMPARISON OF RESIN SYSTEMS}

\subsubsection{Results at Low Doses}

Three sets of data discussed above present results of comparative adhesion tests with different resin systems (Figures A.11-3, A.11-4, and A.11-5). However, no conclusions can be drawn from these comparisons because other parameters of the reinforcement system were not kept constant when the resin was varied. Both $B T$ resin and epoxy resin systems reinforced with $R$ or $T$ glass exhibited resistance to radiation damage at the highest doses at which they were tested for adhesion (Figures A.11-3, A.11-6, A.11-7, and A.11-8), but these doses were at least an order of magnitude below the dose expected at the ITER TF coils. (Considerable degradation of a DGEBA/MNA system was found when the dose was increased to $5 \times 10^{7} \mathrm{~Gy}$, as shown in Figure A.11-9, even though the fast neutron component was only about $1 \times 10^{7} \mathrm{~Gy}$. Schmunk et al. [1981] made shear bond tests of fiber-glass-reinforced DGEBA resin systems with and without flexibilizer. Figure A.11-10 shows that the flexibilizer caused no discernable difference in the shear strength, but the ambient reactor irradiation was carried out only to about $1.2 \times 10^{6} \mathrm{~Gy}$. The specimen is depicted in Figure 11.1b.

\subsubsection{Results at High Doses}

Only three groups have tested adhesive bond systems at doses approaching or comparable to the ITER TF magnet dose. The preliminary results of Katheder et al. at a fast neutron fluence of $10^{22} / \mathrm{m}^{2}$ were discussed above (\$11.2). Coltman et al. [1979] tested two of the DGEBA resin systems studied by Kernohan et al. [1979]. The same reactor irradiation conditions and specimen types and tests were used, but the copper substrate was sanded beforehand, which increased the initial shear strengths by about a factor of three, as Figure A.11-11 indicates. However, these high strengths were degraded to very low values after the maximum dose of $10^{8}$ Gy was administered.

The other group that conducted bond adhesion tests in shear after doses above $1 \times 10^{8} \mathrm{~Gy}$ found that no degradation outside the standard deviation was observed at a reactor dose of $4 \times 10^{8} \mathrm{~Gy}$ [McManamy et al., 1991b]. (About 408 of this dose was from neutrons.) These results, at ambient irradiation and 
test temperatures, were obtained with a styrene-modified epoxy resin system (Figure A.11-12) on sandwich-type specimens (Figure 11.4). No differences in shear strength were found for specimens that were thermally shocked at $77 \mathrm{~K}$ before and after radiation and those that were not thermally shocked. Since a polyurethane-based primer (Conastic AD-20) was used on the grit-blasted copper substrate, but no significant degradation was observed, these results are not in accord with other results indicating radiation sensitivity of the chemical bond achieved with a primer $(\$ 11.1)$. Perhaps the radiation resistance of the polyurethane (isocyanate-derived) primer-epoxy bond is higher than that of other primers, in accord with a small amount of data that indicates increased radiation resistance of epoxy-isocyanate systems (\$2.10.2, Figure A.2-87; §9, Figures A.9-3 to A.9-5). Other types of primers, including DZ-80, were tested for bond shear strength and generally found to have greater strength than the AD-20 primer. However, these specimens were not tested after irradiation.

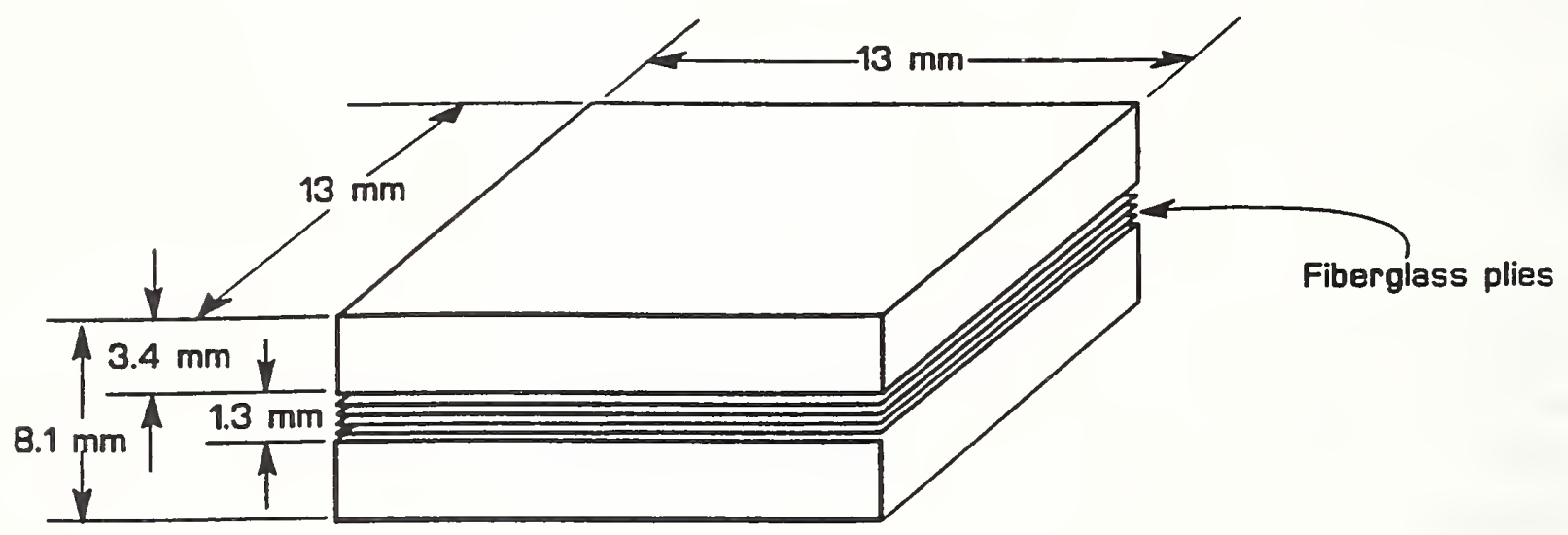

Figure 11.4. Shear/compression specimen, McManamy et al. [1991b]. 


\subsection{SUMMARY OF RESULTS}

Using fiber-glass-reinforced flexural specimens, gamma irradiation, and (usually) holding hardener type constant, a series of investigations at Rutherford Laboratories established the following order of radiation resistance for epoxy resins: TGDM $\geq$ TGPAP > EPN > Cycloaliphatic resins > DGEBA. These investigations were carried out by Price and Sheldon [1965], Sheldon and Stapleton [1968], and Evans et al. [1970], at doses up to $-10^{8} \mathrm{~Gy}$. Investigations at other laboratories on neat resins corroborated the superiority of TGDM, TGPAP, and EPN resins to cycloaliphatic resins and to DGEBA [Pluym and Van de Voorde, 1967; Markley et al., 1969]. Further work by Morgan and Stapleton [1975] on neat resins confirmed the superior radiation resistance of TGDM. The trend of these results followed well-established chemical rules for radiation stability [e.g., Pluym and Van de Voorde, 1967].

When the radiation resistance of various types of hardeners used with the TGDM resin was studied by the same investigators, aromatic amine hardeners such as DDS and DDM conferred greater radiation stability than anhydrides. However, results with the anhydride MNA were also fairly good, and MNA provided lower viscosity. Since the viscosity of resin systems cured with aromatic amines is usually too high to allow their use for vacuum impregnation, Evans et al. [1972a] investigated the use of high (nonstoichiometric) amounts of MNA with TGDM to see if a low viscosity TGDM/MNA system would have adequate radiation resistance. These authors demonstrated that over $100 \%$ of the initial flexural strength in a composite was retained after a gamma dose of $1.5 \times 10^{8}$ Gy with a TGDM/MNA system that had a viscosity of only $2.7 \mathrm{~Pa} \cdot \mathrm{s}$ at $24^{\circ} \mathrm{C}$. Furthermore, Schönbacher and Stolarz-Iźycka [1979] presented data on a TGDM/MNA resin system used in magnets that retained about $80 \%$ of the initial flexural strength at $7 \times 10^{7}$ Gy (Figure A.2-23). However, MNA-cured resins release more gaseous product ( $\mathrm{CO}$ and $\mathrm{CO}_{2}$ ) after irradiation than DDM and DDS-cured resins do. This is a disadvantage for cryogenic systems, since the sudden release of gas upon warm-up may cause problems. Therefore, it is of interest that Morgan and Stapleton [1975] indicated that an aromatic-amine cured TGDM system could also be used for impregnation. They reported that a low 
viscosity, TGDM/aromatic amine resin system that contained DDM and other components was "suitable for impregnating glass fiber reinforced components." Since DGEBA resins have better vacuum-impregnation characteristics than the more radiation-resistant glycidyl amines such as TGDM and TGPAP, many attempts were made to improve their radiation resistance through optimization of variables such as curing conditions and fiber-glass surface finish. Increasing the curing time did not improve the radiation resistance of DGEBA systems at high radiation doses, $-5 \times 10^{7}$ to $2 \times 10^{8}$ Gy (\$2.4). Although an aminosilane finish was found to be more radiation-resistant at high doses than a heat-cleaned finish, the improvement was not large enough to raise the resistance of DGEBA composites to the levels exhibited by other resin systems (Figure A.2-13, [Sheldon and Stapleton, 1968]). An increase in volume fraction, which of course, contributes to the strength of an nonirradiated specimen, just as an increase in curing time or heat-cleaning of the glass may do, was likewise found to be ineffective in raising radiation resistance at high doses, $-5 \times 10^{7}$ to $10^{8} \mathrm{~Gy}$, in a DGEBF system (Figure A.2-11, [Egusa et al., 1988]). Similar results would probably be found with DGEBA systems. The most satisfactory radiation resistance shown by a DGEBA resin composite at $10^{8}$ Gy was obtained with a proprietary resin system, Orlitherm and a "special" finish on the glass tape reinforcement (Figure A.2-25, [Schönbacher and Stolarz-Iźycka, 1979]). Other Orilitherm systems tested by these authors had retained flexural strengths that were considerably below the 60 retention that this special system exhibited. However, Lipták et al. [1985] reported 50 to 608 retention of strength at $10^{8}$ Gy with two fiber-glass reinforced Orlitherm resins with different type designations that may have varied slightly in composition from those tested by Schönbacher and Stolarz-Iźycka (Figure A.2-88). DGEBA resins reinforced with granular fillers, such as alumina and dolomite, had initial strengths lower than those of fiber-glassreinforced systems and did not exhibit as high a radiation resistance as TGDM and EPN systems.

Ambient-temperature testing has indicated that another way to increase radiation resistance is to use mica in addition to glass as a reinforcement. Although systems such as Samica contain semi-organic binders (silicone), the resistance of some resin-rich (prepreg), mica/glass-reinforced systems to irradiation composed mainly of gamma rays was very good (Figure A.2-77, [Lipták et al., 1985]). The chemical character of the proprietary (Isola) 
[Lipták et al., 1985]). The chemical character of the proprietary (Isola) resin systems used with mica/glass reinforcement was apparently EPN [Brandenberger, 1992], a system that often does not have properties suitable for vacuum impregnation. Since these resin systems were not tested independently with other reinforcements or as neat resins, it is not clear how much of the superior radiation resistance should be attributed to the resin system and how much to the additional mica reinforcement. Schönbacher and Stolarz-Iźycka [1979] also reported good radiation resistance of some mica/glass-reinforced epoxy resins [retention of $-60 \%$ of flexural strength at $10^{8}$ Gy (Figure A.2-75)]. However, Lipták et al. cautioned that gas buildup that caused separation of mica layers could be a problem at high dose rates.

Most of the ambient-temperature investigations have given little guidance in choosing resins for vacuum impregnation of magnets, because viscosity and pot life were usually not reported. Other parameters, such as contraction upon solidification and thermal shock resistance, that must meet certain criteria if a system is to be practical for cryogenic magnet use, were also rarely reported. For these reasons, Pöhlchen [1992] has suggested that only resins that had been successfully used for impregnating large magnets should be studied for radiation resistance. Hacker et al. [1984] reported good radiation resistance for a one-component resin system that was used in the European coil for the LCT (Table 2.1 and \$2.10.1). Although the chemical nature of this resin system was not given, the flexural strength was reported to be unchanged after $3 \times 10^{9}$ Gy of gamma irradiation. Numerical data from the irradiation measurements were not supplied, but other parameters relevant to impregnation of cryogenic magnets were discussed. Viscosity, gel time, pot life, and fracture energy were also reported for several magnet resins and other resins suitable for vacuum impregnation by Phillips et al. [1981] (Table 2.2). However, irradiation testing was carried out only on the resin in neat form, only with gamma radiation, and only to a dose of $5 \times 10^{7} \mathrm{~Gy}$. Considerable degradation was exhibited by all resins at this dose, but with reinforcement, the radiation resistance would be better. The resin used in JET coils was reported to retain over $60 \%$ of initial flexural strength at $10^{8}$ Gy [Banford, 1984]. Lipták et al. [1985] reported the retention of about $50 \%$ of initial strength at $10^{8}$ Gy with a magnet resin containing isocyanate, an EPN resin used with glass mat, and Orlitherm composites. Also, the mica- 
reinforced systems tested by Lipták et al. had applications to magnets or to other electrical machinery.

\subsection{DISCUSSION}

Since only a few of the epoxy-resin composite systems that have been tested appear to be sufficiently radiation-resistant to satisfy the criteria of the ITER conceptual design, attention should be directed toward optimization of systems that are known to have good radiation resistance. For example, the cryogenic properties of TGDM systems with low viscosity could be explored further, and there may be opportunities for more optimization of TGDM or TGPAP systems. Replicate experiments should be done to confirm the apparent high radiation resistance at $10^{8}$ Gy of a few proprietary resin systems, such as the Siemens one-component LCT resin, the JET resin, the Orlitherm variants, and the Samica systems. If ambient-temperature replication is successful, additional testing with neutron irradiation at cryogenic temperatures should be done.

A major shortcoming of the results of ambient-temperature testing is that most of it was carried out with gamma irradiation. Even when some of the dose was from neutrons, it was usually only a small portion. The IPNS and RTNS neutron irradiations were not carried out to doses high enough to meet the ITER conceptual design requirements, and very few magnet insulation materials were tested in these facilities before they were decommissioned. The use of gamma irradiation (and some electron irradiation) for so much of the work has one advantage, as $\mathrm{E}$ glass, rather than boron-free glass, was almost invariably used as a reinforcement. However, there is no theoretical or experimental proof that neutron damage at a given dose level is equal to gamma-ray damage at the same dose level. In fact, one experiment suggested that gamma doses should be downgraded by almost a factor of ten for comparision to neutron damage [Abe et al., 1987]. Therefore, the more promising systems noted above should be tested in a predominately neutron environment, with a fluence of fast neutrons ( $\mathrm{E}>0.1 \mathrm{MeV}$ ) equal to $10^{23} / \mathrm{m}^{2}$ or $10^{8}$ Gy by the usual conversion factor. The first systems for which further validation testing is suggested are the glycidyl amines, TGDM and TGPAP, with hardeners such as MNA that lower the viscosity to the range where vacuum impregnation is feasible [Evans et al., 1972a; Morgan and Stapleton, 1975]. Aromatic hardeners that offer higher 
radiation resistance could be tested if alternate modes of magnet fabrication are adopted, as the ITER design is modified from the conceptual design requirements. Second, more testing should be carried out on the specific resin systems (usually proprietary) that have demonstrated high strength retention, such as Orlitherm variants, the Siemens LCT and the JET magnet resins, the class "H" resin containing isocyanate, and the mica/glassreinforced systems. The extension of testing for these resins should include standardized measurements of surface tension viscosity, pot life, gel time, resin shrinkage in both liquid and solid phases and cryogenic characteristics such as resistance to thermal shock and thermal contraction.

In addition, other modes of testing that simulate magnet operating conditions better than flexural tests should be used to test these composites. These tests include true interlaminar shear measurements, combined compression/shear tests, and metal-bond adhesion tests. The degree to which interlaminar shear and/or fiber-matrix interface failures can occur in the test may dramatically influence the observed response to radiation. If simultaneous shear and compressive loading will occur in the magnet, radiation tests that simulate this operating condition should be employed. Similarly, if adhesion to the conductor sheath alloy is required, tests that simulate this condition should be used. Very preliminary data indicate that a chemical bond between a metal and composite epoxy insulation is more vulnerable to radiation damage than a predominately mechanical bond, although isocyanate-derived primers may offer higher radiation resistance ( $(11)$. Therefore, the use of primers and other parameters that affect chemical adhesion also requires investigation. An example of this type of parameter is the time interval between metal abrasion by sand-blasting and the application of epoxy insulation. 


\subsection{CRYOGENIC TESTING: AMBIENT TEMPERATURE IRRADIATION}

Egusa demonstrated that the cryogenic properties of fiber-glass reinforced composites were more sensitive to irradiation damage than the ambient properties were, even when the irradiation was carried out at ambient temperature (Figures 1.26 and 1.27 ). However, the radiation sensitivity of neat resin flexural properties was high at both ambient and cryogenic temperatures (Figure A.3-2). Although this result could be used to argue that ambient-temperature tests of neat resins could suffice to screen resin systems for radiation resistance, there is a high degree of scatter in tests of neat resins because specimens are more sensitive to surface flaws. Because scatter is high, tests of a series of neat resin systems may not correctly rank the radiation resistance of the same series of resins when tested with reinforcement in a composite. Therefore, results of ambient-temperature tests should not be used for selection or design of ITER magnet insulation.

Flexural tests of fiber-glass reinforced composites at both 77 and $4 \mathrm{~K}$ indicated a considerable superiority of a TGDM/DDS resin system compared to several DGEBA resin systems, when ambient gamma radiation was used (Figure A.3-6, [Egusa and Hagiwara, 1986]). However, negligible differences were found between one multifunctional resin system (similar to TGDM or TGPAP) and a DGEBA system when torsional specimens were tested in shear at $77 \mathrm{~K}$ and the irradiation had a substantial neutron component (Figure A.3-7 [Munshi, 1991, 1992; Munshi and Weber, 1992]). At an intermediate radiation dose of $-3 \mathrm{x}$ $10^{7} \mathrm{~Gy}$, Egusa and Hagiwara reported some differences between flexural strengths of two commercial laminates with DGEBA resin systems, G-10CR and G-1ICR, and a DGEBA/DDM composite, but at a higher dose of $-6 \times 10^{7}$ Gy the flexural strengths of all three DGEBA systems were below $10 \%$ of the initial value (Figure A.3-6). However, one of the DGEBA systems tested by Munshi retained about 458 of the initial shear strength at a dose of $2.3 \times 10^{8}$ Gy. These uniaxially reinforced specimens that were tested in torsion may exhibit radiation sensitivities different from those of 2 -dimensional reinforced composites tested in flexure, because different properties are measured in the two tests. The different results for DGEBA systems could also be due to differences in hardners and other additives. 
Comparative cryogenic testing of reinforcement parameters showed that the use of a plain fiber-glass weave of lower areal density resulted in a higher initial flexural strength than a weave of higher density (higher fiber diameter and higher number of fibers per yarn of the weave, Figure A.3-9). Since composites prepared with both fabric weaves had equal dose dependence with gamma irradiation, the composite with the less dense weave had a somewhat higher flexural strength after a dose of $-10^{8}$ Gy [Egusa, 1991a]. A higher glass volume fraction or a smaller specimen thickness also were shown to increase the initial flexural strength of specimens, but there was an important distinction between these effects and the effect of areal density: the initial advantage of volume fraction or thickness disappeared after a dose of about $10^{8} \mathrm{~Gy}$, whereas it was maintained for areal density, although only to a slightly higher dose of about $1.8 \times 10^{8} \mathrm{~Gy}$. Overall, however, the failure to significantly improve radiation resistance by varying the glass reinforcement parameters gave weight to the hypothesis of Egusa that the predominant radiation damage mechanism is the change in matrix ultimate strain with irradiation. Even if this hypothesis is not confirmed by further research, it seems clear that changes of resin type generally affect radiation performance much more strongly than changes in reinforcement parameters.

Empiricaliy then, the results of reinforcement tests suggest that to improve radiation resistance of epoxy composites, greater effort should be put into resin system selection and optimization, rather than into reinforcement optimization. There may be one exception to this rule: ambient-temperature testing of glass/mica-reinforced systems gave promising results in some cases $(\S 2.8)$. However, such systems have not yet been irradiated and tested at cryogenic temperatures, and gas accumulation upon warm-up (\$1.4.2) could lead to separation of mica layers. Furthermore, comparative irradiation testing of different types of glass weaves has been very limited. Recently, 3dimensional weaves were found to perform better than 2-dimensional weaves when tested in simultaneous shear/compression after irradiation (\$10). Threedimensional weaves may not have acceptable dielectric properties, however. Nevertheless, some glass or mica reinforcement variants might offer significant improvement in radiation resistance since this area has not been fully explored. 
In contrast to the ambient temperature studies of Morgan et al. [1970] on DGEBA/DDM neat resins, Egusa [1991a] found that there was no difference in the degradation of 77-K flexural properties of DGEBA/DDM composites under irradiation, whether or not oxygen was present during the irradiation process. This difference was attributed to the lack of surface-flaw sensitivity in composites. (Effects of oxygen upon radiation resistance have been demonstrated only for neat resins [Evans et al., 1971; Morgan et al., 1970].) Since the lack of oxygen is supposed to improve radiation resistance under cryogenic conditions, the insensitivity of glass-reinforced epoxy composites to oxygen means that ambient irradiation results should not be taken as conservative indicators of cryogenic irradiation results.

\subsection{CRYOGENIC TESTING: 77-K IRRADIATION}

The major results under these test conditions are those of Weber et al. [1983] on specimens that were vacuum-impregnated with Orlitherm and tested in tension without warm-up. The results of these tensile tests failed to extend the irradiation resistance reported for some Orlitherm composites in ambienttemperature irradiation and flexural testing (see Figures A.2-25 and A.2-88) to cryogenic conditions. Although tensile properties of composites have been shown to be less sensitive to radiation damage than flexural properties (§2.1), cryogenic specimens were broken at a dose of $1.5 \times 10^{8}$ Gy before they could be tested, and specimen damage was also observed at lower doses. The failure to reproduce ambient-temperature results may have resulted from the much larger neutron component in the $77-\mathrm{K}$ radiation dose compared to the ambient doses, since the specimens were reinforced with E glass. But, the results could also indicate that greater damage occurs in 77-K irradiation and testing than at ambient conditions. Since different variants of Orlitherm were apparently tested by the different investigators (the type designations were not the same), it is also possible that different formulations of the basic DGEBA resin system were responsible for the variations of the 77-K results from the ambient temperature results. Another confounding variable was the addition of Kapton film to the glass reinforcement system in some of these 77-K tests. 


\subsection{CRYOGENIC TESTING: 20-K IRRADIATION}

Some of the 20-K reactor irradiation data were obtained with $\mathrm{E}$ glass and, therefore, are of limited use for ITER insulation selection. The volume fraction of $\mathrm{E}$ glass did not affect the interlaminar shear strength after irradiation at $20 \mathrm{~K}$ [Okada et al., 1992]. However, examination of possible volume-fraction effects in neutron irradiation of B-free glass would be of value, since excessive alpha-particle damage may have confounded the results obtained with E glass (Figure A.7-3). Nevertheless, Egusa [1991a] also reported no effect of volume fraction after ambient gamma radiation followed by tests at both 77 and $295 \mathrm{~K}$ (Figure A.3-11).

A 3-dimensional glass weave was found to be superior to a 2-dimensional weave after $20-\mathrm{K}$ reactor irradiation at a maximum dose of $1.5 \times 10^{7}$ Gy [Nishijima et al., 1990]. Various parameters that could have affected the results, such as the glass type, finish, and the resin type, were the same for both reinforcements. The experiment simulated magnet operation conditions better than most experiments because low temperature irradiation was followed by $77-\mathrm{K}$ testing, without warm-up. However, the lack of information on the systems used and the low total dose prevent the information from being useful for ITER insulation selection and optimization, especially since Egusa [1991a] found that at higher doses of about $10^{8} \mathrm{~Gy}$, many reinforcement parameters no longer significantly affect retained strength.

\subsection{CRYOGENIC TESTING: 4- OR 5-K IRRADIATION}

The IPNS neutron irradiations carried out by Hurley et al. [1978; 1983] and Tucker et al. [1985] at $4 \mathrm{~K}$ were at doses too low to show significant degradation ( $<10^{7} \mathrm{~Gy}$ ); therefore, their results on G-10CR, G-11CR, and another DGEBA resin are of limited use to the current ITER program. Tests by Kato and Takamura [1979] on DGEBA resins irradiated at $5 \mathrm{~K}$ were restricted to a maximum dose of $1.1 \times 10^{7} \mathrm{~Gy}$, and are also of limited usefulness.

Kernohan et al. [1979] and Coltman et al. [1979] presented results of gamma irradiation at $5 \mathrm{~K}$ on G-10CR, B-free G-10CR, G-11CR, and two filled epoxies at total doses up to $10^{8} \mathrm{~Gy}$. Some fast neutrons were present, but they constituted only about 0.28 of the total dose. Flexural testing was conducted 
at $77 \mathrm{~K}$, after warm-up to ambient temperature. None of the DGEBA systems tested retained more than 10 to 208 of the initial strength at $10^{8} \mathrm{~Gy}$. This indicated again that DGEBA resins will usually not be satisfactory at doses $\sim 10^{8} \mathrm{~Gy}$, as was found in ambient temperature irradiation tests. None of the resin types that were known from ambient-temperature testing to be more promising than DGEBA were tested in this cryogenic program. Furthermore, information on the suitability of the resins tested for vacuum impregnation was not supplied, perhaps because this fabrication method was not under consideration when the irradiations were carried out.

Egusa et al. [1987b] conducted neutron irradiation at $5 \mathrm{~K}$ on a more radiationresistant resin system, TGDM/DDS, but only to a dose of $2 \times 10^{7}$ Gy. The results are compared with those for ambient-temperature irradiation in Figure 1.24b. In contrast to the poor results on DGEBA systems, this system, which had also exhibited superiority in ambient temperature testing ( $\$ 2.1$ and 2.2), demonstrated a retained flexural strength of about $70 \%$ of the initial value when tested at $77 \mathrm{~K}$ after warm-up. However, the maximum 5-K dose was considerably below the ITER conceptual design requirement.

Takamura and Kato $[1980 ; 1981 ; 1984]$ conducted several in situ tests of specimens that were irradiated at $4 \mathrm{~K}$. Although the conditions of the tests simulated magnet operating conditions, the maximum dose of $1.1 \times 10^{7}$ Gy was considerably below the dose expected at the ITER TF magnets. Primarily, DGEBA resins were tested, but a higher retained percentage of flexural strength was obtained for an isocyanate-hardened epoxy. High radiation resistance was also obtained with a combined isocyanate and epoxy resin system in one test at ambient temperature (Lipták et al. [1985], Figure A.2-87). The viscosity, pot life, and other parameters related to fabrication of cryogenic magnets were not supplied by either Takamura and Kato or Lipták et al.

Comparative testing of a number of different types of resin systems under 4-K neutron and gamma reactor irradiation has been done only by Munshi [1991]. The rod specimens were uniaxially reinforced with S-2 Glass and tested in torsion at $77 \mathrm{~K}$ after warm-up. The three main types of resin systems investigated at ambient temperatures, DGEBA, EPN, and a multifunctional epoxy (similar to TGDM or TGPAP) were included in this test series. As would be expected from ambient-temperature results, the multifunctional resin was 
superior to both the DGEBA and EPN resins at the maximum dose, $1.6 \times 10^{8}$ Gy. However, the interpolated percentage of retained shear strength at $10^{8} \mathrm{~Gy}$, about 508, was much lower than that found in ambient-temperature flexural tests conducted with nearly pure gamma radiation on TGDM (e.g., Figure A.2-3). Differences in both the test temperature and test type, as well as in the radiation species, may have contributed to the dissimilarity in results. The torsion screening test measures chiefly matrix-fiber adhesion in shear, and pure interlaminar shear does not occur in flexural tests because both compressive and tensile stresses are present (except at low span-to-thickness ratios, §1.5.2). Since the flexural test stresses are dominated by the tensile stress along the outside bend radius, the warp or fill fibers play a dominant role in determining the flexural strength.

A bismaleimide resin also tested by Munshi did not retain significantly better shear strength than the multifunctional epoxy after $1.6 \times 10^{8}$ Gy. Other tests of bismaleimide composites are in general agreement with this result: for example, Egusa [1990a] found that the flexural strength of Kerimid 601 was comparable to that of a TGDM/DDS composite at $77 \mathrm{~K}$ after ambient gamma irradiation to about $8 \times 10^{7} \mathrm{~Gy}$.

Information on viscosity and pot life that Munshi furnished for the systems tested indicated that the DGEBA resin system was most suitable for vacuum impregnation. However, the DGEBA resin system retained only about $22 \%$ of the initial shear strength at the maximum dose of $1.6 \times 10^{8} \mathrm{~Gy}$, which included a fast neutron fluence of about $3.1 \times 10^{22} / \mathrm{m}^{2}$.

The cryogenic testing of 4- or 5-K irradiated specimens by Munshi, Egusa, and Coltman et al. was conducted after warm-up to ambient temperature. However, in a limited number of comparative tests, both Takamura and Kato [1981] and Katheder et al. [1992] have reported that specimens that were warmed up exhibited post-irradiation properties significantly higher than those of specimens that were kept at cryogenic temperatures and tested in situ $(\S 1.4 .2)$. These two groups conducted compressive and shear-bond adhesion tests, respectively. Therefore, to predict steady-state cryogenic radiation resistance, it would be conservative to "downgrade" the retained strengths of cryogenically irradiated and tested specimens by at least a factor of 2 or 3 if they were exposed to ambient temperature before testing. 
13.5. DISCUSSION OF CRYOGENIC DATA BASE ON MAGNET RESINS: FURTHER INVESTIGATIONS

Ambient-temperature investigations indicated that a few glass-reinforced epoxy systems suitable for magnet construction retained excellent shear or flexural properties at a radiation dose of $10^{8} \mathrm{~Gy}$. (This dose was usually obtained with gamma irradiation, and is not equivalent to the fast neutron fluence of $\sim 10^{23} / \mathrm{m}^{2}$ at the ITER TF magnet.) These systems and the percentage of initial shear or flexural strength retained at $10^{8}$ Gy include: (1) Orlitherm reinforced with glass with a "special silane finish" ( $60 \%)$; (2) CTD 101 ( $47 \%$ ); (3) Siemens one-component LCT resin (100\%); (4) JET (Joint European Torus) bisphenol A/MNA resin system ( $60 \%)$; (5) TGDM/MNA ( -80 to 1008); (6) resin-rich mica/glass reinforced systems ( -20 to $111 \%)$. [Systems (1) and (2) are DGEBA resin systems; the chemical nature of the proprietary resins of (3) and (6) were not identified.] Only system (2) was tested in shear; all other systems were tested in flexure, which chiefly reflects the tensile strength of the reinforcement and does not simulate magnet operating stresses. Except for (6), all of these systems were reported to be suitable for vacuum impregnation, or were used in magnet construction.

Cryogenic testing of irradiated glass-reinforced epoxy composites has indicated a significantly greater degradation than has ambient-temperature testing. However, only systems (1) and (2) have been irradiated and tested at cryogenic temperatures to doses $\approx 10^{8} \mathrm{~Gy}$. Orlitherm composite specimens ( 1 ) were irradiated and tested in tension at $77 \mathrm{~K}$ without warm-up. However, after a dose of $1.5 \times 10^{8} \mathrm{~Gy}$, the specimens broke apart before they could be tested. Besides the difference in irradiation and test temperatures, a larger portion of the $77-\mathrm{K}$ dose was composed of neutron irradiation ( $-33 \%$ instead of $\sim 5 \%$ at ambient temperature), and the specimens were reinforced with $\mathrm{E}$ glass. The CTD 101 specimens (2) retained -228 of the initial shear strength when tested in torsion at $77 \mathrm{~K}$ after $4-\mathrm{K}$ reactor irradiation to $1.6 \times 10^{8} \mathrm{~Gy}$ and warm-up to ambient temperature. Although the specimen warm-up procedure and type of test were different for the cryogenic tests of systems (1) and (2), the most significant difference may have been the use of an E-glass reinforcement for system (1) and an S-glass reinforcement for system (2). Alloy-adhesion testing at $4 \mathrm{~K}$ to compare these two resin systems is currently in progress at 
Garching ( $(11)$, and B-free glass reinforcement is now being used with both resin systems.

Some glass-reinforced resin systems that are not compatible with fabrication by vacuum impregnation have exhibited satisfactory radiation resistance when tested at cryogenic temperatures. One 77-K test of a TGDM/DDM system showed an increase in the relatively low initial flexural strength at $77 \mathrm{~K}$ after $10^{8} \mathrm{~Gy}$ of electron irradiation. The TGDM/DDS system has been more extensively investigated at $77 \mathrm{~K}$ after ambient irradiation to high doses with neutron, gamma, and electron radiation. However, the neutron-irradiated specimens were reinforced with $\mathrm{E}$ glass, and the maximum neutron dose was $2 \times 10^{7} \mathrm{~Gy}$. At a gamma dose of $10^{8} \mathrm{~Gy}$, the TGDM/DDS system retained about 648 of initial flexural strength when tested at $295 \mathrm{~K}$, about $47 \%$ at $77 \mathrm{~K}$, and about $59 \%$ at $4 \mathrm{~K}$ (at $7 \times 10^{7} \mathrm{~Gy}$ ). This performance was excellent, compared to that of several DGEBA systems tested under the same conditions. CTD 112, a multifunctional epoxy similar to TGDM or TGPAP, retained about $40 \%$ of the initial shear strength when tested under the same cryogenic conditions described above for CTD 101. Appendix B of this report shows that polyimide and bismaleimide resins also demonstrated high radiation resistances when tested at cryogenic temperatures, although the irradiation used did not simulate the dose and neutron spectrum at the TF magnet. Furthermore, since initial strengths of polyimides are often lower than those of epoxies, the strengths after irradiation at high doses were similar, not superior, to those of the best epoxy systems.

With the exception of the tensile tests of Orlitherm at $77 \mathrm{~K}$ by Weber et al. and the low viscosity DGEBA system tested by Munshi after warm-up, cryogenic irradiation and testing of magnet resins and resins suitable for vacuum impregnation has not yet been carried out to doses $-10^{8} \mathrm{~Gy}$. Furthermore, fast neutrons constituted only a fraction of these total doses. Therefore, the present data base does not allow selection of a resin system that would meet the requirements of the ITER conceptual design. 


\section{APPENDIX A: GRAPHS AND TABLES}

\section{NOTES FOR GRAPHS AND SUPPLEMENTARY TABLES}

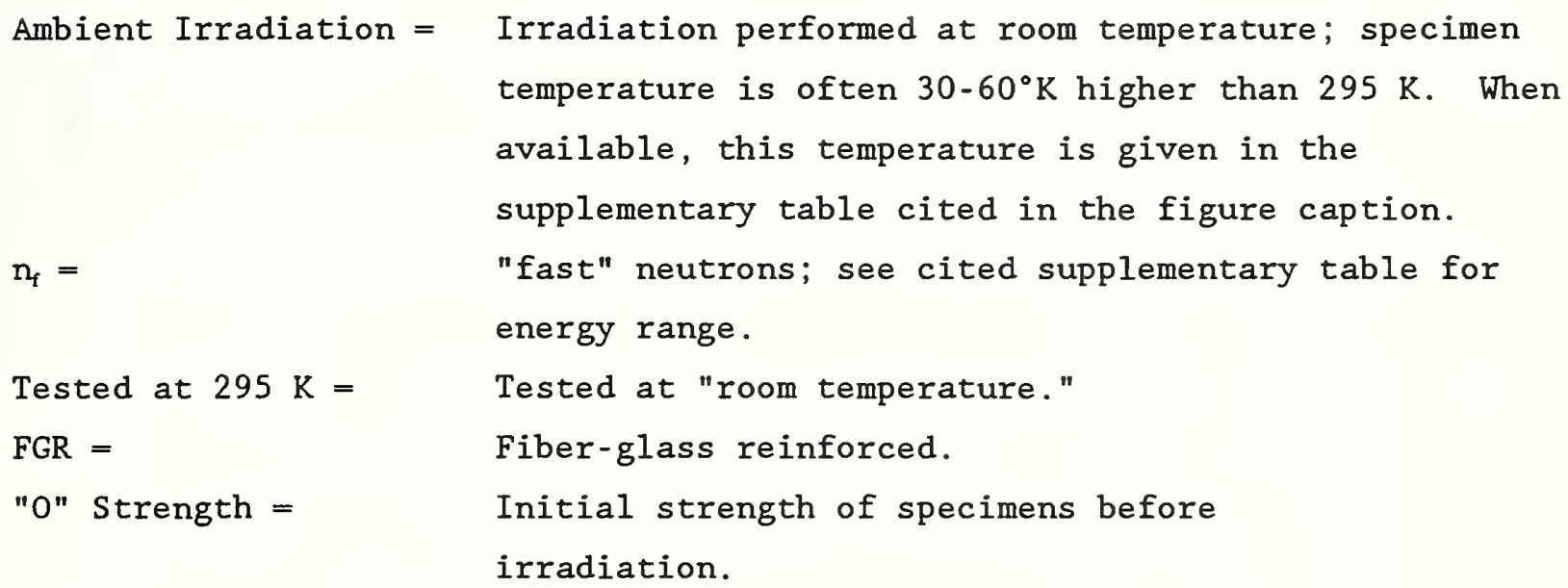

For resin and hardener acronyms and chemical structures, see Figures 1.21, $1.26,1.33,2.1,2.3,2.4 .3 .1$.

Commercial names and suppliers are included for scientific accuracy when available because the purity and exact chemical forms of chemicals may vary with the source.

The available information on irradiation conditions, test procedures, and materials is supplied in the supplementary tables noted in the figure captions. Owing to space limitations, sometimes the information supplied pertains to most, but not all specimens tested by the authors referenced in the table.

The approximate neutron dose-to-fluence conversion rule, 1 Gy $=10^{15} / \mathrm{m}^{2}$ for $E>0.1 \mathrm{Mev}$, has been used when the authors supplied only neutron fluence; otherwise, the authors' values for dose were used whether or not they agreed with this approximate conversion rule. When the conversion rule was used, it is noted in the figure caption. At best, there is often an uncertainty of about $10 \%$ in the determination of dose [Hahn et a1., 1986]. Since different investigators used different methods to determine dose, results in figures from various investigations should be compared with caution. Also note that the radiation species constituting the total dose differed from one investigation to another and that neutron spectra were often quite variable, particularly in their high-energy component. When available, the percentage 
of dose from neutrons and gamma rays is supplied in the upper right corner of the graph.

In the graphs from Egusa and Egusa et al., a missing error bar means that the standard deviation was too small to be shown. 


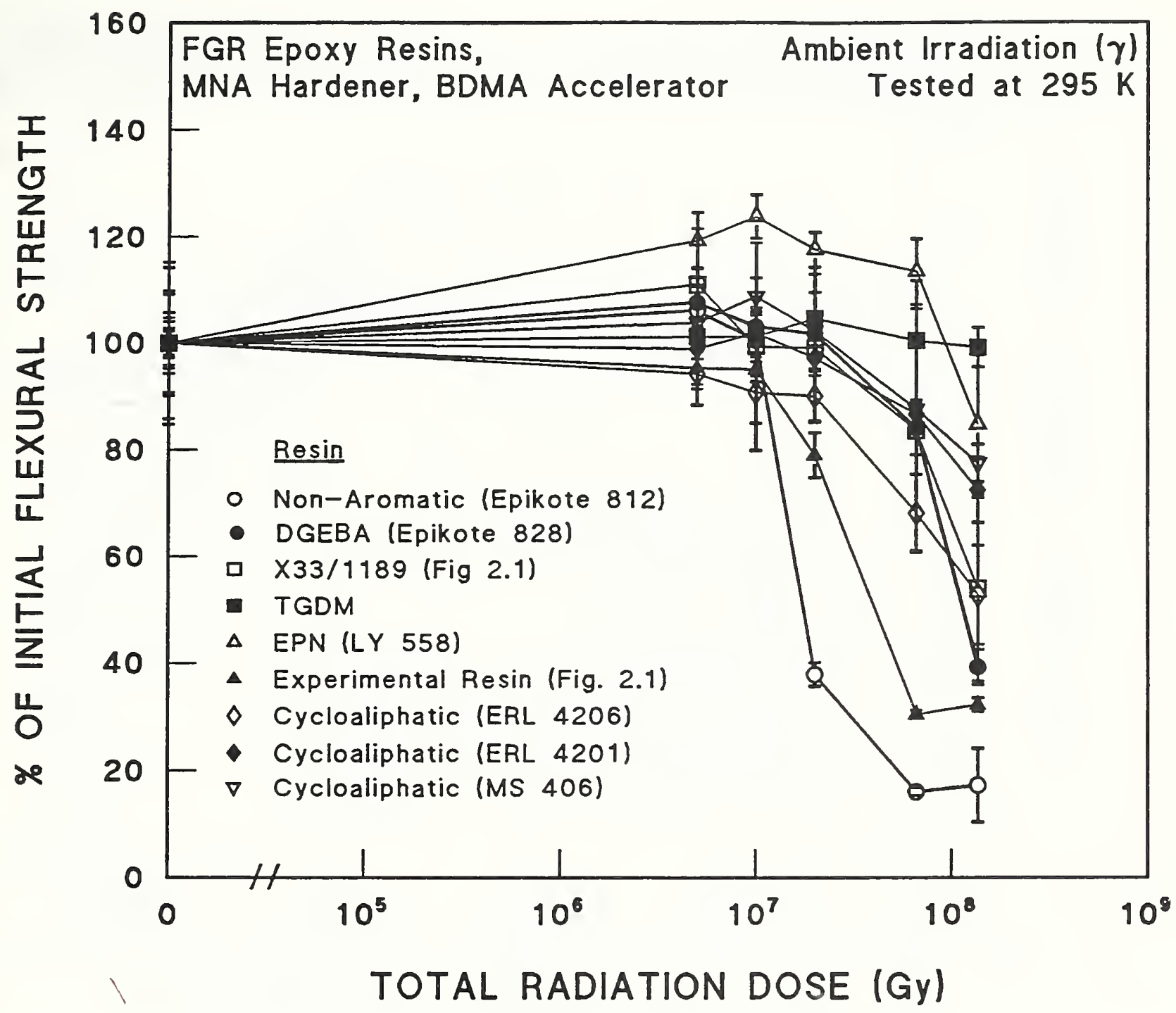

\begin{tabular}{|c|c|c|}
\hline "O" Strength, MPa & Cure Conditions & Supplier \\
\hline$\circ 405.0 \pm 16.7$ & $2 \mathrm{~h},=80^{\circ} \mathrm{C} ; 8 \mathrm{~h}, 150^{\circ} \mathrm{C}$ & Shell \\
\hline - $414.8 \pm 38.2$ & $2 \mathrm{~h}, \leq 150^{\circ} \mathrm{C} ; 2 \mathrm{~h}, 150^{\circ} \mathrm{C}$ & Shell \\
\hline ㅁ $297.2 \pm 41.5$ & $2 \mathrm{~h}, 120^{\circ} \mathrm{C} ; 8 \mathrm{~h}, 250^{\circ} \mathrm{C}$ & Ciba \\
\hline$=319.7 \pm 31.4$ & $3 \mathrm{~h}, 100^{\circ} \mathrm{C} ; 4 \mathrm{~h}, 180^{\circ} \mathrm{C} ; 4 \mathrm{~h}, 220^{\circ} \mathrm{C}$ & Ciba \\
\hline$\Delta \quad 331.5 \pm 47.1$ & $2 \mathrm{~h}, 100^{\circ} \mathrm{C} ; 2 \mathrm{~h}, 138^{\circ} \mathrm{C} ; 8 \mathrm{~h}, 180^{\circ} \mathrm{C}$ & Ciba \\
\hline$\Delta \quad 375.6 \pm 21.6$ & $16 \mathrm{~h}, 120^{\circ} \mathrm{C} ; 16 \mathrm{~h}, 180^{\circ} \mathrm{C}$ & Ciba \\
\hline$\Delta \quad 420.7 \pm 11.8$ & $2 \mathrm{~h}, \leq 150^{\circ} \mathrm{C} ; 8 \mathrm{~h}, 150^{\circ} \mathrm{C}$ & Union Carbide \\
\hline $368.7 \pm 17.7$ & $2 h_{0}=150^{\circ} \mathrm{C} ; 8 \mathrm{~h}, 150^{\circ} \mathrm{C}$ & Bakelite \\
\hline $360.9 \pm 7.8$ & $16 \mathrm{~h}, 120^{\circ} \mathrm{C} ; 8 \mathrm{~h}, 180^{\circ} \mathrm{C}$ & Ciba \\
\hline
\end{tabular}

Figure A.2-1. Flexural strength at $295 \mathrm{~K}$ of FGR epoxy resins with MNA hardener after ambient gamma irradiation. Supplementary Table A.2-1. Data from Price and Sheldon [1965]. 


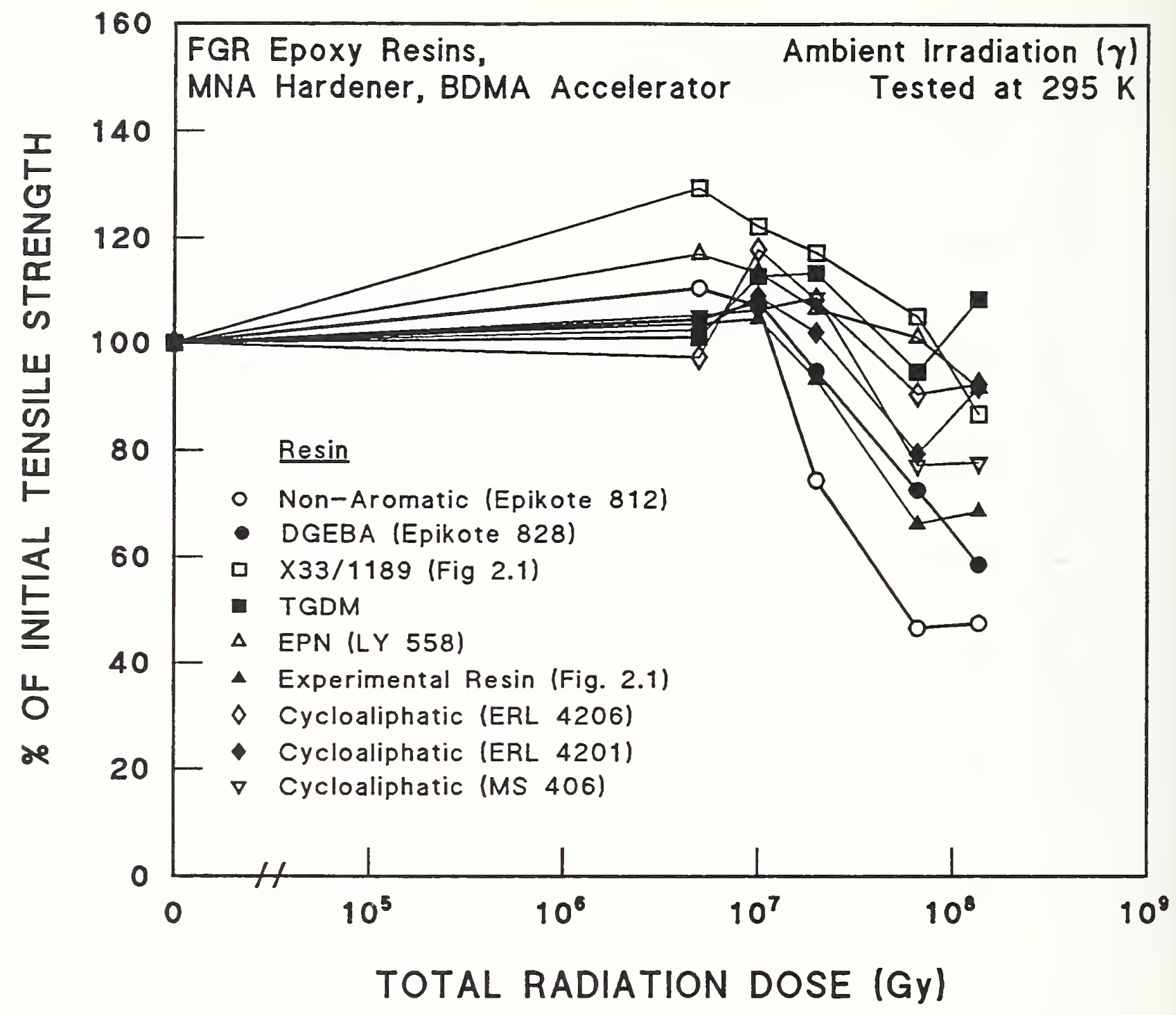

\begin{tabular}{llll} 
"O" Strength, MPa & \multicolumn{2}{l}{ Cure Conditions } & Supplier \\
\hline 233.4 & $2 \mathrm{~h}, \leq 80^{\circ} \mathrm{C} ; 8 \mathrm{~h}, 150^{\circ} \mathrm{C}$ & Shell \\
\hline 339.3 & $2 \mathrm{~h}, \leq 150^{\circ} \mathrm{C} ; 2 \mathrm{~h}, 150^{\circ} \mathrm{C}$ & Shell \\
$\square \quad 176.5$ & $2 \mathrm{~h}, 120^{\circ} \mathrm{C} ; 8 \mathrm{~h}, 250^{\circ} \mathrm{C}$ & Ciba \\
$\square \quad 177.5$ & $3 \mathrm{~h}, 100^{\circ} \mathrm{C} ; 4 \mathrm{~h}, 180^{\circ} \mathrm{C} ; 4 \mathrm{~h}, 220^{\circ} \mathrm{C}$ & Ciba \\
$\Delta \quad 197.1$ & $2 \mathrm{~h}, 100^{\circ} \mathrm{C} ; 2 \mathrm{~h}, 138^{\circ} \mathrm{C} ; 8 \mathrm{~h}, 180^{\circ} \mathrm{C}$ & Ciba \\
$\Delta \quad 214.8$ & $16 \mathrm{~h}, 120^{\circ} \mathrm{C} ; 16 \mathrm{~h}, 180^{\circ} \mathrm{C}$ & Ciba \\
$\Delta \quad 255.0$ & $2 \mathrm{~h}, \leq 150^{\circ} \mathrm{C} ; 8 \mathrm{~h}, 150^{\circ} \mathrm{C}$ & Union Carbide \\
$\nabla 199.1$ & $2 \mathrm{~h}, \leq 150^{\circ} \mathrm{C} ; 8 \mathrm{~h}, 150^{\circ} \mathrm{C}$ & Bakelite \\
$\nabla 202.0$ & $16 \mathrm{~h}, 120^{\circ} \mathrm{C} ; 8 \mathrm{~h}, 180^{\circ} \mathrm{C}$ & Ciba
\end{tabular}

Figure A.2-2. Tensile strength at 295 K of FGR epoxy resins with MNA hardener after ambient gamma irradiation. Supplementary Table A.2-1. Data from Price and Sheldon [1965] . 


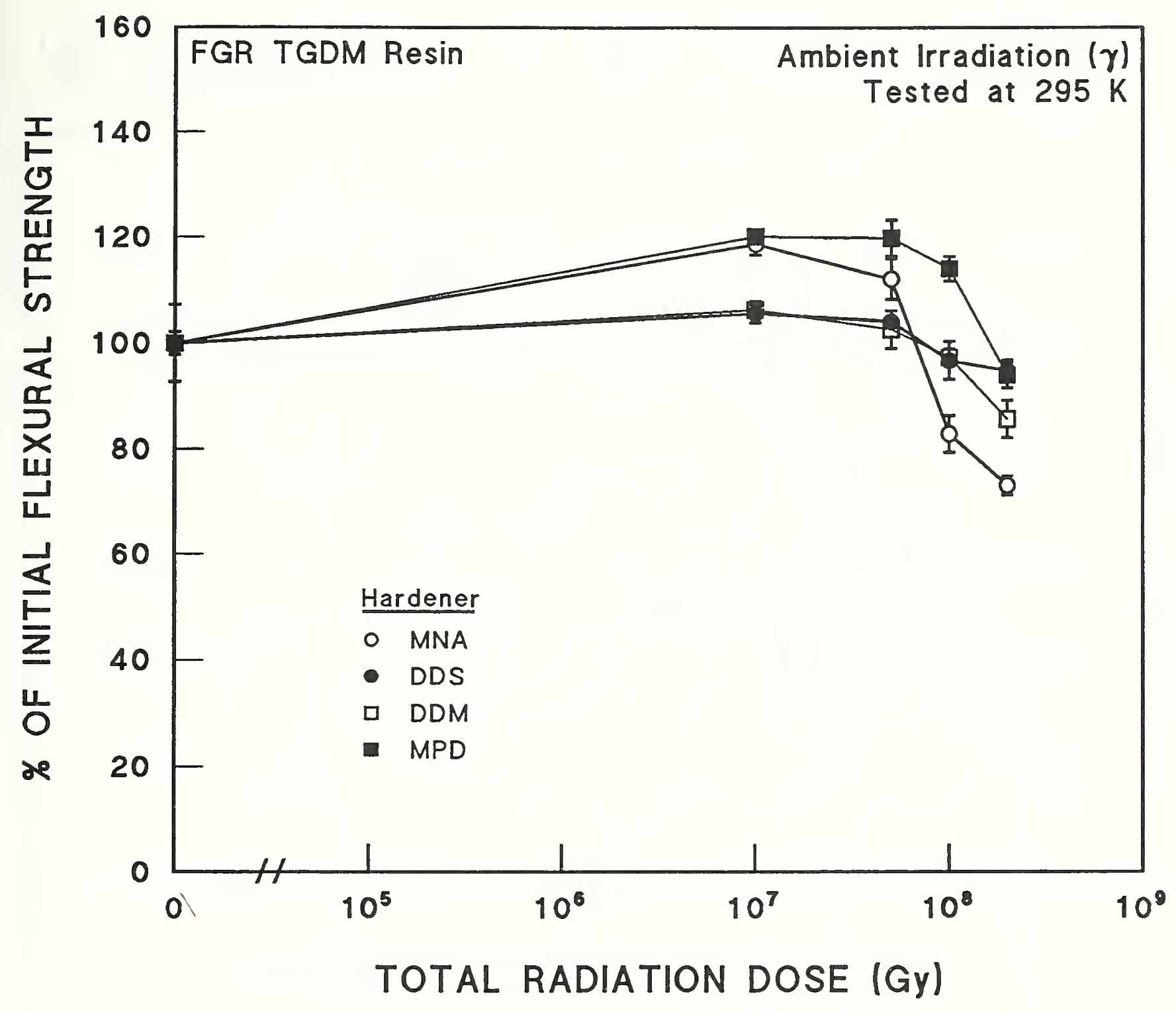

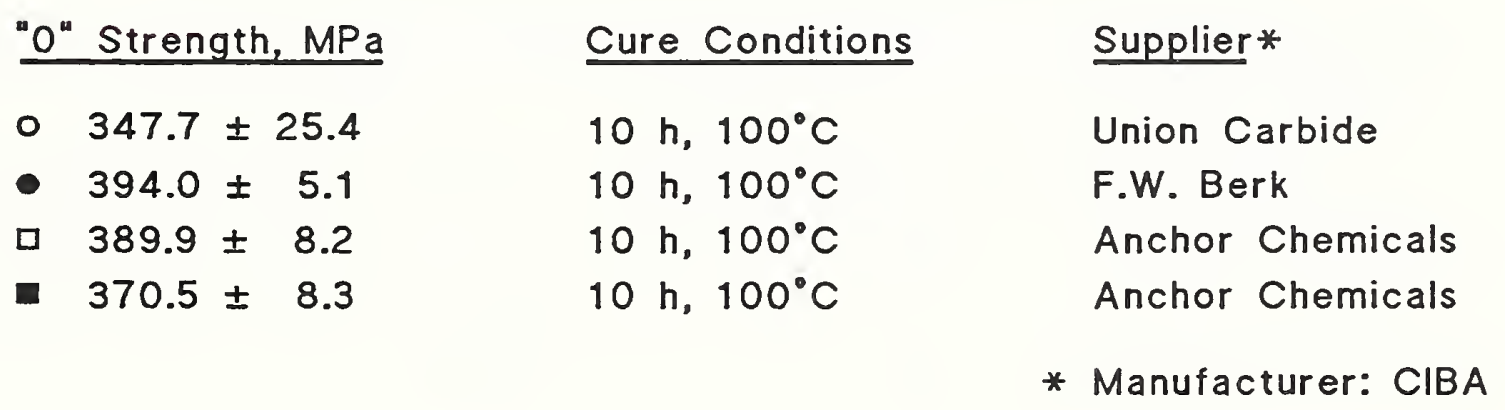

Figure A.2-3. Flexural strength at $295 \mathrm{~K}$ of FGR TGDM resin with several hardeners after ambient gamma irradiation. Supplementary Table A.2-1. Data from Sheldon and Stapleton [1968]. 


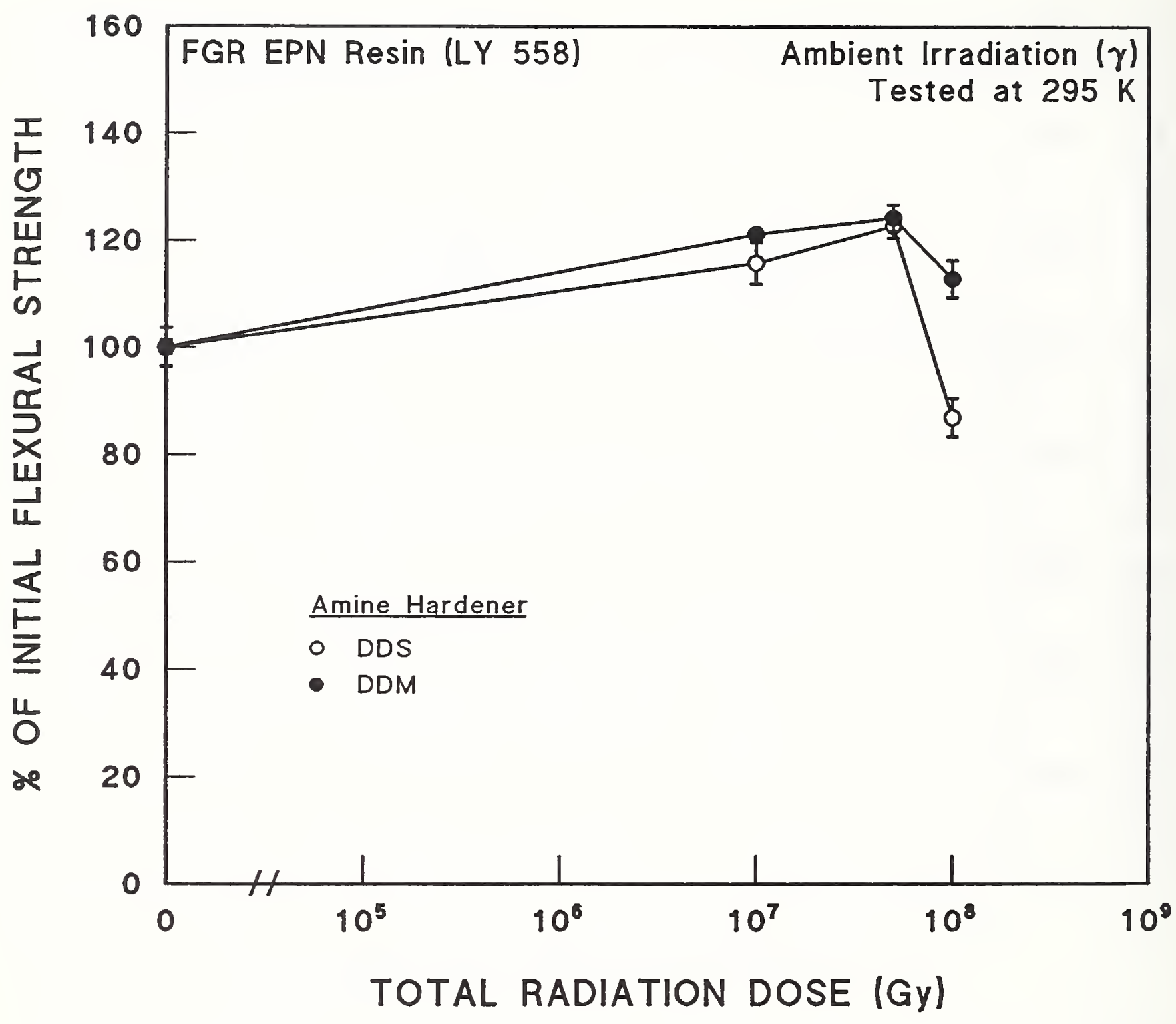

"O" Strength, MPa

- $333.2 \pm 4.3$

- $313.5 \pm 11.3$
Cure Conditions

$24 \mathrm{~h}, 160^{\circ} \mathrm{C}$

$10 \mathrm{~h}, 160^{\circ} \mathrm{C}$
Supplier*

F.W. Berk

Anchor Chemicals

* Manufacturer: CIBA

Figure A.2-4. Flexural strength at $295 \mathrm{~K}$ of FGR EPN resin with two hardeners after ambient gamma irradiation. Supplementary Table A.2-1. Data from Sheldon and Stapleton [1968]. 


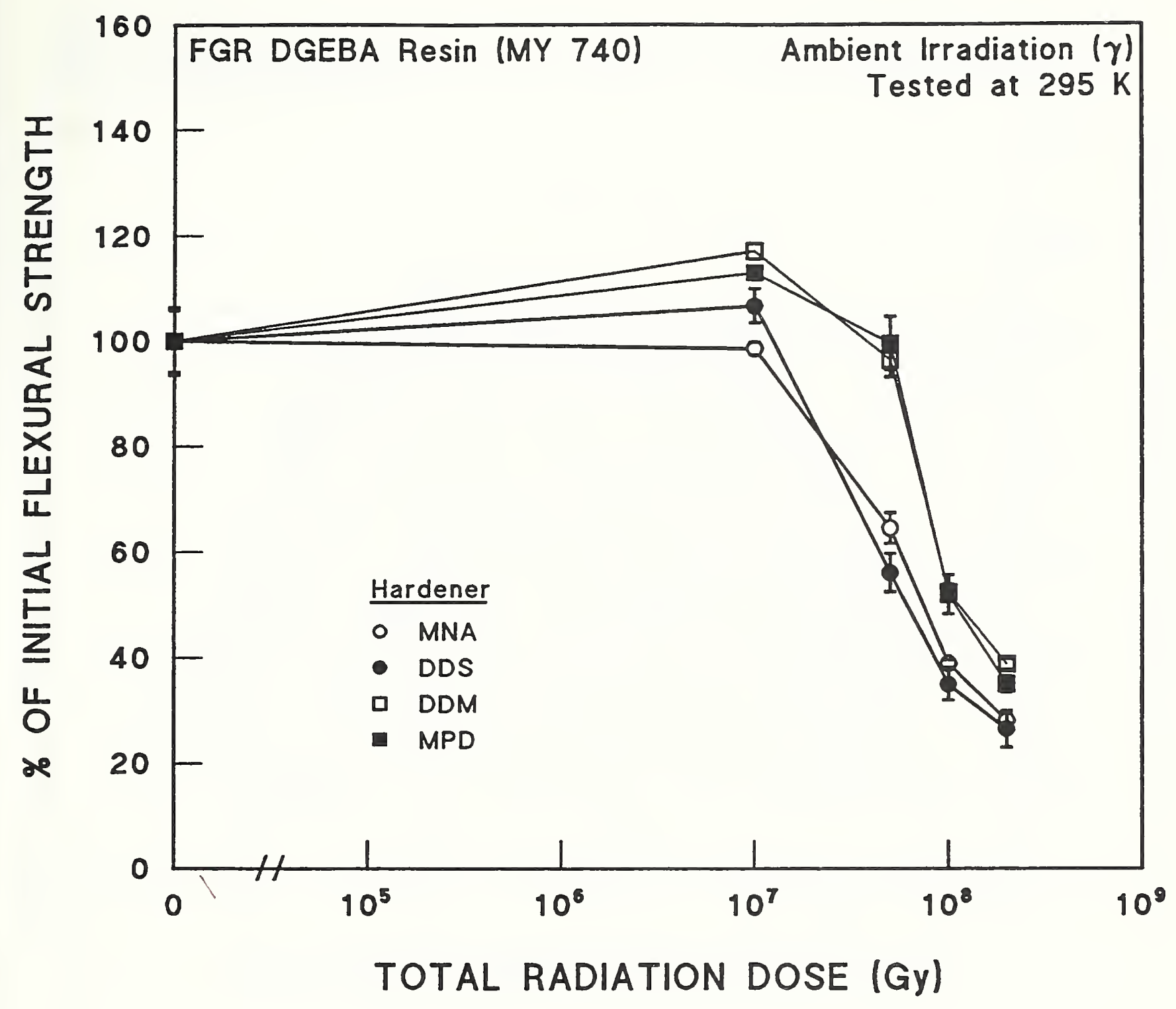

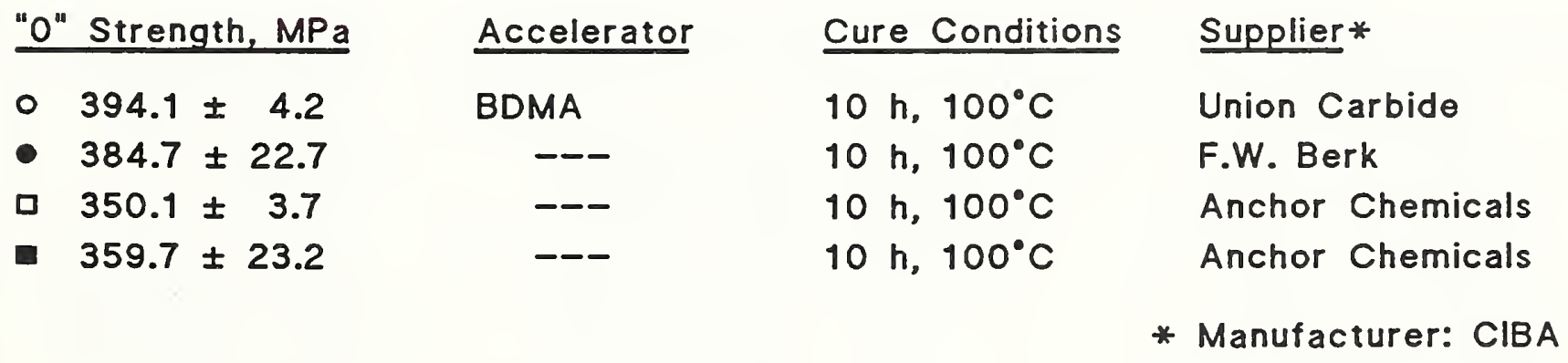

Figure A.2-5. Flexural strength at $295 \mathrm{~K}$ of FGR DGEBA resin (MY 740) with several hardeners after ambient gamma irradiation. Supplementary Table A.2-1. Data from Sheldon and Stapleton [1968]. 


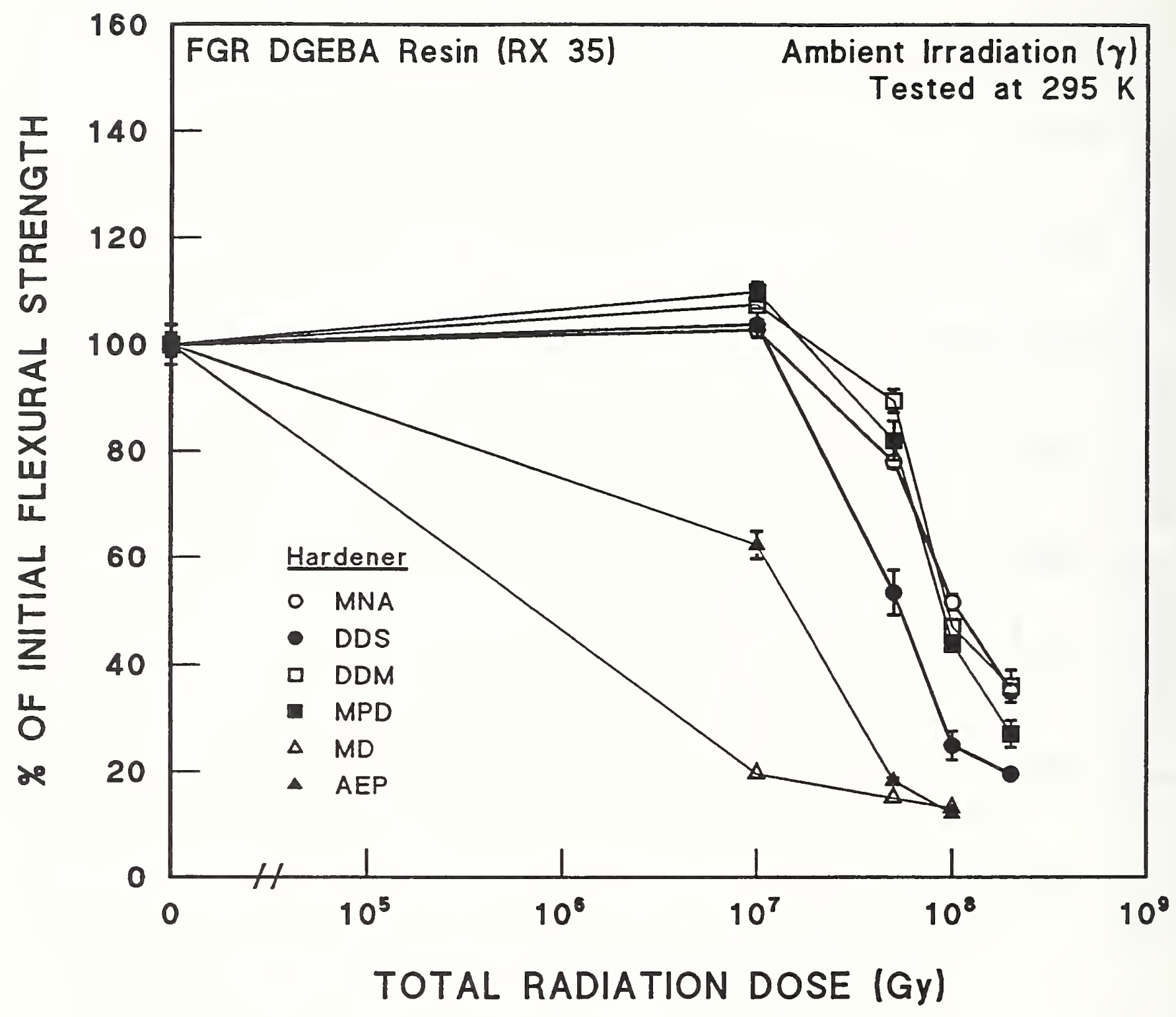

"O" Strength, MPa

- $425.4 \pm 15.9$

- $367.7 \pm 6.3$

ㅁ $400.2 \pm 5.6$

- $387.8 \pm 3.2$

$\Delta 395.3 \pm 8.6$

$\triangle \quad 399.8 \pm 3.6$

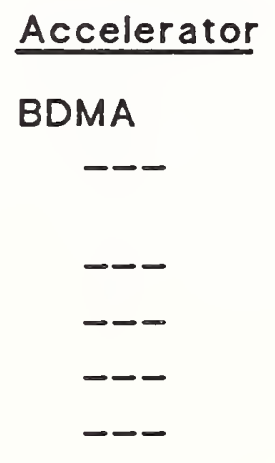

\begin{tabular}{|c|c|}
\hline Cure Conditions & Supplier* \\
\hline $10 \mathrm{~h}, 100^{\circ} \mathrm{C}$ & Union Carbide \\
\hline $\begin{array}{l}4 \mathrm{~h}, 100^{\circ} \mathrm{C} \\
6 \mathrm{~h}, 160^{\circ} \mathrm{C}\end{array}$ & F.W. Berk \\
\hline $10 \mathrm{~h}, 100^{\circ} \mathrm{C}$ & Anchor Chemicals \\
\hline $10 \mathrm{~h}, 100^{\circ} \mathrm{C}$ & Anchor Chemicals \\
\hline $10 \mathrm{~h}, 100^{\circ} \mathrm{C}$ & Lennig Chemicals \\
\hline $10 \mathrm{~h}, 100^{\circ} \mathrm{C}$ & Cyanamid \\
\hline
\end{tabular}

* Manufacturer: Shell

Figure A.2-6. Flexural strength at $295 \mathrm{~K}$ of FGR DGEBA resin (RX 35) with several hardeners after ambient gamma irradiation. Supplementary Table A.2-1. Data from Sheldon and Stapleton [1968]. 


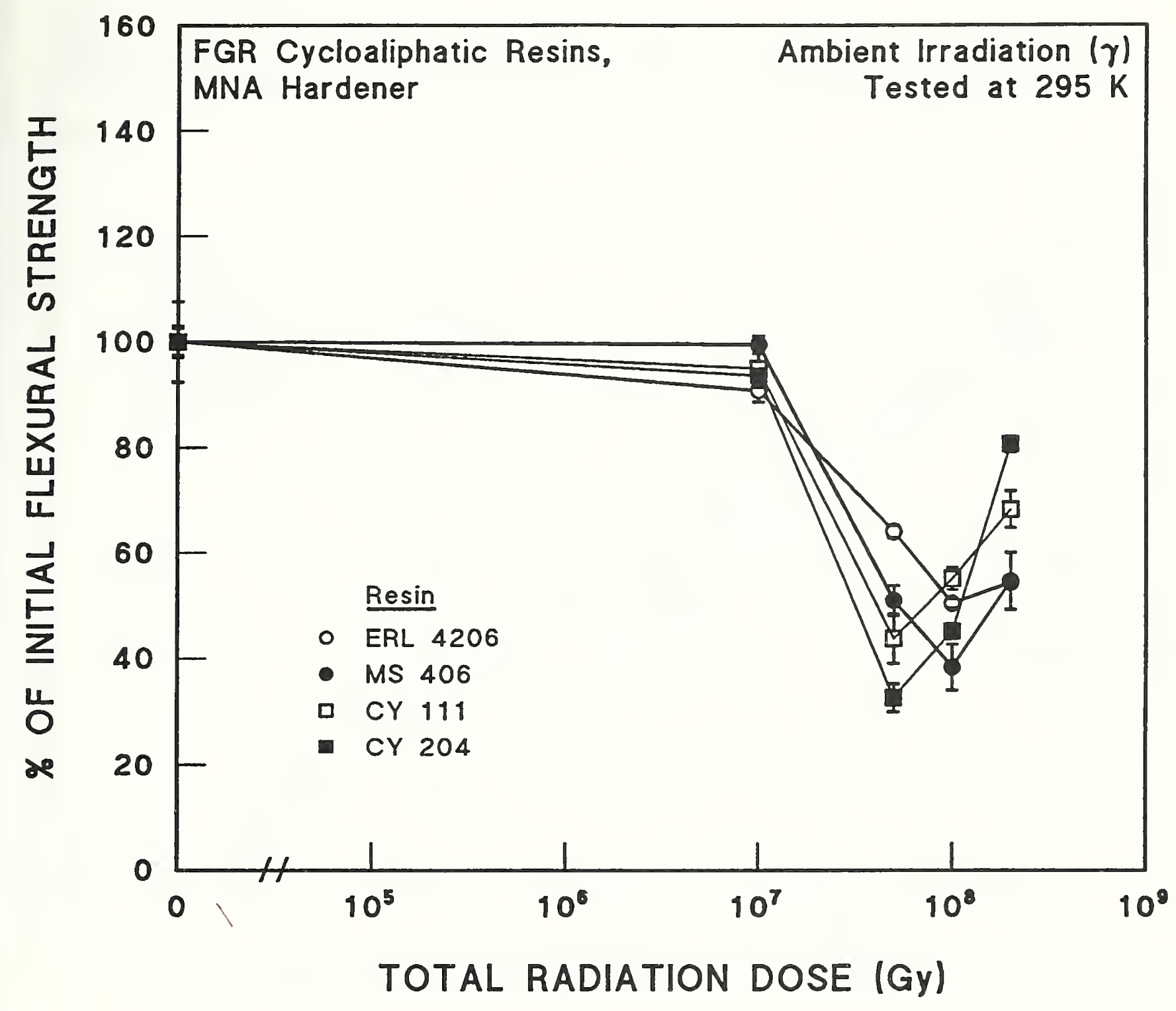

"O" Strength, MPa Accelerator Cure Conditions Manufacturer*

- $422.9 \pm 3.2 \quad$ BDMA $\quad 5 \mathrm{~h}, 100^{\circ} \mathrm{C} ; \quad \mathrm{BXL}$

- $379.4 \pm 9.6 \quad$ BDMA $4 \mathrm{~h}, 100^{\circ} \mathrm{C}$; CIBA

$\begin{array}{lll}459.1 \pm 13.9 \quad \text { BDMA } & 4 \mathrm{~h}, 100^{\circ} \mathrm{C} ; & 6 \mathrm{~h}, 160^{\circ} \mathrm{C}\end{array}$

- $402.3 \pm 30.5 \quad$ BDMA $4 \mathrm{~h}, 100^{\circ} \mathrm{C} \quad$ RUT, Ag.

* Supplier: Union Carbide

Figure A.2-7. Flexural strength at $295 \mathrm{~K}$ of FGR cycloaliphatic resins with MNA hardener after ambient gamma irradiation. Supplementary Table A.2-1. Data from Sheldon and Stapleton [1968]. 


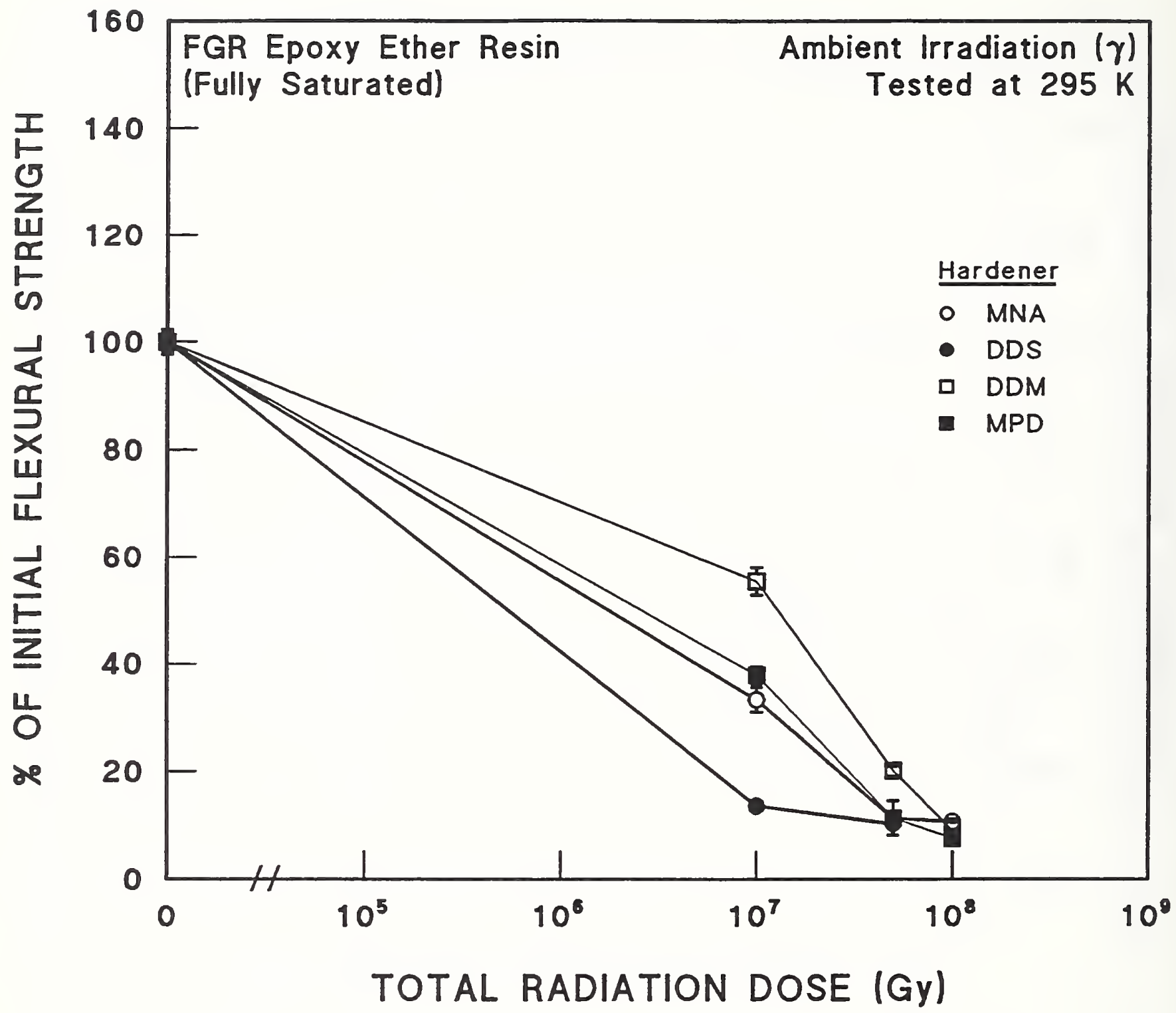

"O" Strength, MPa Accelerator Cure Conditions Supplier*

○ $328.6 \pm 5.8$

BDMA

$10 \mathrm{~h}, 160^{\circ} \mathrm{C}$

Union Carbide

- $370.5 \pm 8.6$

$--\quad 88 \mathrm{~h}, 165^{\circ} \mathrm{C}$

F.W. Berk

ㅁ $345.5 \pm 5.0$

$10 \mathrm{~h}, 160^{\circ} \mathrm{C}$

Anchor Chemicals

- $410.6 \pm 4.4$

$10 \mathrm{~h}, 165^{\circ} \mathrm{C}$

Anchor Chemicals

* Manufacturer: RUT, Ag.

Figure A.2-8. Flexural strength at $295 \mathrm{~K}$ of FGR epoxy ether resin with several hardeners after ambient gamma irradiation. Supplementary Table A.2-1. Data from Sheldon and Stapleton [1968]. 


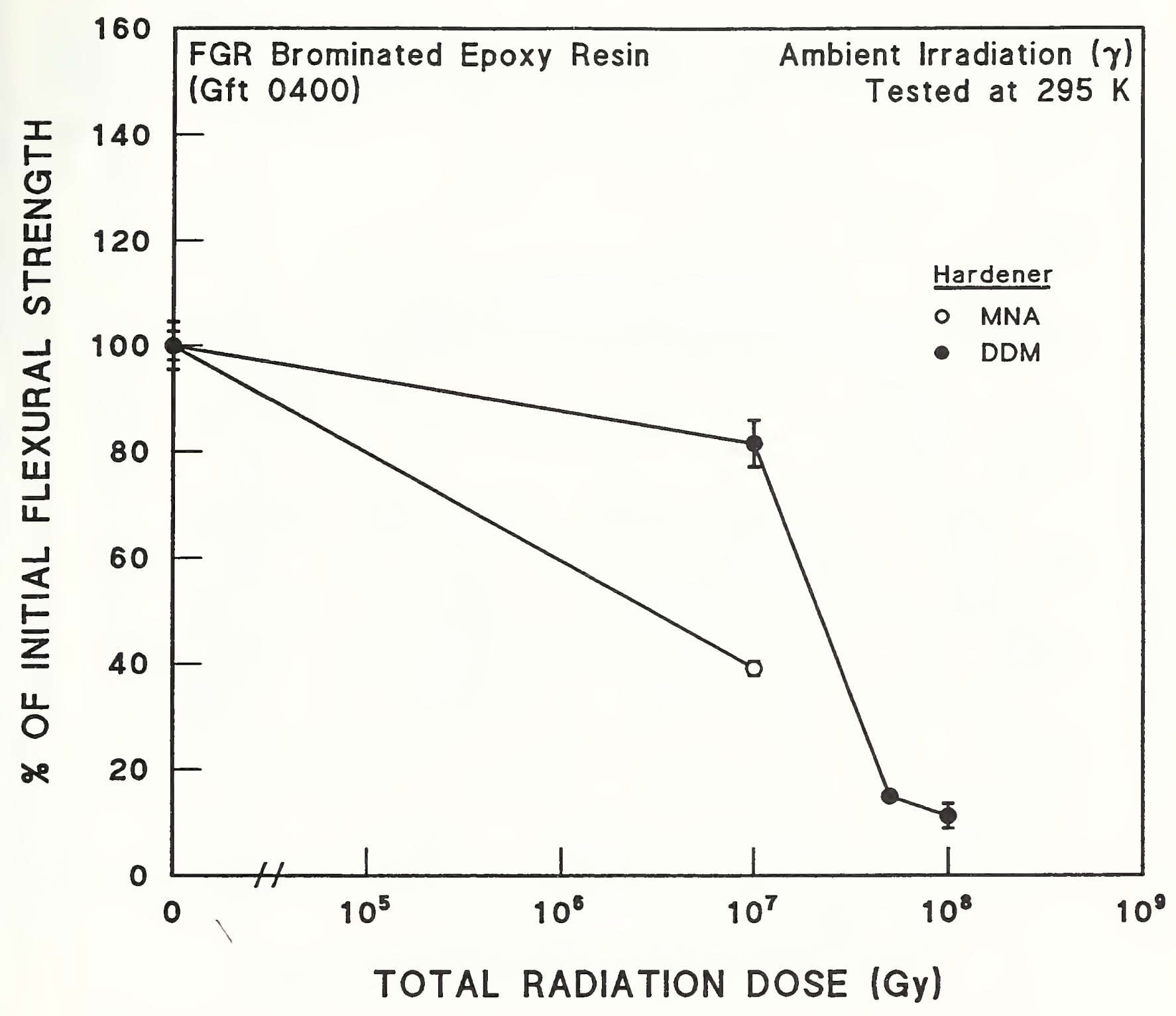

. 


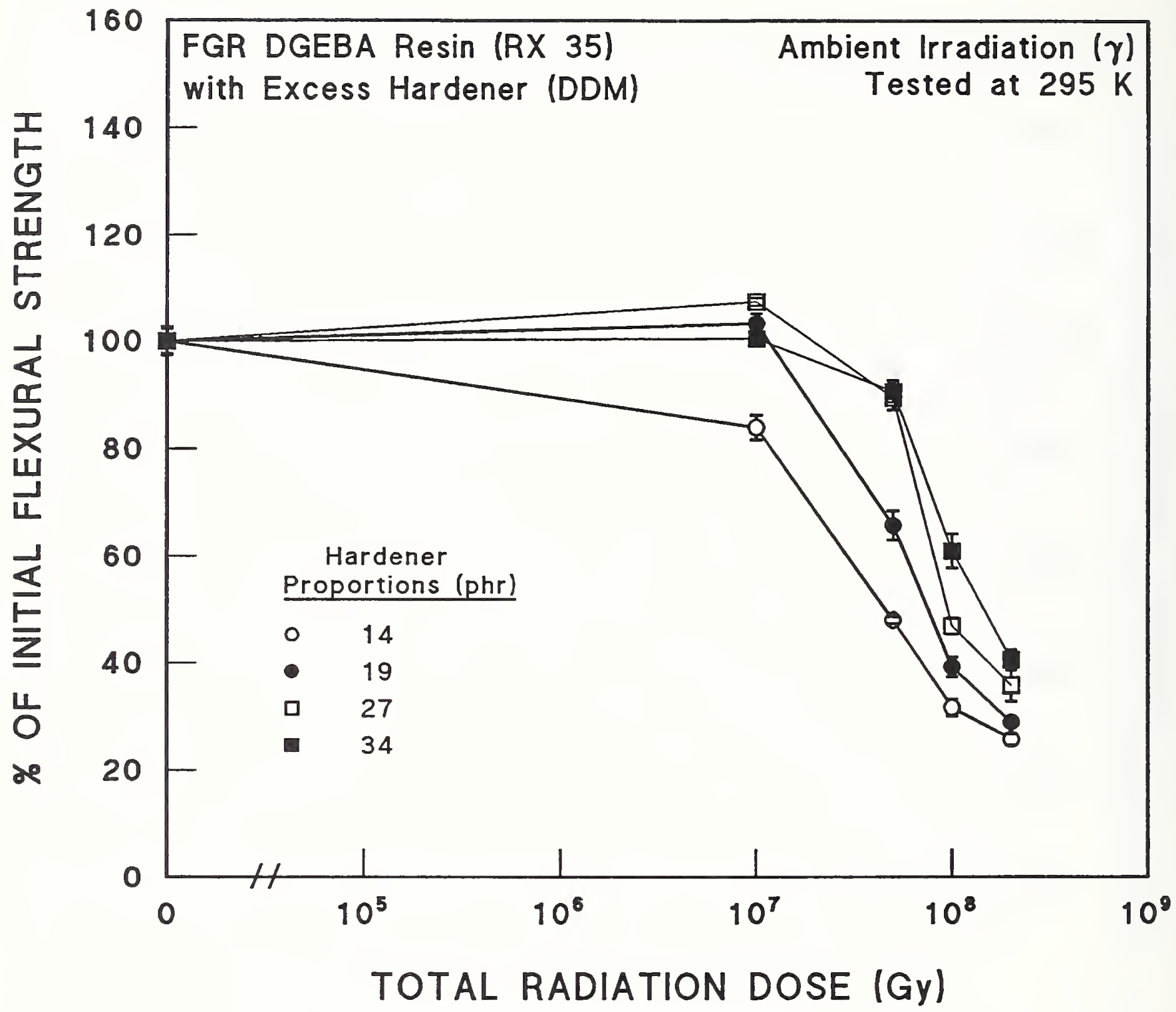

"O" Strength, MPa

- $351.9 \pm 9.5$

- $365.4 \pm 8.4$

ㅁ $400.2 \pm 5.6$

- $374.5 \pm 4.5$

\section{Accelerator}
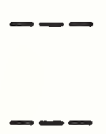

$--$
Cure Conditions

$4 \mathrm{~h}, 100^{\circ} \mathrm{C}$;

$6 \mathrm{~h}, 160^{\circ} \mathrm{C}$

$4 \mathrm{~h}, 100^{\circ} \mathrm{C}$;

$6 \mathrm{~h}, 160^{\circ} \mathrm{C}$

$10 \mathrm{~h}, 100^{\circ} \mathrm{C}$

$4 \mathrm{~h}, 100^{\circ} \mathrm{C}$;

$6 \mathrm{~h}, 160^{\circ} \mathrm{C}$
Supplier*

Anchor Chemicals

Anchor Chemicals

Anchor Chemicals

Anchor Chemicals

* Manufacturer: Shell

Figure A.2-10. Flexural strength at $295 \mathrm{~K}$ of FGR DGEBA resin with excess DDM hardener after ambient gamma irradiation. Supplementary Table A.2-1. Data from Sheldon and Stapleton [1968]. 


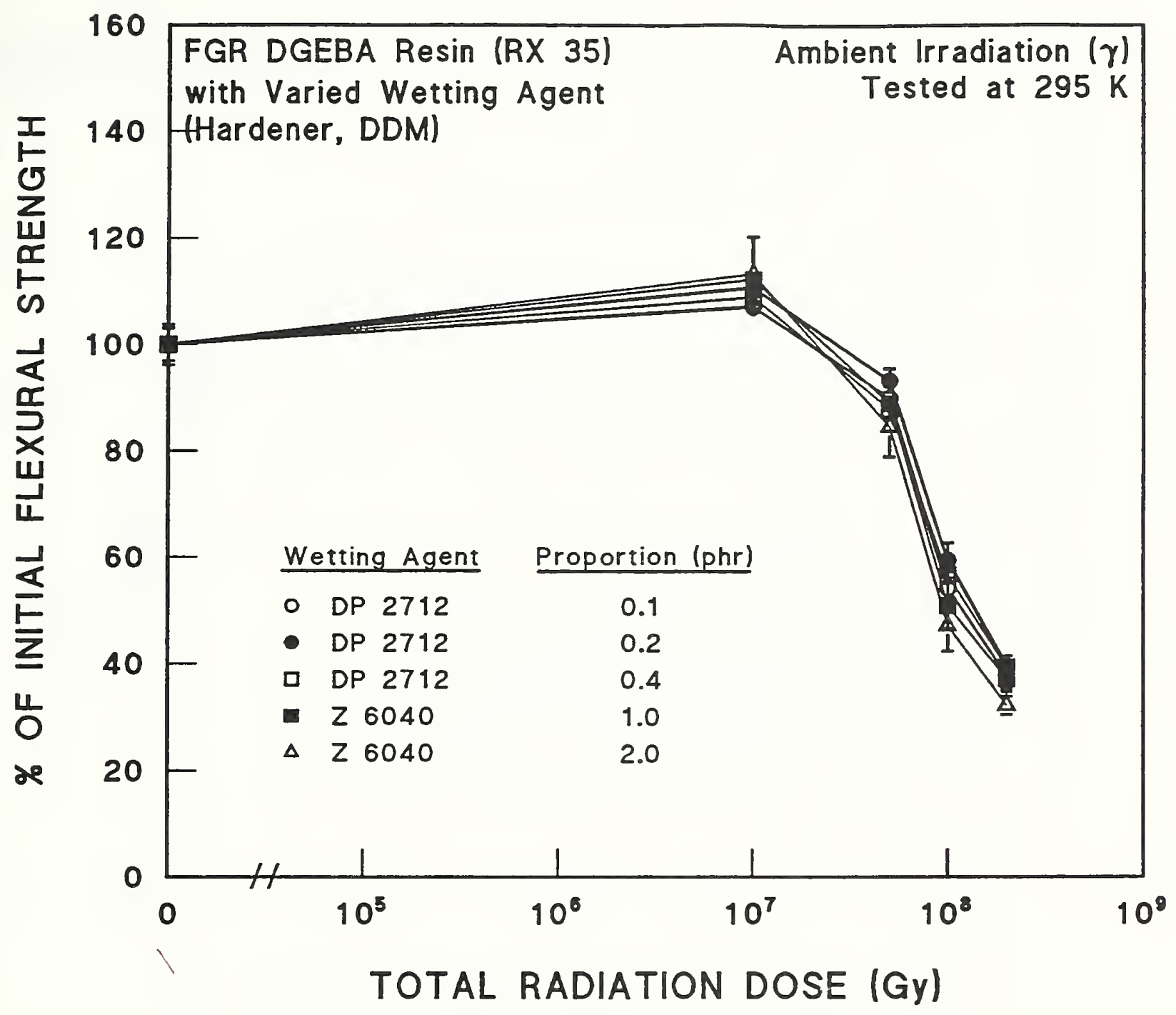

\begin{tabular}{|c|c|c|c|c|}
\hline & Strength, MPa & Accelerator & Cure Conditions & Supplier* \\
\hline 0 & $350.2 \pm 3.2$ & --- & $\begin{array}{l}2 \mathrm{~h}, 100^{\circ} \mathrm{C} \\
10^{\circ} \mathrm{h}, 180^{\circ} \mathrm{C}\end{array}$ & Anchor Chemicals \\
\hline$\bullet$ & $345.6 \pm 13.1$ & -- & $\begin{array}{l}2 \mathrm{~h}, 100^{\circ} \mathrm{C} \\
10^{\mathrm{h}}, 180^{\circ} \mathrm{C}\end{array}$ & Anchor Chemicals \\
\hline$\square$ & $382.7 \pm 11.8$ & -- & $\begin{array}{l}2 \mathrm{~h}, 100^{\circ} \mathrm{C} \\
10 \mathrm{~h}, 180^{\circ} \mathrm{C}\end{array}$ & Anchor Chemicals \\
\hline- & $352.9 \pm 7.2$ & -- & $\begin{array}{l}4 \mathrm{~h}, 100^{\circ} \mathrm{C} ; \\
6 \mathrm{~h}, 160^{\circ} \mathrm{C}\end{array}$ & Anchor Chemicals \\
\hline$\Delta$ & $364.3 \pm 5.3$ & --- & $\begin{array}{l}4 \mathrm{~h}, 100^{\circ} \mathrm{C} \\
6 \mathrm{~h}, 160^{\circ} \mathrm{C}\end{array}$ & Anchor Chemicals \\
\hline
\end{tabular}

Figure A.2-11. Flexural strength at $295 \mathrm{~K}$ of FGR DGEBA resin with DDM hardener and varied wetting agent after ambient gamma irradiation. Supplementary Table A.2-1. Data from Sheldon and Stapleton [1968]. 


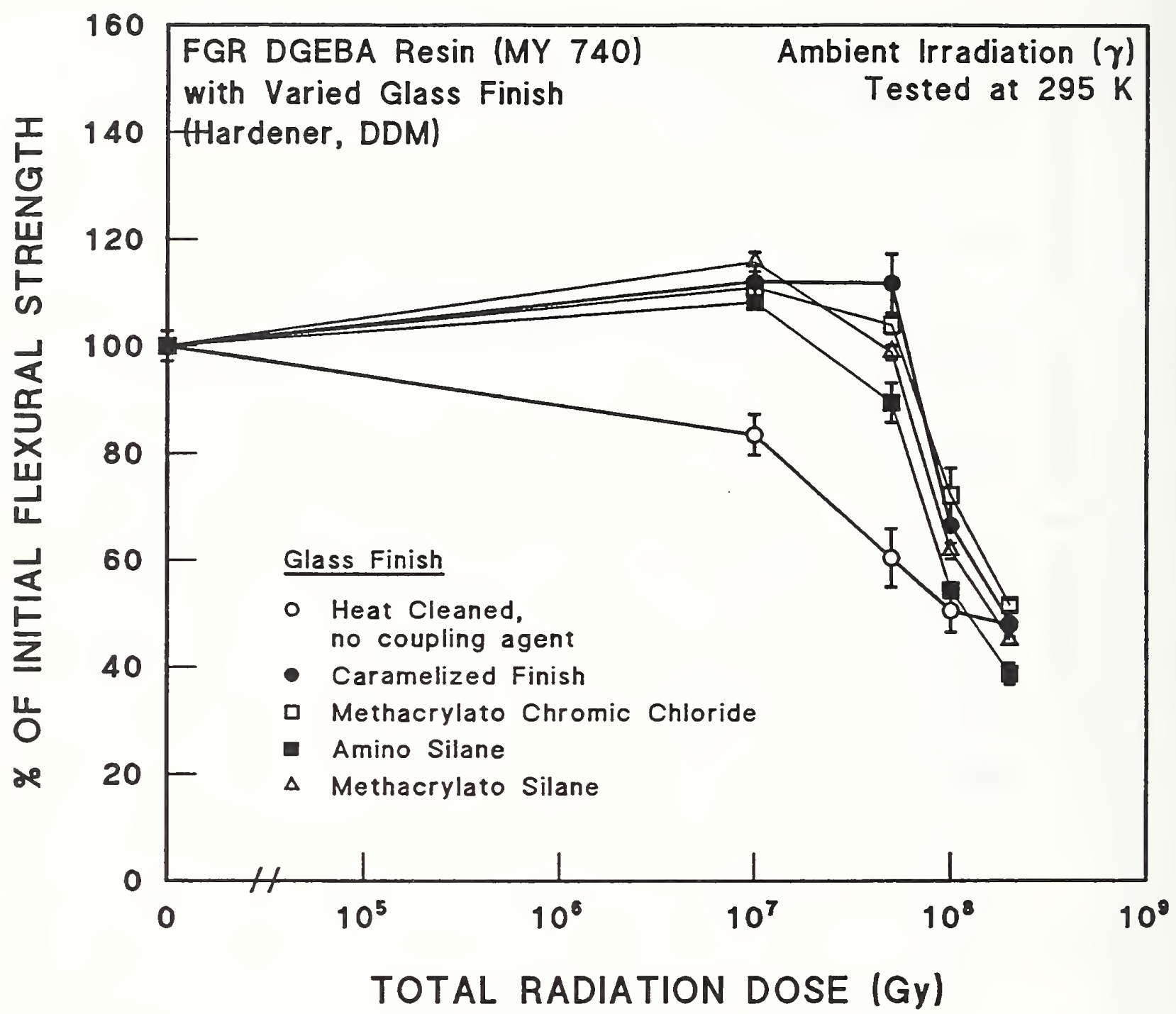

\begin{tabular}{|c|c|c|c|c|}
\hline "O" & Strength, MPa & Accelerator & Cure Conditions & Supplier* \\
\hline 0 & $332.1 \pm 9.4$ & --- & $\begin{array}{l}4 \mathrm{~h}, 100^{\circ} \mathrm{C} ; \\
6 \mathrm{~h}, 160^{\circ} \mathrm{C}\end{array}$ & Anchor Chemicals \\
\hline & $345.6 \pm 4.1$ & --- & $\begin{array}{l}4 \mathrm{~h}, 100^{\circ} \mathrm{C} ; \\
6 \mathrm{~h}, 160^{\circ} \mathrm{C}\end{array}$ & Anchor Chemicals \\
\hline 口 & $333.7 \pm 2.8$ & -- & $\begin{array}{l}4 \mathrm{~h}, 100^{\circ} \mathrm{C} ; \\
6 \mathrm{~h}, 160^{\circ} \mathrm{C}\end{array}$ & Anchor Chemicals \\
\hline 口 & $382.1 \pm 3.3$ & -- & $\begin{array}{l}4 \mathrm{~h}, 100^{\circ} \mathrm{C} ; \\
6 \mathrm{~h}, 160^{\circ} \mathrm{C}\end{array}$ & Anchor Chemicals \\
\hline$\Delta$ & $361.5 \pm 1.6$ & -- & $\begin{array}{l}4 \mathrm{~h}, 100^{\circ} \mathrm{C} ; \\
6 \mathrm{~h}, 160^{\circ} \mathrm{C}\end{array}$ & Anchor Chemicals \\
\hline
\end{tabular}

Figure A.2-12. Flexural strength at $295 \mathrm{~K}$ of FGR DGEBA resin with DDM hardener and varied glass finish after ambient gamma irradiation. Supplementary Table A.2-1. Data from Sheldon and Stapleton [1968]. 


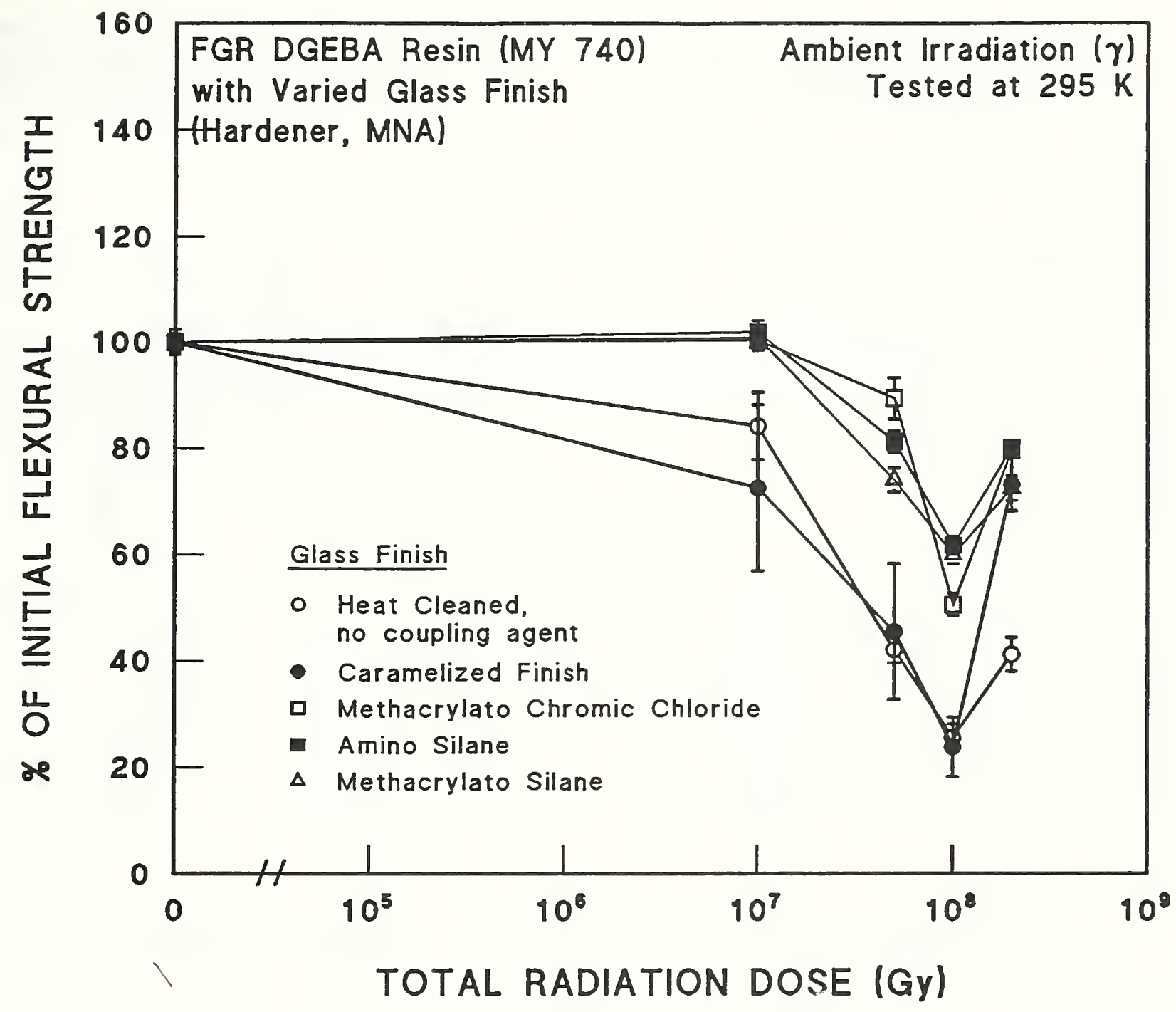

\begin{tabular}{|c|c|c|c|c|}
\hline "O" & Strength, MPa & Accelerator & Cure Conditions & Supplier* \\
\hline o & $369.2 \pm 3.1$ & BDMA & $\begin{array}{l}4 \mathrm{~h}, 100^{\circ} \mathrm{C} \\
6 \mathrm{~h}, 160^{\circ} \mathrm{C}\end{array}$ & Union Carbide \\
\hline - & $396.6 \pm 9.4$ & BDMA & $\begin{array}{l}4 \mathrm{~h}, 100^{\circ} \mathrm{C} \text {; } \\
6 \mathrm{~h}, 160^{\circ} \mathrm{C}\end{array}$ & Union Carbide \\
\hline 口 & $386.6 \pm 6.6$ & BDMA & $\begin{array}{l}4 \mathrm{~h}, 100^{\circ} \mathrm{C} \\
6 \mathrm{~h}, 160^{\circ} \mathrm{C}\end{array}$ & Union Carbide \\
\hline 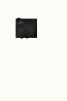 & $394.7 \pm 5.1$ & BDMA & $\begin{array}{l}4 \mathrm{~h}, 100^{\circ} \mathrm{C} ; \\
6 \mathrm{~h}, 160^{\circ} \mathrm{C}\end{array}$ & Union Carbide \\
\hline$\Delta$ & $396.1 \pm 6.8$ & BDMA & $\begin{array}{l}4 \mathrm{~h}, 100^{\circ} \mathrm{C} \\
6 \mathrm{~h}, 160^{\circ} \mathrm{C}\end{array}$ & Union Carbide \\
\hline
\end{tabular}

Figure A.2-13. Flexural strength at $295 \mathrm{~K}$ of FGR DGEBA resin with MNA hardener and varied glass finish after ambient gamma irradiation. Supplementary Table A.2-1. Data from Sheldon and Stapleton [1968]. 


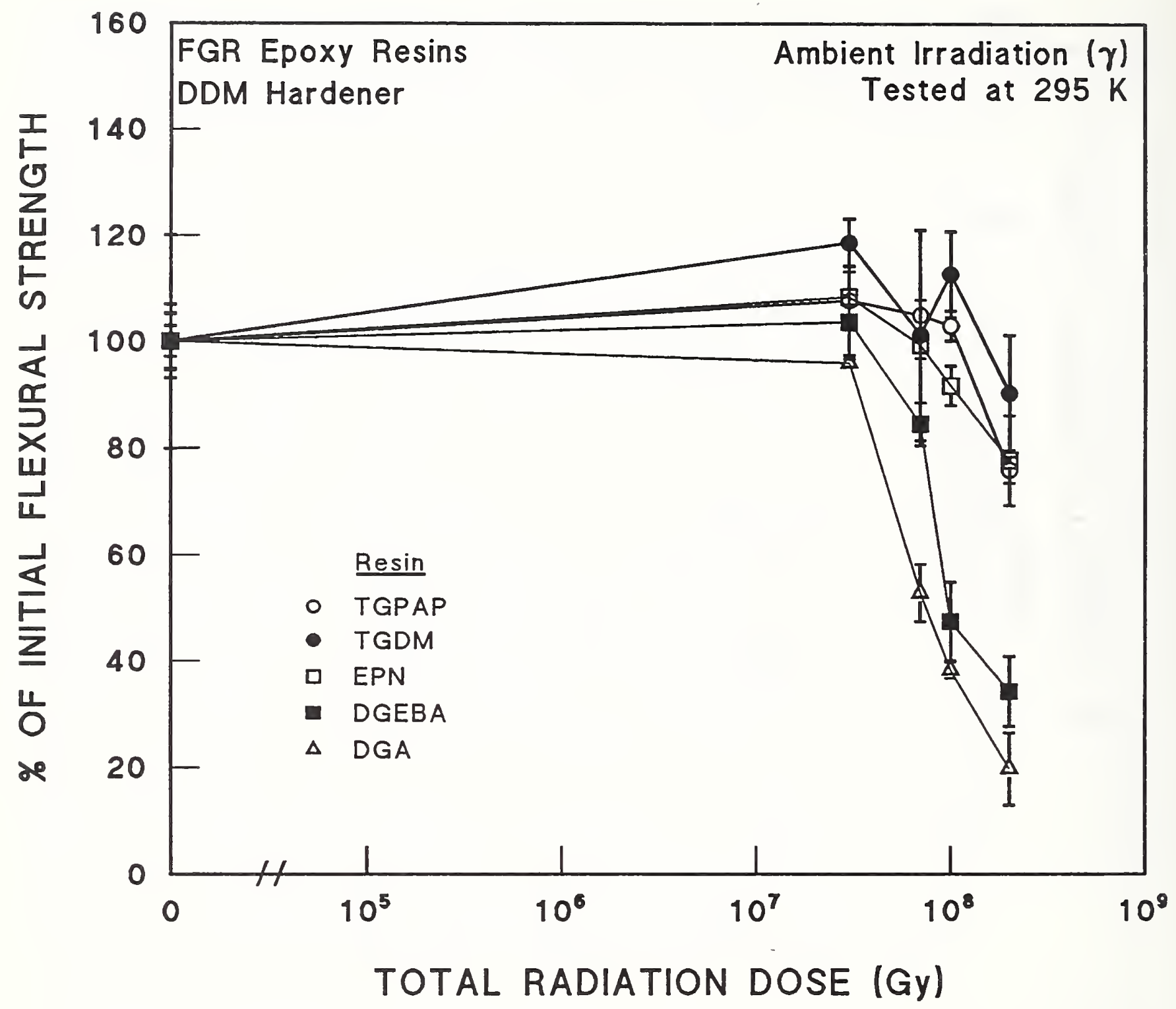

\section{"O" Strength, MPa}

○ $392.7 \pm 11.4$

- $316.5 \pm 63.6$

ㅁ $352.7 \pm 18.7$

- $356.1 \pm 24.6$

$\Delta \quad 437.3 \pm 22.3$

\section{Accelerator Cure Conditions}

$2 \mathrm{~h}, \leq 150^{\circ} \mathrm{C} ; 6 \mathrm{~h}, 150^{\circ} \mathrm{C}$;

$3 \mathrm{~h}, 5120^{\circ} \mathrm{C} ; 5 \mathrm{~h}, 150^{\circ} \mathrm{C}$

$2 \mathrm{~h}, 100^{\circ} \mathrm{C} ; 6 \mathrm{~h}, 150^{\circ} \mathrm{C}$

$2 \mathrm{~h}, \leq 150^{\circ} \mathrm{C} ; 2 \mathrm{~h}, 150^{\circ} \mathrm{C}$

$2 \mathrm{~h}, \leq 100^{\circ} \mathrm{C} ; 4 \mathrm{~h}, 150^{\circ} \mathrm{C}$;

$2 \mathrm{~h}, 180^{\circ} \mathrm{C}$
Supplier

Ciba-Geigy

$B X L$

Ciba-Geigy

Ciba-Geigy

Ciba-Geigy

Ciba-Geigy

Figure A.2-14. Flexural strength at $295 \mathrm{~K}$ of FGR epoxy resins with DDM hardener after ambient gamma irradiation. Supplementary Table A.2-1. Data from Evans et al. [1970]. 


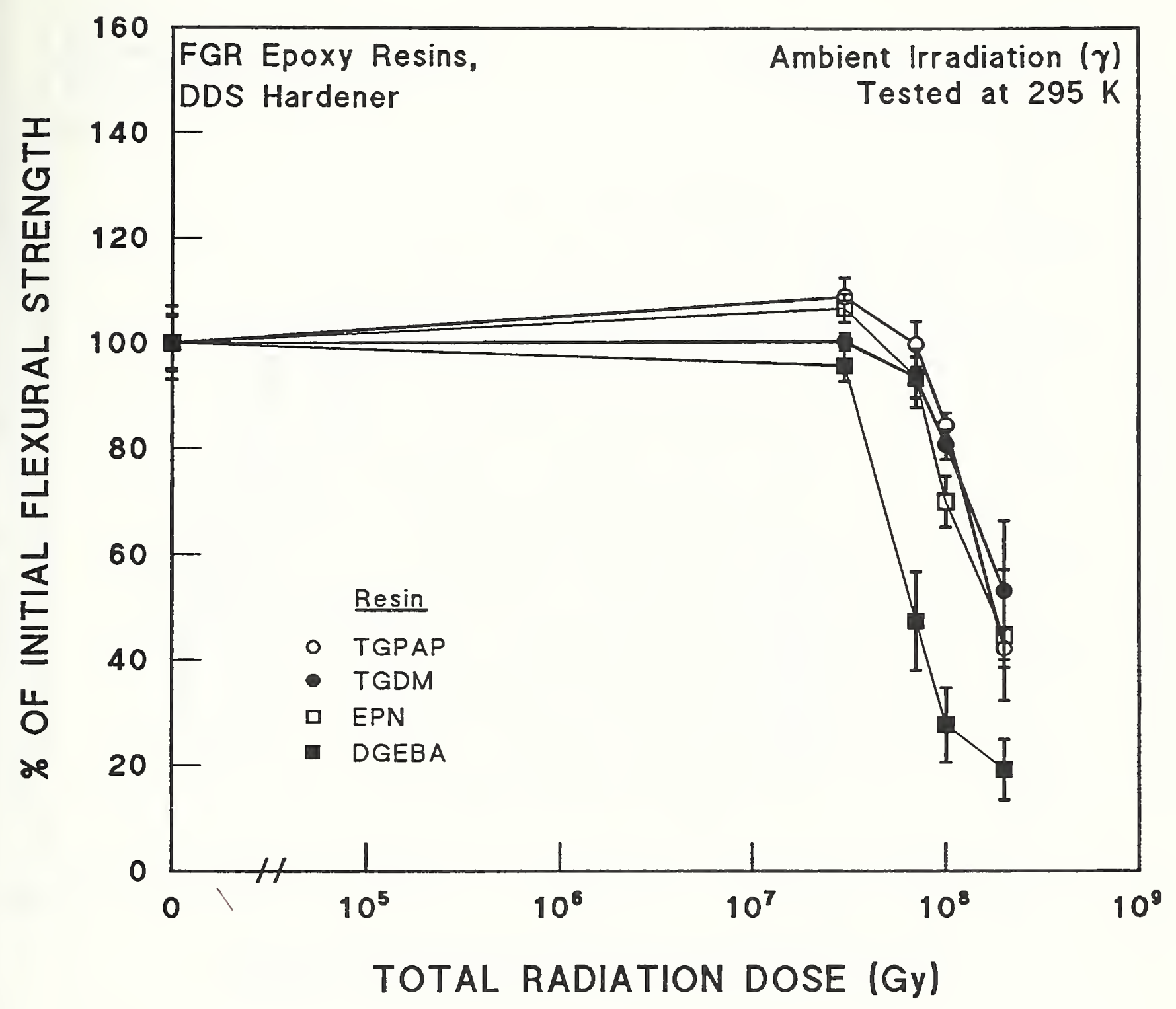

\begin{tabular}{|c|c|c|c|c|}
\hline "0" & Strength, MPa & Accelerator & Cure Conditions & Supplier \\
\hline$\circ$ & $401.4 \pm 20.1$ & -- & $\begin{array}{l}2 \mathrm{~h}, 130^{\circ} \mathrm{C} ; \\
20 \mathrm{~h}, 200^{\circ} \mathrm{C}\end{array}$ & $\begin{array}{l}\text { F.W. Berk } \\
\text { BXL }\end{array}$ \\
\hline & $380.3 \pm 20.9$ & --- & $\begin{array}{l}2 \mathrm{~h}, 130^{\circ} \mathrm{C} ; \\
20^{\circ} \mathrm{h}, 200^{\circ} \mathrm{C}\end{array}$ & $\begin{array}{l}\text { F.W. Berk } \\
\text { Ciba-Geigy }\end{array}$ \\
\hline$\circ$ & $466.8 \pm 24.3$ & -- & $\begin{array}{l}2 \mathrm{~h}, 120^{\circ} \mathrm{C} \text {; } \\
6 \mathrm{~h}, 200^{\circ} \mathrm{C}\end{array}$ & $\begin{array}{l}\text { F.W. Berk } \\
\text { Ciba-Geigy }\end{array}$ \\
\hline & $356.2 \pm 24.9$ & -- & $\begin{array}{l}2 \mathrm{~h}, 130^{\circ} \mathrm{C} \\
20 \mathrm{~h}, 200^{\circ} \mathrm{C}\end{array}$ & $\begin{array}{l}\text { F.W. Berk } \\
\text { Ciba-Geigy }\end{array}$ \\
\hline
\end{tabular}

Figure A.2-15. Flexural strength at $295 \mathrm{~K}$ of FGR epoxy resins with DDS hardener after ambient gamma irradiation. Supplementary Table A.2-1. Data from Evans et al. [1970]. 


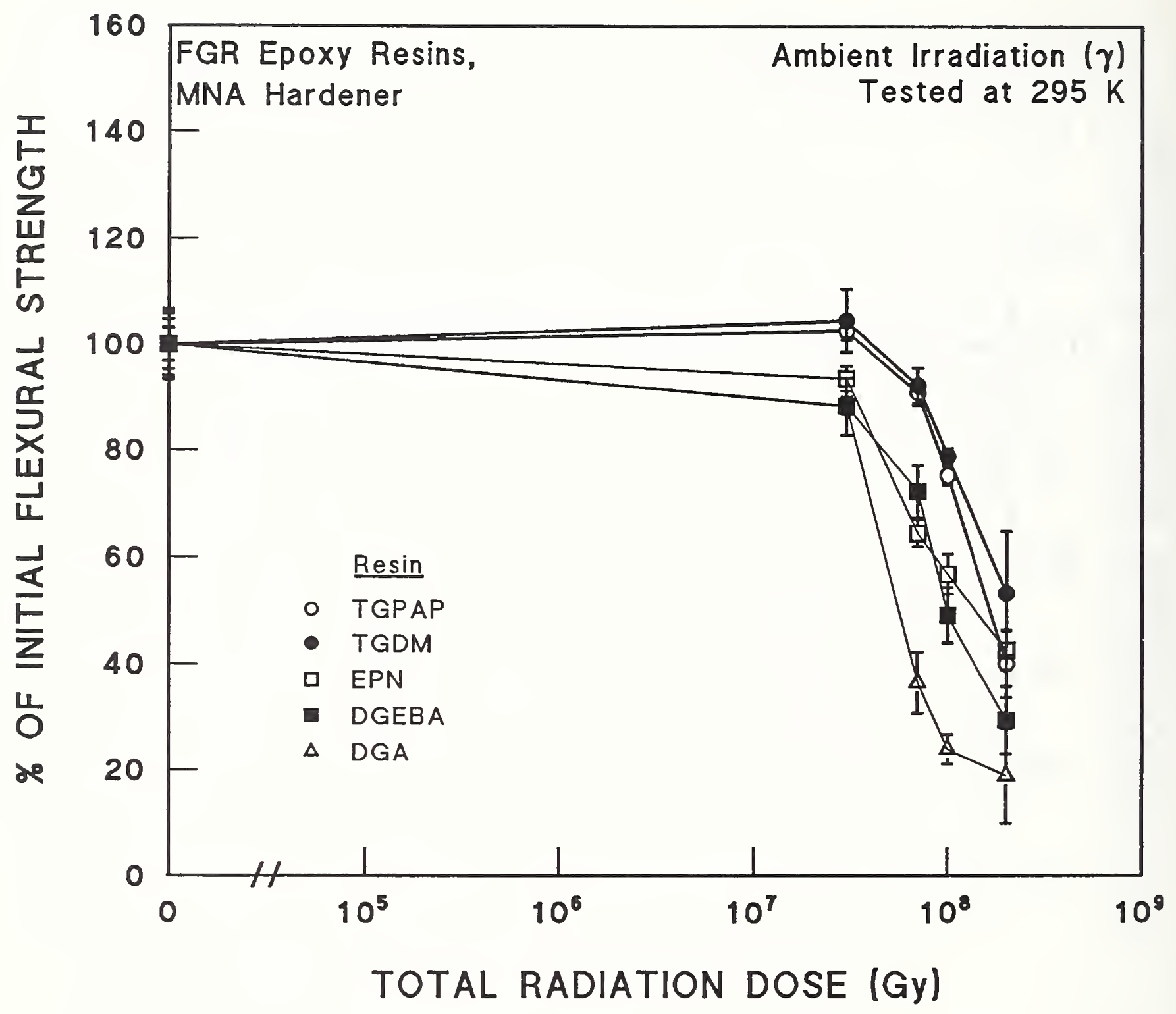

"O" Strength, MPa

○ $378.8 \pm 18.8$

- $357.3 \pm 21.1$

$370.3 \pm 23.0$

- $358.3 \pm 24.0$

$\Delta 403.4 \pm 12.9$

\section{Accelerator Cure Conditions}

$2 \mathrm{~h}, \leq 110^{\circ} \mathrm{C} ; 3 \mathrm{~h}, 150^{\circ} \mathrm{C}$;

$3 \mathrm{~h}, 200^{\circ} \mathrm{C}$

$3 \mathrm{~h}, \leq 120^{\circ} \mathrm{C} ; 5 \mathrm{~h}, 150^{\circ} \mathrm{C}$

$2 \mathrm{~h}, \leq 100^{\circ} \mathrm{C} ; 3 \mathrm{~h}, 150^{\circ} \mathrm{C}$;

$6 \mathrm{~h}, 215^{\circ} \mathrm{C}$

BDMA
Supplier

Ciba-Geigy

$B X L$

Ciba-Geigy

Ciba-Geigy

Ciba-Geigy

Ciba-Geigy

$2 \mathrm{~h}, \leq 100^{\circ} \mathrm{C} ; 4 \mathrm{~h}, 150^{\circ} \mathrm{C}$;

$2 \mathrm{~h}, 180^{\circ} \mathrm{C}$

Figure A.2-16. Flexural strength at $295 \mathrm{~K}$ of FGR epoxy resins with MNA hardener after ambient gamma irradiation. Supplementary Table A.2-1. Data from Evans et al. [1970]. 


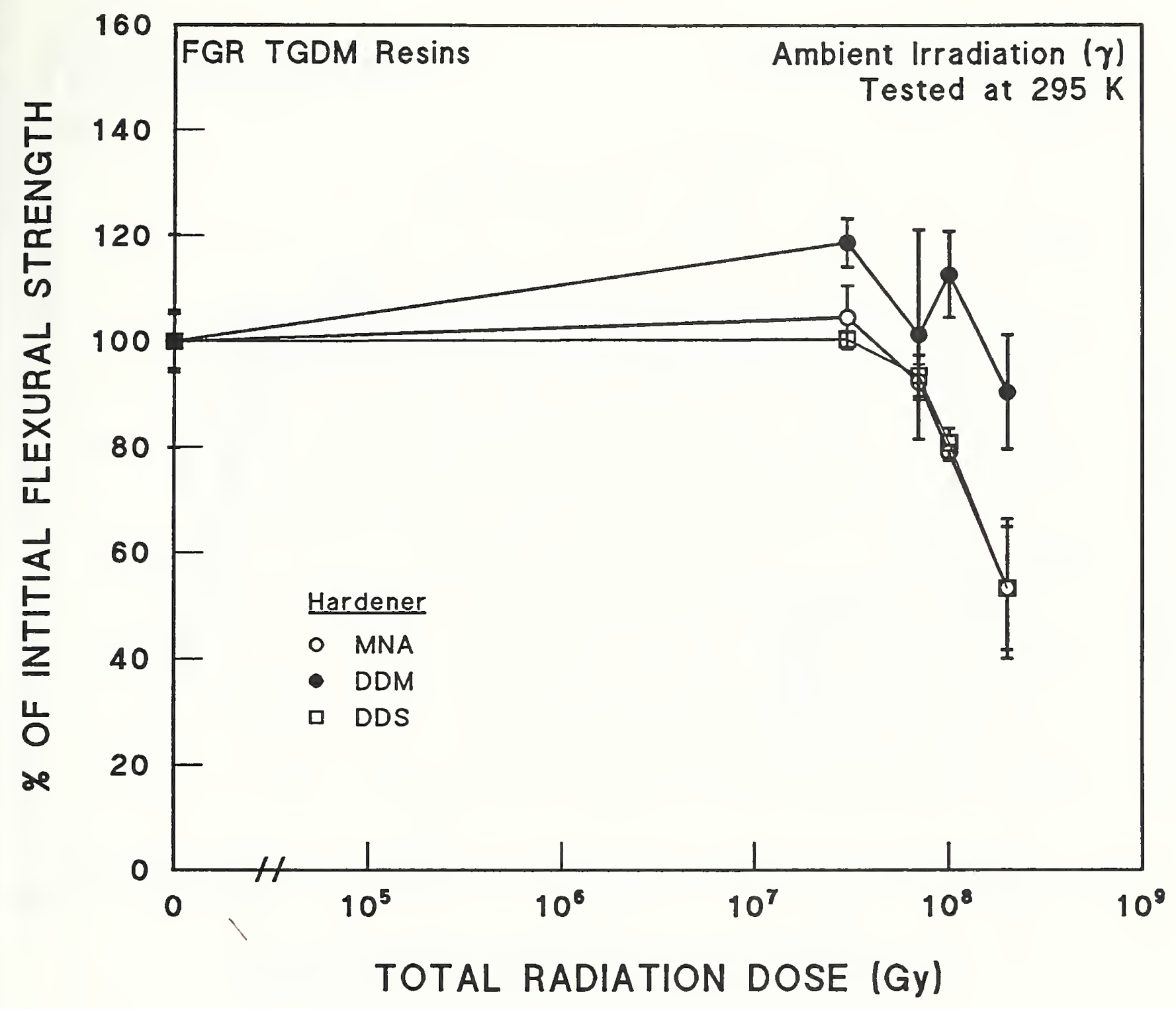

\begin{tabular}{|c|c|c|c|c|}
\hline "0" & Strength, MPa & Accelerator & Cure Conditions & Supplier \\
\hline ○ & $357.3 \pm 21.1$ & -- & $\begin{array}{l}3 \mathrm{~h}, \leq 120^{\circ} \mathrm{C} \\
5 \mathrm{~h}, 150^{\circ} \mathrm{C}\end{array}$ & -Geigy \\
\hline$\bullet$ & $316.5 \pm 63.6$ & --- & $\begin{array}{l}3 \mathrm{~h}, \leq 120^{\circ} \mathrm{C} \\
5 \mathrm{~h}, 150^{\circ} \mathrm{C}\end{array}$ & Ciba-Geigy \\
\hline ם & $466.8 \pm 24.3$ & --- & $\begin{array}{l}2 \mathrm{~h}, 130^{\circ} \mathrm{C} \\
20 \mathrm{~h}, 200^{\circ} \mathrm{C}\end{array}$ & $\begin{array}{l}\text { Ciba-Geigy } \\
\text { F.W. Berk }\end{array}$ \\
\hline
\end{tabular}

Figure A.2-17. Flexural strength at $295 \mathrm{~K}$ of FGR TGDM resin with several hardeners after ambient gamma irradiation. Supplementary Table A.2-1. Data from Evans et al. [1970]. 


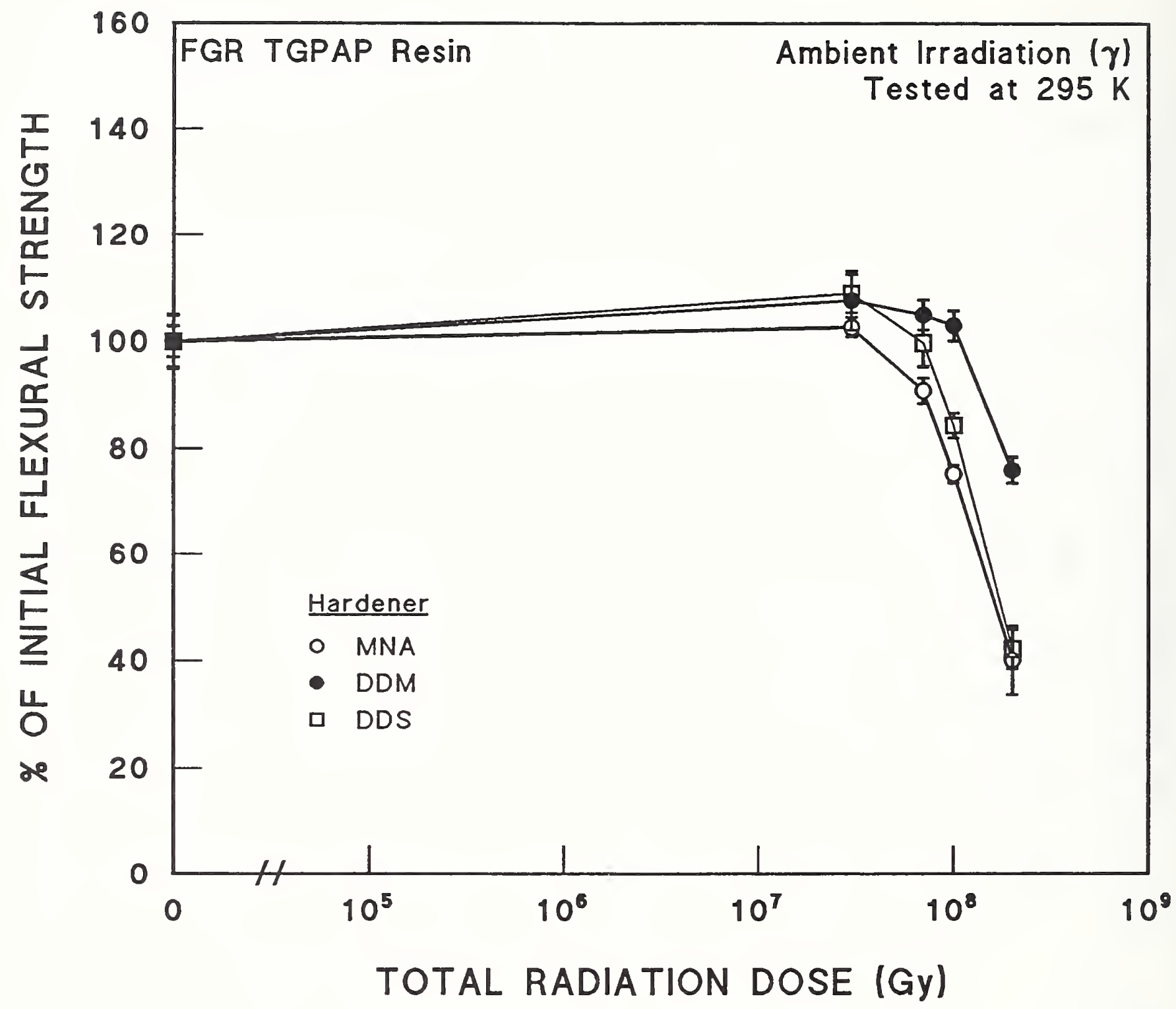

"O" Strength, MPa

Accelerator Cure Conditions

Supplier

○ $378.8 \pm 18.2$

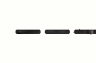

$2 \mathrm{~h}, \leq 110^{\circ} \mathrm{C} ; 3 \mathrm{~h}, 150^{\circ} \mathrm{C}$;

BXL

$3 \mathrm{~h}, 200^{\circ} \mathrm{C}$

- $392.7 \pm 11.4$

--

$2 \mathrm{~h}, 5150^{\circ} \mathrm{C} ; 6 \mathrm{~h}, 150^{\circ} \mathrm{C}$

ㅁ $401.4 \pm 20.1$

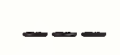

$2 \mathrm{~h}, 130^{\circ} \mathrm{C} ; 20 \mathrm{~h}, 200^{\circ} \mathrm{C}$

Ciba-Geigy

BXL

Ciba-Geigy

$B X L$

F.W. Berk

Figure A.2-18. Flexural strength at $295 \mathrm{~K}$ of FGR TGPAP resin with several hardeners after ambient gamma irradiation. Supplementary Table A.2-1. Data from Evans et al. [1970]. 


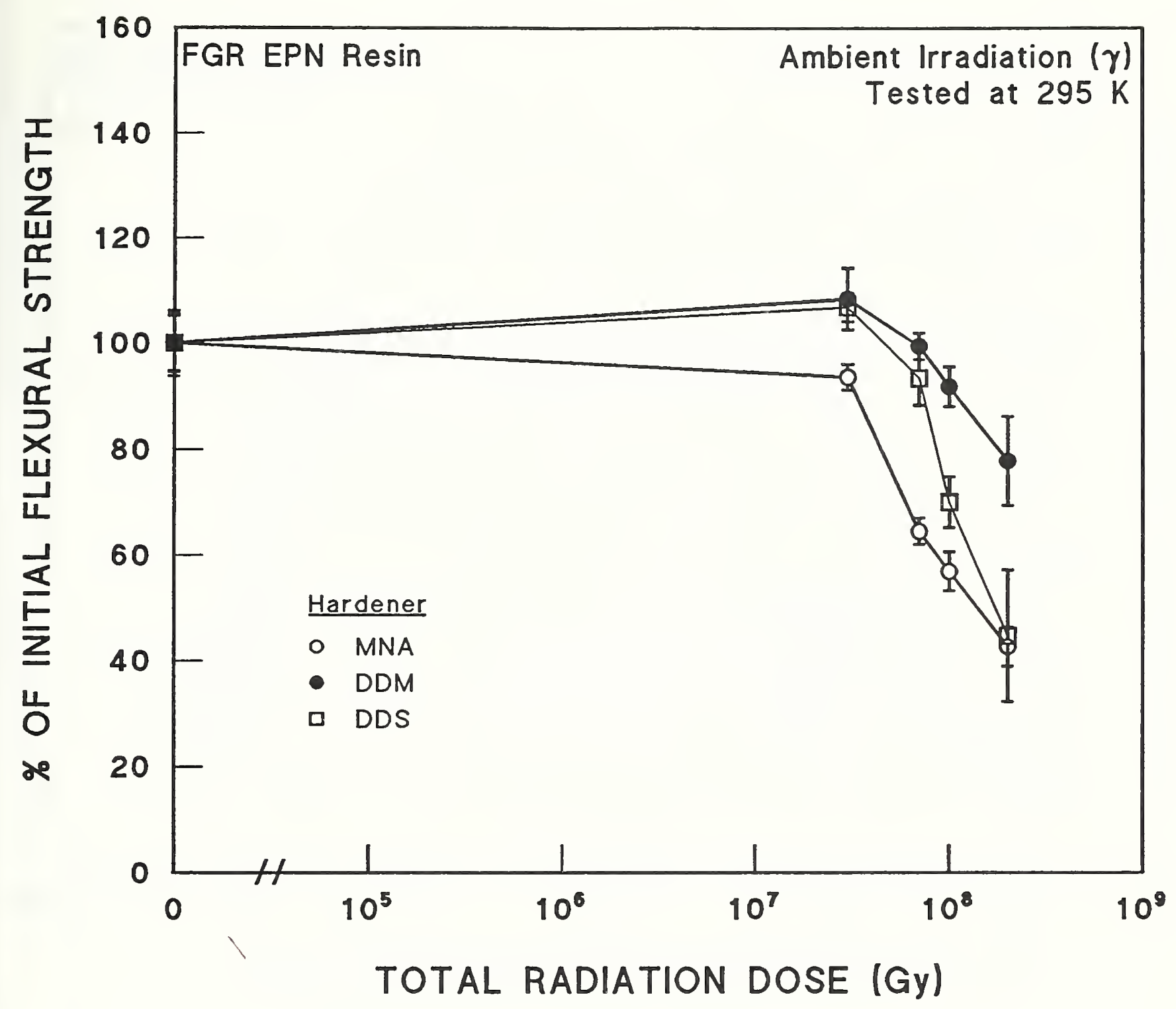

\begin{tabular}{|c|c|c|c|c|}
\hline "O" & Strength, MPa & Accelerator & Cure Conditions & Supplier \\
\hline ○ & $370.3 \pm 23.0$ & -- & $\begin{array}{l}2 \mathrm{~h}, 400^{\circ} \mathrm{C} ; 3 \mathrm{~h}, 150^{\circ} \mathrm{C} \\
6 \mathrm{~h}, 215^{\circ} \mathrm{C}\end{array}$ & Geigy \\
\hline & $352.7 \pm 18.7$ & --- & $2 \mathrm{~h}, 100^{\circ} \mathrm{C} ; 6 \mathrm{~h}, 150^{\circ} \mathrm{C}$ & Ciba-Geigy \\
\hline 口 & $380.3 \pm 20.9$ & --- & $2 \mathrm{~h}, 120^{\circ} \mathrm{C} ; 6 \mathrm{~h}, 200^{\circ} \mathrm{C}$ & $\begin{array}{l}\text { Ciba-Geigy } \\
\text { F.W. Berk }\end{array}$ \\
\hline
\end{tabular}

Figure A.2-19. Flexural strength at $295 \mathrm{~K}$ of FGR EPN resin with several hardeners after ambient gamma irradiation. Supplementary Table A.2-1. Data from Evans et al. [1970] . 


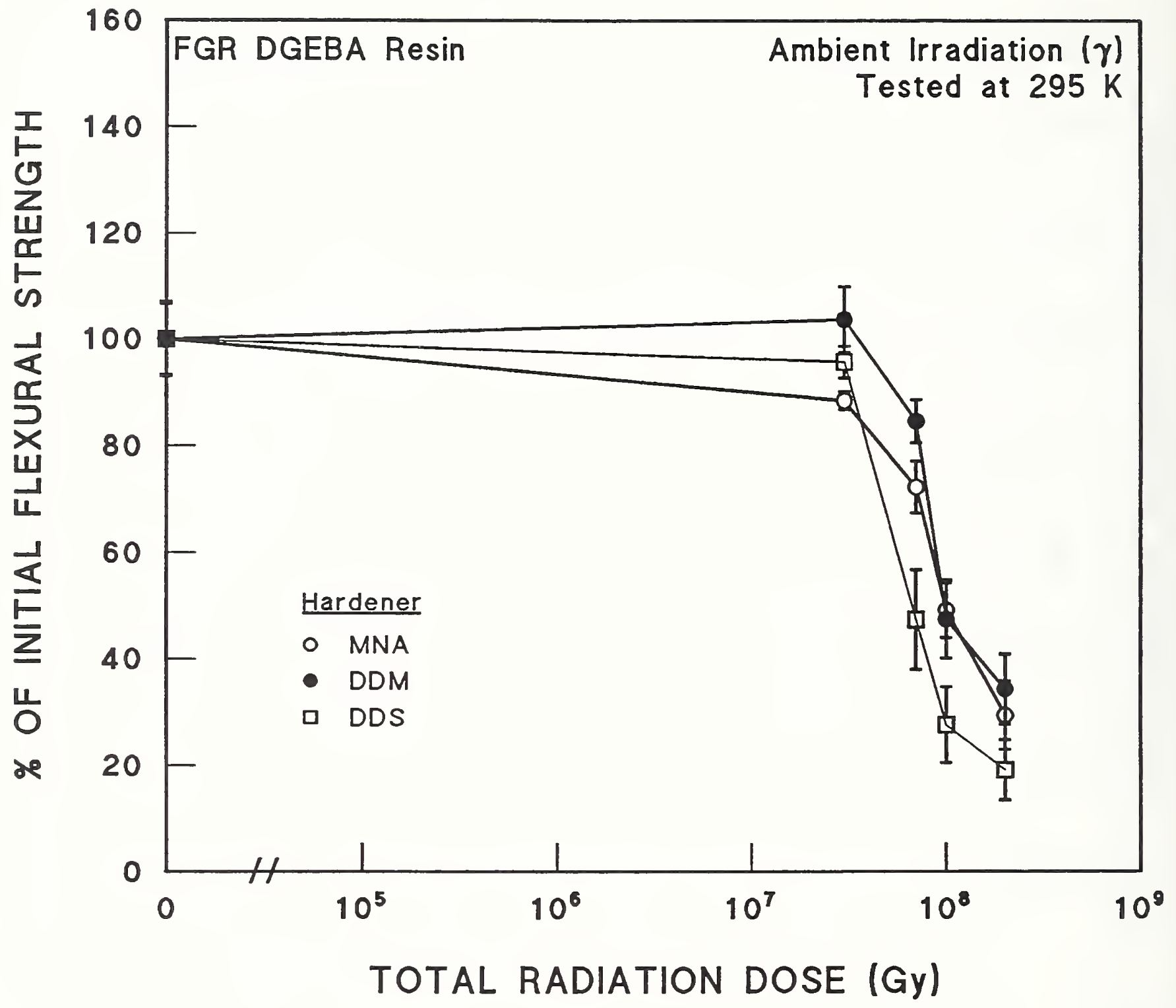

\begin{tabular}{|c|c|c|c|c|}
\hline "O" & Strength, MPa & Accelerator & Cure Conditions & Supplier \\
\hline ○ & $358.3 \pm 24.0$ & BDMA & $\begin{array}{l}2 \mathrm{~h}, \leq 150^{\circ} \mathrm{C} ; \\
2 \mathrm{~h}, 150^{\circ} \mathrm{C}\end{array}$ & Ciba-Geigy \\
\hline$\bullet$ & $356.1 \pm 24.6$ & --- & $\begin{array}{l}2 \mathrm{~h}, \leq 150^{\circ} \mathrm{C} ; \\
2 \mathrm{~h}, 150^{\circ} \mathrm{C}\end{array}$ & Ciba-Geigy \\
\hline$\square$ & $356.2 \pm 24.9$ & --- & $\begin{array}{l}2 \mathrm{~h}, 130^{\circ} \mathrm{C} \\
20 \mathrm{~h}, 200^{\circ} \mathrm{C}\end{array}$ & $\begin{array}{l}\text { Ciba-Geigy } \\
\text { F.W. Berk }\end{array}$ \\
\hline
\end{tabular}

Figure A.2-20. Flexural strength at $295 \mathrm{~K}$ of FGR DGEBA resin with several hardeners after ambient gamma irradiation. Supplementary Table A.2-1. Data from Evans et al. [1970]. 


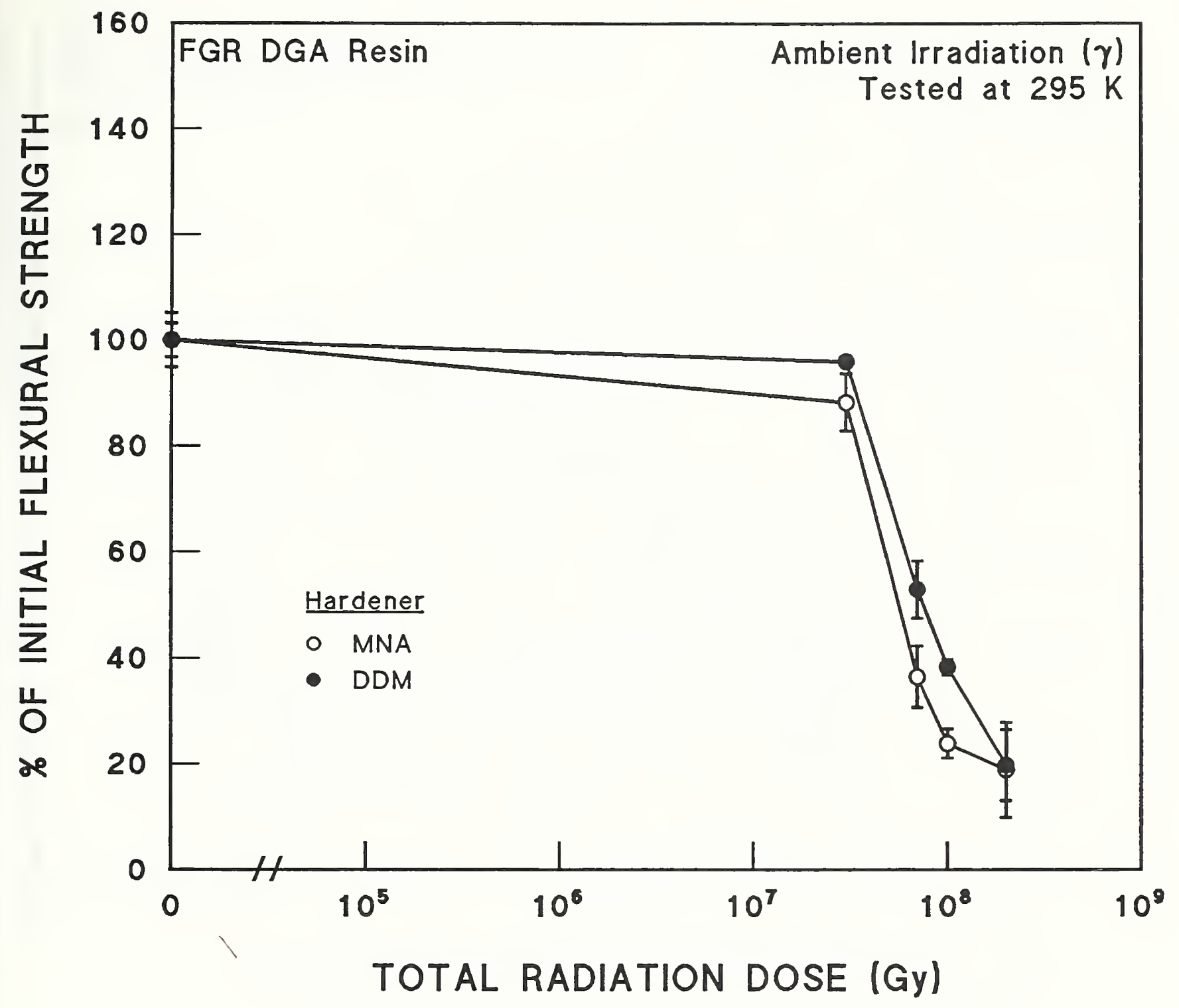

\begin{tabular}{lclll} 
"O" Strength, MPa & Accelerator & & Cure Conditions & Supplier \\
\hline \multirow{2}{*}{$403.4 \pm 12.9$} & --- & & $2 \mathrm{~h}, \leq 100^{\circ} \mathrm{C} ; 4 \mathrm{~h}, 150^{\circ} \mathrm{C} ;$ & Ciba-Geigy \\
& & $2 \mathrm{~h}, 180^{\circ} \mathrm{C}$ & \\
& & $2 \mathrm{~h}, \leq 100^{\circ} \mathrm{C} ; 4 \mathrm{~h}, 150^{\circ} \mathrm{C} ;$ & Ciba-Geigy \\
& $237.3 \pm 22.3$ & --- & &
\end{tabular}

Figure A.2-21. Flexural strength at $295 \mathrm{~K}$ of FGR DGA resin with several hardeners after ambient gamma irradiation. Supplementary Table A.2-1. Data from Evans et al. [1970]. 


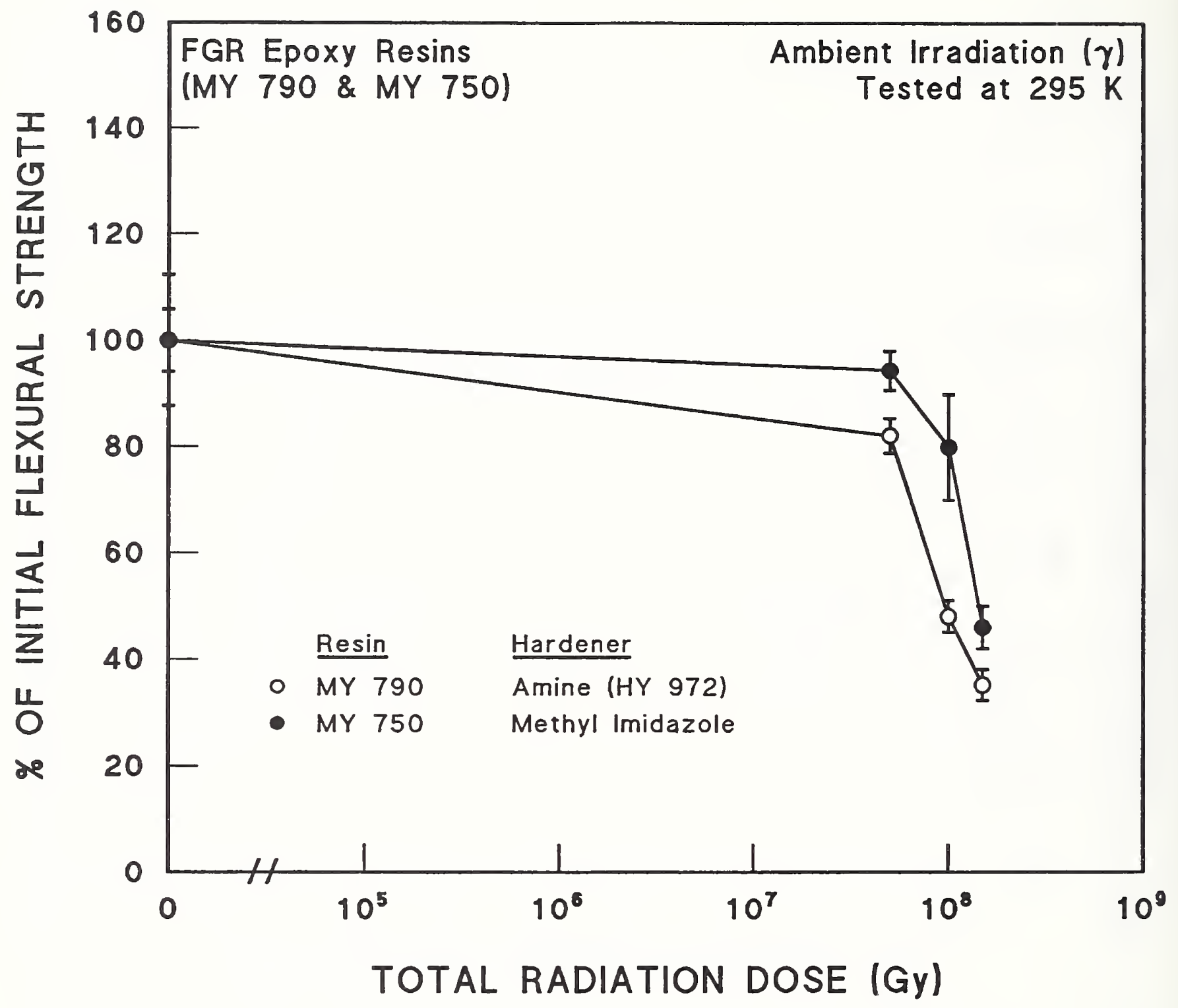

"O" Strength, MPa

- $324.1 \pm 19.1$

- $335.4 \pm 41.3$
Cure Conditions

$8 \mathrm{~h}, 150^{\circ} \mathrm{C}$

$6 \mathrm{~h}, 150^{\circ} \mathrm{C}$
Supplier

Ciba-Geigy

Ciba-Geigy

Figure A.2-22. Flexural strength at $295 \mathrm{~K}$ of FGR epoxy resins (MY 790 and MY 750) after ambient gamma irradiation. Supplementary Table A.2-1. Data from Evans et al. [1972a]. 


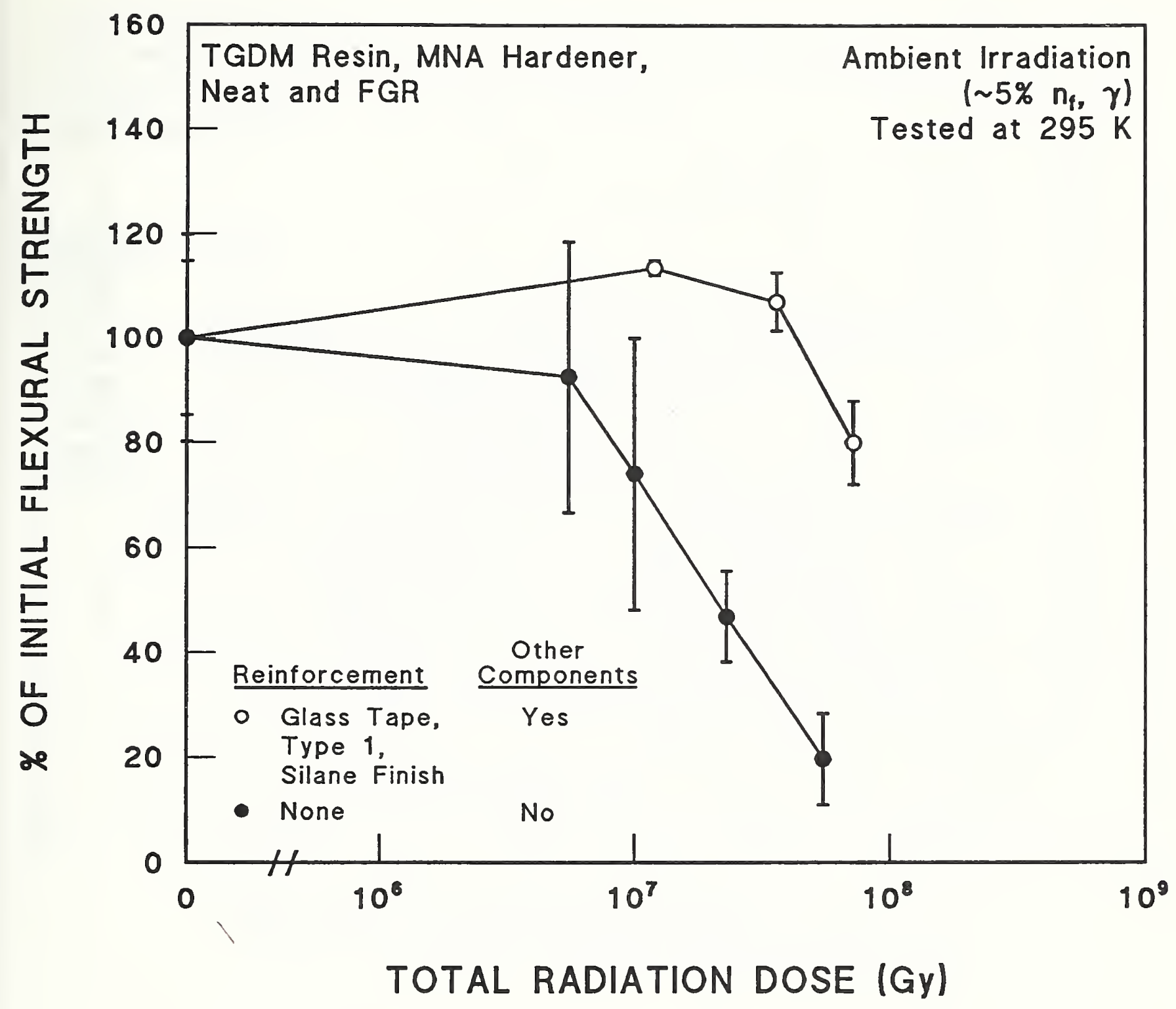

\begin{tabular}{lcll} 
"O" Strength, MPa & & Other Information & Supplier \\
\hline $421.8 \pm 83.4$ & Type B Magnet Coil Resin & BBC Baden \\
- $79.5 \pm 11.8$ & --- & Lintott
\end{tabular}

Figure A.2-23. Flexural strength at $295 \mathrm{~K}$ of neat and FGR TGDM resin, MNA hardener after ambient reactor irradiation. Supplementary Table A.2-2. Data from Schönbacher and Stolarz-Izycka [1979]. 


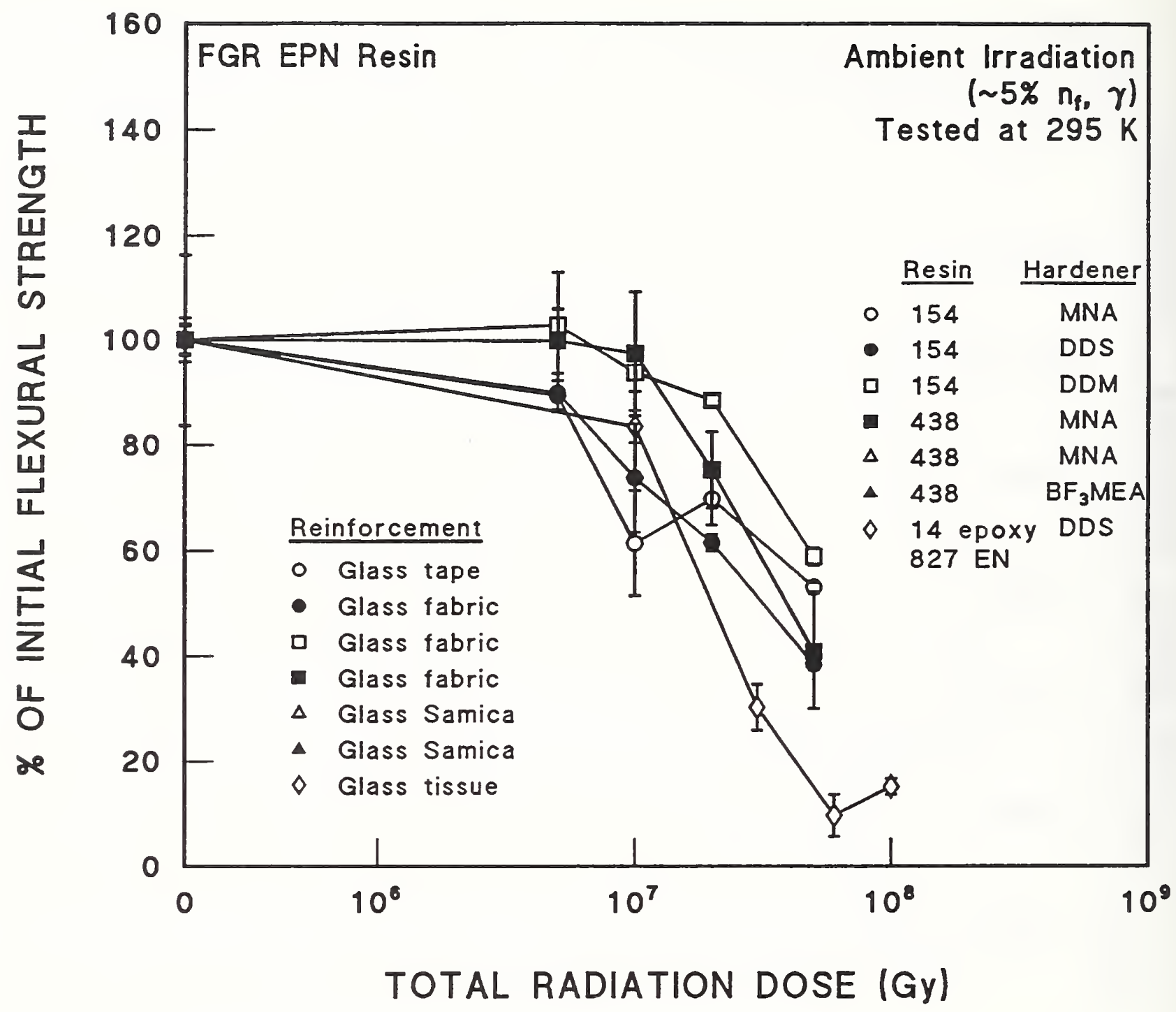

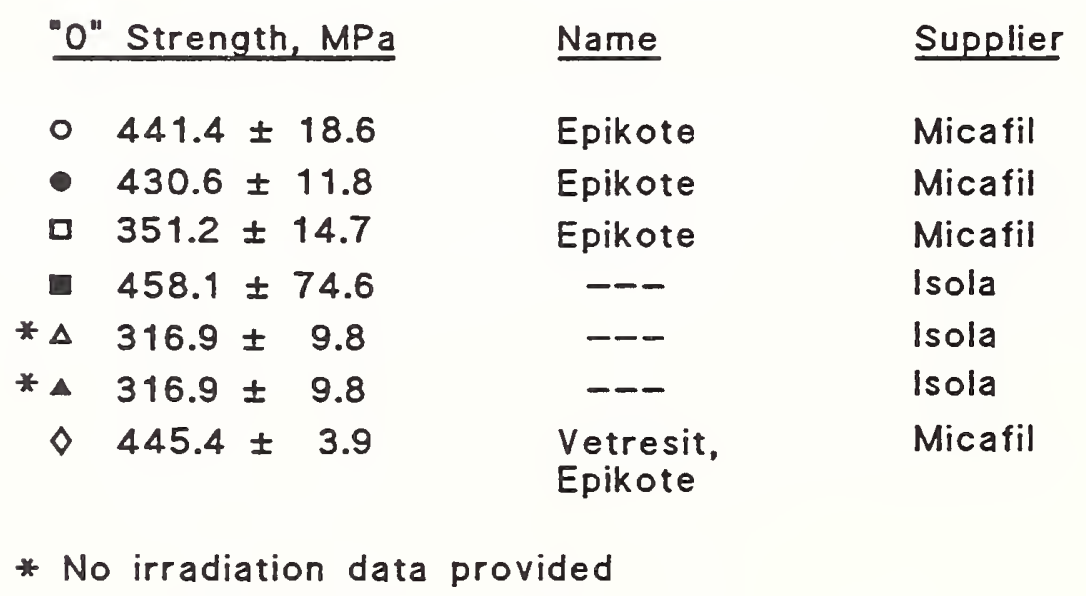

Figure A.2-24. Flexural strength at $295 \mathrm{~K}$ of FGR EPN resin with several hardeners after ambient reactor irradiation. Supplementary Table A.2-2. Data from Schönbacher and Stolarz-Iźycka [1979]. 


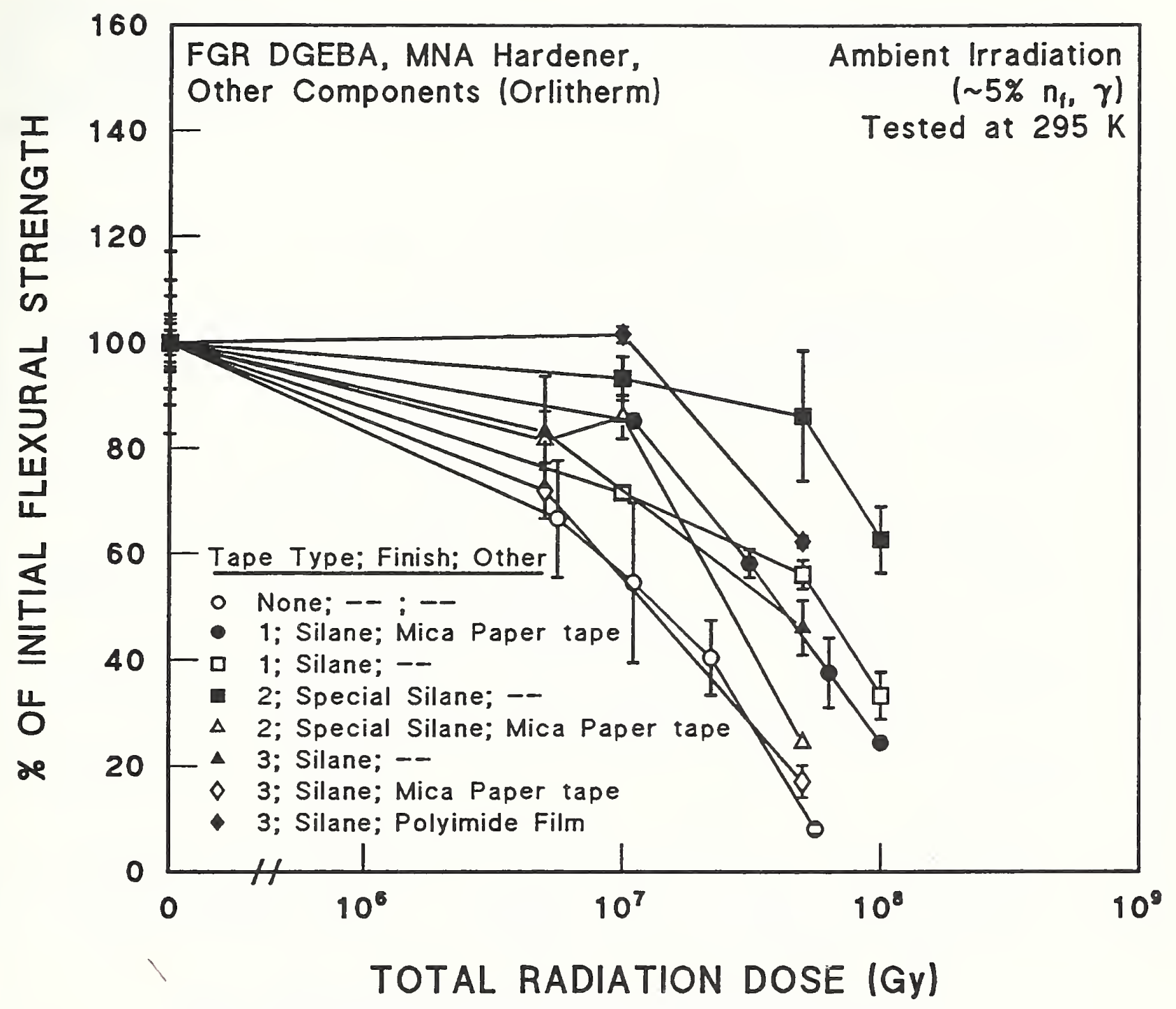

\section{"O" Strength, Mpa}

- $\quad 97.1 \pm 16.7$

- $224.6 \pm 11.7$

a $510.1 \pm 11.8$

- $450.3 \pm 24.5$

$\Delta 264.9 \pm 9.8$

$\triangle 563.1 \pm 25.5$

$\diamond 223.7 \pm 19.6$

- $379.6 \pm 44.7$

Cure Conditions
---
---
$12 \mathrm{~h}, 165^{\circ} \mathrm{C}$
$12 \mathrm{~h}, 165^{\circ} \mathrm{C}$
$5 \mathrm{~h}, 135^{\circ} \mathrm{C} ; 6 \mathrm{~h}, 160^{\circ} \mathrm{C}$
$5 \mathrm{~h}, 135^{\circ} \mathrm{C} ; 6 \mathrm{~h}, 160^{\circ} \mathrm{C}$
$5 \mathrm{~h}, 135^{\circ} \mathrm{C} ; 6 \mathrm{~h}, 160^{\circ} \mathrm{C}$
$16 \mathrm{~h}, 140^{\circ} \mathrm{C}$

Supplier

BBC Baden

BBC Baden

BBC Baden

BBC Baden

BBC Baden

BBC Baden

BBC Baden

BBC Baden

Figure A.2-25. Flexural strength at $295 \mathrm{~K}$ of FGR DGEBA resin, MNA hardener, mica paper tape (Orlitherm) after ambient reactor irradiation. Supplementary Table A.22. Data from Schönbacher and Stolarz-Iżycka [1979]. 


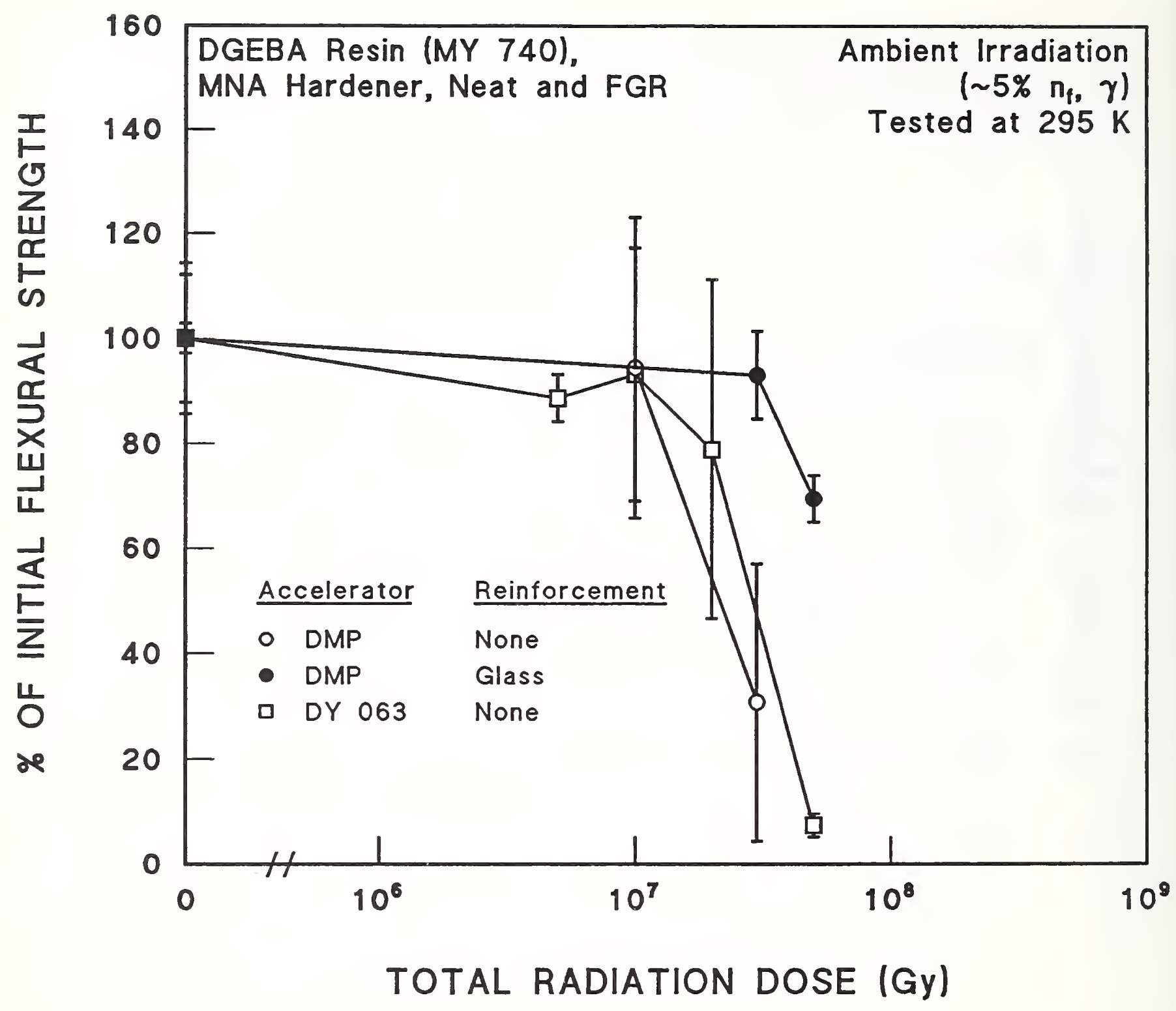

"O" Strength, MPa

- $\quad 89.3 \pm 10.8$

- $283.5 \pm 7.8$

ㅁ $130.5 \pm 18.6$
Supplier

Plessey

Plessey

Lintott

Figure A.2-26. Flexural strength at $295 \mathrm{~K}$ of neat and FGR DGEBA resin with MNA hardener after ambient reactor irradiation. Supplementary Table A.2-2. Data from Schönbacher and Stolarz-Izycka [1979]. 


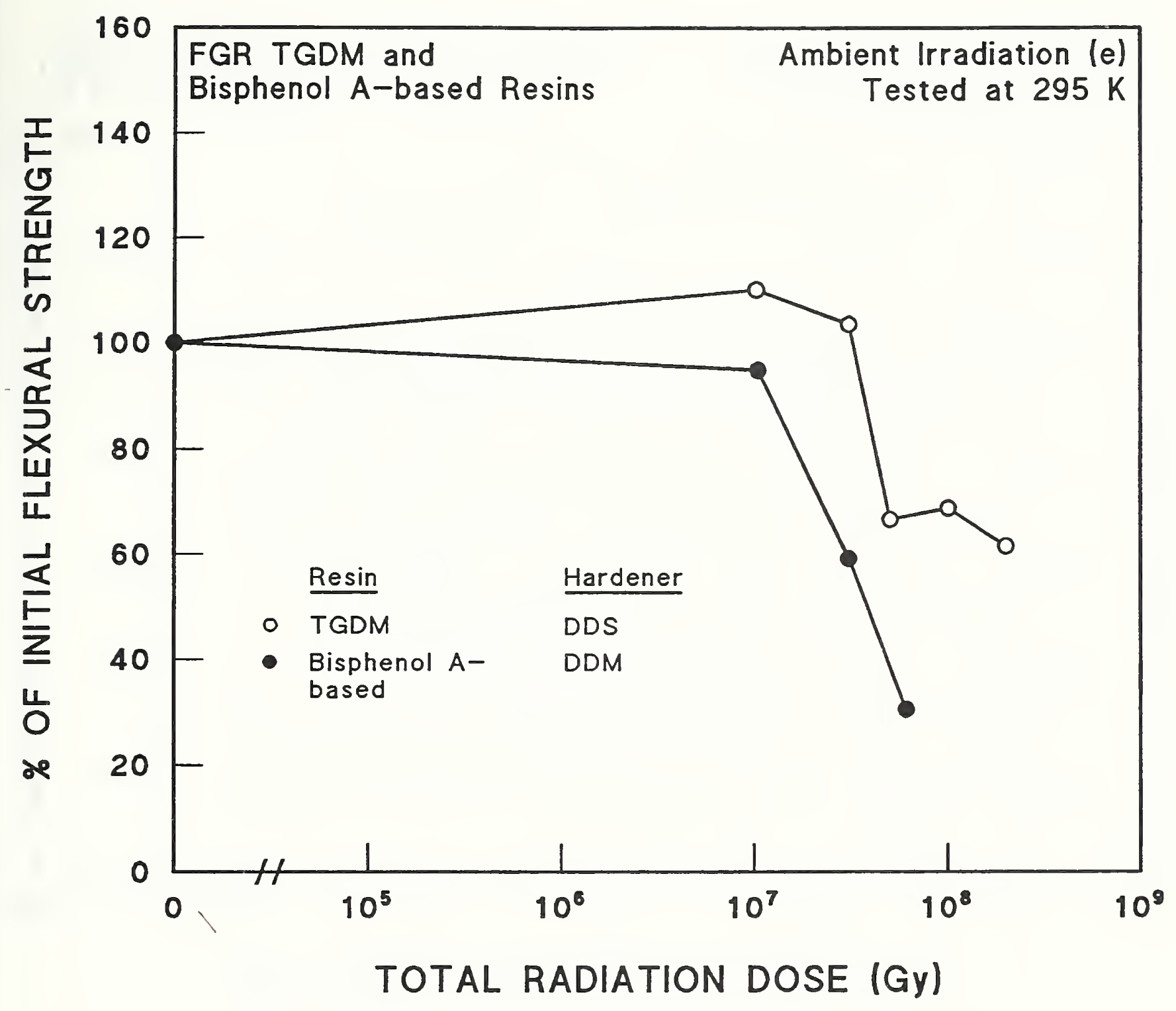

"O" Strength, MPa
o 696.6
$0 \quad 548.1$

Supplier

Sumitomo

Bakelite Co., Ltd.

--

Figure A.2-27. Flexural strength at $295 \mathrm{~K}$ of FGR TGDM and bisphenol A-based resins after ambient electron irradiation. Supplementary Table A.2-3. Data from Hagiwara et al. [1985]. 


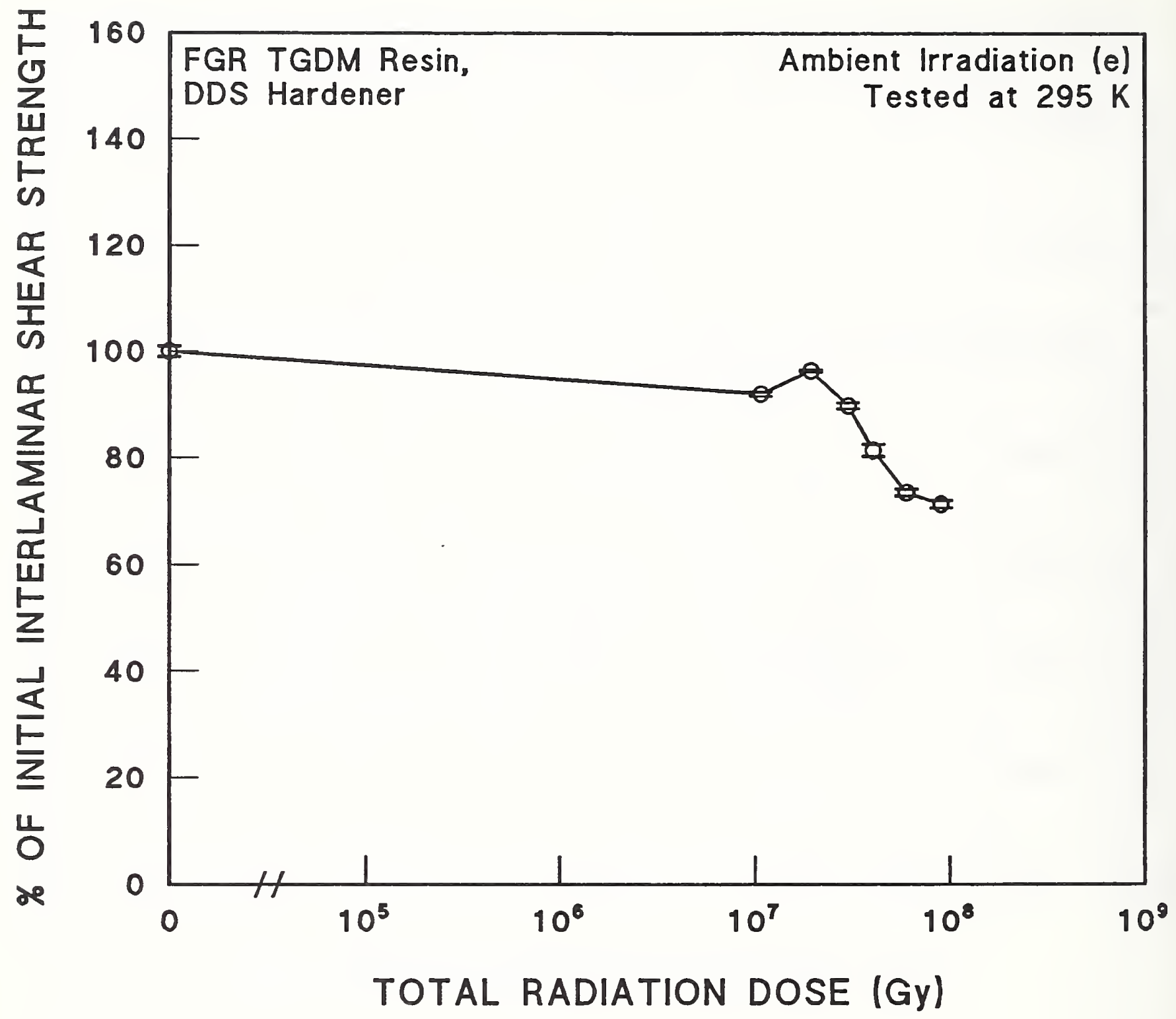

\begin{tabular}{llll} 
"O" Strength, MPa & Weave & Vol. Frac. & Supplier \\
\hline $59.8 \pm 1.6$ & Plain & $54-60 \%$ & $\begin{array}{l}\text { Sumitomo } \\
\text { Bakelite } \\
\text { Co., Ltd. }\end{array}$
\end{tabular}

Figure A.2-28. Interlaminar shear strength at $295 \mathrm{~K}$ of FGR TGDM resin, DDS hardener after ambient electron irradiation. Supplementary Table A.2-3. Data from Hagiwara et al. [1985]. 


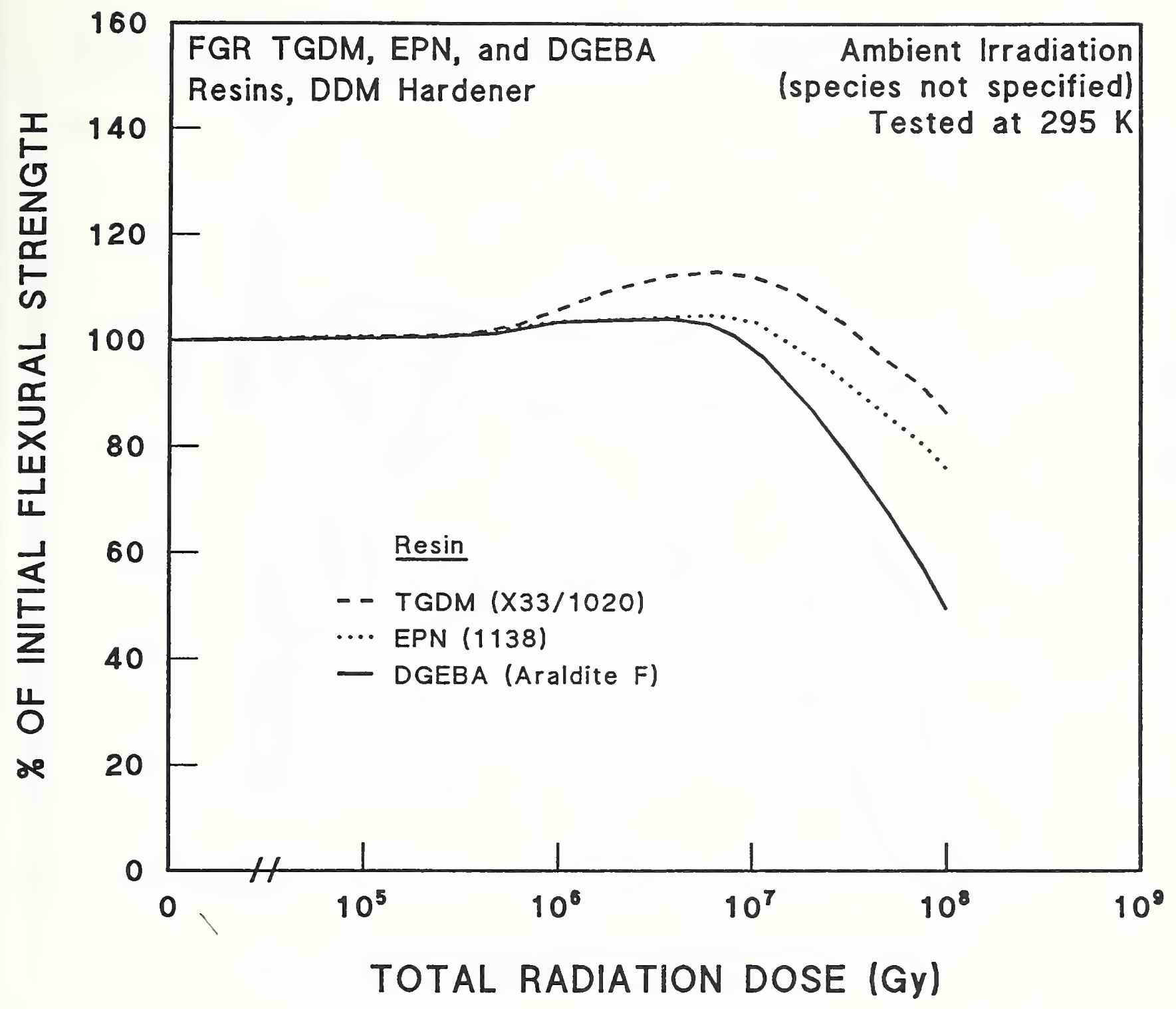

\begin{tabular}{ll} 
"O" Strength, MPa & Supplier \\
\hline-386.4 & -- \\
$\ldots 357.0$ & Ciba-Geigy \\
-383.3 & Ciba-Geigy
\end{tabular}

Figure A.2-29. Flexural strength at $295 \mathrm{~K}$ of FGR TGDM, EPN, and DGEBA resins, DDM hardener after ambient irradiation. No supplementary information. Data from Van de Voorde and Restat [1972]. 


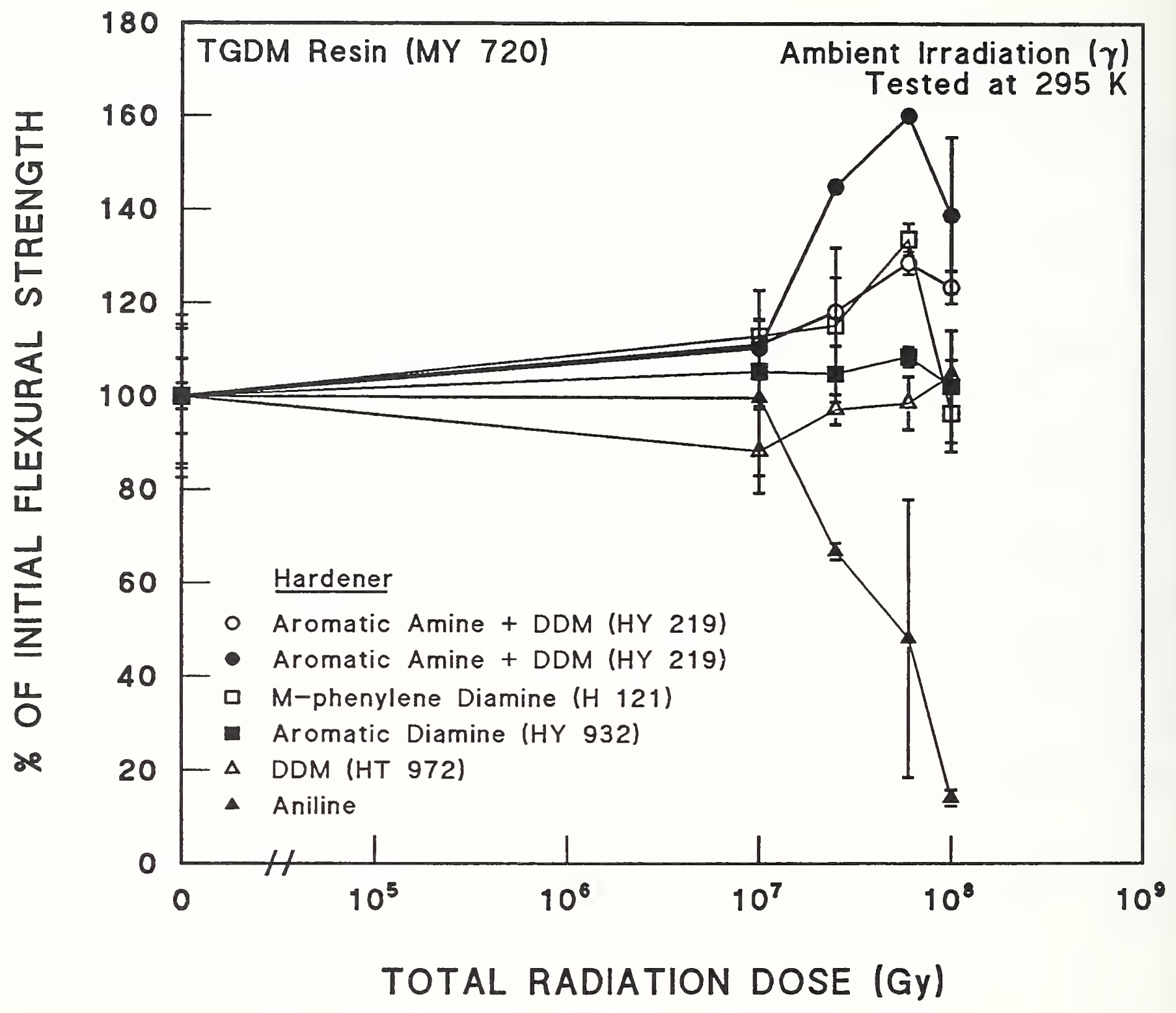

"O" Strength, MPa

- $109.9 \pm 1.3$

- $\quad 87.8 \pm 7.1$

ㅁ $126.6 \pm 22.0$

- $152.0 \pm 22.0$

$\Delta 160.4 \pm 4.5$

$\Delta \quad 162.1 \pm 25.0$
Addl. Hardener
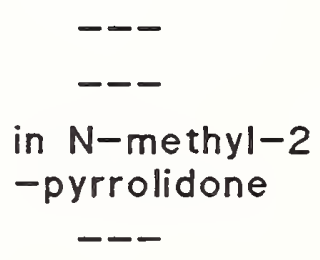

Accelerator

DY 219

- -

$--$

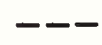

- -
Supplier

Ciba-Geigy

Ciba-Geigy

Ciba-Geigy

Ciba-Geigy

Ciba-Geigy

Ciba-Geigy

Figure A.2-30. Flexural strength at $295 \mathrm{~K}$ of neat TGDM resin with several hardeners after ambient gamma irradiation. Supplementary Table A.2-1. Data from Morgan and Stapleton [1975]. 


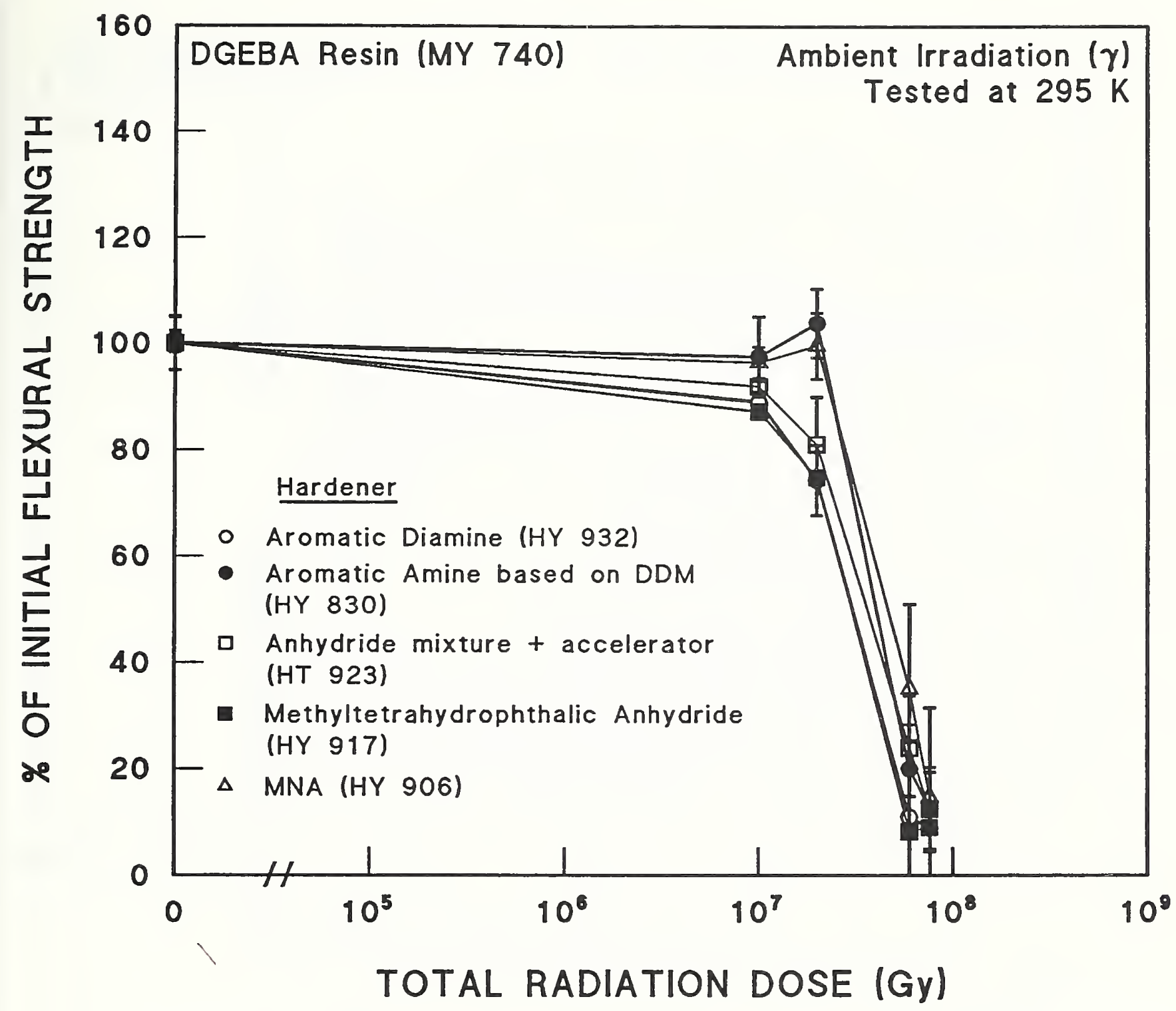

"O" Strength, MPa

- $124.5 \pm 2.5$

- $120.4 \pm 2.0$

ㅁ $147.1 \pm 3.4$

- $141.4 \pm 6.9$

$\Delta \quad 129.1 \pm 6.6$
Accelerator

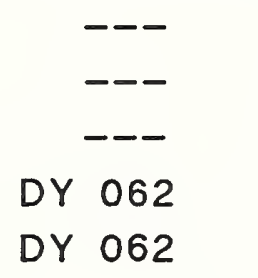

Supplier

Ciba-Geigy

Ciba-Geigy

Ciba-Geigy

Ciba-Geigy

Ciba-Geigy

Figure A.2-31. Flexural strength at $295 \mathrm{~K}$ of neat DGEBA resin with several hardeners after ambient gamma irradiation. Supplementary Table A.2-1. Data from Morgan and Stapleton [1975]. 


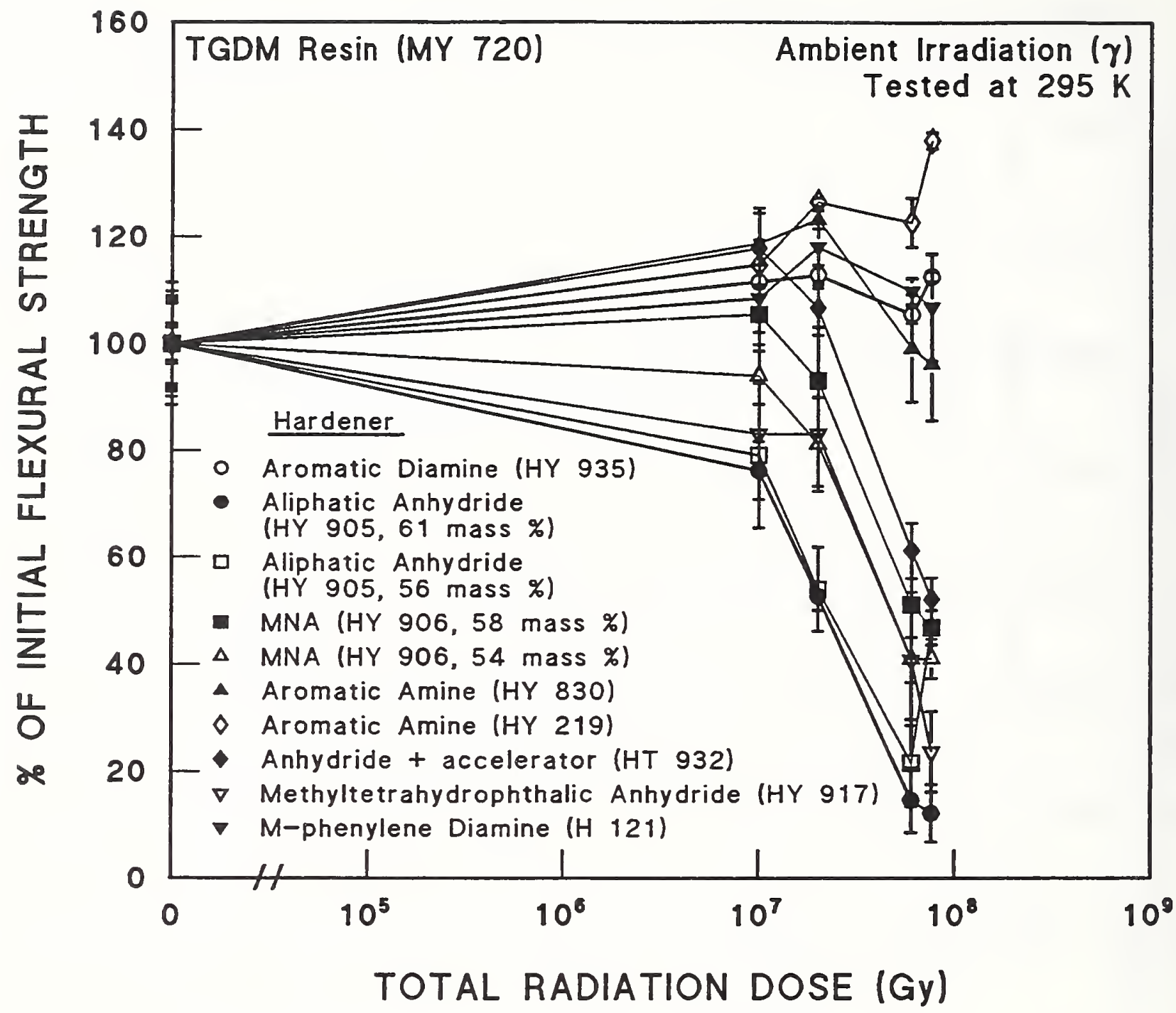

\begin{tabular}{|c|c|c|c|}
\hline "O" Strength, MPa & Addl. Hardener & Accelerator & Supplier \\
\hline - $132.6 \pm 11.3$ & --- & --- & Ciba-Geigy \\
\hline - $140.2 \pm 10.5$ & --- & DY 062 & Ciba-Geigy \\
\hline ㅁ $136.4 \pm 15.6$ & -- & DY 062 & Ciba-Geigy \\
\hline$=111.4 \pm 9.0$ & -- & DY 062 & Ciba-Geigy \\
\hline$\Delta \quad 139.8 \pm 12.4$ & -- & DY 062 & Ciba-Geigy \\
\hline$\triangle 109.3 \pm 0.8$ & Based on DDM & -- & Ciba-Geigy \\
\hline $97.8 \pm 3.0$ & Contains DDM & DY 219 & Ciba-Geigy \\
\hline$-123.4 \pm 12.1$ & -- & --- & Ciba-Geigy \\
\hline $\begin{array}{ll}\nabla & 124.7 \pm 9.9\end{array}$ & -- & --- & Ciba-Geigy \\
\hline$\nabla \quad 134.2 \pm 5.0$ & $\begin{array}{l}\text { in } N \text {-methyl-2 } \\
\text {-pyrrolidone }\end{array}$ & --- & Ciba-Geigy \\
\hline
\end{tabular}

Figure A.2-32. Flexural strength at $295 \mathrm{~K}$ of neat TGDM resin with several hardeners after ambient gamma irradiation. Supplementary Table A.2-1. Data from Morgan and Stapleton [1975]. 


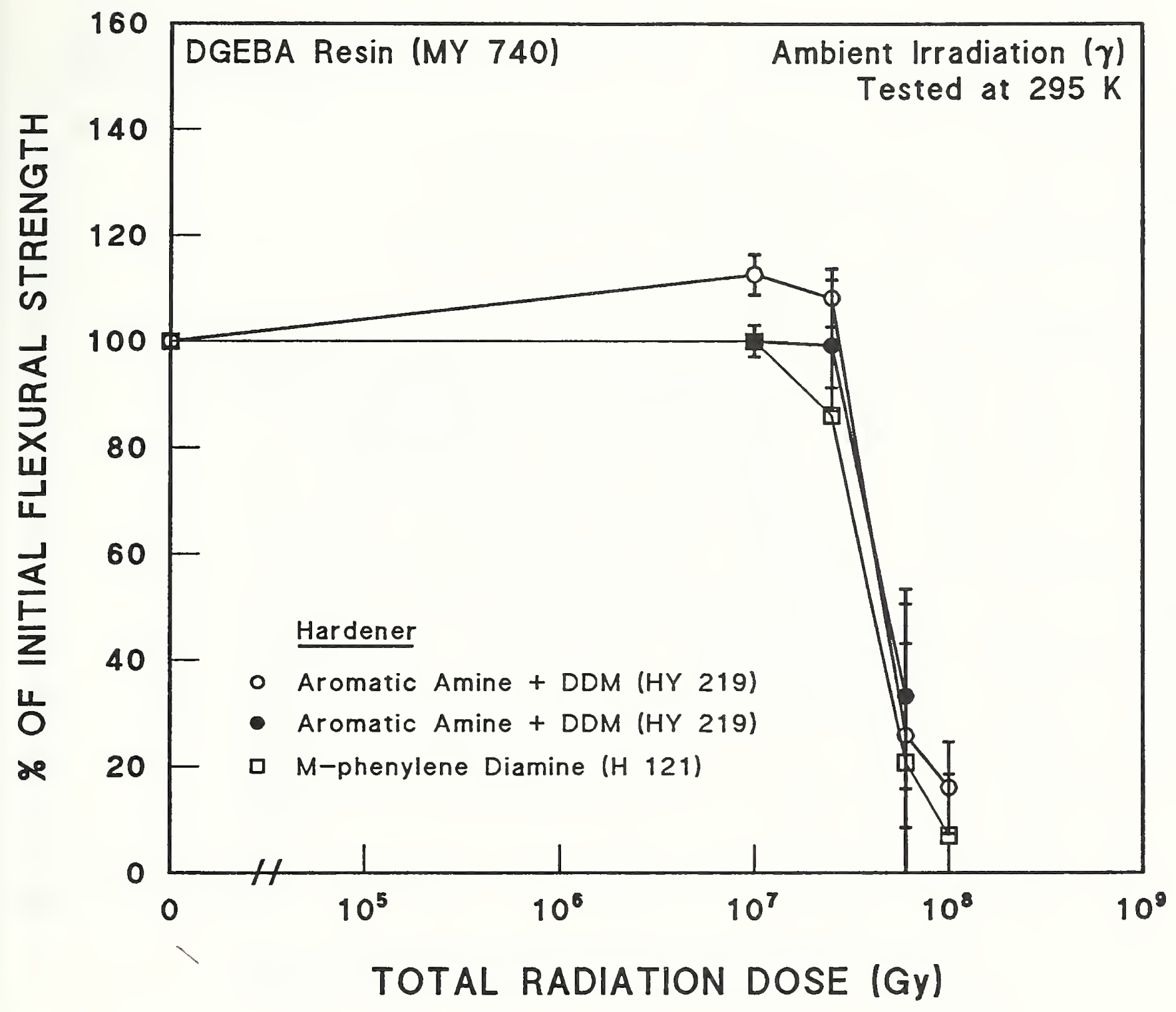

\begin{tabular}{|c|c|c|c|}
\hline "O" Strength, MPa & Addl. Hardener & Accelerator & Supplier \\
\hline o $102.9 \pm 1.2$ & --- & DY 219 & Ciba-Geis \\
\hline - $\quad 116.2 \pm 3.5$ & -- & --- & Ciba-Geigy \\
\hline ㅁ $159.5 \pm 1.9$ & $\begin{array}{l}\text { in } \mathrm{N} \text {-methyl-2 } \\
\text {-pyrrolidone }\end{array}$ & -- & Ciba-Geig! \\
\hline
\end{tabular}

* value at $1 \times 10^{7}$ Gy dose (no "O" dose given)

Figure A.2-33. Flexural strength at $295 \mathrm{~K}$ of neat DGEBA resin with several hardeners after ambient gamma irradiation. Supplementary Table A.2-1. Data from Morgan and Stapleton [1975]. 


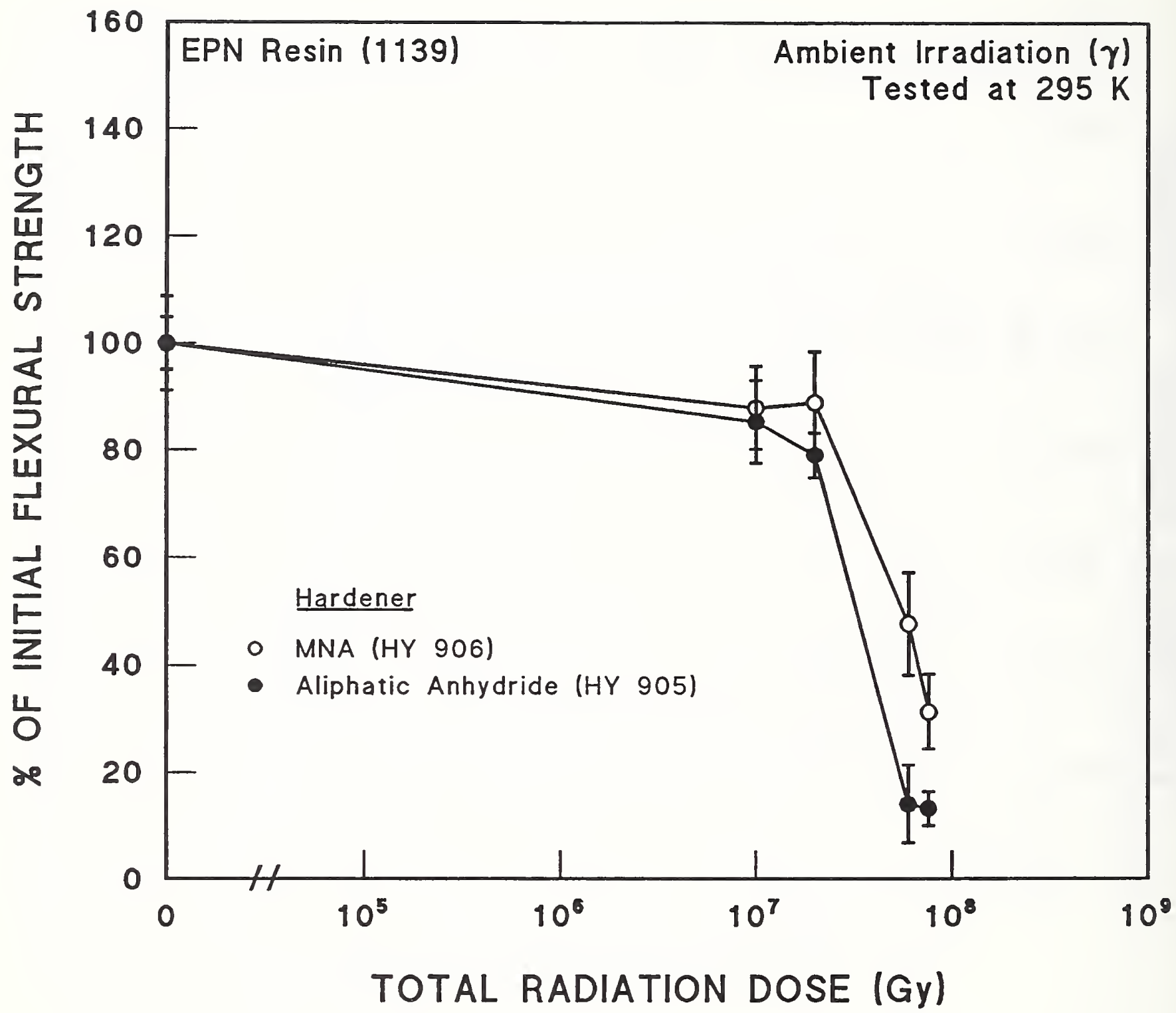

"O" Strength, MPa

- $138.1 \pm 12.2$

- $158.4 \pm 7.8$
Accelerator

DY 062

DY 062
Supplier

Ciba-Geigy

Ciba-Geigy

Figure A.2-34. Flexural strength at $295 \mathrm{~K}$ of neat EPN resin with anhydride hardeners after ambient gamma irradiation. Supplementary Table A.2-1. Data from Morgan and Stapleton [1975]. 


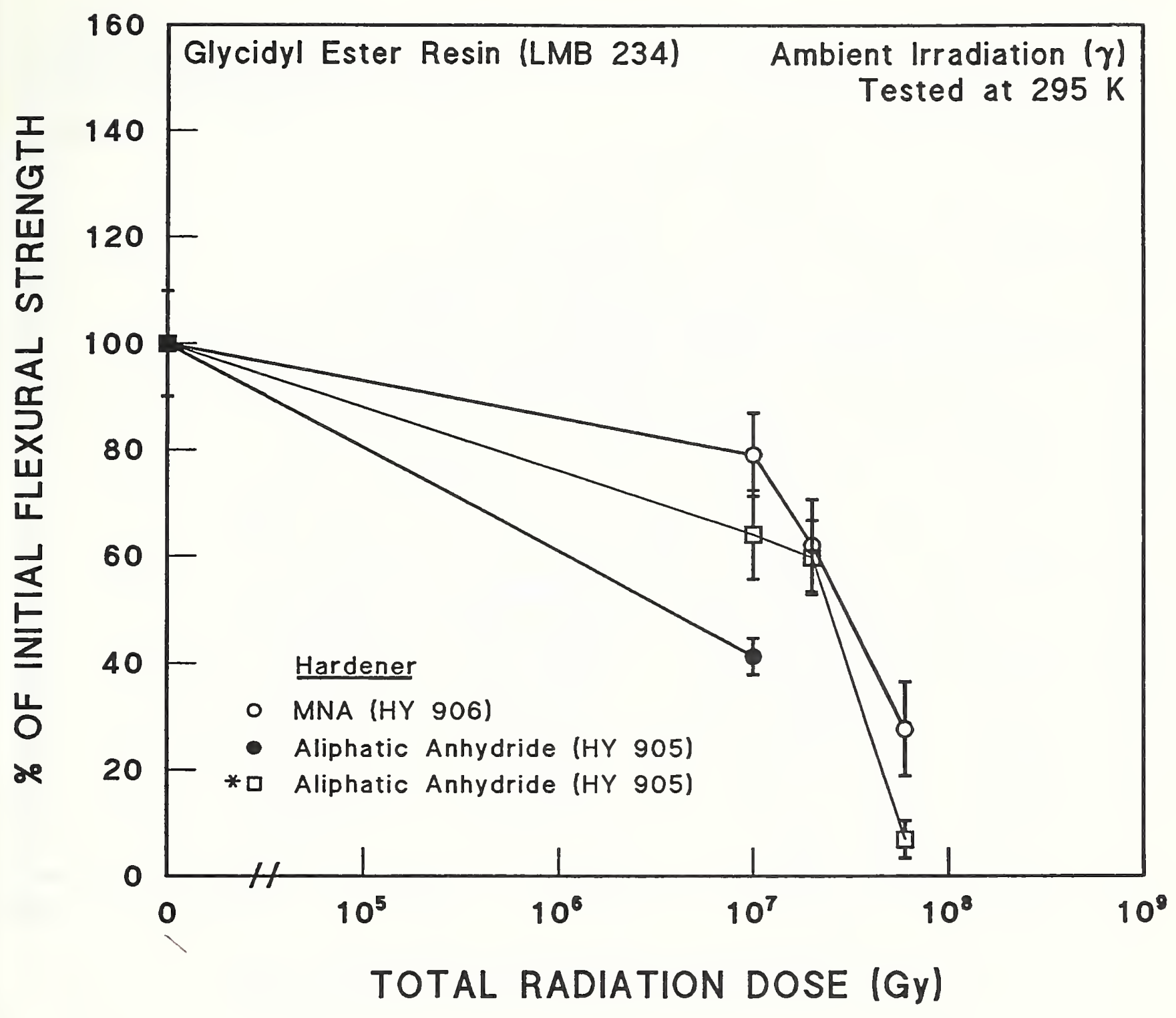

$\begin{array}{lll}\text { "O" Strength, MPa } & \text { Accelerator } & \text { Supplier } \\ 0174.8 \pm 1.7 & \text { DY } 062 & \text { Ciba-Geigy } \\ \text { - } 159.9 \pm 0.5 & \text { DY } 062 & \text { Ciba-Geigy } \\ \text { Ciba-Geigy } & \text { DY } 062 & \\ \text { * Resin is } 50 \text { mass \% LMB 234, and } 50 \text { mass \% MY } 720\end{array}$

Figure A.2-35. Flexural strength at $295 \mathrm{~K}$ of neat glycidyl ester resin with anhydride hardeners after ambient gamma irradiation. Supplementary Table A.2-1. Data from Morgan and Stapleton [1975]. 


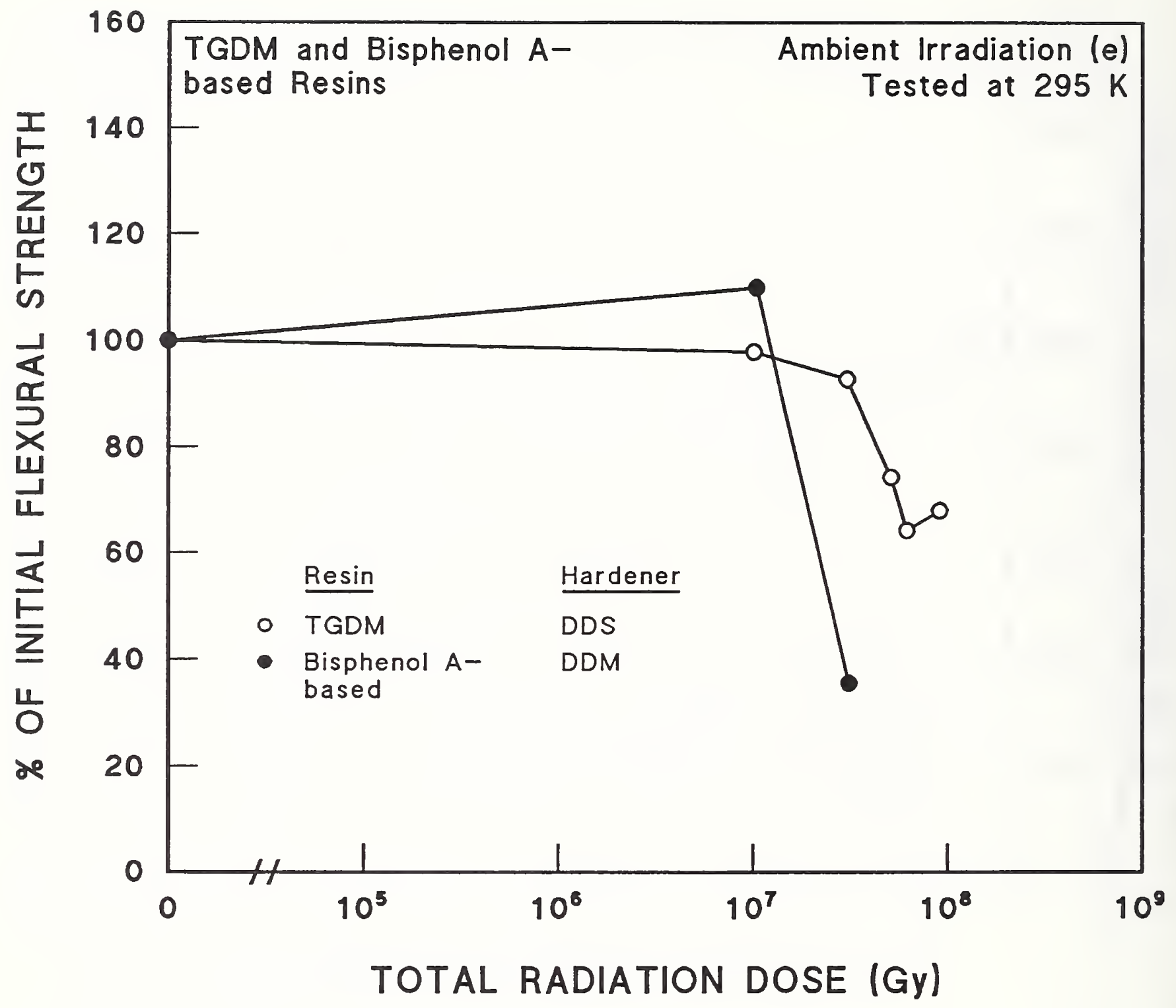

"O" Strength, MPa

- 127.1

- 109.8
Supplier

Sumitomo

Bakelite Co., Ltd.

Figure A.2-36. Flexural strength at $295 \mathrm{~K}$ of neat TGDM and bisphenol A-based resins after ambient electron irradiation. Supplementary Table A.2-3. Data from Hagiwara et al. [1985]. 


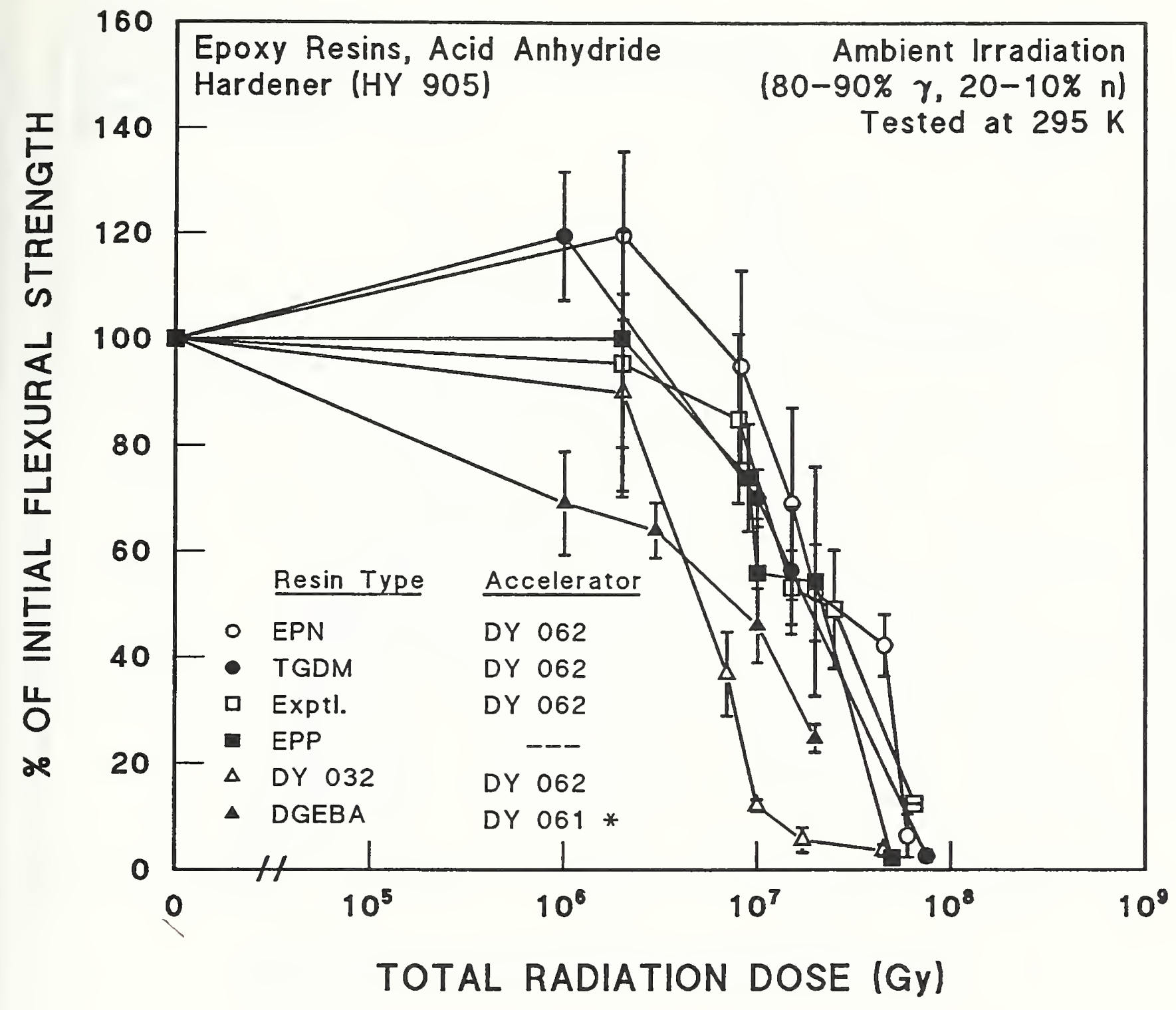

"O" Strength, MPa

- 98.1

- 73.6

ㅁ 103.0

- 83.4

$\Delta \quad 73.6$

$\Delta \quad 153.0$
Resin Name

EPN 1138

$\times 33 / 1020$

$\times 33 / 1189$

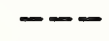

- -

Araldite F
Supplier

Ciba-Geigy

Ciba-Geigy

Ciba-Geigy

Ciba-Geigy

Ciba-Geigy

Ciba-Geigy

* + DY 040 (flexibilizer)

Figure A.2-37. Flexural strength at $295 \mathrm{~K}$ of neat epoxy resins with anhydride hardener after ambient reactor irradiation. Supplementary Table A.2-4. Data from Pluym and Van de Voorde [1967] and Van de Voorde and Restat [1972]. 


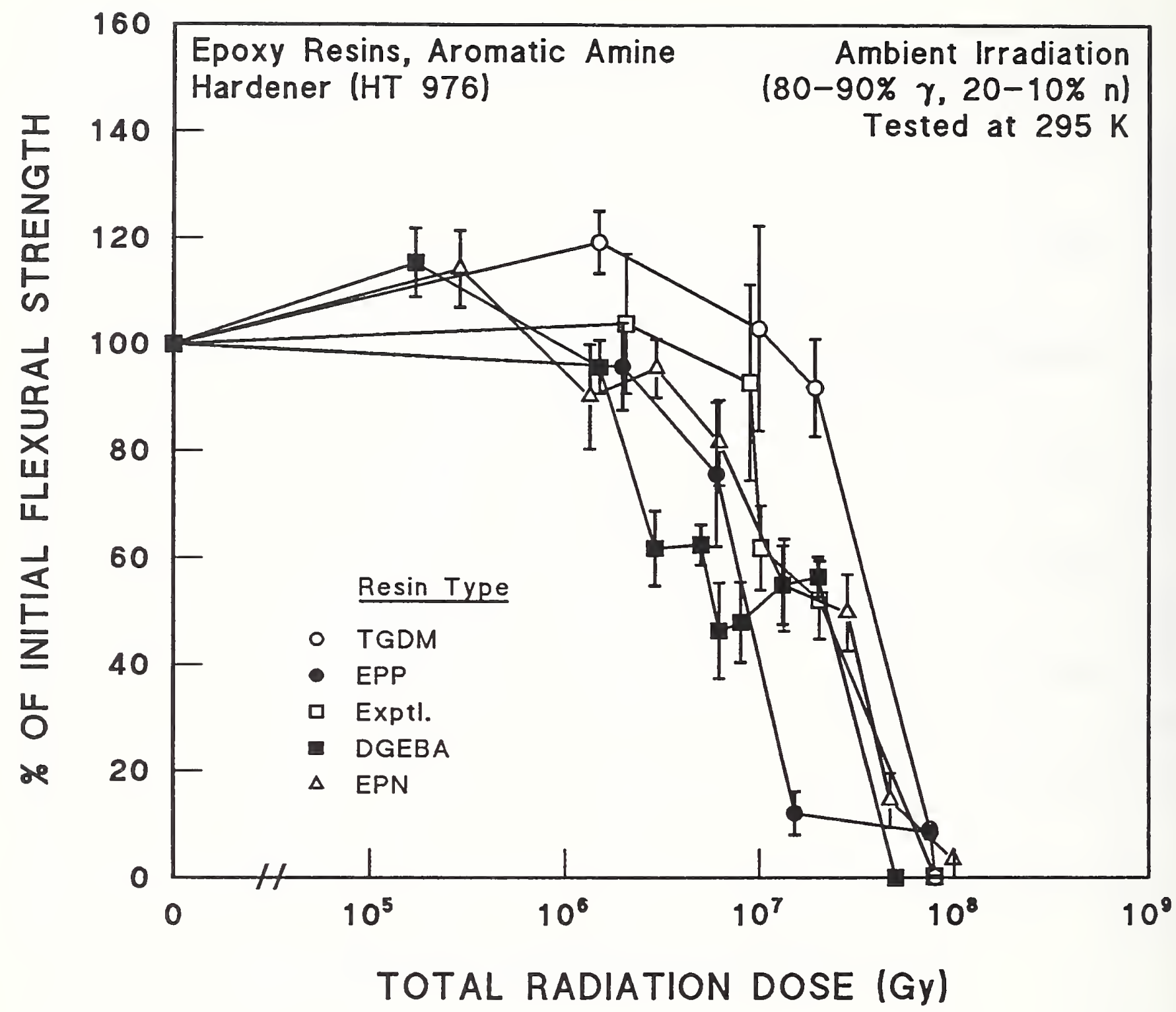

Resin Name
$\times 33 / 1020$
--
$\times 33 / 1189$
Araldite F
EPN 1138

Supplier

Ciba-Geigy

Ciba-Geigy

Ciba-Geigy

Ciba-Geigy

Ciba-Geigy

Figure A.2-38. Flexural strength at $295 \mathrm{~K}$ of neat epoxy resins with aromatic amine hardener after ambient reactor irradiation. Supplementary Table A.2-4. Data from Pluym and Van de Voorde [1967] and Van de Voorde and Restat [1972]. 


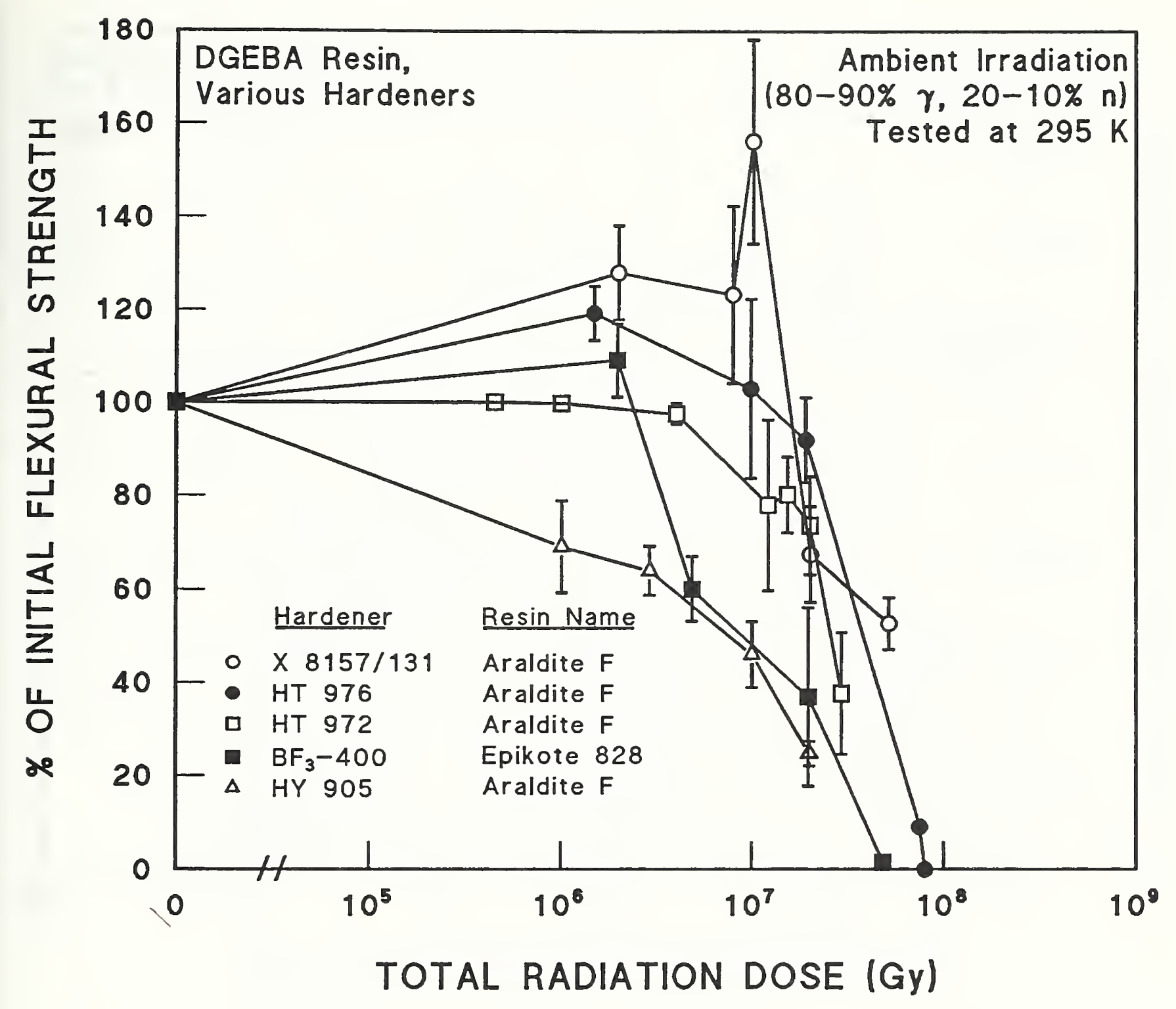

\begin{tabular}{|c|c|c|c|}
\hline "O" & Strength, MPa & Other & Supplier \\
\hline 0 & 98.1 & --- & Ciba-Geigy \\
\hline & 89.2 & --- & Ciba-Geigy \\
\hline$\square$ & 166.7 & --- & Ciba-Geigy \\
\hline (a & 120.6 & -- & Shell \\
\hline & 153.0 & $\begin{array}{l}\text { DY } 061 \text { (accelerator) } \\
\text { DY } 040 \text { (flexibilizer) }\end{array}$ & Ciba-Geigy \\
\hline
\end{tabular}

Figure A.2-39. Flexural strength at $295 \mathrm{~K}$ of neat DGEBA resin with several hardeners after ambient reactor irradiation. Supplementary Table A.2-4. Data from Pluym and Van de Voorde [1967] and Van de Voorde and Restat [1972]. 


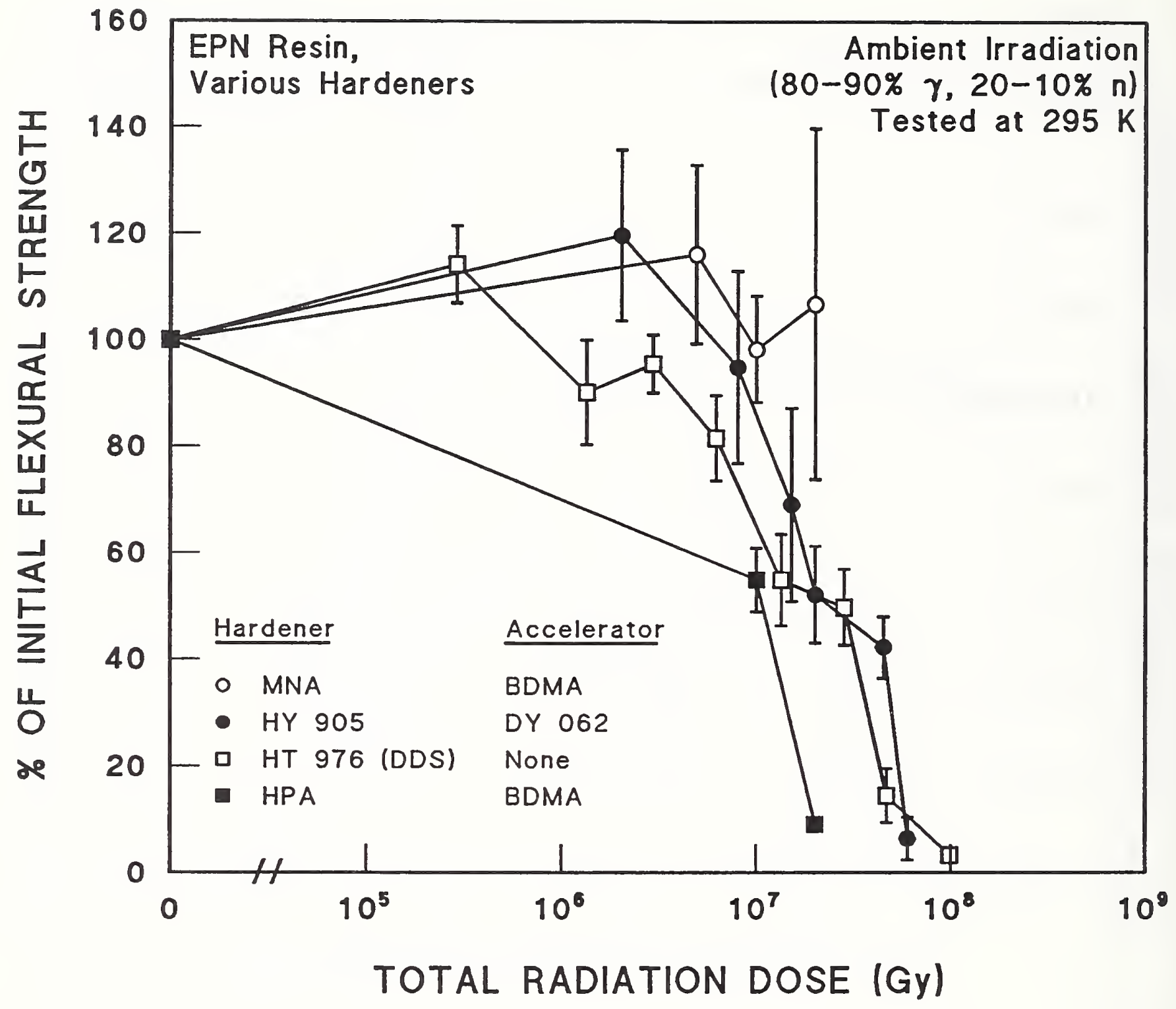

Resin Name
Epikote 154
EPN 1138
EPN 1138
Epikote 154

Supplier

Shell

Ciba-Geigy

Ciba-Geigy

Shell

Figure A.2-40. Flexural strength at $295 \mathrm{~K}$ of neat EPN resin with several hardeners after ambient reactor irradiation. Supplementary Table A.2-4. Data from Pluym and Van de Voorde [1967] and Van de Voorde and Restat [1972]. 


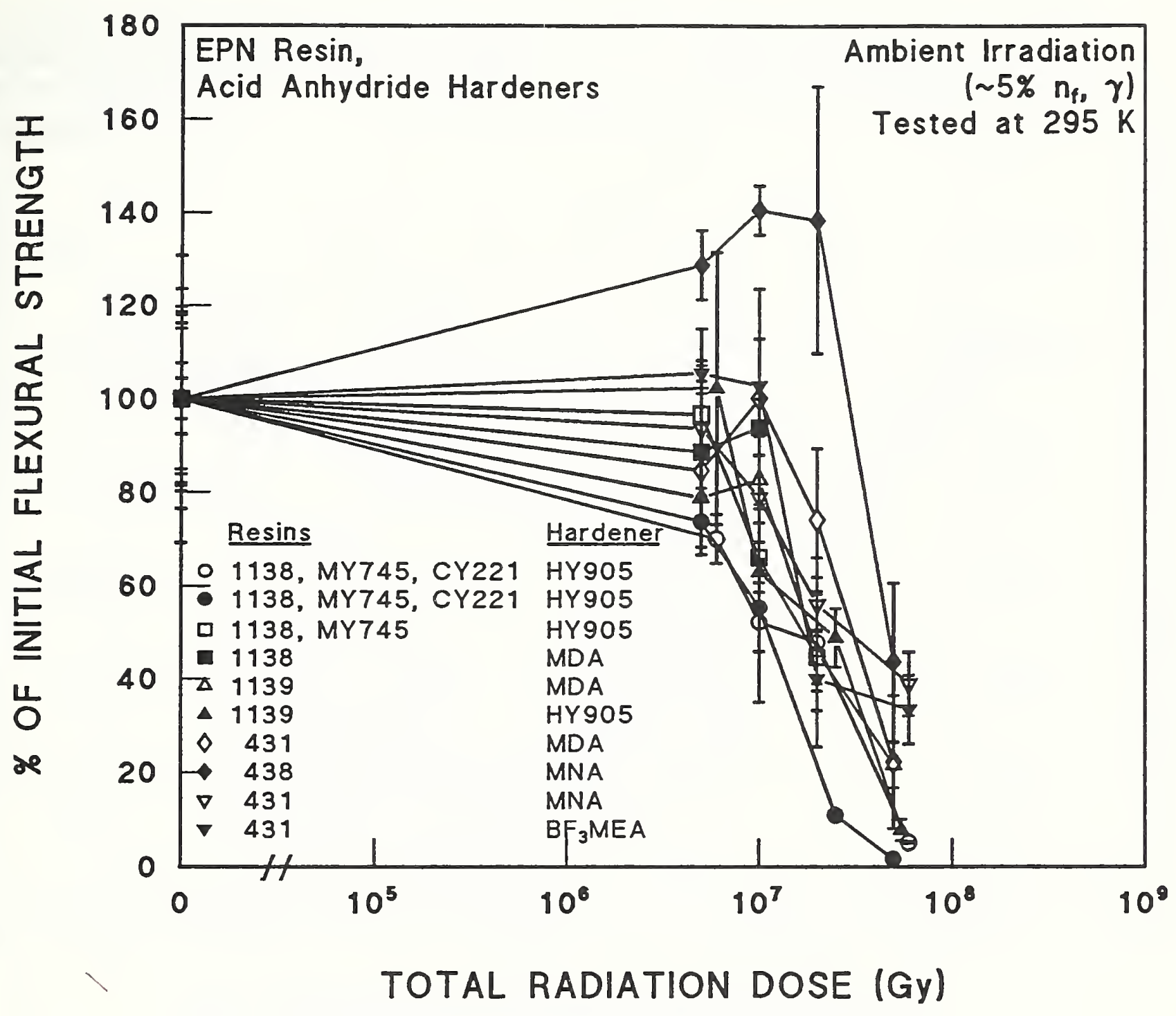

Other Components
Accelerator, DY 063
XB 2687
XB 2687
Accelerator, DY 062
X157/2505
XB 2687
---
---
Accelerator
-- -

Supplier

Als thom

Ciba-Geigy

Alsthom

Ciba-Geigy

Alsthom

Alsthom

DOW

DOW

Isola

Isola

Figure A.2-41. Flexural strength at $295 \mathrm{~K}$ of neat EPN resin with anhydride hardeners after ambient reactor irradiation. Supplementary Table A.2-4. Data from Pluym and Van de Voorde [1967]. 


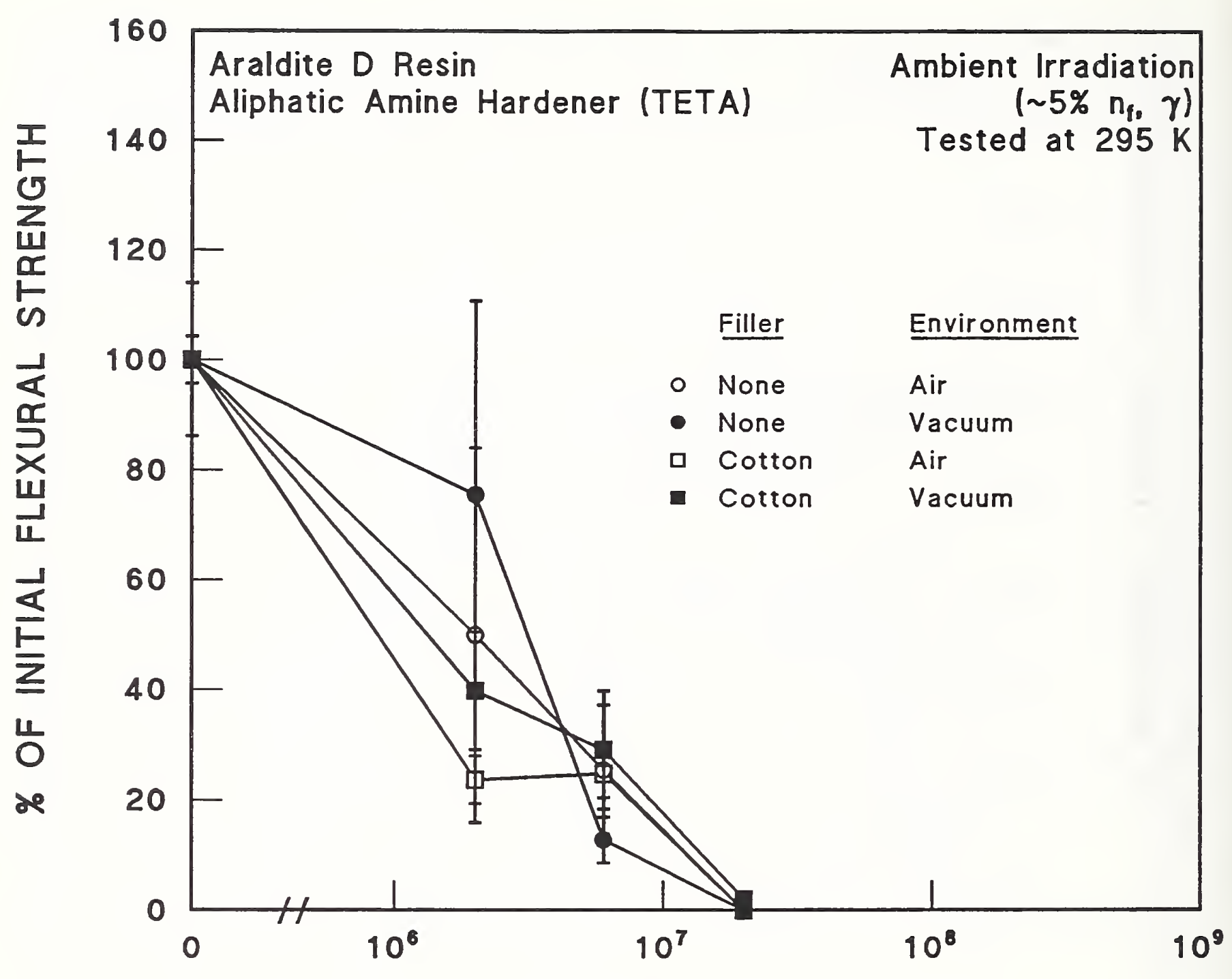

TOTAL RADIATION DOSE (Gy)

"O" Strength, MPa

$\circ \quad 92.2 \pm 12.8$
$-\quad 92.2 \pm 12.8$
口 $91.2 \pm 3.9$
- $91.2 \pm 3.9$

Cure Conditions

Ambient Temp.

Ambient Temp.

Ambient Temp.

Ambient Temp.

\section{Supplier}

SIN

SIN

SIN

SIN

Comments: Samples $(0, \bullet, \square)$ broke after irradiation at doses of $2 \times 10^{7}$ Gy.

Figure A.2-42. Flexural strength at $295 \mathrm{~K}$ of neat Araldite $\mathrm{D}$ resin with TETA hardener after ambient reactor irradiation. Supplementary Table A.2-2. Data from Schönbacher and Stolarz-Iżycka [1979]. 


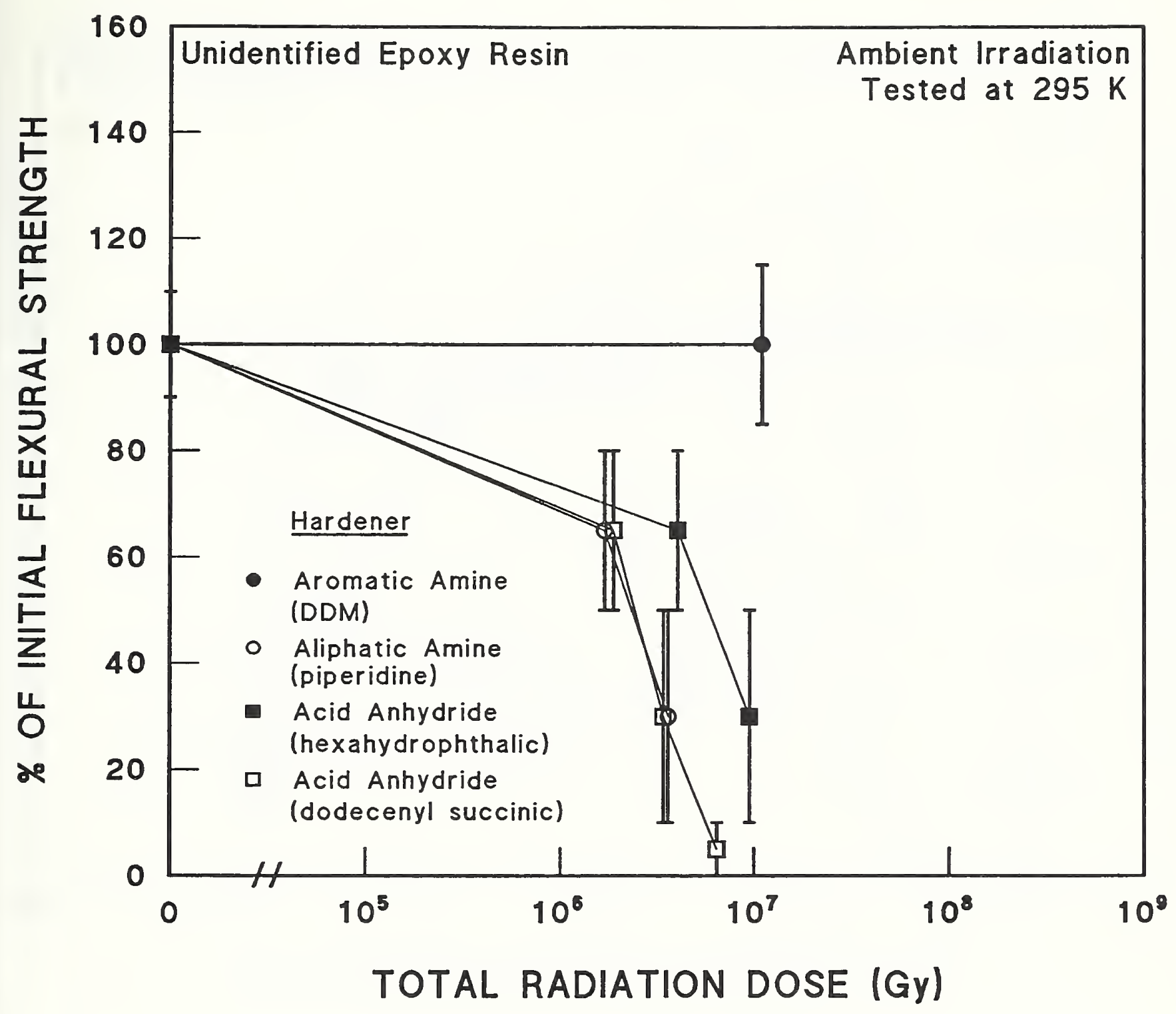

$$
\begin{array}{lr}
\text { "O" Strength, MPa } \\
\hline- & 117.2 \pm 10.0 \\
0 & 127.6 \pm 10.0 \\
\text { a } & 124.1 \pm 10.0 \\
\text { } & 79.3 \pm 10.0
\end{array}
$$

Figure A.2-43. Flexural strength at $295 \mathrm{~K}$ of neat unidentified resin with several hardeners after ambient irradiation. No supplementary information. Data from Parkinson and Sisman [1971]. 


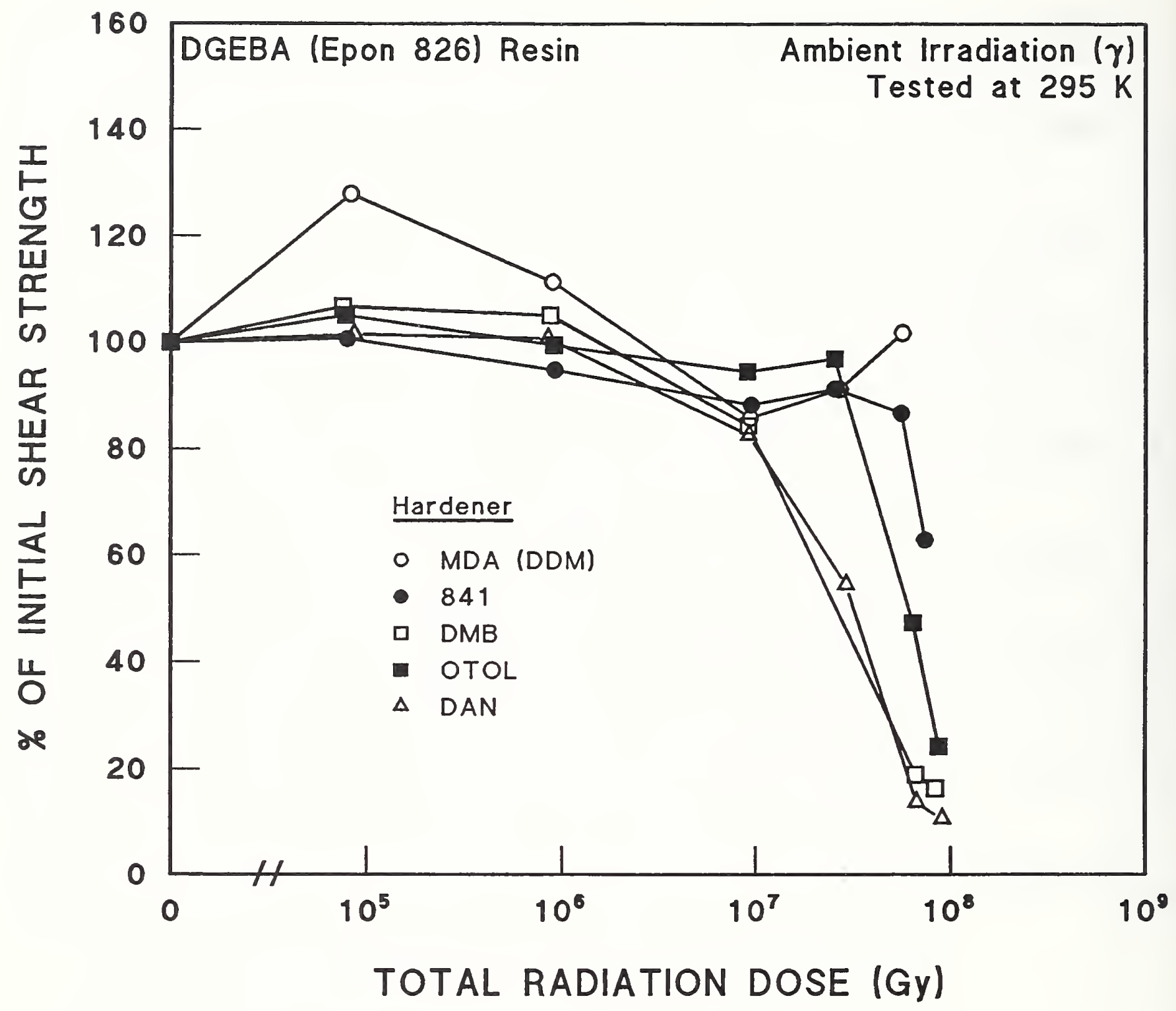

"O" Strength, MPa

- 62.1

- 72.3

ㅁ 70.9

- 64.8

$\triangle \quad 64.1$
Supplier

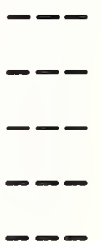

Figure A.2-44. Shear strength at $295 \mathrm{~K}$ of neat DGEBA resin with several hardeners after ambient gamma irradiation. Supplementary Table A.2-5. Data from Markley et al. [1969]. 


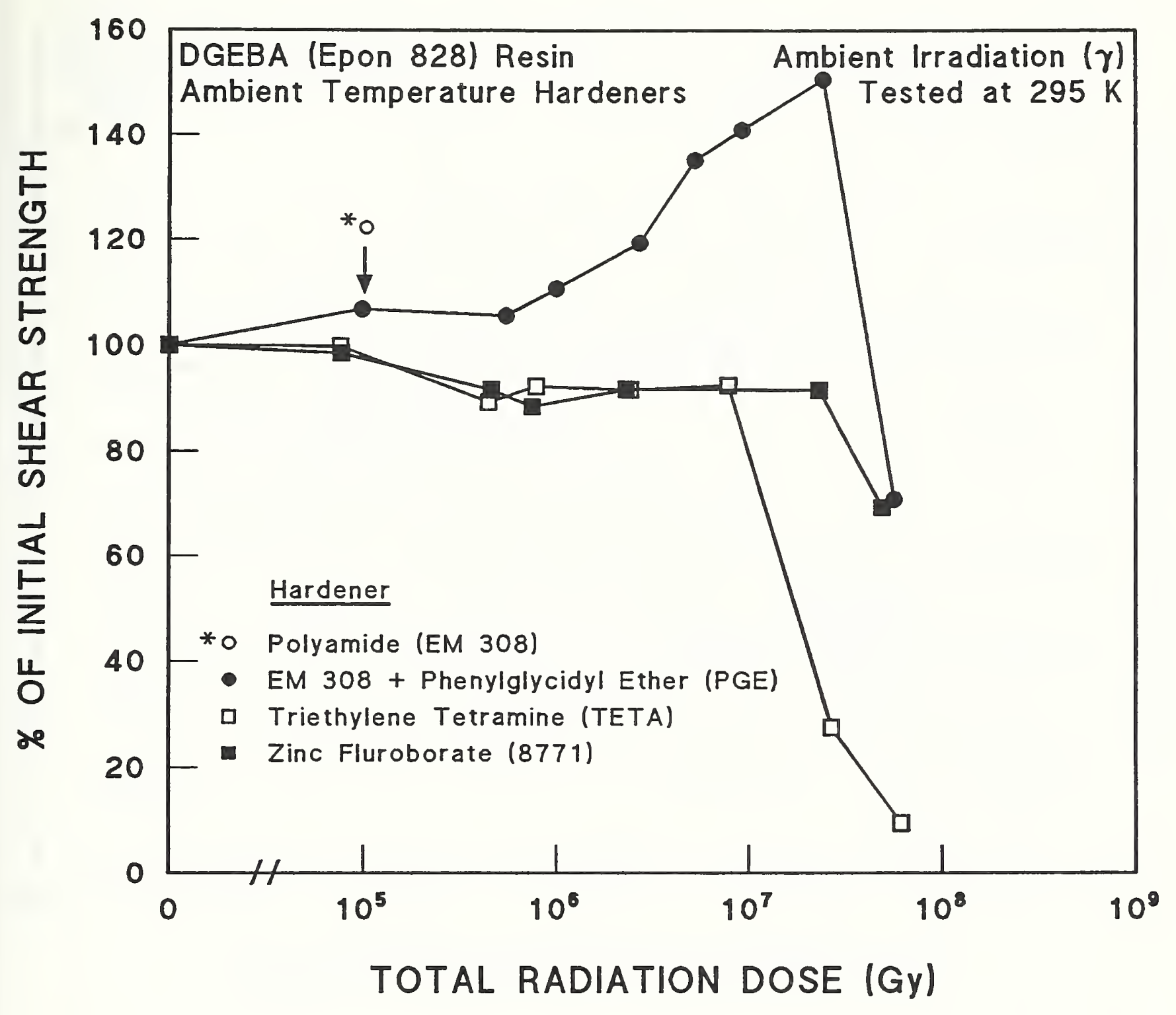

\begin{aligned} & "O" Strength, MPa Supplier \\ & \hline * 36.4-- \\ & - 36.4-- \\ & a 65.6-- \\ & - The $\quad 55.1-- \\ &$ * points overlap the 0 points \end{aligned}

Figure A.2-45. Shear strength at $295 \mathrm{~K}$ of neat DGEBA resin with ambient temperature hardeners after ambient gamma irradiation. Supplementary Table A.2-5. Data from Markley et al. [1969]. 


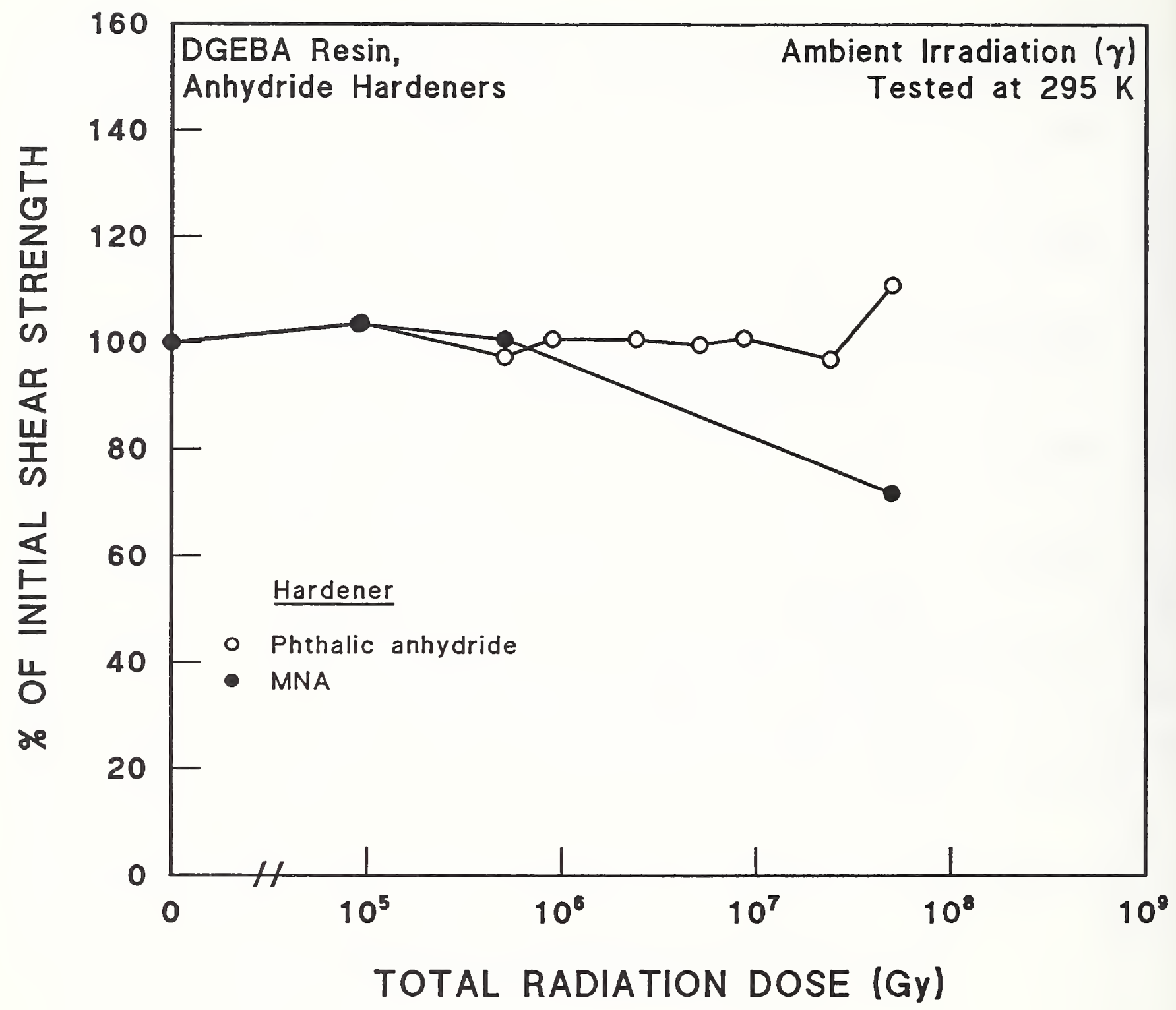

\begin{tabular}{|c|c|c|}
\hline "O" Strength, MPa & Resin Name & Supplier \\
\hline 61.0 & Epon 1001 & -- \\
\hline - $\quad 65.1$ & Epon 828 & --- \\
\hline
\end{tabular}

Figure A.2-46. Shear strength at $295 \mathrm{~K}$ of neat DGEBA resin with anhydride hardeners after ambient gamma irradiation. Supplementary Table A.2-5. Data from Markley et al. [1969]. 


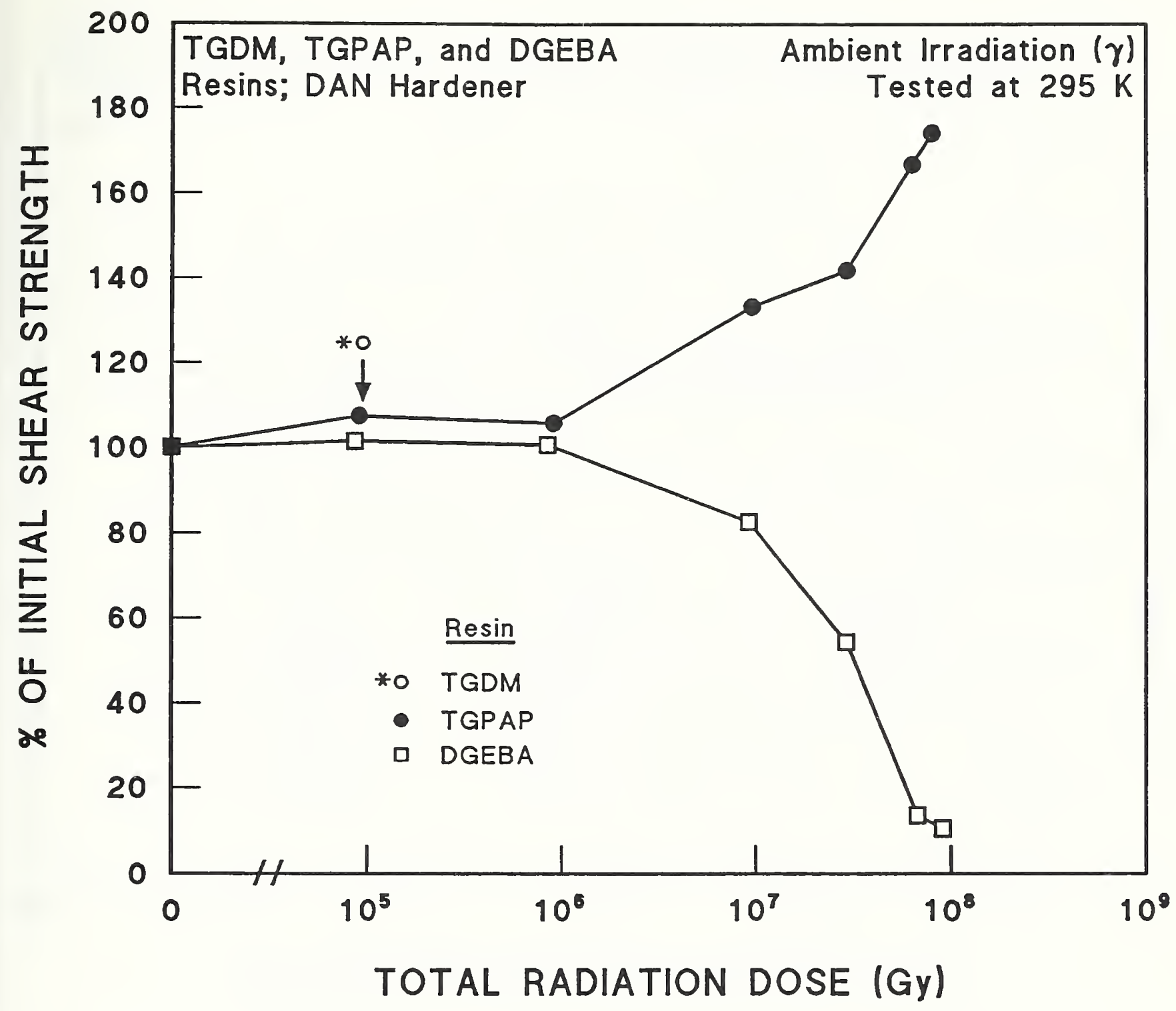

\begin{tabular}{llll} 
"O" Strength, MPa & & Resin Name & Supplier \\
\hline * 37.0 & & X8183/137 & -- \\
- 37.0 & ERL 0510 & -- \\
口 64.1 & Epon 826 & ---
\end{tabular}

* The - points overlap the 0 points

Figure A.2-47. Shear strength at $295 \mathrm{~K}$ of neat TGDM, TGPAP, and DGEBA resins with DAN hardener after ambient gamma irradiation. Supplementary Table A.2-5. Data from Markley et al. [1969]. 


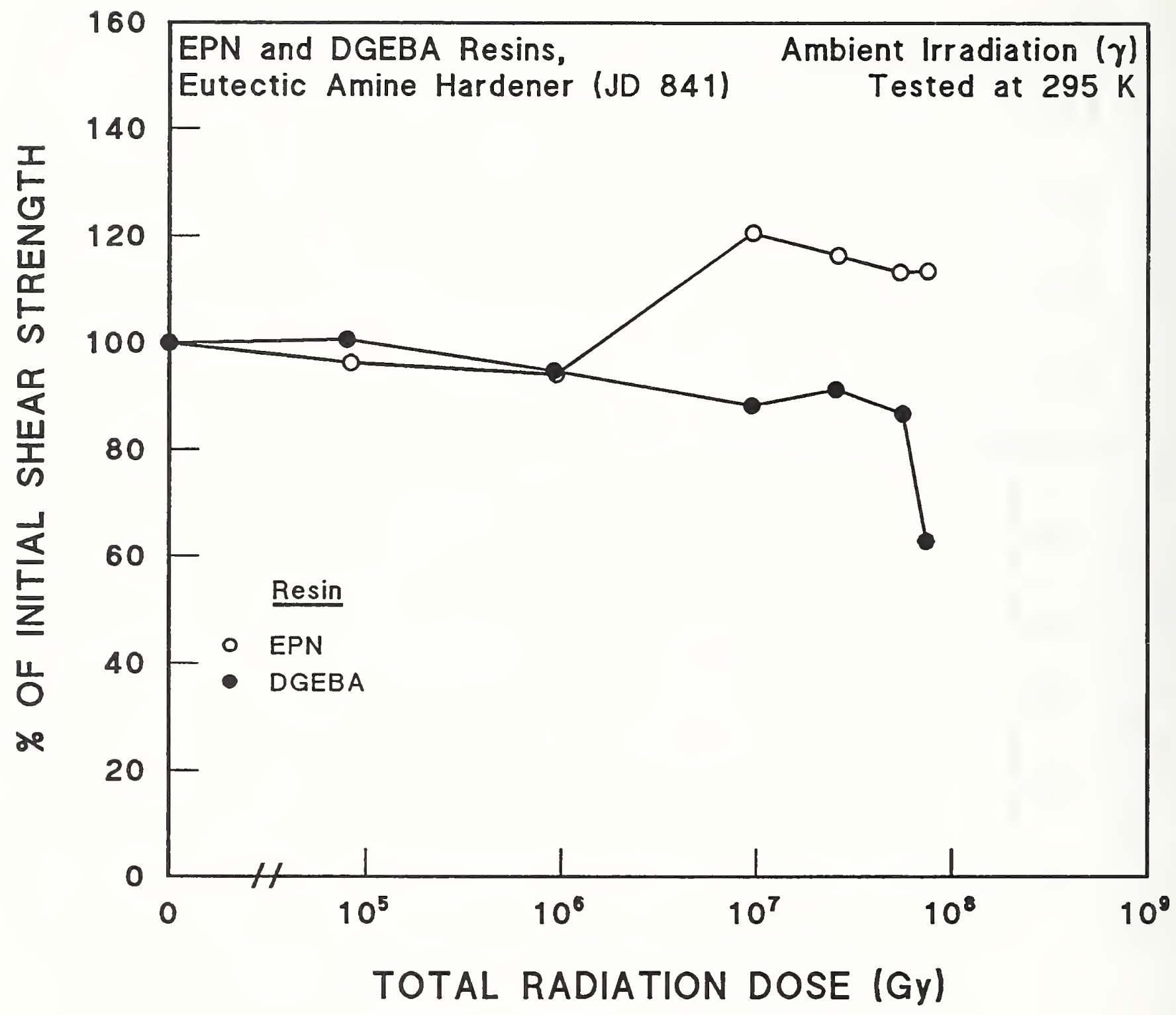

\begin{tabular}{llll} 
"O" Strength, MPa & Resin Name & Supplier \\
\hline 0 & 62.5 & DEN 438 & --- \\
0 & 72.3 & Epon 826 & ---
\end{tabular}

Figure A.2-48. Shear strength at $295 \mathrm{~K}$ of neat EPN and DGEBA resins with eutectic amine hardener after ambient gamma irradiation. Supplementary Table A.2-5. Data from Markley et al. [1969]. 


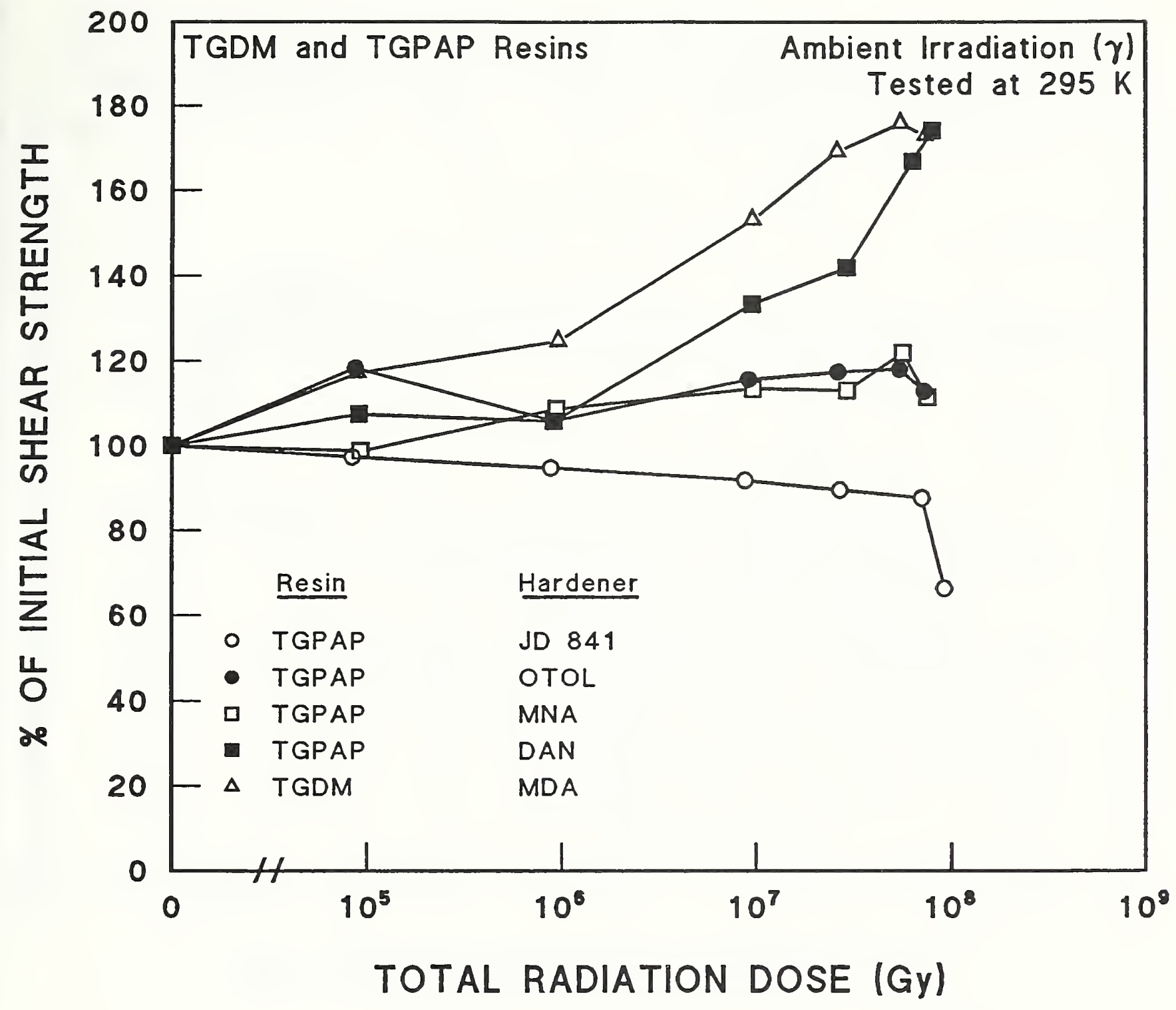

"O" Strength, MPa

- 101.4

- $\quad 71.7$

ㅁ 57.3

- 37.0

$\triangle \quad 41.5$
Resin Name

ERL 0500

ERL 0510

ERL 0510

X8183/137

$\times 8183 / 137$
Supplier

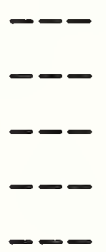

Figure A.2-49. Shear strength at $295 \mathrm{~K}$ of neat TGDM and TGPAP resins with several hardeners after ambient gamma irradiation. Supplementary Table A.2-5. Data from Markley et al. [1969]. 


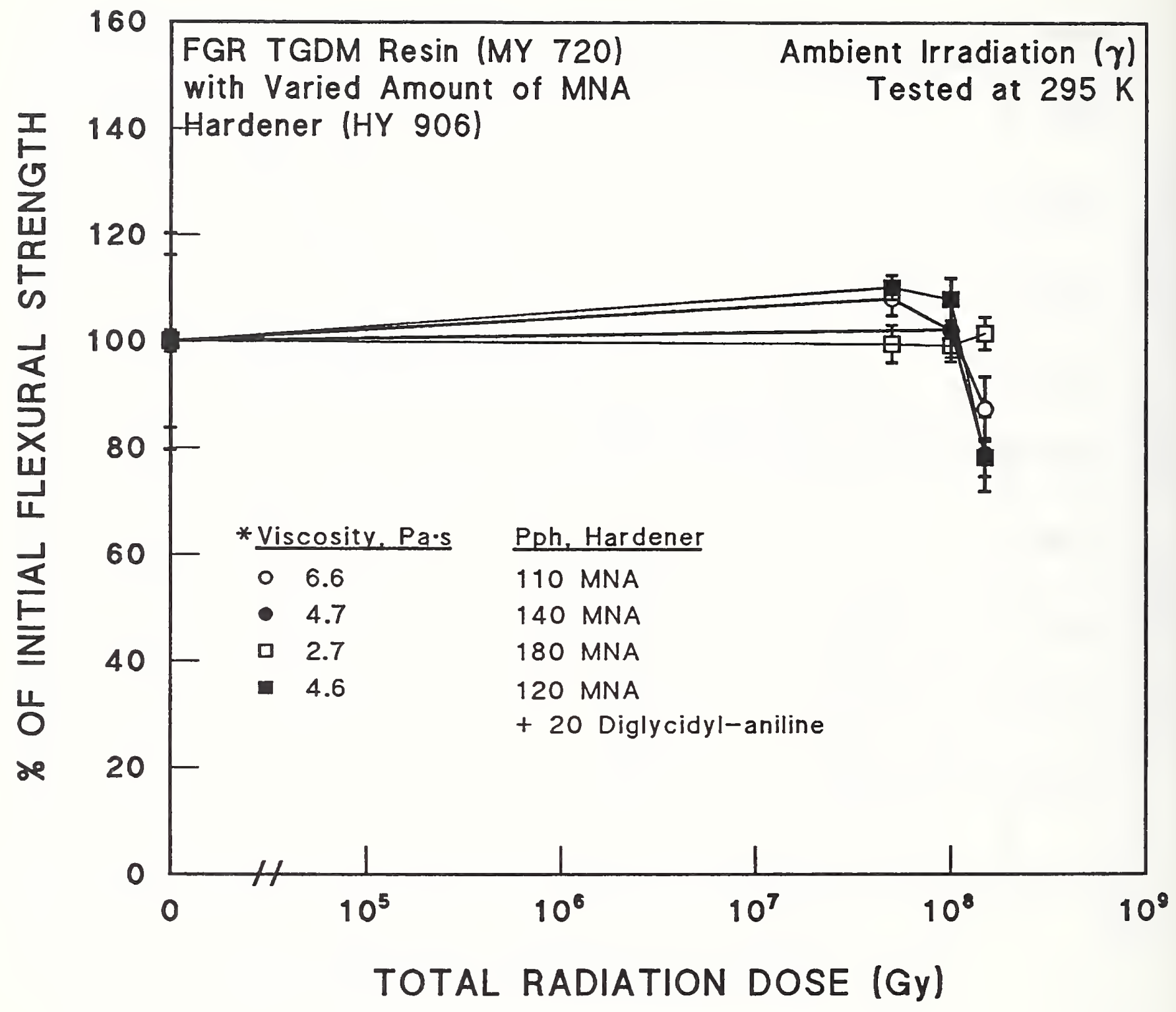

\begin{tabular}{llll} 
"O" Strength, MPa & & Cure Conditions & Supplier \\
\hline $284.3 \pm 5.7$ & $4 \mathrm{~h}, 100^{\circ} \mathrm{C} ; 3 \mathrm{~h}, 150^{\circ} \mathrm{C} ;$ & Ciba-Geigy \\
& $4 \mathrm{~h}, 200^{\circ} \mathrm{C}$ & \\
\hline $298.1 \pm 60.5$ & $4 \mathrm{~h}, 100^{\circ} \mathrm{C} ; 3 \mathrm{~h}, 150^{\circ} \mathrm{C} ;$ & Ciba-Geigy \\
& $4 \mathrm{~h}, 200^{\circ} \mathrm{C}$ & \\
\hline $312.3 \pm 50.6$ & $4 \mathrm{~h}, 100^{\circ} \mathrm{C} ; 3 \mathrm{~h}, 150^{\circ} \mathrm{C} ;$ & Ciba-Geigy \\
& $4 \mathrm{~h}, 200^{\circ} \mathrm{C}$ & \\
$-298.5 \pm 0.6$ & $4 \mathrm{~h}, 100^{\circ} \mathrm{C} ; 3 \mathrm{~h}, 150^{\circ} \mathrm{C} ;$ & Ciba-Geigy \\
& $4 \mathrm{~h}, 200^{\circ} \mathrm{C}$ & \\
$* 24^{\circ} \mathrm{C}$ & &
\end{tabular}

Figure A.2-50. Flexural strength at $295 \mathrm{~K}$ of FGR TGDM resin with varied amount of MNA hardener after ambient gamma irradiation. Supplementary Table A.2-1. Data from Evans et al. [1972a]. 


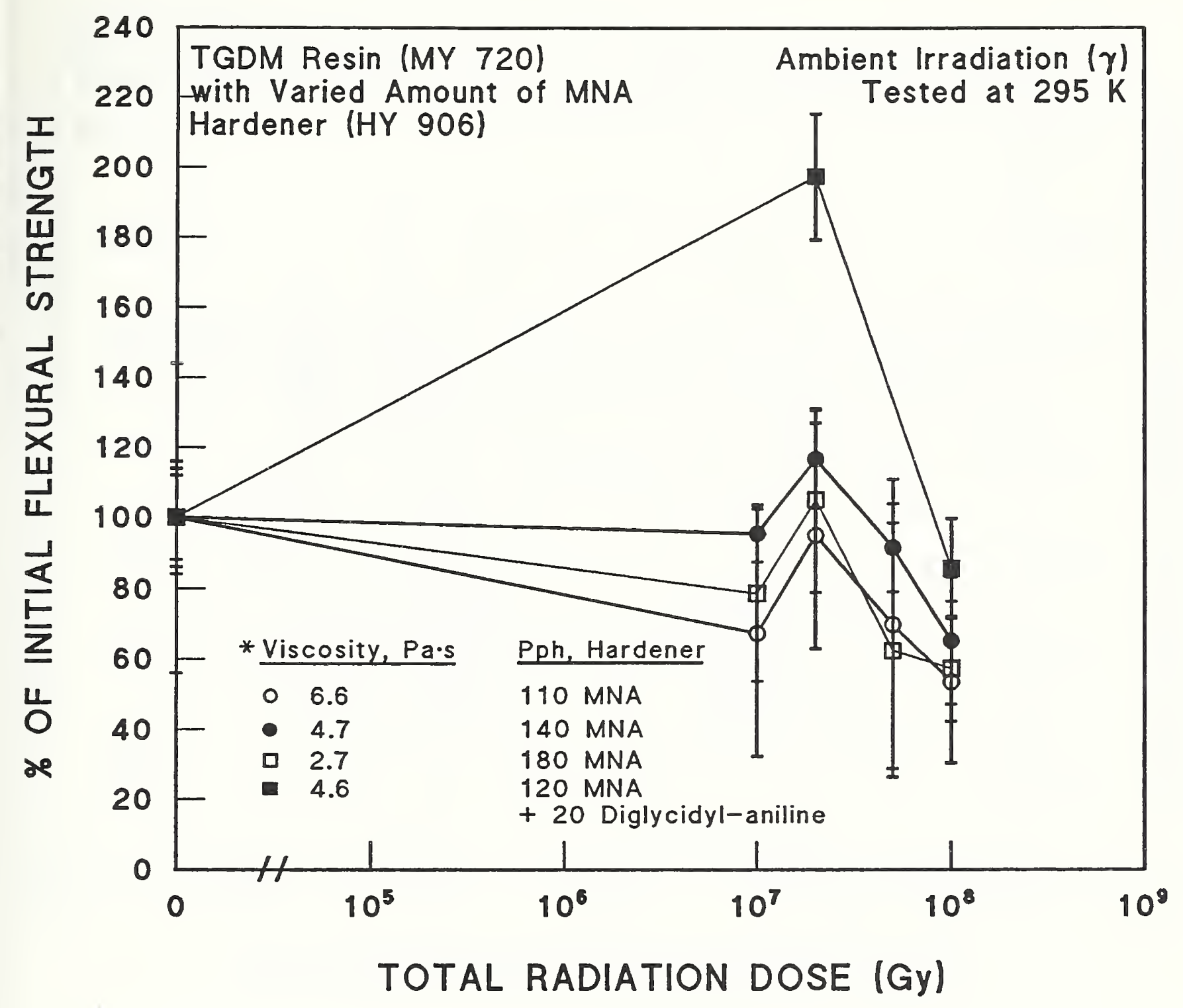

"O" Strength, MPa

- $90.0 \pm 10.8$

- $\quad 83.7 \pm 13.4$

ㅁ $89.8 \pm 12.6$

$54.0 \pm 23.8$
Cure Conditions

$4 \mathrm{~h}, 100^{\circ} \mathrm{C} ; 3 \mathrm{~h}, 150^{\circ} \mathrm{C}$;

$4 \mathrm{~h}, 200^{\circ} \mathrm{C}$

$4 \mathrm{~h}, 100^{\circ} \mathrm{C} ; 3 \mathrm{~h}, 150^{\circ} \mathrm{C}$;

$4 \mathrm{~h}, 200^{\circ} \mathrm{C}$

$4 \mathrm{~h}, 100^{\circ} \mathrm{C} ; 3 \mathrm{~h}, 150^{\circ} \mathrm{C}$;

$4 \mathrm{~h}, 200^{\circ} \mathrm{C}$

$4 \mathrm{~h}, 100^{\circ} \mathrm{C} ; 3 \mathrm{~h}, 150^{\circ} \mathrm{C}$;

$4 \mathrm{~h}, 200^{\circ} \mathrm{C}$
Supplier

Ciba-Geigy

Ciba-Geigy

Ciba-Geigy

Ciba-Geigy

* $24^{\circ} \mathrm{C}$

Figure A.2-51. Flexural strength at $295 \mathrm{~K}$ of neat TGDM resin with varied amount of MNA hardener after ambient gamma irradiation. Supplementary Table A.2-1. Data from Evans et al. [1972a]. 


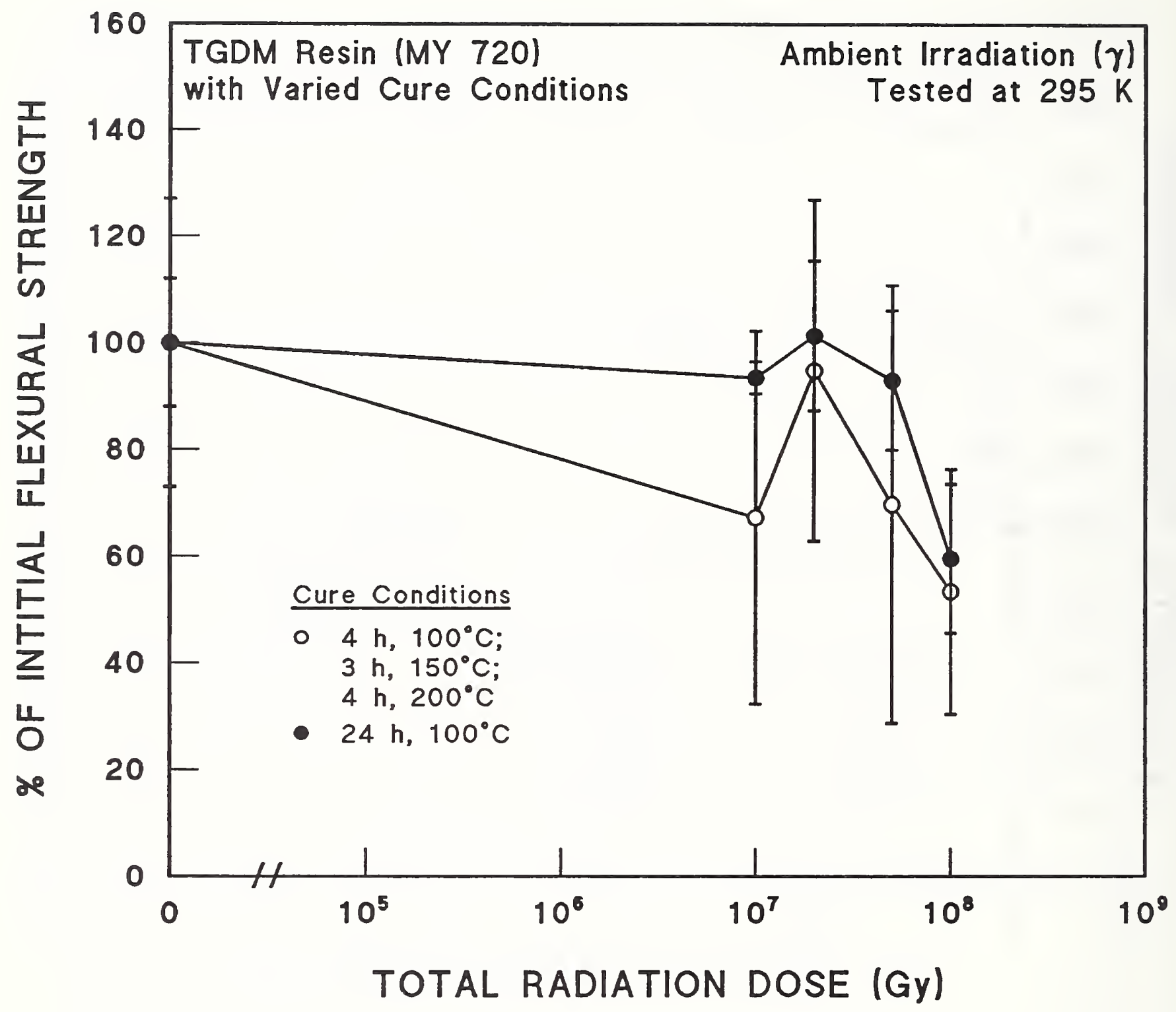

"O" Strength, MPa

- $90.0 \pm 10.8$

- $97.9 \pm 26.4$
Hardener

MNA (HY 906)

MNA (HY 906)
Accelerator

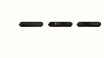

Supplier

Ciba-Geigy

Ciba-Geigy

Figure A.2-52. Flexural strength at $295 \mathrm{~K}$ of neat TGDM resin with varied cure conditions after ambient gamma irradiation. Supplementary Table A.2-1. Data from Evans et al. [1972a]. 


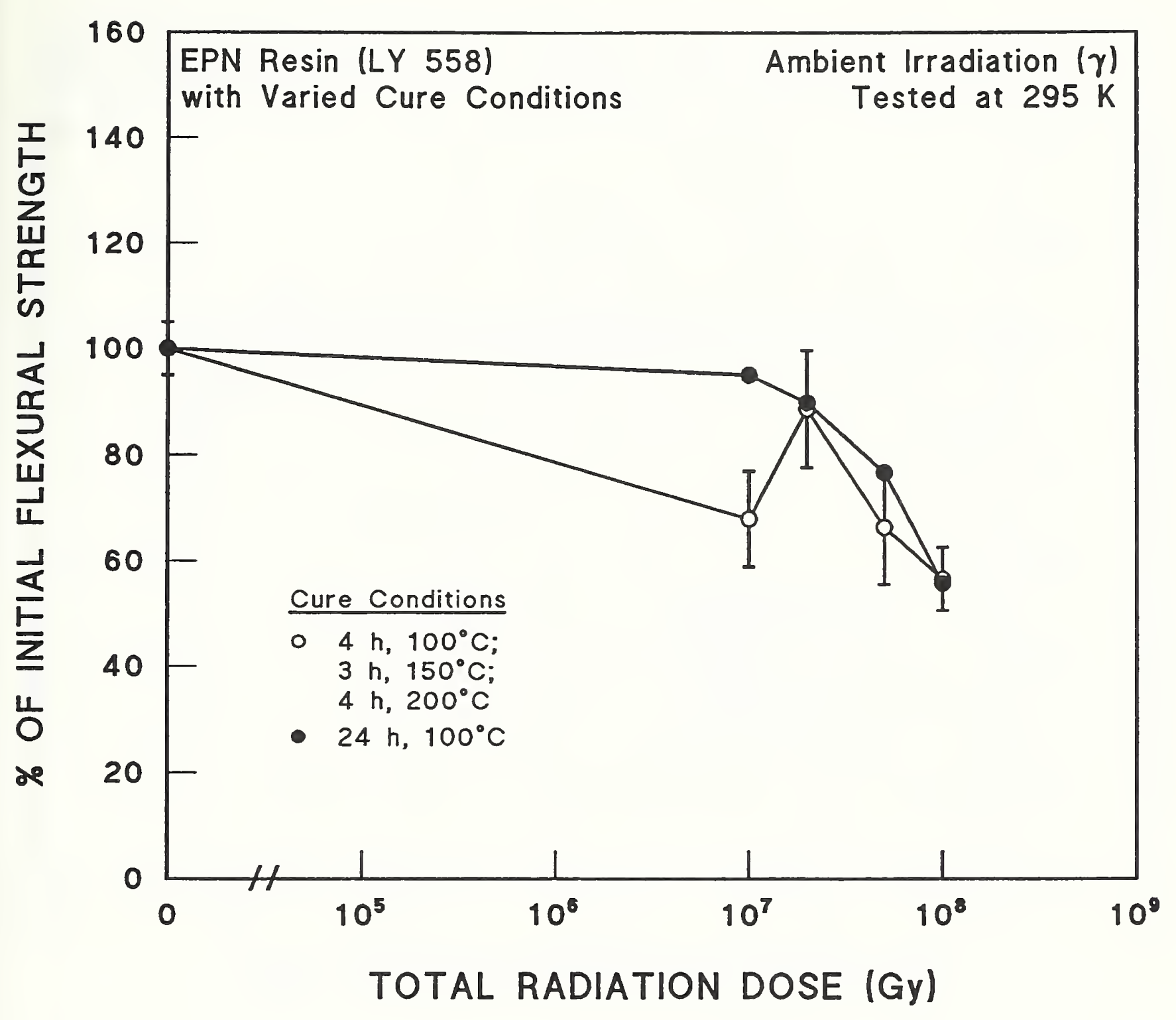

\begin{tabular}{|c|c|c|c|}
\hline "O" Strength, MPa & Hardener & Accelerator & Supplier \\
\hline $149.2 \pm 7.5$ & MNA (HY 906) & BDMA (DY 062) & -Geigy \\
\hline - $132.1 \pm 0.0$ & MNA (HY 906) & BDMA (DY 062) & Ciba-Geigy \\
\hline
\end{tabular}

Figure A.2-53. Flexural strength at $295 \mathrm{~K}$ of neat EPN resin with varied cure conditions after ambient gamma irradiation. Supplementary Table A.2-1. Data from Evans et al. [1972a]. 


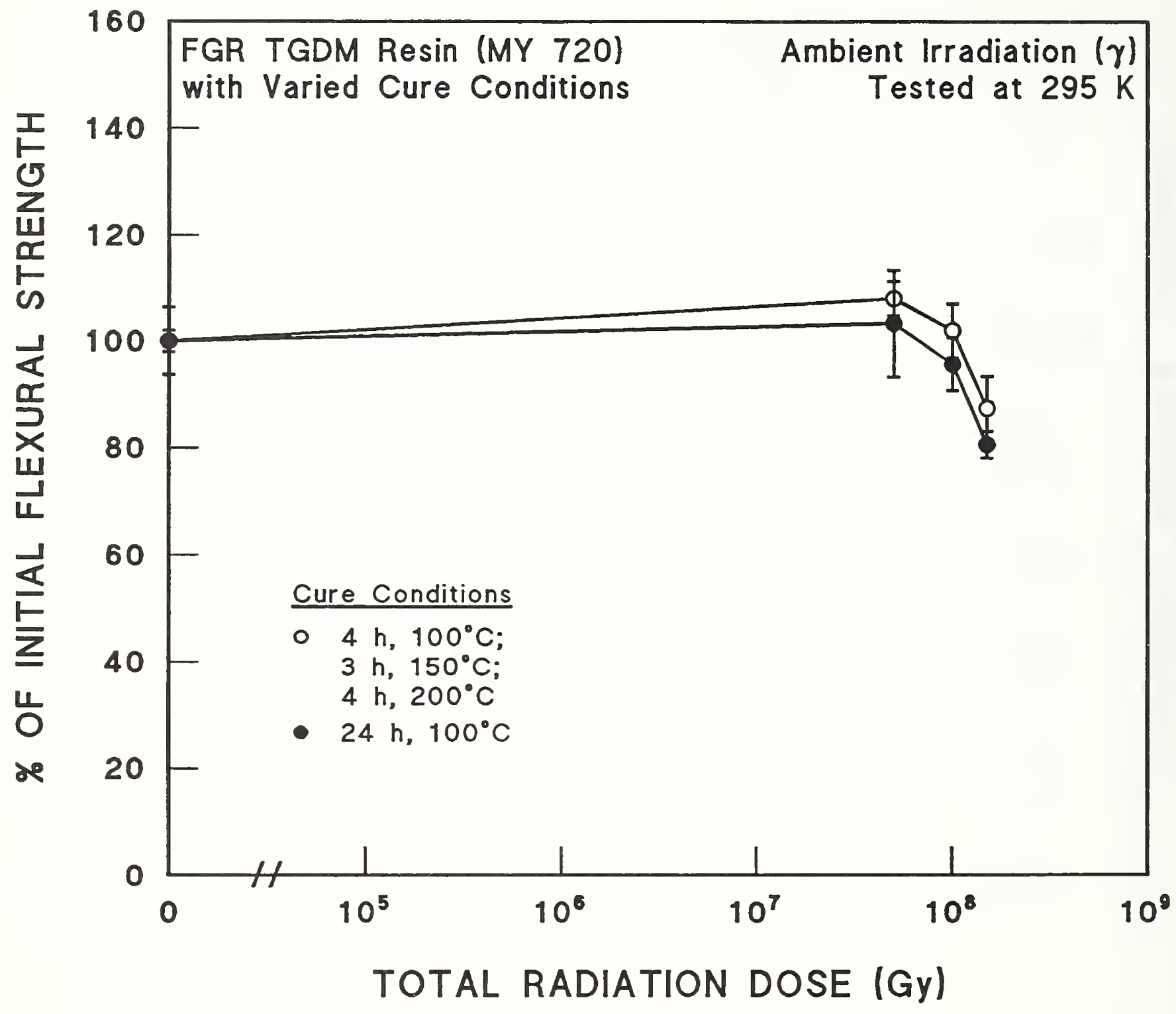

\begin{tabular}{lllll} 
"O" Strength, MPa & & Hardener & Accelerator & Supplier \\
\hline 0 & $284.3 \pm 5.7$ & MNA (HY 906) & -- & Ciba-Geigy \\
$-308.6 \pm 19.4$ & MNA (HY 906) & -- & Ciba-Geigy
\end{tabular}

Figure A.2-54. Flexural strength at $295 \mathrm{~K}$ of neat TGDM resin with varied cure conditions after ambient gamma irradiation. Supplementary Table A.2-1. Data from Evans et a1. [1972a]. 


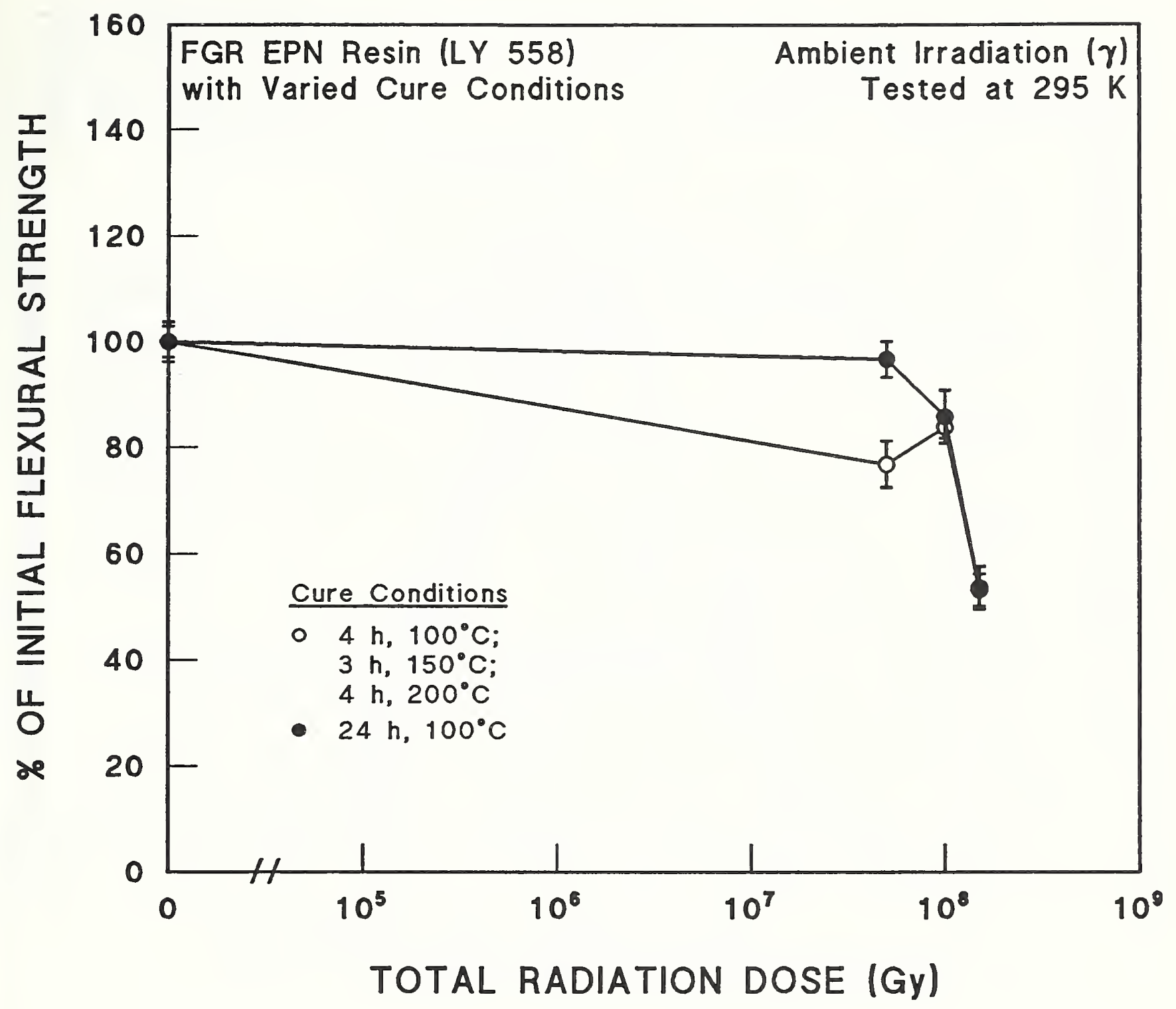

\begin{tabular}{llll} 
"O" Strength, MPa & Hardener & Accelerator & Supplier \\
\hline $0353.1 \pm 10.6$ & MNA (HY 906) & BDMA (DY 062) & Ciba-Geigy \\
$-335.7 \pm 12.8$ & MNA (HY 906) & BDMA (DY 062) & Ciba-Geigy
\end{tabular}

Figure A.2-55. Flexural strength at $295 \mathrm{~K}$ of FGR EPN resin with varied cure conditions after ambient gamma irradiation. Supplementary Table A.2-1. Data from Evans et al. [1972a]. 


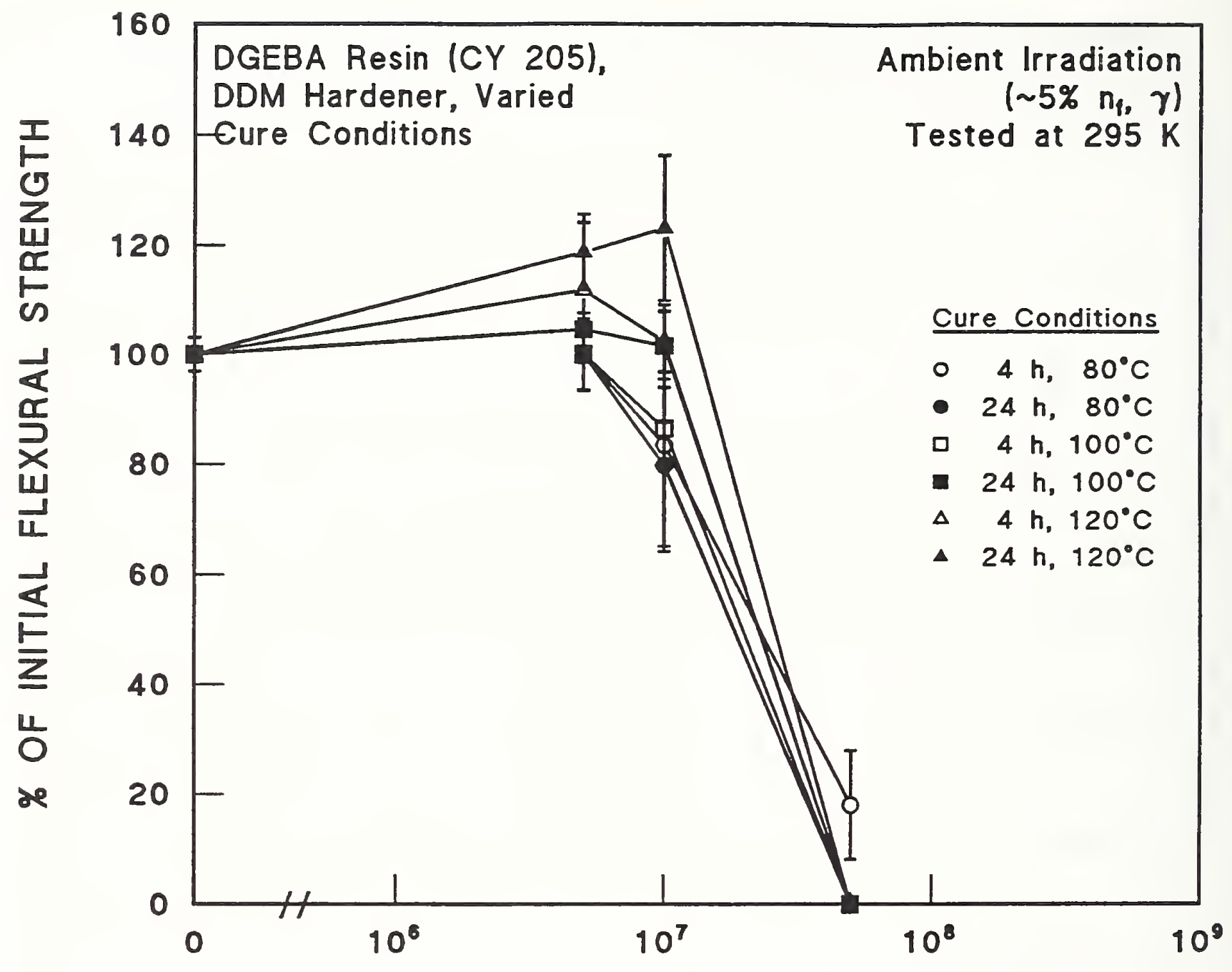

TOTAL RADIATION DOSE (Gy)

\section{"O" Strength, MPa}

* 0 too flexible for testing

* too flexible for testing

* too flexible for testing

- $130.5 \pm 4.0$

$\triangle 125.6 \pm 1.6$

$\Delta \quad 110.8 \pm 1.6$

\begin{tabular}{c}
$5 \times 10^{6} \mathrm{~Gy}$ Strengt \\
\hline $119.7 \pm 7.8$ \\
$131.5 \pm 0.0$ \\
$137.3 \pm 2.0$ \\
NA \\
NA \\
NA
\end{tabular}

Supplier

CERN Workshop

CERN Workshop CERN Workshop CERN Workshop CERN Workshop CERN Workshop

Comments: Samples $(\bullet, \square, \square, \Delta, \Delta)$ were all broken at a dose of $5 \times 10^{7} \mathrm{~Gy}$. * Strength at $5 \times 10^{6} \mathrm{G}$ y was used as the initial value for samples $(0, \bullet, \square)$.

Figure A.2-56. Flexural strength at $295 \mathrm{~K}$ of neat DGEBA resin with DDM hardener and varied cure conditions after ambient gamma irradiation. Supplementary Table A.2-2. Data from Schönbacher and Stolarz-Iżycka [1979]. 


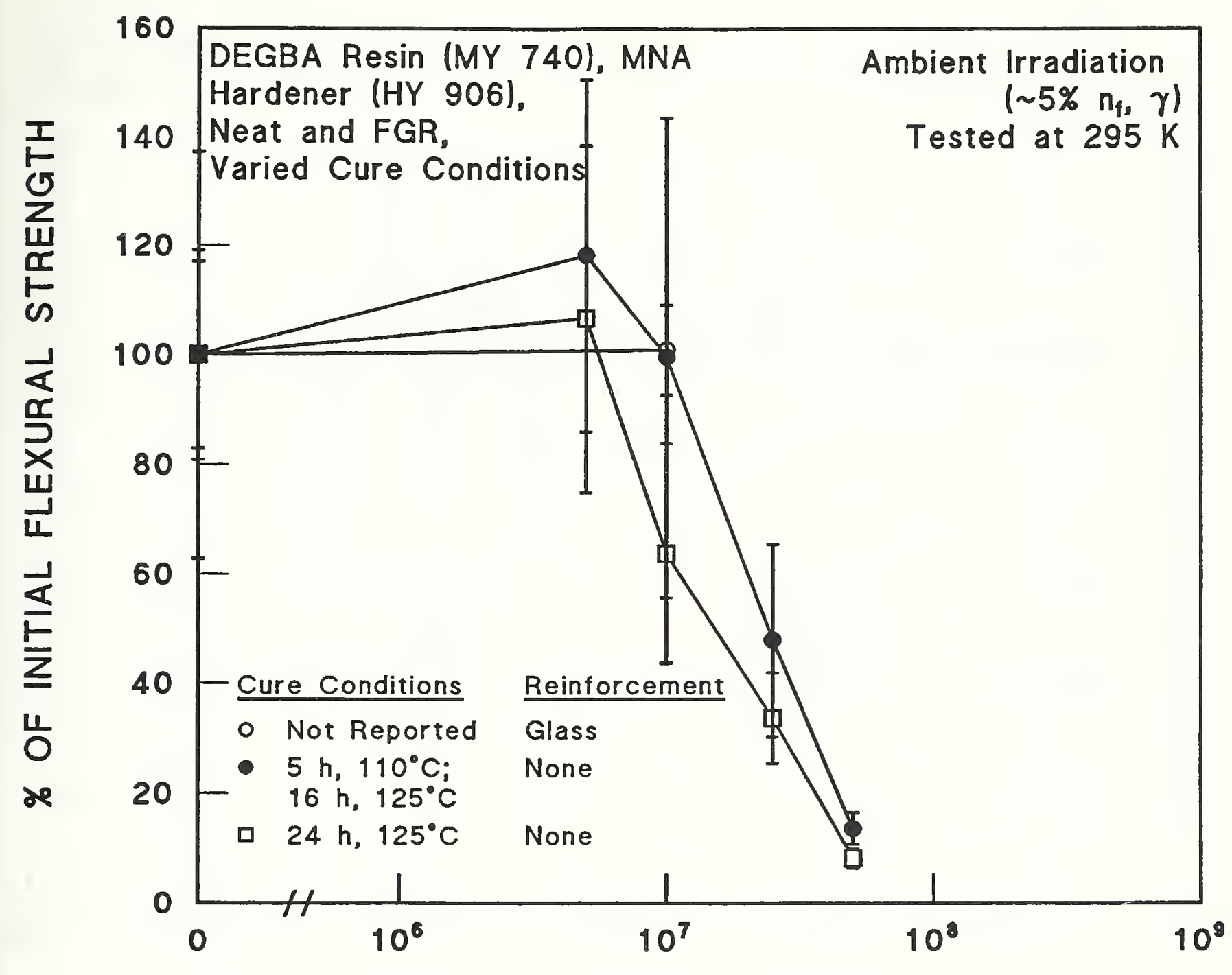

TOTAL RADIATION DOSE (Gy)

"O" Strength, MPa

○ $333.5 \pm 56.9$

- $100.4 \pm 37.3$

ㅁ $107.7 \pm 20.6$
Accelerator

XB 2687

XB 2687

XB 2687
Supplier

Als thom

Ciba-Giegy

Ciba-Giegy

Figure A.2-57. Flexural strength at $295 \mathrm{~K}$ of neat and FGR DGEBA resin with MNA hardener and varied cure conditions after ambient reactor irradiation.

Supplementary Table A.2-2. Data from Schönbacher and Stolarz-Iżycka [1979]. 


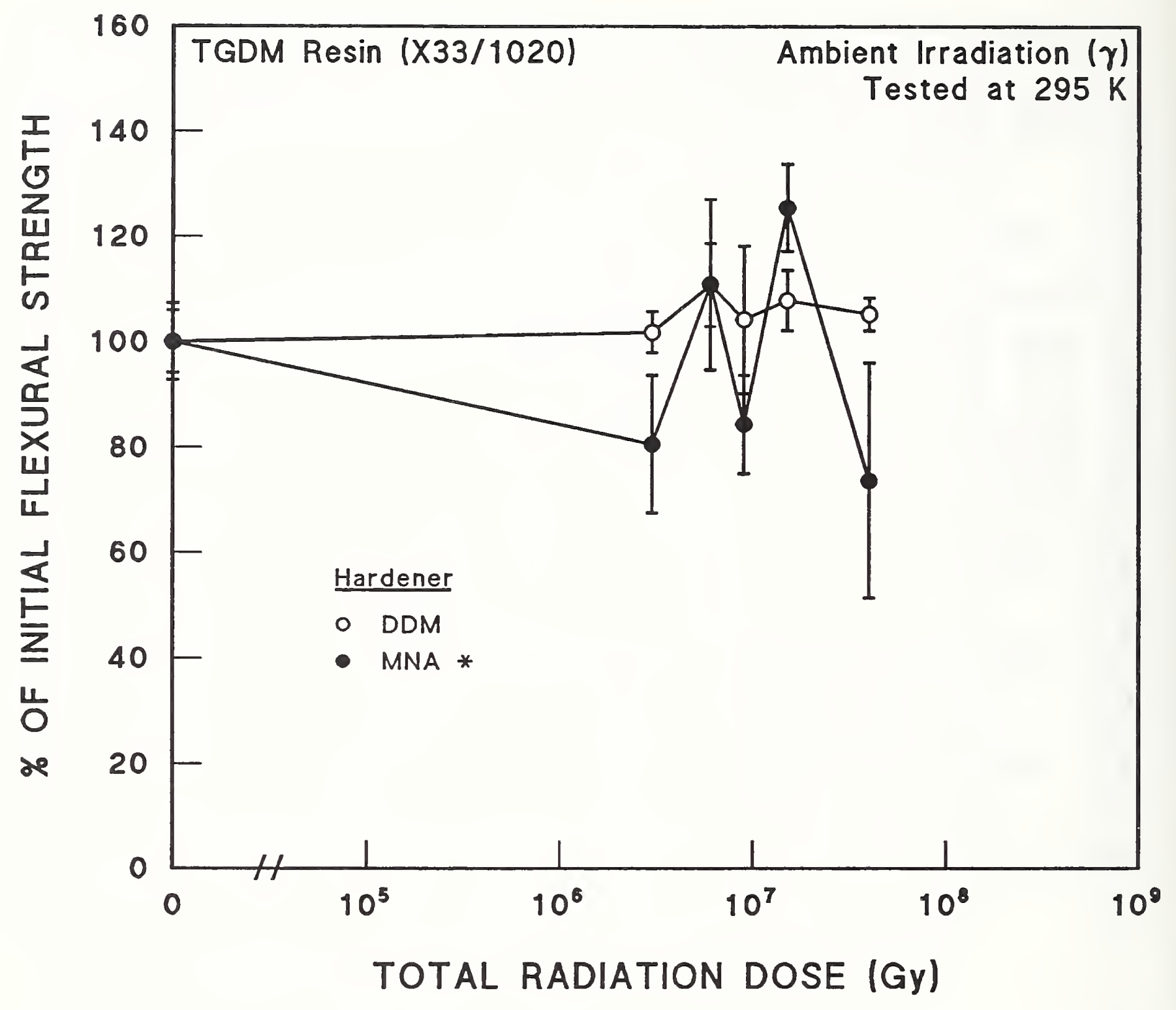

"O" Strength, MPa

○ $138.7 \pm 8.2$

- $105.9 \pm 7.7$

* BDMA Accelerator
Cure Conditions

$10 \mathrm{~h}, 50^{\circ} \mathrm{C}$;

$10 \mathrm{~h}, 100^{\circ} \mathrm{C}$

$10 \mathrm{~h}, 100^{\circ} \mathrm{C}$;
Supplier

Ciba-Geigy

Ciba-Geigy

Figure A.2-58. Flexural strength at $295 \mathrm{~K}$ of neat TGDM resin with two hardeners after ambient gamma irradiation. Supplementary Table A.2-1. Data from Morgan et a1. [1970]. 


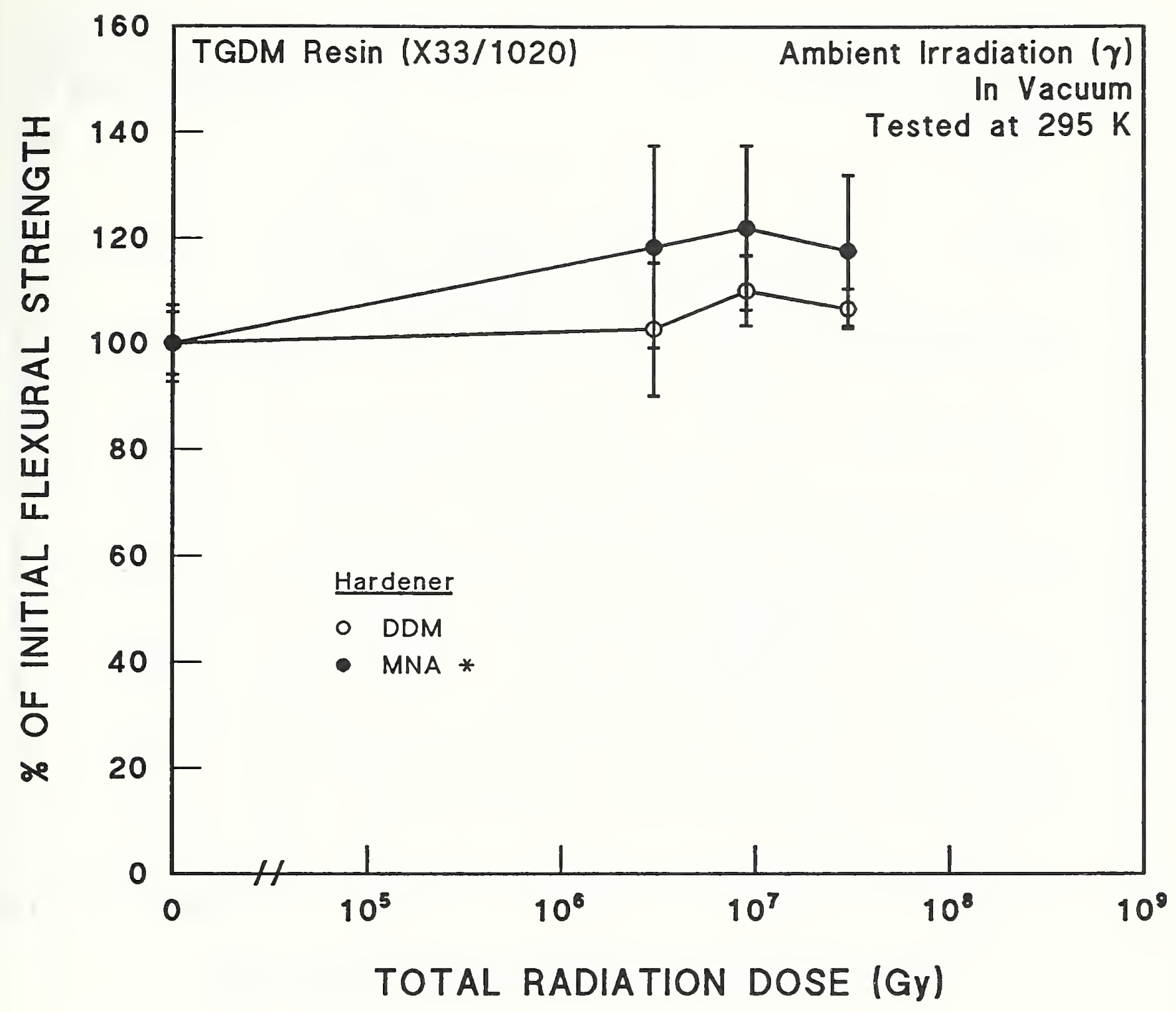

\begin{tabular}{|c|c|c|}
\hline "O" Strength, MPa & Cure Conditions & Supplier \\
\hline - $\quad 138.7 \pm 8.2$ & $\begin{array}{l}10 \mathrm{~h}, 50^{\circ} \mathrm{C} \\
10 \mathrm{~h}, 100^{\circ} \mathrm{C}\end{array}$ & Ciba-Geigy \\
\hline - $105.9 \pm 7.7$ & $10 \mathrm{~h}, 100^{\circ} \mathrm{C}$ & Ciba-Geigy \\
\hline
\end{tabular}

Figure A.2-59. Flexural strength at $295 \mathrm{~K}$ of neat TGDM resin with two hardeners after ambient gamma irradiation in vacuum. Supplementary Table A.2-1. Data from Morgan et al. [1970]. 


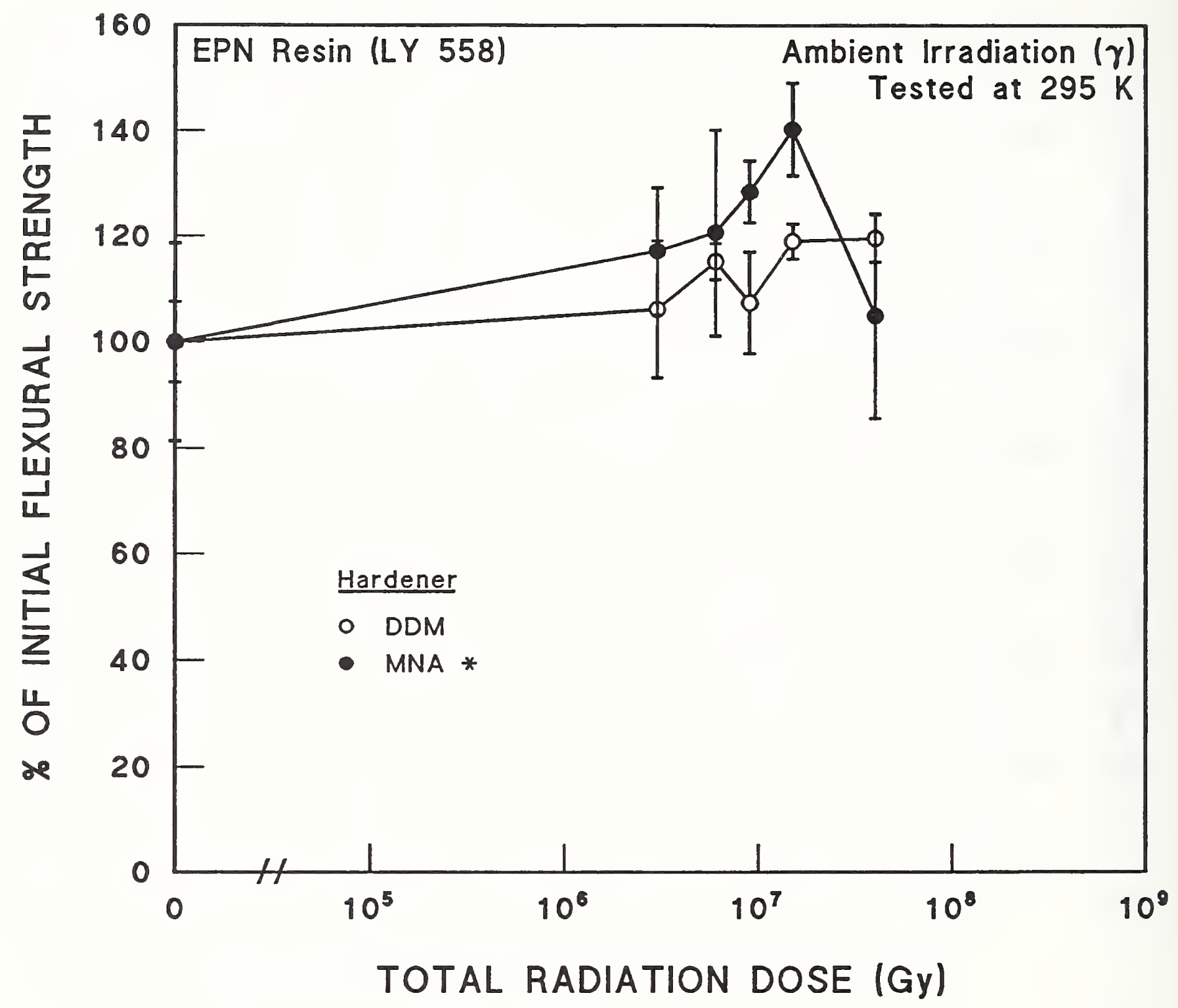

\begin{tabular}{|c|c|c|c|}
\hline "O" & Strength, $\mathrm{MPa}$ & Cure Conditions & Supplier \\
\hline ○ & $119.0 \pm 9.0$ & $\begin{array}{l}2 \mathrm{~h}, 100^{\circ} \mathrm{C} ; \\
10 \mathrm{~h}, 160^{\circ} \mathrm{C}\end{array}$ & -Geigy \\
\hline$\bullet$ & $89.6 \pm 16.7$ & $\begin{array}{l}2 \mathrm{~h}, 100^{\circ} \mathrm{C} \\
10 \mathrm{~h}, 160^{\circ} \mathrm{C}\end{array}$ & Ciba-Geigy \\
\hline
\end{tabular}

* BDMA Accelerator

Figure A.2-60. Flexural strength at $295 \mathrm{~K}$ of neat EPN resin with two hardeners after ambient gamma irradiation. Supplementary Table A.2-1. Data from Morgan et al. [1970]. 


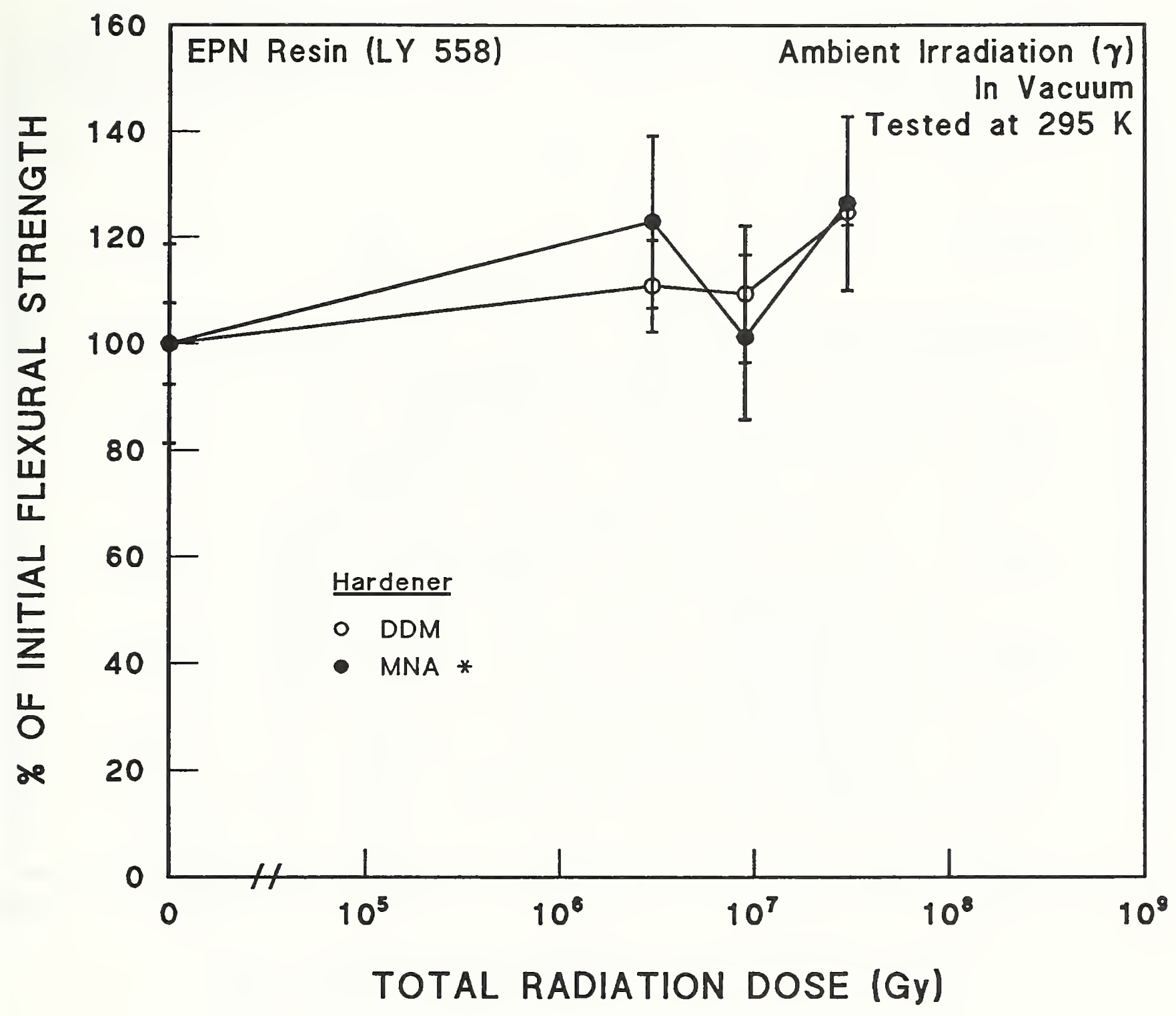

"O" Strength, MPa

- $119.0 \pm 9.0$

- $89.6 \pm 16.7$
Cure Conditions

$2 \mathrm{~h}, 100^{\circ} \mathrm{C}$;

$10 \mathrm{~h}, 160^{\circ} \mathrm{C}$

$2 \mathrm{~h}, 100^{\circ} \mathrm{C}$;

$10 \mathrm{~h}, 160^{\circ} \mathrm{C}$
Supplier

Ciba-Geigy

Ciba-Geigy

* BDMA Accelerator

Figure A.2-61. Flexural strength at $295 \mathrm{~K}$ of neat EPN resin with two hardeners after ambient gamma irradiation in vacuum. Supplementary Table A.2-1. Data from Morgan et al. [1970]. 


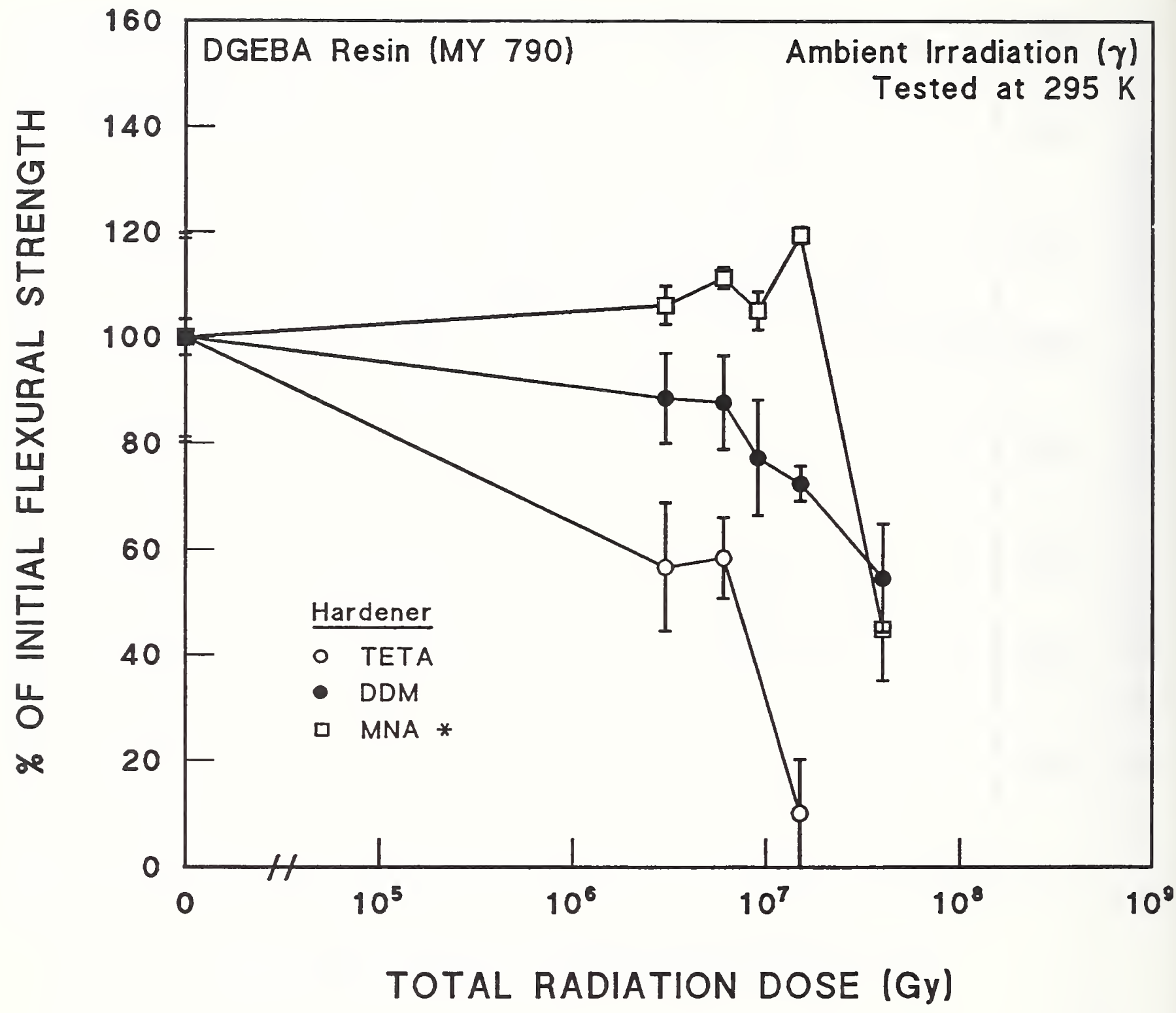

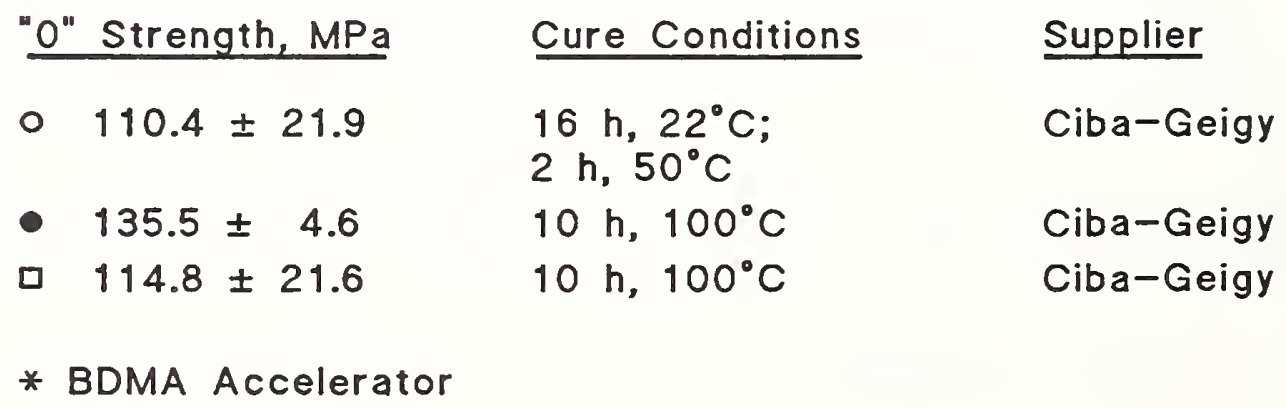

Figure A.2-62. Flexural strength at $295 \mathrm{~K}$ of neat DGEBA resin with three hardeners after ambient gamma irradiation. Supplementary Table A.2-1. Data from Morgan et al. [1970]. 


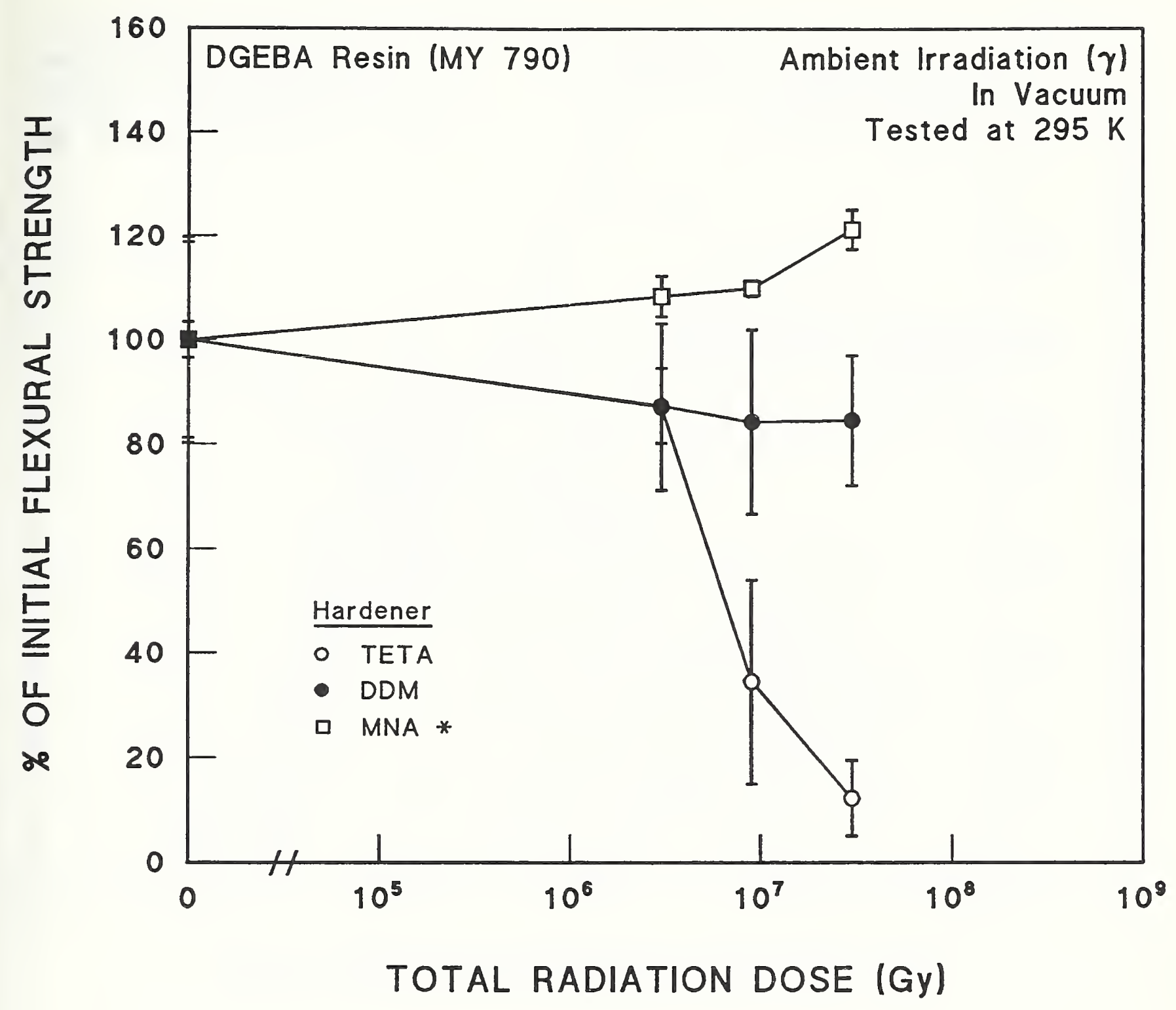

\begin{tabular}{|c|c|c|}
\hline "O" Strength, MPa & Cure Conditions & Supplier \\
\hline - $110.4 \pm 21.9$ & $\begin{array}{l}16 \mathrm{~h}, 22^{\circ} \mathrm{C} \text {; } \\
2 \mathrm{~h}, 50^{\circ} \mathrm{C}\end{array}$ & Ciba-Geigy \\
\hline - $\quad 135.5 \pm 4.6$ & $10 \mathrm{~h}, 100^{\circ} \mathrm{C}$ & Ciba-Geigy \\
\hline ㅁ $114.8 \pm 21.6$ & $10 \mathrm{~h}, 100^{\circ} \mathrm{C}$ & Ciba-Geigy \\
\hline
\end{tabular}

Figure A.2-63. Flexural strength at $295 \mathrm{~K}$ of neat DGEBA resin with three hardeners after ambient gamma irradiation in vacuum. Supplementary Table A.2-1. Data from Morgan et al. [1970]. 


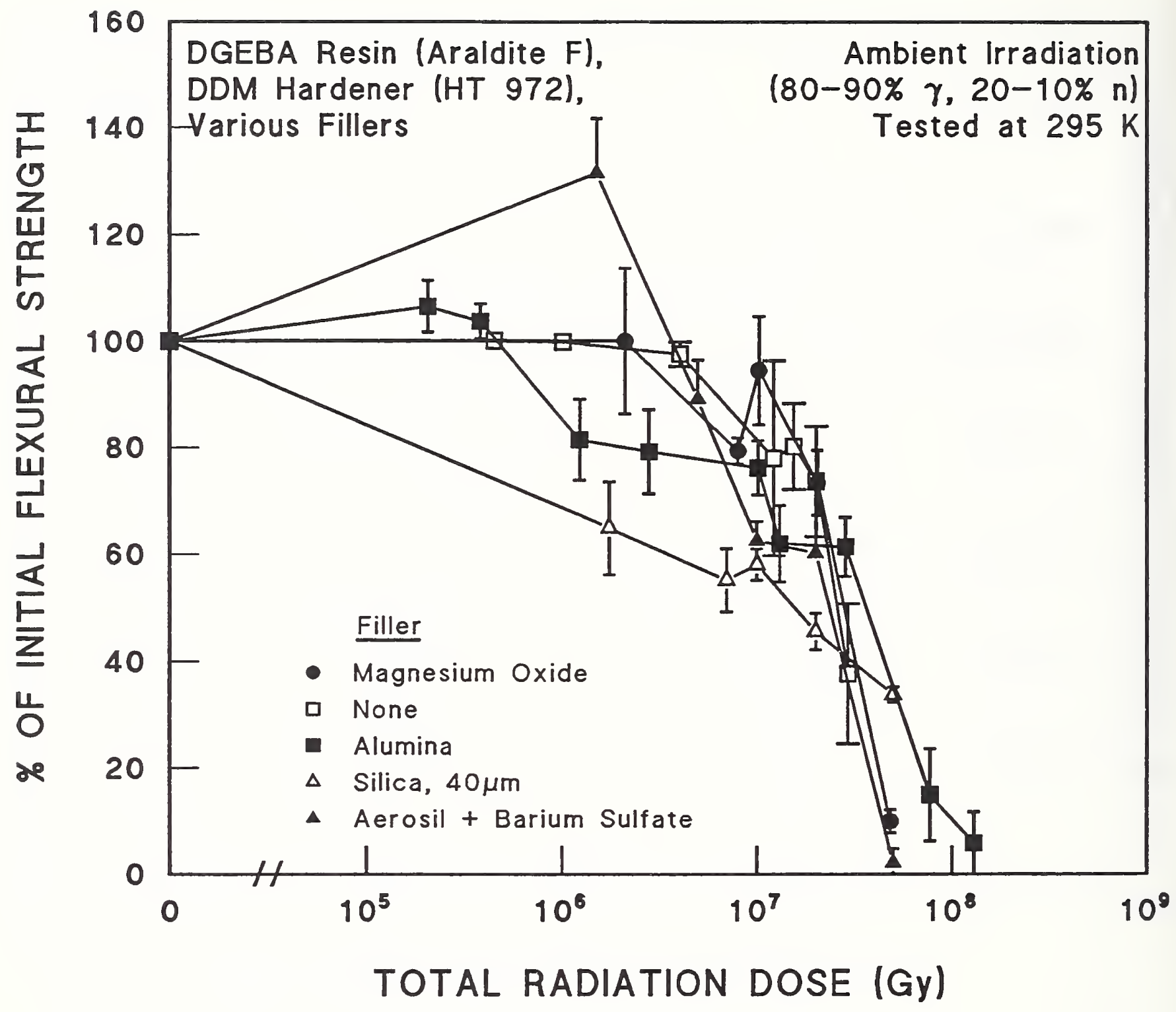

"O" Strength, MPa

- 85.3

ㅁ 166.7

- 111.8

$\Delta \quad 103.0$

$\triangle \quad 56.9$
Supplier

Ciba-Geigy

Ciba-Geigy

Ciba-Geigy

Ciba-Geigy

Ciba-Geigy

Figure A.2-64. Flexural strength at $295 \mathrm{~K}$ of DGEBA resin with DDM hardener and various fillers after ambient reactor irradiation. Supplementary Table A.2-4. Data from Pluym and Van de Voorde [1967] and Van de Voorde and Restat [1972]. 


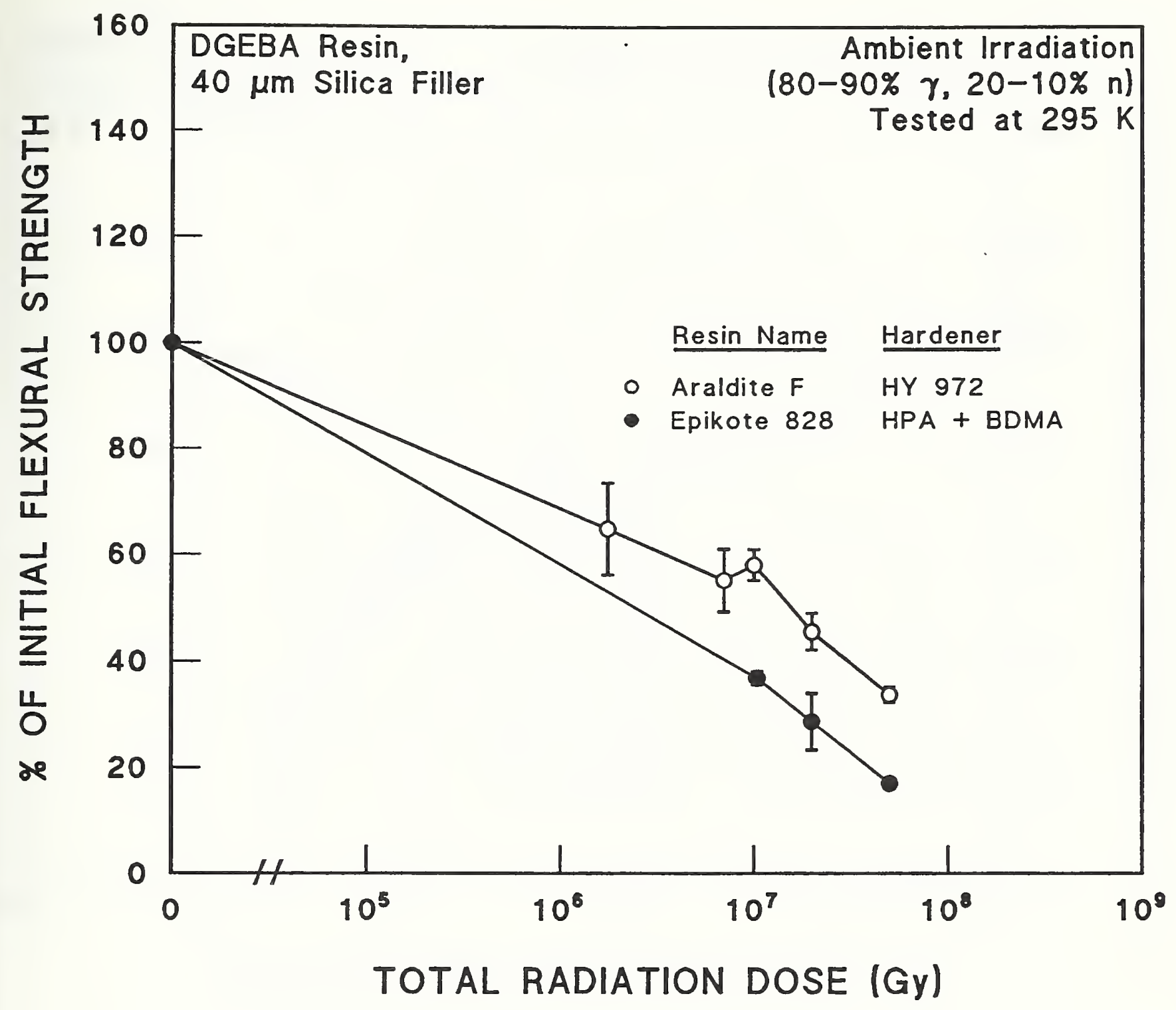

"O" Strength, MPa

- 103.0

- 92.2
Supplier

Ciba-Geigy

Ciba-Geigy

Figure A.2-65. Flexural strength at $295 \mathrm{~K}$ of DGEBA resin with two hardeners and silica filler after ambient reactor irradiation. Supplementary Table A.2-4. Data from Pluym and Van de Voorde [1967]. 


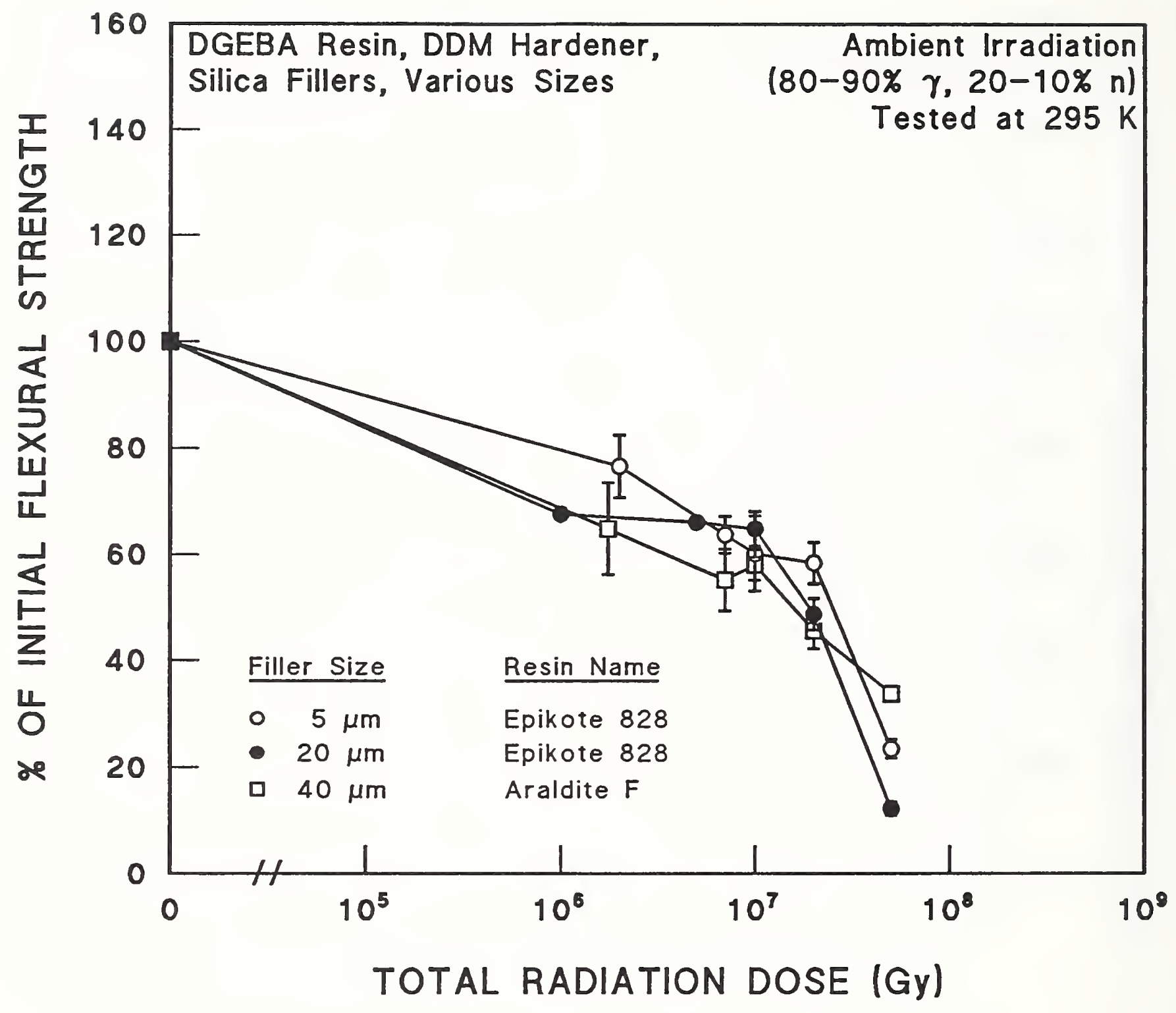

\begin{tabular}{lll} 
"O" Strength, MPa & Supplier \\
\hline 0 & 93.2 & Shell \\
- 91.2 & Ciba-Geigy \\
口 103.0 & Ciba-Geigy
\end{tabular}

Figure A.2-66. Flexural strength at $295 \mathrm{~K}$ of DGEBA resin with DDM hardener and silica fillers after ambient reactor irradiation. Supplementary Table A.2-4. Data from Pluym and Van de Voorde [1967]. 


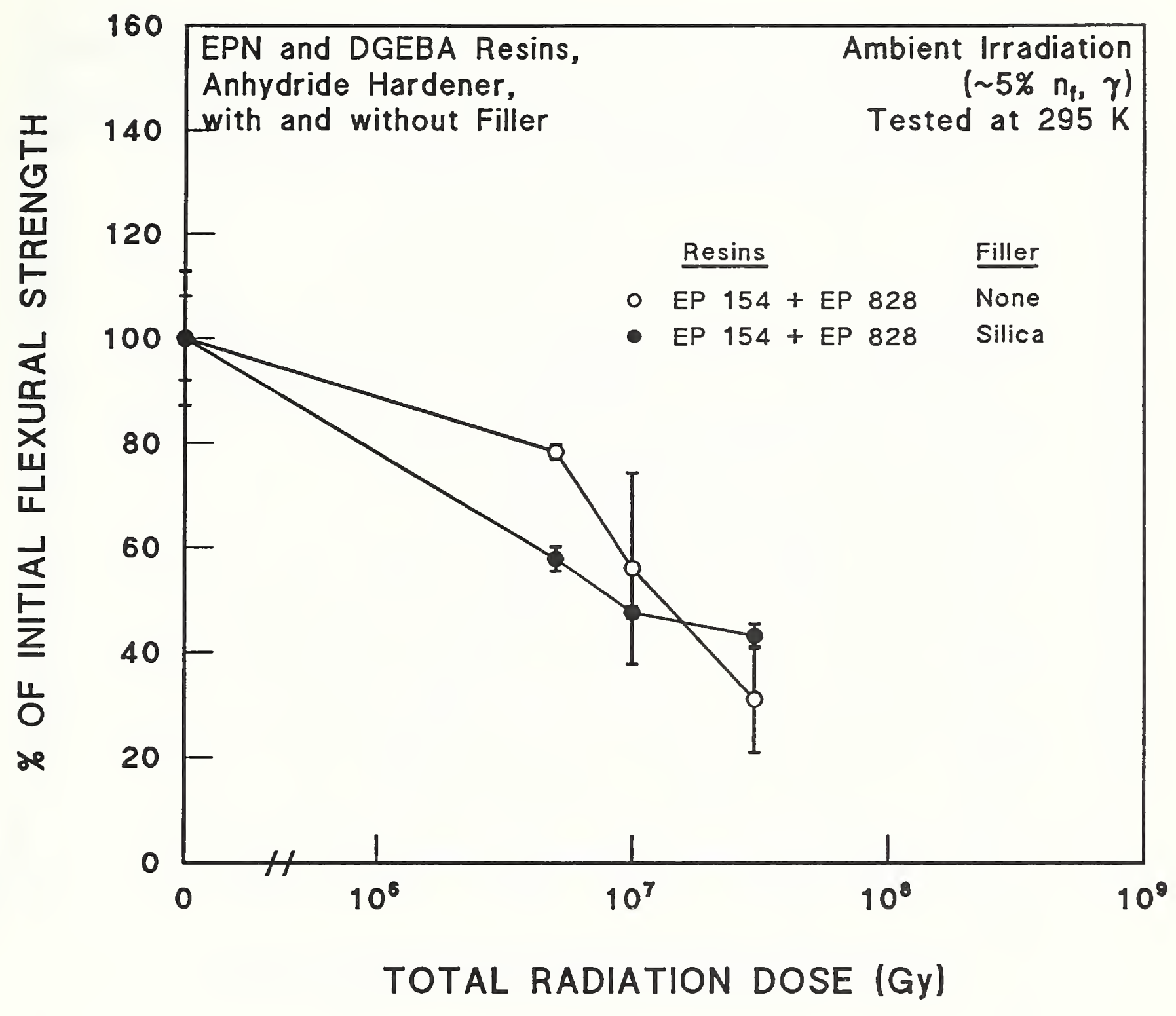

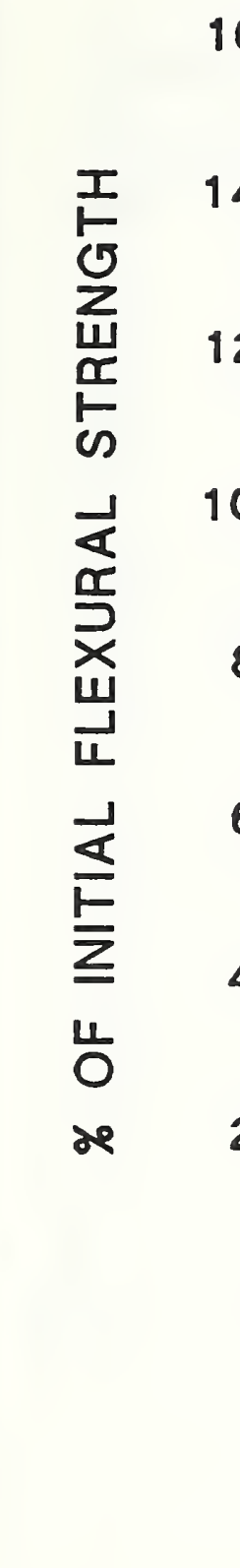

"O" Strength, MPa

- $145.2 \pm 18.6$

- $86.4 \pm 6.9$
Other Components

DX 126, DX 127

DX 126, DX 127
Supplier

Shell

Shell

Figure A.2-67. Flexural strength at $295 \mathrm{~K}$ of EPN and DGEBA resins with and without filler after ambient reactor irradiation. Supplementary Table A.2-2. Data from Schönbacher and Stolarz-Iżycka [1979]. 


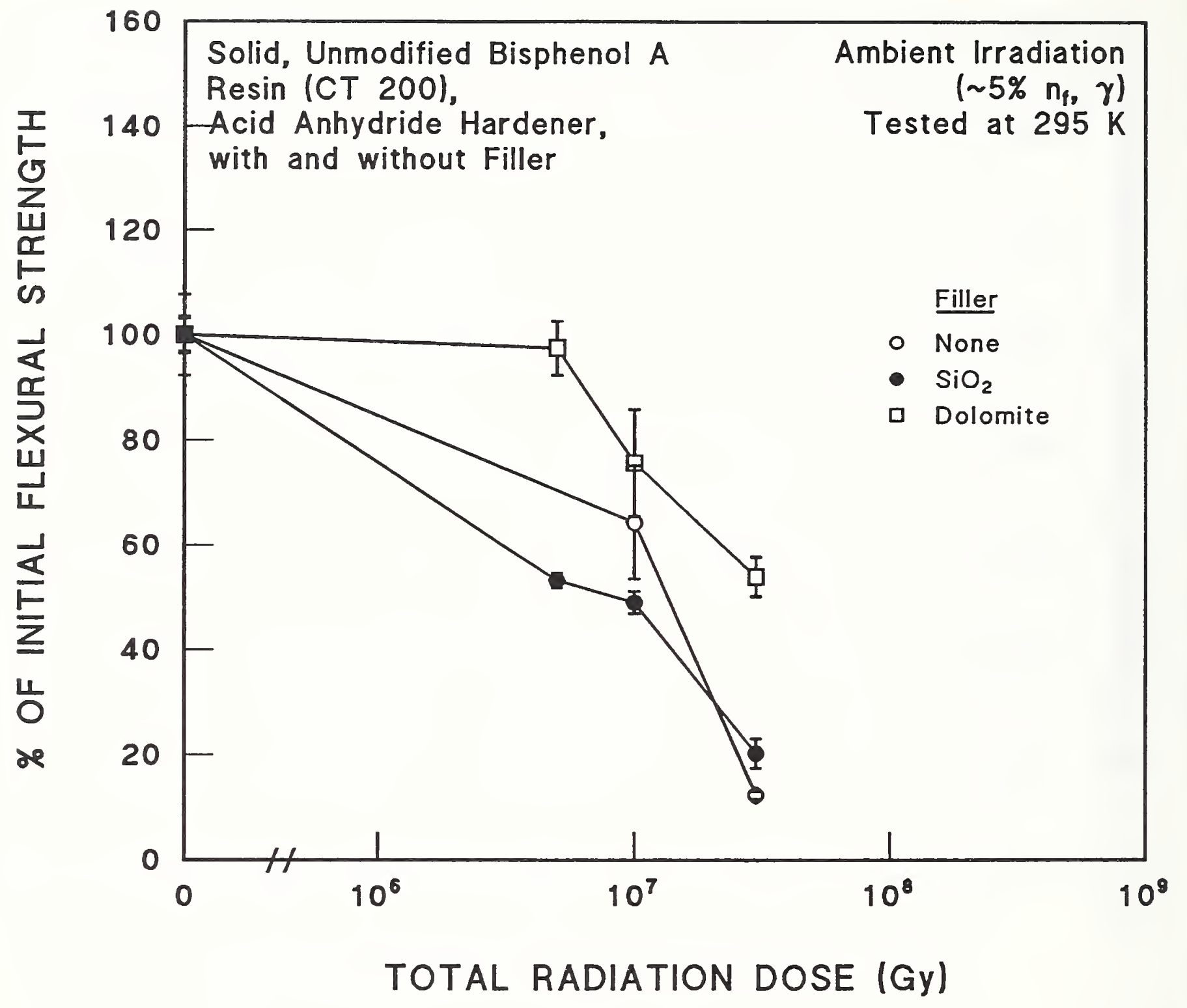

\begin{tabular}{|c|c|c|}
\hline "O" Strength, MPa & Cure Conditions & Supplier \\
\hline ○ $126.5 \pm 3.9$ & $2 \mathrm{~h}, 110^{\circ} \mathrm{C} ; 14 \mathrm{~h}, 140^{\circ} \mathrm{C}$ & Ciba-Geigy \\
\hline - $140.3 \pm 4.9$ & $2 \mathrm{~h}, 110^{\circ} \mathrm{C} ; 14 \mathrm{~h}, 140^{\circ} \mathrm{C}$ & Ciba-Geigy \\
\hline ㅁ $76.5 \pm 5.9$ & $2 \mathrm{~h}, 110^{\circ} \mathrm{C} ; 14 \mathrm{~h}, 140^{\circ} \mathrm{C}$ & Ciba-Geigy \\
\hline
\end{tabular}

Comments: Sample (0) was too flexible for testing at a dose of $5 \times 10^{6} \mathrm{~Gy}$.

Figure A.2-68. Flexural strength at $295 \mathrm{~K}$ of bisphenol A resin with and without filler after ambient reactor irradiation. Supplementary Table A.2-2. Data from Schönbacher and Stolarz-Iźycka [1979]. 


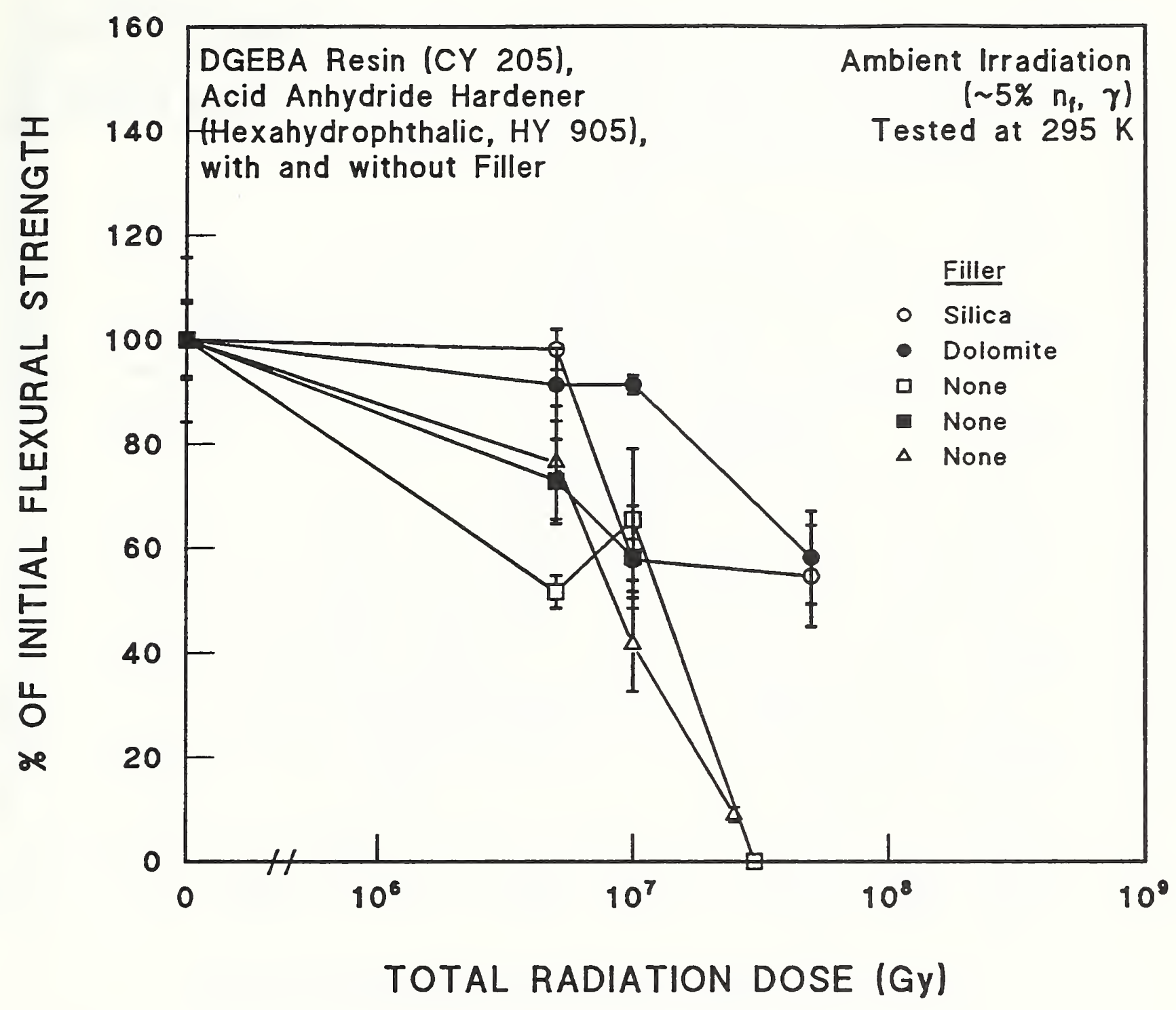

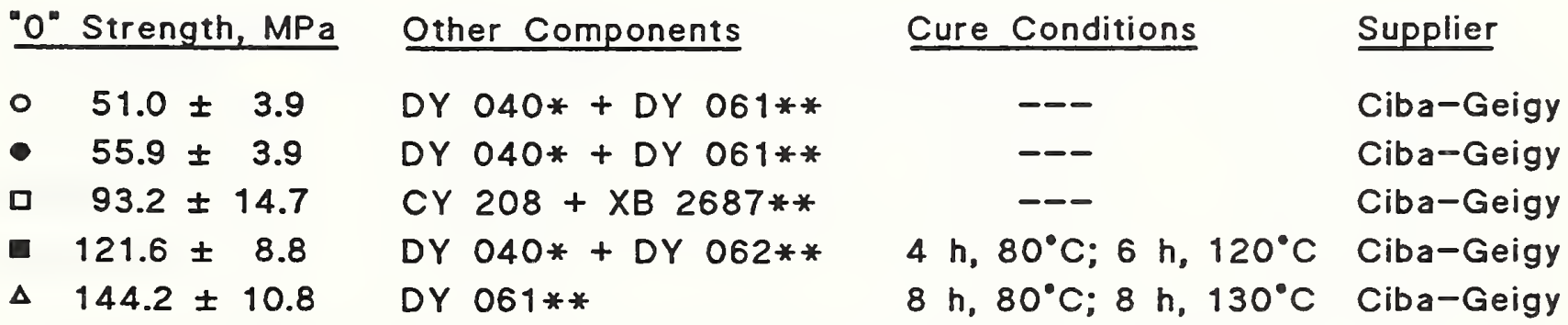

Comments: Sample () broke after irradiation at a dose of $3 \times 10^{7} \mathrm{~Gy}$. * Flexibilizer ** Accelerator

Figure A.2-69. Flexural strength at $295 \mathrm{~K}$ of DGEBA resin, acid anhydride hardener, with and without filler after ambient reactor irradiation. Supplementary Table A.22. Data from Schönbacher and Stolarz-Iżycka [1979]. 


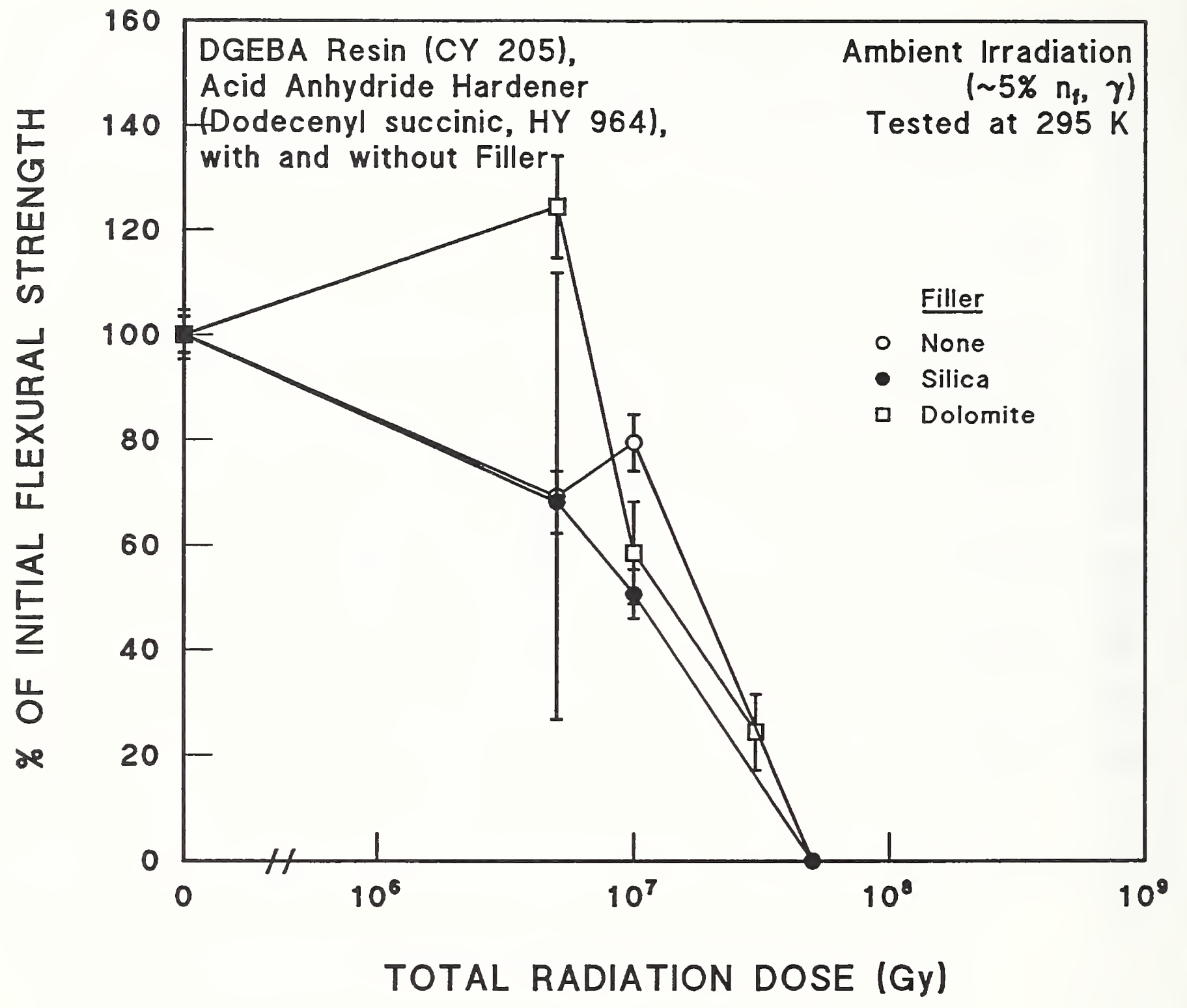

"O" Strength, MPa

Other Components Cure Conditions Supplier

- $71.6 \pm 0.98$

DY $040 *+$ DY $064 * * 40 \mathrm{~h}, 75^{\circ} \mathrm{C}$

Ciba-Gèigy

- $83.4 \pm 2.9$

DY $040 *+$ DY $064 * 40 \mathrm{~h}, 75^{\circ} \mathrm{C}$

Ciba-Geigy

ㅁ $40.2 \pm 1.9$

DY $040 *+$ DY $064 * * 40 \mathrm{~h}, 75^{\circ} \mathrm{C}$

Ciba-Geigy

Comments: Samples $(0, \bullet)$ broke after irradiation at a dose of $5 \times 10^{7} \mathrm{~Gy}$.

* Flexibilizer

* Accelerator

Figure A.2-70. Flexural strength at $295 \mathrm{~K}$ of DGEBA resin, acid anhydride hardener, with and without filler after ambient reactor irradiation. Supplementary Table A.22. Data from Schönbacher and Stolarz-Iżycka [1979]. 


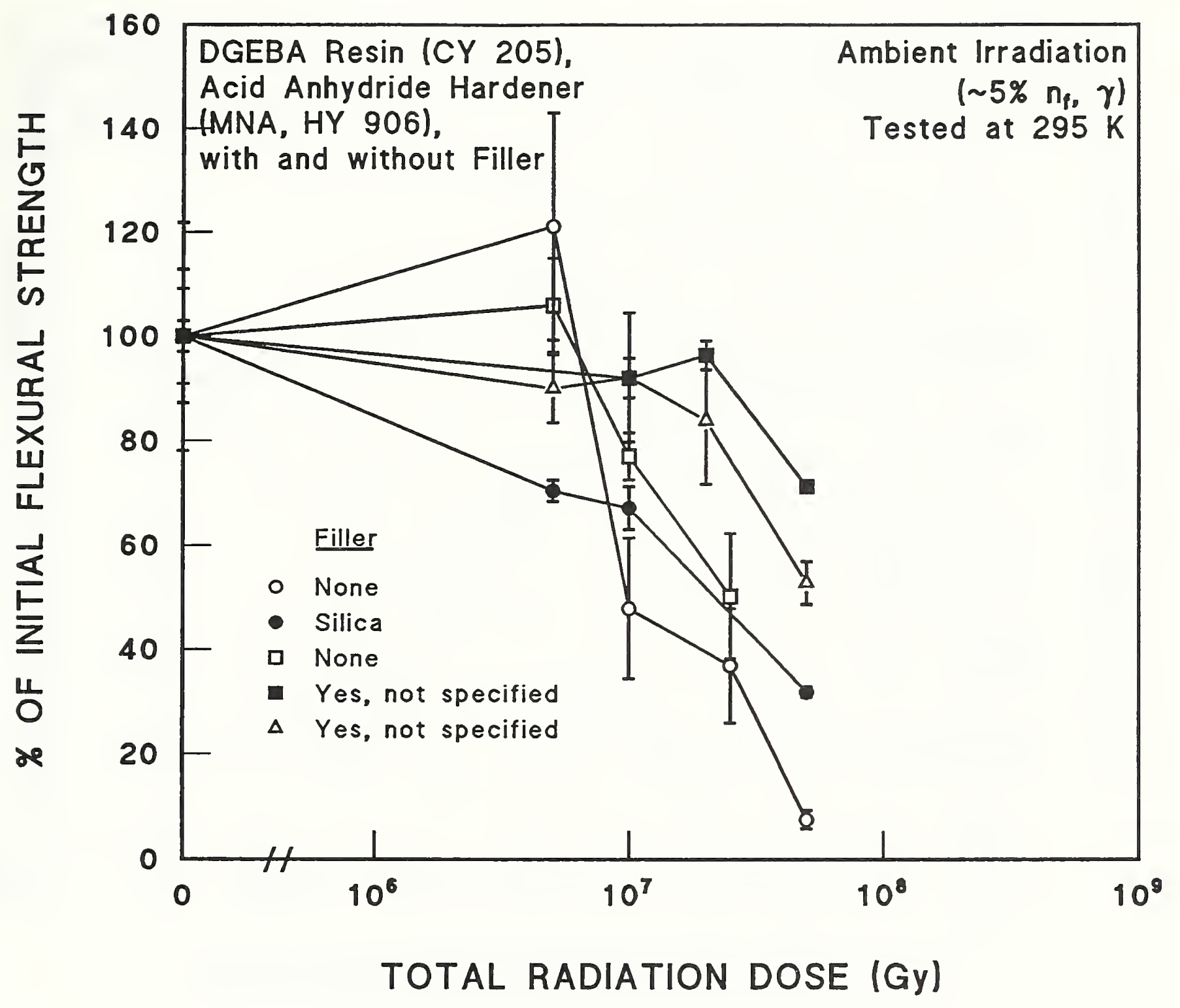

"O" Strength, MPa

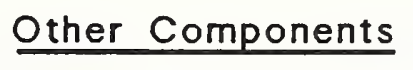

Cure Conditions

Supplier

- $116.7 \pm 25.5$

DY 064*

- $\quad 96.1 \pm 2.9$

DY 061*

$24 \mathrm{~h}, 150^{\circ} \mathrm{C}$

Ciba-Geigy

$64.9 \pm 5.9$

DY 061*

- -

Ciba-Geigy

DMNA

Ciba-Geigy

- $312.9 \pm 2.9$

$---$

Rutherford

Workshop

$\Delta \quad 436.5 \pm 55.9$

-

$--$

Lintott

\section{* Accelerator}

Figure A.2-71. Flexural strength at $295 \mathrm{~K}$ of DGEBA resin, MNA hardener, with and without filler after ambient reactor irradiation. Supplementary Table A.2-2. Data from Schönbacher and Stolarz-Iżycka [1979]. 


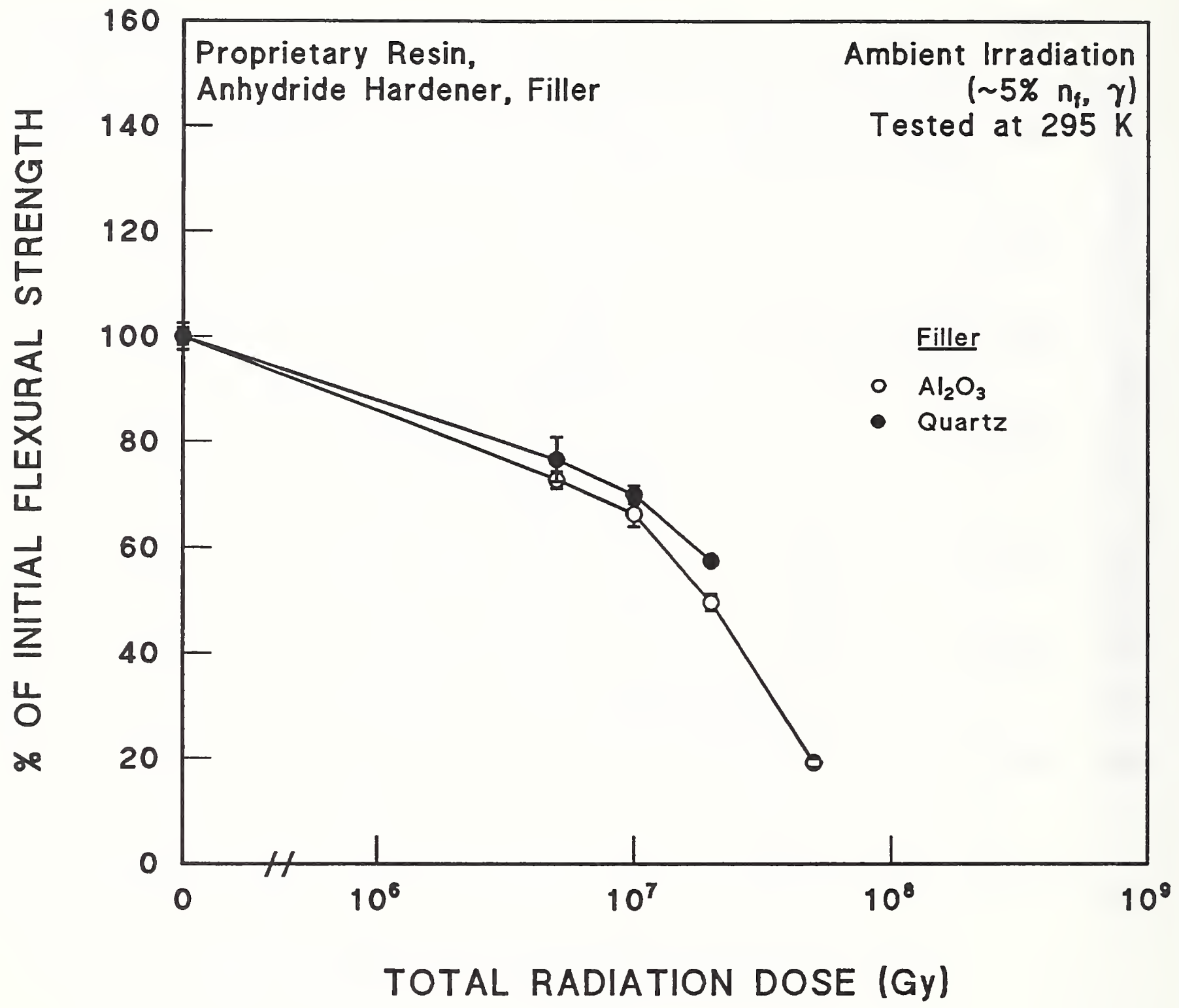

\begin{tabular}{|c|c|}
\hline "O" Strength, MPa & Supplier \\
\hline$\circ \quad 122.6 \pm 2.0$ & BBC Mannheim \\
\hline - $\quad 117.7 \pm 2.9$ & BBC Mannheim \\
\hline
\end{tabular}

Figure A.2-72. Flexural strength at $295 \mathrm{~K}$ of proprietary resin, anhydride hardener, with silica and quartz fillers, after ambient reactor irradiation. Supplementary Table A.2-2. Data from Schönbacher and Stolarz-Iżycka [1979]. 


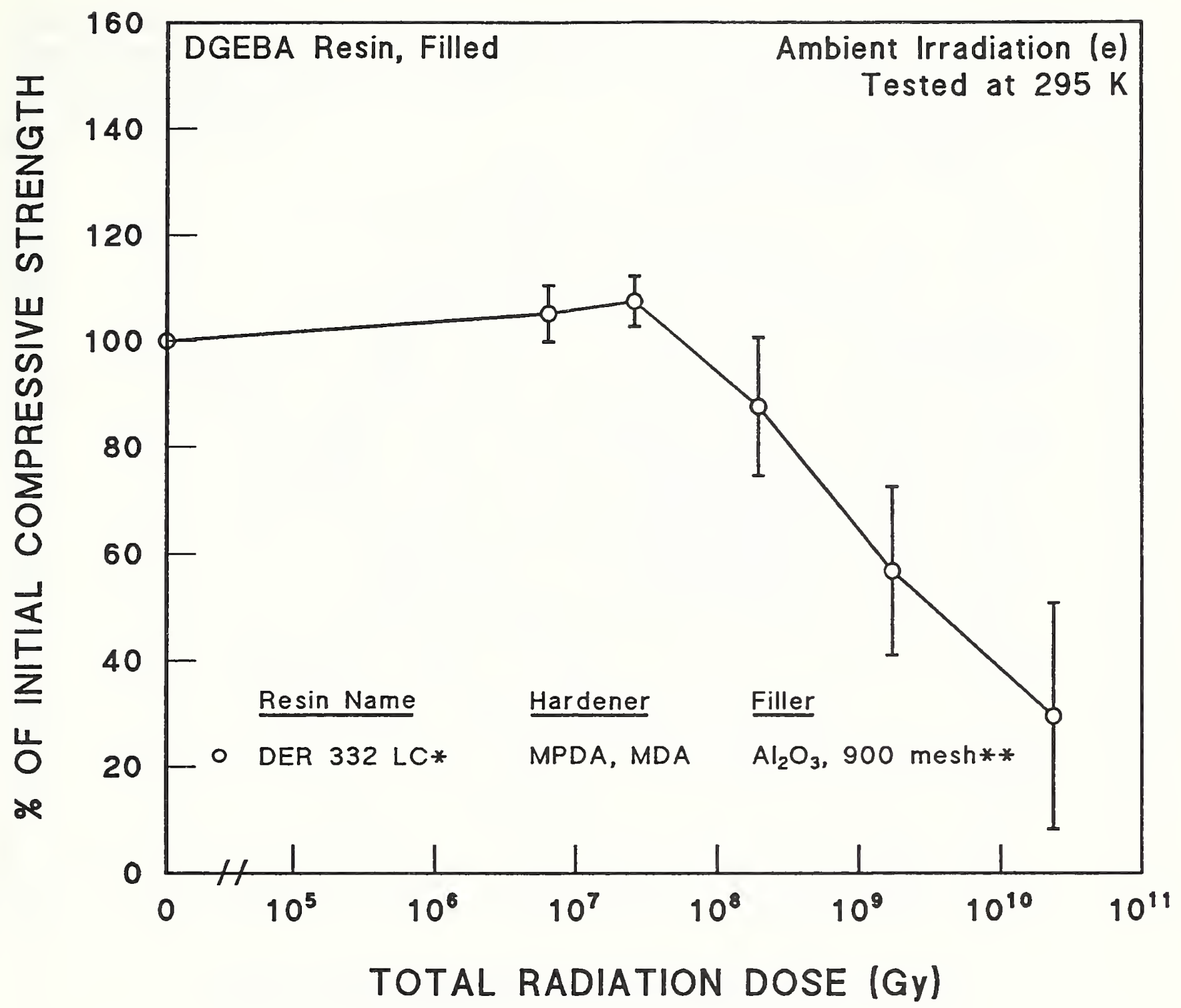

"O" Strength, MPa

- $338.1 \pm 0.0$
Additive

Cab-o-sil
Supplier

DOW

* Low chlorine

* 100-120 parts to 100 parts per mass of resin-hardener system

Figure A.2-73. Compressive strength at $295 \mathrm{~K}$ of DGEBA resin with alumina filler after ambient electron irradiation. Supplementary Table A.2-6. See text for discussion of possible error in dose determination. Data from Brechna [1965]. 


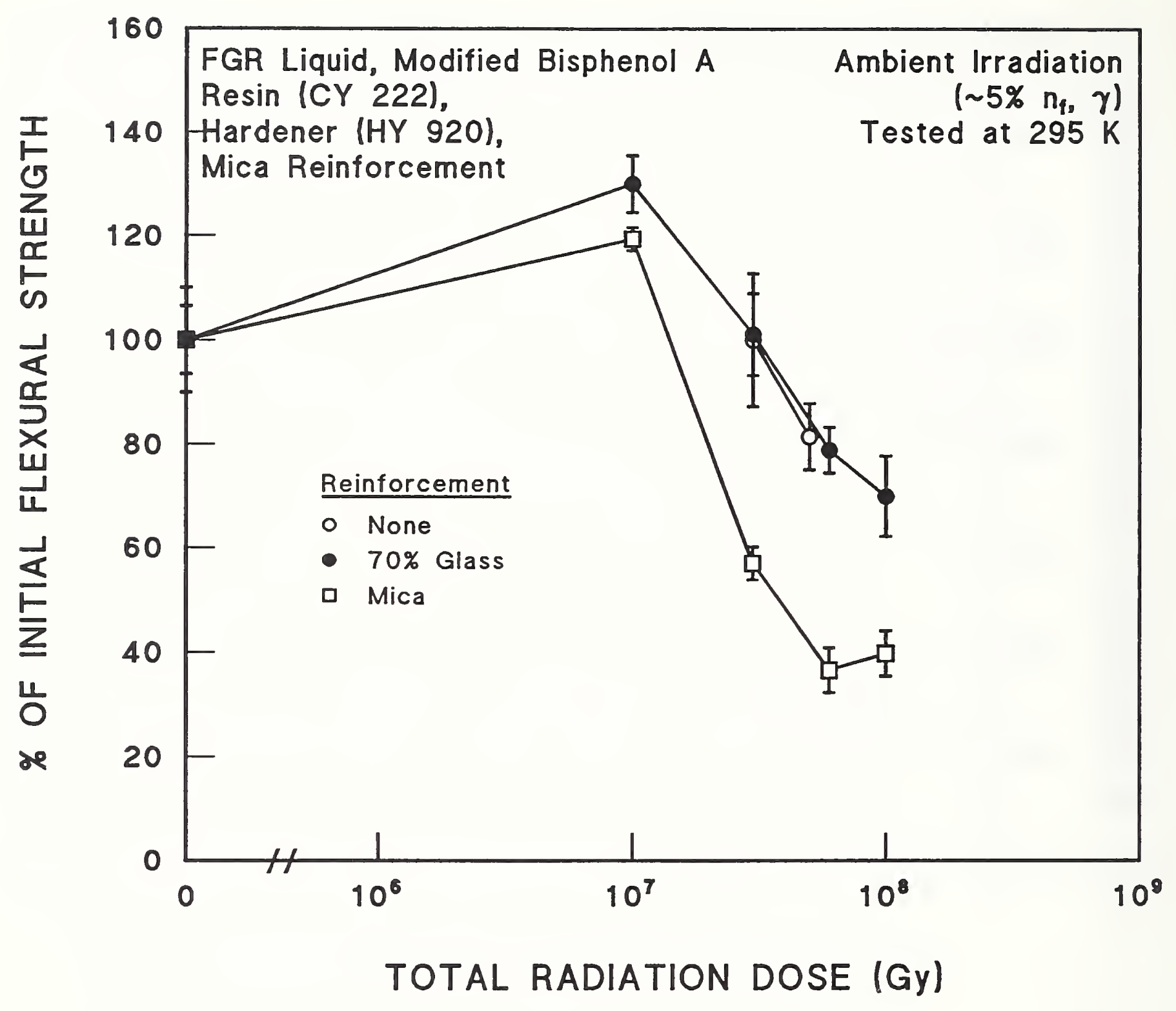

Supplier

BBC Baden

BBC Baden

Micafil

* Strength at $3 \times 10^{7}$ Gy was used as the initial value because specimens were too flexible for testing at doses of $0,5 \times 10^{6}$, \& $1 \times 10^{7}$ Gy.

Figure A.2-74. Flexural strength at $295 \mathrm{~K}$ of neat and glass or mica reinforced bisphenol A resin after ambient reactor irradiation. Supplementary Table A.2-2. Data from Schönbacher and Stolarz-Iżycka [1979]. 


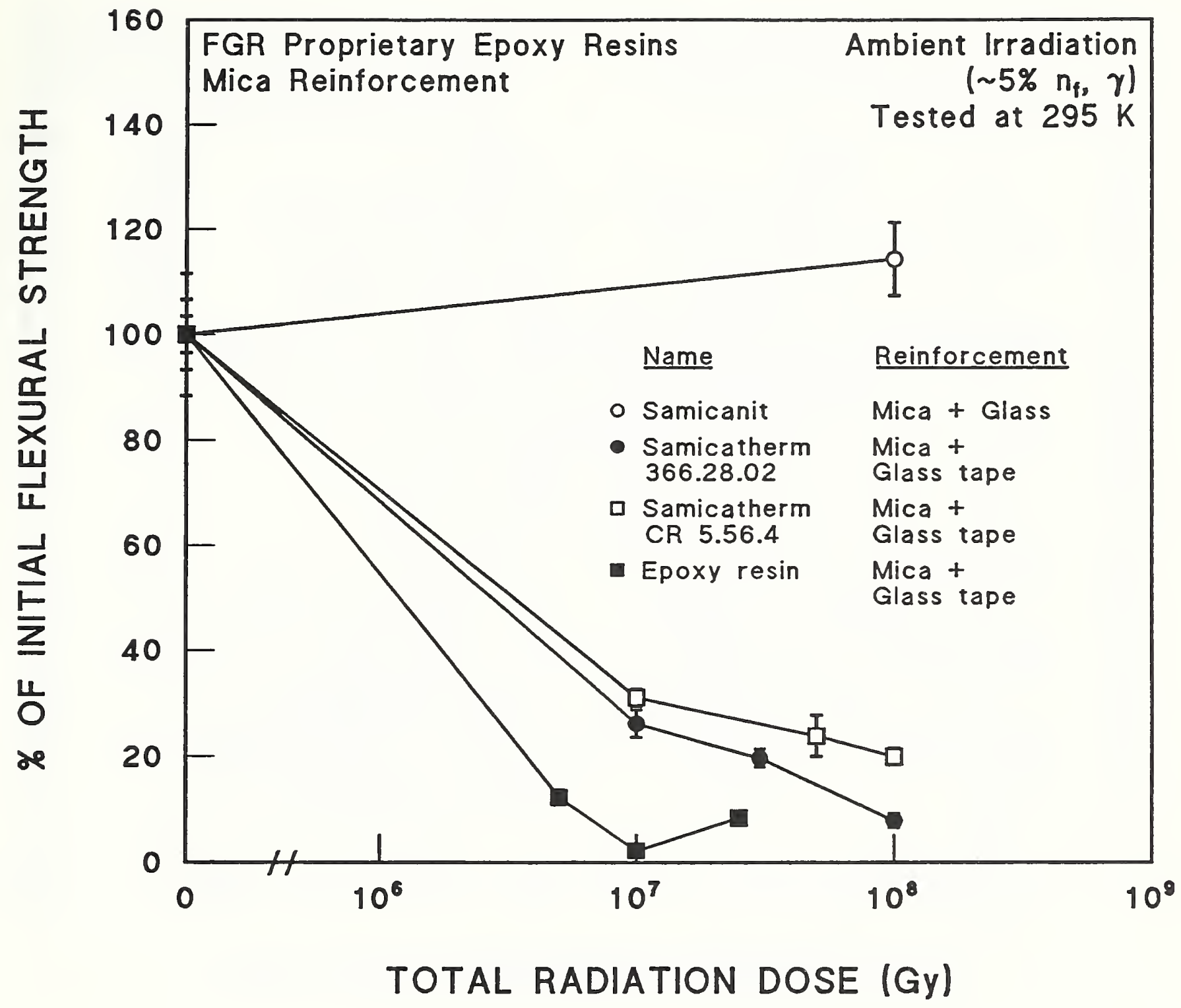

"O" Strength, MPa

○ $160.6 \pm 18.6$

- $224.6 \pm 7.8$

ㅁ $176.6 \pm 11.8$

- $299.2 \pm 19.6$
Supplier

Isola

Isola

Isola

Ciba-Geigy

Figure A.2-75. Flexural strength at $295 \mathrm{~K}$ of FGR/mica proprietary resins after ambient reactor irradiation. Supplementary Table A.2-2. Data from Schönbacher and Stolarz-Iźycka [1979]. 


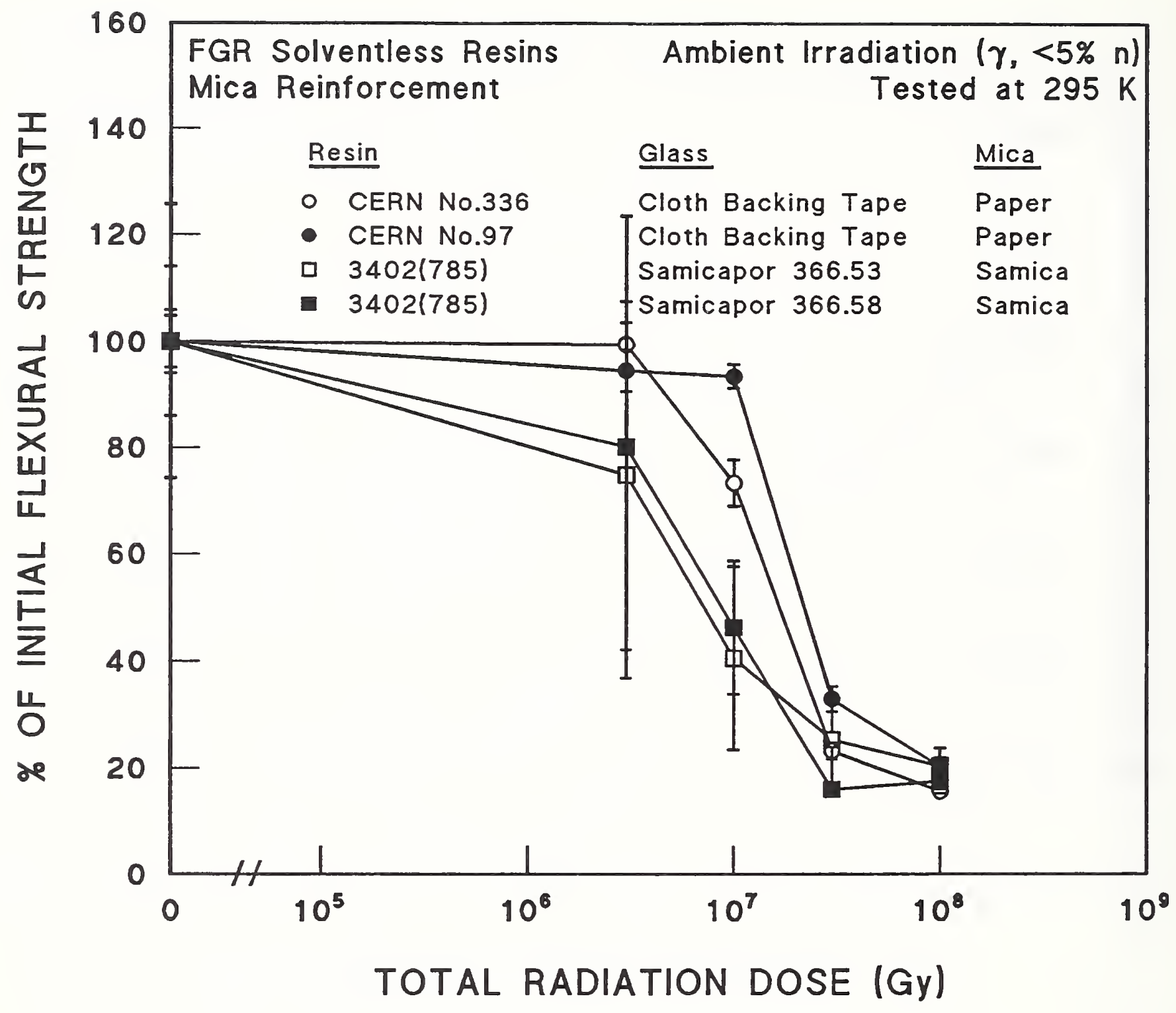

"O" Strength MPa Insulation

$\begin{array}{crc}\circ & 210.4 \pm 12.5 & \text { Micadur/Orlidur } \\ - & 186.0 \pm 9.2 & \text { Orlitherm-S } \\ & & \\ \text { 口 } 187.0 \pm 48.1 & --- \\ \text { - } 164.5 \pm 23.1 & ---\end{array}$

Application

HV machine insulation

Conductor insulationmagnets

VPI HV

VPI HV
Supplier

BBC, Baden

$B B C$, Badèn

Isola

Isola

Figure A.2-76. Flexural strength at $295 \mathrm{~K}$ of FGR/mica solventless resins after ambient reactor irradiation. Supplementary Table A.2-7. Data from Lipták et al. [1985]. 


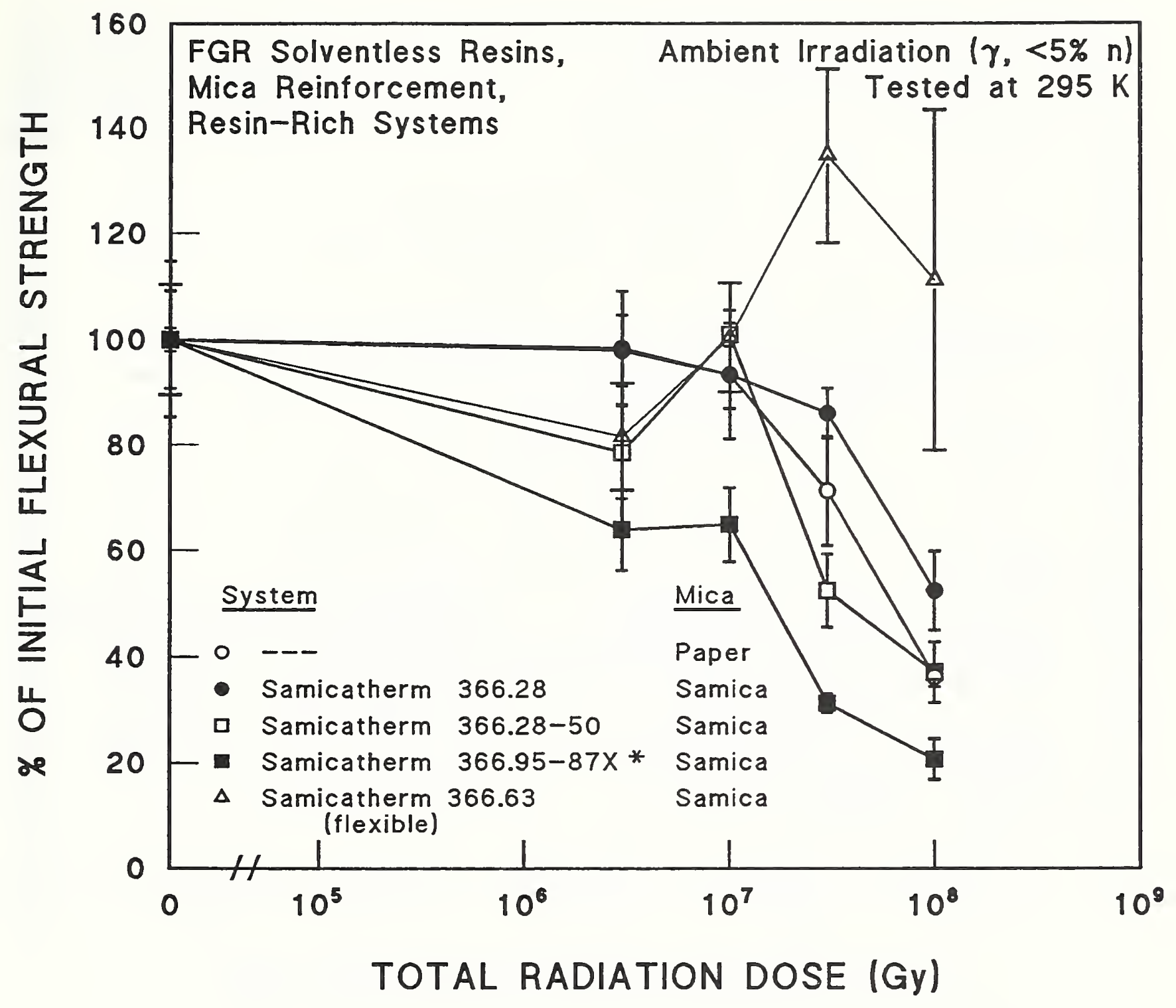

\begin{tabular}{|c|c|c|c|c|}
\hline "O" & Strength $\mathrm{MPa}$ & Glass & Application & Supplier \\
\hline$\circ$ & $114.0 \pm 10.5$ & Cloth Tape & HV - general & BBC, Baden \\
\hline - & $127.0 \pm 1.2$ & Cloth Tape & HV - general & Isola \\
\hline 口 & $128.8 \pm 2.8$ & Cloth Tape & HV - general & Isola \\
\hline E & $119.0 \pm 17.5$ & Cloth Tape & HV - general & Isola \\
\hline$\Delta$ & $89.1 \pm 9.3$ & Cloth Tape & HV - general & Isola \\
\hline
\end{tabular}

* Cycloaliphatic

Figure A.2-77. Flexural strength at $295 \mathrm{~K}$ of FGR/mica solventless, resin-rich systems after ambient reactor irradiation. Supplementary Table A.2-7. Data from Lipták et al. [1985]. 


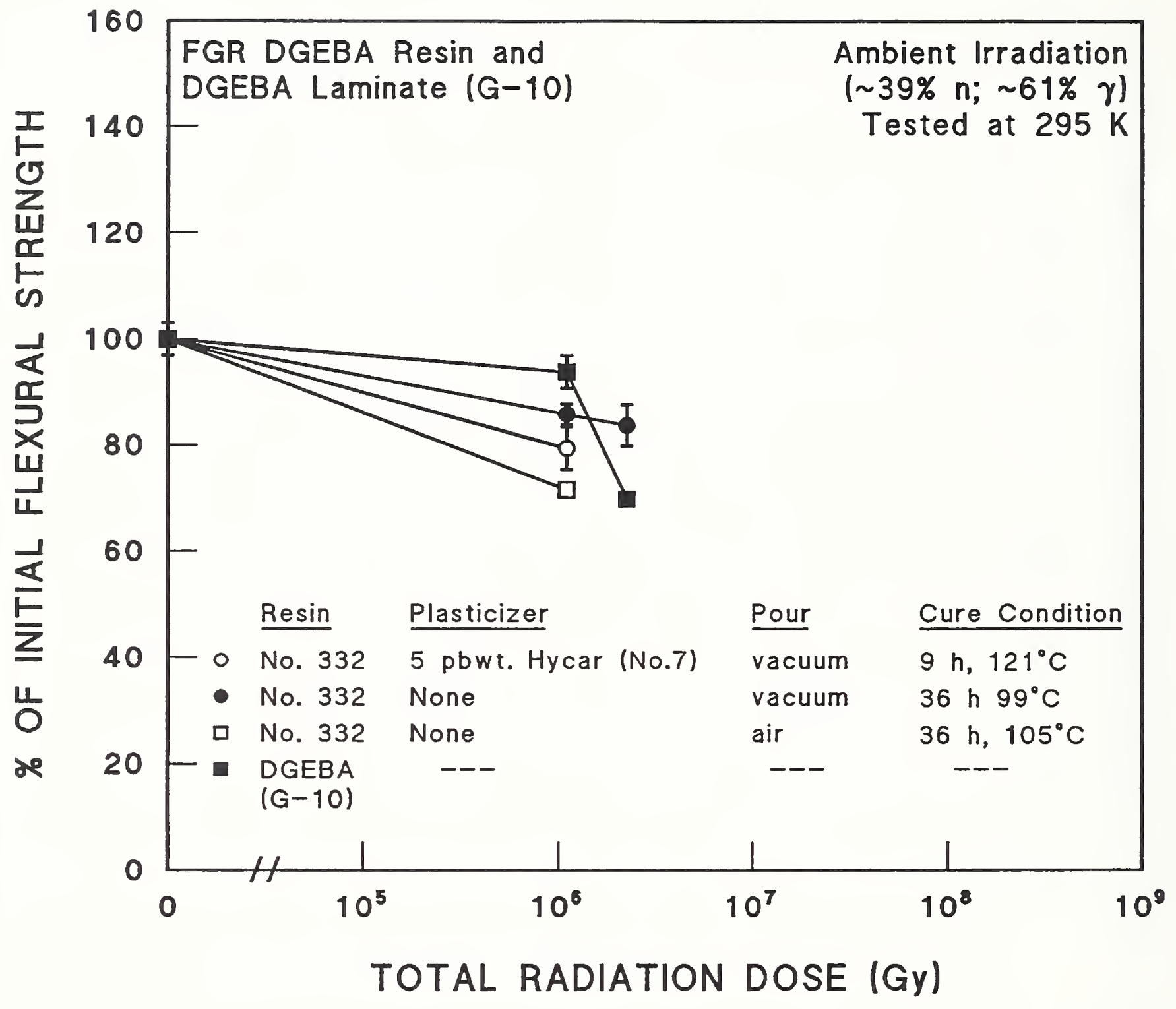

"O" Strength, MPa

- $141.0 \pm 0.0$

- $141.0 \pm 0.0$

ㅁ $141.0 \pm 0.0$

- $291.0 \pm 9.0$
Hardener

NMA

NMA

NMA

DICY

\section{Accelerator}

BDMA

BDMA

BDMA
Supplier

DOW, Goodrich

DOW

DOW

Figure A.2-78. Flexural strength at $295 \mathrm{~K}$ of FGR DEGBA resin and DGEBA laminate (G10) after ambient reactor irradiation. Supplementary Table A.2-8. Data from Imel et al. [1979]. Total dose estimated from conversion provided by Imel et al. and aim glass fraction. 


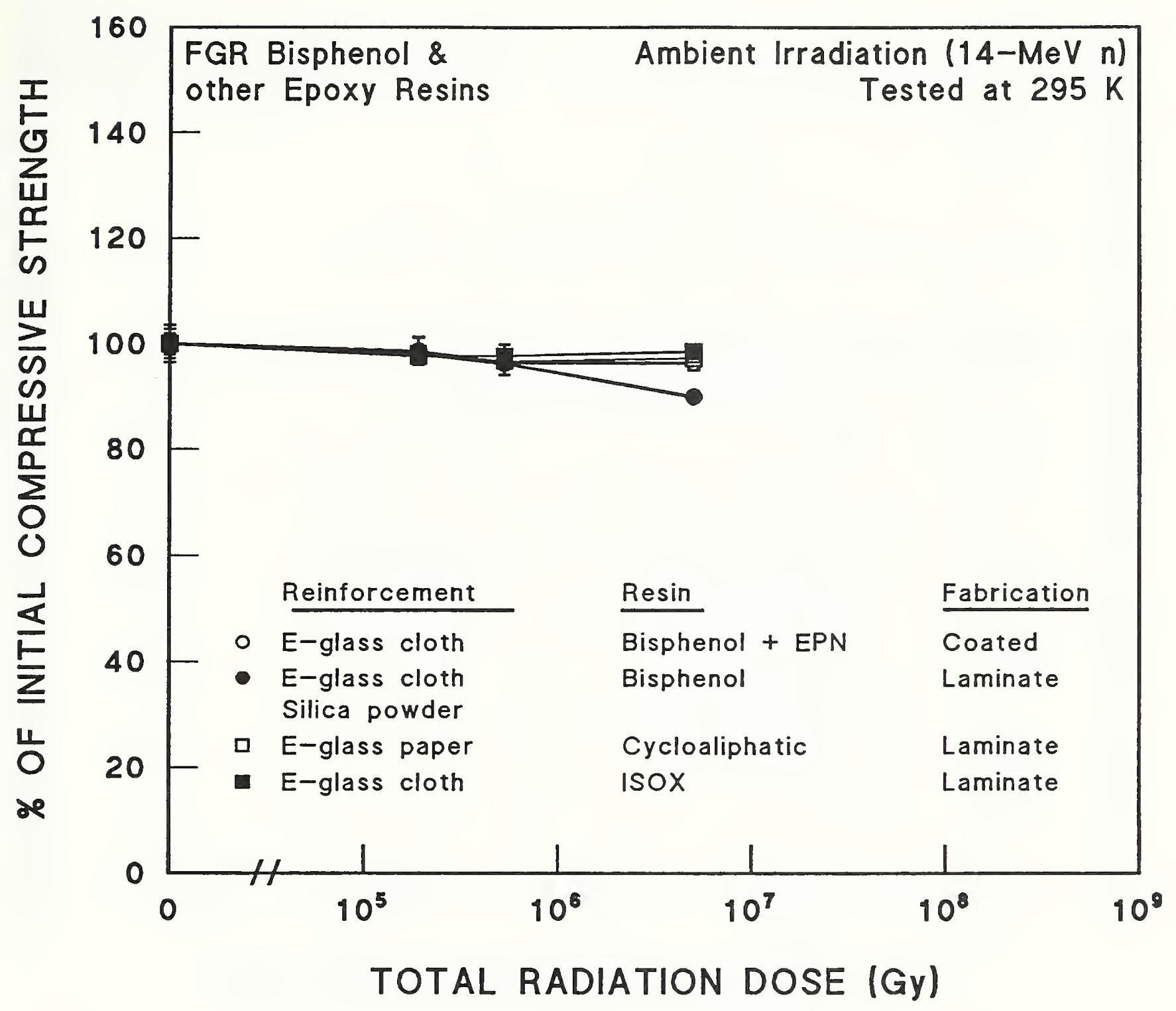

"O" Strength, MPa

- $427.0 \pm 14.8$

- $557.0 \pm 5.0$

$562.0 \pm 15.6$

- $531.0 \pm 10.5$
Hardener

Polyamide amine

Anhydride

Anhydride

Isocyanate
Supplier

Hitachi Co., Ltd.

Hitachi Co., Ltd.

Hitachi Co., Ltd.

Hitachi Co., Ltd.

Comments: Used for Japanese coil in LCT.

Figure A.2-79. Compressive strength at $295 \mathrm{~K}$ of FGR bisphenol $\mathrm{A}$ and other resins after ambient 14-MeV neutron irradiation. Supplementary Table A.2-9. Data from Iida and Sumita [1983]. 

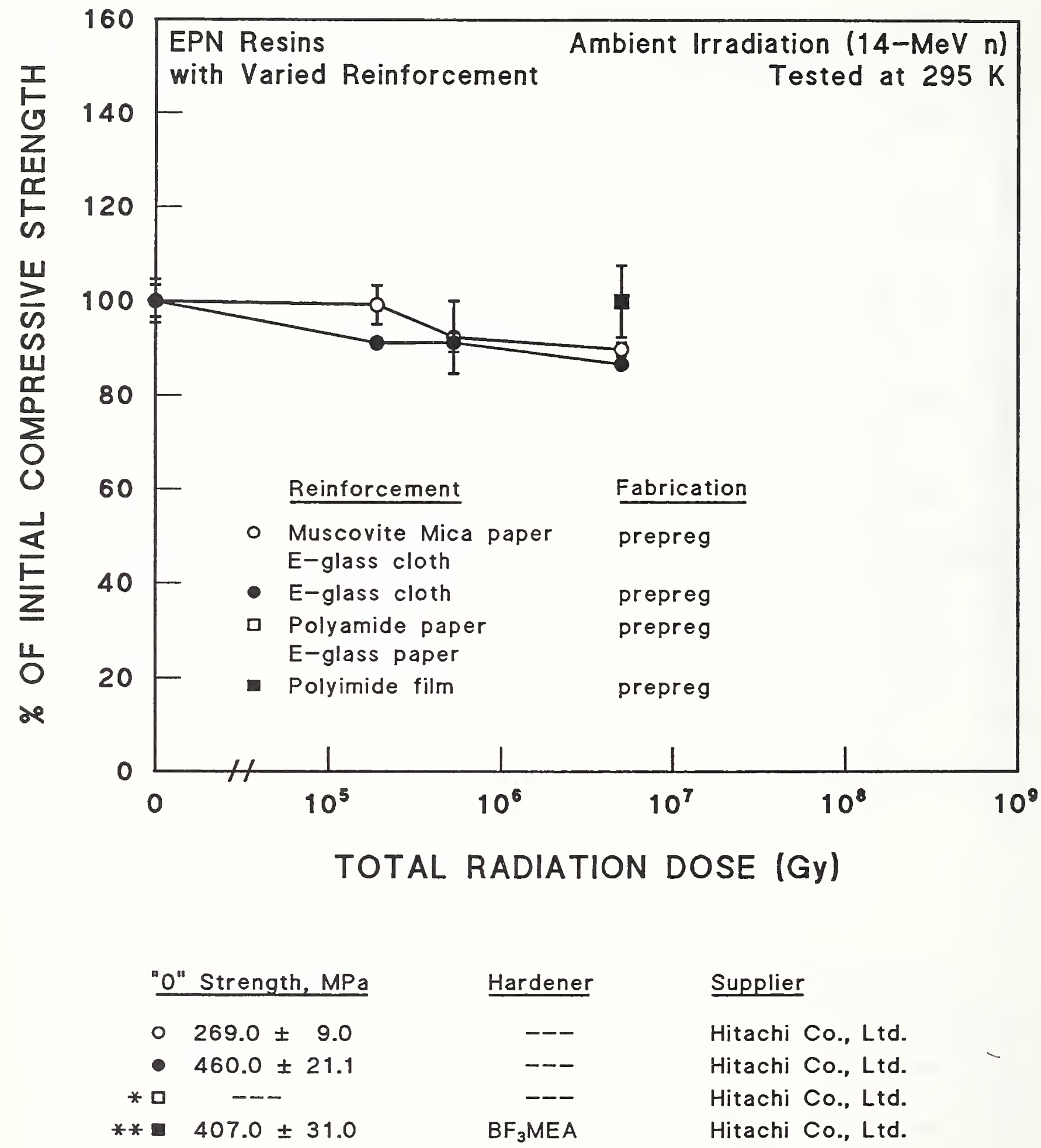

Supplier

Hitachi Co., Ltd.

Hitachi Co., Ltd.

Hitachi Co., Ltd.

Hitachi Co., Ltd.

* Deformed without rupturing

* Strength at $5 \times 10^{6} \mathrm{G}$ y was used as the initial value, owing to deformation.

Figure A.2-80. Compressive strength at $295 \mathrm{~K}$ of EPN resins with varied reinforcement, pre-preg fabrication after ambient $14 \mathrm{-MeV}$ neutron irradiation. Supplementary Table A.2-9. Data from Iida and Sumita [1983]. 


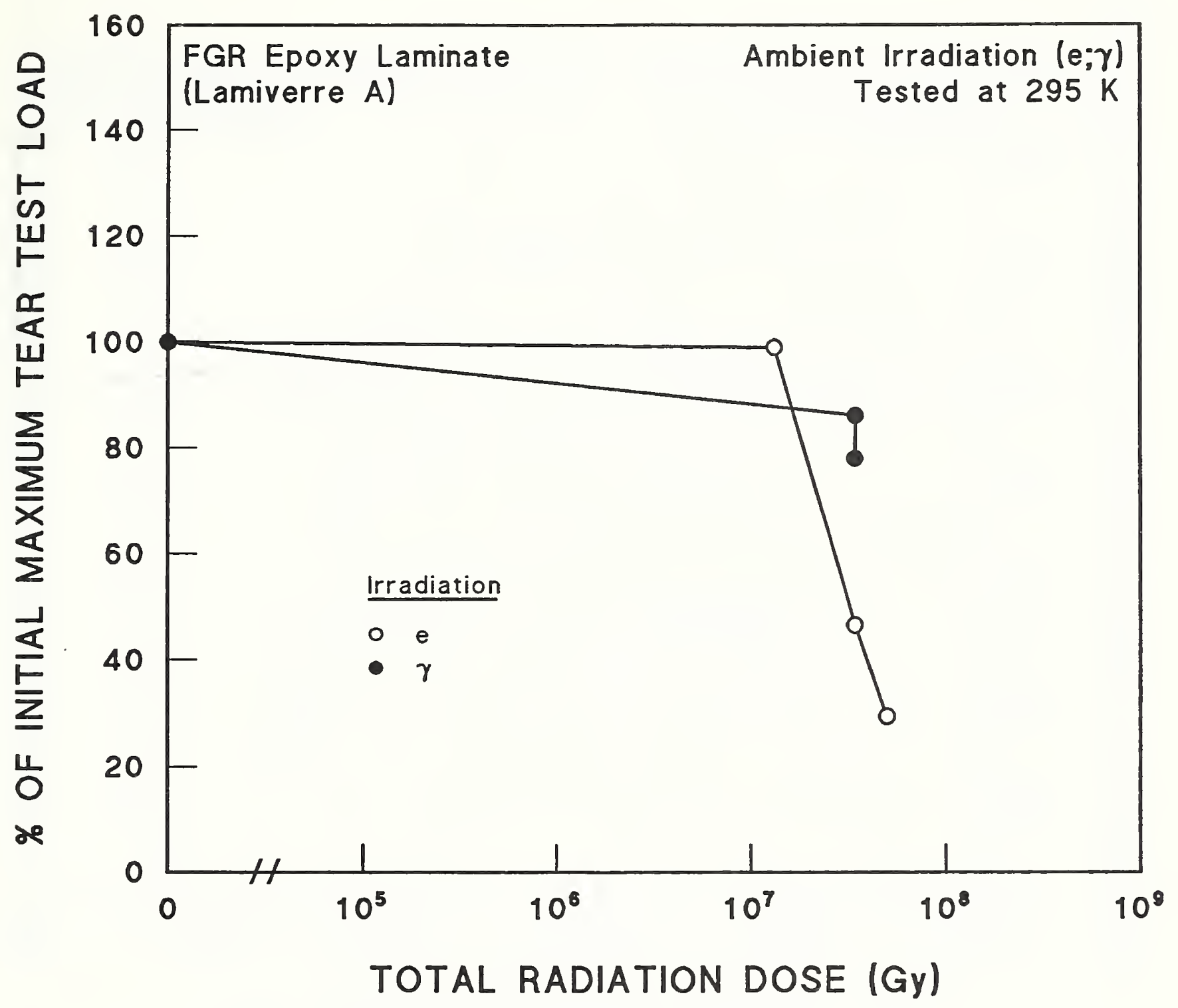

\begin{tabular}{|c|c|c|c|c|}
\hline "O" Load, N & Weave & Finish & Glass & Supplier \\
\hline 84.1 & Plain & Silane & $62-65$ mass $\%$ & -- \\
\hline 84.1 & Plain & Silane & $62-65$ mass $\%$ & --- \\
\hline
\end{tabular}

Figure A.2-81. Maximum tear test load (see text) at $295 \mathrm{~K}$ of FGR epoxy laminate (Lamiverre A) after ambient electron or gamma irradiation. Supplementary Table A.210. Data from Nishiura et al. [1988a]. 


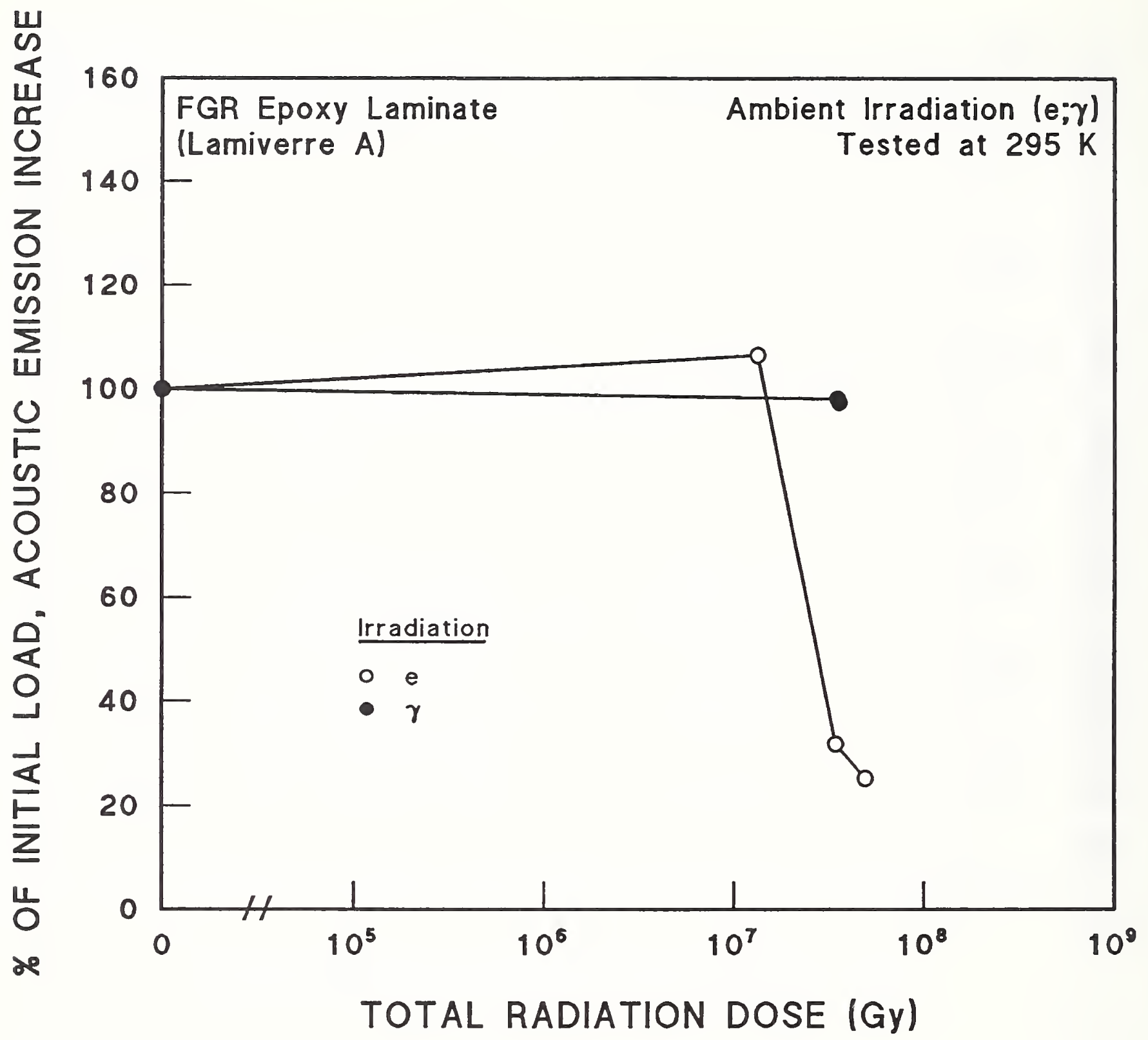

\begin{tabular}{|c|c|c|c|}
\hline "O" Load, N & Weave & Finish & Glass \\
\hline 68.8 & Plain & Silane & $62-65$ mass $\%$ \\
\hline 68.8 & Plain & Silane & $62-65$ mass \\
\hline
\end{tabular}

Figure A.2-82. Acoustic emission increase (see text) at $295 \mathrm{~K}$ of FGR epoxy laminate (Lamiverre A) after ambient electron or gamma irradiation. Supplementary Table A.210. Data from Nishiura et a1. [1988a]. 


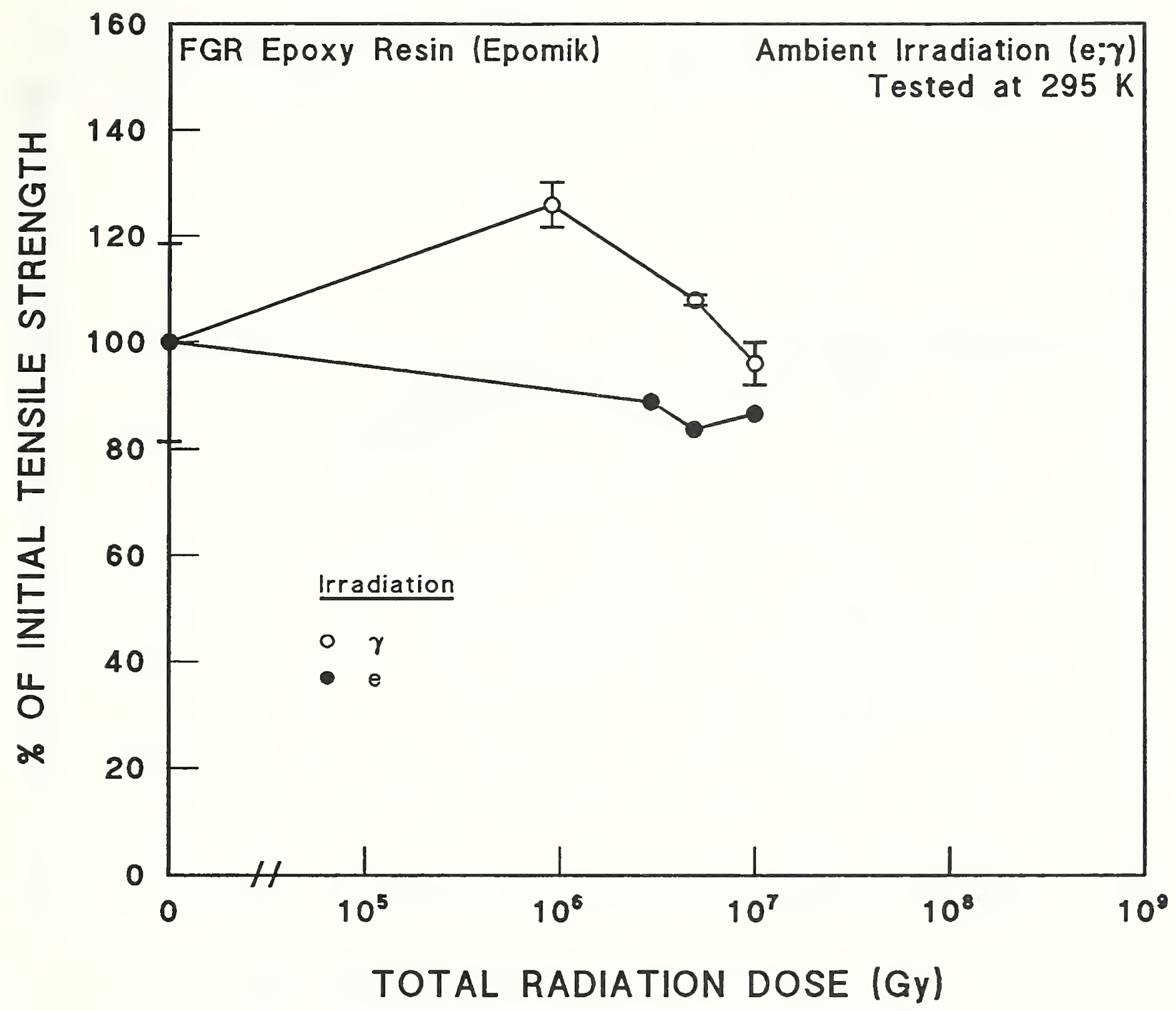

\begin{tabular}{lll} 
"O" Strength, MPa & Hardener & Supplier \\
\hline $0172.7 \pm 32.1$ & Jefermin-D230,30 & Mitubishiyuka Co., Ltd. \\
- $261.3 \pm 0.0$ & Jefermin-D230,30 & Mitubishiyuka Co., Ltd.
\end{tabular}

Figure A.2-83. Tensile strength at $295 \mathrm{~K}$ of FGR epoxy resin (Epomik) after ambient electron or gamma irradiation. Supplementary Table A.2-10. Data from Nishiura et al. [1988b]. 


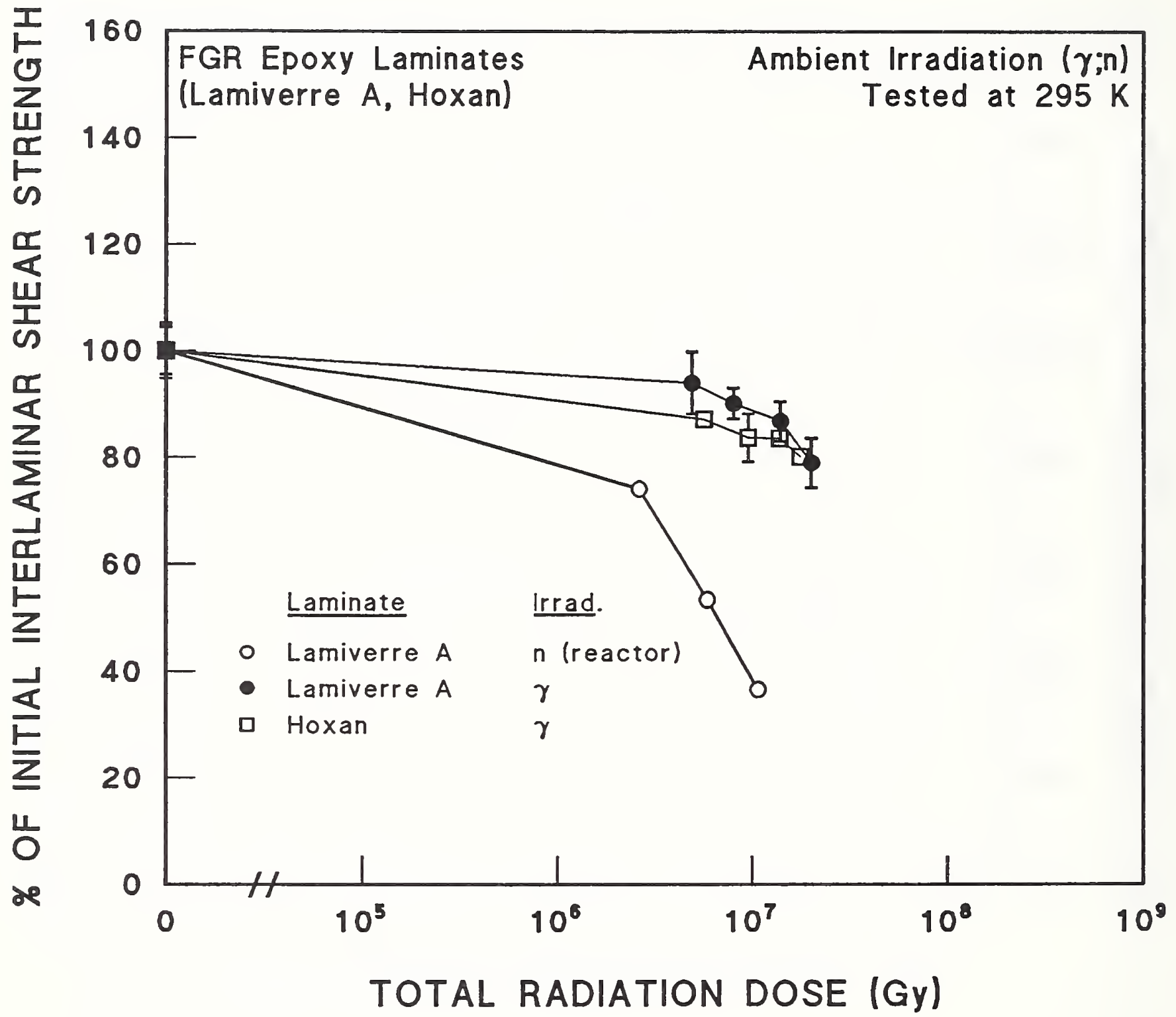

\begin{tabular}{|c|c|c|c|c|c|c|c|}
\hline "O" & Strength, MPa & Weave & Finish & Glass & & & Supplie \\
\hline 0 & $63.7 \pm 0.0$ & Plain & Silane & $60-62$ & mass & $\%$ & -- \\
\hline . & $63.7 \pm 2.8$ & Plain & Silane & $60-62$ & mass & $\%$ & --- \\
\hline ב & $55.3 \pm 2.9$ & Plain & Silane & $60-62$ & mass & $\%$ & - - \\
\hline
\end{tabular}

Figure A.2-84. Interlaminar shear strength at $295 \mathrm{~K}$ of FGR epoxy laminates (Lamiverre A, Hoxan) after ambient neutron or gamma irradiation. Supplementary Table A.2-11. Data from Nishijima et al. [1988]. 


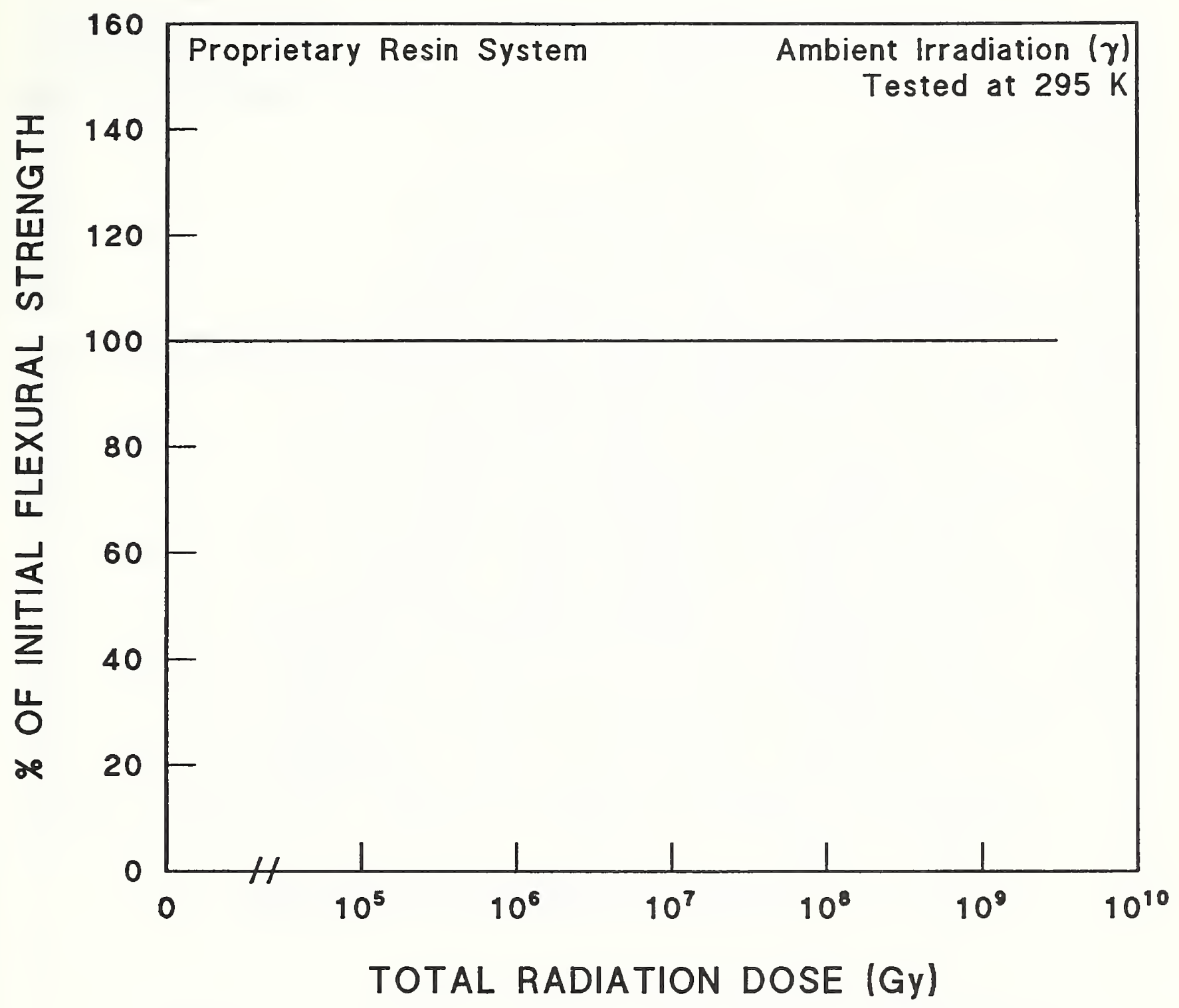

\author{
"O" Strength, MPa $\quad$ Supplier \\ * $138.0 \quad$ Siemens AG
}

* Stress at 3.5\% Strain of the outer fiber (no break)

Comments: Numerical Strength data not presented for irradiation specimens. Resin used for European Comminity coil in LCT.

Figure A.2-85. Flexural strength at $295 \mathrm{~K}$ of proprietary resin after ambient gamma irradiation. Supplementary Table A.2-12. Data from Hacker et al. [1984] 


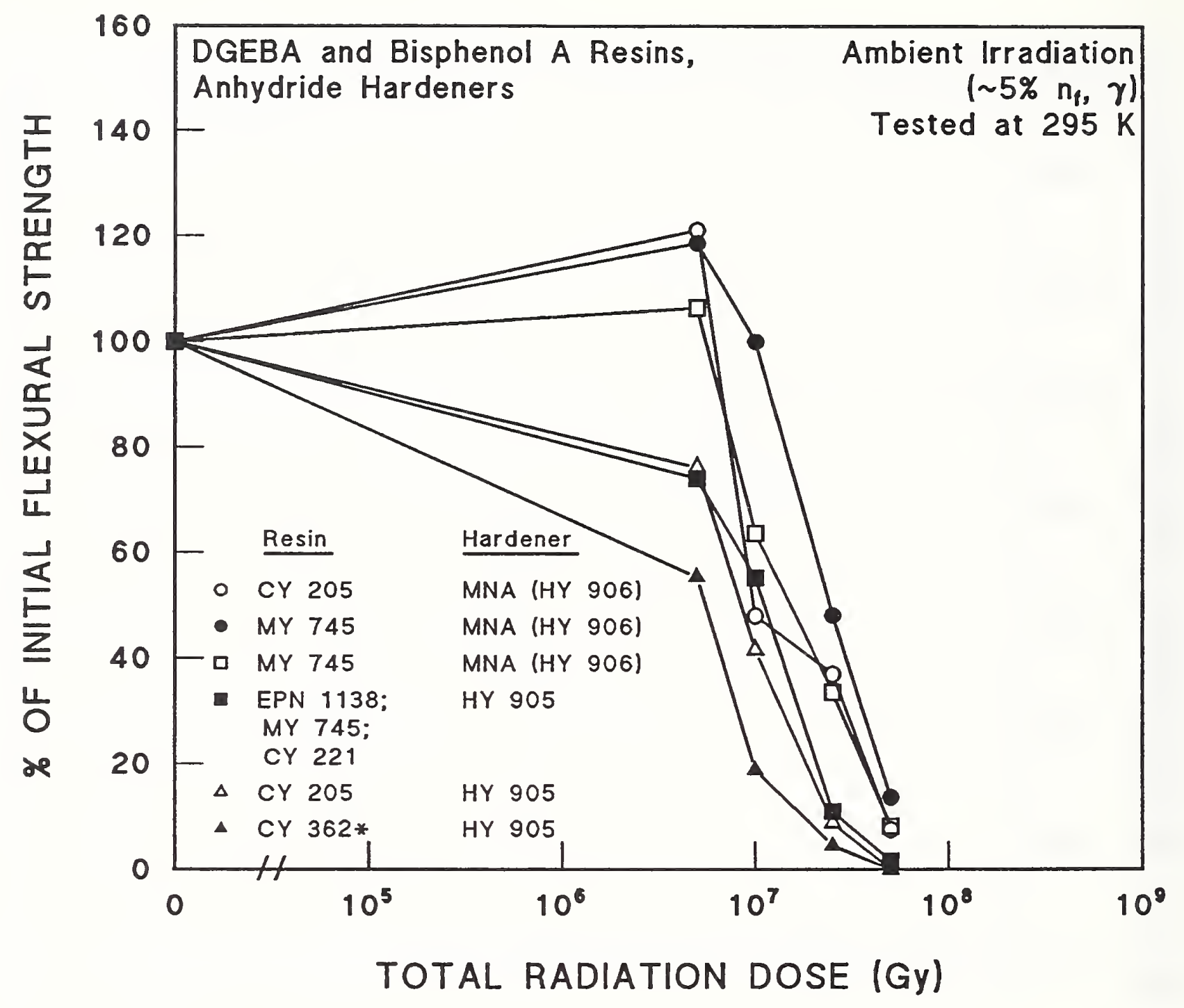

\begin{tabular}{|c|c|c|c|c|c|}
\hline & "O" & Strength, MPa & Accelerator & Cure & Supplier \\
\hline & ○ & 120.0 & DY 064 & $24 \mathrm{~h}, 150^{\circ} \mathrm{C}$ & Ciba-Geigy \\
\hline & - & 100.0 & XB $2687 * *$ & $5 \mathrm{~h}, 110^{\circ} \mathrm{C}: 16 \mathrm{~h}, 125^{\circ} \mathrm{C}$ & Ciba-Geigy \\
\hline & $\square$ & 110.0 & XB 2687 & $24 \mathrm{~h}, 150^{\circ} \mathrm{C}$ & Ciba-Geigy \\
\hline & e & 128.0 & XB 2687 & 24 h $120^{\circ} \mathrm{C}$ & Ciba-Geigy \\
\hline *** & $\Delta$ & 147.2 & DY 061 & $8 \mathrm{~h}, 80^{\circ} \mathrm{C} ; 8 \mathrm{~h}, 130^{\circ} \mathrm{C}$ & Ciba-Geigy \\
\hline$* * *$ & $\Delta$ & 132.0 & XB 2687 & $12 \mathrm{~h}, 90^{\circ} \mathrm{C} ; 18 \mathrm{~h}, 140^{\circ} \mathrm{C}$ & Ciba-Geigy \\
\hline
\end{tabular}

Figure A.2-86. Flexural strength at $295 \mathrm{~K}$ of neat DGEBA and bisphenol A resins with anhydride hardeners after ambient reactor irradiation. Supplementary Table A.2-13. Data from Phillips et al. [1981]. 


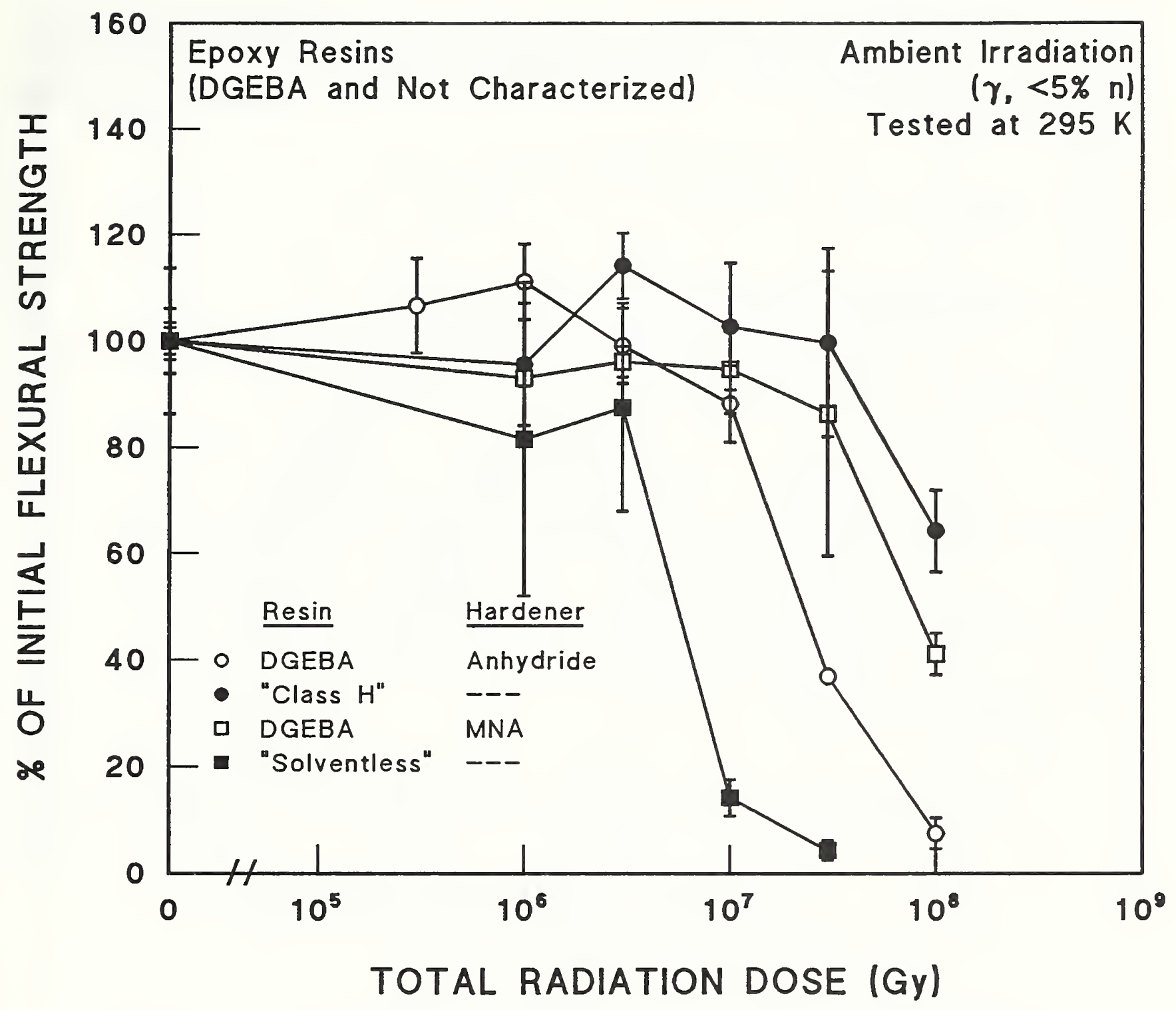

\begin{tabular}{|c|c|c|}
\hline "O" Strength MPa & Additives; Name & Supplier \\
\hline $85.5 \pm 3.0$ & Yes; Micadur & BBC, Baden \\
\hline - $93.8 \pm 5.7$ & Isocyanate; Veridur-K & BBC, Baden \\
\hline $127.4 \pm 3.2$ & Yes; Orlitherm-N & BBC, Baden \\
\hline - $136.2 \pm 18.7$ & --- Art. No. SIB 785 & Isola \\
\hline
\end{tabular}

Figure A.2-87. Flexural strength at $295 \mathrm{~K}$ of neat DGEBA and not characterized resins (includes isocyanate system) after ambient reactor irradiation. Supplementary Table A.2-7. Data from Lipták et al. [1985]. 


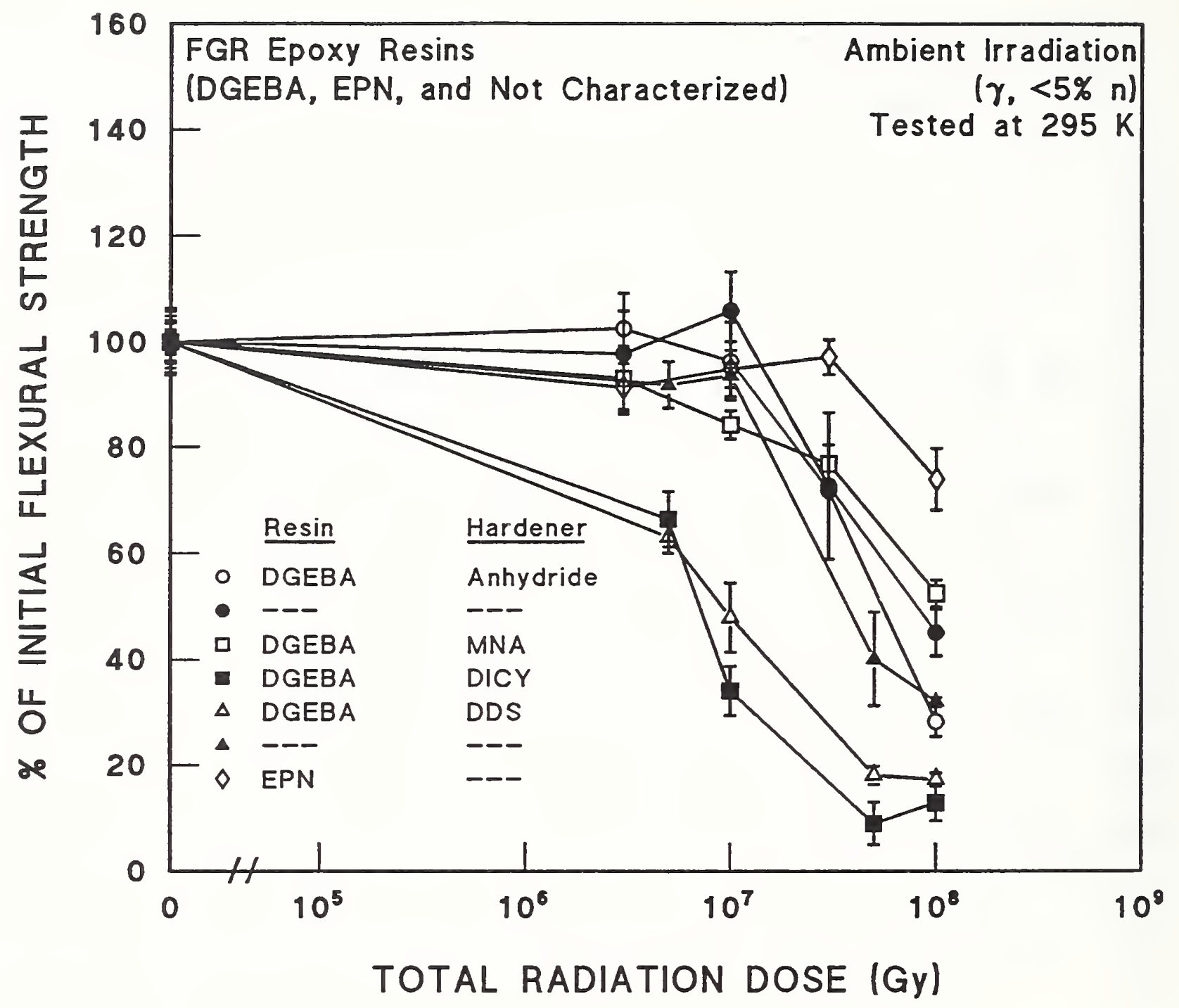

\begin{tabular}{|c|c|c|c|c|}
\hline & Strength MPa & Glass & Additives; Trade Name & Supplier \\
\hline o & $326.4 \pm 20.7$ & Silane Finish & Yes; Micadur & BBC, Baden \\
\hline - & $231.0 \pm 11.4$ & $\begin{array}{l}\text { Thermally } \\
\text { Desized, Type } 5\end{array}$ & --- & BBC, Baden \\
\hline a & 324.0 & $\begin{array}{l}\text { Silane Finish, } \\
\text { Type } 3\end{array}$ & Yes; Orlitherm-D & - Baden \\
\hline - & $559.5 \pm 12.7$ & Cloth & --- ; Vetronit G-10 432.81 & Isola \\
\hline$\Delta$ & $453.0 \pm 16.1$ & Cloth & --- ; Vetronit G-11 432.86 & Isola \\
\hline$\Delta$ & $364.0 \pm 6.8$ & Mat & --- ; Vetonit 541.12 & Isola \\
\hline$\Delta$ & $464.4 \pm 19.0$ & Mat & ---; Delmat 64.841 & Isola \\
\hline
\end{tabular}

Figure A.2-88. Flexural strength at $295 \mathrm{~K}$ of FGR DGEBA, EPN, and not characterized resins (includes Orlitherm systems) after ambient reactor irradiation. Supplementary Table A.2-7. Data from Lipták et al. [1985]. 


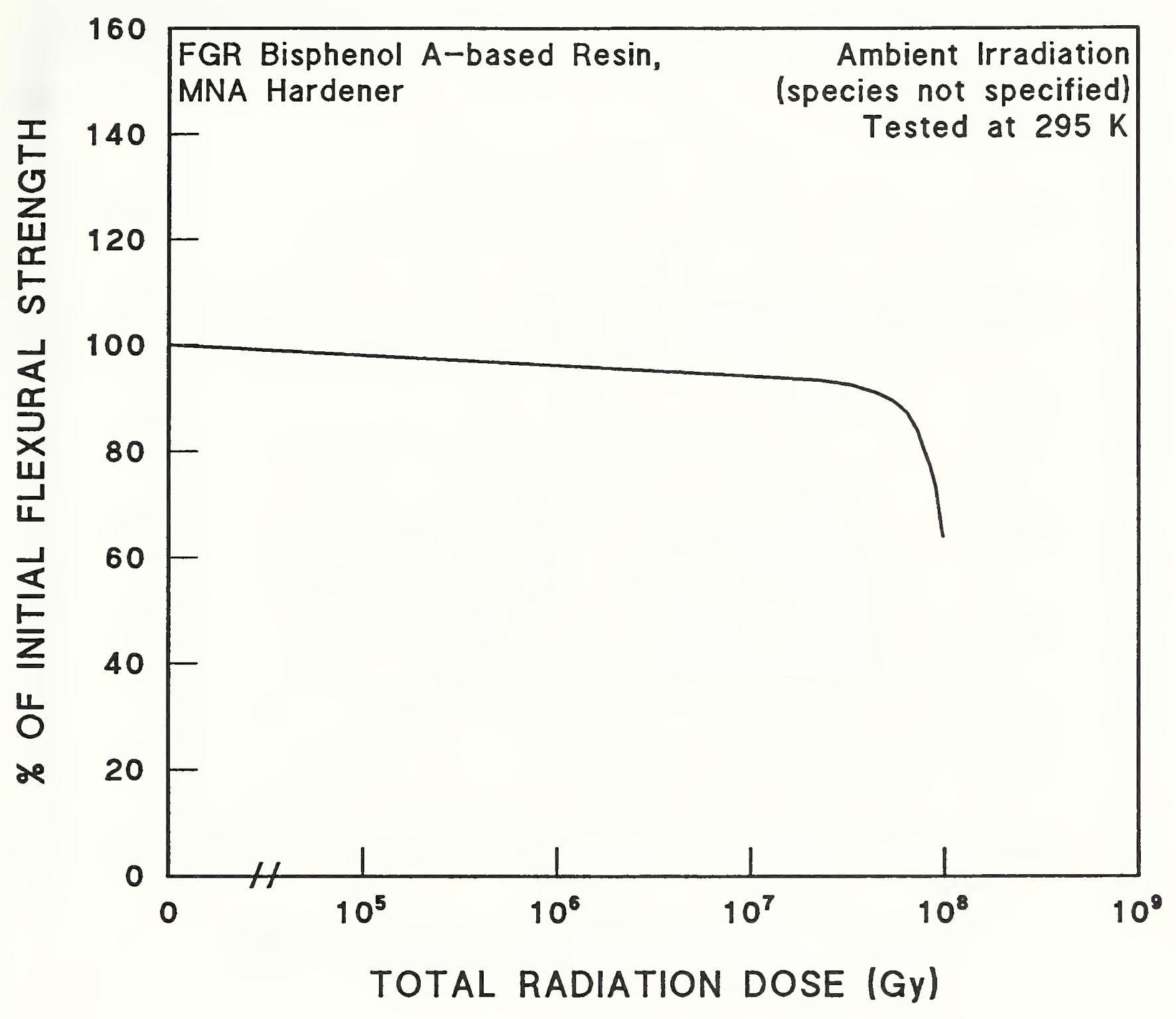

"O" Strength, MPa

$-\quad 455.8$
Supplier

Brown Boveri, Zurich

Figure A.2-89. Flexural strength at $295 \mathrm{~K}$ of FGR bisphenol A-based resin with MNA hardener after ambient reactor irradiation. No supplementary information. Banford [1984] . 


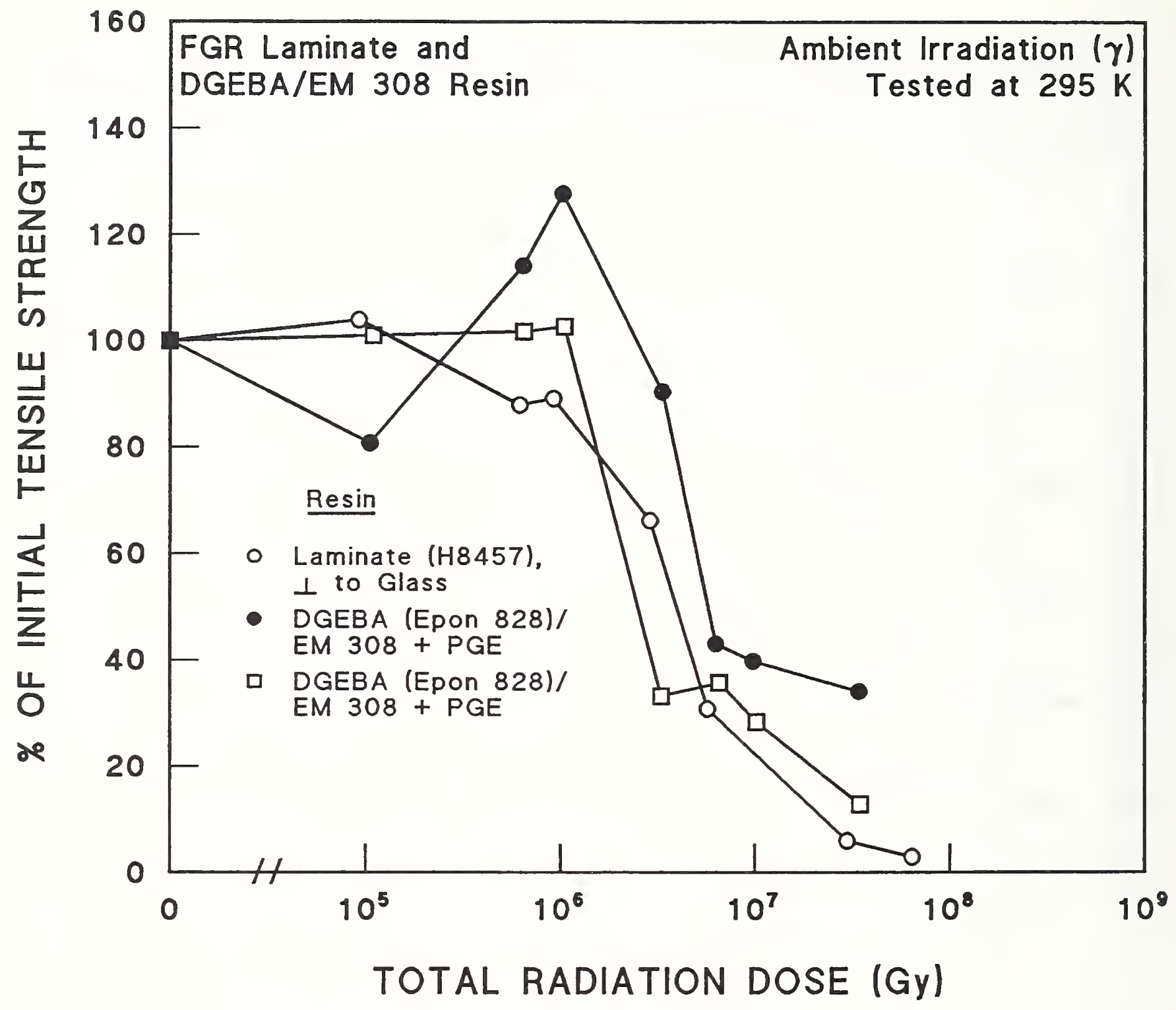

"O" Strength, MPa

$\circ \quad 29.5$

- $\quad 35.1$

ㅁ $\quad 58.3$
Supplier

Westinghouse

Shell

Shell

Figure A.2-90. Tensile strength at $295 \mathrm{~K}$ of FGR laminate and DGEBA resin after ambient gamma irradiation. Supplementary Table A.2-5. Data from Markley et al. [1969]. 


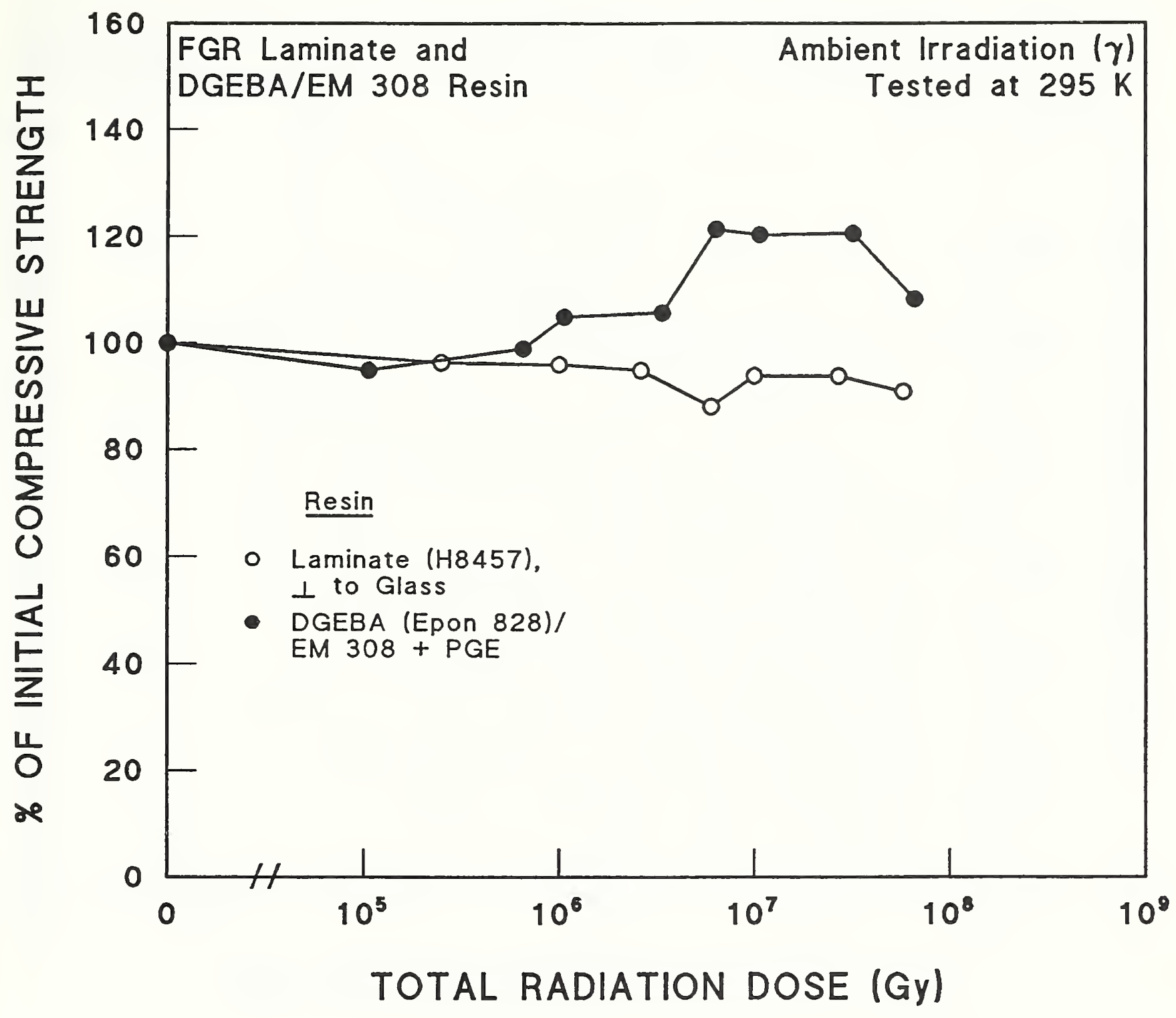

\begin{tabular}{lcl} 
"O & Strength, MPa & Supplier \\
\hline 0 & 523.5 & Westinghouse \\
- & 69.1 & Shell
\end{tabular}

Figure A.2-91. Compressive strength at $295 \mathrm{~K}$ of FGR laminate and DGEBA resin after ambient gamma irradiation. Supplementary Table A.2-5. Data from Markley et al. [1969]. 


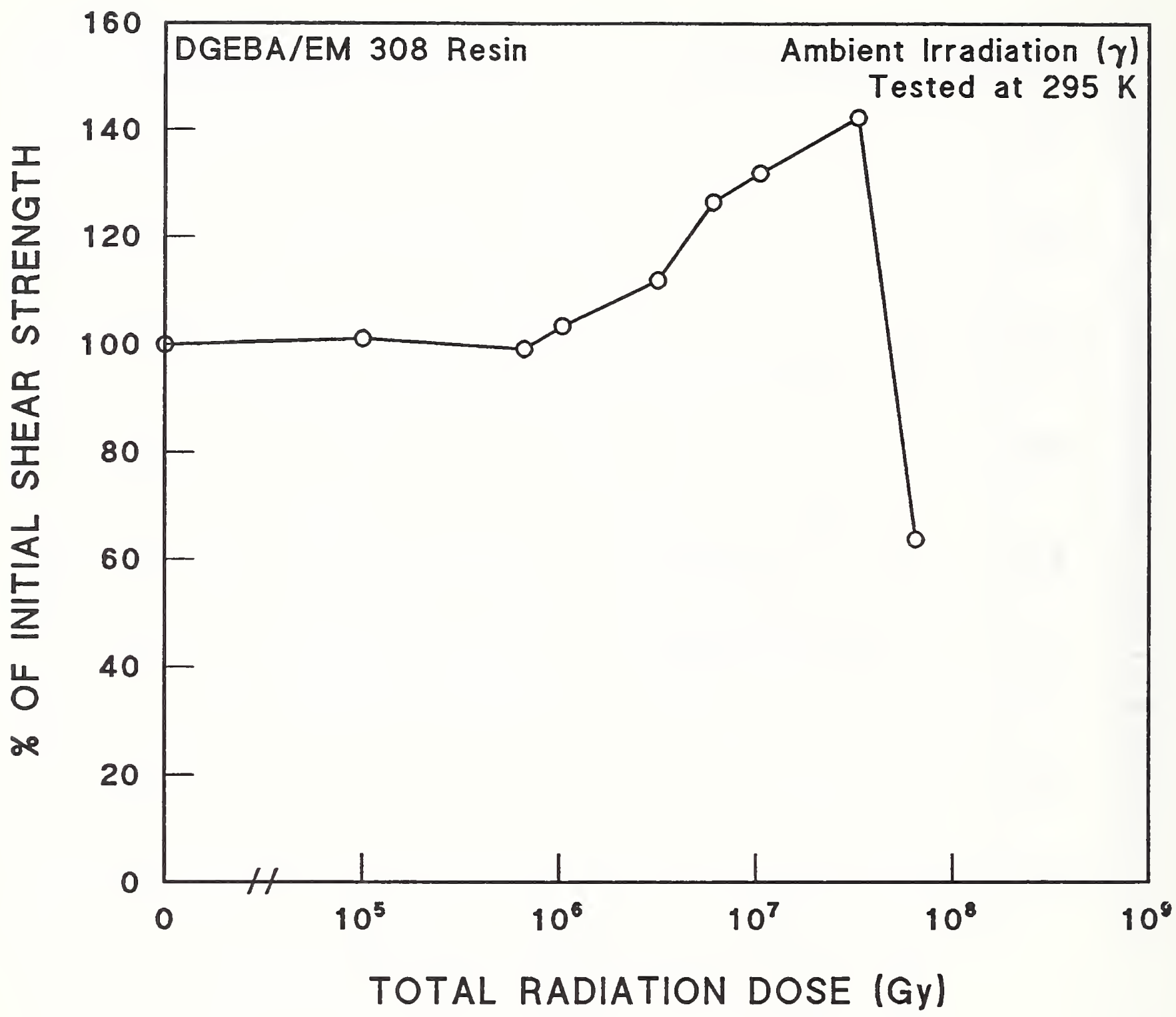

"O" Strength, MPa

- $\quad 38.1$
Supplier

Shell

Comments: Identical results were obtained with a PGE (phenylglycidyl ether) additive.

Figure A.2-92. Shear strength at $295 \mathrm{~K}$ of DGEBA resin after ambient gamma irradiation. Supplementary Table A.2-5. Data from Markley et al. [1969]. 


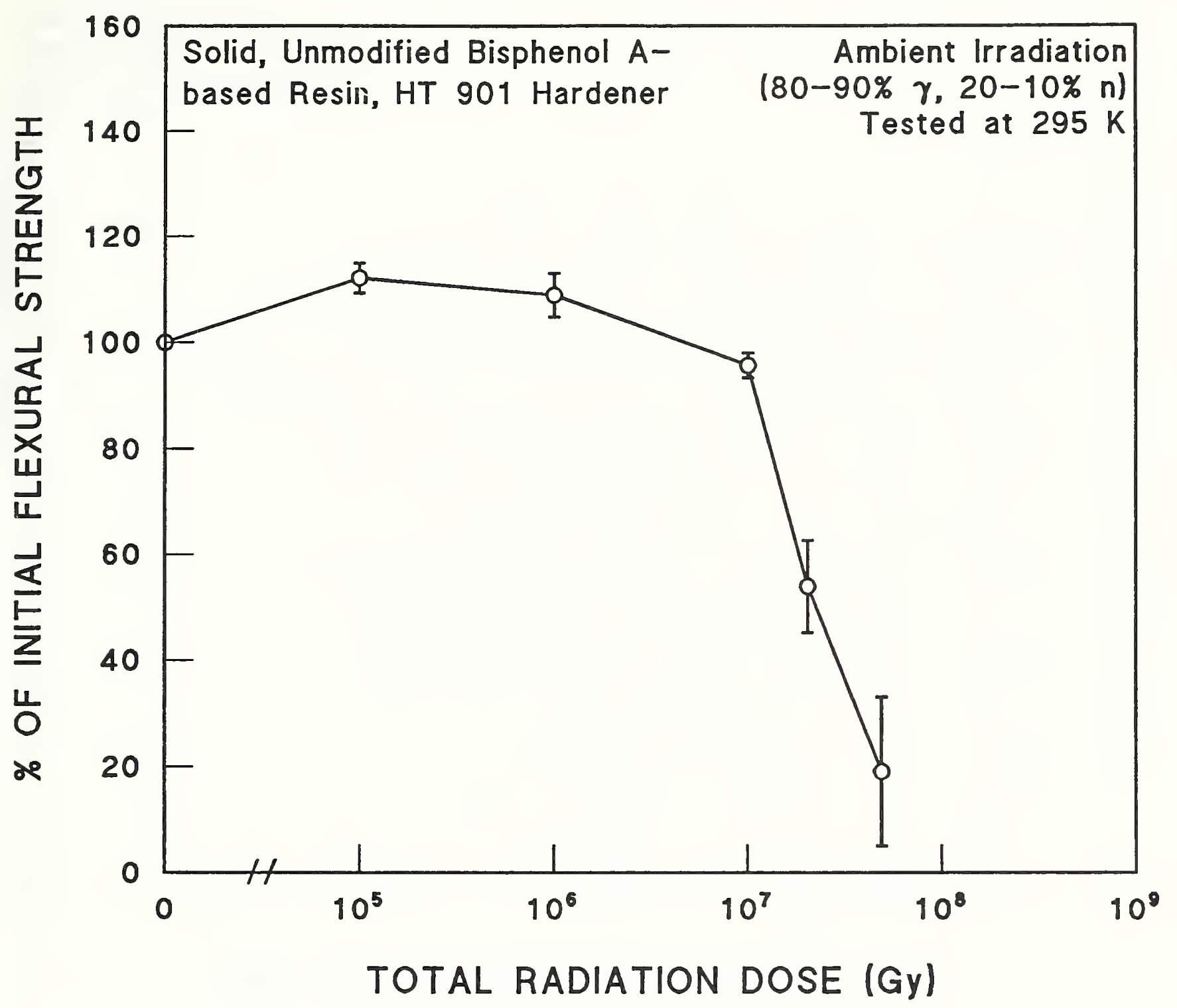

"O" Strength, MPa

Supplier

- 158.9

Ciba-Geigy

Figure A.2-93. Flexural strength at $295 \mathrm{~K}$ of neat bisphenol A-based resin after ambient reactor irradiation. Supplementary Table A.2-4. Data from Pluym and Van de Voorde [1967]. 


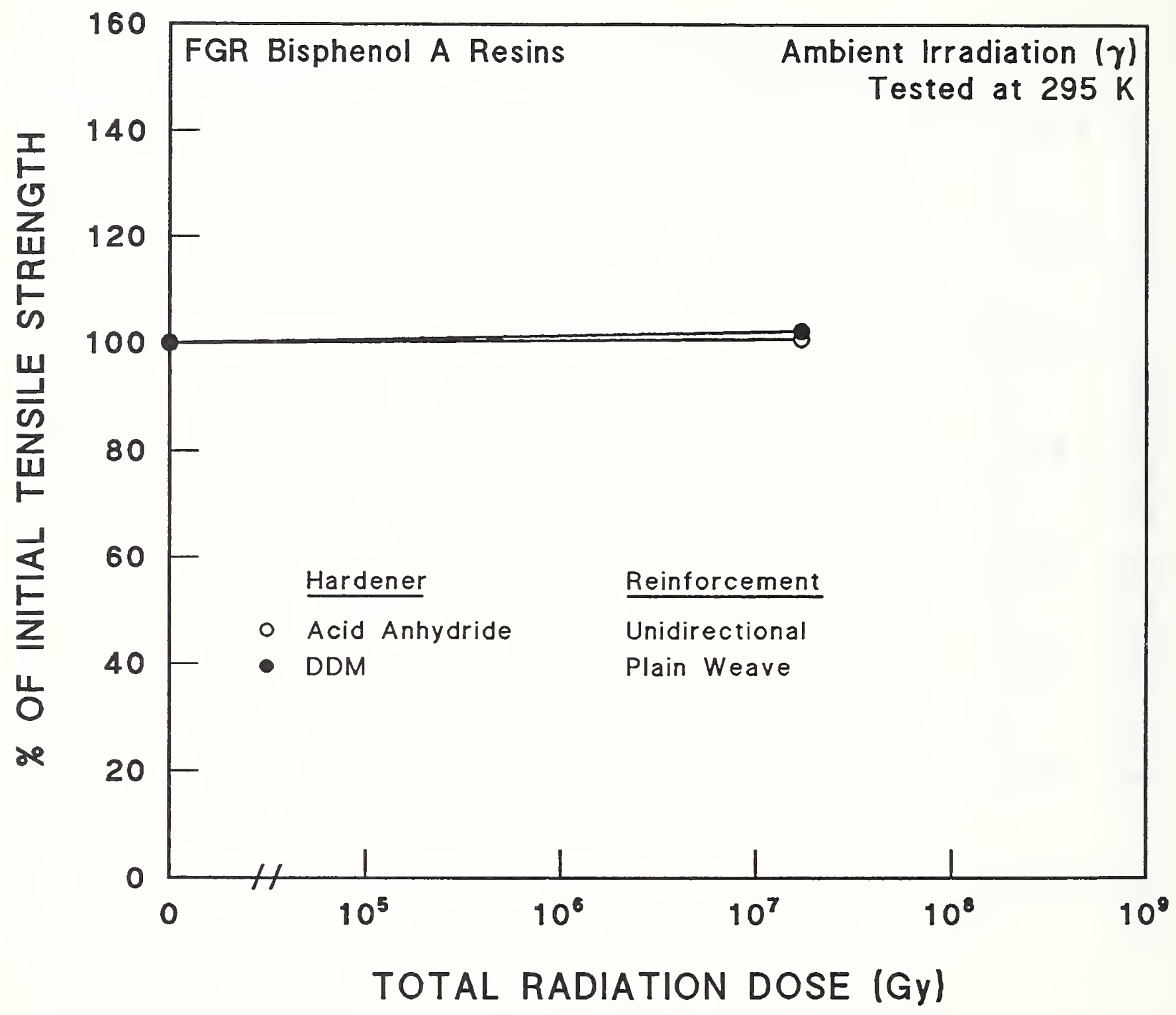

\begin{tabular}{|c|c|c|}
\hline "O" Strength, $\mathrm{MPa}$ & Resin/Laminate & Supplier \\
\hline - 1028.0 & $\begin{array}{l}\text { Araldite } \\
\text { B } 901\end{array}$ & Ciba-Geigy \\
\hline - 497.0 & $\begin{array}{l}\text { Permaglas } \\
\text { TE } 630\end{array}$ & -- \\
\hline
\end{tabular}

Figure A.2-94. Tensile strength at $295 \mathrm{~K}$ of FGR bisphenol A resins after ambient gamma irradiation. Supplementary Table A.2-14. Data from Dahlerup-Peterson and Perrot [1979] and Dahlerup-Peterson [1980]. 


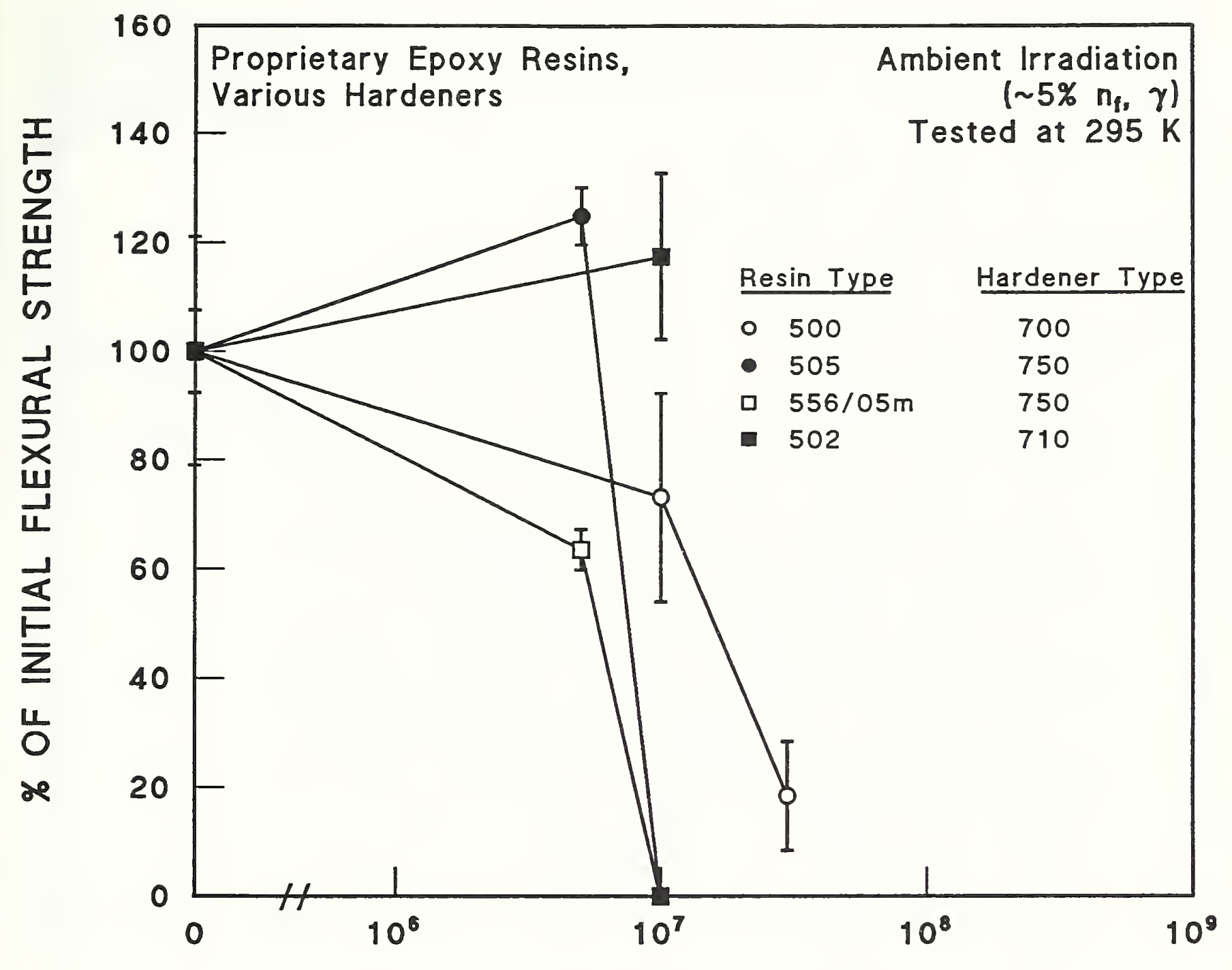

\section{TOTAL RADIATION DOSE (Gy)}

\begin{tabular}{|c|c|c|c|c|c|}
\hline $\mathrm{O}^{\prime \prime}$ & Strength, MPa & Cure & Remarks & Name & Supplier \\
\hline ? & $127.5 \pm 1.9$ & $14 \mathrm{~h}, 140^{\circ} \mathrm{C}$ & $\begin{array}{l}\text { too flexible } \\
\text { at } 5 \times 10^{6} \text { Gy }\end{array}$ & ckot & $\begin{array}{l}\text { BASF Farben } \\
\text { \& Fasern AG }\end{array}$ \\
\hline & $75.5 \pm 1.0$ & $24 \mathrm{~h}, 25^{\circ} \mathrm{C}$ & $\begin{array}{l}\text { samples broken at } \\
1 \times 10^{7} \& 3 \times 10^{7} \mathrm{~Gy}\end{array}$ & Dobeckot & $\begin{array}{l}\text { BASF Farben } \\
\text { \& Fasern AG }\end{array}$ \\
\hline & $51.0 \pm 3.9$ & $24 \mathrm{~h}, 25^{\circ} \mathrm{C}$ & $\begin{array}{l}\text { samples broken at } \\
1 \times 10^{7} \& 3 \times 10^{7} \mathrm{~Gy}\end{array}$ & Dobeckot & $\begin{array}{l}\text { BASF Farben } \\
\text { \& Fasern AG }\end{array}$ \\
\hline & $84.4 \pm 17.7$ & $160^{\circ} \mathrm{C}$ & --- & Dobeckot & Plessey \\
\hline
\end{tabular}

Figure A.2-95. Flexural strength at $295 \mathrm{~K}$ of neat proprietary resins with several hardeners after ambient reactor irradiation. Supplementary Table A.2-2. Data from Schönbacher and Stolarz-Iźycka [1979]. 


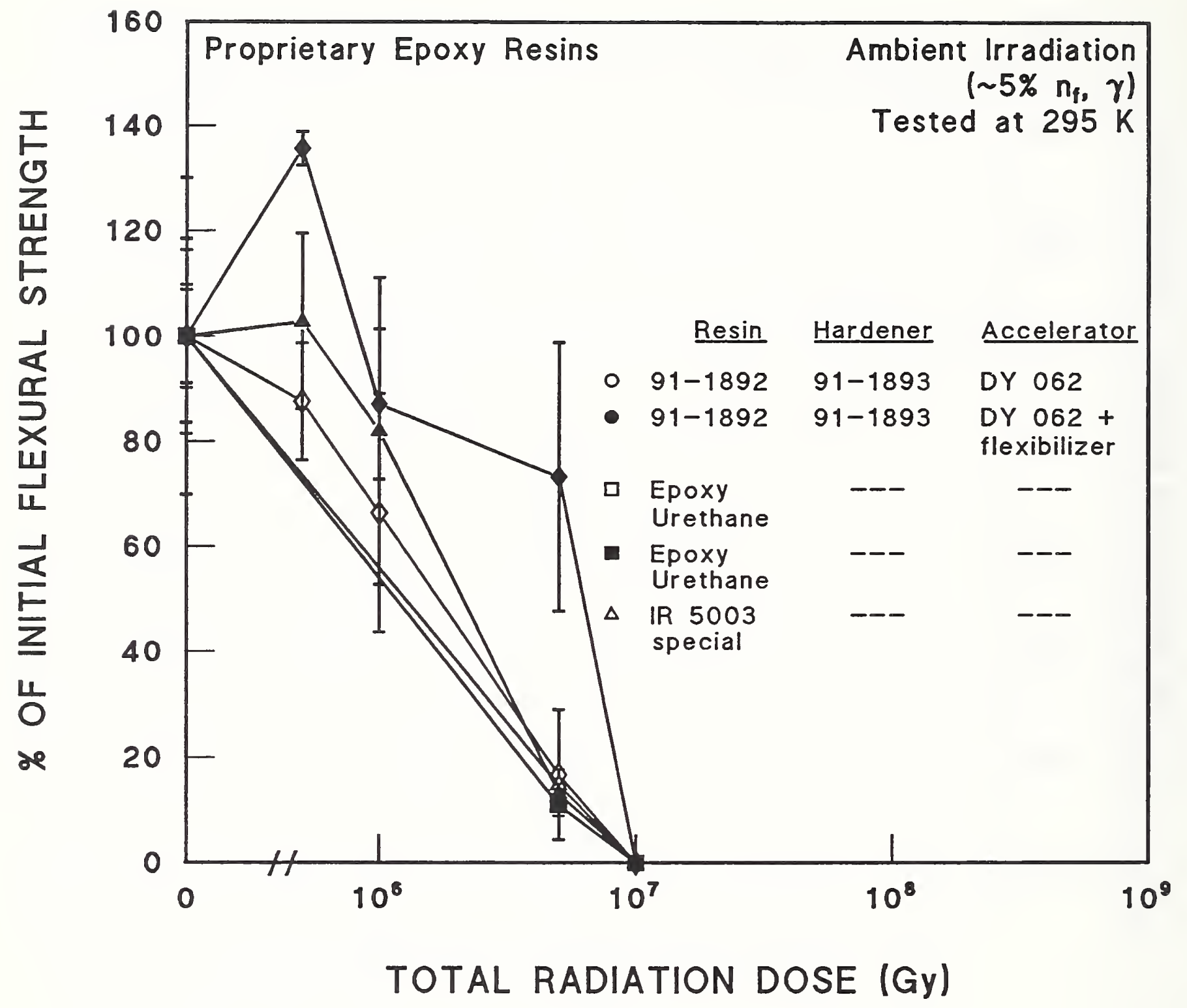

Supplier

Lars Foss Kemi

Lars Foss Kemi

LRCE

LRCE

LRCE

Comments: samples broken after irradiation

Figure A.2-96. Flexural strength at $295 \mathrm{~K}$ of neat proprietary resins with several hardeners after ambient reactor irradiation. Supplementary Table A.2-2. Data from Schönbacher and Stolarz-Iżycka [1979]. 


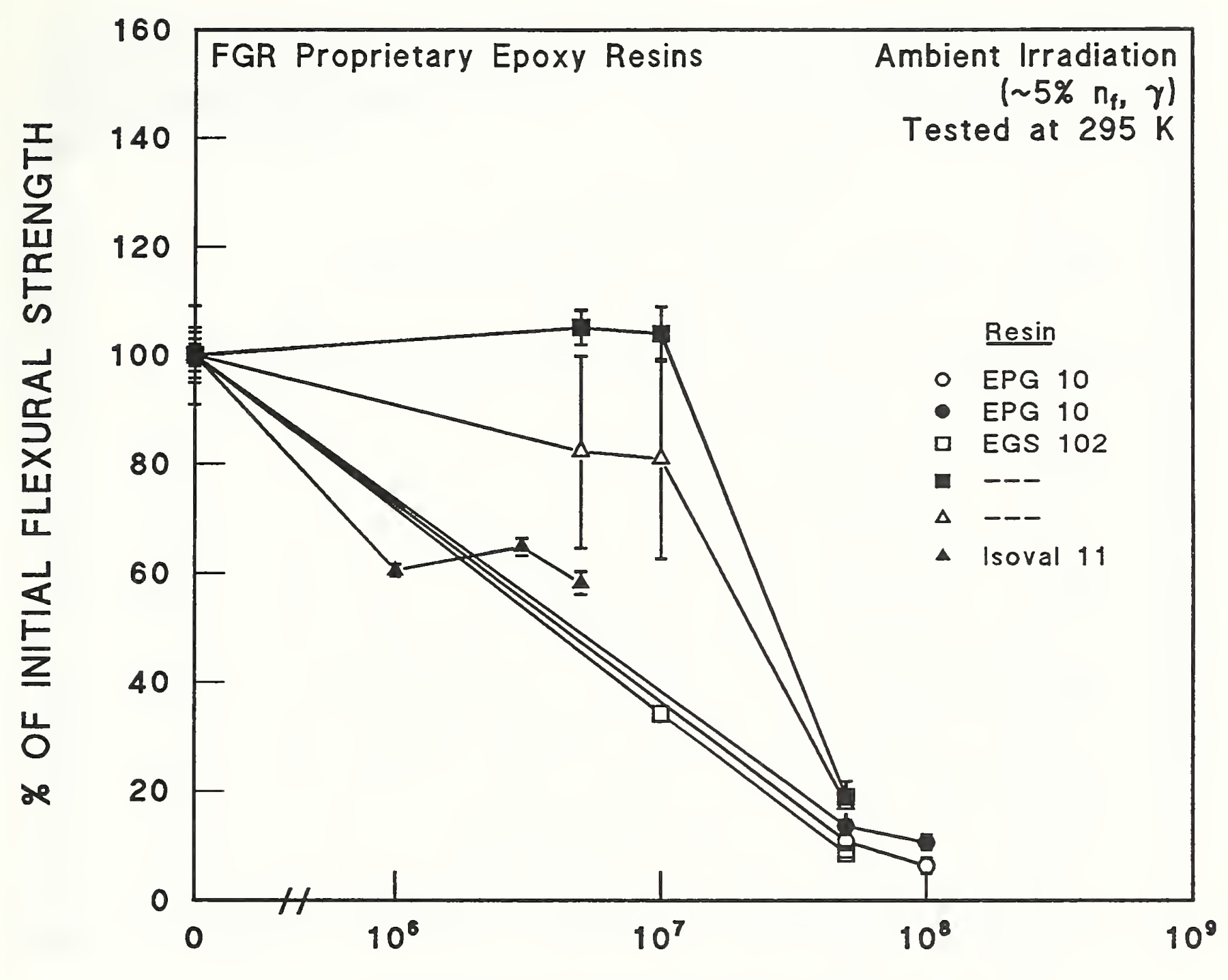

TOTAL RADIATION DOSE (Gy)

\begin{tabular}{llll} 
"O" Strengih, MPa & & Name & Supplier \\
\hline$\circ 510.1 \pm 21.6$ & & Vetronit & Isola \\
$-350.2 \pm 6.9$ & Vetronit & Isola \\
口538.6 \pm 4.9 & Epoxy & Ferrozell \\
M $546.4 \pm 27.5$ & $\begin{array}{l}\text { Birakrit } \\
\text { Laminate 2370.4 }\end{array}$ & UOP \\
$\Delta 508.2 \pm 46.1$ & $\begin{array}{l}\text { Birakrit } \\
\text { Laminate 2372.2 }\end{array}$ & UOP \\
$\Delta \quad 364.9 \pm 10.8$ & $\begin{array}{l}\text { Isoval } \\
\text { Charge 2569 }\end{array}$ & Isovolta
\end{tabular}

Figure A.2-97. Flexural strength at $295 \mathrm{~K}$ of FGR proprietary resins after ambient reactor irradiation. Supplementary Table A.2-2. Data from Schönbacher and StolarzIżycka [1979]. 


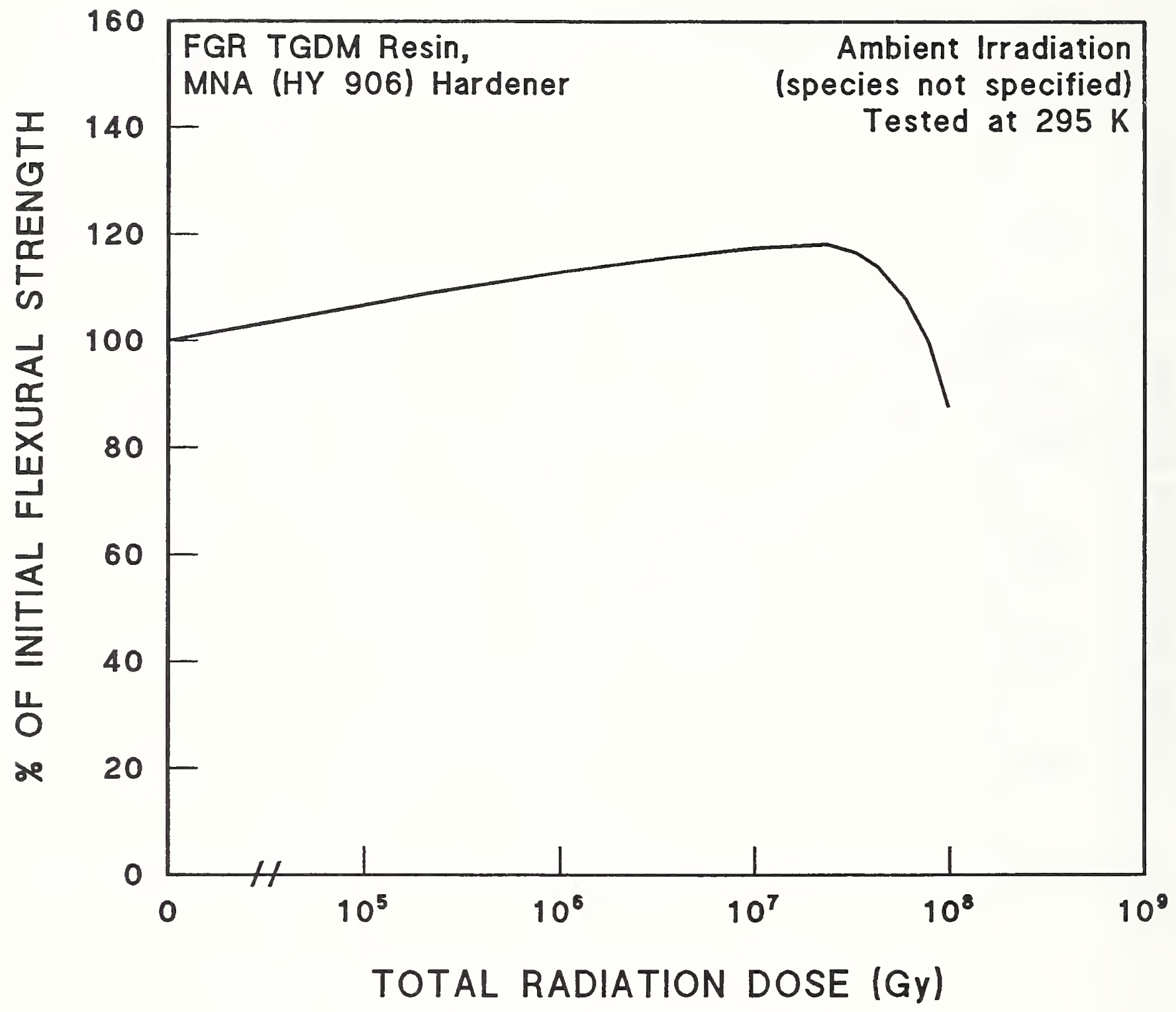

"O" Strength, MPa

$-\quad 313.8$
$-\quad 1$

Figure A.2-98. Flexural strength at $295 \mathrm{~K}$ of FGR TGDM resin with MNA hardener after ambient irradiation. No supplementary information. Data from Van de Voorde and Restat [1972]. 


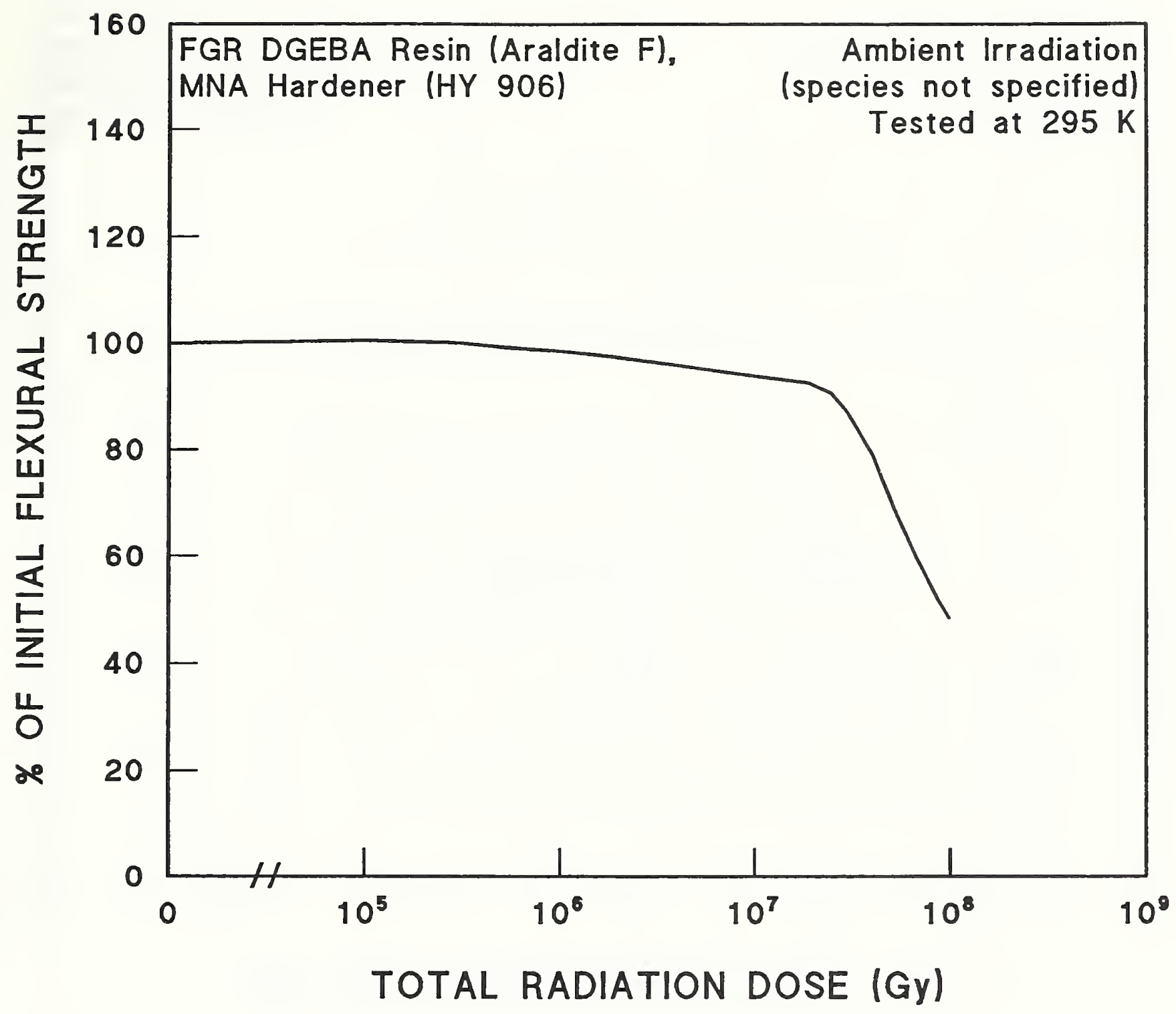

"O" Strength, MPa

$-\quad 353.1$
Supplier

Ciba-Geigy

Figure A.2-99. Flexural strength at $295 \mathrm{~K}$ of FGR DGEBA resin with MNA hardener after ambient irradiation. No supplementary information. Data from Van de Voorde and Restat [1972]. 


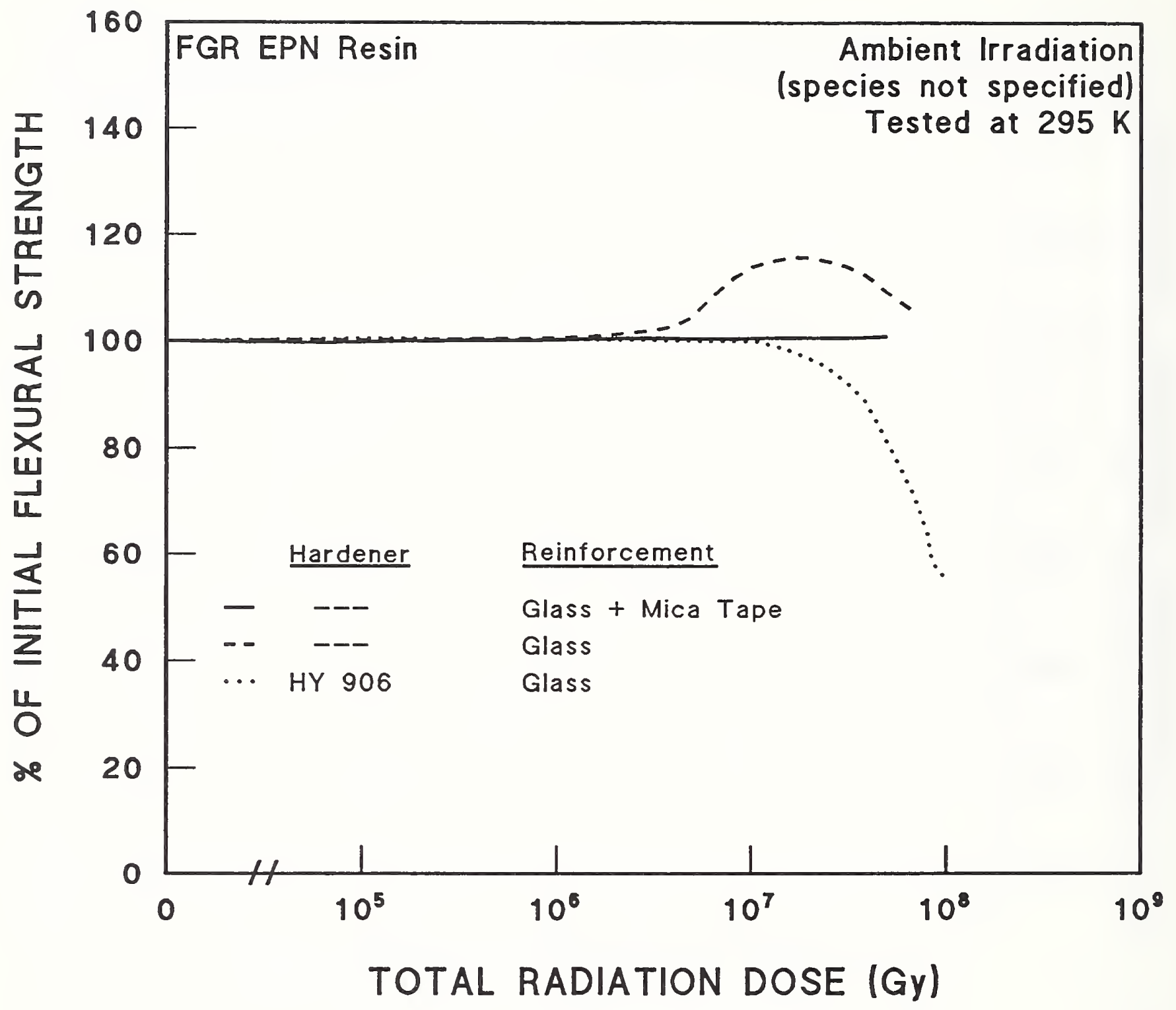

\begin{tabular}{ll} 
"O" Strength, MPa & Insulation Type \\
\hline$\quad 239.3$ & Coil "prepreg" \\
--307.9 & Coil "prepreg" \\
$\ldots \quad 392.3$ & Laminate
\end{tabular}

Figure A.2-100. Flexural strength at $295 \mathrm{~K}$ of FGR EPN resin with mica reinforcement after ambient irradiation. No supplementary information. Data from Van de Voorde and Restat [1972]. 


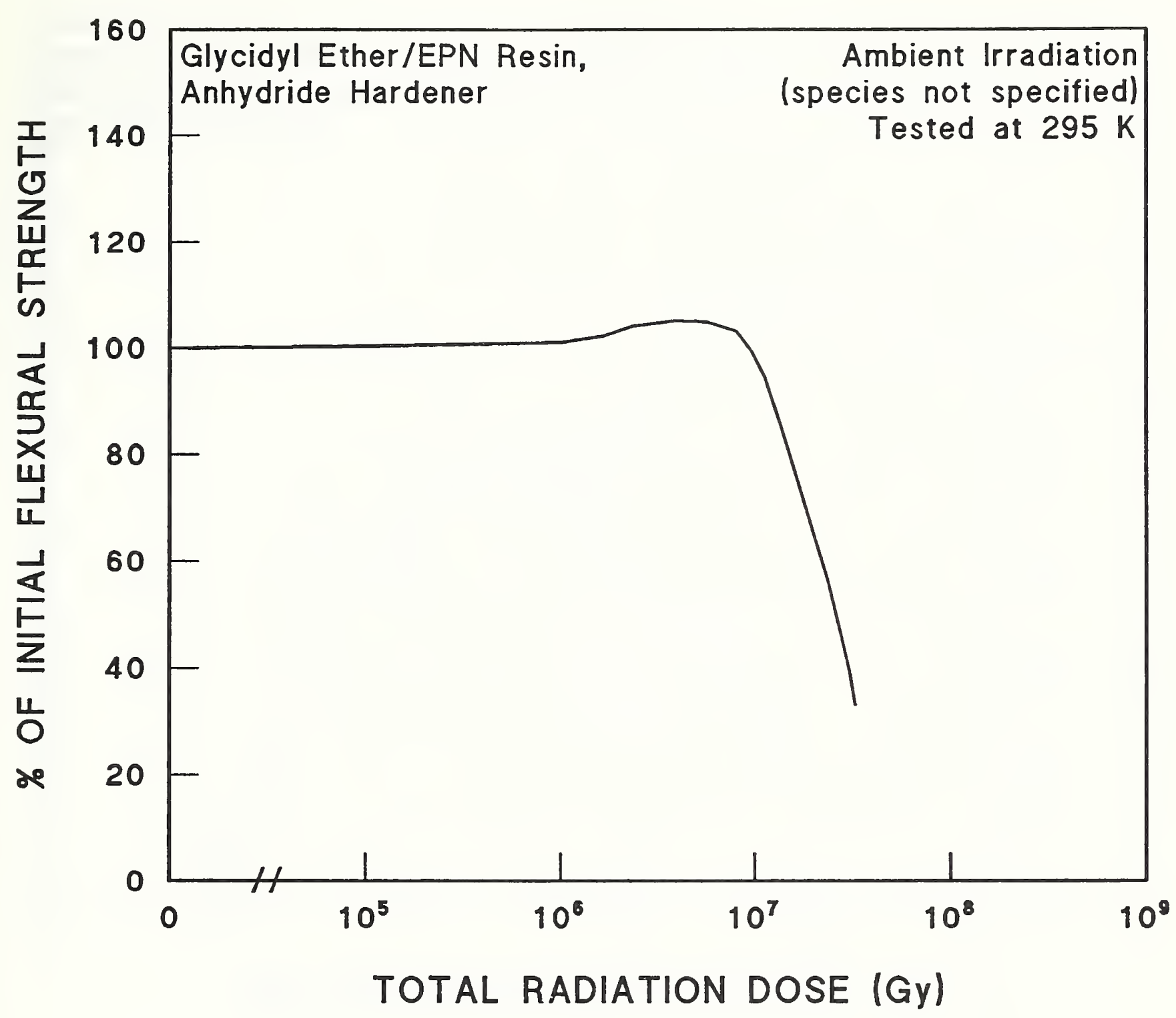

"O" Strength, MPa

$-116.7$

Figure A.2-101. Flexural strength at $295 \mathrm{~K}$ of neat glycidyl ether/EPN resin with anhydride hardener after ambient irradiation. No supplementary information. Data from Van de Voorde and Restat [1972]. 


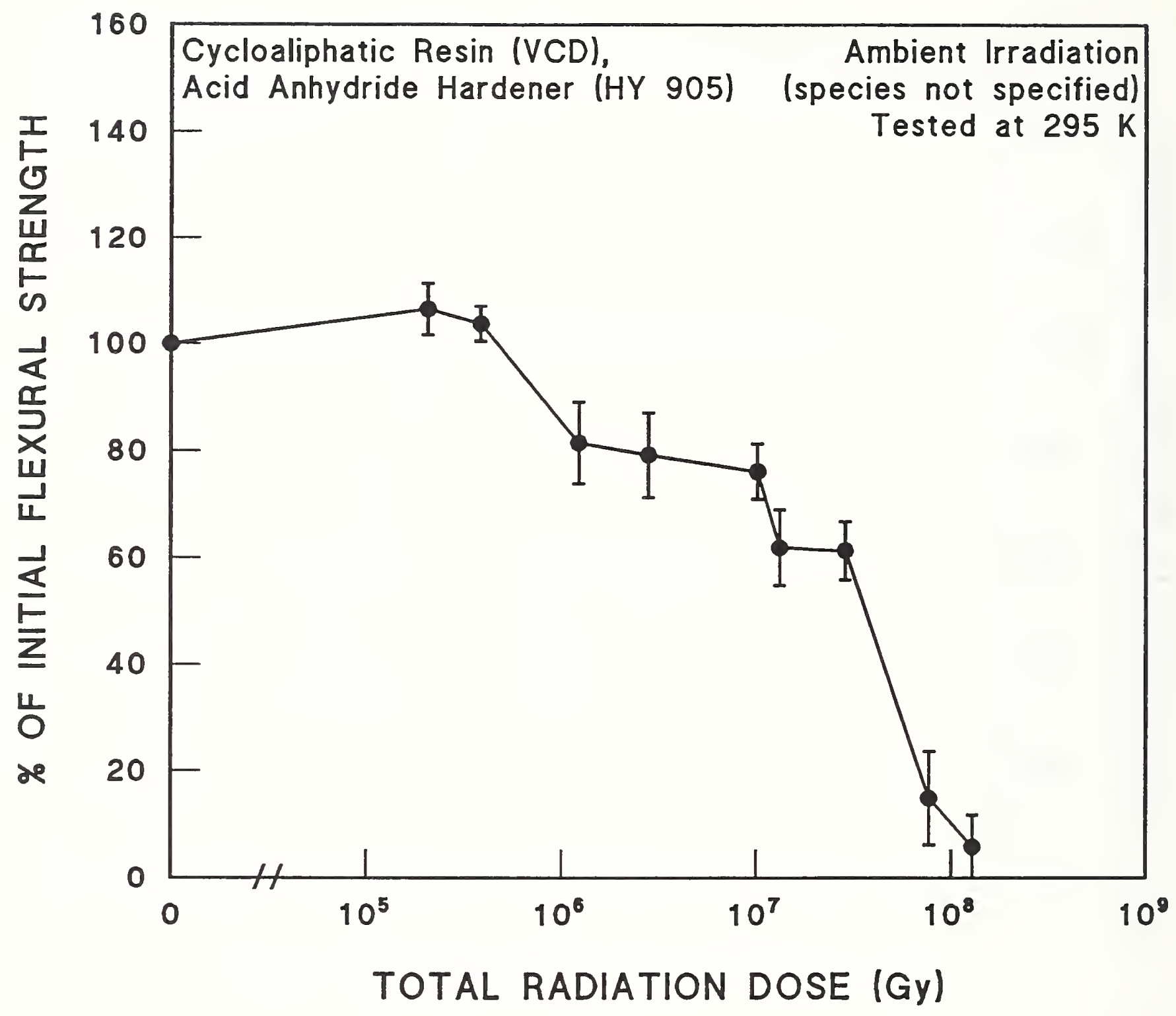

"O" Strength, MPa

- 111.8
Accelerator

DY 061
Supplier

Ciba-Geigy

Figure A.2-102. Flexural strength at $295 \mathrm{~K}$ of neat cycloaliphatic resin with acid anhydride hardener after ambient irradiation. No supplementary information. Data from Van de Voorde and Restat [1972]. 


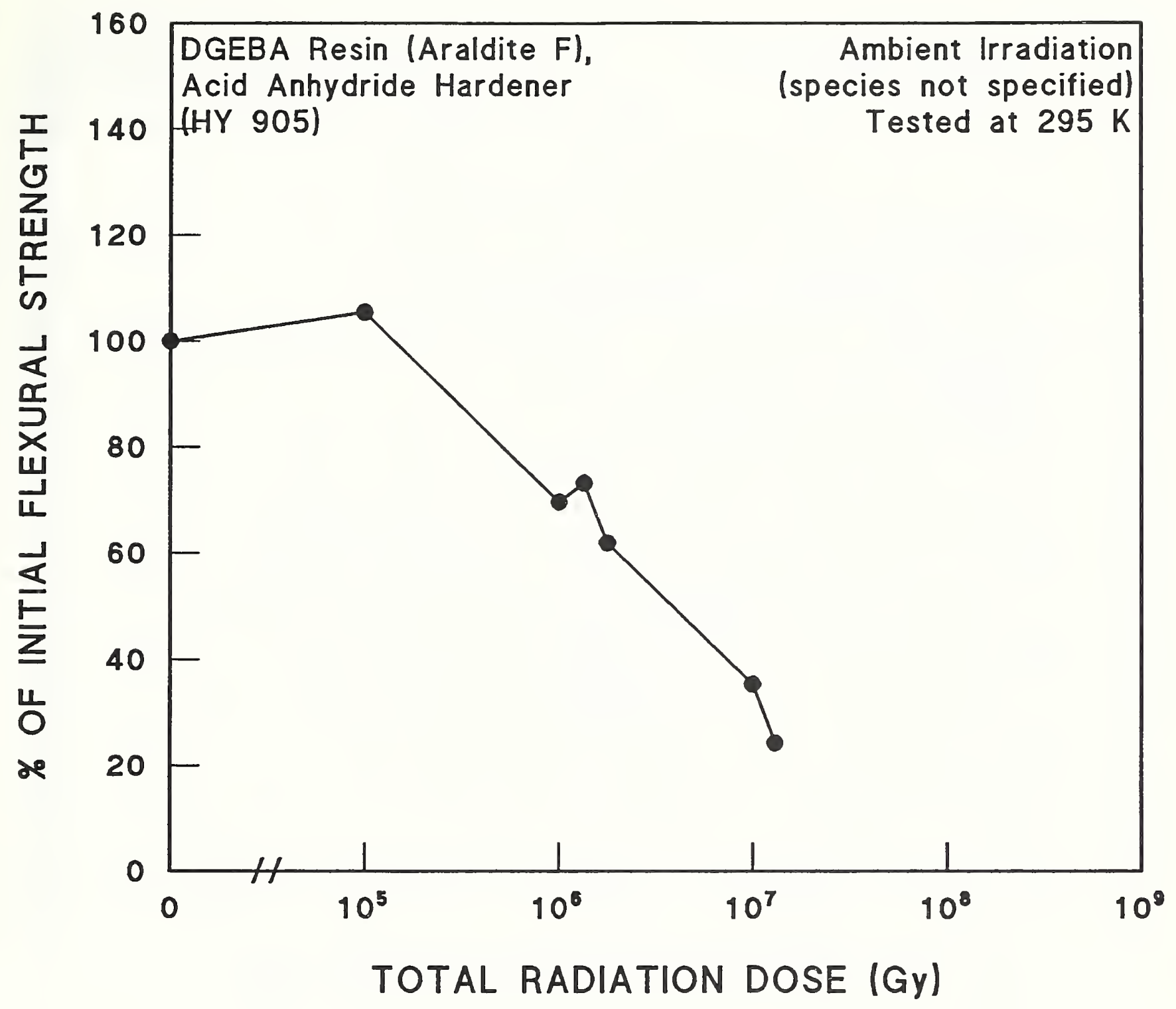

"O" Strength, MPa

- 120.6
Accelerator

DY 063, DY 061
Supplier

Ciba-Geigy

Figure A.2-103. Flexural strength at $295 \mathrm{~K}$ of neat DGEBA resin with acid anhydride hardener after ambient irradiation. No supplementary information. Data from Van de Voorde and Restat [1972]. 


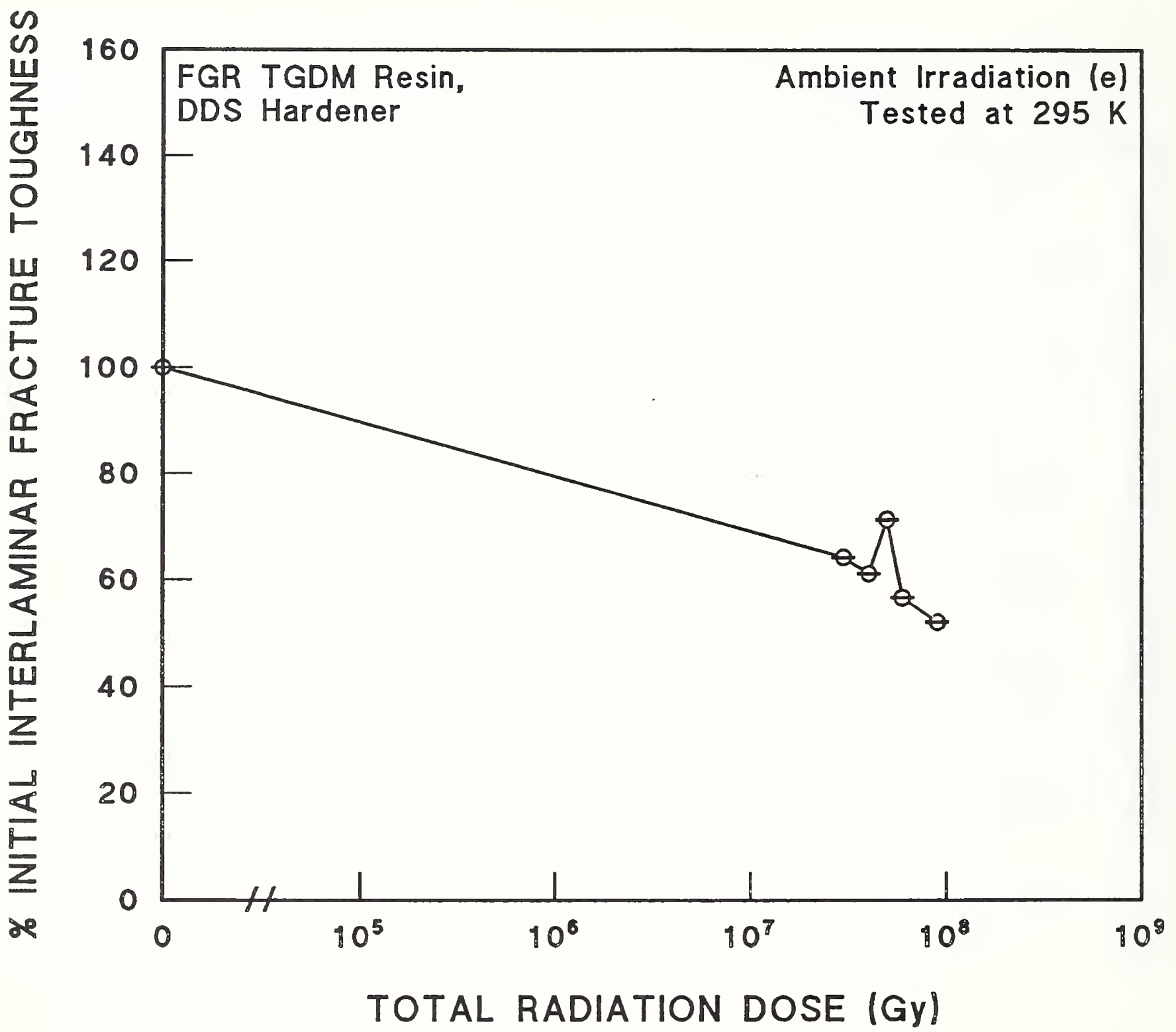

\begin{tabular}{|c|c|c|}
\hline "O" Toughness, $\mathrm{kJ} / \mathrm{mm}^{2}$ & Glass & Supplier \\
\hline$\quad 1.2 \pm 0.1$ & KS $1210 \mathrm{E}$ & $\begin{array}{l}\text { Sumitomo } \\
\text { Bakelite } \\
\text { Co., Ltd. }\end{array}$ \\
\hline
\end{tabular}

Figure A.2-104. Interlaminar fracture toughness at $295 \mathrm{~K}$ of FGR-TGDM resin with DDS hardener after ambient electron irradiation. Supplementary Table A.2-15. Data from Takeda et al. [1989]. 


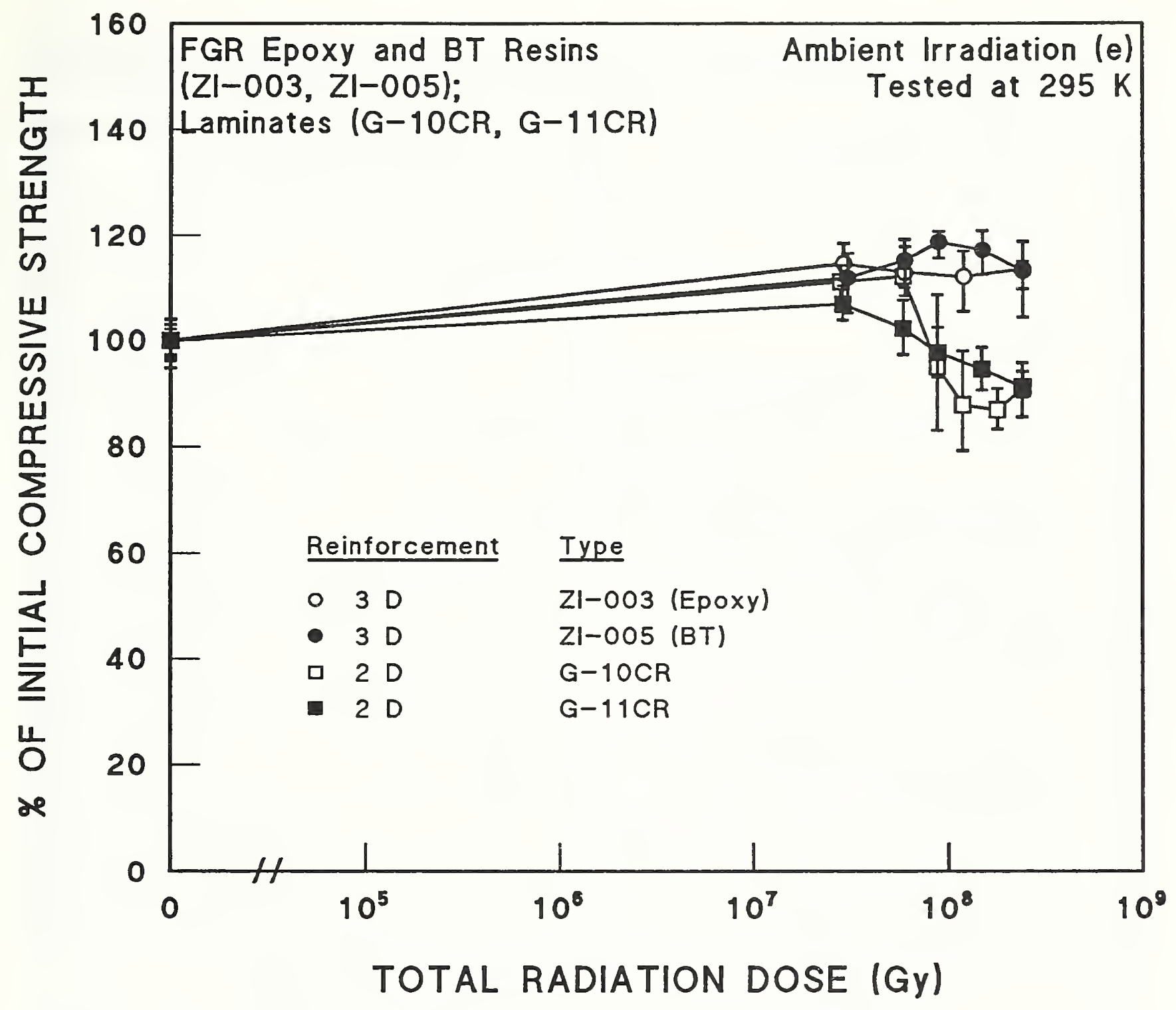

"O" Strength, MPa

- $721.5 \pm 27.9$

- $801.3 \pm 32.4$

ㅁ $485.9 \pm 10.2$

- $594.1 \pm 17.7$
Supplier

Shikishima Canvas Co.

Shikishima Canvas Co.

Spaulding Fiber Co.

Spaulding Fiber Co.

Figure A.2-105. Compressive strength at $295 \mathrm{~K}$ of FGR epoxy and BT resins and laminates (G-10CR, G-IICR) after ambient electron irradiation. Supplementary Table A.2-16. Data from Nishijima et al. [1990]. 


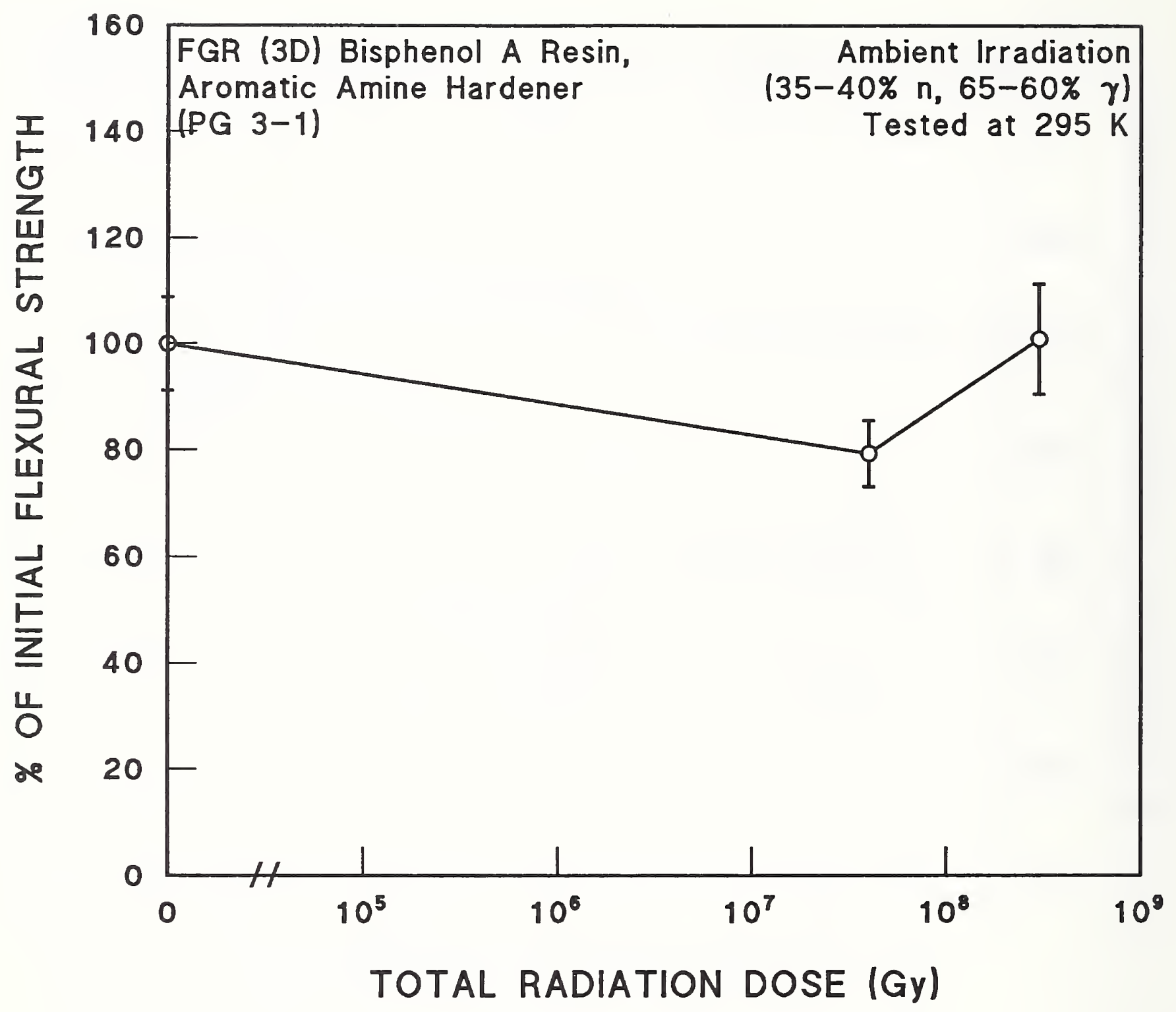

"O" Strength, MPa

$\circ \quad 695.2 \pm 61.8$
Supplier

Shikishima

Canvas Co.

Figure A.2-106. Flexural strength at $295 \mathrm{~K}$ of FGR (3-D) bisphenol A resin with aromatic amine hardener after ambient reactor irradiation. Supplementary Table A.217. Data from McManamy et a1. [1990; 1991]. 
Table A.2-1. Supplementary Information, Price and Sheldon [1965]

and other Rutherford Laboratory references.

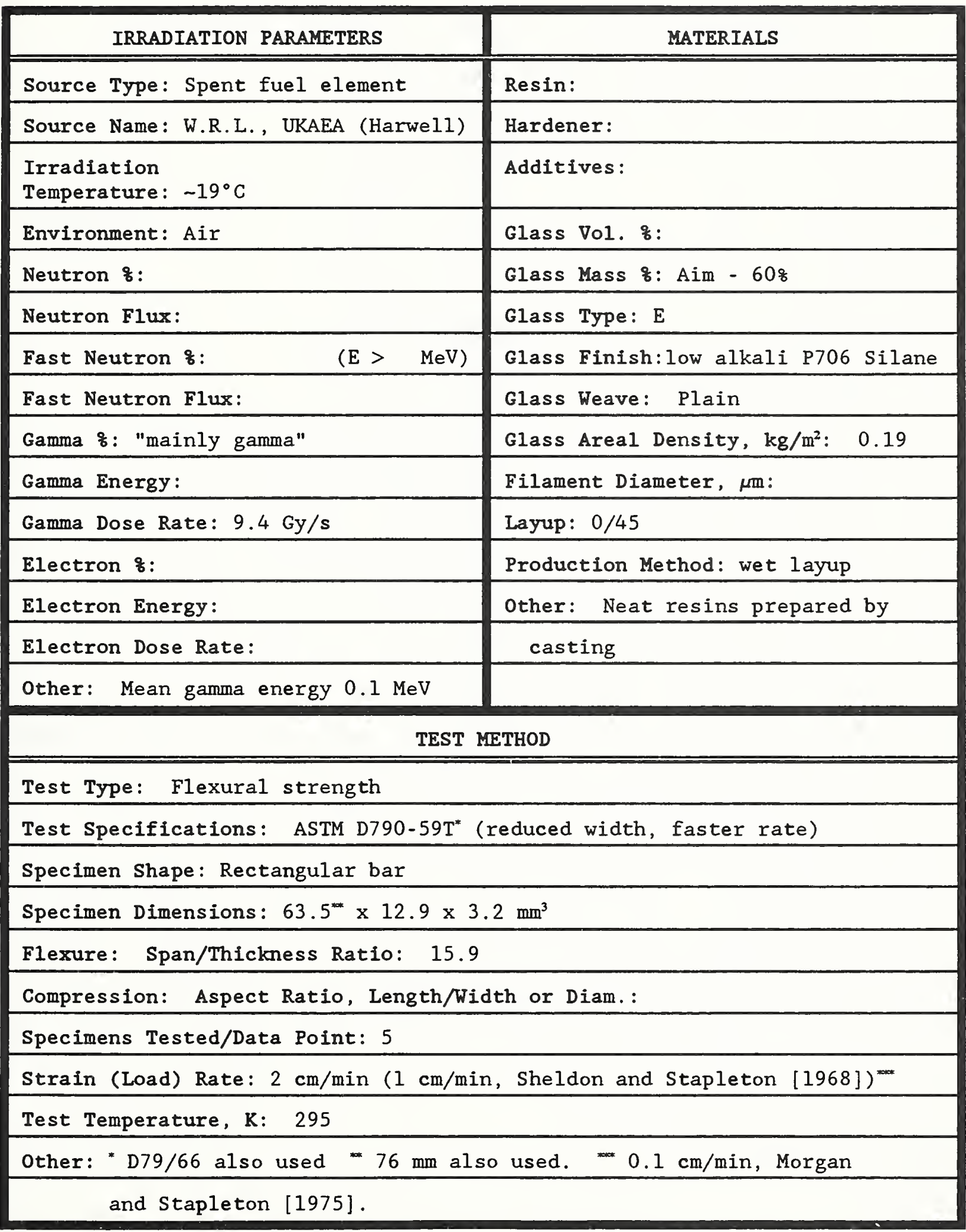


Table A.2-2. Supplementary Information, Schönbacher and StolarzIźycka [1979].

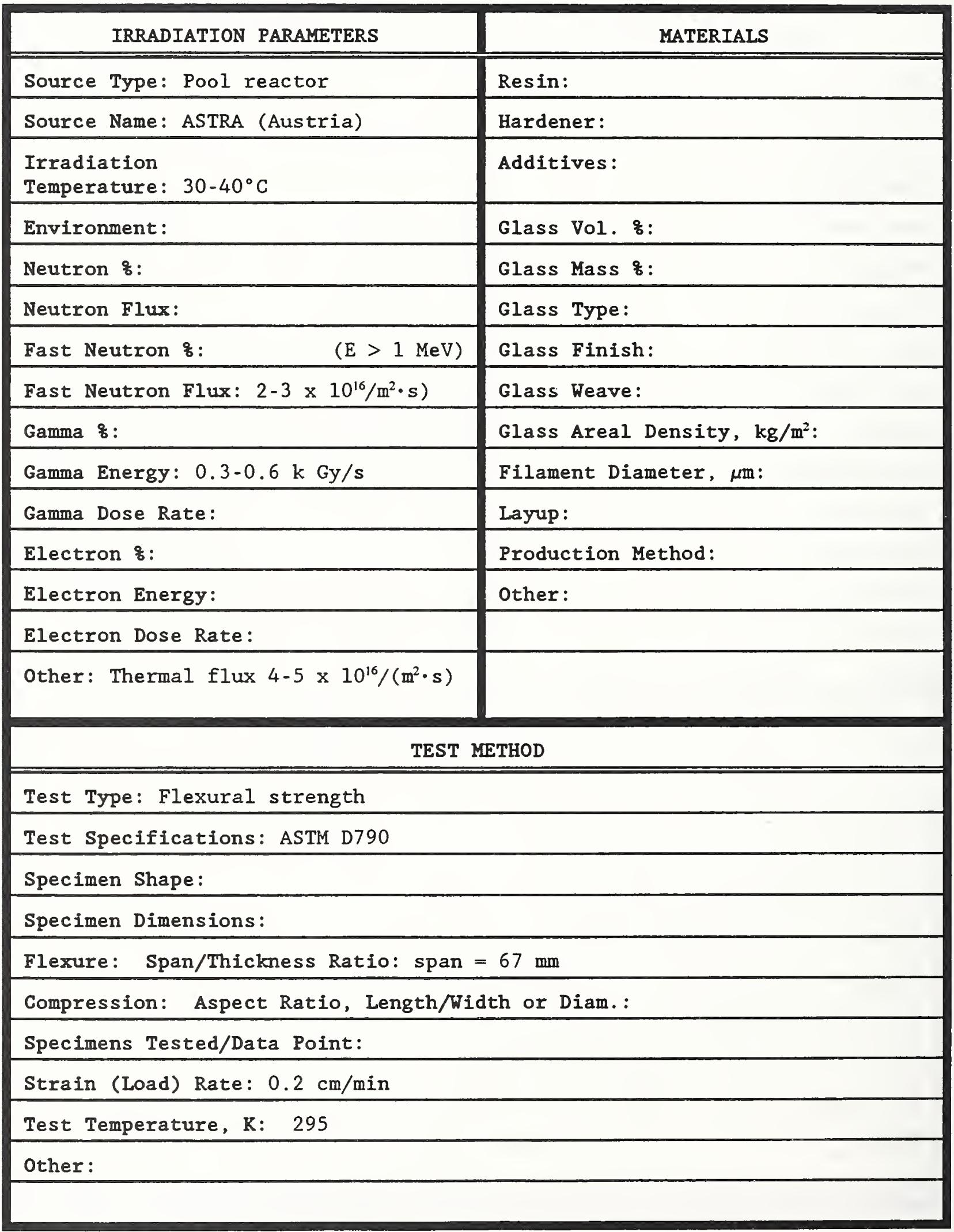


Table A.2-3. Supplementary Information, Hagiwara et al. [1985], Takeda et al. [1985]

\begin{tabular}{|c|c|}
\hline IRRADIATION PARAMETERS & MATERIALS \\
\hline Source Type: & Resin: \\
\hline Source Name: & Hardener: \\
\hline $\begin{array}{l}\text { Irradiation } \\
\text { Temperature: }(70 \pm 5) \text { or }(100 \pm 10)^{\circ} \mathrm{C}\end{array}$ & Additives: \\
\hline Environment: Air & Glass Vol. $8: 54-60$ \\
\hline Neutron $q$ : & Glass Mass ơ: \\
\hline Neutron Flux: & Glass Type: E \\
\hline Fast Neutron $q$ : & Glass Finish: $\begin{array}{c}\gamma \text {-glycidoxypropyl- } \\
\text { trimethoxysilane }\end{array}$ \\
\hline Fast Neutron Flux: & Glass Weave: plain, KS1210 \\
\hline Gamma $q$ & Glass Areal Density, $\mathrm{kg} / \mathrm{m}^{2}$ : \\
\hline Gamma Energy: & Filament Diameter, $\mu \mathrm{m}: 7$ \\
\hline Gamma Dose Rate: & Layup: \\
\hline Electron \&: 100 & Production Method: \\
\hline Electron Energy: $3 \mathrm{MeV}$ & Other: Prepreg \\
\hline \multicolumn{2}{|l|}{ Electron Dose Rate: $0.5-1 \times 10^{4} \mathrm{~Gy} / \mathrm{s}$} \\
\hline \multicolumn{2}{|l|}{ Other: } \\
\hline \multicolumn{2}{|c|}{ TEST METHOD } \\
\hline \multicolumn{2}{|c|}{ Test Specifications: $\quad$ (similar to ASTM D-2733) } \\
\hline \multicolumn{2}{|c|}{ Specimen Shape: Rectangular bar (notched tensile) } \\
\hline \multicolumn{2}{|c|}{ Specimen Dimensions: $70 \times 6.4 \times 2.0 \mathrm{~mm}^{3}$ (varied) } \\
\hline \multicolumn{2}{|l|}{ Flexure: Span/Thickness Ratio: } \\
\hline \multicolumn{2}{|c|}{ Compression: Aspect Ratio, Length/Width or Diam.: } \\
\hline \multicolumn{2}{|l|}{ Specimens Tested/Data Point: } \\
\hline \multicolumn{2}{|l|}{ Strain (Load) Rate: } \\
\hline \multicolumn{2}{|l|}{ Test Temperature, K: 295} \\
\hline Other: & \\
\hline
\end{tabular}


Table A.2-4. Supplementary Information, Pluym and Van de Voorde [1967] .

\begin{tabular}{|c|c|}
\hline IRRADIATION PARAMETERS & MATERIALS \\
\hline Source Type: Pool reactor & Resin: \\
\hline Source Name: (Seibersdorf, Austria) & Hardener: \\
\hline $\begin{array}{l}\text { Irradiation } \\
\text { Temperature: rise of } 11^{\circ} \mathrm{C}\end{array}$ & Additives: \\
\hline Environment: & Glass Vol. \&: \\
\hline Neutron $8: \quad 20-10$ & Glass Mass $q$ : \\
\hline Neutron Flux: & Glass Type: \\
\hline Fast Neutron $8: \quad(E>M e V)$ & Glass Finish: \\
\hline Fast Neutron Flux: & Glass Weave: \\
\hline Gamma $: 80-90$ & Glass Areal Density, $\mathrm{kg} / \mathrm{m}^{2}$ : \\
\hline Gamma Energy: $2.7 \times 10^{2} \mathrm{~Gy} / \mathrm{s}$ & Filament Diameter, $\mu \mathrm{m}$ : \\
\hline Gamma Dose Rate: & Layup: \\
\hline Electron 8 : & Production Method: \\
\hline Electron Energy: & Other: \\
\hline Electron Dose Rate: & \\
\hline \multicolumn{2}{|l|}{ Other: } \\
\hline \multicolumn{2}{|c|}{ TEST METHOD } \\
\hline \multicolumn{2}{|l|}{ Test Type: Flexural strength } \\
\hline \multicolumn{2}{|l|}{ Test Specifications: } \\
\hline \multicolumn{2}{|l|}{ Specimen Shape: } \\
\hline \multicolumn{2}{|l|}{ Specimen Dimensions: } \\
\hline \multicolumn{2}{|l|}{ Flexure: Span/Thickness Ratio: } \\
\hline \multicolumn{2}{|c|}{ Compression: Aspect Ratio, Length/Width or Diam.: } \\
\hline \multicolumn{2}{|l|}{ Specimens Tested/Data Point: 5} \\
\hline \multicolumn{2}{|l|}{ Strain (Load) Rate: } \\
\hline Test Temperature, K: 295 & \\
\hline Other: & \\
\hline
\end{tabular}


Table A.2-5. Supplementary Information, Markley et al. [1969] .

\begin{tabular}{|c|c|}
\hline IRRADIATION PARAMETERS & MATERIALS \\
\hline Source Type: ${ }^{60} \mathrm{Co}$ & Resin: \\
\hline Source Name: & Hardener: \\
\hline $\begin{array}{l}\text { Irradiation } \\
\text { Temperature: }\end{array}$ & Additives: \\
\hline Environment: & Glass Vol. : : \\
\hline Neutron $\&$ : & Glass Mass of: \\
\hline Neutron Flux: & Glass Type: \\
\hline Fast Neutron $8: \quad(E>M e V)$ & Glass Finish: \\
\hline Fast Neutron Flux: & Glass Weave: \\
\hline Gamma \&: 100 & Glass Areal Density, $\mathrm{kg} / \mathrm{m}^{2}$ : \\
\hline Gamma Energy: $-1.2 \mathrm{MeV}$ & Filament Diameter, $\mu \mathrm{m}$ : \\
\hline Gamma Dose Rate: $<28 \mathrm{~Gy} / \mathrm{s}$ & Layup: \\
\hline Electron s: & Production Method: \\
\hline Electron Energy: & Other: \\
\hline \multicolumn{2}{|l|}{ Electron Dose Rate: } \\
\hline \multicolumn{2}{|l|}{ Other: } \\
\hline \multicolumn{2}{|c|}{ TEST METHOD } \\
\hline \multicolumn{2}{|l|}{ Test Type: Shear } \\
\hline \multicolumn{2}{|l|}{ Test Specifications: } \\
\hline \multicolumn{2}{|l|}{ Specimen Shape: Disk } \\
\hline \multicolumn{2}{|c|}{ Specimen Dimensions: $19 \mathrm{~mm}$ diam., $1.6 \mathrm{~mm}$ thick } \\
\hline \multicolumn{2}{|l|}{ Flexure: Span/Thickness Ratio: } \\
\hline \multicolumn{2}{|c|}{ Compression: Aspect Ratio, Length/width or Diam.: } \\
\hline \multicolumn{2}{|l|}{ Specimens Tested/Data Point: } \\
\hline \multicolumn{2}{|l|}{ Strain (Load) Rate: } \\
\hline \multicolumn{2}{|l|}{ Test Temperature, K: 295} \\
\hline Other: & \\
\hline
\end{tabular}


Table A.2-6. Supplementary Information, Brechna [1965].

\begin{tabular}{|c|c|}
\hline IRRADIATION PARAMETERS & MATERIALS \\
\hline Source Type: Electron accelerator & Resin: \\
\hline Source Name: Stanford Mark II & Hardener: \\
\hline $\begin{array}{l}\text { Irradiation } \\
\text { Temperature: }<72^{\circ} \mathrm{C}\end{array}$ & Additives: \\
\hline Environment: & Glass Vol. z: \\
\hline Neutron $8:$ & Glass Mass z: \\
\hline Neutron Flux: & Glass Type: \\
\hline Fast Neutron $z: \quad(E>M e V)$ & Glass Finish: \\
\hline Fast Neutron F1ux: & Glass Weiave: \\
\hline Gamma z: & Glass Areal Density, $\mathrm{kg} / \mathrm{m}^{2}$ : \\
\hline Gamma Energy: & Filament Diameter, $\mu \mathrm{m}$ : \\
\hline Gamma Dose Rate: & Layup: \\
\hline Electron : : 100 & Production Method: Vacuum impreg. \\
\hline Electron Energy: $\quad 60-70 \mathrm{MeV}$ & Other: See original report for \\
\hline Electron Dose Rate: & details of specimen preparation. \\
\hline \multicolumn{2}{|l|}{ Other: } \\
\hline \multicolumn{2}{|c|}{ TEST METHOD } \\
\hline \multicolumn{2}{|c|}{ Test Type: Compressive strength (Bond shear) } \\
\hline \multicolumn{2}{|l|}{ Test Specifications: } \\
\hline \multicolumn{2}{|l|}{ Specimen Shape: } \\
\hline \multicolumn{2}{|l|}{ Specimen Dimensions: } \\
\hline \multicolumn{2}{|l|}{ Flexure: Span/Thickness Ratio: } \\
\hline \multicolumn{2}{|c|}{ Compression: Aspect Ratio, Length/width or Diam.: } \\
\hline \multicolumn{2}{|l|}{ Specimens Tested/Data Point: } \\
\hline \multicolumn{2}{|l|}{ Strain (Load) Rate: } \\
\hline Test Temperature, K: 295 & \\
\hline Other: & \\
\hline
\end{tabular}


Table A.2-7. Supplementary Information, Lipták et al. [1969].

\begin{tabular}{|c|c|}
\hline IRRADIATION PARAMETERS & MATERIALS \\
\hline Source Type: Pool reactor & Resin: \\
\hline Source Name: ASTRA (Austria) & Hardener: \\
\hline $\begin{array}{l}\text { Irradiation } \\
\text { Temperature: } 62^{\circ} \mathrm{C} ; 45^{\circ} \mathrm{C}\end{array}$ & Additives: \\
\hline Environment: Air & Glass Vol. \&: \\
\hline Neutron $:$ : $<5$ & Glass Mass o: : \\
\hline Neutron Flux: & Glass Type: \\
\hline Fast Neutron $q$ : & Glass Finish: \\
\hline $\begin{array}{l}\text { Fast Neutron Flux: } 1.5 \text { or } \\
1.8 \times 10^{14} /\left(\mathrm{m}^{2} \cdot \mathrm{s}\right)\end{array}$ & Glass Weave: \\
\hline Gamma $q:>95$ & Glass Areal Density, $\mathrm{kg} / \mathrm{m}^{2}$ : \\
\hline Gamma Energy: $0.5-6.0 \mathrm{MeV}$ & Filament Diameter, $\mu \mathrm{m}:$ \\
\hline Gamma Dose Rate: 2.8-69 Gy/s & Layup: \\
\hline Electron \&: & Production Method: VPI, prepreg. \\
\hline Electron Energy: & Other: \\
\hline \multicolumn{2}{|l|}{ Electron Dose Rate: } \\
\hline \multicolumn{2}{|l|}{ Other: } \\
\hline \multicolumn{2}{|c|}{ TEST METHOD } \\
\hline \multicolumn{2}{|c|}{ Test Type: Flexural strength (Tensile lap shear) } \\
\hline \multicolumn{2}{|c|}{ Test Specifications: ISO 178 (DIN 53 283) } \\
\hline \multicolumn{2}{|l|}{ Specimen Shape: Rectangular bar } \\
\hline \multicolumn{2}{|l|}{ Specimen Dimensions: $80 \times 10 \times 4 \mathrm{~mm}^{3}$} \\
\hline \multicolumn{2}{|l|}{ Flexure: Span/Thickness Ratio: 16.8} \\
\hline \multicolumn{2}{|c|}{ Compression: Aspect Ratio, Length/Width or Diam.: } \\
\hline \multicolumn{2}{|l|}{ Specimens Tested/Data Point: } \\
\hline \multicolumn{2}{|l|}{ Strain (Load) Rate: $0.2 \mathrm{~cm} / \mathrm{min}$} \\
\hline \multicolumn{2}{|l|}{ Test Temperature, $\mathrm{K}: 295$} \\
\hline Other: & \\
\hline
\end{tabular}


Table A.2-8. Supplementary Information, Imel et a1. [1979] .

\begin{tabular}{|c|c|}
\hline IRRADIATION PARAMETERS & MATERIALS \\
\hline Source Type: Pool reactor & Resin: \\
\hline Source Name: CFRMF (INEL) & Hardener: \\
\hline $\begin{array}{l}\text { Irradiation } \\
\text { Temperature: } 62^{\circ} \mathrm{C}\end{array}$ & Additives: \\
\hline Environment: Air & Glass Vol. $\&:$ \\
\hline Neutron 8 : 39 (65\% glass, $35 \%$ epoxy) & Glass Mass $\%: 65$ (probable aim) \\
\hline Neutron Fluence: $1.4 \times 10^{21} / \mathrm{m}^{2}$, $\max$. & Glass Type: E \\
\hline Fast Neutron $\%$ : $\quad(E>M e V)$ & Glass Finish: \\
\hline Fast Neutron Flux: & Glass Weave: \\
\hline Gamma $z: 61$ & Glass Areal Density, $\mathrm{kg} / \mathrm{m}^{2}$ : \\
\hline Gamma Energy: & Filament Diameter, fm: \\
\hline Gamma Dose Rate: & Layup: \\
\hline Electron $8:$ & Production Method: impregnation \\
\hline Electron Energy: & Other: \\
\hline Electron Dose Rate: & \\
\hline \multicolumn{2}{|l|}{ Other: } \\
\hline \multicolumn{2}{|c|}{ TEST METHOD } \\
\hline \multicolumn{2}{|l|}{ Test Type: Flexural strength } \\
\hline \multicolumn{2}{|l|}{ Test Specifications: ASTM D-790 } \\
\hline \multicolumn{2}{|l|}{ Specimen Shape: Rectangular bar } \\
\hline \multicolumn{2}{|l|}{ Specimen Dimensions: $100 \times 50 \times 13 \mathrm{~mm}^{3}$} \\
\hline \multicolumn{2}{|l|}{ Flexure: Span/Thickness Ratio: 6.4} \\
\hline \multicolumn{2}{|c|}{ Compression: Aspect Ratio, Length/Width or Diam.: } \\
\hline \multicolumn{2}{|l|}{ Specimens Tested/Data Point: } \\
\hline \multicolumn{2}{|l|}{ Strain (Load) Rate: $0.25 \mathrm{~cm} / \mathrm{min}$} \\
\hline Test Temperature, K: 295 & \\
\hline Other: & \\
\hline
\end{tabular}


Table A.2-9. Supplementary Information, Iida and Sumita [1983].

\begin{tabular}{|c|c|}
\hline IRRADIATION PARAMETERS & MATERIALS \\
\hline Source Type: $14 \mathrm{MeV} \mathrm{n}$ & Resin: \\
\hline Source Name: RTNS-II (LLNL) & Hardener: \\
\hline $\begin{array}{l}\text { Irradiation } \\
\text { Temperature: }\end{array}$ & Additives: \\
\hline Environment: & Glass Vol. 8 : \\
\hline Neutron $z: 100$ & Glass Mass \&: \\
\hline Neutron F1ux: & Glass Type: \\
\hline Fast Neutron $8: 100 \quad(E=14 \mathrm{MeV})$ & Glass Finish: \\
\hline Fast Neutron Flux: & Glass Weave: \\
\hline Gamma $8:$ & Glass Areal Density, $\mathrm{kg} / \mathrm{m}^{2}$ : \\
\hline Gamma Energy: & Filament Diameter, $\mu \mathrm{m}$ : \\
\hline Gamma Dose Rate: & Layup: \\
\hline Electron $8:$ & Production Method: \\
\hline Electron Energy: & Other: \\
\hline \multicolumn{2}{|l|}{ Electron Dose Rate: } \\
\hline \multicolumn{2}{|l|}{ Other: } \\
\hline \multicolumn{2}{|c|}{ TEST METHOD } \\
\hline \multicolumn{2}{|l|}{ Test Type: Compression } \\
\hline \multicolumn{2}{|l|}{ Test Specifications: } \\
\hline \multicolumn{2}{|l|}{ Specimen Shape: Cube } \\
\hline \multicolumn{2}{|l|}{ Specimen Dimensions: $3 \times 3 \times 3 \mathrm{~mm}^{3}$} \\
\hline \multicolumn{2}{|l|}{ Flexure: Span/Thickness Ratio: } \\
\hline \multicolumn{2}{|c|}{ Compression: Aspect Ratio, Length/Width or Diam.: I } \\
\hline \multicolumn{2}{|l|}{ Specimens Tested/Data Point: 2 or 3} \\
\hline \multicolumn{2}{|l|}{ Strain (Load) Rate: $0.1 \mathrm{~cm} / \mathrm{min}$} \\
\hline Test Temperature, $\mathrm{K}: 295$ & \\
\hline Other: & \\
\hline
\end{tabular}


Table A.2-10. Supplementary Information, Nishiura et al. $[1988 \mathrm{a}, \mathrm{b}]$.

\begin{tabular}{|c|c|}
\hline IRRADIATION PARAMETERS & MATERIALS \\
\hline Source Type: e or $\gamma\left({ }^{60} \mathrm{Co}\right)$ & Resin: \\
\hline Source Name: Linac (Osaka Univ.) & Hardener: \\
\hline $\begin{array}{l}\text { Irradiation } \\
\text { Temperature: }\end{array}$ & Additives: \\
\hline Environment: & Glass Vol. $q$ : \\
\hline Neutron $q$ : & Glass Mass $8: 62-65$ \\
\hline Neutron Flux: & Glass Type: \\
\hline Fast Neutron $z: \quad(E>M e V)$ & Glass Finish: \\
\hline Fast Neutron Flux: & Glass Weave: \\
\hline Gamma : : 100 & Glass Areal Density, $\mathrm{kg} / \mathrm{m}^{2}$ : \\
\hline Gamma Energy: $-1.2 \mathrm{MeV}$ & Filament Diameter, $\mu \mathrm{m}:$ \\
\hline Gamma Dose Rate: $1.1 \times 10^{3} \mathrm{~Gy} / \mathrm{s}$ & Layup: \\
\hline Electron $8: 100$ & Production Method: \\
\hline Electron Energy: $24 \mathrm{MeV}$ & Other: \\
\hline Electron Dose Rate: $61 \times 10^{3} \mathrm{~Gy} / \mathrm{s}$ & \\
\hline \multicolumn{2}{|l|}{ Other: } \\
\hline \multicolumn{2}{|c|}{ TEST METHOD } \\
\hline \multicolumn{2}{|l|}{ Test Type: Tensile strength (Tear test) } \\
\hline \multicolumn{2}{|c|}{ Test Specifications: $\quad$ (ASTM E-399-78a) } \\
\hline \multicolumn{2}{|c|}{ Specimen Shape: Rectangular bar, notched (Double-cantilever) } \\
\hline \multicolumn{2}{|c|}{ Specimen Dimensions: $85 \times 10 \times 2 \mathrm{~mm}^{3}\left(50 \times 10 \times 5 \mathrm{~mm}^{3}\right)$} \\
\hline \multicolumn{2}{|l|}{ Flexure: Span/Thickness Ratio: } \\
\hline \multicolumn{2}{|c|}{ Compression: Aspect Ratio, Length/Width or Diam.: } \\
\hline \multicolumn{2}{|l|}{ Specimens Tested/Data Point: } \\
\hline \multicolumn{2}{|l|}{ Strain (Load) Rate: $0.16 \mathrm{~cm} / \mathrm{min}$} \\
\hline Test Temperature, $\mathrm{K}: 77,295$ & \\
\hline Other: $3.25-\mathrm{mm}$-deep notches $(0.60-\mathrm{mm}$ & learance slots) \\
\hline
\end{tabular}


Table A.2-11. Supplementary Information, Nishijima et al [1988].

\begin{tabular}{|c|c|}
\hline IRRADIATION PARAMETERS & MATERIALS \\
\hline Source Type: Reactor $\left({ }^{60} \mathrm{Co}\right)$ & Resin: \\
\hline Source Name: Kyoto Univ. Reactor & Hardener: \\
\hline $\begin{array}{l}\text { Irradiation }-20 \mathrm{~K}, 360 \mathrm{~K},(77 \text {, } \\
\text { Temperature: }-295 \mathrm{~K})\end{array}$ & Additives: \\
\hline Environment: & Glass Vol. \\
\hline Neutron $q$ : & Glass Mass $\frac{8}{8}: 60-62$ \\
\hline Neutron Flux: & Glass Type: \\
\hline Fast Neutron $q$ : $\quad(E>M e V)$ & Glass Finish: \\
\hline Fast Neutron Flux: & Glass Weave: \\
\hline Gamma of: & Glass Areal Density, $\mathrm{kg} / \mathrm{m}^{2}$ : \\
\hline Gamma Energy: & Filament Diameter, $\mu \mathrm{m}$ : \\
\hline Gamma Dose Rate: $5.5 \mathrm{~Gy} / \mathrm{s}$ & Layup: \\
\hline Electron oे: & Production Method: laminate \\
\hline Electron Energy: & Other: \\
\hline \multicolumn{2}{|l|}{ Electron Dose Rate: } \\
\hline \multicolumn{2}{|l|}{ Other: $n$ dose $-0.1 \times \gamma$ dose } \\
\hline \multicolumn{2}{|c|}{ TEST METHOD } \\
\hline \multicolumn{2}{|l|}{ Test Type: Interlaminar shear strength } \\
\hline \multicolumn{2}{|l|}{ Test Specifications: } \\
\hline \multicolumn{2}{|c|}{ Specimen Shape: Notched, rectangular bar } \\
\hline \multicolumn{2}{|c|}{ Specimen Dimensions: $16 \times 4 \times 4 \mathrm{~mm}^{3}$, notch depth, width $=2 \mathrm{~mm}$} \\
\hline \multicolumn{2}{|l|}{ Flexure: Span/Thickness Ratio: } \\
\hline \multicolumn{2}{|c|}{ Compression: Aspect Ratio, Length/Width or Diam.: } \\
\hline \multicolumn{2}{|l|}{ Specimens Tested/Data Point: } \\
\hline \multicolumn{2}{|l|}{ Strain (Load) Rate: } \\
\hline Test Temperature, $\mathrm{K}: 77,295$ & \\
\hline Other: & \\
\hline
\end{tabular}


Table A.2-12. Supplementary Information, Hacker et a1. [1984]

\begin{tabular}{|c|c|}
\hline IRRADIATION PARAMETERS & MATERIALS \\
\hline Source Type: ${ }^{60} \mathrm{Co}$ & Resin: \\
\hline Source Name: & Hardener: \\
\hline $\begin{array}{l}\text { Irradiation } \\
\text { Temperature: }\end{array}$ & Additives: \\
\hline Environment: & Glass Vol. $8:$ \\
\hline Neutron $8:$ & Glass Mass of: \\
\hline Neutron Flux: & Glass Type: \\
\hline Fast Neutron $q: \quad(E>M e V)$ & Glass Finish: \\
\hline Fast Neutron Flux: & Glass Weave: \\
\hline Gamma $:$ : 100 & Glass Areal Density, $\mathrm{kg} / \mathrm{m}^{2}$ : \\
\hline Gamma Energy: $-1.2 \mathrm{MeV}$ & Filament Diameter, $\mu \mathrm{n}$ : \\
\hline Gamma Dose Rate: & Layup: \\
\hline Electron $8:$ & Production Method: \\
\hline Electron Energy: & Other: \\
\hline Electron Dose Rate: & \\
\hline \multicolumn{2}{|l|}{ Other: } \\
\hline \multicolumn{2}{|c|}{ TEST METHOD } \\
\hline \multicolumn{2}{|l|}{ Test Type: Flexural strength } \\
\hline \multicolumn{2}{|l|}{ Test specifications: DIN 53452} \\
\hline \multicolumn{2}{|l|}{ Specimen Shape: Bar } \\
\hline \multicolumn{2}{|l|}{ Specimen Dimensions: } \\
\hline \multicolumn{2}{|l|}{ Flexure: Span/Thickness Ratio: } \\
\hline \multicolumn{2}{|c|}{ Compression: Aspect Ratio, Length/Width or Diam.: } \\
\hline \multicolumn{2}{|l|}{ Specimens Tested/Data Point: } \\
\hline \multicolumn{2}{|l|}{ Strain (Load) Rate: } \\
\hline Test Temperature, K: 295 & \\
\hline Other: & \\
\hline
\end{tabular}


Table A.2-13. Supplementary Information, Phillips et al. [1984].

\begin{tabular}{|c|c|}
\hline IRRADIATION PARAMETERS & MATERIALS \\
\hline Source Type: Pool reactor & Resin: \\
\hline Source Name: ASTRA & Hardener: \\
\hline $\begin{array}{l}\text { Irradiation } \\
\text { Temperature: } 30-40^{\circ} \mathrm{C}\end{array}$ & Additives: \\
\hline Environment: & Glass Vol. : के: \\
\hline Neutron of: & Glass Mass of: \\
\hline Neutron Flux: (thermal) $4-5 \times 10^{16} / \mathrm{m}^{2} \cdot \mathrm{s}$ ) & Glass Type: \\
\hline Fast Neutron 8 : & Glass Finish: \\
\hline Fast Neutron Flux: $2-3 \times 10^{16} /\left(\mathrm{m}^{2} \cdot \mathrm{s}\right)$ & Glass Weave: \\
\hline Gamma oे: & Glass Areal Density, $\mathrm{kg} / \mathrm{m}^{2}$ : \\
\hline Gamma Energy: & Filament Diameter, $\mu \mathrm{m}$ : \\
\hline Gamma Dose Rate: $280-560 \mathrm{~Gy} / \mathrm{s}$ & Layup: \\
\hline Electron 8 : & Production Method: Machined (cut) \\
\hline Electron Energy: & Other: \\
\hline \multicolumn{2}{|l|}{ Electron Dose Rate: } \\
\hline \multicolumn{2}{|l|}{ Other: } \\
\hline \multicolumn{2}{|c|}{ TEST METHOD } \\
\hline \multicolumn{2}{|l|}{ Test Type: Flexural strength $\left(G_{\mathrm{KC}}\right)$} \\
\hline \multicolumn{2}{|c|}{ Test Specifications: ISO $178-1975^{*}$ (Double-torsion technique) } \\
\hline \multicolumn{2}{|l|}{ Specimen Shape: Rectangular bar } \\
\hline \multicolumn{2}{|c|}{ Specimen Dimensions: $80 \times 10 \times 4 \mathrm{~mm}^{3}\left(80 \times 30 \times 3 \mathrm{~mm}^{3}\right)$} \\
\hline \multicolumn{2}{|l|}{ Flexure: Span/Thickness Ratio: 16} \\
\hline \multicolumn{2}{|c|}{ Compression: Aspect Ratio, Length/Width or Diam.: } \\
\hline \multicolumn{2}{|l|}{ Specimens Tested/Data Point: 5} \\
\hline \multicolumn{2}{|l|}{ Strain (Load) Rate: } \\
\hline Test Temperature, $\mathrm{K}: 295$ & \\
\hline Other: "Probable specifications and & nsion \\
\hline
\end{tabular}


Table A.2-14. Supplementary Information, Dahlerup-Peterson and Perrot [1979], Dahlerup-Peterson [1980].

\begin{tabular}{|c|c|}
\hline IRRADIATION PARAMETERS & MATERIALS \\
\hline Source Type: ${ }^{60} \mathrm{Co}$ & Resin: Araldite B901 (Permaglas) \\
\hline Source Name: & Hardener: \\
\hline $\begin{array}{l}\text { Irradiation } \\
\text { Temperature: Ambient }\end{array}$ & Additives: \\
\hline Environment: & Glass Vol. $8: 50.6 \quad(55.7)$ \\
\hline Neutron $8:$ & Glass Mass of: \\
\hline Neutron Flux: & Glass Type: E \\
\hline Fast Neutron $s:$ & Glass Finish: \\
\hline Fast Neutron Flux: & Glass Weave: unidirectional (plain) \\
\hline Gamma $8: 100$ & Glass Areal Density, $\mathrm{kg} / \mathrm{m}^{2}$ : \\
\hline Gamma Energy: $-1.2 \mathrm{MeV}$ & Filament Diameter, $\mu \mathrm{m}$ : \\
\hline Gamma Dose Rate: & Layup: \\
\hline Electron $8:$ & Production Method: \\
\hline Electron Energy: & Other: \\
\hline \multicolumn{2}{|l|}{ Electron Dose Rate: } \\
\hline \multicolumn{2}{|l|}{ Other: } \\
\hline \multicolumn{2}{|c|}{ TEST METHOD } \\
\hline \multicolumn{2}{|l|}{ Test Type: Tensile strength } \\
\hline \multicolumn{2}{|l|}{ Test Specifications: ASTM D 3039-74 } \\
\hline \multicolumn{2}{|l|}{ Specimen Shape: } \\
\hline \multicolumn{2}{|c|}{ Specimen Dimensions: Total length, $270 \mathrm{~mm}$; gage $130 \times 12.7 \times 2 \mathrm{~mm}^{3}$} \\
\hline \multicolumn{2}{|l|}{ Flexure: Span/Thickness Ratio: } \\
\hline \multicolumn{2}{|c|}{ Compression: Aspect Ratio, Length/Width or Diam.: } \\
\hline \multicolumn{2}{|l|}{ Specimens Tested/Data Point: } \\
\hline \multicolumn{2}{|l|}{ Strain (Load) Rate: $0.2 \mathrm{~cm} / \mathrm{min}$} \\
\hline Test Temperature, $\mathrm{K}: 77,295$ & \\
\hline Other: Reinforcing tabs used with est & ially designed grip system \\
\hline
\end{tabular}


Table A.2-15. Supplementary Information, Takeda et a1. [1989].

\begin{tabular}{|c|c|}
\hline IRRADIATION PARAMETERS & MATERIALS \\
\hline Source Type: Electron & Resin: \\
\hline Source Name: Dynamitron 1EA & Hardener: \\
\hline $\begin{array}{l}\text { Irradiation } \\
\text { Temperature: }\end{array}$ & Additives: \\
\hline Environment: & Glass Vol. q: \\
\hline Neutron $q$ : & Glass Mass o: \\
\hline Neutron Flux: & Glass Type: \\
\hline Fast Neutron $q$ : & Glass Finish: \\
\hline Fast Neutron F1ux: & Glass Weave: \\
\hline Gamma $8:$ & Glass Areal Density, $\mathrm{kg} / \mathrm{m}^{2}$ : \\
\hline Gamma Energy: & Filament Diameter, $\mu \mathrm{m}$ : \\
\hline Gamma Dose Rate: & Layup: \\
\hline Electron $\&: 100$ & Production Method: \\
\hline Electron Energy: $3 \mathrm{MeV}$ & Other: \\
\hline Electron Dose Rate: $5 \times 10^{3} \mathrm{~Gy} / \mathrm{s}$ & \\
\hline \multicolumn{2}{|l|}{ Other: } \\
\hline \multicolumn{2}{|c|}{ TEST METHOD } \\
\hline \multicolumn{2}{|c|}{ Test Type: Mode II interlaminar fracture toughness } \\
\hline \multicolumn{2}{|l|}{ Test Specifications: 3 -point flexure } \\
\hline \multicolumn{2}{|l|}{ Specimen Shape: End notched flexure } \\
\hline \multicolumn{2}{|c|}{ Specimen Dimensions: $60 \times 6.5 \times 2 \mathrm{~mm}^{3} ;$ notch depth $=15 \mathrm{~mm}$} \\
\hline \multicolumn{2}{|c|}{ Flexure: Span/Thickness Ratio: } \\
\hline \multicolumn{2}{|c|}{ Compression: Aspect Ratio, Length/Width or Diam.: } \\
\hline \multicolumn{2}{|l|}{ Specimens Tested/Data Point: } \\
\hline \multicolumn{2}{|l|}{ Strain (Load) Rate: } \\
\hline Test Temperature, K: 295 & \\
\hline Other: & \\
\hline
\end{tabular}


Table A.2-16. Supplementary Information, Nishijima et al. [1990].

\begin{tabular}{|c|c|}
\hline IRRADIATION PARAMETERS & MATERIALS \\
\hline Source Type: Electron & Resin: 003 (epoxy); 005 (BT) \\
\hline Source Name: (JAERI) & Hardener: \\
\hline $\begin{array}{l}\text { Irradiation } \\
\text { Temperature: Ambient }\end{array}$ & Additives: \\
\hline Environment: & Glass Vol. q : \\
\hline Neutron $\frac{q}{6}:$ & Glass Mass z: \\
\hline Neutron Flux: & Glass Type: 3 D (ZI types) \\
\hline Fast Neutron $\%: \quad(E>M e V)$ & Glass Finish: \\
\hline Fast Neutron Flux: & Glass Weave: \\
\hline Gamma $;:$ & Glass Areal Density, $\mathrm{kg} / \mathrm{m}^{2}$ : \\
\hline Gamma Energy: & Filament Diameter, $\mu \mathrm{m}$ : \\
\hline Gamma Dose Rate: & Layrup: \\
\hline Electron $8: 100$ & Production Method: \\
\hline Electron Energy: $2 \mathrm{MeV}$ & Other: \\
\hline Electron Dose Rate: $10^{5} \mathrm{~Gy} / \mathrm{s}$ & \\
\hline \multicolumn{2}{|l|}{ Other: } \\
\hline \multicolumn{2}{|c|}{ TEST METHOD } \\
\hline \multicolumn{2}{|l|}{ Test Type: Compressive strength } \\
\hline \multicolumn{2}{|l|}{ Test Specifications: } \\
\hline \multicolumn{2}{|l|}{ Specimen Shape: } \\
\hline \multicolumn{2}{|l|}{ Specimen Dimensions: $5 \times 5 \times 1 \mathrm{~mm}^{3}$} \\
\hline \multicolumn{2}{|l|}{ Flexure: Span/Thickness Ratio: } \\
\hline \multicolumn{2}{|c|}{ Compression: Aspect Ratio, Length/Width or Diam.: 0.2} \\
\hline \multicolumn{2}{|l|}{ Specimens Tested/Data Point: } \\
\hline \multicolumn{2}{|l|}{ Strain (Load) Rate: } \\
\hline Test Temperature, $\mathrm{K}: 295$ & \\
\hline Other: & \\
\hline
\end{tabular}


Table A.2-17. Supplementary Information, McManamy et al. [1990, $1991 \mathrm{a}, \mathrm{b}]$.

\begin{tabular}{|c|c|}
\hline IRRADIATION PARAMETERS & MATERIALS \\
\hline Source Type: Reactor & Resin: \\
\hline Source Name: ATR (INEL) & Hardener: \\
\hline $\begin{array}{l}\text { Irradiation } \\
\text { Temperature: }<67^{\circ} \mathrm{C}\end{array}$ & Additives: \\
\hline Environment: & Glass Vol. \&: 57, warp; 9, thickness \\
\hline Neutron $8: 35-40$ & Glass Mass $8: 71$ \\
\hline Neutron Flux: & Glass Type: T \\
\hline Fast Neutron $8: 4($ of $n)(E>1 \mathrm{MeV})$ & Glass Finish: Epoxy silane \\
\hline Fast Neutron Flux: & Glass Weave: $3 \mathrm{D}$ \\
\hline Gamma $\%: 65-60$ & Glass Areal Density, $\mathrm{kg} / \mathrm{m}^{2}$ : \\
\hline Gamma Energy: & Filament Diameter, $\mu \mathrm{m}:$ \\
\hline Gamma Dose Rate: & Layup: \\
\hline Electron 8: & Production Method: \\
\hline Electron Energy: & Other: \\
\hline Electron Dose Rate: & \\
\hline Other: $\operatorname{Max} n_{f}-2.5 \times 10^{22} / \mathrm{m}^{2}$ & \\
\hline TEST & METHOD \\
\hline Test Type: Flexural strength (Shear/ & compression) \\
\hline $\begin{array}{c}\text { Test specifications: } \begin{array}{c}\text { (Compression of } \\
\text { failure) }\end{array} \\
\end{array}$ & $345 \mathrm{MPa}$, then shear load increased to \\
\hline Specimen Shape: Rectangular bar & \\
\hline Specimen Dimensions: $51 \times 5 \times 1.5 \mathrm{~mm}^{3}$ & $\left(12.7 \times 12.7 \times-1 \mathrm{~mm}^{3}\right)$ \\
\hline Flexure: Span/Thickness Ratio: 21 & \\
\hline Compression: Aspect Ratio, Length/Wi & dth or Diam.: \\
\hline Specimens Tested/Data Point: 6 (3) & \\
\hline Strain (Load) Rate: & \\
\hline Test Temperature, K: 295 & \\
\hline Other: & \\
\hline
\end{tabular}





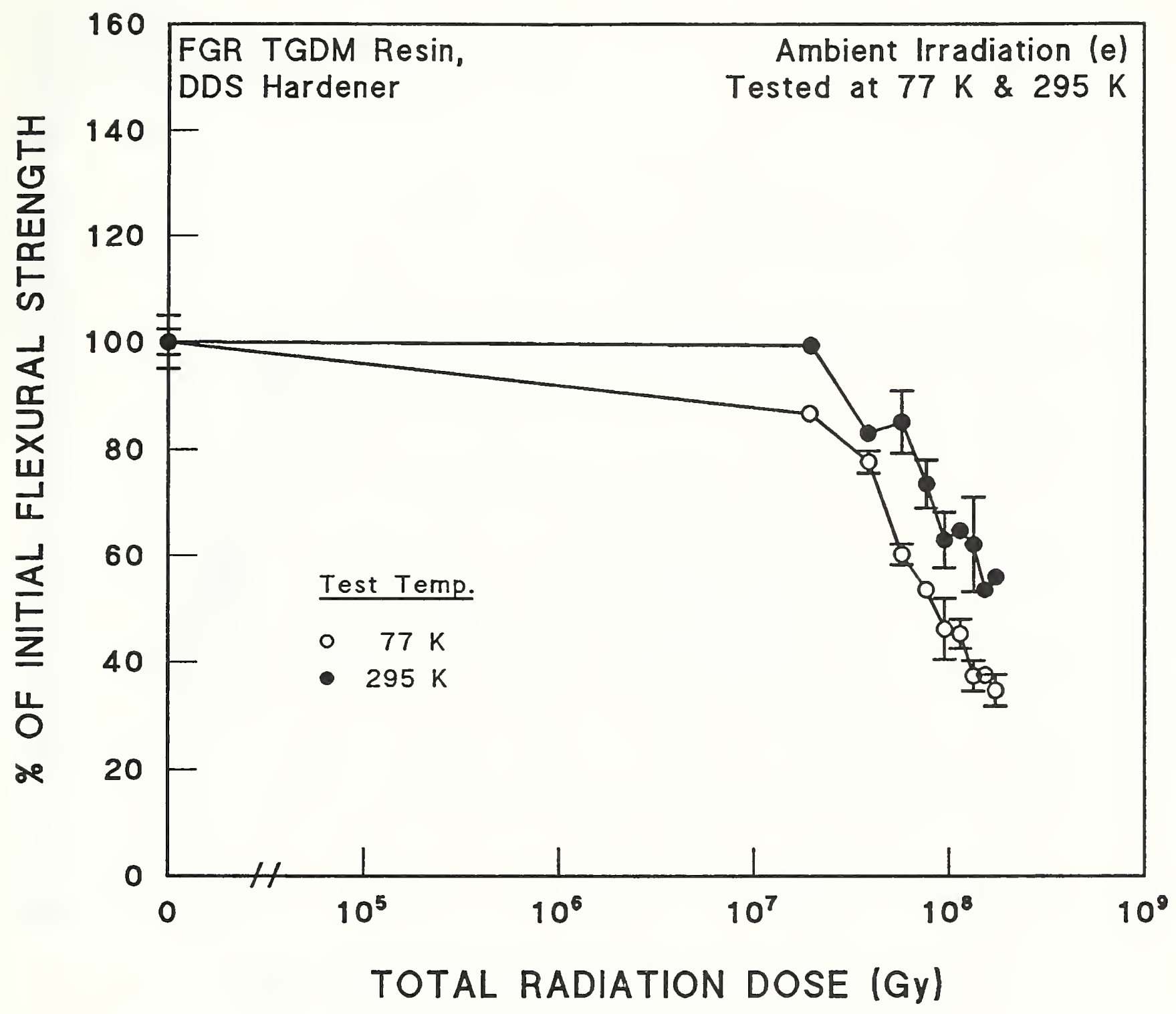

\begin{tabular}{|c|c|}
\hline "O" Strength, MPa & Supplier \\
\hline ○ $1544.8 \pm 36.9$ & $\begin{array}{l}\text { Sumitomo Bakelite } \\
\text { Co., Ltd. }\end{array}$ \\
\hline - $\quad 815.3 \pm 40.6$ & $\begin{array}{l}\text { Sumitomo Bakelite } \\
\text { Co., Ltd. }\end{array}$ \\
\hline
\end{tabular}

Figure A.3-1. Flexural strength at 77 and $295 \mathrm{~K}$ of FGR TGDM resin with DDS hardener after ambient electron irradiation. Supplementary Table A.3-1. Data from Egusa [1988]. 


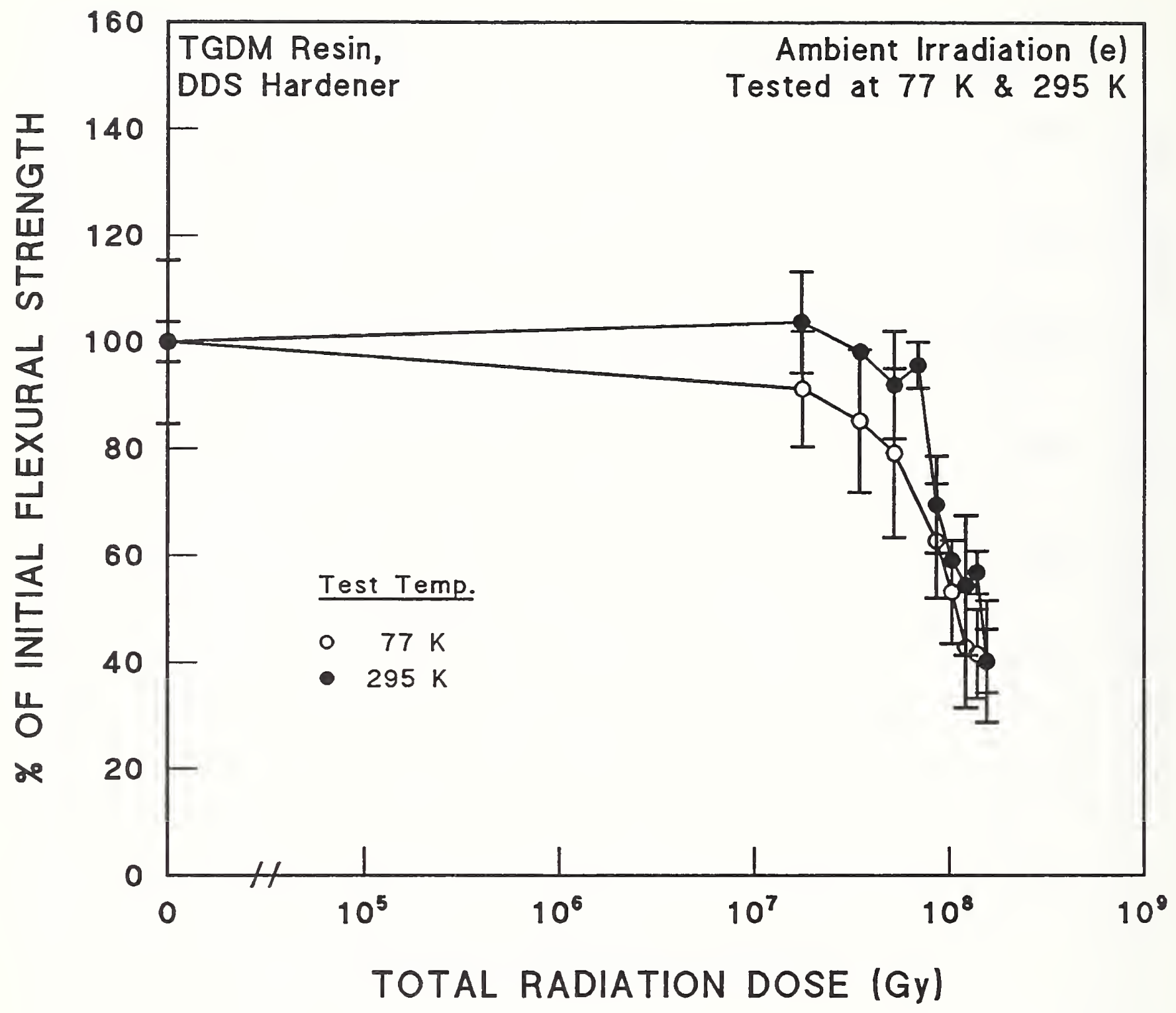

\section{Supplier}

Sumitomo Bakelite Co., Ltd.

Sumitomo Bakelite Co., Ltd.

Figure A.3-2. Flexural strength at 77 and $295 \mathrm{~K}$ of neat TGDM resin with DDS hardener after ambient electron irradiation. Supplementary Table A.3-1. Data from Egusa [1988]. 


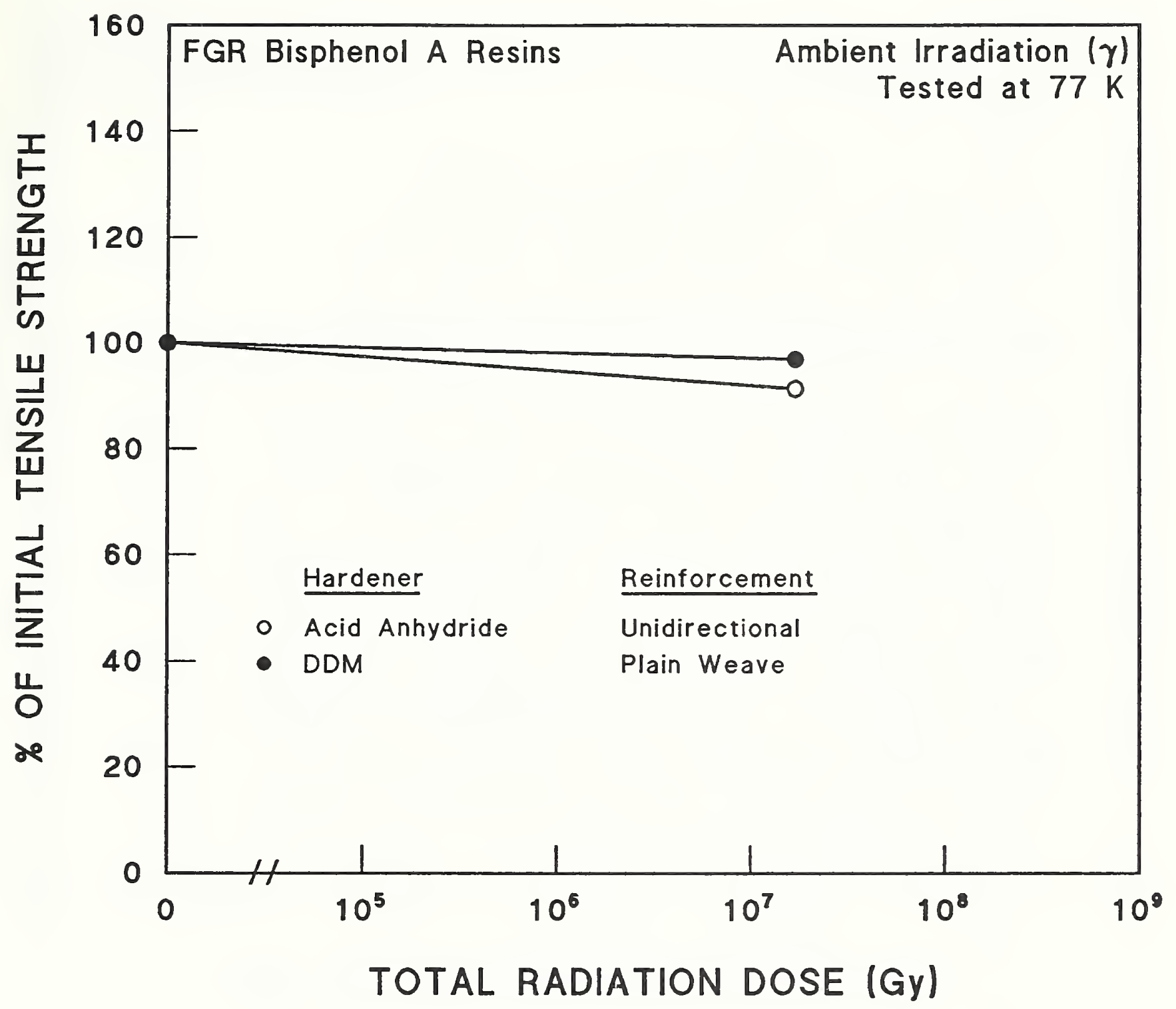

\begin{tabular}{|c|c|c|}
\hline "O" Strength, MPa & Resin/Laminate & Supplier \\
\hline o 1311.0 & $\begin{array}{l}\text { Araldite } \\
\text { B } 901\end{array}$ & Ciba-Geigy \\
\hline - 834.0 & $\begin{array}{l}\text { Permaglas } \\
\text { TE } 630\end{array}$ & --- \\
\hline
\end{tabular}

Figure A.3-3. Tensile strength at $77 \mathrm{~K}$ of FGR bisphenol A resins after ambient gamma irradiation. Supplementary Table A.2-14. Data from Dahlerup-Peterson and Perrot [1979] and Dahlerup-Peterson [1980]. 


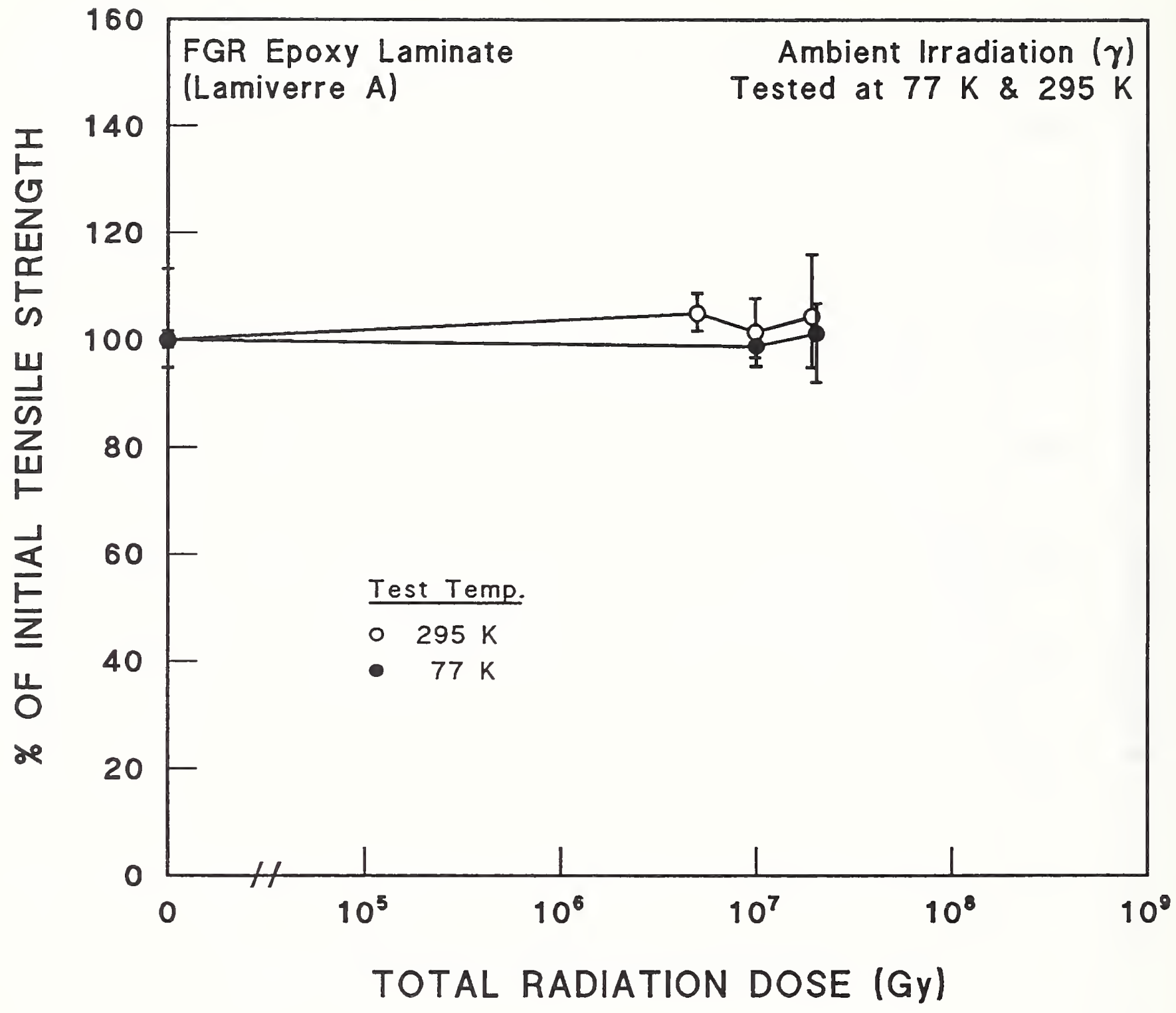

"O" Strength, MPa

Weave

Finish

Glass

Supplier

- $325.4 \pm 29.8$

Plain

Silane

$62-65$ mass $\%$

- $641.9 \pm 19.95$

Plain

Silane

$62-65$ mass $\%$

Figure A.3-4. Tensile strength at 77 and $295 \mathrm{~K}$ of FGR epoxy laminate (Lamiverre A) after ambient gamma irradiation. Supplementary Table A.2-10. Data from Nishiura et a1. [1988a] . 


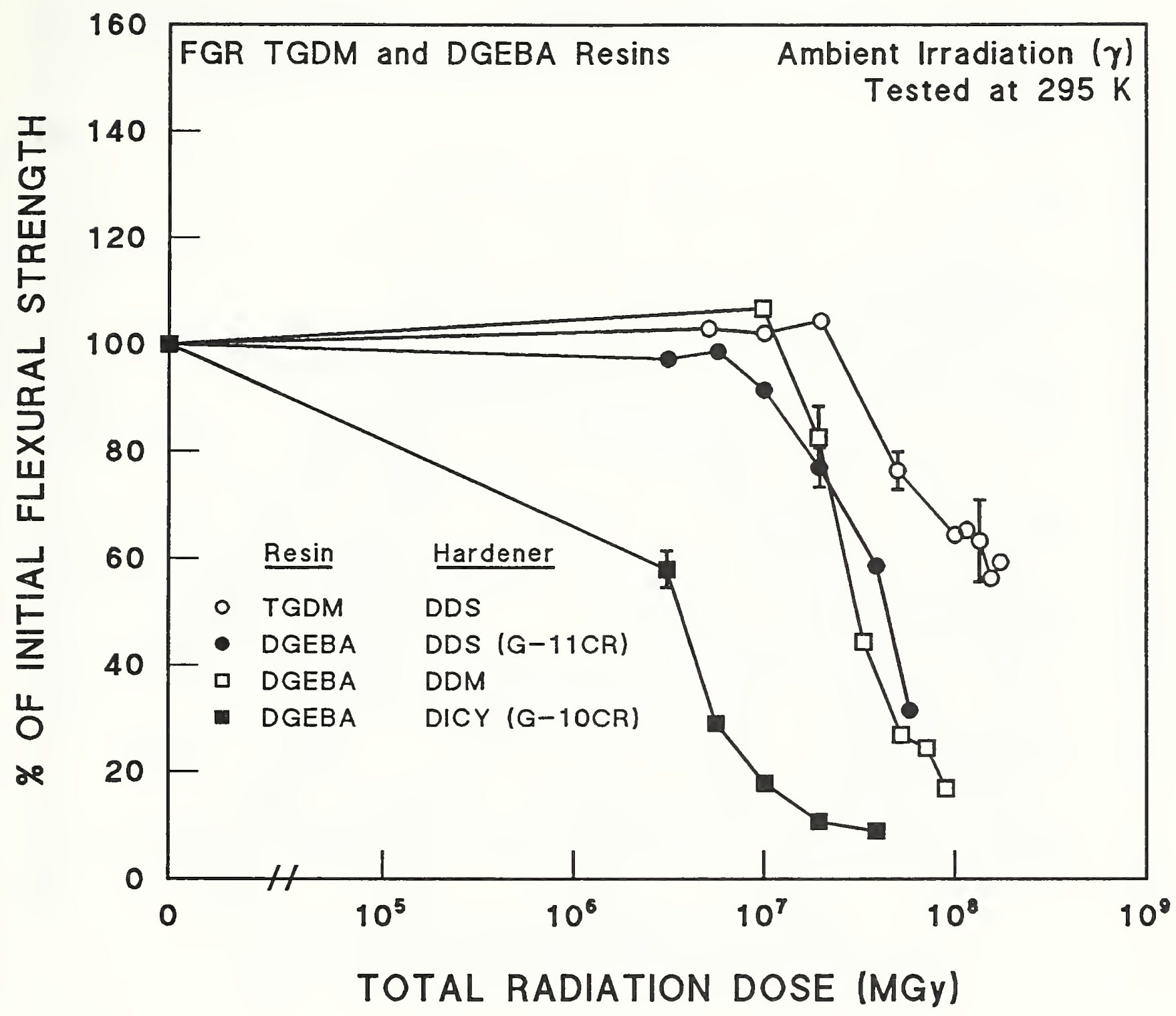

\begin{tabular}{|c|c|c|c|}
\hline "0" & Strength, MPa & Glass Finish & Supplier \\
\hline 0 & $776.7 \pm 0.0$ & $\begin{array}{l}\gamma \text {-glycidoxypropyl- } \\
\text { trimethoxysilane }\end{array}$ & $\begin{array}{l}\text { Sumitomo Bakelite } \\
\text { Co., Ltd. }\end{array}$ \\
\hline - & $645.6 \pm 0.0$ & $\begin{array}{l}\gamma \text {-aminopropyltrieth- } \\
\text { oxysilane }\end{array}$ & Spaulding \\
\hline 口 & $564.8 \pm 0.0$ & $\begin{array}{l}\gamma \text {-glycidoxypropyl- } \\
\text { trimethoxysilane }\end{array}$ & $\begin{array}{l}\text { Sumitomo Bakelite } \\
\text { Co., Ltd. }\end{array}$ \\
\hline a & $582.7 \pm 0.0$ & $\begin{array}{l}\gamma \text {-aminopropyltrieth- } \\
\text { oxysilane }\end{array}$ & Spaulding \\
\hline
\end{tabular}

Figure A.3-5. Flexural strength at $295 \mathrm{~K}$ of FGR TGDM and DGEBA resins and laminates (G-10CR, G-11CR) after ambient gamma irradiation. Supplementary Table A.3-2. Data from Egusa and Hagiwara [1986]. 


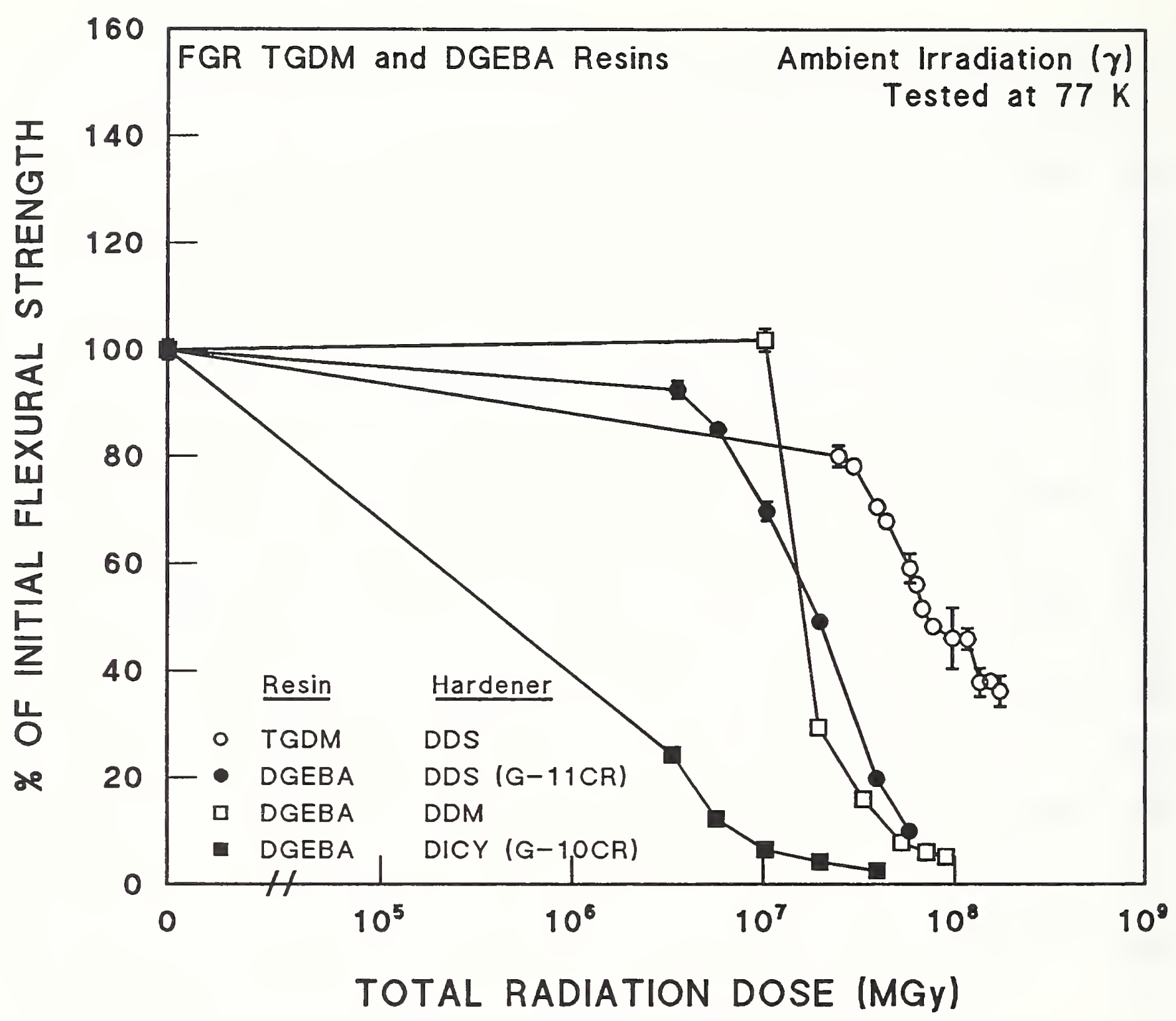

\begin{tabular}{|c|c|c|c|}
\hline "O" & Strength, MPa & Glass Finish & Supplier \\
\hline o & $1529.0 \pm 28.2$ & $\begin{array}{l}\gamma \text {-glycidoxypropyl- } \\
\text { trimethoxysilane }\end{array}$ & $\begin{array}{l}\text { Sumitomo Bakelite } \\
\text { Co., Ltd. }\end{array}$ \\
\hline - & $1123.5 \pm 20.8$ & $\begin{array}{l}\gamma \text {-aminopropyltrieth- } \\
\text { oxysilane }\end{array}$ & Spaulding \\
\hline$\square$ & $1070.6 \pm 0.0$ & $\begin{array}{l}\gamma \text {-glycidoxypropyl- } \\
\text { trimethoxysilane }\end{array}$ & $\begin{array}{l}\text { Sumitomo Bakelite } \\
\text { Co., Ltd. }\end{array}$ \\
\hline ש & $1213.5 \pm 0.0$ & $\begin{array}{l}\gamma \text {-aminopropyltrieth- } \\
\text { oxysilane }\end{array}$ & Spaulding \\
\hline
\end{tabular}

Figure A.3-6. Flexural strength at $77 \mathrm{~K}$ of FGR TGDM and DGEBA resins and laminates (G-10CR, G-11CR) after ambient gamma irradiation. Supplementary Table A.3-2. Data from Egusa and Hagiwara [1986]. 


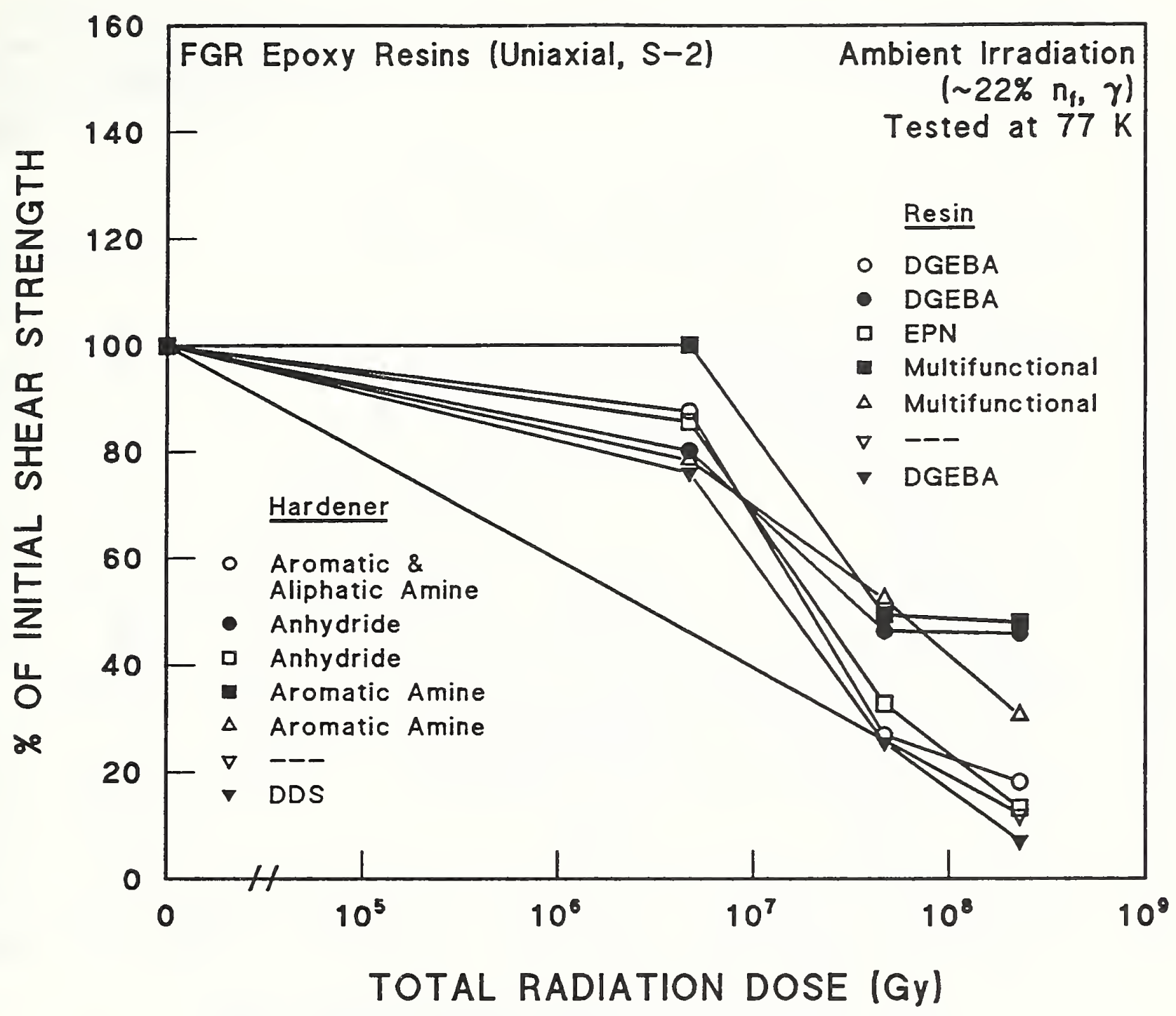

\begin{tabular}{|c|c|c|c|c|}
\hline "O" strength, $\mathrm{MPa}$ & Resin Name & Accelerator & Flexibilizer & Supplier \\
\hline - 160.0 & CTD -100 & No & Yes & CTD, Inc. \\
\hline - 201.0 & CTD-101 & Yes & No & CTD, Inc. \\
\hline 220.0 & CTD-102 & Yes & No & CTD, Inc. \\
\hline - 142.0 & CTD -110 & No & No & CTD, Inc. \\
\hline$\Delta \quad 184.0$ & CTD-112 & No & No & CTD, Inc. \\
\hline$\nabla \quad 100.0$ & CTD-501 & --- & -- & CTD, Inc. \\
\hline$\nabla \quad 196.0$ & G11-CR & -- & -- & $\begin{array}{l}\text { Spaulding } \\
\text { (Resin) }\end{array}$ \\
\hline
\end{tabular}

Comments: $110-125^{\circ} \mathrm{C}$ Cure Temperature

Figure A.3-7. Shear strength at $77 \mathrm{~K}$ of FGR epoxy resins (including G-11CR) after ambient reactor irradiation. Supplementary Table A.3-3. Data from Munshi [1991]. 


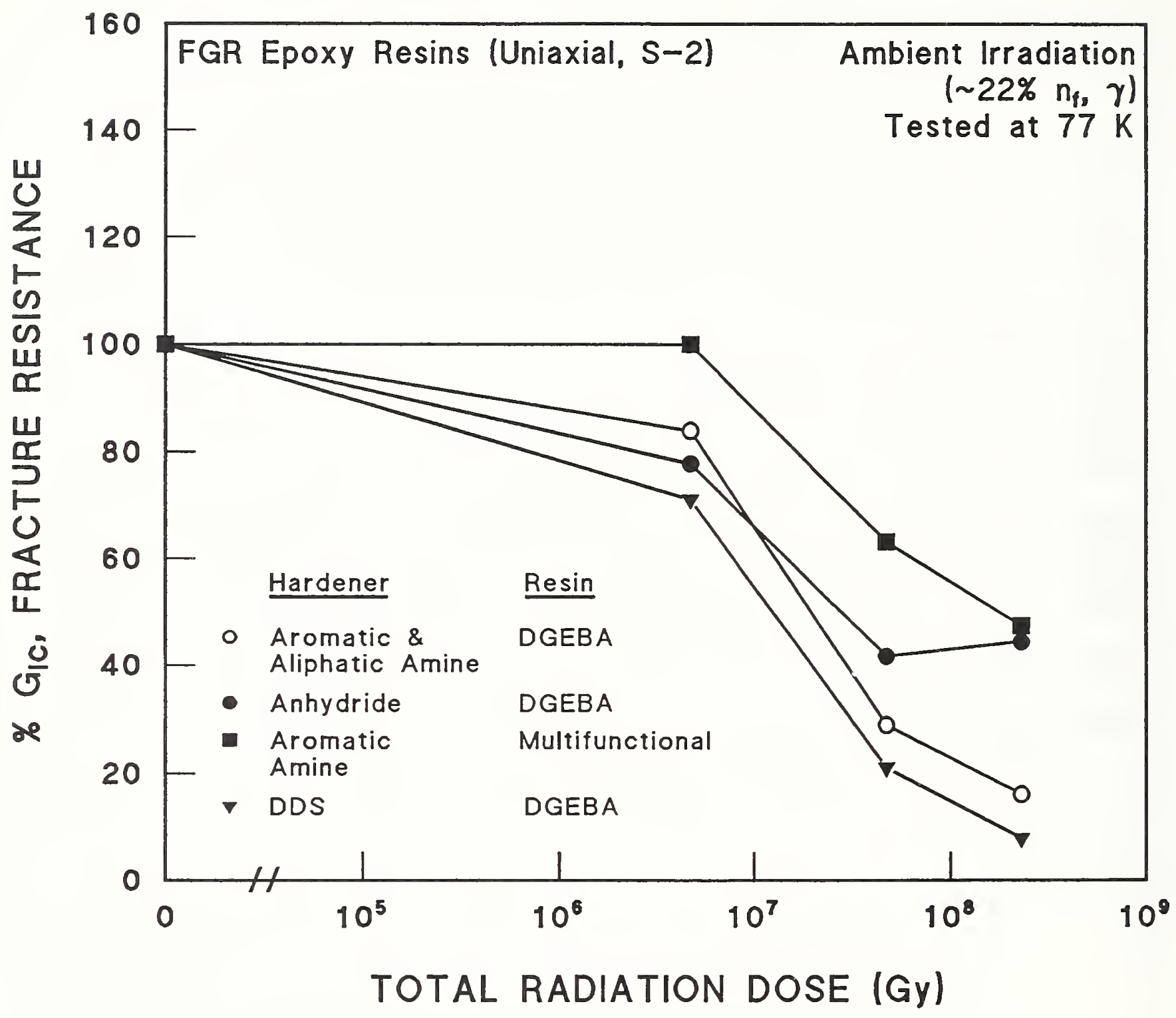

"O" Stren., kJ/m

Resin Name Accelerator

CTD-100

CTD-101

CTD-110

G11-CR

No

Yes

No

-- $\nabla \quad 0.38$

0.31

0.19
Flexibilizer

Yes

No

No
Supplier

CTD, Inc.

CTD, Inc. CTD, Inc.

Spaulding (Resin)

Figure A.3-8. Fracture resistance at $77 \mathrm{~K}$ of FGR epoxy resins (including G-11CR) after ambient reactor irradiation. Supplementary Table A.3-3. Data from Munshi [1991] . 


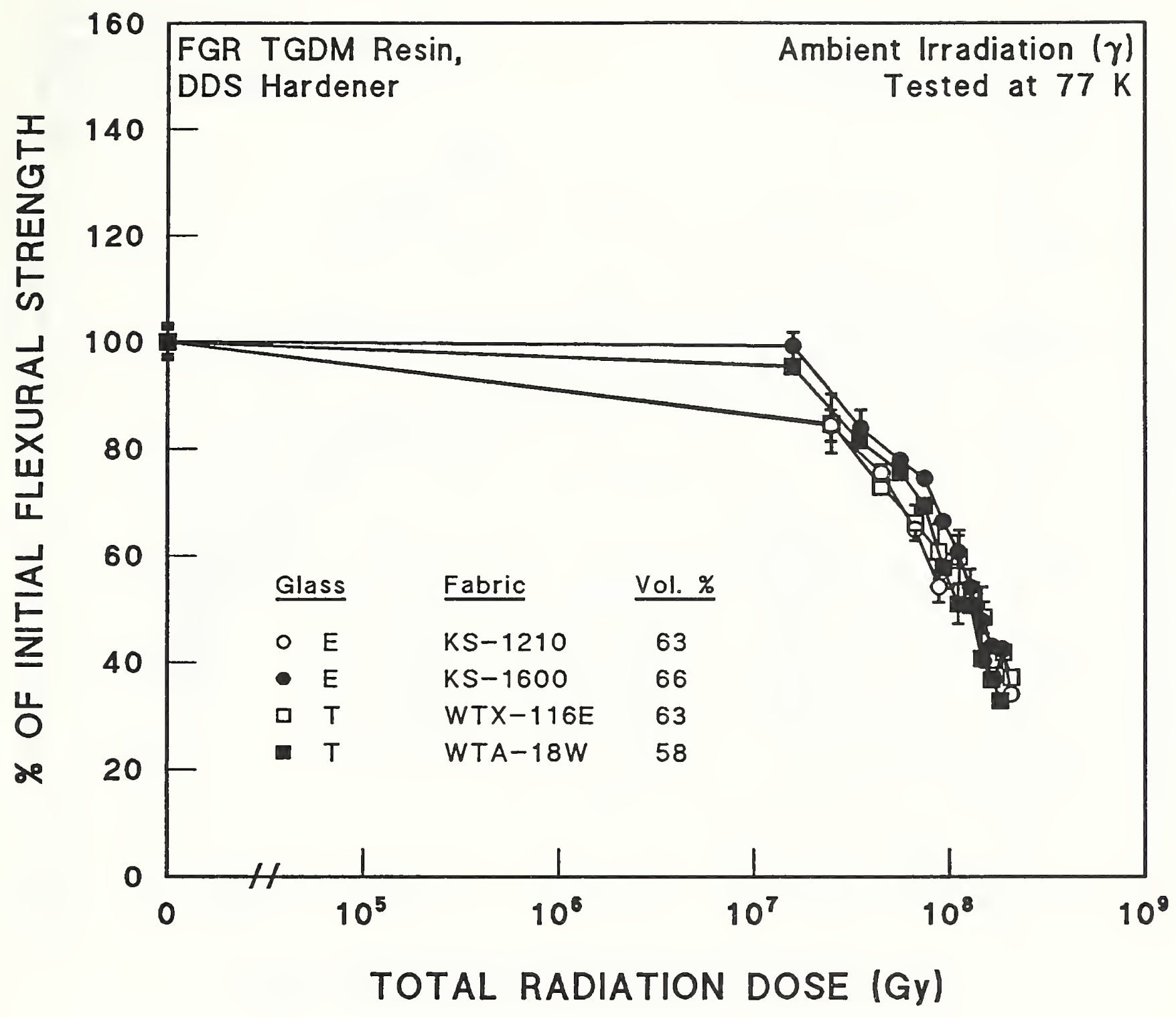

Yarn \#/25mm

"O" Strength, MPa

warp/fill

Fiber\#/yarn

Fiber diam.

Supplier

○ $1517.3 \pm 34.9$

$53 / 48$

200

$7 \mu \mathrm{m}$

Sumitomo

Bakelite

Co., Ltd.

- $1299.2 \pm 0.0$

$41 / 32$

400

$9 \mu \mathrm{m}$

Sumitomo

Bakelite

ㅁ $1087.8 \pm 31.7$

$60 / 58$

200

Co., Ltd.

- $1022.3 \pm 34.6$

$44 / 34$

400

Nikko Kasei

Nikko Kasei

Figure A.3-9. Flexural strength at $77 \mathrm{~K}$ of FGR (E and $T$ glass) TGDM with DDS hardener after ambient gamma irradiation. Supplementary Table A.3-4. Data from Egusa [1991a]. 


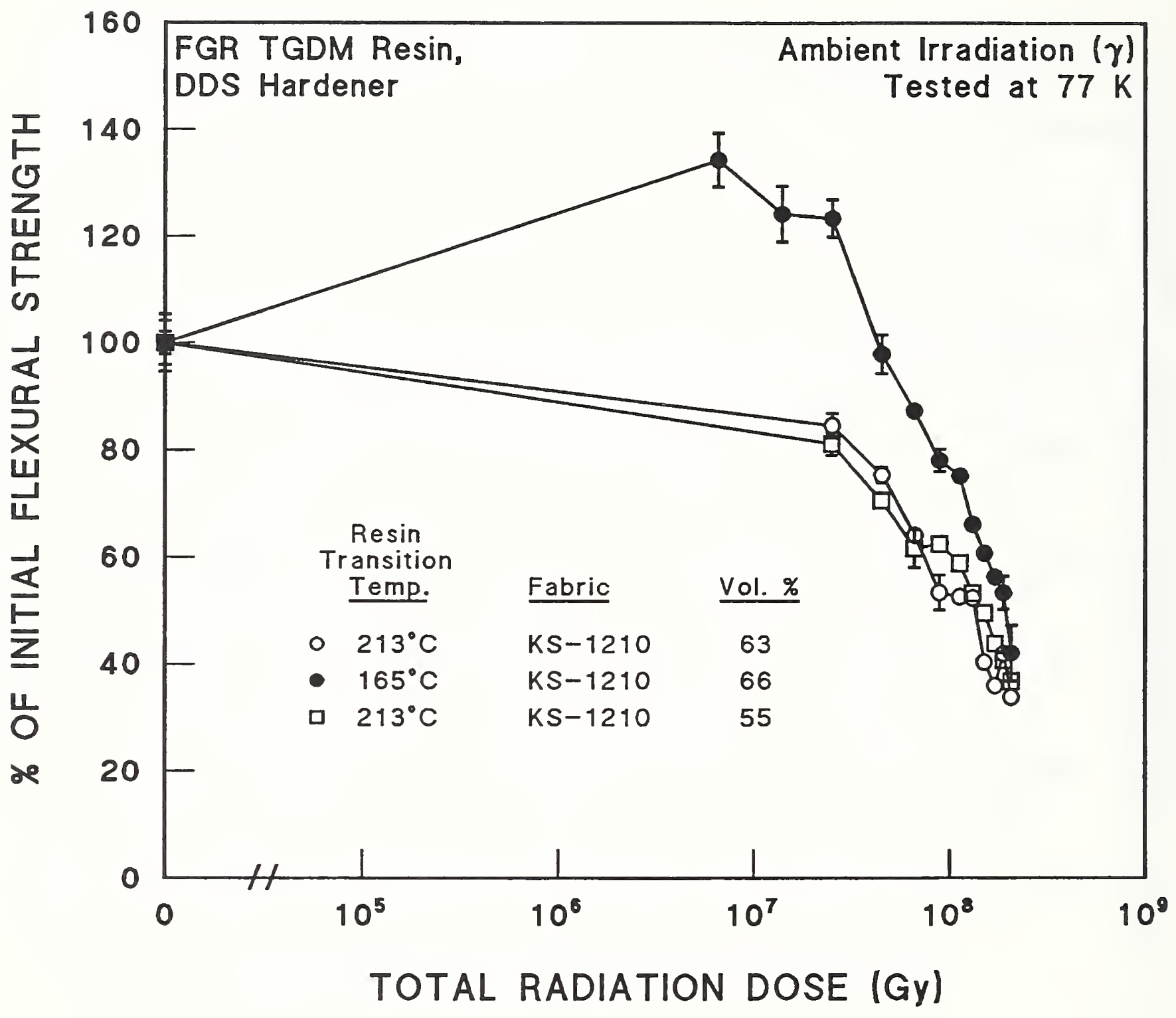

Yarn \#/25mm

"O" Strength, MPa

warp/fill

Fiber\#/yarn

Fiber diam. Supplier

○ $1525.7 \pm 32.5$

$53 / 48$

200

$7 \mu \mathrm{m}$

Sumitomo

Bakelite

Co., Ltd.

- $1097.7 \pm 58.8$

$53 / 48$

200

$7 \mu \mathrm{m}$

Sumitomo

Bakelite

Co., Ltd.

ㅁ $1339.9 \pm 55.8$

$53 / 48$

200

$7 \mu \mathrm{m}$

Sumitomo

Bakelite

Co., Ltd.

Figure A.3-10. Flexural strength at $77 \mathrm{~K}$ of FGR (variable volume fraction) TGDM with DDS hardener after ambient gamma irradiation. Supplementary Table A.3-4. Data from Egusa [1991a]. 


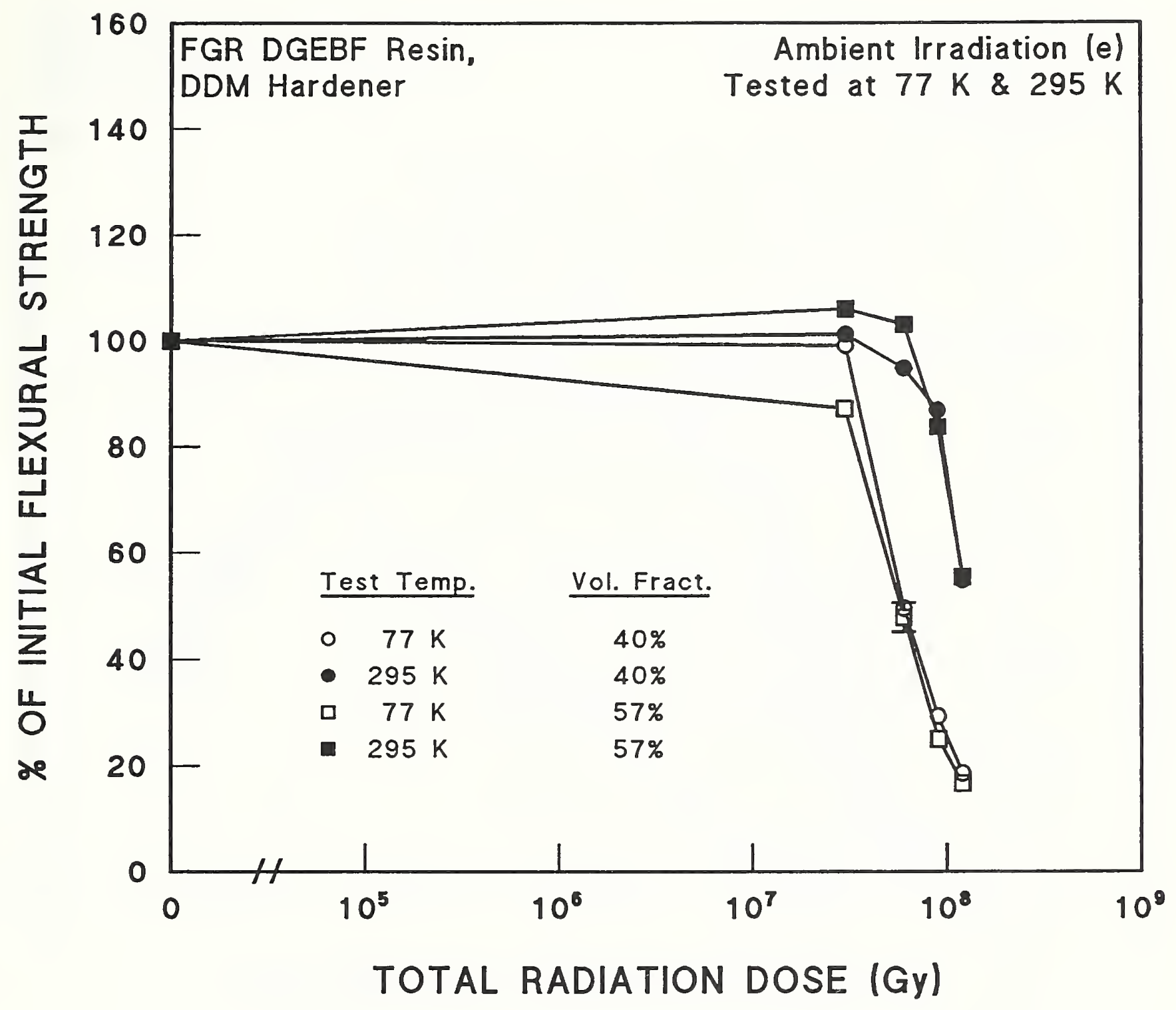

"O" Strength, MPa

Supplier

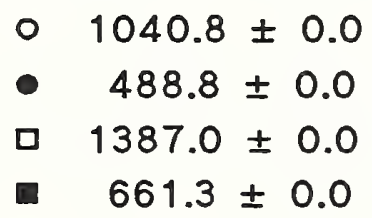

$--$

- -

-ー-

Figure A.3-11. Flexural strength at $77 \mathrm{~K}$ and $295 \mathrm{~K}$ of FGR (variable volume fraction) DGEBF with DDM hardener after ambient electron irradiation. Supplementary Table A.3-5. Data from Egusa et al. [1988]. 


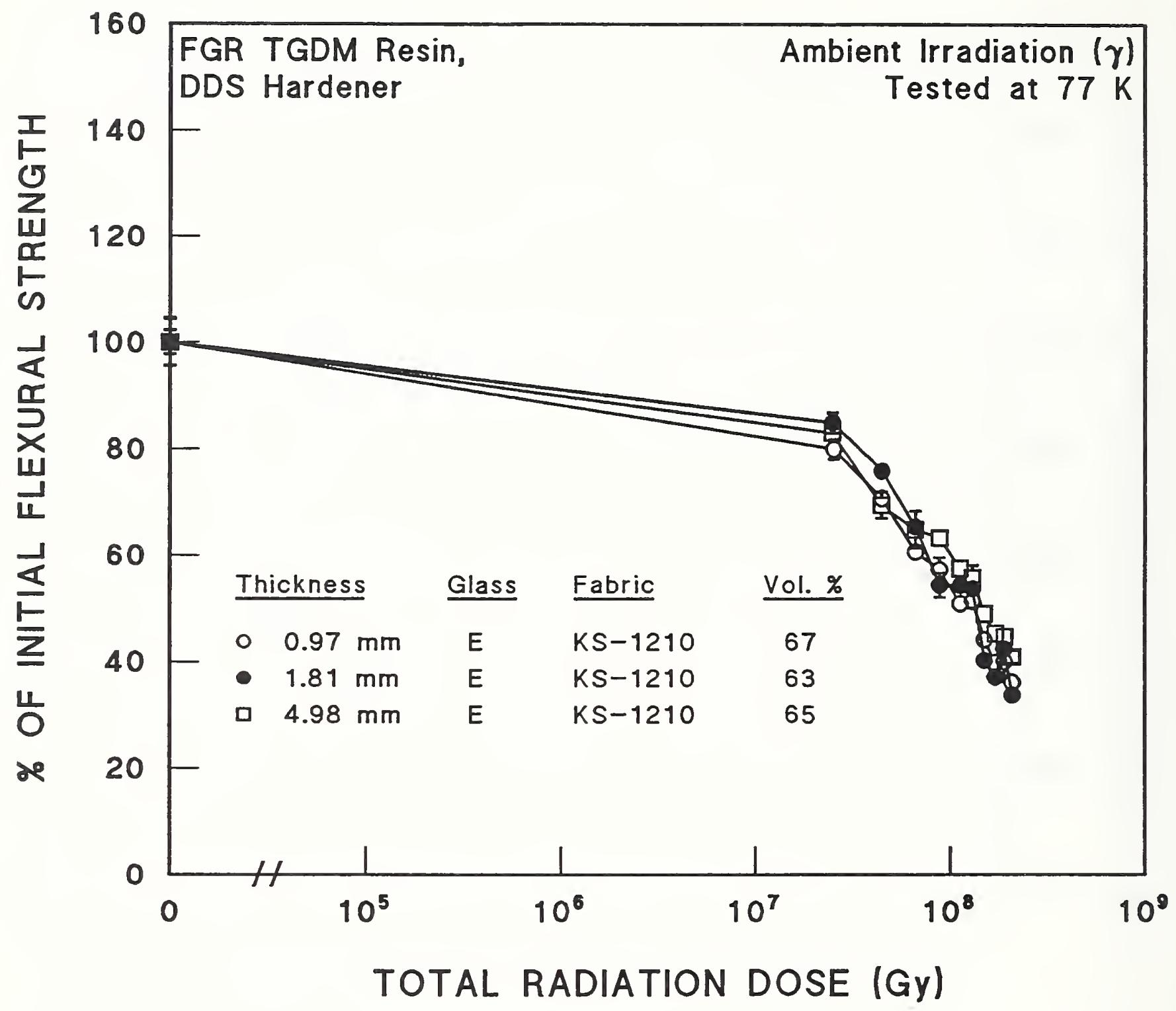

Yarn \#/25mm

"O" Strength, MPa

warp/fill

Fiber\#/yarn Fiber diam. Supplier

○ $1721.7 \pm 77.4$

$53 / 48$

200

$7 \mu \mathrm{m}$

Sumitomo

Bakelite

Co., Ltd.

- $1532.7 \pm 34.4$

$53 / 48$

200

$7 \mu \mathrm{m}$

Sumitomo

Bakelite

Co., Ltd.

ㅁ $1283.9 \pm 56.1$

$53 / 48$

200

$7 \mu \mathrm{m}$

Sumitomo

Bakelite

Co., Ltd.

Figure A.3-12. Flexural strength at $77 \mathrm{~K}$ of FGR TGDM resin (variable thickness) with DDS hardener after ambient gamma irradiation. Supplementary Table A.3-4. from Egusa [1991a]. 


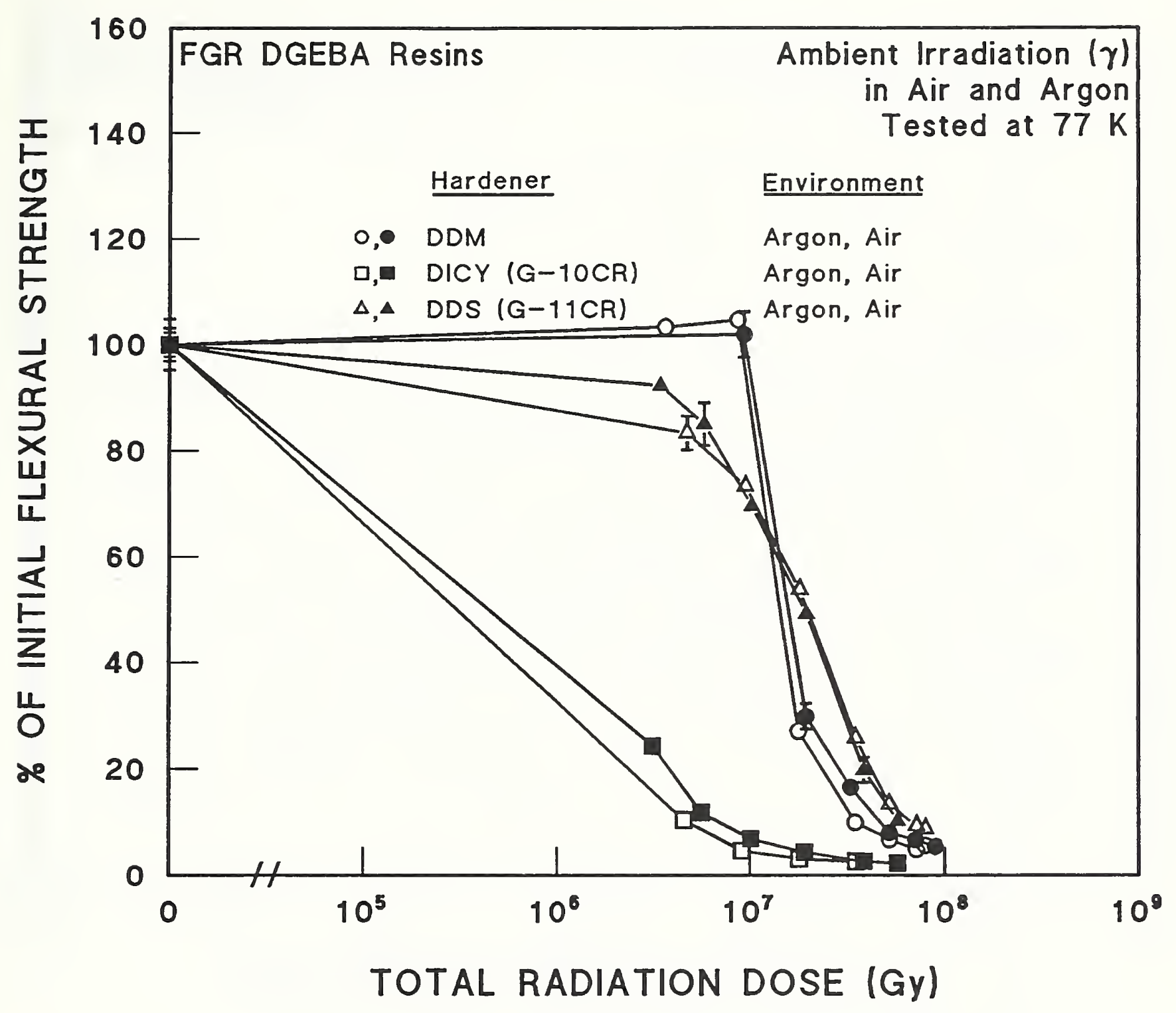

\begin{tabular}{|c|c|c|}
\hline "O" Strength, MPa & Vol. \% & Supplier \\
\hline $0,01067.0 \pm 0.0$ & 55 & $\begin{array}{l}\text { Sumitomo Bakelite } \\
\text { Co., Ltd. }\end{array}$ \\
\hline$\square, \pm 1201.7 \pm 27.2$ & 50 & Spaulding \\
\hline$\Delta \Delta 1112.2 \pm 49.8$ & 52 & Spaulding \\
\hline
\end{tabular}

Figure A.3-13. Flexural strength at $77 \mathrm{~K}$ of FGR DGEBA resins (including G-10CR and G-11CR) after ambient gamma irradiation in air or argon. Supplementary Table A.3-4. Data from Egusa [1991a]. 


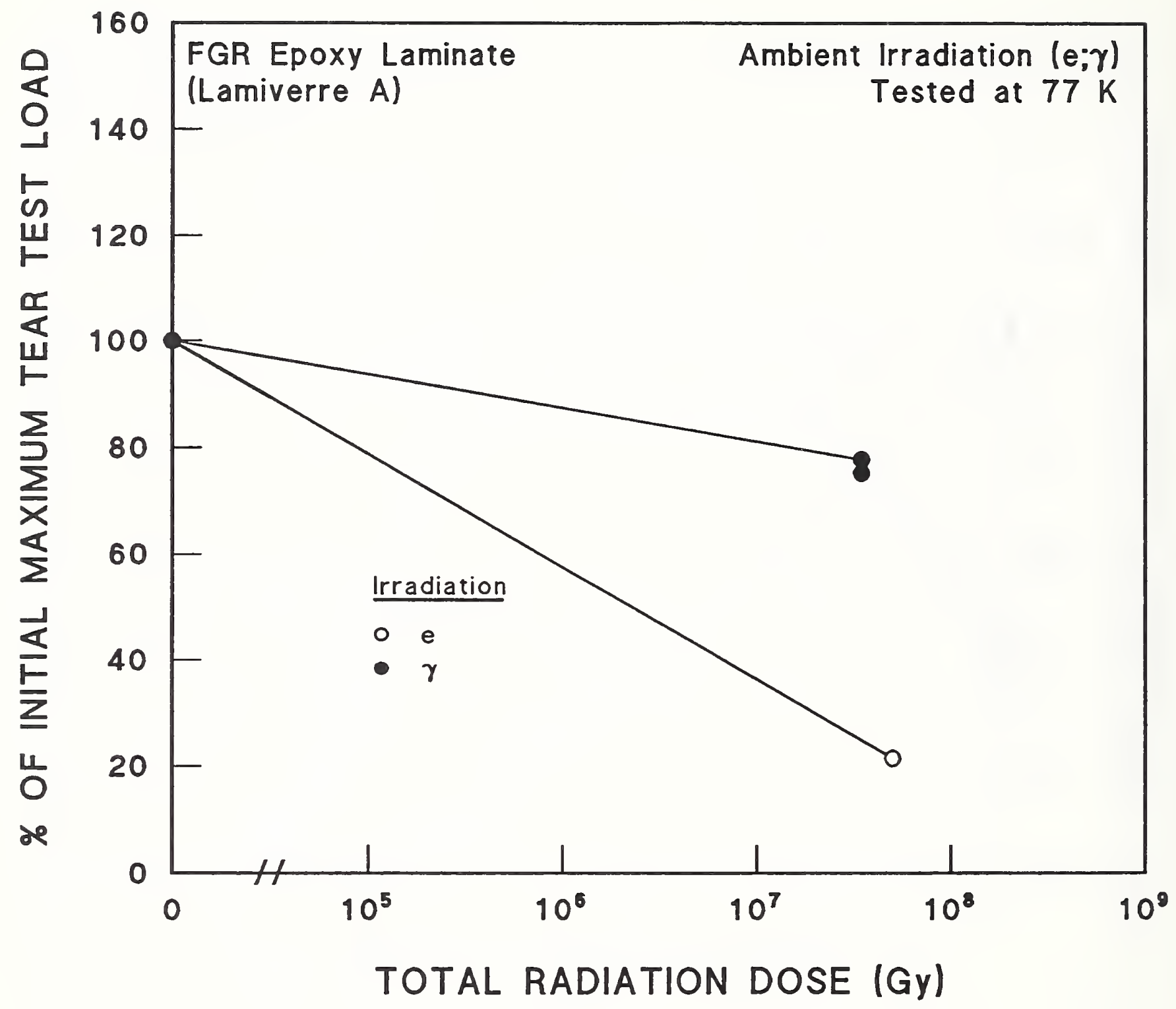

"O" Load, N

Weave

Finish

Glass

Supplier

- 108.0

Plain

Silane

62-65 mass $\%$

- 108.0

Plain

Silane

62-65 mass $\%$

Figure A.3-14. Maximum tear test load at $77 \mathrm{~K}$ of FGR epoxy laminate (Lamiverre A) after ambient gamma or electron irradiation. Supplementary Table A.2-10. Data from Nishiura et al. [1988a]. 


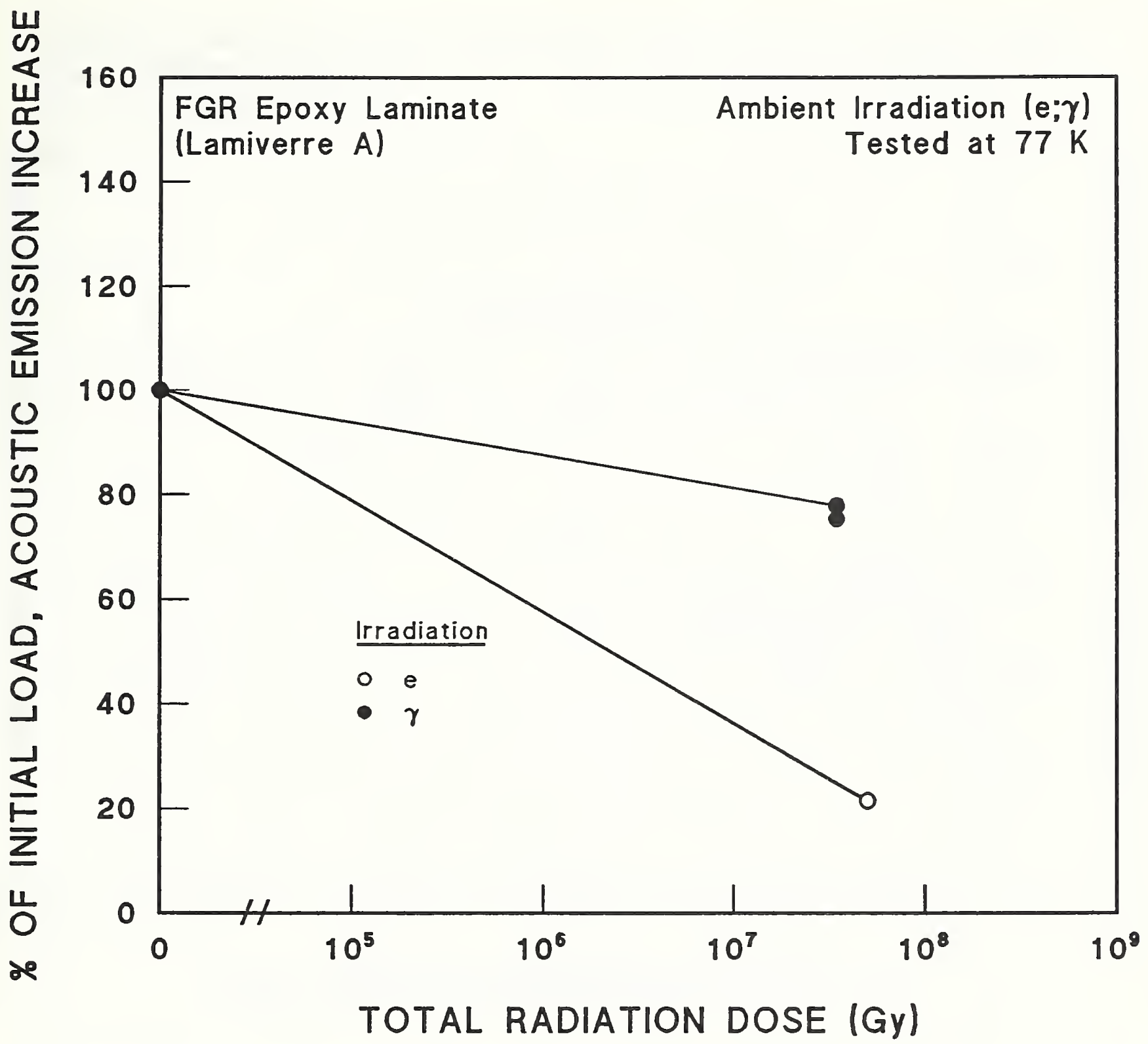

\begin{tabular}{|c|c|c|c|c|}
\hline "O" Load, N & Weave & Finish & Glass & Supplier \\
\hline - 96.2 & Plain & Silane & $62-65$ mass & -- \\
\hline - $\quad 96.2$ & Plain & Silane & $62-65$ mass & -- \\
\hline
\end{tabular}

Figure A.3-15. Load at which acoustic emission count increases steeply at $77 \mathrm{~K}$ of FGR epoxy laminate (Lamiverre A) after ambient gamma or electron irradiation. Supplementary Table A.2-10. Data from Nishiura et al. [1988a]. 


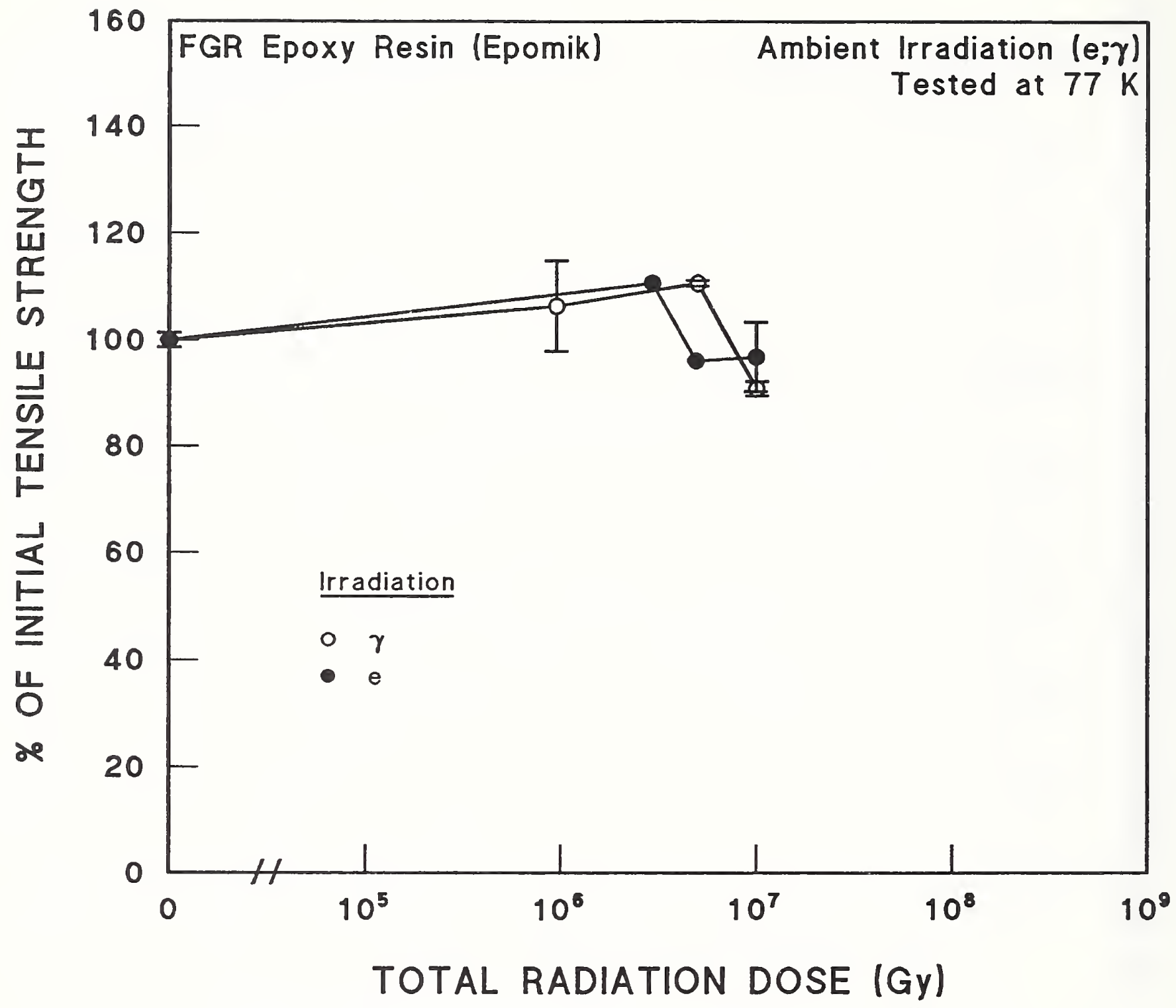

"O" Strength, MPa

- $478.1 \pm 6.4$

- $520.1 \pm 0.0$
Hardener

Jefermin-D230,30

Jefermin-D230,30
Supplier

Mitubishiyuka Co., Ltd.

Mitubishiyuka Co., Ltd.

Figure A.3-16. Tensile strength at $77 \mathrm{~K}$ of FGR epoxy resin (Epomik) after ambient gamma or electron irradiation. Supplementary Table A.2-10. Data from Nishiura et al. [1988b] . 


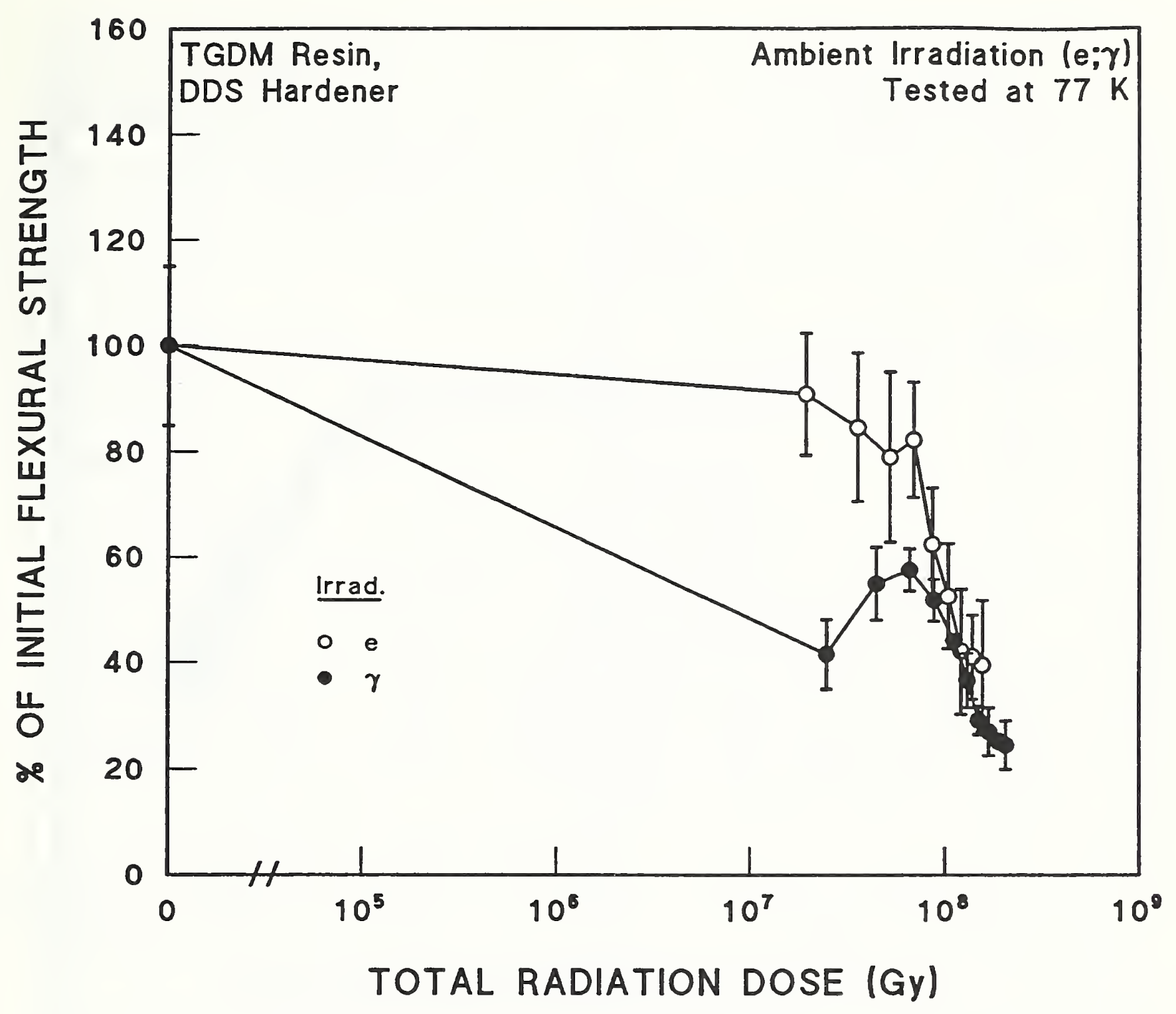

\section{Supplier}

Sumitomo Bakelite

Co., Ltd.

Sumitomo Bakelite Co., Ltd.

Figure A.3-17. Flexural strength at $77 \mathrm{~K}$ of neat TGDM resin with DDS hardener after ambient gamma or electron irradiation. Supplementary Table A.3-4. Data from Egusa [1991a]. 


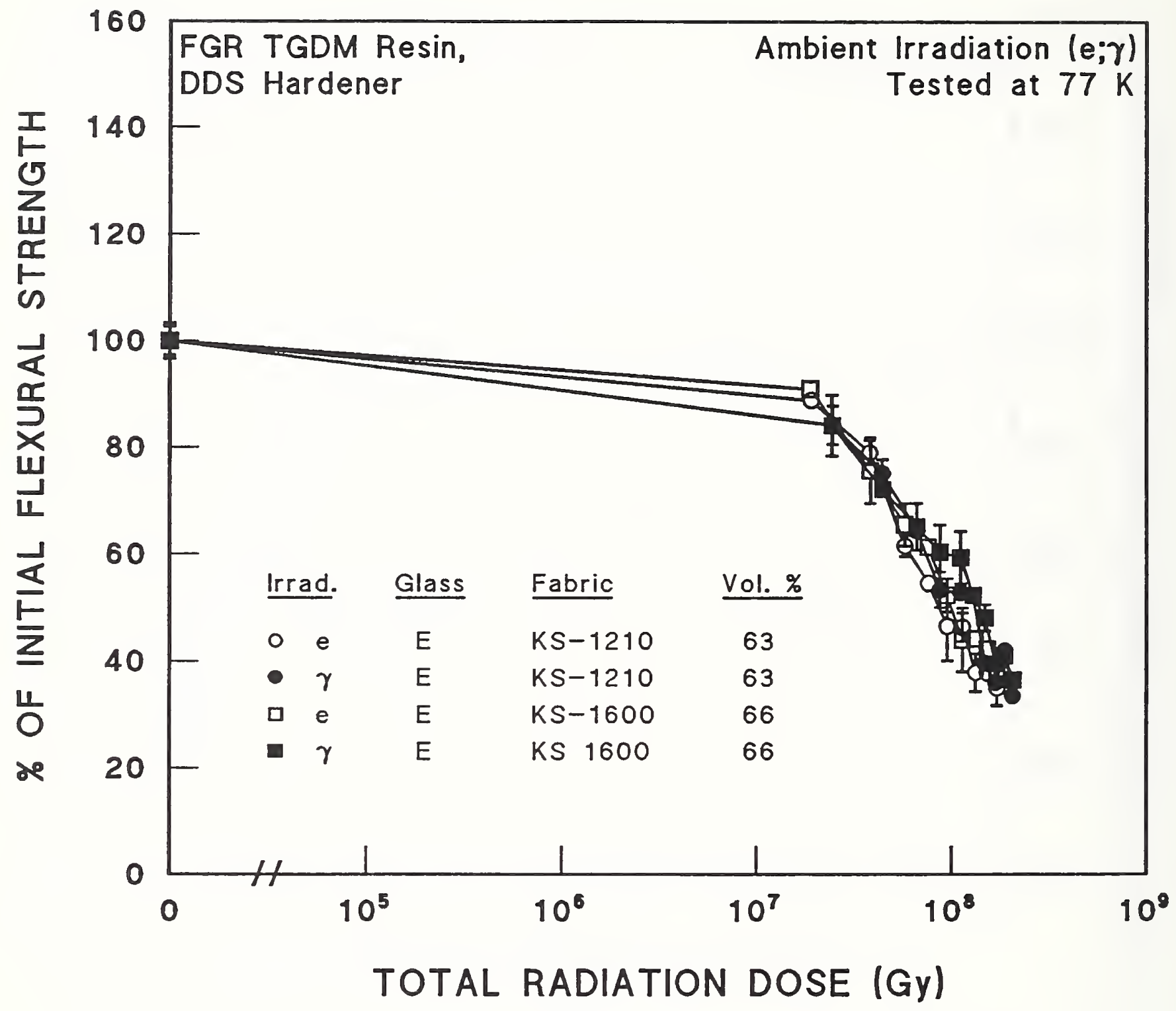

$\begin{array}{lccccc}\text { "O" Strength, MPa } & \begin{array}{c}\text { Yarn \#/25mm } \\ \text { warp/fill }\end{array} & \text { Fiber\#/yarn } & \text { Fiber diam. } & \underline{\text { Supplier }} \\ 0,01525.2 \pm 42.4 & 53 / 48 & 200 & 7 \mu \mathrm{m} & \begin{array}{l}\text { Sumitomo } \\ \text { Bakelite } \\ \text { Co., Ltd. }\end{array} \\ \square, \square 1096.2 \pm 36.4 & 41 / 32 & 400 & 9 \mu \mathrm{m} & \begin{array}{l}\text { Sumitomo } \\ \text { Bakelite } \\ \text { Co., Ltd. }\end{array}\end{array}$

Figure A.3-18. Flexural strength at $77 \mathrm{~K}$ of FGR TGDM resin with DDS hardener after ambient gamma or electron irradiation. Supplementary Table A.3-4. Data from Egusa [1991a]. 
Table A.3-1. Supplementary Information, Egusa [1988] .

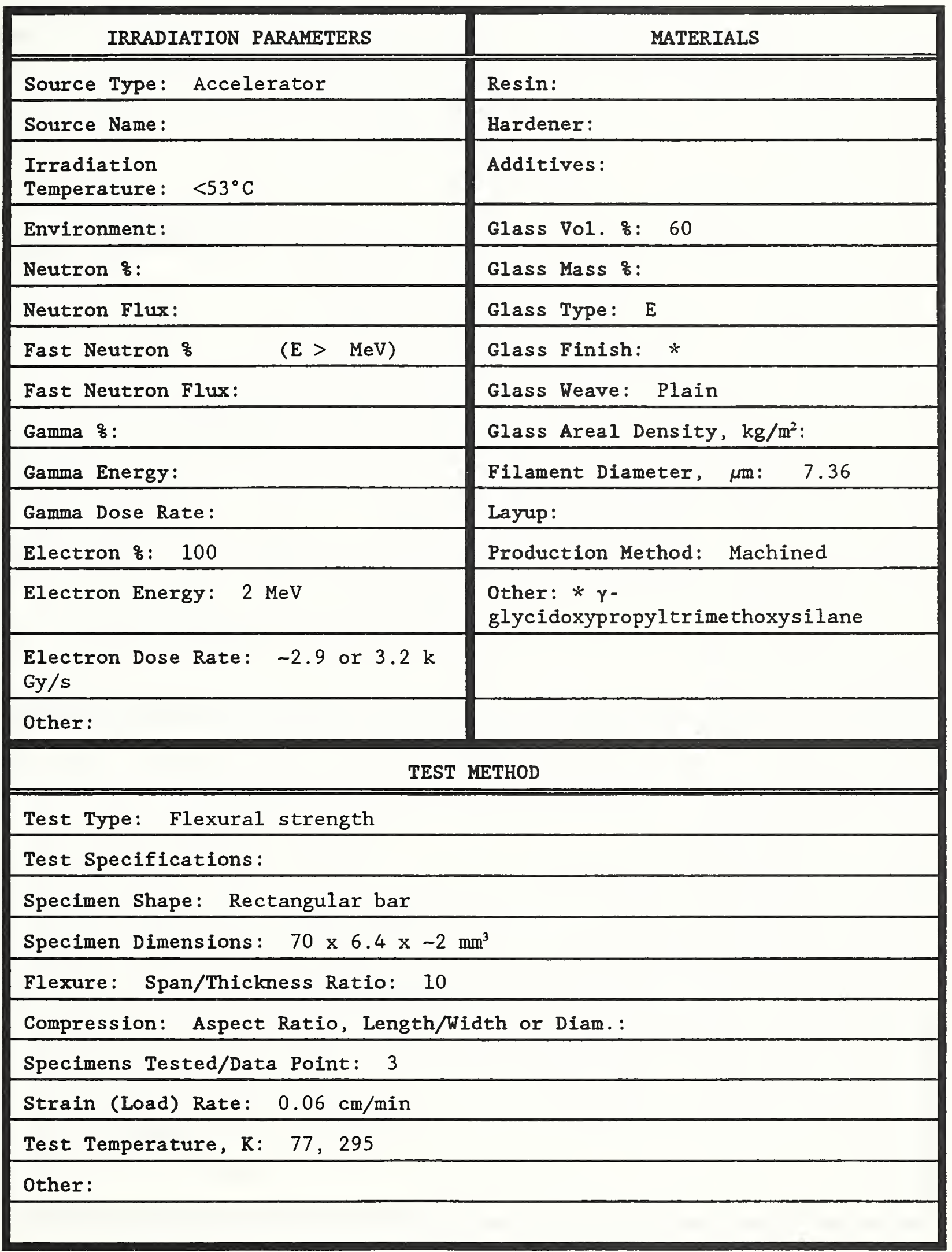


Table A.3-2. Supplementary Information, Egusa and Hagiwara [1986].

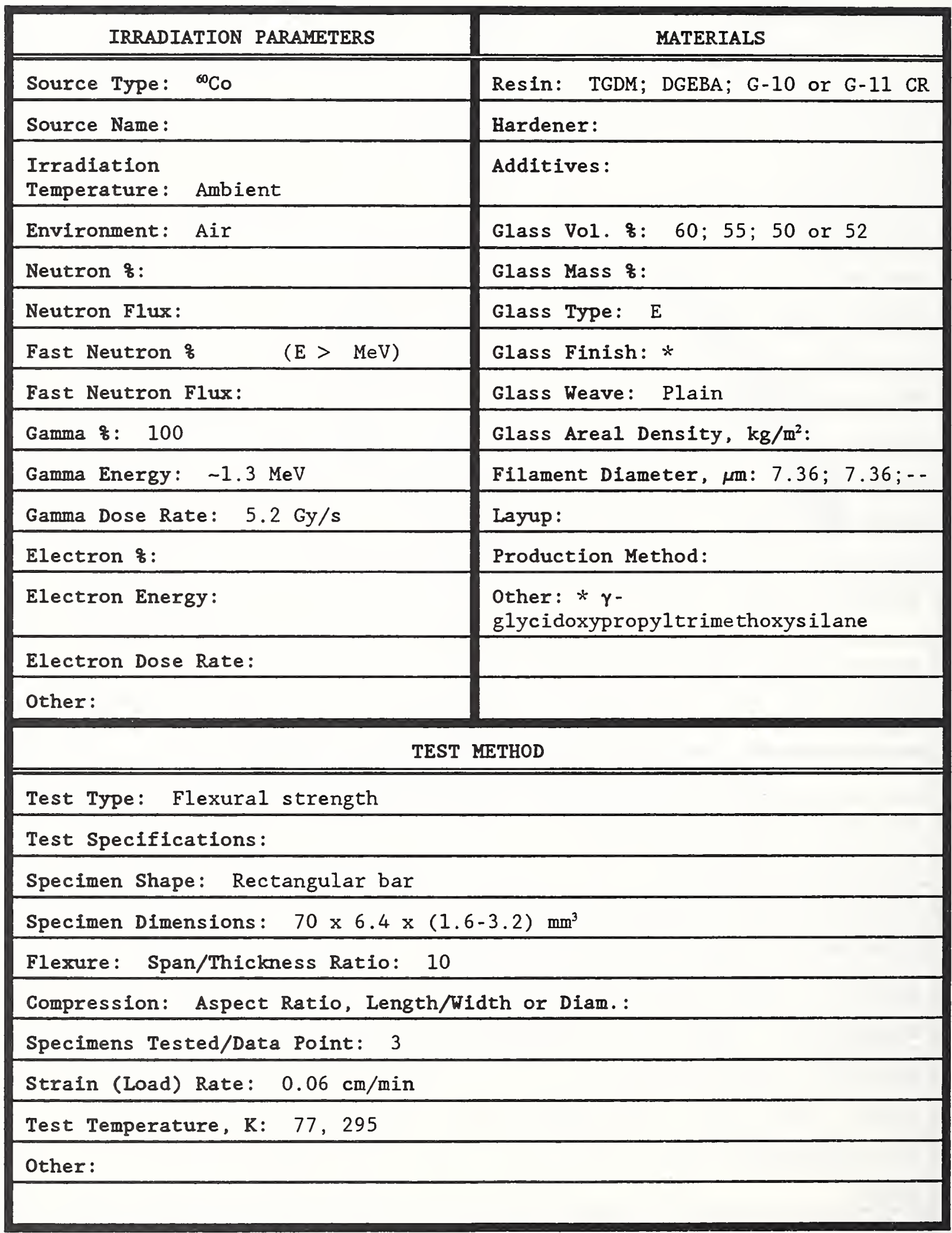


Table A.3-3. Supplementary Information, Munshi [1991], Munshi and Weber [1992].

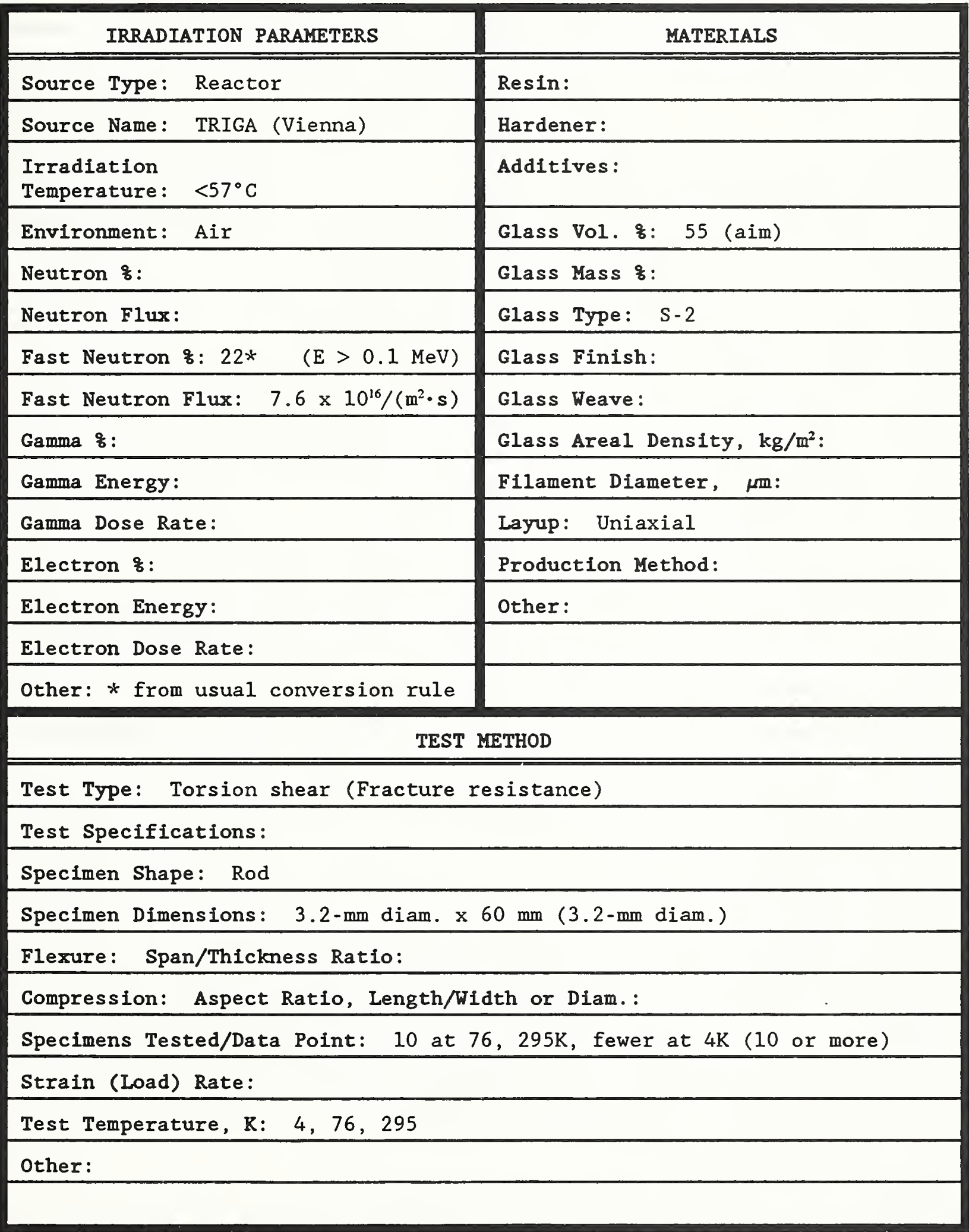


Table A.3-4. Supplementary Information, Egusa [1991a].

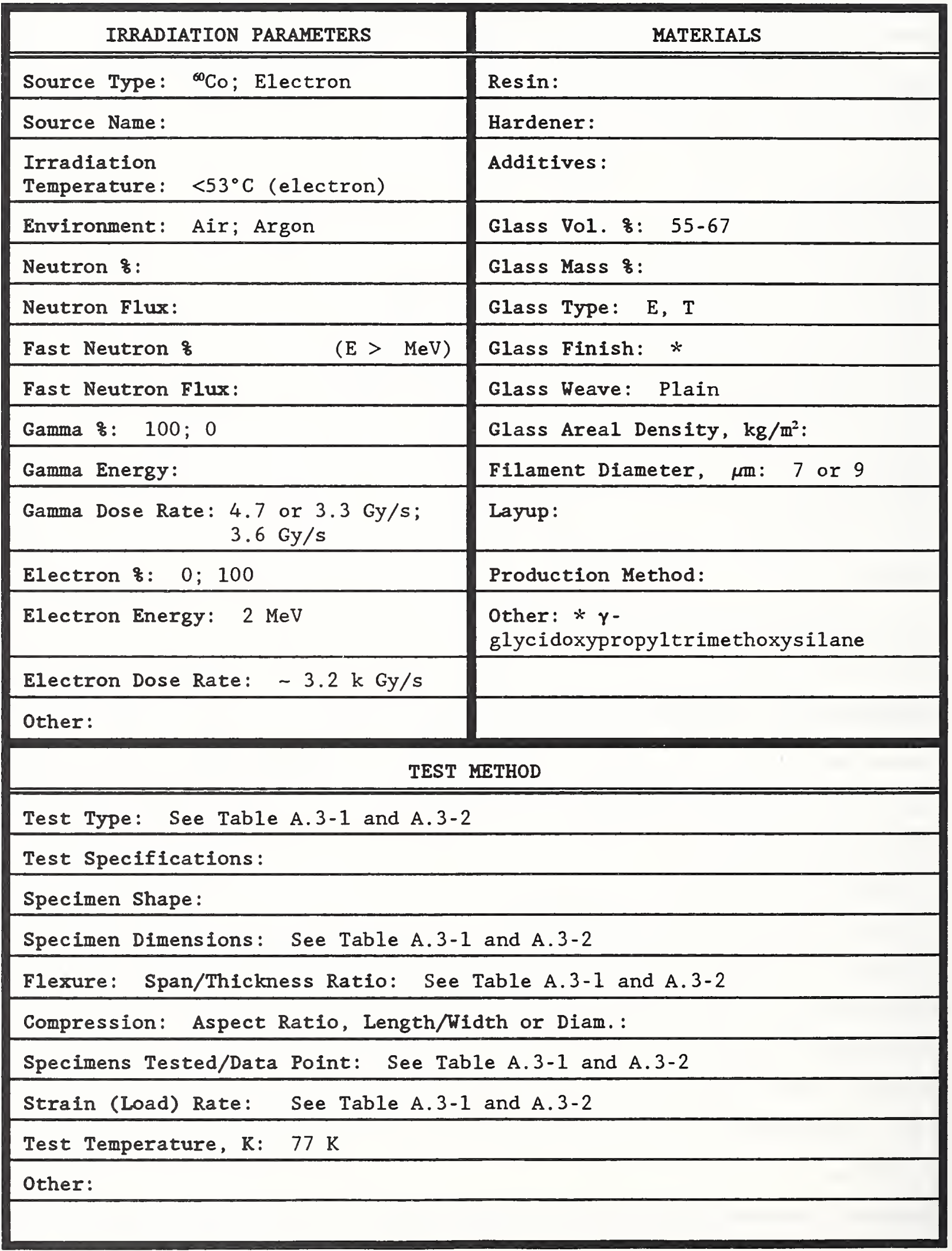


Table A.3-5. Supplementary Information, Egusa et al [1988].

\begin{tabular}{|c|c|}
\hline IRRADIATION PARAMETERS & MATERIALS \\
\hline Source Type: Accelerator & Resin: DGEBF or TGDM; BT/DGEBA \\
\hline Source Name: & Hardener: \\
\hline $\begin{array}{l}\text { Irradiation } \\
\text { Temperature: Ambient }\end{array}$ & Additives: \\
\hline Environment: Air & Glass Vol. $8: 40$ or $57 ; 45$ \\
\hline Neutron $\&$ : & Glass Mass $\%$ : \\
\hline Neutron Flux: & Glass Type: \\
\hline Fast Neutron 8 & Glass Finish: \\
\hline Fast Neutron Flux: & Glass Weave: \\
\hline Gamma of: & Glass Areal Density, $\mathrm{kg} / \mathrm{m}^{2}$ : \\
\hline Gamma Energy: & Filament Diameter, $\mu \mathrm{m}$ : \\
\hline Gamma Dose Rate: & Layup: \\
\hline Electron $q: 100$ & Production Method: \\
\hline Electron Energy: $2 \mathrm{MeV}$ & Other: \\
\hline Electron Dose Rate: $5 \mathrm{k} \mathrm{Gy} / \mathrm{s}$ & \\
\hline \multicolumn{2}{|l|}{ Other: } \\
\hline \multicolumn{2}{|c|}{ TEST METHOD } \\
\hline \multicolumn{2}{|l|}{ Test Type: $\quad$ See Table A.3-2 } \\
\hline \multicolumn{2}{|l|}{ Test Specifications: } \\
\hline \multicolumn{2}{|l|}{ Specimen Shape: } \\
\hline \multicolumn{2}{|l|}{ Specimen Dimensions: $\quad$ See Table A.3-2 } \\
\hline \multicolumn{2}{|c|}{ Flexure: Span/Thickness Ratio: See Table A.3-2 } \\
\hline \multicolumn{2}{|c|}{ Compression: Aspect Ratio, Length/Width or Diam.: } \\
\hline \multicolumn{2}{|c|}{ Specimens Tested/Data Point: See Table A.3-2 } \\
\hline \multicolumn{2}{|l|}{ Strain (Load) Rate: See Table A.3-2 } \\
\hline Test Temperature, $\mathrm{K}: 77,295$ & \\
\hline Other: & \\
\hline
\end{tabular}





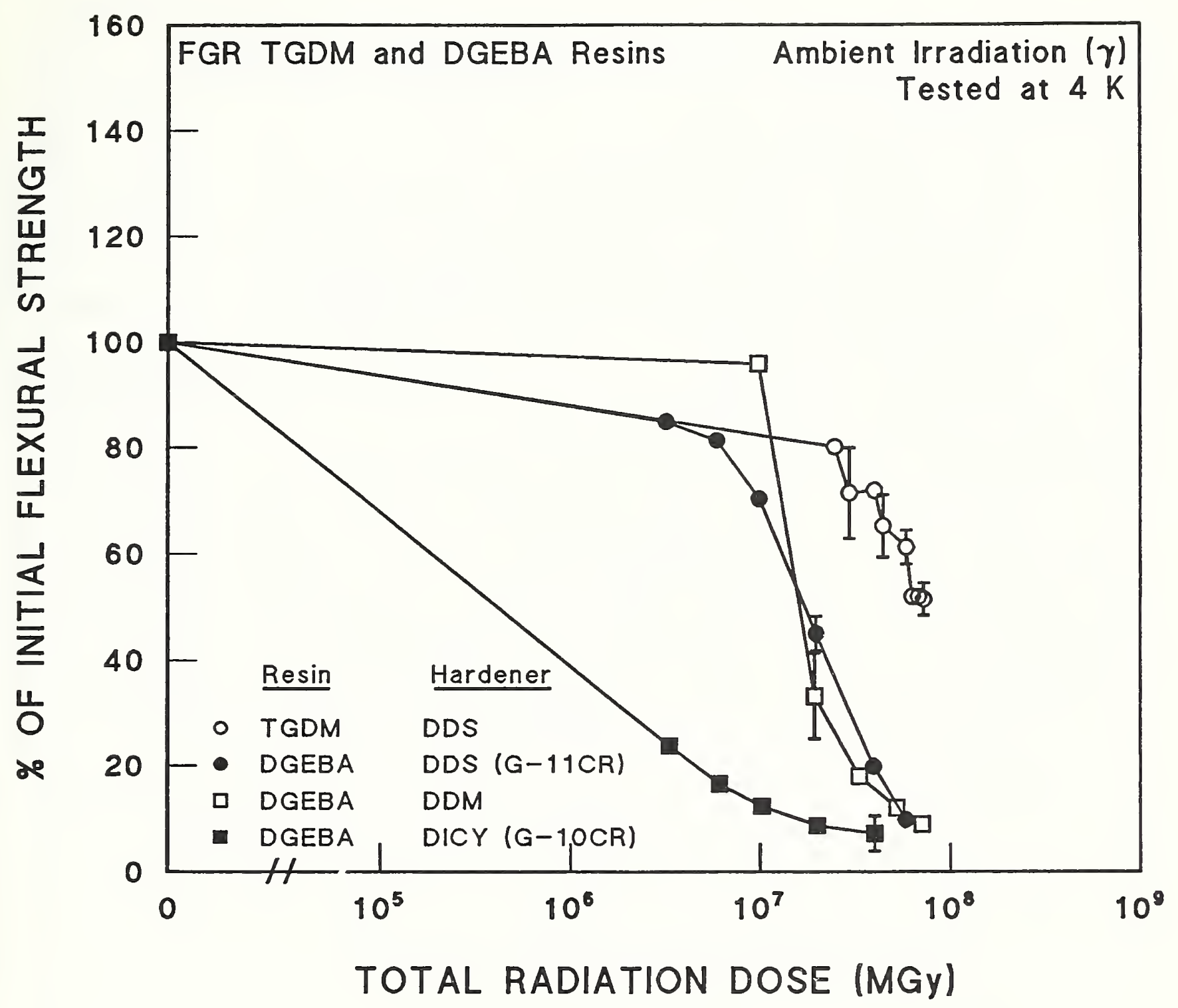

Supplier

Sumitomo Bakelite Co., Ltd.

Spaulding

Sumitomo Bakelite Co., Ltd.

Spaulding

Figure A.4-1. Flexural strength at $4 \mathrm{~K}$ of FGR TGDM and DGEBA resins (including G10CR and G-11CR) after ambient gamma irradiation. Supplementary Table A.3-4. Data from Egusa [1990]. 


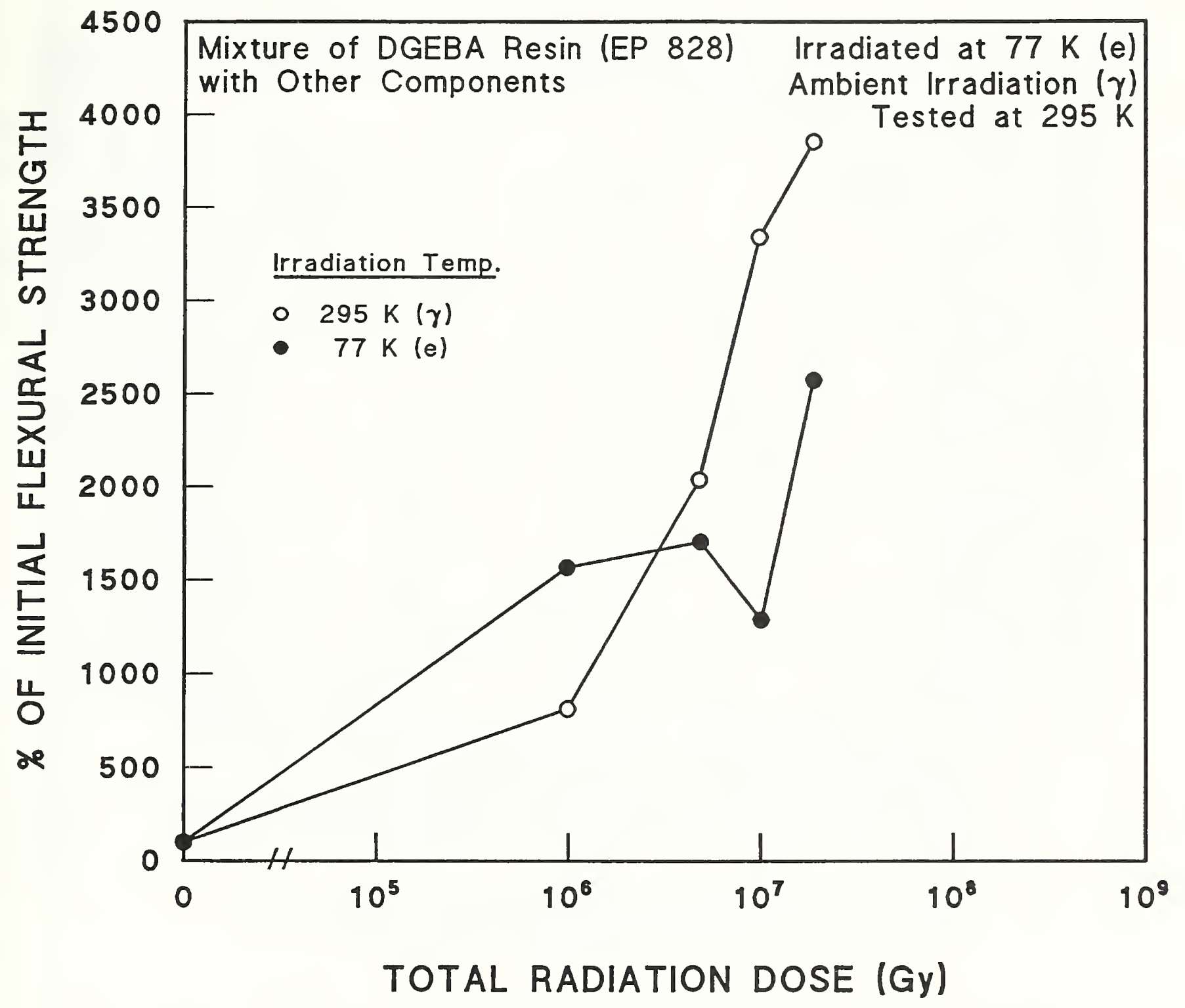

"O" Strength, MPa

Additives (pbmass) Cure Conditions

Supplier

$0, \bullet \quad 0.95$

Adiprene $L 100$

(50)

$5 \mathrm{~h}, 140^{\circ} \mathrm{C}$

Dupont Ltd.

87 (15)

Shell Chemical

MOCA (29.6)

Phenyl Glycidyl

Co., Ltd.

Dupont Ltd.

ether $(10)$

Ciba-Geigy

Figure A.5-1. Flexural strength at $295 \mathrm{~K}$ of a mixture of DGEBA resin with other components after 77-K electron or ambient gamma irradiation. Supplementary Table A.5-1. Data from Evans et al. [1971]. 


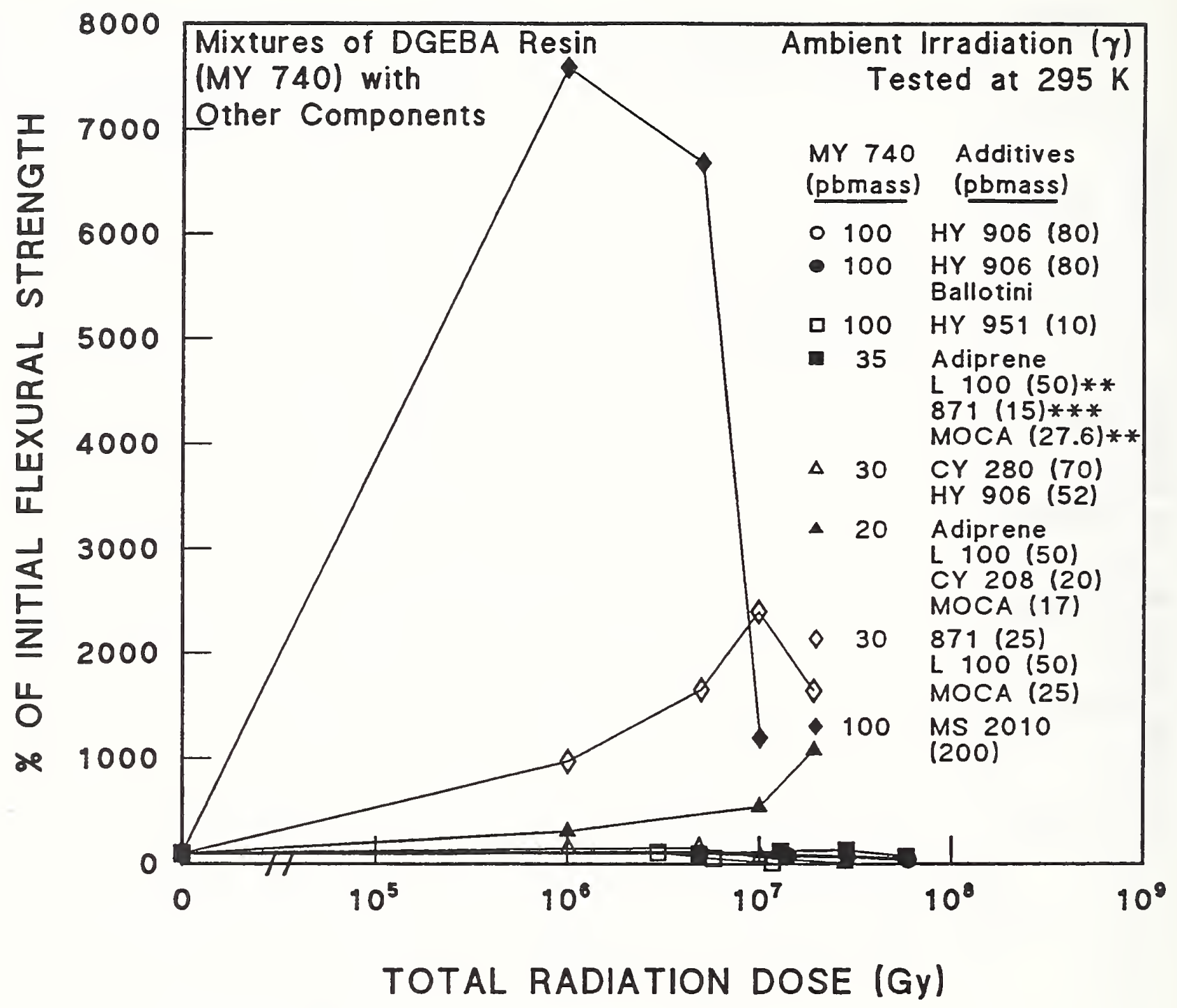

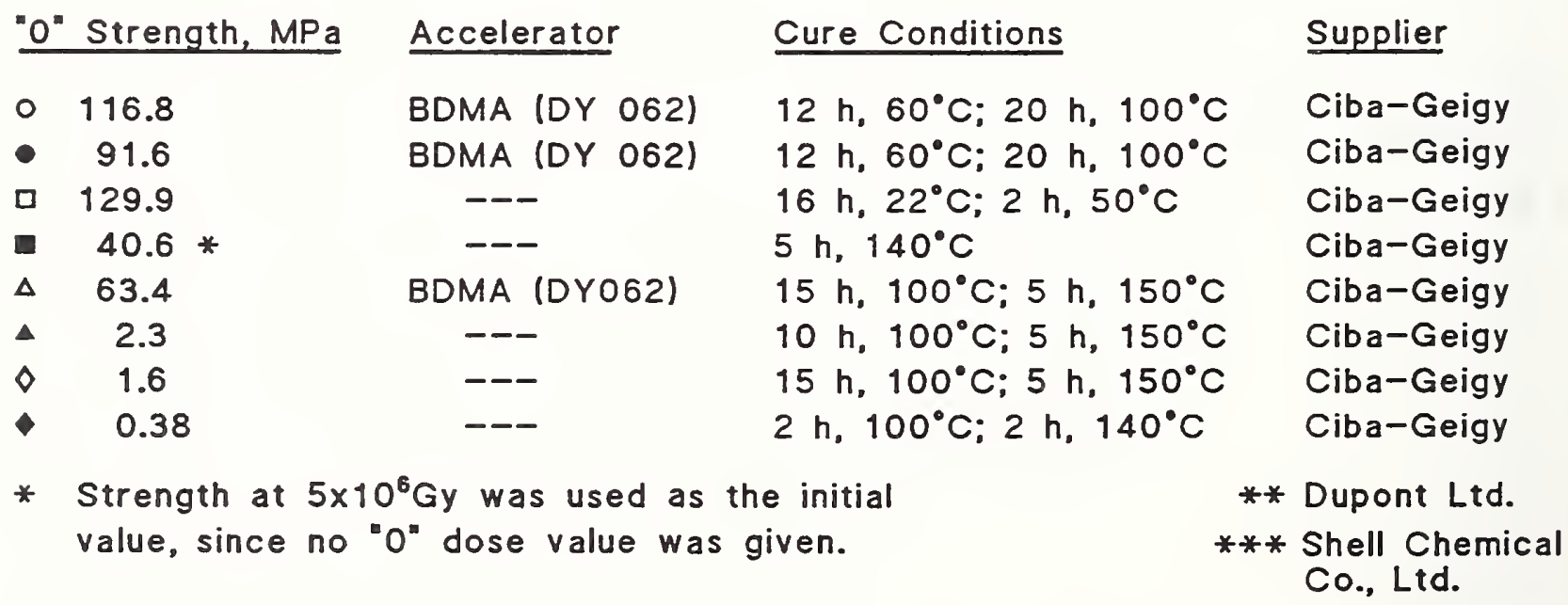

Figure A.5-2. Flexural strength at $295 \mathrm{~K}$ of mixtures of DGEBA resin with other components after ambient gamma irradiation. Supplementary Table A.5-1. Data from Evans et al. [1971]. 


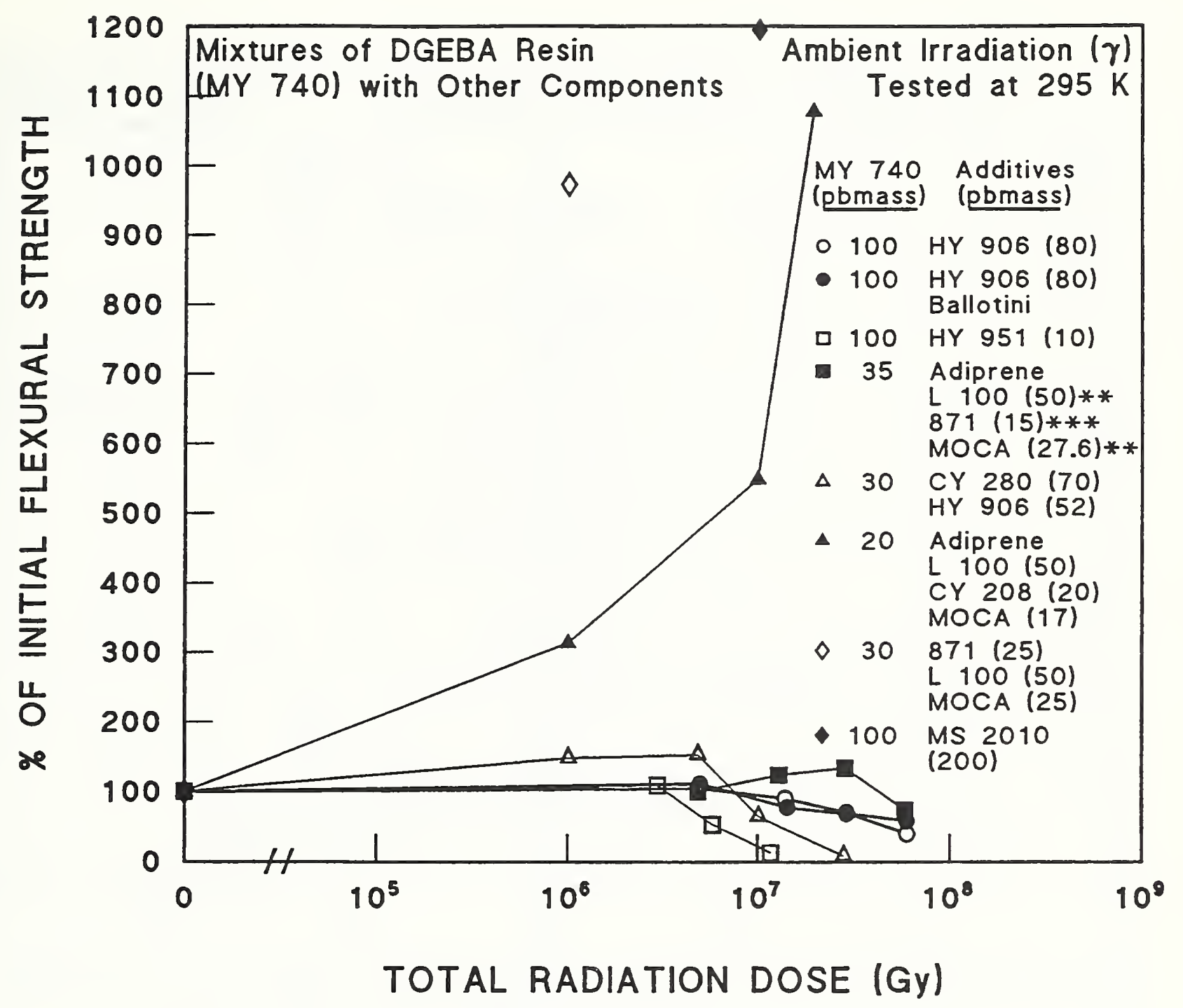

\begin{tabular}{|c|c|c|c|}
\hline "O" Strength, MPa & Accelerator & Cure Conditions & Supplier \\
\hline$\circ \quad 116.8$ & BDMA (DY 062) & $12 \mathrm{~h}, 60^{\circ} \mathrm{C} ; 20 \mathrm{~h}, 100^{\circ} \mathrm{C}$ & Ciba-Geigy \\
\hline - $\quad 91.6$ & BDMA (DY 062) & $12 \mathrm{~h}, 60^{\circ} \mathrm{C} ; 20 \mathrm{~h}, 100^{\circ} \mathrm{C}$ & Ciba-Geigy \\
\hline 129.9 & --- & $16 \mathrm{~h}, 22^{\circ} \mathrm{C} ; 2 \mathrm{~h}, 50^{\circ} \mathrm{C}$ & Ciba-Geigy \\
\hline $40.6 *$ & -- & $5 \mathrm{~h}, 140^{\circ} \mathrm{C}$ & Ciba-Geigy \\
\hline 63.4 & BDMA (DY062) & $15 \mathrm{~h}, 100^{\circ} \mathrm{C} ; 5 \mathrm{~h}, 150^{\circ} \mathrm{C}$ & Ciba-Geigy \\
\hline 2.3 & -- & $10 \mathrm{~h}, 100^{\circ} \mathrm{C} ; 5 \mathrm{~h}, 150^{\circ} \mathrm{C}$ & Ciba-Geigy \\
\hline 1.6 & --- & $15 \mathrm{~h}, 100^{\circ} \mathrm{C} ; 5 \mathrm{~h}, 150^{\circ} \mathrm{C}$ & Ciba-Geigy \\
\hline 0.38 & --- & $2 \mathrm{~h}, 100^{\circ} \mathrm{C} ; 2 \mathrm{~h}, 140^{\circ} \mathrm{C}$ & Ciba-Geigy \\
\hline
\end{tabular}

Figure A.5-3. Flexural strength at $295 \mathrm{~K}$ of mixtures of DGEBA resin with other components after ambient gamma irradiation. (Expanded scale.) Supplementary Table A.5-1. Data from Evans et a1. [1971]. 


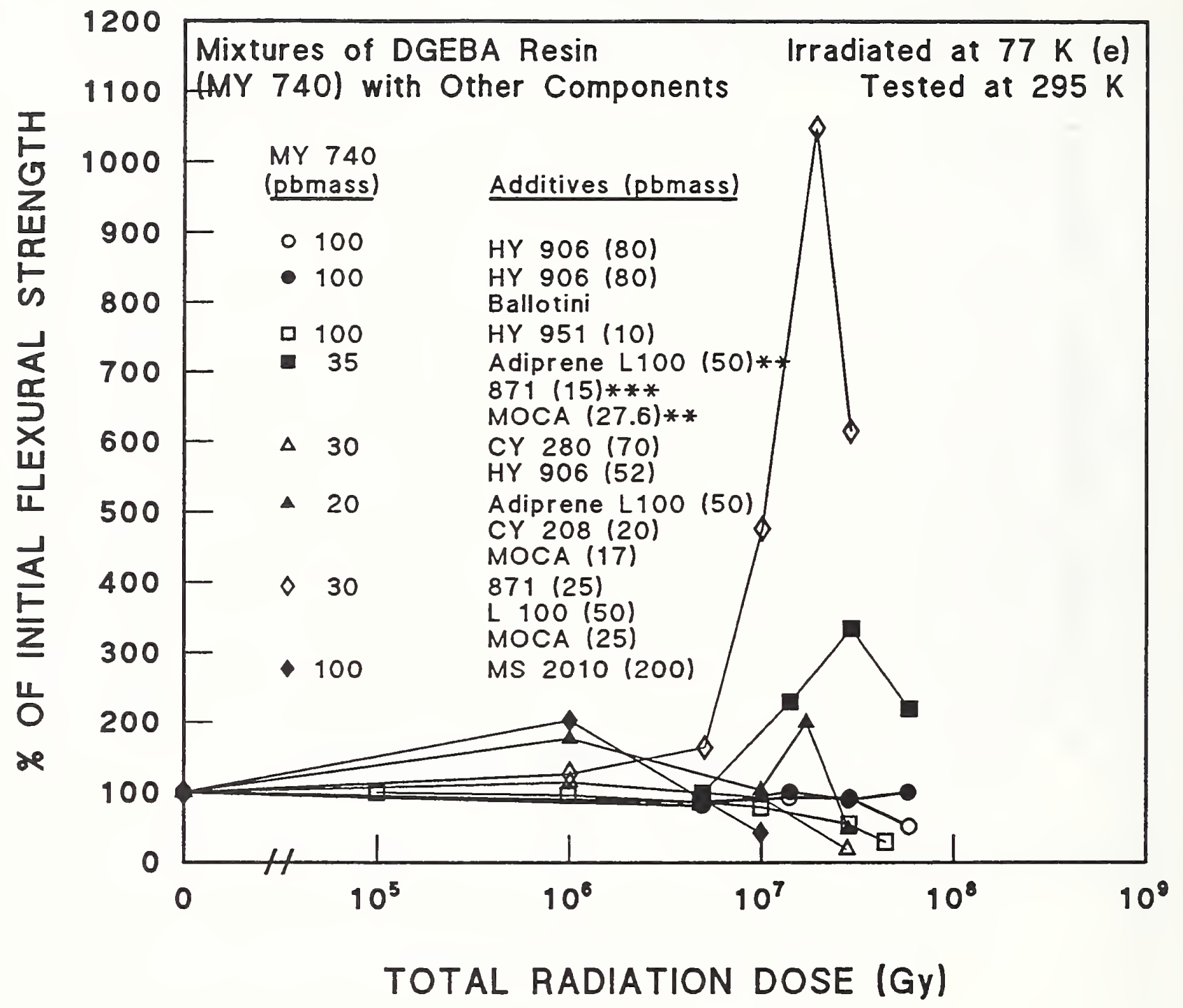

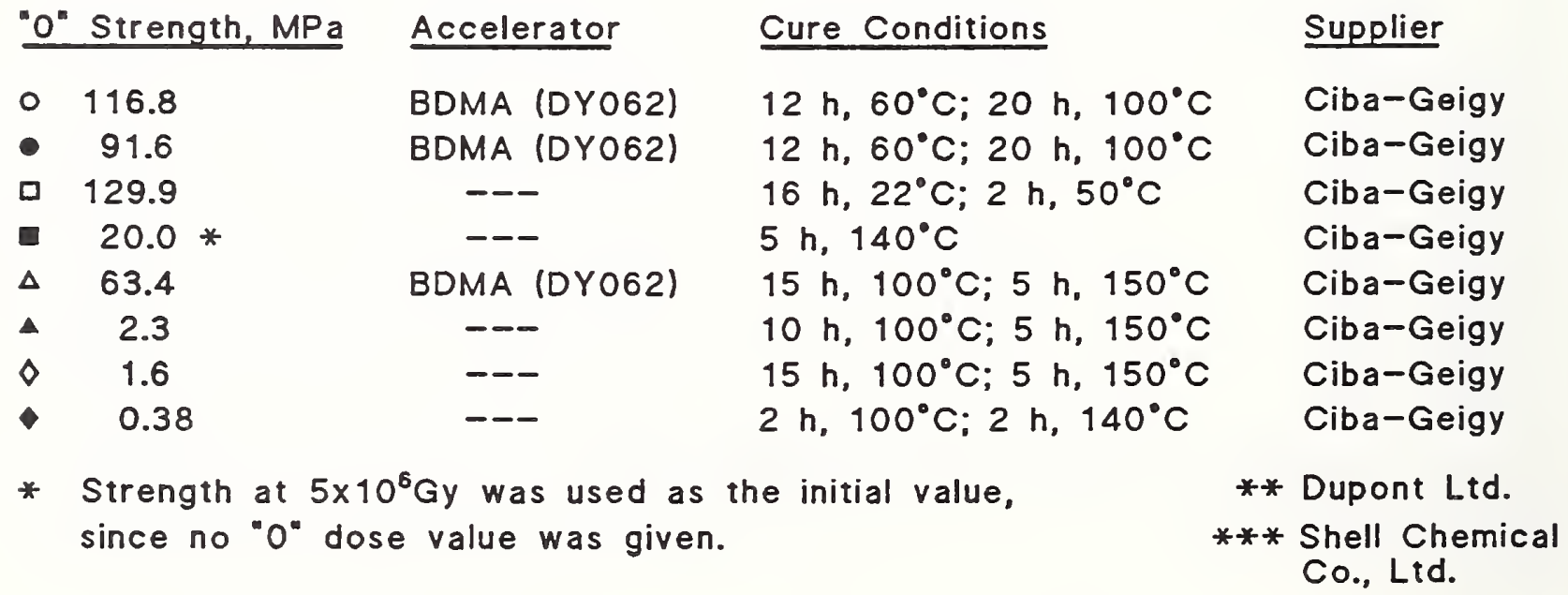

Figure A.5-4. Flexural strength at $295 \mathrm{~K}$ of mixtures of DGEBA resin with other components after 77-K electron irradiation. Supplementary Table A.5-1. Data from Evans et a1. [1971]. 
Table A.5-1. Supplementary Information, Evans et al. [1971].

\begin{tabular}{|c|c|}
\hline IRRADIATION PARAMETERS & MATERIALS \\
\hline $\begin{aligned} \text { Source Type: } & \text { Accelerator (e); } \\
& \text { Spent fuel }(y)\end{aligned}$ & Resin: \\
\hline Source Name: & Hardener: \\
\hline $\begin{array}{l}\text { Irradiation } \\
\text { Temperature: } \quad 77 \mathrm{~K} \text {; Ambient }\end{array}$ & Additives: \\
\hline Environment: $\quad \mathrm{LN}_{2}(\mathrm{e})$ & Glass Vol. \&: \\
\hline Neutron 8 : & Glass Mass o: \\
\hline Neutron Flux: & Glass Type: \\
\hline Fast Neutron $q \quad(E>M e V)$ & Glass Finish: \\
\hline Fast Neutron Flux: & Glass Weave: \\
\hline Gamma \&: $\quad-100$ & Glass Area1 Density, $\mathrm{kg} / \mathrm{m}^{2}$ : \\
\hline Gamma Energy: $1 \mathrm{MeV}$ (mean) & Filament Diameter, $\mu \mathrm{m}$ : \\
\hline Gamma Dose Rate: $-8.3 \mathrm{~Gy} / \mathrm{s}$ & Layup: \\
\hline Electron $\%: 100$ & Production Method: Mold \\
\hline Electron Energy: $\quad 4 \mathrm{MeV}$ & other: \\
\hline Electron Dose Rate: $1 \mathrm{k} \mathrm{Gy} / \mathrm{s}$ & \\
\hline \multicolumn{2}{|l|}{ Other: } \\
\hline \multicolumn{2}{|c|}{ TEST METHOD } \\
\hline \multicolumn{2}{|l|}{ Test Type: Flexural strength } \\
\hline \multicolumn{2}{|l|}{ Test Specifications: ASTM D790 166} \\
\hline \multicolumn{2}{|l|}{ Specimen Shape: Rectangular bar } \\
\hline \multicolumn{2}{|l|}{ Specimen Dimensions: $76 \times 12.7 \times 3.2 \mathrm{~mm}^{3}$} \\
\hline \multicolumn{2}{|l|}{ Flexure: Span/Thickness Ratio: } \\
\hline \multicolumn{2}{|c|}{ Compression: Aspect Ratio, Length/Width or Diam.: } \\
\hline \multicolumn{2}{|c|}{ Specimens Tested/Data Point: 1 at $77 \mathrm{~K}$, more at $295 \mathrm{~K}$} \\
\hline \multicolumn{2}{|l|}{ Strain (Load) Rate: } \\
\hline Test Temperature, $\mathrm{K}: 295$ & \\
\hline Other: & \\
\hline
\end{tabular}




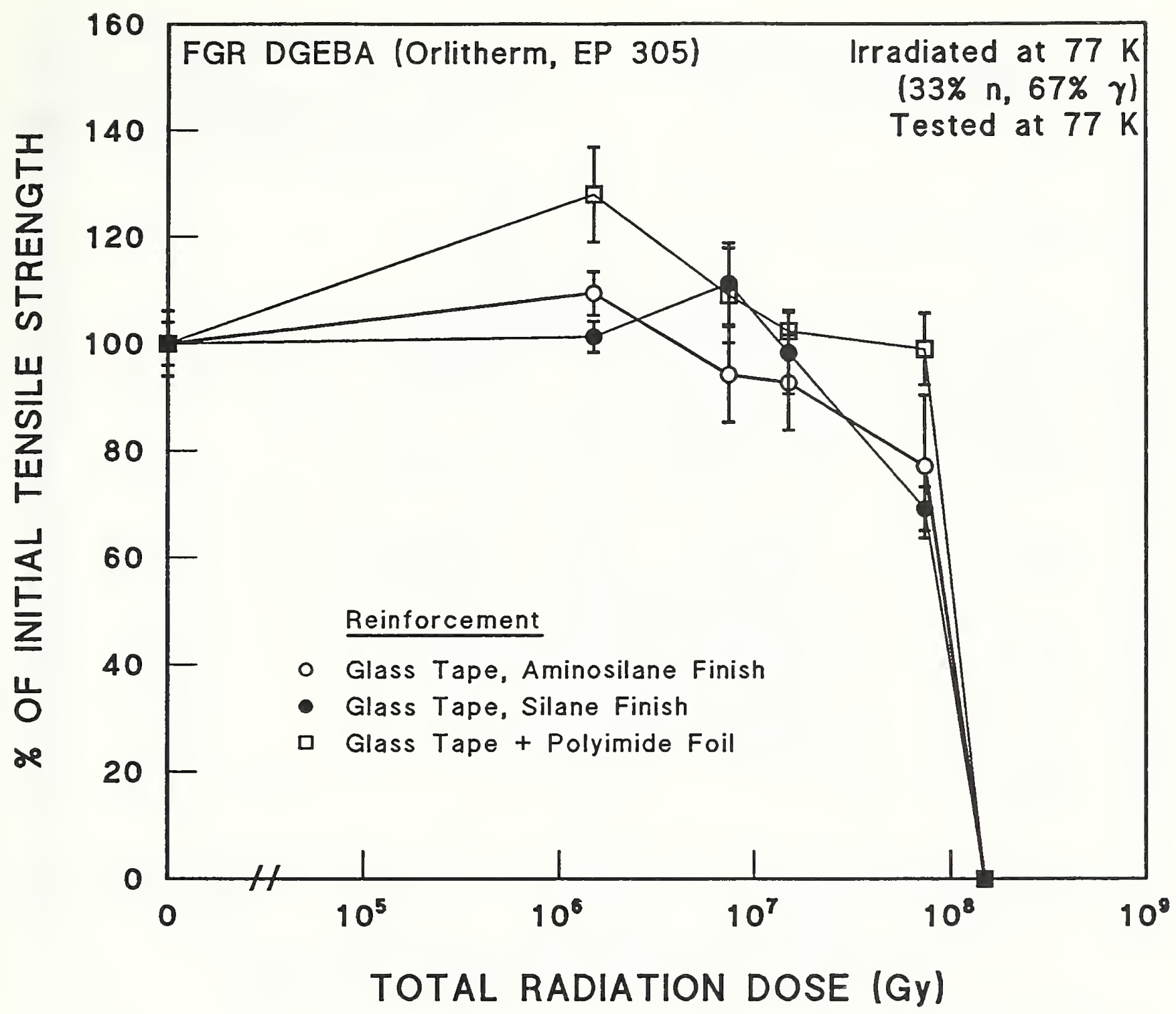

\begin{tabular}{lll} 
"O" Strength, MPa & Supplier \\
\hline $0>1345.0 \pm 53.5$ & Brown, Boveri \& Cie \\
- $855.0 \pm 52.5$ & Brown, Boveri \& Cie \\
口 $900.0 \pm 54.0$ & Brown, Boveri \& Cie
\end{tabular}

Comments: All test samples were broken at doses of $1.5 \times 10^{8} \mathrm{~Gy}$.

Figure A.6-1. Tensile strength at $77 \mathrm{~K}$ of FGR DGEBA resin (Orlitherm) after 77-K reactor irradiation. Supplementary Table A.6-1. Data from Weber et al. [1983]. 


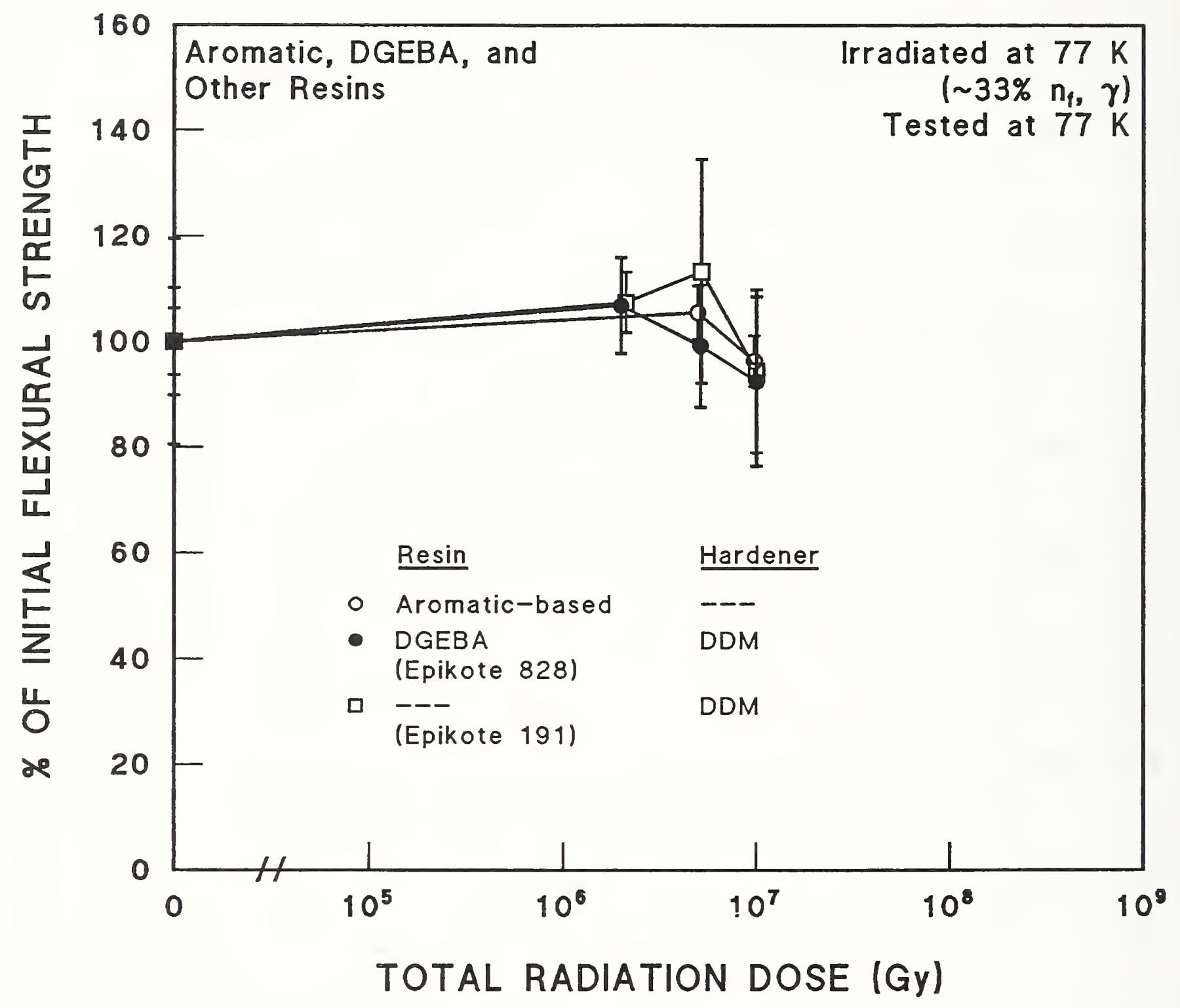

"O" Strength, MPa

○ $201.0 \pm 12.7$

- $211.8 \pm 21.6$

ㅁ $171.6 \pm 33.5$
Supplier

Shell

Shell

Figure A.6-2. Flexural strength at $77 \mathrm{~K}$ of neat aromatic, DGEBA, and other resins after 77-K reactor irradiation. Supplementary Table A.6-2. Data from Van de Voorde [1972]. 


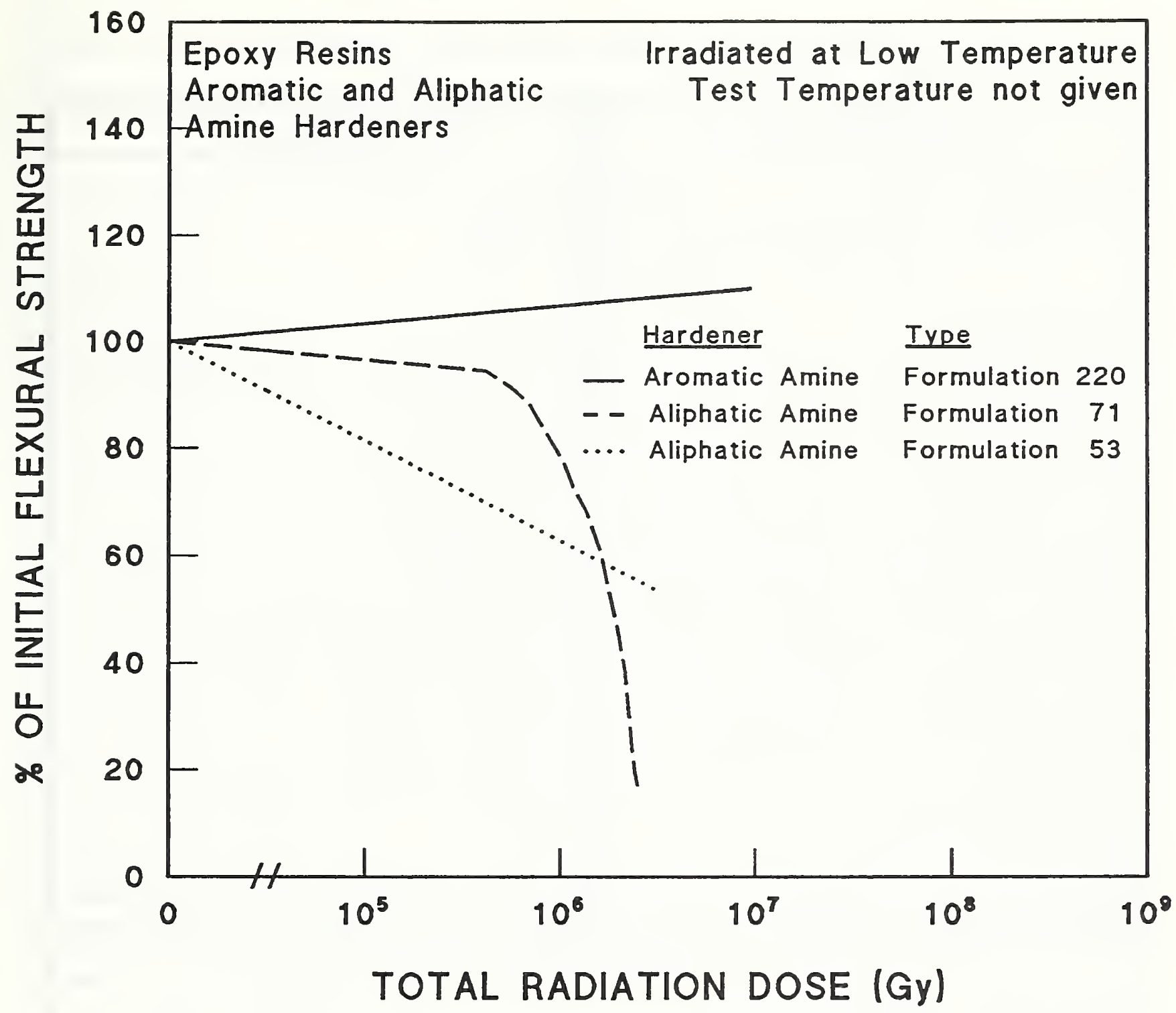

"O" Strength, MPa

- 111.0

- $\quad 97.5$

... 46.9
Supplier

Rutherford

Rutherford

Rutherford

Figure A.6-3. Flexural strength at unspecified temperature of neat epoxy resins irradiated at low temperature. No supplementary information. Data from Evans and Morgan [1982]. 
Table A.6-1. Supplementary Information, Weber et al. [1983] .

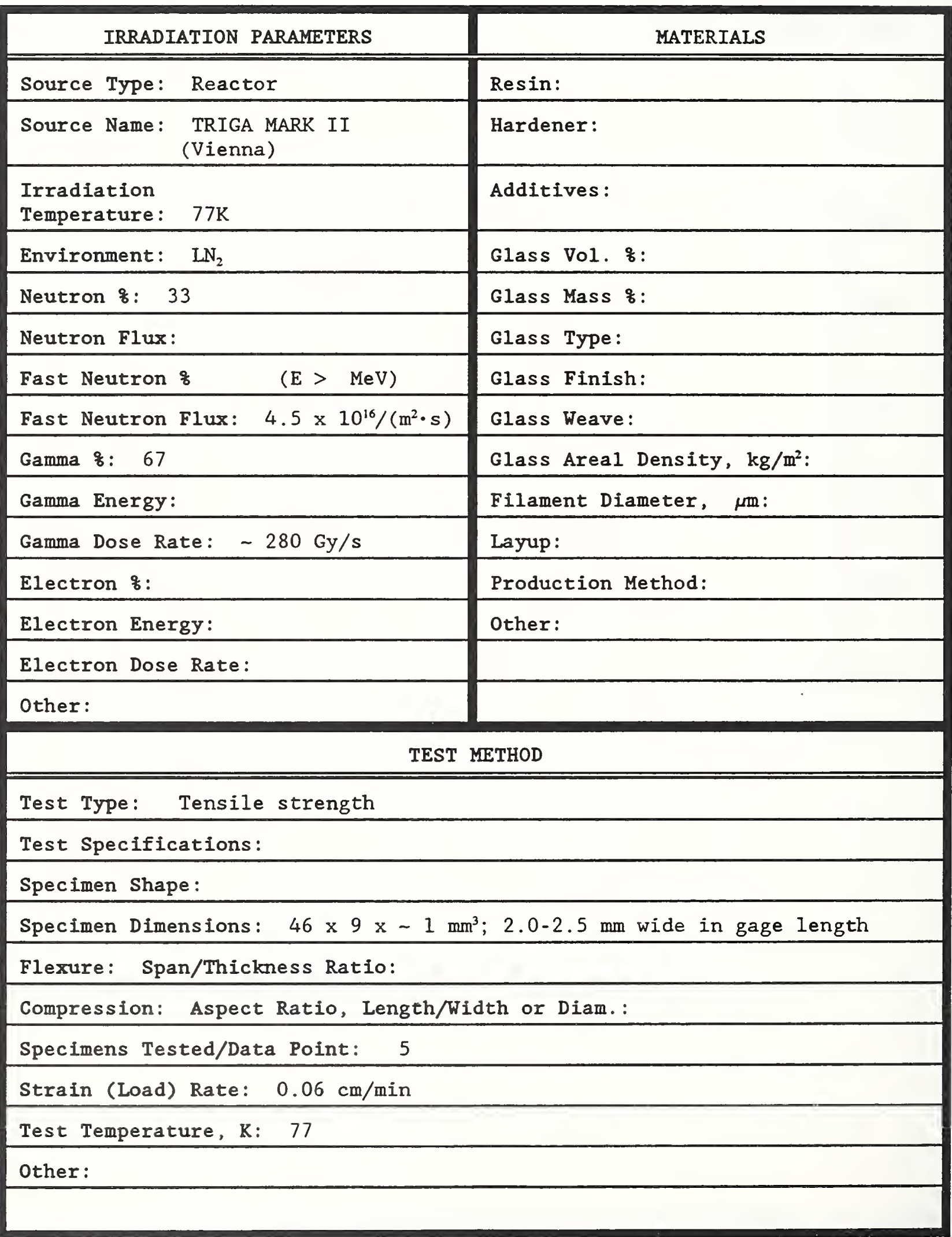


Table A.6-2. Supplementary Information, Van de Voorde [1972].

\begin{tabular}{|c|c|}
\hline IRRADIATION PARAMETERS & MATERIALS \\
\hline Source Type: Pool reactor & Resin: \\
\hline Source Name: & Hardener: \\
\hline $\begin{array}{l}\text { Irradiation } \\
\text { Temperature: } 77 \mathrm{~K}\end{array}$ & Additives: \\
\hline Environment: $\quad \mathrm{LN}_{2}$ & Glass Vol. \%̊: \\
\hline Neutron $q$ & Glass Mass : \\
\hline Neutron Flux: & Glass Type: \\
\hline Fast Neutron of: $\quad(E>1 \mathrm{MeV})$ & Glass Finish: \\
\hline Fast Neutron Flux: $-3 \times 10^{15} /\left(\mathrm{m}^{2} \cdot \mathrm{s}\right)$ & Glass Weave: \\
\hline Gamma of: & Glass Areal Density, $\mathrm{kg} / \mathrm{m}^{2}$ : \\
\hline Gamma Energy: & Filament Diameter $, \mu \mathrm{m}:$ \\
\hline Gamma Dose Rate: $-83 \mathrm{~Gy} / \mathrm{s}$ & Layup: \\
\hline Electron $8:$ & Production Method: \\
\hline Electron Energy: & Other: \\
\hline Electron Dose Rate: & \\
\hline \multicolumn{2}{|l|}{ Other: } \\
\hline \multicolumn{2}{|c|}{ TEST METHOD } \\
\hline \multicolumn{2}{|l|}{ Test Type: Flexural strength } \\
\hline \multicolumn{2}{|l|}{ Test Specifications: } \\
\hline \multicolumn{2}{|l|}{ Specimen Shape: Rectangular bar } \\
\hline \multicolumn{2}{|l|}{ Specimen Dimensions: $80 \times 10 \times 4 \mathrm{~mm}$} \\
\hline \multicolumn{2}{|l|}{ Flexure: Span/Thickness Ratio: 16.8} \\
\hline \multicolumn{2}{|c|}{ Compression: Aspect Ratio, Length/Width or Diam.: } \\
\hline \multicolumn{2}{|l|}{ Specimens Tested/Data Point: 4} \\
\hline \multicolumn{2}{|l|}{ Strain (Load) Rate: $0.2 \mathrm{~cm} / \mathrm{min}$} \\
\hline Test Temperature, $\mathrm{K}: 77$ & \\
\hline Other: & \\
\hline
\end{tabular}




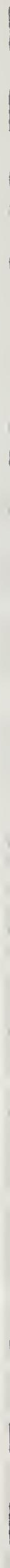




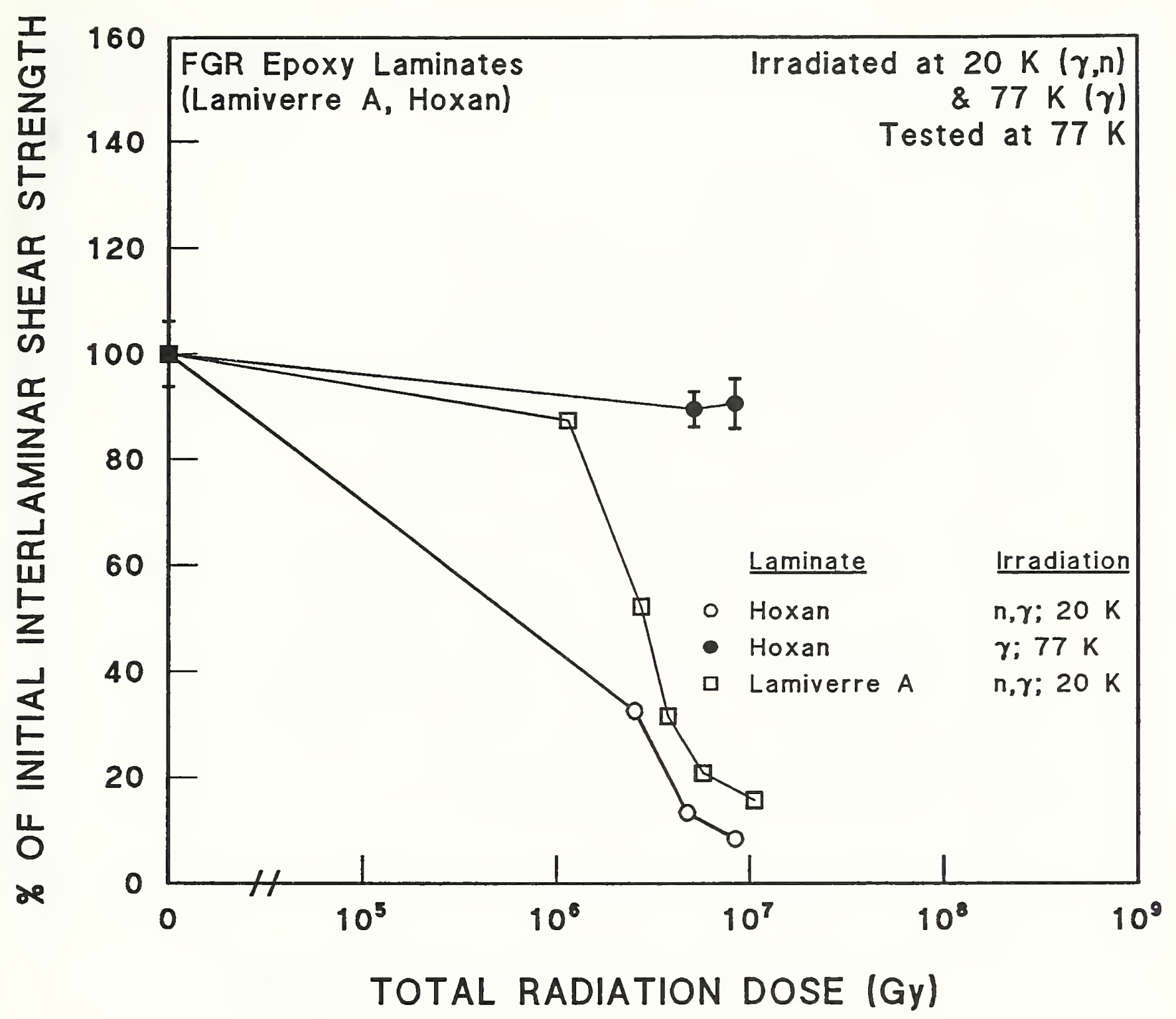

\begin{tabular}{|c|c|c|c|c|c|}
\hline "0" & Strength, MPa & Weave & Finish & Glass & Supplie \\
\hline 0 & $93.7 \pm 0.0$ & Plain & Silane & $60-62$ mass & --- \\
\hline$\bullet$ & $93.7 \pm 5.8$ & Plain & Silane & $60-62$ mass & --- \\
\hline$\square$ & $103.1 \pm 0.0$ & Plain & Silane & $60-62$ mass & --- \\
\hline
\end{tabular}

Figure A.7-1. Interlaminar shear strength at $77 \mathrm{~K}$ of FGR epoxy laminates (Lamiverre A, Hoxan) after 20-K reactor and 77-K gamma irradiation. Supplementary Table A.211. Data from Nishijima et a1. [1988]. 


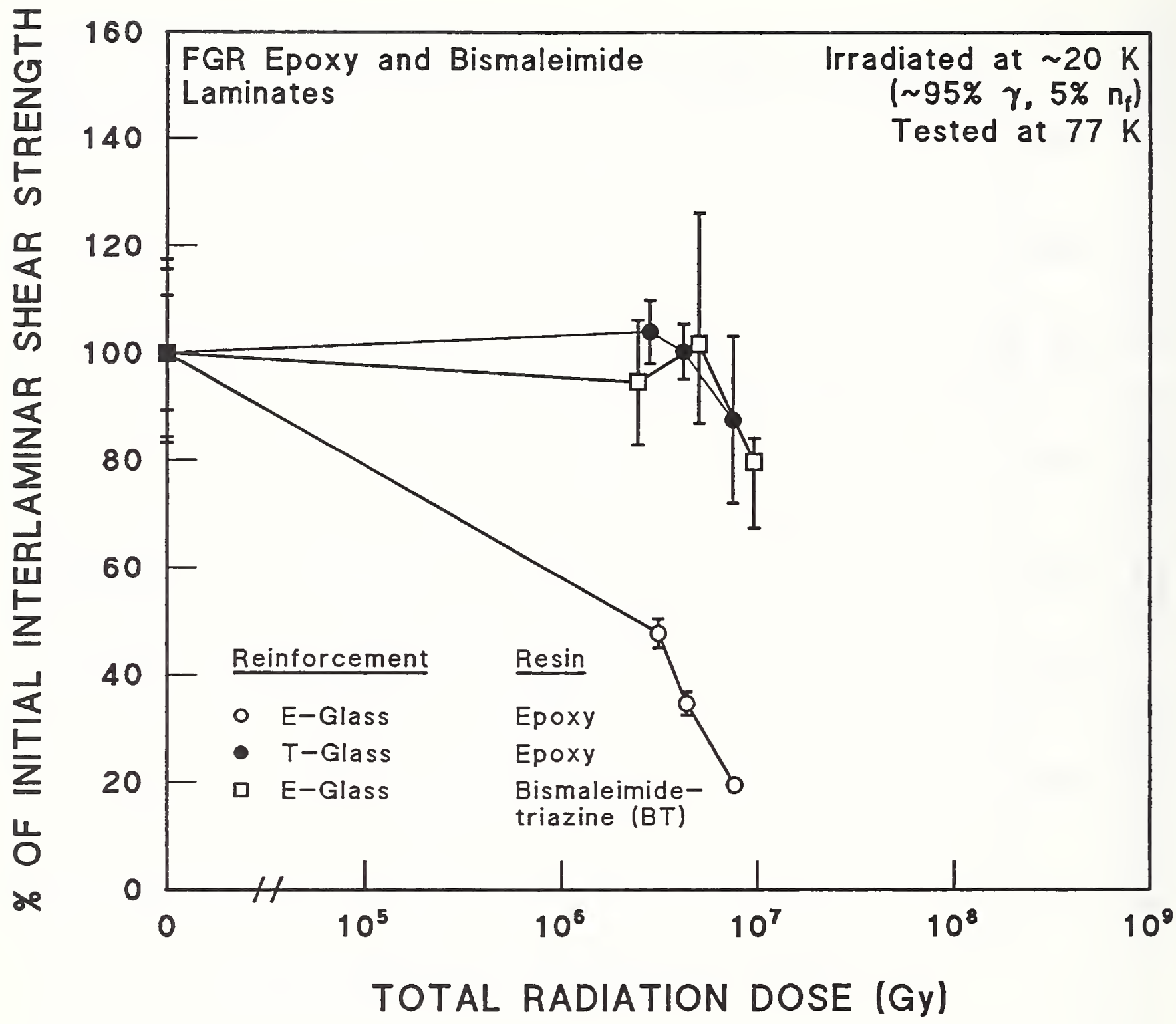

\section{"O" Strength, MPa}

○ $120.6 \pm 18.9$

- $142.7 \pm 16.3$

ㅁ $95.3 \pm 16.3$
Glass Vol. Fr.

$50 \%$
$50 \%$
$50 \%$

Supplier

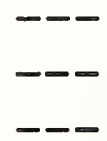

Comments: Identical fabric type, glass surface finish and epoxy matrix for E-Glass and T-Glass laminates.

Figure A.7-2. Interlaminar shear strength at $77 \mathrm{~K}$ of FGR epoxy and bismaleimide laminates after $20-\mathrm{K}$ reactor irradiation. Comparison of E- and T-glass reinforcement. Supplementary Table A.7-1. Data from Nishijima et al. [1991] and Okada et al. [1992]. 


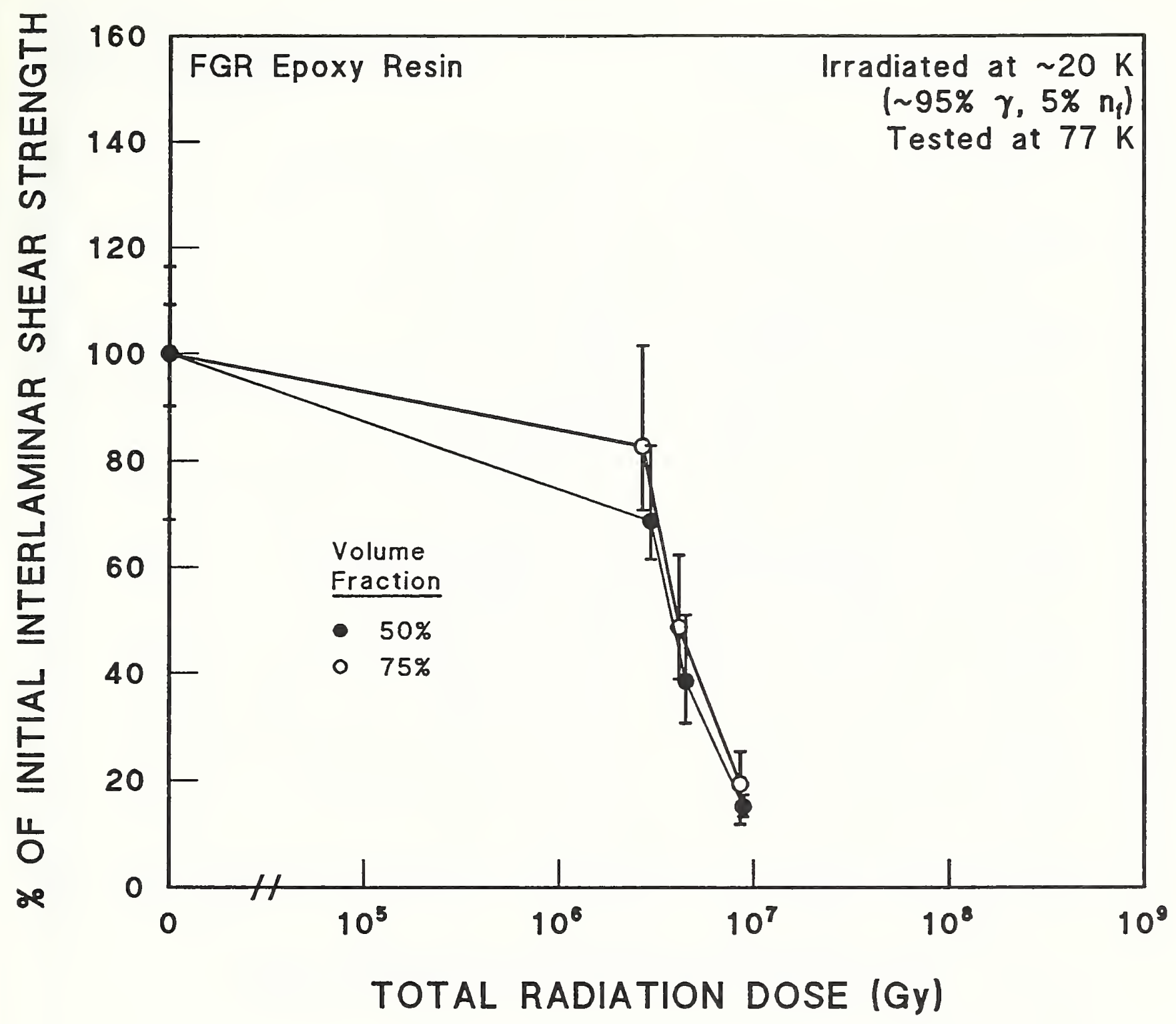

"O" Strength, MPa

- $168.2 \pm 40.0$

- $141.9 \pm 13.5$
Type

Similar to $\mathrm{G}-10$

Same Resin as

$50 \%$ vol. fraction

type
Supplier

Commercially Available

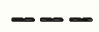

Figure A.7-3. Interlaminar shear strength at $77 \mathrm{~K}$ of FGR (variable volume fraction) epoxy resin after 20-K reactor irradiation. Supplementary Table A.7-1. Data from Okada et al. [1992]. 


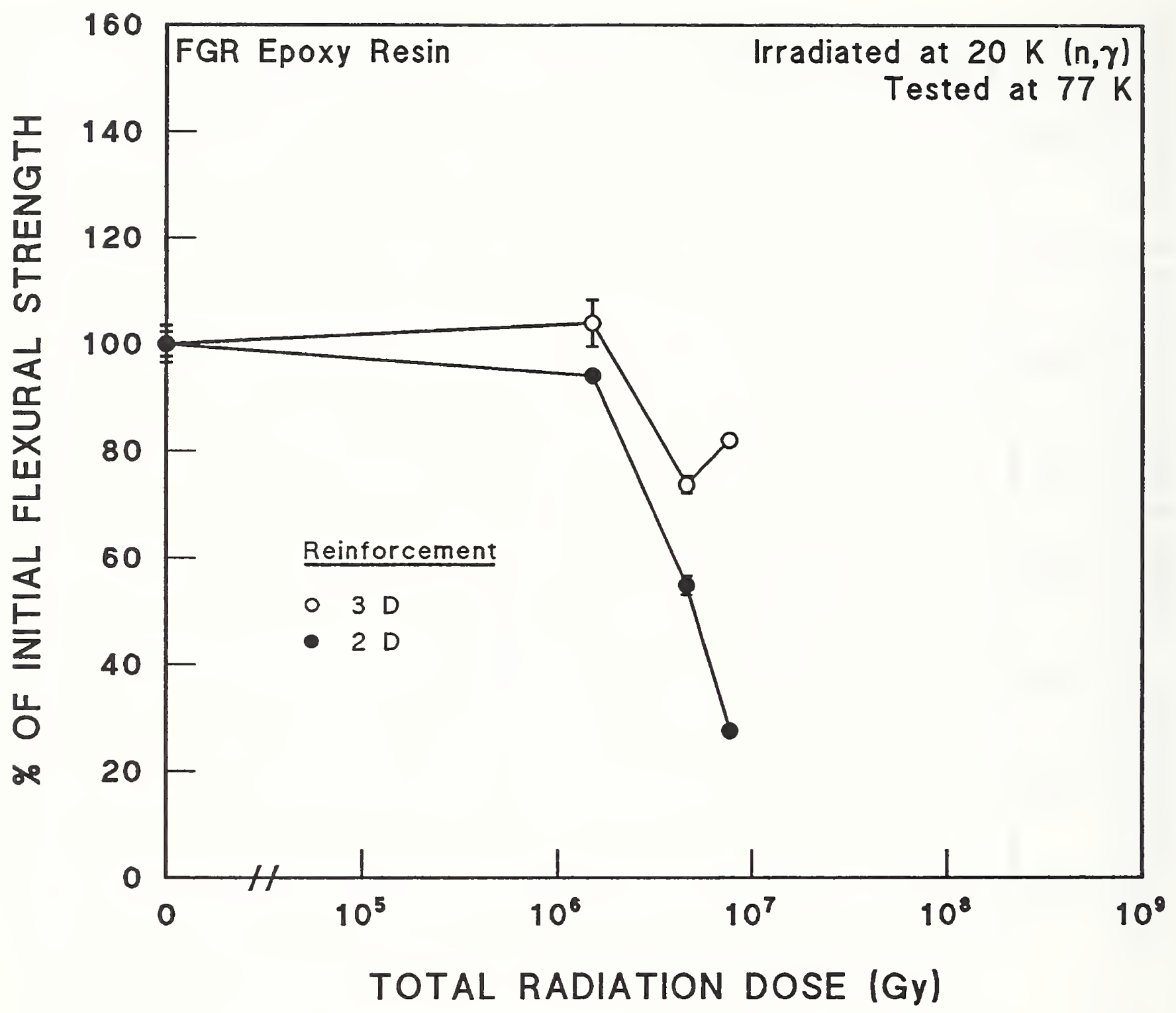

\begin{tabular}{ll} 
"O" Strength, MPa & Supplier \\
\hline $0 \quad 1160.0 \pm 27.0$ & Shikishima \\
& Canvas Co. \\
$-940.7 \pm 32.4$ & $\begin{array}{l}\text { Shikishima } \\
\text { Canvas Co. }\end{array}$
\end{tabular}

Figure A.7-4. Flexural strength at $77 \mathrm{~K}$ of FGR (2- and 3-D) epoxy resin after 20-K reactor irradiation. Supplementary Table A.2-16. Data from Nishijima et al. [1990]. 


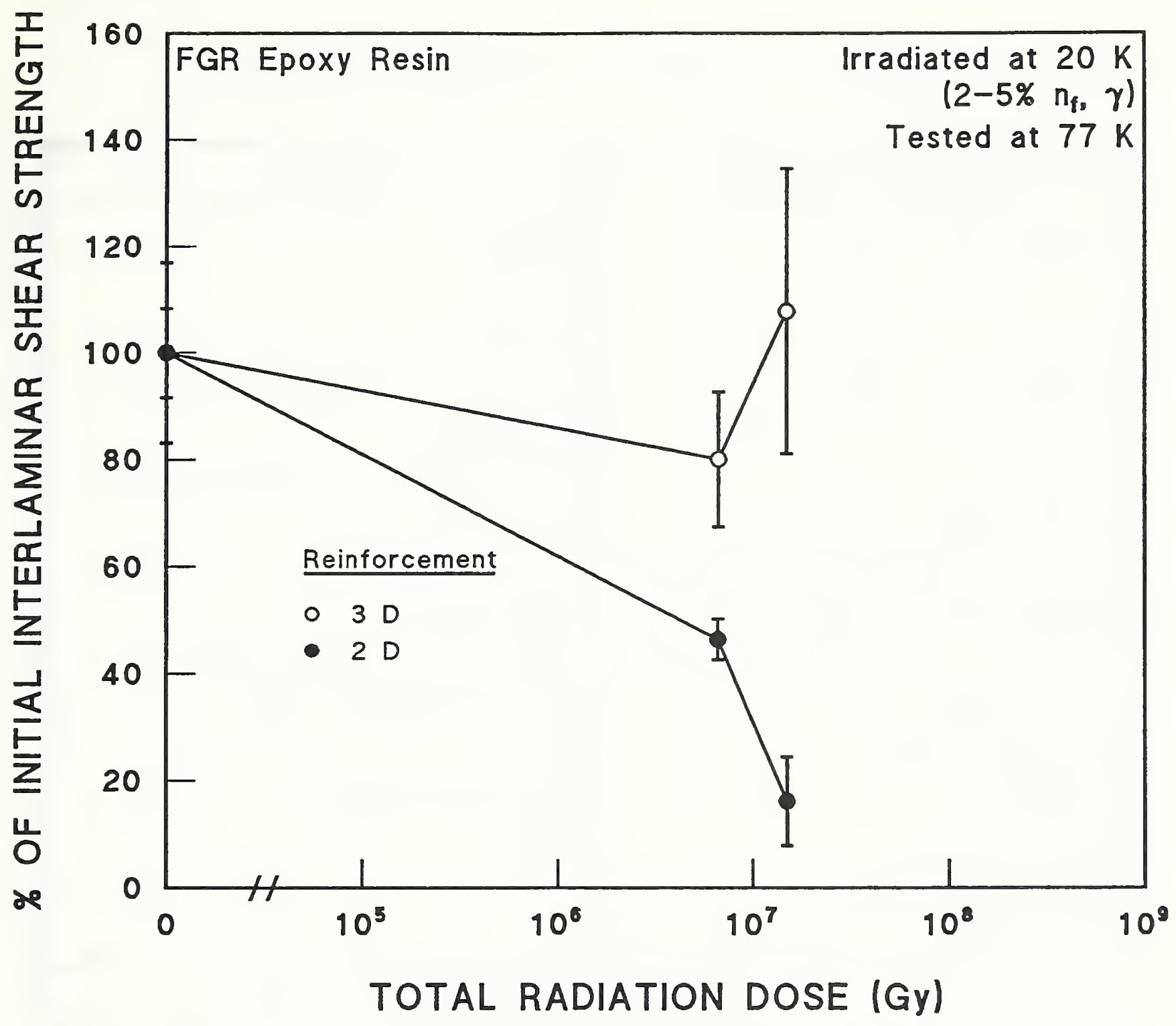

\begin{tabular}{|c|c|c|}
\hline & Strength, MPa & Supplier \\
\hline 0 & $62.7 \pm 10.6$ & $\begin{array}{l}\text { Shikishima } \\
\text { Canvas Co. }\end{array}$ \\
\hline & $47.3 \pm 4.0$ & $\begin{array}{l}\text { Shikishima } \\
\text { Canvas Co. }\end{array}$ \\
\hline
\end{tabular}

Figure A.7-5. Interlaminar shear strength at $77 \mathrm{~K}$ of FGR (2- and 3-D) epoxy resin after 20-K reactor irradiation. Supplementary Table A.2-16. Data from Nishijima et al. [1990]. 
Table A.7-1. Supplementary Information, Nishijima et a1. [1991], Okada et al. [1992].

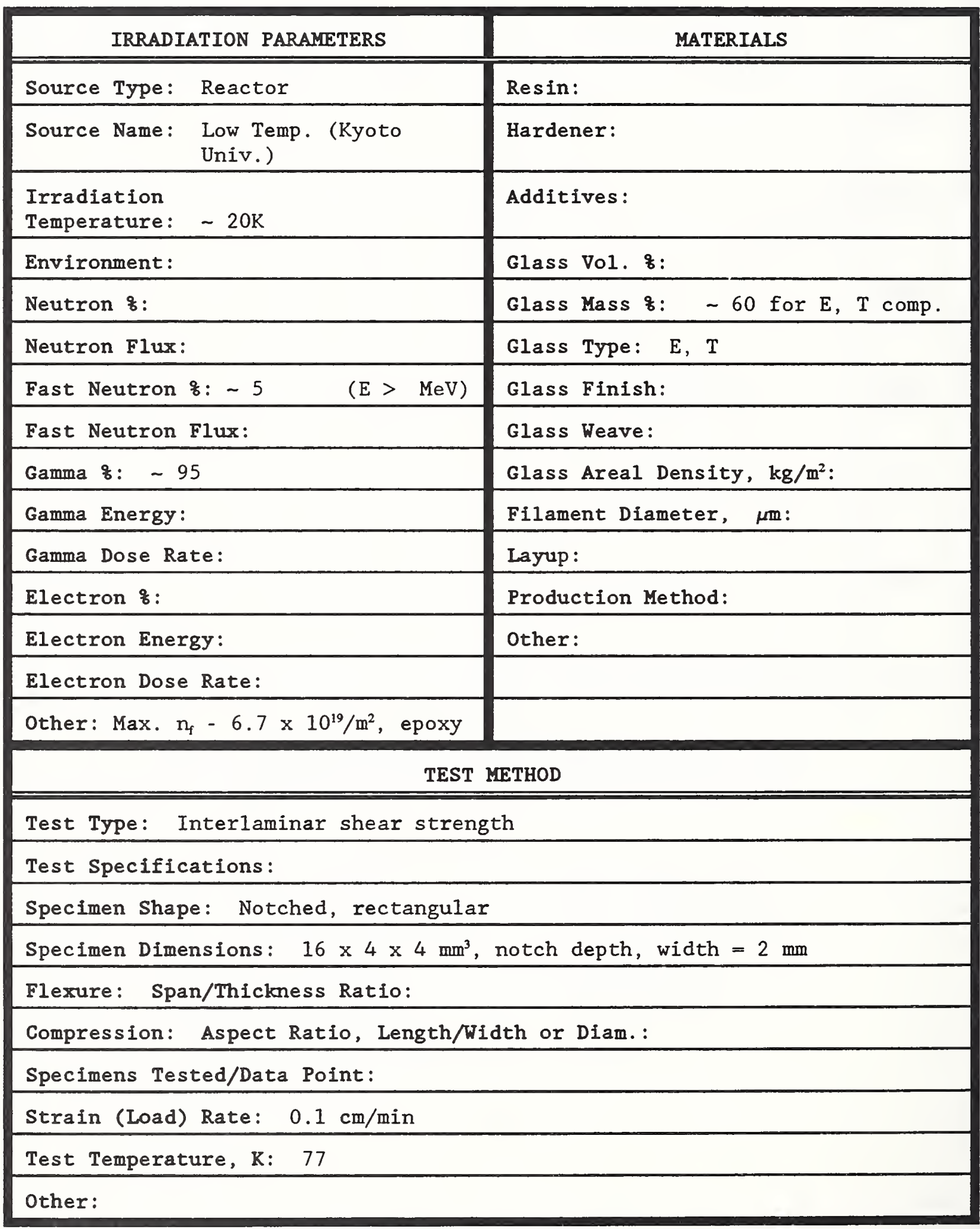




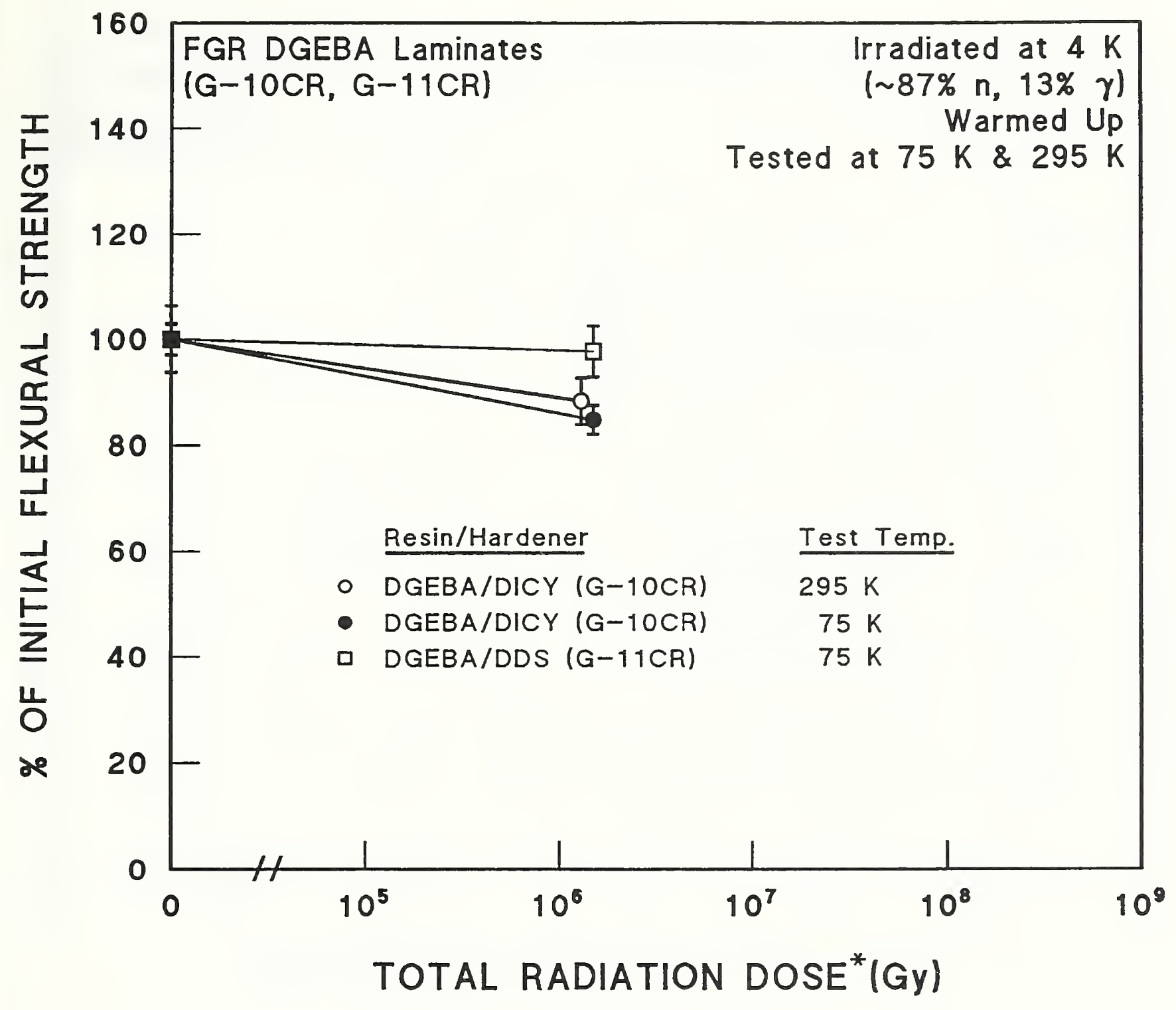

\begin{tabular}{ll} 
"O" Strength, MPa & Supplier \\
\hline $0 \quad 604.4 \pm 18.0$ & - \\
- $1162.9 \pm 73.1$ & -- \\
口 $1394.0 \pm 41.1$ & --
\end{tabular}

* Neglects $\gamma$ contribution, estimated at $\sim 15 \%$ of neutron dose

Figure A.8-1. Flexural strength at 75 and $295 \mathrm{~K}$ of FGR DGEBA 1aminates (G-10CR, GllCR) after 4-K reactor irradiation. Supplementary Table A.8-1. Data from Hurley et al. [1978; 1983]. 


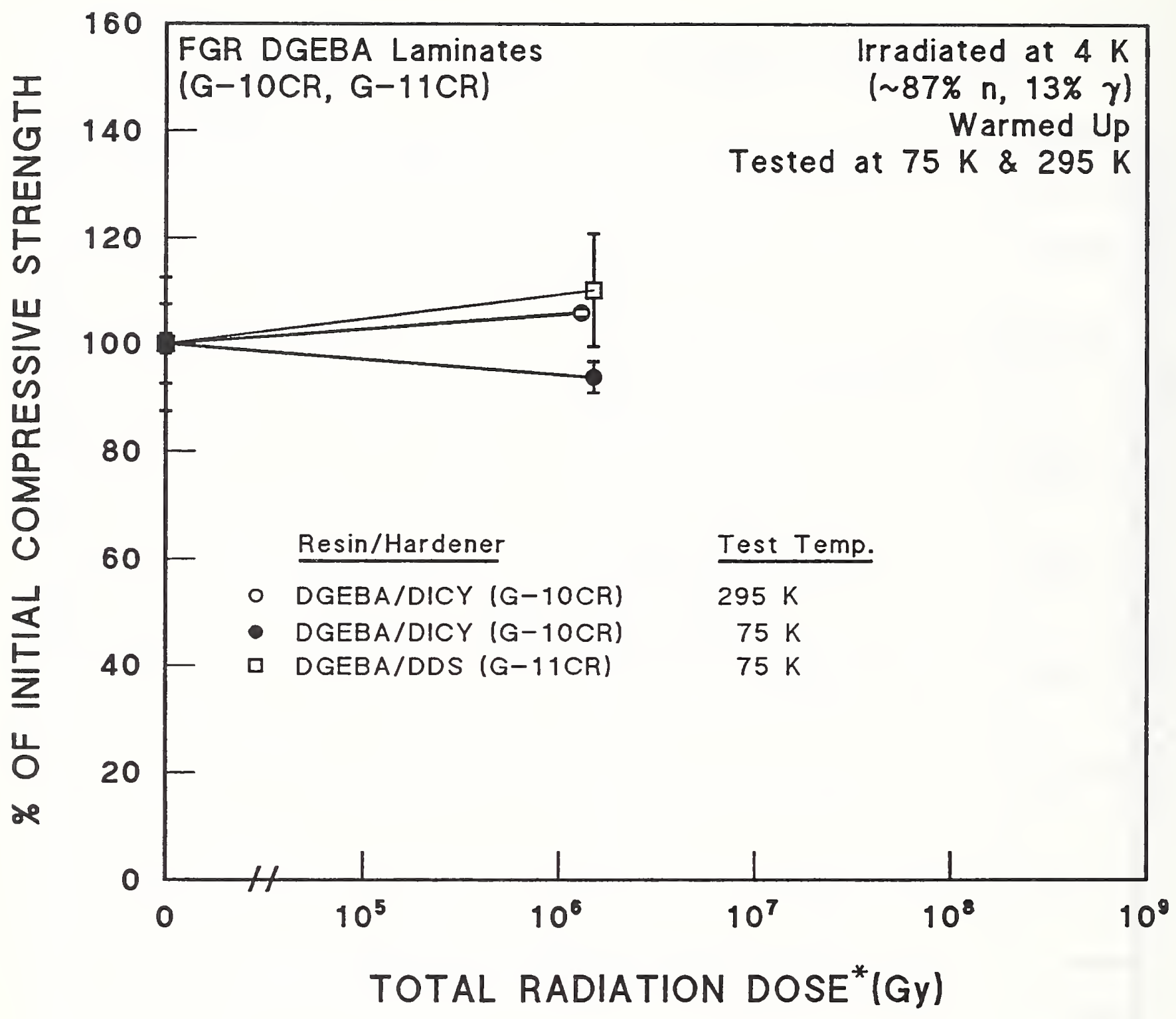

"O" Strength, MPa

- $396.9 \pm 7.3$

- $838.0 \pm 62.9$

ㅁ $809.0 \pm 101.9$
Supplier

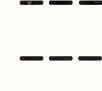

* Neglects $\gamma$ contribution, estimated at $\sim 15 \%$ of neutron dose

Figure A.8-2. Compressive strength at 75 and $295 \mathrm{~K}$ of FGR DGEBA laminates (G-10CR, G-11CR) after 4-K reactor irradiation. Supplementary Table A.8-1. Data from Hurley et a1. [1978; 1983]. 


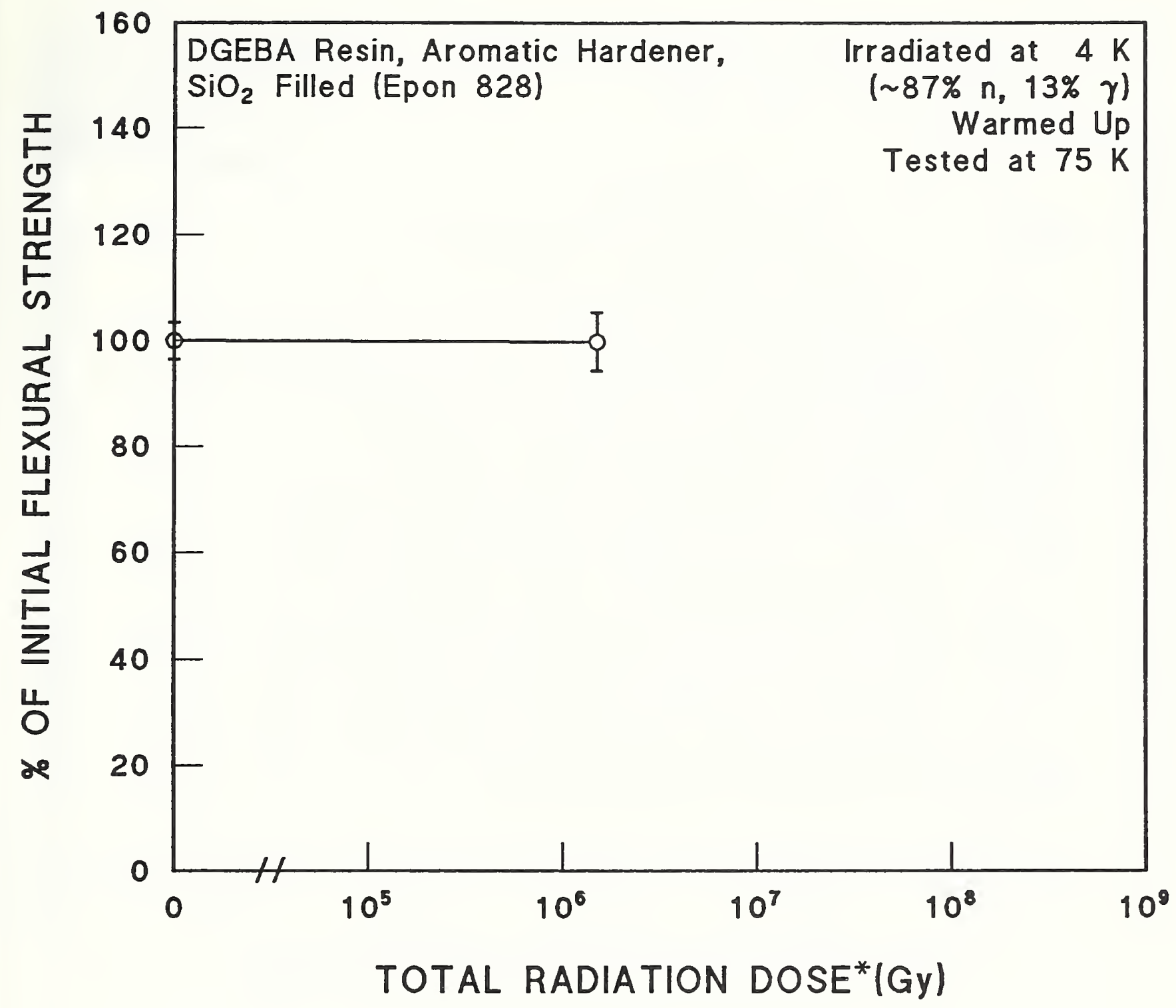

"O" Strength, MPa

Supplier

○ $250.0 \pm 8.8$

Shell

* Neglects $\gamma$ contributions estimated at $\sim 15 \%$ of neutron dose

Figure A.8-3. Flexural strength at 75 and $295 \mathrm{~K}$ of DGEBA resin with silica filler after 4-K reactor irradiation. Supplementary Table A.8-1. Data from Hurley et al. [1978]. 


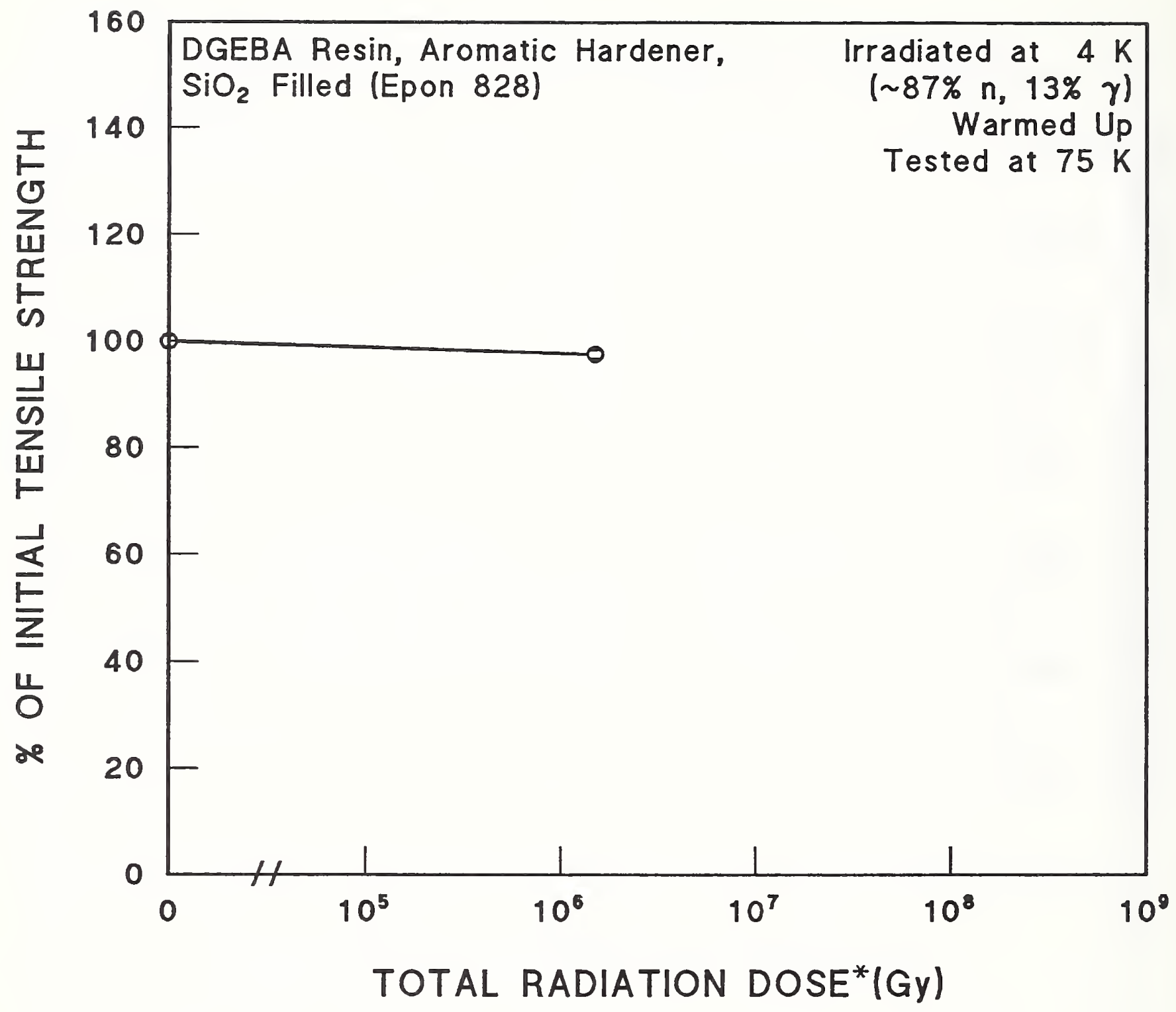

"O" Strength, MPa

○ $593.8 \pm 6.5$
Supplier

Shell

* Neglects $\gamma$ contributions estimated at $\sim 15 \%$ of neutron dose

Figure A.8-4. Tensile strength at 75 and $295 \mathrm{~K}$ of DGEBA resin with silica filler after 4-K reactor irradiation. Supplementary Table A.8-1. Data from Hurley et al. [1978]. 


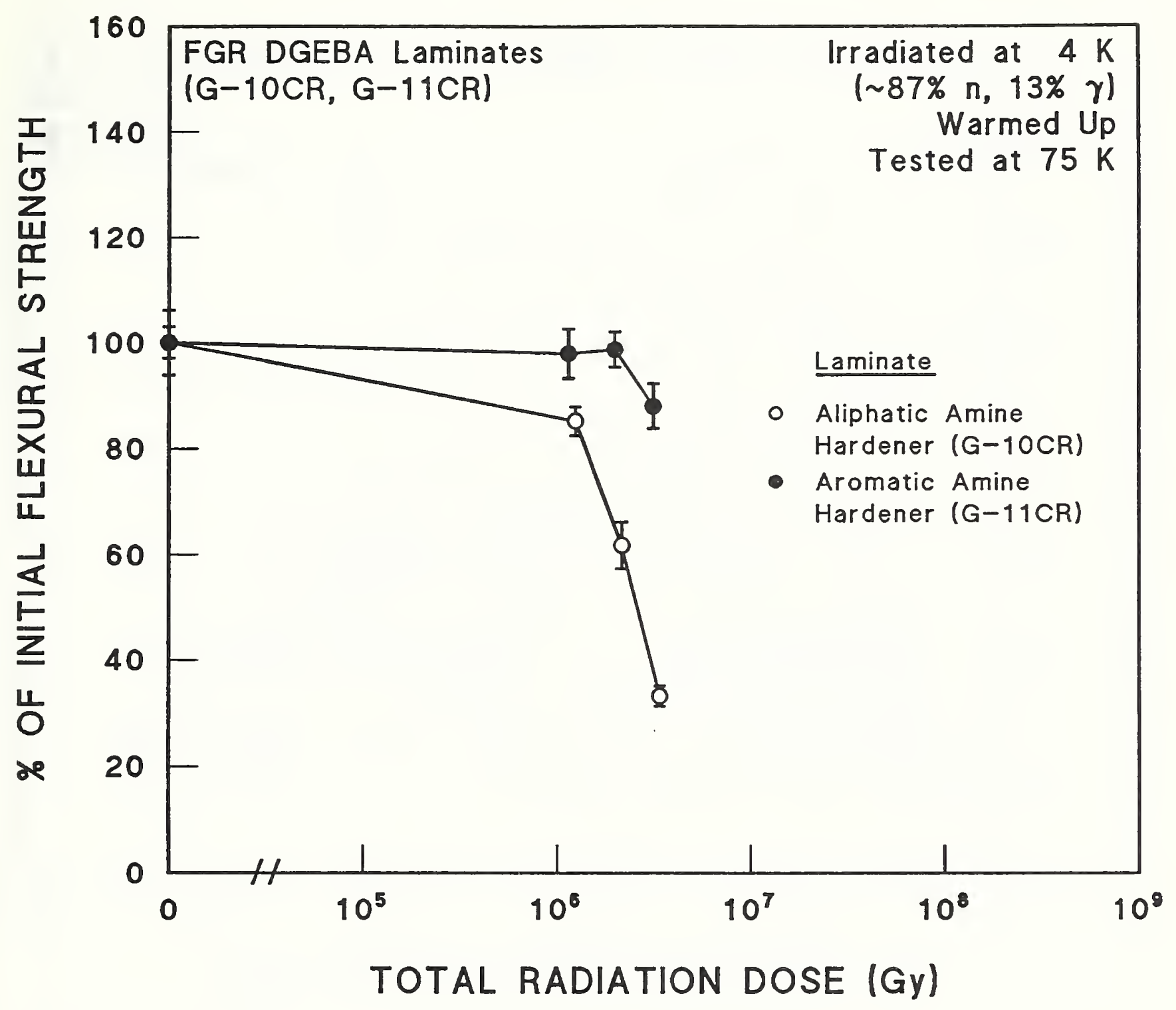

\begin{tabular}{l} 
"O" Strength, MPa \\
\hline $\begin{array}{l}\text { Supplier } \\
\text { 1167.0 } 1393.0 \pm 72.0\end{array}$
\end{tabular}

Figure A.8-5. Flexural strength at $75 \mathrm{~K}$ of FGR DGEBA laminates (G-10CR, G-11CR) after 4-K reactor irradiation. Supplementary Table A.8-1. Data from Tucker et al. [1985]. 


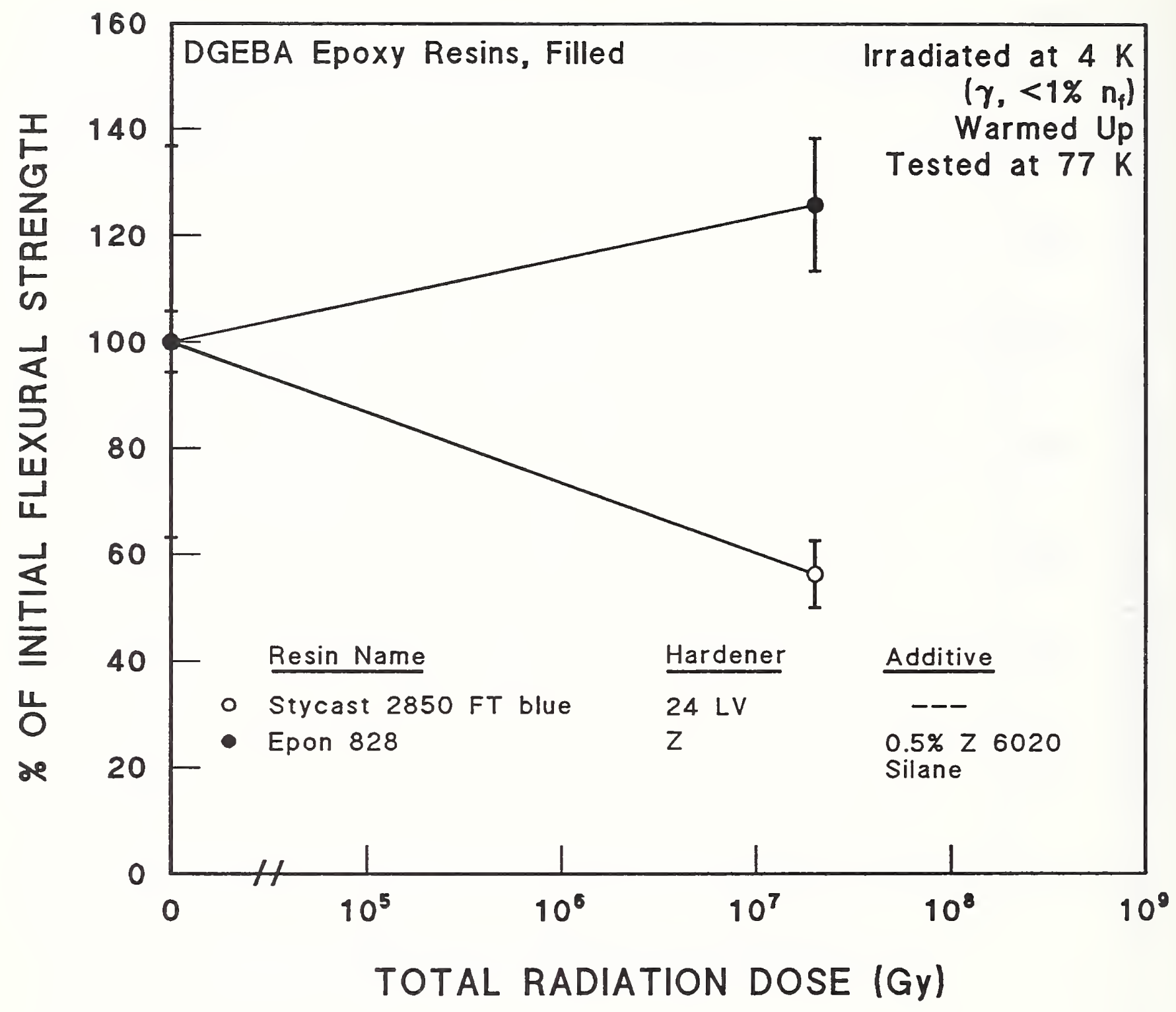

"O" Strength, MPa

Cure Condition

Filler

Supplier

○ $254.0 \pm 14.6$

$24 \mathrm{~h}, 22^{\circ} \mathrm{C}$

not specified

$2 \mathrm{~h}, 80^{\circ} \mathrm{C}$;

40 mass $\%$

400-mesh $\mathrm{SiO}_{2}$

Emerson-Cumming Shell

Figure A.8-6. Flexural strength at $77 \mathrm{~K}$ of DGEBA resins, filled, after 4-K reactor irradiation. Supplementary Table A.8-2. Data from Kernohan et al. [1979]. 


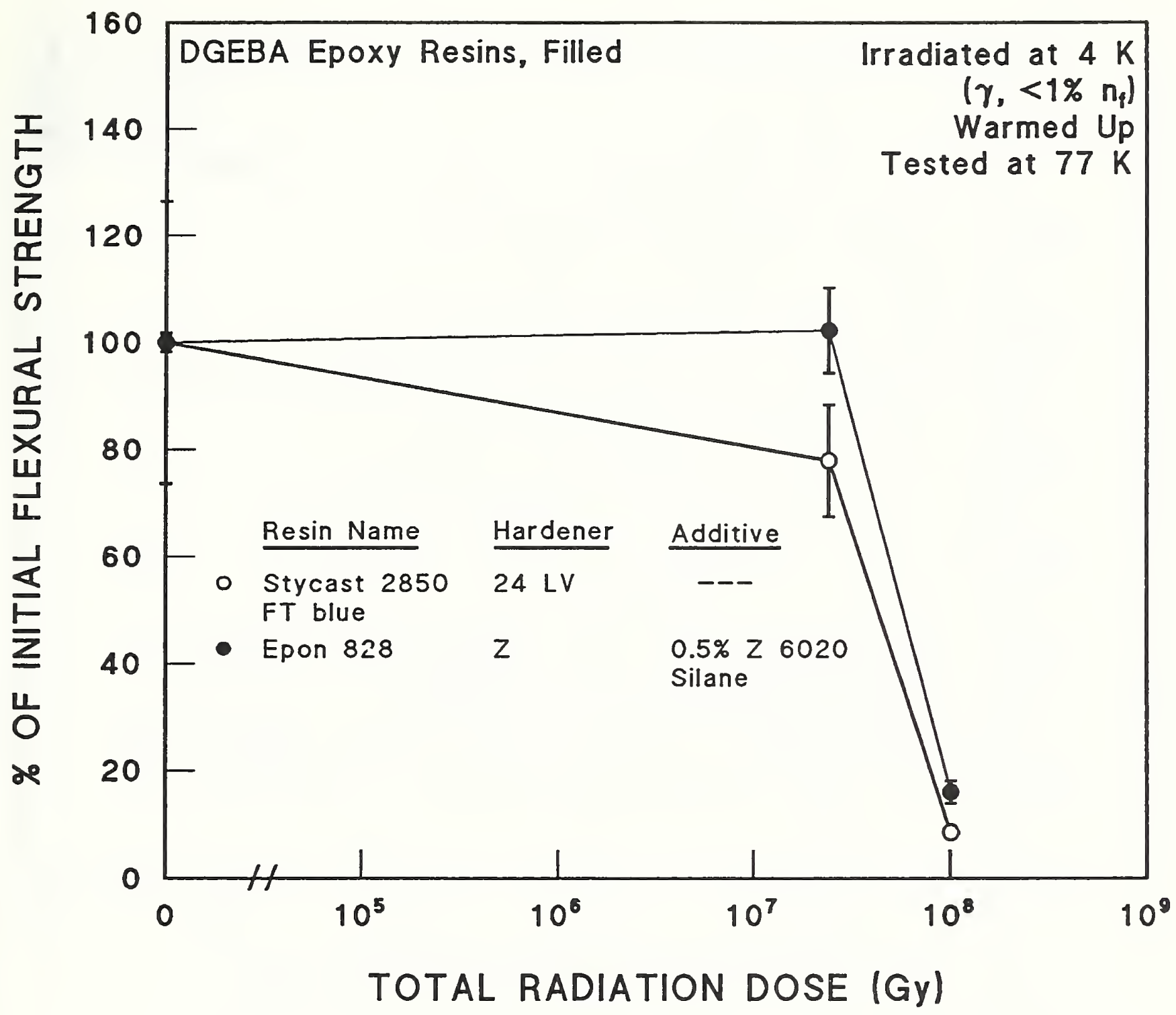

"O" Strength, MPa

Cure Condition

Filler

Supplier

- $262.3 \pm 18.3$

$24 \mathrm{~h}, 22^{\circ} \mathrm{C}$

not specified

$2 \mathrm{~h}, 80^{\circ} \mathrm{C}$;

$2 \mathrm{~h}, 150^{\circ} \mathrm{C}$

40 mass \% 400-mesh $\mathrm{SiO}_{2}$

Emerson-Cumming Shell

Figure A.8-7. Flexural strength at $77 \mathrm{~K}$ of DGEBA resins, filled, after 4-K reactor irradiation. Supplementary Table A.8-2. Data from Coltman et al. [1979]. 


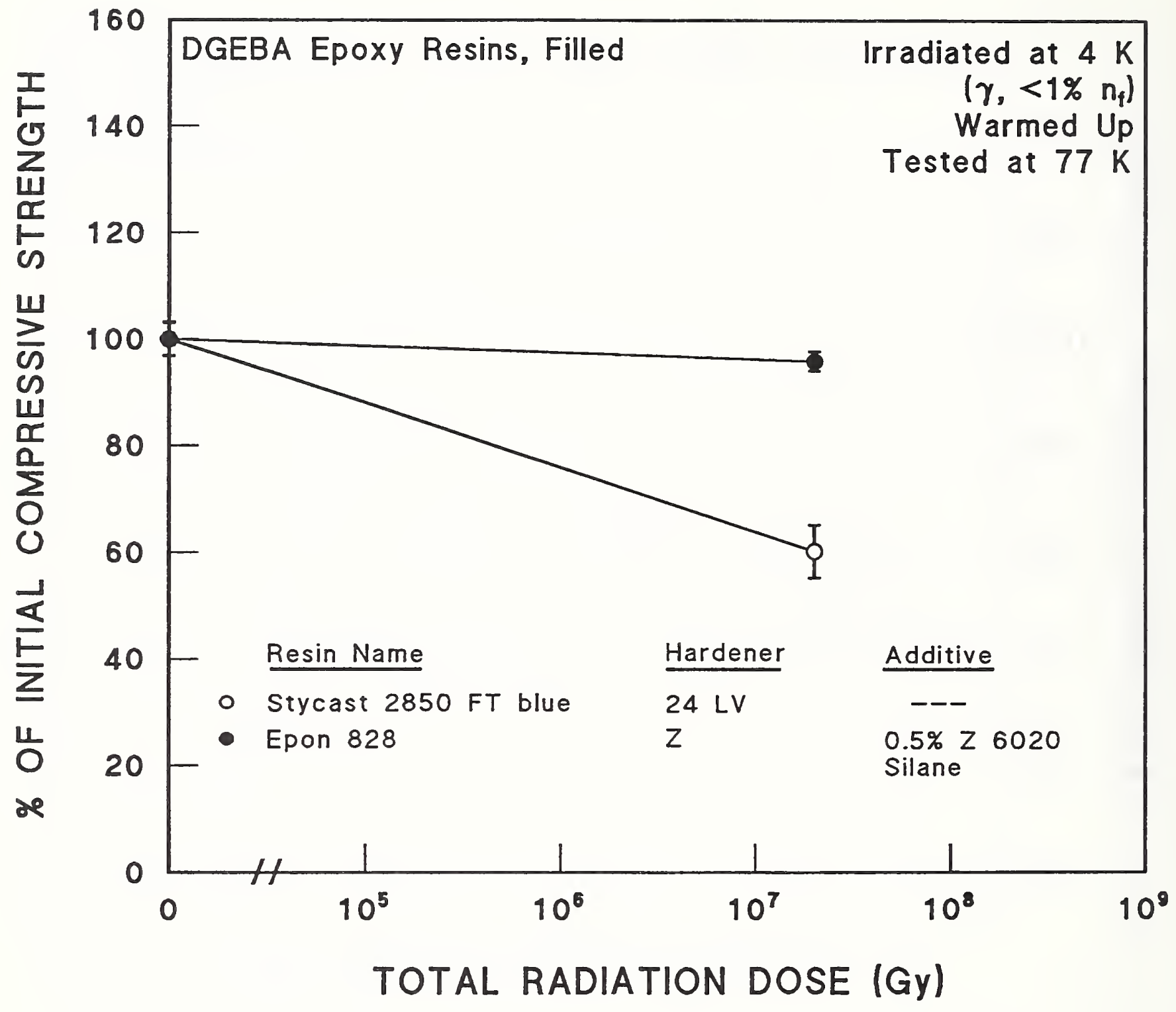

"O" Strength, MPa

- $494.5 \pm 5.5$

- $495.0 \pm 16.0$

\section{Cure Condition}

$24 \mathrm{~h}, 22^{\circ} \mathrm{C}$

$2 \mathrm{~h}, 80^{\circ} \mathrm{C}$;

$2 \mathrm{~h}, 150^{\circ} \mathrm{C}$
Filler

not specified

40 mass $\%$

400-mesh $\mathrm{SiO}_{2}$
Supplier

Emerson-Cumming Shell

Figure A.8-8. Compressive strength at $77 \mathrm{~K}$ of DGEBA resins, filled, after 4-K reactor irradiation. Supplementary Table A.8-2. Data from Kernohan et al. [1979]. 


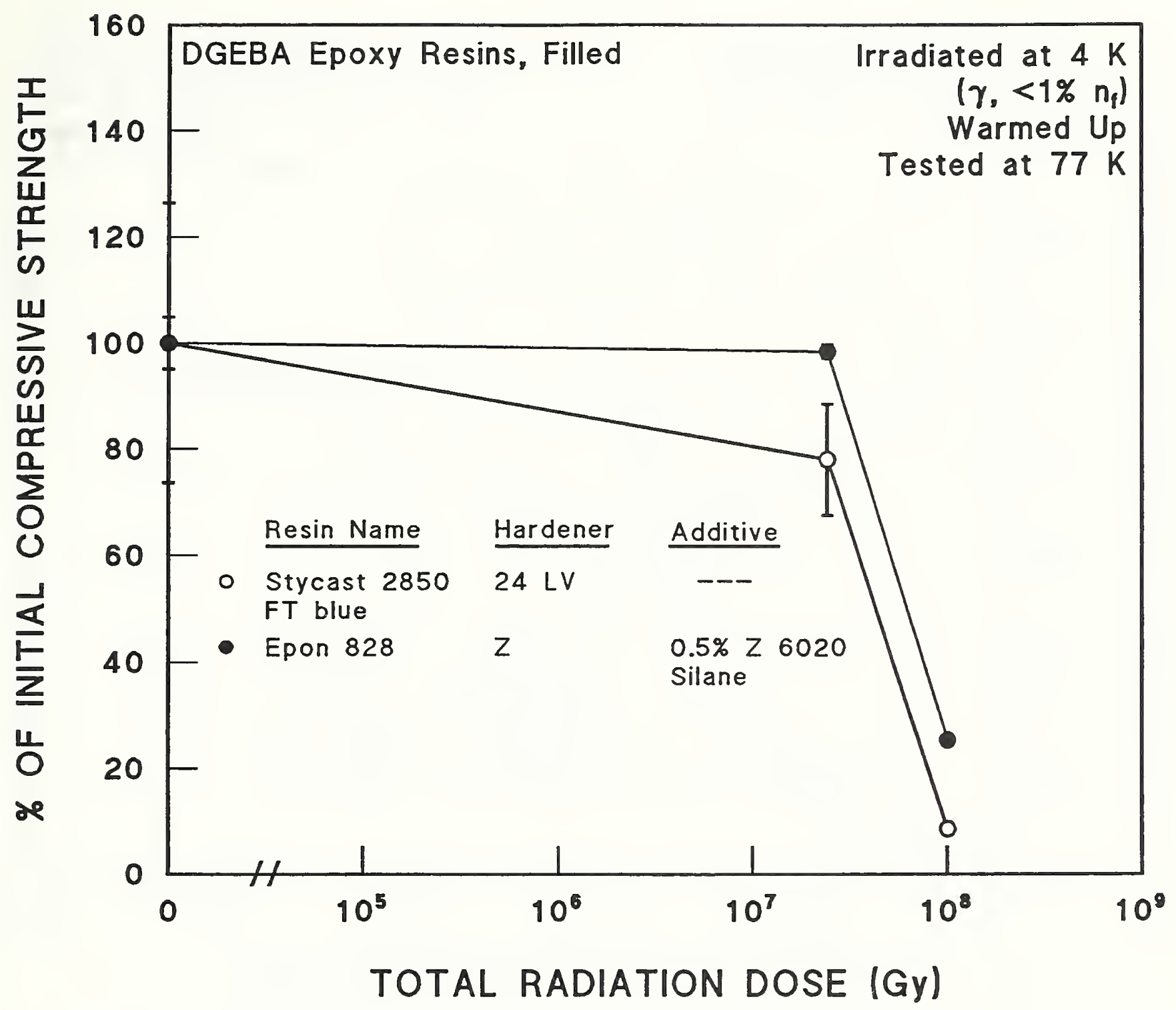

\begin{tabular}{|c|c|c|c|}
\hline "O" Strength, MPa & Cure Condition & Filler & Supplier \\
\hline $497.7 \pm 131.2$ & $24 \mathrm{~h}, 22^{\circ} \mathrm{C}$ & not specified & Emerson-Cumming \\
\hline - $503.3 \pm 24.8$ & $\begin{array}{l}2 \mathrm{~h}, 80^{\circ} \mathrm{C} ; \\
2 \mathrm{~h}, 150^{\circ} \mathrm{C}\end{array}$ & $\begin{array}{l}40 \text { mass } \% \\
400-\text { mesh } \mathrm{SiO}_{2}\end{array}$ & Shell \\
\hline
\end{tabular}

Figure A.8-9. Compressive strength at $77 \mathrm{~K}$ of DGEBA resins, filled, after 4-K reactor irradiation. Supplementary Table A.8-2. Data from Coltman et al. [1979]. 


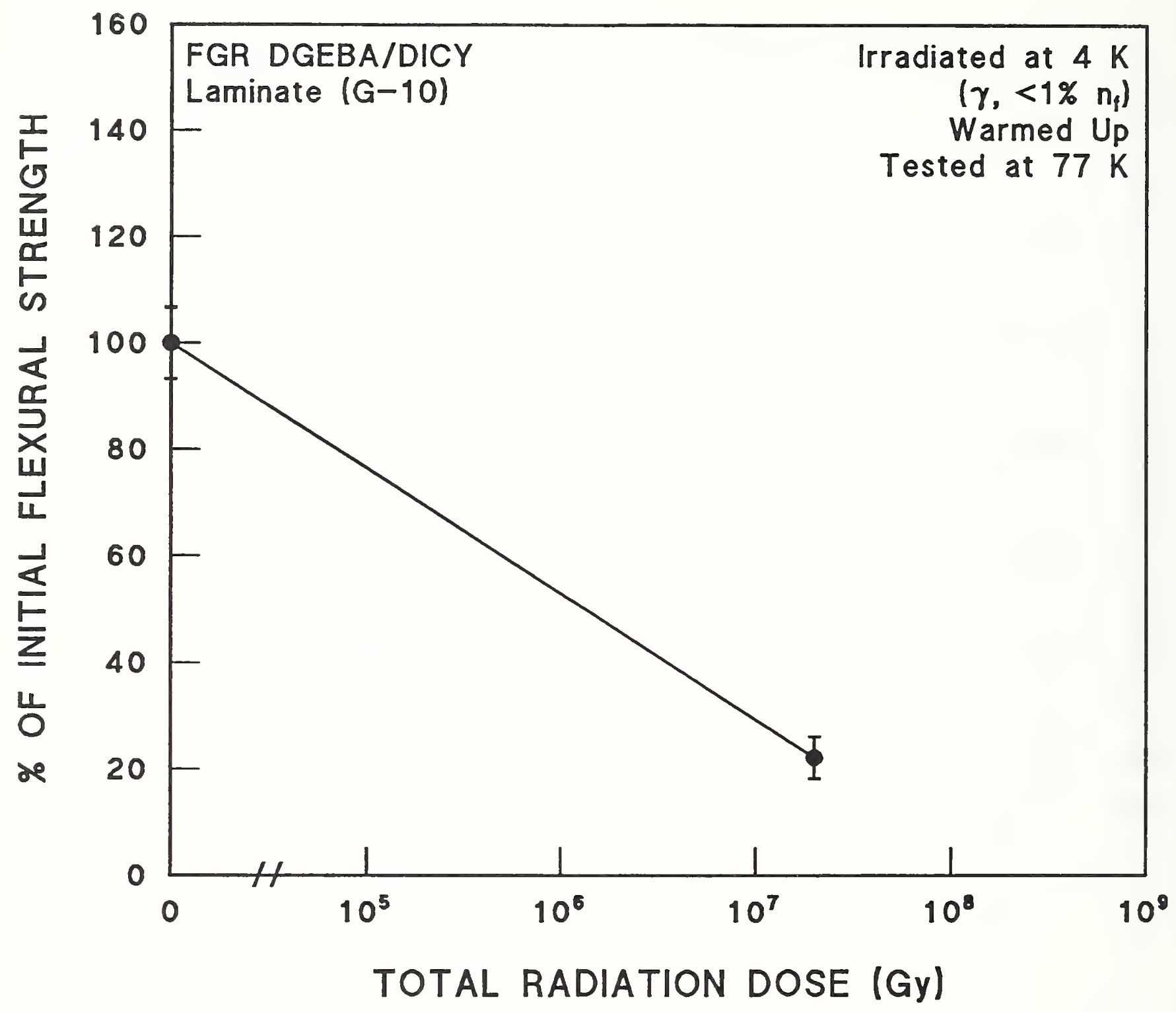

"O" Strength, MPa

Supplier

- $862.0 \pm 58.0$

Spaulding

Figure A.8-10. Flexural strength at $77 \mathrm{~K}$ of FGR DGEBA laminate (G-10) after 4-K reactor irradiation. Supplementary Table A.8-2. Data from Kernohan et a1. [1979]. 


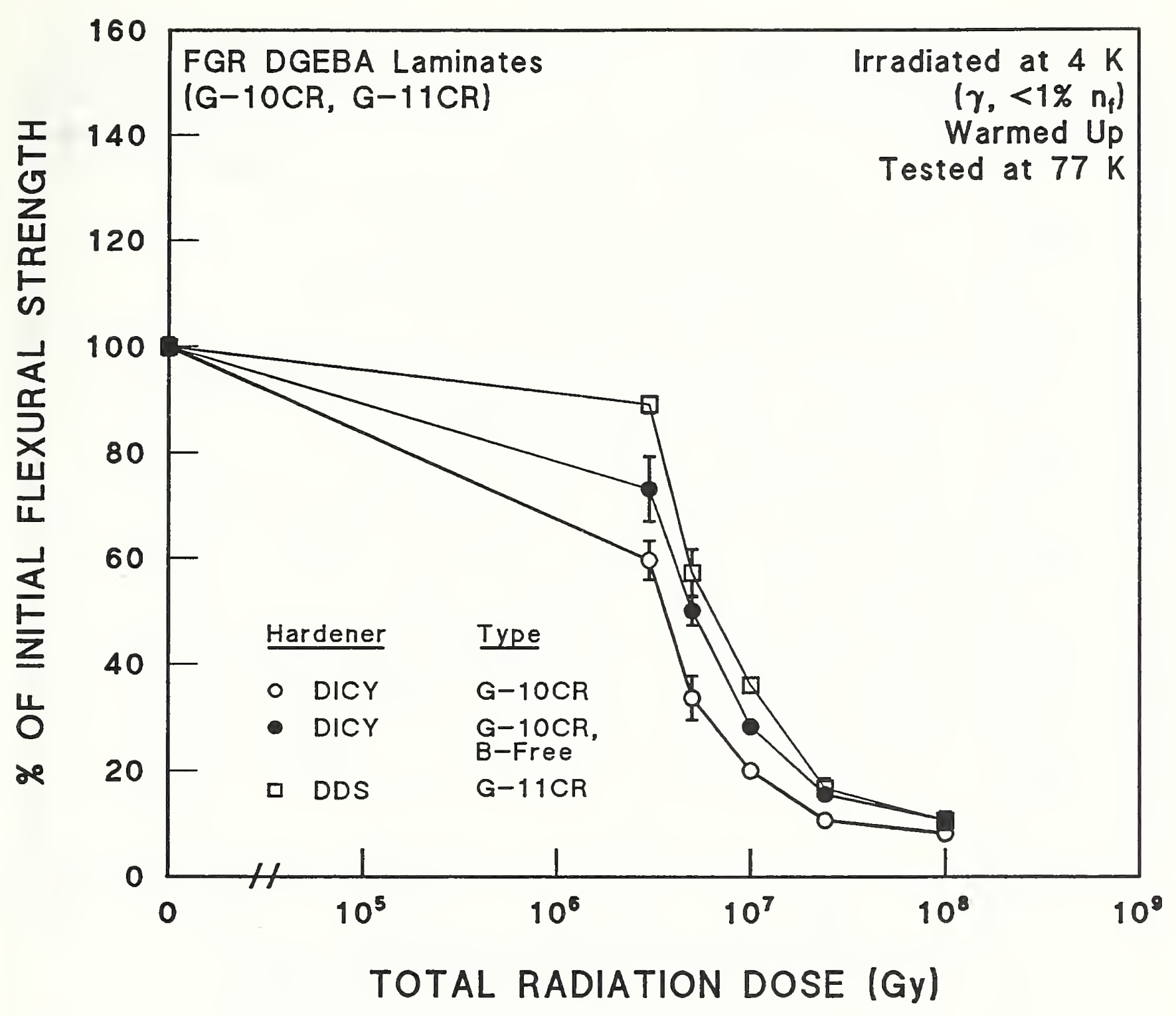

"O" Strength, MPa

- $1260.3 \pm 0.0$

- $1070.7 \pm 0.0$

$1171.6 \pm 18.8$
Glass Finish

Silane

Silane

Silane
Supplier

Spaulding

Spaulding

Spaulding

Figure A.8-11. Flexural strength at $77 \mathrm{~K}$ of FGR DGEBA laminates (G-10CR, G-10-11CR) after 4-K reactor irradiation. Supplementary Table A.8-2. Data from Coltman et al. [1979] and Coltman and Klabunde [1983]. 


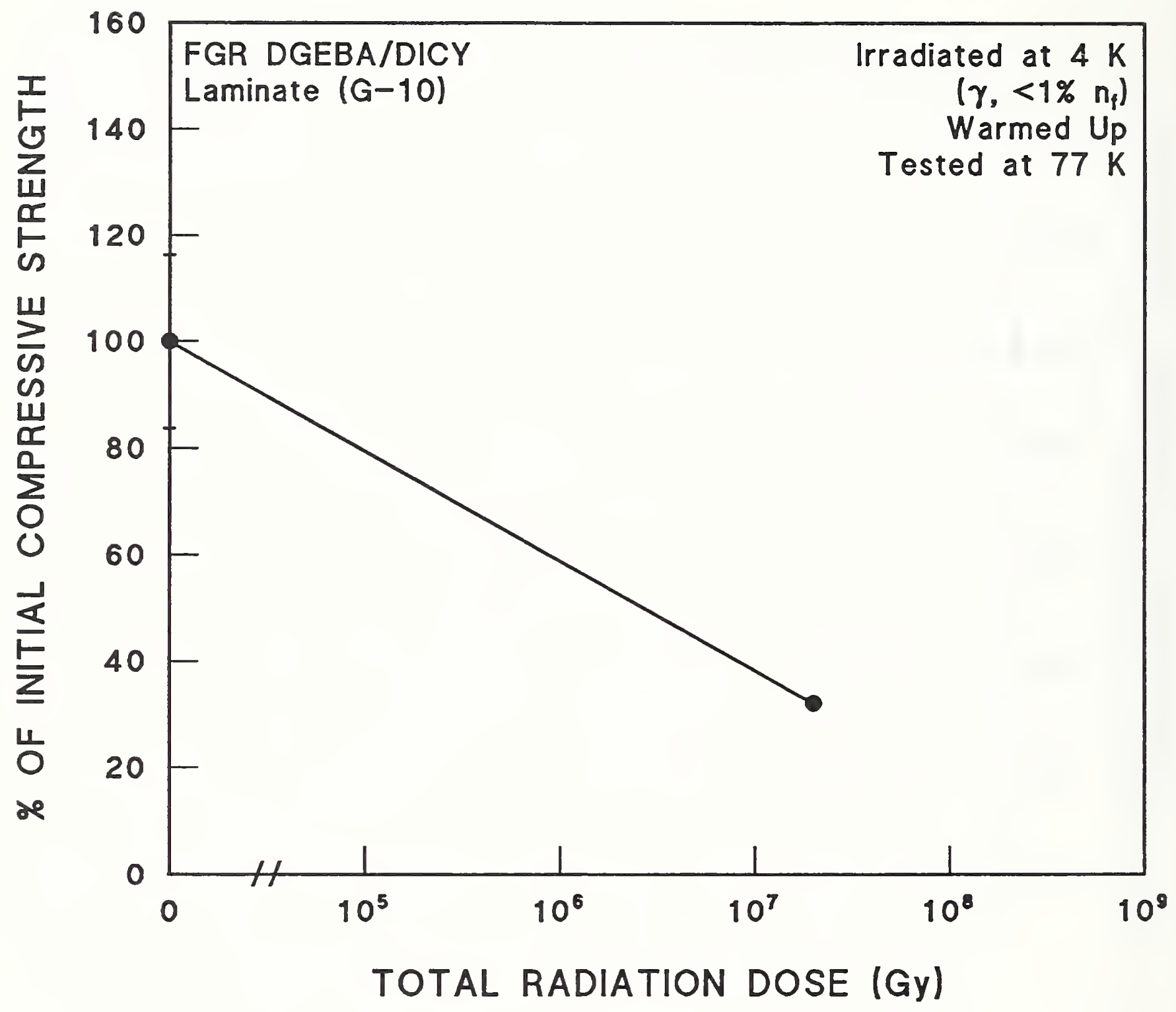

"O" Strength, MPa

- $719.5 \pm 117.5$
Supplier

Spaulding

Figure A.8-12. Compressive strength at $77 \mathrm{~K}$ of FGR DGEBA laminate (G-10) after 4-K reactor irradiation. Supplementary Table A.8-2. Data from Kernohan et al. [1979]. 


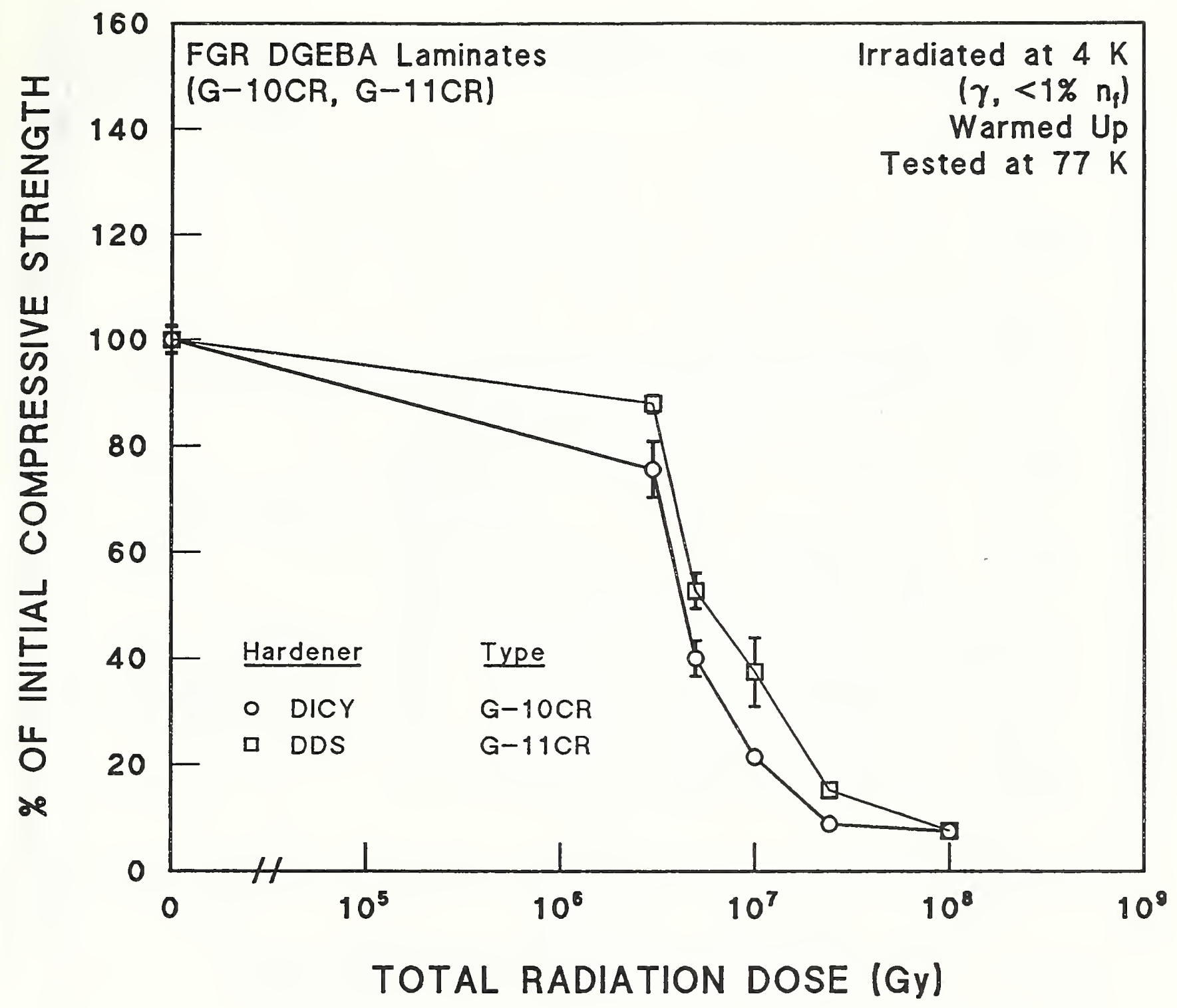

"O" Strength, MPa

- $899.5 \pm 21.2$

ㅁ $1008.5 \pm 27.1$
Glass Finish

Silane

Silane
Supplier

Spaulding

Spaulding

Figure A.8-13. Compressive strength at $77 \mathrm{~K}$ of FGR DGEBA laminates (G-10CR, G-10$11 C R$ ) after 4-K reactor irradiation. Supplementary Table A.8-2. Data from Coltman et al. [1979] and Coltman and Klabunde [1983]. 


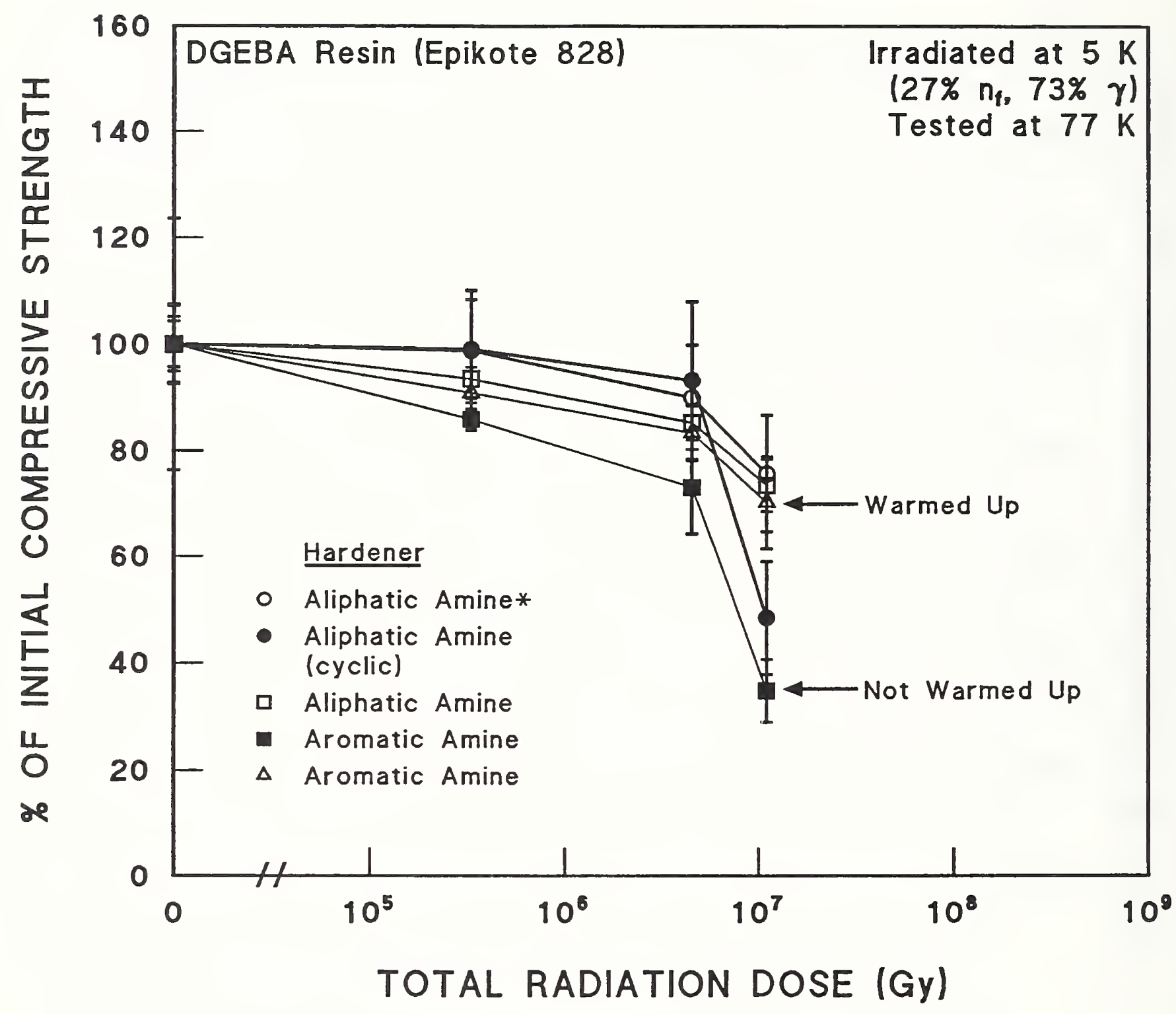

"O" Strength, MPa

- $301.7 \pm 12.9$

- $561.1 \pm 40.5$

ㅁ $565.1 \pm 42.2$

- $506.4 \pm 25.8$

$\Delta 504.4 \pm 119.0$

\section{Supplier}

Shell

Shell

Shell

Shell

Shell

* Filled

Figure A.8-14. Compressive strength at $77 \mathrm{~K}$ of DGEBA resin with various hardeners after 5-K reactor irradiation. Supplementary Table A.8-3. Data from Kato and Takamura [1979]. 


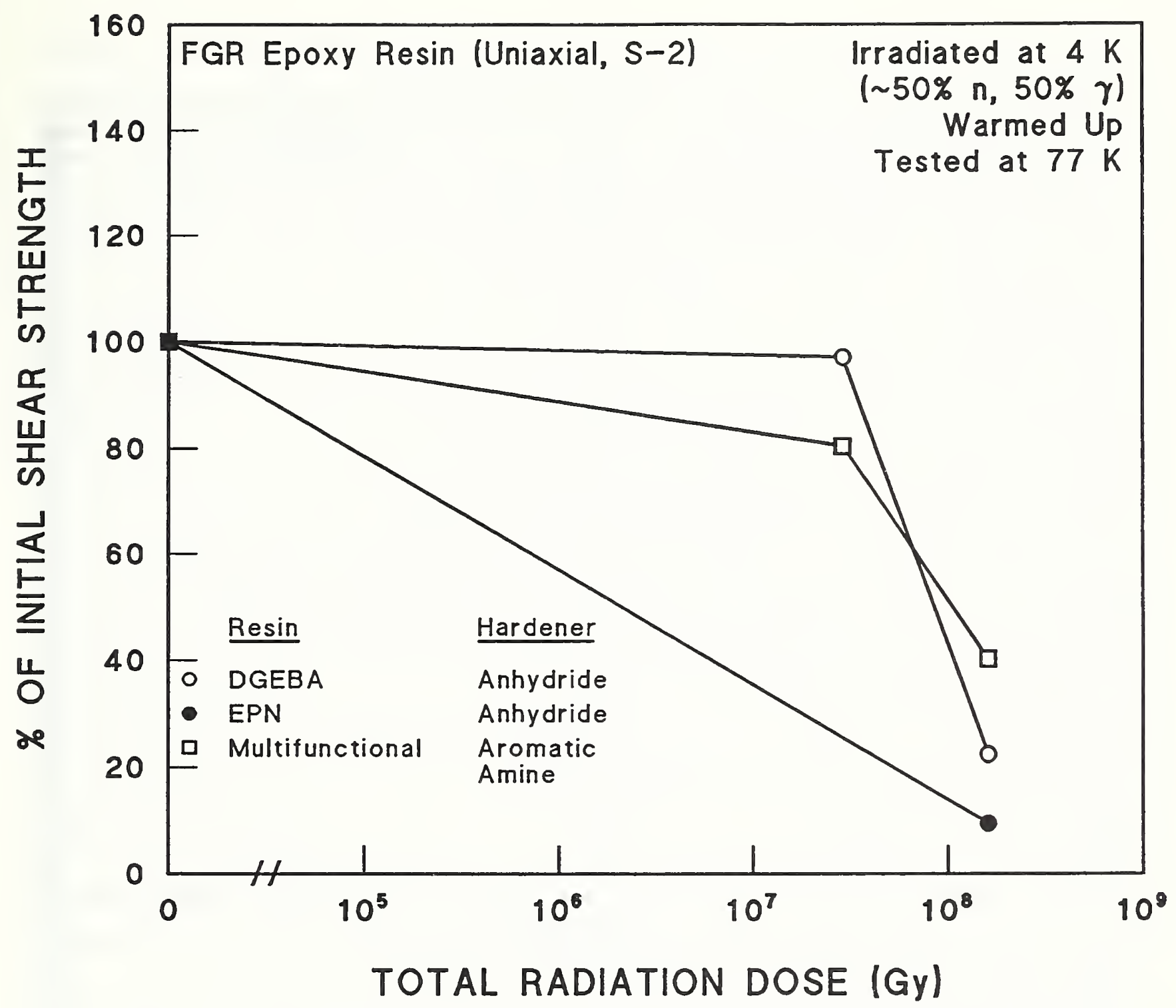

\begin{tabular}{|c|c|c|c|c|}
\hline "O" Strength, MPa & Resin Name & Accelerator & Flexibilizer & Supplier \\
\hline - 201.0 & CTD-101 & Yes & No & CTD, Inc. \\
\hline - 220.0 & CTD-102 & Yes & No & CTD, Inc. \\
\hline ㅁ 184.0 & CTD -112 & No & No & CTD, Inc. \\
\hline
\end{tabular}

Figure A.8-15. Shear strength at $77 \mathrm{~K}$ of FGR epoxy resins after 4-K reactor irradiation. Supplementary Table A.8-4. Data from Munshi [1991]. 
Table A.8-1. Supplementary Information, Hurley et al. [1978; 1983].

\begin{tabular}{|c|c|}
\hline IRRADIATION PARAMETERS & MATERIALS \\
\hline Source Type: Neutron Spallation & Resin: \\
\hline Source Name: IPNS (Argonne) & Hardener: \\
\hline $\begin{array}{l}\text { Irradiation } \\
\text { Temperature: } 4 \mathrm{~K}\end{array}$ & Additives: \\
\hline Environment: LHe & Glass Vol. \%: \\
\hline Neutron $\%:-85$ & Glass Mass ơ: \\
\hline Neutron Flux: & Glass Type: E \\
\hline Fast Neutron $8: 69 *(E>0.1 \mathrm{MeV})$ & Glass Finish: \\
\hline Fast Neutron Flux: & Glass Weave: \\
\hline Gamma $8:<15$ & Glass Areal Density, $\mathrm{kg} / \mathrm{m}^{2}$ : \\
\hline Gamma Energy: & Filament Diameter, $\mu \mathrm{m}$ : \\
\hline Gamma Dose Rate: & Layup: \\
\hline Electron $\%$ & Production Method: \\
\hline Electron Energy: & Other: \\
\hline \multicolumn{2}{|l|}{ Max. $n_{p}-1.5 \times 10^{21} / \mathrm{m}^{2}$} \\
\hline \multicolumn{2}{|l|}{ Other: * of total neutron fluence } \\
\hline \multicolumn{2}{|c|}{ TEST METHOD } \\
\hline \multicolumn{2}{|c|}{ Test Type: Flexural strength (Compressive strength) } \\
\hline \multicolumn{2}{|l|}{ Test Specifications: ASTM D790-80 } \\
\hline \multicolumn{2}{|l|}{ Specimen Shape: Rectangular bar } \\
\hline \multirow{2}{*}{\multicolumn{2}{|c|}{$\begin{array}{l}\text { Specimen Dimensions: } 25 \text { (span) } \times 3.2 \times 1.6 \mathrm{~mm}^{3}(12.8 \mathrm{~mm} \times 6.4-\mathrm{mm} \mathrm{di} \\
\text { Flexure: Span/Thickness Ratio: } 15.6\end{array}$}} \\
\hline & \\
\hline \multicolumn{2}{|c|}{ Compression: Aspect Ratio, Length/Width or Diam.: 2} \\
\hline \multicolumn{2}{|l|}{ Specimens Tested/Data Point: 3 (3) } \\
\hline \multicolumn{2}{|l|}{ Strain (Load) Rate: $0.13 \mathrm{~cm} / \mathrm{min}$} \\
\hline Test Temperature, $\mathrm{K}: 75,300$ & \\
\hline Other: Control specimens cooled in L & \\
\hline
\end{tabular}


Table A.8-2. Supplementary Information, Kernohan et al. [1979], Coltman et al. [1979], Coltman and Kalubunde [1983].

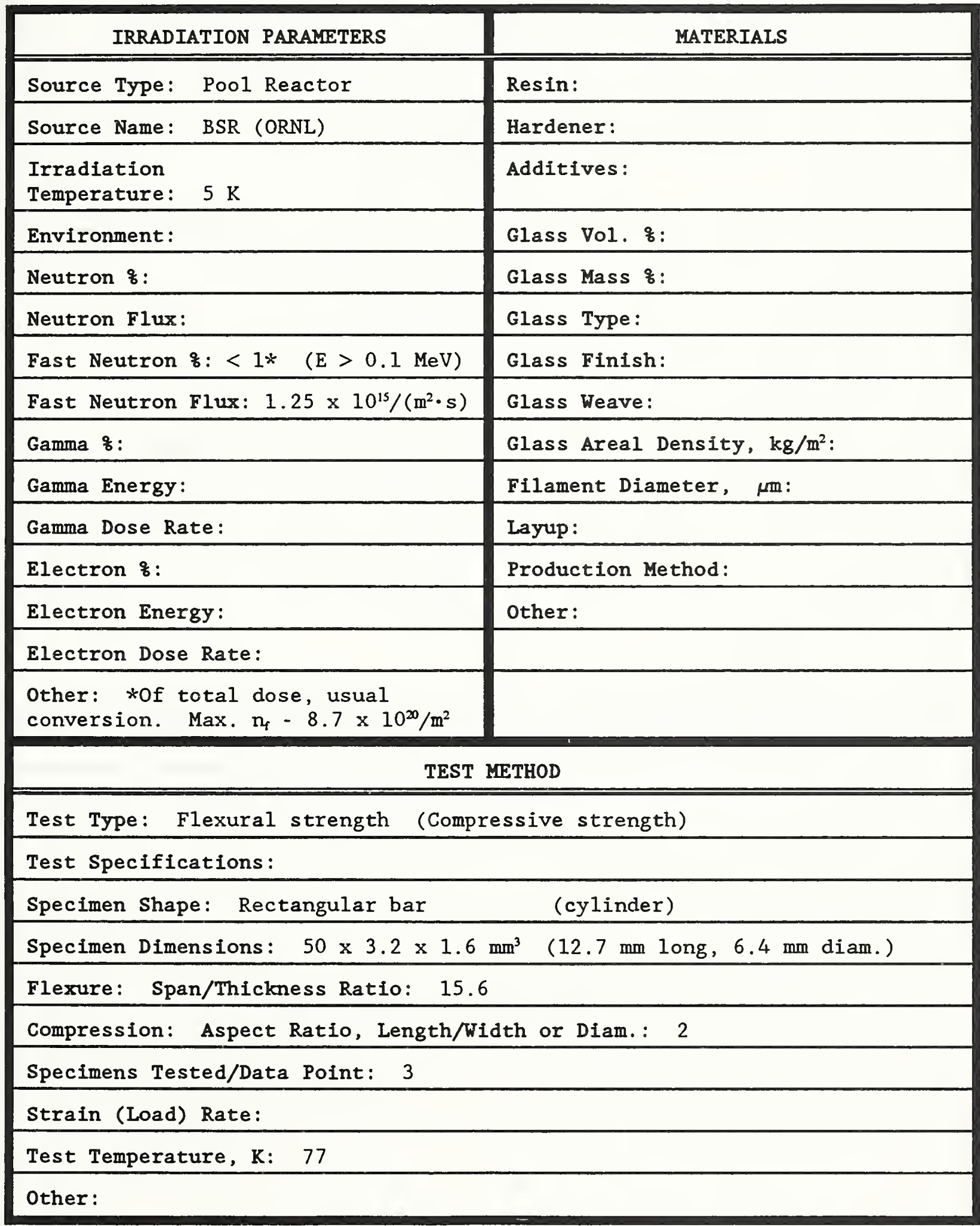


Table A.8-3. Supplementary Information, Kato and Takamura [1979], Takamura and Kato $[1979 ; 1980]$.

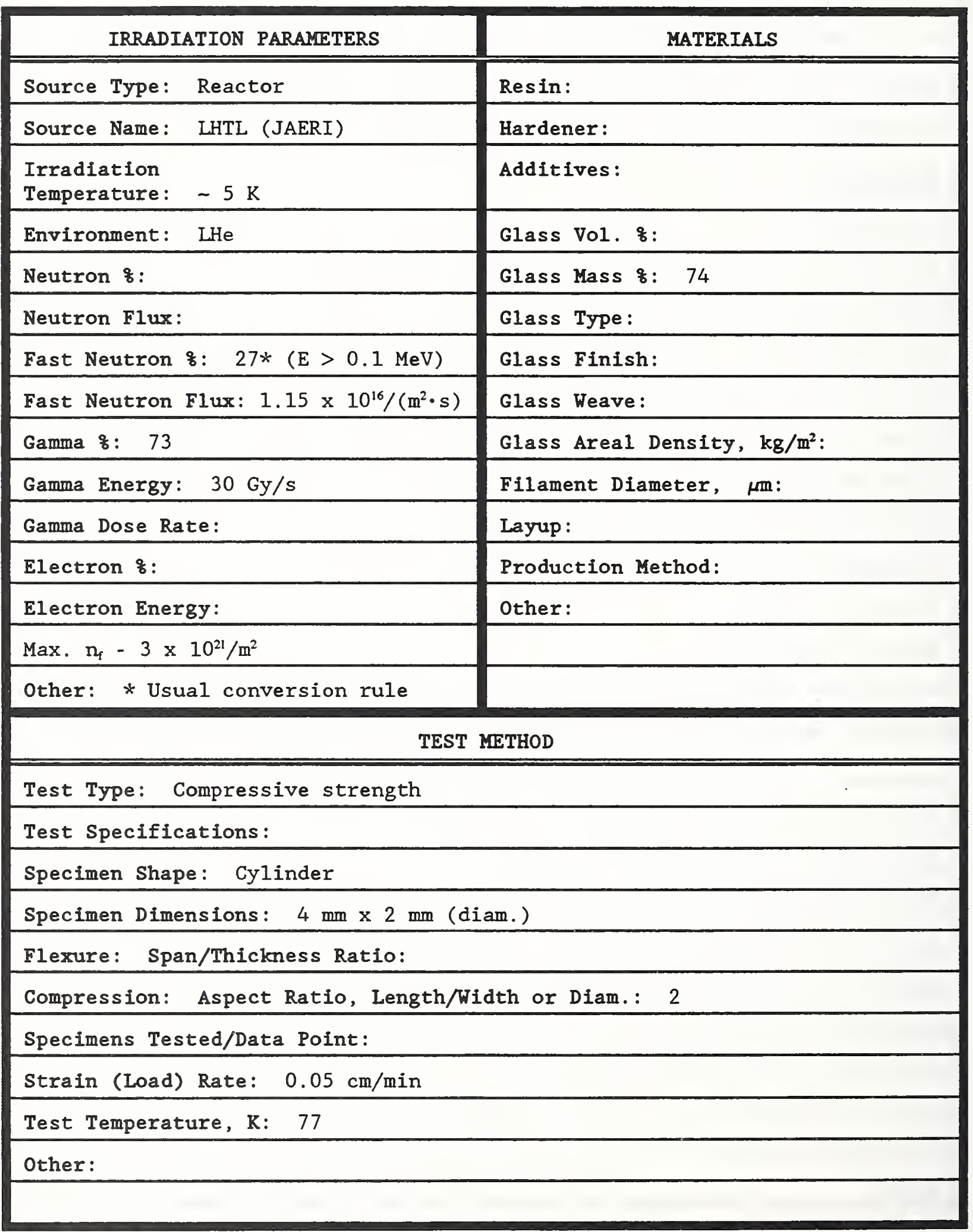


Table A.8-4. Supplementary Information, Munshi [1991; 1992] .

\begin{tabular}{|c|c|}
\hline IRRADIATION PARAMETERS & MATERIALS \\
\hline Source Type: Pool reactor & Resin: Same as Table A.3-3 \\
\hline Source Name: TTB, FRM (Munich) & Hardener: \\
\hline $\begin{array}{l}\text { Irradiation } \\
\text { Temperature: } \quad<6 \mathrm{~K}\end{array}$ & Additives: \\
\hline Environment: LHe & Glass Vol. \&: \\
\hline Neutron $8:-50 *$ & Glass Mass $8:$ \\
\hline Neutron Flux: & Glass Type: \\
\hline $\begin{aligned} \text { Fast Neutron } \%:-20 * \text { usual } \\
\text { conversion }(\mathrm{E}>0.1 \mathrm{MeV})\end{aligned}$ & Glass Finish: \\
\hline Fast Neutron Flux: $-2.9 \times 10^{15} /\left(\mathrm{m}^{2} \cdot \mathrm{s}\right)$ & Glass Weave: \\
\hline Gamma $\&: \quad-50 *$ & Glass Areal Density, $\mathrm{kg} / \mathrm{m}^{2}$ : \\
\hline Gamma Energy: & Filament Diameter, $\mu \mathrm{m}$ : \\
\hline Gamma Dose Rate: & Layup: \\
\hline Electron $q$ : & Production Method: \\
\hline Electron Energy: & Other: \\
\hline \multicolumn{2}{|l|}{ Electron Dose Rate: } \\
\hline \multicolumn{2}{|l|}{$\begin{array}{l}\text { Other: *based on Katheder et al. } \\
\text { [1992] }\end{array}$} \\
\hline \multicolumn{2}{|c|}{ TEST METHOD } \\
\hline \multicolumn{2}{|l|}{ Test Type: Same as Table A.3-3 } \\
\hline \multicolumn{2}{|l|}{ Test specifications: } \\
\hline \multicolumn{2}{|l|}{ Specimen Shape: } \\
\hline \multicolumn{2}{|l|}{ Specimen Dimensions: } \\
\hline \multicolumn{2}{|l|}{ Flexure: Span/Thickness Ratio: } \\
\hline \multicolumn{2}{|c|}{ Compression: Aspect Ratio, Length/Width or Diam.: } \\
\hline \multicolumn{2}{|l|}{ Specimens Tested/Data Point: } \\
\hline \multicolumn{2}{|l|}{ Strain (Load) Rate: } \\
\hline Test Temperature, $\mathrm{K}: \quad 77$ & \\
\hline Other: & \\
\hline
\end{tabular}





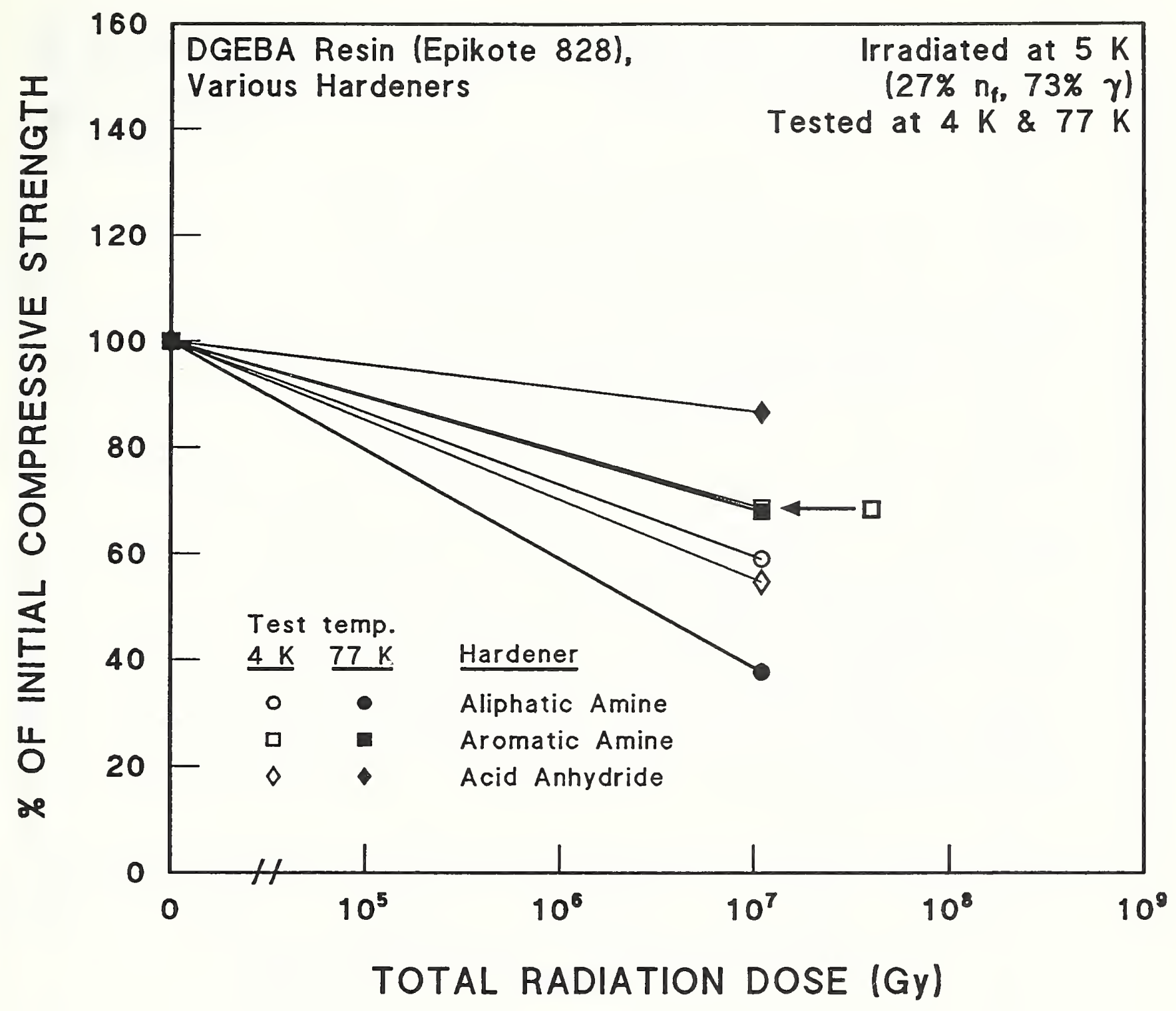

"O" Strength, MPa

- 500.7

- 532.3

ㅁ 461.6

- 529.7

○ 443.5

$4 \quad 405.7$
Supplier

Shell

Shell

Shell

Shell

Shell

Shell

Figure A.9-1. Compressive strength at 4 and $77 \mathrm{~K}$ of DGEBA resin with various hardeners after 5-K reactor irradiation. Supplementary Table A.8-3. Data from Takamura and Kato [1980]. 


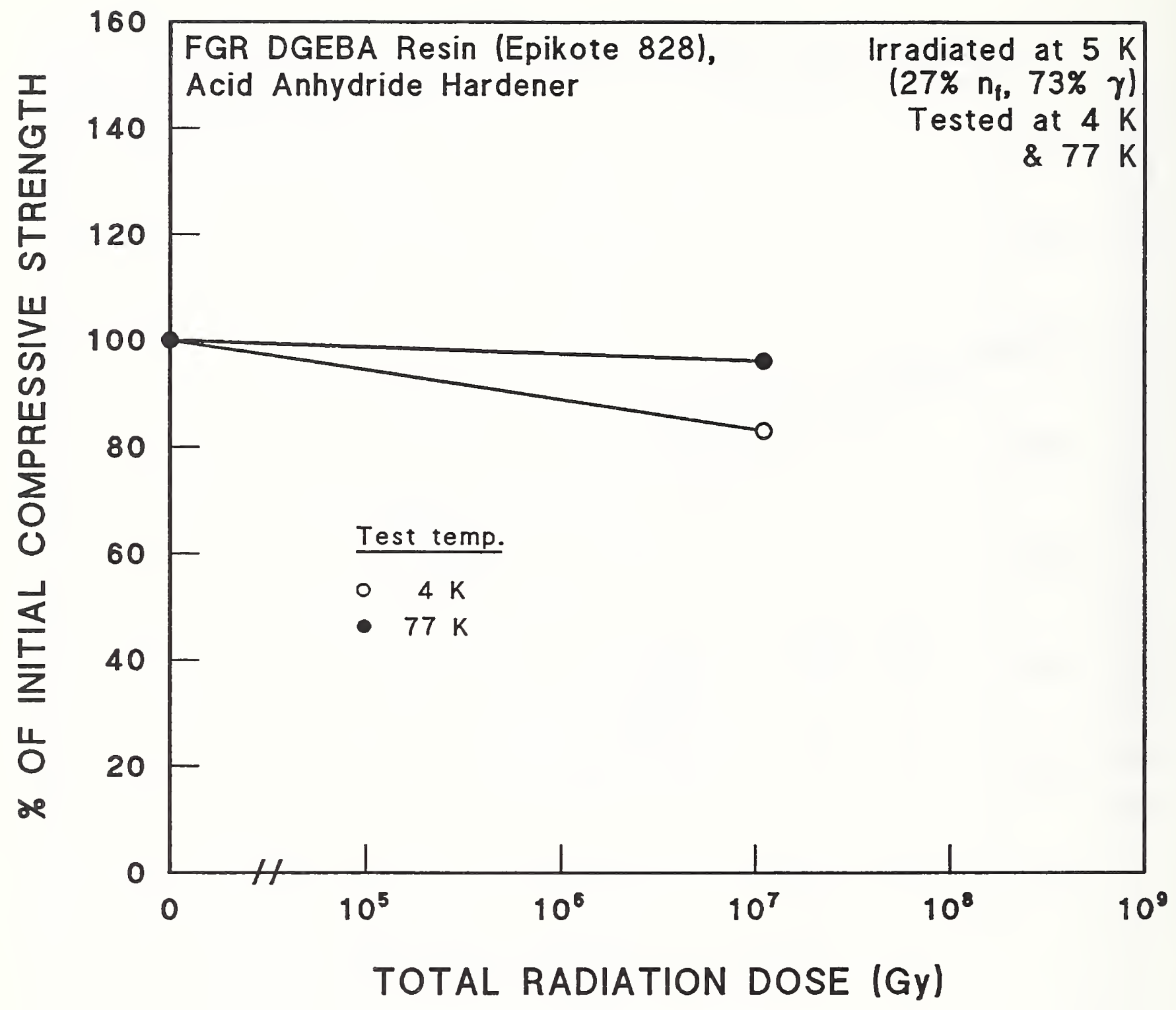

"O" Strength, MPa

- 685.6

- 673.2
Supplier

Shell

Shell

Figure A.9-2. Compressive strength at 4 and $77 \mathrm{~K}$ of FGR DGEBA resin with acid anhydride hardener after 5-K reactor irradiation. Supplementary Table A.8-3. Data from Takamura and Kato [1980]. 


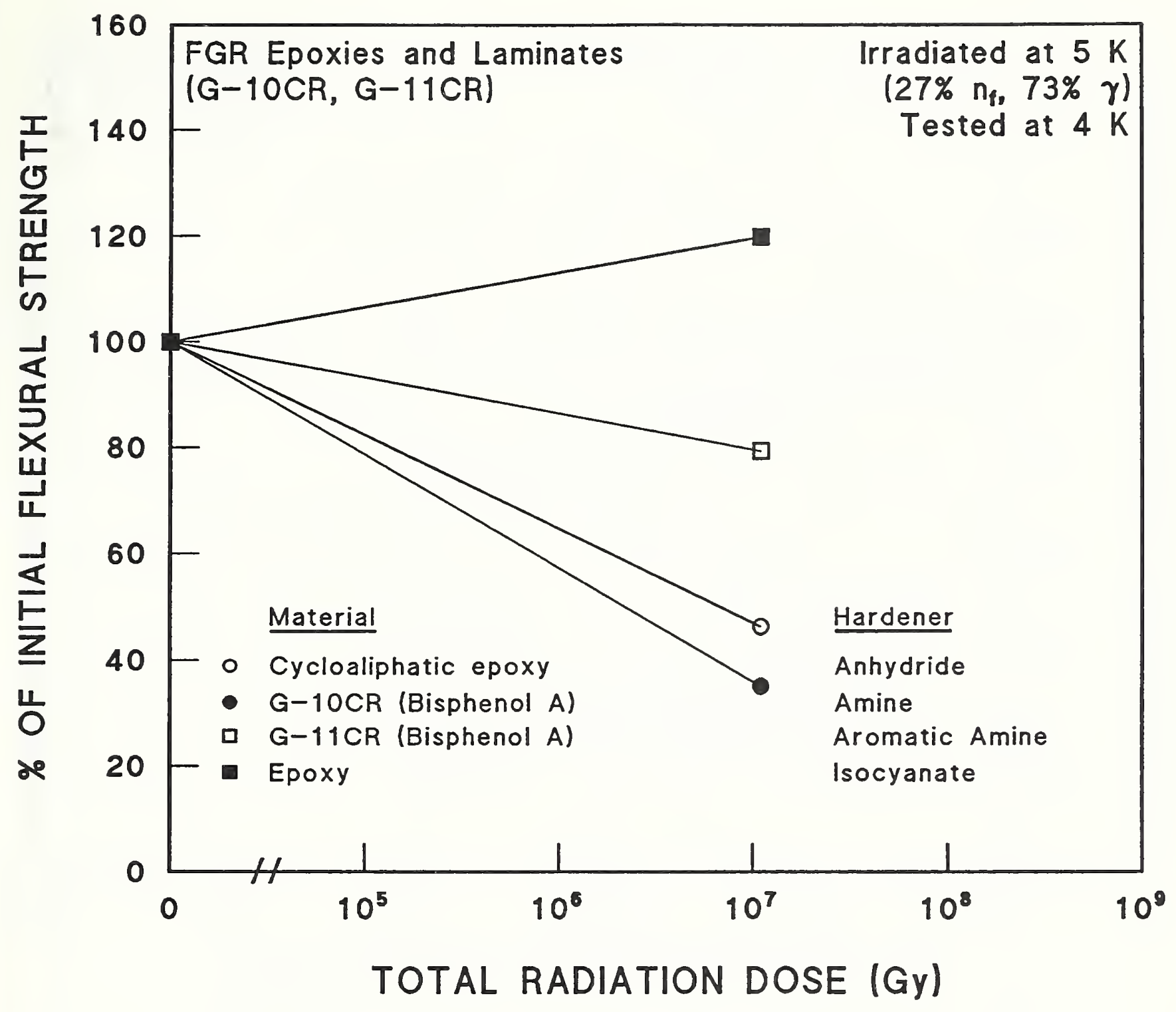

"O" Strength, $\mathrm{MPa}$

○ 1077.4

- 1073.2

ㅁ 887.6

- $440.0 * *$
Supplier *

--

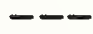

---

* Some specimens supplied by Kobayashi Kigata, Ltd. and Hitachi, Ltd.

** ISOX

Figure A.9-3. Flexural strength at $4 \mathrm{~K}$ of FGR epoxies and laminates (including G$10 C R$ and $G-11 C R$ and isocyanate hardener) after 5-K reactor irradiation.

Supplementary Table A.9-1. Data from Takamura and Kato [1984]. 


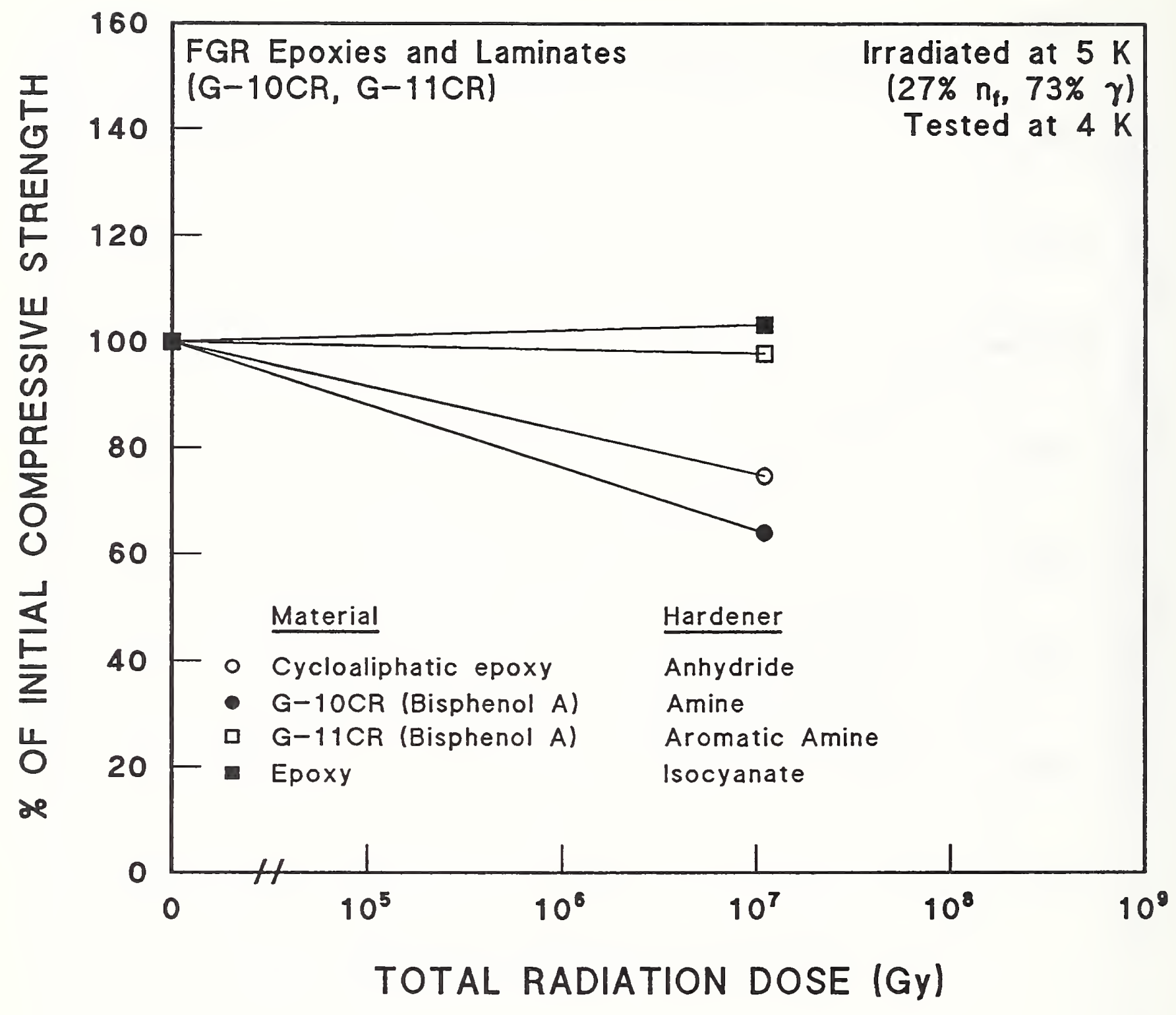

"O" Strength, MPa

- 850.7

- 898.2

ㅁ 1063.7

- $790.0 * *$
Supplier *

-- radiated at $5 \mathrm{~K}$ Tested at $4 \mathrm{~K}$ 


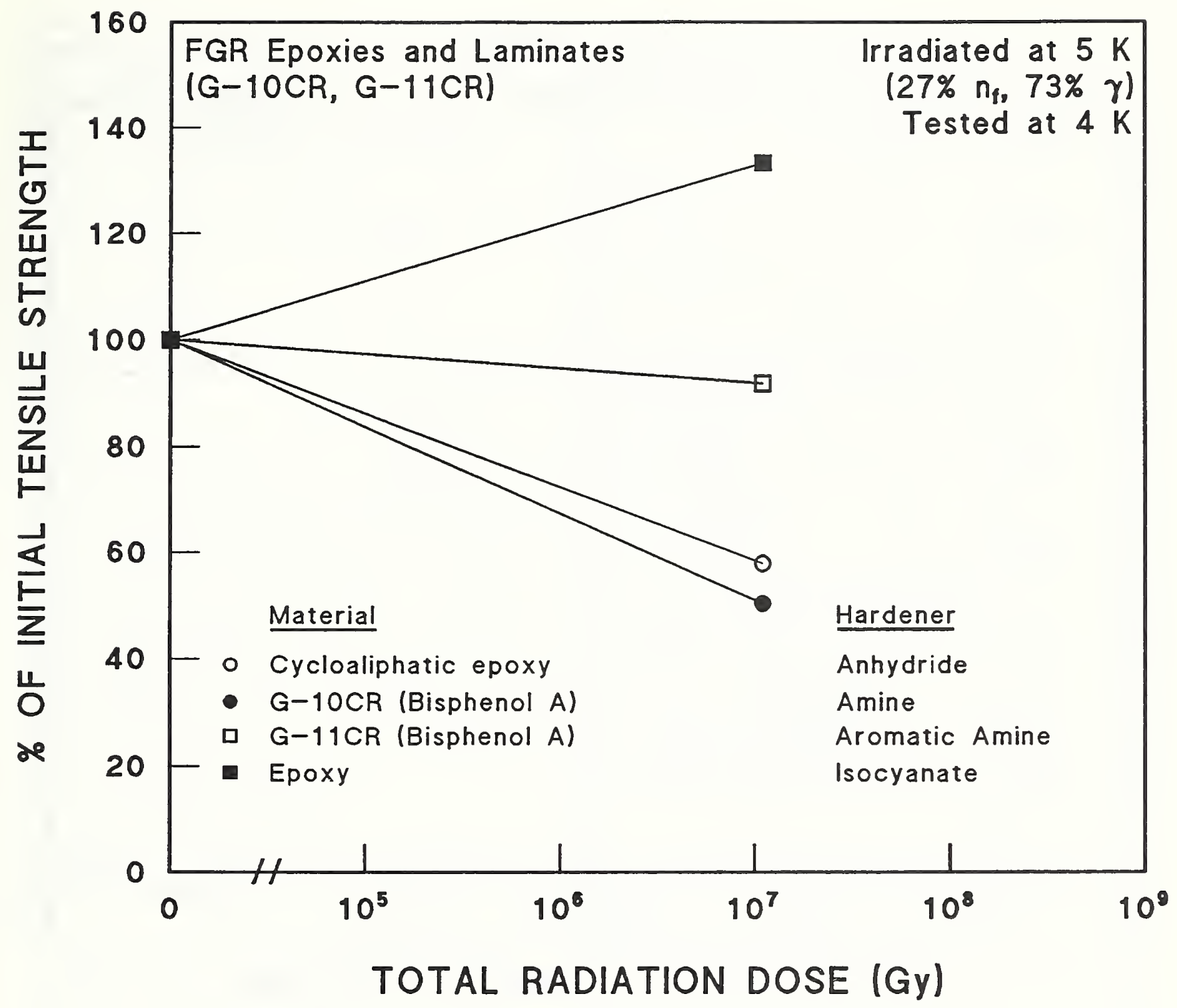

"O" Strength, MPa

- 839.9

- 833.0

$\quad 515.4$
Supplier *

$---$

$---$

-ー-

\footnotetext{
- $255.0 * *$

* Some specimens supplied by Kobayashi Kigata, Ltd. and Hitachi, Ltd. ** ISOX
}

Figure A.9-5. Tensile strength at $4 \mathrm{~K}$ of FGR epoxies and laminates (including G$10 C R$ and $G-11 C R$ and isocyanate hardener) after $5-K$ reactor irradiation.

Supplementary Table A.9-1. Data from Takamura and Kato [1984]. 


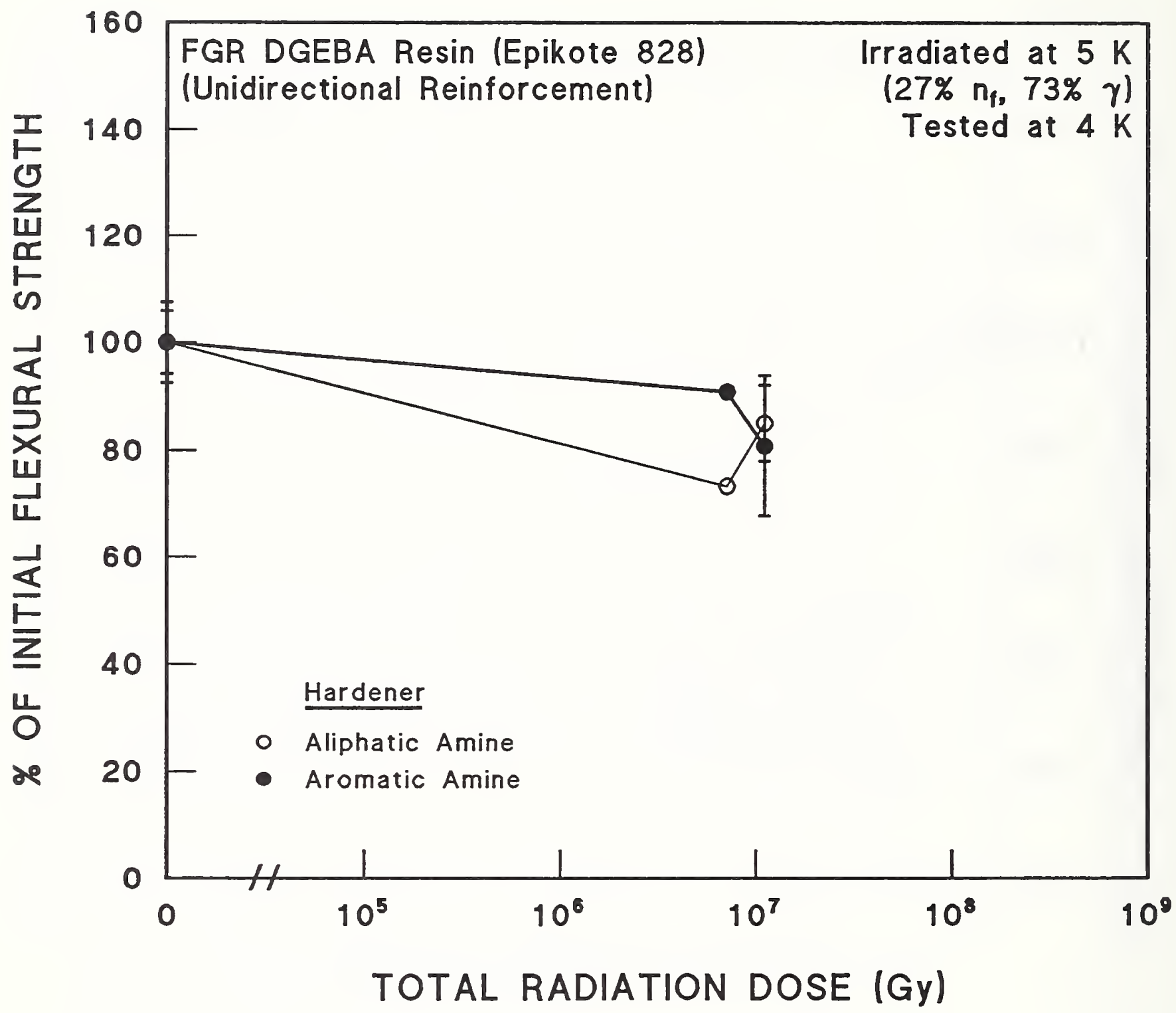

$$
\begin{aligned}
& \text { "O" Strength, MPa } \\
& \hline \text { - } 1849.6 \pm 105.9 \\
& \text { - } 1392.5 \pm 80.9
\end{aligned}
$$

Supplier *

* Some specimens supplied by Kobayashi Kigata, Ltd. and Hitachi, Ltd.

Figure A.9-6. Flexural strength at $4 \mathrm{~K}$ of FGR DGEBA resin with two hardeners after 5-K reactor irradiation. Supplementary Table A.9-1. Data from Takamura and Kato $[1981 ; 1984]$. 
Table A.9-1. Supplementary Information, Takamura and Kato [1984].

\begin{tabular}{|c|c|}
\hline IRRADIATION PARAMETERS & MATERIALS \\
\hline Source Type: Reactor & Resin: \\
\hline Source Name: LHTL (JAERI) & Hardener: \\
\hline $\begin{array}{l}\text { Irradiation } \\
\text { Temperature: } \quad-5 \mathrm{~K}\end{array}$ & Additives: \\
\hline Environment: LHe & Glass Vol. $8:$ : $67-70$ \\
\hline Neutron $q$ : & Glass Mass o: : \\
\hline Neutron F1ux: & Glass Type: \\
\hline Fast Neutron $8: 27 \quad(E>0.1 \mathrm{MeV})$ & Glass Finish: \\
\hline Fast Neutron Flux: $1.15 \times 10^{16} /\left(\mathrm{m}^{2} \cdot \mathrm{s}\right)$ & Glass Weave: \\
\hline Gamma $8: \quad 73$ & Glass Areal Density, $\mathrm{kg} / \mathrm{m}^{2}$ : \\
\hline Gamma Energy: & Filament Diameter, $\mu \mathrm{m}:$ \\
\hline Gamma Dose Rate: $30 \mathrm{~Gy} / \mathrm{s}$ & Layup: \\
\hline Electron के: & Production Method: \\
\hline Electron Energy: & Other: \\
\hline \multicolumn{2}{|l|}{ Electron Dose Rate: } \\
\hline \multicolumn{2}{|l|}{ Other: $\quad$ Max. $\mathrm{n}_{\mathrm{f}}-2.9 \times 10^{21} / \mathrm{m}^{2}$} \\
\hline \multicolumn{2}{|c|}{$\begin{array}{r}\text { TEST METHOD } \\
\end{array}$} \\
\hline \multicolumn{2}{|c|}{ Test Type: Flexural strength (Tensile strength) * } \\
\hline \multicolumn{2}{|l|}{ Test Specifications: } \\
\hline \multicolumn{2}{|c|}{ Specimen Shape: Rectangular bar (Dumbe11) } \\
\hline \multicolumn{2}{|c|}{ Specimen Dimensions: $21 \times 2 \times 2 \mathrm{~mm}^{3}\left(18 \times 5 \times 2 \mathrm{~mm}^{3}\right.$; gage $\left.10 \mathrm{~mm}\right)$} \\
\hline \multicolumn{2}{|l|}{ Flexure: Span/Thickness Ratio: 8.5} \\
\hline \multicolumn{2}{|c|}{ Compression: Aspect Ratio, Length/Width or Diam.: } \\
\hline \multicolumn{2}{|l|}{ Specimens Tested/Data Point: } \\
\hline \multicolumn{2}{|l|}{ Strain (Load) Rate: $0.05 \mathrm{~cm} / \mathrm{min}$} \\
\hline \multicolumn{2}{|l|}{ Test Temperature, $\mathrm{K}: 4$} \\
\hline Other: *See Table A.8-3 for compres & ive strength \\
\hline
\end{tabular}




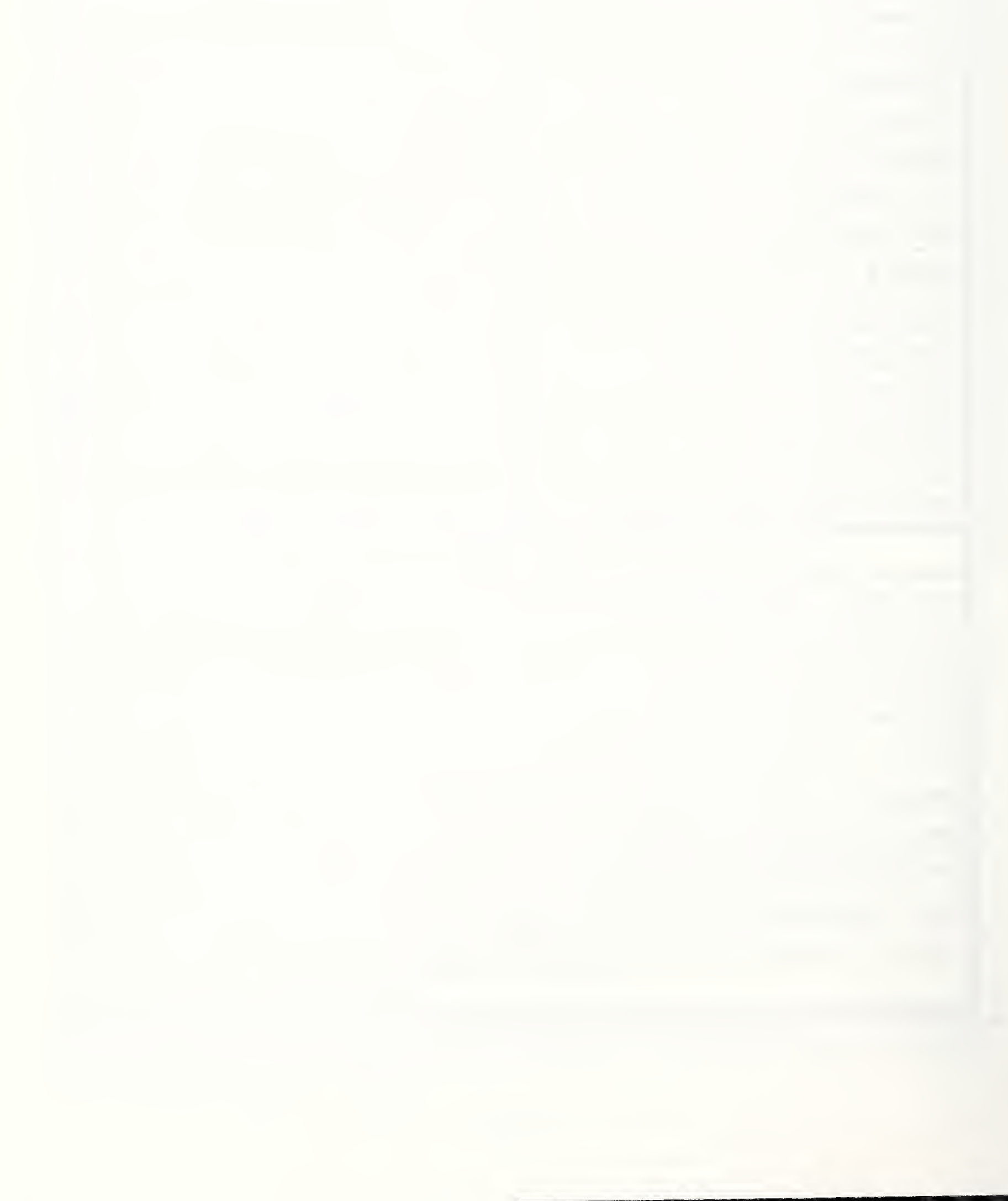




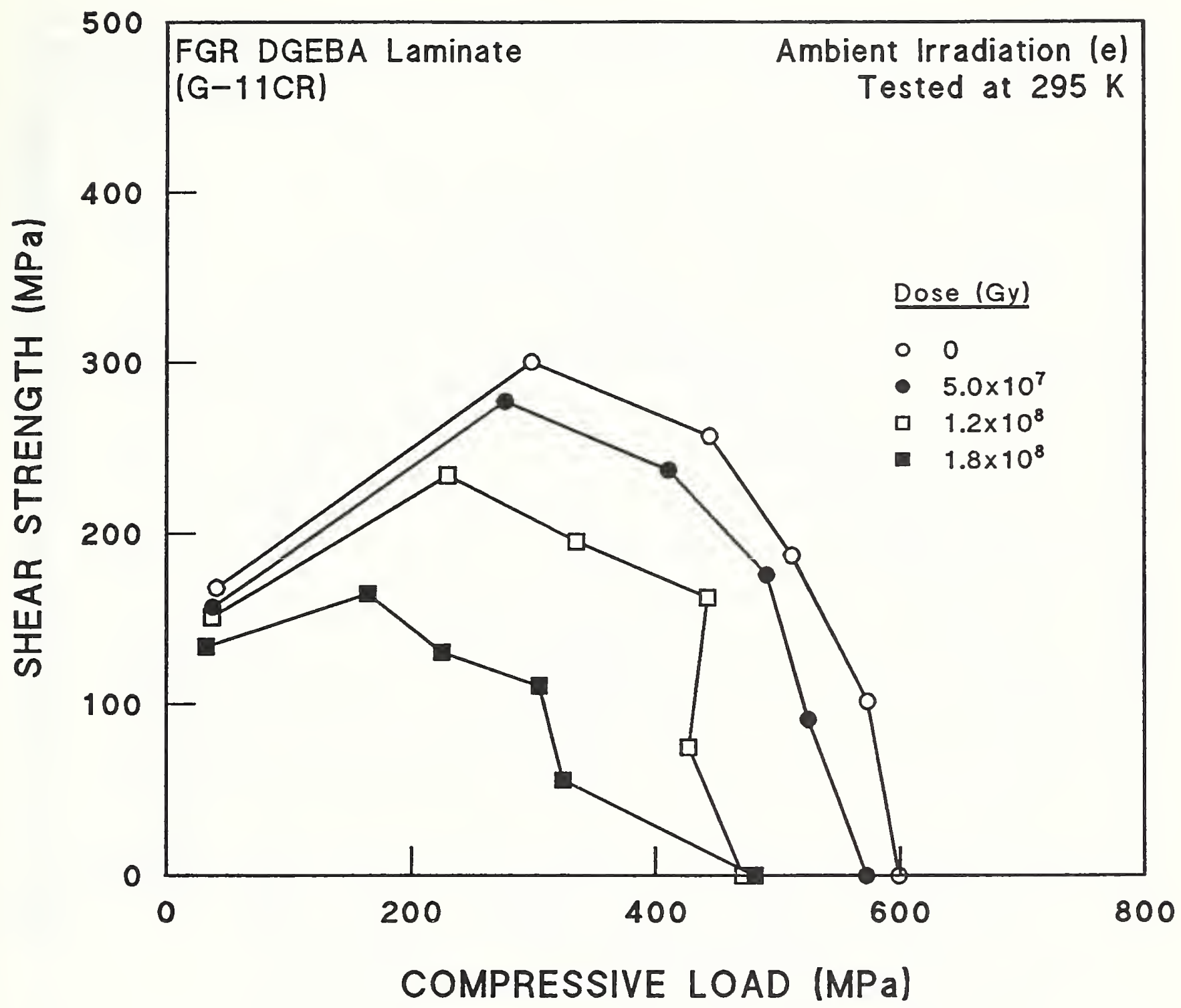

Weave Type

- 2 D

- $2 \mathrm{D}$

口 $2 \mathrm{D}$

- $2 \mathrm{D}$
Supplier

- -

- -

---

Figure A.10-1. Shear/compression failure envelope at $295 \mathrm{~K}$ of FGR DGEBA laminate (G-10CR) after ambient electron irradiation. Supplementary Table A.10-1. Data from Nishijima et al. [1990]. 


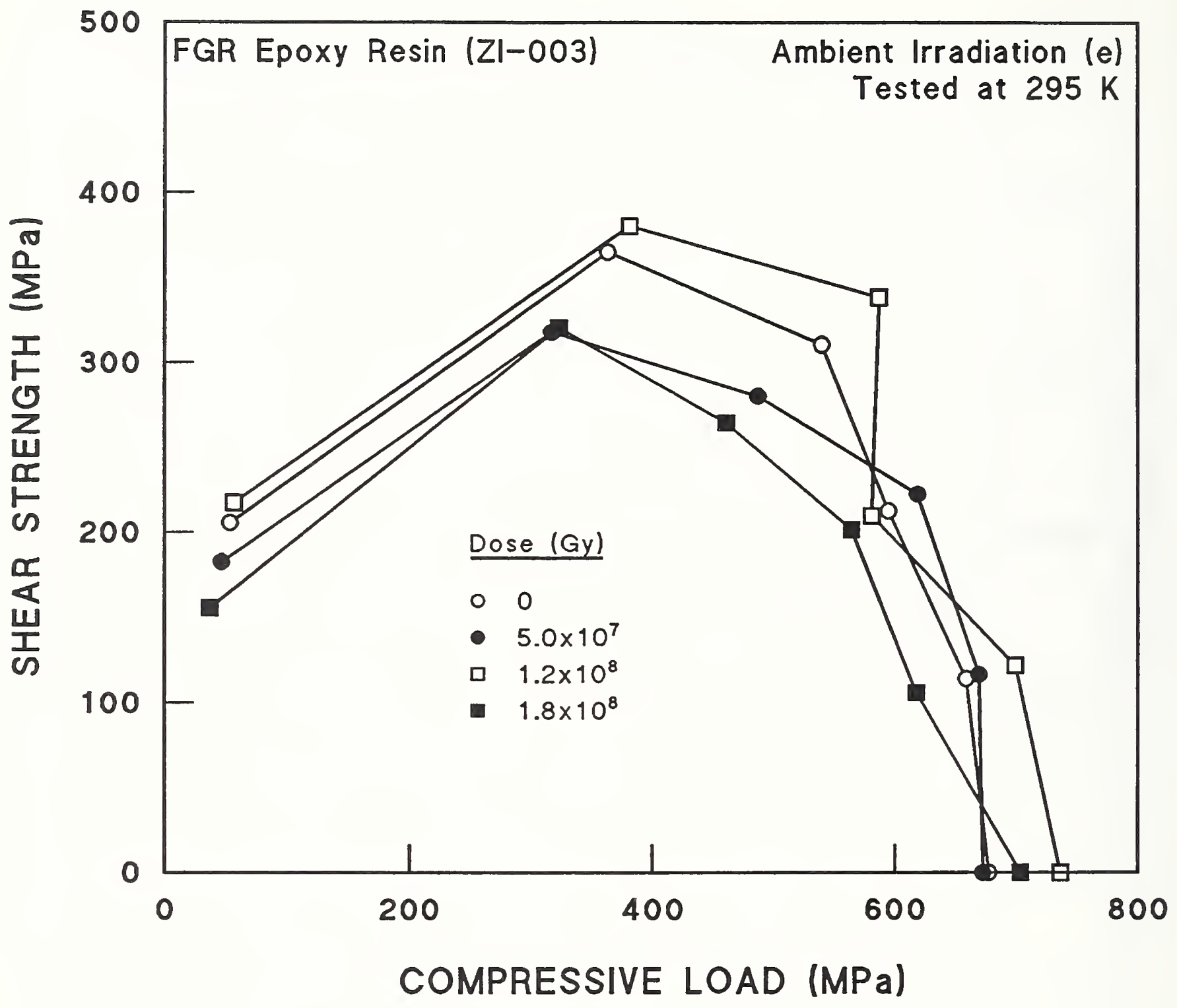

Weave Type

- 3 D

- $3 \mathrm{D}$

$3 \mathrm{D}$

- $3 D$
Supplier

Shikishima Canvas Co.

Shikishima Canvas Co.

Shikishima Canvas Co.

Shikishima Canvas Co.

Figure A.10-2. Shear/compression failure envelope at $295 \mathrm{~K}$ of FGR epoxy resin after ambient electron irradiation. Supplementary Table A.10-1. Data from Nishijima et al. [1990] . 


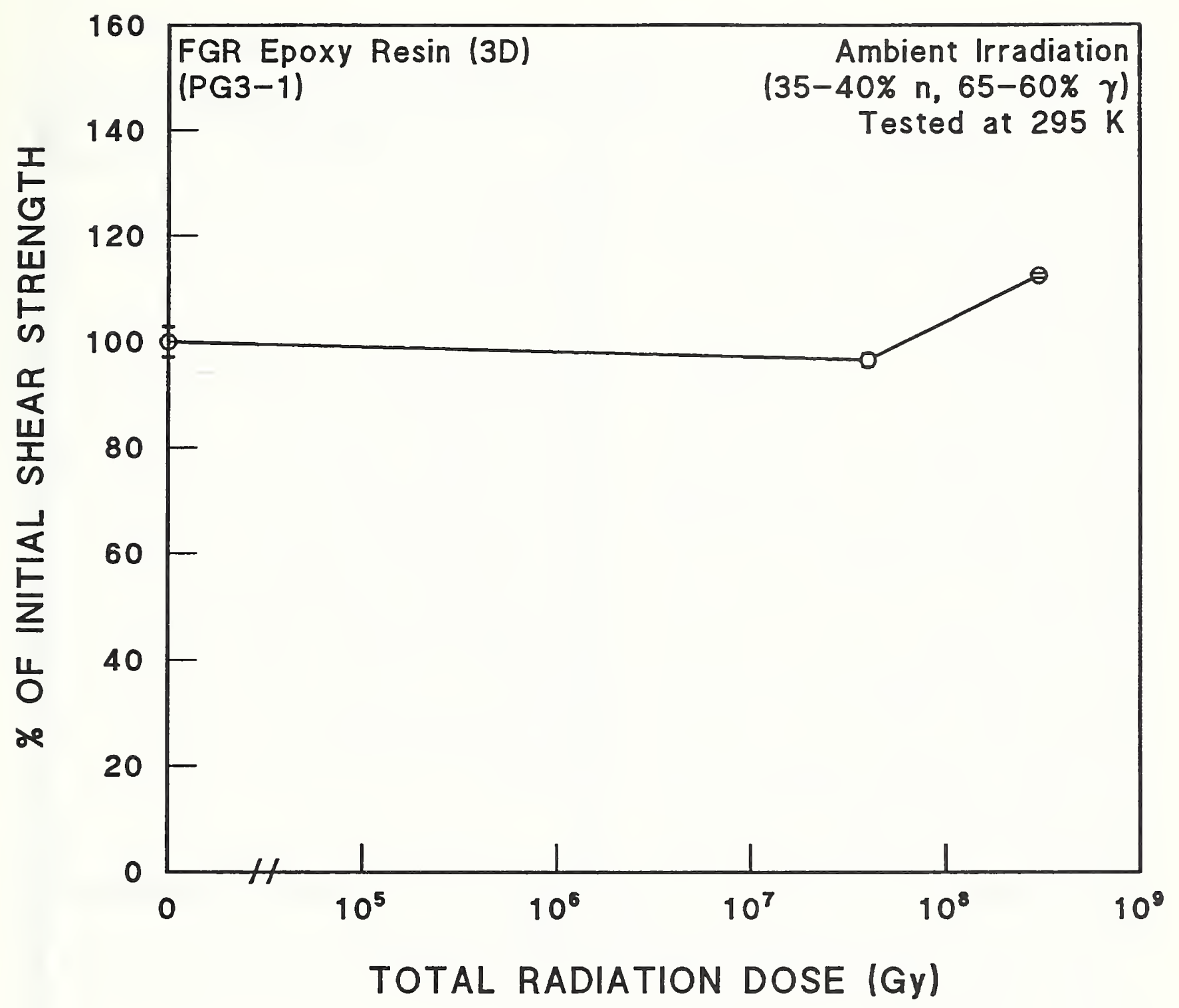

\begin{tabular}{|c|c|}
\hline "O" Strength, MPa & Supplier \\
\hline o $\quad 123.0 \pm 3.5$ & $\begin{array}{l}\text { Shikishima } \\
\text { Canvas Co. }\end{array}$ \\
\hline
\end{tabular}

Figure A.10-3. Shear strength with compressive load of $345 \mathrm{Mpa}$ at $295 \mathrm{~K}$ of FGR (3D) epoxy resin after ambient reactor irradiation. Supplementary Table A.2-17. Data from McManamy et al. [1990; 1991a]. 
Table A.10-1. Supplementary Information, Nishijima et al. [1990].

\begin{tabular}{|c|c|}
\hline IRRADIATION PARAMETERS & MATERIALS \\
\hline Source Type: Electron & Resin: \\
\hline Source Name: ISIR & Hardener: \\
\hline $\begin{array}{l}\text { Irradiation } \\
\text { Temperature: } 10^{\circ} \mathrm{C}\end{array}$ & Additives: \\
\hline Environment: & Glass Vol. of: \\
\hline Neutron 8 : & Glass Mass ơ: \\
\hline Neutron Flux: & Glass Type: \\
\hline Fast Neutron 8 & Glass Finish: \\
\hline Fast Neutron Flux: & Glass Weave: $3 \mathrm{D}(\mathrm{ZI})$ \\
\hline Gamma 8 : & Glass Areal Density, $\mathrm{kg} / \mathrm{m}^{2}$ : \\
\hline Gamma Energy: & Filament Diameter, $\mu \mathrm{m}$ : \\
\hline Gamma Dose Rate: & Layup: \\
\hline Electron \&: 100 & Production Method: \\
\hline Electron Energy: $20 \mathrm{MeV}$ & Other: \\
\hline Electron Dose Rate: $2.8 \mathrm{k} \mathrm{Gy} / \mathrm{s}$ & \\
\hline \multicolumn{2}{|l|}{ Other: } \\
\hline \multicolumn{2}{|c|}{ TEST METHOD } \\
\hline \multicolumn{2}{|l|}{ Test Type: Shear/compression } \\
\hline \multicolumn{2}{|c|}{ Test Specifications: V-shaped compressive jig } \\
\hline \multicolumn{2}{|l|}{ Specimen Shape: } \\
\hline \multicolumn{2}{|l|}{ Specimen Dimensions: 1 -mm thick } \\
\hline \multicolumn{2}{|l|}{ Flexure: Span/Thickness Ratio: } \\
\hline \multicolumn{2}{|c|}{ Compression: Aspect Ratio, Length/Width or Diam.: } \\
\hline \multicolumn{2}{|l|}{ Specimens Tested/Data Point: } \\
\hline \multicolumn{2}{|l|}{ Strain (Load) Rate: } \\
\hline Test Temperature, $\mathrm{K}: 295$ & \\
\hline other: & \\
\hline
\end{tabular}




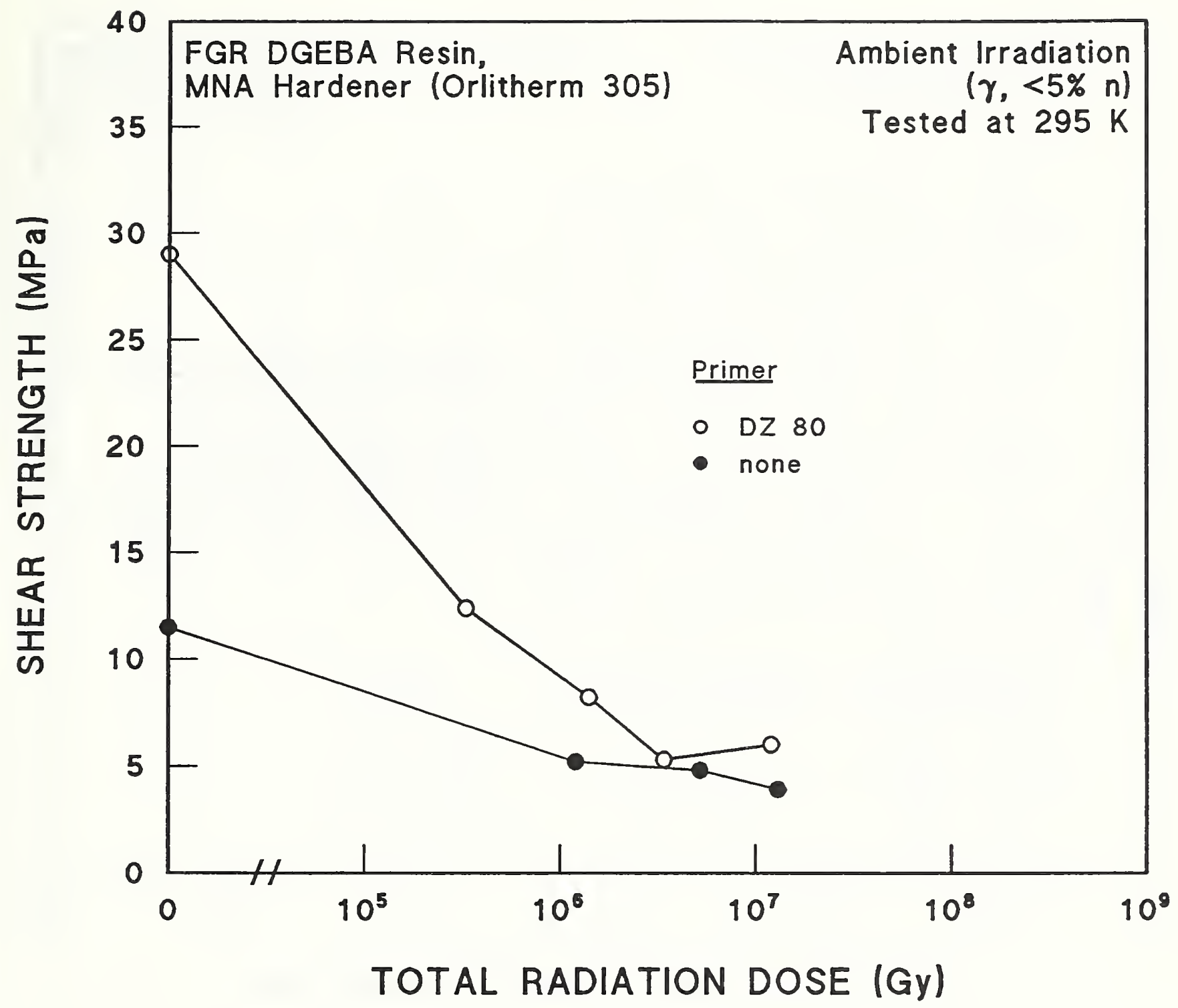

Supplier

- BBC, Baden

- BBC, Baden

Comments: Copper Substrate

Figure A.11-1. Shear strength at $295 \mathrm{~K}$ of FGR DGEBA resin (Orlitherm) to copper substrate, with and without primer, after ambient reactor irradiation.

Supplementary Table A.2-7. Data from Lipták et al. [1985]. 


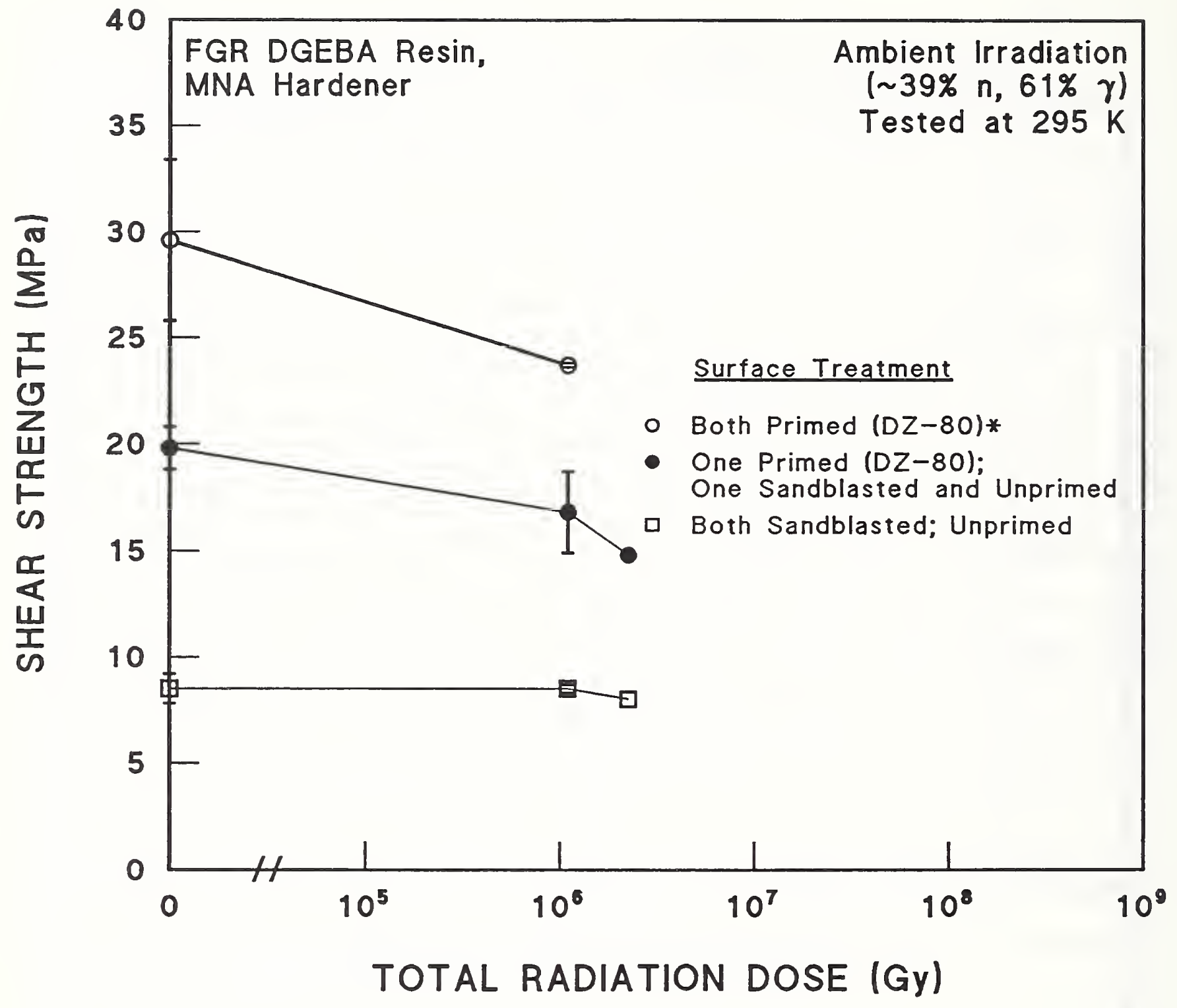

\begin{tabular}{lll} 
Resin Name & Accelerator & Supplier \\
\hline No. 332 & BDMA & DOW \\
- No. 332 & BDMA & DOW \\
№. 332 & BDMA & DOW \\
* Ciba-Geigy & & \\
Comments: Copper Subtrate &
\end{tabular}

Figure A.11-2. Shear strength at $295 \mathrm{~K}$ of FGR DGEBA resin to copper substrate, with and without primer and sandblasting, after ambient reactor irradiation.

Supplementary Table A.2-8. Data from Imel et al. [1979]. Total dose estimated from conversion provided by Imel et al. and aim glass fraction. 


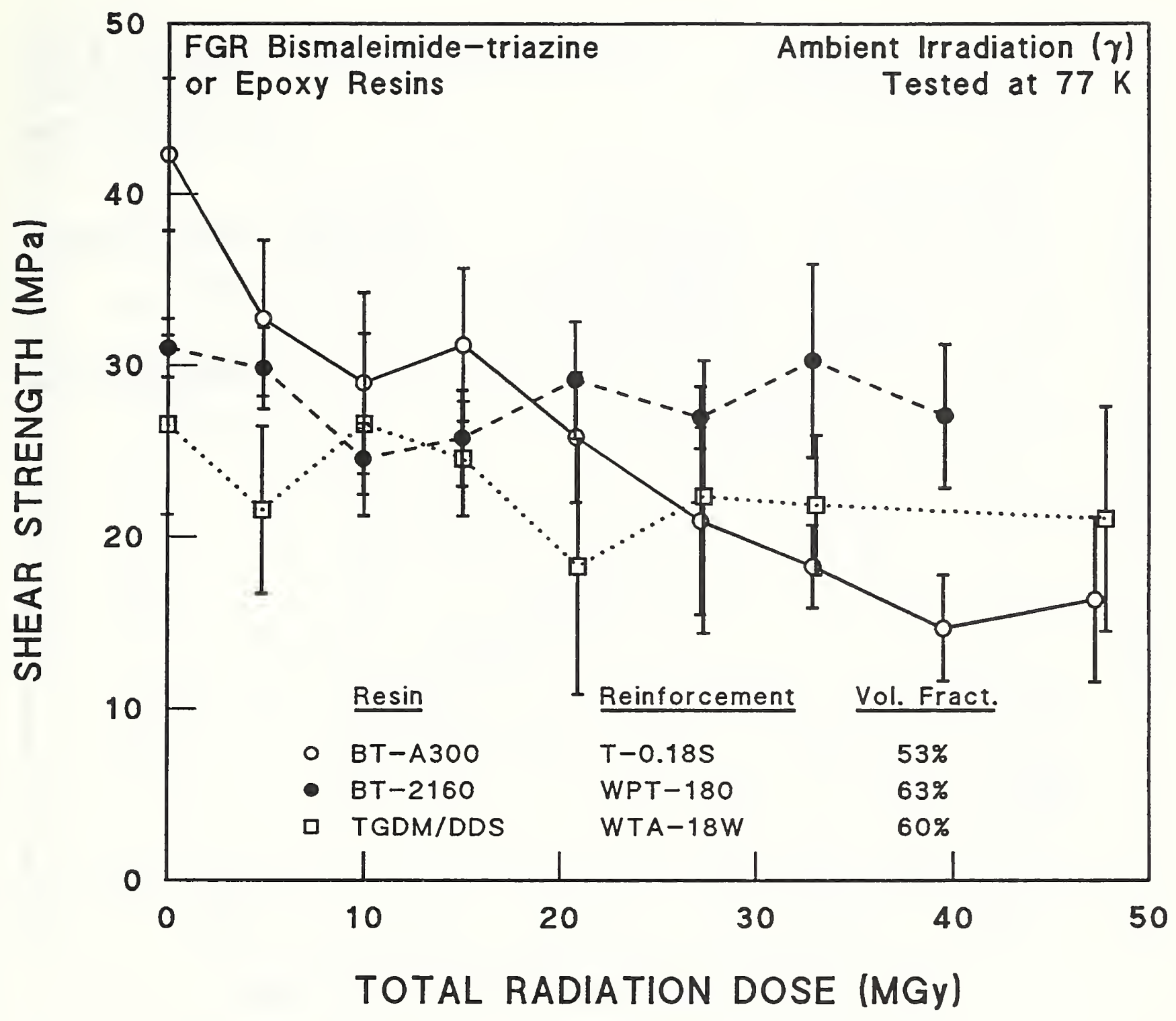

\section{Weave Style}

- Satin

- Satin

口 Plain
No. of Plies

3

3

5

\section{Supplier}

Arisawa Mfg.

Hitachi Chemical

Nitto Boseki

Comments: Stainless Steel Substrate, all reinforcements of $T$ Glass

Figure A.11-3. Shear strength at $77 \mathrm{~K}$ of FGR epoxy or BT resins to stainless steel substrate after ambient gamma irradiation. Supplementary Table A.11-1. Data from Egusa et al. [1992]. 


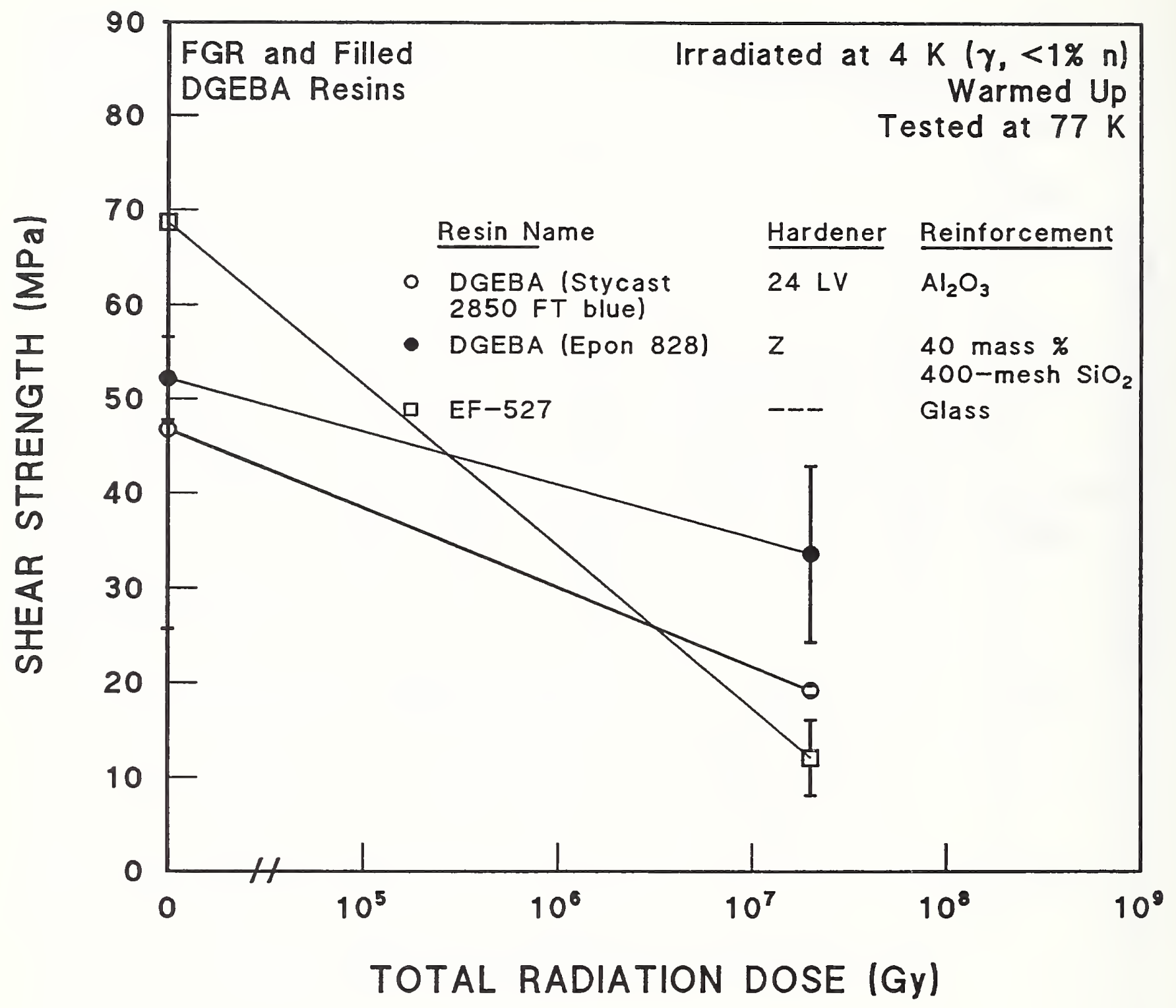

"O" Strength, MPa

- $46.8 \pm 21.1$

- $52.2 \pm 4.4$

ㅁ $\quad 68.7 \pm 22.4$
Additive

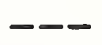

$0.5 \%$ Z 6020

Silane

---

\section{Cure Condition}

$24 \mathrm{~h}, 22^{\circ} \mathrm{C}$

$2 \mathrm{~h}, 80^{\circ} \mathrm{C}$;

$2 \mathrm{~h}, 150^{\circ} \mathrm{C}$

B Staged;

$1 \mathrm{~h}, 175^{\circ} \mathrm{C}$

\section{Supplier}

Emerson-Cumming

Shell

Synthane-Taylor

Comments: Copper Substrate, not Abraded

Figure A.11-4. Shear strength at $77 \mathrm{~K}$ of FGR and filled DGEBA resins to copper substrate (not abraded) after 4-K reactor irradiation. Supplementary Table A.8-2. Data from Kernohan et a1. [1979]. 


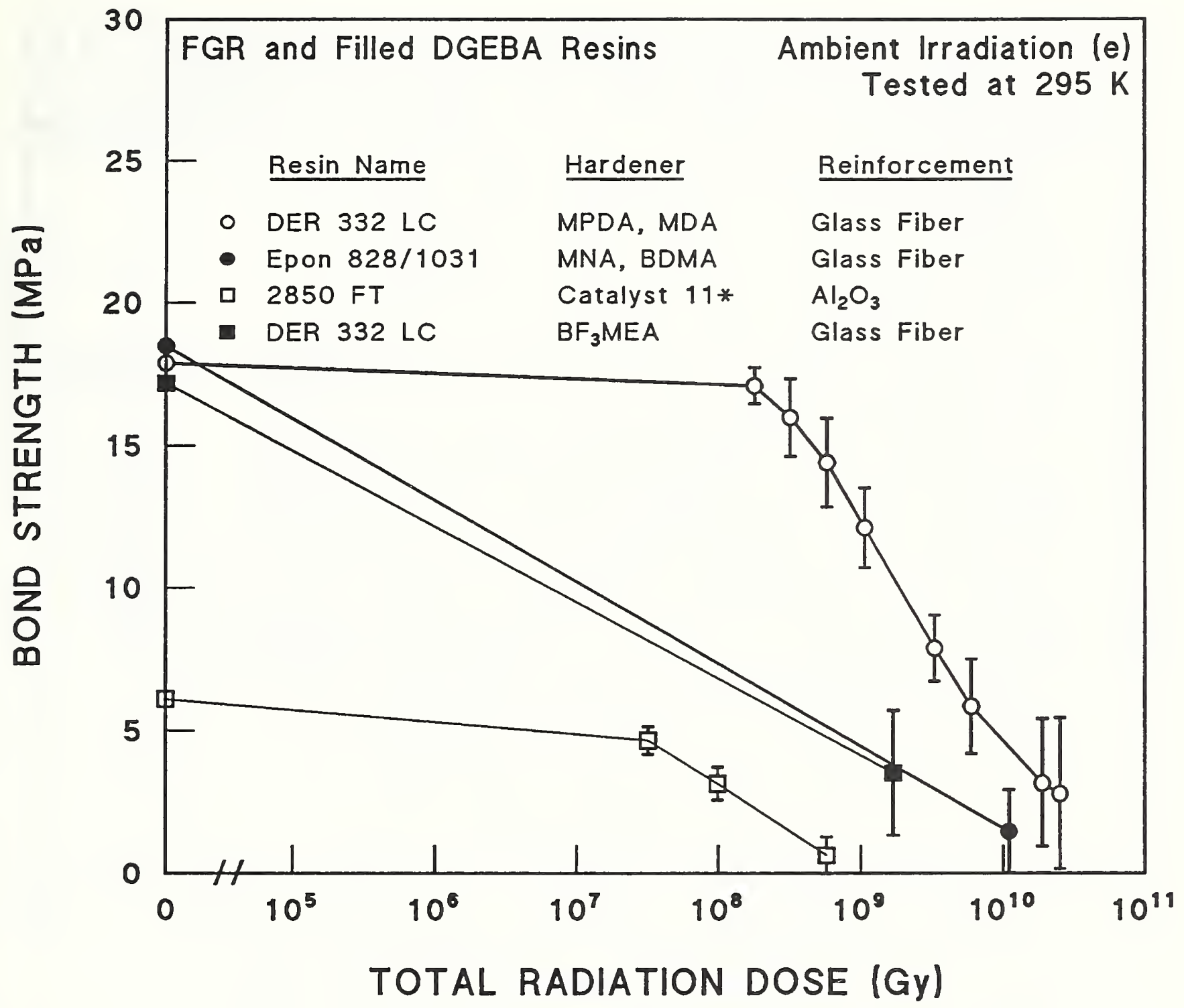

\begin{tabular}{|c|c|c|}
\hline "O" Strength, MPa & Wetting Agent & Supplier \\
\hline $17.9 \pm 0.0$ & $z-6040 * *$ & DOW \\
\hline - $\quad 18.5 \pm 0.0$ & --- & Shell \\
\hline $6.1 \pm 0.0$ & --- & Emerson-Cumming \\
\hline - $\quad 17.2 \pm 0.0$ & --- & DOW \\
\hline
\end{tabular}

* Major component, MPDA ** Glycidoxyl-propyl-trimethoxy silane

Comments: Copper Subtrate, sanded

Figure A.11-5. Shear strength at $295 \mathrm{~K}$ of FGR and filled DGEBA resins to sanded copper substrate after ambient electron irradiation. Supplementary Table A.2-6. See discussion in text of a possible error in dose determination. Data from Brechna [1965]. 


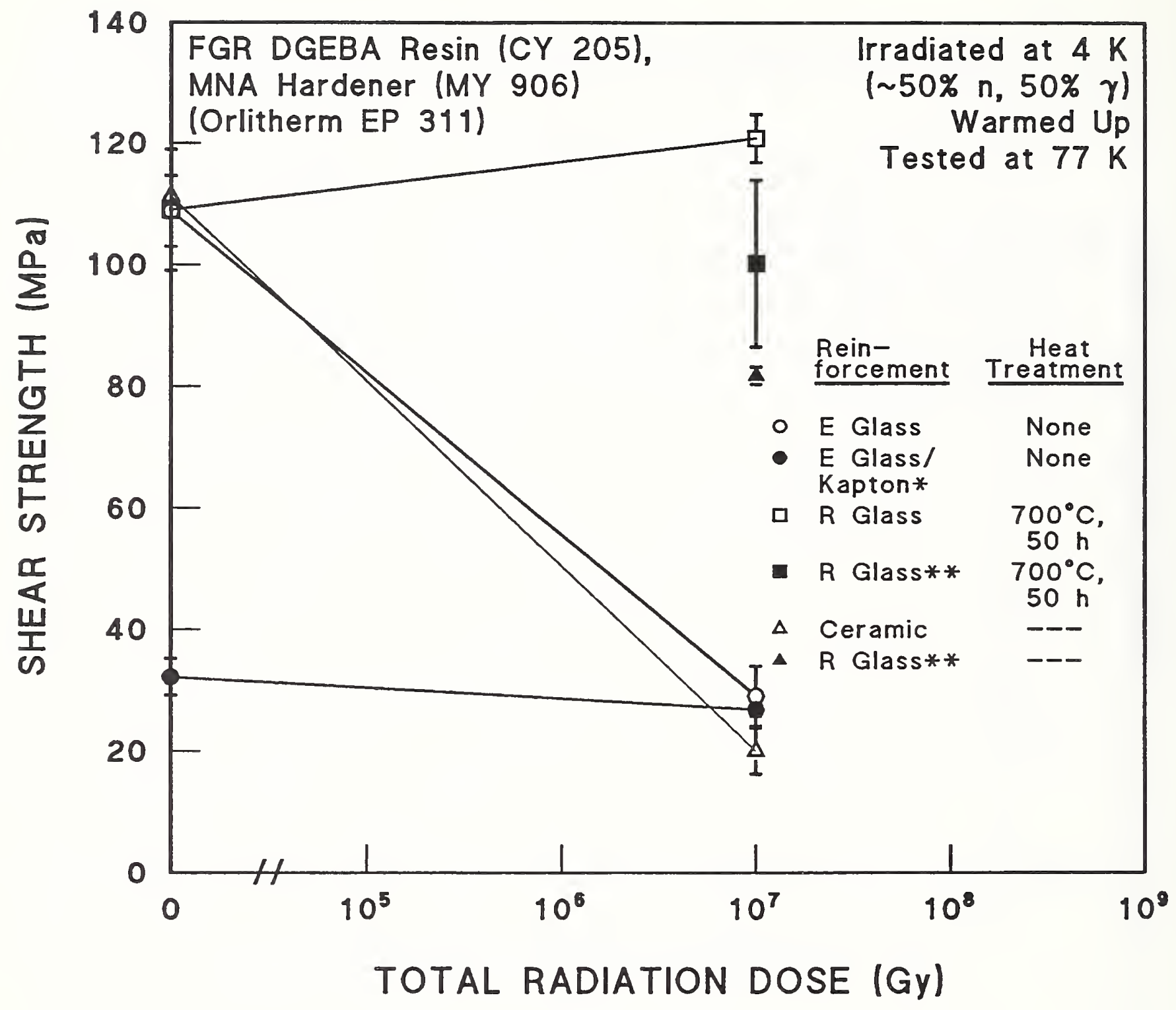

\author{
Supplier \\ $\odot, \bullet, \square,=$ ASEA Brown Boveri; Ciba Geigy \\ * Trivoltherm \\ * * Unirradiated shear strength not reported
}

Comments: $=$ Resin system without flexibilizer, other data obtained on resin system with flexibilizer.

$\triangle \mathrm{OH} 68$

Stainless Steel Substrate, Sandblasted

Figure A.11-6. Shear strength at $77 \mathrm{~K}$ of FGR DGEBA resin (Orlitherm) to stainless steel substrate after 4-K reactor irradiation. Supplementary Table A.11-2. Data from Poehlchen et al. [1990] and Pöhlchen [1992]. 


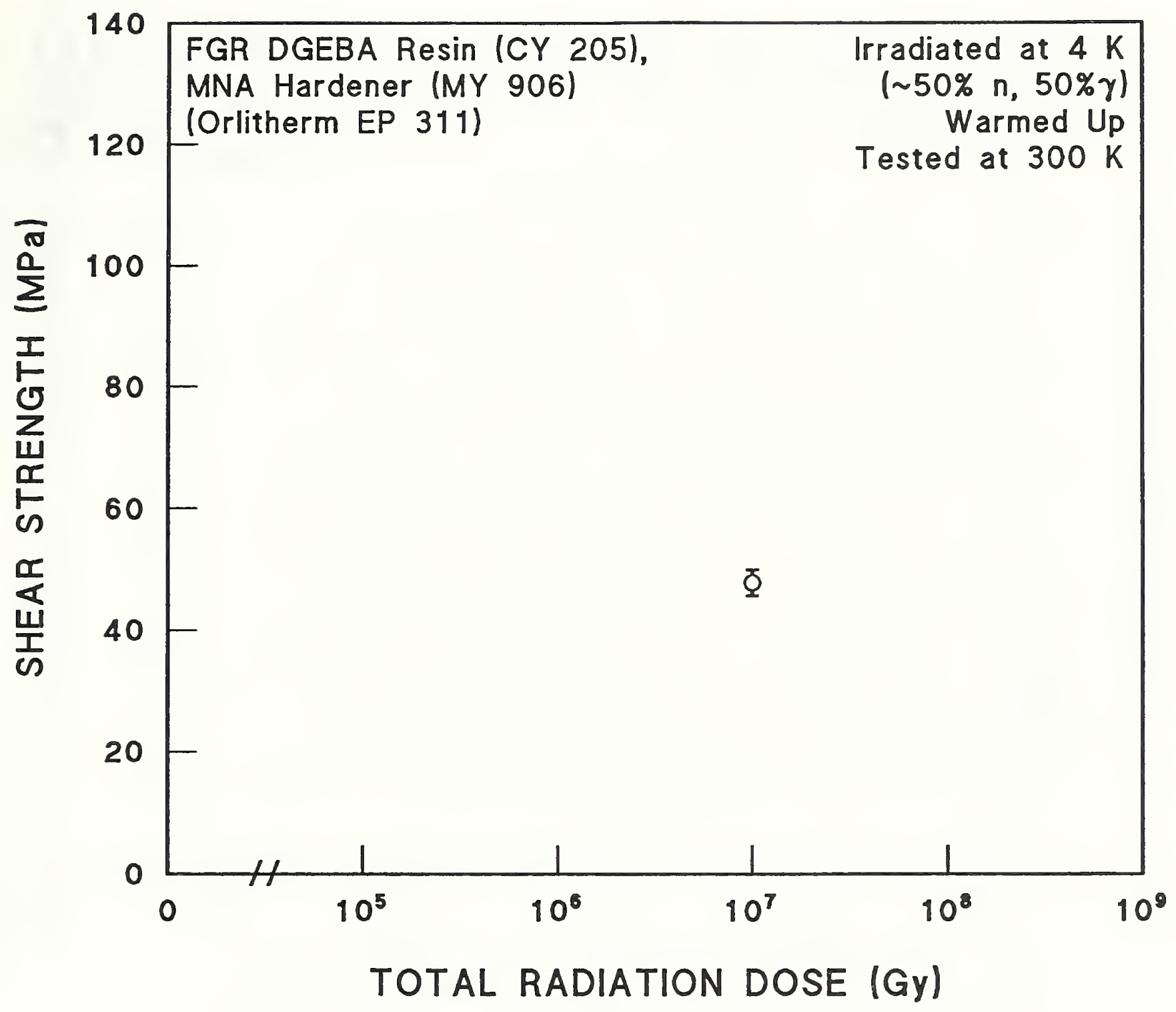

Heat

Reinforcement

- R Glass
Treatment

$700^{\circ} \mathrm{C}, 50 \mathrm{~h}$
Supplier

ASEA Brown Boveri; Ciba Geigy

Comments: Stainless Steel Substrate, Sandblasted

Figure A.11-7. Shear strength at $300 \mathrm{~K}$ of FGR DGEBA resin (Orlitherm) to stainless steel substrate after 4-K reactor irradiation. Supplementary Table A.11-2. Data from Poehlchen et al. [1990] and Pöhlchen [1992]. 


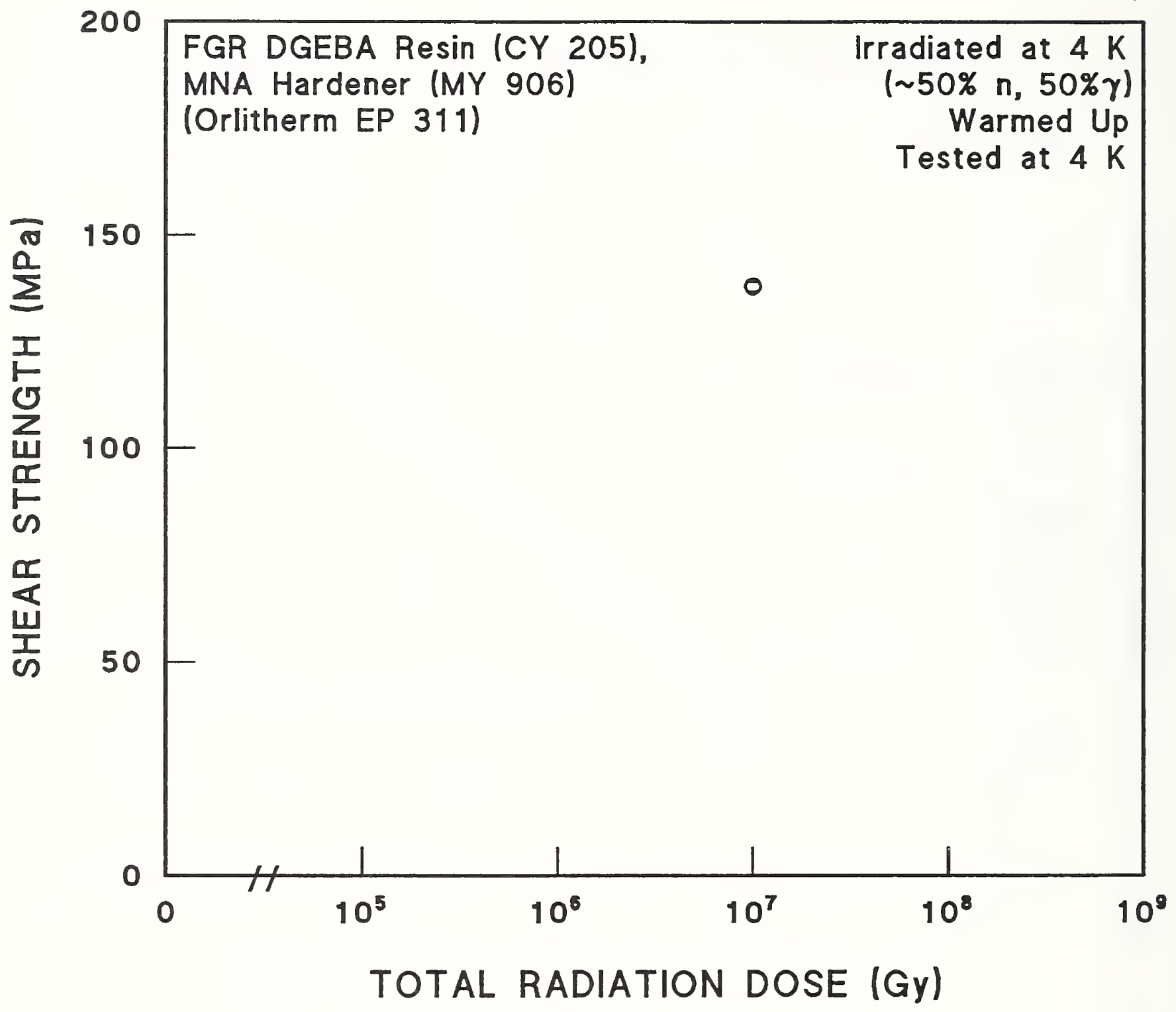

$\begin{array}{lll}\text { Reinforcement } & \begin{array}{c}\text { Heat } \\ \text { Treatment }\end{array} & \text { Supplier } \\ \text { - R Glass } & 700^{\circ} \mathrm{C}, 50 \mathrm{~h} & \begin{array}{l}\text { ASEA Brown Boveri; } \\ \text { Ciba Geigy }\end{array}\end{array}$

Comments: Stainless Steel Substrate, Sandblasted

Figure A.11-8. Shear strength at $4 \mathrm{~K}$ of FGR DGEBA resin (Orlitherm) to stainless steel substrate after 4-K reactor irradiation. Supplementary Table A.11-2. Data from Poehlchen et al. [1990] and Pöhlchen [1992]. 


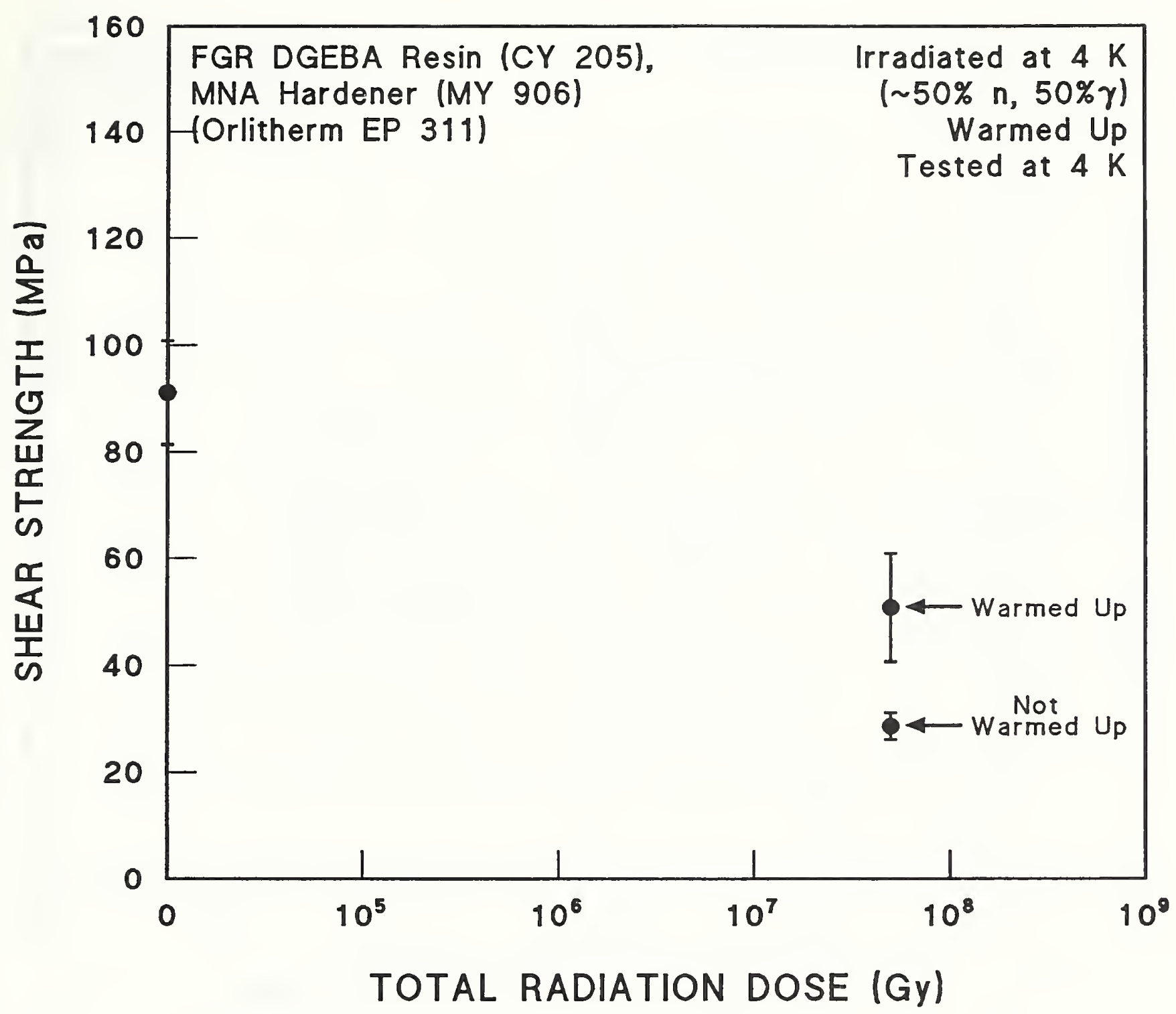

Heat

Treatment

Desized
Supplier

ASEA Brown Boveri; Ciba Geigy

Comments: Stainless Steel Substrate, Sandblasted

Figure A.11-9. Shear strength at $4 \mathrm{~K}$ of FGR DGEBA resin (Orlitherm) to stainless steel substrate after $4-\mathrm{K}$ reactor irradiation. Supplementary Table A.11-2 (except $\mathrm{n}_{\mathrm{f}}$ fluence $\sim 10^{22} / \mathrm{m}^{2}$ ). Data from Katheder et al. [1992]. 


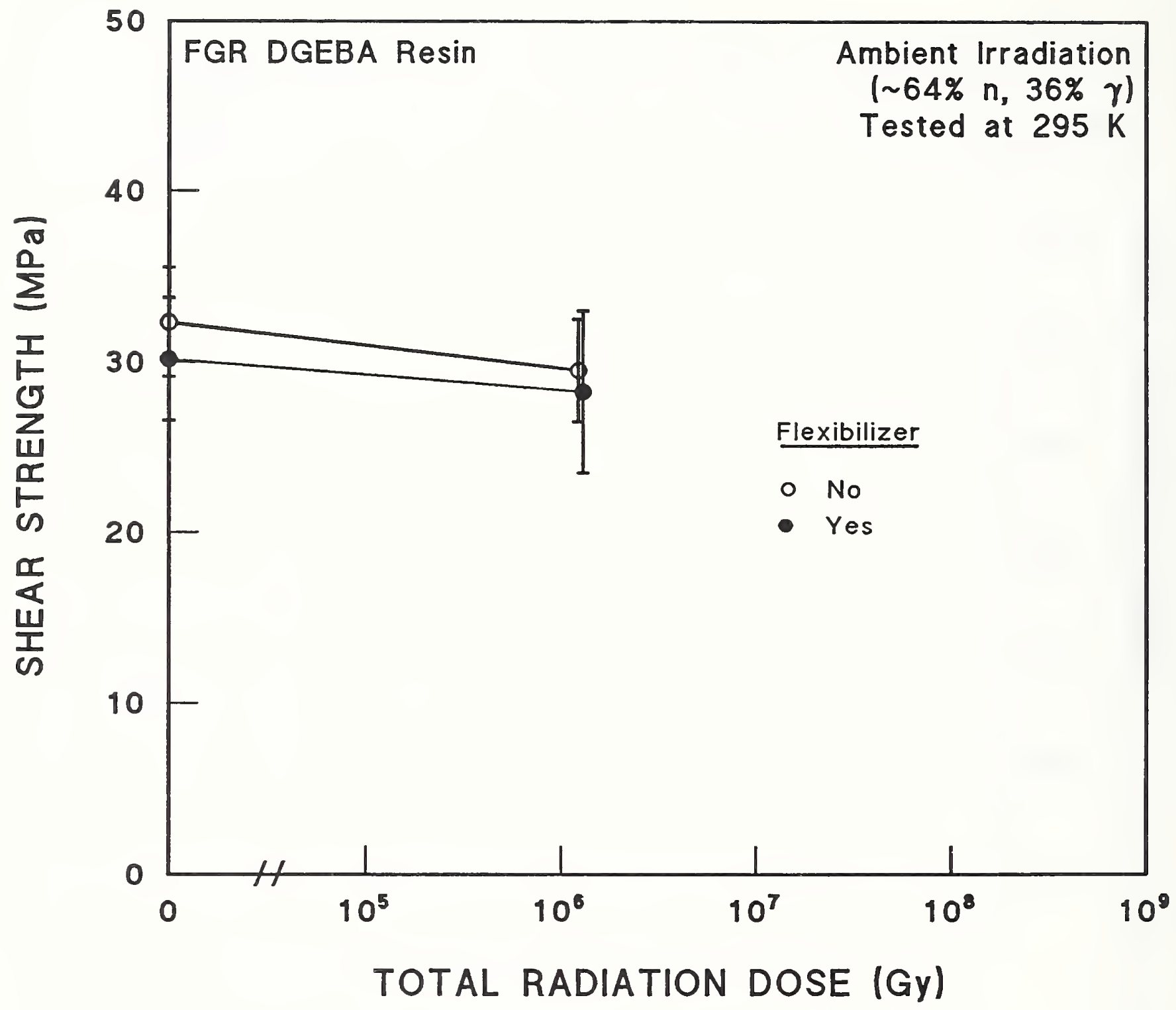

Supplier

- Princeton Plasma Physics Lab

- Princeton Plasma Physics Lab

Comments: Copper Substrate, Failure in composite

Figure A.11-10. Shear strength at $295 \mathrm{~K}$ of FGR DGEBA resin to copper substrate after ambient reactor irradiation. Supplementary Table A.11-3. Data from Schmunk et a1. [1981]. Dose determined from usual conversion factor. 


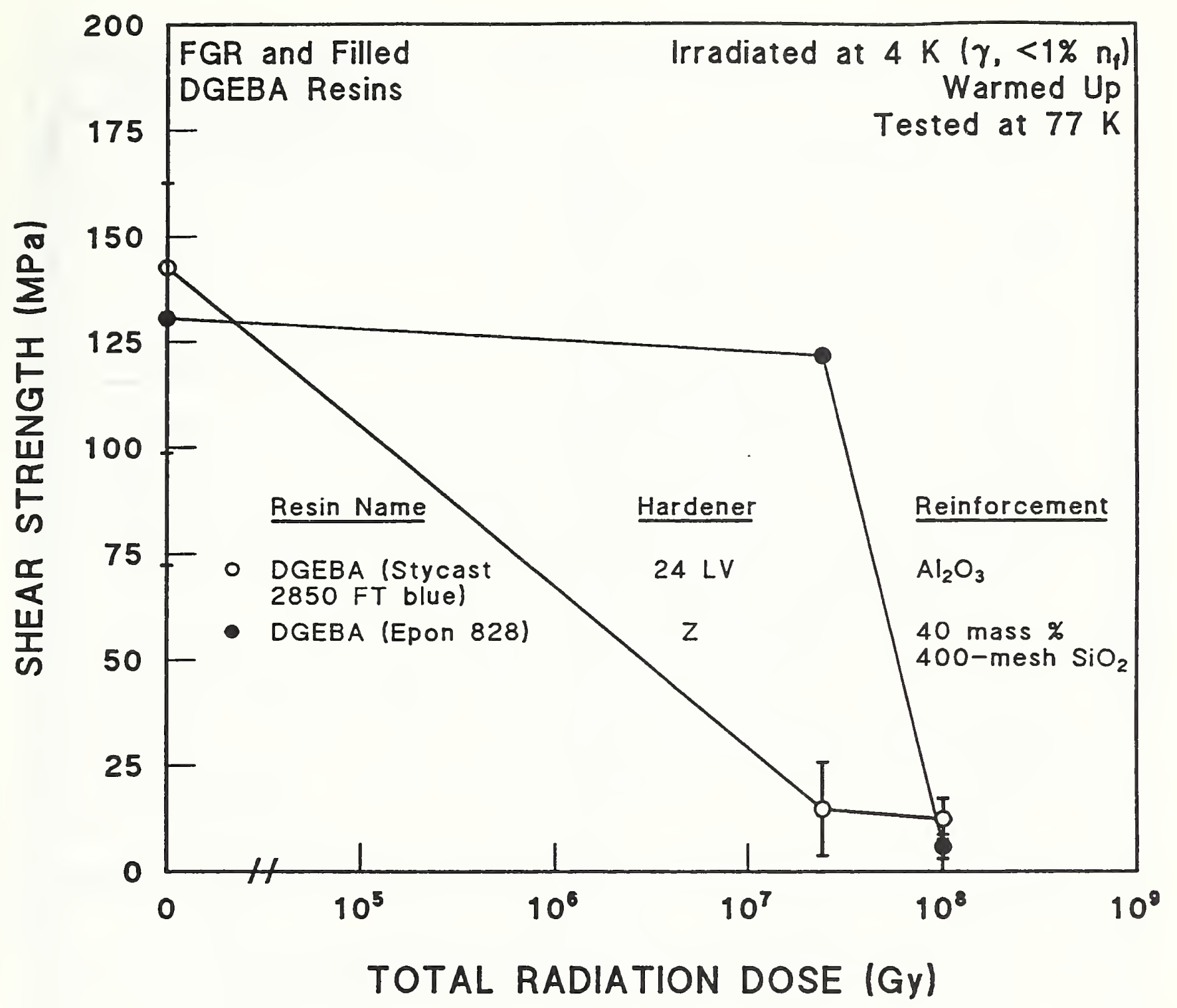

\begin{tabular}{|c|c|c|c|c|}
\hline & Strength, MPa & Additive & Cure Condition & Supplier \\
\hline o & $142.8 \pm 70.4$ & --- & $24 \mathrm{~h}, 22^{\circ} \mathrm{C}$ & Emerson-Cumming \\
\hline$\bullet$ & $130.7 \pm 32.0$ & $\begin{array}{l}0.5 \% \text { Z } 6020 \\
\text { Silane }\end{array}$ & $\begin{array}{l}2 \mathrm{~h}, 80^{\circ} \mathrm{C} \\
2 \mathrm{~h}, 150^{\circ} \mathrm{C}\end{array}$ & Shell \\
\hline
\end{tabular}

Comments: Copper Substrate, Abraded

Figure A.11-11. Shear strength at $77 \mathrm{~K}$ of FGR and filled DGEBA resins to copper substrate (abraded) after 4-K reactor irradiation. Supplementary Table A.8-2. Data from Coltman et al. [1979]. 


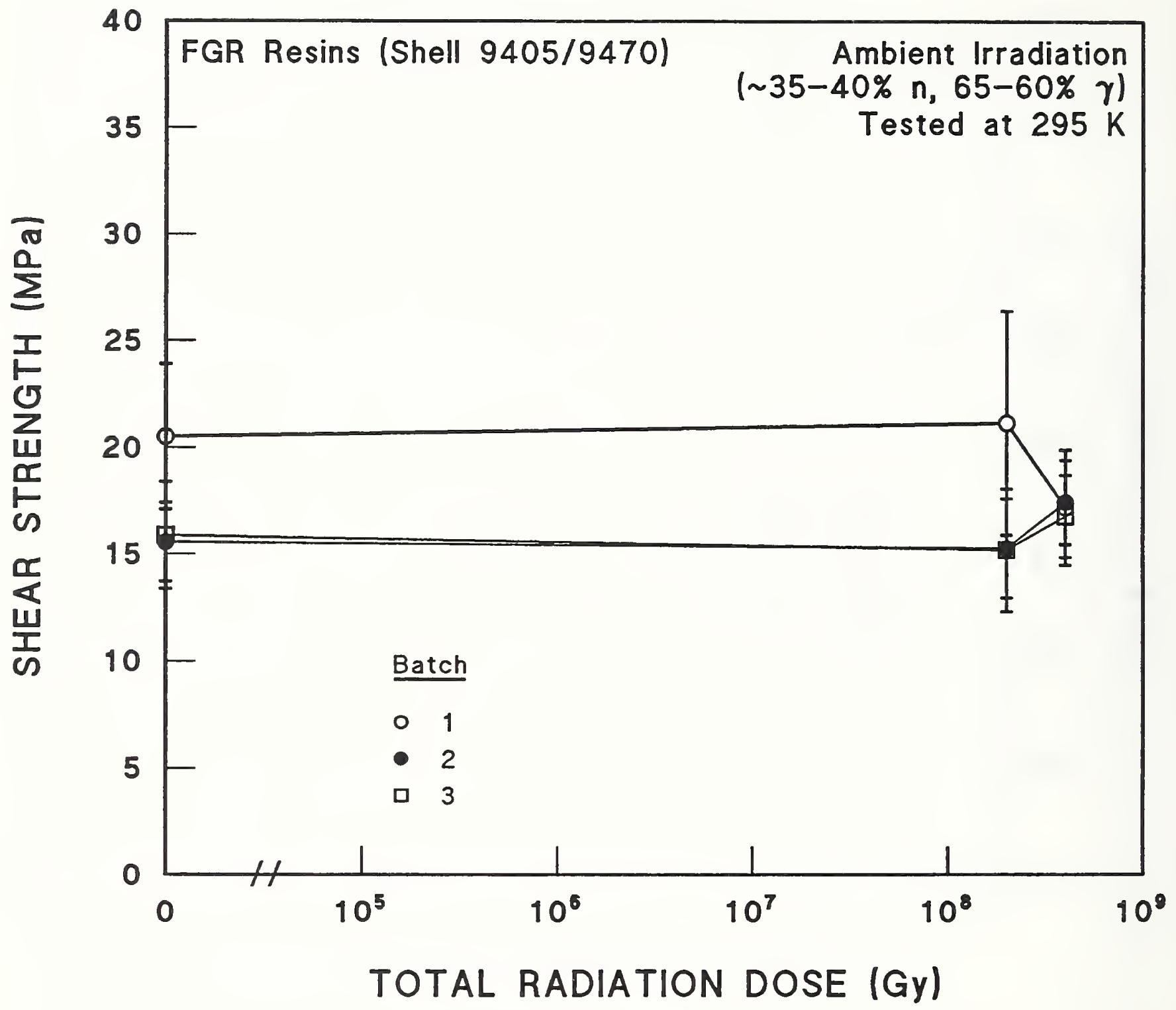

Supplier

$0, \bullet, \square$ Shell

Comments: Copper Substrate, sandblasted, primed with Polyurethane-based Conastic AD-20

Figure A.11-12. Shear strength at $295 \mathrm{~K}$ of FGR resin to copper substrate, sandblasted and primed, after ambient reactor irradiation. Supplementary Table A.217. Data from McManamy et al. [1991b]. 
Table A.11-1. Supplementary Information, Egusa et al. [1992].

\begin{tabular}{|c|c|}
\hline IRRADIATION PARAMETERS & MATERIALS \\
\hline Source Type: ${ }^{60} \mathrm{Co}$ & Resin: \\
\hline Source Name: & Hardener: \\
\hline $\begin{array}{l}\text { Irradiation } \\
\text { Temperature: Ambient }\end{array}$ & Additives: \\
\hline Environment: Air & Glass Vol. $8:$ \\
\hline Neutron $q$ : & Glass Mass $8:$ \\
\hline Neutron Flux: & Glass Type: $\mathrm{T}$ \\
\hline Fast Neutron $\%$ : $\quad(E>M e V)$ & Glass Finish: \\
\hline Fast Neutron Flux: & Glass Weave: \\
\hline Gamma $\&: 100$ & Glass Areal Density, $\mathrm{kg} / \mathrm{m}^{2}$ : \\
\hline Gamma Energy: & Filament Diameter, $\mu \mathrm{m}$ : \\
\hline Gamma Dose Rate: $2.7 \mathrm{~Gy} / \mathrm{s}$ & Layup: \\
\hline Electron $8:$ & Production Method: Prepreg plies \\
\hline Electron Energy: & Other: \\
\hline \multicolumn{2}{|l|}{ Electron Dose Rate: } \\
\hline \multicolumn{2}{|l|}{ Other: } \\
\hline \multicolumn{2}{|c|}{ TEST METHOD } \\
\hline \multicolumn{2}{|l|}{ Test Type: Lap shear } \\
\hline \multicolumn{2}{|l|}{ Test Specifications: } \\
\hline \multicolumn{2}{|c|}{ Specimen Shape: Substrate: semicircular rods (Figure 11.3) } \\
\hline \multicolumn{2}{|c|}{ Specimen Dimensions: Bond area: $13 \times 30 \mathrm{~mm}^{2}$, thickness $0.41-0.57 \mathrm{~mm}$} \\
\hline \multicolumn{2}{|l|}{ Flexure: Span/Thickness Ratio: } \\
\hline \multicolumn{2}{|c|}{ Compression: Aspect Ratio, Length/Width or Diam.: } \\
\hline \multicolumn{2}{|l|}{ Specimens Tested/Data Point: } \\
\hline \multicolumn{2}{|l|}{ Strain (Load) Rate: $0.06 \mathrm{~cm} / \mathrm{min}$} \\
\hline Test Temperature, $\mathrm{K}: 77$ & \\
\hline Other: & \\
\hline
\end{tabular}


Table A.11-2. Supplementary Information, Poehlchen et al. [1990], Pöhlchen [1992].

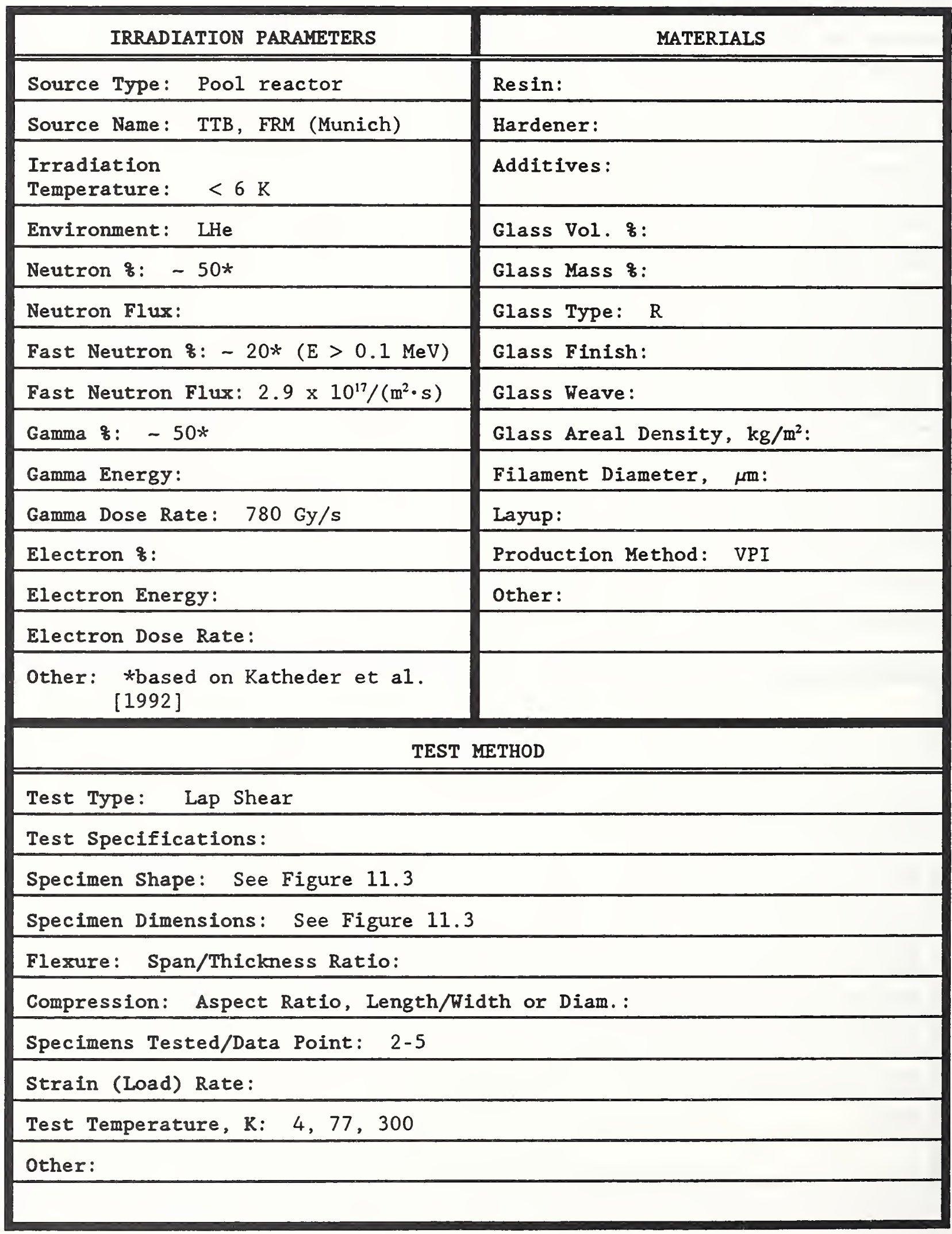


Table A.11-3. Supplementary Information, Schmunk et al. [1981] .

\begin{tabular}{|c|c|}
\hline IRRADIATION PARAMETERS & MATERIALS \\
\hline Source Type: Pool reactor & Resin: \\
\hline Source Name: CFRMF (INEL) & Hardener: \\
\hline $\begin{array}{l}\text { Irradiation } \\
\text { Temperature: }<3 \mathrm{~K} \text { above ambient }\end{array}$ & Additives: \\
\hline Environment: & Glass Vol. ơ: \\
\hline Neutron $\%$ : & Glass Mass $\frac{\circ}{2}$ : \\
\hline Neutron F1ux: $8.3 \times 10^{15} /\left(\mathrm{m}^{2} \cdot \mathrm{s}\right)$ & Glass Type: \\
\hline Fast Neutron $\%$ : $\quad(E>0.1 \mathrm{MeV})$ & Glass Finish: \\
\hline Fast Neutron Flux: & Glass Weave: \\
\hline Gamma $\frac{q}{2}:$ & Glass Areal Density, $\mathrm{kg} / \mathrm{m}^{2}$ : \\
\hline Gamma Energy: & Filament Diameter, $\mu \mathrm{m}$ : \\
\hline Gamma Dose Rate: & Layup: \\
\hline Electron 8 : & Production Method: \\
\hline Electron Energy: & Other: \\
\hline Electron Dose Rate: & \\
\hline \multicolumn{2}{|l|}{ Other: } \\
\hline \multicolumn{2}{|c|}{ TEST METHOD } \\
\hline \multicolumn{2}{|l|}{ Test Type: Shear bond } \\
\hline \multicolumn{2}{|l|}{ Test Specifications: } \\
\hline \multicolumn{2}{|l|}{ Specimen Shape: See Figure 11.4a } \\
\hline \multicolumn{2}{|l|}{ Specimen Dimensions: See Figure $11.4 \mathrm{a}$} \\
\hline \multicolumn{2}{|l|}{ Flexure: Span/Thickness Ratio: } \\
\hline \multicolumn{2}{|c|}{ Compression: Aspect Ratio, Length/Width or Diam.: } \\
\hline \multicolumn{2}{|l|}{ Specimens Tested/Data Point: 8} \\
\hline \multicolumn{2}{|l|}{ Strain (Load) Rate: $0.0021 \mathrm{~cm} / \mathrm{s}$} \\
\hline Test Temperature, $\mathrm{K}: 295$ & \\
\hline Other: & \\
\hline
\end{tabular}




\section{APPENDIX B: POLYIMIDES AND BISMALEIMIDES}

\section{B. INTRODUCTION}

Polyimide and bismaleimide resins often appear more resistant to radiation than epoxy resins; even when polyimide resins are reinforced with E-glass, the glass-polyimide or bismaleimide interface sometimes appears to be less susceptible to alpha- particle damage from neutron irradiation than does the glass-epoxy interface. Polyimide resins, however, cannot be used in vacuumimpregnation processes, and their reinforced flexural and interlaminar shear strengths are usually about half those of similarly reinforced epoxy resins. Furthermore, Hartwig [1987] noted that polyimides were more radiation-stable than other polymers but that this property was necessarily combined with brittleness at cryogenic temperatures.

Many of the same basic studies on the effects of radiation species, radiation temperature, radiation environment, and test temperature that were done for epoxy resins have also been done for polyimides, with similar results. These studies are discussed first, to give a basis for assessment of the polyimide data in relation to the ITER requirements. Then, the results of irradiating various polyimides are presented, sometimes in comparison with epoxies. Although there is some basis for preferring polyimides to epoxies as matrix materials, adequate data do not exist to ensure that polyimides could meet ITER requirements. Furthermore, the strengths of reinforced polyimides and bismaleimides are often lower than those of the most radiation-resistant epoxy composites after high-dose irradiation.

\section{B.1. ASSESSMENT OF DATABASE ON IRRADIATION OF POLYIMIDE INSULATION}

\section{B.1.1. Comparison of Neutron and Gamma Irradiation Damage}

Egusa et al. [1987b] tested E-glass-reinforced specimens in flexure that had been irradiated at ambient temperature with either neutrons from the IPNS or ${ }^{60} \mathrm{Co}$-gamma rays. The resin was polyaminobismaleimide (Kerimid 601), diagrammed in Figure B.1.1a. At the highest neutron dose, $4 \times 10^{7} \mathrm{~Gy}$, the damage from low-energy neutron irradiation (H2) was more severe than damage from the same gamma dose: only $30 \%$ of the initial flexural strength was 
a<smiles>O=C1C=CC(=O)N1c1ccc(Cc2ccc(N3C(=O)C=CC3=O)cc2)cc1</smiles>

Kerimid 601

b<smiles>Cc1ccc(Cc2ccc(N3C(=O)c4ccc(C(=O)c5ccc6c(c5)C(=O)N(C)C6=O)cc4C3=O)cc2)cc1</smiles>

Figure B.1.1. (a) The chemical structure of (a) a polyaminobismaleimide (b) a polyimide.

retained, as compared to about $85 \%$ with the gamma dose (Figure B.1-1). Damage from neutrons with a high energy component ("Rabbit" irradiation thimble) was intermediate. These results are similar to those obtained with a TGDM/DDS system (Figure 1.18, §1.3.2.4), but the difference between the neutron and gamma doses is more pronounced for the bismaleimide system. However, as noted in $\$ 1.3 .2 .4$, these results are confounded by the use of E-glass reinforcement that allows production of alpha particles by thermal neutrons and He and tritium by high energy neutrons; perhaps a system reinforced with B-free glass would not exhibit the same degree of difference between neutron and gamma irradiation. 
Fortunately, Abe et al. [1987] made comparative neutron and gamma irradiation tests on the polyimide PPMI (polypyromellitimide Kapton) in neat resin form (Figure B.1.1b). The RTNS-II facility furnished 14-MeV ambient-temperature neutron irradiation, and ambient tensile test results were compared with those of specimens irradiated with ${ }^{60} \mathrm{Co}$-gamma rays. The fluence-to-dose conversion factor was $1015 / \mathrm{m}^{2}=3.25 \mathrm{~Gy}$, calculated for the molecular constituents of PPMI. It was found that the average elongation after $8.6 \times 10^{7}$ Gy of gamma irradiation was $76 \%$, but that an equivalent elongation required only $1.1 \mathrm{x}$ $10^{7}$ Gy of neutron irradiation (Figure B.1-2). The authors concluded that, for a given dose, the $14-\mathrm{MeV}$ neutrons were about eight times more effective than ${ }^{60} \mathrm{Co}$-gamma rays in producing changes in mechanical properties.

Conservatively, this implies that gamma doses should be downgraded by a factor of eight to equate the damage to that produced by very high energy neutrons. But note that these results were found for miniature tensile specimens of film

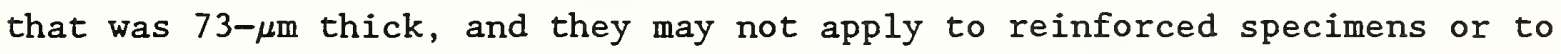
other types of resins.

These results for irradiation of Kapton by $14-\mathrm{MeV}$ neutrons are supported by earlier, qualitative studies with the IPNS, which has a substantial fast neutron component and less than a $13 \%$ gamma component. When Hurley et al. [1978] compared IPNS irradiation of Kapton with BSR gamma irradiation, they found that IPNS irradiation of $-2 \times 10^{6}$ Gy discolored specimens significantly, whereas a dose of $1 \times 10^{8}$ Gy of gamma irradiation was required to produce the same degree of discoloration. Gamma-irradiated Kapton was visibly indistinguishable from the nonirradiated material after a dose of $10^{7} \mathrm{~Gy}$. Environmental conditions for the two types of irradiation at $4 \mathrm{~K}$ were reported to be similar. When infrared spectroscopy was used to examine the specimens, the spectra after gamma irradiation were distinctly different from those after neutron irradiation. This indicated that different types of chemical changes occurred during the two types of irradiation. However, discoloration does not necessarily correlate precisely with changes in mechanical properties, according to Abe et al. [1987] (tensile properties) and Iida and Sumita [1983] (compressive properties). 


\section{B.1.2. Comparison of Gamma and Electron Irradiation Damage}

Comparative flexural tests of polyaminobismaleimide resin were made after irradiation by $2-\mathrm{MeV}$ electron and ${ }^{60} \mathrm{Co}$-gamma rays. Ambient-temperature irradiation was followed by 77-K tests [Egusa, 1991a]. In accord with the results found for a neat TGDM resin system (Figure A.3-17, §1.2.3.5), the gamma irradiation degraded the initial bismaleimide strength much more than the electron irradiation at doses of about $1 \times 10^{8}$ Gy (Figure B.1-3). Theoretically, damage from electrons and gamma rays in this energy range would be expected to be similar; Egusa ascribed the disparity to the much lower dose rate of the gamma irradiation, which would permit greater diffusion of oxygen and, therefore, more reactions of free radicals with oxygen and superficial radiation damage. Thus, this experiment did not provide a conclusive test of the relative effects of electron and gamma irradiation.

\section{B.1.3. Effect of Irradiation Temperature}

When results of 4-K reactor irradiation were compared with ambient-temperature irradiation, the 77-K shear strength of polyimide torsion specimens (CTD-310) was slightly less degraded after the 4-K dose [Munshi, 1991]. Figure B.1-4 shows that similar tests of a bismaleimide resin also indicated little difference in the degree of degradation of initial shear strength after either ambient temperature or 4-K irradiation. Another polyimide (CTD-300) exhibited excellent retained strength after ambient temperature irradiation, but was not irradiated at $4 \mathrm{~K}$, owing to space restrictions. Specimens of both resins were uniaxially reinforced with $S-2$ glass. Except for the $4-K$ dose to the polyimide, all of the maximum doses in these tests were above $10^{8} \mathrm{~Gy}$. The high energy irradiation spectrum in the fission reactor used for the 4-K irradiation was somewhat different from that of the fission reactor used for the ambient irradiation [Weber et al., 1986]; differences in this spectrum and possible differences in the ratio of neutrons to gamma rays may have confounded the results of this investigation to some degree. Clearly, additional tests of irradiation-temperature effects carried out with sources that were more nearly identical would be of interest, as would in-situ 4-K tests. 
The experiments of Egusa [1990a] on irradiation temperature are more uniform in regard to radiation source because both ambient temperature and 5-K irradiations were conducted with irradiation thimbles in the IPNS that had similar neutron spectra. Figure B.1-5 shows the results of flexural tests at ambient temperature of E-glass reinforced polyaminobismaleimide specimens [Egusa et al., 1984a], and Figure B.1-6 shows the results of 77-K flexural tests, after warm-up, of this material [Egusa, 1990a]. There is very little effect of irradiation temperature, up to a dose of about $3 \times 10^{7} \mathrm{~Gy}$. These results tend to corroborate those of Munshi [1991] on a bismaleimide resin, discussed above. However, the highest dose was about a factor of 5 lower, and the use of E-glass may have complicated the results, owing to the effects of energetic alpha particles in the resin-glass interfacial region, since the sources used were characterized as having a "degraded fission spectrum with a high-energy component."

\section{B.1.4. Effect of Oxygen During Irradiation}

Both an E-glass reinforced polyaminobismaleimide and a commercial polyimide laminate were irradiated in an air and in an argon atmosphere at ambient temperature [Egusa, 1991a]. Gamma ray doses up to $10^{8}$ Gy were employed. Flexural tests at $77 \mathrm{~K}$ showed that the irradiation environment did not affect the percent of flexural strength that was retained and that both composites retained a similar percentage of initial strength, about $60 \%$, at $10^{8} \mathrm{~Gy}$ (Figure B.1-7). Neat polyimide or bismaleimide resins have apparently not been tested after irradiation in argon. When both neat and reinforced epoxy resins were tested after irradiation with and without oxygen, it was found that only neat resins exhibited a degradation in properties in the oxygen environment $(\S 2.5)$.

\section{B.1.5. Effect of Test Temperature with and without Warm-up}

E-glass-reinforced polyaminobismaleimide resin (Kerimid 601) was irradiated at ambient temperature with gamma rays and tested at both ambient temperature and $77 \mathrm{~K}$ in flexure [Egusa, 1988]. The 77-K flexural strength deteriorated more rapidly with irradiation than did the ambient flexural strength, after doses up to $1.4 \times 10^{8}$ Gy (Figure B.1-8). The initial 77-K flexural strength was 
also considerably higher than the ambient flexural strength. In contrast, when neat polyimide resin (Kerimid 1000) was irradiated with 2-MeV electrons and similarly tested at $77 \mathrm{~K}$ and ambient temperature, there was less difference in the initial flexural strength and essentially no difference in the retained strength after a dose of about $1.55 \times 10^{8}$ Gy (Figure B.1-9). Thus, the decrease in flexural strength after irradiation was much more pronounced at $77 \mathrm{~K}$ than at ambient temperature for composites, but this effect was not observed for neat resins. A similar distinction between reinforced and neat resins was observed (Egusa, 1988] when TGDM/DDS systems were tested in both composite and neat form $(\$ 1.4 .1)$.

The same E-glass-reinforced polyaminobismaleimide resin system was tested at ambient temperature and $77 \mathrm{~K}$ after $2-\mathrm{MeV}$ electron irradiation at ambient temperature [Egusa et al., 1985a]. Apparently, this experiment was similar to that reported above (Figure B.1-8), except that electron irradiation was used instead of gamma irradiation. The results were very similar (Figure B.1-10), except that the electron irradiation produced less damage than the gamma irradiation in the $295-\mathrm{K}$ tests.

The effect of different test temperatures was also investigated by Takamura and Kato [1980]. They measured the compressive strength of the polyimide, Vespel, at 4 and $77 \mathrm{~K}$, after reactor irradiation at $5 \mathrm{~K}$. (Vespel is the bulk form of the polyimide, Kapton, Figure B.1.1b.) Tests were conducted without warm-up. Figure B.1-11 shows that there was no difference in the results at the two test temperatures. However, the dose was only $10^{7} \mathrm{~Gy}$, and the compressive-strength test often does not reveal radiation damage at this level.

\section{B.1.6. Effect of Annealing}

Investigations on the effect of annealing radiation-damaged bismaleimide systems were carried out by Egusa et a1. [1985b]. These investigations were similar to those carried out on a TGDM/DDS resin system that were discussed above $(\S 1.4 .2 .3)$. After ${ }^{60} \mathrm{Co}$-gamma irradiation in air at ambient temperature to $2 \times 10^{8} \mathrm{~Gy}$, composite specimens were annealed for $2 \mathrm{~h}$ at $180^{\circ} \mathrm{C}$ in vacuum. Although the results of the epoxy flexural tests indicated that annealing caused or activated further damage, no significant deterioration in flexural 
properties after annealing was noted for the polyaminobismaleimide resin composite, as shown in Figure B.1-12. Both the epoxy and the bismaleimide systems were reinforced with the same E-glass plain weave. Since radiation effects on the shear modulus were correlated with effects on the flexural strength, Egusa et al. suggested that glass-resin interfacial strength was decreased by the annealing, perhaps as a result of increased gas evolution. Evidently, then, gas evolution and other forms of interfacial damage may be less severe for irradiated bismaleimide than for irradiated epoxy composites.

\section{B.1.7. Effect of Glass-Reinforcement Parameters}

The effect of substituting boron-free glass reinforcement for E glass improved radiation resistance in one investigation [Egusa, 1991a] but made no difference in another experiment [Egusa and Hagiwara, 1986]. When similar weaves of $T$ and $E$ glass were used to reinforce polyaminobismaleimide, the initial 77-K flexural strengths were similar, but the E-glass-reinforced composite deteriorated more severely under gamma irradiation of $-10^{8}$ Gy than did the T-glass-reinforced composite. Figure B.1-13 shows this result: fabric type KS-1210 (E glass) should be compared with WTX-116E (T glass), since these weaves are similar. A denser T-glass weave was also tested (WTA18W). There was little difference between the dose-dependent radiation resistances of the two types of T-glass-reinforced composites tested, although the initial and high-dose flexural strengths of the composite fabricated with the thicker, denser fabric were lower. Since both T-glass composites were prepared by the same manufacturer, whereas the E-glass composite was prepared by a different manufacturer, Egusa suggested that fabrication differences could have been primarily responsible for the difference in radiation resistance. When similarly reinforced $\mathrm{E}-$ and $\mathrm{T}-\mathrm{glass}$ epoxy composites were irradiated, both $\mathrm{E}-$ and $\mathrm{T}$-glass composites exhibited the same radiation dose dependence (Figure A.3-9, §3.3). Since the manufacturer of the T-glassreinforced epoxy composites was not the same as the manufacturer of the T-glass-reinforced bismaleimide composite, fabrication techniques may have been different enough to account for the effect.

On the other hand, when E- and S-glass-reinforced polyaminobismaleimides, Spaulrad and Spaulrad-S, were supplied by the same manufacturer, the same dose dependence was observed for both composites [Egusa and Hagiwara, 1986]. 
Figure B.1-14 shows that when these composites were irradiated with gamma rays at ambient temperature and tested in flexure at $77 \mathrm{~K}$, the initial strength of the S-glass composite was slightly higher, but the percentage of retained strength at $1.6 \times 10^{8}$ Gy was similar for both types of glass reinforcement. Egusa and Hagiwara noted that since the fiber surface finishes were different, the similarity in radiation response suggested that the matrix properties dominated the overall composite radiation resistance.

\section{B.1.8. Effect of Test Method}

Little data are available on the use of different test methods to determine the irradiation properties of polyimide and bismaleimide resins. Egusa [1990a; 1990b] conducted three-point bend tests on E-glass-reinforced polyaminobismaleimide specimens that were cut at either an angle of $0^{\circ}$ or $45^{\circ}$ to the warp. For the $0^{\circ}$ orientation, he obtained a flexural failure mode (Figure $1.41 \mathrm{~d}$ ), but the $45^{\circ}$ specimens failed in shear, providing an estimate of the interlaminar shear strength. Figures B.1-15 and B.1-16 show that the results of the tests at $0^{\circ}$ and $45^{\circ}$ for each temperature are similar; for example, about 508 of the initial $77-\mathrm{K}$ strength is retained at $1.4 \mathrm{x} 10^{8} \mathrm{~Gy}$ for both specimen orientations, Similar results were also obtained for both specimen orientations when an epoxy resin, TGDM, was used instead of a polyimide (Figures 1.42 and 1.43 ).

McManamy et al. [1991a] tested a commercial polyaminobismaleimide laminate, Spaulrad S, and bismaleimide triazine (BT) in two modes: flexure and combined shear/compression ( $\$ 1.5 .3$ and 10.2). The Spaulrad was reinforced with a 2dimensional, S-glass weave while the BT was reinforced with a 3-dimensional, T-glass weave. Specimens were exposed to a maximum dose of $3 \times 10^{8}$ Gy ( $42 \%$ from neutrons) at a temperature near ambient and were tested at ambient temperature. As Figures B.1-17 and B.1-18 show, the results of the two types of tests are somewhat different. The specimens tested in shear after a compressive load of $345 \mathrm{MPa}$ was applied appeared much less-sensitive to the radiation than the specimens tested in flexure. However, in both tests, the $B T$ resin reinforced with a 3 -dimensional weave had the higher initial strength and a higher percentage of retained strength after irradiation than the commercial laminate. As noted above, 3-dimensional weaves may have dielectric properties that make them unsuitable for turn-to-turn magnet insulation. 


\section{B.2. COMPARISON OF POLYIMIDE AND EPOXY RADIATION RESISTANCE}

Because fluence-to-dose conversion factors are not always accurately known or reported, and because it is difficult to compare studies that used different irradiation species and spectra, the best assessments of the relative radiation resistance of polyimides, bismaleimides, and epoxies occur when both resin types are exposed to the same radiation source.

\section{B.2.1. Low-Dose Comparisons ( $\left.\$ 10^{7} \mathrm{~Gy}\right)$}

A number of comparative studies in the literature have been made at doses far below the ITER conceptual design requirements. Nevertheless, such studies have demonstrated superior radiation resistance of polyimides and bismaleimides, although often the comparisons have been made only to DGEBA resins, which have radiation resistances below those of many other epoxies.

An example is the comparison by Takamura and Kato [1980] of a polyimide (Vespe1) with a DGEBA resin cured with three different hardeners. Figure B.21 shows that the polyimide retained a significantly higher fraction of initial compressive strength than any of the DGEBA epoxy resin-hardener combinations at a dose of $1.1 \times 10^{7} \mathrm{~Gy}$. The neat resin specimens were irradiated at $5 \mathrm{~K}$ in a reactor and tested at 4 and $77 \mathrm{~K}$ without warm-up.

In another study, Takamura and Kato [1984] compared a different polyimide laminate (T16-G100) with DGEBA laminates (G-10CR and G-11CR), but also tested two E-glass reinforced epoxy composites, a cycloaliphatic and an isocyanatehardened system. The irradiation conditions were the same as in the earlier work, but in-situ tests of flexural and tensile strength were also carried out. As would be expected from the results reported in $\$ 2.1$, the cycloaliphatic epoxy composite did not exhibit a high radiation resistance. However, the radiation resistance of the isocyanate-hardened epoxy system (ISOX) was nearly as high as that of the polyimide although the initial flexural and tensile strengths were much lower (Figures B.2-2, B.2-3, and B.24). Of the three types of properties measured, tensile, compressive, and flexural, the flexural properties appeared to be most sensitive to radiation. 
Hurley et al. [1978] also compared polyimide laminates with DGEBA laminates (Figures B. 2-5 and B.2-6) and the comparisons were extended to higher doses by Tucker et al. [1985] (Figures B.2-7 and B.2-8). Specimens were irradiated at $4 \mathrm{~K}$ in the IPNS, warmed up, and tested at both $75 \mathrm{~K}$ and ambient temperature. Hurley et al. performed both compressive and flexural tests; Tucker et al. carried out flexural tests only. The DGEBA laminates tested were G-10CR and G-11CR, and the polyaminobismaleimide systems were Kerimid 601, Norplex 530, and Spaulrad. As expected, both bismaleimides retained a higher percentage of 77-K flexural strength at the higher doses (still below $10^{7} \mathrm{~Gy}$ ) than the G10CR and G-11CR. Since E-glass fiber layups were probably different for the bismaleimide systems, the comparisons with the DGEBA composites did not test only resin effects.

Okada et al. [1992] compared BT composites reinforced with E-glass with a similarly reinforced composite prepared with an unidentified epoxy. In interlaminar shear tests with a notched specimen, the BT resin showed a higher percentage of retained strength than the epoxy, at a dose just below $10^{7}$ Gy (Figure B.2-9). Reactor irradiation was carried out at $20 \mathrm{~K}$; testing was done at $77 \mathrm{~K}$ without warm-up to ambient temperature. In this case, the E-glass/BT interface appeared less susceptible to secondary alpha particle damage than the epoxy interface, in contrast to results obtained by Egusa et al. (§B.1.1).

\section{B.2.2. High-Dose Comparisons $\left(-10^{8} \mathrm{~Gy}\right)$}

Hagiwara et al. [1985] compared one of the more radiation-resistant epoxy systems, TGDM/DDS, as well as a bisphenol-A based epoxy, with a polyimide resin. Both the $3-\mathrm{MeV}$ electron irradiation and flexural tests were carried out at ambient temperature. Figure B.2-10 shows that the polyimide retained above $100 \%$ of the initial strength and the TGDM system retained less than $70 \%$ of the initial strength at a dose of $10^{8} \mathrm{~Gy}$. The performance of the bisphenol-A resin was considerably worse.

The TGDM/DDS resin system was compared with polyaminobismaleimide and several DGEBA resin systems by Egusa and Hagiwara [1986] and Egusa [1990a]. Ambienttemperature gamma irradiation was administered to a dose of $1.6 \times 10^{8} \mathrm{~Gy}$; flexural tests on glass-reinforced composites were done at ambient temperature and $77 \mathrm{~K}$ (Figures B.2-11 and B.2-12) and at $4 \mathrm{~K}$ (Figure B.2-13). As noted 
above (§B.1.5), the cryogenic flexural properties were much more sensitive to deterioration under radiation than the ambient- temperature properties were. Although the bismaleimide retained the highest percentage of initial strength at all temperatures, the magnitude of its initial strength was so low that the flexural strength of the more rapidly degraded TGDM/DDS system was approximately equal to it after a dose of $8 \times 10^{7} \mathrm{~Gy}$. Since tensile stresses predominate at the outside bend radius in the flexural strength test, perhaps the flexural strengths of both systems become equal at high doses because both matrix resins are degraded and the measurements chiefly reflect the tensile strength of the reinforcement.

Egusa et al. [1988] showed that the 77-K flexural strength of a mixed bismaleimide/epoxy system, BT/DGEBA, was about equal to that of the TGDM/DDS system after an ambient temperature dose of $1.2 \times 10^{8}$ Gy of 2-MeV electron irradiation (Figures B.2-14 and B.2-15). However, the TGDM/DDM system actually increased in strength under irradiation, up to the maximum dose of $1.2 \times 10^{8} \mathrm{~Gy}$. Egusa et al. suggested that the increase could be due to crosslinking under irradiation.

Coltman and Klabunde [1981] tested several bismaleimide laminates at doses up to $10^{8} \mathrm{~Gy}$; none, however, were increasing in flexural strength at the maximum dose as was the TGDM/DDM epoxy system. An increase in strength with radiation dose was observed in compressive strength tests, but compressive strength is a much less sensitive indicator of radiation damage. These results on the commercial laminates Spaulrad and Norplex NP 530 and also for the neat polyimide resin Vespel are presented in Figures B.2-16 through B.2-21. (Spaulrad and Norplex NP 530, also termed Kerimid, are polyaminobismaleimides.) Irradiation composed predominately of gamma rays was carried out at $4 \mathrm{~K}$; tests were performed at $300 \mathrm{~K}$ and at $77 \mathrm{~K}$ after warm-up. The retained flexural strengths at $77 \mathrm{~K}$ of both of the bismaleimide laminates were about 60 to 708 at $10^{8}$ Gy (Figure B.2-17); this is considerably worse than the 77-K performance of the best epoxy system TGDM/DDM (Figure. B.2-15), but the epoxy system was irradiated at ambient temperature, whereas the bismaleimides were irradiated at $4.9 \mathrm{~K}$ and were warmed up before testing. Also, there was some neutron component in the dose administered by Coltman and Klabunde, and their laminates were reinforced with E-glass, so some alpha-particle damage may have occurred in the glass-matrix interfacial region. A comparison of 
Figure B.2-20 with B.2-17 shows that Vespel retained a higher percentage of flexural strength at $77 \mathrm{~K}$ than the laminates. However, the initial flexural strength of this polyimide resin was much lower than that of either of the laminates, and it evidently was not tested in reinforced form.

In comparison with the results of Coltman and Klabunde, Schönbacher and Stolarz-Iżycka [1979] obtained even lower retained flexural strength at $10^{8}$ Gy for E-glass-reinforced polyaminobismaleimide (Kerimid 601). Figure B.2-22 shows that only about $28 \%$ of the initial strength was retained, although the material appeared promising at $5 \times 10^{7} \mathrm{~Gy}$. Perhaps the disparity with the results of Coltman and Klabunde, and with several experiments of Egusa's group, was due to the neutron component ( -148$)$ of the radiation used by Schönbacher and Stolarz-Iżycka, which would cause alpha-particle damage at the glass-matrix interface. At lower doses, Egusa et al. [1987b] showed that Kerimid 601 exhibited much more degradation after neutron irradiation than after gamma irradiation (Figure B.1-1). Most of the other polyimides tested by Schönbacher and Stolarz-Iżycka were not irradiated to $10^{8}$ Gy; therefore, the other data given in Figure B.2-22 are not relevant to ITER requirements. However, the Kerimid 601 data can be compared with other data obtained by the same authors on E-glass-reinforced epoxy resins; Figures A.2-23 to A.2-26 indicate that several of these are more radiation-resistant than this bismaleimide.

McManamy et al. [1991a] compared a commercial S-glass-reinforced polyaminobismaleimide (Spaulrad $S$ ) with an S-glass-reinforced, styrenemodified epoxy system (Shel1 9405/9470). Ambient- temperature flexural tests were conducted after reactor irradiation up to $4 \times 10^{8} \mathrm{~Gy}$. The results indicated that about $55 \%$ of the initial strength of the Spaulrad $S$ polyimide was retained at $2 \times 10^{8} \mathrm{~Gy}$, and about 488 at $4 \times 10^{8}$ Gy (Figure B.2-23). These results for Spaulrad appear to correspond roughly to those of Coltman and Klabunde, who observed about $60 \%$ retention of the initial flexural strength for Spaulrad at a gamma dose of $10^{8} \mathrm{~Gy}$, but under-cryogenic irradiation and testing conditions (with warm-up). The styrene-modified epoxy composite that was also irradiated and tested by McManamy et al. retained nearly the same percentage of initial strength as the Spaulrad $S$, but had initial and post-irradiation strengths much lower than those of the Spaulrad $\mathrm{S}$. 
Comparative testing of epoxy resins, bismaleimides, and polyimides with neutron irradiation was also done by Munshi [1991]. Reactor irradiation was carried out at both ambient temperature and $4 \mathrm{~K}$. Since the fast neutron component in these irradiations was about $20 \%$ of the total dose, the radiation environment better simulated ITER conditions than did the electron and gamma irradiations of Egusa et al. and the predominately gamma irradiations of Coltman and Klabunde. Furthermore, these irradiations were conducted on specimens reinforced with S-2 Glass, so alpha-particle damage did not confound the results as in the investigations of Schönbacher and Stolarz-Izycka. Of course, since the torsion/shear and fracture resistance tests employed by Munshi were carried out on uniaxially reinforced rod specimens of small diameter, screening test data, rather than design data, were obtained, but this comment also applies to the results of flexural tests, since interlaminar shear is not measured in such tests ( $\$ 1.5 .2)$.

The results of Munshi's ambient-temperature irradiations show that both polyimides (CTD-300 and 310) and a bismaleimide (CTD-200) were superior in retained shear strength to the various epoxy resin systems tested, which included a multifunctional epoxy, EPN, and DGEBA (Figure B.2-24). Figure B.225 shows, however, that CTD-300, the polyimide resin that had the highest retained strength at $2.3 \times 10^{8} \mathrm{~Gy}$, had a relatively low fracture resistance. Fracture properties have not been measured in most investigations, so comparable data are not available for most resin systems. Owing to space limitations, the 4-K irradiations were conducted on a smaller number of resin systems, and the CTD-310 polyimide was not exposed to the highest dose. However, Figure B.2-26 shows that at the maximum dose of $1.6 \times 10^{8} \mathrm{~Gy}$, the bismaleimide retained about $70 \%$ of the initial shear strength, a higher percentage than those of the three types of epoxy resins tested. But since the initial shear strength of the multifunctional epoxy was higher than that of the bismaleimide, its post-irradiation strength was only about 128 lower.

\section{B.2.3. Polyimide Films}

A few miscellaneous results have been obtained on polyimide films, sometimes identified as Kapton. Banford [1984] reported that the radiation resistance near $10^{8}$ Gy decreased when Kapton was added to glass fabric tape as a reinforcement for a bisphenol-A based resin (Figure B.2-27). Van de Voorde 
and Restat [1972] reported retained tensile strength above $100 \%$ for a polyimide film after $10^{8}$ Gy of irradiation (Figure B.2-28). Testing and irradiation were conducted at ambient temperature. Takamura and Kato [1979; 1981] irradiated a 50- $\mu \mathrm{m}$ Kapton film to $1.1 \times 10^{7} \mathrm{~Gy}$ at $5 \mathrm{~K}$, and found $88 \%$ retention of the initial 77-K tensile strength (Figure B.2-29). Beynel et al. [1982] reported a significant deterioration in the peeling force required for Kapton $\mathrm{T}$ film at $10^{7} \mathrm{~Gy}$. This polyimide was not recommended for use at $10^{8} \mathrm{~Gy}$ by these authors. 


\section{B.3. SUMARY OF IRRADIATION TESTING OF POLYIMIDES AND BISMALEIMIDES}

In basic studies on polyimides and bismaleimides of the effects of irradiation species, temperature, and environment, and the effects of test temperature, results were generally similar to those discussed above for epoxy resins with the following notable exceptions.

(1) A unique comparison of the effects on a polyimide of pure ${ }^{60} \mathrm{Co}$-gamma irradiation with $14-\mathrm{MeV}$ neutron irradiation showed that the neutrons produced about eight times as much damage as the gamma rays at the same dose (§B.1.1).

(2) In shear tests of uniaxially reinforced torsion specimens, little difference was found between degradation from ambient-temperature irradiation or 4-K irradiation for a polyimide and a bismaleimide. In contrast, the same tests of DGEBA (but not EPN and a multifunctional epoxy) resins showed more degradation from 4-K irradiation. However, in this comparison the ambienttemperature and $4-\mathrm{K}$ reactor sources had somewhat different spectra ( $\$ B .1 .3$ ). (3) Annealing at $180^{\circ} \mathrm{C}$ did not appear to activate or cause further postirradiation damage for a polyaminobismaleimide resin, although it did for a TGDM epoxy system ( $\$$ B.1.6).

Egusa's group made several comparisons of a relatively radiation-resistant epoxy resin, TGDM, with polyimide and bismaleimide systems. Although they showed that the polyimide or bismaleimide retained a high percentage of initial flexural strength, the post-irradiation flexural strengths at $-10^{8} \mathrm{~Gy}$ of a polyaminobismaleimide (Kerimid 601) was about equal to that of TGDM/DDS and below that of TGDM/DDM, which was about $850 \mathrm{MPa}$ at $77 \mathrm{~K}$. All irradiations were at ambient temperature and consisted of gamma rays or electrons; the dose was therefore not equivalent to that expected for ITER magnet insulation.

Coltman and Klabunde also tested polyaminobismaleimide laminates (Spaulrad and Norplex NP 530, also called Kerimid) with predominantly gamma doses of $10^{8} \mathrm{~Gy}$ delivered at $4 \mathrm{~K}$; their flexural test results were similar to Egusa's with post-irradiation strengths of about $600 \mathrm{MPa}$ at best at $77 \mathrm{~K}$. Although Schönbacher and Stolarz-Iźycka tested only at ambient temperature, their irradiation dose consisted of -148 neutrons, and at $10^{8} \mathrm{~Gy}$, the percentage of retained flexural strength for Kerimid 601 had fallen to about 35\%. Egusa et al. have shown that Kerimid 601 exhibits more degradation after neutron irradiation than after gamma irradiation; unfortunately, the results of all of these neutron irradiations are confounded by the use of E-glass reinforcement. 
McManamy et al., however, irradiated Spaulrad $S$ with neutrons and found that it retained about 558 of the initial flexural strength at $2 \times 10^{8} \mathrm{~Gy}$. Although this dose consisted of $40 \%$ neutrons and $60 \%$ gamma rays, both irradiation and testing were done at ambient temperature. Polyimide (Kapton) films have shown very good retention of tensile strength after irradiation, but dose components and irradiation temperatures have not approached ITER requirements.

The comparative irradiations of epoxies, polyimides, and bismaleimides conducted by Munshi were carried out with doses that had a significant fast neutron component, and some irradiation was done at $4 \mathrm{~K}$. The subsequent shear-torsion tests at $77 \mathrm{~K}$ on S-glass-reinforced material showed that a bismaleimide was somewhat superior to a multifunctional epoxy at a dose of 1.6 $x 10^{8}$ Gy. Although the percentage of retained shear strength, -708 , was much higher for the bismaleimide, the higher initial shear strength of the multifunctional epoxy gave it a post-irradiation strength only 128 below that of the bismaleimide.

In summary, except in the work of Munshi, none of the polyimides or bismaleimides were tested at doses and temperatures that approached ITER requirements. The post-irradiation strengths of polyimides and bismaleimides were lower than those of the most radiation-resistant epoxies, such as multifunctional or TGDM systems, except in one study by Munshi. In this case, the shear strength of a bismaleimide system was only slightly higher than that of a multifunctional epoxy. 


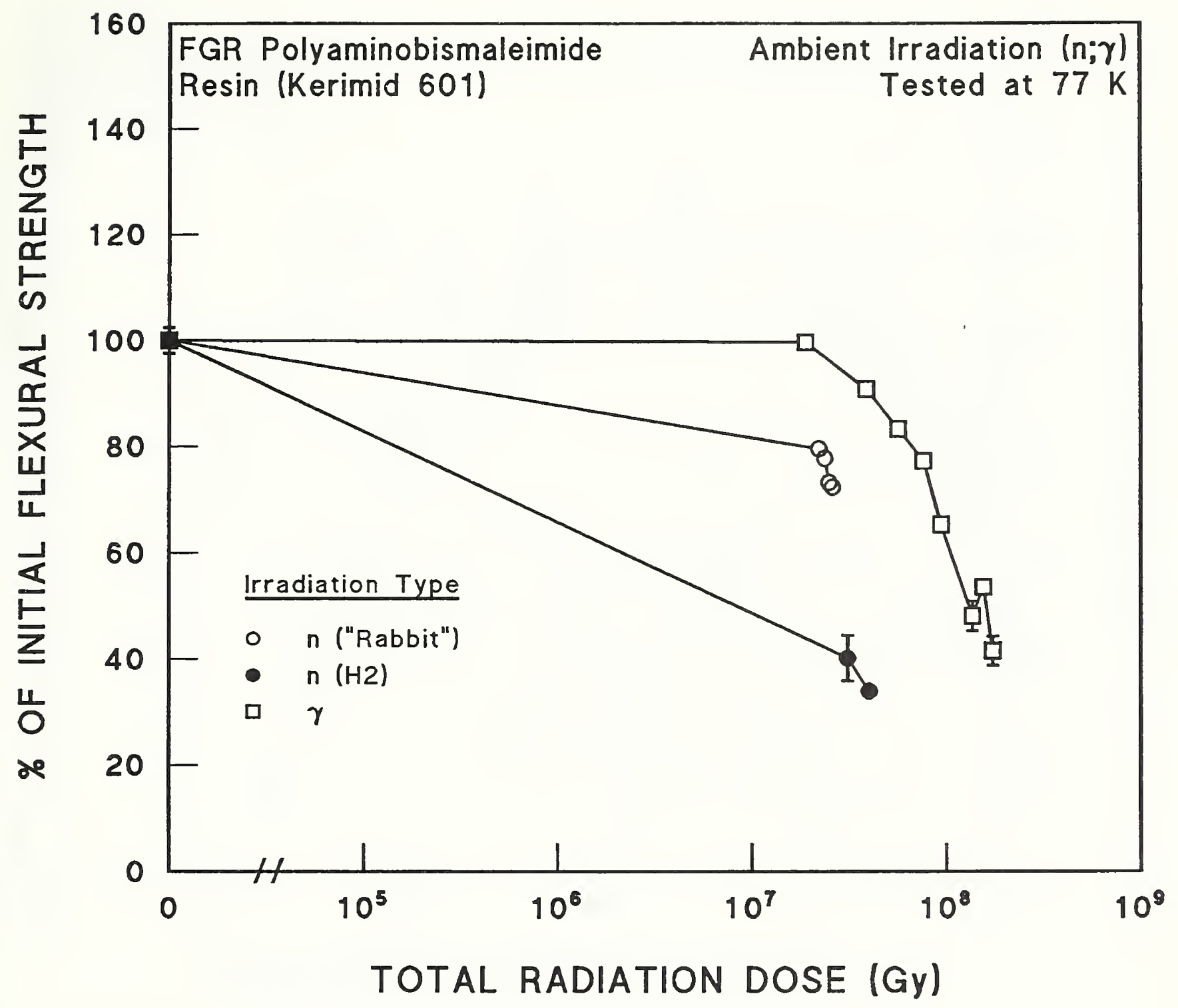

"O" Strength, MPa
$0,0944.7 \pm 22.8$
$909.4 \pm 0.0$

Supplier

Sumitomo Bakelite Co., Ltd.

Sumitomo Bakelite Co., Ltd.

Figure B.1-1. Flexural strength at $77 \mathrm{~K}$ of FGR polyaminobismaleimide resin after ambient neutron (IPNS) or gamma irradiation. The spectrum at the "rabbit" irradiation thimble was described as degraded fission with a high energy component; that at $\mathrm{H} 2$ consisted of a large flux of low energy neutrons. Supplementary Table B.1-1. Data from Egusa et al. [1987b]. 


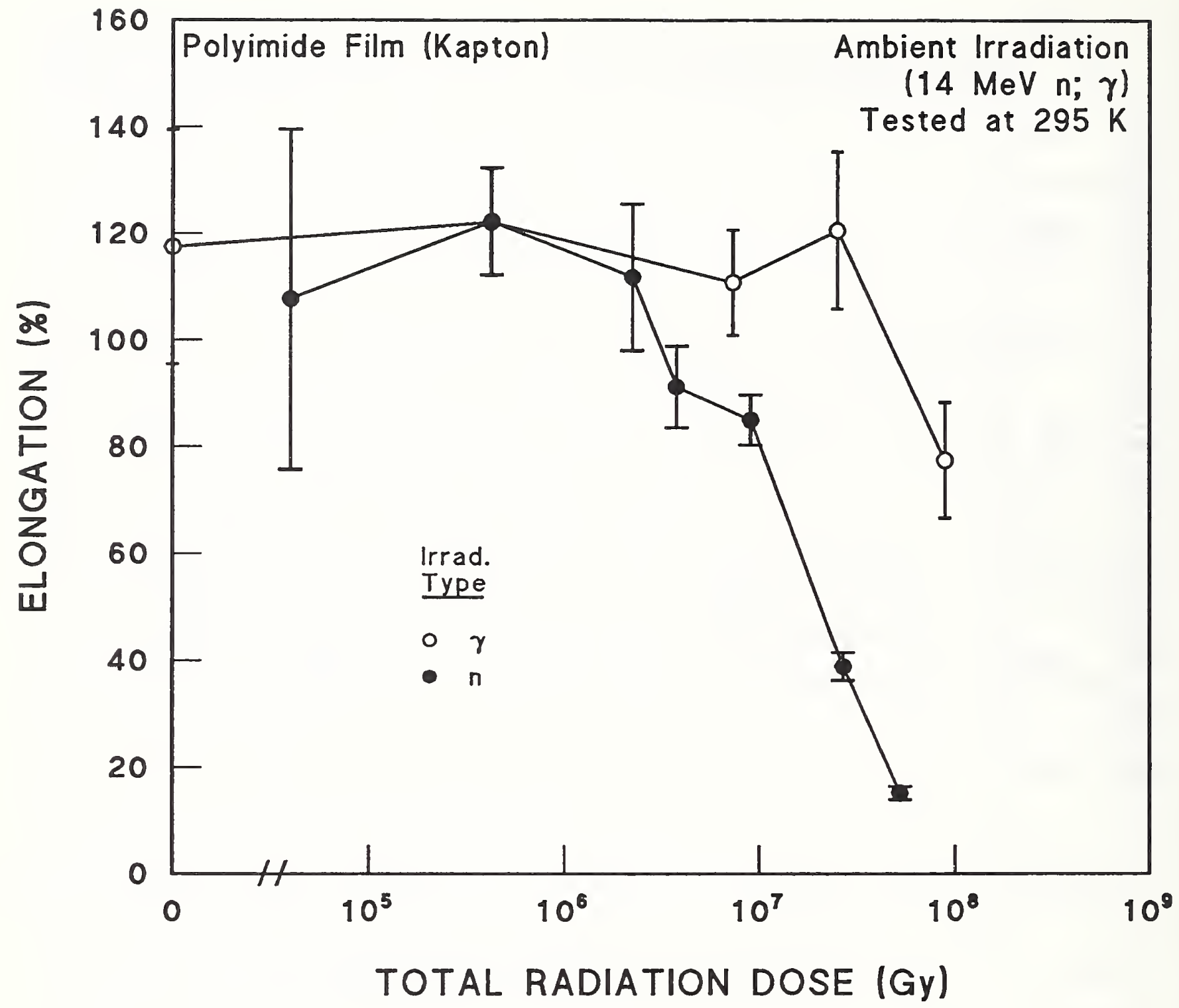

Supplier

$0, \bullet$ DuPont Co.

Figure B.1-2. Elongation at $295 \mathrm{~K}$ of polyimide film after ambient 14-Mev neutron (RTNS) or gamma irradiation. Supplementary Table B.1-2. Data from Abe et al. [1987]. 


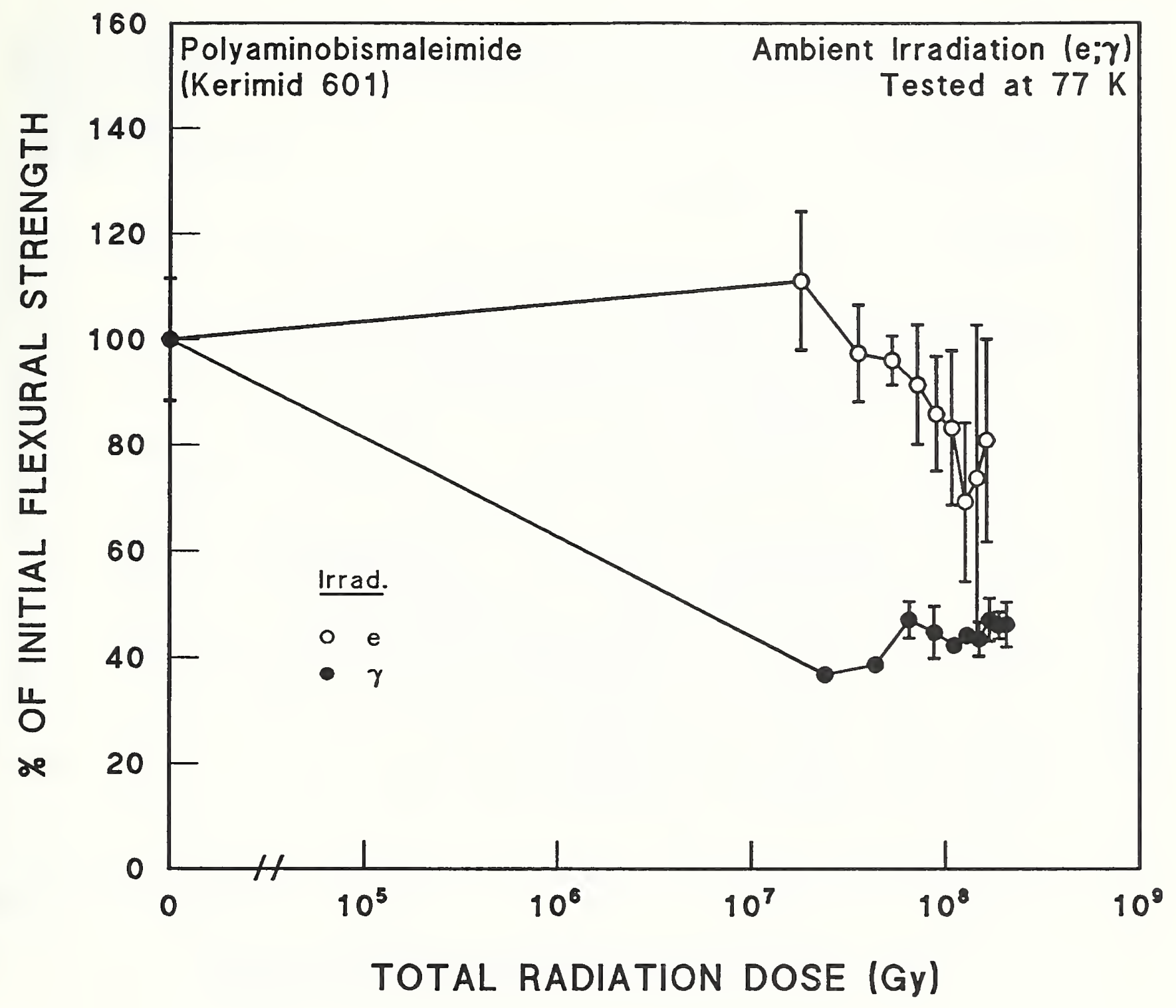

Supplier

Sumitomo Bakelite Co., Ltd.

Sumitomo Bakelite Co., Ltd.

Figure B.1-3. Flexural strength at $77 \mathrm{~K}$ of FGR polyaminobismaleimide resin after ambient electron or gamma irradiation. Supplementary Table A.3-4. Data from Egusa et al. [1991a]. 


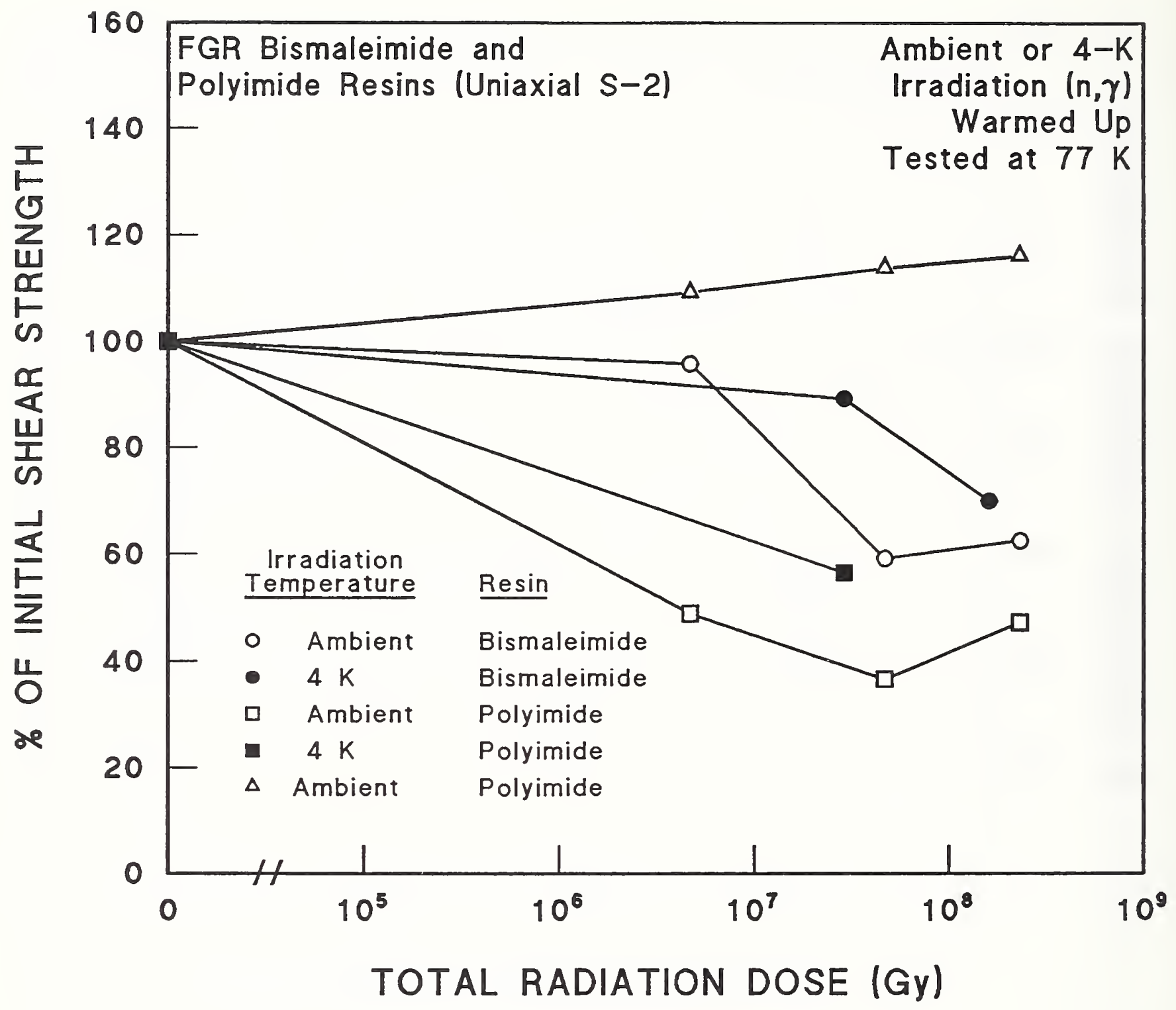

"O" Strength, MPa

$0,0 \quad 120.0$

口, 131.0

$\triangle \quad 87.0$
Type

CTD -200

CTD -310

CTD -300
Supplier

CTD, Inc.

CTD, Inc.

CTD, Inc.

Figure B.1-4. Shear strength at $77 \mathrm{~K}$ of FGR bismaleimide and polyimide resins after ambient or 4-K reactor irradiation. Supplementary Tables A.3-3 and A.8-4. Data from Munshi [1991]. 


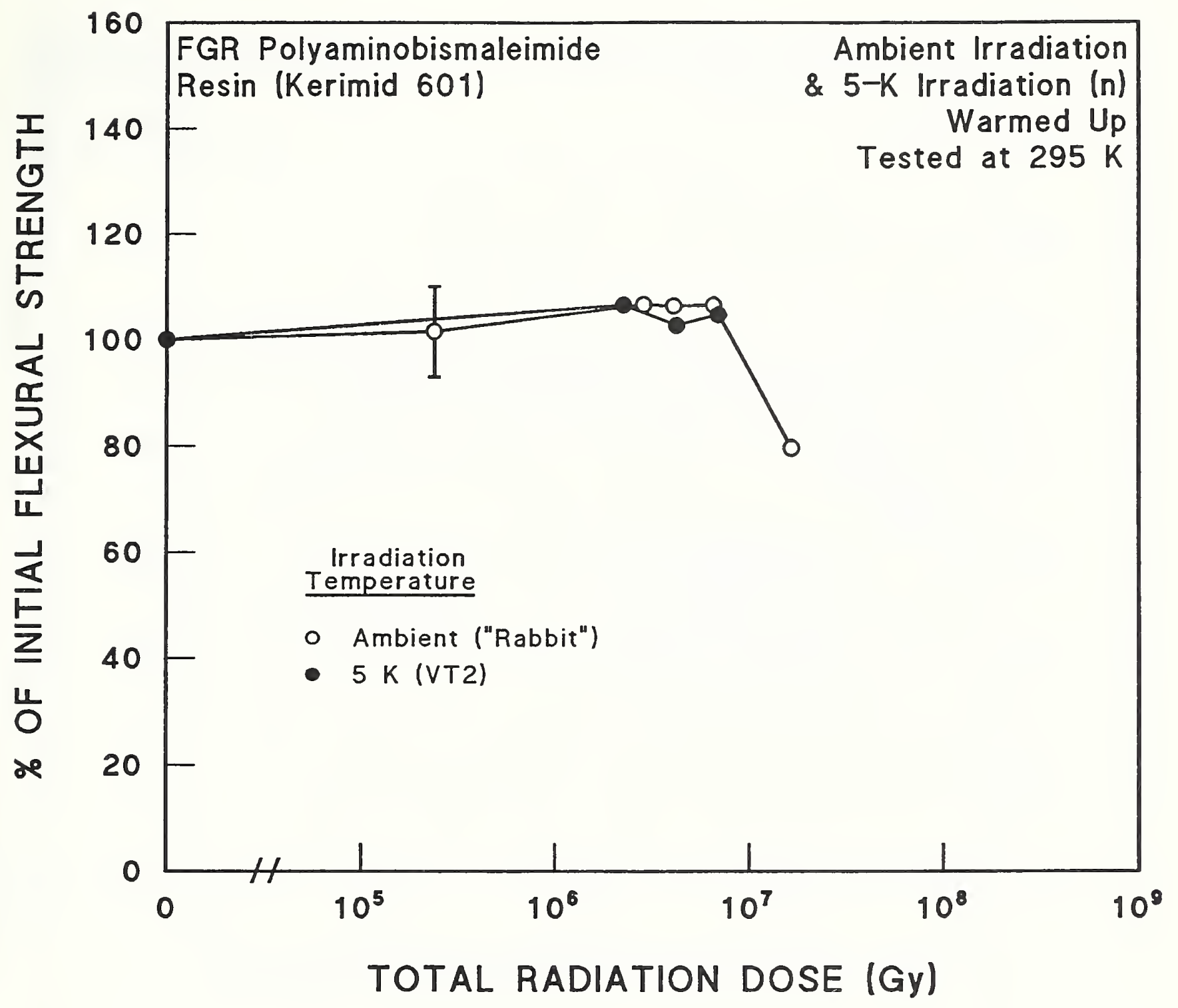

Supplier

Sumitomo

Bakelite

Co., Ltd.

Figure B.1-5. Flexural strength at $295 \mathrm{~K}$ of FGR polyaminobismaleimide resin after ambient or 5-K neutron (IPNS) irradiation. The spectrum at both irradiation thimbles was described as degraded fission with a high energy component. Supplementary Table B.1-1. Data from Egusa et al. [1984a]. 


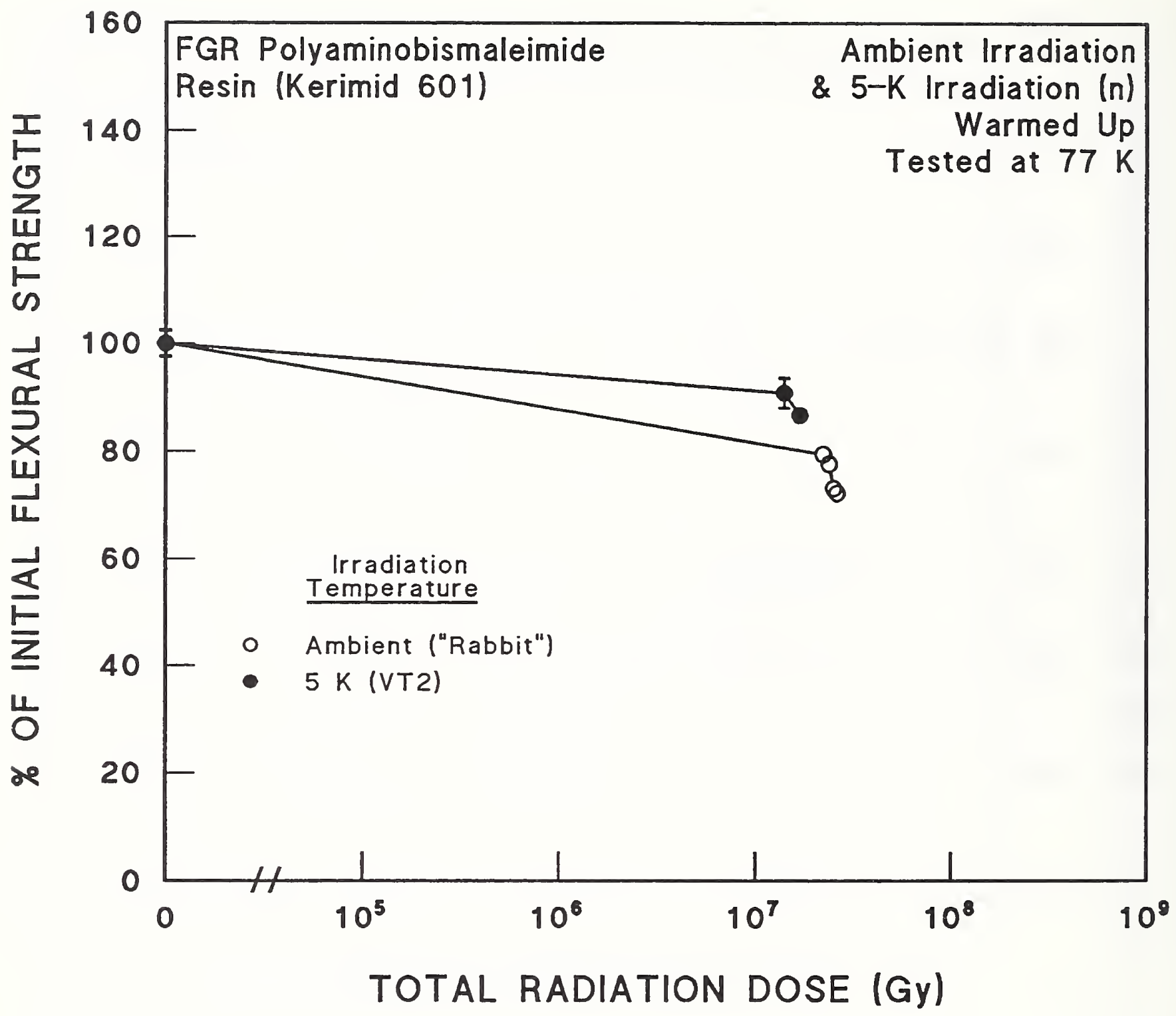

"O" Strength, MPa

$0, \bullet \quad 944.7 \pm 22.8$
Supplier

Sumitomo

Bakelite

Co., Ltd.

Figure B.1-6. Flexural strength at $77 \mathrm{~K}$ of FGR polyaminobismaleimide resin after ambient or $5-\mathrm{K}$ neutron (IPNS) irradiation. The spectrum at both irradiation thimbles was described as degraded fission with a high energy component. Supplementary Table B.1-1. Data from Egusa et al. [1987b]. 


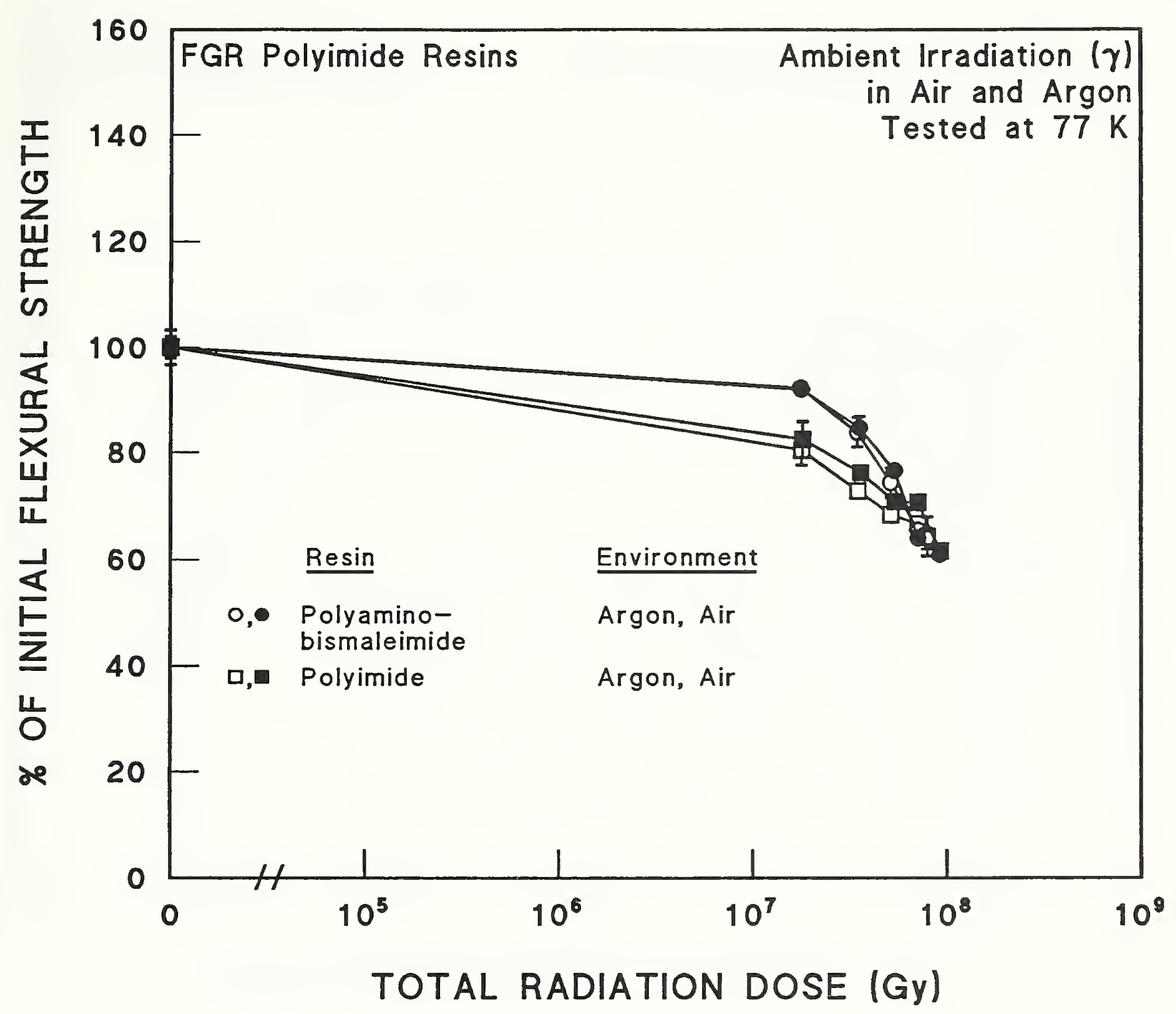

$\begin{array}{llcl}\text { "O" Strength, MPa } & \text { Type } & \underline{\text { Vol.\% }} & \underline{\text { Supplier }} \\ 0,01073.9 \pm 21.4 & \text { Kerimid } 601 & 62 & \begin{array}{l}\text { Sumitomo Bakelite } \\ \text { Co., Ltd. }\end{array} \\ \square, \pm 88.9 \pm 29.1 & \text { TIL G1000 } & 50 & \text { TORAY }\end{array}$

Figure B.1-7. Flexural strength at $77 \mathrm{~K}$ of FGR polyimide resin after ambient gamma irradiation in either air or argon. Supplementary Table A.3-4. Data from Egusa et al. [1991a]. 


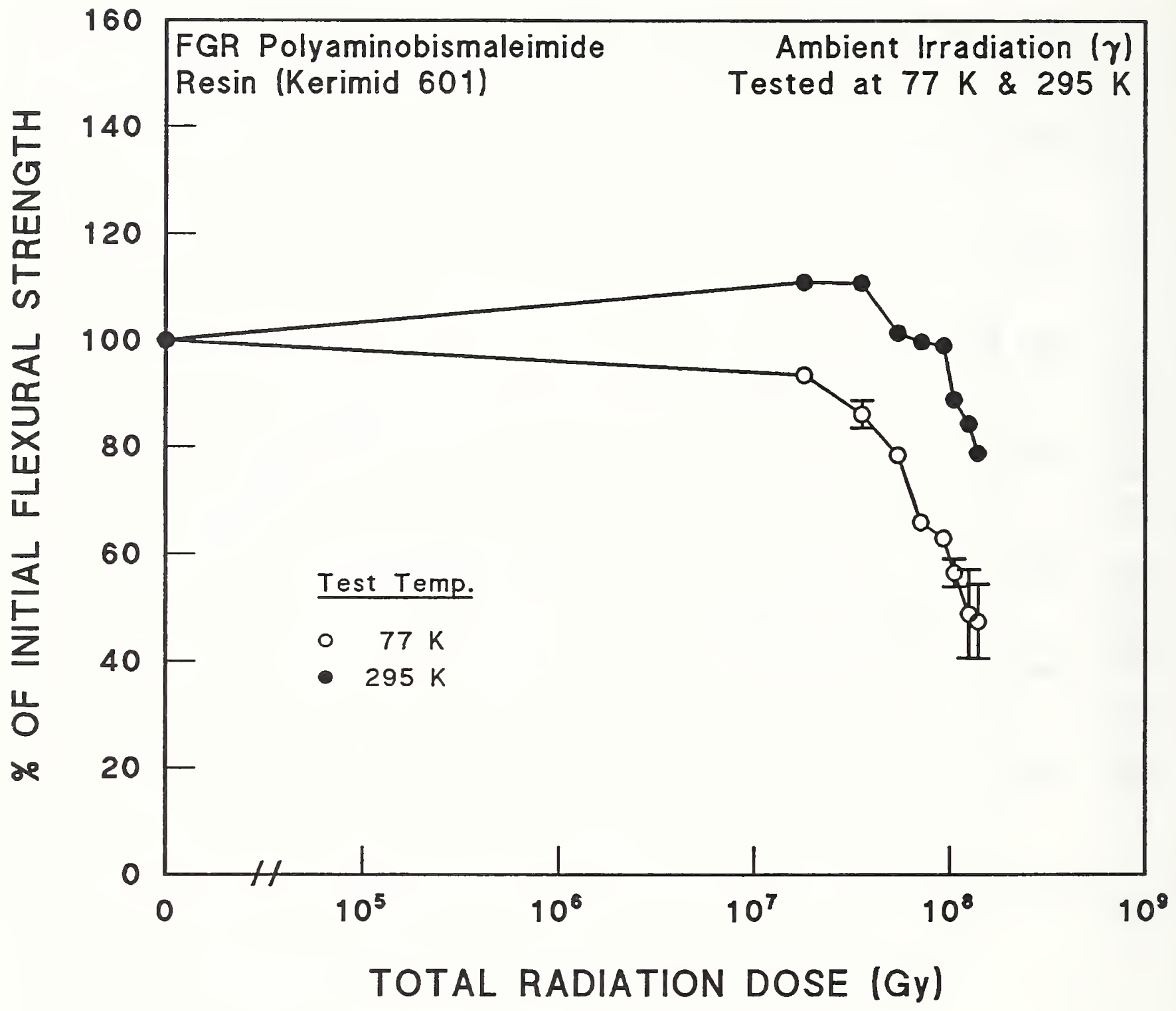

"O" Strength, MPa

- $1055.4 \pm 0.0$

- $\quad 524.4 \pm 0.0$
Supplier

Sumitomo Bakelite

Co., Ltd.

Sumitomo Bakelite Co., Ltd.

Figure B.1-8. Flexural strength at 77 and $295 \mathrm{~K}$ of FGR polyaminobismaleimide resin after ambient gamma irradiation. Supplementary Table A.3-1. Data from Egusa [1988] . 


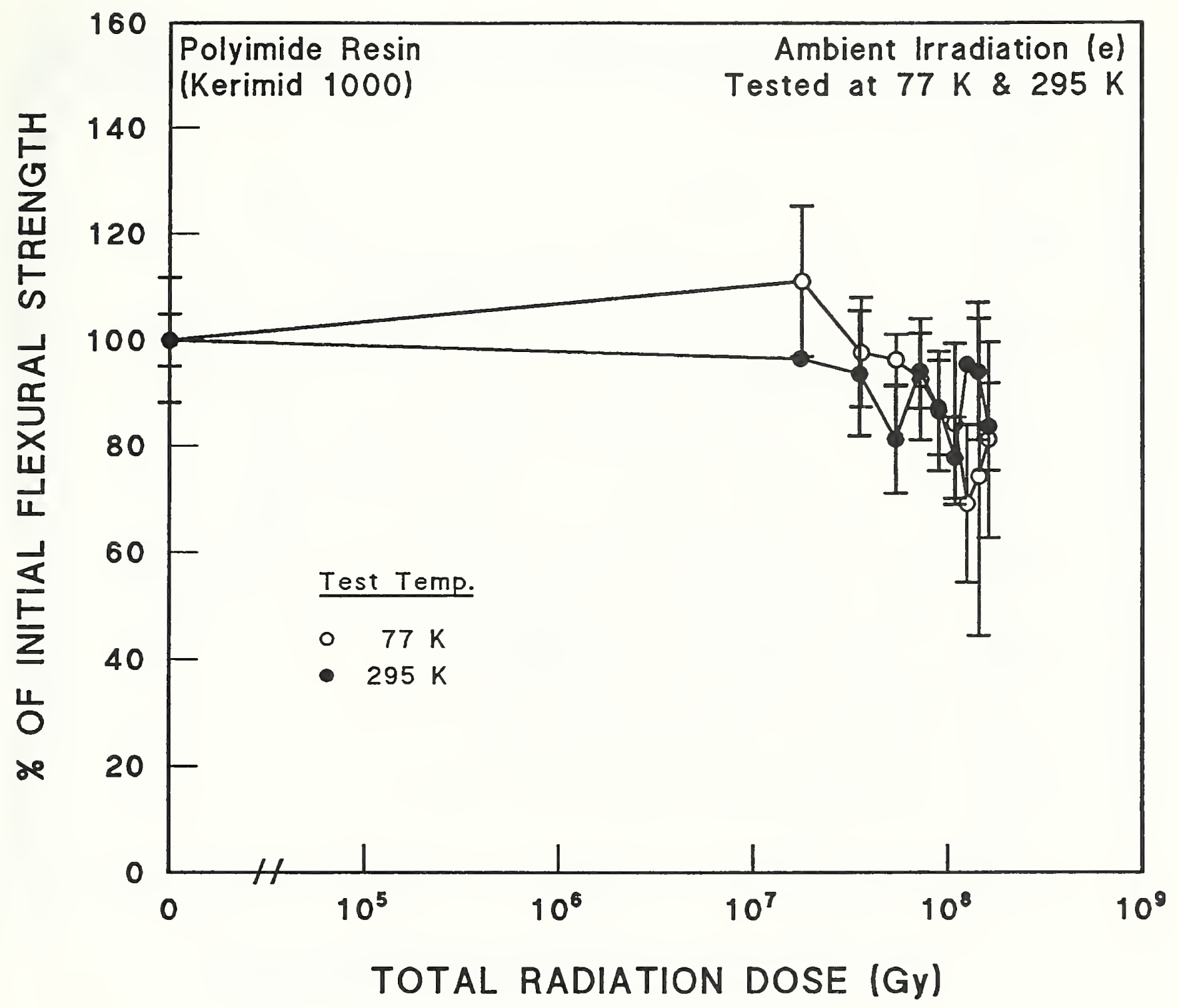

Supplier

Nippon Polyimide

Co., Ltd.

Nippon Polyimide

Co., Ltd.

Figure B.1-9. Flexural strength at 77 and $295 \mathrm{~K}$ of neat polyimide resin after ambient electron irradiation. Supplementary Table A.3-1. Data from Egusa [1988]. 


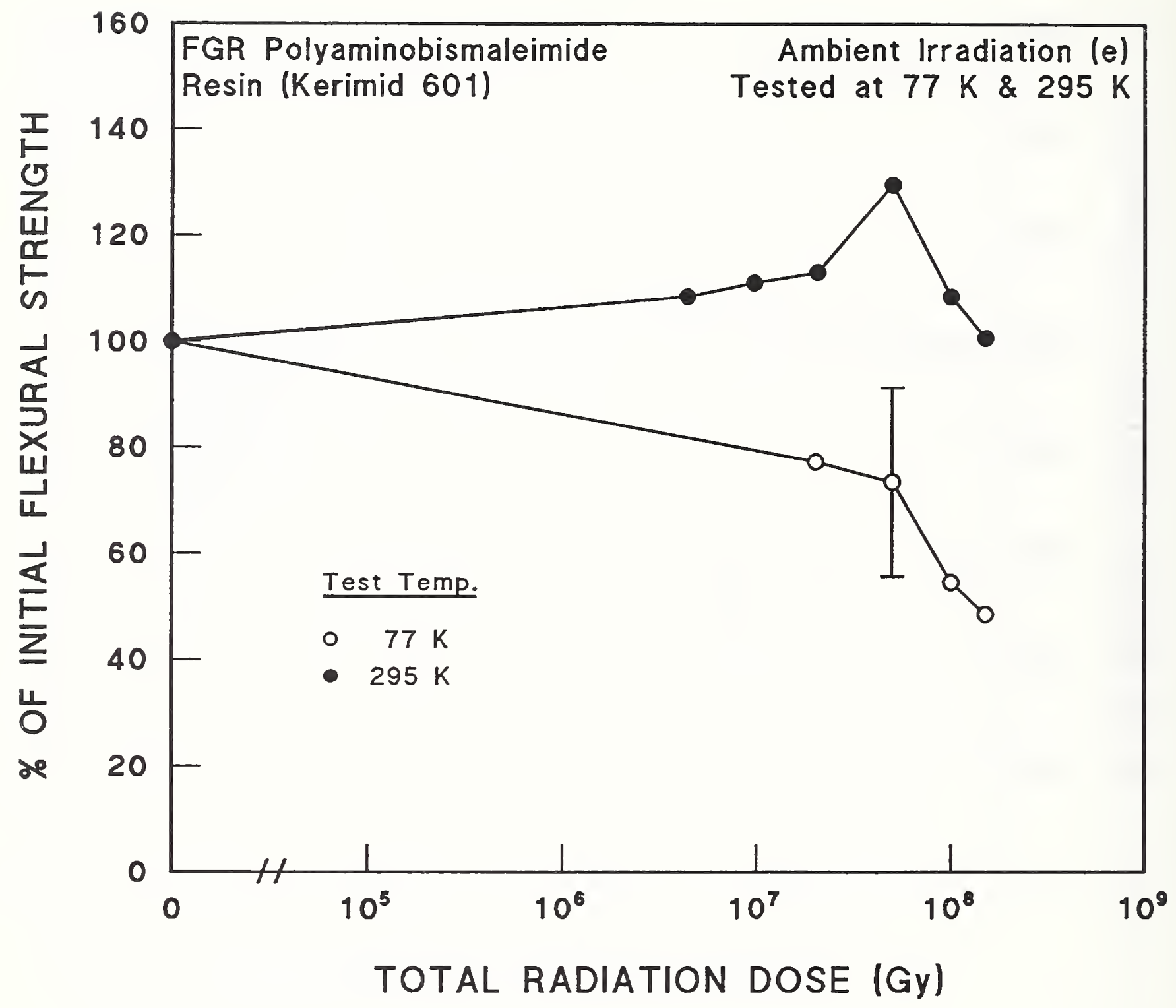

"O" Strength, MPa

$\circ \quad 935.3 \pm 0.0$

- $\quad 491.0 \pm 0.0$
Supplier

Sumitomo Bakelite Co., Ltd.

Sumitomo Bakelite Co., Ltd.

Figure B.1-10. Flexural strength at 77 and $295 \mathrm{~K}$ of FGR polyaminobismaleimide resin after ambient electron irradiation. Supplementary Table B.1-1. Data from Egusa et a1. [1985a]. 


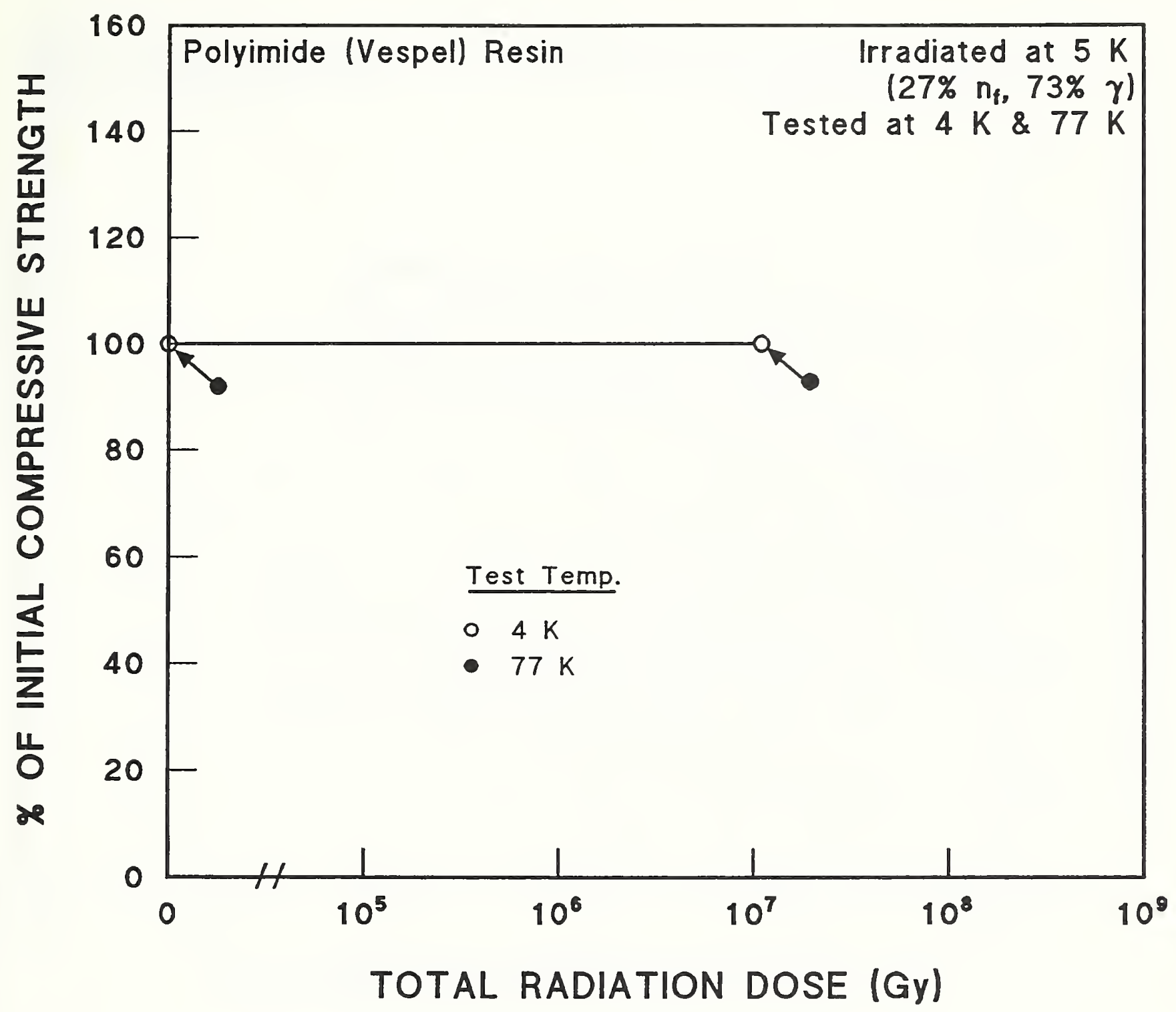

"O" Strength, MPa

- 432.4

- 680.6
Supplier

---

Figure B.1-11. Compressive strength at 4 and 77 of polyimide (Vespel) resin after 5-K reactor irradiation. Supplementary Table A.8-3. Data from Takamura and Kato [1980] . 


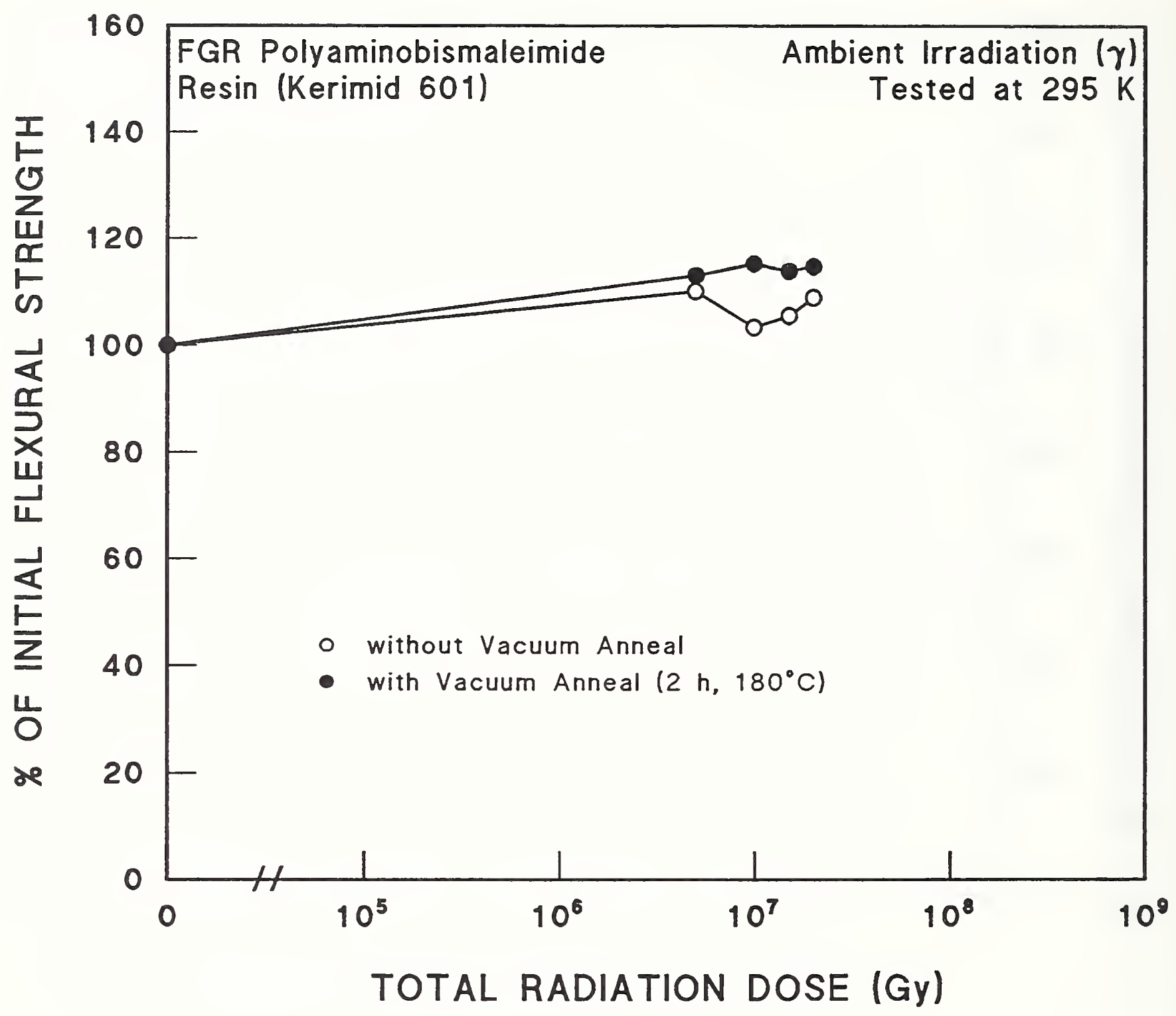

"O" Strength, MPa

$0495.6 \pm 0.0$

- $459.4 \pm 0.0$
Supplier

Sumitomo Bakelite

Co., Ltd.

Sumitomo Bakelite Co., Ltd.

Figure B.1-12. Flexural strength at $295 \mathrm{~K}$ of FGR polyaminobismaleimide resin, with and without vacuum anneal, after ambient gamma irradiation. Supplementary Table B.1-1. Data from Egusa et al. [1985b]. 


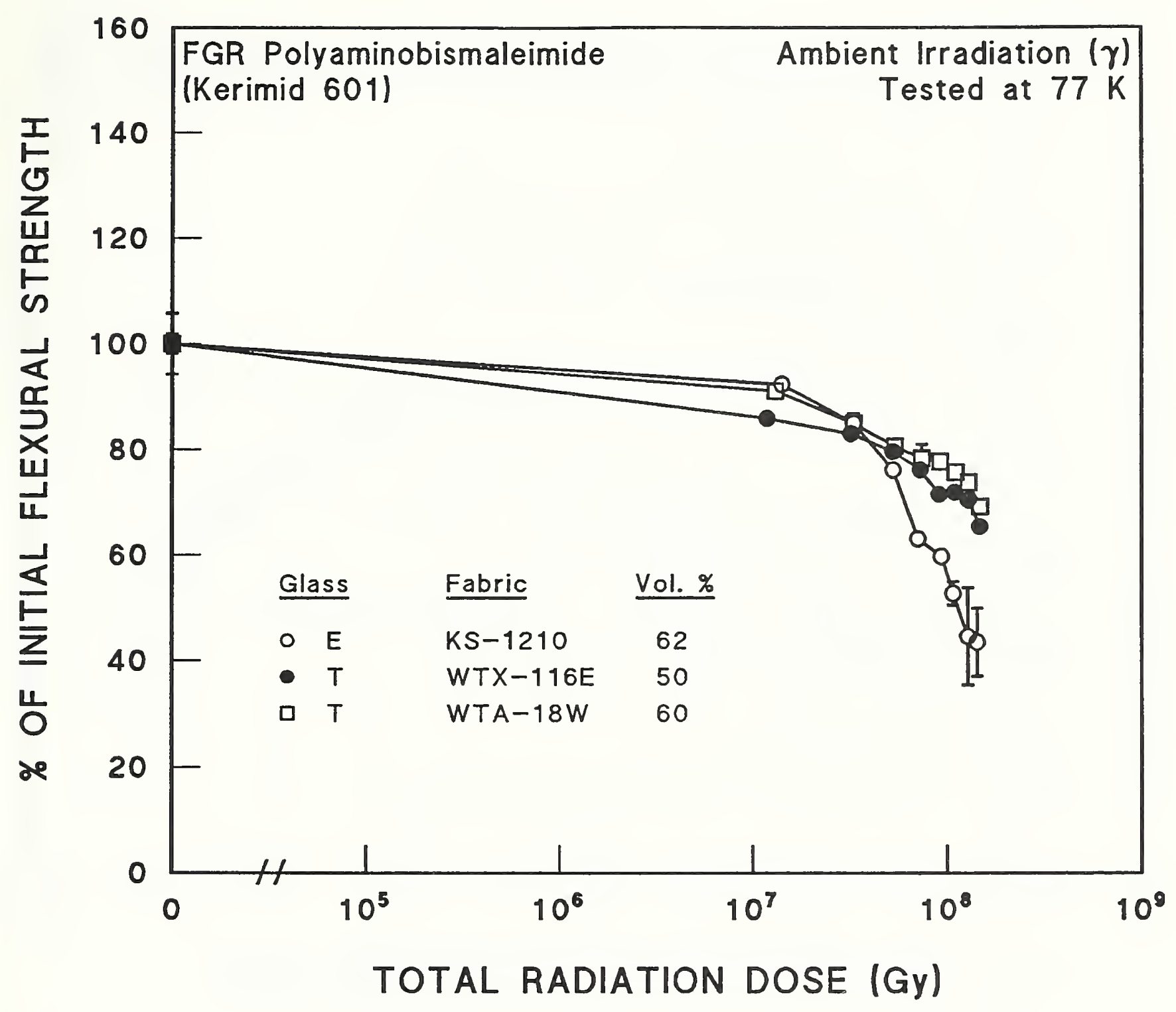

Yarn \#/25mm

"O" Strength, MPa

warp/fill

Fiber\#/yarn

Fiber diam. Supplier

- $1093.4 \pm 20.0$

$53 / 48$

200

$7 \mu \mathrm{m}$

Sumitomo

Bakelite

Co., Ltd.

- $1152.2 \pm 0.0$

$60 / 58$

200

$7 \mu \mathrm{m}$

Mitsui

ㅁ $760.0 \pm 44.0$

$44 / 34$

400

$9 \mu \mathrm{m}$

Petrochemical

Mitsui

Petrochemical

Figure B.1-13. Flexural strength at $77 \mathrm{~K}$ of FGR polyaminobismaleimide resin after ambient gamma irradiation. Comparison of $\mathrm{E}$ - and $\mathrm{T}$-glass reinforcement.

Supplementary Table A.3-4. Data from Egusa et al. [1991a]. 


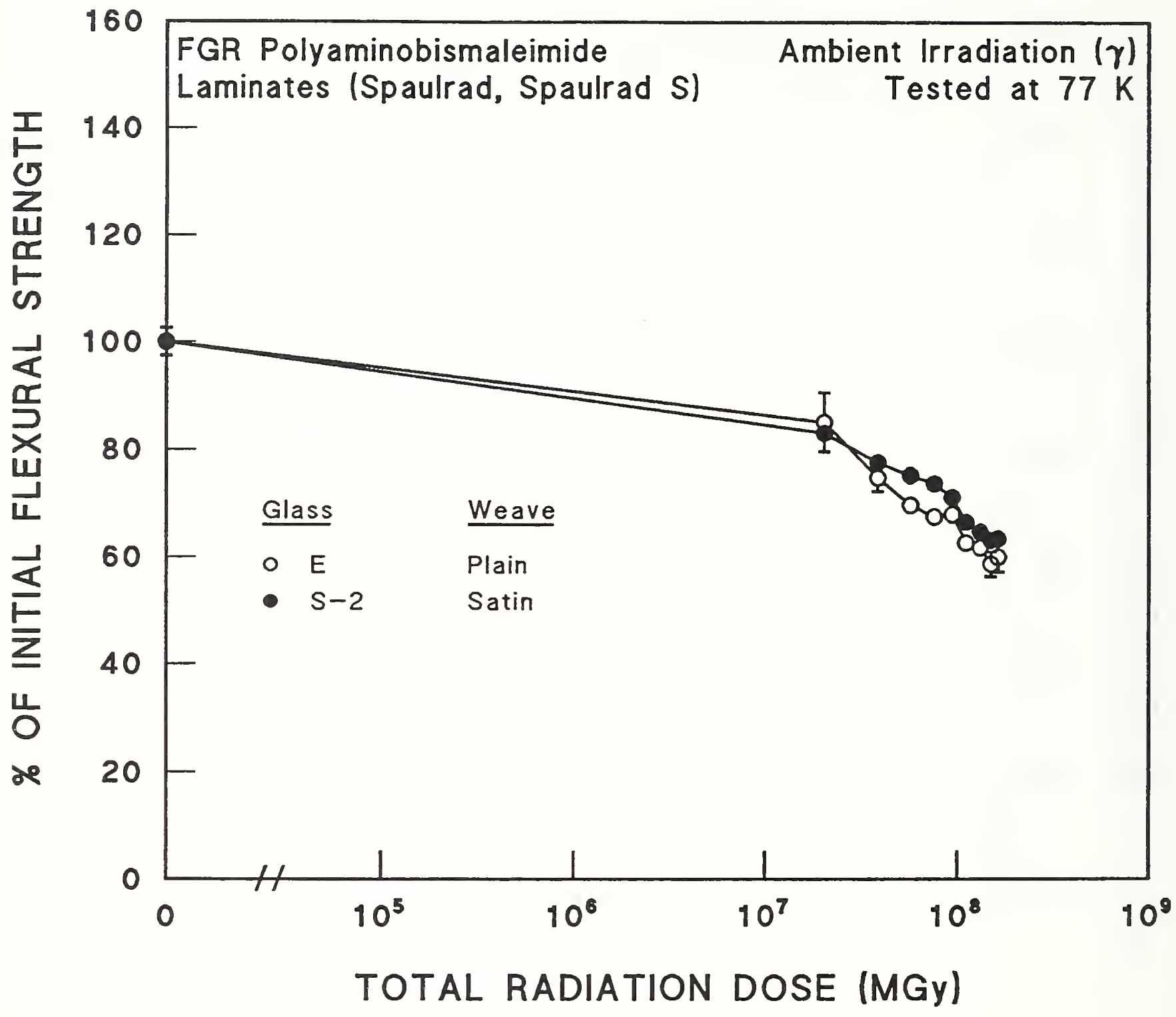

\begin{tabular}{|c|c|c|c|}
\hline "O" & Strength, MPa & Laminate Type & Supplier \\
\hline 0 & $976.1 \pm 25.0$ & Spaulrad & $\begin{array}{l}\text { Spaulding } \\
\text { Fiber Co. }\end{array}$ \\
\hline$\bullet$ & $1070.3 \pm 0.0$ & Spaulrad S & $\begin{array}{l}\text { Spaulding } \\
\text { Fiber Co. }\end{array}$ \\
\hline
\end{tabular}

Figure B.1-14. Flexural strength at $77 \mathrm{~K}$ of FGR polyaminobismaleimide laminates after ambient gamma irradiation. Comparison of E- and S-2-glass reinforcement. Supplementary Table A.3-4. Data from Egusa et al. [1991a]. 


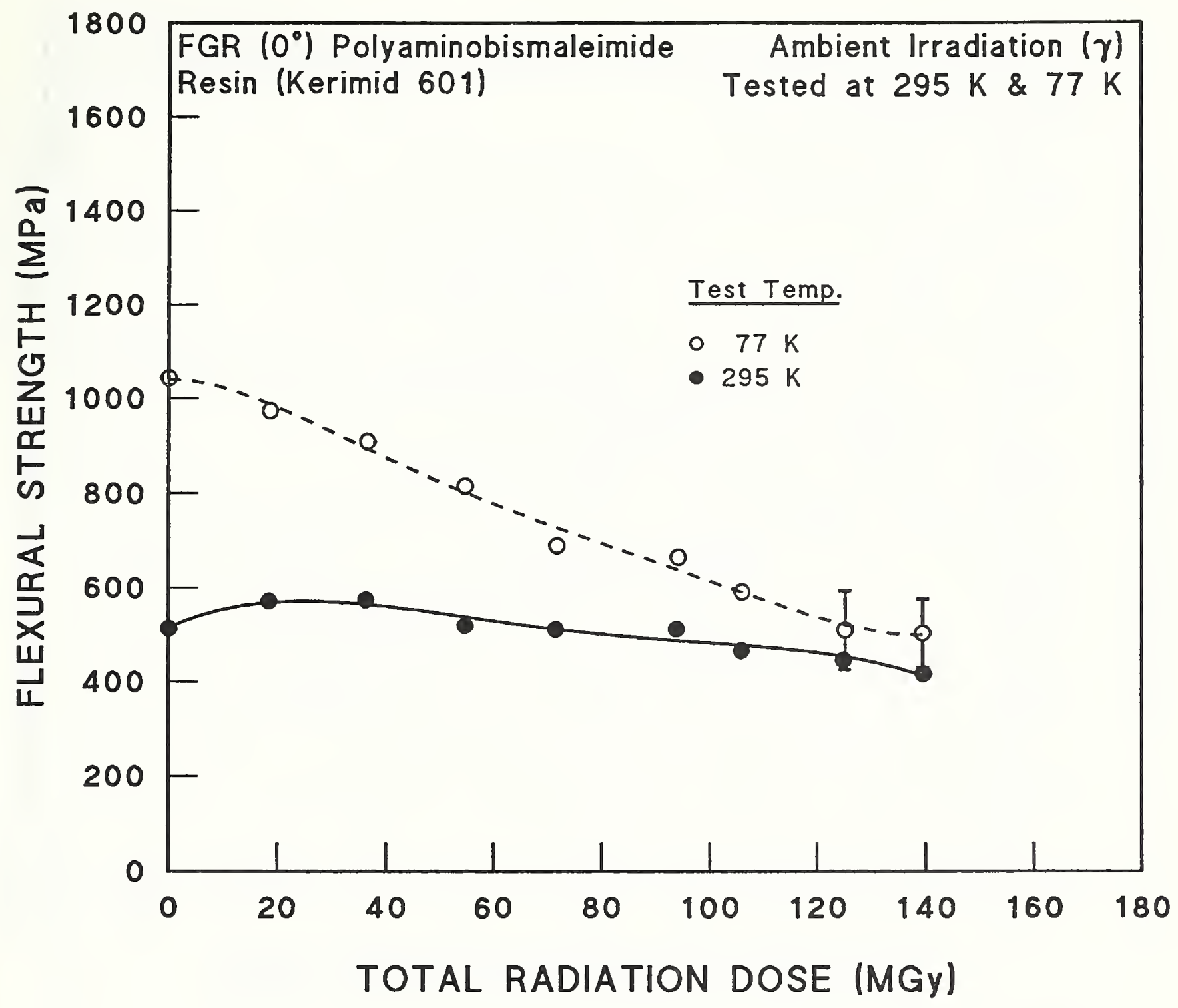

Supplier

0,0 Sumitomo

Bakelite Co., Ltd.

Figure B.1-15. Flexural strength at 77 and $295 \mathrm{~K}$ of FGR polyaminobismaleimide resin after ambient gamma irradiation. Specimen orientation at $0^{\circ}$ to warp. Supplementary Table B.1-1. Data from Egusa [1990a]. 


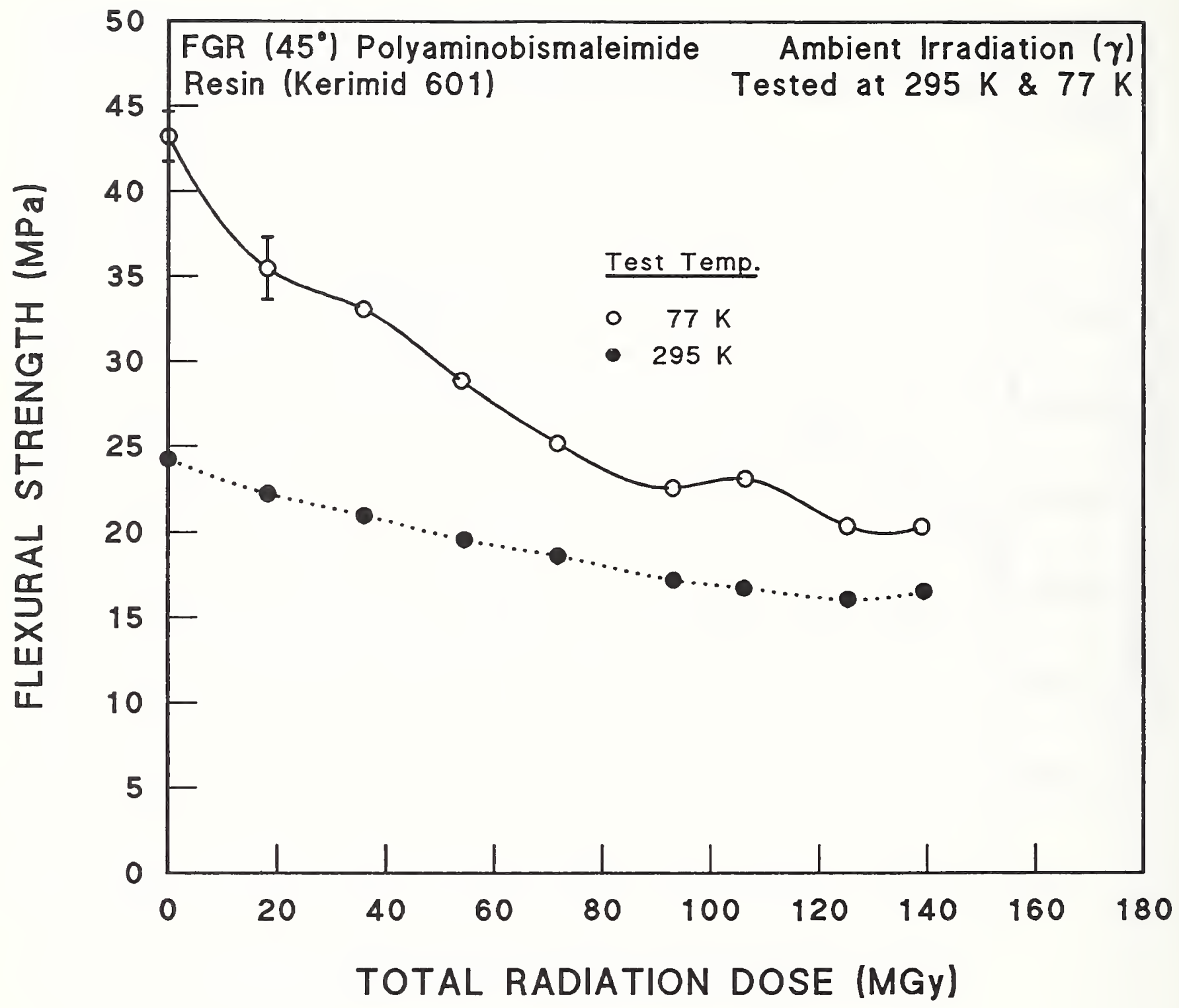

Supplier

0,0 Sumitomo

Bakelite Co., Ltd.

Figure B.1-16. Flexural strength at 77 and $295 \mathrm{~K}$ of FGR polyaminobismaleimide resin after ambient gamma irradiation. Specimen orientation at $45^{\circ}$ to warp.

Supplementary Table B.1-1. Data from Egusa [1990a]. 


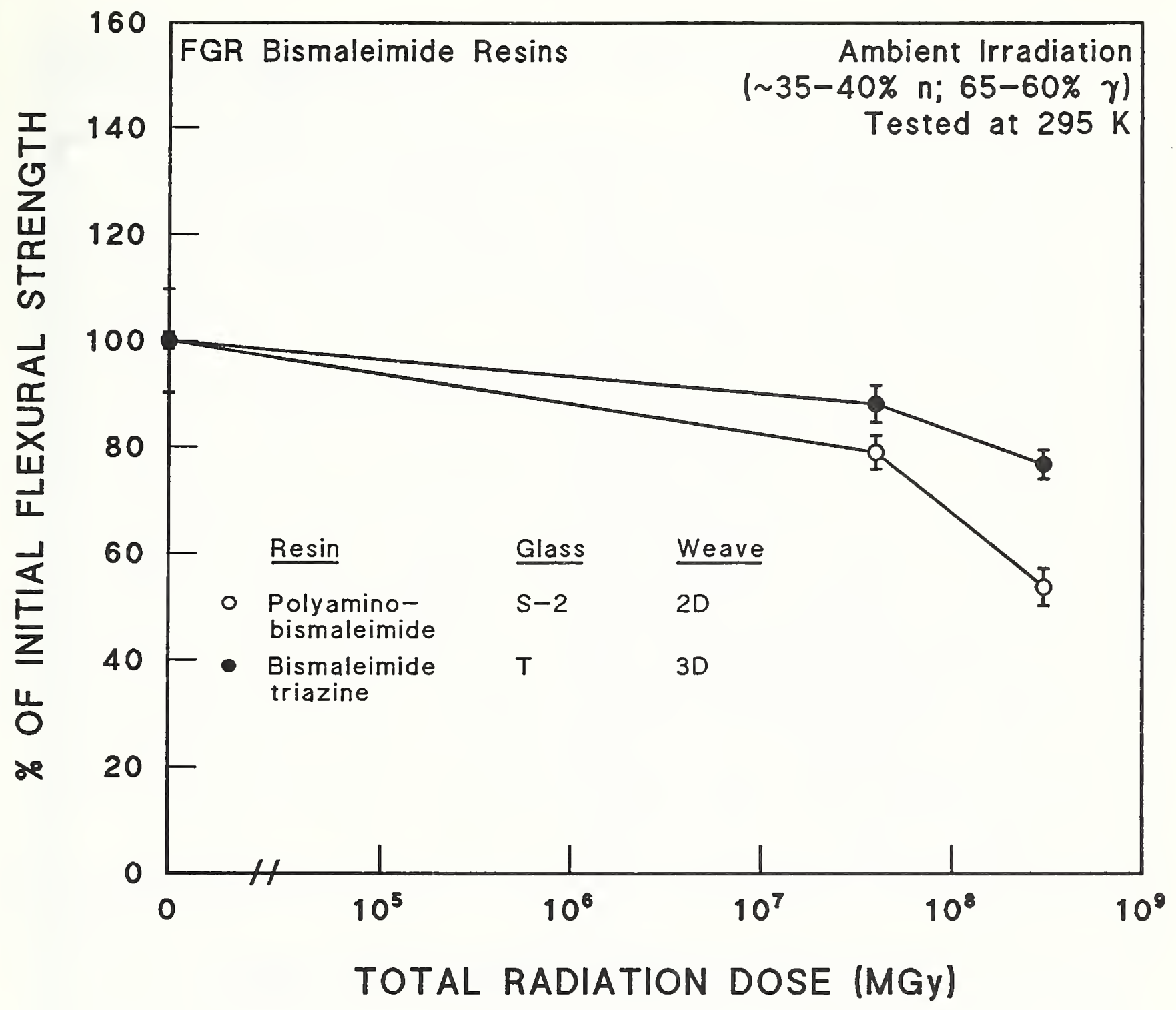

\begin{tabular}{llll} 
"O" Strength, MPa & & Laminate Type & Supplier \\
\hline $0675.1 \pm 66.0$ & & Spaulrad S & Spaulding \\
- $946.4 \pm 14.1$ & PG 5-1 & Shikishima
\end{tabular}

Figure B.1-17. Flexural strength at $295 \mathrm{~K}$ of FGR bismaleimide resins after ambient reactor irradiation. Supplementary Table A.2-17. Data from McManamy et al. [1990; 1991a]. 


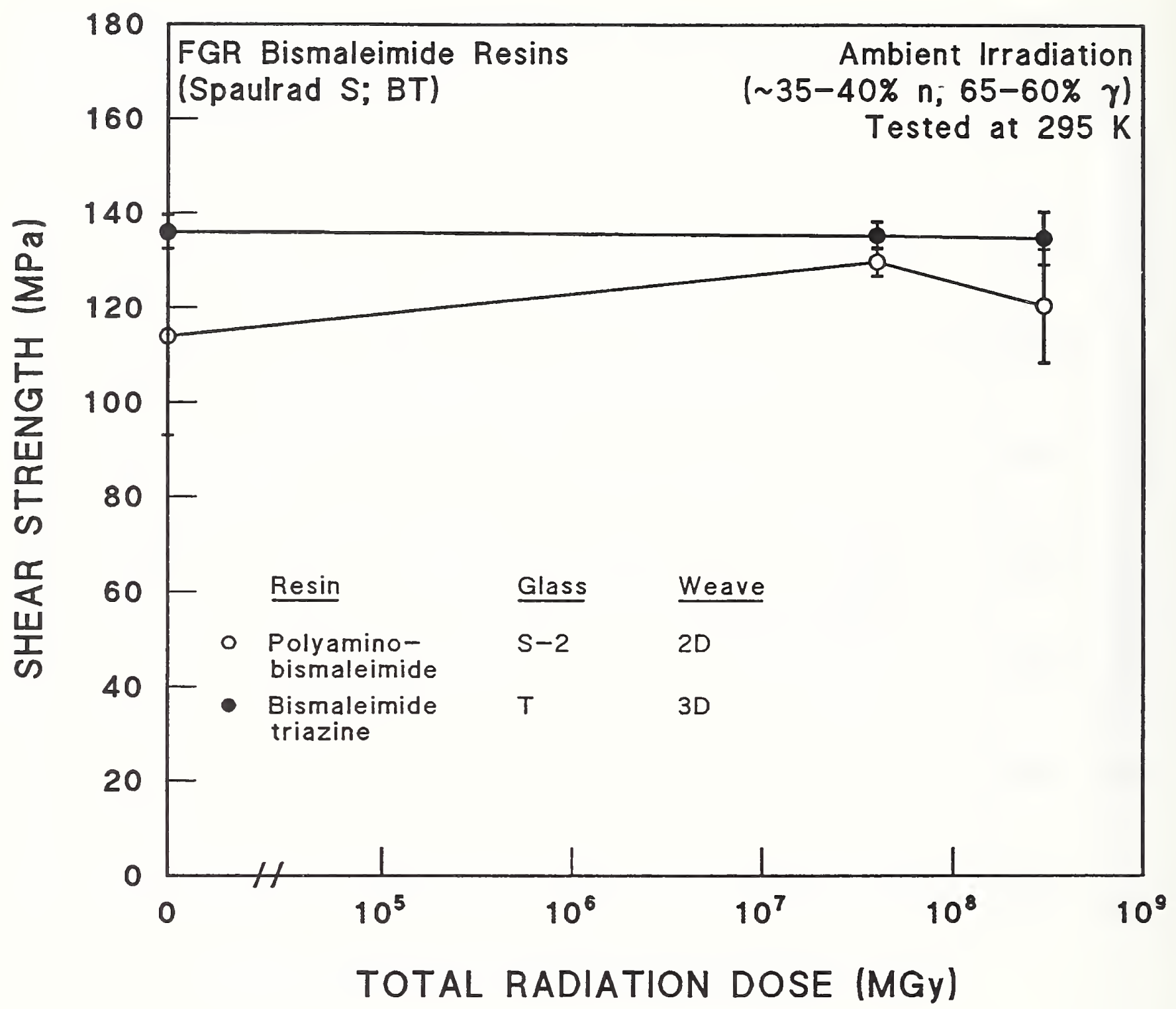

$\begin{array}{ll}\text { Laminate Type } & \text { Supplier } \\ \text { Spaulrad S } & \text { Spaulding } \\ \text { PG 5-1 } & \text { Shikishima }\end{array}$

Figure B.1-18. Shear strength with compressive load of $345 \mathrm{Mpa}$ at $295 \mathrm{~K}$ of FGR bismaleimide resins after ambient reactor irradiation. Supplementary Table A.2-17. Data from McManamy et al. [1990; 1991a]. 
Table B.1-1. Supplementary Information, Egusa et al. [1984a, b; 1985a, b; 1987b].

\begin{tabular}{|c|c|}
\hline IRRADIATION PARAMETERS & MATERIALS \\
\hline Source Type: Spallation neutron & Resin: \\
\hline Source Name: IPNS (ANL) & Hardener: \\
\hline $\begin{array}{l}\text { Irradiation } \\
\text { Temperature: } 5 \mathrm{~K} \text {, Ambient }\end{array}$ & Additives: \\
\hline Environment: $\mathrm{LHe}$ & Glass Vol. \&: \\
\hline Neutron $\&$ : high & Glass Mass \&: \\
\hline Neutron Flux: & Glass Type: $E$ \\
\hline Fast Neutron $\%$ : 70 of $n(E>M e V)$ & Glass Finish: * \\
\hline Fast Neutron Flux: & Glass Weave: Plain \\
\hline Gamma $q$ : low & Glass Areal Density, $\mathrm{kg} / \mathrm{m}^{2}$ : \\
\hline Gamma Energy: & Filament Diameter, $\mu \mathrm{m}: 7.36$ \\
\hline Gamma Dose Rate: & Layup: \\
\hline Electron $q$ : & Production Method: Machined \\
\hline Electron Energy: & $\begin{array}{l}\text { Other: } *^{*}- \\
\text { glycidoxypropyltrimethoxysilane }\end{array}$ \\
\hline \multicolumn{2}{|l|}{ Electron Dose Rate: } \\
\hline \multicolumn{2}{|l|}{ Other: } \\
\hline \multicolumn{2}{|c|}{ TEST METHOD } \\
\hline \multicolumn{2}{|l|}{ Test Type: Flexural strength } \\
\hline \multicolumn{2}{|l|}{ Test Specifications: } \\
\hline \multicolumn{2}{|l|}{ Specimen Shape: Rectangular bar } \\
\hline \multicolumn{2}{|c|}{ Specimen Dimensions: $70 \times 6.4 \times 2.0 \mathrm{~mm}^{3}$} \\
\hline \multicolumn{2}{|l|}{ Flexure: Span/Thickness Ratio: 10} \\
\hline \multicolumn{2}{|c|}{ Compression: Aspect Ratio, Length/width or Diam.: } \\
\hline \multicolumn{2}{|l|}{ Specimens Tested/Data Point: 3} \\
\hline \multicolumn{2}{|l|}{ Strain (Load) Rate: $0.06 \mathrm{~cm} / \mathrm{min}$} \\
\hline Test Temperature, $\mathrm{k}: 77,295$ & \\
\hline Other: & \\
\hline
\end{tabular}


Table B.1-2. Supplementary Information, Abe et a1. [1987].

\begin{tabular}{|c|c|}
\hline IRRADIATION PARAMETERS & MATERIALS \\
\hline Source Type: ${ }^{60} \mathrm{Co}(14-\mathrm{MeV} \mathrm{n})$ & Resin: \\
\hline Source Name: (RTNS II) & Hardener: \\
\hline $\begin{array}{l}\text { Irradiation } \\
\text { Temperature: } 295 \mathrm{~K} \quad(293-298 \mathrm{~K})\end{array}$ & Additives: \\
\hline Environment: He gas & Glass Vol. z: \\
\hline Neutron $\%: \quad 100$ & Glass Mass $q$ : \\
\hline Neutron F1ux: & Glass Type: \\
\hline Fast Neutron $\frac{8}{8}$ : & Glass Finish: \\
\hline Fast Neutron Flux: & Glass Weave: \\
\hline Gamma : : $\quad 100$ & Glass Areal Density, $\mathrm{kg} / \mathrm{m}^{2}$ : \\
\hline Gamma Energy: $\quad-1.2 \mathrm{MeV}$ & Filament Diameter, $\mu \mathrm{m}:$ \\
\hline Gamma Dose Rate: $10 \mathrm{~Gy} / \mathrm{s}$ & Layup: \\
\hline Electron $z:$ & Production Method: \\
\hline Electron Energy: & Other: \\
\hline \multicolumn{2}{|l|}{ Electron Dose Rate: } \\
\hline \multicolumn{2}{|l|}{ Other: } \\
\hline \multicolumn{2}{|c|}{ TEST METHOD } \\
\hline \multicolumn{2}{|l|}{ Test Type: Tensile (miniature) } \\
\hline \multicolumn{2}{|l|}{ Test Specifications: } \\
\hline \multicolumn{2}{|l|}{ Specimen Shape: } \\
\hline \multicolumn{2}{|c|}{ Specimen Dimensions: $10 \times 4 \mathrm{~mm}^{2} \times 73 \mu \mathrm{m}$; Gage length $2 \mathrm{~mm}$} \\
\hline \multicolumn{2}{|l|}{ Flexure: Span/Thickness Ratio: } \\
\hline \multicolumn{2}{|c|}{ Compression: Aspect Ratio, Length/Width or Diam.: } \\
\hline \multicolumn{2}{|l|}{ Specimens Tested/Data Point: 5 (?) } \\
\hline \multicolumn{2}{|l|}{ Strain (Load) Rate: $0.04 \mathrm{~cm} / \mathrm{min}$} \\
\hline \multicolumn{2}{|l|}{ Test Temperature, $\mathrm{K}$ : } \\
\hline Other: & \\
\hline
\end{tabular}




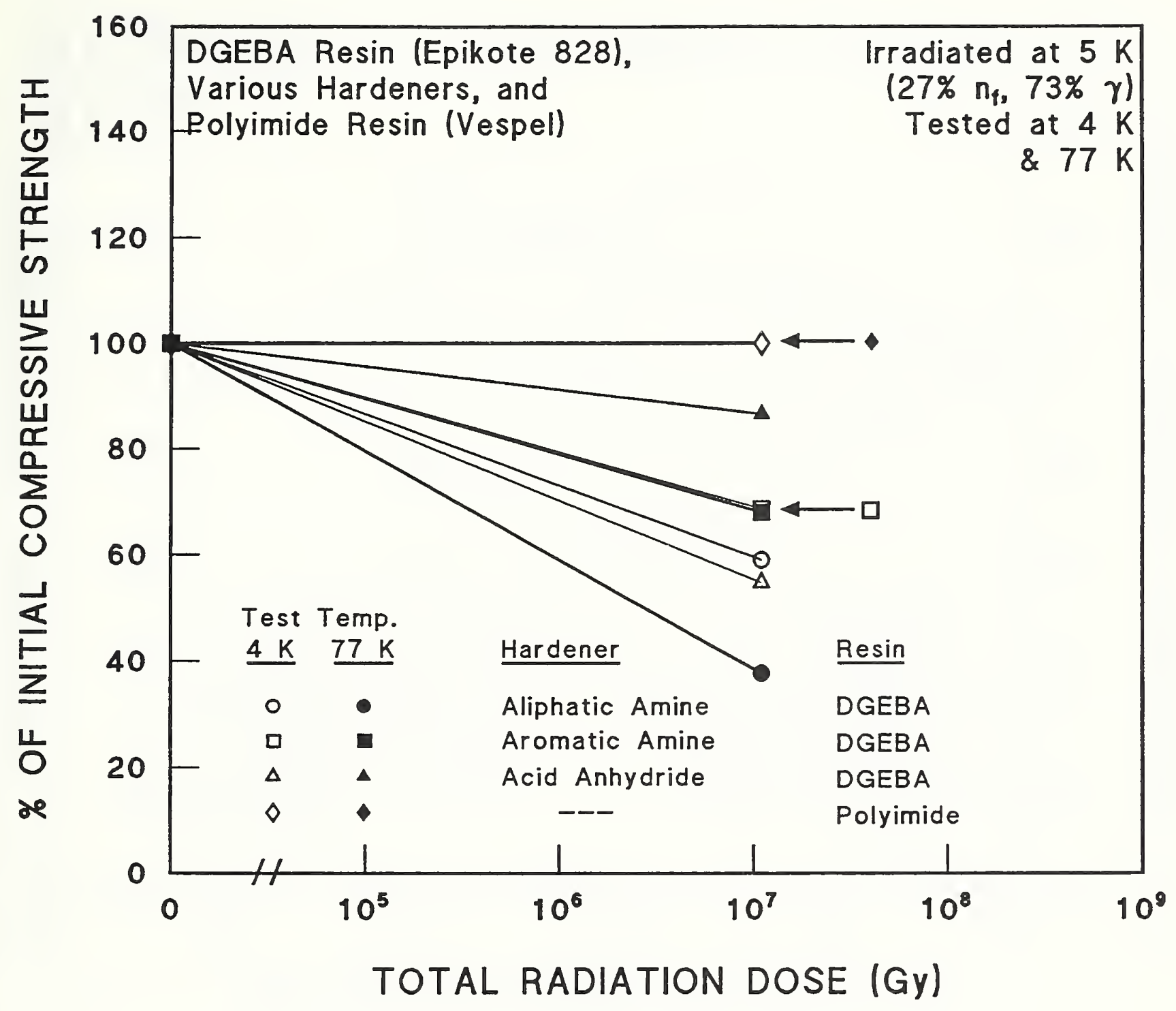

\begin{tabular}{lll} 
"O" Strength, MPa & Supplier \\
\hline$\circ \quad 500.7$ & Shell \\
- 532.3 & Shell \\
461.6 & Shell \\
D 529.7 & Shell \\
$\Delta \quad 443.5$ & Shell \\
$\Delta \quad 405.7$ & Shell \\
$\diamond \quad 432.4$ & Shell \\
& 680.6 & Shell
\end{tabular}

Figure B.2-1. Compressive strength at 4 and $77 \mathrm{~K}$ of FGR DGEBA and polyimide (Vespel) resins after 5-K reactor irradiation. Supplementary Table A.8-3. Data from Takamura and Kato [1980]. 


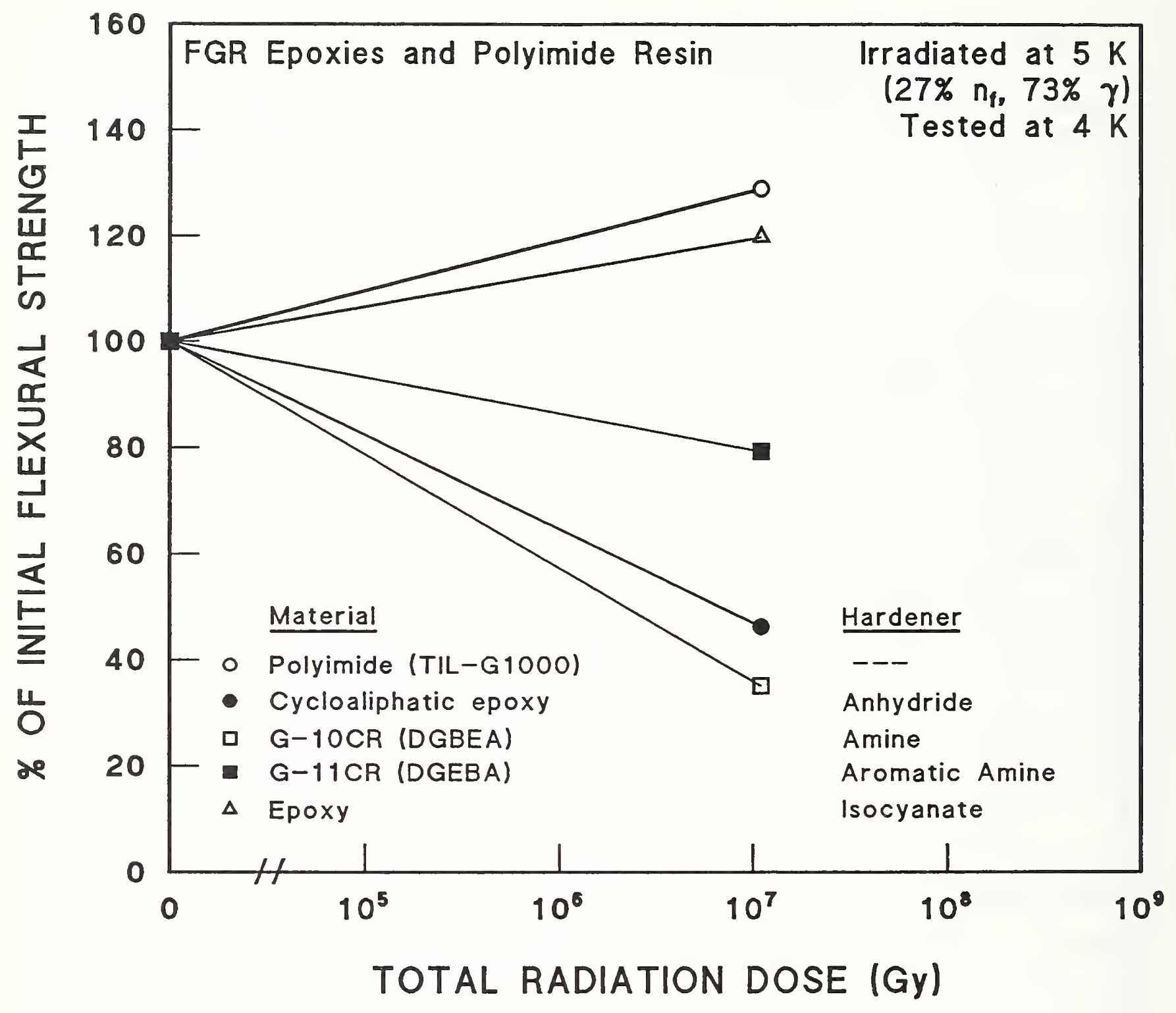

"O" Strength, MPa

- 846.5

- 1077.4

ㅁ 1073.2

- 887.6

$\triangle \quad 440.0 * *$
Supplier * $^{*}$

- -

-

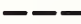

- -

$--$

* Some specimens supplied by Kobayashi Kigata, Ltd. and Hitachi, Ltd. * ISOX

Figure B.2-2. Flexural strength at $4 \mathrm{~K}$ of FGR epoxies and polyimide (TIL-G1000) resin after 5-K reactor irradiation. Supplementary Table A.9-1. Data from Takamura and Kato [1984]. 

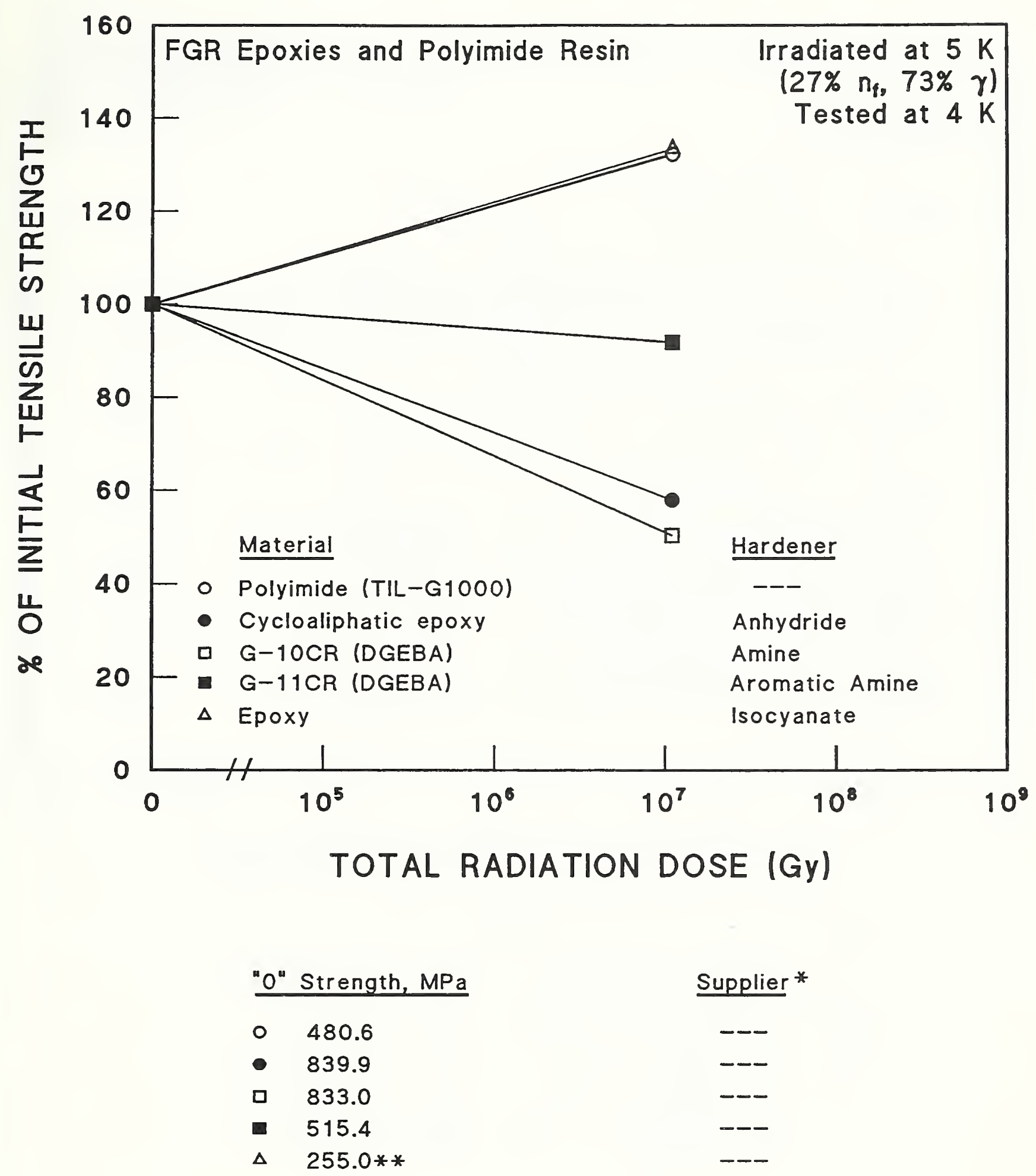

Supplier *

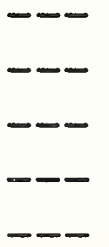

* Some specimens supplied by Kobayashi Kigata, Ltd. and Hitachi, Ltd. * ISOX

Figure B.2-3. Tensile strength at $4 \mathrm{~K}$ of FGR epoxies and polyimide (TIL-G1000) resin after 5-K reactor irradiation. Supplementary Table A.9-1. Data from Takamura and Kato [1984]. 


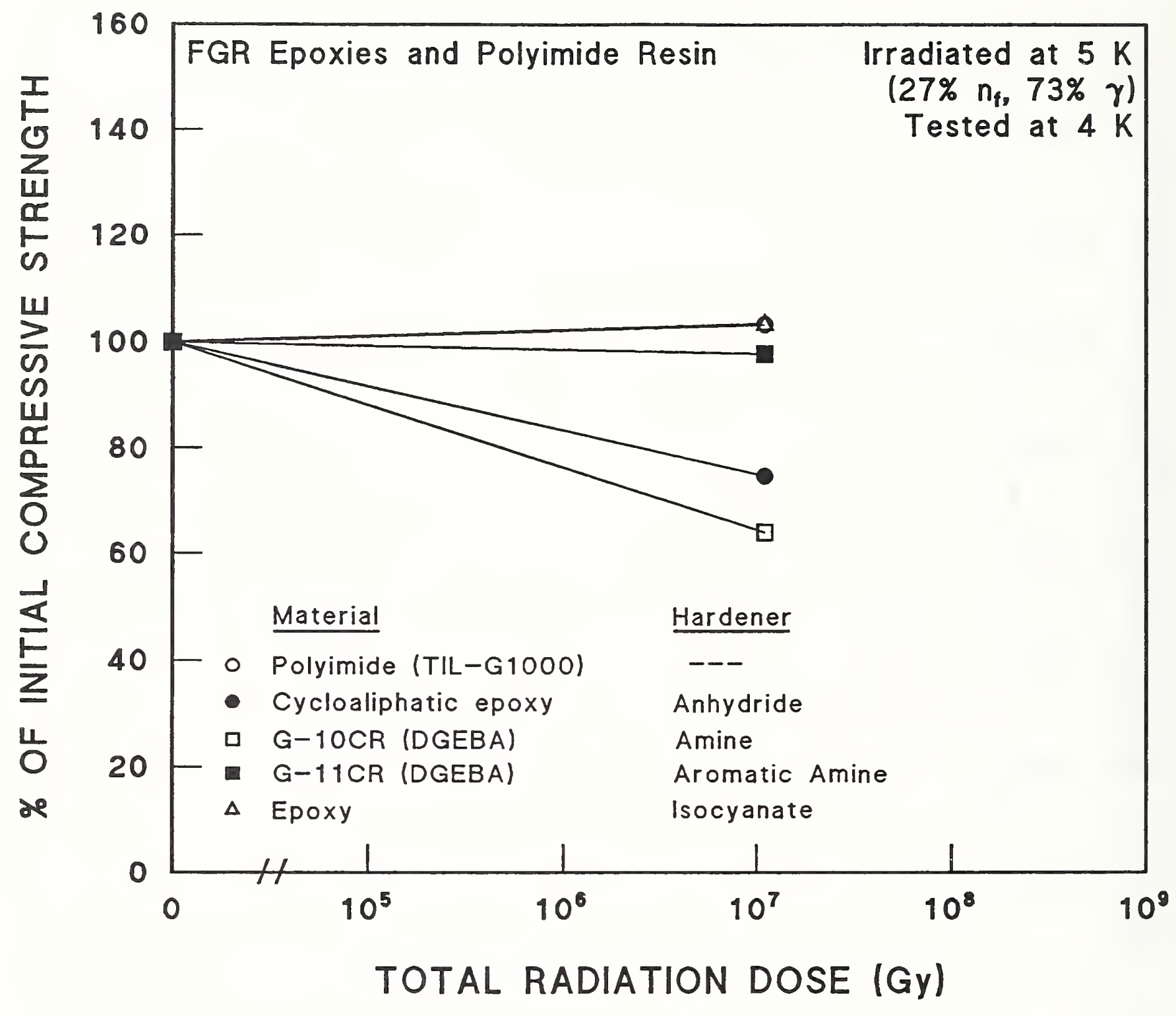

"O" Strength, MPa

- 844.7

- 850.7

ㅁ 898.2

- 1063.7

$\Delta$ 790.0**
Supplier *

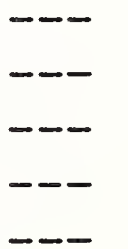

* Some specimens supplied by Kobayashi Kigata, Ltd. and Hitachi, Ltd. ** ISOX

Figure B.2-4. Compressive strength at $4 \mathrm{~K}$ of FGR epoxies and polyimide (TIL-G1000) resin after 5-K reactor irradiation. Supplementary Table A.9-1. Data from Takamura and Kato [1984]. 


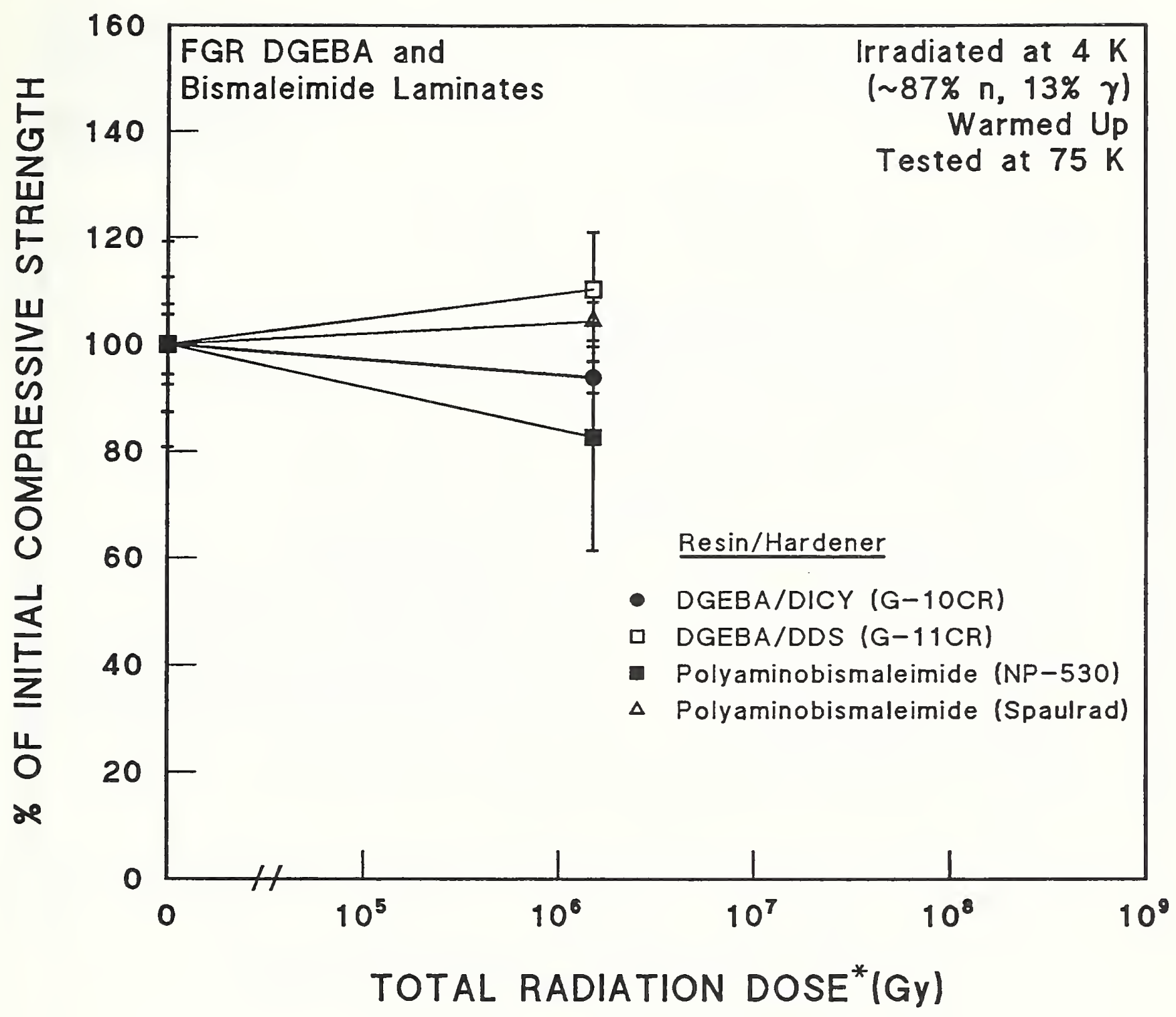

"O" Strength, MPa

- $838.0 \pm 62.9$

ㅁ $809.0 \pm 101.9$

- $512.0 \pm 28.7$

$\Delta \quad 523.0 \pm 100.2$
Supplier

$---$

Norplex

Spaulding

* Neglects $\gamma$ contribution, estimated at $\sim 15 \%$ of neutron dose

Figure B.2-5. Compressive strength at $75 \mathrm{~K}$ of FGR DGEBA and bismaleimide laminates (G-10CR, G-11CR, NP-530, and Spaulrad) after 4-K reactor irradiation. Supplementary Table A.8-1. Data from Hurley et al. [1978; 1983]. 


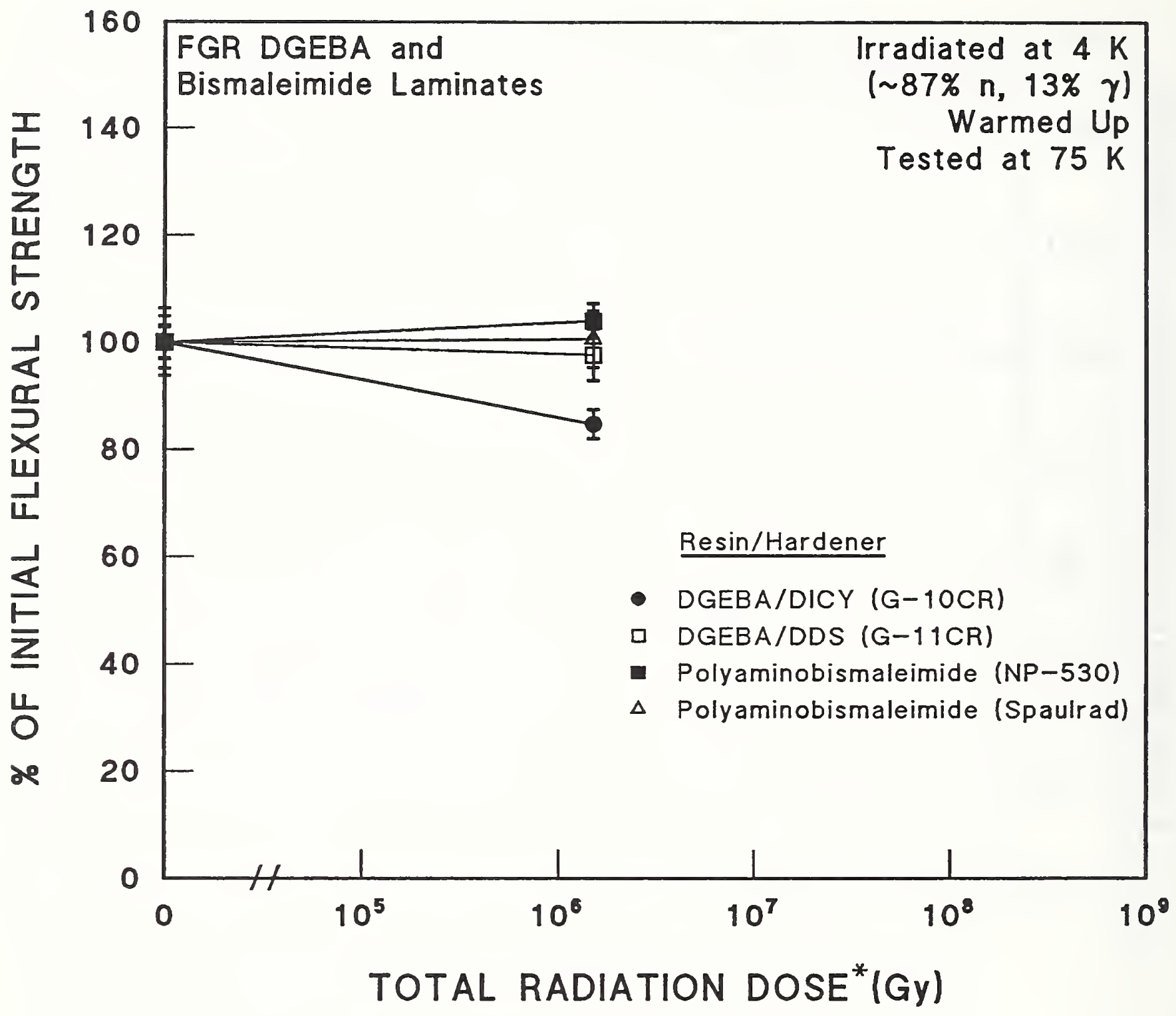

\begin{tabular}{|c|c|}
\hline "O" Strength, MPa & Supplier \\
\hline - $1162.9 \pm 73.1$ & --- \\
\hline ㅁ $1394.0 \pm 41.1$ & --- \\
\hline $734.9 \pm 36.2$ & Norplex \\
\hline $846.0 \pm 26.7$ & Spaulding \\
\hline
\end{tabular}

* Neglects $\gamma$ contribution, estimated at $\sim 15 \%$ of neutron dose

Figure B.2-6. Flexural strength at $75 \mathrm{~K}$ of FGR DGEBA and bismaleimide laminates (G10CR, G-1ICR, NP-530, and Spaulrad) after 4-K reactor irradiation. Supplementary Table A.8-1. Data from Hurley et al. [1978; 1983]. 


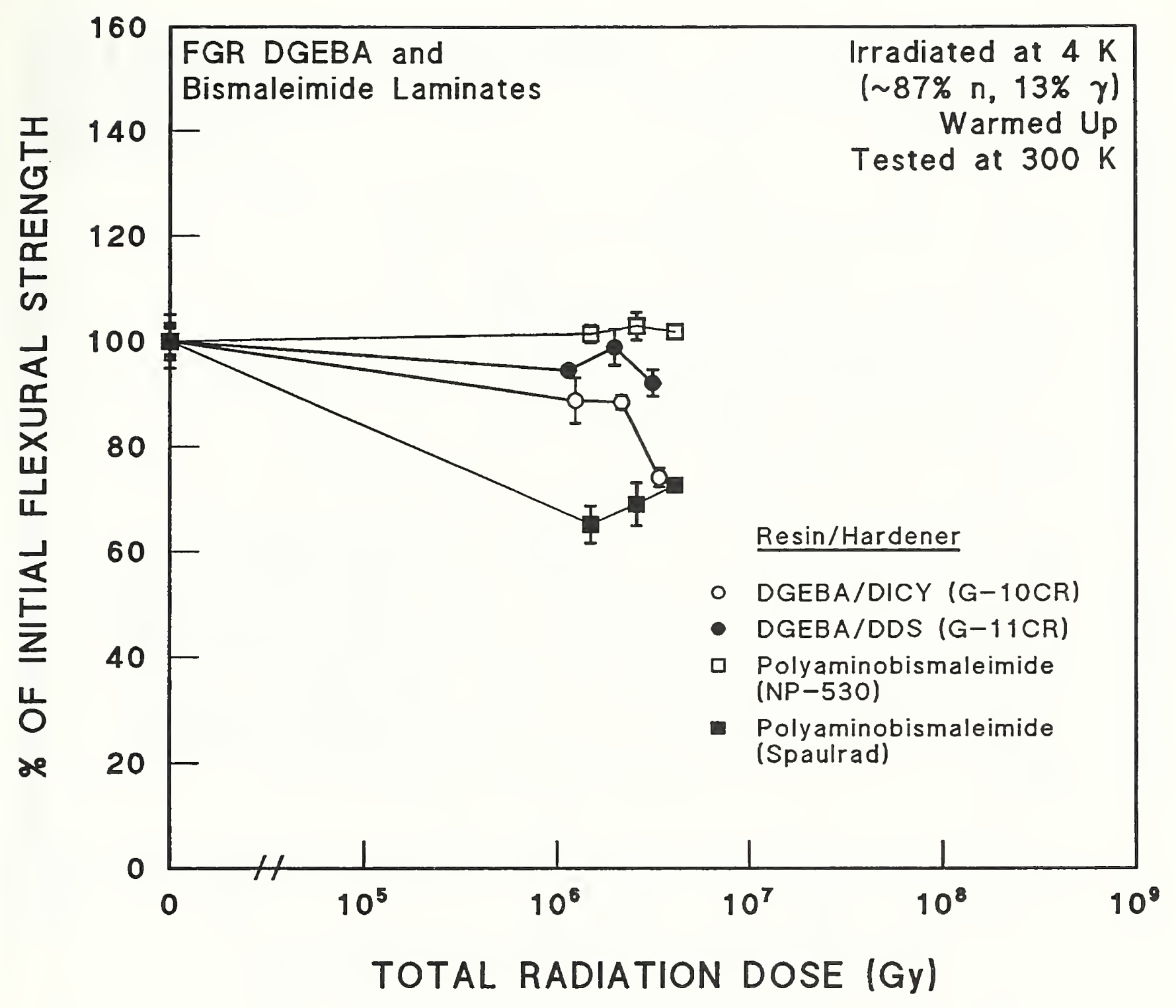

"O" Strength, MPa

- $603.0 \pm 15.0$

- $727.0 \pm 27.0$

ㅁ $538.0 \pm 19.0$

- $835.0 \pm 24.0$
Supplier

$---$

---

Norplex

Spaulding

Figure B.2-7. Flexural strength at $300 \mathrm{~K}$ of FGR DGEBA and bismaleimide laminates (G-10CR, G-11CR, NP-530, and Spaulrad) after 4-K reactor irradiation. Supplementary Table A.8-1. Data from Tucker et al. [1985]. 


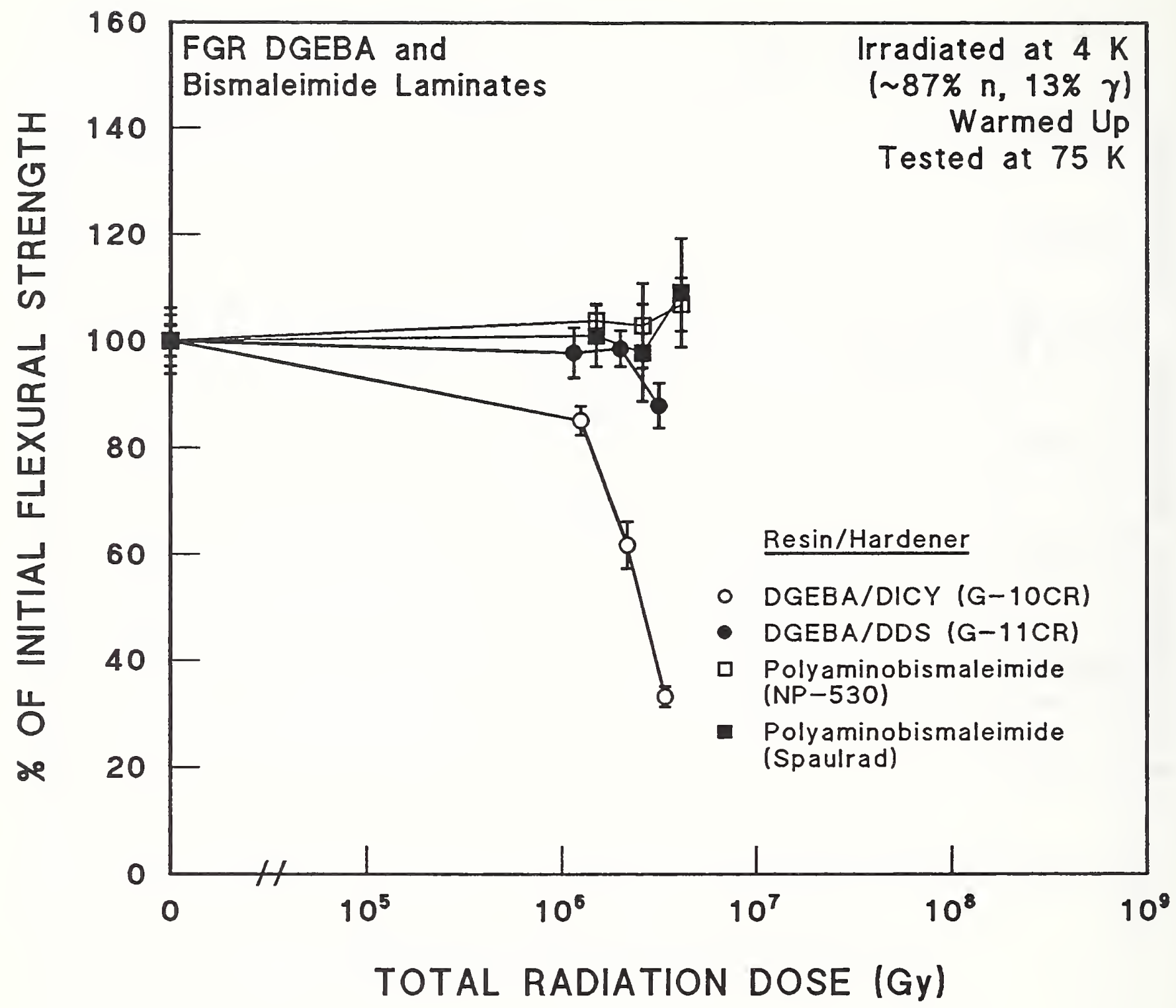

"O" Strength, MPa

- $1167.0 \pm 72.0$

- $1393.0 \pm 42.0$

$734.0 \pm 35.0$

- $835.0 \pm 24.0$
Supplier

--

Norplex

Spaulding

Figure B.2-8. Flexural strength at $75 \mathrm{~K}$ of FGR DGEBA and bismaleimide laminates (G10CR, G-11CR, NP-530, and Spaulrad) after 4-K reactor irradiation. Supplementary Table A.8-1. Data from Tucker et al. [1985]. 


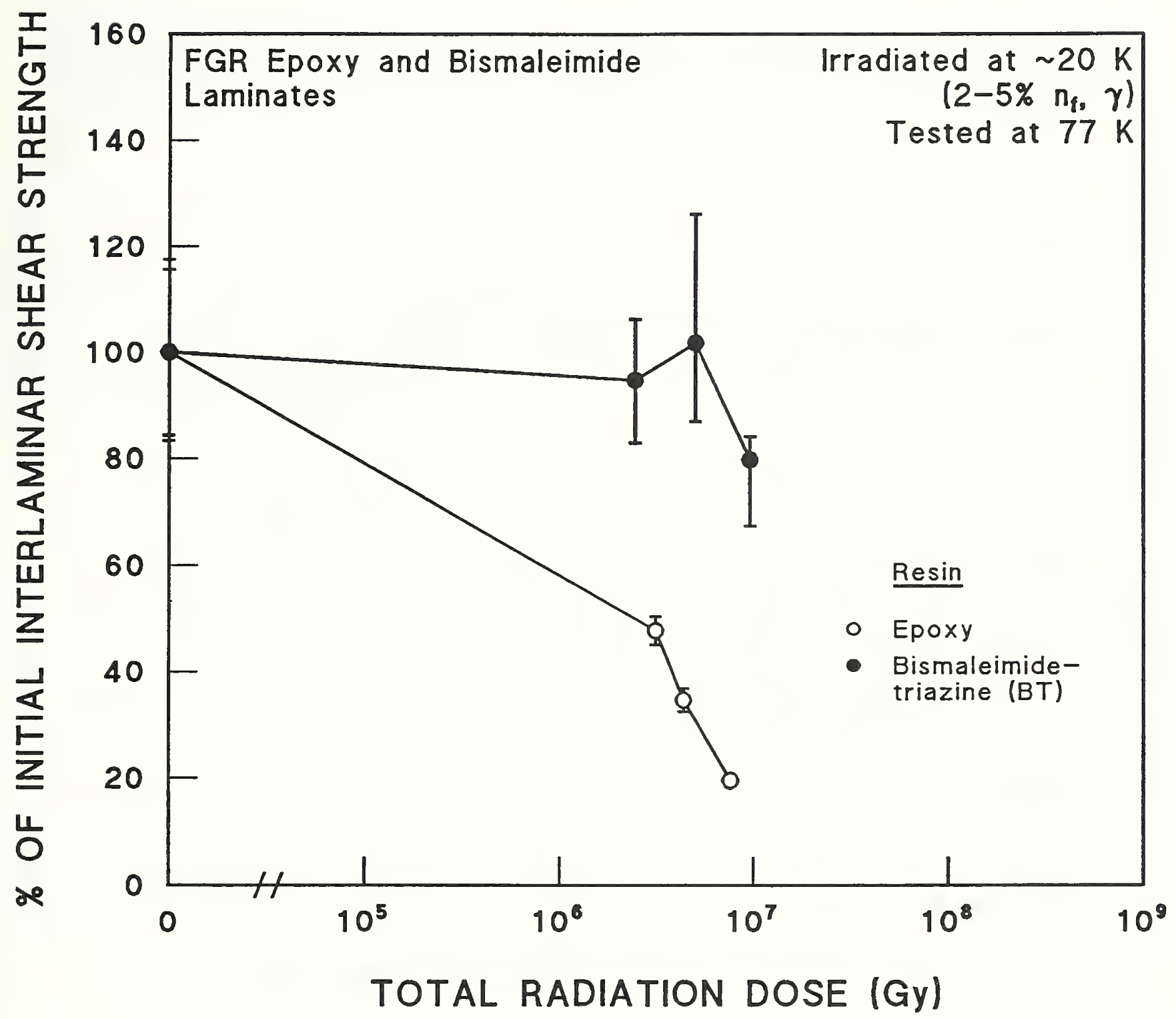

"O" Strength, MPa

- $120.6 \pm 18.9$

- $95.3 \pm 16.3$
Glass Yol. Fr.

$50 \%$

$50 \%$
Supplier

---

Comments: Identical E-Glass fabric type and glass surface finish for both laminates.

Figure B.2-9. Interlaminar shear strength at $77 \mathrm{~K}$ of FGR epoxy and bismaleimide laminates after $20-\mathrm{K}$ reactor irradiation. Supplementary Table A.7-1. Data from Okada et a1. [1992]. 


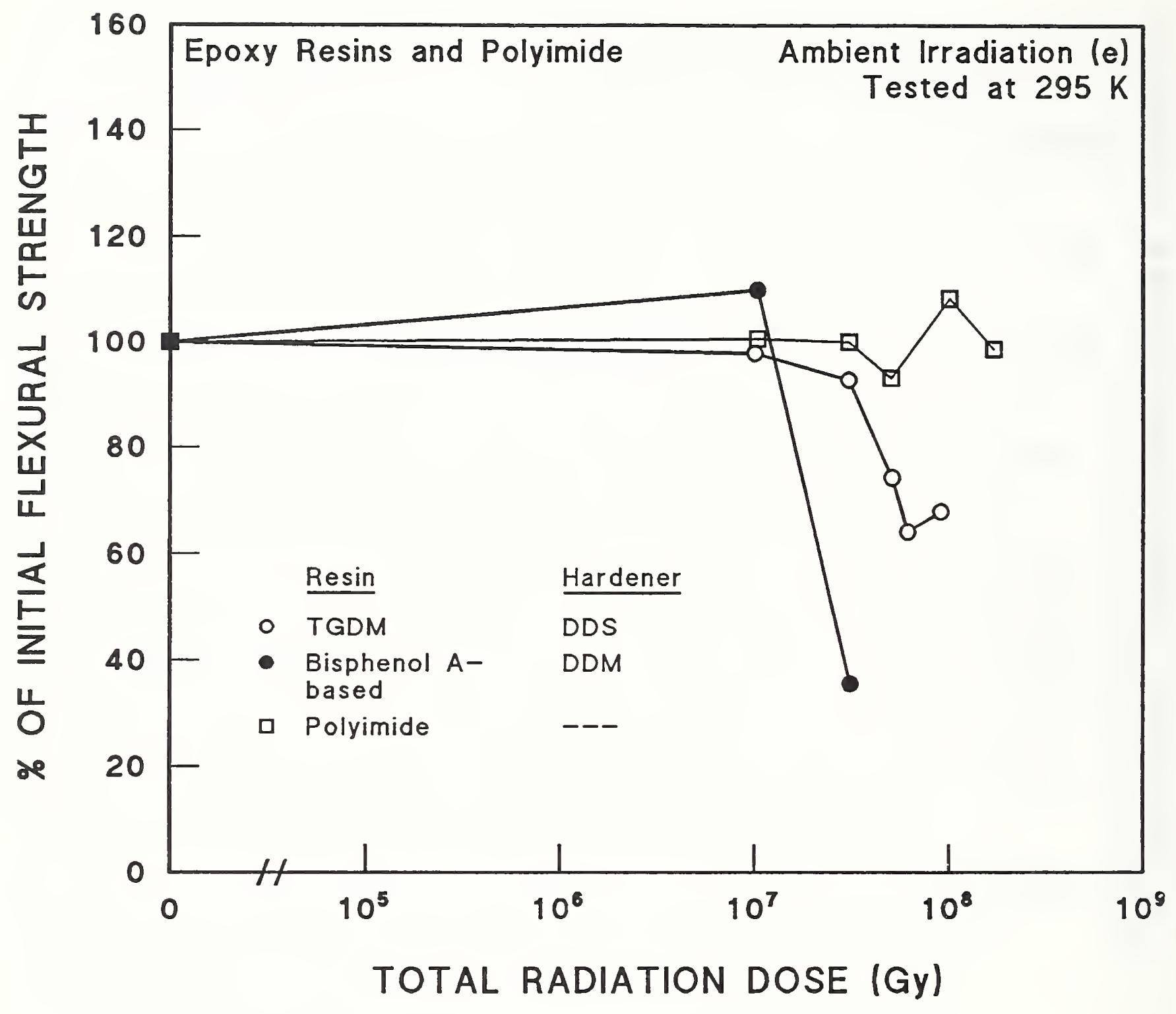

\begin{tabular}{l} 
"O" Strength, MPa \\
\hline O 127.1 \\
- 109.8 \\
133.0
\end{tabular}

Supplier

Sumitomo

Bakelite Co., Ltd.

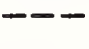

Figure B.2-10. Flexural strength at $295 \mathrm{~K}$ of epoxy resins and polyimide after ambient electron irradiation. Supplementary Table A.2-3. Data from Hagiwara et al. [1985]. 


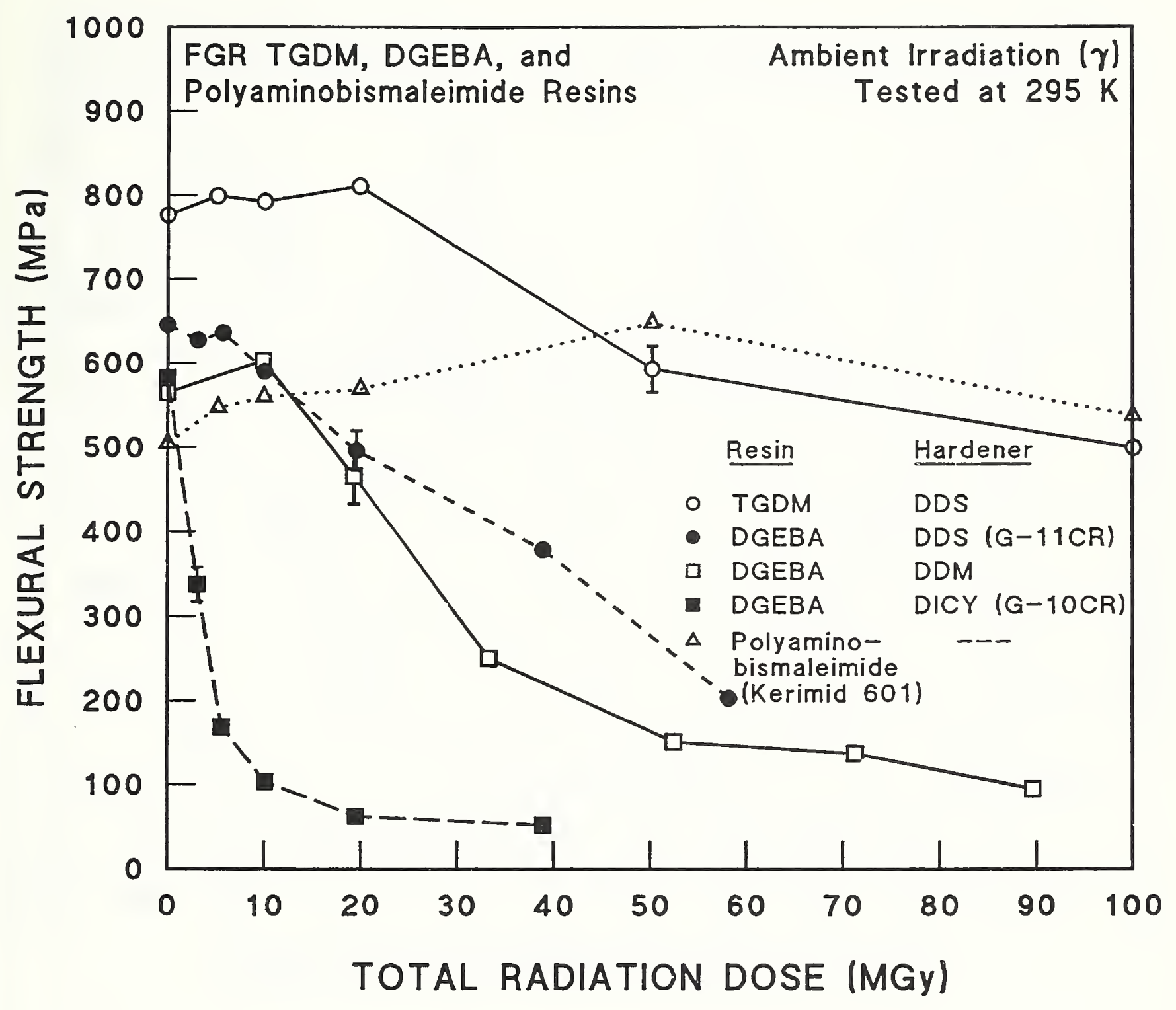

\author{
Supplier \\ - Sumitomo Bakelite \\ Co., Ltd. \\ - Spaulding \\ - Sumitomo Bakelite \\ Co., Ltd. \\ - Spaulding \\ $\Delta$ Sumitomo Bakelite \\ Co., Ltd.
}

Figure B.2-11. Flexural strength at $295 \mathrm{~K}$ of FGR TGDM and DGEBA and polyaminobismaleimide resins and laminates (G-10CR, G-11CR) after ambient gamma irradiation. Supplementary Table A.3-2. Data from Egusa and Hagiwara [1986]. 


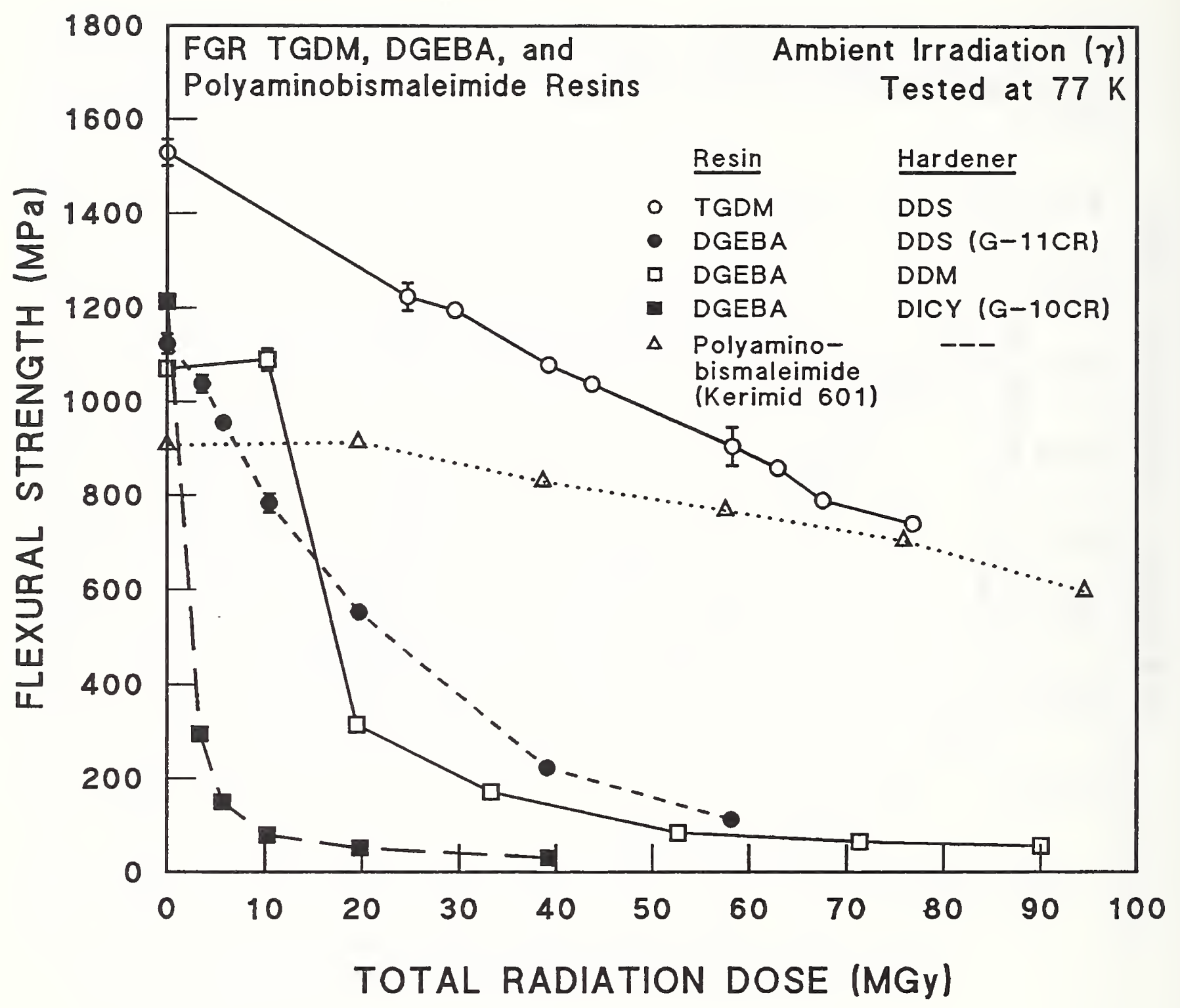

\author{
Supplier \\ - Sumitomo Bakelite \\ Co., Ltd. \\ - Spaulding \\ - Sumitomo Bakelite \\ Co., Ltd. \\ - Spaulding \\ $\Delta$ Sumitomo Bakelite \\ Co., Ltd.
}

Figure B.2-12. Flexural strength at $77 \mathrm{~K}$ of FGR TGDM and DGEBA and polyaminobismaleimide resins and laminates (G-10CR, G-11CR) after ambient gamma irradiation. Supplementary Table A.3-2. Data from Egusa and Hagiwara [1986]. 


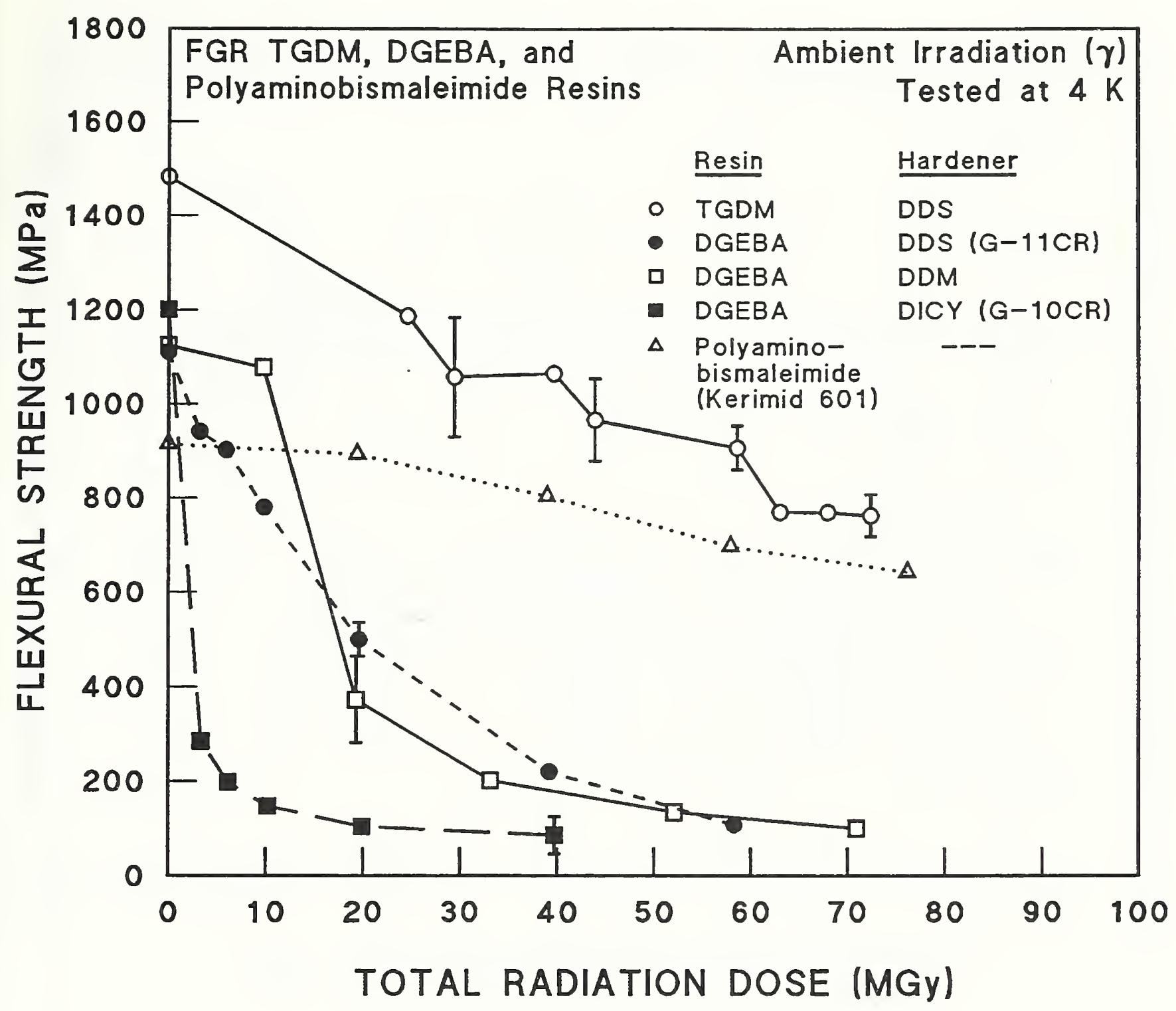

Supplier

- Sumitomo Bakelite

Co., Ltd.

- Spaulding

- Sumitomo Bakelite Co., Ltd.

- Spaulding

$\Delta$ Sumitomo Bakelite Co., Ltd.

Figure B.2-13. Flexural strength at $4 \mathrm{~K}$ of FGR TGDM and DGEBA and polyaminobismaleimide resins and laminates (G-10CR, G-11CR) after ambient gamma irradiation. Supplementary Table B.1-1. Data from Egusa [1990a]. 


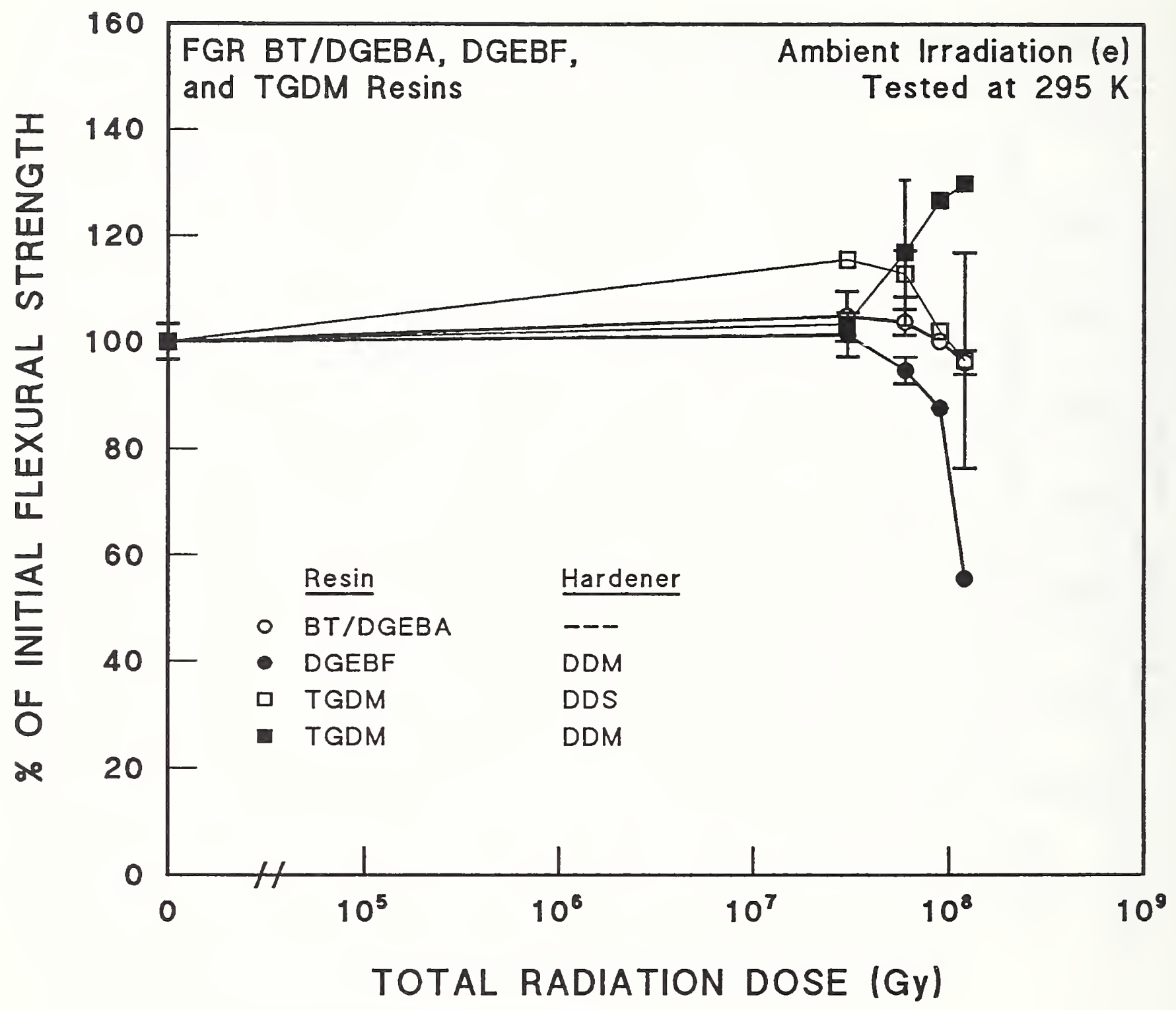

\begin{tabular}{lccc} 
"O" Strength, MPa & & Vol. Fract. & Supplier \\
\cline { 2 - 3 } - $604.4 \pm 19.9$ & & $45 \%$ & -- \\
- $492.1 \pm 16.6$ & & $40 \%$ & -- \\
口 $444.2 \pm 0.0$ & $40 \%$ & -- \\
- $430.4 \pm 0.0$ & $40 \%$ & --
\end{tabular}

Figure B.2-14. Flexural strength at $295 \mathrm{~K}$ of FGR BT/DGEBA, DGEBF, and TGDM resins after ambient electron irradiation. Supplementary Table A.3-5. Data from Egusa et a1. [1988] . 


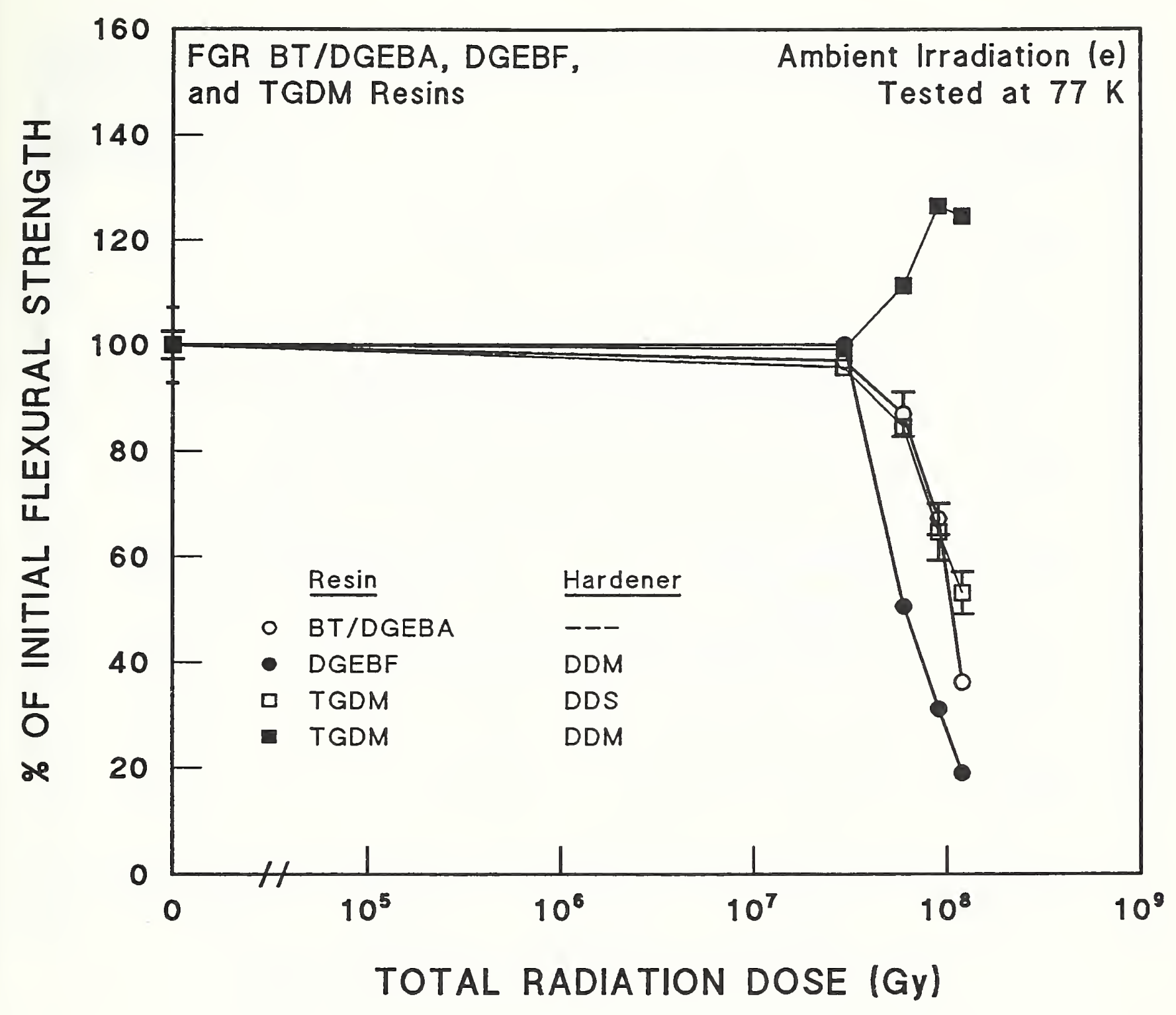

\begin{tabular}{|c|c|c|c|}
\hline & Strength, MPa & Vol. Fract. & Supplier \\
\hline o & $1163.6 \pm 0.0$ & $45 \%$ & -- \\
\hline - & $1040.5 \pm 0.0$ & $40 \%$ & -- \\
\hline$\square$ & $852.3 \pm 22.4$ & $40 \%$ & -- \\
\hline घ & $663.6 \pm 47.4$ & $40 \%$ & --- \\
\hline
\end{tabular}

Figure B.2-15. Flexural strength at $77 \mathrm{~K}$ of FGR BT/DGEBA, DGEBF, and TGDM resins after ambient electron irradiation. Supplementary Table A.3-5. Data from Egusa et al. [1988] . 


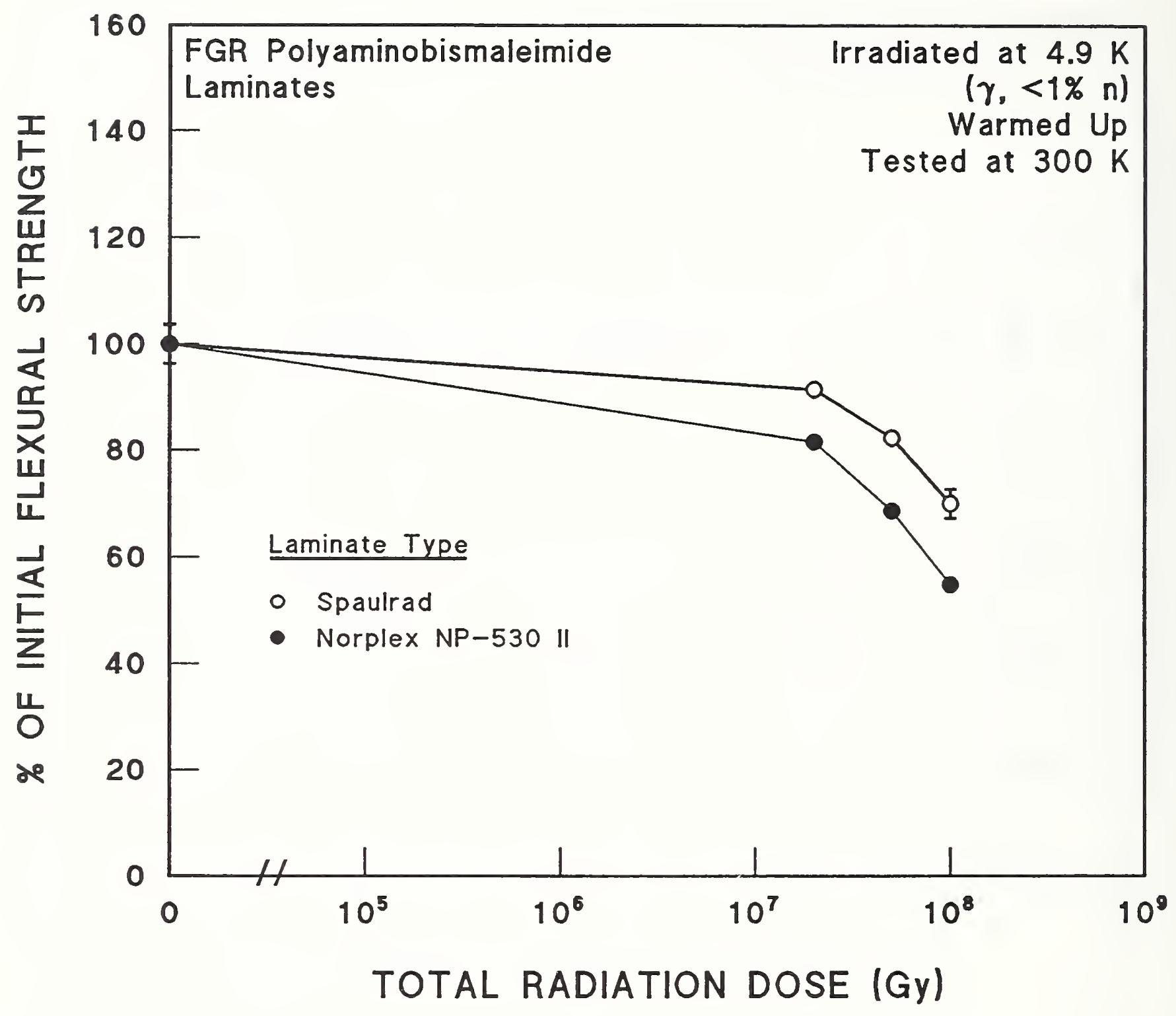

"O" Strength, MPa

○ $544.5 \pm 20.2$

- $439.1 \pm 0.0$
Glass, mass\%

$$
\text { 70-71 }
$$

$40-60$
Supplier

Dupont

Norplex Div., UOP, Inc.

Figure B.2-16. Flexural strength at $300 \mathrm{~K}$ of FGR polyaminobismaleimide laminates after 4-K reactor irradiation. Supplementary Table A.8-2. Data from Coltman and Klabunde [1981]. 


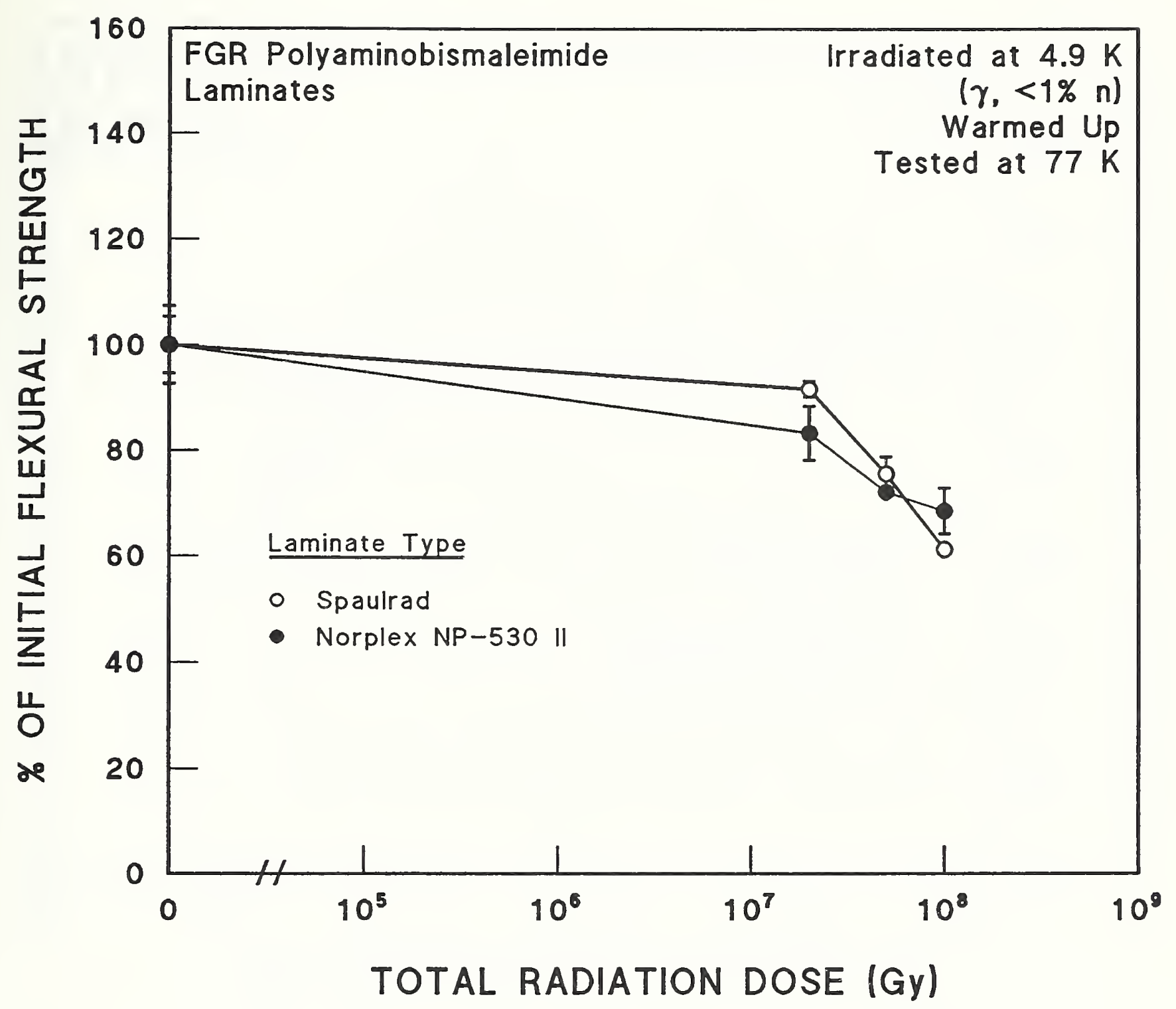

Glass, mass\%
$\begin{gathered}70-71 \\ 40-60\end{gathered}$

Supplier

DuPont

Norplex Div., UOP, Inc.

Figure B.2-17. Flexural strength at $77 \mathrm{~K}$ of FGR polyaminobismaleimide laminates after 4-K reactor irradiation. Supplementary Table A.8-2. Data from Coltman and Klabunde [1981]. 


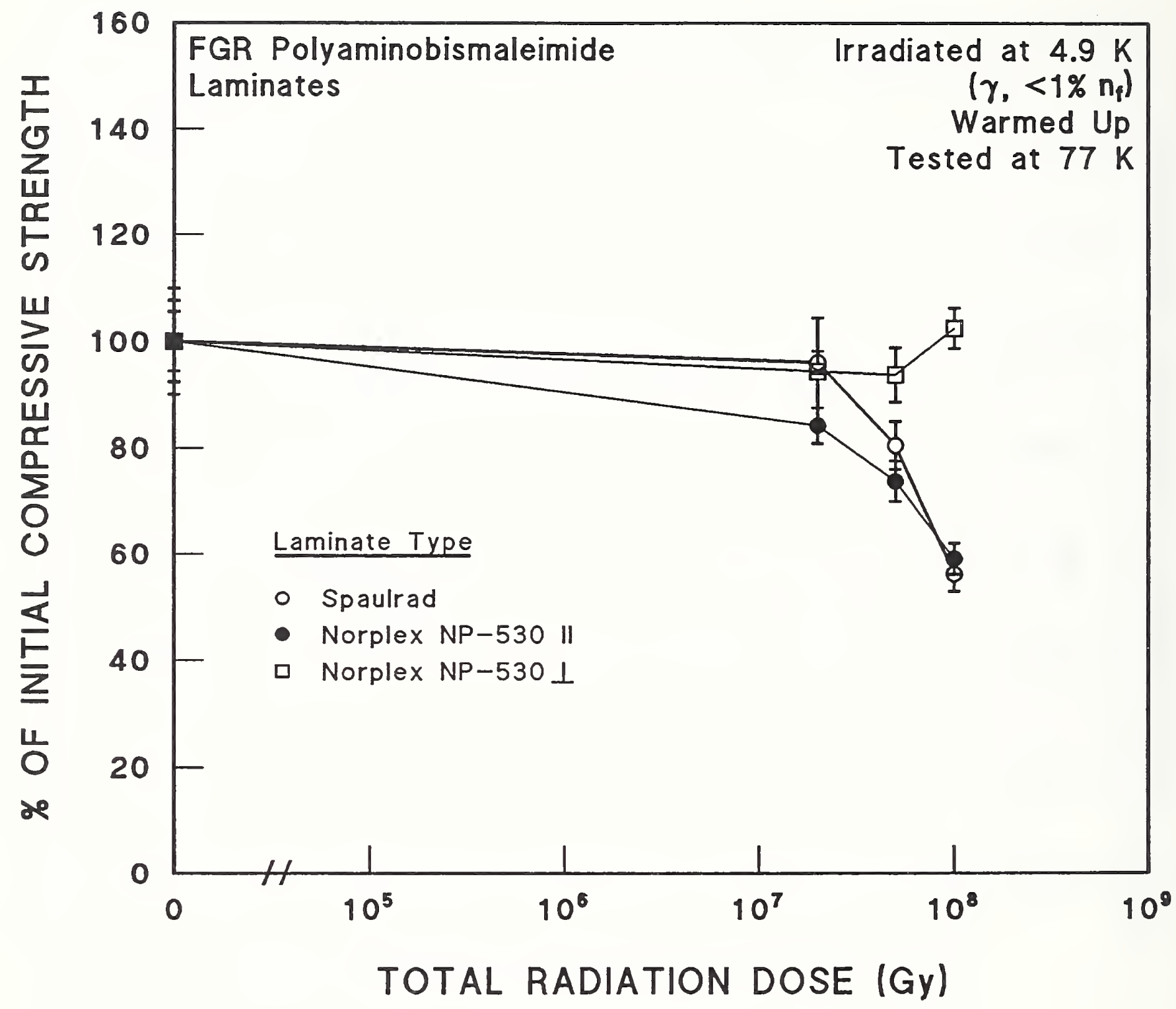

"O" Strength, MPa

- $637.0 \pm 63.3$

- $608.3 \pm 34.0$

ㅁ $879.3 \pm 67.2$
Glass, mass\%

$$
\text { 70-71 }
$$$$
\text { 40-60 }
$$

$40-60$
Supplier

Dupont

Norplex Div., UOP, Inc.

Norplex Div., UOP, Inc.

Figure B.2-18. Compressive strength at $77 \mathrm{~K}$ of FGR polyaminobismaleimide laminates after 4-K reactor irradiation. Supplementary Table A.8-2. Data from Coltman and Klabunde [1981]. 


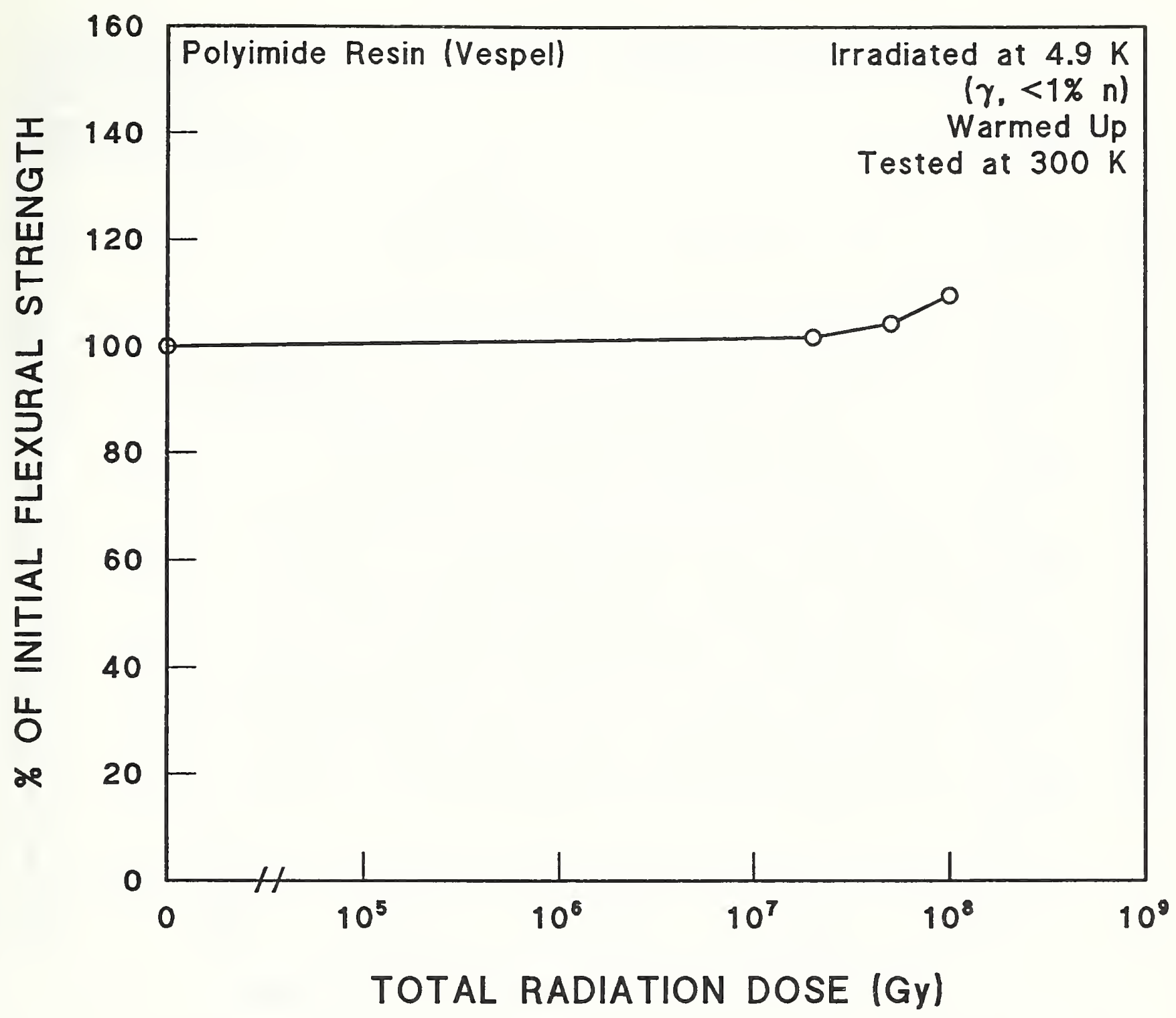

\begin{tabular}{ll} 
"O" Strength, MPa & Supplier \\
\hline $131.8 \pm 0.0$ & DuPont
\end{tabular}

Figure B.2-19. Flexural strength at $300 \mathrm{~K}$ of polyimide resin (Vespel) after 4-K reactor irradiation. Supplementary Table A.8-2. Data from Coltman and Klabunde [1981]. 


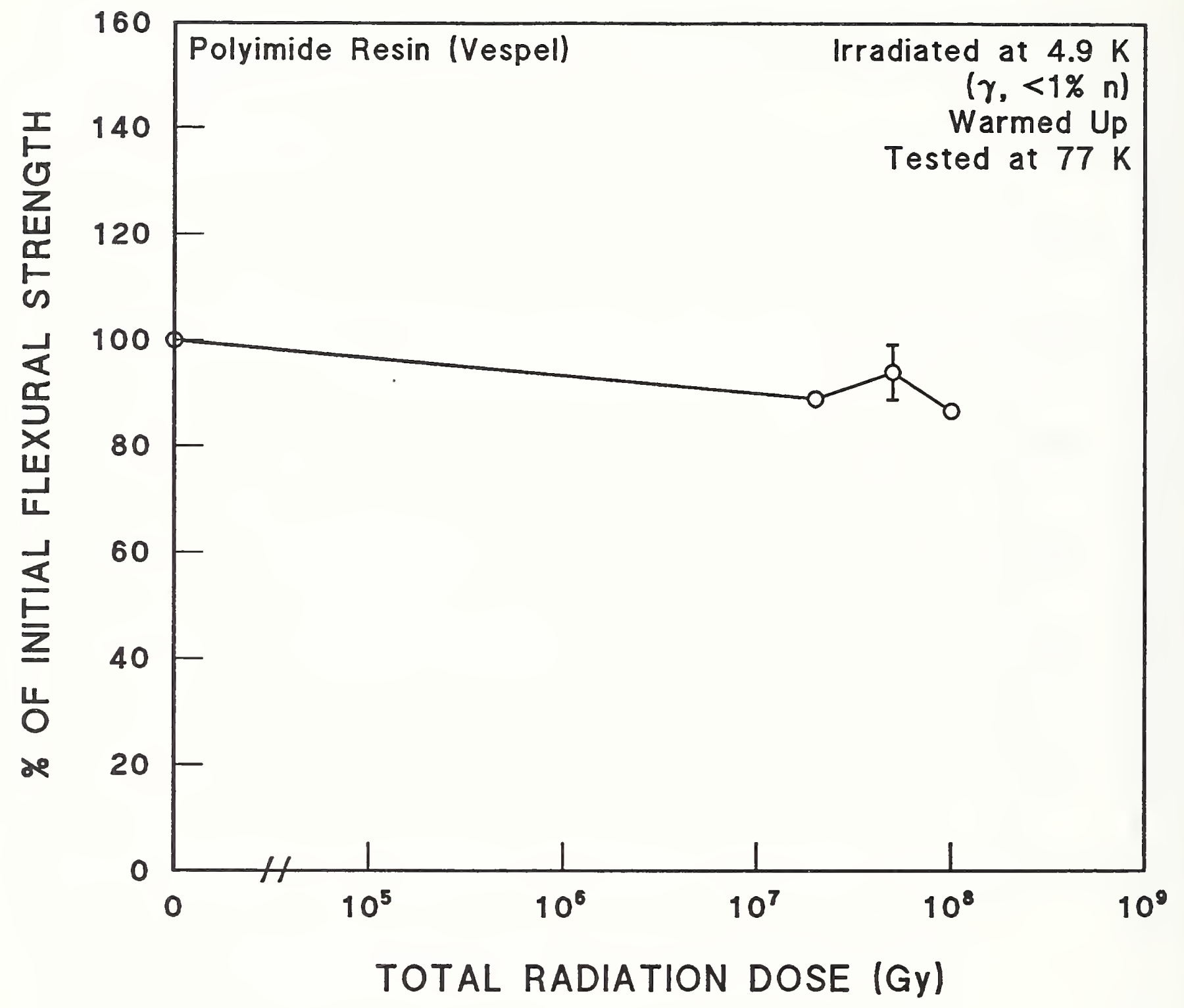

"O" Strength, MPa

○ $323.2 \pm 0.0$
Supplier

DuPont

Figure B.2-20. Flexural strength at $77 \mathrm{~K}$ of polyimide resin (Vespel) after 4-K reactor irradiation. Supplementary Table A.8-2. Data from Coltman and Klabunde [1981]. 


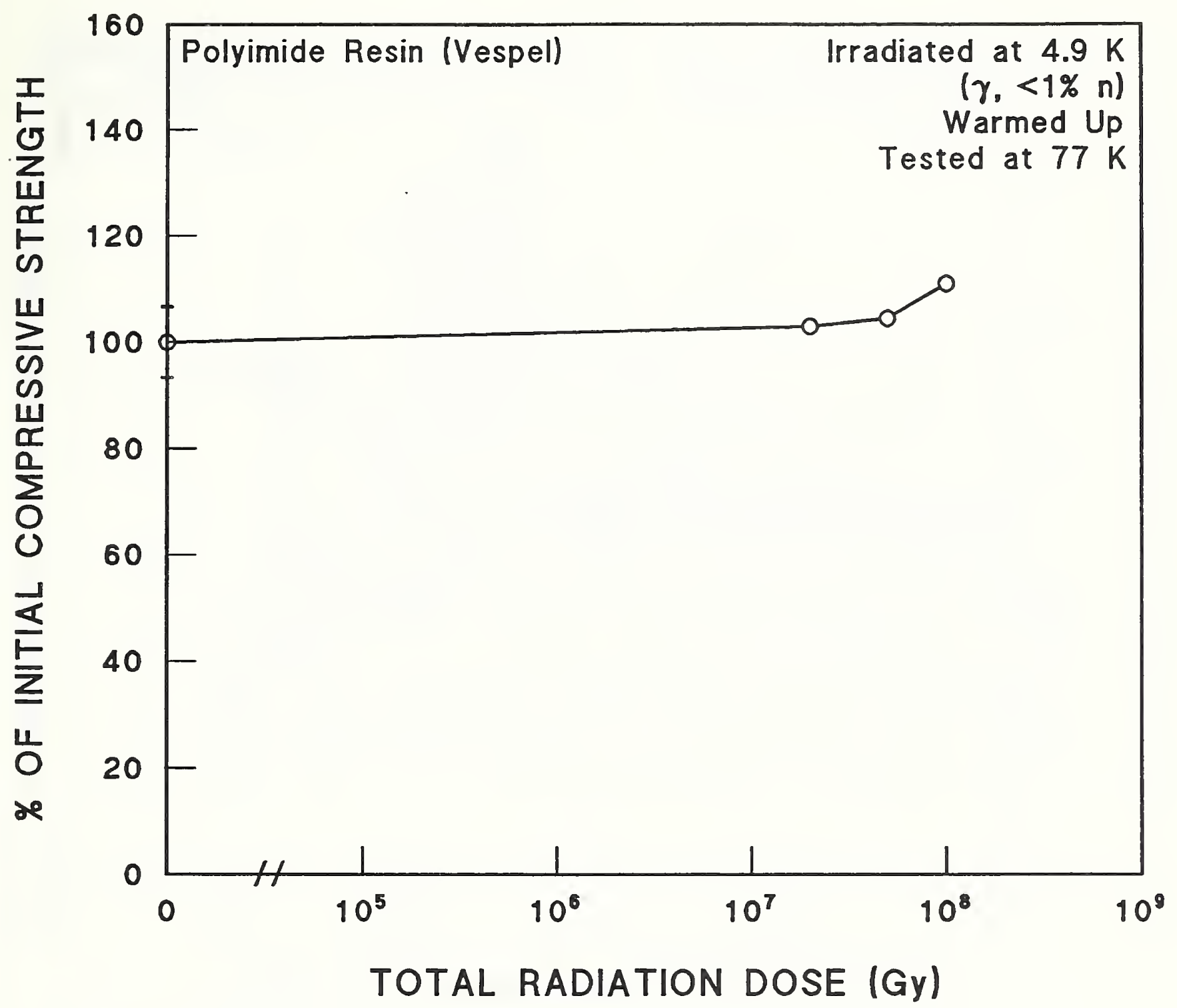

Figure B.2-21. Compressive strength at $77 \mathrm{~K}$ of polyimide resin (Vespel) after 4-K reactor irradiation. Supplementary Table A.8-2. Data from Coltman and Klabunde [1981]. 


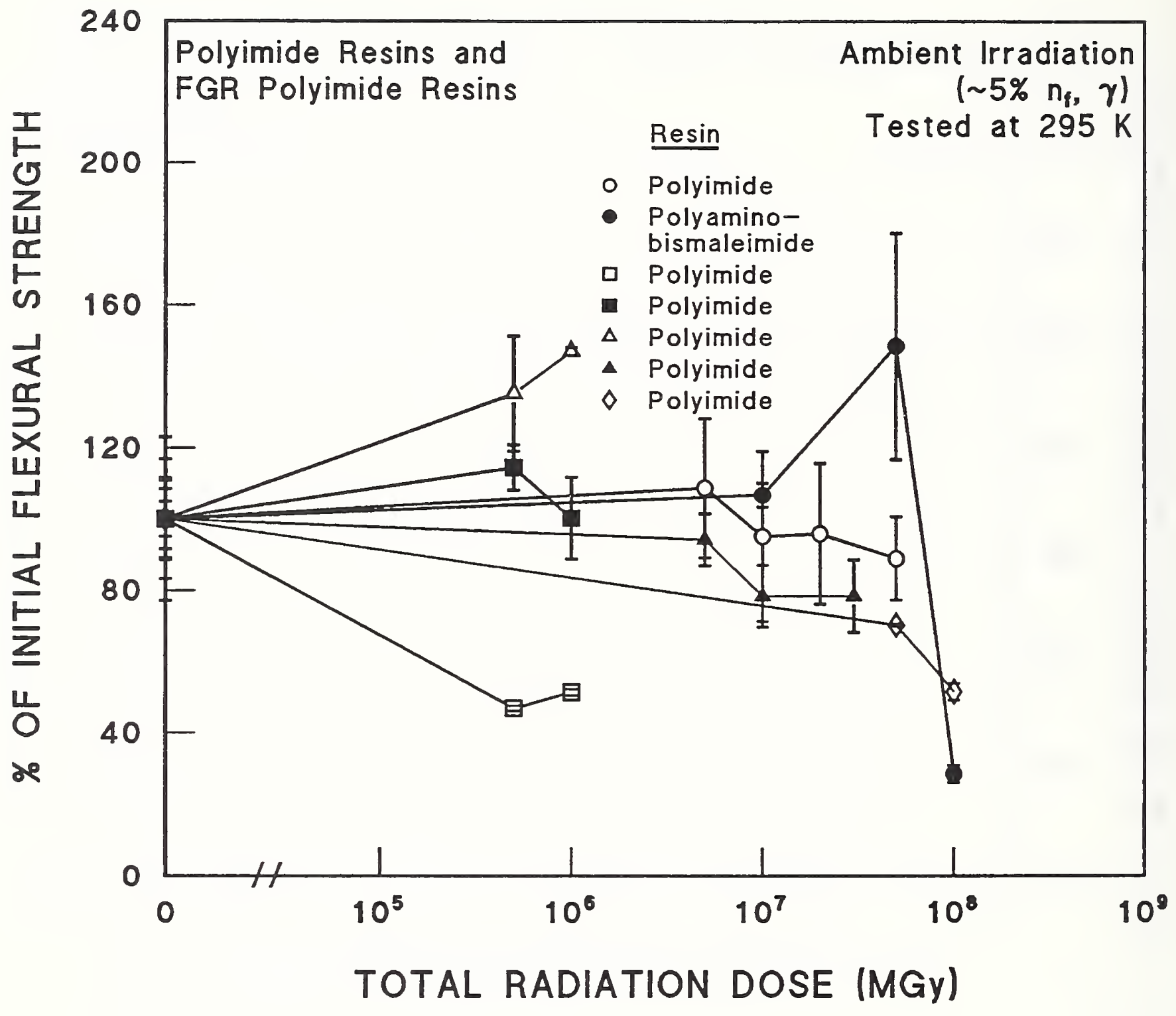

\begin{tabular}{|c|c|c|c|c|}
\hline "O" & Strength, MPa & Reinforcement & Type & Supplier \\
\hline 0 & $426.7 \pm 71.6$ & Glass Fiber & --- & Isola \\
\hline$\bullet$ & $503.3 \pm 42.5$ & Glass Fiber (181E) & Kerimid 601 & Rhône-Poulenc \\
\hline 口 & $108.9 \pm 11.8$ & Glass & Compound 17287 & Shamban \\
\hline - & $144.9 \pm 33.3$ & Glass Fiber or Graphite & Kinel 5.502 & Rhône-Poulenc \\
\hline$\Delta$ & $36.7 \pm 4.2$ & Glass Fiber or Graphite & Kinel 5.504 & Rhône-Poulenc \\
\hline$\Delta$ & $67.7 \pm 7.8$ & -- & Compound $17-286$ & Shamban \\
\hline & $375.7 \pm 18.6$ & --- & Compound 17242 & Shamban \\
\hline
\end{tabular}

Figure B.2-22. Flexural strength at $295 \mathrm{~K}$ of neat and FGR polyimide resins after ambient reactor irradiation. Supplementary Table A.2-2. Data from Schönbacher and Stolarz-Iżycka [1979]. 


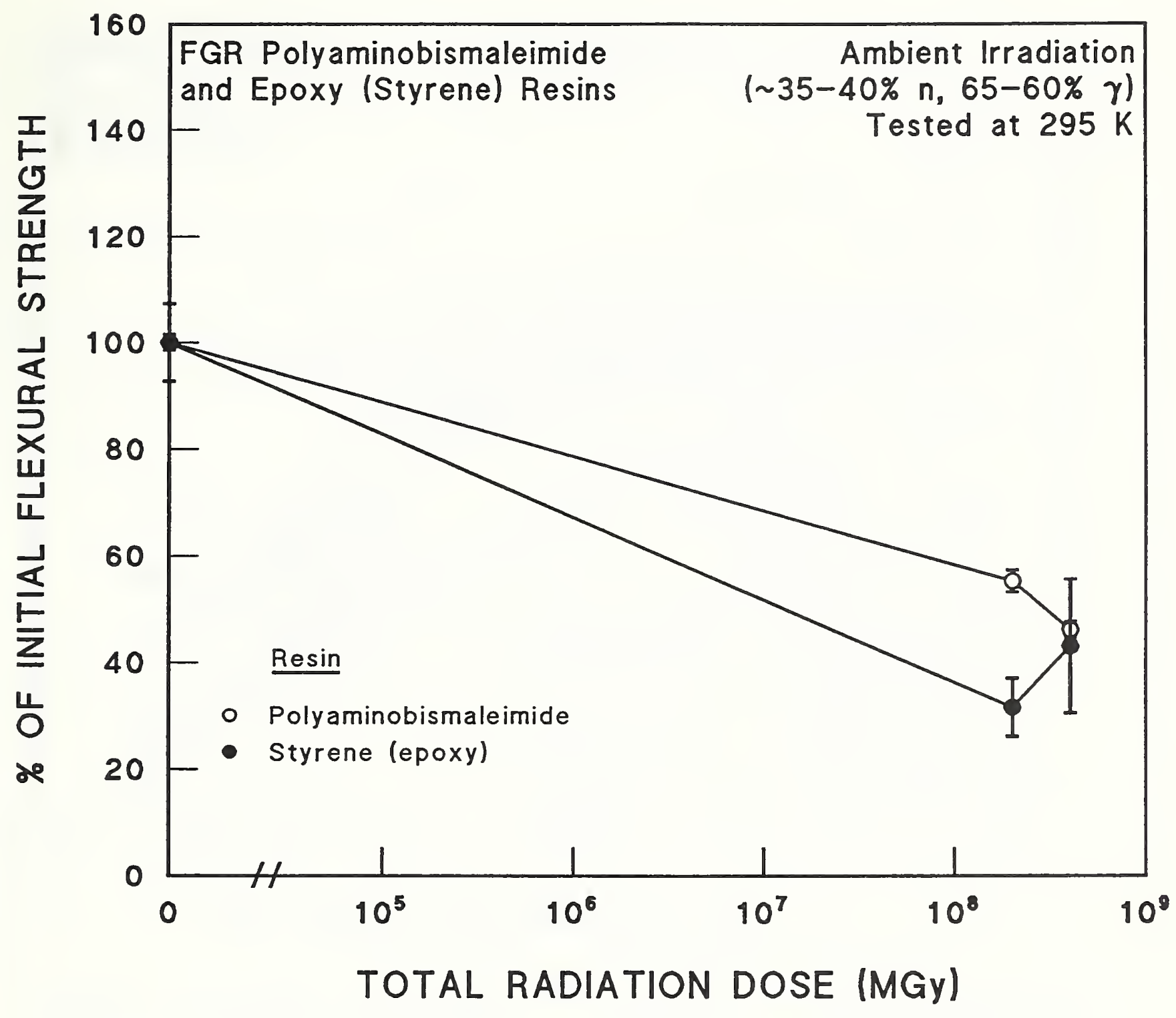

\begin{tabular}{|c|c|c|}
\hline "O" Strength, MPa & Laminate Type & Supplier \\
\hline o $869.8 \pm 13.0$ & Spaulrad $S$ & Spaulding \\
\hline - $556.6 \pm 40.7$ & $\begin{array}{l}9405 \text { resin/ } \\
9470 \text { hardener }\end{array}$ & Shell \\
\hline
\end{tabular}

Figure B.2-23. Flexural strength at $295 \mathrm{~K}$ of FGR polyaminobismaleimide and epoxy resins after ambient reactor irradiation. Supplementary Table A.2-17. Data from McManamy et al. [1991b]. 


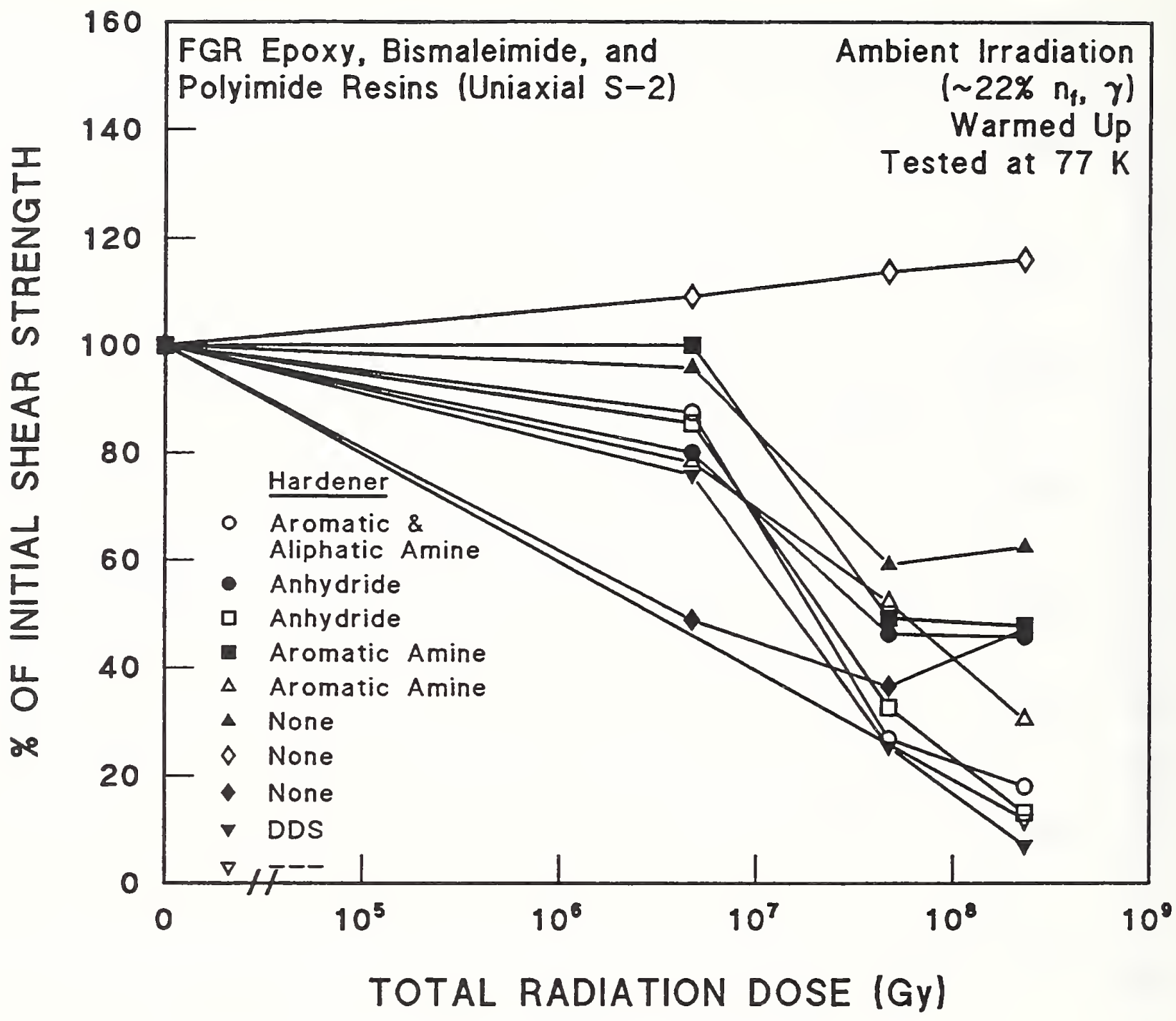

\begin{tabular}{|c|c|c|c|c|c|c|}
\hline "O" & Str., MPa & Type & Resin & Accelerator & Flexibilizer & Supplier \\
\hline 0 & 160.0 & CTD-100 & DGEBA & No & Yes & CTD, Inc. \\
\hline$\bullet$ & 201.0 & CTD-101 & DGEBA & Yes & No & CTD, Inc. \\
\hline$\square$ & 220.0 & CTD-102 & EPN & Yes & No & CTD, Inc. \\
\hline 表 & 142.0 & CTD-110 & Multifunctional & No & No & CTD, Inc. \\
\hline$\Delta$ & 184.0 & CTD-112 & Multifunctional & No & No & CTD, Inc. \\
\hline$\Delta$ & 120.0 & CTD-200 & Bismaleimide & No & No & CTD, Inc. \\
\hline$\diamond$ & 87.0 & CTD-300 & Polyimide & No & No & CTD, Inc. \\
\hline$\diamond$ & 131.0 & CTD-310 & Polyimide & No & No & CTD, Inc. \\
\hline$\nabla$ & 100.0 & CTD-501 & --- & --- & --- & CTD, Inc. \\
\hline$\nabla$ & 196.0 & $G-11 C R$ & DGEBA & --- & --- & $\begin{array}{l}\text { Spaulding } \\
\text { (Resin) }\end{array}$ \\
\hline
\end{tabular}

Figure B.2-24. Shear strength at $77 \mathrm{~K}$ of FGR epoxy, bismaleimide, and polyimide resins after ambient reactor irradiation. Supplementary Table A.3-3. Data from Munshi [1991]. 


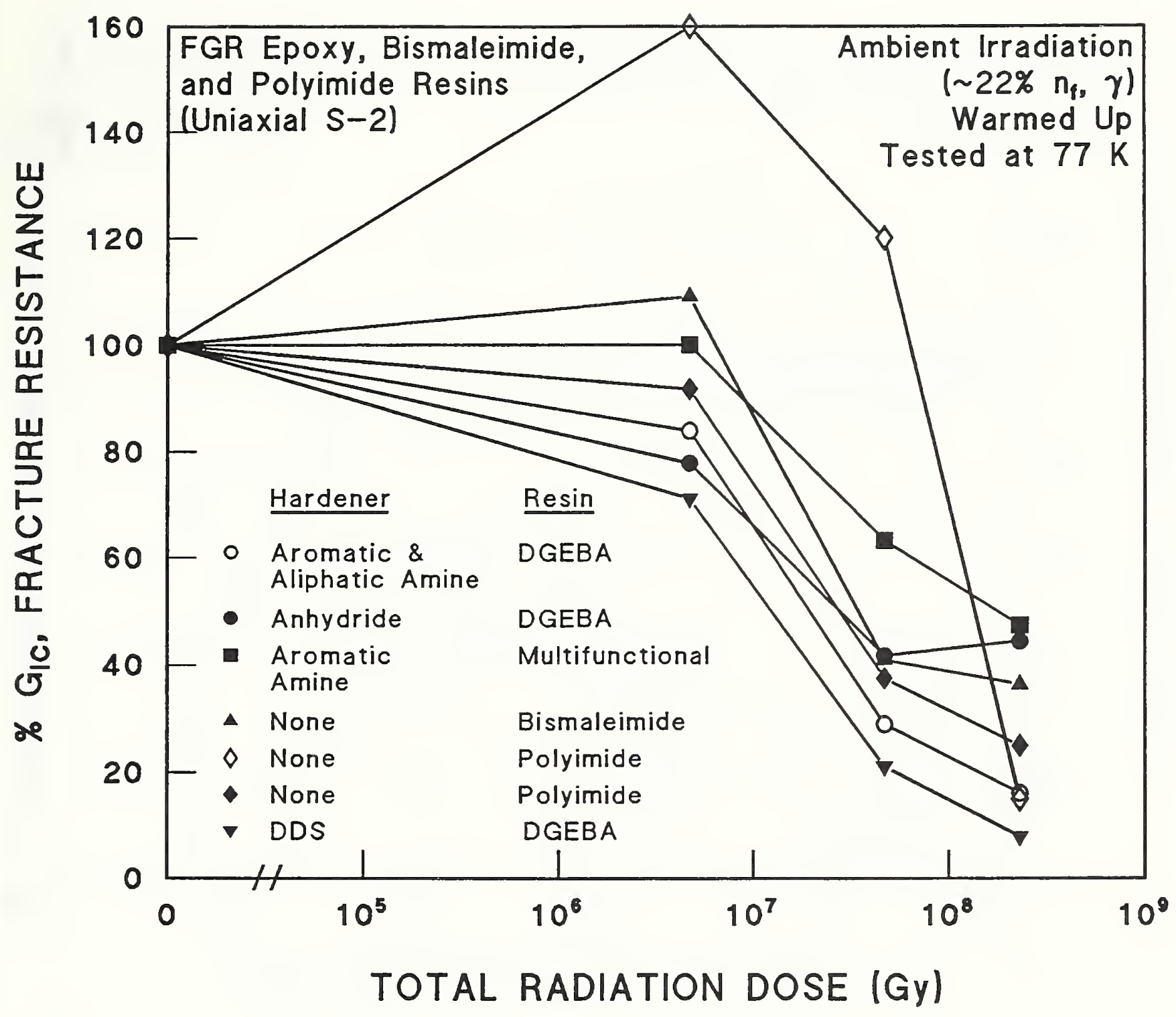

\begin{tabular}{|c|c|c|c|c|c|}
\hline "O" & Stren., $\mathrm{kJ} / \mathrm{m}^{2}$ & Type & Accelerator & Flexibilizer & Supplier \\
\hline 0 & 0.31 & CTD-100 & No & Yes & CTD, Inc. \\
\hline - & 0.36 & CTD-101 & Yes & No & CTD, Inc. \\
\hline - & 0.19 & CTD-110 & No & No & CTD, Inc. \\
\hline$\Delta$ & 0.22 & CTD -200 & No & No & CTD, Inc. \\
\hline$\diamond$ & 0.20 & CTD -300 & No & No & CTD, Inc. \\
\hline$\bullet$ & 0.24 & CTD -310 & No & No & CTD, Inc. \\
\hline$\nabla$ & 0.38 & $G-11 C R$ & --- & -- & $\begin{array}{l}\text { Spaulding } \\
\text { (Resin) }\end{array}$ \\
\hline
\end{tabular}

Figure B.2-25. Fracture resistance at $77 \mathrm{~K}$ of FGR epoxy, bismaleimide, and polyimide resins after ambient reactor irradiation. Supplementary Table A.3-3. Data from Munshi [1991]. 


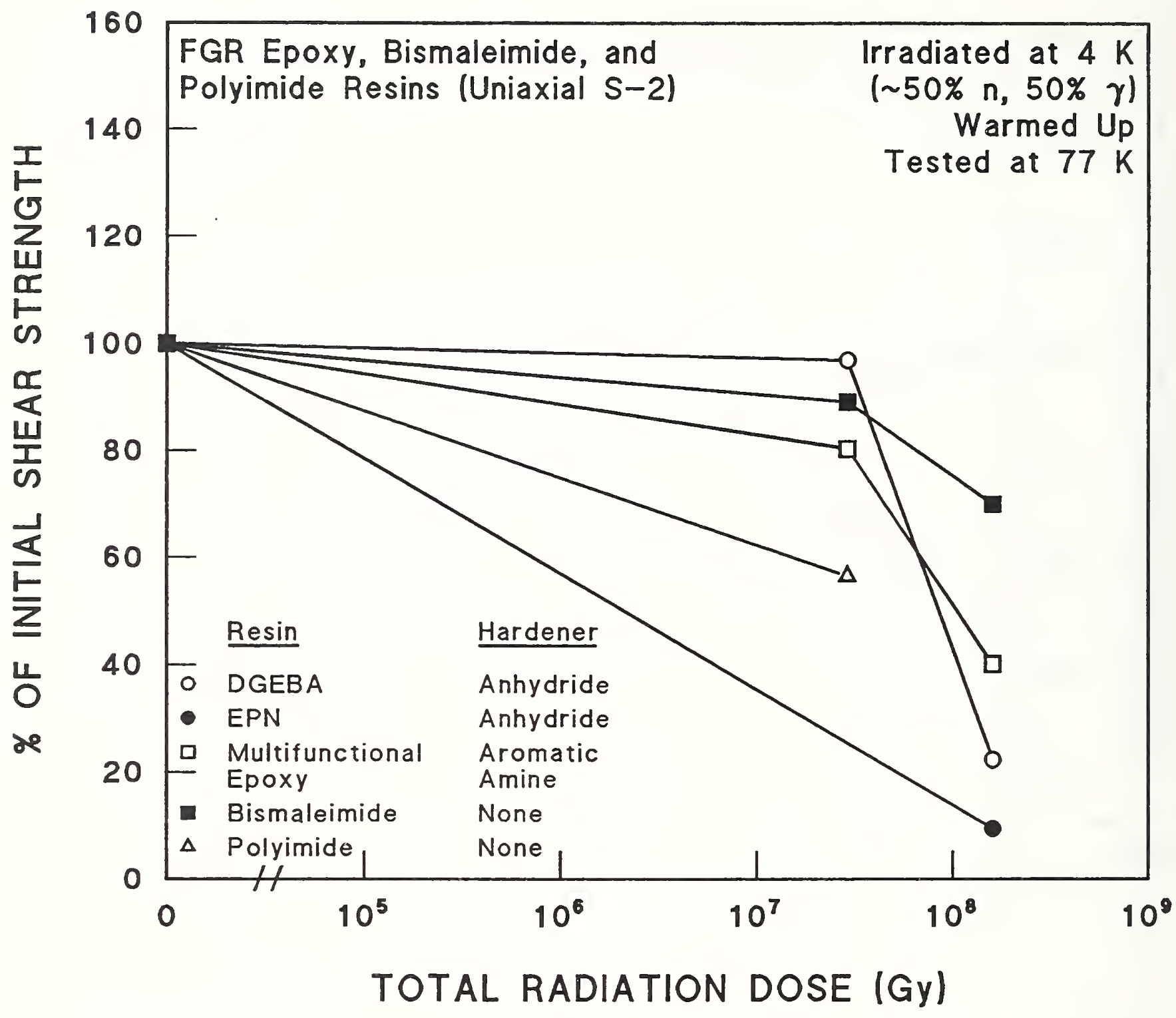

\begin{tabular}{|c|c|c|c|c|}
\hline "O" Strength, MPa & Type & Accelerator & Flexibilizer & Supplier \\
\hline o 201.0 & CTD-101 & Yes & No & CTD, Inc. \\
\hline - 220.0 & CTD-102 & Yes & No & CTD, Inc. \\
\hline ㅁ 184.0 & CTD -112 & No & No & CTD, Inc. \\
\hline - 120.0 & CTD-200 & No & No & CTD, Inc. \\
\hline$\Delta \quad 131.0$ & CTD -310 & No & No & CTD, Inc. \\
\hline
\end{tabular}

Figure B.2-26. Shear strength at $77 \mathrm{~K}$ of FGR epoxy, bismaleimide, and polyimide resins after 4-K reactor irradiation. Supplementary Table A.8-4. Data from Munshi [1991]. 


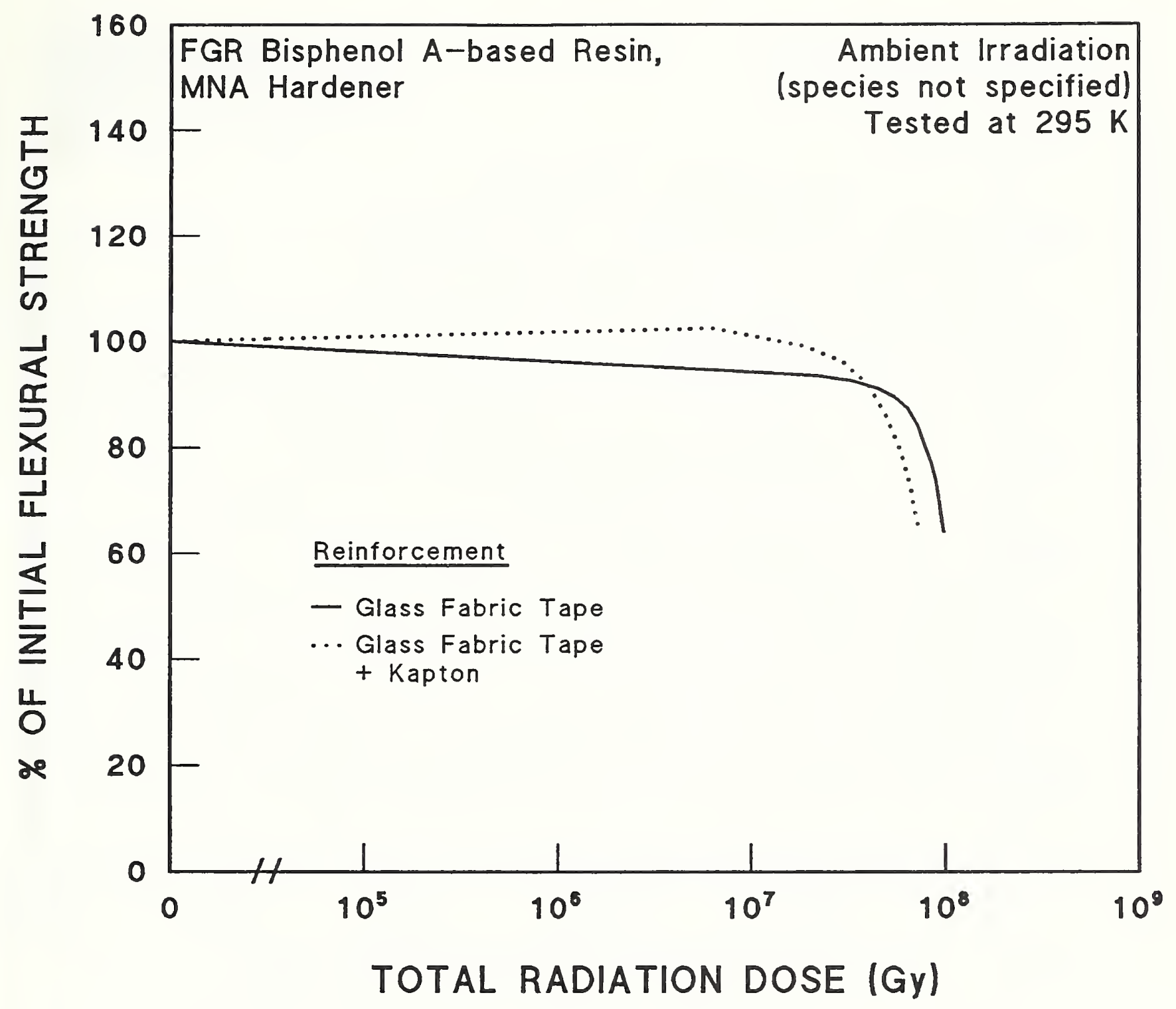

"O" Strength, MPa

$-\quad 455.8$

... 381.4
Supplier

Brown Boveri, Zurich

Brown Boveri, Zurich

Figure B.2-27. Flexural strength at $295 \mathrm{~K}$ of FGR bisphenol resin with MNA hardener, with and without Kapton reinforcement, after ambient reactor irradiation. No supplementary information. Banford [1984] . 


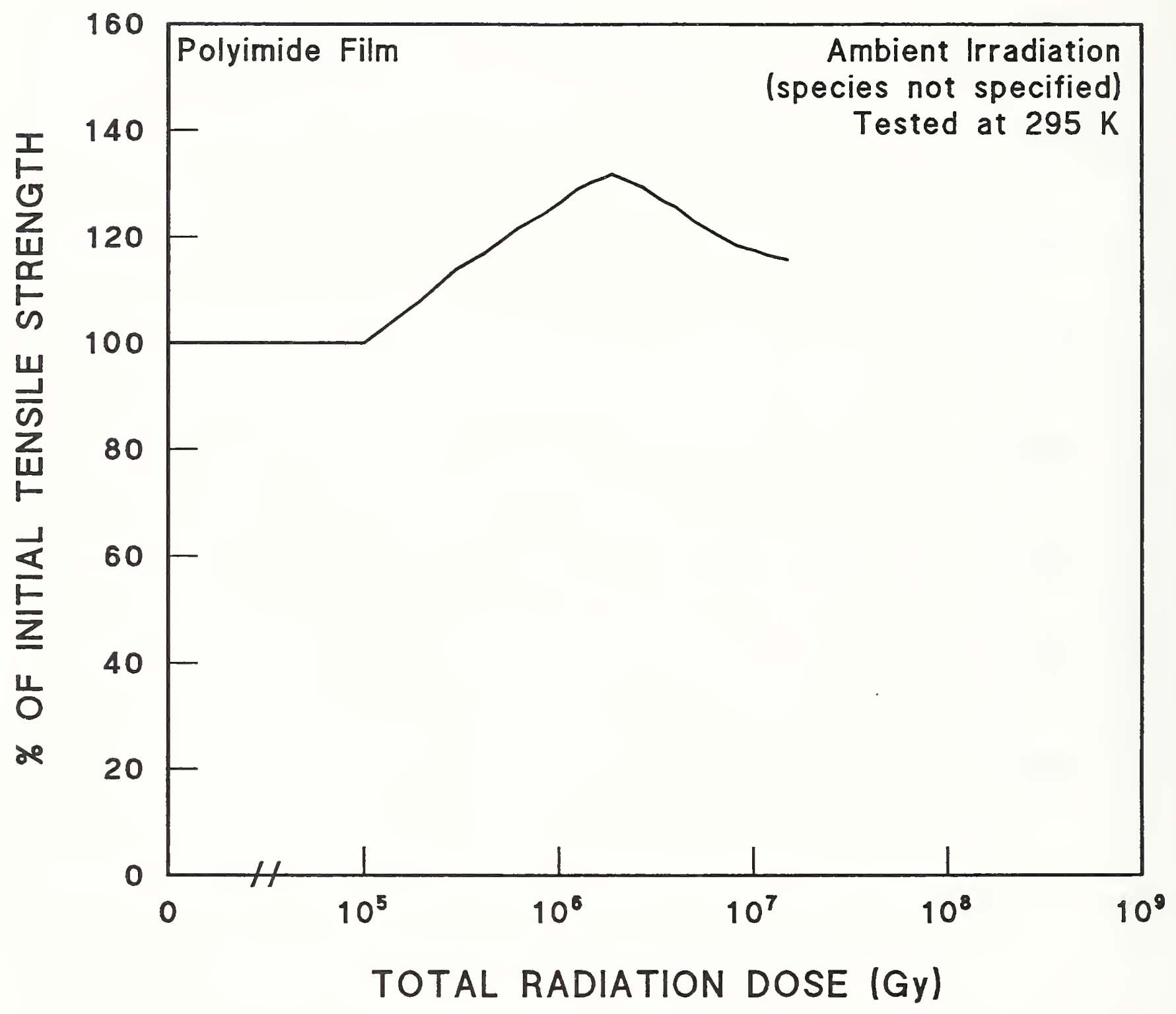

"O" Strength, MPa

- $\quad 156.9$

Figure B.2-28. Flexural strength at $295 \mathrm{~K}$ of polyimide film after ambient irradiation. No supplementary information. Data from Van de Voorde and Restat [1972]. 


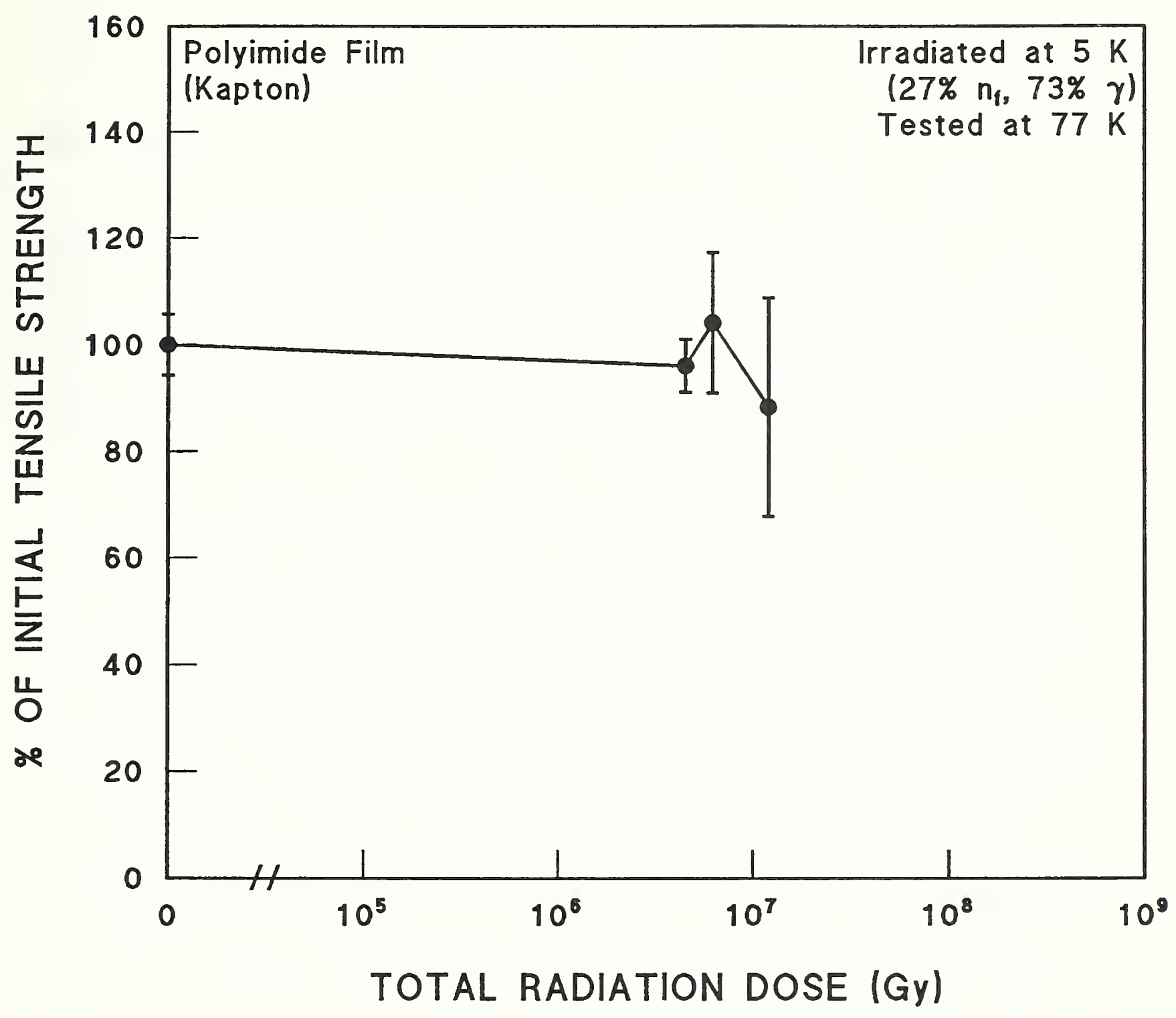

"O" Strength, MPa

Supplier

- $323 \pm 18.5$

Figure B.2-29. Tensile strength at $77 \mathrm{~K}$ of polyimide film (Kapton) after 5-K reactor irradiation. Supplementary Table A.9-1. Data from Takamura and Kato [1979; $1981]$. 


\section{APPENDIX C: FATIGUE}

\section{INTRODUCTION}

The conceptual design document for ITER magnets gives 40000 as the expected number of poloidal field (PF) operational cycles [Bottura et al., 1991]. However, this was a provisional estimate; the wording indicates that new research will modify this number. Since pulsed operation of the PF coil imposes cyclic loading on all magnet structures, TF coils must also withstand the number of cycles needed to attain the ITER fluence goal.

The number of fatigue tests conducted on irradiated specimens is far below the number of static mechanical property tests, and the conditions, as for most static tests, do not match ITER requirements for irradiation and test temperature and neutron fluence. However, some tests were conducted after very high neutron fluences ( $\S C .1$ ). All of the tests have been stresscontrolled, although the turn-to-turn TF insulation is expected to experience strain-controlled fatigue conditions. The compressive, shear/compressive, and tensile fatigue tests that have been performed on several fiber-glassreinforced composites are discussed below. Only the tensile tests and one set of compressive tests were conducted at cryogenic temperatures ( $77 \mathrm{~K})$, and almost no cryogenic irradiation was employed. Radiation doses of reinforced resins have ranged from $10^{6}$ to about $5 \times 10^{9} \mathrm{~Gy}$, and the number of cycles has varied from $3 \times 10^{4}$ to $6.5 \times 10^{5}$. One set of flexural fatigue tests on a neat resin was conducted after cryogenic irradiation without warm-up (above $77 \mathrm{~K}$ ). However, the fast neutron fluence was only $2.5 \times 10^{20} / \mathrm{m}^{2}(\mathrm{E}>1 \mathrm{MeV})$.

\section{C.1. COMPRESSIVE FATIGUE TESTS}

Erez and Becker [1982] and Becker et al. [1985] reported tests of compressive fatigue in $6.4 \mathrm{~mm}$-thick disks at $295 \mathrm{~K}$, after ambient reactor irradiation. For most specimens, the total dose from gamma rays and fast neutrons was about $4 \times 10^{9} \mathrm{~Gy}$, using the customary dose-to-fluence conversion rule for the fast neutron fluence of $1.6 \times 10^{23} / \mathrm{m}^{2}$ (E $\left.>0.1 \mathrm{MeV}\right)$. Some bismaleimide specimens were tested at doses about 3.6 times this dose, and additional specimens were tested at a dose that was about 1/10th of this dose. Five specimens were tested simultaneously in series with individual specimen disks placed between 
hardened steel disks in the specimen cartridge. The load was cycled between zero and the maximum compressive load (perpendicular to plane of disks). Failure was defined as the rapid reduction of stiffness owing to the onset of crushing in one or more disks.

Figure C.1-1 shows that the G-10 specimens tested above a maximum stress of $276 \mathrm{MPa}$ at $295 \mathrm{~K}$ failed below 21900 cycles, but that at $77 \mathrm{~K}$, performance was better. However, 77-K testing was not carried beyond 40000 cycles. G-11 (tested as a single disk) failed at only $10^{4}$ cycles at $295 \mathrm{~K}$ at a maximum stress of $207 \mathrm{MPa}$ and was not tested further. The ambient-temperature performance of Kerimid 601 and DGEBA or TGPAP resins reinforced with $S$ or S-2 Glass was much better. (G-10 and G-11 are reinforced with E glass.)

Schmunk et al. [1984] used the same procedures for fatigue testing. Their total dose was about $4.4 \times 10^{9} \mathrm{~Gy}$, which included a fast neutron fluence of about $3.7 \times 10^{23} / \mathrm{m}^{2}$ ( $\left.\mathrm{E}>1 \mathrm{MeV}\right)$. The laminates G-10, Kerimid 601 (S glass), and Spaulrad-S were tested [Schmunk, 1983]. Two epoxies reinforced with $S$ glass were also investigated. The results of tests at $295 \mathrm{~K}$ are shown in Table C.1.1. Spaulrad-S specimens survived $6.46 \times 10^{5}$ cycles at a much lower fast neutron fluence in the IPNS of $1.9 \times 10^{21} / \mathrm{m}^{2}(E>0.1 \mathrm{MeV})$. The IPNS irradiation temperature was reported as $4.2 \mathrm{~K}$.

\section{C.2. SHEAR/COMPRESSIVE FATIGUE TESTS}

Fatigue tests were carried out with a constant 345-MPa compressive stress and shear stress cycling at $5 \mathrm{~Hz}$ from about $14 \mathrm{MPa}$ to $90 \%$ of the lowest static shear strength of failure [McManamy et al., 1990; 1991a]. The one epoxy and two polyimides tested were reinforced with $\mathrm{S}$ glass, some with a 3-dimensional weave. Figure C.2-1 shows the relative shear strength (with 345-MPa compression) measured after $3 \times 10^{4}$ cycles. No deterioration of the strength was found for specimens irradiated before fatigue. However, in preliminary tests, the epoxy specimens failed at higher cyclic stress levels. 
Table C.1.1. Compression Fatigue. Data from Schmunk et al. [1984] and Schmunk [1983]. Supplementary Table C.I-I (see dose in text).

\begin{tabular}{||l|l|l|l|}
\hline \multicolumn{1}{|c|}{ MATERIAL } & \multicolumn{1}{|c|}{$\begin{array}{c}\text { MAXIMUM STRESS } \\
\text { (MPa) }\end{array}$} & \multicolumn{1}{c|}{$\begin{array}{c}\text { NUMBER OF } \\
\text { CYCLES }\end{array}$} & \multicolumn{1}{c|}{ RESULTS } \\
\hline G-10 & 207 & 13210 & One disk failed \\
\hline & 276 & 6275 & Two disks failed \\
\hline \hline Kerimid 601 & 345 & 440 & One disk failed \\
\hline & 207 & 63750 & No failure \\
\hline & 310 & 83377 & No failure \\
\hline \hline DGEBA & 345 & 226676 & No failure \\
\hline & 310 & 100000 & No failure \\
\hline \hline TGPAP & 345 & 165701 & No failure \\
\hline & 241 & 195413 & No failure \\
\hline \hline Spaulrad S & 345 & 257444 & No failure \\
\hline & 640 & 277500 & One disk failed \\
\hline
\end{tabular}

*Irradiated in IPNS at lower neutron fluence.

\section{C.3. TENSILE FATIGUE TESTS}

Tensile fatigue tests were carried out on G-11CR notched specimens at both 295 and $77 \mathrm{~K}$, after ambient gamma irradiation in air to $10^{6}$ Gy from a ${ }^{60} \mathrm{Co}$ source [Korukonda et al., 1983]. The maximum cyclic stress was set at 90, 75, 60, and 508 of the measured tensile strength. The number of cycles to crack initiation is shown in Figure C.3-1, along with the percentage of tensile strength. At both temperatures, a reversal in the effect of irradiation was found at about 3000 cycles; however, the effect of radiation on fatigue resistance is more pronounced at 77 than at $295 \mathrm{~K}$. Egusa et al. also found that cryogenic $(77-\mathrm{K})$ tests revealed a greater sensitivity to radiation than 295-K tests did (\$1.4.1). However, in their compressive fatigue tests of irradiated thin disks of G-10, Erez and Becker ( $\S \mathrm{C} .1$ ) reported an improvement in fatigue properties at $77 \mathrm{~K}$. Korukonda et al. also reported a change in the failure mode: at $295 \mathrm{~K}$, a through crack propagated normal to the warp fibers; 
at $77 \mathrm{~K}$, a sequential failure occurred that involved delamination, surface crack initiation, and propagation of a dominant surface crack.

\section{C.4. FLEXURAL FATIgUE TESTS}

Flexural fatigue tests were carried out on a neat, bisphenol A-based resin with a polyethanol hardener [Nishijima et al., 1981]. Both irradiation and testing were carried out at cryogenic temperatures; after irradiation below $27 \mathrm{~K}$, specimens were stored at $77 \mathrm{~K}$ and tested at $4 \mathrm{~K}$. Unfortunately, the reactor fast neutron fluence, $2.5 \times 10^{20} / \mathrm{m}^{2}(E>1 \mathrm{MeV})$ was somewhat below ITER goals; however, Figure C.4-1 shows significant degradation of fatigue life properties even at this fluence. Furthermore, the scatter was significantly increased (the correlation coefficient with the equation used to fit the data decreased from -0.97 to -0.13 ). Irradiation also caused a degradation of mechanical properties: the deflection length of the sample before irradiation was about $0.75 \mathrm{~mm}$, but after irradiation it was reduced to about $0.58 \mathrm{~mm}$, and the elastic modulus was reduced to almost $70 \%$ of the initial value.

\section{C.5. SUMMARY OF FATIGUE RESULTS}

In compression tests on G-10, Erez and Becker [1982] found that compressive fatigue resistance was better at $77 \mathrm{~K}$ than at $295 \mathrm{~K}$, and their disk specimens survived 40000 cycles at a maximum applied stress of $241 \mathrm{MPa}$ after about $4 \mathrm{x}$ $10^{9} \mathrm{~Gy}$ of reactor irradiation. The static compressive strength of G-10CR rods at $77 \mathrm{~K}$ is much lower, about $50 \mathrm{MPa}$ after $10^{8} \mathrm{~Gy}$ of predominantly gamma radiation (Figure A.8-13). Compressive fatigue tests on $6.4-\mathrm{mm}$ sheet have been criticized because hydrostatic stress conditions are likely to occur. Although the ITER TF coil insulation will probably be thinner than $6.4 \mathrm{~mm}$, hydrostatic conditions may not occur. Extension of tests on thin disks by Schmunk et al. [1984] and Becker et al. [1985] indicated a lower fatigue life for G-10 at $295 \mathrm{~K}$, but much longer fatigue lives for both bismaleimide and epoxy resins reinforced with $\mathrm{S}$ or $\mathrm{S}-2$ Glass. Although both irradiation and testing were carried out at ambient temperatures, the fast neutron fluences met or exceeded ITER requirements. Furthermore, Becker et al. [1985] suggested that their observed high face compressive strengths appeared to imply interlaminar shear strength values considerable greater than the values of 50 to $60 \mathrm{MPa}$ usually reported (See $\S 11$.). These implied shear strengths 
were said to be necessary to develop the hydrostatic stress fields associated with compression in the test-fixture geometry.

McManamy et al. reported that shear/compressive strengths of three irradiated materials, two polyimides and one unidentified epoxy, did not deteriorate after $3 \times 10^{4}$ cycles. The highest shear load was $117 \mathrm{MPa}$, with a compressive stress of $345 \mathrm{MPa}$. The highest total irradiation dose was over $10^{8} \mathrm{~Gy}$.

Tensile fatigue tests on G-1ICR by Korukonda et al. indicated a deterioration in fatigue life above 3000 cycles after a relatively low irradiation dose of $10^{6} \mathrm{~Gy}$. Performance deteriorated more at $77 \mathrm{~K}$ than at $295 \mathrm{~K}$. Perhaps the relatively low fatigue life found in this investigation was due to the definition of failure as initiation and propagation of a surface crack of a certain size, rather than as a complete failure of the specimen (Supplementary Table (.3-1). Obviously, the test mode and the orientation of the load and reinforcing fibers were also different from those of the other investigations.

Flexural fatigue life tests on a neat bisphenol A-based resin by Nishijima et al. indicated significant degradation after cryogenic irradiation and testing without ambient warm-up. The fast neutron fluence was $2.5 \times 10^{20} / \mathrm{m}^{2}$

$(\mathrm{E}>1 \mathrm{MeV})$. 



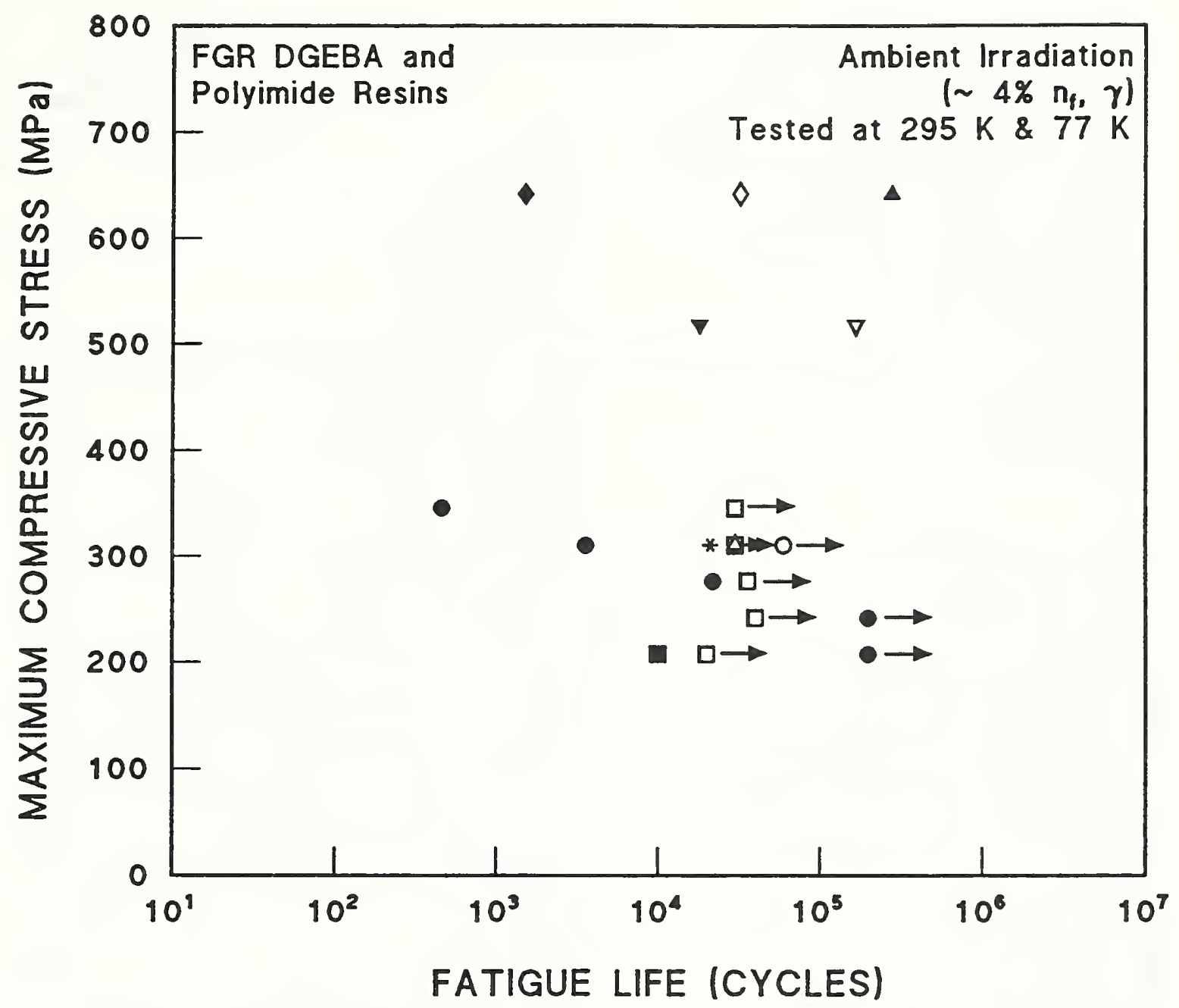

\begin{tabular}{|c|c|c|c|}
\hline Laminate & Test Temp. & Irrad. Dose & Supplier \\
\hline - $G-10$ & $295 \mathrm{~K}$ & 0 & -- \\
\hline - $G-10$ & $295 \mathrm{~K}$ & $4 \times 10^{9}$ Gy & --- \\
\hline 口 $G-10$ & $77 \mathrm{~K}$ & $4 \times 10^{9} \mathrm{~Gy}$ & --- \\
\hline - $G-11$ & $295 \mathrm{~K}$ & $4 \times 10^{9} \mathrm{~Gy}$ & --- \\
\hline$\triangle$ DGEBA or TGPAP** & $295 \mathrm{~K}$ & $4.5 \times 10^{8} \mathrm{~Gy}$ & Ciba, Owens-Corning \\
\hline$\Delta$ Kerimid $601 * *$ & $295 \mathrm{~K}$ & $4 \times 10^{9} \mathrm{~Gy}$ & -- \\
\hline$\nabla . \diamond$ Kerimid $601 * *$ & $295 k$ & $8 \times 10^{9} \mathrm{~Gy}$ & --- \\
\hline$\nabla, \nabla$ Kerimid $601 * *$ & $295 \mathrm{~K}$ & $1.4 \times 10^{10} \mathrm{~Gy}$ & -- \\
\hline
\end{tabular}

Figure C.1-1. Fatigue life at 295 and $77 \mathrm{~K}$ of FGR laminates (G-10 and G-11), FGR DGEBA resin, and FGR polyaminobismaleimide resin (Kerimid 601) after ambient reactor irradiation. Supplementary Table C.1-1. The total dose of gamma and fast neutron irradiation ( $\mathrm{E}>0.1 \mathrm{MeV}$ ) was estimated from the usual conversion rule, except for the Kerimid 601 doses from Becker et al. [1985], in which $3.2 \times 10^{15} / \mathrm{m}^{2}=1$ Gy was assumed. Data from Erez and Becker [1982] and Becker et al. [1985]. 
Table C.1-1. Supplementary Information, Erez and Becker [1982].

\begin{tabular}{|c|c|}
\hline IRRADIATION PARAMETERS & MATERIALS \\
\hline Source Type: Reactor & Resin: \\
\hline Source Name: ATR (INEL) & Hardener: \\
\hline $\begin{array}{l}\text { Irradiation } \\
\text { Temperature: } 49^{\circ}\end{array}$ & Additives: \\
\hline Environment: & Glass Vol. q : \\
\hline Neutron $q$ : & Glass Mass of: \\
\hline Neutron Fluence: $1 \times 10^{24} / \mathrm{m}^{2}$ & $\begin{array}{ll}\text { Glass Type: } & E \text { (G-10, G-11), } \\
& \text { S (others) }\end{array}$ \\
\hline Fast Neutron $8: 4 \quad(E>0.1 \mathrm{MeV})$ & Glass Finish: \\
\hline Fast Neutron Fluence: $\quad 1.6 \times 10^{23} / \mathrm{m}^{2}$ & Glass Weave: \\
\hline Gamma $q$ : & Glass Areal Density, $\mathrm{kg} / \mathrm{m}^{2}$ : \\
\hline Gamma Energy: & Filament Diameter, $\mu \mathrm{m}$ : \\
\hline Gamma Dose: $3.8 \times 10^{9} \mathrm{~Gy}$ & Layup: \\
\hline Electron $8:$ & Production Method: \\
\hline Electron Energy: & Other: \\
\hline \multicolumn{2}{|l|}{ Electron Dose Rate: } \\
\hline \multicolumn{2}{|l|}{ Other: } \\
\hline \multicolumn{2}{|c|}{ TEST METHOD } \\
\hline Test Type: Compressive fatigue & \\
\hline \multicolumn{2}{|l|}{ Test Specifications: } \\
\hline \multicolumn{2}{|l|}{ Specimen Shape: Disk } \\
\hline \multicolumn{2}{|c|}{ Specimen Dimensions: 11.1 -mm diam., 6.4-mm thick } \\
\hline \multicolumn{2}{|l|}{ Flexure: Span/Thickness Ratio: } \\
\hline \multicolumn{2}{|c|}{ Compression: Aspect Ratio, Length/Width or Diam.: 0.58} \\
\hline \multicolumn{2}{|c|}{ Specimens Tested/Data Point: } \\
\hline \multicolumn{2}{|l|}{ Strain (Load) Rate: } \\
\hline \multicolumn{2}{|l|}{ Test Temperature, $\mathrm{K}: \quad 77,295$} \\
\hline Other: $1 \mathrm{~Hz}$ & \\
\hline
\end{tabular}




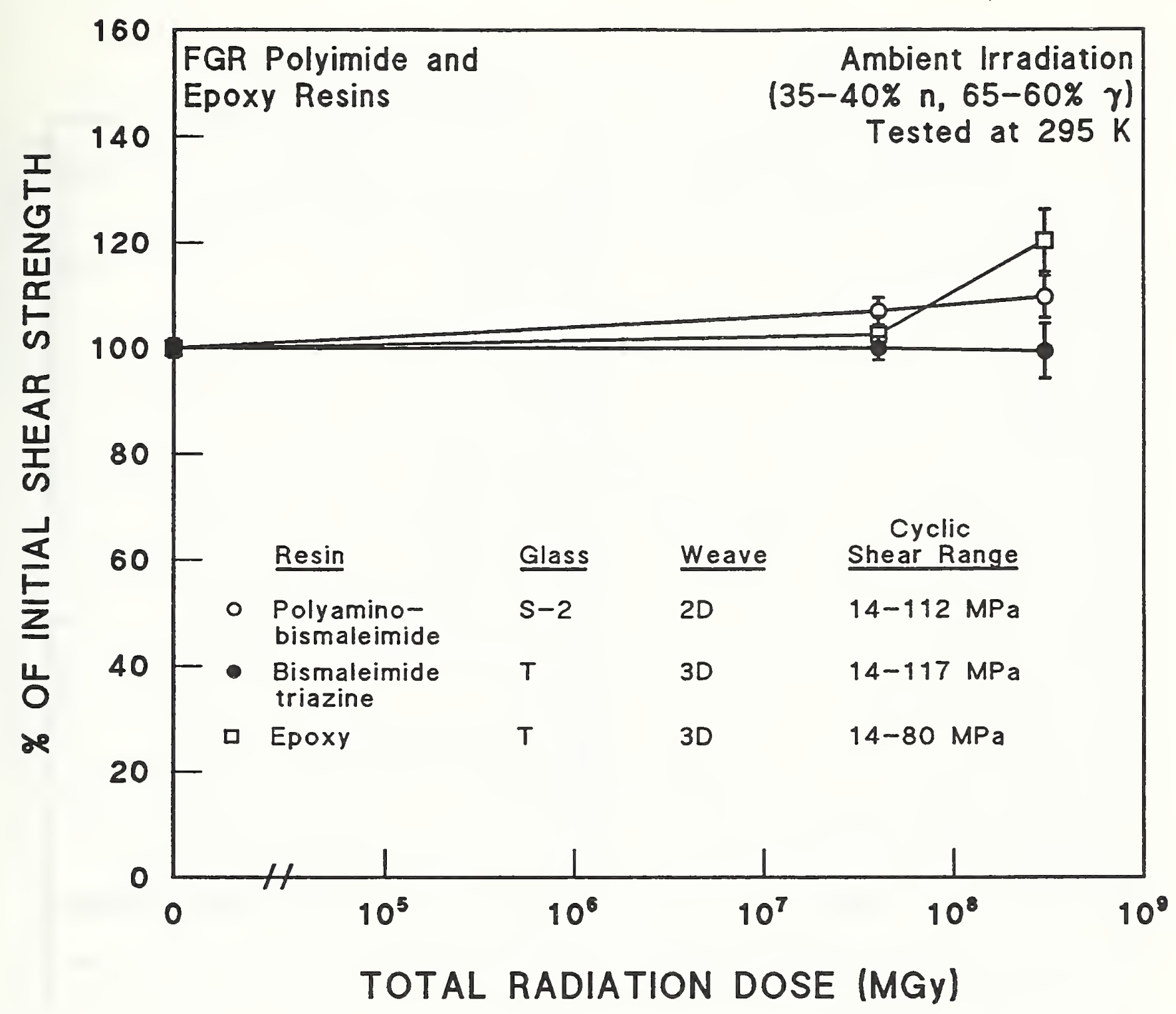

\begin{tabular}{|c|c|c|c|}
\hline & Strength, MPa & Laminate Type & Supplier \\
\hline & $125.9 \pm 2.2$ & Spaulrad S & Spaulding \\
\hline 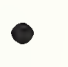 & $131.9 \pm 1.7$ & PG 5-1 & Shikishim \\
\hline 口 & $113.2 \pm 1.8$ & $P G \quad 3-1$ & Shikishima \\
\hline
\end{tabular}

Figure C.2-1. Shear/compressive fatigue at $295 \mathrm{~K}$ of FGR polyaminobismaleimide laminate (Spaulrad S), BT, and epoxy after ambient reactor irradiation.

Supplementary Table C.2-1. Data from McManamy et al. [1991a]. 
Table C.2-1. Supplementary Information, McManamy et al. [1991a].

\begin{tabular}{|c|c|}
\hline IRRADIATION PARAMETERS & MATERIALS \\
\hline Source Type: See Table A.2-17 & Resin: See Table A.2-17 \\
\hline Source Name: & Hardener: \\
\hline $\begin{array}{l}\text { Irradiation } \\
\text { Temperature: }\end{array}$ & Additives: \\
\hline Environment: & Glass Vol. 8 : \\
\hline Neutron $8:$ & Glass Mass $8:$ \\
\hline Neutron Flux: & Glass Type: \\
\hline Fast Neutron $\frac{q}{8}: \quad(E>M e V)$ & Glass Finish: \\
\hline Fast Neutron Flux: & Glass Weave: \\
\hline Gamma : : & Glass Areal Density, $\mathrm{kg} / \mathrm{m}^{2}$ : \\
\hline Gamma Energy: & Filament Diameter, $\mu \mathrm{m}$ : \\
\hline Gamma Dose Rate: & Layup: \\
\hline Electron 8 : & Production Method: \\
\hline Electron Energy: & Other: \\
\hline \multicolumn{2}{|l|}{ Electron Dose Rate: } \\
\hline \multicolumn{2}{|l|}{ Other: } \\
\hline \multicolumn{2}{|c|}{ TEST METHOD } \\
\hline \multicolumn{2}{|c|}{ Test Type: Shear/345 MPa Compression Fatigue } \\
\hline \multicolumn{2}{|l|}{ Test Specifications: } \\
\hline \multicolumn{2}{|l|}{ Specimen Shape: See Figure 11.4} \\
\hline \multicolumn{2}{|c|}{ Specimen Dimensions: See Figure 11.4} \\
\hline \multicolumn{2}{|l|}{ Flexure: Span/Thickness Ratio: } \\
\hline \multicolumn{2}{|c|}{ Compression: Aspect Ratio, Length/Width or Diam.: 0.1} \\
\hline \multicolumn{2}{|c|}{ Specimens Tested/Data Point: } \\
\hline \multicolumn{2}{|l|}{ Strain (Load) Rate: } \\
\hline \multicolumn{2}{|l|}{ Test Temperature, K: 295} \\
\hline other: $\quad 5 \mathrm{~Hz}$ & \\
\hline
\end{tabular}




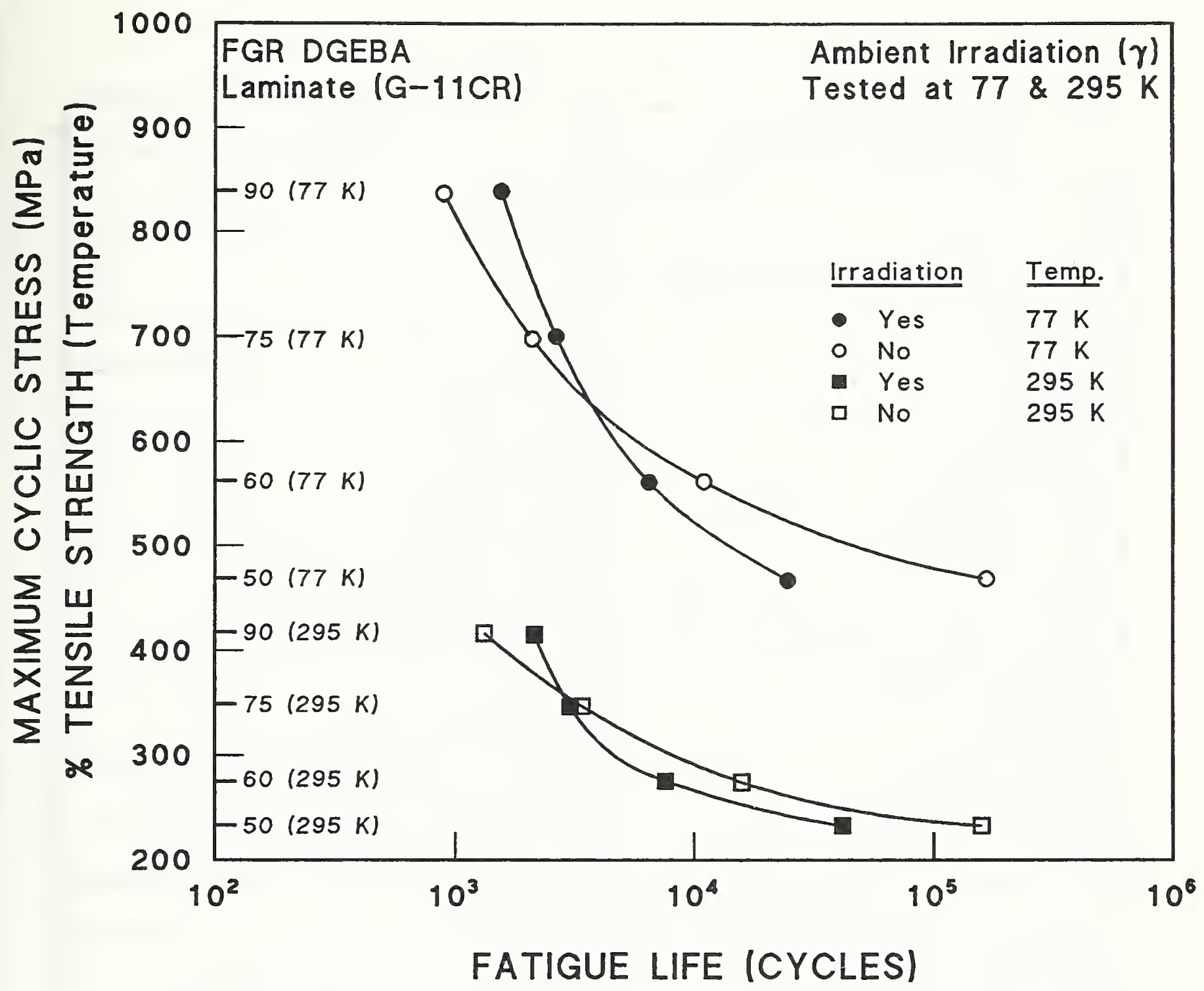

Figure C.3-1. Fatigue life at 295 and $77 \mathrm{~K}$ of FGR laminate, G-11CR, after ambient ${ }^{60}$ Co irradiation. Supplementary Table C.3-1. Data from Korukonda et a1. [1983]. The percent of tensile strength is also shown (463 MPa at $295 \mathrm{~K}, 932 \mathrm{MPa}$ at $77 \mathrm{~K}$ ). The number of cycles to crack initiation is plotted. 
Table C.3-1. Supplementary Information, Korukunda et al. [1983].

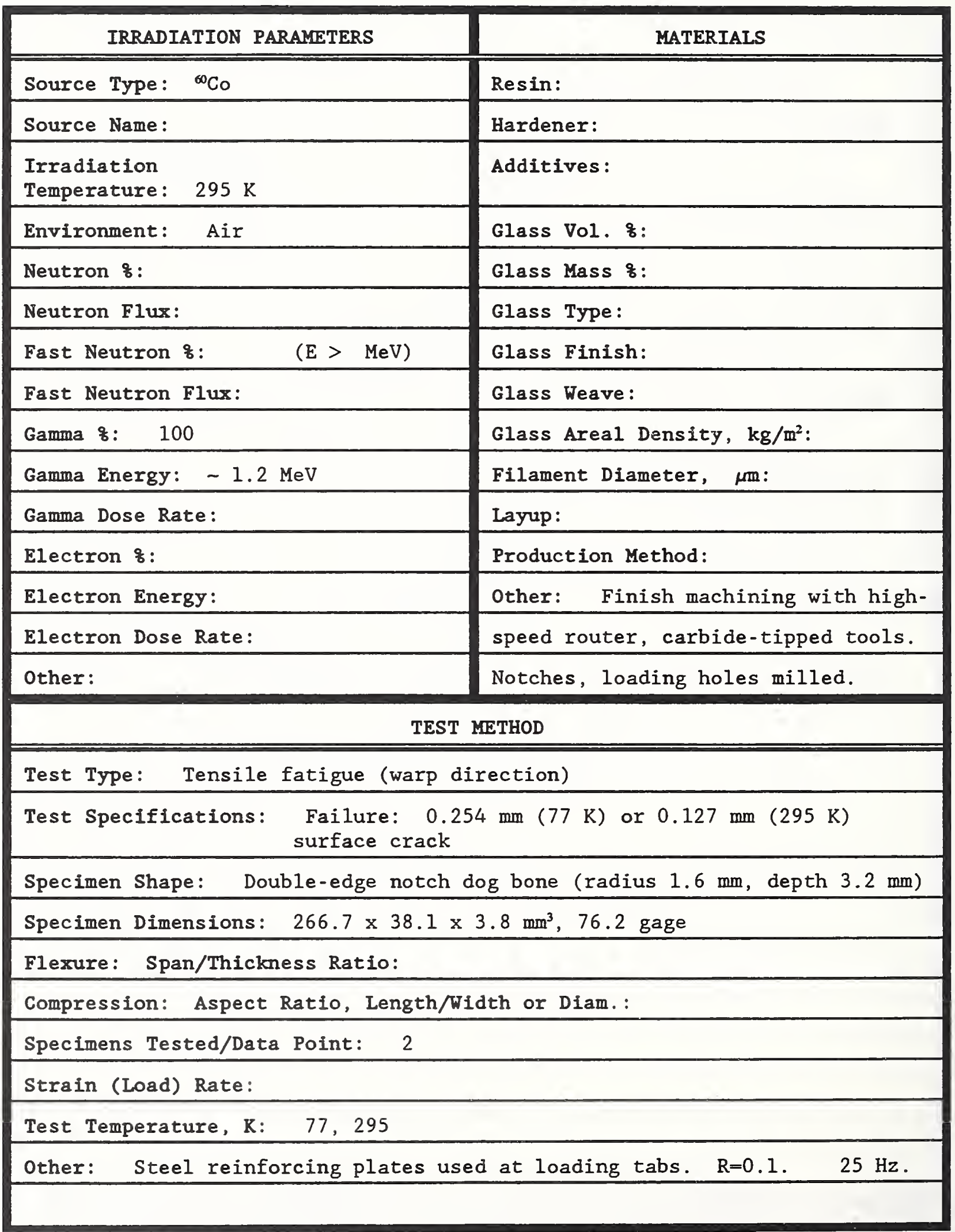




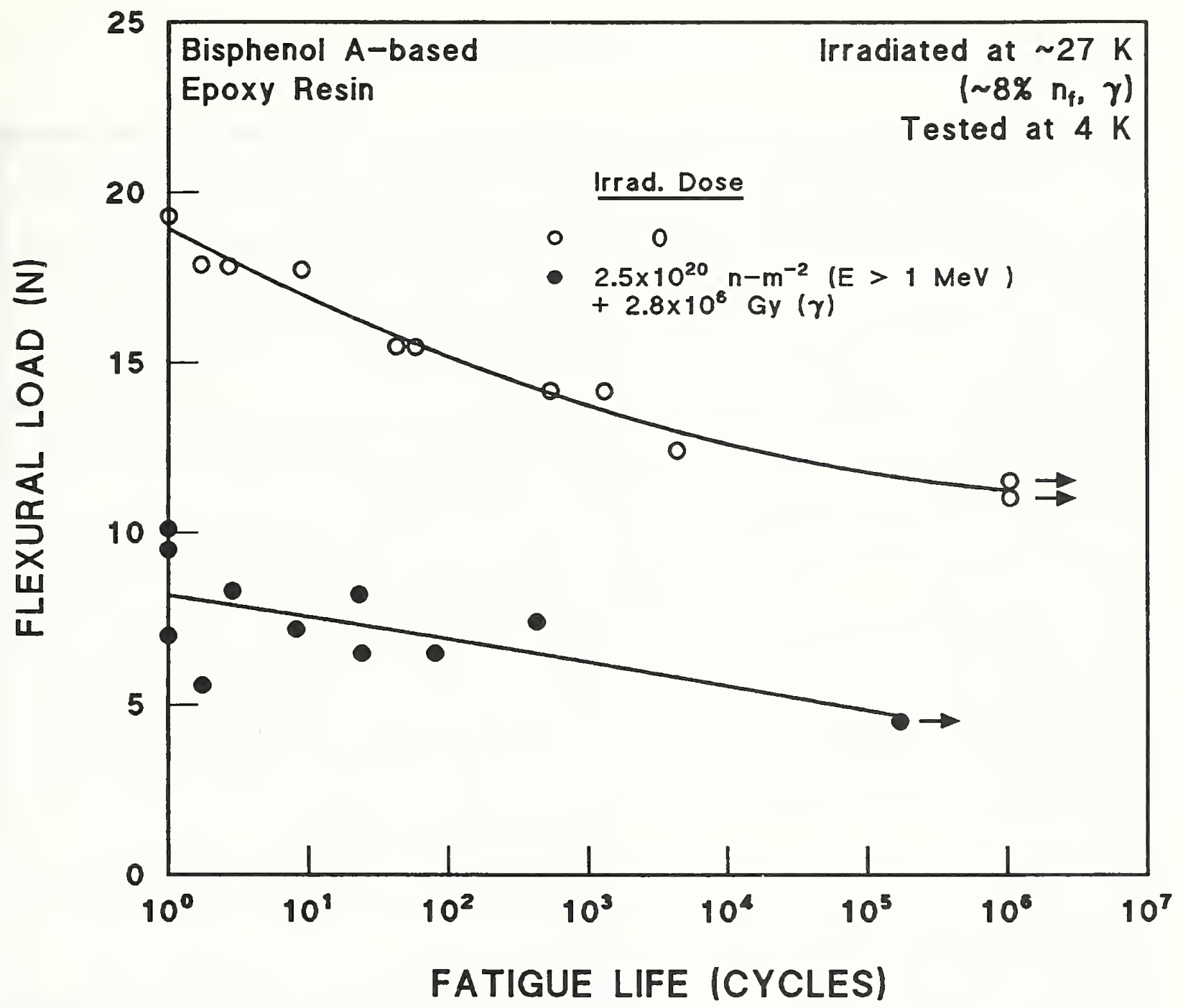

Hardener

0,0 Polyethanol complex of $\mathrm{Ti}$ with phenyl glycidyl ether $(8879-2)$
Supplier

Ryoden Kasei Co., Ltd.

Figure C.4-1. Flexural fatigue life at 4-K of neat bisphenol A-based resin (6861-2) after $-27-\mathrm{K}$ reactor irradiation. Supplementary Table C.4-1. Data from Nishijima et al. [1981]. 
Table C.4-1. Supplementary Information, Nishijima et al. [1981].

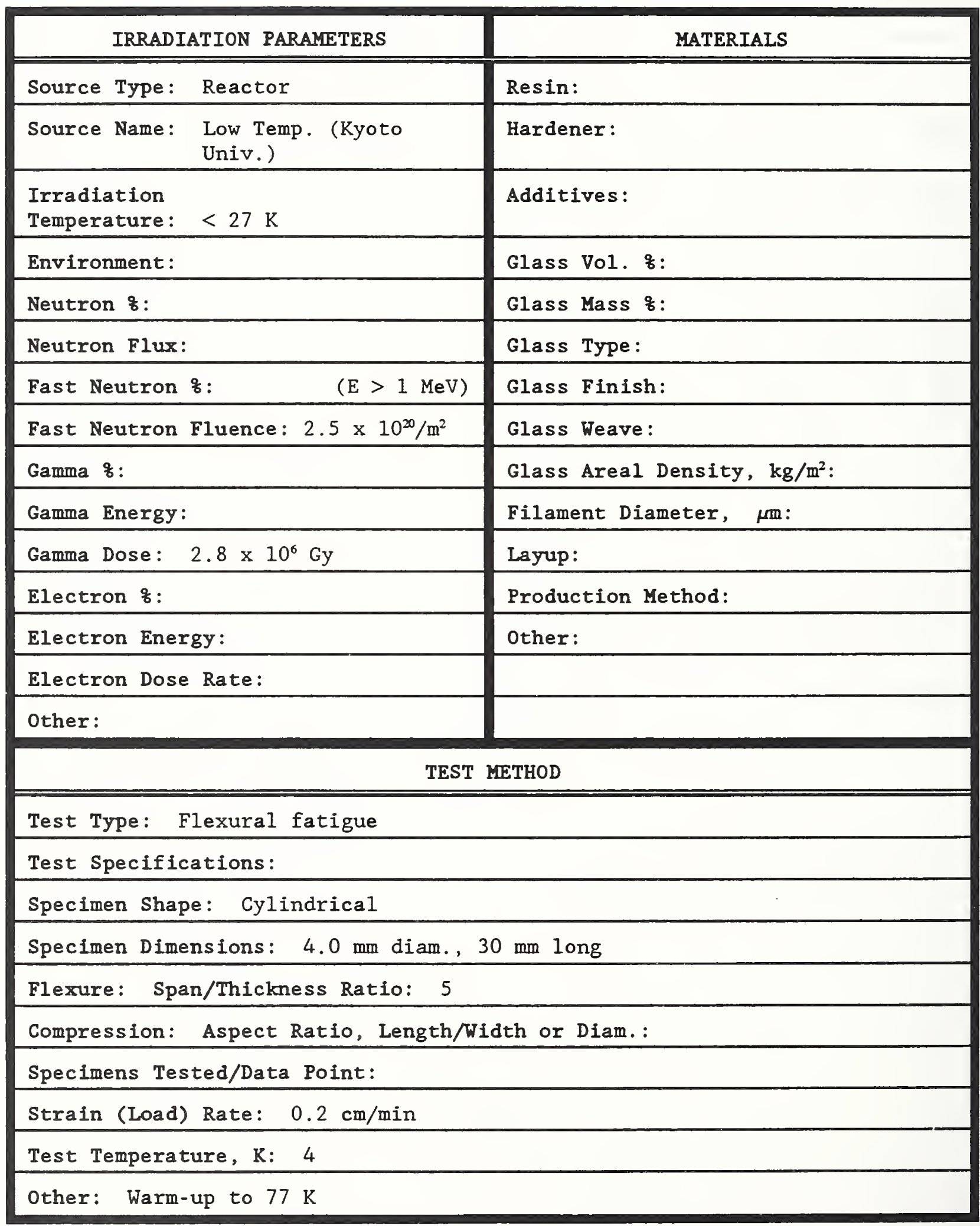


Abdou, M.A. 1978. Radiation Considerations for Superconducting Fusion Magnets. Journal of Nuclear Materials 72. PP. 147-167.

Abe, K.; Logan, C.M.; Saneyoshi, K.; Clinard, F.W., Jr. 1987. Irradiation Effects in KAPTON Polyimide Film from $14 \mathrm{-MeV}$ Neutrons and Cobalt-60 Gamma Rays. In: Influence of Radiation on Material Properties: 13th International symposium (Part II), ASTM STP 956. F.A. Garner, C.H. Henager, Jr., and N. Igata, eds. American Society for Testing and Materials, Philadelphia. pp. 668-681.

Banford, H.M. 1984. Electrical Insulation and Fusion Reactors. In: Advances in Nuclear Science and Technology, Vol. 16. J. Lewins, M. Becker, eds. Plenum Press, New York. pp. 1-73.

Becker, H. 1990. Problems of Cryogenic Interlaminar Shear Strength Testing. In: Advances in Cryogenic Engineering (Materials), Vol. 36. R.P. Reed and F.R. Fickett, eds. Plenum Press, New York. Pp. 827-834.

Becker, H.; Marston, P.G.; Montgomery, D.B.; Schmunk, R.J. ; Tognarelli, D. 1985. Strengths of Unirradiated and Irradiated Thin Sheet Organic Laminate Insulators Loaded Perpendicular to the Laminations. In: Seventh Annual Progress Report on Special Purpose Materials For Magnetically Confined Fusion Reactors. DOE/ER-0113/4. U.S. Department of Energy, Washington, DC. PP. 4041 .

Berger, M.J.; Seltzer, S.M. 1982. Stopping Powers and Ranges of Electrons and Positrons (2nd Ed.). NBSIR 82-2550-A. National Bureau of Standards, Gaithersburg, MD. 169 pp.

Berger, M.J.; Inokuti, M.; Andersen, H.H.; Bichsel, H.; Powers, D.; Seltzer, S.M.; Thwaites, D.; Watt, D.E. 1992. Stopping Powers and Ranges for Protons and Alpha Particles. Preliminary Version of ICRU Report 49. National Institute of Standards and Technology, Gaithersburg, MD. 446 pp.

Beynel, P.; Maier, P.; Schönbacher, H. 1982. Compilation of Radiation Damage Test Data, Part III: Materials Used Around High-Energy Accelerators. CERN 82-10. European Organization for Nuclear Research, Geneva. 339 pp.

Billington, D.S.; Crawford, J.H., Jr. 1961. Radiation Damage in Solids. Investigations in Physics. E.P. Wigner, R. Hofstadter, eds. Princeton University Press, Princeton, NJ.

Birtcher, R.C.; Blewitt, T.H.; Kirk, M.A.; Scott, T.L.; Brown, B.S.; Greenwood, L.R. 1982. Neutron Irradiation Facilities at the Intense Pulsed Neutron Source. Journal of Nuclear Materials $108 \& 109$. pp. 3-9.

Bottura, L. ; Hasegawa, M. ; Heim, J.; Kalinin, V. ; Katheder, H. ; Koizumi, K. ; Kostenko, A.; Miller, J.R.; Mitchell, N.; Roshal, A.; Schultz, J.; Shen, S.S.; Summers, L.; Tada, E.; Yoshida, K. 1991. ITER Magnets. ITER Documentation Series, No. 26. International Atomic Energy Agency, Vienna. 194 Pp. 
Brandenberger, K. 1992. Presentation at Workshop, Magnet Insulation for ITER, Plasma Fusion Center, Massachusetts Institute of Technology, Cambridge, MA, December 9, 10, 1992.

Brechna, H. 1965. Effect of Nuclear Radiation on Organic Materials; Specifically Magnet Insulations in High-Energy Accelerators. SLAC 40, Stanford Linear Accelerator Center, Stanford University, Stanford, CA. 86 pp.

Chabert, J.N.; Marcou, A. 1989. Mechanical Testing of Irradiated Specimens of Magnet Insulation, Rept. No. NOTE SBT/CT/89-39. C.E.A. - C.E.N.G. D.R.F. unpaged.

Chaplin, R.L.; Kerchner, H.R.; Klabunde, C.E.; Coltman, R.R. 1989. Stored Energy in Fusion Magnet Materials Irradiated at Low Temperatures. ORNL/TM-11217. Oak Ridge National Laboratory, Oak Ridge, TN.

Charlesby, A. 1972. Radiation Effects in Polymers. Chapter 23 in: Polymer Science, Vol. 2. A.D. Jenkins, ed. North-Holland Publishing Company, New York. p. 1544.

Coltman, R.R., Jr.; Klabunde, C.E. 1981. Mechanical Strength of Low-TemperatureIrradiated Polyimides: A Five-to-Tenfold Improvement in Dose-Resistance over Epoxies. Journal of Nuclear Materials $103 \& 104$. pp. 717-722.

Coltman, R.R., Jr.; Klabunde, C.E. 1983. The Strength of G-10CR and G-11CR Epoxies after Irradiation at $5 \mathrm{~K}$ by Gamma Rays. Journal of Nuclear Materials 113. pp. 268-272.

Coltman, R.R., Jr.; Klabunde, C.E.; Kernohan, R.H.; Long, C.J. 1979. Radiation Effects on Organic Insulators for Superconducting Magnets. ORNL/TM-7077. Oak Ridge National Laboratory, Oak Ridge, TN. 52 pp.

Daenner, W.; E1-Guebaly, L.; Sawan, M.; Gohar, Y.; Maki, K. ; Rado, V.; Schchipakin, 0.; Zimin, S. 1991. Neutron Shielding and Its Impact on the ITER Machine Design. Fusion Engineering and Design 16. pp. 183-193.

Dahlerup-Petersen, K. 1980. Tests of Composite Materials at Cryogenic Temperatures: Facilities and Results. In: Advances in Cryogenic Engineering (Materials), Vol. 26. A.F. Clark, R.P. Reed, eds. Plenum Press, New York. PP. 268-279.

Dahlerup-Petersen, K.; Perrot, A. 1979. Properties of Organic Composite Materials at Cryogenic Temperatures. CERN ISR-BOM/79-39. European Organization for Nuclear Research, Geneva. unpaged.

Darr, J.B.; Fabian, P.E.; Reed, R.P. 1992. Private communication. Cryogenic Materials, Inc., 2625 Iliff, Boulder, CO 80303.

De11, G.F.; Goland, A.N. 1981. Radiation Damage Parameters in Multicomponent Nonmetals. Journal of Nuclear Materials 102. pp. 246-258. 
Dresner, L.; Fietz, W.A.; Gauss, S.; Haubenreich, P.N.; Jakob, B. ; Kato, T.; Komarek, P.; Lubell, M.S.; Lue, J.W.; Luton, J.N. ; Maurer, W.; Okuno, K. ; Schwenterly, S.W.; Shimamoto, S.; Takahashi, Y.; Ulbricht, A.; Vecsey, G.; Wüchner, F.; Zinchy, J.A. 1989. Results of the International Large Coil Task: A Milestone for Superconducting Magnets in Fusion Power. Cryogenics 29. pp. $875-882$.

Drexler, E.S.; Simon, N.J.; Reed, R.P. 1992. Shear-Compressive Properties of Vacuum-Impregnated Insulation at $76 \mathrm{~K}$. In: Proc. Int. Cryogenic Materials Conference, Kiev, Ukraine, to be published in Cryogenics.

Egusa, S. 1988. Mechanism of Radiation-Induced Degradation in Mechanical Properties of Polymer Matrix Composites. Journal of Materials Science 23. pp. $2753-2760$.

---1990a. Irradiation Effects on and Degradation Mechanism of the Mechanical Properties of Polymer Matrix Composites at Low Temperatures. In: Advances in Cryogenic Engineering (Materials), Vol. 36. R.P. Reed and F.R. Fickett, eds. Plenum Press, New York. Pp. 861-868.

-1990b. Anisotropy of Radiation-Induced Degradation in Mechanical Properties of Fabric-Reinforced Polymer-Matrix Composites. Journal of Materials Science 25. pp. $1863-1871$.

-.-1991a. Radiation Resistance of Polymer Composites at $77 \mathrm{~K}$; Effects of Reinforcing Fabric Type, Specimen Thickness, Radiation Type, and Irradiation Atmosphere. Cryogenics 31. PP. 7-15.

-..-1991b. Effects of Neutrons and Gamma-Rays on Polymer Matrix Composites as LowTemperature Materials. International Journal of Radiation Applications and Instrumentation, Part C, Radiation Physics and Chemistry 37. pp. 147-152.

Egusa, S.; Hagiwara, M. 1986. Mechanical Properties of Polymer Matrix Composites at $77 \mathrm{~K}$ and at Room Temperature after Irradiation with ${ }^{60} \mathrm{Co} \gamma$-Rays. Cryogenics 26. pp. 417-422.

Egusa, S.; Kirk, M.A.; Birtcher, R.C. 1984a. Neutron Irradiation Effects on the Mechanical Properties of Organic Composite Materials. Journal of Nuclear Materials 126. pP. 152-159.

Egusa, S.; Kirk, M.A.; Birtcher, R.C.; Hagiwara, M.; Kawanishi, S. 1984 b. Irradiation Effects on the Mechanical Properties of Composite Organic Insulators. Nuclear Instruments and Methods in Physics Research $B 1$. Pp. 610-616.

Egusa, S.; Kirk, M.A.; Birtcher, R.C.; Hagiwara, M. 1985a. Neutron and $\gamma$-Ray Irradiation Effects in Composite Organic Insulators. Journal of Nuclear Materials $133 \& 134$. pp. 795-799.

Egusa, S.; Kirk, M.A.; Birtcher, R.C.; Hagiwara, M. 1985b. Annealing Effects on the Mechanical Properties of Organic Composite Materials Irradiated with $\gamma$ Rays. Journal of Nuclear Materials 127. pp. 146-152. 
Egusa, S.; Kirk, M.A.; Birtcher, R.C. 1987a. Effects of Neutron Irradiation on Polymer Matrix Composites at $5 \mathrm{~K}$ and at Room Temperature. I. Absorbed-Dose Calculation. Journal of Nuclear Materials 148. pp. 43-52.

Egusa, S.; Kirk, M.A.; Birtcher, R.C. 1987b. Effects of Neutron Irradiation on Polymer Matrix Composites at $5 \mathrm{~K}$ and at Room Temperature. II. Degradation of Mechanical Properties. Journal of Nuclear Materials 148. pp. 53-60.

Egusa, S.; Udagawa, A.; Hashimoto, O.; Ono, T.; Yamamoto, Y.; Sonoda, K. 1988. Preparation and Radiation-Resistance Evaluation of Glass Fibre Composites Having Various Epoxy Matrices. Journal of Materials Science Letters 7. pp. 503-505.

Egusa, S.; Sugimoto, M.; Nakajima, H.; Yoshida, K.; Tsuji, H. 1992. Effects of Fabric Type, Specimen Size, and Irradiation Atmosphere on the Radiation Resistance of Polymer Composites at $77 \mathrm{~K}$. In: Advances in Cryogenic Engineering (Materials), Vol. 38. F.R. Fickett and R.P. Reed, eds. Plenum Press, New York. pp. 247-254.

E1-Guebaly, L.A. 1990. Private communication (memorandum to L. Summers, December 1990), Fusion Technology Institute, University of Wisconsin, Madison, WI.

E1-Guebaly, L.A.; Sawan, M.E. 1989. Tungsten versus Steel in Inboard Shield of ITER: Impact on Magnet Damage, Reactor Size, and Cost. Fusion Technology 15. pp. $881-886$.

Erez, E.A.; Becker, H. 1982. Radiation Damage in Thin Sheet Fiberglass Insulators. In: Nonmetallic Materials and Composites at Cryogenic Temperatures, Hartwig and Evans, eds. Plenum Press, New York. pp. 59-71.

Evans, D.; Morgan, J.T. 1982. A Review of the Effects of Ionising Radiation on Plastic Materials at Low Temperatures. In: Advances in Cryogenic Engineering (Materials), Vol. 28. R.P. Reed and A.F. Clark, eds. Plenum Press, New York. Pp. 147-164.

-.--1984. The Chemistry of Radiation Damage in Epoxide Resins. In: Advances in Cryogenic Engineering (Materials), Vol. 30. A.F. Clark and R.P. Reed, eds. Plenum Press, New York. pp. 89-96.

Evans, D.; Morgan, J.T.; Sheldon, R.; Stapleton, G.B. 1970. Post Irradiation Mechanical Properties of Epoxy Resin Glass Composites. RHEL/R 200. Rutherford Laboratory, Chilton, England. 19 pp.

Evans, D.; Morgan, J.T.; Stapleton, G.B. 1971. The Effect of Ionising Radiation on Synthetic Resins at Very Low Temperature - An Initial Study. RHEL/R 220. Rutherford Laboratory, Chilton, England. 21 pp.

-1972a. Irradiation Damage Studies of some Epoxy Resin Systems of Reduced Viscosity. RHEL/R 249. Rutherford Laboratory. Chilton, England. 19 pp.

---1972b. Epoxy Resins for Superconducting Magnet Encapsulation. RHEL/R 251. Rutherford Laboratory, Chilton, England. 27 pp. 
Fardi, F.; Mitche11, N.; Poehlchen, R. 1989. Comparison of Test Results with Analysis of Glass Fibre Reinforced Epoxy Shear Test Samples. In: $11 \mathrm{th}$ International Conference on Magnet Technology (MT-11), Vol. 1. T. Sekiguchi and S. Shimamoto, eds. Elsevier Applied Science, London and New York. pp. $719-724$.

Fassó, A.; Goebe1, K.; Höfert, M. ; Rau, G.; Schönbacher, H.; Stevenson, G.R.; Sullivan, A.H.; Swanson, W.P.; Tuyn, J.W.N. 1984. Radiation Problems in the Design of the Large Electron-Positron Collider (LEP). CERN 84-02. European Organization for Nuclear Research, Geneva. 93 PP.

Gerstenberg, H.; Gläser, W. 1992. Neutron Irradiations at Temperatures Below $6 \mathrm{~K}$ at the Munich Research Reactor (FRM). Nuclear Engineering and Science 110. pp. $50-55$.

GESSS. 1974. Materials for Use in Superconducting Magnet Construction - A Report of the Work of the GESSS Collaboration. Rutherford Laboratory, Chilton, England. unpaged.

Hacker, H.; Albrecht, C. ; Laupenmühlen, H.; Salzburger, H. ; Ihlein, W. 1984. Epoxies for Low Temperature Application Impregnating Technology. In:

Advances in Cryogenic Engineering (Materials), Vol. 30. A.F. Clark and R.P. Reed, eds. Plenum Press, New York. pp. 51-60.

Hagiwara, M.; Udagawa, A.; Kawanishi, S.; Egusa, S.; Takeda, N. 1985. Degradation Behavior of Fiber Reinforced Composites under Irradiation by $3 \mathrm{MeV}$ Electrons. Journal of Nuclear Materials $133 \& 134$. pp. 810-814.

Hahn, P.A.; Weber, H.W.; Guinan, M.W.; Birtcher, R.C.; Brown, B.S.; Greenwood, L.R. 1986. Neutron Irradiation of Superconductors and Damage Energy Scaling of Different Neutron Spectra. In: Advances in Cryogenic Engineering (Materials), Vol. 32. R.P. Reed and A.F. Clark, eds. Plenum Press, New York. pp. $865-872$.

Hartwig, G. 1987. Radiation Damage to Polymers and Fibre Composites. In: Insulators for Fusion Applications. IAEA-TECDOC-417. International Atomic Energy Agency, Vienna. Pp. 119-157.

Henning, C.D., ed. 1989. Magnet Design Technical Report - ITER Definition Phase. Rept No. UCID 21618. Lawrence Livermore National Laboratory, CA. 117 pp.

Henning, C.D.; Miller, J.R. 1989. Design Considerations for ITER Magnet Systems. Fusion Technology 15. Pp. 915-921.

Hurley, G.F.; Fowler, J.D., Jr.; Liepins, R. ; Jorgenson, B. ; Hammond, J. 1978. Comparison of Neutron and Gamma Irradiation Damage in Organic Insulators. Rept. No. LA-UR 82-1978. Los Alamos National Laboratory, Los Alamos, NM. unpaged.

Hurley, G.F.; Fowler, J.D.; Rohr, D.L. 1983. Low Dose Cryogenic Neutron Irradiation Effects in G-10CR. Cryogenics 23. pp. 415-440. 
Iida, T.; Sumita, K. 1983. Effects of $14 \mathrm{MeV}$ Neutron Irradiation on Polymer Composites. In: 1982 Annual Research Report of Japanese Contributions For US-Japan Collaboration on RTNS-II Utilization. K. Sumita, ed. Osaka University, Yamada-Oka 2-1, Suita, Osaka 565, Japan. pp. 13-20.

Imel, G.R.; Kelsey, P.V.; Ottewitte, E.H. 1979. The Effects of Radiation on IFTR Coil Materials. Journal of Nuclear Materials $85 \& 86$. pp. 367-371.

Iwasaki, Y,; Nishiima, S.; Yasuda, J.; Hirokawa, T.; Okada, T. 1990. Development of 3DFRP for Cryogenic Use. In: Advances in Cryogenic Engineering (Materials), Vol. 36. R.P. Reed and F.R. Fickett, eds. Plenum Press, New York. pp. 969-976.

Kasen, M.B.; Stoddard, R.B. 1986. Screening the Performance of Organic Insulators Under Cryogenic Neutron Irradiation. In: Advances in Cryogenic Engineering (Materials), Vol. 32. R.P. Reed and A.F. Clark, eds. Plenum Press, New York. pp. $153-159$.

Katheder, H.K.; Gerstenberg, H.; Krähling, E.; Söll, M. 1992. Irradiation and Testing at $4.5 \mathrm{~K}$ of Epoxy-Glass-Fibre-Steel Samples. Preprint, The NET Team, Max Planck Institut für Plasma Physik, Boltzmannstrasse 2, D-8046 Garching bei München.

Kato, T.; Takamura, S. 1979. Effect of Low Temperature Reactor Irradiation on Organic Insulators in Superconducting Magnets (II). Cryogenic Engineering (Japan) 14. pp. 178-183.

Kernohan, R.H.; Long, C.J.; Coltman, R.R., Jr. 1979. Cryogenic Radiation Effects on Electric Insulators. Journal of Nuclear Materials $85 \& 86$. pp. 379-383.

Kircher, J.F. 1964. Basic Concepts of Radiation Effects. In: Effects of Radiation on Materials and Components. Kircher,J.F.; Bowman, R.E., eds. Reinhold Publishing Corp. NY. pp. 11-48.

Klabunde, C.E.; Coltman, R.R., Jr. 1983. Debonding of Epoxy from Glass in Irradiated Laminates. Journal of Nuclear Materials 117. pp. 345-350.

Korukonda, B.; Conway, J.C., Jr., Queeney, R.A.; Diethorn, W.S. 1983. The Effect of Radiation and Cryogenic Temperature on the Fatigue Resistance of G-11 CR Glass-Cloth/Epoxy Laminates. Journal of Nuclear Materials 115. pp. 197-202.

Lapp, R.E.; Andrews, H.L. 1954. Nuclear Radiation Physics. Second Edition. Prentice-Hall, Inc.

Leighton, R.B. 1959. Principles of Modern Physics. McGraw-Hill Book Co., NY.

Lipták, G.; Schuler, R.; Maier, P.; Schönbacher, H.; Haberthür, B. ; Müller, H.; Zeier, W. 1985. Radiation Tests on Selected Electrical Insulating Materials for High-Power and High Voltage Application. CERN 85-02. European Organization for Nuclear Research. Geneva. 58 pp.

Long, C.J.; Kernohan, R.H.; Coltman, R.R., Jr. 1979. Radiation Effects on Insulators for Superconducting Magnets. In: Nonmetallic Materials and Composites at Low Temperatures. A.F. Clark, R.P. Reed, and G. Hartwig, eds. Plenum Press, New York. Pp. 141-153. 
Maki, K.; Takatsu, H.; Kuroda, T.; Seki, T.; Nakamura, T.; Mori, S.; Kawasaki, H. 1991. Japanese Contributions to ITER Shielding Neutronics Design. JAERI-M 91-046. Japan Atomic Energy Research Inst. Tokai, Ibaraki, Japan. 163 pp.

Markley, F.; Forster, G.A.; Booth, R. 1969. Radiation Damage Studies of Zero Gradient Synchrotron Magnet Insulation and Related Materials. IEEE Transactions on Nuclear Science NS-16. pp. 606-610.

Maurer, W. 1985. Neutron and Gamma Irradiation Effects on Organic Insulating Materials for Fusion Magnets. Report No. KfK 3974. Kernforschungszentrum Karlsruhe, Karlsruhe, Germany. 68 pp.

McManamy, T.J.; Snook, P. 1989. Shear/Compression Tests of Vendor Supplied Insulation Sheet Material. F-900116-PPL-03, Princeton Plasma Physics Laboratory. unpaged.

McManamy, T.J.; Brasier, J.E.; Snook, P. 1990. Insulation Interlaminar Shear Strength Testing with Compression and Irradiation. In: Proc. IEEE 13th Symposium on Fusion Engineering Vol. 1. IEEE, New York, pp. 342-347.

McManamy, T.J.; Kanemoto, G.; Snook, P. 1991a. Insulation Irradiation Test Programme for the Compact Ignition Tokamak. Cryogenics 31. pp. 277-281.

-.-1991b. BPX Insulation Irradiation Program Test Results. Presented at 14th IEEE Symposium on Fusion Engineering, San Diego, CA, 1991. CONF-910968-18. Oak Ridge National Laboratory, Oak Ridge, TN. 12 pp.

Morgan, T.J.; Stapleton, G.B. 1975. Post Irradiation Mechanical Properties of Radiation Resistant Cast Epoxy Resin Systems. RL-75-136. Rutherford Laboratory, Chilton, England. unpaged.

Morgan, T.J.; Scott, G.; Sheldon, R. Stapleton, G.B. 1970. Gas Evolution from Epoxy Resins by High Energy Radiation. RHEL/R 196. Rutherford Laboratory. Chilton, England. $30 \mathrm{pp}$.

Munshi, N.A. 1991. Cost-Effective Techniques for Development of RadiationResistant Organic Insulators for Superconducting Magnets - Final Report. Rept. No. SBIR Phase II Contract No. DE-AC02-87ER80487. U.S. Department of Energy.

...-1992. A Radiation-Resistant Epoxy Resin System for Toroidal Field and Other Superconducting Coil Fabrication. In: Advances in Cryogenic Engineering (Materials), Vol. 38. F.R. Fickett and R.P. Reed, eds. Plenum Press, New York. pp. 255-259.

Munshi, N.A.; Weber, H.W. 1992. Reactor Neutron and Gamma Irradiation of Various Composite Materials. In: Advances in Cryogenic Engineering (Materials), Vol. 38. F.R. Fickett and R.P. Reed, eds. Plenum Press, New York. PP. 233-239.

Nishijima, S.; Okada, T. 1977. Low Temperature Irradiation Effects on Mechanical Properties of Epoxy Resin Used in Superconducting Magnets. Teion Kogaku 12. PP. 224-229. ORNL-tr-4612. Oak Ridge National Laboratory, Oak Ridge, TN.

-..-1978. Low Temperature Irradiation Effects on Mechanical Properties of Epoxy Used in Superconducting Magnets. Cryogenics 18. pp. 215-219. 
Nishijima, S.; Ueta, S.; Okada, T. 1981. The Effects of Low Temperature Irradiation Effect on the Cryogenic Fatigue Resistance of Epoxy Resin Used in Superconducting Magnets. Cryogenics 21, pp. 312-313.

Nishijima, S.; Okada, T.; Miyata, K.; Yamaoka, H. 1988. Radiation Damage of Composite Materials at Cryogenic Temperatures. In: Advances in Cryogenic Engineering (Materials), Vol. 34. A.F. Clark and R.P. Reed, eds. Plenum Press, New York. pp. 35-42.

Nishijima, S.; Nishiura, T.; Okada, T.; Hirokawa, T.; Yasuda, J.; Iwasaki, Y. 1990. Development of Radiation Resistant Composite Materials for Fusion Magnets. In: Advances in Cryogenic Engineering (Materials), Vol. 36. R.P. Reed and F.R. Fickett, eds. Plenum Press, New York. pp. 877-883.

Nishijima, S.; Okada, T.; Hirokawa, T.; Yasuda, J.; Iwasaki, Y. 1991. Radiation Damage of Organic Composite Material for Fusion Magnet. Cryogenics 31. pp. 273-276.

Nishiura, T.; Katagiri, K.; Nishijima S.; Okada, T.; Nakahara, S. 1988a. Irradiation Effects on the Interlaminar Tear Strength of GFRP at Cryogenic Temperatures. In: Advances in Cryogenic Engineering (Materials), Vol. 34. A.F. Clark and R.P. Reed, eds. Plenum Press, New York. pp. 43-50.

Nishiura, T.; Katagiri, K.; Nishijima, S.; Okada, T. 1988b. Evaluation of Irradiation Effect of FRP by AE Method. In: Proc. of the Osaka University International Symposium on New Developments in Applied Superconductivity. Y. Murakami, ed. World Scientific, Singapore. pp. 431-436.

Okada, T. 1992. Private communication. Institute of Science and Industrial Research, Osaka University, Ibaraki, Osaka, Japan 567.

Okada, T.; Nishijima, S. 1990. Investigation of Interlaminar Shear Behavior of Organic Composites at Low Temperatures. In: Advances in Cryogenic Engineering (Materials), Vol 36. R.P. Reed and F.R. Fickett, eds. Plenum Press, New York. pp. 811-817.

Okada, T.; Nishijima, S.; Nishiura, T.; Miyata, K.; Yamaoka, Y.; Namba, S. 1992. Radiation Damage of Glass-Fiber-Reinforced Composite Materials at Low Temperatures. In: Advances in Cryogenic Engineering (Materials), Vol. 38. F.R. Fickett and R.P. Reed, eds. Plenum Press, New York. pp. 241-246.

Parkinson, W.W.; Sisman, 0. 1971. The Use of Plastics and Elastomers in Nuclear Radiation. Nuclear Engineering and Design 17. pp. 247-280.

Phillips, D.C.; Scott, J.M.; Goebel, K.; Schönbacher, H.; Dieterle, W.; Eichenberger, W.; Maurer, J. 1981. The Selection and Properties of Epoxide Resins Used for the Insulation of Magnet Systems in Radiation Environments. CERN 81-05. European Organization for Nuclear Research, Geneva. 11 pp.

Pluym G.; van de Voorde, M. 1967. Radiation Damage Tests on Epoxy Resins. In: Proc. of the Second International Conference on Magnet Technology. H. Hadley, ed. Oxford. p. 341 . 
Poehlchen, R.; Salpietro, E.; Vassiliadis, M.; Rauch, J.; Koenig, F.; Claudet, G.; Chabert, J.N.; Marangos, J.; Kraehling, E.; Soell, M. 1990. The Mechanical Strength of Irradiated Electric Insulation of Superconducting Magnets. In: Advances in Cryogenic Engineering (Materials), Vol. 36. R.P. Reed and F.R. Fickett, eds. Plenum Press, New York. pp. 893-900.

Pöhlchen, R. 1992. Effects of Radiation on Insulation Materials. In: Advances in Cryogenic Engineering (Materials), Vol. 38. F.R. Fickett and R.P. Reed, eds. Plenum Press, New York. pp. 261-269.

Price, M.J.; Sheldon, R. 1965. The Influence of Structure on the Post-Irradiation Physical Properties of Epoxy Resins. RHEL/R 105. Rutherford Laboratory, Chilton, England. unpaged.

Reed, R.P.; Darr, J.B.; Schultz, J.B. 1992. Short-Beam Shear Testing of Candidate Magnet Insulators. In: Proc. Int. Cryogenic Materials Conference, Kiev, Ukraine. To be published in Cryogenics.

Sawan, M. 1993. Private communication. Memorandum dated March 4, to N. Simon, D. Reed. B. Montgomery, J. Schultz, J. Doggett, B. Hassenzahl. Fusion Technology Institute, University of Wisconsin, Madison, WI.

Schmunk, R.E. 1983. Irradiation and Testing of Spaulrad-S for Fusion Magnet Applications. EGG-FT-6277. Idaho National Engineering Laboratory, EG \& G Idaho, Inc., Idaho Falls, ID. 11 pp.

Schmunk, R.E.; Imel, G.R.; Harker, Y.D. 1981. Irradiation Studies of Magnet Insulator Materials. Journal of Nuclear Materials $103 \& 104$. pp. 723-728.

Schmunk, R.E.; Miller, L.G.; Becker H. 1984. Tests on Irradiated Magnet Insulator Materials. Journal of Nuclear Materials $122 \& 123$. pp. 1381-1385.

Schönbacher, H.; Stolarz-Iżycka, A. 1979. Compilation of Radiation Damage Test Data - Part II: Thermosetting and Thermoplastic Resins. CERN 79-08. European Organization for Nuclear Research, Geneva. 225 pp.

Sheldon, R.; Stapleton, G.B. 1968. The Effect of High Energy Radiation on the Mechanical Properties of Epoxy Resin Systems used for Particle Accelerator Construction. RHEL/R 152. Rutherford Laboratories, Chilton, England. unpaged.

Simon, N.J.; Reed, R.P.; Walsh, R.P. 1992. Compression and Shear Tests of VacuumImpregnated Composites at Cryogenic Temperatures. In: Advances in Cryogenic Engineering (Materials), Vol. 38. F.R. Fickett and R.P. Reed, eds. Plenum Press, New York. pp. 363-370.

Söll, M. 1978. Influence of Radiation Damage on the Maximum Attainable Magnetic Field for Torodial Fusion Magnet Systems. Journal of Nuclear Materials 72. pp. $168-176$.

Takamura, S.; Kato, T. 1979. Effect of Low Temperature Reactor Irradiation on Organic Insulators in Superconducting Magnets. In: Nonmetallic Materials and Composites at Low Temperatures. A.F. Clark and R.P. Reed, Eds. Plenum Press, New York. pp. 155-163. 
-1980. Effect of Low Temperature Irradiation on the Mechanical Strength of Organic Insulators for Superconducting Magnets. Cryogenics 20. pp. 441-444.

1981. Effect of Low Temperature Irradiation on Insulators and Other Material for Superconducting Magnets. Journal of Nuclear Materials $103 \& 104$. pp. $729-734$.

-1984. Mechanical Properties of Organic Insulators for Superconducting Magnets after Low Temperature Irradiation. In: Advances in Cryogenic Engineering (Materials), Vol. 30. A.F. Clark and R.P. Reed, eds. Plenum Press, New York. pp. $41-49$.

Takeda, N.; Kawanishi, S.; Udagawa, A.; Hagiwara, M. 1985. Electron Irradiation Effects on Interlaminar Shear Strength of Glass or Carbon Cloth Reinforced Epoxy Composites. Journal of Materials Science 20. pp. 3003-3010.

Takeda, N.; Tohdoh, M.; Sakurada, Y.; Takahashi, K. 1989. Mode II Interlaminar Fracture of Composite Laminates Subjected to Electron Irradiation. Journal of the Society of Materials Research, Japan 38. pp. 367-371.

Tomabechi, K.; Gilleland, J.R.; Sokolov, Yu.A.; Toschi, R.; ITER Team. 1991. ITER Conceptual Design. Nuclear Fusion 31. pp. 1135-1224.

Tucker, D.S.; Clinard, F.W., Jr.; Hurley, G.F.; Fowler, J.D. 1985. Properties of Ploymers after Cryogenic Neutron Irradiation. Journal of Nuclear Materials $133 \& 134$. pp. 805-809.

Ullmaier, H. 1976. Radiation Damage in CTR Magnet Components, Radiation Effects and Tritium Technology for Fusion Reactors: In: Proc. of the International Conference held at Gatlinburg, TN, Oct. 1-3, 1975. National Technical Information Agency, Springfieid, VA, Vol. 2. pp. II-404 - II-421.

van der Klein, C.A.M. 1975. The Organic Insulation in Fusion Reactor Magnet systems. RCN-240. Reactor Centrum Nederland, Petten.

Van de Voorde, M.H. 1970. Effects of Radiation on Materials and Components. CERN 70-5. European Organization for Nuclear Research, Geneva. 52 pp.

----1972. Mechanical Properties of Non-Metallic Materials at $77^{\circ} \mathrm{K}$ in a Radiation Environment. CERN ISR-MA/72-14. European Organization for Nuclear Research, Geneva. unpaged.

-.-1973. Results of Physical Tests on Polymer Materials at Cryogenic Temperatures. IEEE Transactions on Nuclear Science NS-20. pp. 693-697.

Van de Voorde, M.H.; Restat, C. 1972. Selection Guide to Organic Materials for Nuclear Engineering. CERN 72-7. European Organization for Nuclear Research, Geneva. 134 pp.

Van Konynenburg, R.A. 1981. Relative Radiation Sensitivity of Insulators, Stabilizers, and Superconductors. In: Proc. of Meeting on Electrical Insulators Fusion Magnets, December 2-3, 1980. Germantown, MD. CONF-801237 U.S. Dept. of Energy. 334 pp. 
Wallner, G.; Anand, M.S.; Greenwood, L.R.; Kirk, M.A.; Mansel, W.; Waschkowski, W. 1988. Defect Production Rates in Metals by Reactor Neutron Irradiation at $4.6 \mathrm{~K}$. Journal of Nuclear Materials 152. pp. 146-153.

Weber, H.W.; Kubasta, E.; Steiner, W.; Benz, H.; Nylund, K. 1983. Low Temperature Neutron and Gamma Irradiation of Glass Fiber Reinforced Epoxies. Journal of Nuclear Materials 115. pp. 11-15.

Weber, H..W.; Böck, H.; Unfried, E.; Greenwood, L.R. 1986. Neutron Dosimetry and Damage Caluclations for the Triga Mark-II Reactor in Vienna. Journal of Nuclear Materials 137. pp. 236-240.

Yasuda, J.; Hirokawa, T.; Uemura, T.; Iwasaki, Y.; Nishijima, S.; Okada, T.; Okuyama, H.; Wang, Y.A. 1988. Cryogenic and Radiation Resistant Properties of Three Dimensional Fabric Reinforced Composite Materials. New Developments in Applied Superconductivity 15. pp. 449-454. 


